


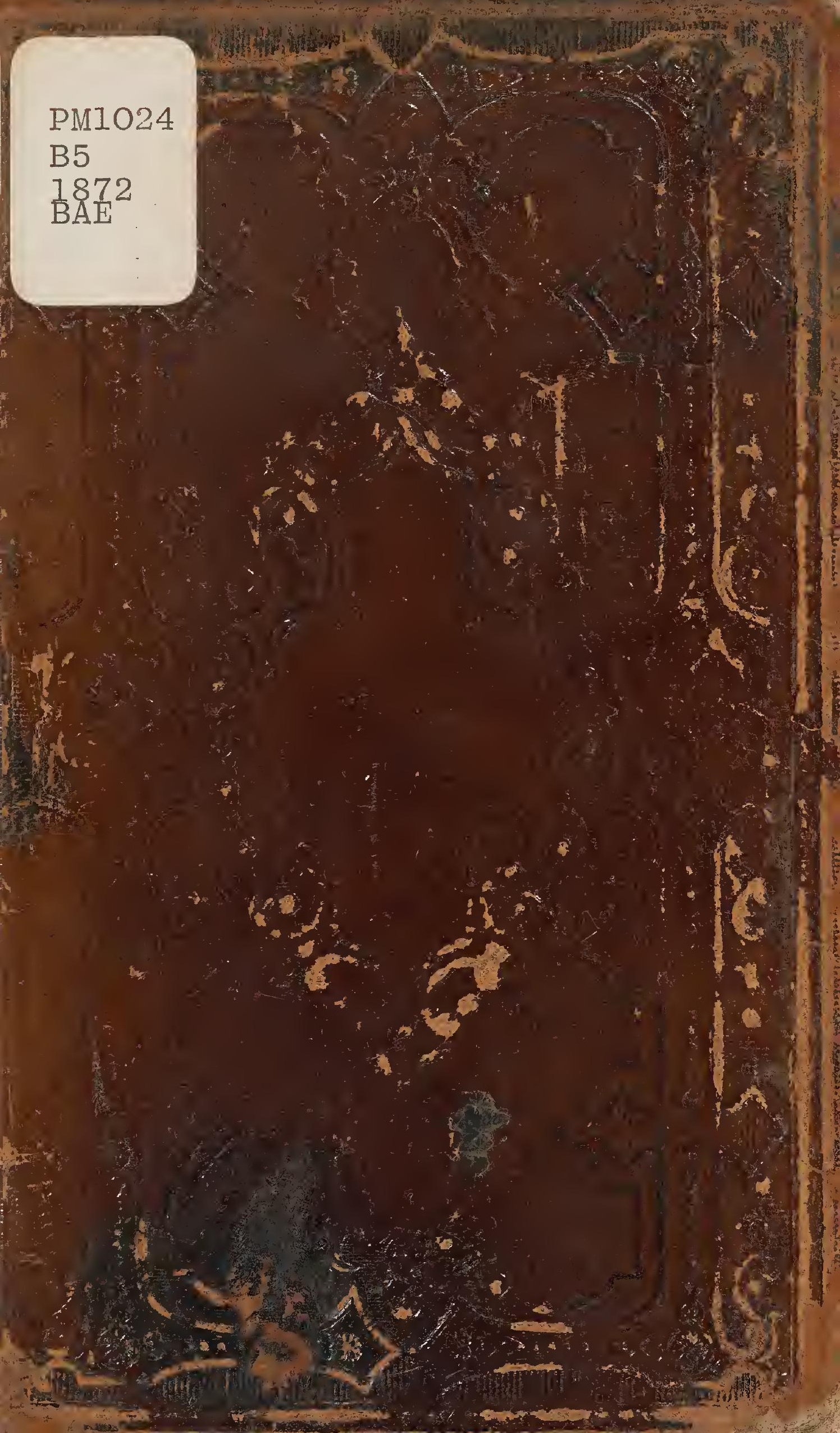


Contents.

Genesis

Lovitías

Numbers

Psalms

Proverbs.

Ecclesiastes

fou g of Solomon

visalá, aud

Tho How Testament 


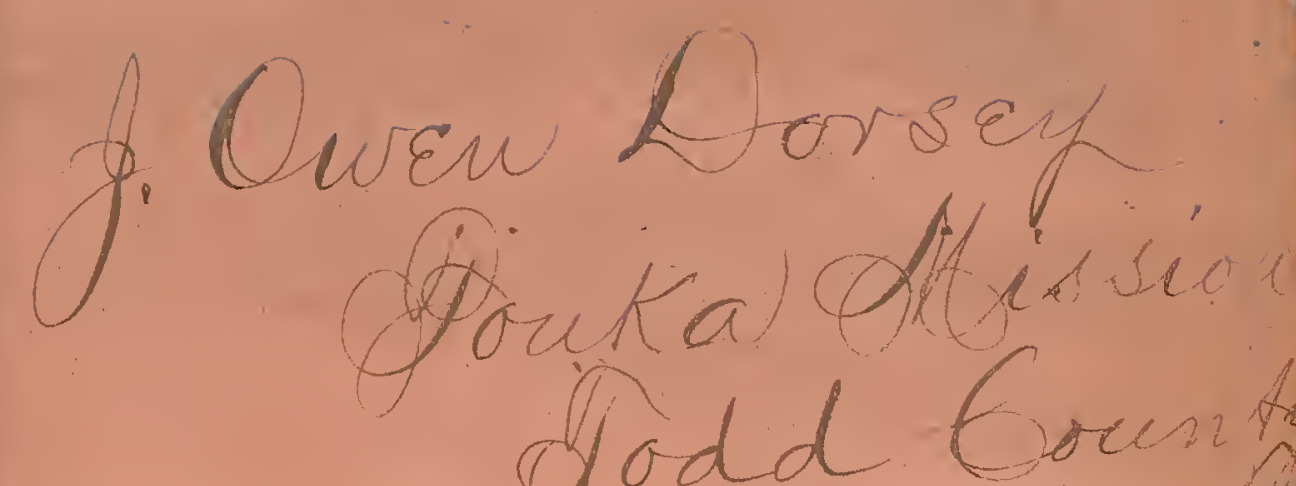
dodd Erunty Latotader orov. 26 in $18 y^{2}$ 


. 




\section{WICOICAGE, HDINANPAPI,

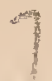

\section{LEWI TOOPE, QA WICAYAWAPI.}

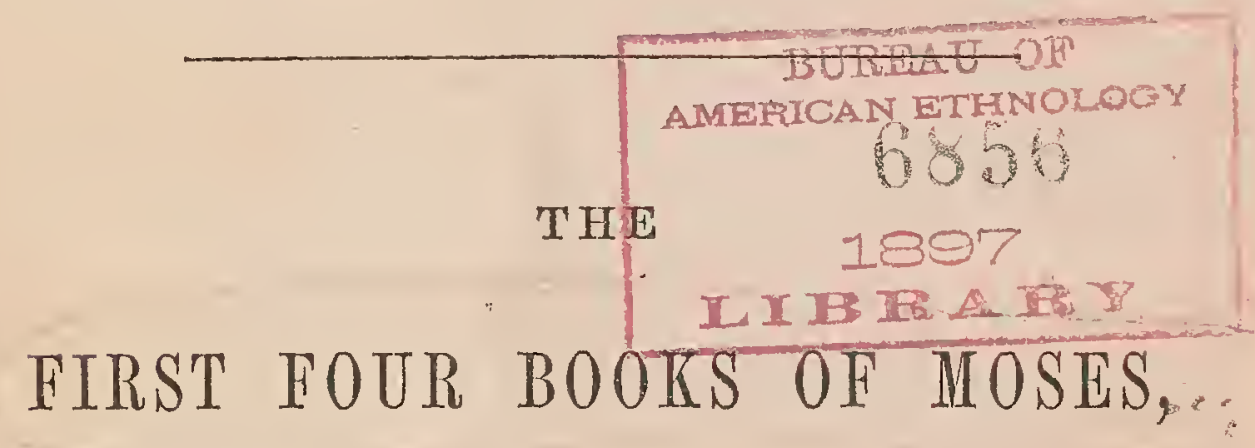

IN THE

\section{DAKOTA LANGUAGE:}

TRANSLATED FROM THE HEBREW, BY

REV. T. S. WILLIAMSON, M. D.

NEW YORK:

AMERICAN BIBLE SOCIETX, INSTITUTED IN THE YEAR MDCCCXVI. 


$$
\begin{aligned}
& P M 1024 \\
& B 5 \\
& 187^{2}
\end{aligned}
$$




\section{Genesir [Jukanwagitai]}

\section{W I C O I C A G E.}

\section{WICOWOYAKF 1.}

1 Otokahe ekta Wakantanka mahpiya kin maka kin ahna kaga.

2 Unkan maka kin tanton śni, qa takudan ohnaka śni, qa otpaza ośbe kin akan wanke ca, Wakantanka Taniya kin mini kin aman.

3 Unkan Wakantanka, Ojanjan kta, eya. Unkan ojanjan.

4 Unkan ojanjan wanke cin, he Wakantanka waśte e wanhdake ça, Wakantanka ojanjan kin otpaze cin kici yukinukan ehnaka.

5 Qa Walkantanka ojanjan kin, Anpetu eciya, otpaze cin iś, Hanyetu eciya. Unkan ohtayetu, qa ohanhanna he anpetu tokaheya kin hee.

6 Unkan, Okotonyan wanke kte, mini kin okitahedan, qa mini kin mini kin yukinukan kte, Wakantanka eya.

7 Unkan okotonyan kin Wakantanka kage ça, mini okotonyan kuyatanhan kin, qa mini okotonyan akantanhan kin kici yukinukan. Unkan ecetu.

8 Unkan okotonyan kin he Wakantanka, Mahipiya eciya. Unkan ohtayetu, qa ohanhanna anpetu icinonpa kin hee

9 Unkan, Mahpiya kin ihukuya mini wanke cin icunonpa kawitaya kte, ca taku puze cin taninyan wanke kte, Wakantanka eya. Unkan ecetu.

10 Unkan taku puze cin he Wakantanka, Maka eciye ca, mini kiwitaya kin he, Miniwanca eciya, ga he Wakantanka waśte e wanhdaka.

11 Hehan Wakantanka heya, Maka kin wato icahye kte, peji kin he su icaliye kte, waskuyeca can kin he waskuyeca icalye lite, waskuyeca mahen su yuke kte, ocaje otoiyohi su kin icaliye kta, maka akan, Wakantanka eya. Unkan ecetu.

12 Unkan maka kin wato aicaga, peji kin ocaje otoiyohi su aicage ca, can kin waskuyeca aicaga otoiyohi mahen su kin yukan ece. Unkan he waśte e Wakantanka wanhdaka.

13 Unkan ohtayetu, qa ohanhanna he anpetu iciyamni kin hee.

14 Unkan Wakantanka heya, Mahpiya okotonyan kin ekta taku iyoyanpa yuke kte, on anpetu hanyetu kici yukinukanyan un kte ca, heon woyaka unpi kte, makonicage, anpetu, omaka, henakiya.

15 Qa malipiya okotonyan kin ekta hena iyoyanpa un kte, maka kin iyoyam ye kte, eya. Unkan ecetu.

16 Wakantanka wiiyoyanpa nom tankinyanyan kaga, tuka uman iyotan tanka kin he anpetu en ounye kte ca uman aoptetu kin he hanyetu en ounye kte; wicanhpi nakun.

17 Hena malipiya okotonyan kin ekta Wakantanka ehnaka, maka kin iyojanjan kta e hecon.

18 Anpetu hanyetu ko ohinniyan ounye wicakiya, qa ojanjan lkin otpaze cin kici yukinukan- 
yan ounye kiya. Unkan he waśte e Wakantanka wanhdaka.

19 Unkan olitayetu, qa ohanhanna he anpetu icitopa kin hee.

20 Unkan Wakantanka heya, Mini kin taku niyake ota icaliye kta, qa taku linyan maka kin iwankam malipiya okotonyan kin ohna okinyanpi kte.

21 Hecen Wakantanka hogan tankinyanyan oicaliye, ça taku niyake, taku mini oicage cin ocaje hiyeye cin owasin, hena mini icalkiye ca, taku kinyan hupahu tonyan ocaje otoiyohi. Unkan he Wakantanka waśte e wanhdaka.

22 Hena Wakantanka yawaśte qa hecen ewicakiya, Icagapi qa ihduotapo, qa miniwanca kin ojudan yakonpo, qa taku kinye cin maka akan ihduotapi kte.

23 Unkan olitayetu, qa ohanlianna he anpetu icizaptan kin hee.

24 Hehan Wakantanka heya, Maka kin taku niyake oicage cin iyecen icaliye kte; woteca, watutka, wamanica, henakiya ocaje otciyuini, eya. Unkan ecetu.

sj Hecen wamanica ocaje otoiyohi, woteca ocaje otoiyohi, qa taku maka asdohan owasin ocaje otoiyohi, henakiya Wakantanka wicakage, ca he waśte e Wakantanka wanhdaka.

26 Hehan Wakantanka hecen eya, Unkiye iyeuncecapi qa ouncage unkitawapi kin en wicaśta unkagapi kta, qa miniwanca ekta hogan oicage cin wicayuhe kte, mahpiya ekta taku kinye cin nakun, qa woteca qa maka kin ocowasin, qa watutka taku maka akan sdohan, ocaje owasin yuhe kta.

27 Wakantanka eyaya iye iyececa wicaśta wan kaga, Wakan- tanka ouncage iyececa kaga; wica winyan kici wicakaga.

$28 \mathrm{Qa}$ Wakantanka wicayawaśte qa, Icagapi, qa ihduotapo, qa maka kin ojudan içiyapo, qa denakiya wicayuha yaunpi kta, miniwanca ekta hogan oicage, ça mahipiya ekta taku kinye cin, qa taku niyake maka aśkanśkan oicage cin owasin, tawa wicaya po; Wakantanka ewicakiya.

29 Unkan, Ito taku maka akan waicaliya suton wojupi owasin cicupi, can waskuyeca suton oicage cin owasin, hena nakun; hena woyute yayapi kta.

30 Tuka taku maka akan ni un kin, mahpiya taku okinye cin, qa taku maka aśkanśkan kin, taku niya un kin owasin, hena peji wato owasin woyute wicawaqu, Wakantanka eya. Unkan ecetu.

31 Unkan Wakantanka taku kage cin owasin wanhdaka ; unkan iyotan waśte. Unkan olitayetu qa ohanlianna he anpetu iciśakpe kin hee.

\section{WICOWOYAKE 2.}

1 Hecen malipiya kin maka kin iyahna, qa taku ohnaka hiyeye cin owasin tanyan yuśtanpi.

2 Unkan anpetu iciśakowin, Wakantanka tohtani kage cin owasin ayuśtan, qa anpetu iciśakowin kin en tohtani kage ciqon etanhan okilipa.

3 Qa Wakantanka anpetu ici-śakowin kin he hdawaśte, qa hduwakan. Tohtani Wakantanka kage cin owasin anpetu kin he en ayuśtan, qa okilipa, heon etanhan.

4 Malipiya maka ko kagapi kin, Jehowa Wakantanka maka mahpiya lko kage cin, anpetu kin he en, woicage oyakapi kin dee. 
5 Can hiyeye cin owasin maka kin en he śni itokam, peji tinta oicage cin owasin uye śni itokam, Jehowa Wakantanka nahahin maka kin amagaju ye śni, qa wicaśta maka kicanye kte cin wanica.

$6 \mathrm{Qa}$ maka kin etanhan po wankan tkiya u, qa maka itohnake kin owancaya acuya.

7 Hehan Jehowa Wakantanka, maka mdu kin etanhan, wicaśta wan kage, ca poge olidoka ohna ipoh iyeya hecen wicaśta nagi ni un kin icaga.

8 Unkan Jehowa Wakantanka, Eden heciya wihinanpe cin ekta, wojupi wan kage ca wicaśta kin he en ehnaka.

9 Qa Jehowa Wakantanka can kin owasin tona owanyag waśte, qa tona woyute waśte aicage cin, maka lkin etanhan icaliya, qa wojupi kin cokaya, can wiconi wan kage ca, can wan on taku waśte taku śica ko sdonyapi kin he nakun en icaliya.

10 Unkan can wojupi kin hpan ye kta e, watpa wan Eden etanhan uye ça hetanhan ointpa tom icaga.

11 Ointpa tokaheya kin Piśon eciyapi; he Hawila makoce kin owancaya ohomniya.

12 Heciya mazaskazi yuke, ca mazaskazi makoce kin he en iyeyapi kin he waśte. Heciya Bedola, qa ihe wicaśake iyececa.

13 Watpa inonpa Gilion eciyapi ; he Kuś makoce kin ohna iyaya.

14 Qa watpa iyamni kin Hedeqel eciyapi; he Aśur makoce kin wiyohiyanpata kiya kaduza. Qa watpa itopa kin Y uphrate hee. -15 Unkan Jehowa Wakantanka Adam icu, qa Eden wojupi kin he kicanye ça awanyake kta $e$, en ehnaka.
16 Qa Jehowa Wakantanka wicaśta kin hecen eciya, Can wojupi kin owasin etanhan icakiś iciye śni yate kta.

17 Tuka can wan, taku waśte taku śica ko on sdonyapi kin de etanhan yate kte śni; tohan hetanhan yate cinhan anpetu kin he en nite lita.

18 Hehan Jehowa Wakantanka, Wicaśta lin de iśnana un kin he śica, wanji iye iyececa wecage lita, eya.

19 Wanna Jehowa Wakantanka, maka kin etanhan tinta wamanica owasin wicakaga, mahpiya etkiya taku kinye cin ko owasin, qa Adam en u wicakiya, token caś wicaton kte cin sdonye lita e heon. Unkan token Adam owasin caś wicaton, hecen taku ni un kin otoiyohi caje wicayatapi.

20 Hecen Adam woteca owasin caś wicakiton, malipiya ektakiya taku kinye cin ko owasin, tinta wamanica nakun owasin; tuka Adam ookiye iye iyececa wanjidan iyeyapi śni.

21 Unkan Jehowa Wakantanka woiśtima tanka wan Adam ahinhipaye ya, unkan iśtima. Unkan Wakantanka cutuhu kin wanji yujon icu, qa conica kin ecen paohduta iyeya.

22 Qa Jehowa Wakantanka cutuhu kin, wicaśta etanhan icu qon on winyan wan kage, ça wicaśta kin en kai.

23 Unkan, Mihu kin etanhan de hu ye ca, micchipi kin etanhan celipi ya. De wicaśta kin etanhan icupi kin, heon de winolinca eciyapi kta, Adam eya.

24. He etanhan wicaśta atkuku hunku kici awicayuśtan, qa tawicu kin en ikoyag içiye kta, hecen cehpi wanjipidan kta. 


\section{WICOICAGE.}

$25 \mathrm{He}$ ehan Adam tawicu kici napin oge içiton śni yaksonpi; tuka iśtecapi śni.

\section{WICOWOYAKE 3.}

1. Wanna wamanica hiyeye cin owasin Jehowa Wakantanka wicakage cin, wamduśka kin hecedan ksapa: qa wamduśka kin winyan kin heciya, Can wojupi kin en can otniyohi etanhan yatapi kte śni awicakehan Wakantanka eya he?

2 Unkan winyan kin wamduśka kin heciya, Can wojupi en can waskuyeca aicage cin hena untapi kta ce.

3 Tuka can wanjidan wojupi cokaya he cin, he taku aicarge cin heceedan, Ihnuhan yatapi qa olutanpi kin, hecanonpi kinhan nițapi k ta ce, Wakantanka eya ce.

4 Unkan wamduśka kin winohinca kin hecen eciya, Nitapi kte śni tuka heya ce;

5 Anpetu kin en he yatapi kinhan niś eya iśta nimdezapi, qa Taku wakan iyenicecapi," taku waśte taku śica ko sdonyayapi kte cin he Wakantanka sdonya, he etanhan heya ce.

6 Unkan can kin waskuyeca woyute waśte, owanyag waśte, qa wicimdeza ksam wicaye kte cin he, winohinca wanyake cehan waskúyeca kin icu, qa yute ca hihnaku kin qu, qa iś eya yuta.

7 Unkan napin iśta mdezapi, qa tancodan yakonpi kin, hehan sdonkiciyapi, qa suken can ape heca kagegepi, qa on oge içitonpi.

8 Unkan anpetu kin cusni hehan, Jehowa Wakantanka ho kin wojupi kin ohna mani kin he nalionpi, qa Wakantanka itohnake kin etanhan wojupi can kin elına naiçilimanpi.
9 Hehan Jehowa Wakantanka Adam kipan, qa heciya, Tukten yaun he?

10 Unkan Adam ayupte ca, Niho kin nawation qa kowakipe ca namicihbe, tancodan waun kin heon etanhan, eya.

11 Unkan tancodan yaun kin tuwe oniciyaka he? Can wanjidan étanhan yute śni ciśi qon, hetanhan yata he? eya.

12 Unkan Adam heya; Winohinca mayaqu hee, qa mici un kin, iye can kin etanhan maqu, qa wata ce.

13 Unkan Jehowa Wakantanka winohinea kin heciya, Tokeca hecanon he? Unkan, Wamduśka kin mahnaye ça wata ce, winohinca kin eya.

14 Unkan Jehowa Wakantanka wamduśka kin hecen eciya, Niye decanon, qa heon woteca watutka ko owasin, niś nana niyaśicapi. Itpi kin on maka kin asdohan yaun qa tohan yani kin hehanyan maka ece yate kta.

15 Qa detanhan niś winohinca kin kici toka kiciya yaunpi kta, qa winohinca cinhintku kin nicinca kici toka kiciya yaunpi kta. He pa kin nicuwa kta, qa niś he siyete kin he yakuwa kta.

16 Hehan winohinca kin heciya, Iyoniciśice ça ihduniśake cin nina mduota kta. Iyokiśinya cinca yaton, qa nihibna cantiheyaye ea iye wowidag niye kta.

17 Únkan wicaśta kin heciya, Nitawin kin token eniciye cin anayagoptan, qa can wan etanhan yute śni ciśi qon, etanhan yate cin heon niye on etanhan maka kin yaśicapi. Taku aicage cin on htayani qa hecen yate kta, tohan yani kin hehanyan. . 18 Wapepeka toka hu ko icah niciye kta, qa peji maka aicage cin yate kta. 


\section{WICOWOYAKE 4.}

19 Niite tenimni kin on aguyapi yahdute kta, maka inicu kte cin hehanyan. Maka kin etanhan inicagapi nakaeś, maka mdu kin henica qa maka kin ekta icicawin yahde kta.

. 20 Hehan Adam iye tawicu kin, Ewe eya caś kiton; taku ni un kin owasin he ina yanpi kin heon etanhan.

21. Unkan Jehowa Wakantanka Adam tawicu kin kici wakihdaka okde wicakicage ça oge wicakicaton.

22 Qa Jehowa Wakantanka heya, Wicaśta kin wanna unkiye wanji he iyeuncecapi, taku waśte taku sica ko sdonya, heon ihnuhan nape yekiye ca wiconi can kin etanhan icu, qa yute ca owihanke wanin ni kte cin.

23 Heon Jehowa Wakantanka wicaśta kin Eden wojupi kin etanhan iyog iyeya; maka kin etashan icage cin he kiçanye kta ece.

24 Hecen wicaśta kin tankan iyeya, qa can wiconi canku kin awanyake kta e, Eden wojupi wiyohiyanpa tanhan Kerubim maza sagye ideya śkanśkan un ko ewicalide.

\section{WICOWOYAKE 4.}

1 Unkan'Adam tawicu kin Ewe sdonya, unkan ihduśake ca Qain he ton, qa, Jehorva etanhan wicaśta wan mduha ce, eya.

2 Qa ake ihduśake ca sunkaku Abel ton. Unkan Abel tahinca wanuyanpi wihan wicakiya, qa Qain maka kin kicanyan.

3 Unkan omaka ihanke kin hehan Qain waskuyeca maka aicage cin hee Jehowa wakiyuśna.

4. Unkan Abel tahinca wanuyanpi tawa tokaheya tonpi wan, ihdi koya wośna. Unkan Jeho- wa Abel taku wakiyuśna kin ko, akicitonwan.

5 Tuka Qain taku wakiyuśna ko akicikta śni. Unkan Qain canteptanye ca itohnake tokeca. 6 Unkan Jehowa Qain heciya, Tokeca canniniye ca ituhnake enicetu śni?

7 Tanyan ecanon kinhan ihaniktapi kte śni he? Tuka tanyan ecanon śni kinhan woahtani tiyopa kin en yanka. Tuka niye qe cantiheniye kta qa niś wowidake yaye kta.

8 Unkan Qain sunkaku Abel okiya, qa wojupi ekta napin yakonpi qehan najin hiyaye ça sunkaku Abel kikte.

9 Unkan Jehowa Qain heciya, Nisunka Abel tukte e he? Unkan, Sdonwaye śni. Ecin misunka kin he awanhdag waun he? eya.

10 Unkan token yalian he? Nisunka we kin maka kin etanhan ho umakiya.

11 Qa wanna maka kin nisunka we kin ninape etanhan icu kta e i kin hdukawa kin be etanhan niyaśicapi ce.

12 Detanhan maka kin yakicanyan eśta, taku ota icah niciye ktc śni. Maka kin de akan onuniyan qa najica yaun kta ce, eya. 13 Unkan Qain Jehowa hecjya, Wawahtani kin he tanka kin kajujupicaśni.

14 Anpetu kin dehan maka kin detanhan iyog iyemayaya, qa nitoye kin inahbe wakiye kta, maka kin akan onuniyan qa najica waun kta, qa tuwe kaśta iyemaye cinhan makte kta.

15 Tuka Jehowa heciya, Tuwe Qain kte kinhan, śakowin akihde wośice akipe kta. Qa Jehowa Qain wapetog ton, hecen on tuwe wanyaka eśta ktepi kte sni. 


\section{WICOICAGE.}

16 Unkan Qain Jehowa itoye tin etanhan inapa, Eden wiyohiyanpa tanhan makuce wan, Nod eciyapi kin, he en ounyan.

17 Qa Qain tawicu kin sdonya, unkan ihduśake ca Henok ton. Unkan Qain otonwe wan kage ca Henok eya caś kiton, cinhintku caje kin iyecen.

18 Unkah Henok cinca wan kicitonpi, Irad hee. Unkan Irad Mecujael cinkśiya. Unkan Mecujael Metuśael cinkśiya. Unkan Metuśael Lamek cinkśiya.

19 Unkan Lamek winohinca nom wicayuza. Tokaheya kin Ada eciyapi, qa iyokihe Zila eciyapi.

20 Unkan Ada Jabel ton; he tona wakeya otipi, qa wanuyanpi awanhdakapi kin, hena ateyapi.

21 Qa sunkaku Jubal eciyapi. Tona candowankiyapi cotanka ko, yahotaninpi kin, hena he ateyapi.

22 Unkan Zila he Tubal Qain ton. Taku maza zi on kagapi, qa maza sapa on kagapi ko owasin, iye onspe qa kaga. Qa Tubal Qain tawinohtin Nahama hee.

23 Unkan Lamek tawicu Ada Zila . Iici hewicakiya, Miho kin nahonpo, Lamek tawicu kin mioie kin anagoptanpo. Wicaśta wan wakte, on camapapi, qa kośka wan on kiunimiciya ce.

24. Qain śakowin akihde tokiconpi kinhan, Lamek wikcemna s̉akowin sanpa śakowin tokiconpi kta ce, eya.

25 Unkan ake Adam tawicu kin sdonya; unkan cinca wan ton, qa, Wakantanka cinca tokeca wan maqu, Abel Qain kte qon hee kiya, eye ça Set eya caś kiton.

26 Unkan Śt iś eya cinca wan kicitonpi, qa Enoś eya caś kiton. Hehan tokaheya wicaśta kin Jehowa caje kin hoyekiyapi.

\section{WICOWOYAKE 5.}

1 Adam wicowazi wowapi kin dee. Wakantanka Adam kage çehan, taku wakan ouncage iyecen icah kiya.

2 Wica winyan ko wicakage ça wicayawaśte, qa wicakage çehan Adam eya caś wicaton.

3 Unkan Adam waniyetu opawinge sanpa wikcemna yamni ni, qa cinca wan iye ouncage iyecen kicitonpi, qa Set eya caś kiton.

4. Qa Śet tonpi kin he iyohakam, Adam waniyetu opawinge śahdogan ni, qa cinca wica winyan ko kicitonpi.

5 Hecen anpetu owasin Adam ni un kin hena waniyetu opawinge napciwanka sanpa wikcemna yamni, hehan ta.

6 Unkan Śet waniyetr opawinge sanpa zaptan ni, qa Euoś kicitonpi.

$7 \mathrm{Qa}$ Set Enoś tonpi kin iyohakam waniyetu opawinge śahdogan sanpa śakowin ni, qa cinca wica winyan ko kicitonpi.

8 Hecen anpetu owasin Śet ni un kin hena waniyetu opawinge napciwanka sunpa ake nonpa, hehan ta.

9 Unkan Enoś waniyetu wikcemna napciwanka ni, qa Qenan kicitonpi.

$10 \mathrm{Qa}$ Qenan tonpi kin iyohakam Enoś waniyetu opawinge śahdogan sanpa wikcemna sanpa zaptan ni, qa cinca wica winyan ko kicitonpi.

11 Hecen anpetu owasin Enoś ni un kin hena waniyetu opawinge napciwanka sanpa zaptan, qa hehan ța. 
WICOWOYAKE 6.

12 Unkan Qenan waniyetu wilicemna śakowin ni, qa Mahalele kicitonpi.

13 Qa Qenan Mahalele tonpi kin iyohakam waniyetu opawinge śahdogan sanpa wikcemna topa ni, qa cinca wica winyan ko kicitonpi.

14 Hecen anpetu owasin Qenan ni un kin hena waniyetu opawinge napciwanka sanpa wikcemna, hehan ța.

15 Unkan Mahalele waniyetu wikcemna śakpe sanpa zaptan ni, qa Jered kicitonpi.

16 Qa Mahalele Jered tonpi kin iyohakam waniyetu opawinge śahdogan sanpa wikcemna yamni ni, qa cinca wica winyan ko kicitonpi.

17 Hecen anpetu owasin $\mathrm{Ma}$ halele ni un kin hena waniyetu opawinge śahdogan sanpa wikcemna napciwanka sanpa zaptan, hehan ta.

18 Unkan Jered waniyetu opawinge sanpa wikcemna śakpe sanpa nonpa ni, qa Henok kicitonpi.

19 Qa Jered Henok tonpi kin iyohakam waniyetu opawinge śahdogan ni, qa cinca wica winyan ko kicitonpi.

20 Hecen anpetu owasin Jered ni un kin hena waniyetu opawinge napciwanka sanpa wikcemna śakpe sanpa nonpa, hehan ta.

21. Unkan Henok waniyetu wikcemna śakpe sanpa zaptan ni, qa Metuśela kicitonpi.

$22 \mathrm{Qa}$ Henok Metuśela tonpi kin iyohakam waniyetu opawinge yamni Wakantanka opeya mani, qa cinca wica winyan ko kicitonpi.

23 Hecen Henok anpetu tawa owasin waniyetu opawinge yamni sanpa wikcemna śakpe sanpa zaptan.
$24 \mathrm{Qa}$ Henok Wakantanka opeya mani, qa en un śni, Wakantanka he ikikcu nakaeś.

25 Unkan Metuśela waniyetu opawinge sanpa wikcemna śakowin sanpa śahdogan ni, qa Lemek kicitonpi.

$26 \mathrm{Qa}$ Lemek tonpi kin iyohakam Metuśela waniyetu opawinge śakowin sanpa trikcemna śahdogan sanpa nonpa ni, qa cinca wica winyan ko kicitonpi.

27 Hecen anpetu owasin Metuśela ni un kin hena waniyetu opawinge napciwanka sanpa wikcemna śakpe sanpa napciwanka, hehan ta.

28 Unkan Lemek waniyetu opawinge sanpa wikcemna śahdogan sanpa nonpa ni, qa cinca wan kicitonpi.

$29 \mathrm{Qa}$, Jehowa maka kin yaśice cin he nape on unkicanyanpi, qa htaunnipi kin en unkikiyapi kte cin dee ce, eye ça Nowa eya caś kiton.

30 Qa Lemek Nowa tonpi kin iyohakam waniyetu opawinge zaptan sanpa wikcemna śakowin sanpa zaptan ni, qa cinca wica winyan ko kicitonpi.

31 Hecen anpetu owasin Lemek ni un kin hena waniyetu opawinge śakowin sanpa wikcemna śakowin sanpa śakowin, hehan ta.

32 Unkan Nowa wanna waniyetu opawinge zaptan, hehan Sem, Ham, qa Japet, kicitonpi.

\section{WICOWOYAKE 6.}

1 Hehan wicaśta maka akan ota ayapi, qa cunwintkupi kicitonpi.

2 Unkan Wakantanka cinkśi wicaye cin wicaśta cunwintkupi kin hena e waśte wanwicayaka- 


\section{WICOICAGE.}

pi, qa tona cinpi kin hena etanhan, winohinca wicayuzapi.

3 Unkan Jehowa heya, Wicaśta kin wicacehpi kin heca heon wahtanipi en mitaniya he kici owihanke wanin ounye kte śni, tuka anpetu tawa kin hena waniyetu opawinge sanpa wikcemna nonpa kta.

4 Unkan anpetu kin hena icunhan, maka kin akan wazise yakonpi, qa Wakantanka cinkśi wicaye cin wicaśta cunwintkupi kin wicayuzapi, qa cinca kicitonpi kin, hena eepi he iyohaliam wicaśta waśakapi, wanakaja tanhan wicaśta nina caje wicayatapi.

5 Unkan wicaśta maka akan wahtanipi kin ota e, Jehowa wanyaka, anpetu osan wicocante oze okah otoiyohi ecedan śica.

6 Unkan wicaśta maka akan icah wicaye cin heon, Jehowa iyopeiçiye ça cante iyokiśin ic̣iya.

$7 \mathrm{Qa}$, Wicaśta icah wicawaye cin, maka itohnake kin etanhan wicawakahinte kta ; wicaśta, qa woteca, qa watutka, qa taku mahpiya ektakiya kinyan un kin henakiya icah wicawaye cin heon iyopemiciya ce, Jehowa eya.

8 Tuka Jehowa Nowa wanyake ca wacin en yuza.

9 Nowa wicoicage oyakapi kin dee. Nowa wicaśta ohan owotanna, qa hecetu wicoicage tawa kin hen, qa Nowa Wakantanka opeya mani.

$10 \mathrm{Qa}$ Nowa cinca yamni kicitonpi, Śem, Ḣam qa Japet.

11 Unkan Wakantanka itokam maka kin honwin ce, qa maka owancaya nina waboticapi.

12 Unkan Wakantanka maka kin wanyaka, unkan honwin; wicacehpi owasin maka alkan iye tacankupi kin honwin kiyapi ce.
13 Unkan Wakantanka Nowa heciya, Taku cehpi yukan owasin owihanke kin mitokam u ce ; iyepi kin on maka kin waboticapi on ojudan; qa maka kin etanhan ihang wicawaye kta.

14 Wazi can on wata wan icicaga wo. Mahentu kin ośkiśkeya yakage kta, qa mahen tanhan akapa tanhan ko, canśin apawinta wo.

15 Qa hecen yakage kta. Wata ohanske cin wiciśpa opawinge yamni, ohdakinyan kin wiciśpa wikcemna raptan, qa obosdatu kin wiciśpa wikcemna yamni kita.

16 Qa wata kin en ojanjan hdepi yakage kta, qa wankantuya wiciśpa wanjidan en yahduśtan kta. Wata tiyopa kin cuwi kin ekta yakage kta, owanka tokaheya, inonpa, qa iyamni yakage kta.

17 Wanna miye maka akan minitan waye lita, heon taku celipi yuke cin niyake iyeye cin owasin mahpiya ihukuya, ihang waye kta. Taku maka akan un kin owasin te kta.

18 Tuka wicotakuye mitawa kin niye en nici wahdusuta kta, wata kin en oyape kta nicinca om, nitawin, nicinca tawicupi kin, henana om.

19 Qa taku niya un kin owasin, taku cehpi yukan hiyeye cin ocaje otoiyohi, nom nom mdoka wiye iyahnahna, en owicayahnake kta; ni wicayaye kta e niye kici wata kin en upi kta.

20 Taku kinye cin token ocaje, woteca token ocaje, wamanica maka akan unpi kin token ocaje, owasin ocaje otoiyohi nom nom niyake wicaduhe kta e niye en upi kta.

21 Qa niye taku yutapi kin ocaje otoiyohi etanhan icu, qa 
mnayan wo; hecen taku yutapi anihduhe, ca woteca kin iś iyacinyan wicayakihduhe kta.

22 Unkan Nowa Wakantanka token eciye cin, owasin anagoptan, qa ecen econ.

\section{WICOWOYAKE 7.}

1 Unkan Jehowa Nowa heciya, Niye, tiyohnaka nitawa kin owasin om, wata kin en opa wo ; wicoicage lin de en mitokam owotanna yaun kin wanciyaka ce.

2 Woteca wakaśoteśni ocaje otoiyohi mdoka wiye ahnahna śakowinwin iyacu kta, qa woteca waaśapapi kin, hena nom nom mdoka wiye kici iyacu kta.

3 Taku mahpiya ektakiya kinye cin nakun śakowinwin, mdoka wiye ahnahna maka akan aicagapi e niyake wicaduhe kta.

4 Ehake anpetu śakowin, hehan maka kin amagaju waye kta, qa taku ni un wakage cin owasin maka kin "owancaya etanhan wakahinte kta ce.

5 Unkan Nowa, Jehowa token eciye cin owasin ecen econ.

6 Nowa tonpi kin ehantanhan waniyetu opawinge śakpe hehan maka kin akan minitan.

7 Qa Nowa cinkśi wicaye om, qa iye tawicu, cinkśi wicaye cin tawicupi ko, hena owasin om minitan mini kin itokapa tanhan wata kin en opa.

8 Woteca wakaśoteśni, qa woteca waaśapapi kin, taku kinye cin, taku maka asdohan unpi ko owasin,

9) Nom nom mdoka wiye ahnahna Nowa en hipi, wata kin en; Wakantanka Nowa econ śi qon he iyecen.

10 Unkan anpetu śakowin hehan maka kin akan minitan aya.
11 Waniyetu opawinge sakpe hehanyan Nowa ni un kin, wi icinonpa, anpetu iake śakowin kin, hehan śbe tanka kin wakoniya hiyeye cin owasin yuomdecapi, qa mahpiya owanyeye hdepi kin owasin yuzamni ehdepi.

12 Qa anpetu wikcemna tom, hanyetu wikcemna tom, hehanyan maka kin ahinheya.

13 Anpetu kin he en Nowa, qa Śsem, qa Ham, qa Japet, Nowa cinkśiwicaye cin, Nowa tawicu kin nakun, cinca tawicupi yamni om, wata kin en hiyupi ;

14 Hena qa wamanica ocaje owasin, woteca ocaje owasin, watutka tona maka asdohan unpi ocaje owasin, taku kinyạ ocaje owasin, zitkadan owasin taku śun yukan owasin,

15 Nom nom hipi, Nowa ekta wata kin en, taku cehpi yuke cin woniya niyake yuke cin owasin,

16 Hena en hiyupi; mdoka wiye ahnahna taku cehpi yuke cin owasin etanhan upi; Wakantanka econ śi qon he iyecetu. Unkan Jehowa tiyopa kin onataka.

17 Unkan anpetu wikcemna tom maka kin ahinheye ca, minitan tanka aya; unkan wata kin okapon maka kin iwankam iyaya.

18 Unkan mini kin waśagya aye ça maka kin akan nina ihduota, unkan wata kin mini kin aokapota.

19 Unkan mini kin nina hin waśagya, maka kin akan, heon he pajodan owasin, tona malipiya ihukuya un kin hena aminitan.

20 Wiciśpa ake zaptan iwankam mini kin aye, ca paha kin aminitan.

21 Unkan taku cehpi yulse cin 
owasin, taku maka aśkanśkan un kin, taku kinyan, qa woteca, wamanica, qa watutka tona maka kin akan sdohanpi kin owasin tapi, wicasta iyulipa nakun tapi.

22 Taku maka puze cin akan ni, taku poge on niya un kin, owasin tapi.

23 Unkan taku hiyeye cin maka akan un kin owasin kahintapi, wicaśta, woteca, watutka, mahpiya zitkadan, owasin maka kin etanhan wicakahinta, qa Nova iśnana qa tona wata kin ohna om un kin henana okaptapi.

24 Anpetu opawinge sanpa wikcemna zaptan mini kin maka kin akan waśagya.

\section{WICOWOYAKE 8.}

1 Hehan Wakantanka Nowa kilksuye, ca wamanica woteca ko owasin, iye kici wata ohna yakonpi kin hena; qa Wakantanka tate wan maka kin akan iyayupte ya, qa mini kin ayuśtan.

2 Qa minihdoka owahinapeye cin onatakapi, mahpiya owanyeye kin hena koya, qa magaju mahpiya etanhan kin he ayuśtan.

3 Unkan mini kin maka kin etanhan kihda, anpetu opawinge sanpa wikcemna zaptan, hehan mini kin oyalie aya.

$4 \mathrm{Wi}$ iśakowin, anpetu iake śakowin wi kin en, hehan wata kin Ararat he kin en ari.

5 Unkan mini kin iyoopta ye ca oyahe aya, wi iwikcemna kin hehanyan; wi iwikcemna kin anpetu tokaheya kin he en, he paha kin taninyan hiyeya.

6 Unkan anpetu wikcemna tom henakeca, hehan Nowa wata kin owanyeye kage ciqon, he yuhidoka.

7 Qa kangi wan tankan iyaye ya, unkan iyaye ça ohnihdeya un, mini kin maka kin etanhan oyahe kin hehanyan.

8 Mini kin maka itohnake kin etanhan wanna pus aya, wanyake kta e wakiyedan wan nakun tankan iyaye ya.

9 Tuka wakiyedan kin tukten sicuha iyahe kte cin iyeye śni, mini kin maka kin owancaya akan un nakaeś; qa Nowa en wata kin ekta hdi; unkan nape kin kiyugatc ca wata kin en ikikeu.

10 Qa ake anpetu śakowin ape, qa hehan ake wakiyedan wan wata kin etanhan iyaye ya.

11 Unkan ihtayetu he ehan wakiyedan kin hdi, qa wihdi can ape wan yapa hdi; unkan Nowa mini kin maka kin etanhan wanna pus aye cin sdonya.

12 Unkan ake anpetu śkowin tokeca ape, qa hehan wakiyedan wan tankan iyaye ya, unkan icimana hdi śni.

13 Unkan Nowa tonpi kin ehantanhan waniyetu opawinge śakpe qa itokaheya kin, wi tokaheya, qa anpetu tokaheya, hehan mini kir maka kin akapatanhan puza, unkan Nowa wata kin akalipe ciqon yuhdoke ça, maka itohnake kin wanna puza wanyake.

14 Wi inonpa kin, anpetu iake sakowin kin, hehan maka kin puza.

15 Unkan Wakantanka Nowa okiye ca,

16 Wata kin etanhan ya wo, niye, qa nitawin, qa nicinca, qa nicinca tawicupi kin niye kici ;

17 Taku niyake niye kici un kin ko owasin, taku cehpi yukan owasin, taku kinyan, woteca, watutka tona maka asdohan unpi kin owasin, tankan awicayaku kte, hecen maka akan cinca 


\section{WICOWOYAKE 9.}

kagapi kte ca icagapi, qa ihduotapi kta ce, eciya.

18 Unkan Nowa tankan iyaye ca, cinca nakun, tawicu, cinca tawicupi koya.

19 Taku niyake owasin, wamanica owasin, qa taku kinyan owasin, taku maka akan sdohan un kin owasin, ocaje otoiyohi wata kin etanhan inanpapi.

20 Unkan Nowa wahna wośnapi wan Jehowa kicaga qa, woteca wakaśoteśni otoiyohi etanhan, qa taku kinyan wakaśoteśni ocaje otoiyohi etanhan icu, qa wawicayuśna, qa liulinali wicaya, wahna wośnapi kin akan.

21 Unkan Jehowa waśtemna kin omna, qa Jehowa cante kin ekta heya, Wicaśta kin heon maka kin ake mdaśice kte śni ; ehaeś wicaśta kin hokśiyoqopa kin ehantanhan cante oze śica ece, tuka icimana taku niya un kin owasin ihang waye kte śni, hecamon qon iyecen.

22 'Tohanyan maka kin wanke cin hehanyan wojupi qa coyapi, qa sni qa didita, qa mdoketu qa waniyetu, qa anpetu qa hanyetu hena ayuśtanpi kte śni ce.

\section{WICOWOYAKE 9.}

1 Unkan Wakantanka Nowa, cincawicaye cin ko, wicayawaśte qa, Icagapi qa ihduótapi, qa maka kin owancaya ojudan yapo ;

2 Qa taku maka aśkanśkan, taku mahpiya okinye cin ko owasin, maka watutka owasin, qa mini owanca hogan owasin, itonnipapi qa ikonipapi kta, hena owasin ninape kin en niçupi ce.

3 Taku niya, qa śkanślkan kin owasin woyute nitawapi kta; peji wato iyecen hena owasin ciçupi.
4 Tuka we, celipi wiconi kin hee, yatapi kte śni.

5 Qa awicakehan we nitawapi kin on yanipi kin awakite kta, wamanica otoiyohi nape kin etanhan awakite kta, wicaśta nape kin ko etanhan, otoiyohi hunka wanjitku nape kin etanhan wicaśta wiconi kin be awakite kta.

6 Wakantanka iye ouncage iyecen wicaśta kin kaga; heon tuwe wicaśta weye cinhan, he wicaśta eciyatanhan weyapi kta.

7 Qa niyepi icagapi, qa maka kin owancaya ounyanpi, qa he ohna ihduotapo, ewicakiya.

8 Qa Wakantanka Nowa, cinca wicaye cin ko, owicakiye ca ; 9 Iho miye wicotakuye mitawa ciciyasuta qa nicinca nihektam unpi kte cin om.

10 Nakun taku niya un kin, niyepi om unpi kin, taku kinyan, woteca, qa maka wamanica owasin, tona niye om wata kin etunhan inanpapi kin, taku maka akan ni un kin owasin om.

11 Niyepi om wicotakuye mitawa wakaga, qa taku cehpi yukan owasin minitan mini on icimana awihnuni waye kte śni, qa detanhan maka ihang ye kta on, icimana minitan kte śni, ewicakiya.

$12 \mathrm{Qa}$, Wicoicage owasin, hehanyan niycpi en, qa taku ni un kin owasin en, wicotakuye wakage cin wowakta kin dee.

13 Mitinazipe kin mahpiya śoka en ewehnake ca he wicotakuye maka kin waqu kin, wowakta kin hee kta.

11 Qa tohan maka kin malipiya soka wan ahanzi waye cinhan, mahipiya solka kin ekta mitinazipe kin tanin kta.

15 Qa wicotakuye mitawa weksuye $\mathbf{k t a}$, niyepi om, qa taku 
ni un kin owasin om, qa taku cehpi yuke cin owasin awihnuni lita e, detanhan icimana minitan lite śni.

16 Itazipa kin malipiya soke cin en yanke kta, qa wicotakuye Wakantanka kici, qa taku maka akan niya un kin taku cehpi yuke cin owasin om, owihanke śniyan un kte cin, he weksuye kta e itazipa kin wanwahdake lita ce, Wakantanka eya :

17 Qa, Taku cehpi yuke cin maka akan owasin, wicotakuye wicawaqu kin wowakta kin hee kta, Wakantanka Nowa eciya.

18 Unkan Nowa cinca wicaye cin, wata qon etanhan inanpapi kin deepi, Śenı, qa Ham, qa Japet. Unkan Ham Kanan atkuku kin hee.

19 Hena yamni Nowa cinca kin eepi, qa hena etanhan maka kin owancaya enanakiya iyayapi.

20 Unkan, Nowa maka lin kicanyan aye ça hastanhanka iywi hu hde.

21 Qa hetanhan miniśa yatke ça witko, qa wakeya ohna tancodan un.

22 Unkan Ham Kanan atkuku kin hee, atkuku tancodan un kin he wanhdake ca cincu nom tankan yakonpi kin, owicakiyaka.

23 Unkan Śem Japet kici, śina wan icupi, qa napin hiyetepi kin akan ehnakapi, qa iheyata manipi, qa ateyapi tancodan un kin akahpapi ; itepi kin iheyata unpi, hecen atcyapi tancodan un kin wanhdakapi śni.

24 Unkan Nowa, miniśa kin etanhan kikta, qa cinhintku hakakta kin taku ecakicon qon sdotkiya ;

$25 \mathrm{Qa}$ heya, Kanan yaśicapi kta. Wowidake wowidag yapi lita, cinye wicaye cin he yuhapi $\mathrm{kta}$.

26 Jehowa Śs taku wakan tawa kin yawaśtepi nunwe. Kanan wowidake yuhapi kta.

27 Wakantanka Japet yuota kta, qa Śem tawakeya kin ohna Japet ounye kta, qa Kanan wowidake yuhapi kta.

28 Unkan Nowa, minitan qon iyohakam, waniyetu opawinge yamini sanpa wikcemna zaptan ni.

29 Hecen Nowa anpetu tawa owasin, waniyetu opawinge napciwanka sanpa wikcemna zaptan hehan ta.

\section{WICOWOYAKE 10.}

1 Nowa wicowazi kin dee. Nowa cinca kin, Śem, Ḣam, qa Japet: Unkan minitan qon iyohakam, hena cinca kicitonpi.

2 Japet cinca kin Gomer, qa Magog, qa Madai, qa Jawan, qa Tubal, qa Meśek qa Tiras.

3 Unkan Gomer cinca kin, Aśkenas, qa Ripat, qa 'I'ogarma.

4 Unkan Jawan cinca kin, Eliśa, qa 'Carśiś, Kitim, qa Dodanim.

5 Hena Hetne wita tawapi kin wicakicipamnipi, makoce tawapi kin en, otoiyohi iapi kin, token wicowazi kin, hecen oyate kin en.

6 Ḣam cinca kin, Kuś, qa Mizarim Egupton hee, Put, qa Kanan.

7 Unkan Kuś cinca kin, Seba, qa Cawila, qa Sabta, qa Rahama, qa Sabteca. Unkan Rahama cinca lin Śeba, qa Dedan.

8 Unkan Kuś Nimrod kicitonpi, he tokaheya maka akan'waśaka aýa.

9 Iye wayatesa Jehowa ito- 
kam waśake cin hee; heon etanhan, Waayatesa waśaka Jehowa itokam, Nimrod iyecen, eyapi kin.

10 Unkan wokiconze tawa tokaheya kin Babel hee, qa Erek, qa Akad, qa Kalne Śsinar makoce kin en unpi.

11 Makoce kin he etanhan iyaya, qa Aśur, Niniwe kage ca Rehobot otonwe Kala nakun;

12 Qa Niniwe Kala kici otahedan Resen otonwe tanka wan hee.

13 Unkan Mizarim Egupton hee cinca kicitonpi kin Ludim, qa Anamim, qa Lehábim, qa Naptuhim,

14. Qa Partusim, qa Kasluhim, hetanhan Piliśtim, qa Kaptorim inanpapi.

15 Unkan Kanan cinca kicitonpi Zidon, he tokapa, qa Ḧet,

$16 \mathrm{Qa}$ Jebusi kin, qa Amori kin, qa Girgiśi kin,

17 Qa Ḣiwi kin, qa Hareqi kin, qa Sini kin,

18 Qa Aradi kin, qa Zemari kin, qa Hamati kin ; qa hehan Kanani wicowazi kin yuomden iyayapi.

19 Qa Kanani makoce tawapi kin dee, Zidon etanhan Gerar ektakiya de cin Gaza etu, qa hetanhan Sodom, qa Gomora, qa Adma, qa Zeboim Lasa ekta.

20 Ḧam cinca wicaye cin hena eepi, wicowazipi kin iyececa, oyatepi tamakocepi kin en, iapi kin iyecen.

21 Unkan Japet tokapa kin sunkaku Śem, he cinca kicitonpi, Heber cinca wicaye cin owasin ateyapi kin hee.

22 Sem cinca wicaye cin, Elam, qa Aśur, qa Arpalśad, qa Lud, qa Aram.

23 Unkan Aram cinca wicaye cin Uz, qa Ḧul, qa Geter, qa Maś.
24. Unkan Arpakśad Śla kicitonpi, qa Śsela Heber kicitonpi. 25 Unkan Heber cinca nonpa kicitonpi ; tokapa anpetu tawa kin en maka kin akipam icupi kin heon Peleg eciyapi, qa sunkaku kin Joktan cajeyatapi.

26 Joktan cinca wicaye cin Almodad, qa Śselep, qa Ḣatsarmawet, qa Jerä;

27 Qa Hadoram, qa Uzal, qa Diqela;

28 Qa Obal, qa Abimahel, qa Śba ;

29 Qa Opir, qa Ḣawila, qa Jobal, hena owasin Joktan cinkśi wicaya.

30 Meśa etanhan de cin, Seper he wan wiyohiyanpata wanke cin; behanyan ounyanpi.

31 Sem cinca wicaye cin hena eepi ; wicowazipi, qa wicoie hduhapi kin iyecen oyatepi kin tamakocepi kin en ounyanpi.

32 Nowa cinca wicaye cin wicowazipi kin heepi, oyatepi kin en. Hena etanhan maka kin owancaya oyate enanakiya iyayapi minitan qon iyohakam.

\section{WICOWOYAKE 11.}

1 Hehan maka kin owancaya wicaiha wanjidan, qa iapi ko wanjidan.

2 Qa wiyohiyanpata ihdaka unpi, qa Śinar makoce kin he en, omdaye wan iyeyapi, qa en eyakonpi.

3 Qa otoiyohi takodaku hekiciyapi, Thopo, maka omdoton unkagapi, qa śpan unkiyapi kta ce, eyapi. Hecen inyan hee kiya maka omdoton kin he icupi, qa upśija hee kiya bitumen icupi.

4 Qa ake hekiciyapi, Ihopo, otonwe wanji unkagapi kte ça nakun conkaślke wan, he ointpa kin mahpiya iyahdeye kta, qa 
caje unkicagapi kte; heconkupi śni kinhan, maka kin owancaya omdecaheya unkiyayapi kta.

5 Unkan, Jehowa otonwe kin, inatake ko, Adam cinca wicaye cin kagapi kin wanyake kta e kun hiyu.

6 Qa, Ehaeś oyate wanjidan, qa owasin iapi wanjidan yuhapi, qa de tokaheya econpi, qa detanhan taku kaga kecinpi owasin takudan tehike dapi kte śni.

7 Iho, kun unyanpi, qa iapi kin icicahi unyanpi kta; kinhan iyakitedan iapi nakicilionpi kte śni, Jehowa eya.

8 Qa hetanhan Jehowa, maka kin owancaya, yuomden ye wicaya'; unkan otonwe kagapi qon ayuśtampi.

$9 \mathrm{He}$ etanhan caje kin Babel eciyapi; heciya Jehowa maka kin owasin iapi kin yuicicahi, qa hetanhan maka kin owancaya yuomden enanakiya ye wicaya.

10 Sem wicowazi wowapi kin dee. Śm waniyetu opawinge sanpa nonpa ni, qa hehan Arpakśad kicitonpi, minitan qon iyohakam waniyetu nonpa.

11 Śem Arpakśad tonpi kin iyohakam, waniyetu opawinge zaptan ni, qa cinca wica winyan ko kiçitonpi.

12 Unkan Arpakśad waniyetu wikcemna yamni sanpa zaptan ni, qa Śela kicitonpi.

13 Qa Arpakśad, Sela tonpi kin iyohakam, waniyetu opawinge tom sanpa yamni ni, qa cinca wica winyan ko kicitonpi.

14 Unkan Śla waniyetu wikcemna yamni ni, qa hehan $\mathrm{He}-$ ber kicitonpi.

$15 \mathrm{Qa}$ Sela Heber tonpi kin iyohakam, waniyetu opawinge tom sanpa yamni ni, qa cinca wica winyan ko kicitonpi.

16 Unkan Heber waniyetu wi- kcemna yamni sanpa topa ni, qa Peleg kicitonpi.

17 Qa Peleg tonpi kin iyohakam, Heber waniyetu opawinge tom sanpa wikcemna yamni ni, qa cinca wica winyan ko kicitonpi.

18 Unkan Peleg waniyetu wikcemna yamni ni, qa Reyu kicitonpi.

19 Qa Peleg, Reyu tonpi kin iyohakam, waniyetu opawinge nonpa sanpa napciwanka ni, qa cinca wica winyan ko kicitonpi: 20 Unkan Reyu waniyetu wikcemna yamni sanpa nonpa ni, qa Serug kicitonpi.

21 Qa Serug tonpi kin iyohakam, Reyu waniyetu opawinge nonpa sanpa śakowin ni, qa cinca wica winyan ko kicitonpi.

22 Unkan Serug waniyetu wikcemna yamni ni, qa Nahor kicitonpi.

23 Qa Nalior tonpi kin iyohakam, Serug waniyetu opawinge nonpa ni, qa cinca wica winyan ko kicitonpi.

24 Unkan Nalior waniyetu wikcemna nonpa sanpa napciwanka ni, qa Tera kicitonpi.

25 Qa Nahor Tera tonpi kin iyohakam, waniyetu opawinge sanpa wikcemna sanpa napciwanka ni, qa cinca wica winyan ko kicitonpi.

26 Unkan Tera waniyetı wikcemna śakowin ni, qa Aberam, qa Nahor, qa Haran kicitonpi.

27 Tera wicowazi wowapi kin dee. Tera iye Aberam, Nahor, qa Haran kicitonpi. Unkan Haran Lot kicitonpi.

28 Unkan Haran, iye atkuku, Tera itokam ta, Ur Kaśdim heciya, makoce en tonpi kin he en ta.

29 Unkan Aberam, qa Nahor winohinca yuzapi. Aberam ta- 
wicu caje kin Sari, qa Nahor tawicu caje kin Milka eciyapi. Haran he cunwintku iye Milka Iska ko ateyapi.

30 Tuka Sari cinca ton śni un, cinca wanica.

31 Unkan Tera iye cinhintku Aberam ikikcu, qa takojatpaku, Lot he Haran cinhintku, qa takośku Sari iye cinhintku Aberam tawicu kin hee. Hena.om $\mathrm{Ur}$ Kaśdim etanhan iyaya, Kanan makoce kin ekta ye kte, ca Taran ehan hipi qa hen hiyakonpi.

32 Qa Tera anpetu tawa kin waniyetu opawinge nonpa sanpa zaptan henakeca, qa Ḧaran hen Tera ta.

\section{WICOWOYAKE 12.}

1 Unkan Jehowa Aberam heciya, Nita makoce kin etanhan ya wo, nitakuye etanhan, qa niyate ti kin etanhan, makoce wan wanyag ciciye kte cin he ekta ya wo.

2 Kinhan oyate tanka icah ciye, ça ciyawaśte, qa nicaje kin tanka wakage kta; qa wowaśte yaun kta ;

3 Qa tona niyawaśtepi kin hena wicamdawaśte, qa tuwe niy aśice cin he mdaśice kta, qa niye en wicowazi tona maka akan unpi kin owasin yawaśtepi kta ce.

4 Unkan Aberam iyaya, Jehowa eciye ciqon he iyecen, qa Lot opa. Aberam wanna waniyetu wikcemna śakowin sanpa zaptan, Haran etanhan iyaye çehan.

5 Qa Aberam tawicu Sari ikikcu, qa iye cincu cinhintku Lot nakun, qa taku içihdepi, taku tonpi kin owasin, wicanagi tona Ḧaran en wicatonpi ko, qa unhdaka yapi, Kanan makoce kin ektakiya, qa Kanan makoce kin en hipi.

6 Qa Aberam makoce kin ohna ya, Śekem ounye cin etu, More utuhu kin en. Unkan hehan Kanani kin makoce kin en unpi.

7 Unkan Jehowa Aberam en tanin iciye ca, Wicowazi nitawa kin makoce kin de waqu kta ce, eciya. Unkan Aberam hen wa. hna wośapi wan, Jehowa tanin içye cin he kiciga.

8 Qa hetanhan unhdaka ya Betel iwiynhiyanpatanhan he wan wanke cin, Betel wiyohpeyata; qa Hai wiyohiyanpata otahedan en e wakeya, qa heci wahna wośnapi wan kage ça Jehowa caje kin hoyekiya.

9 Qa ake Aberam unhdaka itokali kiya ihdaka ya.

10 Unkan makoce kin he en wicaakihan, qa Aberam Egupta ekta apamahde ya, hen yanke kta, makoce en un qon nina wicaakilian nakaeś.

11 Qa Egupta ekta wanna hi kte cehan, tawicu Sari heciya, Winolinca owanyag waśte henica sdonwaya;

12 Qa Egupton oyate kin wanniyakapi kinhan, De tawicu kin ee. ce, eyapi, kta, qa miye maktepi kta qa niye niniyanpi kta.

13 Ceciya ce, tawinolitin ciya keya wo; hecen tanyan waun kte cạ, niye eciyatanhan minagi kin ni kta.

14 Qa Aberam Mizarim, Egupta hee, en hi qehan, Egupton oyate kin tawicu kin owanyag waśte linca e wanyakapi.

15 Unkan Paro taakicita tancan winohinca kin wanyakapi, qa Paro en yatanpi, qa winohinca kin Paro ti kin en un kte cin icupi.

16 Qa iye kin on Aberam tanyan ecakiconpi ; unkan tahin wa17 
WICOICAGE.

nuyanpi yuha, tatanka nakun, qa śuktanka śonśonna mdoka wiye ko qa ookiye wica winyan ko, qa camo hena owasin.

17 Unkan Jehowa Paro oyutan, Sari Aberam tawicu kin on etanhan, Paro tiyohnaka tawa kin koya wowayazan tanka on wicakaśtaka.

18 Unkan Paro Aberam kico, qa heciya, Tokeca hecamiyecon? He nitawin kin tokeca omayakidake śni he?

19 De mitankśi ce, ehe ciqon he tokeca heha he? Hecen miś mduze kta iwacu tuka. 'Tuka nitawin wanhdaka wo. Ehdaku qa hunktiya wo.

$20 \mathrm{Qa}$ Paro wicaśta tawa kin hena he iwahokonwicakiya, unkan hde śipi, tawicu taku yuhc cin ko owasin.

\section{WICOWOYAKE 13.}

1 Unkan Aberam Egupta etanhan iyaya, iye tawicu kici, qa taku yuhe cin ko owasin, Lot nakun opa, itokah makoce kin etu.

2 Qa Aberam wijica hinca, wanuyanpi, mazaska, qa mazaskazi ota yuha.

3 Qa itokah makoce kin oti qon ohna ihdaka ku, Betel etu, wakeya iticage ciqon Betel qa Hai otahedan heciya;

4 Tokag ehan wahna wośnapi kage ciqon he ekta, qa hen Aberam Jehowa caje kin hoyekiya.

5 Unkan Lot Aberam kici ihdaka un kin he tahinca, qa tatanka wakeya ko yuha.

6 Qa witaya tonwanyanpi kte cin makoce kin iyowinwicakiye śni, ehaeś wanuyanpi ota yuhapi, heon witaya ounyanpi kta okitpanipi.
7 Unkan Aberam wanuyanpi wilian wicakiyapi kin qa Lot wanuyanpi wihan wicakiyapi kin akinicapi. Unkan hehan Kanani kin, Perazi kin ko makoce kin en ounyanpi.

8 Unkan Aberam Lot heciya, Unhdokinica kte śni, wilian wicakiya mitawa kin, qa wihan wicakiya nitawa kin nakun akinicapi kte śni, hunka wanjin unkiciye cin.

9 Makoce ocowasin nitokam wanke śni he? Ceciciya ce, miye etanhan kinukankiya ya wo. Niś catka tanhan de cinhan miś etapa tanhan, qa niś etapa tanhan kinhan miś catka tanhan mde kta ce.

10 Unkan Lot wankan etonwe, ça Jordan iyomdaye kin ocowasin Zohar itato owancaya mini yukan, (Sodom Gomora kici Jehowa ihangye cin itokam,) Jehowa tamaga kin iyecen, qa Egupta makoce kin iyecen.

11 Qa Lot Jordan ohna makoce kin owasin kalinige ca, Lot wiyohiyanpata kiya unhdaka ya, hecen otoiyohi hunka wanjitku kin etanhan kinukankiya iyayapi.

12 Aberam Kanan makoce kin en ounyan, qa Lot omdaye kin otonwe kin en ounye, ça Sodom en wakeya iticaga.

13 Unkan Sodom wicaśta kin wicaśtapi śni, qa Jehowa en nina wahtanipisa.

14 Unkan Lot Aberam etanhan kinukankiya iyaye cin, he iyohakam Jehowa Aberam heciya, Iśta kin yuwankan ikikcu, qa toki yaun kin ihdukśan etonwan wo; waziyata, qa itokaga, qa wiyohiyanpata, qa wiyohpeyata.

15 Makoce kin de wandake, cin sitomniyan ciçu kte ca, wicowa- 
zi nitawa nakun owihankeśniyan.

$16 \mathrm{Qa}$ wicoicage nitawa kin maka wiyaka sukaza iyenaka wakage kta. Wicaśta wan maka wiyaka sukaza yawa okihi, hecinhan wicoicage nitawa nakun yawapi kta ce.

17 Najin qa makoce kin ohanske cin, qa ohdakinyan kin ecen omani wo, he ciçu kta ce, eya.

18 Unkan Aberam ihdake ca Mamore utuhu kin he Hebron en wanke cin ekta i, qa en ounye ca hen wahna wośnapi wan Jehowa kicaga.

\section{WICOWOYAKE 14.}

1 Unkan Amrapel Śinar en wicaśta yatapi ounye cin he ehan, iye qa Ariyok Elasar en wicaśta yatapi, qa Kedorlahomer Elam en wicaśta yatapi, qa Tidal Goim en wicaśta yatapi ozuye kagapi.

2 Bera Sodom en wicaśta yatapi, qa Birsa Gomora en wicaśta yatapi, qa Śinab Adma en wicaśta yatapi, qa Ś Semeber Zeboim en wicaśta yatapi, qa nakun Bela, Zohar hee, he en wicaśta yatapi, hena owasin azuwicayapi.

3 Sidim mdaye kin, mini skuya mde kin hee, en hena owasin ecipapi.

4 Waniyetu alke nom, hehanyan Kedorlahomer wowidake wicaya, tuka iake yamni kin hehan kipajinpi.

5 Unkan waniyetu iake topa, hehan Kedorlahomer qa wicaśta yatapi owicape ciqon owasin upi, qa Repaim kin Aśteret Qarnayim en wicakaśtaka, qa Zuzim kin Ham en, qa Emim kin Qiratayim mdaye kin en, hena owasin wicakastakapi.
6 Hori kin nakun, Sehir lie tawapi kin en Paran utuhu kin hetu, he hopuza kin en un.

$7 \mathrm{Qa}$ hdicupi, Miśpat wakoniya, Qadeá hee en, qa Ameleq tamakocepi kin owasin awihnunipi, Amori kin tona Hazazon Tamar en ounyanpi kin nakun.

8 Hehan Sodom en wicaśta yatapi, qa Gomora en wicaśta yatapi, qa Adma en wicaśta yatapi, qa Bela Zohar he en wicaśta yatapi, qa Zeboim en wicaśta yatapi hena owasin zuya iyayapi, qa Sidim mdaye kin en kicizapi.

9 Kedorlahomer Elam en wicaśta yatapi, qa Tidal Goim en wicaśta yatapi, qa Amrapel Ś Sinar en wicaśta yatapi, qa Ariyok Elasar en wicaśta yatapi, wicaśta yatapi ece, uman topa uman zaptan ecipapi.

10 Unkan Sidim mdaye kin ohna bitumen wolia ota, qa Sodom en wicaśta yatapi Gomora en wicaśta yatapi henios najicapi, qa ohinhipayapi, qa umanpi kin, tona nipi kin, he onapapi.

11 Unkan Sodom Gomora ko woyuha, taku yutapi ko yuhapi qon, owasin icupi qa kihdapi.

12 Lot Aberam cincu cinhintku kin Sodom en un kin nakun, taku tawa ko owasin icupi, qa hecen hdapi.

13 Unkan wicaśta wan okicize etanhan u, qa Aberam Heberi kin wokiciyaka, utuhu oju Mamore Amori tawa kin en un; Mamore Eśkol Aner kici hunka wanjin wicaye cin, qa hena yamni Aberam kici dakon kiciyapi.

14 Unkan Aberam sunkaku wayaka ahdapi kin he nakihon, hehan wicaśta opawinge yamni sanpa wikcemna sanpa śahdogan, iye ti kin en icagapi, qa onspewicakiyapi kin hena awi- 
caye ca wicaśta yatapi qon, Dan hehanyan wicakuwa.

15 Qa hanyen taokiye kici anawicatan, qa wicakaśtake ca owicakuwa, Coba Damaskos icatka tanhan wanke cin hehanyan.

16 Qa woyuha qon owasin eyaku; sunkaku Lot, qa taku tawa ko owasin, winolinca oyate ko owasin ahda.

17 $\mathrm{Qa}$ Kedorlahomer, qa wicaśta yatapi owicape ciqon wicakaśtakapi kin etanhan hdi kin, hehan Sodom wicaśta yatapi kin itkokim ya; Kaksiza mdaye kin ekta Wicaśta yatapi takaksiza kin hee.

18 Unkan Melkizedek Salem en wicaśta yatapi, Taku wakan otancanke wakiyuśnapi kin, he aguyapi miniśa ahna Aberam kahi ;

19 Qa yawaśte, qa heya, Wakantanka otancanke mahpiya maka ko ocowasin tawa kin, he $\Lambda$ beram yawaśte nunwe.

20 Qa Wakantanka otancanke kin, toka niyanpi kin nape kin en onihnake cin he yawaśtepi nunwe, eya. Unkan taku owasin etanhan iwikcemna kin qu.

21 Unkan Sodom wicaśta yatapi kin Aberam heciya, Oyate kin micu wo, qa woyuha kin he owasin icu, qa yuha un wo.

22 Unkan A beram Sodom wicaśta yatapi kin heciya, Jehowa Wakantanka otancanke kin malipiya maka ko ocowasin tawa kin, he ekta nape ye wakiya.

23 Taku nitawa kin etanhan hahonta wanjidan, hantpa wanjidan eśta iwacu kte śni, qa Aberam wawokihi wakaga ehe kte śni.

24 Tuka kośka taku yutapi kin hecehnana, qa Aner Eśkol qa Mamore wicaśta omicipapi kin hena eepi, onśpa tawapi kin ikikcupi nunwe, eya.

\section{WICOWOYAKE 15.}

1 Hena henakeca hehan, owanyake wan en Jehowa oie kin Aberam en hi, qa heciya, Aberam ihnuhan koyakipe cin; wahacanka nitawa qa taku iyotan tanka yahduhe kte cin he miye ce.

2 Unkan Aberam heya, Jehowa Itancan taku mayaqu kta he? Miś micinca manica qa wati kin taku ohnaka kin Eliezer Damaskon he yuha.

3 Qa Aberam heya, Miye cinca mayaqu śni, qa wicaśta wan wati kin en tonpi kin, he taku mitawa owasin yuhe kta.

4. Unkan Jehowa oie kin hoyekiya, De taku nitawa owasin yuhe kte śni ce. Tokeśta niteri kin etanhan inanpe kte cin he taku nitawa kin owasin yuhe kta ce, eya.

5 Qa tankan u śi, qa heciya, Mahpiya ekta etonwe ca wicanhipi kin yawa oyakihi hecinhan hena yawa wo. Qa nicinca kin he iyenakecapi kta ce, eciya.

6 Unkan Aberam Jehowa oie kin wicada, qa he woowotanna kiciyawapi.

7 Unkan Jehowa heciya, Makoce kin de ciçu, qa yahduhe lita e Ur Kaśdim etanhan u ciciye cin, Jehowa he miye ce.

8 Unkan, Jehowa Itancan, tokiya tanhan wahduhe kte cin sdonwakiye kta he? eya.

9 Unkan, Pte wan waniyetu iyamni, qa tatokadan wan waniyetu iyamni, qa tamdoka wan waniyetu iyamni, hena owasin imicu wo, qa wakiyedan wan nakun, tin wakiyedan wan iyahna. 


\section{WICOWOYAKE 16.}

10 Unkan hena owasin ikilcu, qa cokaya okiwicakasdece ca wobaśpe kin owasin iciyotakons ehnaka, qa zitkadan kin hena baśpe śni.

11 Unkan taku kinye cin woteca wicakte qon akan kun upi, unkan Aberam ham wicaya.

12 Unkan anpetu wi aisinyan iyaye kte cin, hehan woiśtima tanka wan Aberam ahinhpaye ca nakun otpaza wohitike hinca. 13 Unkan Aberam heciya, Wowicake eciyatanhan he sdonya wo, nicinca makoce wan tawapi śni e en waniyetu opawinge tom ounyanpi kte, unkan wowidag wicayapi, qa iyoyag wicayapi kta.

14 Unkan oyate wan wicayuhe kte cin he mdaco kta, unkan hehan woyuha ota yuha hinanpapi kta.

15 Tuka niye hunkake wicayaye cin ekta tanyan yahde kte ca, pa niska waśte kinahan nihapi kta.

16 Qa wicoicage itopa kin, hehan deciya hdipi kta ce. Amori kin woahtani kin nahahin iyatahdepi śni ce.

17 Unkan wi iyaya hehan nina otpaza, qa oceti izita wan, petijanjan ideya wan ko, taku okicasdece ciqon itoopteya iyaya.

18 Anpetu kin he en Jehowa wicotakuye wan Aberam kicage ca heya, Makoce kin de Egupta watpa etanhan, watpa tanka Yuphrate eciyapi hehanyan nicinca wicawaqu kta.

19 Keni kin, qa Qenizi kin, qa Qadmoni kin,

20 Qa Ceti kin, qa Perazi kin, qa Repaim kin,

21 Qa Amori kin, qa Kanani kin, qa Girgaśi kin, qa Jebusi kin.

\section{WICOWOYAKE 16.}

1 Unkan Aberam tawicu kin Sari cinca kiciton śni, qa Egupton wikośka wan yuha, Hagar eciyapi.

2 Qa Sari Aberam heciya, Tho Jehowa miye onamataka; ceciye, wikośka wan mduhe cin he elta ya ye; okini cinca miciton kta, eya. Unkan Aberam Sari oie kin anagoptan.

3 Unkan Sari Aberam tawicu kin, he wikoślka tawa, Hagar Egupton kin icu, qa Aberam hihnaku kin tawicu kta e qu; wanna waniyetu wikcemna hehanyan, Aberam Kanan makoce en un qehan hecon.

4. Unkan Aberam Hagar en i, unkan ihduśake ca, wanna ihduśaka wanhdake cẹan, yuhe cin he iśta kin on ihakta śni.

5 Unkan Sari Aberam heciya, Ścaya ecamiconpi kin he niye en oiyanihde. Miye wikośka wan mdulic cin he cicu, nimaku kin ekta, unkan ihduśaka wanhdake ca iśta kin on ihamakta śni, miye niye kici unkiyotahedan Jehowa yaco kta ce.

6 Unkan Aberam Sari heciya, Tokeśta wikośka nitawa kin he ninape kin ohna un, token iyonicipi ecen ecakicon wo. Unkan Sari iyoyag ya; unkan najica iyaya.

7 Unkan Jchowa taohnihde wan Hagar tinta kin en iyeya, mini hdoka wan ikiyedan, Śur canku kin ohna mini hdoka he cin hee.

8 Qa heya, Sari tawikośka Hagar, tokiya tanhan yau he? qa toki da he? Unkan, Sari mayuha itoye kin etanhan nawajica ce, eya.

9 Unkan Jehowa taohnihde kin heciya, Niyuhe cin ekta ici- 
cawin hde, ca nape kin ihukuya eicihde wo.

i0 Kinhan nicinca ota wakage kta, qa ota kin heon yawapi kte śni, Jehowa taohnihde kin Hagar eciya.

$11 \mathrm{Qa}$, Iho ihduniśake cin cinca wanji yaton kte, ça Iśmayel eya caś yakiton kta; nicakije cin Jehowa nanition kin heon etanhan.

12 Kinhan iś ikce wicaśta kin heca kta. Wicaśta owasin nape apaha wicayanke kta, qa iś wicaśta owasin nape apaha yankapi kte, ca hunka wanjin wicaye cin owasin itokam ounyc kte, Jehowa ohnihdeye cin eciya.

13 Unkan Wakantanka wanmayake cin he deciya awakita he? eye ca, Jehowa okiye cin, he Wakantanka wanmayadake cin, eya caśton.

1.4 Heon wakoniya kin he, Tuwe ni qa wanmayake cin tawaKoniya eciyapi; he Qadeś qa Bered otahedan wanka.

15 Unkan Hagar Aberam cinca wan kiciton, qa Aberam cinca Hagar ton kin he Iśmayel eya caś kiton.

16 Aberam wanna waniyetu wikcemna śahdogan sanpa śakpe, Hagar Iśmayel Aberam kiciton kin hehan.

\section{WICOWOYAKE 17.}

1 Unkan Aberam wanna waniyetu wikcemna napciwanka sanpa napciwanka, hehan Jehowa Abcram en tanin iciye ca, Taku wakan iyotan waśake cin he niye; mitokam omani qa iyampepica śni un wo.

2 Kinhan odakonciciye kta qa, iyakiçuya ciyuota kta, eciya.

3 Unkan Aberam pustag elipe- içiya ; unkan'Wakantanka okiye ca heya,

4 Odakonciciya qa oyate ota ate niyanpi kta;

5 Qa detanhan Aberam eniciyapi kte śni, tuka Abereyam nicaje ee kta; oyate ota ate niyanpi kte cin cicaga ce.

6 Qa nina yuota ciciye ca, oyate cicage kta, niye etanhan wicaśta yatapi inanpapi kta.

7 Qa miye niye kici wicotakuye cicage $k t a$, qa taku nitawa nihektam u kte cin hena koya, hecen wakage kta, tohanhanyan wiconi kte cin, hehanhanyan hecetu lata, hecen wakan mayada kta, qa nicinca nihektam u kte cin hena owihanke wanin wakan madapi kta.

8 Malroce en ihdaka yaun kin, Kanan makoce kin he owasin cicu kta, qa tona enitanhan u kte cin hena, qa owihanke wanin tawayapi kta qa taku wakan mayanpi kta.

9 Qa, niye wicotakuye mitawa kin yahduhe kta, niye, nicinca nihektam upi kte cin hena koya, wicoicage tawapi kin en.

$10 \mathrm{Qa}$ wicotakuye mitawa miye, qa niye kici, qa nicinca nihektam u kte cin hena koya, yahduhapi kte cin he dee ce. Tona wica un kinhan hena bakihidayapi kte.

11 Ce inkpa ha kin banicilidayapi kta qa odakonciciyapi wowapetokton kin hee kta.

12 Wicoicage nitawapi kin tona wica owasin yatipi kin ohna tonpi, qa nicinca etanhan śni eśta, tuka oyate tokeca cinca, qa mazaska on opetonpi kin owasin, cincatonpi ehantanhan anpetu iśahdogan bahdayapi kta.

13 Tuwe yati kin ohna tonpi qa mazaska nitawa on opetonpi kin he balidayapi kta, qa oda- 
konciyapi, nicelpi kin en he wicotakuye owihanke wanice lkta.

14 Tuwe wica bahdayapi śni, ce inkpa ha kin bakihdayapi śni kinhan, wicotakuye mitawa nalssa, heon wicaśta kin he oyate tawa kin etanhan baśpapi kta, Wakantanka Abereyam eciya.

15 Qa detanhan Sari nitawin kin Sari cajedate kte śni, Sara caje kin hee kta ce.

$16 \mathrm{Qa}$ he mdawaśte kte ca, hetanhan cinca wan ciçu kite ça, cinca niciton kte cin lie nakun mdawaśte kte ça, oyate ota nakun wicaśta yatapi tanka hetanhan inanpapi kta, Wakantanka Abereyam eciya.

17 Unkan Abereyam pustag ehpeiçye ça iha, qa cante kin mahen heya, Wicaśta waniyetu opawinge cinca kicitonpi kta he? qa Sara waniyetu wikcemna napciwanka cinca wan ton kta he?

18 Hehan, Tokin Iśmayel nitokam ni un, Abereyam Wakantanka eciya.

19 Unkan Wakantanka heya, Han; awicakehan Sara nitawin cinca wan niciton kta, qa Izak eya caś yakiton kta, qa wicotakuye mitawa kin he kici wahdusuta kta; cinca wicaye kte cin ihektam u kte cin, hena koya wicotakuye owihanke śniyan.

20 Iśmayel on nakun nacilion, on mdawaśte kta, cinca ota icahye wakiye ça iyakiçuya yuota wakiye kta, wicaśta yatapi ake nom kicitonpi-kte ça, oyate tanka icaliye wakiye kta.

21 Tuka Izak kici wicotakuye mitawa kin wahdusuta kta, detanhan omaka wanjica kinhan, makoncage kin de en Sara ton lita.

22 Wakantanka ie ça ohiniki- ye cin, hehan Abereyam itokam wankan ikiyopta.

23 Unkan Abereyam cinhintku Iśmayel ikikcu, qa nakun tona iye ti kin ohna wicatonpi kin, tona maza ska iye tawa on opewicaton kin hena koya owasin, tona wica iye ti kin ohna yakonpi kin hena, anpetu kin he en ce inkpa ha kin bawicakihdaya, Wakantanka eciye cin he iyecen.

24 Abereyam wanna waniyetu wikcemna napciwanka sanpa napciwanka hehan iye ce inkpa ha kin bakihdayapi.

$25 \mathrm{Qa}$ cinhintku Iśmayel waniyetu ake yamni hehan ce inkpa ha kin bakihayapi.

26 Abereyam cinhintku Iśmayel kici, anpetu wanjidan en bawicahdayapi.

27 Qa tiyohnaka wicaśta owasin, ti kin ohna wicatonpi, qa oyate tokeca etanhan opewicatonpi kin hena koya, owasin Abereyam kici bawicahdayapi.

\section{WICOWOYAKE 18.}

1 Unkan Mamore utuhu kin heciya, Abereyam wakeya tiyopa kin elkta iyotankehan yanke, ca wiyotanhan hehan Jehowa tanin içiya.

2 Unkan Abereyam wankan etonwan, qa wicaśta yamni itokam najinpi wanwicayake ca, walkeya tiyopa kin etanhan itkowicakipe kta e inyang iyaye ca, maka lkin en canpeśka makehdeya inajin.

$3 \mathrm{Qa}$, Itancan ni iśta kin iyokipi ciya hecinhan ceciciya ce, nitaokiye kin etanhan sam iyaye śni wo.

4 Mini onge aupi on siha hdujajapi, qa can kin ihukuya ozi içiyapo. 
5 Kinhan aguyapi onśpa cicaupi kta. Nicantepi kin iwakiśakapo, heon nitaokiyepi kin en yahipi, qa hehan idadapi kta ce, eya. Unkan token ehe cin ecen econ wo, eyapi.

6 Unkan Abereyam wakeya kin en ohankoya hda, Sara ti kin ekta qa, Aguyapi iyutapi yamni kohanna icu qa pasuta qa aguyapi hmiyanyan kaga wo, eya.

7 Hehan Abereyam wanuyanpi ekta inyang ye ca, ptejincadan wan cepa qa waśte ikikcu, qa he kośka wan qu; unkan inaliniyan pata.

8 Unkan pte asanpi qa pte asanpi ihdi, qa ptejincadan pata qon ikikeu, qa wicitokam ehde, qa iye wicikiyedan najin can kin ihukuya. Unkan wotapi.

$9 \mathrm{Qa}$ Sara nitawin kin toki un he? eciyapi. Unkan, Wakeya ohna yanka ce, eya.

10 Unkan, Detanhan omaka akihde linhan, ake niye en cihi kta qa awektunje kte śni. Hehan nitawin Sara cinca wan ton kta. Unkan Sara wakeya tiyopa kin ohna wicaśta kin ihektam yanka tuka he nation.

11 Wanna Abereyam Sara kici wanakaś wotapi, napin kanpi ; winohinca ayakonpi kin he, Sara wanna ecece śni un.

12 Heon Sara cante mahen ilia qa, Wamakanka hehan, qa mayuha iś nakun wicahinca eśta imduśkin kta he? eya.

13 Unkan Jehowa Abereyam heciya, Tokeca e Sara ilia qa, A wicakehan wamakanka hehan cinca waton kta he? eya he?

14. Jehowa taku wanji okitpani tukte e he? Tohantu kte cin ake niye en cihi, qa Sara hokśidan wan ton kta.
15 Hehan Sara kokipe ca heon anakihbe ca, Iwaha śni ce, eya. Unkan, Hiya, iyaha ce, eya.

16 Hehan wicaśta qon heciya tanhan najinpi, qa Sodom ektakiya etonwanpi ; unkan Abereyam awicaye lita e om iyaya.

17 Unkan Jehowa heya, Taku ecamon kte cin he Abereyam anawakifibe kta lie?

18 Abereyam iś oyate tanka, qa waśaka un kte ca iye eciyatanhan oyate maka ohnaka owasin yawaśtepi kta;

19 Qa de sdonwakiye, cinca wicaye cin, tiyohnaka kin iyahna taku owotanna qa ecetu econ wicaśi kta, qa Jehowa tacảnku kin okicipapi kta, hecen Jehowa Abereyam token wahoye cin owasin kicicahi kta.

20 Unkan Jehowa heya, Sodom qa Gomora ceyapi kin he tanka, qa wahtanipi kin tehika e nawahon, heon

21 Nakaha kun mde ça ceyapi kin mahiyohi kin ocowasin iye-" ceca econpi kinhan sdonwaye kta, qa ecece śni kinahan sdonwaye kta ce, eya.

22 Unkan wicaśta qon hetanhan iyayapi, qa Sodom en ipi, qa Abereyam nahahin Jehowa itokam yanka.

23 Qa Abereyam ikiyadan hinajin qa, Tona olian owotanna wahtanipisa om ihang wicayaye kta he?

24 Okini wicaśta owotanna wikcemna zaptan otonwe kin en unpi eśta ihang yaye kta he? Wicaśta owotanna wilkcemna zaptan kin hena on, otonwe en unpi kinhan, otonwe kin itonyape kte śni he?

25 Wicaśta ohan owotanna wahtanipisa om ihang wicayaye kta seca ; tokadan hecanon kte śni. Tona owotanna wahtani- 
pisa iyecen ecawicakiconpi kin he iyonicipi śni. Maka kin owancaya wayaco un kin he woyaco econ kte śni he? eya.

26 Unkan Jehowa heya, Wicaśta owotanna wikcemna zaptan Sodom otonwe en iyewaye cinhan, hena on otonwe kin ocowasin itonwape kta ce.

27 Unkan Abereyam ayupte ca, Maka mdu qa cahota hemaca qeyaś mayuhe cin taku ewakiye kta on iapi iwacu.

28 Okini . wicaśta owotanna wikcemna zaptan kin en zaptan ope kte śni seca; zaptanna kin hena on otonwe kin ocowasin ihang yaye kta he? eya. Unkan, Ihang waye kte śni, hen wikcemna tom sanpa zaptan iyewaye cinhan, eya.

29 Unkan ake ie kta e hda, qa, Okini hen wikcemna tom iye-. wicayapi kta, eya. Unkan, Wikcemna topapi on ecamon kte śni, eya.

30 Unkan, Mayuha ceciciye canniye maye śni wo; iwae kta ce. Okini wikcemna yamni hen iyeyapi kta seca, eya. Unkan, Wikcemna yamni hen iyewicawaye cinhan ecamon kte śni, eya.

31 Unkan, Tokin Mayuhe cin taku ewakiye kta e epen. Okini wikcemna nonpa hen iyeyapi kta seca, eya. Unkan wikcemna nonpa on ihang waye kte śni ce, eya.

32 Unkan, Itancan ceciciye canniye maye śni wo; wancadan iwae kta ce. Okini wikcemna en iyeyapi kta seca, eya. Unkan wikcemna on ihang waye kte śni ce, eya.

33 Unkan Jehowa Abereyam okiya ohinikiye cin hehan toki iyaya, qa Abereyam ekta hda.

\section{WICOWOYAKE 19.}

1 Unkan hitayetu ehan ohnihde wicaye cin nom Sodom en hipi ; he icunhan Lot Sodom tiyopa kin en iyotankehan yanka, unkan Lot wanwicayake ca itkowicakipe kta najin hiyaya, qa wicitokam patuś inajin.

$2 \mathrm{Qa}$ hewicakiya, Itancan ceciciyapi, wati kin en yapo, he wowidake mayaduhapi, heon hanyetu kin de wati kin en yukanpo. Siha kin hdujaja po, qa hanhanna yektapi, qa tokiya dapi hecinhan ekta katinyan yapo, ewicakiya. Unkan, Hiya, ocanku kin de ohna hanyetu osan unyakonpi kta, eyapi.

3 Tuka nina cinwicakiya ; unkan kici kipi. Ti kin ti mahen ipi, unkan aguyapi napohye codan śpan ye ca nakun wowicakihan; unkan yutapi.

4 Iwankapi kta itokam, Sodom otonwe wicaśta kin owasin tipi kin he aohduteyapi, hokśidan wicalinca ko owasin, otonwe ihanke anokatanhan, henana 0tonwe ohnaka kin owasin kawitaya iheya.

5 Qa Lot kipanpi, Wicaśta nom banyetu kin de yati en hipi kin tukte epi he? Tankan u wicaśi wo; sdonwicunyanpi kte, eyapi.

6 Unkan Lot ti kin etanhan inanpe, ca tiyopa kin ecen icu, $7 \mathrm{Qa}$, Hunka wanjin ciyapi, ceciciyapi ce, ihnuhan hecen śicaya ecanonpi kin.

8 Micunkśi nonpapi kin nahahin wicaśta sdonye śni yakonpi, heniyos eiçupi, qa token yacinpi ecen wicayakuwapi kta, tuka wicaśta kin dena conkaśke mitawa ohanzi ihukuya hipi kin heon takudan ecawicakiconpi śni po, ewicaliya. 


\section{WICOICAGE.}

9 Unkan, Tokan hiyaya wo. Wicaśta kin de oyate tokeca, tuka den un kta wacin, qa hi qa he unyacopi wacin. Heon ihomeca niye iyotan śicaya unnicuwapi kta, oyate kin eyapi, qa Lot nina paśkicapi qa tiyopa kin kaptujapi kta e en yapi.

10 Unkan ohnihde unpi kin tiyopa kin yuganpi, qa Lot ti mahen hdicu yapi, qa tiyopa lin onatakapi.

11 Unkan oyate kin wicaśta cistina wicaśta tanka ko, oyate otonwe ohnaka kin owasin, tiyopa kin en hiyeye cin hena iśta gonge wicayapi, heon tiyopa kin yutantan akitapi.

12 Unkan ohnihde unpi kin Lot heciyapi, Nakun den takuyaya yukan he? takoś yaye cin nicinkśi, nicunkśi, qa taku tona otonwe kin ohna duhe cin, de etanhan om tankata inanpa wo.

13 Otonwe kin de ihang unyanpi kta, heon unhipi.; oyate kin de owasin woahtani hoyapi, qa Jehowa itokam oiyahde iciyapi, heon otonwe kin de owihanke ye unhipi.

14 Unkan Lot tankata inanpe, ca wicaśta takoś wicaye kte cin, hena hewicakiya, Otonwe kin de etanhan tankata inanpapo; Jehowa otonwe kin de ihanke ye kta ce. Tuka takoś wicaye kte cin Lot cetunhdapi.

15 Anpao hinanpa, unkan ohnihde unpi kin Lot inahni yapi, qa, Najin wo; nitawin ehdaku wo, qa nakun liicunkśi nonpa den unpi kin, okini otonwe wahtani kin en awihnuniniyanpi kta nace.

16 Tuka ihduwiyeyapi śni; unkan ohnihde unpi nape etanhan wicayuzapi, Lot, qa tawicu, qa cunwintlku nonpa nape kin wicayuzapi; Jehowa hena onśi26 wicakida, he etanhan otonwe kin tankan ewicayayapi.

17 Otonwe tankan awicaipi qa uman heciya, Napa wo, ni içiya wo. Ihnuhan nihektam eyatonwe cin. Omdaye kin ohna tuktedan inajin śni. He kin heciya onapa wo ; hecen yani kta ce.

18 Unkan Lot hewicakiya, Hiya, Itancan ceciciya ce :

19 Wowidake yaya nitokam wopida iyeiciya, qa nitowaonśida kin icantoyahnake cin he miye. Tuka he onawape kte cin owakitpani kta. Okini taku śica hiyohimaye ca mațe kta, kowakipa.

20 Heon ceciciya, otonwe wan cistina den aśkadan he cin, hee iyowahi kta owakihi kta. Ceciciya, heci ye mayan wo; cistina tuka hecen on minagi kin ni kta.

21 Unkan, He wopida kin aciyupte kta; otonwe cistina kin he ihang waye kte śni.

22 Onapa wo; inahni wo. Tohan ehan yai śni liehanyan token wahian lite śni, eciya. He etanhan otonwe kin he $\mathrm{Z}_{0}$ har eciyapi.

23 Wi toka hinanpa hehan Lot Zohar iyohi.

24 Unkan hehan Jehowa magaju ya, Sodom Gomora heniyoza kiya inyan zi peta ko Jehowa mahpiya eciyatanhan ahinhe wicaya.

25 Otonwe kin hena owihanke wicaya, omdaye ko ocowasin, qa tona otonwe kin en ounyanpi, qa makoce kin hen peji koya.

26 Tuka Lot tawicu kin hakikta, unkan tancan kin tokeca hinhde ca miniskuya icu.

27 He icunhan, hanhanna Abereyam kikta, qa Jehowa okiye ciqon en etkiya ya. 


\section{WICOWOYAKE 20.}

28 Qa Sodom, Gomora, makoce ihdukśan koya atonwan ; unkan makoce kin he etanhan śota wan, maza śdoyapi oceti śota iyececa, wankantkiya inanpe cin wanyaka.

29 Jehowa Omdaye otonwe kin hena, owikanke ye cin he icunhan, Abereyam kiksuye ca otonwe ohna Lot ounye cin ihang wicaye cin hetanhan Lot tankan iyaye ya.

30 Unkan Lot Zohar etanhan itawankanhde ye,ca he wan akan cunwintku napin om e ounyan, Zohar he en un kte cin he wikopa heon, qa makohdoka wan cunwintku napin om oti.

31 Unkan tokapa kin he tankaku kin hecen eciya, Ate unye cin wanna wicahinca, qa maka ohnaka wanna wicaśta wanica, maka akan econpi qon iyecen, tuwedan unkiye en lii kte śni.

32 Iho we, ate miniśa yatke unkiye kta, qa kici unwanke lite; hecen on ate etanhan wicoicage unhdoyapi kta.

33 Hanyetu, unkan atkuku miniśa yatke kiyapi. Tokapa kin he tolkaheya atkuku kici iwanka, tuka atkuku kin cunwintku en hiwanke cin he sdotkiye śni, nakun kikta kin.

34. Ihanhanna tokapa kin he tankaku kin heciya, Hinhan ate kici imunka; ecin ake hanyetu kinhan, miniśa yatke unkiye kte ça niś ito kici nunke kte, hecen on ate etanhan wicoicage unhdoyapi kta.

35 Unkan ake ihanyetu, unkan atkuku miniśa yatke kiyapi ; iś ito hakakta kin atkuku kici iwanka. Iś ake cunwintku en hiwanke cin sdotkiye śni, qa kikta kin nakun.

36 Hecen Lot cunwintku napin atkuku etanhan hokśin itpihnakapi.

37 Tokapa kin hokśidan wan ton qa Moab eya caś kiton. He Moab oyate ate yapi anpetu kin dehan aiyahdeya.

38 Unkan hakakta kin iś eya hokśidan wan ton qa, Benami eya caś kiton. He Amon cinca kin anpe dehan aiyahdeya ate yapi.

\section{WICOWOYAKE 20.}

1 Hehan Abereyam makoce itokaga kin ekta ihdaka ye ca, Qadeś qa Śur otahedan iyotanke ça Gerar en ounyan.

$2 \mathrm{Qa}$ Abereyam tawicu Sara ke ca, Mitankśi kin dee ce, eya. Unkan Gerar wicaśta yatapi Abimelek, he ekta ye wicaśi qa Sara icu.

3 Unkan hanyen, Wakantanka Abimelek ekta hi, qa wowihanmde wan en heciya, Iho nițe, winohinca iyacu kin he wicaśta wan tawicu kin heca nakaeś.

4 Tuka Abimelek nahahin Sara ikiyedan wanke śni, qa heya; Itancan oyate owotanna wan he nakun te wicayaye kta he?

5 Mitankśi kin dee, emakiye śni he? qa winyan kin iś, Timdo waya ce, emakiye śni he? Micante kin ecedan qa minape iyecetu kin on hecamon.

6 Unkan Wakantanka wowihanmde kin en heciya, Miś nakun nicante ecedan lin en hecanon kin sdonwaya qa, heon ecihdaku, miye en wayahtani kte śni, qa dutan kte cin iyowin ciciye śni.

7 Qa wanna iho, wicaśta qon tawicu kin kicu wo iścwicaśta wokcan kin léca, kinhan niye on cekiye kta, hecen yani kta; tuka ake yecu śni kinahan nite kte ca nakun taku nitawa kin owasin he sdonya wo. 
8 Unkan Abimelek hanhanna hin kikta, qa ookiye wicayuhe cin owasin wicakico, qa hena owasin owicakiyaka: Unkan wicaśta lkin nina kokipapi.

9 Unkan Abimelek Abereyam kico qa, Tokeca e hecaunyeconpi ? Qa niye en wawahtani kin tukte e on woahtani tanka wan miye qa oyate mitawa ko iyahde unyayapi? Woccon wan econpi kta iyecece śni tuka hecamiyecon, eciya.

10 Qa, Taku wandaka on hecen ecanon he? Abimelek Abereyam, eciya.

11 Unkan Abereyam heya, Tuwedan deciya Wakantanka ohoda śni qa mitawin on maktepi kta kepce cin heon hecamon;

12 Qa awicakehan tankśi waya ce. Ate waye cunwintku kin hee, tuka iṇa tawa śni, qa mitawin ee lita mduza.

13 Qa Wakantanka ate ti kin etanhan ihdaka ye maye cehan winyan qon hewakiya; Taku waśte ionśimayada kte cin dee ce. Makoce owasin tokiya kaśta en unki kinhan, Timdo emakiya wo, epe ciqon, eya.

14 Hehan Abimelel tahin wanuyanpi ikikcu, qa tatanka qa hokśidan wicinyanna ko iwicakikcu qa Abereyam qu; tawicu Sara nakun kicu.

15 Qa Abimelek heya, Mitamakoce nitokam wanka ce ; tukten niiśta iyonicipi kinhan hen iyotanka wo.

16 Qa Sara heciya, Tho nitimdo mazaska kektopawinge waqu; iho he niiśta akahipe kte cin ee, tona nici unpi kin owasin en, qa tokan nakun owasin en ; hecen Sara iyopeyapi.

17 Hehan Abereyam Wakantanka icekiya; unkan Wakantanka Abimelek asni yan, tawicu kici qa wikośka yuhe cin owasin. Unkan cinca tonpi.

18 Jehowa, Sara Abereyam tawicu kin on etanhan, Abimelek tiyohnaka tawa owasin tamnipi kin suksuta onawicataka tuka qon.

\section{WICOWOYAKE 21.}

1 Unkan Jehowa heye ciqon, he iyecen Sara ekta hi, qa Jehowa, eciye ciqon, he iyecen ecakicon.

2 Unkan Sara ihduśake ca Abereyam okanta cinca wan kiciton; Wakantanka eye ciqon iyehantu qehan.

3 Unkan Abereyam cinhintku kicitonpi, Sara ton kin he, Izak eya caś kiton.

$4 \mathrm{Qa}$ Abereyam Izak bakilidaya anpetu iśahdogan tonpi kin ehantanhan, Wakantanka econ śi qon iyecen.

5 Abereyam wanna waniyetu opawinge, hehan cinhintku Izak kicitonpi.

6 Unkan Sara heya, Wakantanka iha mayan, qa tona nahonpi kin imiciyuśkinpi kta.

7 Qa Sara hokśiyoqopa azin kiye kte cin, tuwe Abereyam eciye kta he? wicahinca tulka cinca weciton kin, eya.

8 Unkan hokśidan kin tanka aye ça azin ayaśtan kiyapi. Unkan Izak azin ayaśtan kiyapi anpetu kin he en, Abereyam wohe ca wicota wicakico.

9 Unkan Hagar Egupton kin cinhintku Abereyam kiciton kin he, wowilakia kin he Sara wanyake ca;

10 Wikośka kin de cinhintku kin iyahna tankan ye wicaya ye. Wikośka kin de cinhintku kin, micinkśi Izak kici woyuha ta. waye kte śni, eya. 
WICOWOYAKE 21.

11 Unkan oie kin he, Abereyam iśta kin en telike hinca, cinhintku kin heon etanhan.

12 Unkan Wakantanka Abereyam heciya, Wikośka duhe cin, qa hokśidan kin on etanhan, niiśta kin en tehike śni nunwe. Taku Sara eniciye cin owasin ecen ho kin anagoptan wo. Izak he en wicoicage nitawa kin caje yatapi kta.

13 Wikośka cinhintku kin nicinkśi kin hee, heon he nakun oyate wan icah wakiye kta ce.

14 Unkan Abereyam hanhanna hin kikta, qa aguyapi mini apalita wan ahna icu, qa Hagar qu, tahpa kin akan ehnaka, hokśidan nakun qa toki ye śi. Unkan iyaye ça Berśeba tinta kin onuni.

15 Unkan mini apałta kin etanhan mini wanna yaliepapi, qa hokśidan kin canthaka wan ihukuya elipekiya ;

16 Qa iyaye ca iyotakons iyotanka kitana tehan, wanhinkpe kihiyeyapi hehanyan: Hokśidan te kte cin wanwahdake kte śni ce, eya; heon aisinyan iyotanke ca hoyeye ça ceya.

17 Unkan Wakantanka hokśidan ho kin nahon, qa Wakantanka taohnihde malipiya eciyatanhan Hagar kipan, Hagar taku yaka he? Wakantanka he oyanke kin etanhan hokśidan ho kin nahon. Kokipe śni wo.

18 Najin qa hokśidan kin ikikcu qa, nape kin on hduha wo. Iye oyate tanka wan icah wakiye kta ce, eya.

19 Qa Wakantanka winyan iśta kin yuhidoka, unkan minilidoka wan wanyake ça ekta i, qa mini apahta kin iyatahde ye ça hokśidan kin yatke kiya.

20 Unkan Wakantanka hokśidan kin kici un; hecen tanka icage ça tinta kin en ounye ça itazipa on waoka.

21. Qa Paran tinta kin en ounye ca hunku kin Egupta makoce kin etanhan, tawicu kte cin kiciyuza.

22 Unkan hehan Abimelek qa taakicita tancan Pikol henioza Abereyam heciyapi; Taku ecanon kin owasin en Wakantanka oniciya.

23 iho hecen eya wo, Wakantanka namahon; cihnaye kte śni, nicinkśi nitakoja ko wicawahnaye kte śni, taku waśte ecamiyecon kin iyecen ecacicon kta, makoce en ounwaye cin nakun.

24 Unkan, Miś ecen eya wakonze kta ce, Abereyam eya.

25 Hehan minihidoka wan Abimelek taokiye kin kipi qon, he Abereyam cajeyate ca he on Abereyam Abimelek iyopeya.

26 Unkan, Tuwe hecon kin sdonwaye śni, qa niś nakun omayakidake śni, nakun miś he nawalion śni, nakaha heceyedan, eya.

27 Unkan Abereyam tahinca wanuyanpi pte wanuyanpi ko ikikcu, qa hena Abimelek qu; unkan napin odakota wan kicicagapi.

28 Hehan Abereyam tahinca cinca wiyedan śakowin akipam ewicahde.

29 Unkan Abimelek Abereyam heciya, Tahinca wiyedan śakowin kinukan ewicayahde kin he taku he.

30 Unkan, Minilidoka kin de miś waศe cin he yuotaninpi kte cin, heon tahinca wiyedan śakowin kin hena minape kin etanhan iyacu kta, eya.

31 Unkan heon etanhan makoce kin he Berśeba eciyapi, napin heciya Wakantanka namahon eya, ekiciyapi. 
32 Unkan Berśeba hen odakota wan kicicagapi; hehan Abimelek najin taakicita tancan nakun, qa Piliśtim tamakocepi kin elita hdapi.

33 Unkan Abereyam Berśeba heciya taśkoju wan oju, qa hen Jehowa Taku wakan owihanke wanica hoyekiya.

34 Qa Abereyam Piliśtim tamakocepi kin en anpetu ota ounyan.

\section{WICOWOYAKE 22.}

1 Unkan hena owasin iyohakam, Wakantanka Abereyam iyutan ye ça heciya, Abereyam, eya houkiya. Unkan, Tho miye ce, eya.

2 Unkan, Nicinkśi heceyedan waśte yakidake cin Izak he icu, qa Moriya makoce kin ekta ye ca heciya he wan ociciyake kte cin he akan petan wahduśna wo, eya.

3 Unkan Abereyam hanlianna hin kikta, qa śuktanka śonśonna aqin kiton, qa kośka nom iwicakikcu cinhintku Izak nakun, can on wośna kte cin he kakse ca, najin qa hena om, makoce tukte en Wakantanka cajeyate ciqon heciya iyaya.

4 Anpetu iyamni Abereyam wankan etonwe ca, tukten ke cin he tehan tanhan wanyaka.

5 Qa Abereyam kośka wicayuhe cin hewicakiya, Den iyotankapo śuktanka śonśonna iyahna, qa miś hokśidan kici kan unkipi kinhan canpeśka makehdeya unkanpi, qa den dukanpi kin en unhdipi kta.

6 Qa Abereyam can on wośna kte cin icu, qa cinhintku Izak akan ehnake ça peta isan ko nape kin en icu, qa sakim iyayapi.

7 Unkan Izak atkuku Abereyam kici okiye ca, Ate, eya.
Unkan, Micinkśi nawahon ce, eya. Unkan, tho peta can ko yukan, tuka woteca kin wośnapi on tukte e he? eya.

8 Unkan, Micinkśi Wakantanka tahin wanuyanpi walkiyuśnapi on okide kta, eya. Hecen sakim yapi.

9 Qa wanna tukten Wakantanka ye śi qon hen hiyohipi, qa hen Abereyam wahna wośnapi wan kage ca, cinhintku Izak iyakaśke ça wahna wośnapi kin akan ekihnaka, can kin iwankam.

10 Qa cinhintku te kiye kta e nape kiyugate ça isan kin ikikeu.

11 Hehan malipiya kin eciya tanhan Jehowa ohnihde ye cin kipan, Abereyam, Abereyam, eya. Unkan, Nawahon ce, eya. 12 Unkan, Hokśidan kin elkta ninape kin ye kiye śni, qa takudan ecakicon śni wo. Wanna niye Wakantanka ikoyape cin sdonwaya ce ; nicinkśi heceyedan, tuka mayaqu kta wicayada he etanhan, eya.

13 Hehan Abereyam wankan etonwe ca, inyun iheyata tamdoka wan otehi ohna he kin on ikoyag najin, he wanyake ca, Abereyam ekta i, qa tamdoka kin icu, qa cinhintku kin hee kiya hee petan wośna.

14. Qa Abereyam makoce kin he, Jehowa wiwanhdake kta, eya caś ton; heon Jehowa he tawa kin akan tanin içiye kta ce, nahahin eyapi.

15 Unkan mahpiya kin eciya tanhan Jehowa ohnihde ye cin inonpa akihde Abereyam kipan ; 16 Miye kaeś caje mihdate Jehowa eya; Taku ecanon kin de nicinca wanjidan tuka itonyakipe śni ;

17 Heon wowaśte on ciyawa- 


\section{WICOWOYAKE 23.}

śte kta, nicinca iyakiçuya yuota wakiye kta. Mahipiya wicanhipi iyena, qa miniwanca kahda wiyaka sukaza iyeye cin iyena, taku nitawa iyacin kte ça, nicinca toka wicayapi kin otonwe tawapi kin yuhapi kta.

$18 \mathrm{Qa}$ mioie kin anayagoptan kin heon nicinca kin on maka kin oyate owasin wicayawaśtepi lita ce, eya.

-19 Unkan Abereyam kośka wicayuhe cin ekta hda; unkan najinpi qa yuwitaya Berśeba ekta hdapi; qa Abereyam Berśeba en ounyan.

20 Hena owasin iyohakam A bereyam hecen eya okiyakapi ; Milka he nakun nicinye Nahor he cinca kiciton.

, $21 \mathrm{Uz}$ tokapa kin hee, qa sunkaku Buz, qa Qemuel Aram he atkuku ;

$22 \mathrm{Qa}$ Kesed, qa Ḣazo, qa Pildaś, qa Idlap, qa Betuel.

23 Unkan Betuel he Rebeka kicitonpi. Hena śahdogan kin Milka he Nahor Abereyam cincu kin kiciton.

24 Unkan tawicu tokeca kin, Ruma eciyapi he Teba, qa Gaham, qa Tahos, qa Mahaka hena wicaton.

\section{WICOWOYAKE 23.}

1 Unkan Sara waniyetu opawinge sanpa wikcemna nonpa sanpa śakowin henakeca, waniyetu kin hena Sara ni.

2 Hehan Sara ta, Arba otonwe kin heciya, Ḣebron Kanan makoce kin etu hee. Unkan Abereyam Sara on iyokiśice ca akiceye kta e en hi.

3 Hehan Abereyam tawicu te cin itokapa tanhan najin qa, Het cinca wicaye cin owicaliya.

$4 \mathrm{Qa}$, Miś wicaśta ihdaka waun - qa aśkatudan nicipi wahimdo- tanka, wicaśta hnakapi mduhe kta e niyepi om maqupo; kinhan makite cin mitokapa tanhan wehnalse kta ce, eya.

5 Unkan Het cinca wicaye cin ayuptapi, qa Abereyam heciyapi ;

6 Itancan anaungoptanpo: Wicaśta yatapi wakan unkiyepi cokaya henica. Wicaśta hnakapi unhapi kin tukten iyonicipi kin nicite cin en kihnaka wo. Unkiś wanjidan kaśta wicaśta hnakapi unhapi kin hen nicite cin yehnake kta e tehinda kte śni.

7 Unkan Abereyam najin qa Het cinca wicaye cin oyate makoce yuhapi kin wicitokam apatuja.

8 Qa owicakiye ca, Makița mitokapa tanhan wehnake kte cin he iyonicipipi hecinhan anamagoptanpi, qa Epron Zohar he cinhintku cin makiyapo.

9 Kinhan Makpela imnija olidoka yuhe cin, tamaga ihanke en un kin, mazaska iyenakeca kin heon maqu kte cinhan, niyepi cokaya wicaśta hnakapi wan mduhe kta ce, ewicakiya.

10 Unkan Epron Ḧt cinca wicaye wicehna ounyan; qa Epron Heti kin Abereyam ayupta; Het cinca wicaye cin, otonwe tiyopa ohna upi kin hena owasin nahonpi;

11. Hiya, Itancan namahon wo. Maga kin ciç ; imnija ohdoka en un kin he nakun cicu. Mitaoyate cinca wicaye cin wanmayakapi he cicu. Nicite cin kihnaka wo, eya.

12 Unkan Abereyam oyate makoce tawapi kin wicitokam patuja.

$13 \mathrm{Qa}$ ia, Tho amayadupte cinahan, namahion wo. Maga kin on mazaska ciçu kta. Icu wo; kinhan makite cin wehnake lita, 31 
Epron eciya, oyate makoce yuhapi kin hena nalionpi.

14 Unkan Epron Abereyam ayupte ca heciya ;

15 Itancan namahon wo. Maga kin mazaska opawinge topa iyehantu ce; tuka unkiyotahedan he taku? Nicite cin ekihnaka wo.

16 Unkan Epron ie cin he Abereyam anagoptan, qa mazaska opawinge tom wopeton icupi kin heca Abereyam aspe ye ton qa qu, he Epron Het cinca wicaye cin nahonpi cajeyate ciqon hee.

17 Unkan Epron tamaga Makpela en wanka, Mamore iyotakons he qu; maga kin imnija olidoka he en wanke cin lie nakun, qa can tona he en han qa tona ihdukśan he cin he nakun owasin ;

18 Abereyam tawa kte cin, Het cinca wicaye cin tona otonwe tiyopa kin ohna yapi kin he wanyakapi.

19 Hehan Abereyam tawicu Sara elihnaka; Makpela maga imnija ohdoka kin he en, Mamore ikiyedan, Ḣebron Kanan makoce kin etu hee.

20 Unkan maga imnija olidoka iyahna, Abereyam wicaśta hnalkapi on yuhe kte cin, Ḧet cinca wicaye cin qupi.

\section{WICOWOYAKE 24.}

1 Unkan Abereyam wanna wicafinca omaka ota; unkan Jehowa taku owasin en Abereyam yawaśte.

2 Unkan Abereyam he taokiye tiyohnaka tawa tokapa kin, taku yuhe cin owasin awanyag un kin heciya ; Ninape kin miceca ihukuya ekihnaka wo;

3 Kinhan, Jehowa Taku wakan malipiya maka ko yuhe cin namalion, eye ciciye kta, Kanani oyate om manke cin cunwintkupi kin etanhan micinkśi on winoliinca iyacu kte śni.

4 Tuka mitamakoce qa mitakuye kin hena ekta de kte ca, hetanhan micinkśi Izak on winohinca iyac kta, kehe kta ce, eya.

5 Unkan, taokiye kin heya, Okini winolinca kin makoce kin de ekta omapé kta tawatenye lkte śni ; hecinhan makoce etanhan yau qon, nicinkśi ake ekta ye wakiye kta he?

6 Unkan, Ihnulıan micinkśi icicawin heciya ye yakiye cin.

7 Taku Wakan mahpiya yuhe cin, ate ti kin etanhan qa makoce wan en matonpi kin etanhan, imacu qa omakiye ça, Makoce kin de nicinca waqu kta ce, eya konza, he taohnihde kin nitokam ye śi kta, kinlıan micinkśi kin on heciya tanban winohinca wan iyacu kta.

8 Winohinca kin onipe kta tawațenye śni, hecinhan iyakonze kin de on iyaonpepicaśni yaun kta, tuka micinkśi heciya ye yaye kte śni ce.

9 Unkan taokiye qon nape kin iye yuhe cin Abereyam ceca kin ihukuya ekihnake ca ecen eya konza.

10 Qa ookiye kin, iye yuhe cin camo tawa kin etanhan, camo wikcemna, qa iye yuhe cin taku waśte tawa kin owasin etanhan $\mathrm{icu}$, qa hena yuha iyaye ca $\mathrm{Na}$ hor otonwe kin ekta i, Aram watpa otahedan etu.

$11 \mathrm{Qa}$ otonwe kin itankan minihdoka ikiyedan, camo kin canpeśka makehdeya inajin wicakiya, litayetu mini huwe ipi eca iyehantu.

12 Qa heya, Jehowa Abereyam. 


\section{WICOWOYAKE 24.}

mayuhe cin Wakan nida ceciciya, nakaha tanyan ihuni makiye ca Abereyam mayuhe cin he wowaśte ecakicon ye.

13 Minihdoka kin ekta nawajin ; qa otonwe kin wicaśta cunwintkupi kin mini huwe upi kta.

14 Qa wikośka wan tuwe kaśta hewakiye kta; Wakiśkotpa nitawa kin au wo, mini mdatke kta ce; unkan, Yatkan ye, qa camo nitawa kin nakun yatke wicawakiye $\mathrm{kta}$, eye cinhan, tokin nitaokiye Izak yuhen yakalinige cin hee, kinhan mayuhe cin wowaonśida ecayecon sdonwayen.

15 Unkan iś ia nahahin ayaśtan śni ehan, Rebeka u, Abereyam cincu Nahor tawicu Milka he cinhintku Betuel he cunwintku kin hee, wakiśkotpa hiyeta onpe ca $\mathrm{u}$.

16 Wikośka owanyag waśte hinca, hokśivinà, qa wicaśta tohini sdonye śni, minilidoka kin ekta kun ye ça wakiśkotpa kin ojudan icu qa wankan hiyu.

17 Unkan ookiye qon itkokim inyang ye ça, Wakiśkotpa duhe cin etanhan mini onge yatke makiya wo, eya.

18 Unkan, Itancan yatkan ye, eye ça wakiśkotpa kin nape kin on kun akihde, qa yatke kiya.

19 Unkan ayaśtan hehan, Camo nitawa kin nakun hena on mini awau kta, yatkan ayaśtanpi kin hehanyan, eya.

$20 \mathrm{Qa}$ inahini qa wakiśkotpa kin cankaśkotpa tanka kin okaśtan, qa inyanka minilidoka kin ekta mini huwe ya heon, qa camo kin owasin mini wicakahi.

21 Unkan wicaśta kin yuśinyaye ca taku eye śni, canku ohna $\mathrm{u}$ kin he Jehowa ohinniyan tanyan ikiyuśtan kta hecinhan he sdonye kta.
22 Unkan camo kin mini yatkan ayaśtanpi, hehan wicaśta qon mazaskazi maza oin tanka, mazaska hanke iyecen tke, he icu qa nakun maza huhu nom mazaska wikcemna iyecetu, hena owasin wikośka nape kin en ye kiya;

$23 \mathrm{Qa}$, Tuwe hunkake yaya he? Omakiyaka wo, niyate ti kin ohna unkiwankapi kta okan he? eya.

24 Unkan, Milka cinhintku Betuel Nalior kiciton kin he cunwintku kin miye ce, eya.

$25 \mathrm{Qa}$, Peji puze ca taku yutapi ko ota, qa nakun wankapi kta okan ce; eciya.

26 Unkan wicaśta qon pamahdedan yanke ca Jehowa cekiye ca,

27 Jehowa Abereyäm mayuhe cin taku wakan da kin he yawaśtepi nunwe. Towicake towaonśida ko mayuhe cin ektakiya ayuśtan śni, tuka mayuha sunkaku ti kin canku kin ohna Jehowa amau ce, eya.

28 Unkan wikośka kin dus hde ca iye hunku ti kin en hena owasin oyaka.

29 Rebeka timdoku wan yukan, Laban eciyapi, qa Laban iś dus iyaya wicaśta qon ekta minilidoka kin en.

30 Laban oin tanka, qa maza huhu tankśitku nape kin akan wanyake ça Rebeka tankśitku oie ye cin, Wicaśta kin hecen emakiya eye cin he nation qa, hehan wicaśta qon minilidoka kin ekta, camo kin icahda najin kin en hi.

31 Qa heya, Jehowa he niyawaśte kin u wo. Tokeca tankan nayajin he? Miśs tipi kin wanna wiyeya wakarga, camo yukanpi kte cin nakun.

32 Unkan wicaśta qon tipi kin 33 


\section{WICOICAGE.}

ekta hi ; unkan camo kin kiyuśke ca peji puza taku yutapi ko camo kin qu, qa mini on siha hdujaja kta wicaśta om hi kin nakun hdujajapi kta e au.

33 Qa woyute nakun qu. Unkan, Taku on wahi kin owahdake śni kiuhan onśpadan wate kte śni ce, eya. Unkan, Ia wo, eya.

34 Unkan, Abereyam tahokśidan wan he miye :

35 Qa Jehowa he mayuhe cin nina yawaśte, qa tahin wanuyanpi qu, qa pte, qa mazaska, qa mazaskazi, qa wicaśta winyan ko wowindag wicaye cin, qa camo, qa śuktanka śonśonna, hecen wicaśta okinihan.

36 Qa mayuhe cin tawicu kin Sara cinca wan ton, mayuha wanna wicahinca hehan he kiciton, unkan taku yuhe cin owasin kicu.

37 Qa Kanani makoce tawapi kin en manke cin he cunwintkupi kin etanhan micinkśi on winohinca duze kte śni :

38 Tuka hunkake wicawaye cin tipi kin ekta de kte ca, takuwicawaye cin etanhan micinkśi on winohinca wan duze kta ce, may uhe cin eya konza, keye maśi.

39 Unkan, Okini winohinca kin mici u kte śni, mayuhe cin ewakiya.

40 Unkan, Taku wakan wada Jehowa itokam mawani kin, he taohnihde wan nici ye śi kte ca, mitakuye qa ate ti kin etanhan, winohinca wan micinkśi on duze kta e, canku yaye cin tanyan ihuni niciye lita ce.

41 Tuka taku eya konze ciciye cin nihiyahde kte śni, takuwicawaye cin ekta de cinhan, qa nicupi śni kinhan, hehan taku eya konze ciciye cin iyahde niciye kte śni, emakiya.

42 Unkan nakaha minihdoka 34 kin ekta wahi qa hepa, Jehowa Abereyam mayuhe cin he wakan nida, canku ohna wau kin tanyan ihuni mayakiya hecinhan;

43 Iho miś minihdoka kin icahda nawajin qa wikośka wan mini huwe hiyu qa, Wakiśkotpa duhe cin etanhan mini onge yatke makiya ye, ewakiye kta.

44 Unkan, Yatkan ye, qa nakun camo nitawa kin mini wicawakahi kta ce, emakiye cinhan, winohinca wan mayuhe cin cinhintku yuze kta e Jehowa kahinige cin hee kte.

45 Miś cante mahen ia nahahin mdaśtan śni, unkan inyun Rebeka hinanpe ca, minilidoka kin ekta kun i qa mini icu; unkan, Tokin yatke mayakiyen, ewakiya.

46 Unkan tawakiśkotpa kin inaliniyan kun ahi qa, Yatkan ye, qa camo nitawa kin nakun woyatke wicawaqu kta ce, eya; unkan mdatke ca camo kin nakun yatkanpi.

47 Unkan imonge ca, Tuwe cunwintku niyan lie ? cwakiya. Unkan Betuel cunwintku be miye, Nahor cinhintku Milka kiciton kin hee. Hehan oin tanka ite kin akan ewahnaka, maza huhu nakun nape kin akan.

48 Qa pamahdedan manke ca Jehowa cewakiya, Jehowa Abereyam mayuhe cin taku wakan da kin he mayuhe cin cinhintku kin on, mayuhe cin sunkaku cunwintku iwacu kta e canku wicaka ohna amau kin he mdatan.

49 Qa niyepi wanna wowaonśida wowicake ko mayuhe cin ecayeconpi kte cinhan omakiyakapo, qa ecayeconpi kte śni kinhan omakiyakapo: etapa tanhan mduhomni kta qa iś catka tanhan, eya. 


\section{WICOWOYAKE 25.}

50 Unkan Laban Betuel ko ayuptapi qa Taku kin de Jehowa etanhan u qa taku śica qa iśs taku waśte unkeniciyapi kta unkokitpanipi ce, eyapi.

51 Tho Rebeka nitokam un; icu qa hda wo. He niyuhe cin cinhintku kin yuze kta, Jehowa oie ye cin iyececa.

52 Unkan Abereyam taokiye kin iapi hena nahon qehan, Jehowa ohoda kta e makata patuś iyaya.

53 Hehan ookiye qon mazaska oin qa mazaskazi oin ikikcu, wokoyake nakun, qa hena Rebeka - qu, timdoku hunku ko taku tehika wacaqu.

54 Hehan wotapi qa wayatkanpi, iye wicaśta opapi kin hena koya. Wankapi qa hanhanna kiktapi qa, Mayuhe cin ekta hdicu mayan wo, eya.

55 Unkan timdoku hunku ko, Wikośka unkicipi yanke kte, wikcemna can hehanyan, hehan ye kta ce, eyapi.

56 Unkan, Jehowa iś canku mitawa kin tanyan ihuni mayan heon mayutehanpi śni po. Mayuhe cin elita kihde mayanpo, ewicakiya.

57 Unkan, Wikośka kin unkicopi qa, iye i kin etanhan unkiwangapi kta ce, eyapi.

58 Qa Rebeka kipanpi, qa, Wicaśta kin de kici de kta he? eciyapi. Unkan, Mde kta ce, eya.

59 Unkan Rebeka tawinohtinpi kin ye yapi, qa azinkiye ciqon, qa Abereyam taokiye, qa wicaśta tawa nakun.

60 Qa Rebeka yawaśtepi qa, Unkitawinohtinpi kin he niye, kektopawinge oyawa tanka icaga wo, qa cinca wicayaye kte cin toka wicayapi otonwe kin yuhapi kte, eciyapi.

61 Hehan Rebeka najin, wi- kośka yuhe cin nakun, qa camo akan iyotankapi, qa wicaśta qon kici iyayapi. Hecen ookiye qon Rebeka ica qa kihda.

62 Wanna Izak minihdoka, Tuwe ni un kin wanmayaka tawa kin hetanhan $u$, qa makoce puze cin en iyotanka.

63 Qa hitayetu ca Izak cekiye kta e manin ya, hehan wankan etonwe ca camo upi kin wanwicayaka.

64 Rebeka nakun wankan etonwe ca Izak wanyake ca camo kin etanhan ipsica.

65 Qa ookiye kin heciya, Wicaśta wan itkounkipapi hewoskan omani kin he tuwe? Unkan, Mayuhe cin hee, ookiye kin eya. Unkan ite akahpe wan icu qa aihdahpa.

66 Unkan ookiye kin, taku token econ kin owasin Izak okiyaka.

67 Hehan Izak iye hunku Sara tawakeya kin mahen Rebeka tin ye śi, qa yuze ça tawicu kin waśte kidaka. Hecen lzak iye hunku Sara te cin iyohakam iwacintonhnag yapi.

\section{WICOWOYAKE 25.}

1 Hehan ake Abereyam winohinca wan yuza, Qetura eciyapi. 2 Unkan cinca kiciton kin dena eepi, Zimran, qa Jokśan, qa Medan, qa Midian, qa Iśbak, qa Śuha.

3 Unkan Jokśan iś Śba qa Dedan kicitonpi. Unkan Dedan iś Aśurim, qa Letuśim, qa Lumim cinkśi wicaya.

4 Unkan Midian cinca wicaye cin, Epa, qa Heper, qa Hanok, qa Abida, qa Eldaha. Hena owasin Qetura cinkśi wicaya.

5 Unkan Abereyam taku yuhe cin iyuhpa Izak qu. 
6 Tuka winolinca uman cinca Abereyam kieitonpi kin hena Abereyam taku wicaqu, qa nahahin iye ni un kiı wiyohiyanpata makoce kin ekta ye wicaśi, cinhintku Irak etanhan.

7 Abereyam anpeta tawa kin waniyetu ni un kin dena ee, waniyetu opawinge sanpa wikcemna śakowin sanpa zaptan, hehanyan ni.

8 Hehan Abereyam niya śni ta iyaya, pa ska waśte, qa wicahinca imna; hehan taoyate kin ekta okipa yapi.

9 Qa cinca wicaye cin Tzak Iśmayel kici kihnakapi; Makpela imnija ohdoka kin en Mamore itokam, Zohar Heti he cinhintku Epron tamaga qon en.

10 Maga wan Abereyam Het cinca wicaye cin opewicakicaton, he en Abereyam hnakapi, tawicu Sara kici.

11. Unkan Wakantanka, Abereyam te cin iyohakam, cinhintku kin Izak yawaśte, qa Izak 'Iuwe ni un qa wanmayake cin minihdoka tawa kin ekta oun$y$ an.

12 Hagar Egupton kin Sara tawikośka kin he Abereyam cinca wan kiciton, Abereyam cinhintku Iśmayel wicoicage tawa kin dee.

13 Iśmayel cinca wicaye cin cajepi kin deepi, wicowazipi qa cajepi kin iyececa. Iśmayel cinca tokapa kin he Nebayot, hehan Qedar, qa Adbel, qa Mibśam;

14 Qa Miśma, qa Duma, qa Hasa:

15 Hada, qa Teman, Jetur, Napiś qa Qedma.

16 Hena Iśmayel cinkśi wicaya; toki tipi qa tonwanyanpi kin ecen eciyapi, wicaśtayatapi indawapi ake nom, taoyatepi kin om.
17 Iśmayel ni un kin waniyetu kin dena ee, waniyetu opawinge sanpa wikcemna yamni sanpa śakowin, henakeca; hehan niya śni ta iyaye ca taoyate kin ekta okipa yapi.

18 Hunka wanjin wicave cin owasin wicehna ta. Hawila etanhan Śur itokam un kin ektakiya de cin qa hetanhan Aśur etu makoce kin he en ounyanpi.

19 Unkan Izak Abereyam cinhintku wicoicage tawa kin dee, Abereyam he Izak kicitonpi.

20 Unkan Izak wanna waniyetu wilcemma topa, hehan Rebeka yuza, he Betuel Arami kin he cunwintku, Aram Mdaye kin etanhan, Laban Arami kin tawinohtin kin hee tawicu kta e yuza.

21 Qa Izak tawicu kin cinca ton śni kin heon, Jehowa icekiya. Unkan Jehowa anakicigoptan, hecen Rebeka Izak tawicu kin ihduśaka.

22 Unkan hokśiyoqopa Rebeka malen akinicapi; unkan, De taku hwo hecetu kta hecinhan token on hemaca he? eye ca Jehowa iwange kta e ekta i.

23 Unkan, Nitamni kin mahen oyate nonpa, qa nitezi kir etanhan oyate nonpa inanpapi kta, qa oyate wan waśaka keś oyate uman sanpa waśake kta, qa hakakta kin he tokapa kin wowidake yuhe kta ce, Jehowa eciya. 24 Unkan anpetu cinca ton kte cin wanna henakeca, qa inyun tamni kin mahen cekpa yukan. 25 Unkan tokapa kin hiyu owancaya gi, pte ha śina iyecen; unkan Esa eya caśtonpi.

26 Hehan sunkaku kin hiyu, qa nape kin Esa siyete kin yus un; heon Jakob eya caś kiton. Hena tonpi kin ehan, Izak waniyetu wikcemna śakpe. 


\section{WICOWOYAKE 26.}

27 Unkan hokśipidan tanka icagapi, qa Esa wotihni wayupika, wicaśta hewoskan un kin heca, tuka Jakob wicaśta wahbadan wakeya oti.

28 Unkan Izak Esa waśte kidaka, taku o kin yuta heon. Tuka Rebeka Jakob waśte kidaka.

29 Unkan Jakob wahanpi ohe cin icunhan Esa tinta kin etanhan u qa watuka un ;

30 Qa, Akihan mate kta heon wahanpi śa kin de onge maqu wo, mdatke kta ce, Esa Jakob eciya. Heon Edom eya caje yatapi.

31 Unkan, Watonikapa kin he nakaha maqu wo, Jakob eya.

32 Unkan Esa, Wanna mate kta qa watomakapa kin de taku okihi kta he? eya.

33 Unkan Jakob, Nakaha Wakantanka icajeyan emakiya wo, eya. Untran Wakantanka icajeyan eciya. Hecen Esa watokapa tawa kin he Jakob qu.

34 Unkan Jakob omnica hanpi aguyapi kici Esa qu, unkan yute ca yatkan qa najin, qa toki iyaya. Hecen Esa. watokapa kin ihakta śni.

\section{WICOWOYAKE 26.}

1 Unkan makoce kin he en wicaakihan tokeca wan, Abereyam anpetu tawa icunhan wicaakihian tokaheya qon he iyohakam. Unkan Izak Piliśtim tawicaśta yatapi Abimelek eciyapi ekta i, Gerar etu.

2 Unkan Jehowa iye en tanin içiye ca heciya, Egupta ekta de kte śni ce, makoce wan ociciyake kte cin hen yanka wo.

3 Makoce kin de en ounyan wo, kinhan nici waun qa ciyawaśte, qa makoce kin dena owasin ciçu kta, nicinca ko wokonze Abere- yam niyate konza ewakiye cin he wahdusuta;

4. Qa nicinca yuota wakiye kta mahpiya wicanhipi sukaza iyenaka, qa makoce kin dena owasin nicinca wicawaqu kta, qa oyate maka kin ohnaka owasin nicinca kin on yawaśtepi kta.

5 Nakaeś Abereyam miho kin anamagoptan qa mitawoahope, mitawokonze, mioie, qa mitoope kin hena yuhe cin heon etanhan. 6 Unkan Izak Gerar en ounyan.

7 Unkan wicaśta heciya tipi kin tawicu kin on iwangapi ; unkan, Mitankśi, kin dee ce, eya. Rebeka owanyag waśte, qa iye on okini wicaśta deciya yakonpi kin maktepi kta ecin heon, Mitawin dee eye kte cin itonpa.

8 Unkan wanna anpetu ota hen yanke cehan, Piliśtim wicaśta yatapi Abimelek ojanjan hdepi wan etanhan eyokasin qa Izak tawicu Rebeka kici śkata e wanwicayaka.

9 Hehan Abimelek Izak kico qa, Awicakehan nitawin hee. Tokeca, Mitawinohtin dee ehe ciqon he? eya. Unkan okini iye on mațe kta epce cin heon hepa ce, Izak eya.

10 Unkan, De taku ecaunyeconpi he? Wanna ecadan oyate etannan wicaśta wan nitawin kici iwanke kta unkanś, taku śica iyahde unyayapi kta tuka ce, Abimelek eya.

$11 \mathrm{Qa}$ Abimelek oyate tawa owasin iwakta wicaya, Tuwe wicaśta kin de yutan, qa iś tawicu yutan kinhan te kta ce, eya.

12 Unkan, Izak makoce kin he en woju, qa omaka kin he en opawinge akihde kamna. $\mathrm{He}$ cen Jehowa Izak yawaśte.

13 Unkan wicaśta kin tanka 37 


\section{WTCOICAGE.}

qa ohinniyan tanka aya, wijica hinca.

14. Qa oicihde, tahinca, qa pte wanuyanpi, qa ookiye ota yuha; heon Piliśtim icekinpi.

15 Unkan minihdoka, tona athuku taokiye qapi qon atkuku Abereyam ni qelran, hena owasin Piliśtim aohduteyapi, maka okatapi.

16 Unkan Abimelek Izak heciya, Unkiyepi etanhan toki iyaya wo, waunśakapi keś niś sam iyeya waniśaka ce.

17 Unkan Izak hetanhan iyaye ca Gerar kaksiza kin he en eti, qa hen iyotanka.

18 Qa minihidoka atkuku Abereyam ni kin icunhan qapi qon, qa Abereyam te cin iyohakam Piliśtim lkin hena aohduteyapi kin, hena ake Izak qe ça Abereyam caś ton qon hena iyecen Izak caje yata.

$19 \mathrm{Qa}$ Izak holsśidan wicayuhe cin kaksiza ohna qapi qa, en minicaduza minihdoka wan iyeyapi.

20 Hehan Gerar wanuyanpi wilian wicakiyapi tawa kin, Izak wanuyanpi wihan wicakiyapi tawa kin om akinicapi qa, Mini kin he unkitawapi ce, eyapi; heon minilidolka kin he Esek Izak caś ton, heciya kiyuśepi kin heon.

21 Unkan ake minilidoka tokeca wan qapi; unkan he on nakun akinicapi, heon he Sitna caśton.

$22 \mathrm{Qa}$ hetanhan ihdaka ye ça minilidoka tokeca wan qapi, qa he on alkinicapi śni. Unkan he Rehobot eya caś kiton; Wanna Jehowa tankaya unkiyukanpi kaga makoce kin en, heon unkicagapi kta cc, eya.

23 Unkan hetanhan itawankanhde ye ca Berśeba ekta i.
24 Unkan hanyetu kin he en Jehowa tanin iciye ca heciya, Niyate Abereyam taku walkanda kin he miye ce. Wikopeśni un wo; niyate Abereyam mitaokiye kin heon nici waun, qa ciyawa'śte, qa nicinca yuota wakiye kta ce.

25 Unkan kakiya wahna wośnapi wan kage ca Jehowa caje kin hoye kiye ca, kakiya wakeya oti, qa kan Izak taokiye kin minilidoka wan qapi.

26 Hehan Abimelek Gerar etanhan Izak en hi ; iye takodaku Ahuzat, Pikol taakicita tancan koya.

27 Unkan, Śice mayadakapi qa niyepi kin etanhan toki iyaye mayaśipi, qa tokcea en mayahipi he? Izak ewicakiya.

28 Unkan, Unkanitonwanpi qa Jehowa nici un kin he wanunyakapi qa, tho wokonze yuke kte, unkiyotahedan qa odakonkiciyapi unnicagapi kta ce, unkeyapi.

29 Hecen unkiś unniyutanpi śni, qa taku waśte eccdan ecaunniconpi, qa wounkiyapi iyaye unniśipi kin he iyecen, niś dehan Jehowa niyawaśte, taku śica ecaunyeconpi kte śni, eyapi.

30 Unkan wowicakihe ça wotapi qa wayatkanpi.

31 Qa ihanhanna kiktapi qa konza ekiciyapi, napin hunka wanjitku kici heconpi qa, Izak kihde wicaśi. Unkan wookiyc iyayapi.

32 Anpetu kin he en Izak taokiye kin en hipi, qa minilidoka qapi kin he okiyakapi qa, Mini iyohi unyanpi ce, eyapi.

33 Unkan, Konzapi eya caś kiton. Heon anpetu lkin de hehanyan otonwe kin heciya un kin Berśeba eciyapi.

34 Unkan Esa wanna waniye- 


\section{WICOWOYAKE 27.}

tu wikcemna topa, hehan Beri Ḧeti kin he cunwintku Judit, qa Elon Heti he cunwintku Basmat, henios wicayuza. Hena napin on, Izak Rebeka ko cante śicapi.

\section{WICOWOYAKE 27.}

1 Unkan Izak wanna wicahinca iśta kin mdeze śni, hecen wawanyake śni ; hehan Izak cinhintku tokapa kin Esa kipan qa, Micinkśi, eya. Unkan, Iho miye ce, eya.

2 Unkan, Wanna wicamaliinca, qa tohan mațe kte cin sdonwakiye śni.

3 Ceciya ce, nakaha wipe ikikcu wo, wanju itazipa ko ikikeu, qa hewoskan ye ça tado imakihni wo.

4. Qa mate kte cin itokam, minagi kin niyawaśte kta e, tado skumna taku waśte wadaka heca micage, ça makau wo. Wate kta ce, eya.

5 Unkan Rebeka Izak cinhinthu Esa okiye cin he nation. Unkan Esa atkuku kin on wotihni kta e hewoskan iyaya.

6 Hehan Rebeka cinhintku Jakob heciya, Tho niyate Esa nicinye okiya nawahon;

$7 \mathrm{Qa}$, Tado makau qa taku skumna waśte micaga wo; wate kta qa mațe śni kin itokam Jehowa itokam ciyawaśte kta ce, eciya.

8 Heon micinkśi token econ ciśi miho kin anamakigoptan ye.

9 Ecadan wanuyanpi ekta ye ca tatokadan cinca nom waśte imakicu ye, kinhan tado skumna wecage kta, niyate waśte daka iyececa.

10 Unkan niyate te cin itokam niyawaśte kta e en ayai kta; unkan yute kta ce.

11 Unkan, Jakob hunku kin
Rebeka heciya; Ecali micinye Esa wicaśta hin śma heca, qa nuiś wicaśta śduśduta hemaca.

12 Okini ate mayutan kte cinhan wicahnayesa wan iyececa iyemaye kta, hecinhan mayaśicapi kin he iyahde miciye kta, mayawaśtepi kin hee kte śni ce.

13 Unkan, Micinkśi niyaśicapi kin he miye en amaupi nunwe. Tokeśta miho kin anamakigoptan, qa imakicu ye, hunku kin eciya.

14. Unkan ekta ye ca ikikcu qa hunku kin en au; unkan hunku kin tado skumna atkuku waśte dake cin iyecen kaga.

15 Hehan Rebeka śina waśte wan, cinhintku tokapa Esa he tawa ti kin ohna yuhe cin he icu, qa cinhintku hakakta Jakob in kiya.

16 Qa tatokadan cincadan ha kin he Jakob nape kin, qa tahu śduśduta kin akan ehnaka.

17 Qa tado skumna kage ciqon aguyapi koya cinhintku Jakob nape kin en qu.

18 Unkan atkuku kin en au qa, Ate, eya. Unkan, Tho micinkśi ni tuwe he? eya.

19 Unkan Jakob atkuku kin heciya, Esa nicinkśi tokapa kin he miye ce. Token emayakiye cin ecen ecamon ce. Ceciciya najin qa iyotanka wo; qa ninagi kin mayawaśte kta e tado awahi kin yuta wo, eya.

20 Unkan, Micinkśi token inahniyan iyeyaya he? Izak cinhintku kin eciya. Unkan Jehowa wakanyada kin he itkomakim u kiya heon etanhan, eya.

21 Unkan, Micinkśi mikiyedan u wo, ciyutan kta ce, Esa micinkśi kin de niye qa iś dee śni uman tukte sdonwaye kta, Izak Jakob eciya.

22 Hehain Jakob atkuku Izak 
ikiyedan hinajin, unkan yutan qelian, Ho kin Jakob ho kin hee, tuka nape kin Esa nape kin eepi, eya.

23 Nape kin hin śma cincu Esa nape kin iyececapi; heon etanhan iyekiye śni, qa Jakob yawaśte.

24 Esa micinkśi kin de niye he ? eya iwanga. Unkan, Han he miye ce, eya.

25 Unkan, Mikiyedan u wo. Minagi kin niyawaśte kta e, taku micinkśi o kin wate kta ce, eya. Unkan ikiyedan hinajin, unkan wota, miniśa nakun en au; unkan yatkan.

26 Hehan atkuku Tzak, Tho micinkśi mikiyedan u qa i imaputaka wo, eciya.

27 Unkan en ye ça i iputaka, unkan wokoyake onna kin he omna, qa yawaśte qa, Micinkśi omna kin he tinta wan Jehowa yawaśte omna kin he iyececa.

$28 \mathrm{Qa}$ Wakantanka nıahpiya cu kin nic̣u kte ca nakun maka kin wasna qa aguyapiqa miniśa ko ota.

29 Oyate wowindag wicayaye kta nitokam wicaśta yatapi makata ehpeiciyapi kta. Nihunka wanji yuha un wo, qa nihun cinca wicaye cin nitokam patujapi kta, tuwe niyaśice cin yaśicapi kte, qa tuwe niyawaśte kin yawaśtepi kta ce, eya.

30 Wanna Izak Jakob yawaśte hdaśtan, unkan Jakob atkuku Izak itoye kin etanhan kitana aisinyan iyaya, hehan cincu Esa wotihni kin etanhan hdi.

$31 \mathrm{Qa}$ iśnakun tado skumna kage ça atkuku kin en ahi, qa atkuku kin heciya, Ate najin qa nicinkśi taku o kin etanhan yuta wo ninagi kin mayawaśte kte cin heon etanhan.

32 Unkan atkuku Izak, Ni tu- we he? eciya. Unkan, Nicinkśi tokapa Esa he miye ce, eya.

33 Unkan Izak woyuśninyaye tanka hinca on nina yuśinyaya ; Token tuwe tado ihni qa makahi, unkan owasin etanhan wata, yahi śni ehan, qa mdawaśte qa nakun iye yawaśtepi kta ce, eya.

34 Esa atkuku oie kin nahon hehan nina ceye ça śicahowaye ca, Ate miś nakun mayawaśte ye, atkuku kin eciya.

35 Unkan, Nisunka hi qa wohnaye kin eciya tanhan niyawaśtepi kin icu, eya.

36 Unkan, Jakob eciyapi caje kin iyecetu śni lie? Wanna nonpa yuśna mayan; watomaliapa kin he tokaheya ki qa nakaha mayawaśtepi kin icu; eye ca, Woyawaśte wạ miyecipatan śni he, eya.

37 Unkan Izak ayupte ca, Ecah ohiniciye kte he wakage, ca hunka wanjin wicaye cin owasin wicayuhe kta waqu, aguyapi miniśa ko yuhe kta waqu, qa dehan micinkśi taku ecacicon lita he? Esa eciya.

38 Unkan, Ate woyawaśte kin wanjidan duha he? Miś nakun mayawaśte wo, ate, Esa atkuku kin eciye ca, Esa panyanhan ceya.

39 Unkan atkuku Izak ayupte ca, Maka wasna kin ohna ounyaye kte ca mahpiya cu kin aniu kte; 40 Qa nitacanhipi kin on yani lite ça nisunka wowidag niye kta ; tuka tohan waśaka inicage cinhan iye tawanapin nitahu kin etanhan dukse kta ce, eciya.

41 Unkan Esa Jakob śice kidaka, woyawaśte kin on atkuku kin yawaśte kin heon etanhan, qa Esa cante kin mahen heya; Ate aceyapi kte cin anpetu kin hena wanna ikiyedan qa, hehan misunka Jakob wakte kta ce. 
42 Unkan Rebeka he cinhintku tokapa token ie cin he okiyakapi, unkan ek ta ye śi qa, cinhintku hakakta Jakob kico qa, Iho nicinye Esa niye tokiçon qa nikte kta e oiçiya ce.

43 Iho micinkśi miho kin anamakigoptan ye. Najin qa timdo Laban en Haran ektakiya najica ya ye.

$44 \mathrm{Qa}$ timdo kici nanke kta anpetu tonana; nicinye canniye niye cin ayuśtan kin hehanyan.

45 Nicinye wocanniye aniyuśtan qa, taku ecayecon akiktonje cin hehantu, kinhan hetanhan icicu kta e wahociye kta ce. Ehaeś token on etanhan anpetu wanjidan en niye napin makitaninpi kte śni he? eciya.

46 Unkan Rebeka Izak heciya, Wani kin he wanna owihanketa, Het cunwintku wicaye cin on etanhan, Jakob winolinca wan dena iyececa, Het cunwintku kin etanhan, yuze cinhan token on wani kta he, eya.

\section{WICOWOYAKE 28.}

1 Hehan Izak Jákob kipan, qa yawaśte qa wahokonkiye ca, Kanan cunwinkupi kin etanhan winohinca duze kte śni.

2 Najin Arạm Mdaye kin ekta ya wo, nihun atkuku Betuel ti kin ekta, qa hetanhan timdoku Laban cunwintku kin etanhan winohinca wan yuza wo.

$3 \mathrm{Qa}$ Taku wakan iyotan waśake cin niyawaśte, qa iyakiçuya yuota niciye $k$ ta, qa oyate ota yaun kta.

4 Qa Abereyam yawaśtepi kin niçu, makoce wan en ounyaye cin Wakantanka Abereyam qu qon he nicinca om yahduhe kta, eciya.

5 Hecen Izak Jakob ye śi; unkan iyaya Aram Mdaye kin ekta, Betuel cinhintku Laban Arami kin en, Jakob Esa kici hunkupi kin Rebeka timdoku kin hee.

6 Unkan Izak Jakob yawaśte, qa Aram Midaye kin ekta ye śi kin, heciya tanhan winolinca wan yuze kta, qa yawaśte qehan, Kanan cunwintkupi kin etanhan winolinca duze kte śni ce, eciye cin he Esa wanyaka.

7 Unkan Jakob atkuku hunku kici anawicagoptan, qa Aram Mdaye kin ektakiya ya.

8 Unkan Kanan cunwintkupi kin iye atkuku Izak iśta kin en śicapi e Esa wanyake çehan;

9 Esa Iśmayel ekta i, qa Abereyam cinbintku Iśmayel cunwintku Mahalat, Nebayot tawinohitin kin he yuza, tawicu nonpa tuka he nakun yuza.

10 Unkan Jakob Berśeba etanhan iyaya Haran ektakiya.

11 Qa toki i kin hen iwanka, wi wanna iyaya nakaeś, qa inyan hen yanke cin etanhan icu, qa ipahin ekihnake ça heciya iśtima.

12 Qa wihanmde ca inyun taku iyadipi wan makata han, qa-pa kin mahpiya kin ekta iyohiya; unkan Wakantanka ohnihde wicaye cin adipi qa kun hiyupi.

13 Qa inyun Jehowa iwankam najin qa, Niyate Abereyam taku wakan da qa Izak taku wakan da kin Jehowa he miye ce. Makoce akan nunke cin he ciçu kta nicinca koya.

14 Qa nicinca maka watuśelkśeca sukaza kin iyenakapi kta, eniciciya idade kta wiyohpeyata, qa wiyohiyanpata, qa waziyata, qa itokaga; qa niye nicinca koya en wicowazi tona maka akan unpi kin owasin yawaśtepi kta.

15 Tho miś nici waun, qa toki 
da eśta awanciyake kta, qa makoce kin de ekta acihdi kta, ehpeciye kte śni, tohan taku ociciyaka wahduśtan śni kin hehanyan, eya.

16 Unkan Jakob wihanmde cin etanhan lkikta qa, Awicakehan Jehowa den yanka, tuka miś sdonwaye sni, eya.

17 Hecen kokipe ça, Oyanke kin de itonpapi lita tuka ce, Wakantanka ti kin qa mahipiya tiyopa kin dee śni he? eya.

$18 \mathrm{Qa}$ hanhanna hehan Jakob najin qa inyan wan ipahin ehnake ciqon he icu, qa wokiksuye kte cin ehde, qa he pa kin akan wihdi akaśtan.

19 Qa oyanke kin he Betel, (Taku Wakan ti kin) eya caśton, tuka tokag chan otonwe kin he Luz eciyapi.

20 Unkan Jakob wokonze wan iciconze ca heya, Wakantanka mici un kinhan, qa canku kin de ohna mde cin he ohna awanmayake cinhan, qa aguyapi wate kta maqu qa wokoyake komayake kta nakun;

21 Qa ate ti kin ekta tanyan wahdi hecinhan, Jehowa eceedan wakanwada kta ce.

2.2 Qa inyan wokiksuye ewehde kin de Wakantanka tipi tawa kta ce, qa taku mayaqu kte cin owasin etanhan, iwikeemna kin ciẹu kta ce.

\section{WICOWOYAKE 29.}

1 Hehan Jakob Wiyohiyanpata cinca tamakōepi kin ektakiya siha on mani iyaya.

$2 \mathrm{Qa}$ etonwe ça inyun tinta ohna minilidoka wan, qa tahin wanuyanpi optaye yamni heciya minilidoka kin icahda wankapi, minihdoka kin he etanhan optaye kin mini wicaqupi ece. Un- kan minilidoka i kin akan inyan tanka wan yanka.

3 Unkan hen optaye kin owasin mniciyapi eca, minihdoka i kin akan tanhan inyan kin yuptanyanpi qa, tahinca kin mini wicaqupi ca inyan kin hen yanke kte cin minilidoka i kin akan ehnakapi.

1 Unkan, Hunka wanjin ciyapi tokiya tanhan yaunpi he? Jakob ewicakiya. Unkan, Haran unketanhanpi ce, eyapi.

5 Unkan, Laban Nahor cinhintku sdonyayapi he? ewicakiya. Unkan, Han he sdonunyanpi ce, eyapi.

6 Unkan, Zaniyan un he? ewicakiya. Unkan, Zaniyan un, nakun wanyaka wo; cunwintku Raliel tahin wanuyanpi kin om hiyu ce, eyapi.

7 Unkan, Ampetu nahahin tanka ihan. Wanuyanpi mniciyapi kte cin nahahin iyehantu śni. Tahinca mini wicaqupi qa wilian wicakiyapo, eya.

8 Unkan, Unkokitpanipi ce ; optaye kin owasin mniciyapi kin, hehan inyan kin minihdoka i kin etanhan yuptanyanpi kinhan, hehan tahinca kin mini wicunqupi kta ce, eyapi.

$9 \mathrm{Om}$ wohdaka icunhan, Rahel atkuku talinea tawa om hi ; wihan wicakiya nakaeś.

10 Hecetu unkan Jakob, iye hunku kin he timdoku Laban cunwintku Raliel he wanyake ça, hunku timdoku kin Laban tahinca tawa nakun, qa Jakob ikiyedan i qa, inyan qon mini lidoka i kin akan tanhan yuptanye ca, hunku kin he timdoku Laban tahinca tawa mini wicaqu.

11 Qa Jakob Rahel i iputake ca ho taninyan ceya.

12 Qa Jakob Rahiel heciya, 


\section{WICOWOYAKE 29.}

Niyate sunkaku lin hemaca, Rebeka cinhintku kin he miye ce. Unkan inyang hde ca atkuku okiyaka.

13 Unkan Laban he tarvinohtin cinhintku kin Jakob taku ohdake cin nalion qehan, itkokim inyang ye ca, poskin yuze ca, $i$ iputake ca ti kin timahen u śi. Unkan Jakob Laban hena taku kin owasin okiyaka.

14. Unkan, Mihu, qa micehpi kin he niye ce, Laban Jakob eciya. Unkan anpetu tona wi wanjidan en un kin kici yanka.

15 Unkan Laban Jakob heciya, Hunka wanjin ciye cin on ituya wowidag ciye kta he? Taku iyuwin yacin kin he omakiyaka wo, eya.

16 Wanna Laban cunwintku nonpa, tokapa kin Leha eciyapi, qa hakakta kin Raliel eciyapi.

17 Qa Leha iśta kin wankadan; tuka Rahel okah waśte owanyag waśte hinca.

18 Unkan Jakob Rahel waśte dake ca, Rahel nicunkśi hakakta kin he on waniyetu śakowin wowidag mayaye kta, eya.

19 Unkan he cicu kinhan waśte kta ; wicaśta tokeca waqu kinhan waśte lite śni. Mici ti wo, Laban eya.

20 Unkan Jakob Rahel on waniyetu śakowin okiya; tuka he waśte dake cin on iśta kin en anpetu tonana yawa.

21 Hehan, Mitawin maqu wo, kici imunke kta, anpetu kin wanna henakeca ce, Jakob Laban eciya.

22 Unkan Laban otonwe kin wicaśta owasin mnawicaye ça wowicakihan.

23 Qa otpaza hehan cunwintku Leha he icu, qa Jakob en au. Unkan kici wanka.

24 Unkan Laban wikoślka wan yuhe cin Zilpa, he icu qa cunwintku Leha wikośka yuhe kta e qu.

25 Thanlianna inyun Leha hee, qa Jakob Laban heciya, Rahel on ociciye śni he? Tokeca mayahnayan he?

26 Unkan, Hakakta kin tokaheya qupi, qa hehan tokapa kin deciya hecen econpi śni ;

27 Anpetu śakowin kin de ihuniyan wo; kinhan he nakun cicu kta, tokata waniyetu śakowin tokeca omayakiye kte cin heon etanhan, Laban eya.

28 Unkan Jakob ecen econ, qa śakowin kin he ihuniyan. Unkan Laban cunwintku Rahel he tawicu kta e qu.

29 Qa wikośka wan yuhe cin Bilha, he Laban cunwirtku Rahel wikośka yuhe kta e qu.

30 Unkan Jakob Rahiel kici wanke ca, nakun Rahel waśte dake ca, ake waniyetu śakowin uman Laban cajeyate ciqon. he yuśtan.

31 Unkan Leha śice dakapi kin he Jehowa wanyakc ca, Leha tamni kin yuhdoka, tuka Rahel cinca ton śni.

32 Unkan Leha ihduśake ca, cinca ton, qa Ruben eya caś kiton; Makiyuśepi kin Jehowa wanyaka, unkan dehan mihihna waśte madake kta ce, eya.

$33 \mathrm{Qa}$ ake ihduśake ca cinca ton, qa, Śicc madapike cin he Jehowa nahon, qa cinca kin de nakun maqu, eye ça, Śimeyon eya caś kiton.

34 Qa ake ihduśake ca cinca ton qa, Wanna detanhan mihihna tinsa mahduze kta: Wanna cinca yamni weciton, eye, ca Lewi eya caś kiton.

35 Unkan ake ihduśake ca cinca ton qa, Dehan Jehowa mdatan kta ce, eye ça, Juda eya caś 


\section{WICOICAGE.}

kiton. Hehan cinca ton kin ayuśtan.

\section{WICOWOYAKE 30.}

1 Unkan Rahel Jakob cinca kiciton śni e wanhdake ca, $\mathrm{Ra}$ hel he cunku inakiwizi qa Jakob heciya, Cinca maqu ye, hecanon śni kinhan mațe kta ce.

2 Unkan Jakob canniye ye ca, Rahel heciya, Miye Wakanmatanka kecanni he? Iye taku tamni ton kin onanitaka ce.

3 Unkan, Iloo wikośka mduhe cin Bilha he ekta ya ye ; unkan miśiyoto kin akan cinca ton kinhan, jye etanhan cinca waton kta ce ;

4 Eye ca wikośka yuhe cin Bilha he tawicu kta e qu. Unkan Jakob he en i.

5 Unkan Bilha ihduśake ca Jakob cinca kiciton.

6 Unkan Wakantanka mayaco qa, nakun houwaye cin namalion, qa cinca wan maqu, Rahel eye ca, Dan eya caś ton.

7 Ủnkan ake Rahel tawikośka Bilha ihduśake ca cinca icinonpa Jakob kiciton.

8 Unkan Wakantanka omakiye ca micun kici htawani, qa ohiwaya ce, Rahel eye ca, Neptali eya caś ton.

9 Unkan Leha cinca sanpa ton śni e wanhdake cehan wikośka yuhe cin Zilpa icu, qa Jakob tawicu kta e qu.

10 Unkan Leha wikośka yuhe cin Zilpa, he Jak ob cinca kiciton.

11 Unkan, Wicota kta, Leha eye ca, Gad eya caś tọn.

12 Unkan Leha wikośka yuhe cin Zilpa Jakob cinca icinonpa kiciton.

13 Unkan, Wopida mayanpi, cunwintku unpi kin mayawaśtepi kta ce, Leha eye ca, Aśer eya caś ton.
14. Unkan aguyapi baksapi kin icunhan Ruben magata iyaye ca, dudaim iyeye ca hena hunku kin ekta kai. Unkan Rahel Leha heciya ; Nicinkśi dudaim au kin etanhan maqu ye.

15 Unkan, Mihihna yaki kin he taku cistina naceca, micinkśi dudaim tawa kin, he nakun iyacu kta he? eciya. Unkan, Nicinkśi dudaim tawa kin he on hanyetu kin de nici iwanke kta ce, cya.

16 Unkan htayetu hehan, Jakob maga tanhan hdi; unkan Leha itkokim inanpe ça, Miyahna yau kta, micinkśi dudain tawa kin hena on opeciton ce, eya. Unkan hanyetu kin he kici wanka.

17 Unkan Wakantanka Leha anagoptan: unkan ihduśake ca cinca izaptan Jakob kiciton.

$18 \mathrm{Qa}$, Wikokśa mduhe cin he mihihna waqu kin heon, Wakantanka iyunwin maqu, Leha eyc ca, Isakar eya caś kiton.

19 Unkan ake Leha ihduśke ca cinca iśakpe Jakob kiciton.

$20 \mathrm{Qa}$, Nakaha Wakantanka taku waśte itumakihan, mihihna cinca śakpe weciton, heon detanhan mici ti kta ce, eye ca, Zebulon eya caś kiton.

$21 \mathrm{Qa}$ he iyohakam cinca winyan ton qa, Dina eya caś kitón. 22 Unkan Wakantanka Rahel kiksuye ca Wakantanka anakicigoptan, qa tamni kin yuhdoka.

23 Unkan ihduśake ca cinca wan ton qa, Taku iyamaonpapi qon Wakantanka wanna emicihdaku ;

24. Qa Wakantanka cinca tokeca wan nakun maqu kta, eye ca, Josep eya caś kiton.

25 Unkan Raliel Josep ton kin hehan, Jakob Laban heciya, 
WICOWOYAKE 30.

Wanna hde mayan wo. Mitamakoce qa toki manke kte cin ekta wahde kta.

26 Mitawin micinca ko maqu wo, wahde kta ce; hena on token htawani, qa wowindag mayaye cin niś sdonyaya ce.

27 Unkan, Tokin niiśta kin en iyocicipin, niye on etanhan Jehowa mayawaśte wahdukcan.

28 Taku on omayakiye kta atayedan omakiyaka wo; hecece cinhan cicu kta ce, Laban eciya. 29 Unkain, Niś token ociciye ca, nitawanuyanpi kin token mici unpi qon sdonyaya.

30 Wahi kin itokam taku cistina duhe ciqon, qa wanna ota aye ça, misiha kin eciya tanhan Jehowa niy awaśte. Unkan wanna tohan wati ohnaka kin on taku miçicage kta he? eya.

31 Unkan, Taku cicu kta he? eya. Unkan Jakob heciya, Takudan mayaqu kte śni, tuka taku kin de ecanon kinhan ake tahinca nitawa kin awanwicamdake kta.

32 Nakaha wanuyanpi owasin ehna mawani kta, qa tona hdehdega qa hdehdeze cin iyog iyewicawaye kta, tahinca wanuyanpi kin tona gigi kin owasin, qa tatokadan tona hdehdege ca lidehdeze cin heniyos akipam ewicawahde kta; qa tokata taliin wanuyanpi ehna tona gigi, qa tatokadan ehna tona hdehdega hdehdeza ko, hena iyunwin mitawa kta.

33 Unkan tokata miohan owotanna mihduotanin-kta; taku mitawa nitokam u kinhan, tatokadan tona hdehdegapi śni, qa hdehdezapi śni, qa tahin wanuyanpi ehna tona gigi śni kin hena owasin mawanon kehe kta.

34 Unkan, Tokin token ehe cin hecetun, Laban eya.
$35 \mathrm{Qa}$ anpetu kin he en tatokadan mdoka tôna hdehdeza hdehdega ko, qa tatokadan wiye tona hdehdege ca hdeśkaśka lin owasin, tona akan hin san kin hena koya owasin, akipam ewicahdaku, tahin wanuyanpi ehna tona gigi kin nakun owasin, qa hena cinkśi wicaye cin nape kin en wicaqu.

$36 \mathrm{Qa}$ anpetu yamni imani, iye Jakob kici ihehanyan kaga. Unkan Laban woteca optaye ihe cin hena Jakob wilian wicakiya.

37 Qa Jakob can sakadan icu, waga teca qa can su, qa uma hena owasin etanhan icu, qa ha kin pago hdeza, ska tanin kta e hecen hdehdeza kaga.

$38 \mathrm{Qa}$ watpadan tukten wanuyanpi mini yatkanpi kin hena ohna, can sakadan pago qon ehde, wanuyanpi itokapa tanhan, unkan wanuyanpi yatke upi ell kiyuliapi ece.

39 Hecen can sakadan qon ekta wanuyanpi kin kiyuhapi, qa wanuyanpi hdehdeza hdehdega hdeślkaśka ko wicatonpi.

40 Unkan Jakob tacincadan yukinukan ewicahnake ca, wanuyanpi itepi kin taku hdeza ektakiya ewicahde, Laban wanuyanpi gigi kin owasin, qa wanuyanpi tawa kiṇ yukinukan ewicahde, Laban tawanuyanpi kin kici ewicahde śni.

41 Qa tohan wanuyanpi waśakapi kiyuhapi eca, Jakob can sakadan qon watpadan ohna wanuyanpi iśta kin wicitokam ehde ; can sakadan lin ekta kiyuliapi kta e hecon.

42 Tuka wanuyanpi wankapidan eca, ehde śni, hecen tona wankapi kin hena Laban tawa, qa tona waśakapi kin hena Jakob tawa. 
43 Hecen wicaśta kin tanka hinca icage ça wanuyanpi ota ton, qa nakun winyan taokiye, qa hokśidan, qa camo, qa śuktanka śonśonna.

\section{WICOWOYAKE 31.}

1 Hehan, Taku ate unyanpi tawa qon he owasin Jakob icu, qa taku ate yuhe ciqon etanhan, woyuha tanka kin de owasin icicaga, Laban cinksśi wicaye cin eyapi kin Jakob nahon.

2 Qa Laban ite kin he nakun Jakob wanyaka, qa iye ektakiya wanna tokeca.

3 Nakun, Niyate makoce tawapi kin ekta, qa takuwicayaye cin ekta hda wo, kinhan nici waun lita ce, Jehowa Jakob eciya.

4 Heon Jakob tuwe ekta ye śi qa, tawicu napin Rahel Leha kici, tinta kin heciya, tawanuyanpi kin ekta wicakico.

5 Qa, Niyatepi kin wanna tolieca amatonwe cin wanmdaka, tuka ate taku wakan da kin mici un.

$6 \mathrm{Qa}$ token owakihi ohinniyan niyatepi kin owakiya sdonyayapi ;

7 Tuka niyatepi kin mahnaye ca, wikcemna akihde taku maqu kte cin yutokeca, tuka kiunni maye kte cin Wakantanka iyowinye śni.

8 Kaken, Hdehdegapi kin hena iy unwin duhe kta ce, eye cehan, waunyanpi kin owasin hdehdegapi kin heca ece wicatonpi ; qa, Idehdezapi kin hena iyunwin nitawa kta eye cehan, hehan wanuyanpi owasin hdehdezapi heca ece wicatonpi ece.

9 Hecen Wakantanka niyatepi tawanuyanpi kin ki ecc, qa he maqu.
10 Wanuyanpi kin kiyuhapi eca wiwahanmde ca, ewatonwe ca, inyun tamdoka hdehdeza hdehdega hdeśkaślka hecapi ko, wanuyanpi akan adipi e iśta on wan mdaka.

11 Nakun wiwahanmde ca, Wakantanka taohnihde ye cin hemakiya ; Jakob. Unkan, Iho miye ce, epa.

12 Unkan, Ista yuwankan ikikcu qa, tamdoka wanuyanpi adipi hin awicatonwan wo; hdehdeza hdehdega hdeśkaśka hecapi; token Laban ecanicon kin he owasin wanmdaka ce.

13 Taku wakan Betel en tanin içiye cin he miye, hen wokiksuye sdayaye ca, hen taku konza emayakiya. Wanna najin, qa makoce kin de etanhan, iyaye ca taku wicayaye cin ekta hda wo, eya.

14 Unlian Rahel Leha ko ayuptapi; A te ti kin ohna taku unhapi kte ça taku unkitawapi kte cin yukan he?

15 Oyate tokeca unhdawapi śni he? Wiyopeunyanpi, qa nakun mazaska unkitawapi kin owasin yusota.

16 Heon woyuha ate unyanpi kin etanhan Wakantanka ki kin he iyuhpa unkiye unkitawapi, qa unkicincapi tawapi ce. Iho wanna token Wakantanka econ niśi kin owasin ecen econ ye, eyapi.

17 Unkan Jakob najin, qa cinca tawicu ko owasin camo akan ewicahnaka,

18 Qa atkuku Izak en Kanan makoce kin ekta kihde kta e tawanuyanpi kin owasin awicaya, woyuha içicage cin, qa taku tonpi kiton kin, taku Aram Mdaye kin en ihdamna kin owasin.

19 Wanna, Laban tahinca kin hduśda iyaye çehan, Rahel he 
atkuku terapim tawa kin manon.

20 Unkan Jakob Laban A rami kin cante kin manon, najica iyaya, okiyake śni nakaeś.

21 Hecen najica, taku tawa kin owasin hduha najin, qa watpa kin iyuwege ca, Gilead he kin ektakiya etonwan.

22 Unkan anpetu iyamni kin hehan, Jakob wanna najica Laban okiyakapi.

23 Unkan hunka wanjin wicaye cin wicakico, hena om okide ye ca, śakowin can icimanipi hehan, Gilead he kin en kihdegápi.

24 Unkan, Wakantanka hanyen Laban Arami kin en hi qa, wihanmde cin en heciya, Ihnuhan taku waśte taku śica uman tukte kaśta eyakiye cin, eya.

25 Unkan Laban Jakob kihdege cehan, Jakob he kin en wakeya oti, qa Laban hunka wanjin wicaye cin om Gilead he kin hiyahanpi.

26 Qa Laban Jakob heciya, Taku tokanon micante kin mayahnaye ça, micunkśi canłipi on wayaka iyecen awicayahda.

$27 \mathrm{He}$ tokeca e hecanon he? Anamayakilibe ca, maniçinon, omayakidake śni. Omayakidaka unkanś wiyuśkinyan iyaye ciye ça, cicidowan, qa cancega candowankiyapi ko ciciyahoton kta tuka hecanon.

28 Micinca wica winyan ko i iwicawaputake kte, iyowin mayakiye śni. Wanna witkoya hecanon.

29 Dehan taku śica ecacicon kta nape wamaśaka, tuka niyate taku wakan da kin hinhan hemakiya; Ihnuhan Jakob taku waśte taku śica uman tukte kaśta eyakiye cin, eya.

30 Wanna niyate ti kin ekta yahde kte hinca, tuka tokeca
Taku wakan mitawa kin maya." non he? eya.

31 Unkan Jakob waayupte ca, Kowakipe cin heon, ga okini nicunkśi kin hena mayaki kta naceca ce epe cin heon hecamon; 32 Taku wakan nitawa kin tuwe kici iyeyalkiye cinhan, he ni kte śni. Hunka wanji unye cin wicitokam, taku nitawa mici un kin, tukte e he, iyukcan qa ikikcu wo. Rakel hena manon tuka Jakob he sdonye śni, qa heciya. 33 Hehan Laban Jakob tawakeya kin en ti mahen i, Leha tawakeya kin nakun, qa wikośka nonpa tawakeyapi kin ; tuka iyekiye śni ; hehan Laban Leha tawakeya kin etanhan hdinanpe ca, Rahel tawakeya kin en i.

34 Tuka Rahel terapim kin icu, qa camo aqinpi kin en ehnake ciqon, qa akan iyotanka. Unkan Laban wakeya kin owancaya yutan qa iyekiye śni.

35 Unkan atkuku kin heciya, Itancan nitokam nawajin owakitpani, hececa śta canniye maye śni ye. Winohinca ayakonpi kin hemaceca ce. Unkan nina akita tuka terapim kin iyekiye śni.

36 Unkan Jakob canniye ça, Laban kige, Taku sica ecamon qa wawalitani kin tukte e he, on hecen mayakuwa?

37 Mitawahipaya kin owasin mayakidutan, wakśica yati kin etanhan wanji iyeyaye cin tukte e. he? takuwicunye cin wicitokam ehnaka wo; hecece cinhan uman tukte unkitawa kta yukcanpi nunwe.

38 Waniyetu wikcemna nonpa kin dena nici waun, tuka tawiyedan nitawa kin kpapi śni, qa, nitawanuyanpi tamdoka kin hena wate śni.

39 Taku yahdecapi kin niye 47 
WICOICAGE.

en cicai śni, he miye piya wakaga ce. Hanyen manonpi kin, qa anposkan manonpi kin he koya, minape kin etanhan mayakida.

40 Kaken waun, anposkan ipuza mața, qa hanyen osni ; woiśtima miiśta lin amayuśtan.

41 Miye waniyetu wikcemna nonpa kin yati kin en wowidag mayaye; waniyetu ake topa nicunkśí nonpa on, qa waniyetu śakpe nitawanuyanpi kin on, ociciye ca, taku mayaqu kte cin wikcemna akihde mayadutokan. 42 Ate taku wakan da kin, Abereyam taku wakan da kin qa Izak Tokokipe kin, mici un kin; hecetu śni unkanś wanna cokadan kihde mayaśi kta tuka ; mawahbadan kin qa minape litanipi kin he Wakantanka wanyake ca, hinhan iyopeniyan ce, Jakob Ĺaban eciya.

43 Unkan Laban waayupte ca, Jakob heciya, Cinca winyan kin dena micunkśi eepi, qa hokśidan kin dena micinca eepi, qa wanuyanpi kin dena wanuyanpi mitawa eepi, qa taku niye wanyahdake cin he iyulipa mitawa ce. Tuka de micunkśi qa iś cinca wicatonpi kin dena, nakaha taku ecawicawecon kta he?

44 Tokin wanna wicoun unkicagen, miye qa niye, hecen waayatanin unkiyotahedan yuke kta.

45 Unkan Jakob inyan wan icu, qa wokiksuye e pasdata:

$46 \mathrm{Qa}$, Inyan icupo, Jakob hunka wanjin wicaye cin ewicakiya. Unkan inyan icupi, qa paha wan kagapi qa, hen paha kin akan wotapi.

47 Unkan paha kin he, Igar sahaduta Laban eya caśton ; tuka Jakob he Galed eya caśton.

48 Unkan Laban heya, Anpe dehan paha kin de unkiyotahedan waayatanin kta; Galed Waayatanin paha kin he ka, paha kin he eciyapi.

49 Qa, Micunkśi wicayakiduse, qa winohinca tokeca duze cinhan, wicaśta tuwe he atonwe kta unkici un śni, unkiyotahedan Wakantanka he waayatanin kta.

50 Tohan kinukankiya unkiyaye cinhan Jehowa unyacopi kta, Laban eye ça, Wayaco caś ton.

51 Qa, Wanyaka wo, paha kin de, wokiksuye unkiyotahedan ewehde kin de ;

52 Paha kin waayatanin, wokiksuye kin de waayatanin, taku śica on paha kin de kapeya imdamde ça niye en cii kte śni, qa niś kapeya yahihdu qa hiyohi mayaye kte śni.

53 Abereyam taku wakan da kin, Nahor taku wakan da kin, hunkake wicayapi taku wakan dapi kin unyacopi kta, Laban Jakob eciya. Unkan Jakob iye atkuku Izak Tokokipe kin cajeyan konza.

54 Hehan Jakob he kin akan wośnapi wan wakiyuśna, qa aguyapi yutapi kta e hunka wanjin wicaye cin wicakico. Unkan aguyapi yutapi qa he kin en wankapi.

55 Unkan ihanhanna Laban kikta qa iye cinca wica winyan ko $\mathrm{i}$ iwicaputake ca, wicayawaśte qa, hehan Laban tohe kin ekta kihda.

\section{WICOWOYAKE 32.}

1 Unkan Jakob tacanku kin ohna iyaye ca, Wakantanka ohnihde wicaye cin iktokim hipi.

2 Unkan Jakob wanwicayake çehan, Wakantanka wicobe ta- 


\section{WICOWOYAKE 32.}

wa kin dee ce, eye ca oyanke kin lıe Mahanaim caś ton.

3 Hehan Jakob Edom wojupi kin Sehir makoce kin etu, cincu Esa ti kin ekta, ohnihde wicaye cin ye wicaśi.

$4 \mathrm{Qa}$ wahokonwicakiye ca, Itancan mitawa Esa hecen eyakiyapi kta ce, eya; Laban kici wati qon dehantu.

5 Qa pte, qa śuktanka śonśonna, qa tahinea, qa hokśidan wikośka ko, ota wicamduha, qa onśimayada kta e itancan ociciyag ye wicawaśi, nitaokiye Jakob eya.

6 Unkan Jakob ohnihde wicaye cin hdipi qa, Nicinye Esa ekta unkipi unkan iś nitkokim u qa, wicaśta opawinge tom opapi ce, eyapi.

7 Hehan Jakob nina kokipe ca, oyate kici un kin nonpakiya ewicakihde, tahinca, pte, camo, ko owasin ośpaye nonpa wicakaga.

8 Qa Esa ośpaye wanji en hi qa hena wicakte kinhan, ośpaye uman okapte cin inaśdoke kta ce, eya.

9 Hehan Jakob heya, Abereyam ate waye wakan nida, qa ate Tzak wakan nida Jehowa niye, Nitamakoce, qa takuwicayaye cin ekta hda wo, kinban taku waśte ecacicon kta ce, emayakiye ciqon ;

10 Miye nitowaonśida qa iewicayake cin owasin nitaokiye ecayecon kin aopten waun; sagye kin heceyedan hduha Jordan watpa kin de imduwege ça, wanna ośpaye nonpa imacaga.

11 Wanna cinye nape kin etanlian emahdaku wo; Esa nape kin etanhan; hi kinhan cinca liunkupi kin kici mikte kta kowakipa ce.

12 Niye, Ohinniyan taku waśte ecacicon kte ca, wiyaka mini wanca kahda sukaza utkana on yawapica śni, iyenaka nicinca wakage kta ehe ciqon, eya.

13 Unkan hanyetu kin he hen wanka, qa taku cincu Esa qu kte cin ikikcu, taku nape en hiyu;

14. Tatoliadan wiye opawinge nonpa, qa mdoka wilkcemna nonpa, tahin wanuyanpi wiye opawinge nom mdoka wikcemna nonpa ahna ;

15 Camo azinkiyapi wikcemna yamni cincapidan om, pte wikcemna tom, qa tatanka wikcemna, śuktanka-Śonśonna wiye wikcemna nonpa cincadan wikcemna ahna.

16 Qa ookiye wicayuhe cin woteca otoiyohi kinnukan yuhe wicaśi, qa, Optaye otoiyohi yukinukan awicahiyayapo, ewicakiya.

17 Qa tokaheya kin wahokonwicakiya, Tohan cinye itkonicipapi kinhan, qa, Tuwe tawa niyan he? qa toki da qa taku nitokam un kin he tuwe tawa he? eya iniwange cinhan;

18 Hehan, Nitaokiye Jakob dena tawa, tuka itancan mitawa Esa qu kta; unkan iś nakun unkihektam u ce, ehapi kta ce, ewicakiya.

19. Unkan inonpa, qa iyamni, qa nakun owasin tona optaye ihakam yapi kin, Esa iyeyayapi kinhan oie kin hena iyecen eyakiyapi kta ce :

20 'Nâkun; Nitaokiye Jakob unkihektam u ce, ehapi kta ce, ewicakiya. Taku waqu kin mitokapa tanhan ye cin, becen ite kin iyokipi waye kta, qa hehau ite kin iyowinmakiye kta ce, eya.

21 Unkan taku qu kin, he tokata iyokipta; tuka iś hanyetu kin he taobe kin om wanka. 
$22 \mathrm{Qa}$ hanyetu kin he en, najin qa tawicu nonpa kin iwicacu qa cinca wicaye cin alke wanji, qa Jabok oiyuwege kin en iyuwega.

$23 \mathrm{Qa}$ iwicakikcu, qa watpa kin iyuweh wicaye ca, taku tawa kin owasin iyuweh wicakiya.

$24 \mathrm{Qa}$ Jakob iśnana hekta yanka, unkan wicasta wan anpao kin hehanyan kici kiihduśpa.

$25 \mathrm{Qa}$ wicaśta kin tokadan ohiye śni e wanhdake cehan, cecunte okihe kin en yuza; unkan kici kiihduśpapi kin icunhan, Jakob cecuntośtan kin papśon iyaya.

26 Unkan, Amayuśtan wo; wanna anpao hinanpa ce, eya. Unkan, Mayadawaśte śni kinhan, aciyuśtan kte śni ce, eya.

27 Qa, Token eniciyapi he? eya. Unkan Jakob emakiyapi ce, eya.

28 Unkan, Nicaje Jakob eniciyapi kte śni. Detanhan Israel eniciyapi kta, Wakantanka wicaśta ko, kici kinihduśpe ca niye ohiyaya ce, eciya.

29 Unkan, Tokin nicaje kin omayakidaken, Jakob eya iwanga. Unkan, Token on etanhan micaje kin inonga he? eye ça hen yawaśte.

30 Unkan, oyanke kin he, Ite wakan, Jakob eya caś ton. Taku Wakan ite icitkokim wanmdaka eśta minagi kin zaniyan un ce, eya.

31 Unkan Ite wakan iyokipte cehan, anpetu wi hinanpa, qa Jakob ceca kin on huśte.

32 Heon etanhan Israel cinca wicaye cin anpetú kin dehan ceca śkotpa kin akan kan kiksuye śni qon he yutapi śni; Jakob ceca skotpa kin akan kan kiksuye śni kin he oyutan kin heon etanhan.

\section{WICOWOYAKE 33.}

1 Unkan Jakob wankan etonwe ca inyun, Esa u, wicaśta opawinge tom kici wanwicayake ca, hokśiyoqopa kin akipam ewicakihde, Leha kici, qa Rahel kici, qa wikośka nonpapi kin om.

2 Qa wikośka kin cinca wicayapi kin om tokaheya ye wicaśi, qa hena ihektapa tanhan Leha cinca wicaye cin om, qa hena ihektapa tanhan Rahel Josep kici.

3 Qa iye owasin wicitokam ye ca, cincu ikiyedan u kin icunhan śakowin akihde patuja.

4. Unkan, Esa itkokim inyanke ça, nape kin yuze ça tahu kin akan ehpeiciye ca i iputaka, qa napin ceyapi.

5 Hehan Esa wankan etonwe ca, tawicu cinca wicaye cin ko wanwicayake ca, Dena token takuyaya he? eya. Unkan, Wakantanka towaonśida kin on nitaokiye cinca qu kin dena eepi ce, eya.

6 Hehan wikośka kin cinca wicayapi kin om ikiyadan hipi, qa patujapi.

7 Hehan Leha cinca wicaye cin om ikiyadan hi qa patujapi ; qa ehake Josep Rahel kici ikiyadan hipi, qa patujapi.

8 Unkan, Optaye kin dena owasin itkowicawakipe cin, token yaka he? eya. Unkan, Ma. yuhe cin iśta kin iyokipi ciye kta e heon.

9 Unkan, Misunka taku ota wahduha ce. Taku nitawa kin hduha un wo, Esa eya.

10 Unkan, Hiya niiśta kin iyokipi ciye cinhan, minape kin etanhan taku waqu kin iyacu kta, nitohnake Wakantanka itohnake iyecen wanciyalse ça iyocicipi kin heon etanhan. 


\section{WICOWOYAKE 34.}

11 Tokin taku ciyawaśte kin en aniupi kin he iyacun. Wakantaka onśimada ece, qa taku owasin mduha, Jakob kitanyan eya; unkan icu.

$12 \mathrm{Qa}$ heya, Ihdaka unye ca miye tokaheya mde kta ce.

13 Unkan, Hokśiyoqopa wankapidan e mayuhe cin sdonye ca, nakun tahinca pte ko ihduśaka micicapi qa, anpetu wanjidan keś awicapapi kinhan, wanuyanpi owasin țapi kta.

14 Tokin itancan nitaokiye itokam yahden, qa miś wanuyanpi mitokam token manipi, qa hokśiyoqoqa kin token manipi kin, ecen i wahnanna mde kta, mayuhe cin ekta Sehir en wai kin hehanyan.

15 Unkan, Tho oyate mici unpi kin etanhan wanjikśi nici elipewicawaye kta ce, Esa eya. Unkan tokeca $e$, mayuhe cin iśta kin iyocicipi hecinharı, eya.

16 Unkan anpetu kin he en, Esa tacanku kin ohna Sehir ektakiya kihda.

17 'Tuka Jakob Sukot ekta unhdaka i qa, hen tipi içicage ca, taku tawa kin nakun ohanzi hdepi wicakicaga; heon otonwe kin he Sukot eya cajeyatapi.

18 Qa Jakob Aram Mdaye kin etanhan u qehan, Śekem otonwe tawa kin Kanan makoce kin etu zaniyan hi, qa otonwe kin itokam ticaga.

$19 \mathrm{Qa}$ makoce onśpa, tukten wakeya iticage cin, Śsem atkuku Hamor cinca wicaye cin napepi kin etanhan opewicakicaton, mazaska opawinge nom wicaqu.

20 Unkan hen wahna wośnapi wan kage ca, El Israel ta-Wakantanka kin eya caśton.

\section{WICOWOYAKE 34.}

1 Hehan Dina Leha he cunwintku Jakob kiciton kin, he makoce wicinyanpidan wanwicayake kta e toki iyaya.

2 Unkan wicaśta yatapi ihdawa Hamor Hiwi kin he cinhintku Śekem, he, wanyake ça icu qa kici wanka, hecen wieyaku.

3 Qa Śekem nagi kin Dina Jakob cunwintku kin en nina iyaskape ca wikoślka kin waśte dake ça, wikośka cantohnag ye cin ecen okiya.

$4 \mathrm{Qa}$, Wicinyanna kin de miciyuza wo, Śekem iye atkuku $\mathbf{H a -}$ mor eciya.

5 Unkan cunwintku yuaśapapi kin he Jakob nakihon, tuka cinkśi wicaye cin tinta ekta wanuyanpi om yakonpi, qa hdipi kin hehanyan Jakob inina yanka.

6 Unkan Ḣamor Śskem atkuku kin Jakob kici okiye kta e en i.

7 Unkan Jakob cinkśiwicaye cin tinta kin etanhan hdipi, qa he nahonpi, qa iyokiśicapi ; nina canniye yapi, Jakob cunwintku kin kici iwankapi : hecen econpi kte śni tuka, Israel witkoya ecakiconpi heon etanhan.

8 Unkan Hamor hewicakiya, Micinkśi Śskem nagi kin nicunkśipi kin iyaskapa; he tawicu kta e qupo.

9 Kinhan takuunkiciyapi kta; nicunkśipi kin hena unqupo, qa niś cunkśi wicunyanpi kin wicayuzapo.

10 Qa unkiyepi om dukanpi kta, makoce kin nitokam un kta ce, en unpo qa makoce kin en wopetonpi qa he yuhapo.

$11 \mathrm{Qa}$ Sekem wikośka atkuku timdo wicaye cin 'ko hewicakiya ; Cantemakiyapo, kinhan token emayakiyapi kin ecen cicupi kta. 
WICOICAGE.

12 Wikośka kin mitawin un lita e mayaqupi kinhan, wolipayapi iyakicuya tanka mayakidapi, taku qupi tanka eśta; token emayakiyapi kin ecen ciçupi kta.-

13 Unkkan Jakob cinkśi wicaye cin tawinohitinpi kin Dina yuśapapi qon kiksuyapi, qa heon Śkem he atkuku Hamor kici hnayeya awicayuptapi.

$14 \mathrm{Qa}$, Taku hececa econpi unkokitpanipi. Wicaśta wan ha iyotan kin bahdayapi śni kin heca tankśi unyanpi kin unqupi kinhan, he wiśten unkiçiyapi kta naceca.

15 Decehnana on unkaniyuptapi kta; unkiś baunlkihdayapi kin, niś nakun tona wica owasil henicecapi kinhan;

16 Hehan cunkśi wicunyanpi kin unnicupi kta, qa nicunkśipi kin wicunyuzapi kta, qa nicipi oununyanpi qa oyate unwanjipidan kta.

17 Tuka taku de en anaunyagoptanpi śni kinhan, baniçilidayapi śni kinhan, cunkśi unyanpi kin he unkikikeupi, qa toki unkiyayapi kta ce, ewicakiyapi.

18 Unkan, Ḣamor Śsekem Ḣamor cinhintku kici wicoie kin lie waśte dakapi.

19 Qa kośka kin Jakob cunwintku kin cantiheya, heon taku eyapi kin lohanna econ. Iye atkuku ti kin ohna yakonpi kin hena owasin iyakapeya kinihanpi.

20 Hehan Hamor iye cinhintku Sekem kici otonwe tawapi kin ekta kipi, qa otonwe tawapi kin wicaśta om wohdakapi, qa heyapi,

21 Wicaśta kin dena unkiyepi om wokiyapi, heon makoce kin en ounyanpi qa, hen iciyopeyapi kte. Ito makoce kin wicitokam tankaya un kinhan, cunwintkupi kin wicunyuzapi kta, qa cunkśi wicunyanpi kin wicunqupi kta.

22 Tuka decehnana on wicaśta kin wicadapi kta; wiciye bakilidayapi kin he iyecen unkiye tona wica owasin baunkilidayapi kinhan; unkiye om ounyanpi, qa oyate unwanjipidan kta.

23 Decehnana en anawicungoptanpi kinhan, unkiyepi om ounyanpi kta; kinhan tawanuyanpi, qa taku yuhapi kin owasin, taku tonpi koya unkitawapi kte śni he? eyapi.

24 Unkan wicaśta tona otonwe tiyopa kin omanipi kin hena owasin, Hamor iye cinhintku Śekem kici anawicagoptanpi, qa bakicihdayapi, tona wica otonwe tiyopa kin omanipi kin owasin.

25 Unkan anpetu iciyamni kin yazanpi kin icunhan, Jakob cinhintku kin nonpa, Śimeyon Lewi kici Dina timdo wicaya, tacanhpipi kin ikikcupi, qa kokipeśniyan otonwe lin tatpe ipi, qa tona wica owasin wicaktepi.

26 Hamor iye cinhintku Sekem ko canhipi hi kin on wicaktepi, qa Dina Śekem ti kin etanhan ehdakupi qa hdolidapi.

27 Hehan Jakob cinkśi wicaye cin wicakaługapi kin en ipi, qa, otonwe wan tankśitkupi kin yuśapapi qon he ocowasin wicakipi ;

28 Tahinca, qa tatanka, qa śuktanka śonśonna, taku otonwe kin ohna un, taku magata un kin ko owasin icupi.

29 Taku yuhapi qon owasin taśiceca tawicupi ahna nakun wayaka icupi, qa taku tipi kin ohna iyeyapi kin owasin icupi. 
30 Hehan Jakob Śimeyon Lewi kici hewicakiya, Iyoyag mayayapi, śicamna mayakagapi makoce wojutipi kin wicitokam, Kanani, qa Pcrazi kin, qa miye matonana hecen tamatpe mniciyapi, qa maktepi kta, hecen miś wati ohnaka ko owasin awimahnuni kta.

31 Unkan wihomnipi kin iyecen tankśi unyanpi kin ccakiconpi kta iyececa he ? eyapi.

\section{WICOWOYAKE 35.}

1 Hchan Wakantanka Jakob heciya, Najin qa Betcl elkta itawankanhde ye ca, hen ounyan wo. Nicinye Essa itohnake kin ctanhan nayajice çehan, Taku wakan, niye en tanin ic̣iyc cin, he wahna wośnapi wan hen kicaga wo.

2 Unkan Jakob tiyoknaka tawa, qa tona kici unpi kin owasin hewicakiya; Taku wakan tokeca duhapi kin, hena owasin yutokan iyewicayapo; piiciyapi qa wokoyake teca ihduzapo ;

3 Qa najinpo, Betel ekta itawankanhde unyanpi kta ce. Heciya wahna wośnapi wan wakage $\mathrm{kta}$, anpetu wan iyomakiśice cehan, Taku wakan namalion, qa canku ohna imdamde cin mici ye cin, he wecage kta ce, eya.

4 Unkan Taku wakan tokcca yuhapi kin hena owasin Jakob qupi, oinpidan noge oinpi kin hena koya. Unkan hena owasin nalima, Śsizom ekta utuhu wan he cin, he ihukuya.

5 Hehan ihdaka iyayapi, qa Jehowa otonwe ihdukśan unpi kin yuśinye wicaya; hecen Jakob cinkśi wicaye cin wicakuwapi śni.

6 Unkan Jakob, iye oyate ope- ya un kin koya owasin, otonwe wan Luz eciyapi, Betel hee, Kanan makoce kin en, he ekta ipi.

7 Qa hen wahna wośnapi wan kage ca, oyankc kin he El Betel cya caś ton, cincu etanhan najica iyaye cehan, heciya iye en Wakantanka ihdutanin kin, heon etanhan.

8 Hehan Debora Rebeka azin kiye ciqon ta, unkan Betel hukun utuhu he cin, he ihukuya hnakapi, qa hc, Utuhu oceyapi, eciyapi.

9 Wanna Jakob Aram Mdaye kin etanhan hiyu, hehan ake iye en Wakantanka tanin iciyc ça yawaśte.

10 Qa, Tokata Jakob nicajo qon eniciyapi kte śni, detanhan Israel eya cajeniyatapi kta, Wakantanka eciye ça, hecen Israel caś ton.

11 Qa, El Iyotan waśaka he miye ; ihduota qa cinca ota yuha wo; oyate qa, oyate obe ota niye etanhan unpi kta, qa wicaśta yatapi ninite kin etanhan inanpapi kta.

12 Qa makoce wan Abereyam Izak kici wicawaqu qon, he niye ciçu kte ca nicinca nihektam unpi kte cin, makoce kin he wicawaqu kta ce, Wakantanka éciya.

13 Qa oyanke kin en, Walkantanka okiye ciqon hetanhan wankantkiya ikiyopta.

14 Unkan tukten okiye ciqon, he en Jakob wokiksuye wan ehde, inyan wokiksuye qa, wośnapi wan akaśtan, wihdi nakun akaśtan.

15 Qa oyanke kin en, Wakantanka okiye ciqon, he Jakob Betel eya caś ton.

16 Hchan Betel etanhan ihdakapi, qa Eprata ikiyadan hipi, 


\section{WICOICAGE.}

hehan Rahel holsśiksuye ça, kitan seh cinca ton.

17 Wanna kitan ecinyan cinea ton, hehan, Ihnuhan koyalipe cin, cinca kin de wica e nakun duhe kta ce, cincatonkiyesa eciya.

18 Unkan wanna te kta, nagi kin iyaya icunhan, Benoni eya caś kiton ; tuka atkuku kin Benjamin eciya.

19 Hehan Rahel ta, unkan Eprata, Betlehem hee, canku kin ohna hnalkapi.

20 Qa Jakob wokiksuye wan ohna hnakapi kin ekta ehde. Raliel ohna hnakapi wokiksuye kin dehantu eciyapi.

21 Hehan Israel unhdake ca, Eder conkaślke kin akotanhan ewakeya.

22 Unkan Israel makoce kin he en ounye cin icunhan, Ruben iye atkuku tawicu Bilha ekta $i$, qa kici iwanka. Unkan Israel he nakition.

23 Jakob cinkśi wicaye cin ake nonpapi ce. Leha cinkśi wicaye cin, Ruben, Jakob cinhintku tokapa kin hee, Śimeyon, Lewi, Juda, Isakar, Zebulon henakeca. 24 Rahel cinkśi wicaye cin Josep, qa Benjamin.

25 Qa Bilha, Rahel tawikośka, cinkśi wicaye cin Dan, qa Neptali.

26 Qa Zilpa Leha tawikośka kin, cinkśi wicaye cin, Gad, qa Aśer. Henakiya Jakob cinkśi wicaya Aram Mdaye kin en wicatonpi.

27 Hehan Jakob iye atkuku kin elkta ki, Arba otonwe, Hebron hee, ekta Mamore etu hen Abereyam ounyan, qa Izak naliun.

28 Wanna Izak anpetu tawa waniyetu opawinge sanpa wiKcemna śahdogan.
29 Hehan Izak ta, qa taoyate kin en okipapi, wicahinca qa anpetu kin wanna imna. Unkan cinkśi wicaye cin Esa, Jakob henios kihnakapi.

\section{WICOWOYAKE 36.}

1 Esa, Edom hee, wicowazi tawa kin dee.

2 Esa Kanan cunwintku kin etanhan winohinca nom wicayuza, Elon Heti kin cunwintku Ada, qa Zibeon Hiwi kin cunwintku Ana he cunwintku Aholibama henioza.

$3 \mathrm{Qa}$ Iśmayel cunwintku Basmat, Nebayot tanksitku he naliun yuza.

4 Unkan Ada he Esa kiciton Elipaz hee: qa Basmat he Ruhel ton.

5 Qa Aholibama he Jehus, qa Jalam, qa Qora, wicaton. Esa cinkśi wicaye cin, Kanan makoce kin en wicatonpi kin hena eepi.

6 Hehan Esa iye tawicu kin iwicacu, qa cinca wicaye cin wica winyan ko, qa tiyohnaka tawa wicanagi owasin, qa tawanuyanpi taku yuhe cin ko owasin, taku tawa Kanan makoce en ton kin iyulipa, qa sunkaku Jakob itohnalre kin etanhan ya, makoce tokeca wan ekta.

7 Taku yuhapi kin he odota, heon witaya iyotankapi kin okitpanipi, wanuyanpi ota yuhapi kin heon, makoce kin en ounyanpi kte cin iyowinwicakiye śni.

8 Unkan Esa Sehir he kin en iyotanka, Esa he Edom eciyapi. 9 Qa Sehir he kin en Edom oyate ateyapi Esa wicoicage kin dee.

10 Esa cinkśi wicaye cin cajepi kin dena ee, Elipaz lisa ta- 
wicu Ada he cinhintku, qa Ruhel Esa tawicu Basmat he cinhintku.

11 Unkan Elipaz cinkśi wicaye cin dena eepi ; Teman, Omar, Zepo, Gatam, qa Kenaz.

12 Unkan Elipaz Esa cinhintku Timna nahmana yuze cin he Ameleqe Elipaz kiciton. Esa tawicu Ada cinkśi wicaye cin hena eepi.

13 Unkan Ruhel cinkśi wicaye cin dena eepi ; Naliat, qa Zera, qa Śama, qa Miza. Esa tawicu Basmat cinkśi wicaye cin hena eepi.

14 Unkan Aholibama Zibeon cunwintku Ana he cunwintku kin Esa yuza. Unkan he Esa cinca kiciton kin dena eepi; Jehus, qa Jalam, qa Qora.

15 Esa cinca kin itancan unpi kin dena eepi. Esa cinhintku tokapa Elipaz cinkśi wicaye cin, Teman itancan, Omar itancan, Zepo itancan, Qenaz itancan,

16 Qora itancan, Gatama itancan, Ameleqe itancan. Edom makoce kin en Elipaz itancanyan unpi kin hena eepi, qa hena Ada cinkśi wicaya.

17 Unkan Esa cinhintku Ruhel cinca kin dena eepi : Nahat itancan, Zera itancan, Sama itancan, Miza itancan, Ruhel itancanyan unpi kin hena eepi, Edom makoce kin en, Esa tawicu Basmat hena cinkśi wacaya.

18 Unkan Esa tawicu Aholibama he cinca kin dena eepi ; Jehus itancan, Jalam itancan, Qora itancan, Ana cunwintku Aholibama Lsa yuze cin itancan ton kin hena eepi.

19 Esa, Edom hee, cinkśi wicaye cin hena eepi, qa itancan yuhapi kin heepi.

20 Makoce kin he en iyotan- kapi qon Sehir Hori kin cinca dena eepi ; Lotan, qa Śobal, qa Zibeon, qa Ana, qa Diśon, qa Ezer, qa Diśan.

21 Sehir cinkśi wicaye cin, Edom tamakoce kin en, Hori kin itancanyan unpi kin hena eepi.

22 Unkan Lotan cinkśi wicaye cin Hori, qa Heman, qa Lotan tawinohtin kin 'Timna ee.

23 Unkan Śobal cinkśi wicaye cin dena eepi ; Alan, qa Manaliat, qa Ebal, qa Sepo, qa Onan. 24 Unkan Zibeon cinkśi wicaye cin dena eepi, Aya qa Ana; Ana kin de atkuku Zibeon taśunka śonśonna witian wicakiye cehan hopuza en wakoniya kata iyeya.

25 Ana cinhintku kin Diśon hee, qa Aholibama Ana cunwinku kin ee.

26 Qa Diśon cinkśi wicaye cin dena eepi, Ḣemdan qa Éśban, qa Jiteran, qa Keran.

27 Unkan Etzer cinkśi wicaye cin dena eepi, Bilhan, qa Zawan, qa Aqan.

28 Diśon cinkśi wicaye cin dena eepi, Uz qa Aran.

29 Hori kin itancanyan unpi kin dena eepi; Lotan itancan, Sobal itancan, Zibeon itancan, Ana itancan,

30 Diśon itancan, Etzer itancan, Diśan itancan; Hori kin itancanyan unpi kin, token Sehir makoce kin en itancan wicayawapi kin hena eepi.

31 Israel cinca kin nahahin wicaśta yatapi wanica, Edom makoce kin wicaśta yatapi wicayawapi kin dena eepi.

32 Behor cinhintku Bela Edom en wicayuhe cin, qa otonwe tawa Dinaba eciyapi.

33 Qa Bela ta, unkan hee kiya Zera cinhintku Jobab Bozra 


\section{WICOICAGE.}

etanhan wicaśta yatapi yawapi.

34 Qa Jobab ta, unkan hee kiya Huśam Teman makoce kin etanhan, wicaśta yatapi yawapi.

35 Qa Hiusam ța, unkan hee kiya Hadad Bedad cinhintku wicaśta yatapi yawapi. Iye Midian oyate kin wicakaśtaka, Moab tinta kin ohna qa otonwe en ti kin Awit eciyapi.

36 Qa Hadad ta, unkan hee kiya Samla, Masrika etanhan, wicaśta yatapi yawapi.

37 Qa Samla tạ, unkan hee kiya Śaul, Rehobot watpa kin ohna wanke cin he etanhan, wicaśta yatapi yawapi.

38 Qa Śaul ta, unkan hee kiya Balhenan Akbor he cinhintku wicaśta yatapi yawapi.

$39 \mathrm{Qa}$ Balhenan Akbor cinhintku kin ta, unkan hee kiya Hadar wicaśta yatapi yawapi. Otonwe tawa Pahu eciyapi, qa tawicu kin Mehetabel eciyapi, Matred cunwintku Mezahab cunwintku.

40 Esa itancan wicayawapi kin hecen ewicakiyapi, wicowazipi kin iyececa, qa tonwanyanpi kin iyecen cajewicayatapi ; Timna itancan, Alwa itancan, Jetet itancan,

41 Aholibama itancan, Ela itancan, Pinon itancan,

42 Qenaz itancan, Teman itancan, Mibsar itancan,

42 Magdiyel itancan, Iram itancan. Fidom itancan wicayawapi kin hena eepi, tonwanyanpi kin iyecen, tamakocepi kin en; Edom Esa ate yapi.

\section{WICOWOYAKE $3 \%$.}

1 Unkan Kanan makoce kin en Jakob atkuku unhdaka un 56 qon, makoce kin he en Jakob iyotanka.

2 Jakob wicoicage wowapi kin dee, Josep wanna waniyetu ake śakowin, hehan wanuyanpi wihan wicakiya cinye wicaye cin om, qa Bilha qa Zilpa napin atkuku wicayuze cin, hena cinkśi wicayapi kin om, hokśidan kin un qa taku śica eyapi kin ateyapi lin en ahda.

3 Unkan Israel, cinkśi wicaye cin owasin sam iyeya, Josep waśte kidaka wanna wicahinca ehan kicitonpi kin heon etanhan, qa onlidohda hanska wan Josep kicaga.

4 Unkan ateyapi kin, cinkśi wicaye cin owasin sam iyeya Josep waśte kidake cin he cincu wanhdakapi, qa wokiya okiyapi kte cin okihipi śni.

5 Unkan Josep wowihanmde wan ihanmna, qa cincu owicakiyaka; unkan sam iyeya śice kidakapi.

6 Unkan, Ihopo wowihanmde wan iwahanmde cin de nalionpo.

7 Maga ekta aguyapi paunhitapi, unkan inyun pawahte cin bosdan najin hiyaya, unkan payahtapi kin ihdukśan najin hiyaye ca, pawalite cin itokam makata ehpeiciyapi, ewicakiya.

8 Unkan cinye wicaye cin heciyapi, Ehanqon tawannyayapi lita he? qa iś wowidake unyaduhapi kta he? Qa iyakapeya śice kidakapi, wihanmde ciqon oie ko heon.

9 Unkan ake wowihanmde tokeca wan ihanmde ça, ake cincu kin owicakiyaka. Ake wiwahanmde ca inyun anpetu wi, lianyetu wi, qa wicanhipi ake wanji mitokam makata elipeiciyapi, ewicakiya.

10 He atkuku cincu ko owicakiyaka; unkan atkuku he iyo- 
pekiye ca, Wowihanmde wan iyahanmde cin de taku hwo? Miye qa nihun nicinye ko owasin nitokam makipusdiya ehpeunkiciyapi kta he? eciya.

11 Unkan cincu kin icekinpi, tuka atkuku kin wicoie kin he awalkicin un.

12 Hehan cincu kin ateyapi tawanuyanpi kin wihan wicakiyapi kta e Śekem ekta ipi.

13 Unkan Israel Josep heciya, Nicinye Śkem ekta wawilian kiya unpi śni he? Tho wo hena ekta ye ciśi kta ce. Unkan, Ho miye ce, eciya.

14 Unkan, Tho ekta de ca nicinye wanuyanpi ko zaniyan yakonpi hecinhan wanhdake ca, hośi makahdi wo, eciya. Unkan Hebron kaksiza kin etanhan iyaye ca Sekem ekta i.

15 Qa tinta onuni; unkan wicaśta wan wanyake ça, Den taku ayakita he ? eya iwanga.

16 Unkan, Cinye wicawaye cin awicawakita ce. . Ceciye tukten wilian wicakiyapi kin omakiyaka wo, eya.

17 Unkan wicaśta qon, Wanna detanhan iyayapi; Dotan ekta unyanpi kta ce, eyapi nawahion ce, eya. Unkan Josep cinye wicaye cin wicihakam ye ça Dotan en iyewicaya.

18 Unkan tehantanhan wanyakapi qa wicikiyedan u śni kin itokam, nahmana țe yapi kta awacinpi.

19 Qa otoiyohi hunka wanjitku kin hekiciyapi, Wanyakapo ; wihanmdesa kin de wanna $u$.

20 Thopo dehan unktepi, qa makohdoka wan ohna ehpeunyanpi kte ca, Warnanica ocin śica wan he temya ce, unkeyapi kinhan, wihanmde ciqon toketu kte cin wanunyakapi lita ce, ekiciyapi.
21 Ruben he nahon qa, napepi kin etanhan ehdaku kta e, Unktepi kte śni, eya.

22 Napepi kin etanhan ehdaku kte ca, atkuku kin en akiyahde kta e Ruben hewicakiya; Weyapi śnipo. Wolia hewoslrantuya wanke cin de ohña iyeyapo, tuka ninapepi on apapi śnipo.

23 Unkan kaketu, Josep iye cincu najinpi kin en hi qehan, taonhidohda yuśdokapi, onlidohida hanska un kin hee;

$24 \mathrm{Qa}$ Josep icupi, qa woha wan en iyohpeyapi, tuka wohia kin takudan ohnaka śni, mini wanica.

25 Qa aguyapi yutapi kta e iyotankapi, qa pa yuwankan ikikcupi qa, Iśmayeli optaye wan Gileyad eciyatanhan u kin wanwicayakapi, camo yuhapi kin taku waśtemna canśin pejihuta koya qinpi, Egupta ekta kun yapi kta.

26 Unkan Juda hunka wanjin wicaye cin hewicakiya, De sunka unyanpi unktepi kinhan, we kin naunlimanpi eśta taku unkihdamnapi kta he?

27 Ihopo, Iśmayeli kin dena en wiyopeunyanpi kte. De sunka unyanpi qa kici celipi unwanjipidan kin heon, nape on unkațapi kte śni, eya. Unkan hunka wanjin wicaye cin anakigoptanpi.

28 Hehan wicaśta wopeton hecapi Midiani en hiyayapi. Unkan Josep yuzapi, qa woha qon etanhan yuwankan ehdakupi, qa mazaska wikcemna nonpa on Josep wiyopeyapi. Unkan Iśmayeli kin Josep opetonpi, qa Egupta ekta ayapi.

29 Unkan Ruben wolia qon ekta hi, unkan Josep en un śni, heon tawnkoyake hdupota,

$30 \mathrm{Qa}$ hunka wanjitku kin en 57 
wicahdi qa, Hoksidan kin tanin śni ce, miś toki imdamde kta hwo, ewicakiya.

31 Unkan Josep taonhidohda qon icupi qa tatokadan mdoka wan ktepi, qa we kin on oputkanpi.

32 Hehan tuwe onhidolida hanske cin ahde sipi qa ateyapi kin kahipi qa heciyapi; De iyeunyanpi ce. Ito de nicinkśi onlidolida tawa qa is tawa śni uman tukte hecetu hecinhan iyukcan wo.

33 Unkan iyekiye ça, Micinkśi taonlidolida qon dee. Wamanica sica wan he temya. Ehanqon Josep wanna yapotapi ce, eya.

34 Unkan Jakob tawokoyake hdupote ça, waśihda qa anpetu ota cinhintlku kin akiceya.

- 35 Unkan cinca wicaye cin owasin wica winyan lo magagaye wacinpi, tuka iś magaga yapi cin śni, qa, Iyomakiśica ecen micinkśi en Hades ekta mde kta ce, eye ca cinhintku kin akiceya.

36 Unkan Midiani kin Egupta en Josep wiyopeyapi ; unkan Potipar Paro taakicita tancan opeton.

\section{WICOWOYAKE 38.}

1 Hena owasin hecetu, unkan Juda hunka wanjin wicaye cin etanhan apamahde ye ça, Adulami wicaśta wan Hira eciyapi ekta i ;

2 Qa hen Juda Kanani wicaśta wan, Suha eciyapi, he cunwintku wanyake ca yuze ça en i.

3 Unkan ihduśake ça cinca wan ton; unkan Er eya caś kiton.

4 Unkan ake ihduśake ca, cinca wan ton qa, Onan eya caś kiton.

5 Qa ake cinca wan ton qa,
Sela eya caś kiton. He ton kin ehan Kezib en un.

6 Unkan Juda iye cinhintlru tokapa kin $\mathrm{Er}$, winohinca wan, Tamar eciyapi yus kiya.

7 Unkan Er Juda cinhintku tokapa kin he sica e Jehowa wanyake ca, Jehowa he te ya.

8 Hehan Juda Onan heciya, Nicinye tawicu qon ekta ye ça, he yuza wo. Hecen nicinye cinkśi ye kte cin yakage kta ce.

9 Unkan cinca kin he iye tawa yawapi kte śni Onan sdonye, ca cincu cinca kicage kte śni e cincu tawicu qon en i eca, maka kin atpapson.

10 Unkan taku econ kin he sica e Jehowa wanyake ca, he nakun te ya.

11 Hehan Juda iye takośku kin Tamar heciya, Wiwazica niyate ti kin en yanka wo, micinkśi Śsela tanka icage cin hehanyan. Okini iś nakun cinye wicaye cin iyecen te kta kecin, heon hecen eya. Ünkan Tamar kihde ça atkuku ti kin en yanka.

$12 \mathrm{He}$ iyohakam anpetu ota, qa Suha cunwintku Juda tawicu kin wanna ța ; unkan Juda wanna magaga yapi, qa Timnat ekta tahin wanuyanpi tawa yuśdapi kin ekta itawankanhde ya, iye takodaku Hira Adulami kin kicica.

13 Unkan Tamar heciyapi, Tho nitunkan tahin wanuyanpi tawa hduśda kta e Timnat ekta itawankanhde ya ce, eyapi.

14 Unkan Tamar iyoyakeca wokoyake hduśdoke ca, ite ahalipe on aihdahpe ca, Timnat canku iyahde kin he icahda taninyan iyotanka; Sela wanna tanka, tuka qupi śni he wanyaka, heon hecon.

15 Juda he wanyaka ite aihda- 
lipe cin heon winohinca wihomni heca kecin ;

16 Qa canku kin etanhan ekta $i$, qa takośku kin hee kin sdonye śni heon, Tokin unwanken, eciya. Unkan unwanke ca, taku mayaqu kta he? eya.

17 Unkan, Miś wanuyanpi kin etanhan tatokadan cincadan wan nicahipi waye kta, eya. Unkan, Au wicayakiye cin itokam taku mayaqu kinhan, eya.

18 Unkan, He cicu kta e sdonyaye kte cin on etanhan taku e nakaha cicu kta yacin he? eya. Unkan, Maza huhu duhe cin ikan iyahna, qa nakun nitacanlipi ninape ohna duhe cin, eya. Unkan hena qu qa, kici wanka; unkan iye etanhan ihduśaka.

19 Qa najin qa hde ca ite akahpe hduśdole ça ake waśihda.

20 Unkan Juda taku qu qon, winolinca nape kin etanhan ehdaku kta e, takodaku Adulami kin tatokadan cincadan aye śi, tuka iye winohinca qon iyeye sni.

21 Qa wicaśta heciya yakonpi kin iwicawanga; Witkowin can$\mathrm{ku}$ ohna taninyan iyotanke ciqon tukte e he? eya. Unkan, Tohini witkowin wan deciya yanke śni, eyapi.

22 Unkan ihdamna, qa Juda ekta i qa heciya; Winohinca qon iyewaye śni qa, nakun wicaśta heciya yakonpi kin, Witkowin wanjidan tohini deciya un śni ce, eyapi.

23 Unkan, Yuha un nunwe. Tatokadan cincadan kin de aye ciśi tuka iyeyaye śni; sanpa unkakite cinhan, okini wowihahia unke kta, Juda eya.

24 Unkan hetanhan wi yamni hehan, Nitakoś Tamar wawicihaha econ, qa wanna wawicilia- ha kin etanhan ihduśaka ce, Juda eciyapi. Unkan, Tankan aupo ; huhnage kta ce, eya.

$25 \mathrm{He}$ aupi kin icunhan tunkanku hecen wahoya, Tokin maza huhu kin de ikan iyahna, qa canlipi kin de, dena tuwe tawa imiyecidukcan. Tuwe dena tawa ye cin, wicaśta kin he etanhan ihdumaśaka ce, eya.

26 Unkan Juda hena iyekiye ca, Winohinca kin he ohan owotanna miś hemacece śni ; he micinkśi Ś́la waqu śni kin heon etanhan, eye ça hetanhan icimana winohinca qon sdonye śni. 27 Unkan wanna cinca ton kte cehan, inyun tezi mahen cekpa yukan.

28 Qa tonpi kin icunhan, wanji nape kin qu; unkan cinca tonkiye cin he nape kin icu, qa taku duta iyakaśke ça, De tokaheya hinanpe kta ce, eya.

29 Unkan nape kin ehdaku, hehan inyun cincu hiyu. Unkan winohinca kin heya, Token payahdeca; Opahdeca kin de anicipe nunwe, eye ca, Parez eya caś ton.

30 Hehan sunkalku taku duta nape kin akan yruhe cin he hiyu; unkan he Zara eya caś ton.

\section{WICOWOYAKE 39.}

1 Unkan Iśmayeli kin Josep Egupta ekta ayapi. Unkan Potipar Egupton wicaśta kin heca, Paro taakicita tancan kin he aupi kin Josep opewicakicaton.

2 Unkan Jehowa Josep kici un. Unkan taku econ kin owasin tanyan ihuni ye ca iye yuhe cin Egupton ti kin en yanka.

3 Unkan Jehowa kici un, qa taku nape econ kin owasin Jehowa tanyan yustan kiye cin he yuhe cin wanyaka. 
4 Unkan Josep yuhe cin okiye ça iyokipi ya; unkan tiyohnaka tawa owasin awanyag śi, qa talu tawa owasin nape kin en qu.

5 Unkan tipi tawa, qa taku tawa owasin awanyag si kin ehantanhan, Jehowa Josep on etanhan Egupton tiyohnaka kin yawaśte, qa taku ti ohnaka, qa tamaga ekta wanke cin ko owasin, Jehowa yawaśte kin akan un.

6 Hehan taku tawa owasin Josep nape kin en ehpeye ça, iś takudan awalkicin śni, aguyapi hdute cin heceedan. Unkan Josep owanyag waśte, tancan ite lio waśte.

7 Hena owasin hecetu; unkan hehan yuhe cin tawicu kin Josep a tonwe ca, Mici wanka ye, eya.

8 Tuka wicada śni qa yuhe cin tawicu kin heciya; Hiya, mayuhe cin taku tawa owasin minape kin en qu, qa taku tipi ohnaka kin mici un kin iye sdonye śni.

9 De tipi kin ohna tuwedan iyamakapa śni ; qa nihihna miye etanhan takudan tpatan śni, niś nana niyuze cin heon etanhan, qa token woalitani tanka kin de ecamon, qa Wakantanka en wawahtani kta he? eya.

10 Unkan winolinca qon anpetu owasin heciya, qa kitan hinca, tuka Josep anagoptan śni, kici iwanke śni qa ecaca kici un śni.

11 Unkan kaketu, anpetiı wan Josep taku tokon kta e ti mahen i ehan, wicaśta hen tipi kin tuwedan timahen un śni.

12 Unkan winohinca qon Josep taśina kin iyahpaye ça, Mici iwanka ye, eya; tuka śina kin winohinca nape kin etanhan elipeya naśdog hdicu, qa tankan iyaya.
13 Unkan nape kin etanhan śina ehpeya tankan naśdog hdicu kin he winyan kin wanyake ça,

14 Hehan wicaśta tona ti kin ohna tipi kin hena wicakipan, qa hewicalkiya, Wanyakape, Heberi wicaśta wan den unkahdipi, unkan wiśtenunyanpi cin. Manke cin en mahi qa mici wanke kte hinca, tuka ho tankakiya nina waśicahowamda.

15 Nina ho tankakiya waśicahowamde cin he nahon, qa wapan qehan, mitankiyedan śina ehpeya naśdog tankan iyaya ce, eya.

16 Qa yuhe cin hdi kte cin hehanyan śina qon, itankiyedan ehnaka.

17 Qa eye ciqon owasin ake ecen okiyaka, Heberi hokśidan unyakahdipi kin lie imalia kta e mikiyadan $u$.

18. Qa ho tankakiya waśicahowamde cehan mitankiyedan śina ehpeya naśdog tankan iyaya ce, eya.

19 Unkan yuhe cin he tawicu oie kin, Hokśidan nitawa kin hecen ecamicon, eciye cin he nahon hehan cantiyahdeya.

$20 \mathrm{Qa}$ yuhe cin Josep icu, qa wicaśta yatapi tona kaśka wicahnake cin tipi wan en kaśka wicahnakapi kin he en Josep kaśka ehnaka. Unkan wicakaśka tipi kin en yanka.

21 Unkan Jehowa Josep kici un qa, towaonśida kin hiyohiya qa wicalkaśka tipi kin itancan kin he Josep wacin en yus kiya. 22 Unkan wicakaśka tipi itancan kin, tona wicakaśka tipi kin en kaśka wicahnakapi kin owasin, Josep nape kin en qu. Unkan taku hen econpi kin owasin Josep econ wicakiya.

23 Hecen Jehowa Josep Kici 
un, qa taku econ kin owasin tanyan yuśtan kiya; heon wicakaśka tipi itancan kin, taku Josep nape en yuhe cin hena takudan awanhdake śni.

\section{WICOWOYAKE 40.}

1 Hena owasin hecetu, unkan hehan Egupta wicaśta yatapi tawayatkekiye tawohekiye ko, wicayuhe cin Egupta wicaśta yatapi kin en walitanipi.

2 Hecen Paro akicita nom wicayuhe cin canniye wicaya, wayatkekiyapi tancan, qa wohekiyapi tancan kin henioza.

3 Qa akicita tancan tipi tawa wicakaślka tipi kin tukten Joscp kaśka hnakapi yanke cin he en awanyag wicakiyapi kta e ewicahnaka.

4 Unkan akicita tancan kin he Josep hena awanwicayag kiya, unkan owicakiya. Unkan anpetu ota kaśka hnakapi ecen yukanpi.

5 Hehan Egupta wicaśta yatapi tawayatkekiye, tawohekiye ko, wicakaśka tipi kin ohna kaśka wicahnakapi, hanyetu wanjidan en kinukan wowihanmde ihanmnapi, otoiyohi wihanmnapi kin iyukcanpi kin iyecen.

6 Hehan ihanlianna Josep en wicahi qa iyokiśicapi kin wanwicayaka.

7 Qa Paro taakicita wicakaślka tipi kin, iye yuhe cin tipi tawa kin he en iye kici unpi wiwicawanga ; 'Tokeca nakaha ite iyoyag dukanpi he? eya.

8 Unkan wowihanmde wiunhanmnapi, tuka tuwe iyukcan kte cin den wanica ce, eciyapi. Unkan wiyukcan kin Wakantanka hee śni he? Wiyahanmnapi kin omakiyakapo, Josep ewicakiya.
9 Hehan wayatkekiyapi tancan kin wihanmde ciqon ohdaka qa, Wiwahanmde cin en hastanhanka iyuwi wan mitokam han.

10 Qa adetka yamni wiyuwi aicage ça ape walica ko icali ye, qa iyage has tanhanka suton yapi.

11 Hehan Paro wiyatke tawa kin minape ohna "mduha, qa 'hastanhanka kin iwacu, qa Paro wiyatke tawa kin ohna mduśkice ca Paro nape kin en wiyatke kin ew ehde, eya.

12 Unkan Josep heciya, Wiyahanmde cin he kaken ka. Adetka yamni qon anpetu yamni ee.

13 Ebake yamni can Paro nipa kin yuwankan inicu kte ca nanke ciqon ake en anihde kta; unkan tokáheya wayatkeyakiye ciqon wicohan kin ake ecen ecanon kta, Paro nape kin en tawiyatke kin yaqu kta.

14. Hena hecetu kinhan milksuya wo. Tokin tanyan yaun kinhan wowaśte ecamiyecon, Paro en caje mayadaten qa tipi kin de etanhan hinanpe mayayen.

15 Awicakehan Heberi tamakocepi kin etanhan mamanonpi, qa deciya takudan ecamon śni on makolidoka kin den emahnakapi kta, eya.

16 Hehan wohekiyapi tancan kin wiyukcan waśte e wanyake ça Josep heciya; Miś nakun wiwahanmde ça inyun, aguyapi makanopiye yamni mapa kin akan.

17 Qa makanopiye akantu kin ohna Paro tawoyute ocaje owasin wohekiyapi kage cin. Unkan zitkadan hena temyapi, makanopiye mapa kin iwankam un kin hetanhan.

18 Unkan Josep ayupta; He kaken ka. Makanopiye yamni kin anpetu yamni eepi: 
19 Ehake yamni can Paro pa kin yuwankan inicu qa, can akan otke niye kta; hehan zitkadan nicehpi kin etanhan yutapi kta.

20 Unkan iyamni can, anpetu en Paro tonpi kin he kiksuyapi kta e wowidag wicaye cin owasin wowicakihe, ca wayatkekiyapi tancan wohekiyapi tancan henioza wowidake wicaye cin wicehna hdinanpe wicaya.

$21 \mathrm{Qa}$ wayatkekiyapi tancan kin wayatke wicakiye ciqon wicolian ake hecon si ; unkan wiyatke kin Paro nape kin en qu.

22 Tuka wohekiyapi tancan kin otke ya, Josep iwicakiciyukcan qon iyecen.

23 Unkan wayatkekiyapi tancan kin Josep kiksuye śni, tuka akiktonja.

\section{WICOWOYAKE 41.}

1 Hehan tanhan waniyetu nonpa hehan Paro wihanmna. Unkan iho wakpa kahda inajin.

2 Qa inyun wakpa kin etanhan, pte śakowin owanyag waśte qa cepa heca hinanpapi, qa tinta en wilianpi.

3 Qa hena ihektapa tanhan pte tokeca śakowin owanyag śica qa tamaheca hecapi, wakpa etanhan hinanpapi, qa pte umanpi qon ehna wakpicahda najinpi.

$4 \mathrm{Qa}$ pte owanyag śica tamaheca hena pte owanyag waśte cepapi śakowin qon temwicayapi. Unkan Paro kikta.

5 Ake iśtima qa inonpa wihanmna. Unkan inyun wamnaheza hu wanjidan wahuwapa śakowin suton, qa waśteśte aicaga.

6 Qa hena iyohakam wahuwapa tokeca śakowin hinampa, tuka śikśica qa wiyohiyanpa tanhan tate on śnija.
7 Qa wahuwapa conica ton śni kin wahuwapa suton qa ojugjudan qon owasin temya. Paro hecen wihanmde ca hehan liikita.

8 Unkan ihanlianna qehan Paro nagi kin iyokiśice ça, Egupton en pejihuta wicaśta qa wicaśta ksapapi kin owasin wicakico, qa wihanmde ciqon Paro owasin owicakiyaka; tuka tuwedan yukcan okihi śni.

9 Hehan Paro wayatkekiyapi tancan kin ie ca, Anpe dehan wawahtani kin weksuya ce.

10 Paro taokiye kin canniye wicaye ça, akicita tancan tipi kin en kaśka mahnaka, miye qa wohekiyapi tancan kin he nakun.

11 Unkan hanyetu wanjidan en wiunhanmnapi, miye iye kici kinukan, wihanmnapi iyukcanpi kin iyecen wiunhanmnapi.

12 Unkan hen unkicipi kośka wan Heberi kin heca, akicita tancan tahokśidan kin he wiunhanmnapi kin unkokiyakapi, unkan iye hena iyukcan otoiyohi wiunhanmnapi kin iyecen unkiciyukcanpi.

13 Unkan unkiciyukcanpi kin ecetu; miye ake wiyatke yuhe makiye ça uman kin otke ya.

14 Hehan Paro.Josep huwe ye wicaśi, unkan wicakaśka tipi kin etanhan kiyuśkapi, hehan putin hin hdasan, qa wokoyake tokeca kiçun qa Paro yanke cin en hi.

15 Unkan Paro Josep heciya, Wowihanmde wan iwahanmna, unkan he yukcan kta okihi tuwedan iyewaye śni, tuka wihanmnapi niś nayalion unkan idukcan oyakihi keyapi nawalion.

16 Unkan Josep ayupte ça, Miye ee śni, Wakantanka eqe toketu kin tanyan oniciyake kta ce, eciya. 


\section{WICOWOYAKE 41.}

17 Unkan Paro ie ça, Wiwahanmde ca watpa hutata kin nawajin ;

$18 \mathrm{Qa}$ inyun wakpa kin etanhan, pte śakowin cepa, owanyag waśte hinanpapi, qa tinta kin en wilianpi.

19 Unkan hena iyohakam pte tokeca śakowin tamaheca, owanyag śikśica hinanpapi ; tamahenheca sica hena iyececa, Egupta makoce kin en tohini wanmdake śni.

$20 \mathrm{Qa}$ pte tamahenheca śice cin pte śakowin cepa tokaheya qon temwicayapi.

21 Unkan tan mahen iyayapi kin hehan, tan mahen iyayapi kin sdonye pica śni; otokahe ekta śikśica taninpi qon nahahin hececapi. Unkan hehan wekta. 22 Qa ake wiwahanmde ça inyun wamnaheza hu wanjidan wahuwapa śakowin hanskaska waśteśte aicage cin wanmdaka.

23 Hehan ake wahuwapa śakowin cikcistina, wiyohiyanpata tateyanpa kin on śnijapi, qa co wanica hinanpapi.

24 Qa wahuwapa cikcistina wahuwapa śakowin waśteśte qon owasin temyapi. Unkan wicaśta wiyukcan heca owicawakimdaka, tuka miciyukcanpi śni.

25 Hehan Josep Paro heciya, Paro wihanmde cin hena okonwanjidan, Wakantanka token econ kte cin he Paro okiyaka. 26 Pte śakowin waśte qon, hena waniyetu śakowin ee; qa wahuwapa śakowin waśteśte qon, hena nakun waniyetu śakowin ee, wowihanmde kin hena wanjidan.

$27 \mathrm{Qa}$ pte śakowin tamaheca, qa śice cin umanpi kin iyohakam ninanpapi kin, hena waniyetu śakowin ee, qa wahuwapa śako- win cikcistina wiyohiyanpata tateyanpa kin on śnija kin hena nakun, waniyetu śakowin wicaakilian kte cin hee.

28 Paro taku ewakiye cin he dee. Wakantanka taku econ kte cin he Paro wanyag kiya.

29 Hecetu kta, tokata waniyetu sakowin hehanyan, Egupta makoce kin owancaya taku owasin iyakicuya icage kta.

30 Qa hena iyohakam waniyetu śakowin wicaakilian $\mathrm{kta}$, qa Figupta makoce kin en taku iyakicuya icage kte ciqon akiktunjapi kte ca, wicaakilian kte cin makoce kin awihnuni kta.

31 Unkan hehan wicaakihan kte cin iyotan tehike kte cin makoce kin en, heon wipipi qon sdonyapi kte sni.

32 Qa wicoie kin de Wakantanka yuecetu kte ça, wanna kohanna Wakantanka econ kta, heon Paro nonpa akihde wihanmna.

33 Qa wanna Paro wicaśta wan ksape ça wayupika akite ca, Egupta makoce kin yuhe kiya nunwe.

$34 \mathrm{Qa}$ Paro akicita wicakage ca makoce kin en wicahdusuta kte; unkan waniyetu śakowin wipipi kta hena icunhan, taku makoce aicage kte cin owasin izaptanptan icupi nunwe.

35 Hecen waniyetu sakowin waśteśte kte cin hena icunhan, woyute ocaje owasin kamnapi kte, Paro nape kin ihukuya, tonwanyanpi kin en aguyapi pahipi kte cin he woyute awanyakapi nunwe.

36 Qa waniyetu śakowin wicaakilian kte cin Egupta makoce kin en hena on, taku yutapi kihnakapi kinhan, makoce kin wicaakilian lite cin on awihnuni kte śni, eya. 
37 Unkan Paro ookiye wicaye cin ko wicoie kin he waśte dakapi.

38 Hehan Paro iye taokiye kin hewicakiya; Wicaśta wan Wakantanka taniya en un kin de iyececa iyeunyanpi pica he ?

39 Qa Paro Josep heciya, Wakantanka de owasin sdonye niciye cin, nis̉ nana widukcan qa yaksapa, tuwedan iyenicece śni.

40 Niś wati kin awanmiyecidake kta, qa oyate mitawa owasin oie ananigoptanpi kta. Oiyotanke kin en hecedan iciwankam waun kta.

$41 \mathrm{Qa}$ wanyaka wo, Egupta makoce kin owasin yuhe ciciya ce, eya.

42 Hehan Paro maza napcupe hduśdoke ca Josep nape kin ohna iyeye ca, minihuha ska waśte koyag ye ca, mazaskazi wanapin wan napin kiya.

43 Qa canpahmihma yuhe cin etanhan canpahmihma inonpa kin he ohna iyotang ye ca, Itokam canpeśka makehde inajinpo, ieyanpaha eyapi, qa Egupta makoce kin owasin yuhe kiyapi.

44 Qa Paro Josep heciya, Paro miye ce. Oyate kin tuwedan ive cinka takudan econ kte śni. Niś nana token econ wicayaśi kin hecetu kta ce.

45 Qa Paro Josep, Wicoicage nikiye cin, eyá caś ton, qa On atonwe kin en wośnapi kage cin, Potipera he cunwintku Asenat tawicu kta e qu. Unkan Josep Egupta makoce kin ehna iyaya.

46 Josep, Egupton wicaśta yatapi Paro itokam najin qehan, wanna waniyetu wikcemna yamni qa, hehan Josep Paro itokapa tanhan inanpe ca Egupta makoce kin owancaya iyoptapta iyaya.
47 Unkan waniyetu wipipi kin hena icunhan, makoce kin napohnahnaka icali ya.

48 Qa waniyetu śakowin Egupta makoce kin en, taku yutapi owasin pahipi, qa tonwanyanpi kin en kihnakapi; otonwe otoiyohi ohna maga ihdukśan un kin etanhan woyute otonwe kin en kihnakapi.

49 Qa Josep, miniwanca kahdaya wiyaka wanke cin iyecen, aguyapi ota hinca pahi, yawapica śni nakaeś yawapi kte cin ayaśtanpi.

50 Unkan Josep cinca wica nonpa kicitonpi, waniyetu wicaakihan kin itokam, Asenat Potipera On en wośnapi kage cin he cunwintku kin hena Josep kici ton.

51 Iyomakiśice ciqon owasin, qa ate ti kin owasin Wakantanka akiktunś mayan, eye ca tokapa kin Manaśe eya cás kiton.

$52 \mathrm{Qa}$, Makoce en kakiś mayanpi qon, he en Wakantanka taku icah mayan, eye ca iyokihe Epraim eya caś kiton.

53 Unkan waniyetu śakowin wipipi kin Egupta makoce kin en wanna henakeca.

$54 \mathrm{Qa}$ waniyetu śakowin wicaakilian kte cin wanna en au, Josep cye ciqon hecetu. Unkan makoce owasin en wicaakihan, tuka Egupta makoce kin en owancaya aguyapi yukan.

55 Unkan Égupta makoce kin owancaya akihanpi kte cehan, oyate kin aguyapi on Paro hoyekiyapi. Unkan, Josep ekta yapi qa, token econ niśipi kin ecen econpo, Paro Egupton owasin ewicakiya.

56 Wanna maka ohnaka owancaya wicaakilian, hehan Josep aguyapi opiye kin owasin hdu- 
hdoke ça, Egupton taku yutapi wicaqu ; Egupta makoce kin, en wicaakihan tehika aye nakaś.

57 Unkan maka owan caya etanhan, Egupta ekta Josep en taku yutapi opeton hipi, maka kin owancaya nina wicaakihan ece.

\section{WICOWOYAKE 42.}

1 Hehan Egupta makoce kin en aguyapi yuke cin Jakob wanyake ca cinkśi wicaye cin hewicakiya, Tokeca ecin opakicihita dukanpi he?

2 Ihopo Egupta heciya aguyapi yukan keyapi nawation. Ecin kutkiya ekta yapi qa heciya tanhan woyrite opeunkicatonpo, kinhan unnipi qa unțapi kte śni ce, eya.

3 Unkan Josep cinye wicaye cin wikcemna qon aguyapi opetonpi kta e Egupta makoce kin ekta kutkiya ipi.

4 Tuka Benjamin Josep sunkaku kin ope śni, Okini taku śica akipe kta naceca, Jakob eye ca hecen ye śi śni.

5 Únkan Kanan makoce kin en wicaakikan, heon uman hipi kin om, Israel cinkśi wicaye cin woyute opeton hipi.

6 Wanna makoce kin en Josep itancanyan un, qa makoce kin ohna oyate hiyeye cin owasin taku yutapi wicaqu kin Josep hee. Unkan cincu qon en hipi, qa itokam ite makipusdiya makata elipeiçiyapi.

7 Unkan Josep wanwicayake ca cincu iyewicakiya, tuka wicitokam ihdutokeca, qa-icanksiya owawicakiya qa, Tokiyatanhan yaupi he? eya iwicawanga. Unkan Kanan makoce kin etanhan taku yutapi opeton unhipi ce, eyapi.

8 Hecen Josep eqe cíncu kin E. iyewicakiya, tuka iye Josep iyekiyapi śni.

9 Hehan Josep wowihanmde ihanmde ciqon kiksuye ça, Makoce iwanyakapi kin henicapi, qa makóce wanna śdayehna wanke cin he iwanyag yahipi ce, ewicakiya.

10 Unkan, Hiya itancan hecetu śni. Hokśidan nitawa woyute opeton unhipi.

11 Wicaśta wanijidan owasin cinkśi unyanpi. Unkiye iewicaunkapi ; hokśidan nitawa kin makoce iwanyakapi kin heuncapi śni ce, eciyapi.

12 Unkan, Hiya makoce śdayehna wanke cin he iwanyag yahipi ce, ewicakiya.

13 Unkan, Hokśidan nitawa hunka wanjin unkiciyapi ake unnonpapi ; Kanan makoce kin en wicaśta wanjidan ate unyanpi, qa hakakta kin he anpe dehan ate unyanpi kin kici yanka, qa wanjidan tanin śni, eyapi.

14 Unkan, Makoce iwanyakapi kin henicapi ; eya eciciyapi qon he hecetu ce.

15 Deciya tanhan niyukcanpi kta. Paro ni un kin nisunkaji cistinna kin he hi sni kinhan detanhan tokidan dapi kte śni.

16 Ihopo, niyepi kin wanjidan sunka yayapi kin hduwe ye śipo, qa nioiepi kin yahduwicakapi kin hehanyan kaśka dukanpi kta. Hecen niyukcanpi kta, hecetu śni kinhan, Paro ni un kin makoce iwanyakapi kin henicapi kta, Josep ewicakiya.

17 Qa iyamni can hehanyan witaya onatag wicahnaka.

18 Anpetu iciyamni hehan Josep hewicakiya, Wakantanka kowakipa ce. Kaken e econpo qa ni unpo.

19 Wicayakapi kinhan, hunka wanjin yayapi kin wanjidan ka- 


\section{WICOICAGE.}

śka yanke kta, tipi kin de onatag nihnakapi kin he en, qa niye umanpi kin ihopo, tiyohnaka nitawapi kin wicaakitian kin on woyute akiyahdapo.

$20 \mathrm{Qa}$ sunka yayapi cistina kin he miye en makaupo, hecen nioie yahduwicakapi kinhan nitapi kte śni. Unkan ecen econpi.

$21 \mathrm{Qa}$ iyakitedan hekiciyapi, Ehanqon waunhtanipi. Sunka unyanpi wacin iyokiśin wanunyakapi qa ceunkiyapi, tuka anaungoptanpi śni, heon wocante śice cin de unhiyahdepi ce, eyapi.

22 Unkan Ruben ayupte ca, Hokśidan kin en wahtanipi śnipo eciciyapi śni he? tuka anamayagoptanpi śni; unkan ecin iye we kin wanna wiunwangapi kin he dee ce, eya.

23 Unkan Josep he nalion sdonyapi śni. Taku ewicakiya eca wicaśta wan ie ska kiya ece nakaeś.

24. Tuka Josep wicitokapa tanhan toki ye ca ceya. Hehan icicawin en wicahdi qa, taku ewicakiye ca, hehan umanpi kin etanhan Śineyon icu qa, iśtapi kin wicitokam pakilita.

25 Hehan Josep wojuha tawapi kin aguyapi on ojuwicakicitonpi kta, qa mazaska tawapi otoiyolii wojutonpi kin en owicakicihuakapi kta; waneya nakun wicaqupi kta, keya. Unkan hecen ecawicakicon.

26 Unkan śuktanka śonśonna kin taku yutapi qin wicakiyapi qa hdapi.

27 Qa tukten kiwankapi kin, wanji taśunke śonśonna wo qu kta e wojutonpi kin hdulidoka; unkan inyun wojutonpi i kin en tamazaska kin yanka.

28 Unkan hunka wanjin wica- ye cin hewicakiya, Mazaska mduhe ciqon ake micupi. Wanyakapo, wojutonpi mitawa kin en ohnakapi ce. Unkan owasin icanwankapi qa ikopapi qa, De taku Wakantanka ecaunkiconpi hwo, ekiciyapi.

29 Qa Kanan makoce kin ekta ateyapi Jakob en hdipi, qa token akipapi qon owasin okiyakapi.

30 Makoce yuhe cin he ihnuhana icanksiya taku unkekiciyapi, qa makoce kin iwanyag unkipi iyecen ecaunkiconpi.

31 Tuka, Olian unkowotanpi qa makoce iwanyakapi kin heuncapi śni ;

32 Hunka wanjin unkiciyapi ake unnonpapi liena owasin ate unwanjipidan, wanjidan tanin śni, qa hakakta lin he anpe dehan Kanan makoce kin en ate unyanpi kici yanka ce, unkekiyapi.

33 Unkan, Ohan oniwotanpidan kinhan, detanhan sdonwaye kta ce. Hunka wanjin yayapi kin wanjidan den mici yanke kta, qa yatipi kin en wicaakitian kin on, woyute icupi qa ekta hdapo.

34 Qa sunka yayapi hakakta kin he makaupo. Kinhan makoce iwanyakapi kin henicapi śni, qa ohan oniwotanpidan kin sdonwaye kta ce, qa hehan, hunka wanjin yayapi kin de cicupi kta; kinhan makoce kin den wopeyatonpi kta ce, niakoce yuhe cin unkekiciyapi ce, eyapi.

35 Hehan wojutonpi kin tokokadapi; unkan inyun mazaska apahtapi qon, owasin wojutonpi kin en yanka. Owasin ateyapi kin kici mazaska apalitapi qon wanhdakapi, qa inihanpi.

36 Hehan Jakob ate yapi kin hewicakiya; Miye micinca ma- 
yakipi, Josep tanin śni, qa Śsimeyon tanin śni, qa Benjamin iyacupi kta; hena owasin iyokisin mayanpi.

37 Unkan Ruben he atkuku kin heciya, He minape kin en maqu wo. Miś niye en awahdi kta ce. He niye en awahdi śni kinhan, micinca nom kața iyewicaya wo, eya.

38 Tuka, Micinkśi kin de nicipi ye kte śni ce. Cincu wanna te ça iś nana okaptapi, qa canku ohna dapi kin he tahepi Benjamin taku śice cin akipe cinhan; mapa ska kin Hades ekta iyokiśin yemayayapi kta ce, eya.

\section{WICOWOYAKE 43.}

1 Wanna makoce kin en wicaakilian teliika aya.

2 Unkan aguyapi Egupta etanhan ahdipi qoul temyapi, hehan ateyapi kin hewicakiya, Ake ekta yapi qa taku yutapi onge opeunkicitonpo.

3 Unkan Juda ayupte ca, Wicaśta qon, Nisunka nicipi $h i$ śni kinhan miite kin wanmayadakapi kte śni ce, nina kitanyan unkekiciyapi.

4 Hecen niś sunka unyanpi kin unkicipi ye yaśi kinhan kun unyanpi, qa woyute opeunnicitonpi kta.

5 Tuka, niś.ye yaśi kte cin tawatenya ye śni kinhan, kun unyanpi kte śni. Nisunkapi kin ope śni kinhan, miite kin wandakapi kte śni ce, wicaśta kin unkekiciyapi ce, eya.

6 Unkan, Tokeca nis̄unka wanji yuke cin, wicaśta kin oyakidakapi qa śicaya ecamiyeconpi he? Israel eya.

7 Unkan, Wicaśta kin atayedan wicowazipi kin unkiwangapi, qa heya, Niyatepi kin naha- hin ni he? Nisunka wanji yukan he? Unkan owasin wicoie kin hena ecen unkokiyakapi. Ecin token on, Nisunkapi kin makaupo, eye kte cin sdonunyanpi lita he ? eyapi.

8 Qa, Koślka kin mici ye śi wo, kinhan naunjinpi qa unyanpi kta; hecen unnipi kta, unkiye niye unkicincapi ko owasin untapi kte śni.

9 Miś lie awanmdake ca minape kin etanhan mayakida kta. Niye en cicicahdi śni, qa nitokam ewehde śni kinhan, anpetu owasin, Niye en wawahtani kemakiyapi kta.

10 Ye unkapinpi śni unkanś, wanna dehan inonpa unhdipi kta tuka ce; Juda atkuku Israel eciya.

11 Unkan, Hecetu hecinhan kaken e econpo. Makoce kin de waskuyeca tukte iyotan waśte kin wojuha duhapi kin ohna yuha yapo, canśin onge, qa hastanhanka hanpi tiktica onge, qa taku waśtemna, qa pejihuta yatan, hma qa uma, henakiya wicaśta kin yaqupi kta.

12 Mazaska nakun oiyopeye nom, nape kin ohna icupo, mazaska wojutonpi ohna yahdohdipi qon he koya, nape ohna adapi lita, he wanun econpi naceca.

13 Nisunkapi kin nakun icupi, qa najinpi qa wicaśta qon ekta ake yapo.

14. Qa wicaśta kin cante niciyapi qa hunka wanjin yayapi kin, uman kin he ku śi kte, Benjamin koya, Taku wakan Otancanke ionśinidapi kte. Miś micinca makipi kinhan hecen makipi kta, ateyapi Israel ewicakiya.

15 Hecen wicaśta kin taku ikce qupi kte cin he icupi, qa mazaska nape ohna icupi, qa 
Benjamin kici inajinpi, qa Egupta ekta kun yapi qa Josep itokam inajinpi.

16 Unkan Josep wanwicayaka, Benjamin kici, qa taokiye wan tipi awanyake cin he heciya; Wicaśta kin dena tin awicaye ça, wanuyanpi wan pata, qa śpanyan wo. Wiyotanhan hehan wicaśta kin dena om wawate kta ce.

17 Unkan wicaśta kin token Josep eye cin owasin ecen econ, qa Josep ti kin ti mahen u wicaśi.

18 Unkan Josep ti kin ti mahen awicaipi kin heon wicaśta kin kokipapi qa, Mazaska 'tokaheya wojutonpi unkitawapi kin ohna unhdohdapi qon, heon anauntanpi, qa taku iyaunkonpapi kła e unkicopi, qa wayaka unyuzapi kta unkiye śuktanka śonśonna unhapi koya, eyapi.

19 Qa Josep ti kin awanyake cin he en ikiyedan hinajinpi, qa tipi tiyopa kin en okiyapi.

$20 \mathrm{Qa}$, Itancan otokahe ekta taku yutapi opeuntonpi kta e kun unhipi.

$21 \mathrm{Qa}$ owanka qon, en unkiwankapi, qa wojutonpi unhduhdokapi, unkan mazaska unkahipi qon otoiyohi wojutonpi unkitawapi i kin ohna yanka, mazaska aspeunkiye unkitonpi qon he iyececa, he owasin nape ohna unnicicahipi.

$22 \mathrm{Qa}$ mazaska tokeca on taku yutapi opeuntonpi lkta e nape ohna yuha unhipi. Tuwe mazaska unkahipi qon, unkitawojutonpi kin ohna iyolipeye cin sdonunyanpi śni ce, eyapi.

23 Unkan, Tanyan yaunpi kta ce. Wikopapi śni po. Taku wakan yadapi kin niyatepi ta Wakantankapi kin hee wojutonpi nitawapi kin ohna woyuha niçupi. Mazaska nitawapi qon miye en makahipi, eye ca hehan Simeyon wicitokam hinanpe ya. 24 Qa Josep ti kin ti mahen u wicaśi, qa mini wicaqu; unkan siha hdujajapi, qa śuktanka śonsonna tawapi kin nakun taku yutapi wicaqu.

25 Unkan, Hen aguyapi yutapi kta keya nahonpi, heon wiyotanhan hehan Josep hdi kte cin he itokam taku ikce qupi kte cin owasin wiyeya ehnakapi.

26 Unkan Josep tiyata hdi hehan taku ikce qupi kte cin, nape ohna Josep en ti mahen aupi, qa itokam makipusdiya ehpeiçiyapi.

27 Unkan, tanyan unpi hecinhan iwicawange ca, Niyatepi kin wicalinca cajedatapi qon nahahin ni he? tanyan un he? eya:

28 Unkan, Nitaokiye ate unyanpi kin he nahahin ni, qa tanyan un ce, eyapi qa patujapi qa makata ehpeiciyapi.

29 Unkan Josep en etonwe ca, sunkaku Benjamin kici hunku wanjidan kin he wanhdake ca, Sunkayayapi cistina miye en cajedatapi qon he dee he? eye ca, Micinkśi Wakantanka cantonihnake kte, eya.

30 Qa sunkaku kin en cante kin śkanśkan he cin heon Josep inahniyan tukten ceye kta alite ca wankan tipi ekta $i$, qa ceya.

31 Hehan ite kin hdujaja qa hdinanpe ça ihduhe ça, Aguyapi ehnakapo, eya.

32 Unkan aiyog Josep qupi, qa hunka wanjitku kin iś aiyog wicaqupi, qa Egupton tona hen wotapi kin aiyog wicaqupi ; Egupton Heberi om aguyapi yutapi okihipi śni nakaeś, heconpi kinhan iśten iciyapi kta.

33 Unkan josep itokam iyotankapi, tokapa kin watokapa kin iyecen, qa hakakta kin ci- 
WICOWOYAKE 44.

stina un kin iyecen, qa wicaśta qon iyalritedan yuśinyayapi.

34 Taku yutapi iyacinpi otoiyohi wicaqu, tuka Benjamin taku tawa tanka umanpi taku tawapi zaptan akihde heon. Unkan kici yatkanpi, qa iyuśkinpi.

\section{WICOWOYAKE 44.}

1 Unkan Josep tuwe tipi tawa awanyake cin heciya, Wicaśta kin wojuha yuhapi kin en taku yutapi oju wo; tohan qin okihipi kin hehanyan, qa mazaska aupi kin owasin otoiyohi tawojutonpi i kin ohna ehnaka wo.

2 Qa hakakta kin tawojutonpi i kin ohna mazaska wakśica mitawa kin he oyahnake kta, mazaska woyute iyopeye ahi kin he iyahna. Unkan token Josep eciye cin, ecen econ.

3 Anpao hehan hde wicaśipi, wicaśta taśunke śonśonna koya. 4 Wanna otonwe kin etanhan kinanpapi kitanna tehan hdapi, hehan Josep wicaśta tipi tawa awanyake cin heciya; Najin qa wicaśta qon tawicapa wo, qa wicayehdege cinhan, He tokeca tanyan ecaniconpi kin itkom śicaya ecanonpi?

5 Mayuhe cin taku ohna wayatise ça on ayate cin dee śni he? Taku ecanonpi kin he śica, ewicakiya wo.

6 Unkan ewicakihdege ca wicoie kin hena ewicakiya.

7 Unkan, Itancan tokeca e hecen eha he? Hokśidan nitawa token he conkupi lita he?

8 Mazaska wojutonpi i kin ohna iyeunyanpi qon he ake, Kanan makoce kin etanhan unnicicahipi kin sdonyaya. Hehan ecin token on etanhan niyuhe cin ti kin etanhan mazaska qa iś mazaskazi maunnonpi kta he?
9 Hokśidan nitawa uman tukte kaśta he yuha iyeyaye cimban te kta, qa unkiye owasin wowindag unyayapi kte, eyapi.

10 Unkan, Wanna token ehapi kin hecetu kte. Tuwe he yuha iyewakiye cinhan wowldake waye kta, tuka umanpi kin iyaonpepicaśni yaunpi kta, eya.

1. Unkan owasin inahniyan wojutonpi kin makata pahpapi qa, otoiyohi wojutonpi hduhdokapi.

12 Unkan tokapa kin tawojutonpi kin en tokaheya akite ca hakakta kin ecen owecinhan yuśtan. Qa Benjamin tawojutonpi kin ohna wakśica kin iyeya.

13 Unkan wokoyake kin hdupotapi qa owasin śuktanka śonśorna qin wicakiyapi, qa icicawin otonwe kin ekta ipi.

14 Hehan Juda, hunka wanjin wicaye cin om, Josep ti kin en ipi. Unkan iye nahahin hen yanka, qa he itokam owasin makata ehipeiciyapi.

15 Unkan woecon kin de taku ecanonpi hwo? Wicaśta tuwe hemacece cinhan ayate cin okihi sdonyayapi śni he? Josep ewicakiya.

16 Unkan Juda heya, Mayuha token unkaniyuptnpi kta he? Taku unkeyapi kta, qa tokiya tanhan unhduecetupi kta he? Waunhtanipi qon wanna Wakantanka iyeunyanpi. Tho, Itancan wayaka unyaduhapi, wakśica kin yuha iyeyapi qon qa unkiyepi owasin nakun, eya.

17 Unkan, Hecamon kte cin Wakantanka iyomaki śni nunwe. Wiyatke yuha iyeyapi qon wicaśta kin he, hecedan wowindag waye kta; tuka niyepi wookiye on niyatepi kin elkta hdapo, Josep eya.

18 Unkan Juda ikiyedan hina- 


\section{WICOICAGE.}

jin qa heya, Itancan ceciya ce, hokśidan nitawa canniye maye śni wo. Mayuhe cin Paro iyeniceca. Tokin hokśidan nitawa taku epe cin nayahon.

19 Mayuhe cin hokśidan nitawa wiwicanonga; Niyatepi yuke ca nisunkapi yukan he? ehe ciqon.

20 Unkan itancan hecen unkeniciyapi; Han ate' unyanpi kin wanna wicahinca, qa cinhintku cistina kin wicalinea ehan tonpi kin he kici yanka, qa cincu kici hunku wanjidan qon he ț, hecen iśnana okaptapi, unkan atkuku cantekiya ce, unkeyapi.

21 Unkan, Nisunkapi kin makaupo, wanmdake kta ce, hokśidan nitawa kin ewicayakiye ciqon.

22 Unkan, Itancan hokśidan kin atkuku kin ehpeye kte cin okitpani, he atkuku ehpeya u kinhan, atkuku kin te kta ce, unkeniciyapi qon.

23 Unkan, Nisunkapi cistina kin he kici kun yaupi śni kinhan, ake mirte kin icimana wandakapi kte śni, hoksidan nitawa unkeyakiyapr.

24. Unkan hokśidan nitawa kin ate unyanpi kin ekta itawankanhde unhdapi, qa he en token iyae cin owasin unkokiyakapi.

25 Unkan, Ake ekta yapi, qa woyute onge opeunkicatonpo, ate unyanpi kin eya.

26. Unkan, Sunka unyanpi cistina kin de ope cinhan unyanpi lrta ce ; tuka iye ope śni kinhan apamahde unvanpi kte cin unkokitpanipi. Sunka unyanpi hakakta kin kici unyanpi śni kinhan, wicaśta qon itobnake kin wanunyakapi kte śni ce, unkeyapi.

27 Unkan hokśidan nitawa ate 70 unyanpi kin heunkiciyapi, Mitawin cinca nom nana niciton e sdonyayapi.

28 Tuka uman kin mitan tanhan toki iyaye ca, Ehanqon yapotapi ce, epe ciqon ehantanhan wanwahdake śni.

29 Qa wanna de nakun mitan tanhan toki adapi kta, qa tahepi taku śice cin akipe cinhan pa maska iyokiśica Hades ekta amayadapi kta ce, eya.

30 Unkan dehan kaketu kta ce. Nitahokśidan ate unyanpi kin ekta unhdapi, qa iye nagi kin hokśidan nagi kin en ihdaśke ca hokśidan kin ope śni kinhan ;

31 Ate unyanpi kin hokśidan tanin śni kin wanhdake cinhan te kta. Hecen wowindag unyayapi kin hokśidan nitawa ate unyanpi kin pa ska kin iyokiśica Hades ekta kun unkayapi kta.

32 Hokśidan nitawa miye hokśidan kin ate wakida qa hewakiya; Niye cicicahdi śni kinhan, ate niye en wawahtani ohinniyan kecanni kta ce, epa.

33 Unkan wanna ceciciye, hokśidan kin hee kiya den manke ça mayuhe cin wowindag mayaye kta, qa hokśidan kin cinye wicaye cin om itawankanhde hde kta.

34. Hokśidan kin omicipe śni kinhan ate ekta itawankanhde wahde kte cin owakitpani. 'Taku śica ate ahinlipaye kte cin he wanmdake kte cin tawațenwaye śni, eya.

\section{WICOWOYAKE 45.}

1 Hehan Josep tona en najinpi kin owasin wicitokam ihduhe kta okihi śni, qa panyanhan hewicakiya, Wicaśta owasin mitokapa tanhan tankan iyayapo. Hecen Josep hunka wanjin wi- 
caye cin en sdonye içiye cehan, turvedan kici yanke śni.

2 Hehan Josep hotaninyan ceya. Unkan Egupton he nahonpi, Paro tiyohnaka tawa kin nakun nalonpi.

3 Unkan Josep hunka wanjin wicaye cin hewicakiya, Josep he miye ce, A te nahahin ni he? eya ; tuka cincu itolrapa tanhan yuśinyayapi qa ayuptapi kte cin okitpanipi.

4 Unkan, Ihopo, mitan kiyedan upo, Josep hurka wanjin wicaye cin, ewicakiya. Unkan kiyadan hinajinpi. Unkan, Josep nisunka Egupton ekta wiyopeyayapi qon he miye ce.

5 Qa dehan wiyopemayayapi kin on iyokiśin içyapi śnipo, qa icanniye kiciyapi śnipo. Wanikiye makage kta e Wakantanka u makiya ce.

6 Wanna waniyetu nonpa wicaakihan maka kin ohna, qa tokata waniyetu zaptan yumdupi kte śni, qa woksapi kte śni ce.

7 Taku maka akan ecicihnakapi, qa taku iyotan teliika etanhan ni ciyapi kta e Wakantanka nitokam u makiya ce.

8 Wanna deci u makiyapi kin he niyepi ee śni. Wakantanka liee hecon qa, Paro ate maye ça tiyohnaka tawa kin owasin itancan makiyapi, qa Egupta makoce kin owancaya wowindag wicawaye kta e emahde.

9 Inahnipo, qa ate ekta itawankanhde hdapi, qa heciyapo; $\mathrm{Ni}$ cinkśi Josep hecen eya; Wakantanka Egupta owasin yuhe makiya ce. Tho liun en mahi qa yutehan śni wo.

10 Kinhan Gośen en ounyaye ca mitan kiyedan yaun kta ce. Niye nicinca qa nicinca cinca wicayapi, tahin wanuyanpi nita- wa, pte nitawa ko, qa taku duhe cin owasin;

11 Kinhan wo ciç kta: nahahin tokata waniyetu zaptan wicaakilian kte cin heon, yau śni kinhan, okini niwahpanica lkta nace, niye yati ohnaka kin, qa taku nitawa ko owasin.

12 Ihopo, niiśtapi kin misunka Benjamin iśta kin nakun mii kin on ociciyakapi kin he wanmayahdakapi.

13 Egupta en wowitan mduhe owasin, qa taku wandakapi ko owasin, ate okiyakapo, qa inahiniyan ate deciya kun aupo ;

14 Eye ça sunkaku Benjamin poskin yuze ca ceya. Unkan Benjamin cincu tahu kin akan ceya.

15 Hehan hunka wanjin wicaye cin owasin i iwicaputake ca wicahduha ceya. Unkan cinye wicaye cin kici wohdakapi.

16 Wanna Josep hunka wanjin wicaye cin hipi ce, eya oyakapi kin, Paro tiyohnaka tawa kin nahonpi, qa Paro ookiye wicaye cin ko he waśte dakapi.

17 Qa Paro Josep heciya, Hunka wanjin wicayaye cin hecen ewicakiya wo. Kaken econpo; wanuyanpi duhapi kin qin wicalkiyapi, qa Kanan makoce kin ekta hdapo.

18 Qa niyatepi tiyohnaka nitawapi kin lo owasin, icupi qa en maupo ; kinlian Egupta makoce kin en taku waśte yuke cin cicupi kta qa, taku iyotan waśte makoce aicage cin yutapo.

19 Qa niye hecen econ niśipi. Nitaśicecapi nitarvinpi lio ol Egupta makoce kin etanhan canpahmihma yuha hdapi, qa niyatepi kin kici wicahduha upo.

20 Tuka wahpaye duhapi kin he awakicinpi śnipo. Taku wa- 


\section{WICOICAGE.}

śte Egupta makoce ohnaka kin owasin nitawapi kta ce.

21 Unkan Israel cinca kin ecen econpi, qa Josep canpahmihma wicaqu, Paro eya econ śi qon iyecen, waneya tahepi yutapi lita nakun wicaqu.

$22 \mathrm{Qa}$ owasin wokoyake iyohi wicaqu, tuka Benjamin mazaska opawinge yanni qu, qa wolroyake owasin zaptanptan nakun.

$23 \mathrm{Qa}$ suktanka śonśonna wilicemna taku Egupta aicage cin qin wicakiya, qa nakun śuktanla śonśonna wiye wikcemna wamnaheza, qa aguyapi śpan, woyute atkuku waneya yuhe kte cin qin wicakiye ca, hena owasin atkuku kin en ahde wicaśi.

$24 \mathrm{Qa}$ hunka wanjin wicaye cin kihde wicaśi qa, Ihunhan tahepi taku ayakinicapi kin, ewicakiya. Unkan hdapi.

25 Egupta makoce kin etanhan itawankanhde hdapi, qa Kanan makoce kin ate yapi Jakob yanke cin ekta lipi.

26 Qa heciyapi, Josep nahahin ni, qa Egupta makoce kin owasin yuha ce, eyapi. Unkan cetonwicahda qa wacin hnuni.

27 Hehan Josep oie ewicakiye cin owasin okiyakapi, qa canpahmihma ohna iye ihdaka ye lita e Josep ahde wicaśi qon hena wanyake cehan, Jakob ate yapi cante kin limdeza.

$28 \mathrm{Qa}$ Israel heya, Tho henana Josep micinkśi nahaliin ni. Mațe śni wanhdag mde kta ce.

\section{WICOWOYAKE 46.}

1 Hehan Israel ihdaka, iye taku tawa ko owasin, qa Berśeba en hi qa, atkuku Izak taku wakan da kin wośnapi wakiyuśna.
2 Unkan Wakantanka hanyetu wowanyake wan en Israel heciya, Jakob, Jakob, eya. Unkan, De miye ce, eya.

3 Unkan, El niyate ta Wakantanka kin he miye. Egupta en de kte cin ikope śni wo. Hen oyate tanka ical ciciye kta ce.

4. Miye Egupta ekta ciyahna kun mde ca ake acihdi kta, qa Josep nape kin niiśta kin akan aniputake kta ce, eya.

5 Unkan Jakob Berśeba etanhan inajin. Unkan Israel cinkśi wicaye cin taśicecapi, qa tawicupi, qa Jakob ate yapi kin, henakiya canpahmihma Jakob aupi kta e Paro wicaqu qon, hena ohna yuha yapi.

6 Qa tawanuyanpi, qa tawahpayapi, taku Kanan makoce kin en tonpi kin ikikcupi qa Egupta ekta ihdaka ipi. Jakob taku tawa ko owasin;

7 Cinkśsi wicaye cin, qa sanpa cincapi, cunkśi wicaye cin qa cinhintku cunkśi wicayapi kin, taku tawa ko owasin, Jakob kici Egupta elita ipi.

8 Israel cinca kin Egupta en ipi kin, decen ewicakiyapi, Jakob qa iye cinca, Jakob cinhintku tokapa kin Ruben eciyapi.

9 Qa Ruben cinkśi wicaye cin Hanok, qa Palu, qa Hezron, qa Karmi.

10 Unkan, Śimeyon cinkśi wicaye cin; Jemuel, qqa Jamin, qa Ohad, qa Jakin, qa Zohar, qa Saul, he Kanani winohinea wan ton.

11 Unkan Lewi cinkśi wicaye cin Gerśon, qa Qehat, qa Merari.

12 Unkan Juda cinkśi wicaye cin Er, qa Onan, qa Śla, qa Perez, qa Zera; tuka Er Onan kici Kanan makoce kin en tapi ; 
qa Perez cinkśi wicaye cin Hezron, qa Ḣamul.

13 Unkan Isakar cinkśi wicaye cin Tola, qa Puwa, qa Job, qa Śmron.

14 Unkan Zebulon cinkśi wicaye cin Sered, qa Elon, qa Jalel.

15 Hena Leha cinkśi wicaye ca Jakob kiciton, Aram Mdaye kin hen, cunwintku Dina nakun, cinca wica winyan ko wicanagi owasin wikcemna yamni sanpa yamni.

16 Gad cinkśi wicaye cin Zipeyon, qa Ḣagi, qa Śuni, qa Ezebon, Eri, qa Arodi, qa Areli.

17 Unkan Aśser cinkśi wicaye cin Jimma, qa Jiśwa, qa Jiświ, qa Beriya, qa Sera tawinohinpi. Unlkan Beriya cinkśi wicaye cin Heber, qa Malkiyel.

18 Hena Zilpa, Laban iye cunwintku Leha qu qon, cinkśi wicaya. Hena Zilpa Jakob kiciton wicanagi ake śakpe.

19 Rahel Jakob tawicu kin cinkśsi wicaye cin Josep, qa Benjamin.

20 Unkan Josep cinkśi wicaye cin Manaśe, qa Epraim hena Asenat, Potipera On otonwe kin en wośnapi kage cin he cunwintku, Egupta makoce kin en Josep kiciton.

21 Unkan Benjamin cinkśi wicaye cin, Bela, qa Bered, qa Aśbel, Gera, qa Naaman, Eri, qa Roś, Mupim, qa Ḣupim, qa Ared.

22 Hena Rahiel cinkśi wicaye ca Jakob kiciton, wicanagi owasin ake topa.

23 Unkan Dan cinkśi wicaye cin Ḣuśim.

24 Unkan Neptali cinkśi wicaye cin Jahzel, qa Guni, qa Jezer, qa Śilem.

25 Bilha, he Laban iye cunwintku Rahel qu qon, hena Ja- kob kiciton, wicanagi cinkśi wicaye cin owasin śakowin.

26 Wicanagi Jakob nite kin etanhan inanpapi, (Jakob cinca tawicupi kin hena yawapi śni) Jakob kici Egupta ekta ipi kin wicanagi owasin wikcemna śakpe sanpa śakpe.

27 Unkan Josep cinkśi wicaye cin Egupta en kicitonpi kin, wicanagi nonpa. Jakob wicowazi Egupta en hi qon, wicanagi kin owasin wikcemna śakowin.

28 Wanna Jakob Juda tokata ye śi, Josep en, Gośen etu itkokim u kte cin heon. Unkan Gośen makoce kin en hipi.

29 Unkan Josep śuktanka canpahmihma koyag wicakiye ca atkuku Israel itkokipe kta e Gośen etu i, qa wanhdake ca poskin hduze ça tahu akan tehan ceya.

30 Unkan Israel Josep heciya, Nahahin yani kin, nitohnake kin he wanciyaka, heon dehan mața eśta tehilke kte śni.

31 Unkan Josep cinye wicaye cin, qa atkuku tiyohnaka tawa kin owasin hewicakiya; Wahde ca Paro owakimdake kta, Hunka wanjin wicawaye cin, qa ate tiyohnaka tawa kin Kanan makoce kin en unpi qon, wanna en mahipi.

32 Qa wicaśta kin wanuyanpi wihan wicakiyapi kin hecapi, wicaśta wanuyanpi yuha unpi kin iś hecapi, qa talinea pte ko, qa taku tawapi kin owasin awicahipi.

33 Hecen tohan Paro nicopi, qa winiwangapi qa, Wicohtani tukte wanji opiniçiya he? eye cinhan ;

34 Hehan kaken ehapi kta ce, Hokśidan unyaduhapi kin, wanuyanpi awanwicnnyakapi ece ; 
unkiś uncistinpidan ehan tanhan heuncapi, qa hunkake wicunyanpi kin hecen opiiçiyapi ece, ehapi kta; hecinhan Gośen en idotankapi lita. Tona wanuyanpi wihan wicalkiyapi kin hena owasin Egupton walite wicadapi śni.

\section{WICOWOYAKE 47.}

1 Hehan Josep hde ca Paro okiyake ça, Ate qa hunka wanjin wicawaye cin, qa tahinca tawapi, pte tawapi ko, qa taku tawapi owasin, Kanan makoce kin etanhan upi, qa wanna Gośen makoce kin en ahi tipi, eya.

$2 \mathrm{Qa}$ hunka wanjin wicaye cin etanhan wicaśta zaptan iwicacu, qa Paro itokam ewicahde.

3 Unkan, Wicohtani tukte wanji opiniçiyapi he? Paro Josep hunka wanjin wicaye cin ewicakiya. Unkan, Hokśidan nitawa wanuyanpi wilian wicunkiyapi, unkiś hunkake wicunyanpi koya, Paro eciyapi.

$4 \mathrm{Qa}$, Makoce kin en ouny anpi lita e unhipi ce, Kanan makoce kin heciya wicaakihan wanna tehika; wanuyanpi unhapi ko takudan yutapi śni, peji wanica; heon ceunniciyapi Gośen makoce kin en hokśidan nitawa unkiyotankapi kte, Paro eciyapi.

5 Unkan Paro Josep heciya, Wanna niyate qa hunka wanjin wicayaye cin niye en hipi ;

6 Egupta makoce kin owasin nitokam wanka. Makoce kin tukte iyotan waśte kin, hen niyate hunka wanjin wicayaye cin ko iyotanke wicakiya wo. Guśen makoce kin he en iyotankapi kte ça, wanjikśi minihecapi sdonwicayaya hecinhan, hena wanuyanpi mitawa kin awanyag wicakiya wo.
7 Unkan Josep iye atkuku Jakob u kiye ca Paro itokam ehde. Unkan Jakob Paro yawaśte.

8 Unkan, Waniyetu anpetu tona yani he? Paro Jakob eciya.

9 Unkan, Waniyetu anpetu ihdaka wani kin hena waniyetu opawinge sanpa wikcemna yamni. Waniyetu anpetu wani kin hena tonana qa śikśica. Hunkake wicawaye cin waniyetu anpetu ihdaka nipi qon iyehan wani śni ce, Jakob Paro eciya.

10 Hehan Jakob Paro yawaśte, qa Paro itokapa tanhan kinanpa.

11 Unkan Josep atkuku hunka wanjitku ko, makoce tukte iyotan waśte kin, Rameses makuce kin he en, iyotang wicaśi, qa Egupta makoce kin en maga wicaqu, Paro hecen econ śi qon, he iyecen.

$12 \mathrm{Qa}$ Josep atkuku, hunka wanjin wicaye cin, qa atkuku wicowazi kin owasin, taku yutapi wicaqu, taśiceca iyacinyan aguyapi wicaqu.

13 Unkan wicaakilian kin iyo$\tan$ tehika aye cin, heon makoce kin owancaya aguyapi wanica. Wicaakihan kin on Egupta makoce, Kanan makoce ko, awihnuni aya.

14 Unkan aguyapi opetonpi kin he Josep wiyopeye cin, hecen mazaska taku Egupta makoce kin en iyeyapi, Kanan makoce en nakun mazaska iyeyapi kin owasin, Josep kamna, qa mazaska kin Paro ti kin en ahiju.

15 Unkan wanna Egupta makoce kin etanhan, Kanan makoce kin etanhan ko, mazaska kin wanna henakeca, hehan Egupton Josep en ahi qa, Aguyapi unqupo. Tokeca e nitokam 
untapi kta he? Ecin mazaska wanna unnicapi kin, eciyapi.

16 Unkan, Wanuyanpi duhapi kin maqupo. Mazaska wanice cinhan nitawanuyanpi kin on aguyapi ciçupi kta ce, Josep eya.

17 Unkan tawanuyanpi kin Josep en ahipi ; śuktanka, tahinca, pte, qa śuktanka śonśonna, henakiya on, waniyetu kin he aguyapi wicaqu.

18 Waniyetu kin he wanna ihunipi, qa waniyetu iyokihe ake Josep en hipi qa, Itancan anaunkilimapi kte śni, mazaska wanna ecaca wanice ça woteca wanuyanpi owasin itancan duha. Unyuhapi kin itokam takudan unkohdaptapi śni, tancan maga kici ehalie hecehnana unhapi.

19 Tokeca awiunhnunpi. kin wandake kta unkiye, maga unhapi ko owasin he? Opeuntonpo unkiye unkitamagapi koya. Aguyapi unyaqupi kinhan Paro wowindag unyanpi kta, unkiś maga unhapi koya. Taku unkojupi kta nakun unqupo; kinhan unnipi qa unțapi kte śni, qa unkitamagapi kin hewoskan wanke kte śni ce, eyapi.

20 Hecen Egupton wicaakilian kin on iyotan iyekiyapi, qa wicaśta otoiyohi owasin tamagapi kin wiyopekiyapi; unkan Josep Egupta makoce kin owasin $\mathrm{Pa}-$ ro opekiciton, qa Paro makoce kin owasin tawa ya.

21 Qa oyate kin he Egupta makoce ihanke kin anokatanhan otonwe tokeca ohna iyotang wicakiya.

22 Wośnapi kagapi kin henana tamagapi kin wiyopeyapi śni ece. Wośnapi lragapi kin taku yutapi onge Paro wicaqu ece, qa taku Paro wicaqu kin he yutapi, heon tamagapi kin opeton śni.
23 Qa Josep oyate kin hewicakiya, Ihopo nakaha opecitonpi niyepi nitamagapi ko, Paro tawa niyanpi kta. Ihopo de wojupi kin cicupi qa makoce kin oyajupi kta.

24. Qa kaketu kta, tohan taku aicaga eca, owasin idutapi qa iyutapi tomtom yahduhapi kta, hetanhan ake maga kin woyajupi qa he yatapi kta, niye, nitaśicecapi qa tona yatipi kin ohna yakonpi lin hena owasin he yutapi kta; tuka iyutapi izaptanptan kin owasin Paro yaqupi kta ce, eya.

25 Unkan, Itancan ni unyayapi iyounnicipipi kte; hecen $\mathrm{Pa}$ ro wowindag unyanpi kta.

26 Unkan wicoope kin de Josep ope wicakiya, qa anpe dehan hececa, Egupta makoce kin en wiyutapi izaptanptan kin Paro tawa ya. Wośnapi kagapi tamagapi kin henana hecapi śni, henana Paro tawa ye śni.

27 Unkan Israel Egupta makoce kin ohna, Gośen makoce kin en iyotanke ca, maga yuhapi qa icagapi qa nina ihduotapi. $28 \mathrm{Qa}$ Jakob Egupta makoce kin en waniyetu ake śakowin ni, hecen Jakob anpetu tawa kin waniyetu ni un kin, waniyetu opawinge sanpa wikcemna topa sanpa śakowin henakeca.

$29 \mathrm{Qa}$ anpetu en Israel te kte cin he wanna aśkadan, liehan cinhintku Josep kico qa, Ceciciya iyocicipi kinhan ninape miceca kin ihukuya kiyugata wo ; kinhan wowaonśida wowicake ko ecamiyecon, qa Egupta makoce kin en mayahnake kte śni; 30 Tuka Egupta makoce kin etanhan amayade ca, ate wicawaye cin ohna hnakapi kin ohna mayahnake kta; hecen hena om imunke kta ce, eciya. Unkan, 


\section{WICOICAGE.}

Miś token ehe cin ecen ecamon kta ce, eya.

31 Unkan Wakantanka cajeyate ca emakiya wo, eya. UnIran Wakantanka cajeyate ca eciya. Unkan Israel can sagye ipa kin akan pamahdedan wocekiye eya:

\section{WICOWOYAKE 48.}

1 Unkan hena owasin iyohakam hehan, Iho niyate wayazanka ce, Josep eciyapi, qa cinlintku napin Manaśe Epraim kici.hduha elkta i.

2 Unkan Jakob okiyakapi, Nicinkśi Josep niye en u ce, eyapi. Unkan Israel wakiśag ic̣iye ça owinja kin skan iyotanka.

$3 \mathrm{Qa}, \mathrm{El}$ Iyotan waśake cin Kanan makoce kin heciya Luz ekta miye en ihdutanin, qa mayawaśte,

4. Qa hemakiya, Miye wawicahye cicage, yuota ciciye ça, oyate obe ota cicage ca makoce l:in de nakun ciçu kta, nicinca nihakam upi kte cin owihankeśniyan tawapi kta ce, emakiya.

5 Qa wanna nicinca nonpa, Egupta makoce kin en nicitonpi, Egupta ekta niye en cihi śni kin itokam, Epraim Manaśe kici, hena mitawa ce, Ruben Śimeyon kici iyecen, tawa wicawaye kta ce.

6 Qa taku nitawa kin hena iyohakam nicitonpi kte cin hena nitawapi kta, tuka cinye wicayapi kin eciyatanhan, makoce tawapi kin en caś wicatonpi kta.

7 Miye Aram Mdaye kin etanhan wau qon, hehan Rakiel makița, Kanan makoce kin en, canku ohna Eprata etanhan makoce iyutapi kin wanjidan, qa heciya owehnaka Eprata, Betle- hem eyapi kin hee, canku kin ikiyedan, eya.

8 Hehan Israel Josep cinlsśi wicaye cin wanwicahdake ça, Dena tuwepi he? Jakob Josep eciya.

9 Unkan, Wakantanka cinca deciya maqu kin dena eepi, Josep atkuku kin eciya. Unkan, Ceciciya ce, napin makau wo, wicawahdawaśte kta ce, eya.

10 Wanna Israel wicahinca heca on iśta gonga, wanyaka okitpani. Unkan en awicahi, unkan poskịn wicahduze ça i iwicaputaka. 11 Israel Josep heciya, Niite kin eqeś wanwahdake kte cin epce śni, unkan wanna icali wicayaye cin hena, Wakantanka wanwicahdag mayan.

12 Unkan Josep hupahu kin etanhan inanpe wicaye ca, ite kin maka kin etkiya patuja.

13 Qa napin wicahduza, Epraim Josep nape etapa kin en, Israel catka kin ektakiya, qa Manaśe iye napé catka kin en, Israel etapa kin ektakiya; hecen atkuku kin en awicai.

14. Unkan Israel kiyugate ca nape etapa kin Epraim pa kin aputaka, he hakakta tuka, qa nape catka kin Manaśe pa kin akan he tokapa tuka, nape yukcanyan ye kiya.

15 Qa Josep yawaśte qa heya, Wakantanka ate wicawaye Abereyam Izak kici itokam manipi qon, he Wakantanka matonpi lin ehantanhan anpe dehanyan won mayan kin;

16 Ohnihde un kin taku sica owasin etanhan opemakiton kin he, hokśipidan kin wicaya waśte kte, micajeyan qa ate wicawaye cin Abereyam Izak kici icajeyan ewicakiyapi, qa maka kin ohna nina ihduotapi kte, eya.

1.7 Unkan atkuku nape etapa 


\section{WICOWOYAKE 49.}

kin Epraim pa kin aputake cin he Josep wanyake ca, śice dake ca atkuku nape kin yuze ca, Epraim pa kin etanhan Manaśe pa kin etkiya ye kiye kta tuka ;

18 Qa, Hecetu śni ate, de tokapa kin ee. Iye pa kin nape eni.tapa kin on aputaka wo, Josep atkuku kin eciya.

19 Unkan sdonwaya. Micinkśi sdonwaya ce. He nakun oyate kta qa he tanka kta, qeyaś sunkaku iyotan tanka kta qa cinca wicaye kte cin oyate ota kta ce ;

20 Eye ça anpetu kin hehan wicayawaśte qa, Nicajeyan Israel ihdawaśtepi qa, Wakantanka Epraim qa Manaśe iyecen icah niyan nunwe, eyapi kta ce, eya. Hecen Epraim Manaśe itokam cajeyata.

21 Hehan Israel Josep heciya, Anagoptan wo. Miye mațe kta, tuka Wakantanka nicicapi, qa ate wicayayapi tamakocepi kin ekta anihdapi kta.

22 Qa miye makoce onśpa, Amori nape kin etanhan mitacanhpi mitinazipe kin iyahna on iwacu qon, he hunka wanjin wicayaye cin iyakapa ciçu, eciya.

\section{WICOWOYAKE 49.}

1 Qa Jakob cinkśi wicaye cin wicakico qa heya, Mniciyapo, tokata anpetu yeye cin token nihiyahdepi kte cin ociciyakapi kta.

2 Jakob cinkśi wicaye cin witaya upi qa anagoptanpo. Israel niyatepi kin he anakicigoptanpo.

3 Ruben micinkśi tokapa kin he niye, mitowaśake mitowookihi tokaheya kin iyotan wicimdeza iyotan suta.

4 Mini iyecen nihduhepicaśni, lıeon initancan kte śni. Niyate ohehde kin ayadi qa hehan mitowinja kin duśapa ce.

5 Simeyon Lewi kici hunka wanjin kiciyapi. Tacanlipipi kin wipe wicakisa kin heepi.

6 Canteptanyanpi qehan wicaśta wan ktepi, qa iyokipi ic̣iyapi kta e oinatake wan ogapi; heon wohdakapi kin en minagi kin ye kte śni, qa omniciye tawapi kin en wowitan mitawa un śni nunwe.

\% Wicocanniye tawapi kin yaśicapi nunwe, waditaka ce, towalianiçidapi kin he nakun, wohitika ce ; Jakob en yukinukan wicawakiye ça Israel ohna yuomden iyaye wicawakiye kta.

8 Juda niye hunka wanjin wicayaye cin niyatanpi kta. Ninape kin toka niyanpi tahu kin akan niyate cinkśi wicaye cin nitokam patujapi kta.

9. Mraja cincadan wan Juda heca. Wicayapotapi kin etanhan micinkśi nayajin. Mnaja iy ecen canpeśka makehde wanka, qa mnaja wiye wan iyecen, tuwe he pahice $\mathrm{k}$ ta he?

10 Juda etanhan maza sagye yutokanpi kte śni, tuka cadotahedan wicaśta yatapi yuke kta, Ślo hi kin helınyan, qa iye en oyate kin mniciyapi kta.

11 Taśunke śonśonna hastanhanka iyuwi kin en iyakaśkahan, qa taśunke sonśonna cinca kin hastanhanka waśte hinca iyuwi kin ekta, taonlidolida kin miniśa on hdujaja kta qa taśina hastanhanka we kin on.

12 Iśta kin miniśa on śa kte ça hi kin pte asanpi on ska kta.

13 Zebulon miniwanca huta kin en ti qa iye en wita wata oihuni kta, qa Zidon otonwe kin hehanyan yuhe kta.

14 Isakar sulktanka śonśonna 


\section{WICOICAGE.}

waśake cin heca wan, conkaśke otahedan makan wanka.

15 Okihpapi kin he waśte kin, qa makoce kin wiciyokipi kin he wanyake ca qin kta e tahpa kin $q u$, hecen wayaka wicohtani kin econ.

16 Dan Israel wicoun wan iyecen taoyate kin wicayaco kta.

17 Wamduśka wan canku tukte ohna yapi kte cin ohna naicilibe ça, śuktanka siyete kin yalitaka on tuwe akan yotanke cin itunkam hinhpaye kta Dan hecece kta.

18.Jehowa woyuśke nitawa kin awape.

19 Ozuye wan Gad azuye kta, tuka ihakam iye azuwicaye kta.

20 Aśer taaguyapi kin waśte qa wicaśta yatapi woyute wiciyokipi kin wicaqu kta.

21 Neptali tamdoka kiyuśkapi kin heca iapi wicimdeza ia.

22 Josep adetka waicahya ece, adetka waicahya mini hidoka icahda, taadetka kin conkaśke iyadipi.

23. Wanhinkpe yuhapi kin hena iyaonpapi qa kigepi qa śicaya kuwapi.

24 Tuka tinazipe kin sutaya han, Jakob waśagye cin towaśake kin eciya tanhan nape isto kin yuwaśakapi ce. Heciya tanhan Israel taimnija $q a$ wo wicaqu kin hee;

25 Niyate Taku wakan da kin eciya tanhan. Iye oniciye ça Iyotan waśake cin iye niyawaśte kta, mahipiya wankan tanhan wowaśte kin, ośbe kin ihukuya wanke cin wowaśte kin, aze tezi ko wowaśte kin niçu kte.

26 Niyate wicayawaśte kin he wawolihika, he ohinniyan he cin isanpa. Paha owihanke wanice cin taku wiciyokipi aicage cin he Josep pa lrin en, qa tuwe hunka wanjin wicaye cin etanhan yukinukanpi qon, pesdete kin en unpi kte.

27 Benjamin sunktokeca yațesa heca anpao kte cin hehanyan yute kta ece; hitayetu eca wawicaki kin pamni kta.

28 Israel wicoun ake nonpa kin hena owasin heepi, qa hecen ate yapi kin ewicakiye ca wicahdawaśte, otoiyohi towaśte kin iyecen wicayawaśte.

29 Qa hecen wahokonwicakiya, Miś oyate mitawa kin en ope mayanpi kta, ate wicawaye cin om emahnakapo, imnija olidoka maga wan Epron Ḣeti kin tawa qon en un kin, he en.

30 Kanan makoce kin etu, Mamore itokam, Makpela maga kin en imnija ohdoka wanke cin he ohna. Abereyam wicaśta ohna hnakapi kte cin he tawa kta e maga kin he Epron Heti kin he opekicaton.

31 Hen Abereyam tawicu Sara kici wicahnakapi, hen Izak tawicu Rebeka kici wicahnakapi, qa hen miś Leha wehnaka.

32 Maga kin he imnija ohdoka ohna wanke cin ko, Het cinca wicaye cin opewicakicaton qon, ewicakiya.

33 .Hecen Jakob cinkśi wicaye cin wahokonwicakiye cin ayaśtan qehan, siha kin ohehdepi kin en ehdaku qa ta; unkan taoyate kin ekta okipe yapi.

\section{WICOWOYAKE 50.}

1 Hehan Josep a tkuku ite kin akan ehpeiçiye ca i iputake ca ceya. 2 Qa Josep taokiye peji huta wicaśtapi kin atkuku yuliwin śni waśtemna ye wicaśi. Unkan peji huta wicaśta kin Israel waśtemna yanpi; anpetu wikcemna tom hecakiconpi. 


\section{WICOWOYAKE 50.}

3 Tona yuhwin śni waśtemna yanpi eca hehanyan yuśtanpi ece. Unkan Egupton anpetu wikcemna śakowin Jakob aceyapi.

4 Anpetu hena aceyapi kin wanna iyokiptapi, hehan Josep Paro tiyohnaka tawa kin hewicakiya, Wanna waśte mayadapike cinhan miye makapi qa $\mathrm{Pa}$ ro itokam hecen eyapo.

5 Ate hecen emakiya, Iho miś wanna mate kta ce. Kanan makoce kin heciya ohna hnakapi wan micice cin he ohna mahnaka wo, eye ca Wakantanka cajeyate maśi. Unkan tokin wanna itawankanhde mde ca ate wehnaken, kinhan wahdi kta.

6 Unkan Paro ayupte ca, Itawankanhde ye ca niyate kihnaka wo, konza eye niśi qon iyecen. .7 Unkan Josep atkuku kihnake kta e itawankanhde ye ca Paro taokiye kin owasin opapi, tiyohna hunka wicayapi, qa Egupta makoce kin hunka wicayapi ko owasin.

8 Qa Josep tiyohnaka tawa, qa hunka wanjin wicaye cin atkuku tiyohnaka tawa owasin; taśiceca qa tahinca, pte ko yuhapi kin henana Gośen makoce kin en kihnakapi.

9 Canpahmihma qa wicaśta śuktanka akan yotankapi ko opapi, hecen ośpaye tanka hinca.

10 Unkan Atad tahocoka kin ekta ipi he Jordan akasanpa wanka, qa heciya woceye tanka anpetu śakowin atkuku kin nina aceyapi.

11 Unkan makoce wojutipi kin - Kanani hena Atad tahocoka ohna ceyapi kin he wanyakapi qa, Egupton de nina ceyapi ce, eyapi; heon oyanke kin he $\mathrm{E}$ gupton oceyapi kin eciyapi, he Jordan akasanpa wanka.
12 Hecen cinkśi wicaye cin token econ wicaśi qon, ecen ecakiconpi.

13 Qa cinkśi wicaye cin, hena Kanan makoce lkin ekta hduha yapi, qa Makpela maga kin en imnija ohdoka wanke cin he ohna kihnakapi; Mamore itokam; maga kin he Abereyam Epron Heti kin etanhan opekicaton wicaśta ohnahnakapi tawa kta e hecon.

14 Unkan Josep Egupta ekta hda, atkuku hnakapi kin iyohakam; iye hunka wanjin wicaye cin, qa tona iye om atkuku hnakapi kta e itawankanhde yapi qon owasin.

15 Unkan Josep cinye wicaye cin ate yapi kin te cin, heon wikopapi qa heyapi, Okini Josep śicaya unkuwapi qa iye en taku śica ecaunkiconpi qon iś owasin tokiçon kta.

16 Qa hecen Josep okiyag wicaśipi ; Niyate te cin itokam hecen wahokonunkiyapi.

17 Josep kaken eyakiyapi kta, Tokin nicinye wahtanipi kin wicayecicajujun. Śsicaya ecaniconpi tuka niyate ta Wakantanka kin wowindag wicaye cin walitanipi qon kajujun; eya ce, eyapi. Unkan Josep wicoie okiyakiapi kin on ceya.

18 Hehan Josep cinye wicaye cin iye en ipi, qa itokam ehpeiçiyapi qa, Iho unyaduhapi ce, eciyapi.

19 Unkan, Ihnuhan ininihanpi kin. Miś Wakantanka hemaceca he?

20 Niyepi kin śicaya ecamicon wacanipi, tuka Wakantanka waśte kta è cin, oyate ota ni wicaye kta anpe dehan iyecen hecon ce.

21 Heon nakaha inihanpi śnipo. Miś wo ciçupi kta niyepi $7 \dot{9}$ 


\section{WICOICAGE.}

nitaśicecapi ko, ewicakiye ca, | kin de etanhan itawankanhde ye hecen wicakihna qa cantowi- niyanpi kta; makoce wan Abecahnaka.

22 Josep Egupta en ounyan; iye atkuku tawicoun koya, qa Josep waniyetu opawinge sanpa wikcemna ni.

23 Qa Josep Epraim cinca takojatpaku, qa sanpa cinca wanwicahdake ca nakun Manaśe cinhintku Makir cinca kin hena Josep śiyoto akan wicahduha.

24 Hehan Josep hunka wanjin wicaye cin hewicakiya; Miś mate kta tuka Wakantanka niyepi en mihipi kte ca, makoce reyam Izak Jakob ko konza ewicakiye cin he ekta.

25 Qa Josep Israel cinca kin konza ewicakiya, Awicakehan Wakantanka niyepi kin en nihipi kta, qa mihu kin detanhan ayahdapi kta ce, eya.

26 Hehan Josep ta iyaya. Wanna waniyetu opawinge sanpa wikcemna hehan. Unkan iye tancan yuhwin śni waśtemna yanpi, qa Egupta makoce kin en can ohna knakapi wan ohna hnakapi. 80

JEHOWA TATANPI KTE. 


\section{Exodus
Saxibithigoi)}

HD IN ANPAPI.

\section{WICOWOYAKE 1.}

1 Wanna Israel cinca Jakob kici Egupta ekta hipi cajepi dena eepi, otoiyohi tiyohnaka om hipi.

2 Ruben, Simeon, Lewi Juda,

3 Isakar, Zebulun, qa Benjainin,

4 Dan qa Naptali, Gad qa Aśer.

5 Unkan wicanagi Jakob ceca etanhan inanpapi kin owasin wicanagi wikcemna śakowin, qa Josep eqe wanna Egupta en un. 6 Unkan Josep ta cincu ko owasin, nakun tona kici icagapi kin owasin.

7 Unkan Israel cinca kin nina icagapi, qa ihduotapi, qa waśaka hinca ayapi, qa nina ota ayapi, on makoce kin owancaya unpi.

8 Unkan Egupta en wicaśta yatapi tokeca wan najin, he Josep sdonye śni.

9 Unkan oyate tawa hewicakiya, Inyun, Israel cinca oyate kin unkiyepi sam iyeya otapi qa waśakapi.

10 Ito wacinksamya econ wicunkiyapi kta, okini ihduotapi, qa ozuye wan tatpe unhipi kinhan is eya toka unyanpi om kokijuyapi kta, qa azuunyanpi qa makoce kin etanhan hdinanpapi kta, eya.

11 Unkan tawaqinpi on waśaka śni wicakiyapi kta e wohtani itancan yuhe wicakiyapi. Unkan opiye otonwe, Piton qa Rameses Paro kicagapi.

12 Unkan token iyokiśin wicayapi kin hecen ihduotapi, qa icaexod. gapi, qa Israel cinca kin on etanhan nina kokipapi.

13 Unkan Egupton Israel cinca kin nina htani wicakiyapi.

$14 \mathrm{Qa}$ wohtani tehilka owasin econ wicakiyapi on wiconi tawapi pa wicakagapi upśija en, qa maka omdoton en qa wohtani owasin tehiya econ wicakiyapi.

15 Unkan Egupton wicaśta yatapi Heberi cincatonwicakiyapi wanji Ś Sipera eciyapi qa unman Pua eciyapi kin hena hewicakiya;

16 Heberi winohinca cinca ton wicayakiyapi eca, qa can akan iyotankapi wanwicadakapi eca cinca wica kinhan te yayapi kta, tuka he winyan kinhan eqe ni kta.

17 Tuka cincatonwicakiyapi kin Wakantanka kokipapi qa token Egupton wicaśta yatapi kin econ wicaśi ecen econpi śni, tuka cinca wica kin ni wicayapi.

18 Unkan Egupton wicaśta yatapi kin cincatonwicakiyapi kin wicakico, qa hewicakiya, Tokeca decen ecanonpi qa cinca wica kin ni wicayayapi he? eya.

19 Unkan cincatonwicakiyapi kin Paro heciyapi, Heberi winohinca kin Egupton winohinca kin iyececapi śni ye, tuka minihecapi qa cincatonwicakiye cin en wicahi śni itokam cincatonpi heon heconkupi ye.

20 Unkan Wakantanka cincatonwicakiyapi tanyan ecawicakicon, unkan oyate kin ihduotapi qa waśaka hinca ayapi.

21 Unkan cincatorrwicakiyapi 


\section{HDINANPAPI,}

kin Wakantanka kokipapi lıeon tipi wicakicaga.

22 Unkan Paro oyate tawa owasin taku wanji econ wicaśi, Cinca wica lin owasin walipa kin cn elipewicayayapi kta, tuka winyan kin, owasin niwicayayapi kta ce, eya,

\section{WICOWOYAKE 2.}

1 Unkan wicaśta wan Lewi tiyohnaka etanhan.Lewi cunwintku wan ekta i qa yuza.

2 Unkan winolinca kin ihduśake ca cinca wan tón, qa hokśidan waśte e wanhdake ca wi yamni nakilima.

3 Hehan sanpa nakihma okihi śni, qa huliba wakiśkotpa wan icu qa bitumen canśin ko on apawinte ca he ohna hokśiyoqopa kin okihnake ca wakpaicahda psa en ehnaka.

4. Unkan tankeku token ecakiconpi kte cin sdonye kta e itehanyan inajin.

5 Unkan Paro cunwintku ihdujaja kta wakpa ekta i, qa tawikośka wakpa hutata kin omanipi; qa wakiśkotpa kin psa ehna wanyake ca tawikoślka kin he huwe ye śi.

$6 \mathrm{Qa}$ yuhdoke ça hokśiyoqopa wanyaka. Unkan inyun hokśiyoqopa kin ceya. Unkan onśida qa, Heberi cinca wan dee, eya.

7 Unkan, Heberi winohinca waazinkiye cicipan mde ca hokśiyoqopa azin niciye kta ye, tankeku Paro cunwintku kin eciya.

8 Unkan, Ya ye, Paro cunwintku eciya. Hecen wiciyanna kihde ca hokśiyoqopa hunku kin liipan.

9 Unkan, Hokśiyoqopa kin de ahda, qa azin miciciya ye, kinhan cicicajuju lite ye, eya. Un- kan winohinca kin hokśiyoqgopa ikikcu qa azinkiya.

10 Unkan hokśidan tanka icaga. Unkan Paro cunwintku kin kahi, hecen he cinisśi ya, qa miniyatanhan iwacu eya, heon Mowis eya cajehdata.

11 Unkan Mowis tanka icage cin he iyohakam kaketu; Mowis hunkawanjitlku wicaye cin ekta $i$, qa token qin wicakiyapi atonwan, qa Egupton wicaśta wan Heberi wicaśta iye sunkaku wan kte iwanyaka.

$12 \mathrm{Qa}$ anokatanhan ihduhomni, qa wicaśta wanica wanyake cehan, Egupton kin he kte, qa wiyalka mahen nahma.

13 Qa inonpa can tankan i, unkan inyun Heberi wicaśta nom kicizapi, unkan, Tokeca nitakoda ayapa he? śicaya econ kin eciya.

14. Unkan, Tuwe wicaśta yatapi, qa wayaco tawa unyayapi kta e nicaga he? Ḧtanihan Egupton yakte kin he iyecen mayakte kta he? eya. Unkan Mowis kokipe ca awicakehan wicoie kin de sdonyapi ce, eya.

15 Unkan. Paro wicoie kin de nahon qa Mowis kte kta e akita, unkan Mowis najica, Paro ite kin etanhan qa Midian makoce kin en yanke ca miniyowe wan akan iyotanka.

16 Unkan Midian wawayuśna kin cunwintku śakowin atkukupi tahinca tawa mini yatke wicakiyapi kta e hipi, qa etanhan icupi, qa wakiśkotpa ojudan yapi.

17 Unkan wihanwicakiyapi heca en hipi qa nape wicayapi ; unkan Mowis najin, qa owicakiye ca tahinca tawapi mini wicaqu.

18 Unkan atkukupi kin Ruel en hdipi; unkan, Tokeca nakaha ecadan yahdipi he? eya. 


\section{WICOWOYAKE 3.}

19 Unkan, Egupton wicaśta wan wihan wicakiyapi etanhan eunhdakupi, qa nakun mini unkicicupi, qa tahinca kin mini wicaqu, eyapi.

20 Unkan tukte he? tokeca wicaśta kin ehpe yayapi he? kicopo, aguyapi yute kta ce, eya.

21 Unkan wicaśta kin he kici ounye kta Mowis iyokipi ; unkan cunwintku Zipora tawicu kta e qu.

22 Unkan cinca wan kiciton, unkan, Oyate tokeca tamakoce kin en manka ce, eye ca heon Gersom eya caśkiton.

23 Unkan anpetu ota iyohalam, Egupton wicaśta yatapi ta. Unkan Israel cinca kin śica howayapi wohtanipi kin on, qa wolitanipi kin on ceyapi kin he wankantuya Wakantanka ekta $i$. 24 Unkan Wakantanka śica howayapi kin nahon, qa Abereham, qa Izak, qa Jakob om wicotakuye kage ciqon he kiksuya.

25 Qa Wakantanka Israel cinca kin awicatonwan, qa Wakantanka sdonwicaya.

\section{WICOWOYAKE 3.}

1 Unkan Mowis iye tunkanku Jetro Midian wawayuśna tahinca tawa awanwicayake ca tahinca hopuze cin iheyata awicaye ca Wakantanka paha tawa kin Ḧ̈oreb ekta i.

2 Unkan Jehowa ohnihde kin otehi wan cokaya, peta ide wan en ihdutanin he wanyaka; unkan inyun otehi peta en itkon, tuka hulinage śni.

3 Unkan, Ito ekta mde ca wowanyake tanka kin he, tokeca otehi kin hulinage śni he wanmdake kta, Mowis cya.

4 Unkan wanyake kta e u kin he Jehowa wanyake ca Wakan- tanka otehi cokaya tanhan kipan, qa Mowis, Mowis, eya. Unkan, Tho miye, eya.

5 Unkan, Ihnuhan deciya ikiyedan yahi kinhan. Maka kin tukten nayajin kin he wakan ce; heon nisiha etanhan hanpa hduśdokà wo ;

6 Qa miś nihunkake taku wakandapi kin he miye, Abereham taku wakanda qa Izak taku wakanda kin, qa Jakob taku wakanda kin he miyc ce, eya. Unkan Mowis Taku Wakan atonwe kta kokipa, heon ite kin aihdalipa.

7 Unkan, Egapta en oyate mitawa toiyokiśicapi kin he wanmdake, ca wicakahapapi kin on śicahowayapi kin he nawahon qa wayazanidapi kin sdonwaya.

8 Qa Egupton napepi kin etanhan ewicawahdaku qa makoce kin he etanhan, makoce tanka qa waśte, makoce wan ohna pte asanpi qa canhanpi tiktica kaduze cin he, Kanani, qa Ḧeti, qa Amori, qa Perazi, qa H́iwi, qa Jebusi ounyanpi kin, heciya awicamde kta e kurn wahi ce.

9 Hantuke, Israel ceyapi kin wanna en mahi, qa iyokiśicapi kin token Egupton iyokiśin wicayapi kin he nakun wanmdaka.

10 Heon iho wanna oyate mitawa, Israel cinca lin Egupta etanlian hdinanpe wicayaye kta e Paro ekta ye ciśl kta ce, Jehowa eya.

11 Unkan, Miś matuwe he on Paro ekta mde ca Israel cinca kin Egupta etanhan hdinanpe wicawaye kta, Mowis Wakantanka eciya.

12 Unkan heya, Awicakehan nici waun, qa ye ciśi kin de etanhan sdonyaye kta, oyate kin Egupta etanhan hdinanpe wicayaye iyohakam he kin de akan Wakantanka ohoyadapi kta ce. 


\section{HDINANPAPI,}

13 Unkan Mowis Wakantanka heciya, Iho Israel cinca kin en wai, qa nihunkakepi Taku Wakandapi kin he niyepi ekta u maśi, epe cinhan; hehan, Token eciyapi emakiyapi kinhan, taku epe kta he?

14 Unkan, Taku waun kin he waun ce, qa Israel cinca kin hecen ewicayakiye kta, Waun kin lıe niyepi en u maśi ce, Wakantanka Mowis eciya.

15 Qa nakun Wakantanka Mowis heciya, Israel cinca kin hecen ewicayakiye lita, Jehowa nihunkakepi Takd.,Wakan dapi kin, Aberaham Taku Wakan da kin, qa Tzak Taku Wakan da kin qa Jakub Taku Wakan da kin he niyepi ekta u maśi ce. Micaje kin hee owihanke wanin qa wicuicage yeye cin hecen miksuyapi lita.

16 Ekta hde ca Israel hunkayapi kin mniciye wicayakiye ca hecen ewicayakiye kta; Jehowa nihunkakepi wakandapi kin he miye en ihdutanin, Aberaham qa Izak qa Jakob Taku Wakan dapi kin hemakiya; Awicakehan wanciyakapi qa Egupta en token ecannionpi kin.

17 Qa Egupton iyokiśin niyanpi kin etanhan hdinanpe ciyapi lita, Kanani qa Hiti qa Amori qa Perazi, qa Hiwi qa Jebusi tamakocepi lin ekta, makoce wan pte asanpi qa canhanpi tiktica on kaduze cin he ekta, epa ce.

Is Qa niho kin nanicilionpi kta, qa niś Israel hunkayapi kin om Egupton wicaśta yatapi kin en yaipi kta, qa Heberi T'aku Wakan dapi kin Jehowa unkakipapi, heon tokin anpetu yamni oicimani hopuze elita unyanpi, qa Jehowa Wakan undapi kin taku waunkiyuśnapin, eyakiyapi kta.
19 Unkan miye he sdonwaya, Egupton wicaśta yatapi kin dapi iyowinniciyapi kte śni, qa nape kin waśake kte śni.

20 Qa minape kin ycwakiye ca taku tehilika mitawa Egupton cokaya ecamon kta, hena owasin on awicawape kta he iyohakam ye niśipi lita.

21 Q a Egupton kin oyate kin de cante wicakiyapi he wakage kta, hecen yaupi kinhan cokadan yaupi kte śni.

22 . Tuka winohinca otoiyohi tuwe ikiyedan ti kin, qa tuwe iye ti kin ohna ti kin, he etąnhan mazaska oinpi qa mazaskazi oinpi qa wokoyake da kta, qa nicincapi wica winyan ko hena un wicayakiyapi kta, hecen Egupton wawicayakipi kta.

\section{WICOWOYAKE 4.}

1 Unkan Mowis ayupte ca, Ehaeś wicamadapi kte śni, qa Jehowa niye en ihdutanin śni, eyapi kta, hecen maho kin nahonpi lite śni, cya.

2 Unkan Jehowa heciya, Ninape ohna yanke cin he taku he? Unkan, Cansakadan, eya.

3 Unkan, Makata ehpeya wo, eya. Unkan makata ehpekiya qehan wanmduśka icaga; unkan itokapa tanhan Mowis najica.

4 Unkan, Ninape hdugate ça sinte kin yuza wo, Jehowa Mowis eciya. Unkan nape hdugate ca he yuza, hehan nape kin ohna cansakadan icaga.

5 Heon Jehowa iye hunkakepi Taku Wakan dapi kin, Abereham Taku Wakan da, qa Izak Taku Wakan da, qa Jakob Taku Wakan da kin he niye en ihdutanin kin he wicadapi kta.

6 Qa nakun, Ikpi kin ohna nape ye kiya wo, Jehowa eciya. 


\section{WICOWOYAKE 4.}

Unkan nape ikpi kin ohna ye kiye ca ehdaku; unkan inyun nape kin ska, wa iyececa.

7 Unkan, Ninape ake niikpi ohna ye kiya wo, eya. Unkar ake nape ikpi"kin olna ye kiye ca ikpi etanhan ehdaku, qa inyun ake iye cehpi kin iyececa.

๖ Unkan kaketu lkta, wicanidapi kte śni, qa wowapetokeca tokaheya ho kin wicadapi kte śni eśta, wowapetokeca unman ho kin wicadapi kta.

9 Qa wowapetokeca nonpa kin dena nakun wicadapi śni kinhan qa niho kin nahonpi kte śni kinhan, wakpa kin etanhan mini iyacu, qa maka puze cin ayakaśtan kta, qa mini wakpa etanhan iyacu kin he maka puze cin akan we icu kta ce, eya.

10 Unkan, Itancan ceciciya ce, miś wicaśta wamdapike śni, nitaokiye kici woyahdake cin ehantanhan nakun he itokam; tuka i matke, qa ceji matke, Mowis Jehowa eciya.

11 Unkan Jehowa heciya, Tuwe wicai kin kaga he? qa ia okitpani, qa nogetpa, qa wawanyake cin, qa iśta gonge cin? Jehowa he miye śni he?

12 Heon wanna ekta ya wo, unkan nii kin kici waun, qa token ehe kte cin he onspe ciciye kta ce.

13 Unkan, Itancan ceciciya, Tuwe nape kin on ye yaśi kte cin he ye śi ye, eya.

14 Hehan Jehowa tocanniye kin Mowis aide qa heya, Aaron Lewi kin he nicinye heca śni he? Iye wayapika he sdonwaya, qa nakun, iho wanna itkonicipe kta u ce; qa wanniyake cinhan cante kin iyuśkin kta.

$15 \mathrm{Qa}$ he taku eyakiye kta, qa. i kin ohna wicoie kin yeyakiye kta, qa miś nii kici waun kta, qa iye i kin kici, qa token ecanonpi kta he onspe ciciyapi kta.

16 Qa iye niye on oyate kin taku ewicakiye kta, qa hecen iye nii kin ee yakiye kta, qa iye Taku Wakan niyuhe kta.

17 Unkan cansakadan kin de ninape ohna iyacu kta, qa he on wowapetokeca ecanon kta.

18 Unkan Mowis iye tunkanku Jetro ekta kihde ca heciya, Ito mde ca mihunkawanji Egupta obna unpi kin ekta wahde ça nahanhin ni unpi kinhan wanmdake kta ce. Unkan, Zaniyan hda wo, Jetro Mowis eciya.

19 Wanna Midian ohna Jehowa Mowis heciye ciqon, Tho Egupton ekta hda wo, wicaśta tona ninagi akitapi qon hena owasin tapi ce.

20 Unkan Mowis iye tawicu qa cinhintku napin ewicahdaku, qa śuktanka śonśsouna akan iyotangwicakiye ca Egupta makoce kin ekta kihda; qa Mowis Wakantanka cansakadan tawa kin nape kin ohna icu.

21 Unkan Jehowa Mowis heciya, Egupta ekta yaki kinhan waktaya un wo. Wowapetokeca owasin ninape ohna ciçu kin Paro itokam ecanon kta, qa miś Paro cante kin mdusuta kta, hecen oyate kin kihde wicaye kte śni.

22 Qa Paro hecen eyakiye kta, Jehowa hecen eya, Israel micinkśi tokapa kin hee.

23 Qa heciciya, Micinkśi omakiye kta hde śi wo; qa hde kta teyahinda kinhan nicinkśi tokapa te waye kta ce.

24 Unkan, kaketu tahepi tukten iwankapi kin en Jehowa akipe ca te ye kta cin.

25 Unkan Zipora wanhi wan icu, qa cinhintku ce inkpa ha kin bakihdaya, qa siha kin ekta 


\section{HDINANPAPI,}

ehpeye ca, Awicakehan we wicaśta takuciya ye, eya.

26 Unkan he ayuśtan hehan, Bahday ipi on etanhan we on takuciye ye, eya.

27 U ikan Jehowa Aaron kaken eciye ciqon, Mowis itkokipe kta e.iyr ya wo, hopuze cin ekta, un"kan tyaye ça Wakantanka he tawa kin ekta akipe ca i itputaka.

28 Unkan Jehowa yeśi kin, oie kin owasin, qa wowapetokeca econ śi kin owasin, Mowis Aaion okiyaka.

29 Unkan Mowis iyaya Aaron Kici, qa Israel cinca hunkayapi kin owasin mniciye wicayapi.

30 Qa wicoie Jehowa Mowis eciye ciqon owasin Aaron ewicakiya, qa wowapetokeca kin econ, oyate kin wicitokam.

31 Unkan oyate kin wicadapi; qa Jehorva Israel cinca kin en wicahi, qa iyokiśicapi kin wanyake cin he nahonpi qehan patujapi qa cekiyapi.

\section{WICOWOYAKE 5.}

- Unkan iyohakam Mowis qa Aaron Paro en hipi, qa heciyapi, Oyate mitawa hewotahedan wohanpi wan micicagapi kta e iyowin wicakiya wo, Jehowa Israel Taku Wakan dapi kin eya ce.

2 Unkan Paro heya, Jehowa he tuwe on ho lrin nawahion, qa Israel ye wicawaśi kta he ? Jehowa sdonwaye śni qa Israel amduśtan kte śni ce; Paro eya.

3 Unkan heyapi, Heberi Taku Wakan dapi kin unkakipapi, ceunniciyapi, iyowinunkiyapi, hopuza elita anpetu yamni unyanpi, qa Jehowa Taku Wakan undapi waunkiciyuśnapi kte, okini makośica qaiś canlipi on iyaunkihpayapi kta.

4 Unkan, Tokeca Mowis qa 86
Aaron oyate kin wicohtani ayuśtan wicaya kiyapi he? Nitawaqinpi kin ekta hida po, Egupton wicaśta yatapi kin ewicakiya.

$5 \mathrm{Qa}$, Inyun makoce oyate kin wamna ota ce, qa tawaqinpi kin kpahpe wicayakiyapi, Paro eya. $6 \mathrm{Qa}$ anpetu kin he Paro oyate wicakahapapi, qa itancanpi kin econ wicaśi qa hewicakiya,

7 Dehanyan oyate maka omdoton kagapi kta e peji puza wicayaqupi ece, tokata ecanonpi kte śni. Iye yapi qa peji puza mnakiyapi kta.

$8 \mathrm{Qa}$ dehanyan maka omdoton iyuta kagapi kin he kah wicayakiyapi kta, duoptetupi kte śni; kujapi nakaś, heon ho yeyapi qa, Ito unyanpi qa Taku Wakan undapi kin waunkiciyuśnapi kta, eyapi.

9 Wicaśta kin wohtani tehika econ wicayakiyapi kta, hecen litanipi kta, qa wicoie ecinśniyan ihaktapi kte śni ce.

10 Unkan oyate wicakahapapi kin, qa itancanpi kin tankan ipi, qa oyate kin hewicakiyapi ; $\mathrm{Pa}-$ ro hecen eya, Peji puza ciçupi kte śni ce.

11 Niś hunktiya po, qa peji puza tuk ten iy eyayapi kin hetanhar ikikeu po, qa htayanipi kin takudan yuoptetupi kte śni.

12 Hecen oyate kin Egupta makoce kin owancaya enanakiya iyayapi, qa peji puza hee kiya aguyapi hu kin mnayanpi.

13 Unkan wicakahapapi kin inahni wicayapi, qa Nitohtanipi kin hduśtan po; anpetu otoiyohi peji yuke ciqon he iyecen; eyapi.

14 Unkan Israel cinca itancanpi kin Paro wicakahapapi kin yuhe wicakiyapi kin hena awicapapi qa, Tokeca maka omdoton kah niśipi kin htanihan qa 


\section{WICOWOYAKE 6.}

nakaha duśtanpi śni, hekta ecannonpi qon he iyecen.

15 Hehan Israel cinca itancanpi kin upi, qa Paro ho yekiyapi, qa heyapi, 'Tokeca nitaokiye hecen ecawicayecon he?

16 Peji puza takudan nitaokiye unqupi śni ; Maka omdoton kaga po ; unkekiyapi, qa nitaokiye unkapapi tuka, oyate nitawa kin hena ee taku sica econpi.

17 Unkan, Niś nikujapi, nikujapi ce, heon, Unkiyayapi qa Jehowa waunkiciyuśnapi kta, ehapi.

18 Heon wanna hunktiya po, qa htani po. Peji takudan nicupi kte śni, qa maka omdoton iyenaka yakagapi kta, eya.

19 Unkan, Anpetu otoiyohi maka omdoton yakagapi qon he duoptetupi kte śni, èwicakiyapi qehan, taku śica ehna unpi Israel cinca itancanpi kin wanihdakapi.

20 Unkan Paro etanhan hdinanpapi qehan, Mowis Aaron kici, canku ulna najinpi, unkan itkowicakipapi.

21 Qa hewicakiyapi, Jehorva anitonwanpi qa wayaco kta. $\mathrm{Pa}-$ ro iśta kin ohna qa taokiye iśtapi kin ohna ounmnapi śicamna unyakagapi, qa napepi kin en isan wan on unktepi kta wicayaqupi ce.

22 Unkan Mowis Jehowa ekta ihdamna, qa heya, Itancan tokeca oyate kin de, śicaya ecawicayecon, qa tokeca ekta ye mayaśi he?

23 Qa nicajeyan Paro takewakiya ekta wahi kin ehantanhan oyate kin de taku śica ecawicakicon, qa dehanyan oyate nitawa ecaca wicayakiduśke śni, eya.

WICOWOYAKE 6.

1 Unkan Jehowa Mowis heciye, Ito token Paro ecawecon kte cin he wandake kta. Nape waśaka on ye wicaśi kta, qa makoce tawa etanhan nape waśaka on wicakahape kta.

2 Unkan Wakantanka Mowis kici wohdaka, qa heciya, Jehowa he miye.

3 Qa Abereham qa Izak qa Jakob en Taku Wakan waśaka mihdutanin, tuka micaje Jehowá he sdonmayanpi śni.

4 Qa nakun wicotakuye mitawa wicaweciyusuta; makoce tawapi śni ohna ihdakapi kin he, Kanan makoce kin he wicawaqu kta.

5 Nakun Israel cinca śicahowayapi kin he nawahon, Egupton wowidag wicayapi kin he on, qa wicotakuye mitawa kin weksuya.

6 Heon Israel cinca kin hecen ewicakiya wo, Jehowa he miye, qa Egupton waqin niciyapi kin ihukuya tanhan hdinape ciyapi kte, ça htani niciyapi kin etanhan ecihdakupi kta; qa isto hdugata qa woyaco tanka on opecitonpi kta.

7 Qa oyate mitawa kta e ciyuzapi kta, qa Taku Wakan yadapi kin he miye kta, qa Jehowa wakan yadapi Egupton waỵin niciyapi ihukuya tanhan hdinape niyanpi kin he miye, sdonyayapi kte.

8 Qa makoce wan Abereham qa Izak qa Jakob wicawaqu kta kepa, qa minape wankan yewakiya, he elsta aciupi, qa tawayayapi kta e cicupi kta ce, Jehowa he miye.

9 Unkan Mowis Israel cinca. kin hecen ewicakiya; tuka toksape kicaksapi, qa wicohtan tehika, heon Mowis anaguptanpi śni.

10 Unkan Jehowa ie ça Mowis heciya; 
11 Paro Egupton wicaśta yatapi kin ekta ya wo; qa iye tamakoce kin etanhan Israel cinca kin ye wicaśi kta, keciya wo.

12 Unkan Mowis Jehowa itokam ie ca, Inyun, Israel cinca kin namałionpi śni, qa iha bamakilidayapi śni hecen token Paro namahon kta he? eya.

13 Unkan Jehowa Mowis Aaron kici Israel cinca kin ekta, qa $\mathrm{Pa}$ ro Egupton wicaśta yatapi kin ekta ye wicaśi, qa Egupta makoce kin etanhan hdinape wicaye kta, kewicakiya.

14 Hunkake wicayapi tiyohna. ka itancanpi kin dena eepi. Israel cinca tokapa kin Ruben, he cinca kin Ḧanok, qa Pallu, qa Hezron, qa Karmi ; Ruben wicowazi kin hena eepi.

15 Simeon cinca kin, Jemuel, qa Jamin, qa Ohad, qa Jakin, qa Zohar, qa Kanani winohinca wan cinca Śaul; Simeon wicowazi kin dena eepi.

$16 \mathrm{Qa}$ Lewi cinca cajepi wicoicagepi kin iyececa; Gerśom, qa Qohat, qa Merari. Qa waniyetu Lewi ni kin opawinge sanpa waniyetu wikcemna yamni sanpa śakowin.

17 Gerśom cinca kin Libni, qa Simi wicowazi tawapi kin iyececa.

18 Qa Qohat cinca kin Amram, qa Izhar, qa Hebron, qa Uziel; qa waniyetu Qohat ni kin waniyetu opawinge sanpa wikcemna yamni sanpa yamni.

19 Qa Merari cinca kin, Mahli, qa Muśi. Lewi wicowazi kin hena eepi wicoicage tawapi kin iyececa.

20 Unkan Amram iye tunwicu Jokebed e yuza; unkan he Aaron, qa Mowis kiciton; qa waniyetu Amram ni kin hena wariyetu opawinge sanpa wikcemna yamni sanpa śakowin.
21 Qa Izhar cinca kin Qora, qa Nepeg, qa Zikri.

22 Unkan Uziel cinca kin Miśael, qa Elizapan, ya Sitri.

23 Unkan Aaron Aminadab cunwintku, Nahison tawinohitin, Eliśba yuza; unkan Nadab, qa Abihu, qa Eleazar, qa Itamar hena kiciton.

24 Unkan Qora cinca kin Aśer qa Elqana, qa Abiasap; Qorahi wicowazipi kin hena eepi.

25 Unkan Aaron cinhintku Eleazar, Putiel cunwintku kin etanhan wanji yuza; unkan he $\mathrm{Pi}$ nehas kiciton. Lewi ateyapi kin itancanpi kin hena eepi, wicowazipi kin iyececa.

26 Egupta makoce etanhan Israel cinca kin obe tawapi ecekcen hdinape wicaya po, Jehowa tuwe ewicakiye ciqon, he Aaron qa Mowis dena eepi.

27 Tuwe Israel cinca kin Egupta etanhan hdinape wicaye kta, Paro, Egupton wicaśta yatapi eciyapi qon, Mowis qa Aaron hena eepi.

28 Qa anpetu wan Jehowa Mowis Egupta makoce kin ohna taku eciya he en kaketu.

29 Unkan Jehowa ie ca, Mowis heciya, Jehowa he miye, taku eciciye cin owasin Paro Egupton wicaśta yatapi eciya wo, eya.

30 Urkan, Inyun iha bamakihdayapi śni, lieon Paro namalion kte śni, Mowis, Jehowa itokam, eya.

\section{WICOWOYAKE 7 .}

1 Unkan Jehowa Mowis heciya, Wan Paro taku wakan niye kta e cicaga. qa Aaron nicinye ie yakiye kta.

2 Niś taku eye ciśi kin owasin ecen ehe kta, qa nicinye Aaron Paro iye tamakocepi kin etanhan 
Israel cinca kin kihde wicaśi kta, eciye kta.

3 Tuka miś Paro cante kin mdusuta kta, qa Egupta makoce kin ohna wowapetogtokeca mitawa, qa taku woyuśinya wahduota kta.

4 Tuka Paro nanilionpi kte śni, hecen Egupton akan minape ewahnake kte ca woyaco tankinkinyan on Israel cinca kin, oyate mitawa, obe mitawa kin, Egupta makoce kin etanhan hdinape wicawaye kta.

5 Hecen Jehowa he miye Egupton sdonyapi kta,minape Egupton akan ye wakiye ca wicacokaya tanhan Israel cinca kin hdinape wicawakiye cinhan.

6 Unkan Mowis qa Aaron Jehowa token econ wicaśi kin owasin ecen econpi.

7 Unkan Mowis waniyetu wikcemna śahdogan, qa Aaron waniyetu wikcemna śahdogan sanpa yamni, hehan Paro en wohdakapi.

8 Unkan Jehowa ie cá Mowis Aaron kici hewicakiya;

9 Tohan, Wowapetokeca kin kpazo po, Paro eniciyapi kinhan, Cansakadan ikikcu, qa Paro itokam ehpe kiya wo; Aaron eyakiye kta; unkan wamduśka icage kta.

10 Unkan Mowis qa Aaron Paro en hipi; qa token Jehowa econ wicaśi kin ecen econpi, qa Aaron cansakadan kin ehpe kiya, Paro itokam, qa taokiye wicitokam; unkan wamduśka icaga.

11 Unkan Paro nakun wicaśta ksapa qa pejuta wicaśta wicakizo, unkan Egupton pejuta wicaśta iś eya taku wakanyan olianyanpi on iyecen econpi.

$12 \mathrm{Qa}$ otoiyohi cansakadan ehpe kiya; unkan wamduśka ica- gapi, tuka Aaron cansakadan tawa kin he cansakadan tawapi kin napca.

13 Unkan Paro cante kin ihdusuta, qa nawicalion śni, Jehowa eye ciqon he iyecen.

14 Unkan Jehowa Mowis heciya. Paro cante kin tke, oyate kin ye wicaśi wicada śni.

15 Tho hanhanna Paro mini ekta i kta; ekta ya wo, qa wakpa hutata itkokim nayajin kta, qa cansakadan wamduśka icage ciqon he ninape ohna iyacu kta.

$16 \mathrm{Qa}$ heyakiye kta, Jehowa Heberi Taku Wakan dapi kin niye ekta u maśi qa, Mitaokiye, oyate mitawa kin, hopuze cin ekta ye wicaśi wo, eniciye maśi ; tuka dehanli warrayahon śni.

17 Ito Jehowa hecen eya, Jchowa he miye, de etanhan sdonyaye kta. Miś cansakadan minape ohna mduhe cin on mini wakpa ohna un kin awape kta; hecen mini kin we icu kta.

$18 \mathrm{Qa}$ hogan walrpa ohna yakonpi kin tapi kta, qa wakpa kin śicamna kta, hecen Egupton wakpa mini kin yatkanpi hitihdapi kta.

19 Unkan Jehowa Mowis heciya, Aaron heciya wo, Cansakadan ikikcu; qa Egupton mini tawapi ektakiya nape hdugata wo; wakpa tawapi ektakiya, qa "tawakpadanpi ektakiya, qa colsan tawapi ektakiya, qa minikiwitaya tawapi ektakiya, hecen hena we icu kta; qa Egupta makoce kin owancaya we yuke kta; nakun can-opiye ohna qa inyan opiye ohna.

20 Unkan Mowis qa Aaron token Jehowa econ wicaśi kin he iyecen econpi, qa cansakadan qon yuwankan icu, qa mini wakpa ohna un kin apa, he Paro wanyaka, qa taokiye wanyakapi; he- 
cen mini wakpa ohna un kin owasin we icu.

$21 \mathrm{Qa}$ hogan wakpa ohna yakonpi hin tapi, qa wakpa kin śicamna, qa Egupton mini wakpa etanhan yatkanpi okihipi śni ; qa Egupta makoce kin owancaya we yukan.

22 Unkan Egupton pejuta wicaśta iś eya taku wakanyan ohanyanpi on iyecen econpi, hecen Paro cante kin ihdusuta, qa nawicalion śni, Jehowa eye ciqon he iyecen.

23 Unkan Paro ihdamna, qa ti kin ekta kihda, qa he nakun ekta cante kin ye kiye śni.

24. Unkan Egupton owasin mini wakpa etanhan yatkanpi okihipi śni, heon mini yatkanpi kta e wakpa icahda qapi.

25 Unkan Jehowa wakpa kin apa ehantanhan anpetu śakowin wanna henakeca.

26 Unkan Jehowa Mowis heciya, Paro ekta ya, qa heciya wo ; Jehowa hecen eya, Oyate mitawa omakiyapi kta ye wicaśi wo.

27 Tuka yapi kta teyalida kinhan, inyun makoce nitawa owancaya hnaśka on awape kta.

$28 \mathrm{Qa}$ wakpa kin hnaśka ota icah ye kta, hecen hinanpapi qa yati kin en hiyupi kta, qa tipi ohna inonke cin mahentuya, qa owinja nitawa akan, qa nitaokiye tipi kin mahen, qa onna aguyapi śpanyayapi kin en; qa oyaptapi wopiye nitawapi kin en ;

29 Qa niye en, qa nitaoyate kin en, qa nitaokiye owasin en, linaśka wankan hiyupi kta.

\section{WICOWOYAKE 8.}

1 Unkan Jehowa Mowis heciya, Aaron hecen eciya wo; Cansakadan hduha ninape hdugata wo, wakpa kin ektakiya, qa wakpadan kin ektakiya, qa cokan eltakiya, hecen hnaśka kin Egupta makoce kin akan hinanpapikta.

2 Unkan Aaron Egupton mini tawapi ektakiya nape hdugata, unkan hnaśka kin hinanpapi qa Egupton tamakocepi kin akahpapi.

3 Unkan pejuta wicaśta wakanyan olianyanpi ece on hecen econpi, unkan hnaśka kin Egupta makoce kin akan hinanpapi.

4 Unkan Paro Mowis Aaron kici wicakico qa, Jehowa cekiyapo, hecen hnaśka kin yutokan kta ; miye etanhan qa oyate mitawa etanhan; kinhan oyate kin ye wicawaśi kta, qa Jehowa wakiciyuśnapi kta, eya.

5 Unkan Miye on ihdatan wo; tohantu kte, niye qa nitaokiye, qa nitaoyate wocekiye eciciyapi kta, qa hnaśka awihnunipi kta; niye etanhan qa yati kin etanhan; wakpa kin heceedan ohna ihanpi kta? Mowis Paro eciya. 6 Unkan, Heyakecinhan, eya; Unkan heya, Jehowa Wakan undapi kin he tuwedan iyececa śni sdonyaye kta e heon token elie cin hecetu kta;

7 Qa hnaśka kin aniyuśtanpi kta, qa yati kin, qa nitaokiye, qa oyate nitawa, wakpa heceedan ohna ihanpi kta.

$8 \mathrm{Qa}$ Mowis qa Aaron Paro etanhan inanpapi, qa Mowis Jehowa hoyekiya; hnaśka Paro en hiyuye ciqon heon etanhan.

9 Unkan Mowis eye cin he iyecen Jehowa econ, qa hnaśka kin tapi, tipi kin etanhan, qa tonwanyanpi kin etanhan qa maga etanhan.

10 Unkan hena mnayanpi paha ota, unkan makoce kin śicamna. 11 Unkan iyozi yukan he Paro 
wanyake ca cante kin ihdutke, qa nawicakion śni, Jehowa eye ciqon he iyecen.

12 Unkan Jehowa Mowis heciya, Aaron heciya wo, Cansakadan ye kiye ça makamdu kin apa wo, hecen Egupta makoce kin owancaya hoponkadan icage kta.

13 Unkan hecen econpi, qa Aaron cansakadan hduha nape lidugate ca makamdu lin apa, hecen hoponkadan icaga, wicaśta akan qa woteca akan, Egupta makoce kin owancaya makamdu kin owasin hoponkadan icaga.

14. Unkan Pejuta wicaśta taku wakanyan ohanyanpi on he iyecen econpi hoponkadan hinanpe yapi kta, tuka okihipi śni; hececa eśta hoponkalan yukan, wicaśta akan qa woteca akan.

15 Unkan Pejuta wicaśta lkin Wakantanka napsukaza kin dee, Paro eciyapi; tuka Paro cante kin ihdusuta, qa nawicahon śni; Jehowa éye ciqon he iyecen.

16 Unkan Jehowa Mowis heciya, Hanhanna hin lkikta, qa $\mathrm{Pa}-$ ro itokam inajin wo, iho mini elita inanpe lkta, qa heyakiye kta; Jehowa hecen eya, Oyate mitawa ye wicaśi wo, hecen omakiyapi kta.

17 'Tuka niye mitaoyate kin ye wicayaśi śni kinhan, ito miś tatawamduśka ye wicawaśi kta, niye en, qa nitaokiye en, qa nitaoyate en, qa yatipi kin en; hecen tatawamdnśka kin Egupton tipi kin ojun yapi kta, qa maka akan yakonpi kin he nakun.

18 Qa anpetu kin he en, Gośen makoce kin akan mitaoyate yakonpi kin he mdukinukan kte, he ohna tatawamduśka wanice kta; hecen maka cokaya kin Jehowa he miye sdonyaye kta.
19 Qa oyate mitawa oyate nitawa iyececapi kte śni wakage kta. Heyakecinhan wowapetokeca kin de tanin kta.

20 Unkan Jehowa hecen econ, qa tatawamduśka tehika Paro ti kin ekta hipi; qa taokiye tipi kin, qa Egupta makoce owancaya en, makoce kin awihnuni tatawamduśka kin on.

21 Hehan Paro Mowis Aaron kici wicakico, qa lieya, Hunktiya po, makoce kin ohna Taku Wakan yadapi kin wakiciyuśna po.

22 Unkan Mowis heya, Hecen econkupi kta iyecetu śni. Jehowa Wakan undapi waunkiciyuśnapi kin he Egupton wahte dapi śni. Inyun Egupton taku wahte dapi śni iśtapi kin itokam waunyuśnapi kinhan inyan on unkininpi kta.

23 Anpetu yamni imanipi hewoskantu kin ohna unyanpi, qa Jehowa Wakan undapi kin waunkiciyuśnapi kta, unkekiyapi qon he iyecen.

24 Unkan Paro heya, Ye ciśipi kta qa hewoskantu ohna Jehowa Wakan yadapi kin wayeciduśnapi kta, tuka sanpa tehan idadapi kte śni. Wocekiye emiciciya po.

25 Unkan Mowis heya, Ito miś niye etanhan inawape ca Jehowa cewakiye kta; unkan tatawamduśka, kin yutokan kta, Paro etanhan, qa taokiye etanhan, qa taoyate etanhan; tuka ihnuhan Paro ake wahnayan, qa oyate kin Jehowa wakiyuśnapi kta ye wicaśi śni.

26 Hehan Mowis Paro etanhan tankan inanpe ca Jehowa cekiya. 27 Unkan Jehowa Mowis eye ciqon he iyecen econ, qa tatawamduśka kin yutokan, Paro etanhan, qa taokiye etanhan, qa 


\section{HDINANPAPI,}

taoyate etanhan, wanjidan ihe śni.

28 Tuka Paro dehan nakun cante kin ihdutke, qa oyate kin ye wicaśi śni.

\section{WICOWOYAKE 9.}

1 Unkan Jehowa Mowis heciya, Paro ekta ya wo, unkan heyakiye kta; Jelowa Heberi Wakan dapi kin hecen eya, Oyate mitawa omakiyapi kta ye wicaśi wo.

2 Qa yapi kta teyahinda, qa nahanhin sutaya wicaduze cinhan;

3 Ito Jehowa nape kin wanunyanpi nitawa tinta ohna yakonpi kin akan un kta, śuktanka en, śuktanka śonśonna en, camo en, pte en, qa tahinca en makośice tehika hinca kta.

$4 \mathrm{Qa}$ Jehowa Israel tawanunyanpi, qa Egupton tawanunyanpi otahedan yukinukan kta, hecen Israel cinca taku tawapi kin owasin etanhan takudan te kte śni.

5 Nakun tohantu kte Jehowa yaotanin, qa heya; heyakecinhan Jehowa wicoie kin de econ kta, makoce ohna.

6 Unkan ihanhanna wicoie kin he econ; unkan Egupton tawanunyanpi kin owasin tapi, tuka Israel cinca tawanunyanpi wanjidan te śni.

7 Unkan Paro tuwe ekta ye śi, unkan inyun, Israel tawanunyanpi kin wanjidan te śni, tuka cante kin ihdutke qa oyatc kin ye wicaśi śni.

8 Unkan Jehowa Mowis Aaron kici hewicakiya, Maza śdoyapi oceti cahota kin ninape ohnakapi kin icu po, qa Mowis he mahpiya ektakiya kadada iyeye kta, Paro iśta kin itokam.

9 Qa he watuśekśeca kta, Egup- ta makoce kin owancaya, qa śiyaka wicao ton icaliye cin heca kta, wicaśta akan, qa woteca akan, Egupta makoce kin owancaya.

10 Unkan Maza śdoyapi oceti etanhan cahota icupi, qa Paro itokam inajinpi, qa Mowis he mahpiya ektakiya kadada iyeya. Unkan he siyaka wicao ton icali ye cin heca, wicaśta en, qa woteca en.

11 Unkan śiyaka wicao on etanhan pejuta wicaśta kin Mowis itokam najin okitpanipi ; nakaś śiyaka wicao pejuta wicaśta en, qa Egupton owasin en.

12 Tuka Jehowa Paro cante kin yusuta, hecen nawicahon śni, Jehowa Mowis eciye ciqon he iyecen.

13 Unkan Jehowa Mowis heciya, Hanhannahin kikta, qa Paro itokam inajin wo, qa heyakiye kta; Jehowa Heberi Wakan dapi kin hecen eya, Oyate mitawa ye wicaśi wo, unkan ohomadapi lita.

14 Dehan wowayazan mitawa owasin nicante ekta hiyu waye kta, qa nitaokiye en, qa nitaoyate en, hecen maka kin owancaya tuwedan iyemaceca śni sdonyaye kta, heon.

15 Nakaha minape ye wakiye ca acipe kta, qa oyate nitawa makośice on, qa maka kin etanhan nitanin kte śni ;

$16 \mathrm{Qa}$ awicakehan heon najin ciye, mitowaśake kin niye en wahdutanin kta; qa micaje kin maka kin owancaya oyakapi kte.

17 Dehanyan niś nihduwankantuya, oyate nitawa en, qa yapi kta iyowin wacayakiye sni.

18 Ito miś heyakecinhan dehantu Egupta ohna hinhewaye kta, wasu tehika hinca anpetu tokaheya en ounyanpi ehantan-

92 
han ecen dehanyan, tohinni hececa śni.

19 Heon wanna ye wicaśî, qa nita wanunyanpi kin nape wicaya wo, qa taku nitawa tinta en un kin owasin, wicaśta, qa woteca tona tinta ohna yakonpi, qa tipi en mnawicayapi śni, hena owasin wasu ahinhewicaye kta, unkan țapi kta.

20 Paro taokiye tuwewe Jehowa oie kin ikope cinhan iye taokiye, qa tawanunyanpi tipi kin ekta awicahdi.

21 Tuka tuwe Jehowa oie kin ekta cante kin ye kiye śni kinhan, taokiye qa tawanunyanpi kin tinta ohna elipe wicaya.

22 Unkan Jehowa Mowis heciya, Malipiya ek takiya nape hdugata wo, hecen Egupta makoce owancaya wasu yuke kta, wicaśta akan, qa woteca akan, qa tinta wato owasin akan, Egupta makoce ohna.

23 Unkan Mowis cansakadan hdugata makipiya ektakiya, unkan Jehowa hoton, qa wasu hiyuya, qa peta maka ainyanka, hecen Jehowa wasu Egupta makoce ahinhe kiya.

24 Unkan wasu, qa peta wasu kici icicahiya tehika linca, Egupton oyate yukan ehantanhan makoce owancaya ohna tohinni hececa śni.

25 Qa Egupta makoce kin owancaya wasu kin taku tankan un kin owasin apa, wicaśta, qa woteca, qa tinta wato kin owasin apa, qa wasu kin can iheye cin owasin kawega.

26 Tuka Gośen makoce kin Israel cinca ohna yakonpi kin, heceedan ohna wasu wanica.

27 Unkan Paro tuwe ekta ye śi, qa Mowis Aaron kici wicakico, qa hewicakiya; Dehan wawahtani, Jehowa hee ohan owo- tanna tuka miś qa oyate mitawa unśicapi.

28 Jehowa cekiya po, qa henakeca kta, sanpa Wakantanka hoton kte śni, qa wasu wanice kta'; unkan ye ciśipi kta, qa sanpa owanji nayajinpi kte śni.

29 Unkan Mowis heciya, Otonwe etanhan inanwape cinhan Jehowa ektakiya nape wahdumdaye kta hecen hoton kin henakeca kta, qa wasu kin sanpa wanice kta; heon Jehowa maka kin tawaya sdonyaye kta.

30 Tuka niye qa nitaokiye dehanyan Jehowa Wakantanka yakinihanpi kte śni sdonwaya.

31 Unkan hahonta on kagapi; qa barle kin hena kawankapi; nakaś barle kin wanna itka hinanpa, qa hahonta on kagapi wahica.

32 Tuka aguyapi hu kin qa wamnaheza kin hena kawankapi śni, nakaś hena oicah tehan.

33 Unkan Mowis Paro etanhan otonwe itankan inanpe ca nape hdumdaya, Jehowa ektakiya ; unkan hotonpi kin qa wasu kin anapta, qa magaju maka kin sanpa ahinhe śni.

34 Unkan magaju kin qa wasu kin qa hotonpi kin anaptapi, $\mathrm{Pa}$ ro wanyake ca ake wahtani, qa cante kin ihdutke ; iye qa taokiye nakun.

35 Hecen Paro cante kin ihdusuta, qa Israel cinca kin ye wicaśi śni, Jehowa Mowis on eye ciqon he iyecen.

\section{WICOWOYAKE 10.}

1 Unkan Jehowa Mowis heciya, Paro ekta ya wo, miś iye cante kin mdutke, qa taokiye cantepi kin nakun; hecen wicacokaya wowapetokeca kin dena wahulatanin kta, heon etanhan. 


\section{HDINANPAPI,}

2 Qa hecen Egupta ohna taku wakanyan owahanmda, qa wowapetokeca mitawa ecawicawecon kin hena nicinkśi, qa nitakoja owicayakidake kta, nogepi ohna, qa Jehowa he miye sdonyayapi kta.

3 Unkan Mowis qa Aaron Paro en hipi, qa heciyapi, Jehowa Heberi Wakan dapi kin hecen eya, Tohanyan mitokam nihduhukuya tawatenyaye kte sni he? Oyate mitawa ye wicaśi wo, unkan omakiyapi kta.

4. Tuka mitaoyate yapi kta teyahinda kinhan, ito heyakecinhan miś psipsicadan ota nitamakoce en hiyu waye kta.

5 Unkan maka itohnake kin akahpapi kta, hecen tuwe maka kin wanyake kte śni, qa taku nahanhin ecen han, taku wasu kin onicicaptapi kin he temyapi kta, qa maga ohna can nitawapi kin tona camni uye cin owasin temyapi kta.

6 Qa yati kin ohna otapi kta, qa nitaokiye tipi kin owasin, qa Egupton owasin tipi kin ohna otapi kta, niyatepi qa niyate ateyapi kin takudan he iyececa wanyakapi śni, maka akan unpi ehantanhan ecen anpetu kin de iyehantu; eya qa ihduhomni qa Paro etanhan inanpa.

7 Unkan Paro taokiye heciyapi, Tohanyan wicaśta kin de unhmunkapi kta he? Wicaśta kin Jehowa wakan dapi kin okiyapi kta e ye wicaśi wo ; Egupta awihnuni nahanhin sdonyaye śni he?

8 Unkan ake Mowis, Aaron kici Paro en hiyuwicayapi, unkan hewicakiya, Hunktiyapo, Jehowa Wakan yadapi kin okiya po ; tuwewe dapi kta he?

9 Unkan Mowis heya, Kośka, wicahinca ko unyanpi kte, unki- cincapi wica winyan ko opapi kta, talinca pte ko hduba unyanpi kta, Nakaś Jehowa wounkicihanpi kta.

10 Unkan hewicakiya, Niś nitaśicecapi ko ye ciśipi kta e Jehowa nicipi un kte. Wanyaka po ; nitokam taku śica yukan.

11 Hecetu śni do. Wicaśta tanka kin wanna yapo, qa Jehowa okiyapo yacinpi qon hee. Unkan Paro itokapatanhan ham wicayapi.

12 Unkan Jehowa Mowis heciya, Egupta makoce ektakiya nape hdugata wo, psipsicadan on, unkan Egupta makoce kin akan hiyupi kta, qa makoce wato kin owasin temyapi kte, taku wasu kin okapte cin iyulipa.

13 Unkan Mowis cansakadan kin hdugate, Egupta makoce kin ektakiya; unkan Jehowa wiyohiyanpa tanhan tateyanpa makoce en hiyuya, anpetu kin he osan, qa hanyetu osan, qa ihanhanna wiyohiyanpa tanhan tateyanpa kin psipsicadan hiyuya.

14 Unkan psipsicadan kin Egupta makoce owancaya iwankam iyayapi, qa Egupta ihdukśan owancaya iyahanpi, tehika hinca, hena itokam psipsicadan hececa wanica, qa iyohakam hececapi kte śni.

$15 \mathrm{Qa}$ makoce itohnake kin owancaya akahpapi, qa makoce kin aotpas yapi, qa makoce wato kin owasin temyapi, qa can waskuyeca wasu okapte cin nakun owasin; hecen Egupta makoce owancaya can akan, qa tinta peji olna takudan to ihe śni

16 Unkan Paro inahniyan Mowis Aaron kici wicakico, qa heya, Wawahtani, Jehowa Wakan yadapi kin en, qa niyepi nakun en. 
17 Qa wanna tokin wawahtani kin nakaha heceedan miyecicajujun, qa Jehowa Wakan yadapi kin cekiya po, hecen wiconte kin de heceedan miciyutokan kta.

18 Unkan Paro etanhan inanpe ca Jehowa cekiya.

19 Unkan Jehowa tateyanpa kin yuhomni wiyohpeya tanhan suta hinca, qa he psipsicadan icu, qa Mde śa en iyolipe wicaya, Egupta makoce owancaya psipsicadan wanjidan ihe śni.

20 Unkan Jehowa Paro cante kin yusuta unkan Israel cinca kin ye wicaśi. śni.

21 Unkan Jehowa Mowis heciya, Mahpiya ektakiya nape hdugata wo, hecen Egupta makoce akan otpaza kta, qa otpaza kin yutanpica kta.

22 Unkan Mowis mahpiya ektakiya nape hdugata, hecen Egupta makoce kin owancaya otpaza soka hinca yıkan, anpetu yamni.

23 Tuwedan sunkaku wanhdake śni, qa tuwedan towinja kin etanhan najin śni, anpetu yamni ; tuka Israel cinca owasin tipi kin ohna ojanjan wicayukan.

24 Unkan Paro Mowis kico qa heya, Hunktiyapo, Jehowa okiyapo nitaśicecapi nakun onipapi kta. Tahinca nitawapi qa pte nitawapi kin heceedun owanji yakonpi kta.

25 Unkan Mowis heya, Wośnapi, qa wohuknahyapi nakun hduhe unyakiyapi kta, hecen Jehowa Wakan undapi waunkiciyuśnapi kta.

26 Qa nakun wanunyanpi unkitawapi opapi kta, taśake wanjidan ihe kte śni. Hena etanhan Jehowa Wakan undapi kin ounkiyapi kta. Nakaś unkiś elkta unkipi śni hehanyan taku on Jehowa ounkiyapi kta sdon unkiyapi śni.
27 Unkan Jehowa Paro cante kin yusuta; unkan ye wicaśi tawatenye śni.

28 Qa Paro heciya, Mitan tanhan hunktiya wo. Ihnunhan miite kin ake wandake cinhan. Tohan miite wandake cinhan anpetu kin he en nite kta:

29 Unkan Mowis heya, Token ehe cin hecetu kta. Niite kin ake icimana wanmdake kte śni.

\section{WICOWOYAKE 11.}

1 Wanna Jehowa Mowis heciye ciqon, Ehake wowayazan wanjidan Paro akan hiyu waye kta, qa Egupton akan. He iyohakam detanhan ye niśipi kta, tohan ye niśipi kinhan ocowasin detanhan nicahapapi kta.

2 Wanna oyate nogepi ohna kewicakiya wo, qa wicaśta otoiyohi takodaku etanlıan, qa winohinca tuwe kodaye cin etanhan mazaska oinpi, qa mazaskazi oinpi kidapi kta.

3 Unkan Egupton kin oyate kin cante wicakiye Jehowa kaga. Nakun Mowis wicaśta kin tanka hinca, Egupta makoce ohna, Paro taokiye wicitokam, qa oyate kin wicitokam.

4. Unkan Mowis heya, Jehowa hecen eya, Hanyetu cokaya miś Egupton cokaya inanwape kta.

5 Qa tuwe caske owasin Egupta makoce ohna tapi kta Paro cinhintku tokapa oiyotanke tawa akan iyotanke kte cin etanhan, wikośka wiyukpan icahda iyotanke cin cinhintku tokapa kin aiyahdeya; nakun woteca cinca tokaheya wica tompi kin owasin.

6 Qa Egupta malroce owancaỳa woceye tanka kta; he iyececa hektapatanhan"wanica, qa tokata ake he iyecece kte sni. 
7 Tuka Israel cinca owasin ekta śunka wanjidan keś ceji hduśkan kte śni, wicaśta qa woteca unman tukte ekta. Hecen Egupton, qa Israel cinca kin otahedan Jehowa yukinukan sdon yayapi kta.

8 Qa nitaokiye dena owasin en mahipi qa miye en patujapi kta, qa Toki iyaya ye niye, qa oyate nihukuya unpi kin owasin, emalkiyapi kta, qa he iyohakam toki mde kta, eye ca Paro nina canniye cin etanhan inanpa.

9 Wanna Jehowa Mowis heciye ciqon, Mita wowapetokeca Egupta makoce ohna yuotapi kta; heon Paro nanihonpi kte śni.

10 Unkan Mowis Aaron kici wowapetokeca kin dena owasin Paro itokam econpi, unkan Jehowa Paro cante kin yusuta, heon iye tamakoce etanhan Israel cinca kin ye wicaśi śni.

\section{WICOWOYAKE 12.}

1. Wanna Jehowa Mowis Aaron kici Egupta makoce kin ohna hewicakiye ciqon.

2 Wi kin de wi itancan dawapi kta; omaka wi kin de tokaheya dawapi kta ce.

3 Israel omniciye owasin hecen ewicakiya po; Wi kin de anpetu iwikcemna kin tipi otoiyohi on tacincadan ikikcupi kta, ateyapi tipi kin iyececa.

4 Tuka tiyohnaka tonana on tacincadan yutapi okihipi śni kinhan, iye qa tuwe ikiyedan ti kin he kici icu kta, wicanagi yawapi iyecen, wicaśta otoiyohi token yute kta okihi, tacincadan on dawapi kta.

5 Tacincadan waśte, mdoka waniyetu wanjidan $\mathrm{kta}$, tahin wanunyanpi, qa iś tatokadan etanhan iyekcupi kta.
6 Qa wi kin de anpetu iaketopa kin, hehanyan kaśka yahduhapi kta, qa htayetu otahedan, Israel omniciye owasin he patapi kta.

7 Qa we kin etanhan icupi qa tośu tiyopa anokatanhan, qa iwankam amnimnipi kta, tipi ohna he yutapi kte cin he akan.

8 Qa hanyetu kin he en cehpi kin peta on ceonpapi yutapi kta, aguyapi onapohye codan, qa peji pa kici he yutapi kta.

9 Hetanhan ongedan saka qa mini ohna ohanpi yatapi kte śni, tuka petan ceonpapi; pa kin qa huha śupe kici yatapi kta.

10 Hetanhan ihanhanna hehanyan oyahdaptapi kte śni; qa ihanhanna hehanyan ongedan oyaptapi kinhan, he peta on huhnah yayapi kta.

11. Qa hecen yatapi kte, nite ipiyag yakitonpi, qa siha hanpa oyakihanpi, qa sagye nape ohna yahduhapi kta, qa inabniyan yatapi kta; Jehowa toacakśin kin hee.

12 Qa hanyetu kin de Egupta makoce opta mde ca tona tokapa Egupta makoce ohna, wicaśta woteca ko owasin awicawape kta, qa Egupton taku wakan dapi kin owasin akan woyaco ecamon kta. Jehowa he miye.

13 Unkan tipi ohna dukanpi kin hena we kin on wapetog yakitonpi kta, hecen we kin wanmdake ca niwankam acakśin mde ca Egupta makoce kin awape cinhan wowayazan on nițapi lrta iyonihipi kte śni.

14 Unkan anpetu kin de wokiksuye duhapi kta, qa he ohna wohanpi Jehowa woyecihanpi kta; nitoicagepi ohna wokage, owihanke wanica wohanpi yayapi kta.

15 Anpetu śakowin aguyapi 
onapoliye codan yatapi kta ; anpetu tokaheya awicakehan yatipi kin etanhan onapohye dutokanpi kta. Nakaś anpetu tokaheya etanhan anpetu iśakowin kiil hehanyan tuwe kaśta onapohye yute cinhan, wicanagi kin he Israel etanhan yutokanpi kta.

16 Anpetu tokaheya kin en wicakicopi wakan, qa anpetu iśakowin en wicakicopi wakan duhapi kte, anpetu kin hena en wohtani takudan econpi kte śni, tuka wicanagi iyohi taku yute kta wiyeya kagapi kta, heceedan ecanonpi kta.

$17 \mathrm{Qa}$ onapohye codan wohanpi kin yapatanpi kta, nakaś anpetu kin de en obe nitawapi kin Egupta makoce etanhan hdinanpe ciyapi, heon anpetu kin de yapatanpi kta, nitoicagepi yeye cin wokage owihanke wanica.

18. Wi tokaheya, anpetu iaketopa, litayetu etanhan. aguyapi onapohye codan yatapi kte, wi anpetu wikcemna nonpa sanpa wanjidan htayetu kta hehanyan.

19 Anpetu śakowin onapohye yatipi kin ohna iyeyapi kte śni, nakaś hehanyan tuwe taku napoliyapi yute cinhan wicanagi kin he oyate tokeca qa iś makoce he en tonpi kin unman tukte kaśta, Israel omniciye etanhan yutokanpi kta.

20 Takudan napohyapi yatapi kte śni. . Yatipi kin owasin ohna aguyapi napohyapi śni yatapi kta, eya.

21 Unkan Mowis Israel hunkayapi kin owasin wicakico, qa hewicakiya, Ekta hdapo, qa wicoun nitawapi on tacincadan ikikcupo, qa woacakśin.kin patapo.

$22 \mathrm{Qa}$ peji hota opahte wan iyacupi kta, qa we kin tiyopa icahda un kin he en oyaputkanpi, qa tośu tiyopa anokatanhan, qa iwankapatanhan ayamnimnipi kta, we tiyopa icahda un kin he on. Unkan ihanhanna kin hehanyan tuwedan iye tiyopa etanhan inanpe kte śni.

23 Hecen Jehowa Egupton wicakaśtaka hiyaye kta, qa tośu tiyopa anokatanhan qa iwankam hena akan we kin wanyake cinhan Jehowa tiyopa kin acakśin kta, qa waihang ye cin he anipe kta e yatipi en hiyu iyowinkiye kte śni.

24 Unkan wicoie kin de yapatanpi kta, he wokage owihanke wanica niye qa nicinca duhapi kta.

25 Qa hecetu kta, tohan makoce wan Jehowa niçupi kin ekta yaipi, eye ciqon iyecen, wohtani kin de oyapapi kta.

$26 \mathrm{Qa}$ tohan nicincapi kin, Wohtani kin de ecannonpi kin he taku he? eniciyapi kinhan;

27 Hehan hehapi kta, Woacakśin Jehowa wakiyuśnapi kin dee, Iye Egupton wicakaśtake cehan, Egupta makoce ohna Israel cinca tipi kin acakśin, qa untipi kin patan ce. Unkan oyate kin patujapi qa ohodapi.

28 Unkan Israel cinca kin ekta hdapi, qa token Jehowa Mowis Aaron kici econ wicaśi kin he iyecen econpi.

29 Unkan kaketu, hanyetu cokaya hehan Jehowa tona Egupta makoce ohna caske owasin wicakaśtaka, Paro cinhintku tokapa toiyotanke akan iyotanke kta etanhan, wayaka wicakaśka tipi ohna yanke cin cinhintku tokapa aiyahdeya, qa woteca cinca tokapa nakun owasin.

30 Unkan Paro hanyen kikta, iye qa taokiye owasin, qa Egupton owasin. Unkan Egupta 


\section{HDINANPAPI,}

ohna śicahowayapi tanka, nakaś tipi tukte tuwedan țe śni wanica.

31 Unkan hanyen Mowis, Aaron kici wicakico, qa heya, Najinpo, oyate mitawa wicehnatanhan inanpapo, niye qa nakun Israel cinca kin owasin, qa hunktiyapo, Jehowa okiyapo ehapi qon iyecen.

32 Nakun talinca nitawapi qa pte nitawapi kin ikikcupo, ehapi qon iyecen; nakun miś eya mayawaśtepo.

33 Unkan Egupton iyowicapaśtakapi, ga owasin unțapi se eyapi, heon oyate kin makoce etanhan ye wicayapi kta inalinipi.

34 Unkan oyate kin aguyapi mdu kin nahanhin napoliyapi śni ikikcupi, wakśica towokoyakepi ohna pahtapi tahpapi kin akan.

$35 \mathrm{Qa}$ Israel cinca kin Mowis eye ciqon he iyecen econpi, qa Egupton etanhan mazaska oinpi, qa mazaskazi oinpi, qa wokoyake dapi.

36 Unkan Egupton kin oyate kin cante wicakiyapi kin he Jehowa kaga; heon wicaqupi, unkan taku Egupton tawapi yuha iyayapi.

37 Qa Israel cinca kin Rameses etanhan ihdakapi, Sukot ekta, wicaśta kektopawinge opawinge śakpe Huninyunkenken manipi qa śicecapi kin hena wicayawapi śni.

$38 \mathrm{Qa}$ oyate tokeca wicota opapi qa tahinca qa pte, wanuyanpi ota hinca.

39 Qa aguyapi mdu Egupta etanhan yuha hdinanpapi kin he śpanyanpi, aguyapi hmiyanyan he napoliyapi śni, ecin Fgupta etunhan nape wicayapi, qa owanji unpi okihipi śni, qa nakun waneya içicagapi śni, nakaś.

40. Israel cinca Egupta en iyo98 tankapi kin wanna waniyetu opawinge topa sanpa wikcemna yamni en ounyanpi.

$41 \mathrm{Qa}$ waniyetu opawinge topa sanpa wikcemna yamni owihanketa, anpetu kin he en, Jehowa obe tawa owasin, Egupta etanhan hdinanpapi.

42 Hanyetu kin de Jehowa on awacinpi kta; Egupta makoce etanhan hdinanpe wicaya, heon Israel cinca kill owasin wicoicage yeye cin ecekcen Jehowa hanyetu tawa kin he awacinpi kta.

43 Unkan Jehowa Mowis Aaron kici hewicakiye ciqon; Wuacakśin wokonze kin dee, oyate tokeca cinca kin tuwedan hetanhan yute kte śni.

44 Tuka wicaśta otoiyohi taokiye mazaska on opetonpi, he bayahdaye cinhan, hehan hetanhan yute lita.

45 Tuwe oyate tokeca makoce en ti, qa iś tolitani opeyaton eśta he yute ktc śni.

46 Tipi wanjidan ohna he yutapi kta, qa cehpi kin ongedan tipi kin he etanhan tankan adapi kte śni, qa huhu kin wanjidan duwegapi kte śni.

47 Israel omniciye ocowasin heconpi kta.

$48 \mathrm{Qa}$ tuwe oyate tokeca niye kici ounye ça woacakśin Jchowa on ope kite cinhan, tona wica iye ti kin ohna owasin bahdayapi kte, hehan ikiyedan hi, qa hecon kta; hecen tuwe makoce ohna tonpi kin iyececa kta; tuka balidayapi śni tuwedan hetanhan yute kte śni.

49 Tona makoce en tonpi, qa oyate tokeca niyepi ehna ounyanpi kin wicoope wanjidan yuhapi kta.

50 Unkan token Jehowa Mowis qa Aaron econ wicaśi kin he iye- 


\section{WICOWOYAKE 13.}

cen Israel cinca kin owasin econpi.

51 Unkan anpetu kin he Jehowa Israel cinca kin obe tawapi kin ecekcen, Egupta makoce kin etanhan hdinanpe wicaya.

\section{WICOWOYAKE 13.}

1 Unkan Jehowa ie ca Mowis heciya,

2 Tona caske owasin miciyuwakan wo, Israel cinca kin ehna tona winyan tamni nalidoke cin wicaśta woteca ko owasin mitawa.

3 Unkan Mowis oyate kin hewicakiya, Anpetu kin de kiksuya po, he en Egupta makoce kin etanhan, wayaka tipi kin etanhan yahdinanpapi, Jehowa nape waśaka on hetanhan hdinanpe niyanpi, heon takudan napohyapi yutapi kte śni.

4 Aguyapi itka hinanpe, Abib wi kin anpetu kin de yahdinanpapi.

5 Unkan makoce wan Jehowa nicu kta e niyate wokonze on ewicakiya, Kanani qa Hiti kin, qa Amori kin qa Hiwi kin qa Jebusi kin tamakocepi kin he en Jehowa hiyuniye cinhan, makoce wan ohna pte asanpi qa canhanpi tiktica kaduze cin he ohna, wi kin de en wicohan kin de ecannon kta.

6 Anpetu śakowin hehanyan aguyapi napohyapi śni yate $k$ ta, qa anpetu iśakowin kin Jehowa wohanpi kicagapi kta.

7 Aguyapi napohyapi śni, anpetu śakowin yutapi kta, qa aguyapi napoliyapi nicipi wanyakapi kte śni, qa onnapohyapi kin makoce nitawapi kin ohna wanyakapi kte śni.

$8 \mathrm{Qa}$ anpetu kin he nicinkśi oyakidake ça heyakiye kta,
Egupta makoce etanhan wau qehan Jehowa taku ecamicon heou de ecamon.

9 Unkan he ninape akan wowapetokeca duhe kta, qa niiǵta otahedan wokiksuye kta, hecen Jehowa toope kin nii lsin ohna un kta; nakaś nape waśaka on Jehowa Egupta etanhan hiyu niyan.

10 Heon wokonze kin de omaka iyehantu ca oyape kta, waniyetu otoiyohi.

11 Unkan tohan Jehowa Kanani makoce kin ekta anıye cinhan, qa he niçu, niś qa nihunkakepi eniciya qon he iyecen ;

12 Hehan winyan cinca tokapa kin owasin, Jehowa on dutukan kta, qa wanunyanpi nitawa cinca tokapa mdoka kin owasin Jehowa tawa kta.

13 Qa śuktanka śonśonna cinca tokapa kin owasin opeyakiton $k t a$, tahinca cinca wan he iyopeyakiye kta, qa opeyakiton śni kinhan pakin yakakse kta; qa wicaśta nicincapi kin tona caskepi owasin opeyakiton kta.

14 Qa kaketu kta, tokata tohan nicinca, De taku hwo? eya iniwange cinhan, hehan heyakiye kta, Nape waśaka on Egupta etauhan, wayaka tipi kin etanhan, Jehowa hdirranpe unyanpi.

15 Unkan unhdapi Paro katinyan tehinda; heon Jehowa tona caskepi Egupta makoce ohna owasin wicakte, wicaśta cinca tokapa, qa nakun woteca cinca tokapa, heon tona caskepi owasin Jehowa wawakiyuśna, qa micinca caske opewakiton, ehe kta.

16 Qa he ninape akan wowapetokeca kta, qa ite akahpe niiśta otahedan niciyuke kta, nakaś nape waśaka on Jehowa Egupta etanhan hdinape niyanpi. 


\section{HDINANPAPI,}

17 Unkan kaketu, Paro oyate ye wicaśi qehan Wakantanka Pilistim tamakocepi canku kin ohna awicaye śni, he ptecedan tuka okini oyate ozuye wanyakapi kinhan iyope içiyapi qa Egupta ekta hdapi kta, eya.

18 Heon Wakantanka oyate kin wicayuhomni, qa Mde-śa lopuze canku kin ohna awicaya; unkan Israel cinca kin ipiyag kitonpi Egupta makoce kin etanhan inaupapi.

19 Unkan Mowis Josep huhu kin yuha hda, nakaś Israel cinca kin 'Taku Wakan icajeyan wicaśi qa heya, Awicakehan Wakantanka en nihipi kta, qa huhu mitawa yuha detanhan inayapapi kta.

20 Unkan Sukot etanhan ihdakapi, qa hopuze kin icahda Elam en etipi.

21 Unkan Jehowa wicitokam anpa eca mahpiya bosdata wan ohna, canku kin ohna ye wicaya, qa hanyetu eca peta busdata, hecen anpetu hanyetu ko, mani okihipi ece.

22 Anpetu eca mahpiya bosdata ewicakicihdaku śni, qa hanyetu eca peta bosdata wicitokapa tąnhan.

\section{WICOWOYAKE 14.}

1 Unkan Jehowa ie ca Mowis heciya,

2 Israel cinca kin decen ewicakiya wo ; Ihdamnapi qa Hahirot i kin itokanı etipi kta, Migdol qa Mde kin otahedan, Baal Zepon itokapatanhan he iyotakons, mde icahda eyatipi kta.

3 Unkan, Israel cinca kin makoce ohna nunipi hewoskan onawicataka, Paro eye kta.

4. Hecen Paro cante kin mdusuta kta, unkan tawicape kta; he- cen Paro qa ozuye tawa kin owasin etanhan wowitan waton kta; qa Jehowa he miye Egupton sdonyapi \%ta, eya. Unkan hecen econpi.

5 Unkan oyate kin najicapi kin Egupton wicaśta yatapi kin okiyakapi, hehan Paro cante kin, qa taokiye cantepi kin oyate ekta ayuhomnipi, qa tokeca de econkupi, qa Israel wowidag wicunyanpi qon tokan ye wicunśipi hwo? eyapi.

6 Qa canpahmihma aqin kicaton qa taoyate kin hduha eyaya.

7 Qa canpahmihma opawinge śakpe kahinili icu, qa Egupton tacanpahmihma owasin, qa otoiyohi ohna wicaśta yamni.

8 Unkan Jehowa Egupton wicaśta yatapi kin Paro cante kin yusuta; hecen Israel cinca kin tawicapa ; tuka Israel cinca kia nape hduwankan iyayapi.

9 Unkan Egupton lkin tawicapapi, qa Paro śuktanka tawa owasin, canpahmihma qa sulktanka akan iyotankapi, qa ozuye tawa kin wicakihdegapi, mde icahda wankapi, Hahirot i kin ekta Baal Zepon iyotakons.

10 Unkan Paro ikiyedan hi qehan, Israel cinca iśta wankan ikikcupi, unkan inyun Egupton wicihakam natan au, heon nina kokipapi, qa Israel cinca kin Jehowa hoyekiyapi.

11 Qa Mowis heciyapi, Egupta ekta wicahapi wanica nace, heon Ḣewoskan ohna untapi kta e hetanhan hiyu unyayapi he? Tokeca de ecaunyeconpi, qa Egupta etanhan hiyu unyayapi he?

12 Oie wan Egupta en unkenjciyapi quon dee seca. Unkayuśtanpo, hecen Egupton wowidag unyanpi kta, unkeyapi. Egupton wowidag unyanpi kin hee 
waśte, qa Ḣewoskan unțapi kin hee śica.

13 Unkan Mowis oyate kin hewicakiya, Ihnuhan koyakipapi kin. Owanji najîn po, hecerı Jehowa woehdaku ecanionpi kte cin he wandakapi kta ; Egupton token nakaha wandakapi, he iyecen ake icimana wanwicadakapi kte śni.

14 Jehowa niyepi on kize kta, qa niś inina dukanpi kta.

15 Unkan Jehowa Mowis heciya, Tolkeca miye hoyemayakiya he? Israel cinca kin ihdag wicaśi wo.

$16 \mathrm{Qa}$ niśs cansakadan yuwankan ikikcu, qa ninape kin mde kin iwankan ye kiya wo; qa he yuakipam iyeya wo, hecen Israel cinca kin mde cokaya maka puza ohna manipi kta.

17 Unkan miś Egupton cantepi kin mdusuta, kta, hecen vicapasipi kta, qa Paro on, qa ozuye tawa ocowasin, canpahmihma qa śuktanka akan yotankapi on mayutanpi kta.

18 Qa Paro on, canpahmihma tawa on, qa śuktanka akan yotankapi on, wowitan waton kinhan hehan Jehowa he miye, Lgupton sdonyapi kta, eya.

19 Unkan Wakantanka ohnihde kin Israel ihdakapi wicitokam mani qon śkanśkan, qa wicihektam u, qa malipiya bosdata wicitokapatanhan iyaya, qa wicihakam najin.

20 Qa Egupton owanka kin, qa Israel owanka kin otahedan $u$, qa malipiya śapa qa otpaza heca, tuka dena hanyen iyoyamya, hecen hanyetn osan icikiyedan upi śni.

21 Unkan Mowis mde kin iwankam nape hdugata, unkan Jehowa hanyetu osan wiyohiyanpatanhan tateyanpa waśaka on mde kin yutokan, mini kin kicaśpe ca mde kin puza kaga.

22 Unikan Israel cinca kin mde colsaya ipi, puze rin ohna, qa etapa tanhan catka tanhan ko mini kin aconkaślke wicaya.

23 Unkan Egupton kuwa awicayapi, qa Paro śuktanka tawa owasin, canpahmihma, suktanka akan yotankapi koya, mde kin cokaya wicinakam ipi.

24 Unkan hanhanna irvaktapi hehan, Jehowa Egupton ihdakapi ekta etonwan, peta bosdata qa malipiya śapa ohna, qa Égupton ihdakapi kin yuśinye wicaya.

25 Qa canpahmihma huha kin yuśdoka hecen tketkeya tukśupi; unkan Jehowa Israel on Egupton wicakiza, heon etanhan Israel etanhan naunjicapi kta, Egupton eyapi.

26 Hehan Jehowa Mowis heciya, Mue kin ek takiya ninape kiyugata wo, hecen mini kin ecen hdiwanke kta ligupton akan, canpahmihma tawapi, qa śuk tanka tawapi akan.

27 Unkan Mowis mde kin iwankam nape yekiya; unkan hanhanna hehan mde kin token un qou he iyecen hdiwanka; unkan Ligupton najicapi kin he itkokipapi, hecen Jehowa Hguptun kin mde cokaya ehpe wicaya.

28 Unkan mini hdiwanka qa canpahmihnıa, śuktanka akan yotankapi ko awicakahpa, Paro ozuye tawa owasin, tona wicihakam mde kin en hipi wanjidan kaś ni śni.

29 Unkan Israel cinca kin mde cokaya puze cin amanipi, qa etapa tanhan catka tanlian ko mini kin aconkaśke wicaya.

30 Unkan Jehowa anpetu kin he en Israel niwicaya, Egupton napepi etanhan. Unkan Israel 101 


\section{HDINANPAPI,}

Egupton țapi wanwicayaka, inde kin hutata ekta.

31 Qa Israel wicohan tanka Jehowa Egupton ecawicakicon kin he wanyakapi, qa oyate kin Jehowa kokipapi, qa Jehowa wacinyanpi, taokiye Mowis nakun.

\section{WICOWOYAKE 15.}

1 Hehan Mowis Israel cinca ko odowan kin de Jehowa kahiyayapi, qa heyapi.

Jehowa iwadowan kta, wowitan tanka içicaga.

Śuktanka qa tuwe akan iyotanke cin mde kin en elipe wicaya.

2 Ja mitowaśake qa mitadowan lzin hee, qa nakaha nimayan.

Taku wakan wada kin hee, heon mdatan kta.

A te taku wakanda, heon, Imdawankantu ye kta.

3 Jehowa wicaśta kicizapi itancan kin heca, caje tawa kin Jehowa ee.

4 Paro tacanpahmihma, ozuye tawa kol mde en elipe wicaya.

Unkan taakicita kahnigapi mde śa ohna minin țapi.

5 Wośbe kin awicakahpa; mini śbe kin ohna kun iyayapi; inyan wan iyecen.

6 Jehowa ninape etapa kin wowaśake on yutanpi ce.

Ninape etapa, Jehowa, toka kin wicakawanka.

₹ Nitowitan tanka on tona nicipajinpi kin ihang wicayaya.

Nitocanniye kin yeyaliye cin he peji śeca iyecen huhnahwicaya.

8 Nipoge oniye kin on mini kin kiwitayapi.

Minitan kin paha iyecen bosdan najin. ieu.

Mde cokaya. wośbe kin tasag
9 Toka kin heya, Wicawakuwa kta ewicawehdege ktal woyuha wapamni kta, hena on minagi imnayanpi kta, isan wahduśdoke kta, minape ihang wicaye kta.

10 Tate nitawa ipoh yalkiya,

Mde kin awicakahpa.

Mini waśaka aohdute wicaya, máza su iyecen. [!

11 Jehowa taku wakan ehna tuwe iyeniceca?

Tuwe iyeniceca wowakan on yutanpi kta? I"

Yatanpi kin nicinihanpi kta, wowape tokeca ecannon.

12 Ninape etapa kin yeyakiya, maka kin nawicapca.

13 Oyate kin de opewicayakiton, nitocantekiye ón awicada.

Nitowaśake on oyanke wakan nitawá kin en/ híyuwícayáye kta.

I4 Oyate nahonpi qa kokipapi kta, [Palestina ounyanpi kin wokokipe wícayưze kta.

15 Hehan Ldom itancanpi kin wicáyuśin yayápi kta.

Moab wicaśta waśaka wocancan wicayúze kta.!

Kanan ounyanpi iyuhpa skánpi kta.

16 Nibinciyapi, wokokipe ko awicáhinhipaye kta.

Niisto tanka kin on tasag tapi kta, inyan iyecen.

Oyate nitawa iyoopta iyaye kte cin heharyan, Jehowa;

Oyate kin de opeyakiton iyoopta iyaye kte cin héhanyan.

17 He nitawa kin he ekta hiyu wicáyaye ça ohna hu bde wícayaye kta.

Oyanke ohna yakidótanké lrta elwiyeya yakaga, Jehowa.

Tipi wakan, I tancan, ninape niçicage kte cin he ekta.

18 Jehowa owihanke wanin wicaśtá yatápi kta. 
19 Hecen Paro suktanka tawa, canpahmihma qa śuktanka akan yotankapi kin mde lkin en hipi, unkan Jehowa mde mini kin hena akan awicahdi; tuka Israel cinca kin mde cokaya taku puze cin amanipi.

20 Unkan Miriam, winyan wokcan, Aaron tankeku cancega wan nape ohna icu, unkan winohinca owasin cancega yuha opapi, qa wacipi.

21 Unkan Miriam awicayupta, qa heya, Jehowa idowanpo, wowitan tanka icicaga, suktanka qa akan yotanka kin he mde en elipe wicaya.

22 Unkan Mowis Israel Mdeśa etanhan ihdag wicaya, unkan Śur hopuze kin elkta ipi, qa anpetu yamni Hopuza ohna manipi, qa mini iyeyapi śni.

$23 \mathrm{Qa}$ Mara en hipi qa Mara mini yatkanpi okihipi śni, ecin pa ce, heon he Mara (pa hee) eciyapi.

24 Unkan oyate kin Mowis kipajinpi qa taku unyatkanpi kta hwo? eyapi.

25 Unkan Jehowa hoyekiya, unkan Jehowa he can wan sdonyekiya; he mini kin en elipe ye cin hehan mini waśte icu. Hen wokage wan qa woyaco wan wicaqu, qa hen iyutan wicaya.

26 Qa heya, Jehowa wakan yada ho kin ohinniyan anayagoptan, qa taku iye iśta kin ohna ecetu kin hecannon, qa woahope tawa kin owasin nayahion, qa wokage tawa owasin duhe cinhan, wowayazan tona Egupton awicawahnaka wanjidan acihnake kte śni, nakaś Jehowa asniniye cin he miye.

27 Unkan Elim en hipi, hen miniyowe ake nonpa, qa tamar can. wikcemna śakowin, qa hen etipi mini icahda.
WICOWOYAKE 16.

1 Unkan Elim etanhan ihdakapi, qa Egupta makoce etanhan iyayapi, wi inonpa anpetu iake zaptan hehan Israel cinca omniciye owasin Sin Hopuze cin elkta hipi; he Elim qa Sina otahedan.

2 Qa Israel cinca omniciye ocowasin Hopuze cin ohna Mowis qa Aaroll en kipajinpi.

3 Qa Israel cinca kin hewicakiyapi, Tokin Egupta makoce ohna Jehowa nape on untapin, cehpi cega icahda iyotankehan aguyapi imnahan untapi qehan; omniciye kin de ocowasin akihan te unyayapi kta e Hopuze cin de ekta hiyu unyayapi.

4. Unkan Jehowa Mowis heciya, Inyun, malipiya etanhan agruyapi amagaju ciciyapi kta, qa oyate kin tankan yapi, qa anpetu otoiyohi taku anpetu wan iyohiye cin he mnayanpi kta; hecen iyutan wicawaye kta, mitoope omanipi kta, qaiś omanipi kte śni.

5 Qa anpetu iśakpe hehan taku anpetu otoiyohi mnayanpi kin he nonpa akihde ahdipi kta, qa he śpanyanpi kta.

6 Unkan Mowis Aaron kici Israel cinca kin owasin hewicakiya, Ḣtayetu kinhan Egupta makoce etanhan hdinanpe niyanpi kin Jehowa hee sdonyayapi kta. 7 Qa hanhanna eca Jehowa towitan kin wandakapi kta; Jehowa en wiyahnupi kin he nation, tuka unkiyepi untakupi hwo? on wiunyahnupi he?

8 Qa Mowis heya, Hitayetu eca Jehowa cehpi yatapi kta e nicupi, qa hanhanna ca aguyapi inimnapi kta, heon etanhan Jehowa wiyahnupi kin iś iye en wiya103 
hnupi kin he nalion sdonyayapi kta ; tuka unkiś untakupi he? unkiyepi en wiunyahnupi śni, tuka Jehowa he en.

9 Unkan Mowis Aaron heciya, Israel cinca omniciye ocowasin hewicakiya wo; Jehowa wiyahnupi kin nahon, heoll ikiyedan upo.

10 Unkan Aaron Israel omniciye ocowasin ewicakiye cin he icunhan, Hopuze cin elrta ihduhomnipi, qa inyun, Jehowa towitan kin malipiya soka kin ohna wanyakapi

11 Unkan Jehowa ie ca Mowis heciya ;

12 Israel cinca wihnupi kin nawahon, hecen ewicakiya wo, Htayetu eca celipi yatapi ece $k t a$, qa hanlianna eca aguyapi inimnapi kta, qa Jehowa wakan yadapi kin he miye sdonyayapi kta.

13 Unkan htayetu śiyo en hipi qa owanke kin akahpapi, qa hanhanna eca owanke ihdukśan cu kin makata wanka ece.

$14 \mathrm{Qa}$ cu makata wanke cin akan hiyu qehan, inyun liopuze ite akan taku cistinna wa suta iyecen, hewanke iyecen cistinna maka akan wanka.

15 Unkan Israel cinca kin he wanyakapi, qa wicaśta otoiyohi hunkawanjitku kin hekiciyapi, Man hu (de taku hee:) eya; nakaś he taku sdonyapi śni. Unkan Mowis hewicakiya, Aguyapi yutapi kte Jehowa niçupi kin dee.

16 Taku Jehowa econ niśipi kin dee, Hetanhan mnayanpo, wicaśta otoiyohi token yute lita iyacinyan wicaśta iyohi, wicanagi tona wakeya tawa ohna yakonpi, pahu otoiyohi on omer iyutapi wanjidan icu kta.

17 Unkan Israel cinca kin ecen econpi, qa hunli ota mnayanpi, qa hunh aoptetu mnayanpi.

$18 \mathrm{Qa}$ omer ohna iyutapi qehan tuwe ota mnaye cin he taku iyakicuya yuhe śni, qa tuwe cistinna mnaye cin he aokpani yuhe śni; otoiyohi token yute kta iyececa hecen mnayan.

19 Unkan Mowis hewicakiya; Ihnuhan tuwe hetanhan ihanhanna hehanyan onge oyapte cinhan.

20 Unkan Mowis nahonpi śni, qa wicaśta wanjikśi ihanhanna kin hehanyan onge oyaptapi; unkan he śicamna qa ohna wamdudan icagapi; unkan Mowis canniye wicaya.

$21 \mathrm{He}$ hanhanna otoiyohi mnayanpi, wicaśta otoiyohi token yute kta iyececa; tuka anpetu wi kin kate cin hehan skan.

22 Unkan anpetu iśakpe hehan aguyapi nonpa akihde mnayanpi, o toiyohi on omer iyutapi nonpa ; unkan omniciye itancan kin owasin en hipi, qa Mowis okiyakapi. 23 Unkan hewicakiya, Jehowa eye ciqon dee, Heyakecinhan Sabaton Jehowa okilipapi wakan tawa kin hee. Taku aguyayapi lte cin he aguyapo, qa taku cega en oyahanpi kte cin he ohanpo, qa taku ihe cin owasin kihnakapo; heyakecinhan hehanyan he yahduhapi kta.

24 Unkan lianhanna hehanyan kihnakapi, Mowis econ wicaśi kin he iyecen; tuka śicamna śni, qa ohna wamdudan yuke śni.

25 Unkan Mowis heya, Nakaha he yutapo, anpetı kin de Jehowa okihpapi tawa kin ee. Ecin tinta ekta he iyeyayapi kte śni.

26 Anpetu śakpe he mnayayapi kta, qa anpetu iśakowin kin he okilipapi, he icunhan wanice kta. 
27 Unkan anpetr iśakowin kin hehan oyate etanlun wanjikśi mnayanpi kta tankun ipi, tuka takudan iyeyapi śni.

28 Unkan Jehowa Mowis heciya, Tohanyan mitawoahope, qa mitoope oyapapi tawaten yayapi kte śni he.

29 Wanyakapo, Jehowa okihipapi nicupi kin, heon anpetu iśakpe kin he en nonpa can on woyute nicupi, owanji yankapo, otoiyohi tohe kin ohna. Anpetu iśakowin icunhan tuwedan oyanke tawa etanhan tankan iyaye kte śni, eya.

30 Unkan oyate kin anpetu iśakowin kin he en okilipapi.

31 Qa Israel ti kin he Manna eya caje yatapi, he taku su kin iyececa ska, qa he utapi kin he aguyapi zibzipedan canhanpi tiktica icicahiyapi iyececa.

32 Unkan - Mowis heya, Taku Jehowa econ niśipi kin dee, hetanhan omer wanjidan ojuyapo he inicagapi yeye cin yuha yaunpi kta; hecen Egupta makoce etanhan hdinanpe ciyapi qehan, Hopuze cin ohna aguyapi yun ciyapi kin he wanyakapi kta.

33 Unkan Mowis Aaron heciya, Cega wan icu qa he ohna manna omer-iyutapi wan yeya wo, qa he Jehowa itokam kihnaka wo, Inicagapi yeye cin he yulia unpi kta.

34 Unkan Aaron he woyaatanin kin itokam, he yuhapi kta e kihnaka, Jehowa Mowis econ śi qon he iyecen.

35 Unkan Israel cinca kin unanna yutapi, waniyetu wikcemna tom hehanyan, makoce ohna woju tipi wan en ipi kin hehanyan, Kanan makoce ihanke kin en ipi kin hehanyan manna kin yutapi. 36 Unkan omer iyutapi wikcemna ca epah wanji ojunyanpi.

\section{WICOWOYAKE 17.}

1 Unkan Israel cinca kin omniciye owasin Jehowa oie kin eciyatanhan ihdakapi kin hena Sin lopuze etanhan ihdakapi, qa Repidim en etipi tuka oyate yatkanpi kta e mini wanica.

2 Unkan oyate kin Mowis iyope yapi qa, Mini unqupo, unyatkanpi kta eyapi ; unkan, Tokeca miś iyope mayayapi? Tokeca Jehowa iyutan yayapi? Mowis ewicakiya.

3 Unkan hen oyate kin mini on ipuzapi, qa oyate kin Mowis i en hiyeyapi, qa, 'De tokeca hwo? Egupta etanhan inape unyayapi qa ipuza te mayaye kta, qa micinca, qa mitawanuyanpi kin, eyapi.

4 Unkan Mowis Jehowa hoyekiya, qa, Oyate kin de taku ecawicawecon kta he? unhanketa e inyan on makininpi kta, eya.

5 Unkan Jehowa Mowis heciya, Oyate kin itokam ya wo, qa Israel hunkayapi etanhan ope wicayaśi kta, qa cansakadan on wakpa ayape ciqon ninape ohna ikikcu, qa iyaya wo.

6 Inyun miś Ḣoreb heciya imnija kin akan nitokam iyawahe kta, qa imnija kin ayape kta, qa hetanhan mini hiyu kta; hecen oyate kin mini yatkanpi kta. Unkan Israel hunkayapi kin wanyakapi, Mowis hecen econ.

7 Unkan oyanke kin he Masa (Iyutanyanpi) qa Meriba (Iyopeyapi) cajeyata, Israel cinca kin iyopeyapi qa Jehowa iyutan yanpi, qa Jehowa unkiyopeya un, qaiś un śni he? eyapi kin heon etanhan.

8 Hehan Amaleqi en hipi, qa Repidim ohna Israel azuya.

9 Unkan Mowis Jośuwa heciya, 


\section{HDINANPAPI,}

Wicaśta unkicicaliniga wo, qa Amaleqi wicakiza wo. Heyakecinhan Wakantanka cansakadan tawa nape ohna yuha paha iyawahe kta.

10 Unkan Jośuwa Amaleqi wicakiza, token Mowis eciye cin he iyecen econ. Unkan Mowis, Aaron qa Ḧur paha akan itanwankanhdeya iyayapi.

11 Unkan tohan Mowis nape kin wankantuya hdugate hehanyan Israel ohiya, qa tohan nape ozikiya ca Amaleqi ohiya.

12 Unkan Mowis nape kin tke lieon iny an wan icupi, qa ihukuya ehnakapi; 'unkan he akan iyotanka; hehan Aaron Hur kici nape en yuzapi, unman etapa tanhan, qa unman catka tanhan; hecen nape kin patin anpetu wi kin iyaya, hehanyan.

13 Unkan Jośuwa tacanlipi hi kin on Amaleqi, qa taoyate wicakawanka.

14 Unkan Jehowa Mowis heciya; Wokiksuye kin de wowapi wan en kage ça Jośuwa noge kin en kihnaka wo. Amaleqi kiksuyapi kin mahpiya ihukuya tanhan wapajuju kta.

15 Unkan Mowis wahna wośnapi wan kage ca, Jehowa Nisi (Mitawiyokihedan) eya caje yata.

16 Qa heya, Nape kin Ja toiyotanke kin akan heon wicoicage yeye cin ohinniyan Jehowa Amaleqi kize kta.

\section{WICOWOYAKE 18.}

1 Unkan Jetro Mowis tunkanku, Midian wawayuśna kin, Wakantanka taoyate Israel, qa Mowis token ecawicakicon owasin nahon, Jehowa Israel Egupta etanhan hdinanpe wicaye cin he.

2 Hehan Jetro Mowis tunkanku kin Zipora Mowis tawicu kin icu, he kihdeye ciqon iyohakam.

$3 \mathrm{Qa}$ cinhintku nonpa, tokapa Gerśom (Hen ounwaya) eciyapi, Makoce tokeca obna ounwaya, eya, heon etanhan.

4 Qa unman Eliezer (Taku wakan wada onnakiya) eciyapi; A te Taku Wakanda kin omakiye ca Paro tacanlipi kin etanhan emahdaku, eya, heon etanhan.

5 Unkan Wakantanka he tawa kin icahda, hopuze ohna Mowis ti kin he ekta Jetro Mowis tunkanku hi, qa cinhintku qa tawicu nakun.

$6 \mathrm{Qa}$ Mowis heciya, Nitunkan Jetro he miye, niye en wahi, nakun nitawill, qa nicinca nonpa opapi.

7 Unkan Mowis, iye tunkanku itkokipe lkta tankan iyaye ca pakicapsan, qa i iputaka, qa Zaniyan unpi liecinhan ikiciwangapi, qa wakeya kin en hipi.

8 Unkan Jehowa taku Paro qa Egupton ecawicakicon, Israel on etanhan owasin Mowis iye tunkanku okiyaka, taku tehika canku ohna awicakipa owasin, qa Jehowa ewicahdaku kin nakun, Mowis iye tunkanku okiyaka.

9 Unkan Jehowa Egupton napepi etanhan Israel ehdaku, qa taku waśte ecawicakicon owa.. sin on Jetro iyuśkin.

10 Qa Jetro heya, Jehowa Egupton napepi etanhan, qa $\mathrm{Pa}-$ ro nape etanhan enihdakupi kin he yawaśtepi nunwe. He Egupton napepi ihukuya tanhan taoyate kin ewicahdaku.

11 Wanna Jehowa taku wakan kin owasin isanpa tanka ee sdonwaya, taku en wahan içidaya śkanpi kin he en ohiwicaya ce.

12 Unkan Mowis tunkanku Jetro wośnapi, qa wohulinah yapi Wakantanka wakiciyuśna. Un-

106 
kan Aaron qa Israel hunkayapi, Wakantanka itokam, Mowis tunkanku kici aguyapi yutapi kta en hipi.

13 Unkan ihanlianna Mowis oyate kin wicayaco kta e iyotankahan yanka. Unkan oyate kin Mowis ihdukśan najinpi, hanhanna etanhan ecen hitayetu.

14 Unkan Mowis tunkanku taku iye taoyate kin ecawicakicon owasin wanyake ca heya, De taku oyate kin ecawicayecon hwo? Tokeca niś nana yakidotanka, qa oyate kin owasin hanhanna etanhan ecen hitayetu nihdukśan najinpi he?

15 Unkan Mowis iye tunkanku heciya, Oyate kin Wakantanka iwangapi kta en mahipi ece.

16 Nakaś taku akinicapi eca en mahipi ece, qa wicaśta wiciyotahedan mdaco, qa Wakantanka tokage, toope, ko sdonye wicawakiya ece.

17 Unkan Mowis tunkanku kin heya, Taku ecannon kin de waśte śni.

18 Ḧanyaye kta, niś qa oyate kin de onipapi owasin nakun; 'Taku kin de tke heon niś nana hecon oyakihi śni nakaś.

19 Wanna maho kin nahon, wahokon ciciya, qa Wakantanka nici un kta. Oyate kin ie ska niyanpi kta, Wakantanka ekta, qa oiepi kin Wakantanka oyakidake kta.

$20 \mathrm{Qa}$ wokage, woope ko onspe wicayakiyé kta, qa canku ohna omanipi kta, qa woecon econpi kte cin hena sdonye wicayakiye kta.

$21 \mathrm{Qa}$ niye oyate kin owasin etanhan wicaśta waditaka yakahnige kta, wicaśta wicakapi, Wakantanka kokipapi qa basminyanpi kin sice dakapi kin hecapi, ua kektopawingege itancanpi, opawingege itancanpi, "qa wikcemna zaptanptan itancanpi, qa wikcemnamna itancanpi kin hena wicayecage kta.

22 Unkan hena ohinniyan oyate kin yacopi kta, qa taku tanka kin owasin en anihipi kta; hecen iyepi niciçinpi kta qa kapojedan nicicagapi kta.

23 Taku kin de ecannon, qa Wakantanka hecon niśi kinhan, najinhan oyakihi kta, unkan oyate kin de owasin oyanke tawapi kin en zaniyan kipi kta.

24 Unkan Mowis iye tunkanku ho kin anagoptan, qa token eye cin owasin ecen econ.

$25 \mathrm{Qa}$ Mowis. Israel owasin etanhan wicaśta waditaka wicakahnige ca oyate pa wicayapi kta, kelktopawingege itancanpi, opawingege itancanpi, wikcemna zaptanptan itancanpi, qa wikcemnamna itancanpi wicakaga.

26 Unkan hena ohinniyan oyate kin yacopi, taku tehike cin he Mowis en ahipi, qa taku cistinna akinicapi kin he iye yacopi.

27 Unkan Mowis iye tunkanku kihde ya; unkan iye tamakoce kin ekta kihda.

\section{WICOWOYAKE 19.}

1 Israel cinca Egupta makoce etanhan inanpapi kin etanhan, wi iyamni, wi tanin kin anpetu kin en Sina ho puze cin en hipi.

2 Nakaš Repidim etanhan ihdakapi qon, qa Hopuze en hipi, qa Hopuze ohna e tipi, qa Israel hen wankapi he itokam.

3 Unkan Mowis itanwankanhdeya Wakantanka ekta i, unkan Jehowa paha kin etanhan hoyekiye ça heya, Jakob tiyohnaka hecen eyakiye ça Israel cinca kin owicayakidake kta; 
4. Taku Egupton ecawicawecon niś wandakapi, qa Wamdi hupahu akan ciyuhapi, qa miye en cicahipi.

5 Qa wanna maho kin katinyan nayahonpi, qa wicotakuye mitawa yapatanpi kinhan, maka kin ocowasin mitawa, tuka oyate owasin isanpa miś tawa ciyapi kta.

6 Unkan niye oyate wakan, qa wicaśta yatapi wawayuśnapi ciyuhapi kta. Wicoie kin hena Israel cinca kin owicayakidake lita.

7 Unkan Mowis en hi, qa oyate hunkayapi kin wicakico, qa wicoie kin hena owasin wicitokam ehde, Jehowa econ śi kin iyecen.

8 Unkan oyate kin owasin okonwanjidan ayuptapi qa heyapi, Token Jehowa eye cin owasin ecen econkupi kta. Unkan Mowis itkom oyate oiepi kin Jehowa okiyaka.

9 Unkan Jehowa Mowis heciya, Inyun mahpiya śoka wan ohna en cihi kta, hecen ociciye cin he oyate kin nahonpi kta, qa owihanke wanin wicanidapi kta. Unkan Mowis oyate oiepi kin Jehowa oliyaka.

10 Unkan Jehowa Mowis lieciya, Oyate kin ekta hda, qa wicayuwakan wo, ecin qa nakun heyakecinhan, qa wokoyake hdujajapi kta.

11 Unkan anpetu iyamni on wiyeya icihnakapi kte, anpetu iyamni kin hehan Jehowa kun u, qa Sina paha akan iyahe kte cin he oyate owasin wanyakapi kta.

12 Unkan ihdukśan oyate kin iwicayecago kta, qa hehe lita, Ihnuhan paha akan idadapi kin, qa ihanke kin eśta odutanpi kin; tuwe kaśta he yutan kinhan ktepi kta.
13 Ihnuhan wicanape wanjidan he yutan kin, tuka inyan on kininpi kta qa iś wanhinkpe on ipahdan iheyapi kte, woteca wicaśta unman tukte laśta ni kte śni. Maza yahotonpi tehan ho ton kinhan iyepi paha en itanwankanhdeya yapi kte.

14. Unkan Mowis paha kin etanhan apamahdedan ku, oyate kin ekta, qa oyate kin wicayuwakan, unkan wokoyake kin hdujajapi.

15 Unkan oyate kin hewicakiya, Anpetu iyamni on wiyeya un po. Ihnuhan winolinca odutanpi kin. 16 Unkan anpetu iyamni hanhanna hehan wakinyan hotonpi, qa wakanhdi, qa paha akan mahpiya wan. śoka hinca, qa maza yahotonpi nina hoton, unkan oyate kin owanka ohna owasin cancanpi.

17 Unkan Mowis oyate kin Wakantanka itkokipapi kta owanke etanhan inanpe wicaya; unkan paha ihukuya inajinpi.

18 Unkan Jehowa peta ohna Sina paha akan kun hiyu, heon paha kin owancaya śotoju, qa śota wanlzan hiyaya, maza śdoyapi oceti śota kin iyececa, qa paha kin owancaya nina cancan.

19 Unkan maza yahotonpi kin tehan nina hoton qehan, Mowis ia, unkan Wakantanka ho wan on ayupta.

20 Unkan Jehowa Sina paha kin akan kun hiyu, he paha kin akan, qa Jehowa Mowis kipan he paha kin ekta, qa Mowis itanwankanhdeya elita $i$.

21 Unkan Jehowa Mowis heciya, kun lida oyate kin wicakiyaotanin, okini wanyakapi kta e Jehowa ekta iyoptapi kta, qa etanhan wicota ilipayapi kta.

$22 \mathrm{Qa}$ wawayuśnapi Jehowa ikiyedan hipi ece kin, iś eya 
ihduwakanpi kte, okini Jehowa wicakaohpe kta.

23 Unkan Ḣe kin yuwakan, qa ihdukśan icago wo, unyalidaotanin, qa eha, heon oyate kin Sina he kin ekta ipi okitpanipi ce, Mowis Jehowa eciya.

24 Unkan, Hunktiya kun hda, qa itanwankanhdeya yau kta niś qa Aaron yecica kta, tuka wawayuśnapi kin, qa oyate kin ihnunhan Jehowa ekta yapi kta e naoksapi, okini wicakaohpe kte, Jehowa eciya.

25 Unkan Mowis oyate ekta kun hde ca owawicakiye.

\section{WICOWOYAKE 20.}

1 Unkan Wakantanka ie ca oie kin dena owasin eya.

2 Jehowa wakanyada, qa Egupta makoce etanhan, wayaka tipi etanhan, inanpe niye cin he miye ce.

3 Mitokam Taku Wakan tokeca duhe lite śni.

4 Ihnunhan wakagapi pagopi nicicage cin, qa mahpiya wankan taku ohnaka, qa ihukuya taku maka kin ohnaka, qa mini maka ihukuya taku ohnaka kin hena ow asin taku iyececa niçicage cin.

5 Hena ohoyada kte śni, qa itokam yapatuje kte śni. Nakaś Jehowa wakanyada, Taku Wakan winawizi he miye, tona śice madapi wicoicage iyamni, qa itopa kin hehanyan ateyapi wahtanipi iye cincapi kin en awicawai ece.

$6 \mathrm{Qa}$ tona waśte madapi qa mitawoahope patanpi kin hena wicoicage kektopawinge hehanyan cantewicawakiya ece.

7. Jehowa wakan yada kin he ilicekceya caje date kte-śni, Jehowa tuwe ikcekceya caje yate cinhan yaowotanna kte śni.
8 Anpetu isakowin wakan yada kta e kiksuya wo,

9 Anpetu śakpe hitayani, qa niton̆tani kin owasin ecannonkta. 10 'Iuka anpetu iśakowin kin Jehowa Wakan yada kin he tawa, he en wohtani takudan ecannon kte śni; niye qa nicinkśi, qa nicunkśi, nitahokśidan, qa wiciyanna nitawa, qa wanuyanpi nitawa, qa tuwe oyate to keca otonwe nitawa ohna un kin. 11 Nakaś anpetu śakpe en Jehowa, mahpiya kin, qa maka kin, miniwanca, qa taku hena ohna un kin owasin kage ca anpetu iśakowin kin en okilipa, heon Jehowa anpetu iśakowin kin he hdawaśte qa hdawakan.

12 Niyate qa nihun wicahduonihan wo, hecen Jehowa wakan yada kin makoce niçu kin he en tehan yani kta.

13 Tin wicayakte kte śni.

14 Wawicihahapi ecannon kte sni.

15 Wamayanon kte śni.

16 Nitakoda on woyaotanin iton śniyan odake kte śni.

17 Nitakoda ti kin cantiheyaye kte śni. Nitakoda tawicu, qa hokśidan tawa, qa wiciyanna tawa, qa tatanka tawa, qa śuktanka tawa, qa taku kaśta nitakoda tawa cantiheyaye kte śni.

18 Unkan oyate owasin taku hotonpi kin qa taku ide kin qa mazayahotonpi kin hoton, qa he sotoju kin he wanyakapi qehan, oyate kin kokipapi, qa cancanpi, qa itehan kinajinpi.

$19 \mathrm{Qa}, \mathrm{Niś}$ unkokiyapo, hecen naunhonpi kta, tuka Wakantanka unkokiyapi kte śni, okini unțapi kta, Mowis eciyapi.

20 Unkan Mowis oyate kin hewicakiya, Inihanpiśnipo; iyutanniyanpi kta on Wakantanka hi, qa tokokipe niitepi itokam un 109 


\section{HDINANPAPI,}

kta, hecen wayahtanipı kte śni, heon etanhan.

21 Unkan oyate kin itehan najinpi, tuka Mowis otpaza śoka ohna Wakantanka yanke cin he ikiyedan i.

22 Unkan Jehowa Mowis heciya, Israel cinca kin hecen ewicayakiye kta, Malipiya etanhan, ociciyapi kin he niś wandakapi.

23 Mitokam mazaska taku wakan, qa mazaskazi taku wakan niçicagapi kte śni.

24 Maka owayuśna miyecage kta, qa he akan peta wośnapi, qa wopida wośnapi nitawa kin, tahinca nitawa qa pte nitawa kin wayaduśna kta; tuktee kaśta micaje kiksuye ciyapí kin he en cihi qa ciyawaśte kta.

25 Qa inyal owayuśna miyecage einhan, wokakanpi on yakage kte śni, maziyape nitawa kin he ekta yeyakiye cinhan duśape kta. 26 Qa owayuśna mitawa kin ekta itanwankunhdeya idada eca, woiyadipi akan mayani kte śni ece, okini tanconikadan tanin kta.

\section{WICOWOYAKE 21.}

1 Unkan woyaco wicitokam eyahde kta dena ee.

2 Heberi ookiye wan opeyaton kinhan waniyetu śakpe wowidag yaye kta, tuka iśakowin hehan iyuwin codan ikceya tankan iyaye kta.

3 Tanśnana en hi kinhan tanśnana tankan ye kta, tawicu ton kinhan, tawicu kici tankan ye kta.

4 Yuhe cin he winolinca wan qu kinhan, qa cinca wica qa iś winyan kiciton kinhan, tawicu cinca ko yuhe cin tawaye kta, qa iye taśnana inape kta.

5 Tuka, Mayuhe cin waśte wa- daka, mitawin, micinca naliun, ikceya inawape kte śni, ookiye kin he katinyan eye cinhan,

6 Yuhe cin he wayaco ekta aye kta, qa tiyopa, qa iś tiyopa icahda tośu kin ekta, qa tahinśpa wan on noge kin pahdoke kta, liecen owihanke wanin he okiye kta.

7 Unkan wicaśta wan iye cunwintku wiciyanna on wiyope kiye cinhan, he hokśipidan tankan yapi kin he iyecen tankan inape kte śni.

8 Yuhe cin he wokihpe śni, qa śicedake cinhan he opekiton wicakiye kta, wanna he hnayan, heon wiyopekiye ca oyate tokeca qu kta okihi śni.

9 Qa iye cinhintku on he wokihpe cinhan, cunwintku yapi woyaco kin he iyecen ecakicon kta.

10 Winohinca tokeca yuza eśta tado tawa, qa wokoyake tawa qa kici un kin he ekiciyaku kte śni.

11 Qa hena yamni ecakicon śni kinhan, mazaska wanica ikceya inape kta.

12 Tuwe wicaśta wan apa, unkan te cinhan, awicakehan te yapi kta.

13 Unkan tuwe he kuwa śni unkanś, tuka nape ekta 'Taku Wakan hiyukiye cinhan, oyanke wan ociciyake kta hen inape kta

14 Tuka tuwe takodaku kte kta e hnayan kicah kuwa kinban, owayuśna mitawa eśta etanhan ktepi kta e iyacu kta.

15 Unkan tuwe iye atkuku qaiś liunku ape cinhan awicakehan te yapi kta.

16 Unkan tuwe wicaśta wan manun qa wiyope ye cinhan, qaiś yuha iyeyapi kinhan te yapi kta.

17 Unkan tuwe atkuku qaiś hunku ihakta śni kinhan awicakehan te yapi kta. 


\section{WICOWOYAKE 22.}

18 Unkan wicaśta nom akinicapi qa wanji tawaśitku inyan on, qaiś nape hdupśunka on ape cinhan, unkan țe śni tuka hinhpaya makan wanka.

19 Najin, qa sagye kiton tankan nrani kinhan, tuwe he ape cin he te kte śni ; tuka okizi yapi kajuju kta, qa nakun makan wanke cin on.

20 Unkan wicaśta wan tahoksidan, qaiś wiciyanna tawa cansakadan on ape cinhan, qa nape kin ihukuya țe cinhan, katinyan tokiconpi kta.

21 T'uka anpetu wanji, qaiś nonpa can najin kinhan, tokiçonpi kte śni, he mazaska tawa nakaś.

22 Unkan wicaśta nom akicipapi qa winohinca ihduśaka wan kiuniyanpi kinhan, unkan cinca hiyu tuka taku śica sanpa ope śni kinhan, mazaska on kajuju kiyapi kta, token winohinca hihnaku eye ca wayaco konzapi kin he qu kta.

23 Tuka taku śica ope cinhan wiconi on wiconi yaqu kta,

24 Iśta on iśta, hi on hi, nape on nape, siha on siha,

25 Guyapi on guyapi kta, capapi on capapi kta, apapi on apapi lita.

26 Unkan wicaśta wan tahokśidan iśta kin, qaiś tawiciyanna iśta kin apa, unkan kpe cinhan, iśta kin on ikceya kiyuśke kta.

27 Qa tahokśidan hi wan qaiś tawiciyanna hi wan kapśun kinhan, hi kin on ikceya kiyuśke kta.

28 Unkan tatanka wan wicaśta qaiś winohinca capa, unkan te cinhan, tatanka inyan on kininpi kta, qa cehpi kin yutapi kte śni ; tuka yuhe cin takudan ecakiconpi kte śni.

29 Tuka he itokam tatanka kin he wacapa, qa yuhe cin okiya- kapi eśta, he hdaśke śni kinhan, tatanka wicaśta qaiśs winohinca te kiye cinhan, inyan on kininpi kta, qa yuhe cin nakun țe yapi kta.

30 Mazaska on kajuju śipi kinhan token nagi kin on kajuju śipi kın owasin qu kta.

31 Cinca wica qaiś winyan cape cinhan, woyaco kin de iyecen ecakiconpi kta.

32 Tatanka kin hokśidan, qaiś wiciyanna cape cinhian, wicayuhe cin mazaska wikcemna yamni kicu kta, qa tatanka inyan on ktepi kta.

33 Unkan wicaśta wan woha wan yulidoke cinhan, qaiś woha wan qe ca akahpe kiton śni kinhan, unkan tatanka wan, qaiś śuktanka śonśonna wan he oiyohipaye cinhan,

34. Woha tawa kin kajuju kta, qa yuhe cin mazaska qu kta, qa taku te cin he tawa kta.

35 Unkan wicaśta wan tatanka tawa tawaśitku tatanka tawa kiunni on te cinhan, tatanka ni kin he wiyope kiyapi kta, qa mazaska kin yuakipam icupi kta, qa te cin nakun cokaya kibaśpapi kta.

36 Tuka tatanka kin wacapa he itokam sdonyapi eśta, yuhe cin he hdaśke śni kinhan, yuhe cin tatanka te cin on tatanka wan qu kte ça te cin he tawaye kta.

\section{WICOWOYAKE 22.}

1. Wicaśta wan tatanka qaiś tahinca manun kinhan, qa he patr qaiś wiyope ye cinhan, tatanka zaptan, tatanka wanjidan on kicu kta, qa tahinca topa tahinca wanjidan on.

2 Wamanun wan tipi kahdokahan iyeyapi, qa apapi on ta eśta he on weyapi wanice kta. 
3 Wi kin iyoyam ye cinhan wokajuju on kajuju kiyapi kte, takudan yuhe śni eśta, womanun kin on wiyope liyapi kta tuka, heon tuwe he kte kinhan we yapi kta. 4 Taku manun kin he niyake nape kin ohna iyeyapi kinhan, tatanka, śuktanka śoñśonna, tahinca eśta, wanjidan on nonpa hdajuju kta.

5 Wicaśta wan taku wilhan ye kte cin tokan tamaga, qaiś tahastanhanka iyuwi oju kin en iyaye ye ça maga qaiś hastanhanka iyuwi oju kin temye cinhan, iye tamaga waśte qa hastanhanka iyuwi waśte kin on hdajuju kta.

6 Cetipi qa tinta ideyapi, qa peji paha, qaiś aguyapi baśdapi śni, qaiś maga wan huhnage cinhan he hdajuju lita.

7 Wicaśta wan tawaśitku kin mazaska qaiś wakśica kicihñăke kta e qu kinhan qa tipi tawa etanhan manunpi kinhan wamanun iyeya kinhan nonpa akihde hdajuju kta.

8 Wamanun kin iyeyapi śni kinhan, tipi yuhe cin he tawaśitku woyuha tawa ekta nape yekiye śni taku wakan ekta ohdag kiyapi kta.

9 Taku akinicapi owasin on, tatanka on, śuktanka śonśonna on, tahinca on, wokoyake on, taku kaśta tuwe tawa tanin śni akinicapi kinhan, napin taku wakan kin ekta ohdag wicakiyapi kta, qa tuwe taku wakan kin yaśicapi kinhan tawașitku nonpa akihde kicu kta.

10 Wicaśta wan tawaśitku śuktanka śonśonna, qaiś tatanka, qaiś talinca wanuyanpi, taku kaśta awanyake kta e qu kinhan, unkan te cinhan, qaiś tuwedan wanyake śni kiunniyanpi, qaiś tokan kałapapi kinhan; 112
11 Tawaśitku taku tawa kin ekta nape kin yekiye śni, Jehowa namahon keya ohdake kta, hecen tawa qon he iyowin kiye. kta, unkan kajuju kte śni.

12 Tuka manunpi kinhan tawa qon taku iyehantri kicu kta.

13 Yahdecapi kinhan onśpa yalidecapi yuotanin kta e ahi kta, qa hecen yahdecapi on takudan kicu kte śni.

14 Unkan wicaśta wan takodaku etanhan taku odote, qa tawa kin kici un śni icunhan kiunniyanpi, qaaiś țe cinhan wokajuju kicu kta.

15 Tawa kin kici un kinhan kajuju kte śni, opeton odote cinhan iyuwin kin he on $u$ ce.

16 Wicaśta wan hokśiwinna wan wahoye kuns okiye ça kici wanke cinhan tawicu kta e awicakehan wicakicida lita.

17 Tuka wikośka atkuku kin kicu kta e katinyan tehinda kinban, wikośka wohpàpi kin he iyecen mazaska qu kte.

18 Wicalimugesa niyakiye kte śni.

19 Tuwe woteca wan kici wanke cinban katinyan ktepi kta.

20 Jehowa heceedan wakiyuśnapi kta, qa tuwe taku wakan tokeca wan wakiyuśna kinhan ocowasin ihangyapi kte.

21 Tuwe oyate tokeca nici ounye cinhan iyokiśin yaye kte śni, qa yakiduśe kte śni, nakaś niś Egupta makoce kin ohna oyate tokeca ounyayapi.

22 Wiwazica qa wamdenica wanjidan duśicapi kte śni.

23 He duśice unkan hoye makiye cinhan ho kin nation manke kta ce.

$24 \mathrm{Qa}$ mitocanniye ideye kta, qa canhipi on ciktepi kta, hecen nitawinpi wiwazicapi kta, qa nicinca wamdenicapi kta. 


\section{WICOWOYAKE 23.}

25 Oyate mitawa tuwe onśika nici un kin mazaska oyaqu kinhan, wayalitakesa iyecen ecayecon kte śni, woyahtake yakidake kte śni.

26 Nitakoda taku oyaqu nicicajuju kta e taśina iyacu kinhan tohan wi iyaye cinhan yecu kta.

27 Taśina uka kin hee, taku hdowin kta heceedan kinhan, ta'ku ohna iśtinbe kta he? hecen loye makiye cinhan nawation kta; wacantkiya waun ce.

28 Taku wakan duşice kte śni, qa oyate nitawa itancan kinihnuhan wahte yada śni kinhan.

29 Nicinkśi tokapa kin mayaqu kte; ca taku icah yaye tokaheya suton kin woyute woyatke ko mayaqu dutehan kte śni.

30 He iyecen pte nitawa qa tahinca nitawa kin ecayecon kte ; anpetu sakowin, hunku kici yanke kte ça iśahdogan can hehan mayaqu kta.

31 Wicaśta wakan mitawa kin yaunpi kta, heon taku tinta ekta yahdecapi kin he yatapi kte śni, he śunka ekta ehpe yayapi kta.

\section{WICOWOYAKE 23.}

1. Taku ecinkta oyakapi kin iyacu kte śni, qa itonśniyan wadaatanin kta e wicaśta śica nape duze kte śni.

2 Wicota taku śica econpi owicayape kte śni, qa taku akinicapi en wicota yaśkopapi eśta yaśkope kta e awicadupte kte śni.

3 Nakun wicaśta wahpanica toakinice kin en duonihan kte śni.

4 Tuwe niciyuse tatanka tawa, qaiś taśunke śonśonna nuni kin ayakipe cinhan, he ekta hdiyakiye kta.

5 Tuwe śice nidake taśunka śonśonna tawaqin ihukuya ma- kata wanka wandake cinhan duśke kta yakapin eśta kici duśke kta.

6 Wicaśta wahpanica nici un kin toakinice kin en woyaco dapemni kte śni.

7 Wicoie itonśni etanhan ilıdutehan wo, ihnuhan wicaśta owotanna qa tuwe taku śica econ śni yakte kinhan nakaś tuwe śice cin he mdaowotanna kte śni.

8 Taku ituhanpi kin iyacu kte śni; Nakaś taku ituhanpi tona iśta mdezapi kin hena iśta gonge wicaye ca ohan owotanpidan eśta oiepi kin wicakiyupemni.

9 Egupta makoce kin ohna ounyayapi qon, heon wicaśta oyate tokeca ohna ounyanpi cantepi kin sdonyayapi, qa tuwe oyate tokeca yakiduśe lite śni.

10 Waniyetu śakpe makoce nitara woyaju kte ca taku aicage cin mnayaye kta.

11 Tuka iśakowin kin he aduśtan kte ca ehpe yaye kta, hecen oyate nitawa tona onsika he yutapi kta, unkan taku ihe cin tinta wamanica kin yutapi kta; hastanhanka iyuwi nitawa qa wihdi can nitawa kin nakun he iyecen ecayecon kta ce.

12 Anpetu śakpe nitohtani ecannon kte ca anpetu iśakowin kin en oziniçiye kta ce, hecen tatanka nitawa qa nitaśunke śonsonna okilipe kta; qa nitawiciyanna cinca, qa tuwe oyate tokeca nakun magaga iciye kta.

13 Taku eciciyapi kin owasin en ikpatanpo, qa taku wakan tokeca caje kin he kiksuye yakiye kte śni nii kin etanhan tuwedan he nalion kte śni.

14 Mdoketu eca yamni akihde womiyecihe ece kta.

15 Aguyapi napoliye codan wohanpi yapatanpi kta, Abib wi 


\section{HDINANPAPI,}

kin (aguyapi itka hinanpe cin wi kin) he en Egupta etanhan yahdinape, heon etanhan iyehantu eca anpetu śakowin aguyapi napohye codan yatapi ece kta, econ ciśipi kin he iyecen, qa wośna codan tuwedan mitokam wanyakapi kte śni.

16 Nakun baśdapi wohanpi kin nitohtani taku maga ohna oyaju tokaheya icage cin hee, qa wamnayanpi wohanpi kin omaka owihanketa, nitohtani kin maga etanhan mnayaye cin iyehantu eca.

17 Mdoketu eca, yamni akihde tona wica owasin Jehowa itancan kin itokam nihdutaninpi ece kta.

18. Taku wanayakiduśna we lin aguyapi napohyapi kici mayaqu kte śni, qa wohanpi mitawa waśin kin ihanlianna kin helanyan ihe kte śni.

19 Maga nitawa taku tokaheya aicage cin he Jehowa wakanyada ti kin en ayahi ece kta. Tatokada cinca kin he hunku asanpi kin ohna oyahe kte śni.

20 Inyun miś olnnilide wan nitoliam ye waśi he canku ohna awanniyake ca oyanke wiyeya cicihnake cin he ekta aniye kta.

21 Ite kin awacin wo, qa ho kin nahon wo, ihnuhan yakipajin kin, wayahtanipi kin tokan aniyanpi kte śni, nakaś he cokaya micaje yanka.

22 Tuka awicakehan ho kin anayagoptan, qa taku eciciye cin owasin ecen ecannon kinhan tona toka niyanpi kin owasin toka waye kta, qa tona nicipajinpi kin hena wicawakipajin kta.

23 Mitaohnihde nitokam ye kta, qa Amori kin, qa Ḧiti kin, qa Perazi kin, qa Kanani kin, qa Hiwi kin, qa Jebuśi kin ekta aniye $\mathrm{kta}$, qa awihnuni wicawaye kta ce.

\section{4}

24. Hena taku wakan dapi kin ekta yapatuje kte śni, qa ohoyada kte śni, qa token econpi kin he iyecen ecannon kte śni ; tuka tawakagapi kin yakawanke kta, qa ocowasin yakamdemdece kte ce.

25 Tuka Jehowa wakan yadapi kin he oyecipapi kte, hecen aguyapi nitawapi kin he yawaśte kta qa mini nitawa kin nakun, qa nicokayatanhan wowayazan mdutokan kta.

26 Qa makoce nitawa kin ohna takudan okaśkan ton kte śni, qa cinca nica yuke kte śni, anpetu nitawa oyawa kin ojuwaye kta.

$27 \mathrm{Qa}$ wokokipe mitawa kin nitokam yewaye ca oyate ekta wicaya i kte cin owasin yuśinye wicawaye kta, qa tona toka niyanpi kin owasin tahupi kin cicu kta.

28 Unkan tulimaga nitokam yewaye kta, unkan hena Hiwi kin, Kanani kin, qa Hiti kin nitokapa tanhan wicakahapapi kta.

29 Waniyetu wanjidan en ham wicawaye kte śni, okini makoce kin hewoskantu kta, qa wamanica ota lita niyepi ehna.

30 Tonana qa hehan tonana nitokapatanhan ham wicawaye kta, ecen nihduota, qa makoce lin tawayaye kta.

31 Qa Mde-śa etanhan Pilistim tamde kin aiyahdeya, qa Hopuze cin etanhan wakpa kin ekta, hehanyan makoce kin ciçu kta, nakaś makoce kin he tona ounyanpi kin hena ninape en waqu lita; unkan nitokapatanhan ham wicayaye kta.

32 Hena om, qa taku wakandapi om wicotakuye yakage kte śni.

33 Makoce nitawa kin en ounyanpi kte śni, okini miye en wahtani niyanpi kta. Taku wa- 


\section{WICOWOYAKE 24.}

kandapi kin oyakiye cinhan he nihmunke kta.

\section{WICOWOYAKE 24.}

1 Unkan Mowis heciya, Jehowa ekta itanwankanhdeya u wo, niś qa Aaron, Nadab qa Abihu, qa Israel hunkayapi kin wikcemna śakowin, qa itehanyan yapatujapi kte.

2 Qa Mowis is nana Jehowa ikiyedan hi kta, tuka hena ikiyedan hipi kte śni ; nakun iye kici oyate kin itanwankanhdeya upi kite śni.

3 Unkan Mowis ekta hda, qa Jehowa oie kin owasin, qa woyaco kin orvasin oyate kin owicakiyaka; unkan oyate kin owasin ayuptapi, qa ho wanjidan heyapi ; wicoie owasin Jehowa eye cin ecen econkupi kte ce.

4. Unkan Mowis Jehowa oie kın owasin owa; qa hanhanna hin kikta, qa wahna wośnapi wan paha ihukuya elıde, qa inyan wokiksuye wikcemna sanpa nonpa Israel wicomn akenonpa on ;

5 Qa Israel cinca kin etanhan kośka ye wicaśi, unkan woh̉uhnahyapi huhnahyapi, qa tatanka wopida wośna Jehowa wa kiyuśnapi.

6 Unkan Mowis we kin hanke ruaza wakśica ohna icu, qa we lin hanke wahna wośnapi akan ehpeya.

7 Qa wicotakuye -wowapi kin icu, qa yawa oyate nogepi kin ohna; unkan, Taku Jehowa eye cin owasin ohinniyan ecen econkupi kta, eyapi.

8 Unkan Mowis-we kin icu qa oyate kin awicamnimni, qa, Inyun wicoie kin dena on Jehowa wicotakuye nicicagapi we kin dee ce, eya.

9 Hehan Mowis qa Aaron, Na- dab qa Abihu, qa Israel hunkayapi kin etanhan wikcemna śakowin itanwankanhdeya yapi.

10 Qa Israel Taku Wakan dapi kin wanyakapi, unkan siha lin ihukuya Sapir inyan kamdayapi qa owanka lagapi kin iyecen, qa mahpiya hinca kamdeza kin he iyecen.

$11 \mathrm{Qa}$ Israel cinca otancanke kin Wakantanka wanyakapi, qa wotapi qa wayatkanpi, tuka nape kin awicahnake śni.

12 Unkan Jehowa Mowis heciya, Itanwankanhdeya kuwa, he kin ekta, miye en, qa hen yanka wo, qa onspe wicayakiye kta e inyan mdaska qa woope worhope akan owawa kin hena ciçu kta ce.

13 Unkan Mowis najin, taokiye Jośuwa kici, qa Mowis Wakantanka he tawa kin ekta itawankanhdeya iyaya.

14 Qa hunkayapi kin hewicakiya, Deciya unkapepo, niyepi en unhdipi kte kin hehanyan, qa ihopo Aaron qa Hur niyepi orn unpi, qa tuwe taku akinice cinhan hena en hi kte ce.

15 Qa Mowis itanwankanhdeya paha akan i, qa mahpiya śoka kin paha kin akahipa.

16 Unkan Jehowa towitan kin Sina paha akan owanji yanka, qa anpetu śakpe kin hehanyan mahpiya śoka kin paha akahpa, qa anpetu iśakowin kin hehan mahpiya śoka cokayatanhan Mowis kipan.

17 Unkan Israel cinca kin Jehowa towitan kin peta wahuhnahye cin he iyecen lie ipa akan wanyakapi.

18 Unkan Mowis mahpiya śoka cokaya $i$, qa he kin akan i, qa Mowis anpetu wikcemna topa, qa hanyetu wikcemna topa lie kin ohna yanka. 


\section{HDINANPAPI,}

\section{WICOWOYAKE 25.}

1 Unkan Jehowa Mowis okiye ca heya;

2 Taku itulianpi kte cin he makahipi kta e lsrael cinca kin hewicakiya wo, wicaśta tona cante kin iyopaśtake cin hena owasin taku itumakihanpi kin he iyacupi kta.

3 Qa hena etanhan taku ituhanpi iyacupi kte cin dee; mazaskazi, qa mazaska, qa mazaśa ;

4 Qa tahinca hin to, qa stan, qa duta, qa minikuha ska, qa tatokadan hin,

5 Qa tamdoka ha śayapi, qa hoka ha, qa śitum can;

6 Wibdi petijanjan on, qa taku waśtemna wihdi sdawicayapi on kagapi kin heon, qa izin yapi kin on.

7 Inyan wicaśake iyececa, qa inyan amdo akatipe, qa maku akahpe otaśpuyapi.

8 Lnkan tipi wakan wan micagapi lita, hecen wicacokaya ounwaye kta.

9 Wakeya olkagapi kin, qa taku wo winyunyanpi owasin okagapi kin hena wanyag ciciye cin owasin, he iyecen yakage kta.

10 Qa can wohnaka wan micagapi k.ta, śitim can kin on, wiciśpa nom sanpa hanke ohanske cin, qa wicispa sanpa harke ohdakinyan kin, qa obosdatu kin wiciśpa sanpa hanke.

$11 \mathrm{Qa}$ mazaskazi ecedan on ayapawinte kta, mahentanlıan, akapatanhan koya ayapawiute kta, qa mazaskazi iwateśdake wan he akan ihdukśan yakage iste.

12 Qa mazaskazi napcupe topa oyecicaśte kta, qa hena oise topa ekta ikoyag yaye kta, cuwi tokaheya ekta napcupe nonpa, qa cuwi inonpa ek ta napcupe nonpa.
13 Unkan śitim can tośu nonpa yakage kte ca hena mazaskazi on ayapawinte kte.

14 Qa canwohnake alkiyuhapi lita e tośu kin napcupe canwahnake cuwi ekta ikoyakapi kin hena ohna iyeyaye kta.

15 Qa tośu kin canwohnaka napcupe kin ohna yakonpi kta, hetanhan icupi k te śni.

16 Unkan woyaatunin ciçu kte cin he canwohnaka kin en yehnake kta.

17 Qa wokajuju iha wan mazaskazi ecedan on yakage kta, wiciśpa nonpa sanpa hanke ohanske cin, qa wiciśpa wall sanpa hanke ohdakinyan kin.

18 Qa Kerubi nonpa mazaskazi pagopi on yakage kta, wokajuju iha ihanke nonpa etanhan hena yakage lita.

19 Hecen Kerubi wanji wokajuju iha ihanke deciyatanhan kaga wo, qa Kèrubi unman ihanke unman eciyatanhan wokajuju iha kin hetanhan, ihanke anokatanhan akan Kerubi kin yakage kta.

20 Qa Kerubi hupahu wankan kamdahi yakonpi kte, hupahupi kin wokajuju íha kin akahipa unpi kta, qa itepi kin napin unman ektakiya kta ; Kerubi itepi kin wokajaju iha kin ektakiya tonwanpi kta.

21 Qa woyaatanin cicu kte cin he canwohnake mahen oyakihnake kta, qa he iwankam canwohnaka akan wokajuju iha kin eyahnake kte.

$22 \mathrm{Qa}$ hen itkocicipe kte ca wokajuju iha kin iwanka-patanhan Kerubi nonpa woyaatanin canwohnaka akan unpi kin hena cokayatanhan ociciye kte, taku Israel cinca kin owicakiyag ciśi kte cin owasin.

$23 \mathrm{Qa}$ sitim can wahna wotapi 


\section{WICOWOYAKE 26.}

wan yakage kta, wiciśpa nom ohanske cin, qa wiciśpa wanjidan ohdakinyan kin, qa obosdatu kin wiciśpa sanpa hanke.

$24 \mathrm{Qa}$ he mazaskazi ecedan on ayapawinte kte, qa ihdukśan mazaskazi wateśdake wan yecage kta.

$25 \mathrm{Qa}$ he ihdukśan iyuskite nape hdakinyan yecage kta, qa iyuskite kin ihdukśan mazaskazi ecedan wateśdake wan yecage kta.

26 Qa mazaskazi napcupe topa he yecage kta qa siha topa kin itepi topa kin akan napcupe kin ikoyag yaye kta.

27 Wahna wotapi akiyuhapi kta e mazanapcupe kin tośu icupi kta on iyuskite iyotakons unpi kta.

$28 \mathrm{Qa}$ tośu kin sitim can on yakage kte ça mazaskazi on ayapawinte kta, hena on wahna wotapi kin akiyuhapi kte.

29 Qa wahna wotapi kin wakśica, qa tukiha, qa wiyatke, qa cega, hena owasin yecage kta, on wakanyan okaśtanpi, qa mazaskazi ecedan on hena yakage kta.

$30 \mathrm{Qa}$ wahna wotapi kin akan aguyapi itokam hnakapi eyahnake kta, mitokam ohinniyan un kite.

31 Unkan mazaskazi ecedan petijanjan ihupa wan yakage kta, pagopi he yakage kta, ceca kin, qa adetka kin, qa tawakśica kin, qa pśonka kin, qa wahca kin.

32 Qa petijanjan ihupa cuwi etanhan adetka śakpe inanpe lita, adetka yamni cuwi wanji etanhan, qa adetka yamni petijanjan cuwi unman kin eciyatanhan.

33 Wakśica yamni uma iyececa, pśonka wan, qa wahca wan kicica adetka wanjidan en, qa wakśica yamni uma iyececa, qa pśonka wan qa wahica wan adetka unman en, adetka śakpe petijanjan ihupa etanhan inanpe cin owasin hececa kta.

34 Qa petijanjan ihupa kin en wakśica topa uma iyececa, otoiyohi pśonka wan qa wahca wan kicica kta.

35 Qa pśonka wan adetka nonpa ihukuya, qa ake pśonka wan adetka nonpa ihu kuya, qa pśonka wan adetka nonpa ihukuya, adetka śakpe petijanjan ihupa etanhan inanpe cin owasin hececa kta.

36 Petijanjan ihupa pśonka tawa, qa adetka tawa kin, okonwanjidan kta, mazaskazi ecedan onśpa wanjidan pagopi on owasin kagapi kta.

37 Unkan petijanjan śakowin yecage kta, qa akan petijanjan ehnakapi kta, qa he itokapatanhan iyoyam ye kta.

$38 \mathrm{Qa}$ petijanjan iynkse, qa ohna cahota icupi mazaskazi ecedan.

$39 \mathrm{He}$ qa on kicanyanpi kin hena owasin mazaskazi ecedan woqin wanjidan on kagapi kta.

40 Qa wak taya wo owasin okagapi paha en wandake cin he iyecen kaga wo.

\section{WICOWOYAKE 26.}

1 Unkan tipi kin ozanpi wikcemna minihuhà ska nonpa kahmupi, to, qa stan, qa duta Kerubi kazuntapi hecen yakage kte.

2 Ozanpi wanji ohanske cin wiciśpa wikcemna nonpa sanpa śahdogan, qa ohdakinyan kin wiciśpa topa, ozanpi owasin otoiyohi hinskokeca kta.

3 Ozanpi zaptan icikoyag yapi kte, qa ozanpi zaptan icikoyag yapi kta, 
4 Qa icikoyag yapi kta e ozanpi tokaheya opapun kin en śunjoyake to yakage kta, apahdate ekta, qa ozanpi inonpa ihanke opapun icikoyag yapi en hecen yakage kta.

5 Śnjoyake wikcemra zaptan ozanpi tokaheya en yakage kta, qa icikoyag yapi kin ekta sunjoyake wikcemna zaptan ozanpi inonpa opapun en yakage kta, śunjoyake iyotakonzapi kta, wanji unman ekta.

6 Unkan mazaskazi taśpu wikcemna zaptan yakage kta, qa maza taśpu kin on ozanpi kin icikoyag yaye kte, hecen wakeya wanjidan kte.

7 Unkan tatokadan hin ozanpi wakeya kin yecage kte, tipi kin iwankam; ozanpi kin ake wanji yakage kte.

8 Ozanpi wanji ohanske cin wiciśpa wikcemna yamni, qa ohdakinyan kin wiciśpa topa ; ozanpi akewanji owasin iakedececa hinskokeca kte.

9 Qa ozanpi zaptan yuwitaya icikoyag yaye kte, qa ozanpi śakpe icikoyag yaye kta, qa ozanpi iśakpe kin wakeya tiyopa kin iwankam yape han kte.

10 Qa icikoyag yapi kta e ozanpi opapun ihanke kin en śunjoyake wikcemna zaptan yakage kte, qa ozanpi inonpa opapun en ikoyagyapi kta e śunjoyake wikcemna zaptan.

11 Qa mazaśa taśpu wikcemna zaptan yakage kte, qa mazataśpu kin śunjoyake ohna yeyaye kte, hecen wakeya kin icikoyag yaye kte, qa wanjidan kte.

$12 \mathrm{Qa}$ wakeya ozanpi etanhan taku ihe cin he ozanpi wan hanke iyaye cin he tipi kin ihektam okazeze he kta.

$13 \mathrm{Qa}$ wakeya anokatanhan ozanpi ohanske cin wiciśpa wanji 118 iyaye cin he tipi cuwi anokatanhan okazeze he kta.

14. Unkan tamdoka wanunyanpi ha śayapi woakahpe wakeya kin yecage kta, qa akapatanhan hoka ha woakahipe wan.

15 Unkan śitim can canmdaska bosdan unpi kta tipi kin yecage lita.

16 Canmdaska otoiyohi ohanske cin wiciśpa wikcemna, qa ohdakinyan kin wiciśpa sanpa hanke.

17 Qa canmdaska wanji nape nonpa yecage kta, on icikoyag yapi kte; tipi canmdaska kin owasin hecen yecage kta.

$18 \mathrm{Qa}$ tipi on canmdaska kin hecen yecage kta, canmdaska wikcemna nonpa itokaga cuwi kin on, wiyotanhan ektakiya.

19 Qa mazaska sicu wikcemua tom canmdaska wikcemna nonpa ihukuya yakage kta, canmdaska wanji nape nonpa kin on maza sicu nonpa, canmdaska wanji ihukuya, canmdaska otoiyohi on hecen maza sicu nonpa kta.

20 Qa tipi cuwi inonpa on wazi ya takiya canmdaska wikcemna nonpa kta,

$21 \mathrm{Qa}$ mazaska sicu wikcemna topa; canmdaska wanji ihukuya sicu nonpa, qa canmdaska iyokihe ihukuya sicu nonpa kta.

$22 \mathrm{Qa}$ tipi catku wiyohpeyata ektakiya canmdaska śakpe yecage kta.

23 Qa tipi catku kahmin nonpa kin on canmdaska nonpa yecage kta.

24 Qa napin iakedececapi kta, ihukuyatanhan, qa napin otoiyohi pa kin ekta maza napcupe tokaheya en ikoyag yapi kte kahmin nonpa kin on hena nonpa okonwanjidan kta.

25 Hecen canmdaska kin śahdogan kta, qa maza sicu kin ake śak- 
re, maza sicu nonpa canmdaska wanịidan ihukuya, qa maza sicu nonpa canmdaska wanjtdan ihukuya.

26 Qa sitim canhdakinyan yaliage kta, zaptan tipi onnaptan tokaheya canmdaska kin on.

27. Qa canhdakinyan zaptan tipi onnaptan inonpa canmdaska on, qa canhdakinyan zaptan tipi ihanke wiyohpeyata ektakiya catkuta canmdaska on.

$28 \mathrm{Qa}$ canhdakinyan cokaya kin canmdaska cokaya ohna iyaye kta, ihanke wanji etanhan ihanke unman ekta iyohi ye kta. 29 Qa canmdaska kin mazaskazi ayapawinte kta, qa mazaskazi napcupe yecage kta, qa hena ohna canhdakinyan iyaye kta, qa canhdakinyan kin mazaskazi ayapawinte kta.

30 Qa tipi kin eyahde kta, he en okagapi kin wanyag niyanpi kin he iyecen.

31 Unkan cokaya ozanpi wan yakage kte, minihuha ska nonpa kahmupi, qa to qa stan, qa duta kazuntapi, Kerubi akan kazuntapi kte.

32 Unikan mazaska sicu topa akan sitim can ipatan tom mazaskazi apawintapi qa mazaskazi yukśanpi tom akan cokaya ozanpi otkeyaye kte.

33 Qa cokaya ozanpi kin maza yukśanpi kin ihulkuya otkeyaye kia, qa colkaya ozanpi kin he mahen woyaatanin can wohnake kin ade kta, qa niyepi on cokaya ozanpi kin tipi wakan qa wakan liinca otahedan yukinukan kta.

$34 \mathrm{Qa}$ wolkajuju iha kin woyaatanin can wohnake akan eyahnake kta, tipi wakanhinca mahen.

$35 \mathrm{Qa}$ wahna wotapi kin he cokaya ozanpi kin itankan eyahde kta, qa petijanjan ihupa kin wahna wotapi iyotakons, tipi cuwi itokaga ekta, qa wahna wotapi kin cuwi waziyata kin ekta eyahnake kta.

36 Qa wakeya tiyopa woakahpe wan yecage $k t a$, miniliuha ska nonpa lahmupi, to qa stan qa duta hdehdega ipatapi.

37 Unkan śitim can ipatan zaptan woakahpe kin yecage lkta, qa hena mazaskazi ayapawinte kta, qa yukśanpi kin mazaskazi kta; qa hena on mazaśa sicu zaptan śdoyakiye kte.

\section{WICOWOYAKE $2 \%$.}

1 Unkan owayuśna wan śitim can on yakage kte, ohanske cin wicispa zaptan, qa ohdakinyan kin wiciśpa zaptan; owayuśna topa omdoton kta; qa obosdata kin wiciśpa yamni kta.

2 Qa oise topa kin akan he yakage kta, he kin owayuśna en ikoyakapi kta, qa owayuśna ocowasin mazaśa ayapawinte kte.

3 Qa cahota wakśica yecage kta, qa maza mdaslia qa wakśica on arrinimnipi, qa mazajata, qa mazaoceti kin hena owasin mazaśa on yakage kta.

4 Qa mazaśa oceti wan ho kagapi iyececa, owayuśna kin yecage kta, qa oceti kin akan mazaśa napcupe topa oise topa kin akan.

5 Qa owayuśna opapun kin ihulkuya oceti kin otkeyaye kta, qa owayuśna cokaya kin iyohi kta.

6 Qa sitim can tośu nonpa owayuśna kin yecage kta, qa tośu kin mazaśa ayapawinte kte.

7 Qa tośu kin mazanapcupe ohna yeyaye kta, qa tośu kin owayuśna cuwi napin akan unpi kta, hena on akiyuhapi kta.

8 Canmdaska on yakage kta 119 


\section{HDINANPAPI,}

hdogeca, paha akan okagapi kin wanyag niyanpi kin he iyecen he kagapi kte.

9 Unkan tipi tahocoka kin yakage kta, cuwi itokaga wiyotanhan ektakiya hocoka cuwi wanjidan on ozanpi wan minihuha ska nonpa kahmupi ohanske cin wiciśpa opawinge kta.

$10 \mathrm{Qa}$ mazaśa ipatan kin wikcemna nonpa, qa mazaśa sicu kin wikcemna nonpa, qa ipatan yukśanpi kin, qa içikoyag yapi kin hena mazaska or kagapi kta.

11 He iyecen waziyata cuwi on ozanpi ohanske cin wiciśpa opawinge, qa mazaśa ipatan kin wikcemna nonpa, qa mazaśa sicu kin wikcemna nonpa, ipatan maza yukśanpi kin, qa icikoyag yapi kin hena maza ska kta.

12 Qa hocoka hdakinyan wiyohpeyata ihanke on ozanpi kin wiciśpa wikcemna zaptan, ipatan wikcemna, qa sicu wikcemina.

13 Qa ihanke wihinanpe ektakiya tiyopata hocoka hdakinyan kin wiciśpa wikcemna zaptan kta.

$14 \mathrm{Qa}$ hiyete wanji on ozanpi kin-wiciśpa akezaptan, ipatan kin yamni qa sicu kin yamni.

$15 \mathrm{Qa}$ hiyete unman kin on ozanpi akezaptan, ipatan kin yamni, qa sicu kin yamni kta.

$16 \mathrm{Qa}$ locoka tiyopa akalipe kin minihuba ska nonpa kahmupi, to qa stan, qa duta ipatapi, wiciśpa wikcemna nonpa, qa ipatan topa, qa sicu topa.

17 Ipatan owasin hocoka ihdukśan mazaska on ikoyag yapi kta, qa yukśanpi kin hena mazaska kta, qa tasicu kin mazaśa kta.

18 Hocoka ohanske cin wiciśpa opawinge, qa anog ihanke ohdakinyan kin wiciśpa wikcemna 120 zaptan, qa obosdatu kin wiciśpa zaptan, anog ihanke ozanpi kin minihulıa ska nonpa kahmupi, qa sicu kin mazaśa kta.

19 Tipi wakśica kin owasin on kicanyanpi, qa wihutipaspe kin owasin, qa hocoka wihutipaspe kin owasin mazaśa kta.

20 Unkan Israel cinca kin can ihdi bopanpi heceedan nicahi wicayaśi kta, petijanjan on, hecen ohinniyan ideye kta.

21 Aaron qa iye cinca kin itkokipapi wakeya kin ohna, cokaya ozanpi kin woyaatanin itokam yanke cin, he itankan petijanjan kin kican yanpi kta ece, htayetu etanhan ihanhanna aiyalideya Jehowa itokam, Israel cinca kin wicoicage yeye cin, wicoope kin de ohinniyan yuhapi kta.

\section{WICOWOYAKE 28.}

1 Unkan niś Israel cinca cokayatanhan nicinye Aaron iye cinca om nikiyedan hiyu wicayakiye kte, wayuśnapi ece omakiyapi kte, Aaron qa iye cinca Nadab qa Abihu, Eleazer qa Itamar.

2 Qa wokoyake wakan wowitan on owanyag waśte hinca nicinye Aaron yecage kte.

3 Qa tona cante ksapapi woniya ksapa ojun wicawaye cin owasin Aaron yuwakanpi kte on wamiciyuśna kta e wokoyake kicagapi kta, ewicayakiye kte.

4 Qa wokoyake kagapi kta dena ee; maku akahpe, qa ámdo akalipe, qa nitośke, qa maherı unpi hdeśkaśka, paipahite, qa ipiyaka, hecen wokoyake wakan nicinye Aaron, qa iye cinca wicakicagapi kte; unkan wayuśnapi ece omakiyapi kte.

5 Unkan mazaskazi kin, qa mimihuha ska kin, qa to kin, qa stan 


\section{WICOWOYAKE 28.}

kin, qa, duta kin hena icupi kta.

$6 \mathrm{Qa}$ amdo akalipe kin kagapi kta, mazaskazi qa minihuha ska nonpa kahmupi to qa stan qa duta kazuntapi kin he kagapi kta.

7 Hiyete icaśke nonpa yuke kta, ihanke nonpa kin ekta hecen ikoyag yapi kta.

8 Qa amdo akahpe ipiyaka kin he akan un kin he iyecen mazaskazi qa miniluha ska qa to qa stan qa duta on kagapi kte.

9 Unkan inyan wicaśake iyececa nonpa iyacu kta, qa hena akan Israel cinca cajepi kin yapago kta.

10 Cajepi kin sakpe inyan tokaheya kin akan, qa cajepi unman sakpe inyan inonpa kin akan, token icagapi kin he iyecen.

11 Inyan apagopi iyecen ipuspe akan owapi he iyecen, Israel cinhintku cajepi kin inyan nonpa kin akan apago kta, mazaskazi ozapi en taśpuyapi kta e hena yakage kta.

12 Qa inyan nonpa kin amdo akahpe hiyete akan eyahnake kta, wokiksuye inyan Israel cinca on, qa wicakiksuye kta e Aaron iye hiyete anokatanhan akan cajepi Jehowa itokam yuha kta.

13 Qa mazaskazi ozapi yakage kta.

14 Qa mazaskazi hecedan maza icicahiha yuhmunpi nonpa yakage kta, kazuntapi qa maza icicahilia yuhmunpi kin ozapi kin ekta ikoyag yaye kta.

15 Unkan woyaco maku akahpe kin yakage kta, amdo akahpe kagapi kin he iyecen, mazaskazi qa minihuha ska nonpa kahmupi, to qa stan qa duta kazuntapi kin he yakage kte.

16 Topa omdoton kta, icitakihna, ohanske cin napapaśdecapi, qa ohdakinyan kin napapaśdecapi kta.

17 Qa inyan taśpuyapi cankuye topa he ohna ojuyaye kta, sardius qa topaz qa petaga canku tokaheya kin hee kta.

18 Qa cankuye inonpa kin he smaragdos, qa sapiros, qa inyan paksa ;

19 Qa canku iyamni kin he ligurion, agate, qa ametustos;

20 Qa cankuye itopa kin krusolitos qa inyan wicaśake iyececa, qa iyaspis, qa mazaskazi ozapi ohna hena taśpuyapi kta.

21 Qa inyan kin hena Israel cinca akenonpa cajepi kin hena iyececa ipuspa pagopi kin he iyecen wicoun akenonpa cajepi kin otoiyohi akan pagopi kta.

22 Qa maku akahpe kin akan mazaskazi ecedan ikan maza icicahiha yuhmunpi yakage kta.

23 Qa maku akahpe kin mazaskazi napcupe nonpa yecage kta, qa maza napcupe nonpa kin hena maku akahpe ihanke nonpa en koyag yaye kta.

24 Qa mazaskazi ikan nonpa kin maza napcupe nonpa maku akahpe oise nonpa akan unpi kin hena ohna iyeyaye kta.

$25 \mathrm{Qa}$ ikan nonpa ihanke nonpa ozanpi nonpa kin en koyag yaye kta, qa amdo akahpe hiyete kin akan itankan eyahnake kta.

26 Qa mazaskazi napcupe nonpa yakage kta, qa hena maku akahpe oise nonpa opapun en ikoyag yaye kta, amdo akahpe mahentuya iyotakonza.

27 Qa mazaskazi napcupe nonpa yakage kte amdo akahipe opapun nonpa kin ihukuya tanhan itokam en ikoyag yaye kta, icikoyag yapi ryotakons, maku akalipe ipiyaka kin iwankapatanhan. 28 Qa maku akahpe kin maza 121 


\section{HDINANPAPI,}

napcupe tawa kin on amdo akalipe napcupe kin en iyakaśkapi kte, halionta to wan on, hecen amdo akahpe ipiyaka kin akan yanke kta, qa maku akalipe kin amdo akahpe kin etanhan yukinukan kte śni.

29 Unkan Aaron Israel cinhintku cajepi kin iye cante kin akan yuha kta, woyaco maku akahpe ohna, tipi wakan en ya eca, Jehowa itokam ohinniyan wokiksuye kte.

30 Unkan iyoyamwicaye cin qa yuśtanwicakiye cin hena woyaco maku akahpe kin ekta ikoyag yaye kta, qa Aaron Jehowa itokam u eca hena cante lin akan yanke kta, hecen Aaron Israel cinca woyacopi kin iyc cante kin akan ohinniyan Jehowa itokam yuha kta.

31 Unkan amdo akahpe nitośke kin ocowasin to yakage kta.

32 Qa he cokaya pa i kin yuke kta, qa i kin ihdukśan apalidate kazuntapi maku akahpe i kin iyececa kta, hecen nahdece lite śni.

33 Unkan upi akan taspan tanka seca, to qa stan qa duta yakage kta, opapun ihdukśan, qa mazaskazi hidahda hena otahedan.

34 Mazaskazi lidalida wan, qa taspan tanka seca wan, mazaskazi hdahda wan, qa taspantanka seca wan, nitośke opapun akan ihdukśan.

$35 \mathrm{Qa}$ Aaron omalkiye kta e he koyake kta, qa Jehowa itokam, tipi wakan en u eca, qa tankan inanpa eca he sna kin nahonpi lita, hecen te kte śni.

36 Qa mazaskazi ecedan mdaska wan yakage kta, qa ipuspe pagopi kin he iyecen, he akan Johowa wakan da wo, yapago kta.
37 Qa ikan to wan ikoyag yaye kta, hecen pa iyuskite akan un kta, pa iyuskite itokapatanhan kta.

$38 \mathrm{Qa}$ Aaron ite kin akan un kta, Israel cinca taku yuwakanpi, woqupi yuwakanpi kin hena owasin woahtani kin yuha kta, qa Jehowa iyokipi yapi lita e he ite kin akan ohinniyan yanke kta.

39 Unkan mahenunpi miniliuha hdehdega yakazonte kta, qa minihuha ska pa iyuskite yakage kta, qa ipiyaka ipatapi yakage kta.

40 Unkan Aaron cinca kin mahenunpi wicayecage kta, qa ipiyaka wicayecage kta, qa wapaha, owanyag waśte qa wowitan on yakage kta.

41 Qa hena nicinye Aaron iye cinca kici koyag wicayaye kta, qa napepi kin ojudan wicayakiye kta, hecen wamiciyuśnapi kta e wicayaduwakan kta.

42 Qa miniliuha onzeoge wicayecage $\mathrm{kta}$, on "cehpi iśtecapi akahpapi lita, wicapakśin etanhan wicaceca ekta iyohiye kta.

43 Qa Aaron qa iye cinca kin hena koyakapi kta, itkokipapi wakey a ekta upi eca, qa tipi wakan ohna omakiyapi kta e owayuśna ikiyedan upi eca, hecen woahtani yuhapi kte śni, qa tapi kte śni ; iye, qa tona iye etanhan icagapi kte, wicoope kin he owihanke wanin yuhapi kte.

\section{WICOWOYAKE 29.}

1 Unkan wamiciyuśnapi kta wicaduwalkan lita e taku ecawicayecon kte cin dee, tatanka wan, pte wanunyan pi cinca, qa tamdoka wanunyanpi nonpa owasin waśte icu wo.

2 Qa aguyapi napolye codan, qa aguyapi hmiyanyanna ona- 
polye codan, wihdi icicahiyapi, qa aguyapi ceguguyapi napoliyapi śni, wihdi on sdayapi; hena owasin aguyapi mdu on yakage kta.

3 Qa makanopiye wanjidan en oyahnalse kta, qa hena makan opiyedan ohna, qa tatanka kin, qa tamdoka nonpa kin ikiyedan awicayahi kta.

4 Qa itkokipapi wakeya tiyopa ikiyedan, Aaron qa iye cinca awicayahi kta, qa mini on wicadujaja kta.

5 Qa wokoyake kin iyacu kta, qa mahen unpi kin, qa amdo akalipe nitośke kin, qa amdo akahipe kin, qa maku alkahpe kin, hena owasin Aaron koyag yaye kta, qa amdo akahpe ipiyaka kin ipiyag yaye kta.

$6 \mathrm{Qa}$ pa iyuskite cin pa akan payalite kta, qa wateśdake wakan kin pa iyuskite akan.

7 Qa wihdi on sdayapi kin he iyacu kta, qa pa akan yakaśtan kta, hecen he sda yaye kta.

$8 \mathrm{Qa}$ cinhintku kin ikiyedan awicayahi kta, qa mahenunpi kin koyag wicayaye kta.

9 Qa ipiyake on ipiyag wicayaye kta, Aaron qa cinhintku, qa wapaha kin iyawicayakicaśke kta, hecen wawayuśna hecapi kta, wicoope owihanke wanin, hecen Aaron iye cinca ko napepi ojudan wicayaye kte.

10 Qa tatanka qon itkokipapi wakeya kin itokam ikiyedan ayahi kta, qa Aaron iye cinca koya napepi kin tatanka pa kin akan aputakapi kta;

11 Qa tatanka qon yàkte kta, Jehowa itokam, itkokipapi wakeya tiyopa ekta.

12 Qa tatanka we kin onge iyacu kta, qa owayuśna he kin akan ehpe yaye $k$ ta, ninapsulkaza on, qa we kin ocowasin owa- yuśna sicu kin ekta oyakaśtan lita.

13 Qa watejikśija kin ocowasin, qa pizi pi kin ikoyake cin he, qa pakśin nonpa kin waśín ikoyake cin iyahna, qa owayuśna izin yaye kta.

14 Tuka tatanka qon cehpi kin, qa ha kin, qa tacesdi kin owanka iheyata peta on huhnali yaye kta, woahtani wośna hee.

15 Unkan tamdoka tokaheya kin he iyacu kta, unkan Aaron qa iye cinca kin napepi kin tamdoka pa aputakapi kta.

16 Unkan niye tamdoka kin yakte kta, qa we kin iyacu kta, qa owayuśna akan ilndukśan elipeyaye kta.

17 Qa tamdoka kin yapate kta wobaśpe iyececa, qa śupe kin huha kin ko dujaja kta, qa hena wobaśpe kin qa pa kin ekta eyahnake kta.

$18 \mathrm{Qa}$ tamdoka kin ocowasin on owayuśna izin yaye kta. Jehowa wokicihuhnagapi wan hee; woomna wiciyokipi wan Jehowa kicagapi hee.

19 Unkan tamdoka inonpa kin iyacu kta, qa Aaron, qa iye cinca kin napepi kin tamdoka pa kin aputakapi kta.

20 Qa niś tamdoka kin yakte kta, qa we kin etanhan iyacu kta, qa Aaron noge inkpa alkan, qa cinhintku nogepi inkpa alkan eyahnake kta, etapa kin eciyatanhan, qa napepi etapa hunka akan qa sipahunkepi etapa akan, qa we kin owayuśna akan ihdukśan ehpe yaye kta.

21 Qa we owayuśna akan un kin he etanhan iyacu kta, qa wihdi on sdawicayapi kin he etanhan, qa hena Aaron iyakiun kta, tawokoyake ko, qa cinhintku tawokoyakepi koya, hecen Aaron duwakan kta, qa tawokoyake, 123 


\section{HDINANPAPI,}

qa cinhintku tawokoyakepi koya.

22 Qa tamdoka etanhan waśin kin iyacu kta, qa sinte, qa watejikśija kin he, qa pi pizi kin, qa pakśin napin, qa waśin hena akan un kin, qa hiyete etapa kin, tamdoka wicayuśtan kin hee.

23 Qa aguyapi wanji, qa aguyapi hmiyanyanna wihdi icicahiyapi wanjidan, qa aguyapi ceguguyapi wanji aguyapi napohye codan makan opiye kin Jehowa itokam yanke cin hetanhan.

24 Qa hena owasin Aaron nape kin en eyahnake kta, qa cinhintku napepi kin en, qa Jehowa itokam hena yakoze kta, wokozapi wan hee.

25 Qa napepi kin etanhan hena iyacu kta, qa owayuśna kin izin yaye kta, wohuhnahyapi woomna wiciyokipi Jehowa itokam, peta wośnapi wan Jehowa kicagapi hee.

$26 \mathrm{Qa}$ tamdoka Aaron on yuśtanpi kin he maku kin iyacu kta, qa Jehowa itokam he yakoze kta, qa obaśpe kin he nitawa kta.

27 Hecen tamdoka on Aaron ojunyapi, qa iye cinca nakun ojun wicayapi kin he maku kozapi qa hiyete yuwintapi, taku kozapi, qa taku yuwintapi kin he duwakan kta.

$28 \mathrm{Qa}$ he Aaron qa cinhintku tawapi lita, Israel cinca kin etanhan wicoope owihanke wanice kta, he yuwintapi heon Israel cinca wopida wośnapi etanhan he yuwintapi kta, Jehowa ekta yuwintapi kta.

29) Unkan wokoyake wakan Aaron tawa kin he cinhintku, iye iyohakam tawapi kta, hena ohna sdawicayapi kta, qa napepi kin ojun wicayapi kta.

30 Cinhintku etanhan tuwe heekiya wawayuśna kta, qa tipi wa- kan ohna omakiye kta e itkokipapi wakeya ekta hi kinhan, anpetu śakowin hena koyake kta.

31 Unkan tamdoka ojunwicaye cin he iyacu kta, qa cehpi kin oyanke wakan kin ohna oyahe kta.

32 Unkan Aaron qa cinhintku tamdoka cehpi kin yutapi kte, aguyapi makan opiye olna un kin he nakun, itkokipapi wakeya tiyopa kin ekta.

33 Taku on woakahpe kagapi napepi ojun wicayapi, wicayuwakanpi kte cin, hena yutapi kta, tuka tuwe tokeca hena yute lite śni, nakaeś hena wakan.

34 Unkan ojun wicayapi cehpi kin onge oyaptapi kinhan qaiś aguyapi kin onge ihanhanna hehanyan, taku oyaptapi kin he huhilah yaye kta hee wakan heon yutapi kte śni.

35 Ünkan Aaron qa cinhintku hecen ecawicayecon kta, token econ ciśi kin owasin iyecen, anpetu śakowin napepi kin ojun wicayaye kta.

$36 \mathrm{Qa}$ woakahpapi on anpetu otoiyohi tatanka woahtani wośnapi yapate kta, hecen owayuśna kin duteca kta, woakahpe akan yakage cin on, qa duwakan kta e sdayaye kta.

37 Anpetil śakowin owayuśna on woakahpe yakage kta, qa he duwakan kta, hecen owayuśna kin wakan hinca kta, taku kaśta he yutan kinhan wakan kta.

38 Unkan owayuśna akan taku waduśna kte cin dee; amnos (tahin wanuyanpi) waniyetu wanjidan, nonpa anpetu otoiyohi ohinniyan.

39 Hanhanna eca amnos tokaheya yapate kta, qa amnos inonpa kin olitayetu otahedan yapate kta.

40 Qa amnos wanjı kici aguya-

124 
pi mdu iyutapi iwikcemna (wiyatke ohnaka śakpe) qa wihdi bopanpi hin itopa kin (wiyatke ohnaka nonpa) icicahiyapi qa woakaśtan qupi miniśa hin itopa kin.

41 Unkan tahinca inonpa kin oltayetu otahedan yapate kta hanhanna minha kin qa woakaśtan kin he iyecen ecayecon kta peta wośnapi woomna Jehowa iyokipi ye kta.

42 Jehowa itokam itkokipapi wakeya tiyopa kin ekta wicoicage nitawapi kin yeye cin he peta wośna wan ohinniyan un kte; hen ociciye kta e heciya itkocicipapi kta.

$43 \mathrm{Qa}$ hen Israel cinca kin itkowicawakipe kta, qa mitowitan kin he yuwakanpi kta.

44 Qa itkokipapi wakeya kin, qa owayuśna kin mduwakan kta, qa wamiciyuśnapi kta e Aaron cinhintku ko wicamduwakan kta.

$45 \mathrm{Qa}$ Israel cinca kin wicehna ounwaye kta, qa taku wakan dapi kin he miye kta.

$46 \mathrm{Qa}$ Jehowa wakandapi Egupta makoce etanhan hdinanpe wicaye cin he miye sdonyapi kta, wicehna ounwaye heon etanhan; Jehowa Wakandapi kin he miye.

\section{WICOWOYAKE 30.}

1 Unkan taku waśtemna izin yapi kta e wizinya owayuśna wan yakage $\mathrm{kta}$, sitim can on yakage kta.

2 Ohanske cin he wiciśpa wanjidan kta, qa ohdakinyan kin wiciśpa wanjidan, topa omdoton kta, qa obosdatu wiciśpa nonpa, he kiyuke kta.

3 Qa mazaskazi ecedan on ayapawinte kta, toakahpe kin, qa cuwi kin ihdukśan, qa he kin ; qa ihdukśan mazaskazi wateśdake wan yecage kta.

4 Qa mazaskazi napcupe nonpa yecage kta, wateśdake ihukuya cuwi nonpa akan anokatanhan hena yakage kta, qa hena tośu on akiyuhapi icupi kta e yuke kta.

$5 \mathrm{Qa}$ śitim can tośu kin yakage kta, qa mazaskazi ayapawinte kta.

6 Qa cokaya ozanpi kin itokam woyaatanin can ohnaka iyotakons, tukten itkocicipe kta, wokajuju iha woyaatanin can wohnake kin akan yanke cin he itokam eyahde kta.

$7 \mathrm{Qa}$ he akan Aaron pejuta waśtemna izinye kta, hanhanna otoiyohi petijanjan pikiya eca he izinye kta.

8 Qa Aaron petijanjan wankan ehnaka eca olitayetu otahedan taku waśtemna he akan izinye kta, ohinniyan Jehowa itokam nitoicagepi yeye cin.

$9 \mathrm{He}$ akan wizinyapi tokeca, qa wohulinahyapi, qa minlia hulinah yaye kte śni, qa woakaśtan ayakaśtan kte śni.

10 Tuka he kin akan ptanyetu otoiyohi wancadan Aaron woakahpe kage kta ; nitoicagepi yeye cin ptanyetu eca wancadan woalitani kajujupi we on he akan waakalipe kta. He Jehowa tawa wakan hinca.

11 Unkan Jehowa ie ca Mowis heciya,

12 Tohan Israel cinca kin wicadawa eca, wicayawapi kta e wicaśta otoiyohi nagi kin on woakalipe Jehowa qu kta, wicayawapi icunhan, hecen wicay awapi icunhan makośice awicakipe $\mathrm{kte}$ śni.

13 Hecen qupi kta, tona wicayawapi kin iyoopta ya otoiyohi 125 


\section{HDINANPAPI,}

śeqel hanke tipi wakan śeqel iyececa (omnica wikcemra nonpa śeqel ee ce) śeqel hanke Jehowa qu kta.

14 'Tona wicayawapi iyoopta ya wica waniyetu wikcemna nonpa qa iwankam owasin woahi Jehowa qupi kta.

15 'Tuwe wijica eśta śeqel hanke yuota kte śni, qa tuwe onsika eśta yuoptetu kte śni, ninagipi on hdajujupi kta woahi wan Jehowa qupi eca.

16 Qa woakalipe mazaska kin Israel cinca etanhan iyacu kta, qa itkokipapi wakeya kicanyanpi on yaqu kta, qa Israel cinca wokiksuye yapi kte, Jehowa itokam ninagipi kin on wokajuju kta.

17 Unkan Jehowa ie ca Mowis heciya ;

18 Nakun mazaśa koka wan yakage kta, qa mazaśa cetetahe lin, ekta ihdujajapi kta, qa itkokipapi wakeya qa owayuślıa otahedan eyahde $\mathrm{kta}$, qa he ohna mini oyakaśtan kta.

19. Unkan Aaron iye cinca koya hetanhan napepi sihapi ko ibdujajapi kta.

20 Itkokipapi wakeya kin en yapi eca mini on ihdujajapi kta ece, hecen tapi kte śni, qa tohan Jehowa ókiyapi owayuśna ikiyedan upi, peta wośna izin kiyapi ece kta.

21 Napepi sihapi ko ihdujajapi kta, hecen tapi kte śni; he wokage owihanke wanice kta, iye qa cinca kin wicoicagepi ecen econ kta.

22 Unkan Jehowa ie ca Mowis heciya,

23 Nakun niś pejuta waśtemna itancan icu wo, mur śbuśbu śeqel opawinge zaptan, qa canbaśa waśtemna he hanke, opa winge nonpa sanpa wikcemna zaptan, 126 qa sinkpe tawote waśtemna opawinge nonpa sarupa hanke,

24 Qa kasia opawinge zaptan, tipi wakan śeqel ohna iyutapi kta, qa can ihdi hina wanjidan.

$25 \mathrm{Qa}$ hena sdayapi wakan wihdi yakage kta, pejuta wicaśta taku waśtemna kagapi kin he iyecen, sdayapi wakan wihdi waśtemna hee kta.

$26 \mathrm{Qa}$ he on itkokipapi.wakeya kin sdayaye $\mathrm{kta}$, qa woyaatanin canwohnaka kin,

$27 \mathrm{Qa}$ wahna wotapi kin, qa wakśica tawa kin owasin, qa petijanjan ihupa, qa on kicanyanpi kin, qa izin yapi owayuśna kin.

28 Qa wohulinahyapi owayuśna kin, qa taku on kicanyanpi owasin, qa koka kin qa tacetetahe koya.

29 Hecen hena duwakan kta; qa wakanhincapi kta, taku kaśta hena yutan kinhan wakan kta.

30 Unkan Aaron qa iye cinca kin sdawicayaye kta, qa wamiciyuśnapi kta e wicaduwakan kta. $31 \mathrm{Qa}$ Israel cinca kin becen ewicayakiye kta, wakan sdayapi wihdi mitawa kin dee kta, nitoicagepi yeye cin ohna.

32 Wicaśta cehpi kin akan he akaśtanpi kte śni qa he icicahiyapi kin, qa taku on kagapi iyecen yakagapi kte śni, hee wakan, qa wakanyan yadapi kte.

33 Tuwe he iyecen wihdi waśtemna ye cin, qa tuwe wicaśta tokeca akan he akaśtan kinhan, oyate tawa kin etanhan kaśpapi kta.

34 Unkan Jehowa Mowis heciya, Taku waśtemna icu wo, canśin qa tukiha waśtemna, ya galbanum oomna suta, qa canśin ska, otoiyohi iakedenakeca.

$35 \mathrm{Qa}$ hena wizinyapi yakage kta, pejuta wicaśta taku icicahiyapi kin he iyecen yakage kta 
miniskuya ecedan icicahiyapi wakan kta.

$36 \mathrm{Qa}$ hetanhan boyapan kta, mduhinca yakage kta, qa onge woyaatanin itokam itkokipapi wakeya ohna tukten itkocicipe kta eyahnake kta, he wakanhinca dawapi kta.

37 Qa taku waśtemna kin de yakage cin he iyecen takudan niçicagapi kte śni; he Jehowa tawa, he wakan dawa kta.

38 Tuwe omna kta e taku he iyecen kage cinhan, oyate tawa kin etanhan kaśpapi kta.

\section{WICOWOYAKE 31.}

1 Unkan Jehowa ie ca Mowis lieciya,

2 Tho wo Betzalel Uri cinhint$\mathrm{k} u$, Hur cinhintku, Juda wicoun etanhan caje kin on weco.

3 Qa taku wakan toniya on ojuwaya woksape qa wookahinige, qa wosdonye on, qa wolitani owasin en.

4 Taku wayupiya kagapi iyukcan kta, mazaskazi, qa mazaslia, qa mazaśa on kage kta.

5 Qa inyan ojuyapi kin pago kta, qa can pago kta, qa taku kagapi owasin kage kta.

$6 \mathrm{Qa}$ he kici miś miye Aholiab Alisamak cinhintku, Dan wicoun kin etanhan waqu, qa tona cante ksapapi cantepi ohna woksape wicawaqu, hecen taku kali ciśi kin owasin kagapi kta.

7 Itkokipapi wakeya kin, qa canwohnake woyaatanin on, qa he akalipe cin, wokajuju iha kin, qa taku wakeya kicanyanpi owasin.

8 Qa wahna wotapi wakśica tawa iyahna, qa petijanjan ihupa ecedan kin, qa taku on kicanyanpi owasin, qa wizinyapi owayuśna kin.
$9 \mathrm{Qa}$ wohulinaliyapi owayuśna kin, qa taku on kicanyanpi owasin, qa maza koka kin, qa tacetetahe kin.

10 Qa śina ozanpi kin, qa Aaron wawayuśna tawokoyake wakan kin, qa cinhintku tawokoyakepi ohna wayuśnapi kte.

11 Qa wihdi on sdayapi kin, qa wizinyapi waśtemna kin tipi wakan kin on; token econ ciśi kin owasin iyecen econpi kta.

12 Unkan Jehowa ie ca Mowis heciya,

13 Niś Israel cinca kin hecen ewicakiya wo, Tokeśta anpetu wakan mitawa kin yapatanpi kta, nakaś nitoicagepi yeye cin niyepi qa miye unkiotahedan, he wowapetokeca on ciyuwakanpi ; Jehowa he miye sdonyapi kta.

*14 Qa anpetu okilipapi kin he yapatanpi kta, nakaś niyepi on he wakan, tuwe he yuśape cinhan he ktepi kta, heon tuwe he icunhan wolitani taku kaśta econ kin he taoyate kin cokayatanhan kaśpapi kta.

15 Anpetu śakpe wohtani econpi kta, tuka anpetu iśakowin kin he anpetu wakan, okihpapi wakan Jehowa tawa, tuwe kaśta anpetu okihpapi en wohtani econ kinhan he awicakehan ktepi kta.

16 Heon Israel cinca kin anpetu okilipapi kin he patanpi kta, wicoicagepi yeye cin wicotakuye owihanke wanica, anpetu olkihpapi kin kagapi kta.

17 Miye qa Israel cinca unkiotahedan he wowapetokeca owihanke wanice kta, nakaś anpetu śakpe en Jehowa mahpiya kin qa maka kin kage ça anpetu iśakowin kin en okilipe ca oziiciye.

18 Unkan Sina he kin olina Mowis kici okiye enakiye cehan inyan mdaska woyaatanin qu, in- 


\section{HDINANPAPI,}

yan mdaska nonpa Jehowa napsukaza on owapi.

\section{WICOWOYAKE 32.}

1 Unkan oyate kin Mowis he kin etanhan apamahdeya hiyu hdutehan kin he wanyakapi qehan, oyate kin Aaron en mniciyapi, qa heciyapi, Najin wo, taku wakan unkiyepi itokam yapi kta unkicicagapo. Ecah Mowis wicaśta Egupta makoce etanhan hinanpe unyanpi kin he toki un kin sdonunyanpi śni ce.

2 Unkan Aaron hewicakiya, Mazaskazi oinpi kin nitawinpi qa nicincapi wica winyan ko, nogepi ikoyakapi kin hena yuksapi qa en makahipo.

3 Unkan oyate kin"owasin mazaskazi oinpi kin nogepi kin etan" han hduksapi, qa Aaron en kahipi.

4 Unkan Aaron hena napepi lkin etanhan icu, qa ojuha wan en palita qa ptejicadan śdoyapi wan kaga. Unkan, Israel taku wakan nitawa Egupta makoce etanhan hinanpe niyanpi kin dena ee ce, eyapi.

5 Unkan Aaron wanyalke ça he itokam owayuśna wan kage ca, Heyakecinhan Jehowa wakan wokicihanpi kta, Aaron ieyanpaha, eya.

6 Unkan ihanlianna hin kiktapi, qa wohuhnahyapi hulnahyapi, qa wopida wośnapi ahipi, qa oyate kin wotapi qa wayatkanpi kta e iyotankapi, qa ślkatapi kta e najinpi.

7 Unkan Jehowa Mowis heciya, Kun hda, oyate nitawa Egupta makoce etanhan hinanpe yaye cin wanna ihduhowinpi ce.

8 Canku wan ope wicawaśi qon he etarhan kohanna ihdutokanpi, ptejicadan śdoyapi wan içi128 cagapi, qa ekta pakicapsanpi, qa wakjciyuśnapi, qa Israel taku wakan yada, Egupta makoce etanhan hdinanpe niye cin dena ee ce, eyapi.

9 Unkan Jehowa Mowis heciya, Oyate kin de wanmdake ca inyun oyate tahu patin hecapi.

10 Qa wanna micuni wo, hecen mitocanniye kin awicalrate kta, qa ihang wicawaye kta, qa niś oyate tanka cicage kta ce.

11. Unkan Mowis Jehowa taku iye wakan da kin cin kiye ca heya, Jehowa, tokeca oyate nitawa kin canniye yaya he? hena Egupta makoce etanhan hdinanpe wicayaya, wowaśake tanka on, qa nape waśaka on.

12 Tokeca Egupton heyapi kta, Taku sica on inanpe wicaya, he kin ohna wicakte kta, qa maka ite kin etanhan awihnuni wicaye kta heon etanhan, eyapi kta; nitocanniye kate cin etanhan ihduhomni, qa oyate nitawa en taku śice cin de on iyope içya ye.

13 Nitaokiye Aberaham, qa Izak, qa Israel wicakiksuya ye, token caje nihdate ca konze hewicayakiya, Nicincapi kin mduota kta, mahpiya wicanhipi kin iyenakecapi kta, qa makoce kin de cajemdate cin ocowasin nicincapi kin wicawaqu kta, qa owihanke wanin tawayapi kta.

14 Unkan Jehowa taku śica taoyate kin ecawicakicon $\mathrm{kta}$, eye ciqon on iyope iciya.

15 Unkan Mowis ihdamna, qa he kin etanhan apamahdeya hda, qa woyaatanin inyan mdaska nonpa qon nape kin ohna hdohda, inyan mdaska kin hena anokatanhan owapi, deciyatanhan qa unman eciyatanhan nakun, inyan mdaska owapj ce.

16 Inyan mdaska kin hena Wakantanka kaga, qa owapi kin lie 
Wakantanka owa ce, inyan mdaska kin akan pago.

17 Unkan Jośuwa oyate oqoyapi kin nation qa, Owanka ohna kicizapi iyecen oqoyapi ce, Mowis eciya.

18 Unkan, Wicaśta ohiyapi, qa panpanpi ho kin hee śni, qa wicaśta wicaktepidan howayapi kin hee śni ce, tuka wicaśta dowanpi ho kin nawalion, eya.

19 Qa owanka kin ikiyedan u qehan, qa ptejicadan, qa wacipi kin wanyake cehan, Mowis sinhda, qa inyan mdaska qon nape etanhan elipeya, qa kamdeca, lie kin ihukuya.

20 Qa ptejicadan kagapi qon he icu qa peta on hulinaga, qa yukpan, hecen mdu kage ça mini kin akada; qa Israel cinca kin yatke wicakiya.

21 Unkan Mowis Aaron heciya, Oyate kin de taku ecannionpi kin on woalitani tanka kin de awicayakahi he?

22 Unkan Aaron heya, Itancan, ihnuhan śinyahda kin. Niś oyate kin de śicapi ce, sdonwicayaya.

$23 \mathrm{Qa}$ hemakiyapi; Taku wakan unkitokam yapi kta unkicicagapo, Mowis wicaśta kin he Egupta makoce kin etanhan, itanwankanhde mani unyanpi kin, tuktee he sdonunyanpi śni ce.

24 Unkan, Tuwe mazaskazi onge yuhe cinhan hdukse kta, ewicawakiya, unkan he maqupi, unkan petan ehpewaya, qa ptejicadan kin de inanpa ce.

25 Hehan oyate kin śdokahanpi e Mowis wanyaka, nakaś tokawicayapi iwicahahapi kta Aaron wicayuśdoka ;

26 Qa owanka tiyopa kin ohna Mowis najin qa, Tuwe Jehowa ope cin en mahi wo, eya. Unkan Lewi cinca kin owasin en liawitavapi.
27 Unkañ hewicakiya, Wicaśta otoiyohi tamazasagye kihnake kta, ceca akan qa owanka ohna tiyopa iyaza ye kta, qa wicaśta otoiyohi iye sunkaku qa takodaku, qa tuwe iye ikiyedan ti kin wicakik te kta, Jehowa Israel wakandapi kin he eya ce.

28 Unkan Lewi cinca kin Mowis eye cin he iyecen econpi ; unkan anpetu kin he oyate kin etanhan wicaśta kektopawinge yamni ccetu ilipayapi.

29 Nakaś Mowis hewicakiye ciqon, Jehowa on anpetu kin de ninapepi kin ojunkiyapo, wicaśta iye cinhintku qaiś sunkaku kin on, hecen anpetu kin de woyawaśte wan nicupi kta ce.

30 Unkan ihantianna Mowis oyate kin hewicakiya, Woahtani tanka wan wayalitanipi, heon wanna itanwankanhdcya mde kta, Jehowa ekta, okini wayahtanipi kin akahpe waye kta ce.

$31 \mathrm{Qa}$ Mowis icicawin Jehowa ekta ye ca heya, Hehe, oyate kin de woalitani tanka walitanipi, qa mazaskazi taku wakan icicagapi ;

32 Qa wanna tokin wahtanipi kin wicakiciyutokan, qa hecetu śni kinhan wowapi wan oyakiwa kin hetanhan mapajujuye, ceciciya.

33 Unkan Jehowa Mowis heciya, Tuwe amawahtani kin he wowapi mitawa kin etanhan wapajuju kta ce.

$34 \mathrm{Qa}$ wanna ekta hda, tokiya caje mdata qon heciya oyate kin awicaya wo, ito ohnihde wakiye cin he nitokam ye kta, tuka, tohan en wahi eca wahtanipi kin awicawahi ece kta.

35 Unkan Jehowa ptejicadan kagapi on, Aaron kage cin hee, on etanhan oyate kin wicakaśtaka. 


\section{WICOWOYAKE 33.}

1 Wanna Jehowa Mowis hecen eciye ciqon, Hunktiya detanhan itan wankanhdeya ya wo, niś qa oyate Egupta makoce etanhan bdinanpe wicayaye cin, makoce wan nicinca kin he waqu kta ce, Aberaham, qa Izak, qa Jakob iwicawakonze ciqon he elkta.

$2 \mathrm{Qa}$ ohnihde wakiye cin he nitokam ye waśi kta, qa nitokapatanhan Kanani kin, qa Amori kin, qa Hiti kin, qa Perazi kin, qa Hiwi kin, qa Jebusi kin, ham wicawaye kta;

3 Makoce wan asanpi qa canhanpi tiktica kaduze cin he ekta; oyate tahu patin henicapi heon niyepi cokaya mde kte śni, okini tahepi tem ciyapi kta.

4 Unkan oyate kin wicoie śice cin he nahonpi, qa iyokiśin ic̣iyapi, qa tuwedan oinpi kin ikoyag kiye śni.

5 Nakaś Jehowa Mowis heciye ciqon, Niś oyate tahu patin henicapi, ihnuhanna niyepi cokaya inawape kte ça ihang ciyapi kta ; beon wanna oinpi nitawa kin niye etanhan makata ehpe kiya wo, hecen taku ecacicon kta sdonwakiye kta, Israel cinca kin ewicakiya wo.

6 Hecen Israel cinca kin oinpi kin hduśdokapi, Horeb he kin ihukuya.

7 Unkan Mowis wakeya kin icu, qa owanka kin itankan ehde, owanka kin itehan, qa itkokipapi wakeya kin, eya cajeyata. Unkan tona Jehowa alkitapi kin hena owasin itkokipapi wakeya kin ekta owanka kin itankan inanpapi.

8 Unkan Mowis wakeya kin ektakiya inanpa eca oyate kin owasin najinpi ece, otoiyohi ta130 wakeya tiyopa kin ekta qa Mowis ihakam etonwanpi wakeya kin ekta i kin hehanyan.

9 Unkan Mowis wakeya kin ekta i eca mahpiya śoka bosdata kin kun hiyu, wakeya tiyopa kin ekta, qa Mowis okiya ece.

10 Unkan oyate kin owasin malipiya bosdata qon, wakeya tiyopa kin ekta najin e wanyakapi, qa oyate kin owasin najinpi qa cekiyapi, otoiyohi tawakeya tiyopa ekta.

11 Unkan Jehowa Mowis kici wohdaka, kaicitkokim, wicaśta wan takodaku kici wohdaka he iyececa, unkan owanka kin ekta kihda, qa taokiye Jośuwa Nun cinhintku kośka kin, tuka mahpiya bosdata wakeya cokayatanhan tokan ye śni.

12 Unkan Mowis Jehowa heciya, Tho oyate kin de itanwankanhdeya awicaya wo, eha tuka tuwe mici ye yaśi kta sdonye mayakiye śni, tuka nicaje on sdon ciya, qa nakun miiśta kin iyokipi yaya ce, eha.

13 Qa wanna niiśta kin iyokipi waye cinhan nitacanku kin sdonye makiya ye; hecen niiśta kin ohna woiyokipi iyewaye cin he sdotciye kta, qa oyate kin de oyate nitawa kin ee, awacin wo. 14 Unkan, Miite kin ye kta qa oziciciye lta ce, eya.

15 Unkan heciya, Niite kin ye śni kinhan, detanhan itanwankanhdeya unkayapi śni ye.

$16 \mathrm{Qa}$ wanna niiśta kin ohna woiyokipi iyewaye, miye qa oyate nitawa kin tokiyatanhan sdonyapi lita he? unkicipi idade cin hee śni he? hecen miye qa oyate nitawa kin oyate maka itohnake akan unpi kin owasin etanhan unyukinukanpi kta.

17 Unkan Jehowa Mowis hec1ya, Taku ehe cin he nakiun eca- 


\section{WICOWOYAKE 34.}

mon kta, nakaś miiśta kin ohna woiyokipi iyeyaya, qa nicaje kin on sdonciya.

18 Unkan, Tokin nitowitan he wanyag makiya ye, eya.

19 Unkan, Mitowaśte kin owasin niite kin itokam iyaye wakiye kta, qa Jehowa caje kin eyanwapaha kta, nitokam, qa tuwe cante wakiye cin he cante wakiye kta; qa tuwe onśiwada kin he onsiiwakidake kta ce.

20 Tuka miite kin wandaka oyakihi śni, nakaś wicaśta tuwe wanruayake cinhan ni lite śni ce, eya.

21 Nakun Jehowa heya, Inyun oyanke wan mikiyedan, qa imnija kin akan ayahe kta.

$22 \mathrm{Qa}$ mitowitan kin ahiyaye cin hehan imnija ohdoka wan olidateya ecihde kta, qa awahimdamde cin icunhan minape on akalipe ciye kta.

23 Qa minape kin mihdutokan kta, hecen initapete tanhan wandake lsta, tuka miite (wicaśta) wanyakapi kte śni.

\section{WICOWOYAKE 34.}

1 Unkan Jehowa Mowis heciya, Inyan mdaska nonpa kakan wo, tokaheya qon he iyececa, qa inyan mdaska tokaheya ya kamdeca, oie akan un qon, hena inyan mdaska kin akan owawa kta.

2 Qa hanhanna on ihduwiyeya wo, qa hanlianna Sina he kin ekta itanwankanhdeya yau kta, qa heciya he paha kin akan iyamiyecihe kta.

3 Qa wicaśta tuwedan niciu kte śni, qa nakun he kin owancaya wicaśta tuwedan ihdutanin kte śni; nakun tahinca qa pte kin he kin be icahda wihanpi kte śni.
4 Unlsan inyan mdaska nonpa kakan, tokaheya qon hena iyececa, qa Mowis hanhannahin kikta, qa Sina he kin ekta itanwankanhdeya i, Jehowa econśi qon he iyecen, qa inyan mdaska nonpa kin nape kin ohna yuha.

5 Unkan Jehowa mahpiya soka ohna kun hiyu, qa kici hen iyahan, qa Jehowa caje kin ieyanpaha.

6 Qa Jehowa he itokam ahiyaye ca ieyanpaha, Jehowa, Jehowa, Taku Wakan waonśida, qa wacantkiya, tehan śihda śui, tocantekiye, towicake ko ota.

7 Wicoicage yeye kektopawinge cantewicalkiya ece, wicohan śica qa wokipajin qa woahitani kajuju ece, tuka ohinniyan kajuju kte śni, ateyapi wicuhan śicapi cincapi qa sanpa cincapi kin en awicahi ece, wicoicage iyamni qa itopa kin hehanyan. 8 Unkan Mowis inalini, qa makata pakicapsan, qa cekiya.

9 Qa heya, I tancan wanna niiśta kin ohna woiyokipi iyewaye cinhan, Itancan kin unkiyepi cokaya ye kta ceciciya, qa wicohan śica unkitarvapi, qa waunhtanipi kin unkicicajuju miye, qa tawa unkiya miye, nakaś oyate kin tahu patin hecapi.

10 Unkan heya, Inyun miye wicotakuye wakaga, oyate nitawa kin owasin itokam, wicohan wowinihan ecamon kta, hena iyececa maka kin owancaya ikcewicaśta owasin ehna tuwedan tohini econ śni, qa oyate wicehna yaun kin he owasin Jehowa ohan kin wanyakapi kta nakaś taku wokokipe hinca nici ecamon kta.

11 Taku nakảha econ ciśi kin awacin wo. Ito nitokapa tanhan Amori kin, qa Kanani kin qa Ḣiti kin qa Perazi kin, qa 131 


\section{HDINANPAPI,}

Ḣiwi kin, qa Jebusi kin nape wicawaye kta.

12 Waktaya wo. Ihnuhan oyate makoce ekta idade cin he ounyanpi kin om wicotakuye yakage cinhan okini the niyepi cokaya wihmunke kta.

13 Tuka owayuśnapi tawapi kin ihang ya yapi kta, qa wakagapi tawapi kin yakamdecapi kta, qa can pasdatapi kin hena yakawankapi kta.

14 Taku wakan tokeca ekta payecapsan kte śni; Jehowa winawizi eciyapi kin he Taku Wakan winawizi kin ee.

15 Okini makoce en ounyanpi kin om wicotakuye yakage cinhan, qa taku wakandapi kin ókiyapi, qa taku wakandapi kin wakiyuśnapi, qa nicopi qa taku wośnapi kin he yate kta.

16 Qa nicinkśi kin cunwintkupi kin etanhan yus yakiya, qa cunwintkupi kin taku wakandapi ókiyapi qa nakun nicinkśi taku wakandapi kin ókiye yapi kta.

17 Taku wakan śdoyapi kin niçicage kte śni.

18 Aguyapi napoliyapi śni wohanpi kin yapatan kta. Anpetu śakowin aguyapi napohyapi śni yate kta, econ ciśi kin he iyecen. Aguyapi itka hinanpa wi kin iyehantu kin hehan, nakaś aguyapi itka hinanpe cin wi kin he en Egupta etanhan yahdinanpa ce.

19 Winyan cinca tokaheya ton kin hena owasin mitawa, qa wanunyanpi nitawa, pte, qa tahinca cinca tokapa owasin mdoka kinhan.

20 Tuka śuktanka śonśonna cinca tokapa kin he opeyakiton kta ; talinca cinca kin iyope yakiye kta, qa opeyakiton kte śni kinhan tahu kin bayakse kta. 132
Qa nicinca tokapa kin opeyakiton kta. Qa tuwedan mitokam cokadan ihdu tanin kte śni.

21 Anpetu śakpe litayani kta, qa anpetu iśakowin en oziniçiye kta, maga yumdupi, qa woksapi iyehantu eśta oziniciye kta.

22 Anpetu wakan íśakowin wohanpi kin nakun miyecagapi kta, aguyapi baksapi tokaheya ahipi kin hee, qa womnayanpi wohanpi kin, omaka ihuniyanpi kinhan.

23 Waniyetu otahedan yamni akihde nicinca wica kin owasin Itancan Jehowa Israel Taku Wakan dapi kin he itokam ihdutaninpi lata.

24 Nitokapa tanhan Ikcewicaśta kin tokan ye wicawaye kta ; qa nitamakoce kin mdutanka kta, qa waniyetu otahedan Jehowa itokam nihdutanin kta, yamni akihde, itanwankanhdeya idade cinhan tuwedan nitamakoce kin cin kte śni.

25 Taku wamayakiduśna we kin onapohye ayakaśtan kte śni. Qa acakśsin wohanpi wośna kin ihanlianna kin en yanke kte śni.

26 Maga nitawa taku tokaheya icahya tokapa kin Jehowa wakan yada ti kin ekta ayahi kta. Tatokadan cincadan hunku asanpi kin en oyahe kte śni.

27 Unkan Jehowa Mowis heciya, Wicoie kin dena owa wo; nakaś wicoie kin dena i kin iyececa wicotakuye cicicaga, Israel kici nakun.

28 Unkan Jehowa kici hen yanke anpetu wikcemna tom, qa hanyetu wikcemna tom, aguyapi yute śni, qa mini yatke śni. Unkan wicotakuye oie kin inyan mdaya kin akan owa wicoie wikcemna kin.

29 Unkan Mowis woyaatanin inyan mdaska nonpa napohua yuha apamahdeya u, qa Mowis Si- 
na he kin etanhan apamahdeya u qehan, he kici wohdaka icunhan ite uka lin iyege cin he sdotkiye śni.

30 Unkan Aaron qa Israel cinca kin owasin Mowis ite kin wanyakapi ; unkan inyun ite uka kin iyega, unkan ikiyedan upi kin ilkopapi.

31 Unkan Mowis wicakipan, unkan Aaron qa omniciye itancan owasin en hdipi, unkan hena om Mowis wohdaka.

32 Unkan he iyohakam Israel cinca kin owasin ikiyedan hipi, unkan Jehowa Sina paha ohna taku eciye cin owasin econ wicaśi.

33 Qa Mowis owicakiye hdaśtan kin hehanyan ite akahpe wan ite kin akan ehnaka.

34. Qa Mowis Jehowa kici wohdaka en i qehan, ite akahpe kiı hdutokan, tankan i kin hehanyan, qa tankan inanpe ca taku econ śipi kin owicakiyaka.

35 Unkan Israel cinca kin Mowis ite kin wanyakapi, Mowis ite uka kin iyega; heon Mowis ite akahpe kin ake ite akan hiyuya, kici wohdake kta en i kte cin hehanyan.

\section{WICOWOYAKE 35.}

1 Unkan Mowis Israel cinca omniciye ocowasin yuwitaya u wicakiye, ca hewicakiya, Wicoie Jehowa econ niśipi dena ee.

2 Anpetu śakpe wohtani ecannon kta, tuka anpetu iśakowin kin he wakan yada kta, anpetu okihpapi wakan Jehowa tawa, he icunhan tuwe kaśta wohtani econ kinhan te yapi kta.

3 Ounyayapi kin owasin ohna anpetu okihpapi icunhan ceyatipi kte śni.
4. Unkan Mowis ie ca Israel cinca omniciye ocowasin hewicakiya, Taku Jehowa econ niśipi kin dee, qa heya ;

5 'Taku Jehowa ituyakilianpi kta he icupo. Tuwe cante kin iyopaśtaka, taku Jehowa itukihan kte cin he ahi kta, mazaskazi, qa mazaska, qa mazaśa ;

6 Qa tahinca hin to, qa stan, qa duta, qa minihiuha ska, qa tatokadan hin ;

7 Qa tamdoka ha śayapi, qa hoka ha, qa śitim can;

$8 \mathrm{Qa}$ wihdi petijanjan on, qa taku waśtemna wihdi sdayapi kin on, qa wizin yapi waśtemna on ;

$9 \mathrm{Qa}$ inyan wicaśake iyececa, qa inyan ojuyapi kta, amdo akahpe ohna, qa maku akahpe ohna.

10 Qa niyepi ehna tona cante ksapapi kin owasin upi kta, qa taku Jehowa kah śi kin owasin kagapi kta ;

11 Tipi kin, tawakeya, qa toakahpe kin nakun, tamaza taśpu kin, qa canmdaska kin, canhdakinyan kin, can bosdata tawa qa sicu tawa kin ;

12 Can wohnake kin, qa tatośu kin, wokajuju iha kin, qa oakahpe ozanpi kin ;

13 Wahna wotapi, qa tatośu lkin, qa taku on kicanyanpi kin owasin, qa aguyapi itokam hnakapi kin ;

14 Qa petijanjan ihupa kin, qa taku on kicanyanpi kin, qa wihdi on iyoyam yapi kin ;

15. Qa wizin yapi owayuśna kin, qa tośu on akiyuhapi kin, qa wihdi on sdawicayapi kin, qa taku waśtemna on wizin yapi kin ; qa ozanpi tipi tiyopa akahpe cin he.

16 Qa peta wośnapi owayuśna kin, qa mazaśa oceti tawa; qa 133 
tos̊u on akiyuhapi kin, qa taku on kicanyanpi kin, qa koka kin, tacetetahe iyahna;

17 Hocoka ozanpi kin, ipatan kin, qa sicu kin, qa hocoka tiyopa akalipe kin;

18 Tipi wihutipaspe kin qa hocoka wihutipaspe, qa ikan kin owasin ;

19 Qa tipi wakan ohna taku wəka11 ohodapi kta e ohodapi wokoyake, wokoyake wakan, Aaron wawayuśna kin on, qa cinhintku tawokoyakepi ohna wayuśnapi kta.

20 Unkan Mowis itokapa tanhan Israel cinca omniciye ocowasin inanpapi.

21 Unkan tuwe cante kin iyopaśtaka, qa tuwe taniya kin iyokipi kin hena owasin upi ; qa taku Jehowa itukihanpi ahipi, itkokipapi wakeya kin kagapi kta e, qa taku kicanyanpi on, qa wokoyake wakan on.

22 Unkan wicaśta qa winohinca upi, tona cantepi iyokipipi owasin maza taśpudan, qa oinpi, qa mazanapcupe, qa maza śipto, mazaskazi oinpi owasin hiyuyapi ; qa wicaśta tona taku kosyapi kin hena owasin Jelıwa mazaskazi kos yapi.

$23 \mathrm{Qa}$ wicaśta tona hin to, qa stan, qa duta, qa niiniliuha ska, qa tatokadan hin, qa tamdoka ha śayapi, qa lioka ha yuha iyewicayapi kin hena ahipi.

24 Qa tona mazaska qa mazaśa wankan hiyuyapi kin hena Jehowa taku itukihanpi kin he kahipi, qa tuwe sitim can on taku kahi wicaśi kagapica he kahipi.

$25 \mathrm{Qa}$ winolinca tona cante ksapapi owasin hin to, qa stan, qa duta, qa minihuha ska napepi on kahmupi, qa taku kahmupi kin he kahipi.
$26 \mathrm{Qa}$ winohinca tona cantepi kin iyowicapaśtakapi woksape en, tatokadan hin kahmupi.

27 Unkan wicaśta itancanpi kin iny an wicaśake iyececa ahipi, qa inyan ojuyapi kta ahipi, amdo akahpe kin en, qa maku akalipe kin en.

28 Qa taku waśtemna, qa wihdi petijanjan on, qa wihdi sdawicayapi on, qa wizin yapi waśtemna on.

29 Wicaśta qa winohinca owasin tona cantepi kin taku kahipi kta iyowicapaśtake, taku kagapi Jehowa Mowis nape on kah wicaśi kin hena owasin on, Israel cinca kin iyokipiya Jehowa itukihanpi.

30 Unkan Mowis Israel cinca kin hewicakiya, Jehowa Betzaleel Uri cinhintku Hur cinhintku Juda wicoun etanhan he caje kin on kipan.

31 Qa Taku Wakan woniya ojunya, woksape, qa wookalinige erl, qa wosdonye, qa wohtani ocaje owasin en.

32 Taku wayupiya kagapi iyukcan kta, mazaskazi, qa mazaska, qa mazaśa kage kta.

33 Qa inyan ojuyapi kta e pago kta, qa can pago kta, taku wayupiya kagapi kin owasin kage kta.

$34 \mathrm{Qa}$ waonspe kiye kta e rye cante en qu, iye qa Aholiyab A hisamak cinhintku Dan wicoun etanhan.

35 Hena cante woksape kin ojun wicaya, wapagopi wicohan, qa taku wayupiya kagapi owasin econpi kta, qa to qa stan, qa duta, qa miniliuha ska on wipatapi kta, qa kazuntapi kte, taku kagapi owasin, qa taku wayupiya econpi iyukcanpi kta. 


\section{WICOWOYAKE 36.}

1 Unkan Betzaleel qa Aholiyab htanipi, nakun wicaśta tona cante ksapapi, tona tipi wakan token Jehowa kah wicaśi, taku kagapi owasin sdonyapi kta, qa kagapi kta e Jehowa woksape qa wookahnige yuhe wicakiya.

2 Nakaś Mowis Betzaleel qa Aholiyab wicakipan qon, nakun tona cante ksapapi, tona cante mahen Jehowa woksape yuhe wicakiya, tona taku kagapi kage kta e cante litani kta e iyowicapaśtaka owasin ikiyedan hiyu wicakiya.

3 Unkan Mowis itokapa tanhan taku ituhanpi kin icupi, tipi wakan kagapi kta, qa kicanyanpi kta e taku Israel cinca kin ahipi kin, nakun nabanhin hahanna otoiyohi taku iyokipiya ahipi.

4 Unkan tona ksapapi tipi wakan wohtani econpi kin owasin wohtani econpi etanhan en hipi ;

5 Qa Mowis heciyapi, Taku kagapi kta, Jehowa kah unśipi kin iyehantu, qa iyakicuya oyate kin ahipi ce, eyapi.

6 Hehan Mowis wicoti ohna ieyanpaha wicaśi, Wicaśta winohinca unmanna tipi wakan kagapi itukihanpi kta e tokata taku kage kte śni, eya, hecen oyate taku ahipi kin ayuśtan wicakiya.

7 Taku kah wicaśi owasin kagapi kta iyohiya qa nakun ihan.

8 Unkan tipi wakan kin htanipi wicehna tona cante ksapapi kin hena ozanpi wikcemna kagapi, minihuha ska nonpa kahmupi, qa to, qa stan, qa duta, kerubi kazuntapi hena kagapi.

9 Ozanpi wanji ohanske cin wiciśpa wikcernna nonpa sanpa śa- hdogan, qa ozanpi wanji ohdakinyan kin wiciśpa topa, ozanpi owasin hinskoskocecapi.

10 Unkan ozanpi zaptan icikoyag ya, qa ozanpi zaptan icikoyagya.

11 Hahonta to śunjoyake ozanpi wanji opapun ekta kaga, apahidate etanhan icikoyag yapi en, he riyecen ozanpi unman icikoyag yapi kta e opapun apahdate ekta kaga.

12 Śnjoyake wikcemna zaptan ozanpi tokaheya en kage ça śunjoyake wikcemna zaptan ozanpi inonpa opapun ekta, tokaheya kici ikoyag yapi kta e śunjoyake kin iciyotakonzapi.

13 Unkan mazaskazi taśpu wikcemna zaptan kaga, qa maza taśpu kin on ozanpi wanji unman ekta ikoyag ya; hecen wakeya wanjidan.

14 Unkan tatokadan hin ozanpi wakeya tipi akapatanhan un kin kicaga, hena ozanpi ake wanji kaga.

15 Ozanpi wanji ohanske cin, wiciśpa wikcemna yamni, qa ozanpi ohdakinyan kin wiciśpa tom, ozanpi ake wanji kin owasin iyakehinskokeca.

16 Unkan ozanpi zaptan icikoyag ya, qa ozanpi śakpe icikoyag ya.

17 Unkan icikoyag yapi kta e śunjoyake wikcemna zaptan ozanpi tokaheya opapun akan kaga, qa śunjoyake wikcemna zaptan ozanpi inonpa opapun ekta ikoyag yapi kta e kaga.

18 Qa mazaśa taśpu wikcemna zaptan wakeya icikoyag ye kta e kaga, hecen wanjidan kta.

19 Unkan tamdoka ha sayapi woakahpe wan wakeya kin kicaga, qa he akapatanhan kta hoka ha woakahpe wan kaga. 


\section{HDINANPAPI,}

20 Unkan tipi kín śitim can can mdaska bosdan unpi kta e kicaga.

21 Can mdaska wan ohanske cin wiciśpa wikcemna, qa ohdakinyan kin wiciśpa wan sanpa wiciśpa hanke.

22 Can mdaska ekiciyuzapi kta e otoiyolii nape nonpa kicaga, tipi can mdaska owasin hecen kicaga.

23 Unkan tipi can mdaska kin ti onnaptan itokaga wiyotanhan ektakiya can mdaska wikcemna nonpa tipi kin kicaga.

24 Qa mazaska sicu wikcemna tom kaga, can mdaska wikcemna nonpa ihukuya, sicu nonpa, canmdaska wanji nape nonpa yuke cin on he ihukuya un kta, canmdaska otoiyohi nape nonpa kiyuke cin on, he ihukuya sicu nonpa kta.

$25 \mathrm{Qa}$ tipi onnaptan inonpa waziyata ekta-kiya canmdaska wikcemna nonpa kicaga.

26 Qa mazaska sicu wikcemna topa, canmdaska otoiyohi ihukuya maza sicu nonpa.

27 Qa tipi catku kin wiyohpeyata ektakiya kin he canmdaska śakpe kicaga.

28 Qa canmdaska nonpa tipi kahmi catkutanhan kin hena kicaga.

29 Unkan napin iyakedececapi ihukuya tanhan, qa napin otoiyolii pa kin ekta mazaska napcupe tokaheya en ikoyagyapi, kahmi nonpa on hena nonpa okonwanjidan.

30 Hecen canmdaska kin śahdogan, qa mazaska sicu kin ake śakpe, canmdaska otoiyohi ihukuya sicu nonpa.

31 Qa tipi onnaptan tokaheya canmdaska kin on śitim can hdakinyan zaptan kicaga.

32 Qa tipi onnaptan inonpa can136 mdaska kin on canhdakinyan zaptan, qa tipi catku kin wiyohpeya ta ekta-kiya canmdaska kin canhdakinyan zaptan kicaga.

33 Qa canhdakinyan wanji cokaya un kin he canmdaska cokaya. ohna iyopte kta e kaga, ihanke wanji etanhan ihanke unman ekta kiya.

34 Qa canmdaska kin mazaskazi on apawinta, qa maza napcupe ohna canhdakinyan yakonpi kin hena mazaskazi on kaga, qa canhdakinyan kin mazaskazi apawinta.

35 Qa cokaya ozanpi kin miniliuha ska, qa to, qa stan, qa duta kerubi kazuntapi he kaga.

36 Qa śitim can bosdata tom he kicage ca hena mazaskazi apawinta, qa mazaskazi yukśanpi hena kicage ca mazaska sicu tom śdoyapi kicaga.

37 Qa wakeya tiyopa kin oakalipe wan, minihuha ska, qa to qa stan, qa duta wipatapi kin he kicaga.

38 Qa he on can bosdata zaptan mazayukśanpi iyahna, qa hena pa kin, qa can icikoyagyapi kin hena mazaskazi apawinta, tuka sicu zaptan kin hena mazaśa.

\section{WICOWOYAKE $3 \%$}

1 Unkan Betzaleel canwohnake kin śitim can on kaga, wiciśpa nonpa sanpa hanke ohanske cin, qa wiciśpa sanpa hanke ohdakinyan kin, qa wiciśpa sanpa hanke obosdatu kin.

$2 \mathrm{Qa}$ he mazaskazi ecedan on apawinta, mahentuya, qa akapatanhan, qa ihdukśan mazaskazi wateśdake wan kicaga.

3 Qa mazaskazi śdoye ca maza napcupe tom he kicaga, siha topa kin akan, maza napcupe nonpa onnaptan wanji akan, qa 


\section{WICOWOYAKE $3 \%$.}

maza napcupe nonpa onnaptan unman akan.

4 Qa śitim can tośu kageça mazaskazi on apawinta.

$5 \mathrm{Qa}$ canwohnake kin akiyuhapi kta e maza napcupe canwohnake anokatanhan onnaptan ikoyag yapi kin hena ohna tośu kin iyopte ya.

6 Unkan woahtani kajuju iha kin mazaskazi ecedan on kaga, ohanske cin wiciśpa nonpa sanpa hanke qa ohdakinyan kin wiciśpa sanpa hanke.

7 Qa kerubi nonpa kaga wanji wokajuju iha ihanke anokatanhan akan ; mazaskazi pagopi hena kaga.

8 Kerubi wanji ihanke deciyatanhan qa kerubi unman kin ihanke kakiya tanhan, woahtani kajuju iha kin etanhan kerubi kin kaga; ihanke nonpa kin etanhan.

9 Unkan kerubi kin wankantuya hupahu kamdah yakonpi, hupahupi on woahtani kajuju iha kin ahanzipi, qa itepi kin otoiyohi unman ekta kiya, kerubi itepi kin wokajuju iha ekta kiya yakonpi.

10 Unkan wahna wotapi śitim can on kaga, ohanske cin wiciśpa nonpa, qa ohdakinyan kin wiciśpa, qa obosdatu kin wiciśpa sanpa hanke.

11 Qa he mazaskazi ecedan on apawinta, qa ihdukśan mazaskazi wateśdake wan kicaga.

12 Nakun ihdukśan iyuskite wan nape mdaska hinskokeca kicaga; qa iyuskite akan mazaskazi wateśdake wan kicaga.

13 Qa mazaskazi śdoye ça maza napcupe topa kicage ça oise topa siha topa yuke cin hena ekta maza napcupe kin ilroyag ya.

14 Wahna wotapi akiyuhapi kta e maza napcupe iyuskite iyotakons kagapi, qa hena ohna tośu kin iyeyapi.

15 Unkan śitim can tośu kin kage ca mazaskazi apawinta hena on wahna wotapi kin akiyuhapi.

$16 \mathrm{Qa}$ taku wahna wotapi akan un taku on kicanyanpi kin, tawakśica, qa maza tukiha tawa, qa wiyatke on waakaśtanpi kin hena owasin mazaskazi ecedan on kaga.

17 Unkan petijanjan ihupa kin he kaga, mazaskazi ecedan pagopi petijanjan ceca kin, taadetka kin, tawakśica kin, tapśonka kin qa tawalica kin, hena owasin he etanhan kaga.

18 Qa adetka śakpe petijanjan ihupa cuwi kin etanhan inanpapi, petijanjan ihupa cuwi wanji etanhan adetka yamni, qa petijanjan cuwi unman kin etanhan adetka yamni.

19 Adetka wanjidan en wakśica yamni hma iyececa, otoiyohi pśonka wan, qa wahica wan kicica adetka wanjidan en, qa ake wakśica yamni otoiyohi hma iyececa pśonka wan qa wahica wan kicica adetka wanjidan en, adetka śakpe petijanjan ihupa etanhan inampapi, owasin hececapi. 20 Qa petijanjan ihupa en wakśica topa hma iyececa, otoiyohi pśonka wan qa wahca wan kicica.

21 Hecen petijanjan adetka nonpa ihukuya pśonka wanji, qa adetka nonpa ihukuya pśonka wanji, qa adetka nonpa ihu kuya pśonka wanji petijanjan ihupa etanhan adetka śakpe inanpapi owasin hececapi.

22 Petijanjan ihupa tapśonka, qa taadetka okonwanjidan, mazaskazi ecedan onśpadan pagopi on owasin kagapi. 


\section{HDINANPAPI,}

23 Qa mazaskazi ecedan petijanjan śakowin petijanjan ihupa kin kicaga, nakun petijanjan iyukse qa ohna cahota icupi kin.

24 Mazaskazi ecedan woqin wanjidan on he kage ça tawakśica owasin.

25 Unkan wizinya owayuśna kin sitim can on kaga; ohanske cin wiciśpa, qa ohdakinyan wiciśpa, topa omdoton, qa obosdatu kin wiciśpa nonpa; he kicagapi.

26 Qa mazaskazi ecedan on apawinta iwankam qa onnaptanyan kin ihdukśan, qa he kin; qa ihdukśan mazaskazi ecedan wateśdake wan kicaga.

27 Qa mazaskazi napcupe nonpa he kicaga, wateśdake kin ihuluya onnaptan nonpa akall, tośu on akiyuhapi kin icupi kta e hena owayuśna cuwi nonpa akan kaga.

28 Qa tośu kin śitim can on kage, ça mazaskazi on apawinta.

29 Unkan wihdi wakan on sdawicayapi kaga, nakun taku waśtemna on wizinyapi ecedan kin, pejuta wicaśta kagapi kin iyececa.

\section{WICOWOYAKE 38.}

1 Unkan śitim can peta wośnapi owayuśna kin kaga, ohanske cin wiciśpa zaptan, qa ohdakinyan kin wiciśpa zaptan, topa omdoton, qa obosdatu kin wiciśpa yamni.

2 Qa oise topa kin alkan he kicaga, owayuśna on he kin kaga, qa mazaśa on apawinta.

3 Unkan owayuśna taku on kicanyanpi kin owasin kaga; cega kin, qa maza mdaska kin, qa wakśica kin, qa mazajanjata kin, qa petaga wakśica kin, owayuśna taku on kicanyanpi kin owasin mazaśa on kaga.

4 Qa mazaśa oceti wan ho kagapi iyececa owayuśna kin kicaga, opapun ihukuya, olidateya cokaya ekta.

5 Qa oceti oise topa kin en mazaśa śdoyapi napcupe tom kaga, hena ohna tośu iyeyapi kta.

6 Unkan śitim can tośu kin kage, ça mazaśa on apawinta.

7 Qa owayuśna akiyuhapi kta e maza napcupe kin owayuśna onnaptan en unpi kin ohna tośu kin iyeya; owayuśna canmdaska on kaga, hdogeca.

8 Unkan mazaśa koka kin he kaga, tacetetahe mazaśa on kaga, winohinca itkokipapi wakeya tiyopa ekta obe itokto yuwitaya cekiyapi taihdiyomdasinpi on hena laga.

9 Unkan hocoka kin kaga, itokah onnaptan wiyotanhan ektakiya on minihuha ska nonpa kahmupi ozanpi wan wiciśpa opawinge.

10 Ipatan wilicemna nonpa, qa sicupi wikcemna nonpa, mazaśa on kicaga, tuka ipatan yukśanpi kin qa satapi kin hena mazaska on kaga.

11 Unkan waziyata onnaptan ozanpi kin wiciśpa opawinge, ipatan kin wikcemna nonpa, qa sicu wikcemna nonpa, mazaśa on kaga, tuka ipatan yukśanpi kin, qa satapi kin hena mazaska.

12 Unkan ihanke wiyohpeyata ektakiya ozanpi kin wiciśpa wikcemna zaptan, ipatan wikcemna, qa sicu kin wikcemna, tuka ipatan yukśanpi kin qa sa tapi kin hena mazaska.

13 Qa ihanke wihinanpe ektakiya wiciśpa wikcemna zaptan.

14 Hiyete tokaheya ozanpi kin wiciśpa wikcemuna sanpa zaptan, ipatan yamni, qa siçu yamni. 


\section{WICOWOYAKE 39.}

15. Qa hiyete inonpa ozanpi kin wiciśpa wikcemna sanpa zaptan, ipatan yamni, qa sicu yamni, hocoka tiyopa kin anokatanhan hececa.

16 Hocoka ihdukśan ozanpi kin owasin miniliuha ska nonpa kahmupi.

17 Ipatan sicupi kin hena mazaśa, qa ipatan yukśanpi kin, qa satapi kin mazaska, qa ipatan pahapi kiu mazaska on apawinta ; qa hocoka ipatan kin owasin mazaska on icikoyag yapi.

$18 \mathrm{Qa}$ hocoka tiyopa toakahpe kin he minihuha ska nonpa kahmupi, qa to, qa stan qa duta ipatapi ; qa ohanske cin wiciśpa wikcemna nonpa, qa obosdatu ohdakinyan kin hee wiciśpa zaptan hocoka ozanpi kin iyececa.

19 Qa ipatan topa, qa sicupi topa mazaśa; qa yukśanpi kin mazaska; qa pahapi qa satapi kin mazaska on apawintapi.

-20 Qa tipi wihutipaspe, qa hocoka ihdukśan wihutipaspe kin owasin mazaśa.

21 Tipi wakan woyakapi kin hee, woyaatanin tipi kin, Mowis i kin oyaka, Lewi kin kicanyanpi kta Aaron wawayuśna cinhintku Itamar ihukuya.

22 Unkan Betzaleel Uri cinhintku, Hur cinhintku, Juda wicoun etanhan, taku Jehowa Mowis kali śi kin owasin kaga.

23 Qa he kici Aholiyab, Ahisamak cinhintku, Dan wicnun etanhan, he way upiya wapago, qa wapazunta, qa wipata, minihuha ska, qa to, qa stan, qa duta on.

24 Taku kagapi owasin tipi wakan kin on kagapi, mazaskazi ocowasin mazaskazi itulianpi kin lıe woqin wikcemna nonpa sanpa napciwanka, qa śeqel opawinge śakowin, qa wikcemna yamni, tipi wakan seqel he iyececa.
$25 \mathrm{Qa}$ omniciye wicayawapi kin etanhan mazaska kin woqin opawinge, qa śeqel kektopawinge sanpa opawinge śakowin, sanpa wikcemna śakowin sanpa zaptan tipi wakan śeqel iyececa.

26 Kibaśpapi wan wicapa otoiyohi on śeqel hanke kin hee, tipi wakan śeqel iyececa, tona wicayawapi kta ahiyayapi, waniyetu wikcemna nonpa qa iwankam, kektopawinge opawinge śakpe qa kektopawinge yamni, qa opawinge zaptan qa wikcemna zaptan.

27 Unkan maza ska woqin opawinge kin hena śdoyapi qa tipi wakan sicu kin, qa cokaya ozanpi sicu kin kaga, woqin opawinge on sicu opawinge, woqin wan on sicu wanjidan.

$28 \mathrm{Qa}$ śeqel kektopawinge sanpa opawinge śakowin sanpa wikcemna śakowin sanpa zaptan on ipatan papi kin, qa satapi kin apawintapi qa ipatan yukśanpi kin kaga.

29 Qa mazaśa ituhanpi kin woqin wikcemna śakowin, qa śeqel kektopawinge nonpa qa opawinge topa.

$30 \mathrm{Qa}$ he etanhan itkokipapi wakeya tiyopa sicu kin kage ça mazaśa owayuśna kin, qa mazaśa oceti kin, qa owayuśna tawakśica kin owasin.

$31 \mathrm{Qa}$ hocoka ihdukśan sicu kin, qa hocoka tiyopa sicu kin, qa tipi wihutipaspe owasin, qa hocoka ihdukśan wihutipaspe owasin.

\section{WICOWOYAKE 39.}

1 Unkan hahonta to, qa stan, qa duta on ohodapi wokoyake kin tipi wakan ohna wohtani econpi kin hena heyake wicakicagapi qa wokoyake wakan 


\section{HDINANPAPI,}

Aaron on Kagapi, Jehowa Mowis econ śi qon he iyececa.

2 Unkan amdo akahpe kin kaga mazaskazi, qa hahonta to qa stan qa duta, qa minihuha ska.

3 Qa mazaskazi kin zibzipedan kamdayapi qa basosopi, hecen hahonta to qa stan qa duta, qa miniliuha ska icicahiya kazontapi kta.

4 Qa amdo akahpe kin, icikoyag yapi kta e hiyete ikan kin kicagapi, ihanke napin ekta icikoyag yapi.

5 Qa amdo ipiyake kin he akapatanhan un kin he iyecen kagapi, mazaskazi qa hationta to, qa stan qa duta, qa minihuha ska, nonpa kahmupi on kagapi, Jehowa Mowis econśi kin he iyecen.

6 Unkan inyan wicaśake iyecen hena kicanyanpi Israel cinhintku cajepi kin hena akan apagopi, ipuspe pagopi kin he iyecen, qa mazaskazi ozepi ohna iyeyapi.

$7 \mathrm{Qa}$ amdo akahpe hiyete akan hena ahnaka, wokiksuyè Israel cinca kin on, Jehowa Mowis econ śi kin he iyecen.

8 Unkan maku akahpe kin kaga, mazaskazi, hahonta to, qa stan, qa duta, qa minifiuha ska nonpa kahmupi kin hena kazuntapi, amdo akahpe kin he iyecen.

9 He topa omdoton, icitakihna he kagapi, ohanske cin napapaśdecapi qa ohdakinyan kin napapaśdecapi, icitakihna.

$10 \mathrm{Qa}$ he ohna inyan ocankuye topa ojuyapi, ocankuye wan, sardius, qa topaz qa petaga, ocankuye tokaheya kin hee.

11 Qa ocankuye inonpa kin he, smaragdos, qa sapiron, qa diamon ;

12 Qa ocankuye iyamni kin he ligurion, qa agate, qa ametuston;

$13 \mathrm{Qa}$ ocankuye itopa kin he krusolitos, (mazaskazi inyan) qa inyan wicaśake iyececa, qa iyaspis; hena ihdukśan mazaskazi ozepi en ojuyapi.

$14 \mathrm{Qa}$ inyan kin hena Israel cinca ake nonpa cajepi kin iyececa, ipuspe pagopi kin he iyecen, wicoun ake nonpa cajepi kin otoiyohi inyan wanji akan apagopi.

15 Qa maku akahpe kin ihanke anog ekta mazaskazi ecedan icicahilia yuhmupi kagapi.

16 Qa mazaskazi ozepi nonpa kagapi, qa mazaskazi napcupe nonpa, qa maku akahpe oise nonpa kin en, maza napcupe nonpa ikoyag yapi.

17 Qa maza napcupe nonpa maku akahpe oise en unpi kin hena ohna mazaskazi ikan nonpa kin iyeyapi.

18 Qa ikan nonpa ihanke nonpa maza ozepi nonpa en iyakaśkapi qa amdo akahpe hiyete akan ite ekta-kiya iyakaśkapi.

19 Qa mazaskazi napcupe nonpa kagapi, qa maku akahpe oise nonpa en iyakaśkapi, mahentuya amdo akahpe ektakiya opapun en. 20 Unkan mazaskazi napcupe nonpa kagapi, qa amdo akahpe hiyete nonpa en ikoyag yapi, ihukuya ite ekta kiya, icikoyag yapi iyotakons, amdo akatipe, ipiyaka iwankam.

$21 \mathrm{Qa}$ hahonta to wan on maku akahpe maza napcupe ekta iyakaśkapi, hecen amdo akahpe ipiyaka akan yanke kta, qa maku akahpe kin amdo akahpe kin etanhan yukinukan kte śni.

22 Unkan amdo akahpe nitośke kin ocowasin to kazuntapi kin he kaga.

23 Qa nitośke i kin he nitośke cokaya, minihuha maku akahpe i kin he iyececa, i kin ihdukśan apahdan tonpi, nalidece kte śni heon etanhan. 
24 Unkan nitośke upi akan taspantanka seca to, qa stan qa duta nonpa kahmupi on kagapi.

25 Unkan mazaskazi ecedan hdahda kicagapi, qa mazahdahda kin taspan tanka seca ehna kaśkapi, nitośke upi akan ihdukśan taspantanka seca otahedan.

26 Mazahdahda qa taspantanka seca, maźahdalida qa taspantanka seca,nitośke ohna ohoda kin upi kin ihdukśan akan, Jehowa Mowis econ śi kin he iyecen.

27 Unkan mahen unpi Aaron on, qa iye cinhintku, wicakicagapi, minihuha ska kazuntapi.

28 Unkan miniliuha pa ipahte kin qa miniliuha wapaha waśte, qa miniliuha onzeoge miniliuha ska nonpa kahmupi.

$29 \mathrm{Qa}$ ipiyaka miniliuha ska nonpa kahmupi, qa to, qa stan, qa duta, ipatapi he kagapi, Jehowa Mowis econśi qon he iyecen.

30 Unkan mazaskazi ecedan mdaskà wateśdake wakan kin kagapi, qa Jehowa wakandapo, akan owapi, ipuspe pagopi kin he iyecen owapi.

31 Qa hahonta to wan ekta iyakaśkapi, he on pa ipahte kin akan akapatanhan iyakaśkapi ; Jehowa Mowis econśi qon he iyecen.

32 Hecen itkokipapi wakeya tipi kagapi kin ocowasin yuśtanpi, token Jehowa Mowis econśi kin owasin he iyecen Israel cinca kin yuśtanpi.

33 Unkan tipi kin Mowis ekta lkahipi ; wakeya kin, qa taku on kicanyanpi kin owasin, tamaza taśpu, tacanmdaska, tacanhdakinyan, qa taipatan, qa tasicu kin ;

$34 \mathrm{Qa}$ tamdoka ha śayapi on akahpe kin, qa loka ha on aka hpe kin, qa cokaya ozanpi kin ; 35 Qa woyaatanin canwohnaka kin, qa tośu kin, qa wokajuju iha kin ;

36 Wahna wotapi tawakśica koya owasin, qa aguyapi itokam hnakapi kin;

37 Petijanjan ihupa ecedan kin, petijanjan kin koya, petijanjan kicanyanpi kin hena, qa taku on kicanyanpi kin owasin, qa wihdi on iyoyam yapi kin;

38 Qa mazaskazi owayuśna kin qa sdawicayapi wihdi kin, qa taku waśtemna on wizin yapi kin, qa wakeya tiyopa akahpe kin ;

39 Mazaśa owayuśna kin, qa mazaśa oceti tawa kin, qa tatosu kin qa taku on kicanyanpi kin .owasin; mazaśa koka kin, tacetetahe koya.

40 Hocolka ozanpi kin, can bosdáta kin qa sicu kin, qa hocoka tiyopa oakalipe kin, taikan kin, qa tawihutipaspe kin qa itkokipapi walkeya ohna ohodapi kin, tawakśica koya owasin;

41 Ohodapi wokoyake kin on tipi wakan ohna ohodapi, Aaron wawayuśna tawokoyake wakan kin, qa cinhintku wokoyalie unpi wawayuśnapi ece kta.

42. Taku Jehowa Mowis econ śi kin owasin, he iyecen Israel cinca kin taku owasin kagapi.

43 Unkan Mowis taku kagapi kin owasin wanyaka; unkan inyun taku Jehowa kali wicaśi kin owasin he iyecen kagapi, unkan Mowis wicayawaśte.

WICOWOYAKE 40.

1 Unkan Jehowa ie ca Mowis heciya,

2 Wi tokaheya, anpetu tokahe141 
ya en itkokipapi wakeya tipi kin eyahde kta.

$3 \mathrm{Qa}$ he ohna woyaatanin can wohnaka kin eyahnake kta, qa can wohnaka kin akapatanhan cokaya ozanpi kin otkeyaye kta. 4 Qa wahna wotapi kin he en hiyuyaye kta, qa taku akan hnakapi kin hena akan eyahnake kta, qa petijanjan ihupa kin yakahi kta, qa petijanjan kin ideyaye kta.

5 Qa mazaskazi owayuśna kin wizinyapi kta e woyaatanin can wohnaka itoliam eyahnake kta, qa tipi tiyopa oakahpe otkeyaye kta.

$6 \mathrm{Qa}$ peta wośnapi owayuśna kin itkokipapi wakeya tipi tiyopa itokam eyahde kta.

7 Qa koka kin he itkokipapi wakeya kin, qa owayuśna kin otahedan eyahde kta, qa he ohna mini oyakaśtan kta.

$8 \mathrm{Qa}$ he ihdukśan hocoka kin eyahde kta, qa hocoka tiyopa oakahpe kin otkeyaye kta.

9 Qa sdawicayapi wihdi kin he iyacu kte ça tipi kin, qâ taku ohna un kin, owasin sdayaye kta, hecen he qa taku on kicanyanpi kin owasin duwakan kta, unkan wakan kta.

10 Qa peta wośnapi owayuśna kin he sda yaye kta, taku on kicanyanpi nakun owasin; hecen owayuśna kin duwakan kta; qa owayuśna kin wakan hinca kta.

11 Qa koka kin he sdayaye kta, tacetetahe nakun, hecen he duwakan kta.

12 Unkan Aaron, iye cinhintku om, itkokipapi wakeya tiyopa kin ikiyedan hiyu wicayaye kta, qa mini on wicadujaja kta.

13 Qa wokoyake wakan kin he Aaron koyag yaye kta, qa sda yaye kta, hecen wamiciyuśna kta e duwakan kta.
14 Unkan cinhintku kin ikiycdan hiyu wicayaye kta, qa mahen unpi kin hena koyag wicayaye kta.

15 Qa atkukupi kin sda yaye ciqon he iyecen sda wicayaye kta, hecen wamiciyuśnapi kta, qa sdawicayapi kin on owihanke wanin wawayuśna hecapi kta, wicoicagepi yeye cin ohna.

16 Unkan token Jehowa Mowis econśi kin owasin he iyecen econ.

17 Unkan kaketu wetu inonpa wi tokaheya, anpetu tokaheya kin he ehan tipi wakan kin ehdepi.

$18 \mathrm{Qa}$ Mowis tipi kin ehde qehan tasicu kin eju, qa tacanmdaska kin en iyeya, qa tacanhdakinyan ohna iyoopte iyeya, qa taipatan kin ehde.

19 Qa tipi ıwankapatanhan wakeya kin yumdaya, qa wakeya oakahpe kin he wakeya akapatanhan iwankam yumdaya, Jehowa Mowis econśi qon he iyecen.

20. Unkan woyaatanin kin he icu, qa canwohnaka mahen ehnaka, qa tośu kin canwohnaka ekta iyeya, qa wokajuju iha kin canwohnaka akan ehnaka iwankapatanhan.

21 Qa canwohnake kin tipi kin mahen hiyuye ça cokaya ozanpi kin otkeya, hecen woyaatanin canwohnaka kin akahpa, Jehowa Mowis econ śi kin he iyecen.

$22 \mathrm{Qa}$ wahna wotapi kin he itkokipapi wakeya ohna ehde, tipi cuwi waziyata ekta kiya, cokaya ozanpi itankan.

$23 \mathrm{Qa}$ he akan aguyapi itokam hnakapi kin heca tanyan ehnaka, Jehowa itokam, Jehowa Mowis econ śi kin he iyecen.

$24 \mathrm{Qa}$ petijanjan ihupa kin itkokipapi wakeya mahen ehde, wahna wotapi kin iyotakons, tipi cuwi itokaga ekta kiya.

25 Qa petijanjan kin hena ide- 
ya, Jehowa itokam, Jehowa Mowis econ śi kin lie iyecen.

26 Unkan mazaskazi owayuśna kin he itkokipapi wakeya mahen ehde, cokaya ozanpi kin itokam. 27 Qa he akan taku waśtemna wizinyapi kin izin ya, Jehowa Mowis econ śi kin he iyecen. 28 Qa tipi tiyopa akahipe kin he otkeya.

29 Qa wahuhnah yapi owayuśna kin he itkokipapi wakeya tipi tiyopa kin ikiyedan ehde, qa he akan wohuhnahyapi qa minha kin huhnaliya.

30 Qa koka kin itkokipapi wakeya kin, qa owayuśna kin otahedan ehde qa mini on yujajapi kte hen okaśtan.

31 Unkan hetanhan Mowis qa Aaron iye cinhintku om napepi sihapi ko ihdujajapi.

32 Itkokipapi wakeya en ipi eca; qa owayuśna ikiyedan ipi eca ihdujajapi ece, Jehowa Mowis econ śi kin he iyecen.

33 Unkan tipi kin owayuśna kin koya ihdukśan hocoka kin ehde, qa hocoka tiyopa akahpe kin otkeya, hecen Mowis wohtani kia hduśtan.

34. Hehan mahpiya soka qon itkokipapi wakeya kin akalipa, qa Jehowa towitan kin tipi kin ojunya.

35 Unkan Mowis itkokipapi wakeya en i kta okihi śni, mahpiya qon he akan ounyan qa Jehowa towitan kin tipi kin oju ya nakaś.

36 Unkan malipiya kin wankan iyaya eca, Israel cinca kin ihdakapi ece, unhdakapi kin owasin en hececa.

37 Tuka mahpiya kin wankan iyaye śni kinhan, ihdakapi sni ece, wankan iyaye śni kin hehanyan.

38 Jehowa mahpiya soka kin tipi kin akan yanka, anposkantu eca, tuka hanyetu ca he ohna peta icaga, Israel cinca tipi kin owasin unhdakapi owasin en he wanyakapi.

HDINANPI IHANKE KIN. 


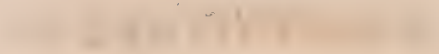

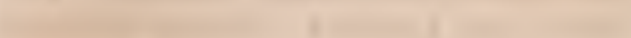

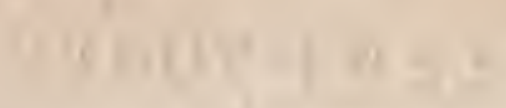

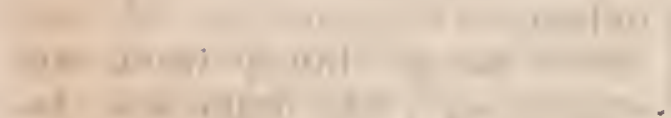

$+10+100$

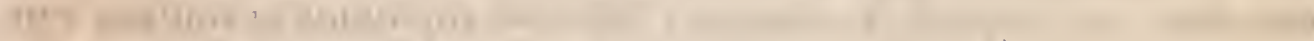

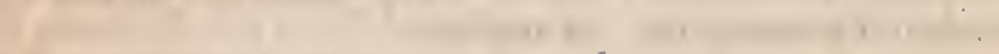

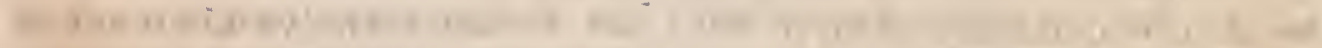

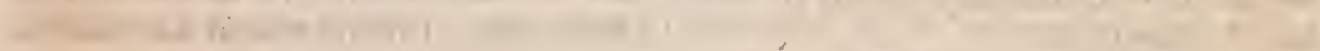

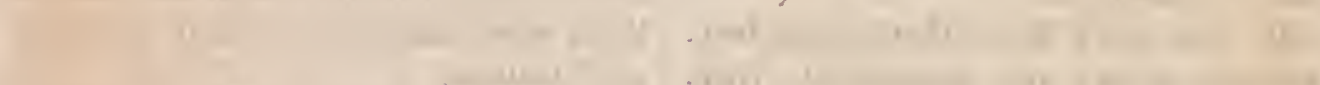

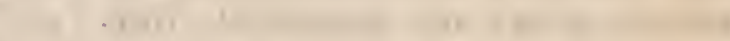

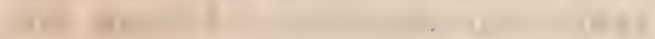

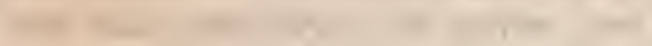

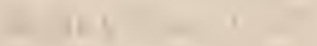
15.

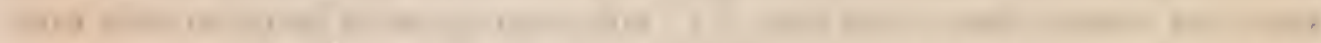

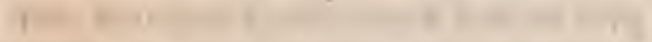

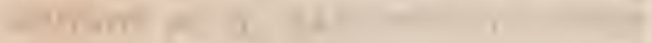
1. $y_{-2}+2$

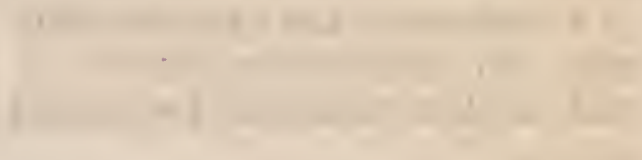




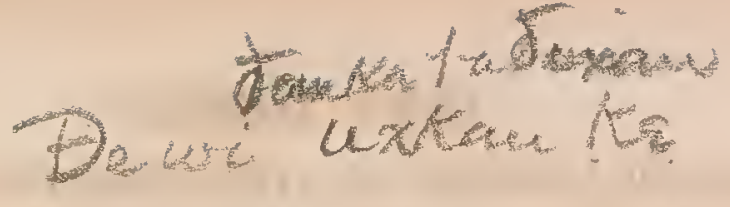

\section{LEWI TOOPE.}

WICOWOYAKE 1.

1 Unkan Jehowa Mowis kipan, qa itkokipapi wakeya etanhan heciya,

2 Israel cinca kin hecen ewicakiya wo, Niyepi etanhan wicaśta tuwe woahi wan Jehowa kahi kte cinhan, woteca etanhan woahi nitawapi yakahipi kta, pte wanunyanpi etanhan, qaiś talinca wanunyanpi etanhan.

3 Pte wanunyanpi etanhan woliulinaliyapi on toahi kinhan, mdoka zanika ahi kte, iyokipiye kta e Jehowa itokam, itkokipapi wakeya tiyopa ekta kahi kta.

4 Hehan wohulinahyapi pa akan nape kin aputake kta, hecen iye walitani kin akahpe kta e iyokipi ye kta.

5 Unkan Jehowa itokam tatankadan kin patapi kta; unkan Aaron cinca wawayuśnapi kin we kin ahipi kta, qa wạhna wośnapi kin itkokipapi wakeya tiyopa ekta yanke cin akan we kin aihipe yapi k ta.

6 Unkan wohuhnahyapi ha kin yuze kta, qa wobaśpe kin baśpuśpu kta.

7 Unkan wawayuśna Aaron cinca kin wahna wośnapi akan peta ehnakapi kta, qa peta akan can aonpapi kta.

8 Unkan Aaron cincā wawayuśnapi kin wobaśpe kin, pa kin, qa waśin kin, can akan peta wahna wośnapi akan yanke cin ahnakapi kta.

9 Unkan śupe kin, qa huha kin mini on yujaja kta, qa ahi kta, unkan wawayuśna kin he ocowasin izin ye kta, peta on wohuhnahyapi wan waśtemna Jehowa iyokipi kin hee.

10 Unkan tahinca etanhan wnhubnahyapi taku ahi kinhan, tahin wanunyanpi etanhan qa iś tatokadan etanhan mdoka zanika wan ahi kta.

11 Unkan Jehowa itokam wahna wośnapi icahda, iwaziya tanhan he pate kta. Unkan Aaron cinca wawayuśnapi kin we kin amnimnipi kta, wahna wośnapi aihdukśan.

12 Unkan wobaśpe kin baśpuśpu kta, qa pa kin, qa waśin kin, unkan wawayuśna kin hena can akan ehnake kta, peta wahna wośnapi akan yanke cin he akan. 13 Unkan śupe kin qa huha kin mini en yujaja kta. Unkan hena owasin wawayuśna kin ahi kta, qa wahna wośnapi izin ye kta. Peta on wohuhinahyapi wan waśtemna Jehowa iyokipi kin hee.

14 Unkan taku kinye cin etanhan wohuhnahyapi wan Jehowa kahi kte cinhan, wakiyedan cinca qaiś tinwakiyedan etanhan toahi kin ahi kta.

15 Unkan wawayuśna kin he wahna wośnapi ikiyedan kahi kta, qa pa kin cape lita; qa wahna wośnapi kin izin ye kta; tuka. we kin yuśkice kta, wahna wośnapi cuwi kin ekta.

16 Qa śupe kin, cesdi ohna yuke cin koya icu kta, qa wahna wośnapi icahda iwiyohinyanpatauhan cahota yanke cin ekta ehpe ye kta. 
17 Unkan maku bamdaze kta, hupahu en, tuka kibaśpe kte śni, qa wawayuśna kin he on wahna wośnapi kin izin ye kta, can akan peta wahna wośnapi akan yanke cin he akan. Peta on wohuhnahyapi wan waśtemna Jehowa iyokipi hee.

\section{WICOWOYAKE 2.}

1 Unkan wicanagi wan minha woahi Jehowa kahi kte cinhan, aguyapi mdu kin kahi kta, qa wihdi he akaśtan kta, qa canśin waśtemna akan ehnake kta.

2 Qa he Aaron cinca wawayuśnapi kin wicakahi kta, qa he etanhan aguyapi mdu qa wihdi nape ohnaka wanjidan, qa canśin ocowasin icu kta; unkan wawayuśna kin wokiksuye kin he on wahna wośnapi kin izin ye kta, peta wośnapi waśtemna wan Jehow a iyokipi kin hee.

3 Unkan minha kin he etanhan taku okapte cin he Aaron qa iye cinca kin tawapi, Jehowa peta wośnapi tawa wakan hinca hee.

4. Unkan taku makacega ohna śpanyanpi minha woahi wan yaliahi kte cinhan, he aguyapi onnapoliye codan wihdi icicahiyapi, qaiś aguyapi zipzibedan onapohye codan wihdi on sdayapi kin heca kta.

5 Unkan minha woahi nitawa mazawiceonpe akan śpanyanpi kinhan, he aguyapi onapoliye codan wihdi on sdayapi heca kta.

6 He duśpaśpa lta qa onśpaśpa akan wihdi ayakaśtan kta, minlia wan hee.

7 Unkan minha woahi nitawa kin he cega ihupa tonna ohna śpanyanpi kinhan, he aguyapi on wihdi en yakage kta.

8 Qa minha hena etanhan kagapi kin he Jehowa ekta hiyu. yaye kta, unkan wawayuśna en kahipi kinhan iye he wahna wośnapi ekta kahi kta.

9 Unkan wawayuśna kin minha kin etanhan wokiksuye wan icu kta, qa heon wahna wośnapi kin izin ye kta, he peta wośnapi waśtemna Jehowa iyokipi kin heca. 10 Unkan minha etanhan taku okaptapi kin he Aaron qa iye cinhintku tawapi kta, taku peta on Jehowa wakiyuśnapi wakan hinca hee.

11 Minha Jehowa yecicahipi kin wanjidan onnapoliye on yakage kte śni. Onnapohye qa canhanpi tiktica etanhan peta on Jehowa izinyaye kte śni.

12 Taku tokaheya suton kin woahi kin hena'Jehowa yakahi kta, tuka wahna wośnapi akan waśtemnayanpi iyokipi kte śni.

13 Qa minha woahi kin hena owasin miniskuya kici icicahiyaye kta, qa taku wakan yada miniskuya on takuyayapi kin he minha kin he etanhan miniskuya teyahinda kte śni. 'Woahi nitawa kin owasin akan miniskuya yakahi kta.

14 Unkan taku tokaheya suton etanhan minha wan Jehowa yakahi kte cinhan, aguyapi itka śtonkadan petan ceonpapi, bopanpi kin heca taku tokaheya suton minha nitawa yakahi kta.

$15 \mathrm{Qa}$ he akan wihdi ayakaśtan kta, qa canśin waśtemna ayakakada kta, minha wan hee.

16 Unkan wawayuśna kin taku bopanpi etanhan, qa wihdi etanhan onge canśin ocowasin iyahna wokiksuye on izin ye kta. Peta on Jehowa wakiyuśnapi wan hee.

\section{WICOWOYAKE 3.}

1 Unkan wopida wośnapi wan kahi kte cinhan, pte wanunyanpi 
etanhan moloka qaiś wiye unman tukte kaśta wanji zanika, Jehowa kahi kta.

2 Qa taku kahi kin pa akan nape kin aputake kta. Hehan itkokipapi wakeya tiyopa ekta he pate kta ; unkan Aaron cinca wawayuśnapi kin we kin amnimnipi kta, wahna wośnapi aihdukśan.

3 Unkan wopida wośnapi etanhan peta wośnapi wan Jehowa kahi kta tejikśije qa śupe śin kin owaśin.

4 Qa pakśin kin napin, qa pakśin śin kin, mica en yanke cin he, nakun pizi pi ikoyake cin he icu kta.

5 Unkan Aaron cinca kin he on wahna wośnapi kin izin yapi kta, wohuhnahyapi kin iyahna, he can peta akan yanke cin he akan, peta wośnapi waśtemna Jehowa iyokipi wan hee.

6 Unkan taliinca etanhan wopida wośnapi Jehowa kahi kte cinhan, mdoka qaiś wiye unman tukte kaśta zanika kahi kta.

7 Woahi tawa amnos wan kahi kinhan, he Jehowa itokam kahi kta.

8 Qa woahi tawa pa kin akan iye nape kin aputake kta, qa itkokipapi wakeya tiyopa kin ekta pate ktá, unkan Aaron cinca kin we kin amnimnipi kta, wahna wośnapi aihdukśan.

9 Unkan wopida wośnapi etanhan peta wośnapi wan Jehowa kahi kte, waśin kin, sinte ocowasin nite kin ekta baśpe kta, qa watejikśije, qa śupe śin kin owasin,

10 Qa pakśin napin, qa pakśin śin kin mica en yanke cin he, qa pizi kin pi ikoyake cin he icn kta.

11 Unkan wawayuśna kin he on wahna wośnapi izinye kta, peta wośnapi Jehowa tawote kin hee.
12 Unkan tatokadan wan kahi kinhan, he Jehowa itokam kahi kta.

13 Qa pa kin akan iye nape kin aputake kta, qa itkokipapi wakeya tiyopa kin ekta he pate kta, unkan Aaron cinca kin we kin amnimnipi kta, wahna wośnapi aihdukśan.

14. Unkan he etanhan toahi peta wośnapi wan Jehowa kahi kte, watejikśije kin he, qa śupe sin kin owasin ;

15 Qa pakśin kin napin, qa pakśin śin kin mica en yanke cin he, qa pizi kin pi ikoyake cin pakśin iyahna icu kta.

16 Unkan wawayuśna kin hena on walına wośnapi kin izin ye kta, woyute peta wośnapi waśtemna wiciyokipi, waśin kin owasin he Jehowa tawa.

17 Nitoicagepi yeye cin tukte e kaeś yatipi kin owasin ohna waśin qaiś we takudan yatapi kte śni, he wokage owihanke wanice kta.

\section{WICOWOYAKE 4.}

1 Unkan Jehowa ie ca Mowis heciya,

2 Israel cinca kin hecen ewicakiya wo; Wicanagi wan ecinśni wahtani kinhan, qa Jehowa tawoahope wan taku econ śni wicaśi kin hena wanji econ kinlıan :

3 Wawayuśna sdayapi kin he wahtani, qa oyate kin wahtani wicaye cinhan, woahtani wahtani kin ou tatankadan pte cinca wan zanika Jehowa kahi kta, woah̉tani wośnapi kin on.

4 Qa tatankadan itkokipapi wakeya tiyopa kin ekta hiyuye kta, Jehowa itokam, qa tatankadan pa kin nape kin aputake kta, qa tatankadan kin pate kta, Jehowa itokam. 


\section{LEWI TOOPE.}

5 Qa wawayuśna sdayapi "kin he tatankadan we kin onge icu kta, qa itkokipapi wakeya tiyopa kin ekta hiyuye kta.

6 Qa wawayuśna kin napsukaza wan we en oputkan qa sakowin akihde we onge elipeye kta, Jehowa itokam, tipi wakan cokaya ozanpi kin ektakiya.

7 Unkan wawayuśna kin we kin etanhan onge wahna wośnapi izinyapi waśtemna he kin akan iun kta; he itkokipapi wakeya ohna yanke cin, Jehowa itokam, qa tatankadan we kin ocowasin wohulinahyapi wahna wośnapi sicu elita akaśtan kta, he itkokipapi wakeya tiyopa ekta yanka.

8 Qa woahtani wośnapi tatanka etanhan waśin kin owasin icu kta, watejikśije kin qa śupe sin kin owasin.

9 Qa pakśin kin napin qa pakśin śin mica en yanke cin he, qa pizi pi kin ikoyake cin mica en yanke cin he icu kta.

10 Wopida wośnapi tatanka etanhan icupi qon he iyecen, qa wawayuśna kin hena wohuhnahyapi wahna wośnapi akan izin ye kta.

11 Tuka tatanka ha kin qa cehpi kin ocowasin, pa kici, qa huha kici, qa śupe kin qa tacesdi kin.

12 Tatanka kin ocowasin hewoskantuya ayapi kta, tukten taku śica wanica ekta, cahota okadapi kin heciya, qa can akan peta on hulinahyapi kta, caliota okadapi kin he akan hulinaliyapi kta.

13 Unkan Israel omniciye ocowasin ecinśniyan walitanipi kinhan, qa Jehowa tawoahope taku econśni wicaśi kin wanji econpi, tuka okodakiciye kin he sdonye śni econpi qa wahtanipi kinhan,
14 Tolian woahtani woahope awahtanipi kin he sdotkiyapi kinhan, okodakiciye kin wahtanipi kin on tatankadan wan ahipi kta, qa itkokipapi wakeya itokam hiyuyapi kta.

15 Unkan omniciye honkayapi kin tatanka pa kin akan napepi kin aputakapi kta, Jehowa itokam, qa tatankadan kin patapi kta, Jehowa itokam.

16 Unkian wawayuśna sdayapi kin tatanka we kin etanhan onge itkokjpapi wakeya ekta hiyuye kta;

17 Qa wawayuśna kin napsukaza wan we oputkan kta, ga he etanhan śakowin akihde ehpeye kta, Jehowa itokam cokaya ozanpi ekta kiya.

$18 \mathrm{Qa}$ we kin onge wahna wośnapi he kin hena akan iun kta, he Jehowa itokam itkokipapi wakeya ohna yanka, qa we kin owasin wohuhnahyapi wahna wośnapi kin itkokipapi wakeya tiyopa ekta yanke cin he ceteta kin ekta okaśtan kta.

19 Qa waśin kin owasin he etanhan icu kta, qa on wahna wośnapi kin izin ye kta.

20 Qa woahtani wośnapi tatankadan ecakicon qon he iyecen tatanka kin he ecakicon kta, hecen wawayuśna kin wahtanipi kin akahpe wicakiye kta; unkan wicakicicajujupi kta.

21 Unkan tatankadan kin hewoskantuya ayapi kta, qa tatankadan tokaheya hulnahyaji qon he iyecen de hulinahyapi kta. Okodakiciye woahtani wośnapi kin hee.

22 Wicaśtayatapi kin wahtani kinhan, qa taku iye wakanda Jehowa tawoahope telinda kin wanji ecinśni econ kinhan qa ihduśica, 
23 Tohan woalitani kin hena en walitani kin sdotkiye cinhan toahi kin tatokadan mdoka zanika wan hiyuye kta.

$24 \mathrm{Qa}$ tamdoka pa akan iye nape kin aputake kta, qa Jehowa itokam tukten wohuhnahyapi patapi kin hen he pate kta; woahtani wośnapi wan hee.

25 Unkan wawayuśna kin woahtani wośnapi we kin etanhan napsukaza wan on icu kta, qa wohulinahyapi wahna wośnapi he kin hena akan iun kta, qa we kin wohuhnahyapi wahna wośnapi sicu ekta okaśtan kta;

$26 \mathrm{Qa}$ waśin kin owasin on wahna wośnapi izinye kta, wopida wośnapi waśin kin he iyecen, hecen walitani kin wawayuśna kin -akahpe kiciye kta, unkan kicicajujupi kta.

27 Unkan oyate tokecinyall unpi etanhan wicanagi wanji wahitani, qa Jehowa tawoahope taku econśni wicaśi wan ecinśuiyan econ, qa ihduśice cinhan ;

28 Tohan woahtani wahtani kin he sdotkiye cinhan, woalitani wahtani kin on toahi tatokadan wiye wan zanika hiyuye kta.

29 Qa woalitani wośnapi pa akan iye nape kin aputake kta, qa wohuhnahyapi tukten patapi kin hen woahtani wośnapi kin pate kta.

30 Unkan wawayuśna kin he we kin onge napsukaza wan on icu kta, qa wohulinahyapi wahna wośnapi he kin hena akan iun kta, qa we kin owasin wahna wośnapi sicu ekta okaśtan kta.

31 Qa wopida wośnapi waśin kin icupi qon, he iyecen waśin kin owasin icu kta, qa wawayuśna kin wahna wośnapi kin izinye $\mathrm{kta}$; waśtemnayanpi Je- howa iyokipi, hecen wawayusna kin akahpe kiciye kta, unkan kicicajujupi kta.

32 Unkan woaktani wośnapi on amnos walk toahi hiyuye cinhan, wiyedan zanika wan hiy uye $\mathrm{kta}$. 33 Qa woalitani wośnapi pa akan iye nape kin aputake kta, unkan tukte wohulinahyapi patapi ece kin!hen woahtani wośnapi kin patapi kte.

34 Unkan wawayuśna kin woahitani wośnapi we onge napsukaza wan oll icu kta, qa wohuhnahyapi wahna wośnapi he kin hena akan iun kta, qa we kin ocowasin wahna wośnapi sicu ekta akaśtan kta.

$35 \mathrm{Qa}$ wopida wośnapi amnos waśin kin icupi qon he iyecen waśin kin owasin icu kta; unkan wawayuśna kin heon wahna wośnapi kin izin ye kta, Jehowa peta on wakiyuśnapi he iyecen, hecen wawayuśna kin akahpe kiciye kta, woahtani wahtani kin on, unkan kicicajujupi kta.

\section{WICOWOYAKE 5 .}

1 Unkan wicanagi wan wahtani, qa tuwe 'Taku wakan cajeyata nalion, qa wayaatanin śipi, taku nahon qaiś sdonya kin he, tuka oyake śni kinhan, wahtani kin kicin kta.

$2 \mathrm{Qa}$ iś wicanagi tuwe taku śapa wan icahtake cinhan, wamanica țe śapa, qa woteca țe śapa, qa watutka te śapa unman tukte kaśta sdotkiye śni econ eśta, hee śape kta, qa hdajuju kte.

$3 \mathrm{Qa}$ is tuwe wicaśtๆ śapa wan icahtake cinhan, woaśape taku kaśta on wicaśta wan ihduśape cinhan, he sdonye śni econ eśta, tohan sdonye cinhan hdajuju kta. 
wakan cajeyate ca awacinśniyan iha on taku eye cinhan, taku śica econ kta, qaiś taku waśte econ kta wicaśta kin awacinśniyan eye cinhan, iyukcan śniyan econ eśta, tohan he sdonye cinhan hena wanji on hdajuju kta.

5 Unkan tohan hena wanji on hdajuju kte cinhan, taku en walitani kin he ohdake kta.

6 Qa woahtani wahtani kin on taku on hdajuju kta Jehowa ekta hiyu ye kta, wahtani kin on tahinca etanhan wiyedan wan talinca ska, qaiś tatokadan etanhan, unkan wawayuśna kin he on akahipe kiciye .kta.

7 Unkan nape kin tacincadan wan iyohiye kte śni kinhan, walitani kin hdajuju kta e tinwakiyedan nonpa, qaiś wakiyedan cinca nonpa, Jehowa kahi kta, wanji woahttani wośnapi on, qa unman wohulinahyapi on.

8 Qa hena wawayuślla kin ekta hiyuye kta, unkan woahtani wośnapi lkin he tokaheya ahi kta, qa pa olidateya dote cape kta, tuka baśpe kte śni.

9 Qa woahtani wośnapi kin we onge wahna wośnapi cuwi akan elipe ye kta, qa we okapte cin owasin wahna wośnapi sicu ekta yuślice kta woahtani wośnapi wan hee.

10 Unkan inonpa kin wohulinahyapi wan kage kta, econ wicaśipi kin iyecen, hecen wawayuśna kin woahtani wahtani q̣on akalipe kiciye kta, unkan kicicajujupi kta.

11 Tuka nape kin tinwakiyedan lonpa, qaiśs wakiyedan cinea nompa iyohiye kte śni kinhan, walitani kin on toahi kin aguyapi mdu can wakśica olnnaka wanjidan woahtani wośnapi on hiyuye kta. He akan wihdi 150 akaśtan kte śni, qa canśin waśtemna aehnake kte śni, woahtani wośnapi wan hee nakaeś.

$12 \mathrm{Qa}$ he wawayuśna ekta hiyuye kta. Unkan wawayuśna kin he etanhan napohnaka wanji icu kta, he wokiksuye ee, qa on wahna wośnapi kin izin ye kta, peta on Jehowa wakiyuśna iyecen, woalitani wośnapi wan hee.

13 Hecen hena on woalitani wan wahtani kin on wawayuśna kin akahpe kiciye kta, unkan kicicajujupi kta. Unkan taku okapte cin he wawayuśna tawa $\mathrm{kta}$, minha wan iyecen.

14. Unkan Jehowa ie ça Mowis heciya ;

15 Wicanagi wan sicaya ohanyan, qa Jehowa taku wakanyan tawa cn ecinśniyan wahtani kinhan, tohdajuju kin Jehowa ekta hiyuye kta, tamdoka wan tahinca etanhan mazaska śeqel tona idacin, tipi wakan śeqel iyececa kici on hdajuju kta.

16 Hecen taku wakanyanpi kin manon qon kicu kta, qa izaptan kin he kici aopekiciciye kta, qa wawayuśna kin he qu kta, unkan wawayuśna kin on hdajujupi tamdoka on akahpe kiciye kta, unkan kicicajujupi kta.

17 Unkan wicanagi wan wahtani kinhan, qa taku wanji Jehowa econ śni wicaśi econ kinhan, he sdonye śni eśta he wahtani, qa on hdajuju kta.

18 Qa hdajuju kta e wawayuśna ekta tahinca etanhan tamdoka wan, taku idacin kici hiyuye kta, unkan taku ecinśniyan walitani, qa iyukcanśniyan nuni kin heon wawayuśna kin akahpe kiciye kta, unkan kicicajujupi kta.

19 On hdajujupi wan hee; Jehowa en walitani kin on hdajuju lita. 


\section{WICOWOYAKE 6 .}

20 Unkan Jehowa ie ca Mowis heciya;

21 Wicanagi wan wahtani qa taku Jehowa tawa wan manon kinhan, qaiś tuwe ikiyedan ti en. itonśni, taku yuhe śipi qaiś talru wicakicihnaka on, qaiś taku wicaki kin on qaiś tuwe ikiyedan ti kiyuśe kinhan;

22 Qaiś taku wicakitaninpi śni iyeye ça on itonśni, qa taku kaśta wicaśta econpi kin hena wanji en wahtani, qa itonśniyan on taku wakan caje yate cinhan;

23 Hehan kaketu kta, walitani kin on hdajuju kta e taku wicaki qon kicu kta, qa iś taku wicahnayan on yuha, qa iś taku kicihnakapi qon, qaiś taku wicakitanin śni iyeye ciqon.

$24 \mathrm{Qa}$ taku kaśta on taku wakan cajeyate ca itonśni kin, he ocowasin kicu kta, qa anpetu tohan hdajuju kin hehan izaptan wan aopekiciciye kta, qa tuwe tawa kin kicu kta.

25 Nakun on hdajuju kta Jehowa wakiyuśnapi wan tahinca etanhan, tamdoka wan zanika, taku idacin kici hdajuju kta e wa wayuśna ekta hiyuye kta.

26 Unkan wawayuśna kin Jehowa itokam akahpe kiciye kta, unkan taku kaśta on hdajuju kta econ qon, iyohi kicicajujupi kta.

\section{WICOWOYAKE 6.}

1 Unkan Jehowa ie ça Mowis heciya,

2 Aaron qa iye cinca hecon wicaśi, qa hewicakiya wo; Wohuhnahyapi wośnapi woope kin dee: Hanyetu osan wahna wośnapi akan itkonyan un kta, ihanhanna kin hehanyan, qa wahna wośnapi akan peta kin itkonye cin heon wohuhnahyapi kin eciyapi.

3 Unkan wawayuśna kin mini- liuha ska mahen unpi kin kiçun qa minihuha ska onze oge koyake kta, iye cehpi akan, qa wohuhnahyapi wośnapi caliota peta wahna wośnapi akan hulinage cin he icu kta, qa wahna wośnapi kin icahda ehnake kta.

4 Hehan wokoyake kin hduśdoke kta, qa wokoyake tokeca kicun kta, qa cahota kin liewoskantuya aye kta, tukten taku śica wanica ekta.

5 Unkan peta wahna wośnapi akan yanke cin he ohinniyan itkon yapi kta, he kasnipi kte śni. Unkan he akan hanhanna otoiyohi wawayuśna kin can ideye kta, qa he akan wohuhnaliyapi kin aehnake kta, qa he akan wopida wośnapi waśin kin izin ye kta.

6 Peta kin ohinniyan wahna wośnapi akan itkon yapi kta, tohinni sni kte śni.

7 Unkan minha woope kin dee, Aaron cinhintku wanji he Jehowa itokam wahna wośnapi ekta kahi kta.

8 Qa minha aguyapi mdu qa wihdi etanhan napohnaka wan. icu kta, qa canśin minha akan un kin he ocowasin, qa wahna wośnapi kin izin ye kta, he wokiksuye waśtemna Jehowa iyokipi.

9 Unkan taku minha etanhan okapte cin he Aaron qa iye cinca kin aguyapi onapohye codan yutapi kta, oyanke wakan kin ohna, he yutapi kta, itkokipapi wakeya hocoka kin ohna he yutapi kta.

10 Onnapoliye on śpanyanpi kte śni, tuka peta on wamiciyuśnapi etanhan wopamni kin he wicawaqu. He wakan hinca, woahtani wośnapi kin iyecen, qa wokajuju wośnapi kin iyecen.

11 Aaron cinca kin tona wica kin hena owasin he yutapi kta. 


\section{LEWI TOOPE.}

Nitoicagepi yeye cin wokage kin de owihanke wanice kta, tona wakanpi kin henana taku peta on Jehowa wakiyuśnapi kin yutanpi kta.

12 Unkan Jehowa ie ca Mowis heciya,

13 Aaron qa iye cinca anpetu en wanji sdayapi kinhan, woahi kin de kahipi kta, aguyapi mdu can wakśica ohnaka wanji, minlia ohinniyan wan, hanke hantianna eca, qa hanke htayetu eca.

14 Maza wiceonpa akan wihdi en cegugu yaye kta, śpan hehan hiyu yaye kta, minlia yuśpuśpupi waśtemna Jehowa iyokipi yakahi kta.

15 Qa tuwe iye cinca etanhan hee kiya wawayuśna sdayapi lin he kage kta, he ocowasin Jehowa tawa izinyapi kta, wokage kin de owihanke wanice kta.

16 Hecen wawayuśna minlia tawa kin he ocowasin hulinahyapi k:ta, tuwedan he etanhan yute kte śni.

17 Unkan Jehowa ie ça Mowis heciya,

18 Aaron qa iye cinca kin hecen ewicakiya wo: Woahtani wośnapi woope kin dee, tukten wohuhinaliyapi patapi kin he en woahtani wośnapi kin patapi kta, Jehowa itokam he wakan hinca.

19 Wawayuśna tuwe heon wokajuju kage cin he yute kta, oyanke wakan kin itkokipapi wakeya hocoka kin en he yutapi kta.

20 Taku kaśta he cehpi kin icalitake cinhan wakan kta, qa wokoyake taku kaśta he we kin akan ehpeyapi kin, he akan hinhipaye cin on, oyanke wakan en yujajapi kta.

21 Qa maka cega ohna he śpanyanpi kin he kamdecapi kta, qa mazaśa cega ohna he śpanyanpi kinhan, he payezapi qa mini on yujajapi kta.

22 Wawayuśna tona wicapi kin hena owasin yutapi kta, he wakan hinca.

$23 \mathrm{Qa}$ woahtani wośnapi tona we kin on akalipapi kta e tipi wakan ohna itkokipapi wakeya en hiyuyapi hena yutapi kte śni, peta on bulinahyapi kta.

\section{WICOWOYAKE $\%$.}

1 Unkan on hdajuju wośnapi woope kin dee, he wakan linca. 2 Tukte wohuhnahyapi wośnapi ktepi kin he en on hdajujupi ktepi kta, qa we kin he wahna wośnapi akan ihdukśan eh̉peyapi kta.

3 He etanhan waśin owasin ahi kta, sinte kin qa waśin śupe akalipe cin he;

4 Qa pakśin kin napin, qa pakśin śin mica en yanke cin he, qa pizi pi ikoyake cin, pakśin icahda he yutokan kte;

$5 \mathrm{Qa}$ hena on wawayuśna kin wahna wośnapi kin izin ye kte, peta on Jehowa wakiyuśnapi, on hdajujupi kin hee.

6 Wawayuśna wica kin hena owasin he yutapi kta, oyanke wakan ohna yutapi kta, he wakan hinca.

7 Woahtani wośnapi kin, qa on hdajuju wośnapi kin hena woope kin okonwanjidan; wawayuśna kin tuwe hena on woakahpe kage cin he tawa kta.

$8 \mathrm{Qa}$ wawayuśna tuwe wicaśta wan wohuhnahyapi kicicahi kinhan, wohulinahyapi ha kin he wawayuśna ahi kin he tawa lkta.

$9 \mathrm{Qa}$ minha kin oceti akan śpanyanpi kin he owasin, qa ce-ga ohna qa cega ihupatonna akan kagapi kin he owasin wawayuś- 
na kin tuwe he ahi kin he tawa kta.

10 Tuka minha wihdi icicahiyapi, qa aguyapi mdu kin hena iyuhpa Aaron cinca kin owasin otoiyohi hunkawanjitku iyececa tawapi kta.

11 Unkan wopida wośnapi Jehowa kahipi kta woope kin dee.

12 Tuwe he woyatan on ahi kinhan, woyatan wośnapi kici aguyapi napolyyapi śni wihdi icicahiyapi, qa aguyapi zibzibedan napohyapi śni, wihdi on sdayapi, qa aguyapi ceguguyapi wihdi akaśtanpi ;

13 Aguyapi napohyapi śni kici aguyapi napoliyapi ahi kta, toahi wopida woyatan wośnapi tawa on.

$14 \mathrm{Qa}$ woahi kin ocaje owasin etanhan aguyapi wanjidan wokosyapi Jehowa tawa, wawayuśna wopida we kin amnimni kin he ektakiya kahi kta, he tawa kta.

15 Qa woyatan wopida wośnapi cehpi kin he yutapi kta, anpetu en ahipi kin en, hetanhan ongedan ihanlianna hehanyan okaptapi kte śni.

16 Tuka toahi kin woyakapi wośnapi qaiś, itukilianpi wośnapi kinhan, anpetu en taku wośna ahi kin hen yutapi kta, qa taku okapte cin he ihanhanna nakun yutapi kta.

17 Qa tado wośnapi anpetu ivamni kin hehanyan okapte cin he peta on hulinahyapi kta.

$18 \mathrm{Qa}$ wopida wośnapi tado onge anpetu iyamni en yutapi kinhan, iyokipi kte śni, qa tuwe ahi kin kiciyawapi kte śni, taku śicamna iyecece kta, wicanagi kin tuwe hetanhan yute cinhan wošice cin hduhe lita.

19 Cehipi taku śapa wan icahtake cin he yutapi kte śni, he pe- tan huhnahyapi kta. Unkan cehpi kin tona ecepidan kin hena owasin celipi yutapi kta.

20 Tuka wicanagi tuwe toaśape ikoyake cin, wopida wośnapi cehpi kin Jehowa wakiyuśnapi kin he yute cinhan, wicanagi kin he taoyate kin etanhan kaśpapi lita.

21 Qa wicanagi tuwe taku śapa yutan kinhan, wicaśta towa aśape qaiś woteca towaaśape, qaiś watutka sapa wan, qa wopida wos̉napi Jehowa wakiyuśnapi cehpi etanhan yute cinhan wicanagi kin he taoyate etanhan kaśpapi kta.

22 Unkan Jehowa ie ça Mowis heciya,

23 Israel cinca kin hecen ewicakiya wo; Pte waśin kin, qa tahinca ska waśin kin, qa tatokadan wanunyanpi waśin kin ongedan yatapi kte śni.

24 Taku wayazanka te cin waśin kin, qa taku wamanica yațapi kin waśin kin he wowiyunyanpi kta, tuka he yutapi kte śni.

25 Tuwe kaśta woteca peta on Jehowa wakiyuśnapi kin he etanhan waśin onge yute cinhan wicanagi kin he iye taoyate etanhan kaśpapi kta.

26 Nakun yatipi owasin ohna we ongedan yatapi kte śni, wahupakoza we kin, qa woteca we kin unman tukte kaśta.

27 Wicanagi tuwe kaśta we ongedan yute cinhan wicanagi kin he taoyate kin etanhan kaspapi kta.

28 Unkan Jehowa ie ca Mowis heciya;

29 Israel cinca kin hecen ewicakiya wo, Tuwe kaśta wopida wośnapi wan Jehowa kahi kte cinhan, he wopida wośnapi toahi kin Jehowa ekta hiyuye kta. 


\section{LEWI TOOPE.}

30 Iye nape kin Jehowa peta wośnapi tawa hiyuye kta, waśin niaku iyahna, maku yuwintapi, Jehowa itokam he yuwintapi kta e hiyuye kta.

31 Unkan wawayuśna kin waśin kin on wahna wośnapi kin izinye kta, tuka maku kin he Aaron qa iye cinca kin he tawapi kta.

32 Qa hiyete etapa kin he wopida wośnapi nitawapi etanhan wokozapi wan wawayuśna kin yaqupi kta.

33 Aaron cinca etanhan tuwe wopida wośnapi we kin, qa wa sin kin ahi kinhan, hiyete etapa kin wobaśpe tawa kta.

34 Israel cinca wopida wośnapi tawapi etanhan, maku yuwintapi, qa hiyete kozapi kin hena iwicawacu, qa Aaron qa iye cinca wicawaqu, Israel cinca etanhan, wokage owihanke wanica.

35 Taku peta on Jehowa wakiyuśnapi etanhan Aaron wiyutapi tawa, qa iye cinca wiyutapi tawapi kin hee: Jehowa wawakiyuśnapi kta e en awicahi anpetu kin he en.

36 He Jehowa wicaqu śi kin anpetu en sdawicaye cin he ehan, Israel cinca etanhan wicoicagepi yeye cin, wokage owihanke wanica.

37 Wohulinahyapi on woope kin hee, qa nakun minhia on, qa woahtani wośnapi on, qa wokajuju wośnapi on, qa wojunyapi on, qa wopida wośnapi on.

38 Jehowa Mowis hecon śi, Sina he ohna anpetu wan Israel cinca toahipi Sina hopuze cin en, Jehowa ekta ahi wicaśi qehan.

\section{WICOWOYAKE 8.}

1 Unkan Jehowa ie ça Mowis heciya,
2 Aaron qa iye cinca om iwicacu wo, nakun wokoyake kin, qa wihdi on sdawicayapi kin, qa woahtani tatanka kin, qa tamdoka nonpa kin, qa aguyapi napoliyapi śni wakiśkotpa kin.

3 Qa omniciye owasin itkokipapi wakeya tiyopa ekta wicakico wo.

4 Unkan token Jehowa econ śi kin he iyecen Mowis econ; unkan omniciye owasin itkokipapi wakeya tiyopa ekta mniciyapi.

5 Unkan Mowis omniciye kin hewicakiya, Taku Jehowa econ unśipi kin dee.

6 Hehan Mowis Aaron qa iye cinca kin awicahi, qa mini on wicayujaja.

7 Qa mahenunpi kin koyag ya, qa ipiyake on ipiyag kiciton, qa nitośke kin koyag ya, qa amdo akahpe kin akihnake ca amdo akahpe ipiyake kazuntapi kin on ipiyag kiya,- hecen amdo kin akahpe kiya.

8 Qa maku akahpe kin akihnake ca iyoyam wicaye ca ecetu wicaye cin maku akahpe kin en iyeya.

9 Qa pa akan pa ipahte kin pahta, qa pa ipalite akan ite ektakiya mazaskazi mdaska wateśdake wakan pahta; Jehowa hecon śi kin he iyecen.

10 Unkan Mowis wihdi on sdawicayapi kin icu, qa tipi kin sdaya, nakun taku ohna un kin owasin; hecen hena yuwakan.

$11 \mathrm{Qa}$ he etanhan śakowin akihde wahnawośnapi akan ehpeya, qa wahnawośnapi kin sdaya, nakun taku on kicanyanpi owasin, nakun maza koka kin, qa taceteta kin; hecen hena yuwakan.

12 Qa wihdi on sdayapi onge Aaron pa kin akaśtan, qa he yuwakan kta e sdaya.

13 Unkan Mowis Aaron cinca 
kin awicahi, qa mahen unpi kin ikoyag wicaya, qa ipiyake on ipiyag wicakiciton, Jehowa Mowis hecon si kin he iyecen.

$14 \mathrm{Qa}$ woahtani tatanka kin ikiyedan hiyuya; unkan Aaron qa iye cinca kin tatanka pa akan napepi aputakapi.

15 Unkan pata, qa Mowis we kin icu qa napsukaza wan on wahna wośnapi he kin aihdukśan iun, hecen wahna wośnapi kin wakiyuśna, qa we kin wahna wośnapi sicu ekta kaśtan, hecen he akan akalipapi kta e he yuwakan.

16 Unkan watejikśija kin he owaśin icu, qa pi pizi kin, qa paksin napin, qa pakśin śin, qa Mowis hena on wahna wośnapi kin izinya.

17 Tuka tatanka pa kin, qa cehpi kin peta on huhnaliya, otipi kin itankan, Jehowa Mowis hecon śi kin he iyecen.

18 Hehan woluhinahyapi tamdoka kin ahi, unkan Aaron qa iye cinca kin napepi tamdoka pa kin aputakapi.

19 Unkan he pata, qa Mowis he we kin wahna wośnapi aihdukśan akaśtan.

20 Unkan tamdoka kin token baśpapi kta iyececa baśpaśpa; unkan Mowis tamdoka pa kin, qa obaśpe kin, qa waśin kin izinya.

21 Unkan śupe kin qa huha kin mini on yujaja, unkan Mowis tamdoka ocowasin on wahna wośnapi kin izinya, peta on Jehowa wakiyuśnapi wan woliuknahyapi waśtemna wiciyokipi wan hee, Jehowa Mowis hecon śi kin he iyecen.

22 Unkan tamdoka inonpa kin he ahi, ojunyapi tamdoka kin hee; unkan Aaron qa iye cinca kin napepi kin tamdoka pa kin aputakapi.
23 Unkan he pata, qa Mowis he we kin etanhan icu, qa Aaron noge etapa inkpa akan ikiciun, nakun napahunka etapa akan, qa sipahunka etapa akan.

24. Qa A aron cinca kin awicahi, qa Mowis nogepi etapa inkpapi akan we kin onge iwicakiciun, nakun napahunkepi, qa sipahunkepi etapa akan, qa we ihe cin wahna wośnapi aihdukśan akaśtan.

25 Qa waśin kin icu, nakua sinte kin, qa watejikśija ocowasin, qa pi pizi kin, qa pakśin napin, qa pakśin śin kin qa hiyete etapa kin ;

26 Nakun aguyapi napohyapi śni wakiśkotpa Jehowa itokam yanke cin etanhan aguyapi napoliyapi śni hmiyanyan wanjidan, qa wihdi aguyapi hmiyanyan wanjidan, qa aguyapi zibzibedan wanjidan, qa hena waśin akan, qa hiyete etapa akan ehnaka.

27 Qa hena owasin Aaron nape en qu, nakun cinhintku napepi en ; unkan hena kozapi, Jehowa itokam; wokozapi wan hee.

28 Unkan napepi etanhan Mowis hena icu, qa on wahna wośnapi kin izinya, woliulinaliyapi akan, wojunyapi kin hena eepi, woomna wiciyokipi peta wakiyuśnapi wan hee.

29 Unkan Mowis maku kin icu, qa Jehowa itokam koza, wokosyapi ojunyapi tamdoka etanhan obaśpe kin he Mowis tawa, Jehowa Mowis hecon si kin he iyecen.

30 Unkan Mowis sdayapi wihdi etanhan icu, qa we wahna wośnapi akan un kin etanhan, qa Aaron akan elipeya, nakun wokoyake tawa akan, qa cinhintku akan, qa cinhintku tawokoyakepi akan, hecen Aaron yuwa155 


\section{LEWI TOOPE.}

han, qa tawokoyake, qa cinhintku, qa nakun cinhintku tawokoyakepi.

31 Unkan Mowis Aaron qa iye cinhintku hewicakiya, Itkokipapi wakeya tiyopa kin ekta tado kin ohanpo, qa heciya he yatapi kta, qa aguyapi ojunyapi kin wakiśkotpa ohna un kin he nakun, liecon ciśipi kin he iyecen, Aaron qa iye cinca kin he yutapi kta, epa.

$32 \mathrm{Qa}$ taku okaptapi tado kin e tanhan, qa aguyapi kin etauhan, petan ehpeyayapi kta.

33 Qa śakowin can itkokipapi wakeya tiyopa etanhan inayapapi kte śni, anpetu ojunniyanpi kin henakecapi kte cin hehanyan, anpetu śakowin ninapepi ojunniyanpi kta nakaś.

34 Nakaha econ ciśipi kin he iyecen Jehowa econ unśipi; alkalipe niciyapi kta heon.

$35 \mathrm{Qa}$ anpetu śakowin, anpetu hanyetu koya, itkokipapi wakeya tiyopa ekta nankapi kta, qa Jehowa topatan kin yapatanpi kta, hecen nițapi kte śni, hecen econ ciśipi heon etanhan.

36 Unkan Aaron, qa iye cinca kin taku Jehowa Mowis nape on econ wicaśi kin owasin econpi.

\section{WICOWOYAKE 9.}

1 Unkan anpetu iśahdogan hehan Mowis Aaron qa iye cinca kin wicakico, nakun Israel hunkawicayapi.

$2 \mathrm{Qa}$ Aaron heciya, Ptejicadan wan pte wanunyanpi cinca woalitani wośnapi on icu, qa tamdoka wan wohulinaliyapi on, napin zanipi, qa Jehowa itokam awicahi wo.

$3 \mathrm{Qa}$ Israel cinca kin hecen ewicayakiye kta, Tatokadan mdoka wan woalitani wośnapi on icupo, qa wohuhnahyapi on ptejicadan wan, qa amnos wan, napin waniyetu wanjidan zanipi. $4 \mathrm{Qa}$ tatanka wan, qa tamdoka wan wopida wośnapi on, Jehowa itokam wakiyuśnapi kta; nakun minlia wihdi icicahiyapi; anpetu kin de Jehowa niyepi en ihdutanin kta, heon etanhan.

5 Unkan taku Mowis itkokipapi wakeya tiyopa ekta au wicaśi kin he aupi, qa omniciye kin ocowasin ikiyedan hiyupi qa Jehowa itokam najinpi.

6 Unkan Mowis heya, Taku Jehowa hecon niśipi kin dee, hecen Jehowa towitankin niyepi en ihdutanin kta.

7 Unkan Mowis Aaron heciya, Wahna wośnapi kin ikiyedan $u$, qa woalitani wośnapi nitawa, qa wohulinahyapi nitawa kin kaga wo, hecen niye on, qa oyate kin on akahpe yakiye kta, nakun oyate toahi kin kaga wo, hecen hena on akahpe yakiye kta, Jehowa econ niśi kin he iyecen.

8 Unkan Aaron wahna wośnapi kin ikiyedan hiyu, qa ptejicadan woahtani wośnapi tawa kin pata.

9 Unkan Aaron cinca kin we kin kahipi, unkan napsukaza wan we kin en oputkan, qa wahna wośnapi he akan iun, qa we kin wahna wośnapi sicu ekta kaśtan. 10 Unkan woalitani wośnapi etanhan waśin kin, qa pakśin kin, qa pi etanhan pizi on wahna wośnapi kin izinya, Mowis econ śi kin he iyecen.

11 Unkan tado kin, qa ha kin hulinahya, owanka itankan.

12 Hehan wohuhnahyapi kin pata ; unkan Aaron cinca kin we kin kahjpi, unkan he wahna wośnapi aihdukśan akaśtan.

13 Unkan wohuhnahyapi kin kahipi, obaśpe kin, qa pa kin, unkan izinya wahna wośnapi akan. 


\section{WICOWOYAKE 10.}

14 Qa śtupe kin, qa huba kin, yujaja, qa wohulinaliyapi wahna wośnapi akan izinya.

$15 \mathrm{Qa}$ oyate toahi kin he ahi, qa tatokadan mdoka woahtani wośnapi oyate tawapi kin he icu, qa pate ça wayuśna, tokaheya qon he iyecen.

$16 \mathrm{Qa}$ wohulinaliyapi kin ahi, qa woyaco kin he iyecen ecakicon.

$17 \mathrm{Qa}$ minlia kin he ahi, qa hetanhan napohnaka. wan icu, qa hanlianna wohulinaliyapi iyahna wahna wośnapi akan izinya.

18 Qa tatanka qa tamdoka oyate wopida wakiyuśnapi kin hena pata; unkan Aaron cinca kin we kin kahipi, unkan he wahna wośnapi aihdukśan akaśtan.

19 Unkan tatanka etanhan, qa tamdoka etanhan waśin kin, sinte kin, qa watejikśija, qa pakśin kin, qa pi pizi kin kahipi.

$20 \mathrm{Qa}$ waśin kin maku kin akan ehnalsapi, qa waśin kin on wahna wośnapi kin izinya.

$21 \mathrm{Qa}$ maku, qa hiyete etapa kin Aaron kosya, Jehowa itokam wokosyapi, Mowis econ śi kin he iyecen.

22 Qa Aaron oyate ektakiya nape kin hduwinta, qa wicayawaśte; hehan woałtani wośnapi, qa wohuhnahyapi, qa wopida wośnapi kage cin etanhan hdicu.

23 Unkan Mowis Aaron kici itkokipapi wakeya en ipi, qa tankan inanpapi, qa oyate kin wicayawaśtepi; unkan Jehowa towitan kin oyate kin owasin en ihdutanin.

24 Unkan Jehowa itokapatanhan peta inanpe ca woliulinaliyapi kin, qa waśin kin wahna wośnapi akan liulinaliya. Unkan oyate kin owasin he wanyakapi, qa panpanpi, qa makipusdiya ihpayapi.

\section{WICOWOYAKE 10.}

1 Unkan Aaron cinca kin $\mathrm{Na}$ dab qa Abihu iyohi tamaza mdaska icu, qa hena akan peta ehnakapi, qa he akan wizinyapi kadapi, qa peta tolkeca Jehowa itokam kahipi, hecon wicaśi śni tuka.

2 Unkan Jehowa itokapa tanhan peta inanpe ça hulinali wicaya.

3 Unkan Mowis Aaron heciya, Jehowa heye ciqon he dee, Tona mikiyedan hipi kin, mayawakanpi kta, qa oyate owasin itokam mayutanpi kta, eya, Unkan Aaron inina yanka.

4 Unkan Mowis Aaron ateye Uziel cinca kin Miśael qa Elitzapan wicakipan, qa hewicakiya, Ikiyedan upo, qa nihunkawanjipi kin tipi wakan itokapa tanhan awicayapo, hewoskantuya.

5 Unkan en hipi, qa mahen unpi ohna owanka itankan awicayapi, Mowis eye cin he iyecen.

6 Unkan Mowis Aaron qa iye cinca kin Eleazar qa Itamar hewicakiya, $\mathrm{Pa}$ bakiśdapi śni po, qa wokoyake hdutokecapi śni po; hecanonpi kinhan okini nițapi kta, qa omniciye ocowasin en wocanteptanye $u \cdot k$ ta ; tuka nihunkawanjipi, Israel cinca tipi ocowasin wogu kin Jehowa guye cin he aceyapi kta.

7 Unkan Jehowa tosdaye wihdi aniunpi, heon itkokipapi wakeya tiyopa etanhan inayapapi kte śni, okini ni țapi kta, eya. Unkan Mowis eye cin ecen econpi.

8 Unkan Jehowa ie ca Aaron heciya,

9 Itkokipapi wakeya ekta yahipi eca miniśa, qa miniwakan dathe kte śni, niye qa nakun nicinca, hecen nitapi kte śni. Woka- 


\section{LEWI TOOPE.}

ge kin de owihanke wanica nitoicagepi yeyc cin.

10 Qa hecen taku wakan qa taku wakan śni otahedan dukinukanpi kta, qa taku śapa qa taku ecedan otahedan:

$11 \mathrm{Qa}$ hecen wokage Jehowa Mowis nape eciyatanhan eniciyapi kin, hena owasin Israel cinca kin onspe wicayakiyapi kta.

12 Unkan Mowis Aaron qa iye cinca kin nahanhiin en unpi, Eleazar qa Itamar hewicakiya, Minha Jehowa tapeta wośnapi etanhan taku okaptapi kin he icu, qa napohyapi śni yutapo, wahna wośnapi icahda, he wakan hinca.

13 Qa he oyanke wakan ohna yutapi kta; taku peta on Jehowa wakiyuśnapi etanhan wopamni nitawa, qa nicinca wopamni tawapi kin hee, heon hecen econ ciśipi nakaś.

14. Qa maku kosyapi, qa hiyete yuwintapi kin, hena oyanke wakaśote śni ohna yatapi kta, niye qa nicinkśi qa nicunkśi opapi kta, wopamni nitawa, qa nicinca wopamni tawapi Israel cinca wopida wośnapi kin etanhàn nicupi nakaś.

15 Hiyete yuwintapi kin qa maku kosyapi kin waśin iyahna hiyuyapi kta, wokosyapi Jehowa itokam kosyapi kta heon, qa he nitawa kta, qa nicinca nakun tawapi kta, wokage owihanke wanica, Jehowa econ unśipi kin he iyecen.

16 Unkan Mowis tatokadan woahtani wośnapi kin he nina akita, tuka hulinahyapi ; heon Eleazar qa Itamar Aaron cinca nahahin en unpi canniyc wicaye ça heya,

17 Tokeca woahtani wośnapi kin he yatapi śni, oyanke wakan ohna, he walran hinca, qa omni- ciye sicaya olianyanpi kin duhapi kta e he nicupi, qa akahpe wicayayapi kta e Jehowa itokam.

18 Ito he we kin tipi wakan mahen hiyuyapi śni heon tipi wakan ohna he yatapi kta iyececa, econ ciśipi kin he iyecen.

19 Unkan Aaron Mowis heciya, Ito nakaha woahtani wośnapi tawapi qa tohuhnahyapi kin hena Jehowa itokam ahipi, unkan taku hececa amakipapi, unkan anpetu kin de. woahitani wośnapi wate kte cin he Jehowa iyokipi ye kta hè?

20 Unkan Mowis he nation qehan he iyokipi.

\section{WICOWOYAKE 11.}

1 Unkan Jehowa ie ça Mowis Aaron kici hewicakiya,

2 Israel cinca kin hecen ewicakiyapo. Taku maka akan ni un kin owasin etanhan woteca kin dena yatapi kta.

3 Woteca tona wayatakonza śake jate śake ocowasin kinaśpe cin hena owasin yatapi kta.

4 Tuka dena wayatakonzapi qaiś śake jatapi eśta yatapi kte śni ; camo kin he wayatakonza eśta śake kin kinaśpe śni, he śapa yadakapi kta.

$5 \mathrm{Qa}$ śapan kin he wayatakonza eśta śake kin kinaśpe śni heon he śapa yadakapi lkta.

6 Qa maśtinca kin he wayatakonza eśta śake kinaśpe śni heon he śapa yadakapi kta.

7 Qa kukuśe kin he sake jata, qa śake ocowaśin kinaśpe eśta wayatakonze śni heon he śapa yadakapi kta.

8 Hena cehpi kin ongedan yatapi kte śni; qa țapi tancan dutanpi kte śni, hena sapa yadakapi kta.

9 Unkan taku mini oicage cin 
owasin etanhan dena yatapi kta, 'Taku hoape, qa hoceśpu yukan, mini oicage, mde ohna, qa wakpa ohna, hena owasin yatapi kta.

$10 \mathrm{Qa}$ taku mde ohna qa wakpa ohna hoape qa hocespu wanice cinhan, taku mini oniwan, qa taku nagi ni un kin, hena owasin etanhan yatapi kte śni, hena wowahteśni yadapi kta.

11 Hena wowahteśni yadapi lita, heon cehpipi etanhan yatapi kte śni, qa țapi tancan wahteśni yadapi kta.

12 Taku mini ohna hoape, qa hoceśpu wanice cin hena owasin wowahteśni yadapi kta.

13 Unkan wahupakoza dena wahteśni yadapi lita, huya, qa wamdi, qa anog paska; hena yatapi kte śni, hena wowahte śni.

14 Qa daa, qa canśka wanmdidan ocaje owasin ;

15 Qa kangi ocaje owasin;

16 Qa hinhanska, qa heca, qa canśka, qa cetan ocaje owasin;

17 Qa hinhan kaga, qa huntkan, qa yaneśupa,

18 Qa tinśemet, qa mdega, qa mdoza;

19 Qa hoka, qa pehan, qa upupa, qa hupawakihdaka.

20 'Taku kinyan sdohan, siha topa awinta kin hena owasin wowahteśni yadapi kta.

21 Tuka taku kinyan sdohan siha topa awinta etanhan dena yatapi kta, tona siha iwankam hu nonpa yukanpi on maka aipsicapi kin hena.

22 Hena etanhan dena yatapi kta, psipsicadan qa, hehakacanteyuśniśnija ocaje owasin.

23 Tuką taku kinyan sdohan siha topa yuke śni kin hena owasin wowahteśni yadakapi kta.

$24 \mathrm{Qa}$ dena on niyuśapapi kta, tuwe kaśta dena wanji te cin yutan kinhan litayetu aiyahdeya śape kta.

25 Qa tuwe hena wanji te cin qin kinhan wokoyake hdujaja kta, qa hitayetu aiyahdeya śape lita.

26 Woteca tona śake gangata eśta śake ocowasin kinaśpe śni, qa wayatakonze śni kin hena owasin śapa yadakapi kta, tuwe kaśta hena wanji țe cin yutan kinhan śape kta.

$27 \mathrm{Qa}$ wamanica tona nape a mani, hu topa amani kin hena owasin śapa yadapi kta, tuwe kaśta hena tapi wanji yutan kinhain htayetu aiyahdeya śape kta,

$28 \mathrm{Qa}$ tuwe hena tapi qin kinhan wokoyake hdujaja kta, qa htayetu aiyahdeya śape kta, hena śapa yadapi kta nakaś.

29 Unkan taku maka asdohan ehna dena śapa yadapi kta, napeheyatahdedan kin, qa hitun ka, qa kezunta ocaje owasin.

$30 \mathrm{Qa}$ ahdeśkadan, qa kohia, qa mini wamnuliadan, qa homat, qa tinśamet,

31 Taku sdohan ehna hena śapa yadapi lita, tuwe kaśta hena tapi wanji yutan kinhan hitayetu aiyahdeya śape kta.

32 'Taku kaśta hena etanhan wanji te cin ahinhipaye cinhan śape kta, canwakśica, qa ogepi, qa wakihdaka, qa wojuha, taku kaśta on wohtani econpi kin hena owasin minin iyeyapi kta, qa htayetu aiyahdeya śape kta, hehan ska kta.

33 Qa maka wakśica tona ohna hena wanji ohinlipaye cin taku ohna un kin owasin śape kta, qa wakśica kin kamdecapi kta.

34. Taku yutapi tona hena etanhan mini atpapsonpi kin hena owasin śape kta, qa woyatke wakśica kin hena ohna yatkanpi kin śape kta. 


\section{LEWI TOOPE.}

35 Qa taku kaśta hena te onśpa ahinhipaye cin he śape kta, maka cega qa maka oceti kinhan kamdecapi kta, hena śapapi, qa śapa yadakapi kta.

36 'Tuka wakoniya, qa miniowe mini ota kiwitaye cin he śape kte śni ; tuka taku tancanpi te wan icalitake cinhan śape kta,

37 Unkan tancanpi te etanhan su wojupi ahinhpaye cinhan wojupi kin śape kte śni.

38 Tuka wojupi minin hpanyanpi qa tancanpi te etanhan onśpa ahinhpaye cinhan śapa yadakapi kta.

39 Unkan woteca yatapi kin wanji te cinhan tuwe tancan țe yutan kinhan htayetu aiyahdeya śape kta.

$40 \mathrm{Qa}$ tuwe hena tancan te onśpa yute cinhan wokoyake kin hdujaja kta, qa htayetu aiyahdeya śape kta, qa tuwe tancan te cin onśpa qin kinhan wokoyake hdujaja kta, qa htayetu aiyahdeya śape kta.

$41 \mathrm{Qa}$ watutka maka asdohan kin hena owasin wowahte śni, hena yutapi kte śni.

42 Taku itpi asdohan, qa siha topa eśta sdohan, qa siha ota nakun taku kaśta maka asdohan kin hena yatapi kte śni, hena wowalite śni nakaś.

43 Ihnuhan watutka sdohan kin on ninagipi wowahteśni nic̣icagapi kin, qa ihnuhan hena on nihduśapapi kin, qa hecen hena on niśapapi kta.

44 Jehowa Wakanyadapi kin he miye heon nihduwakanpi kta, qa miś mawakan heon wakanyan yaunpi kta; qa ihnuhan watutka wan taku kaśta maka asdohan on ninagipi kin nihduśapapi kin.

45 Jehowa he miye qa wakanmayadapi kta e heon Egupta makoce etanhan hdinanpe ciya- pi, qa miś mawakan heon wakanyan yaunpi kta.

46 Woteca on, qa wahupakoza on, qa nagi niyake mini ośkanśkan owasin on, qa nagi maka asdohan owasin on woope kin hee.

47 Taku śapa qa taku ecedan otahedan yukinukan kta, qa taku niya yu tapi kta, qa taku niya yutapi kte śni otahedan.

\section{WICOWOYAKE 12.}

1 Unkan Jehowa ie ca Mowis heciya,

2 Israel cinca kin hecen ewicakiya wo, Winohinca wan ihdusake ca cinca wica wan ton kinhan, anpetu śakowin śape kta anpetu iśnati ececa kin hehanyan śape kta.

3 Unkan anpetu iśahdogan en cinhintku ce inkpaha kin bakihdayapi kta.

4 Unkan winohinca kin anpetu wikcemna yamni sanpa yamni we yuskapi kin en yanke kta, taku kaśta yuwakanpi kin icahtake kte śni, qa tipi wakan kin en i kte śni, anpetu yuskapi henakeca kte cin hehanyan.

5 Unkan cinca ton kin he winyan kinhan anpetu aketopa śape kta iśnati kin iyececa, qa anpetu wikcemna śakpe sanpa śakpe we yuskapi en yanke kta.

6 Unkan anpetu yuskapi cinhintku on, qaiś cunwintku an henakeca kinhan, wohuhinahyapi on amnos wan waniyetu wanjidan, qa woahtani wośnapi on wakiyedan cinca wan, qaiś tin wakiyedan wanji itkokipapi wakeya tiyopa ekta wawayuśna ekta kahi kta.

7 Unkan hena Jehowa itokam ahi kta qa winohinca on akahpe kta, unkan we kaduze cin etan- 


\section{WICOWOYAKE 13.}

han ska kta. Winohinca cinca wica qaiś winyan ton kin on woope kin hee.

8 Qa nape kin taku tahinca wan iyohi iyeye śni kinhan tinwakiyedan noupa qaiś wakiyedan cinca nonpa icu kta, wanji wohuhnahyapi on qa wanji woalitani wośnapi on, unkan wawayuśna akahpe kiciye kta, hecen ska kta.

\section{WICOWOYAKE 13.}

1 Unkan Jehowa ie ca Mowis Aaron kici hewicaliya,

2 Wicaśta wan celipi uka ohna oyuhi wan, qaiś hian wan, qaiś wiyega wan tanin, qa he cehpi uka ohna leprosi makośica iyececa kinhan, Aaron wawayuśna qaiś iye cinca wawayuśnapi wan en hiyuyapi kta.

3 Unkan wawayuśna kin makośica cehpi uka ohna wanyake kta; unkan makośica ohna hin kin hota aye cinhan qá makośica kin celipi uka sanpa mahen iyaya tanin kinhan, leprosi makośica hee: heon wawayuśna kin he wanyake ça yaśape kta.

4 Tuka wiyege celipi uka ohna kin he liota qa uka sanpa mahen iyaye śni tanin kinhan, qa hin kin hota aye śni kinhan, wawayuśna kin makośica ececa kin anpetu śakowin kaśkahnake kta.

5 Unkan anpetu iśakowin en wawayuśna kin wanyake kta; unkan makośica kin ecen yanke ca uka ohna sanpa iyaye śni wanyake cinhan, wa yuśna kin he anpetu śakowin inonpa kaśka hnake kta.

6 Unkan anpetu iśakowin inonpa en ake wawayuśna kin wanyake kta, unkan awicakehan makośica kin san, qa uka ohna mảkośica kin kahtanye śni kin- han, wawayuśna kin yaska kta, ceśpu wan hee, heon wokoyake hdujaja kta qa ska kta.

7 'I'uka wawayuśna yuska kta e wanyake cin he iyohakam, ceśpu kin uka ohna kahtan aye cinhan ake wawayuśna kin en ihdutanin kta.

8 Unkan wawayuśna kin wanyake kta, unkan awicakehan ceśpu kin uka en kahtan aye cinhan, wawayuśna kin he yaśape kta; leprosi hee.

9 Tohan leprosi makośica wicaśta wan en un kinhan, he wawayuśna kin en liyuyapi kta.

10 Unkan wawayuśna kin atonwan kta, unkan inyun ha ohna oyuhi liota wan, qa hin kin liota aya, qa oyulii ohna celpi ni tanin kinhan,

11 Leprosi tanina celipi uka ohna hee, unkan wawayuśna kin he yaśape kta, hee śapa heon kaśka hnake kte śni.

12 Unkan leprosi ha ohna nina ahinape ca leprosi kin makośica ececa ha kin ocowasin pa etanhan siha aiyahdeya wawayuśna taku wanyake cin iyulipa akahipe cinhan,

$13 \mathrm{Qa}$ inyun leprosi cehpi iyulipa akahpe cin he wawayuśna kin wanyake cinhan, makośica ececa kin he yaska kta, ocowasin hota icu, hee ska.

14 Tuka tohan he ohna cehpi niyake tanin kinhan śape kta.

15 Qa wawayuśna cehpi niyake cin he wanyake ca yaśape kta, cehpi niyake cin hee śapa, leprosi hee.

16 Tuka celipi niyake ihdutokeca, qa hota icu kinhan, ake wawayuśna en hi kta.

17 Unkan wawayuśna wanyalie kta, unkan makośica kin hota icu kinhan, wawayuśna kin ma- 


\section{LEWI TOOPE.}

kośica ececa kin yaska kta, hee ska.

18 Unkan celipi uka hin ohna siyakao qon okiziyapi,

19 Qa śiyakao yanke ciqon ohna oyuhi hota wan, qaiś wiyega lota kitanna śa kiuhan, wawayuśna kin wanyag yapi kta.

20 Unkan wawayuśna kin he wanyake ça inyun uka kin sanpa mahen iyaye ca hin kin hota icu kinhan; wawayuśna kin he yaśape kta, leprosi makośica śiyakao qon ohna ahinape cin hee.

21 'Tuka wawayuśna kin he wanyaka unkan ohna hin hota wanice ça uka sam mahen iyaye śni qa hee san kinhan, wawayuśna kin he kaśkahnake kta anpetu śakowin.

22 Unkan uka ohna kahtan aye cinhan yaśape kta, makośica wan hee.

23 Tuka wiyege cin ohna yanke ciqon ecen han, qa kahtanye śni kinhan, śiyakao osnaze wan hee, heon wawayuśna kin yaska kta.

24 Unkan tohan celipi uka lin ohna peta ogu yuke ca ogu owe kin wiyega hota kitanaa śa qaiś ocowasin liota kinhan,

25 Wawayuśna kin wanyake lrta, unkan wiyega kin uka sanpa mahen iyaya tanin, qa he ohna hin kin bota aye cinhan, leprosi osnaze ohna ahinanpe cin hee, unkan wawayuśna kin he yaśape kta; leprosi makośica hee.

26 Tuka wawayuśna he wanyaka, unkan awicakehan wiyega ohna hin liota wanice ca uka kin sanpa mahen iyaye śni, tuka hee san kinhan, wawayuśna kin śakowin can kaślka hnake kta.

27 Qa anpetu iśakowin en wawayuśna kin he wanyake kta, uka ohna kahitan aye cinhan wawayuśna kin he yaśape kta, leprosi makośica hee.

28 Tuka wiyega kin ohna yanke ciqon ecen han, ha ohna kahtan śni tuka hee san kinhain, ogu kin oyuli wan hee, heon wawayuśna kin he yaska kta, ogu osnaze wan hee.

29 Unkan wicaśta qaiś winolinca paha ohna, qaiś putinhin ohna makośica yuke cinhan,

30 Wawayuśna kin makośica kin wanyake kta, unkan uka sanpa mahen iyaya tanin kinhan, qa ohna hin zi zibzipedan yuke cinhan, wawayuśna kin he yaśape kta, netaq hee paha qaiś putinhin leprosi hee.

31 Unkan wawayuśna kin netaq makośica wanyaka, uka ihukuya tanin śni kinhan, qa ohna hin sapa wanice cinhan, wawayuśna kin netaq makośica ececa kin anpetu śakowin kaśka hnake kta.

32 Unkan anpetu iśalkowin en wawayuśna kin makośica kin wanyake kta, unkan netaq kin awicakehan kalitan iyaye śni, qa ohna hin zi wanice cinhan, qa netaq uka ihukuya iyaye śni tanin kinhan,

33 Ihdasan kta, tuka netaq kin hdasan kte śni, unkan ake wawayuśna kin netaq ececa kin anpetu śakowin inonpa kin kaśka hnake kta.

34 Qa anpetu iśakowin en wawayuśna kin netaq ececa kin wanyake kta, unkan awicakehan netaq uka ohna sanpa kahtan śni, qa uka ihukuya tanin śni kinhan, wawayuśna kin yaska kta; unkan wokoyake hdujaja kta, qa ska kta.

35 Tuka yaskapi kin iyohakam netaq uka ohna kahitan aye cinhan, 
36 Wawayuśna kin wanyake kta, unkan netaq kin uka okalitan aye cinhan, wawayuśna kin lin zizi kin akite kte śni, hee śapa.

37 Tuka netaq ecen han wanyake cinhan, qa ohna hin sapa inape cillhan netaq wanna okizi yapi, hee ska, unkan wawayuśna kin he yaska kta.

38 Unkan wicaśta wan qaiś winohinca wan celipi uka ohna wiyegaga hota yuke cinhan,

39 Wawayuśna kin wanyake kta; unkan celipi uka hin ohna wiyega kin san kitanna hota kinhan, bohaq uka ohna ahinape wan hee, hee ska.

40 Unkan wicaśta wan pesdete śda kinhan, he pesdete śda eśta hee ska.

41 Unkan ite ektakiya śda kinhan pa śda eśta hee ska.

42 Tuka paśda ohna makośica lota kitanna śa yuke cinhan, leprosi paśda ohna ahinape cin hee.

43 Unkan wawayuśna kin he wanyake kta, unkan awicakehan paśda ohna makośica oyuhi kin hota kitanna śa leprosi cehpi uka ohna tanin kin iyececa kinhan,

44 Wicaśta kin he leprosi ececa, hee śapa, pa kin akan makośica yukan, wawayuśna kin he yaśape kta.

45 Unkan wicaśta tuwe leprosi makośica ececa kin be tawokoyake hdehidecahe kta, qa pa codan kta, qa pute akahipe ton kta, qa, Sapa śapa, pan kta.

46 Tohan makośica ececa hehanyan śape kta, śapa kin heon iśnana ti kta, otonwe itankan ounye kta.

47 Unkan taku koyakapi ohna leprosi makośica yuke cinhan, wokoyake tahinca hin on kagapi qaiś minihuha unman tukte kaśta ohna ;

48 Kazuntapi qaiś kahmupi, halionta on kagapi qaiś tahinea hin etanhan, qaiś wakihdaka, qqaiś taku kaśta taha on kagapi ohna.

$4.9 \mathrm{Qa}$ wokoyake kin ohna makośica kin san, qaiś kitanna śis kinhan, wakihdaka ohna, qaiś taku kazuntapi ohna, qaiś taku kahmupi ohna, qaiś taku kaśta taha on kagapi ohna, leprosi nakośica kin hee; unkan wawayuśna kin wanyake kta.

50 Qa wawayuśna kin makośica kin wanyake cinhan, anpetu śakowin taku makośica ohna un kin kaśka hnake kta.

$51 \mathrm{Qa}$ anpetu iśakowin en wokoyake ohna makośica kalitan aya wanyake cinhan, taku kazuntapi ohna, qaiś taku kahmupi ohna, qa taha ohna, qaiś taku kaśta unpi kta e taha on kagapi ohna, leprosi pahinea makośica hee, hee śapa.

52 Unkan wokoyake kin he hulinahyapi kta kazuntapi qaiś kahmupi, tahinca hin qaiś halionta on kagapi ohna, qaiś taku kaśta taha on kagapi olına, leprosi pahinca hee nakaś peta ohna hulinahyapi kta.

53 Tuka wawayuśna kin wanyaka, unkan awicakeharı makośica kin wokoyake kazuntapi ohna, qaiś kahmupi ohna, qaiś taha ohna, qaiś taku kaśta taha on kagapi ohna kahitan aye śni kinhan ;

54 Wawayuśna kin taku ohna makośica yuke cin yujaja wicaśi kta, qa ake anpetu śakowin kaśka hnake kta.

55 Qa makośica yujajapi iyohakam wawayuśna kin wanyake kta, unkan awicakehan makośica otanin kin ihdutokeca śni, qa makośica kin sanpa kahtan śni 163 


\section{LEWI TOOPE.}

eśta hee sapa, wayaśdoka hee, mahentanhan śla qaiś akapatanhan śda.

56 Unkan yujajapi iyohakam makośica kin hee san, wawayuśna wanyake cinhan, he wokuyake etanhan baśpe kta, kazuntapi etanhan, qaiś kahmupi etanhan, qaiś taha etanhan;

57 Unkan ake wokoyake ohna ihdutanin kinhan, taku kazuntapi ohna, qaiś taku kahmupi ohna, qaiś taku taha on kagapi ohna, makośica icaga wan hee, taku ohna un kin he huhnah ye kta.

58 Tuka wokoyake kahmupi, quiś kazuntapi, qaiś taku kaśta taha on kagapi kin yujajapi iyohakam makośica kin ayuśtan linhan, ake yujajapi kta qa ska kta. 59 Leprosi makośica woope kin hee, wolsoyake tahinca hin, qaiś hahonta on kagapi, kazuntapi qaiś kahmupi, qaiś taku kaśta taha on kagapi on yaskapi kta qaiś yaśapapi kta.

\section{WICOWOYAKE 14.}

1 Unkan Jehowa ie ca Mowis heciya ;

2 Leprosi ececa on woope kin dee, anpetu yuskapi kin en wawayuśna kin en hiyuyapi kta.

3 Unkan wawayuśna kin tonwanyanpi itankan inape kta, qa leprosi makośica ececa qon leprosi etanhan awicakehan akisni kinhan;

4 Yuskapi kta e wawayuśna zitkadan nonpa zanika, ska icu wicaśi kta, nakun hante śa can, qa hin duta, qa pejiliota.

5 Qa wawayuśna kin zitkadan wanji maka wakśica ohna mini kaduze cin iwankam tuwe kte śi kta.

$6 \mathrm{Qa}$ zitkadan ni kin he icu kta, nakun hante śa can, qa hin duta kin, qa pejihota kin, qa hena owasin zitkadan ni un kin iyahna zitkadan ktepi we en oputkan kta, mini kaduza iwankam.

7 Qa tuwe leprosi etanhan yuskapi kin he sakowin akihde amnimni kta, hecen yuska kta, hehan zitkadan ni kin tinta ektakiya ehpeye kta.

8 Unkan tuwe yuskapi kin wokoyake hdujaja kta, qa hin owasin hdasan kta, qa mini en niwe kta, hecen ska kta, hehan otonwe en hi kta; tuka anpetu śakowin wakeya tawa itankan yanke lita.

$9 \mathrm{Qa}$ anpetu iśakowin en hin owasin hdasan kta, paha hin qa putinhin, qa iśtahe lin, qa tancan hin owasin hdasan kta, qa wokoyake hdujaja kta, qa tancan mini en niwe kta hehan ska kta.

10 Unkan anpetu iśahdogan en amnos mdoka zanika nonpa, qa amnos wiyedan wanjidan, waniyetu wanjidan, zanika icu kta, nakun minha on aguyapi mdu can wakśica ohnaka nonpa wihdi icicahiyapi, qa wihdi wiyatke ohnaka wanjidan.

11 Unkan wawayuśna wayaska kin wicaśta yuskapi kte cin he, qa hena owasin Jehowa itokam ehde kta, itkokipapi wakeya tiyopa ekta.

12 Qa wawayuśna kin amnos mdoka wanji icu qa. wohdajuju on ahi kta, nakun wihdi wiyatke ohnaka kin, qa hena koze kta, wokosyapi Jehowa itokam.

13 Qa amnos kin pate kta, tukte woahtani wośnapi, qa wohuhnahyapi kin patapi, oyanke wakan ohna; wohdajuju kin woahtani wośnapi kin he iyececa, wawayuśna kin he tawa, he wakan hinca.

14. Unkan wawayuśna kin wohdajuju we kin etanhan icu.kta, 
qa tuwe yaska kta noge etapa inkpa akan ikiciun kta, qa nape etapa hunka akan, qa sịja hunka etapa akan.

15 Qa wawayuśna kin wihdi wiyatke ohnaka etanhan icu kta, qa wawayuśna nape coka catka kin okaśtan kta.

16 Unkan wawayuśna kin nape apazo etapa kin wihdi nape catka ohna ull kin okputkan kta, qa napc apazo on we kin śakowin akihde ehpe ye kta, Jehowa itokam.

17 Qa nape kin ohna wihdi ihe cin he etanhan wawayuśna kin tuwe yaska kta noge etapa inkpa akan ikiciun kta, nakun nape hunka etapa akan, qa sipahunka e tapa akan, wohdajuju we akan.

18 Qa wihdi okapte cin wawayuśna nape coka ohna kin he tuwe yuska kte cin he pa akan ikiciun kta, qa wawayuśna kin akahpekiciye kta, Jehowa itokam.

19 Qa wawayuśna kin woahtani wośnapi kin kage kta, qa tuwe yaska kte cin toaśape etanhan akahpe kiciye kta, qa he iyohakam wohuhnahyapi kin pate kta. $20 \mathrm{Qa}$ wawayuśna kin wohuhnahyapi kin qa minha kin wahna wośnapi akan hulinahye kta, hecen wawayuśna kin akahpe kiciye kta, unkan ska kta.

21 Tuka wahpanica on nape kin hena iyohi śni kinhan, akahipe kiciye kta e amnos wanjidan icu kta, wohdajuju wokosyapi on, nakun aguyapi mdu can wakśica ohnaka wanjidan wihdi icicahiyapi minha on, qa wihdi wiyatke ohnaka wanjidan.

22 Qa tin wakiyedan nonpa qaiś wakiyedan cinca nonpa, unman tukte nape kin iyohiye kta, wanji woahtani wośnapi on, qa unma wohulinahyapi on.
23 Qa yuskapi kta e anpetu iśahdogan hehan hena wawayuśna kin ekta hiyuye kta, Jehowa itokam itkokipapi wakeya tiyopa ekta.

24 Unkan wawayuśna kin amnos wohdajuju kin icu kta, qa wihdi wiyatke ohnaka kin, qa hena koze kta, wokosyapi Jehowa itokam.

$25 \mathrm{Qa}$ amnos wohdajuju kin pate kta, qa wawayuśna kin wohdajuju we etanhan icu kta, qa noge inkpa etapa akan, qa nape hunka etapa akan, qa sipa hunka etapa akan ikiciun kta.

26 Qa wawayuśna kin wihdi etanhạn wawayuśna napcoka catka kin oihdaśtan $k$ ta.

27 Qa wawayuśna kin napsukaza etapa on wihdi napcoka catka ohna un kin etanhan śakowin akihde ehpeye kta, Jehowa itokam.

28 Qa wawayuśna kin wihdi napcoka ohnaka un kin etanhan, tuwe yuskapi kte cin noge inkpa etapa akan ikiciun kta, qa napahunka etilpa akan, qa sipa hunka etapa akan tukten wohdajuju we ikiciun he akan.

29 Qa wihdi wawayuśna napcoka ohna okapte cin he tuwe yuskapi kte cin he pa akan ikiciun kta, Jehowa itokam akahipe kiciye kta heon etanhan.

30 Qa tin wakiyedan etanhan wanji qaiś wakiyedan cinca etanhan taku nape kin hiyohiya etanhan.

31 Taku nape kin hiyohiye cin wanji woahtani wośnapi kta, qa unman wohuhnahyapi kta, minha iyahna hecen wawayuśna kin tuwe yuskapi kte cin he on akalipe kiciye kta, Jehowa itokam.

32 'Tuwe leprosi makośice ececa, qa taku yuskapi kta iyececa 


\section{LEWI TOOPE.}

nape kin hiyohi śni woope kin hee.

33 Unkan Jehowa ie ca Mowis Aaron kici hewicakiya;

34 Kanan makoce tawayayapi lita e cicupi kin he en yakipi kinhan, qa makoce tawayayapi kin he ohna leprosi makośice tipi wan iyolipewakiye cinhan ;

35 Tipi tawa kin wawayuśna kin en hi kta, qa oyake qa heciye kta, tipi ohna makośice wan iyececa makitanin epca.

36 Unkan wawayuśna kin makośice wanyag en i śni itokam tipi kin yucokaka wicaśi kta, hecen taku tipi kin ohna owasin yuśapapi kte śni, hehan wawayuśna kin wanyake kta en i kta. 37 Qa makośice kin wanyake k ta, qa inyun tipi conkaśke ohna makuśice kin toto, qaiś śaśa śkopa, qa cuwi kin mahen iyaye tanin kinhan;

38 Wawayuśna kin tipi etanhan inanpe kta, tiyopa ekta, qa tipi kin anpetu śakowin natake kta.

39) Qa wawayuśna kin anpetu iśakowin ake cn hi kta, qa wanyake kta, unkan inyun tipi conkaśke en makośice kin sanpa iyaye cinhan,

40 Wawayuśna kin inyan tona makośice en un kin hena icu wicaśi kta, qa otonwe itankan tukten taku śapa elipeyapi heciya hena elipeyapi kta.

41 Qa ihdukśan tipi kin ti mahen paticapi kta, qa maka paticapi kin he otonwe itankan oyanke śapa wan ekta okadapi kta.

42 Qa inyan tokeca icupi kta, qa inyan qon hena ee kiya ehnakapi kta, qa maka tokeca icupi q̨a tipi apawintapi kta.

4.3 Unkan inyan ehpeyapi iyohakam, qa tipi paticapi iyoha- kam, qa apawintapi iyohakam makośice ake en hi, qa tipi ohna ahinanpe cinhan,

44. Wawayuśna kin hi kta, qa wanyake kta, unkan inyun makośice lkin tipi ohna iyaye cinhan, leprosi iyokiśin wicaye cin tipi ohna hee, he yaśape kta.

45 Unkan tipi kin yujujupi kta, qa tipi inyan kin, qa can kin qa maka kin owasin otonwe itankan ayapi kta, oyanke śapa wan ekta. $46 \mathrm{Qa}$ natakapi icunhan tuwc tipi kin he en i kinhan htayetu aiyahdeya śape kta.

47 Qa tuwe tipi kin ohna makan wanke cin he wokoyake hdujaja kta, qa tuwe tipi ohna wote cin he wokoyake hdujaja kta.

48 Tuka wawayuśna kin en i qa wanyaka, unkan inyun tipi apawintapi iyohakam makośice kin tipi en ahinaupe śni linhan, wawayuśna kin tipi yaska kita, makośice kin he wanna okizi nakaś.

$49 \mathrm{Qa}$ tipi woahtani wośnapi on zitkadan nonpa icu kta, nakun hanteśa can, qa tahin duta qa peji hota.

50 Qa zitkadan wan cape kta, makawakśica wan ohna, mini ni iwankam.

51 Qa hanteśa can kin, qa pcji hota kin, qa tahin duta kin, qa zitkadan niyake kin hena icu k ta, hena owasin zitkadan ktepi qon we en oputkan kta, qa mini ni un kin nakun en qa śakowin akihde tipi kin ekta elpeye kta.

52 Hecen tipi toaśape kin akahpe kiciye kta, zitkadan we kin. on, qa mini ni un kin on qa zitkadan niyake on, qa hante śa can on, qa tahinduta on.

53 Tuka zitkadan ni kin he otonwe itankan tinta ekta iyaye ye kta, hecen tipi kin akahpe kiciye kta, unkan ska kta. 
54. Leprosi makośice owasin on woope kin hee.

$55 \mathrm{Qa}$ leprosi wokoyake en, qa tipi en.

56 Qa kapo kin on, qa ceśpu kin on, qa wiyega on;

57 Leprosi woope kin hee, tohan śapa qa tohan ska onspewicakiye kta.

\section{WICOWOYAKE 15.}

1 Unkan Jehowa ie ça Mowis qa Aaron hewicakiya;

2 Israel cinca kin hecen ewicakiya po; Wicaśta tuwe kaśta celipi kin taku kaduze cinhan wolkaduze cin he on hee śapa.

3 Qa tokaduze cin en toihduśape cin dee kta. Cehpi kaduze cin kajo, qaiś cehpi kaduze cin anapte cinhan unman tukte kaśta hee śapa.

4 Tuwe wakaduze cin owinja tona akan iwanke cin hena owasin śape kta, qa taku kaśta akan iyotanke cin hee śape kta.

$5 \mathrm{Qa}$ tuwe towinja kin yutan kinhan wokoyake hdujaja kta, qa minin niwan kta, qa hitayetu kin hehanyan śape kta.

6 Qa wakaduze cin taku akan iyotanke cin tuwe he akan iyotanke cinhan htayetu aiyahdeya śape kta.

7 Unkan tuwe kaśta wakaduze cehpi kin yutan kinhan wokoyake hdujaja kta, qa minin niwan kta, qa ḣtayetu hehanyan śape kta.

8 Unkan wakaduze cin tuwe śape śni atagośe cinhan he wokoyake hdujaja kta, qa minin niwan kta, qa litayetu hehanyan śape kta.

9 Unkan śuktanka aqin tona wakaduze cin akan iyotanke cin hena owasin śape kta.

$10 \mathrm{Qa}$ taku kaśta he ihukuya yanke cin tuwe he yutan kinhan htayetu hehanyan śape kta, qa taku hececa tuwe qin kinhan wokoyake hdujaja kta, qa minin niwan kta, qa htayetu hehanyan śape kta.

$11 \mathrm{Qa}$ tuwe kaśta wakaduze cin nape minin hdujaja śni yutan kinhan, wokoyake hdujaja $k t a$, minin niwan kta, qa htayetu hehanyan śape kta.

12 Qa maka wakśica tona wakaduze cin yutan kin hena owasin kamdecapi kta, qa can wakśica kin hena owasin minin yujajapi kta.

13 Unkan wakaduze cin he tokaduze cin etanhan akisni kinhan, hehan ihduska kta e anpetu śakowin hdawa kta, qa wokoyake hdujaja kta, qa mini ni kin en niwan kta, qa hehan ska kta.

14 Unkan anpetu iśahdogan tin wakiyedan nonpa, qaiś wakiyedan cinca nonpa icu kte ca itkolipapi wakeya tiyopa elita Jehowa itokam hi kte ça hena wawayuśna qu kta.

15 Unkan wawayuśna kin hena wakiyuśna kta, wanji woahtani on qa unman wohuhnaliyapi on, hecen wawayuśna kin Jehowa itokam akahpe kiciye kta, tokaduze etanhan.

16 Unkan wicaśta wan wankapi su inanpe cinhan celipi kin owasin minin hdujaja kta, qa bitayetu hehanyan śape kta.

17 Unkan wokoyake, qa owinja tona wankapi su ahinhpaye cin hena owasin minin yujajapi kta, qa hitayetu hehanyan śape kta.

18 Unkan winolinca wan wicaśta wan wankapi su on kici wanke cinhan niwan kte ca htayetu hehanyan śape kta.

19 Unkan winolinca wan we kaduza, qa celipi olma kaduze cinhan, anpetu śakowin iśnana 
ti kta, qa taku kaśta icalitake cinhan owasin litayetu hehanyan śape kta.

$20 \mathrm{Qa}$ tohanyan iśna ti kin hehanyan taku akan wanke cin owasin śape kta, qa taku alkan iyotanke cin owasin śape kta.

21 Nakun tuwe taku akan iwanke cin he yutan kinhan wokoyake kin hdujaja kta, qa minin niwan kte, ca litayetu hehanyan śape kta.

22 Qa taku kaśta akan iyotanke cin he tuwe yutan kinhan wokoyake kin hdujaja kta, qa minin niwan kte ca htayetu hehanyan śape kta.

23 Nakun towinje qaiś taku akan iyotanke cin tuwe he yutan kinhan htayetu hehanyan śape kta.

24 Unkan wicaśta wan iśna ti kin icunlıan kici iwanke cinhan he anpetu śakowin śape. kta, qa owinja tona akan iwanke cin hena owasin śape kta.

25 Unkan winohinca wan iśna ti kta iyehantu śni anpetu ota we kaduze cinhan, qaiś iśna ti kta iyohakam wakaduze cinhan, tona can toaśape kaduze cin hena owasin śape kta, iśna ti kin anpetu kin iyececa.

26 Anpetu tona wakaduze cin owasin owinja akan iwanke cin hena owasin iśna ti towinja kin iyececa kta, qa taku kaśta akan iyotanke cin śape kta, iśna ti toaśape kin iyececa.

27 Qa tuwe kaśta hena yutan kinhan śape kta, wokoyake hdujaja qa minin niwan qa htayetu hehanyan śape kta.

28 Qa tokaduze cin etanhan akisni kinhan anpetu śakowin ihdawa kte ca iyohakam ska kta.

29 Qa anpetu iśahdogan tin wakiyedan nonpa, qaiś wakiyedan cinca nonpa icu kta, qa hena wa- wayuśna ekta hiyuye kta, itkokipapi wakeya tiyopa ekta.

30 Unkan wawayuśna kin wanji woahtani on wośna kta, qa unman woluhinahyapi on, hecen wawgyuśna kin wakaduze toaśape on akahpe kiciye kta, Jehowa itokam.

31 Hecen Israel cinca kin toaśapapi kin etanhan wicadukinunkan kta, qa wati kin hena cokaya un kin toaśapapi on yuśapapi kte śni, qa tapi.kte śni.

32 Tuwe wakaduze cin on, qa tuwe wankapi su inanpe cin on, ihduśape cin on woope kin hee.

33 Qa nakun tuwe iśna ti wayazanke cin on, qa tuwe wakaduze kaduza, wica qaiś winyan on, qa wicaśta winohinca śapa wan kici wanke cin he on.

\section{WICOWOYAKE 16.}

I Unkan Aaron cinca nonpa Jehowa itokam ikiyedan hipi qa țapi kin hena țapi kin iyohakam Jehowa Mowis kici wohdaka.

2 Qa Jehowa Mowis heciya; Nicinye Aaron tipi wakan cokaya ozanpi mahen wokajuju iha can wohnake akan un kin he ekta anpetu owasin hi kte śni, hecen te kte śni, keciya wo; mahpiya ohna wokajuju iha kin iwankam mihdutanin kta nakaś.

3 Kaken Aaron tipi wakan en hi kta, tatankadan pte wanunyanpi cinca wan woahtani on, qa tamdoka wan wohuhnahyapi on.

4 Minihuha mahenunpi wakan kin koyake kta, qa miniliuha onze oge cehpi akan un kta, qa minihuha ipiyake on ipihdake kta, qa miniliuha pa iyuskite kin palite kta, wokoyake wakan hena ee, heon cehpi minin hdujaja kta, qa hena koyake kta. 
5 Unkan Israel cinca omniciye etanhan tatokadan indoka nonpa icu kta, woahtani wośnapi on; qa tahinca wanunyanpi mdoka wanjidan wohuhnah̆yapi on.

6 Unkan Arron woahtani wośnapi tatanka tawa kahi kta, qa akahpe iciye kta, qa iye ti kin akahpe kiciye kta.

7 Qa tatokadan nonpa kin icu $k$ ta, qa Jehowa itokam ewicahde kta, itkokipapi wakeya tiyopa ekta.

8 Qa tatokadan nonpa on Aaron oeconna econ kta, oeconna on wanji Jehowa tawa lkta, qa oeconna on unman Azazel tawa kta.

$9 \mathrm{Qa}$ Aaron tatokadan oeconna eciyatanhan Jehowa tawa kin ahi kta, qa woahtani on wośna kage lkta.

$10 \mathrm{Qa}$ tatokadan oeconna eciyatanhan Azazel tawa kin niyake Jehowa itokam ehde kta, he on woakahpekiciye kta, qa liewoskantuya ekta iyaye ye lita.

11 Hehan Aaron woahtani tatanka tawa kin ahi kta, qa akahpe içiye kta, qa ti kin alrahpe kiciye kta, qa woahitani tatanka tawa kpate kta.

$12 \mathrm{Qa}$ oizinye wan petaga ojudan owayuśna etanhan Jehova itokapatanhan icu kta, qa nape sakim wizinyapi waśtemna bomdupi on ojudan, qa cokaya ozanpi mahen hiyuye kta.

13 Qa Jehowa itokam wizinyapi kin peta akada kta, unkan wizinyapi sota kin wokajuju iha kin woyaatanin akan yanke cin akahpe kta hecen te kte śni.

14 Qa tatankadan we kin icu kta qa nape apazo on wokajuju iha itokam wiyohiyanpata ektakiya ehpeye kta, hecen śakowin akihde nape apazo on we kin onge wokajuju iha itokam ehpeye kta.
15 Hehan woahitani tatokadan oyate tawapi kin pate kta, qa we kin cokaya ozanpi ti mahen hiyuye kta, qa token tatankadan we kin ecalkicon qon he iyecen we kin he ecalkicon kta, qa wokajuju iha kin akan qa wokajuju iha ektakiya ehpeye kta.

16 Hecen tipi wakan kin akahpe kiciye kta, Israel cinca toaśapapi etanhan qa wahtanipi etanlıan, śicaya ohanyanpi owasin on, qa itkokipapi walkeya hena om yanka toaśapapi ehna hecen ecakicon kta.

17 Unkan tipi wakan akalipe kiciye kta, en hi kin etanhan tankan iyaye cin hehanyan tuwedan itkokipapi wakeya ti mahen un kte śni; hecen akahpe ic̣iye lrta qa iye ti kin on, qa Israel omniciye owasin on akahpe wicakiciye kta.

18 Hehan owayuśna Jehowa itokam yanke cin elkta hinanpe kta, qa he akahpe kiciye kta qa tatankadan we etanhan icu kta, nakun tatokadan we etanhan, qa owayuśna he kin aihdukśan aiun kta.

$19 \mathrm{Qa}$ he ektaliya nape apazo on we kin śakowin akihde ehpeye kta; hecen Israel cinca toaśapapi etanhan he yuska kta, qa yuwakan kta.

20 Unkan tipi wakan kin, qa itkokipapi wakeya kin, qa owayuśna lkin hena akahpe kiciya yuśtan kinhan, hehan tatokadan niyake kahi kta.

$21 \mathrm{Qa}$ Aaron nape napin tatokadan niyake pa kin aputake kta, qa akan Israel cinca śicaya ohanyanpi kin owasin ohdake kta; nakun wokipajinpi owasin, token wahtanipi kin owasin, qa hena tatokadan pa akan ehnake kta; hehan wicaśta wan wiyeya un kin iyaye śi kta. 


\section{LEWI TOOPE.}

22 Unkan tatokadan qon śicaya ohanyanpi kin owasin makoce tuwedan oti śni ekta yuha kta. Unkan tatokadan hewoskan yuśke kta.

23 Unkan Aaron itkolkipapi wakeya ekta hi kta, qa tipi wakan ekta hi qehan minihuha wokoyake koyake ciqon hena hduśdoke kta, qa hen kihnake kta.

24 Qa oyanke wakan ohna cehpi kin hdujaja kta, hehan tawokoyake kohdake kta, qa tawohuhnahyapi, qa oyate tawohuhnahyapi kin wakiyuśna $\mathrm{kta}$, hecen akahpe ic̣iye kta, qa oyate kin akahpe wicakiciye kta.

25 Qa woahtani wośnapi waśin kin orvayuśna kin aizinye kta.

26 Unkan tuwe tatokadan Azazel tawa kin kiyuśke cin he wokoyake hdujaje ca minin niwe kta, qa ohakam owanka en hi kte.

27 Unkan woahtani tatankadan kin, qa woahtani tatokadan kin hena wepi kin akahpe kiciyapi kta e tipi wakan en hiyuyapi qon, owanka itankan ayapi kta, qa hapi kin, qa celipipi kin, qa cesdipi kin, peta on huhnahyapi kta.

28 Unkan tuwe hena hulinahye cin he wokoyake hdujaje ca minin niwe kta, qa ohakam owanka en hdi kta.

29 Qa wokage kin de owihanke wanin duhapi kta, wi iśakowin wi anpetu iwikcemna kin en ninagipi iyokisin niçiyapi kta ; wicaśta makoce tawapi qaiś oyate tokeca niyepi ehna ounye cin unman tukte kaśta wohtani takudan econ kte śni.

30 Anpetu kin he en akahpe niciyapi kta, niyuskapi kta heon etanhan, hecen wayahtanipi owasin etanhan Jehowa itokam niyuskapi kta.
31 Anpetu okilipapi wakan hinca he niciyukanpi kta, qa ninagipi iyokiśín niçiyapi kte, wokage owihanke wanica.

32 Unkan wawayuśna tuwe atkuku heekiya wawayuśna kta e sdayapi, qa nape kin ojunyapi kin he waakahpe kta, qa minihuha wokoyake kin, wokoyake wakan kin loyake kta.

33 Qa tipi wakan hinca akahpe kiciye kta, nakun itkokipapi wakeya kin, qa owayuśna kin akahpe kiciye kta, qa wawayuśnapi kin, qa oyate omniciye owasin akahpe wicakiciye kta.

34 Wokage owihanke wanica he duhapi kta, Israel cinca wahtanipi owasin etanhan akahpe wicakiciyapi kta, waniyetu otoiyohi wancadan. Unkan token Jehowa Mowis econ śi he iyecen econ.

\section{WICOWOYAKE 17.}

1 Unkan Jehowa ie ça Mowis heciya,

2 Ie ca Aaron qa iye cinca qa Israel cinca owasin hewicakiya wo; Taku Jehowa econ unśipi kin dee.

3 Wicaśta Israel ti kin etanhan tuwe kaśta tatanka wan pata, qaiś tahinca, qaiś tatokadan owanka ohna, qaiś owanka itankan pata;

$4 \mathrm{Qa}$ Jehowa ti itokam woahi Jehowa kahi kta e itkokipapi wakeya tiyopa ekta hiyuye śni kinhan, wicaśta kin he tin wicakte wan iyecen yawapi kta, wicaśta kin he weye cin heon oyate tawa wicehna tanhan he kaśpapi kta.

5 Hecen Iśrael cinca kin taku wośnapi, tinta akan wośnapi qon hena Jehowa ekta hiyuyapi kta, qa hena itkokipapi wakeya tiyo- 
pa ekta hiyuyapi kta, wawayuśna ekta, qa hena wopida wośnapi Jehowa wawicakiciyuśnapi kta.

6 Unkan wawayuśna kin we kin. Jehowa owayuśna tawa akan ehpe ye kta, itkokipapi wakeya tiyopa ekta, qa waśin kin izinye kta, woomna Jehowa iyokipi ye kta.

7 Hecen icimanna wośnapi tawapi wamanica wakiciyuśnapi kta, hena okiyapi iyecen wokage kin he owihanke wanica wicoicagepi yeye cin yuhapi kta.

8 Unkan hecen ewicayakiye kta, Wicaśta tuwe kaśta Israel ti kin etanhan, qaiś oyate tokeca niyepi ehna ounye cin etanhan, tuwe kaśta wohuhnahyapi wan, qaiś wośnapi wan wośna kinhan;

9 Qa Jehowa wakiyuśna kta e itkokipapi wakeya tiyopa ekta hiyuye śni kinhan, wicaśta kin he oyate tawa etanhan kaśpapi kta.

10 Qa wicaśta Israel ti etanhan, qaiś oyate tokeca niyepi ehna ounye cin etanhan, tuwe kaśta we onge yute cinhan, wicanagi we yute cin miite awehnake kta, qa oyate tawa etanhan wakaśpe kta.

11 Cehipi nagi kin we kin ohna yanka heon ninagipi akahpe niciyapi kta e owayuśna akan we kin cicupi, wicanagi akahpe kiciye cin we kin hee nakaś.

12 Heon Israel cinca kin hewicawakiya, Niyepi etankan tuwedan we yute kte śni, qa oyate tokeca niyepi ehna ounye etanhan tuwedan we yute $k$ te śni.

13 Qa wicaśta Israel cinca etanhan, qa oyate tokeca niyepi ehna ounye cin etanhan, tuwe kasta wotihni, qa woteca, qaiś wvahupakoza wanji yutapi kta iyececa kte kinhan, we kin papson kta, qa maka on akahpe kta.
14 Cehpi owasin nagi kin we kin hee, nagi kin he ohna yanka, heon Israel cinca kin hewicawakiya, Cehpi owasin etanhan we ongedan yutapi kte śni, cehpi owasin nagi kin we kin hee nakaś; tuwe he yute cinhan kaspapi lita.

15 Tuwe kaśta makoce en icaga, qaiś oyate tokeca taku wayazanka te, qaiś taku yațapi ongedan yute cinhan wokoyake hdujaja lita qa minin niwe kta, qa htayetu aiyahdeya śape kta.

16 Tuka wahdujaja śni qa niwe śni kinhan, tohan śica hduha kta.

\section{WICOWOYAKE 18.}

1 Unkan Jehowa ie ca Mowis heciya,

2 Israel cinca luecen kewicakiya wo, Jehowa taku wakan yadapi kin he miye;

3 Egupta makoce ohna ounyayapi qon, token olanyanpi kiṇ he iyecen oyahandapi kte śni, qa Kanan makoce ekta ye ciyapi ohanyanpi kin he iyecen oyahandapi kte śni, qa tawokagepi omayanipi kte śni.

4 Woyaco mitawa ecen econpo, qa wokage mitawa patanpo, qa hena omanipo, Jehowa taku wakan yadapi kin he miye.

5 Qa wokage mitawa kin yapatanpi kta, woyaco mitawa nakun; wicaśta tuwe hena iyecen econ kinhan hena on ni kta, Jehowa he miye.

6 Tuwedan iye cehpi takuye hinca tancodan yuzamni lita e ikiyedan hi kte śni, Jehowa he miye.

7 Niyate tancodan kin, qa nihun tancodan kin duzamni kte śni, ni hun kin hee heon tancodan kin duzamni kte śni.

8 Niyate tawicu tancodan kin 171 
duzamni kte śni, niyate tancodan kin hee.

9 Nitawinolitin niyate cunwintku, qaiś nihun cunwintku wohipapi qaiś nahimana yuzapi en tonpi unman tukte kaśta tancodan kin duzamni kte śni.

10 Nicinkśi cunwintku qa nicunkśi cunwintku tancodan kin duzamni kte śni, niye tanconikadan kin hee.

11 Niyate tawicu cunwintku niyate cinca kin tancodan kin duzamni kte śni, nitawinolitin kin hee, heon tancodan kin duzamni kte śni.

12 Niyate tawinolitin tancodan kin duzamni kte śni, niyate celipi kin hee.

13 Nihun tankaku tancodan kin duzamni kte śni, nihun celipi kin hee.

14 Niyate sunkaku tancodan lin duzamni kte śni, tawicu ikiye dan yai kte śni, nihun kin hee.

15 Nitakoś tancodan kin duzaṁni kte śni, nicinkśi tawicu kin hee, tancodan kin duzamni kte śni.

- 16 Nicinye tawicu, qa nisunka tawicu tancodan kin duzamni kte śni, nisunka tancodan kin hee.

17 Winohinca wan qa iye cunwintku tancodan kin duzamni k te śni, iye cinhintku cunwintku qaiś cunwintku cunwintku tancodan kin duzamni kta e duze kte śni, he wowicaśta śni, hena cehpi tawa hecapi nakaś.

18 Nakun winolinea wan cunku kici duze kte śni, hecannon kinhan tancodan duzamni, qa tohanyan ni kin iyokiśin yaye lita.

19 Unkan winohinca tohan toaśape on iśna ti kin hehanyan tancodan duzamni kta e ikiyedan yai kte śni.
20 Nalkun tuwe nikiyedan ti tawicu kin kici inonke kte śni. Hecannon kinhan kici nihduśape kta.

21 Nalkun nicinca etanhan wanjidan Molok ekta iyopta yaye kte śni, qa taku wakan yada caje kin duśape kte śni ; Jehowa he miye. 22 Nakun winyan kici wankapi kin he iyecen wica kici inonke kte śni, wowahte śni hee.

23 Nakun woteca taku kaśta kici inonke kte śni, hecannon kinhan kici nihduśape kta, nakun winohinca wan woteca kici kiyulie kta e he itokam wanke kte śni, woaśape hee.

24 Ihnuhan hena on nihduśapapi kin, oyate nitokapa tanhan ye wicawaye cin hena owasin on ihduśapapi.

25 Unkan makoce lkin lie śapa, heon tolian śice cin he akan hiyu waye kta, unkan makoce kin wicaśta en ounyanpi kin hena hdem wicaya.

26 Tuka niyepi wolrage mitawa, qa woyaco mitawa kin yapatanpi kta, qa wowalite śni kin hena ecannonpi kte śni, niye makoce tawayayapi qa wicaśta oyate tokeca niyepi ehna ounye cin unmanna.

27 Wicaśta niyepi itokam makoce $k$ in hen tipi kin wowahte śni kin hena owasin econpi, qa makoce yuśapapi.

28 Hecen makoce kin duśapapi kte śni, qa oyate nitokapa tanhan wicahdepe cin he iyecen nihdepapi kte śni.

29 Wicanagi tona wowahte śni kin hena wanjidan econpi kin, hena owasin taoyatepi etanhan kaśpapi kta.

30 Heon wopatan mitawa yapatanpi kta, qa wowahte śni wokage nitokam econpi kin hena ecannon pi kte śni, hecen hena 


\section{WICOWOYAKE 19.}

on nihduśapapi kte śni, Jehowa wakan yadapi kin he miye.

\section{WICOWOYAKE 19.}

1 Unkan Jehowa ie ca Mowis heciya,

2 Israel cinca omniciye ocowasin ieyanpaha hewicakiya wo, Jehowa wakan yadapi kin he miye, mawakan, heon wakan yaunpi kta.

3 Otoiyohi hunku kin yakinihan kta, nakun atkuku, qa anpetu okilipapi mitawa kin yapatanpi kta, Jehowa wakan yadapi kin he miye.

4 Ihnuhan takuśniśni ekta nihduhomnipi kin, qa ilınuhan taku wakan śdoyapi niçicagapi kin, Jehowa wakan yadapi kin he miye.

5 Unkan wopida wośnapi wan Jehowa wayakiduśnapi kte cinhan token iyokipi yayapi kte cin hecen wayakiduśnapi kte.

6 Anpetu en waduśnapi qa ihanlianna hena en he yatapi kta, tuka taku anpetu iyamni hehanyan oyapte cin, he petan hulinahyapi kta.

7 Unkan anpetu iyamni en onge yutapi kinhan he wośicamna kta, iyokipi kte śni.

$8 \mathrm{Qa}$ tuwe he yute cin he taku Jehowa on yuwakanpi kin yuśape cin heon tohan śice cin hduha kta, qa wicanagi kin he taoyate etanhan kaśpapi kta.

9 Unkan nitamakoce wolksapi bayalksapi eca nitamaga kalimin owasin bayakse kte śni; qa baksapi icunhan taku hinhpaye cin he mnayaye lkte śni.

$10 \mathrm{Qa}$ hastanhanka iyuwi nitawa akta duśpi kte śni, qa hastanhanka makata ihpayapi kin hena mna yaye kte śni, hena wicaśta onśika, qa oyate tokeca ekta elipe wicaya kiye kta, Jehowa wakanyadapi kin he miye. 11 Ihnuhan wamayanunpi, qa ihnuhan iyatonpi śni, qa ihnuhan otoiyohi yecihnayanpi kin.

$12 \mathrm{Qa}$ ihnuhan micaje itonśniyan cajedatapi qa taku wakan yada caje kin daśapapi kin, Jehowa he miye.

13 Ihnuhan nitakoda yakiduśe kin, qa taku tawa yaki kinhan. Ihnuhan tuwe tohtani opeyaton iyuwin tawa ihanhanna hehanyan niciyanke cin.

14 Tuwe nogetpa daśice kte śni, qa tuwe iśtagonge cin itokam taku iboto kta yecihnake kte śni, tuka taku wakan yada kin he ikoyape kta, Jehowa he miye.

15 Wayacopi en wopemni ecannon kte śni, wicaśta wahpanica ite kin ihayakta kte śni, qa wicaśta tanka ite kin yakinihan kte śni. Tuwe nikiyedan ti kin ecetuya daco kta.

16 Ihnuhan oyate nitawa tipi iyaza waiesa mayani kin, qa ihnuhan nitakoda we kin anayajin kin, Jehowa he miye.

17 Nihunka wanji nicante mahen śice yada kte śni ; tuwe niciti kin atayedan iyope yakiye kta, hecen iye on etanhan woahtani duha kte śni.

18 Toyeçon kte śni, qa oyate nitawa cincapi kin canniye wicayaya eśta ecadan enayakiye kta, qa token waśte nicida lin he iyecen tuwe nikiyedan ti kin waśte yadake kta.

19 Wokage mitawa yapatanpi kta. Wanunyanpi nitawa kin ocaje tokeca kici kiyuha yaki ye kte śni. Maga nitawa su ocaje nonpa icicahiya oju yakiye kte śni, qa wokoyake talinca hin qa hahonta onkagapi icicahiya kazuntapi kodake kte śni. 
20 Unkan wikośka wan wayaka heca wicaśta wan he wahoya tuka nahanhin wohpe śni qa kiyuśkapi śni, wicaśta tokeca wan winohinca kin he kici wanke cinhan napin wicakapsinpsintapi $k$ ta, wikośka kin ihduhe śni, heon te wicayapi kte śni.

21 Unkan wicaśta kin wohdajuju Jehowa kahi kte itkokipapi wakeya tiyopa ekta, tamdoka wan on hdajuju kta.

22 Unkan woahtani wahtani kin on wawayuśna kin akahpe kiciye kta wohdajuju tamdoka on, Jehowa itokam, hecen woahtani wahtani kin he kicicajujupi kta.

23 Unkan makoce kin en yakipi, qa waskuyeca can hu yahdepi kinhan, waskuyeca kin mdoketu yamni taku śapa iyecen elipe yayapi kta, śapa yadapi kta, tuwedan he yute kte śni.

24 Unkan mdoketu itopa kinhan waskuyeca kin owasin Jehowa yatanpi kta e wakan kta.

25 Tuka mdoketu izaptan kin waskuyeca kin yatapi kta, hecen waskuyeca ota icah niciyapi kta, Jehowa wakan yadapi kin he miye.

26 Taku we akan un kin yatapi kte śni. Wamduśka etanhan waadatapi kte śni, qa mahpiya etanhan waadatapi kte śni.

$27 \mathrm{~Pa}$ nitawapi ihdukśan ihanke kin yahduśdapi kte śni, qa putinhin nitawa ihanke kin ihang yakiyapi kte śni.

28 Tuwe nagi ye cin on nicicagopi kte śni nicehpipi en, qa akito niçiyapi kte śni; Jehowa he miye

29 Ihnuhan nicunkśi duśape ca wihomni win yakage cin, hecen makoce en witkowin ota kte śni, qa makoce kin wowicaśta śni on ojudan kte śni.
30 Okilipapi mitawa yapatanpi kta, qa tipi wakan mitawa yakinihanpi kta; Jehowa he miye.

31 Ihnuhan wapiyesa ekta nihduhomnipi, qa ihnuhan wakan içidapi kin awicayakite cin, hecannon kinhan hena on nihduśape kta; Jehowa wakan yada kin he miye.

32 Tuwe paska kin he itokam nayajin kta, qa wicahinca ite kin duonihan kta, qa taku wakan yada kin yakinihan kta, Jehowa he miye.

33 Unkan wicaśta oyate tokeca nici ounye cinhan he yakiduśe ḱte śni.

34 Tuwe oyate tokeca nici ounye cin he wicaśta makoce tawa yapi kin iyecen nicipi un kta, qa waśte niçida kin he iyecen waste yadake kta, oyate tokeca Egupta makoce en ounyayapi qon nakaś; Jehowa wakan yadapi kin he miye.

35. Ihnuhan wayacopi en taku owotanna śni ecannopi kin, ohanske iyutapi en, qa aspe yetonpi en, qa taku laśta ohna iyutapi en.

36 Taku on aspeyapi ecetu qa epah ecetu, qa hina ecetu duhapi kta; Jehowa wakanyadapi Egupta makoce etanhan hdinanpe niyanpi kin he miye.

37 Heon wokage mitawa owasin, qa woyaco mitawa owasin yapatanpi kta, qa hena ecanonpi kta; Jehowa he miye.

\section{WICOWOYAKE 20.}

1 Unkan Jehowa ie ça Mowis heciya ;

2 Hecen Israel cinca kin ewicayakiye kta, Wicaśta tuwe kaśta Israel cinca etanhan, qaiś oyate tokeca. Israel om ounye cin etanhan, iye cinca wan Mo- 
lok qu kinhan, awicakehan te yapi kta; makoce oyate kin inyan on ktepi kta.

3 Unkan miś wicaśta kin he ekta ite ye walsiye kta, qa cinhintku Molok qu kin heon oyate tawa etanhan wakaśpe kta, tipi wakan mitawa yuśape ça micaje wakan yuzamni heon etanhan.

4. Unkan makoce oyate kin wicaśta tuwe iye cinhintku Molok qu kin wanyakapi kte śni e iśtapi katinyan hduohmuzapi, qa hecen ktepi śni kinhan,

5 Miś wicaśta kin he miiśta awehnake kta, nakun tona taku wicaya, qa oyate tawapi etanhan wicawakaśpe $\mathrm{kta}$, iye qa tona Molok kici wankapi kta iye opeya okiyapi owasin.

6 Unkan wicanagi tuwe wapiyesapi, qa wicaśta wakan icidapi kin hena opeya okiyapi kta e ekta ihduhomni kinhan, miś miite wicanagi kin he ekta ye wakiye ca taoyate kin wicehna tanhan wakaśpe kta.

7 Unkan Jehowa wakan yadapi kin he miye, heon ihduwakan po, hecen niwakanpi kta.

8 Qa wokage mitawa yapatanpi kta, qa hecanonpi kta; Jelowa niyuwakanpi kin he miye.

9 Hecen tuwe kaśta atkuku qaiś hunku yaśice cinhan awicakehan te yapi kta, atkuku qaiś hunku yaśica, hecen we ic̣iya.

10 Unkan wicaśta tuwe wicaśta tokeca tawicu kici wawicihahapi econ, takodaku tawicu eśta kici wawicihahapi econ kinhan, awicakehan te yapi kța, wicaśta qa winohinca wawiciliahapi econpi napin.

11 Unkan wicaśta wan atkuku tawicu kici wanke cinhan atkuku tancodan kin yuzamni tokeśta napin țe wicayapi kta, iye we içiyapi.
12 Unkan wicaśta wan iye takośku kici wanke cinhan tokeśta sakim te wicayapi kta, wowicaśtaśni ohanyapi iye we içiyapi.

13 Unkan wicaśta wan winolinca kici wankapi kin he iyecen wica wan kici wanke cinhan, sakim wowahte śni econpi tokeśta țe wicayapi kta, iye we ic̣iyapi.

14 Unkan wicaśta wan winohinca wan hunku kici wicayuze cinhan wicaśtapiśniyan ohanyan, iye qa hena nakun, peta ohna huhnah wicayapi kta, hecen niyepi ehna wowicaśtaśni wanicc kta.

15 Unkan wicaśta wan woteca wan kici wanke cinhan tokeśta te kta qa woteca kin nakun yaiktepi kta.

16 Unkan winohinca wan wow teca wan kici wanke kta e ikiyedan.i kinhan, winohinca kin woteca kin kici wicayakte kta, tokeśta tapi kta, iye we içiyapi. 17 Unkan wicaśta wan iye tawinolitin yuze cinhan, atkuku cunwintku qaiś hunku cunwintku tancodan wanyake cinhan, unkan winyan kin wicaśta tancodan kin wanyake cinhan hee wowiśteca wicakaśpapi kta, oyate tawapi cincapi iśtapi itokam, tawinohin tancodan kin yuzamni woalitani kin hduha kta.

18 Unkan wicaśta wan winohinca iśna ti kici wanke cinhan, qa tancodan kin yuzamni kinhan, tawakoniya yutanin; unkan winyan kin we wakoniya kin hduzamni, heon napin oyate tawapi wicehna tanhan wicakaśpapi kta.

19 Nakun nihun tawinohtin, qa niyate tawinohtin tancodan kin duzamni kte śni, tuwe hecon kin he iye celipi kin hdutanin woa-* hitani hduhapi kta.

20 Unkan wicaśta wan iye tun- 
wincu kici wanke cinhan, tunwincu tancodan kin yuzamni, wahtanipi kin hduhapi kta, cinca nica ewicakiyapi tapi kta.

21 Unkan wicaśta wan iye sunkaku tawicu yuze cinhan, wowahte śni hee, sunkaku tancodan kin yuzamni cinca nica ewicakiyapi kta.

22 Hecen wokage mitawa owasin qa woyaco mitawa owasin yapatanpi kta, qa ecanonpi lita, unkan makoce ohna ounyayapi lita e ekta yeciyapi kin he inihdepapi kte śni.

23 Unkan oyate nitokapa tanhan iyaye wicawaye cin hena wokagepi omayanjpi kte śni. Iye hena owasin econpi heon hiti wicawahda.

24 Unkan heciciyapi, Maka ta-wapi kin tawayayapi kta, qa makoce kin he pteasanpi qa canhanpi tiktica kaduze cin he tawayayapi kta e cicupi kta; Jehowa wakanyadapi oyate tokeca etanhan yukinukan ciyapi kin he miye.

25 Heon woteca ska, qa tona śapapi yukinukan yayapi kta, nakun wahupakoza śapa qa ska dukinukanpi kta, ihnuhan ninagipi nihduwalitepi śni woteca on, qaiś taku kinyan on, qaiś taku kaśta maka asdohan on. Hena śapa yadapi k ta e niyepi etanhan mdukinukan.

26 Unkan tawa ciyapi k ta e miye oyate tokeca etanhan ciyukinukanpi, Jehowa mawakan heon miyeci wakanyan yaunpi lita.

$\dot{2} \dot{7}$ Unkan wicaśta wan, qaiś winolinca wan niyepi ehna wapiyesa, qaiś wicaśta wakan ic̣idake cinhan, awicakehan te - lita, tona hecapi inyan on wicayaktepi. kta, iye we içiyapi.

\section{WICOWOYAKE 21.}

1 Unkan Jehowa Mowis heciya, Aaron cinca wawayuśnapi kin hecen kewicayakiye kta; 'Tuwe nagi ye cin on ihduśapapi kte śni, oyate tawapi ehna.

2 Tuka iye celipi takuye hinca hunku on, qa atkuku on, qa cinhintku on, qa cunwintku on, qa sunkaku on,

$3 \mathrm{Qa}$ iye ta winohtin hinca wikośka tuwedan yuze śni, henana on ihduśape kta.

4 Winohinca oyate tawa ehna yuhe cin on ihduśape kte śni, qa ihduikceka kte śni.

5 Papi tuktedan paśda içicagapi kte śni, qa putinhinpi oise kin baśdapi kte śni qa cehpi ic̣icagopi kte śni.

6 Tuwe wakandapi on wakanyan unpi kta, qa tuwe wakandapi caje kin yuilkcekapi kte śni, Jehowa tapeta wośnapi, tuwe wakandapi taaguyapi kin kahipi nakaś, heon wakanyan unpi kta. 7 Witkowin qaiś wakikśanpi wan yuzapi kte śni, nakun winohinca hihnaku elipe kiye cin he yuzapi kte śni ; tuwe wakandapi on wakanpi nakaś.

8 Tuwe wakan yadapi taaguyapi kahipi heon wicaduwakan kta; 'Jehowa niyuwakanpi kin he miye mawakan heon hena niye om wakanpi kta.

9 Unkan wawayuśna cunwintku wan witkowin ohanyan on ihduśape cinhan, he atkuku yuśapa, petan huhnahyapi kta.

10 Unkan wawayuśrı sunka wicaye cin ehna itancan tawokoyake kin koyake kta e sdawicayapi wihdi kin pa akaśtanpi, qa nape ojun yapi kin he pa kin hduśda kte śni, qa wokoyake hdulidece kte śni.

11 Qa tuwedan nagiye tancan 
te cin ekta i kte śni, atkuku on, qa hunku on ihduśape kte śni.

12 Qa tipi wakan etanhan inanpe kte śni, qa taku wakanda toti wakan kin yuikceka kte śni. Tuwe wakanda wateśdake wihdi sdayapi tawa kin he akaśtanpi nakaś, Jehowa he miye.

13 Qa wikośka wan tuwedan kici wanke śni heca yuze kta.

14. Wiwazica qa tuwe ehpe kiyapi qa wakikśanpi, qa witkowin, tona hecapi etanhan winolinca yuze kte śni; tuka iye taoyate etanhan wikośka wan yuze kta.

$15 \mathrm{Qa}$ tona iye etanhan icagapi kin wica hduikceka kte śni taoyate ehna; Jehowa he yuwakan kin he miye.

16 Unkan Jehowa ie ca Mowis heciya,

17 Aaron hecen eciya wo. Nicinca wicoicage yeye cin tuwe ośteka, Taku wakanda taaguyapi kahi kta e ikiyedan hi kte śni.

18 Nakun tuwe kaśta ośteke cin he ikiyedan hi kte śni, wicaśta iśta gonge, qaiś huśte, qaiś poge mdaśka, qaiś huha unman tukte kaśta iyotan hanska,

19 Qaiś siha ksa, qaiś nape ksa, 20 Qaiś cankahu pawegapi, qaiś tanyan icage śni, qaiś iśta aska, qaiś tuwe ayaśpuya, qaiś hahdi, qaiś susu kapopapi.

21 Aaron wawayuśna cinca etanhan wicaśta ośteka tuwedan Jehowa peta wośnapi tawa kahi kta e ilkiyedan hi kte śni, he ośteka heon tuwe wakanda taaguyapi kin kahi kta e ikiyedan hi kte śni.

22 Tuwe wakan da taaguyapi wakan, qa nakun wakan hinca etanhan yute kta;

23 'Tuka cokaya ozanpi kin ekta i kte śni, ośteka nakaś, heon taku miciyuwakanpi kin he yuikceke kte śni ; Jehowa niyuwa- kanpi kin he miye heon etanhan.

24 Unkan Mowis Aaron qa iye cinca kin, qa Israel cinca owasin owicakiyaka.

\section{WICOWOYAKE 22.}

1 Unkan Jehowa ie ca Mowis heciya,

2 Aaron qa iye cinca kewicakiya wo, Israel cinca kin taku hduwakanpi kin on waktaya unpi kta, hecen taku miciyuwakanpi on micaje wakan kin yuikcekapi kte śni, Jehowa he miye.

3 Hecen ewicakiya wo, Nitoicagepi yeye cin nicincapi owasin etanhan wicaśta tuwe toaśape hduhe cin icunhan, taku yuwakanpi Israel cinca taku Jehowa kicihduwakanpi kin ikiyedan hi kinhan, wicanagi kin he miite itokapa tanhan kaśpapi kta ; Jehowa he miye.

4. Aaron cinca etanhan wicaśta leprosi ececa, qaiś wakaduze cin, tohanyan yuskapi śni taku yuwakanpi kin ongedan yute kte śni; nakun tuwe taku nagiye cin on śapa icahtake cinhan, qaiś wicaśta tuwe wanke cin su hiyuye cin.

5 Qaiś tuwe watutka on wicaśta śape kta wan icahtake cinhan, qaiś wicaśta wan taku hececa on śapa, taku kaśta on ihduśape kła icahtake cinhan,

6 Wicanagi tuwe taku hececa icahtake cin litayetu aiyahdeya śape kta, qa mini on cehpi hdujaja śni kinhan, taku yuwakanpi kin yute kte śni.

7 Qa wi kin tohan iyaye cinhan ska kia, qa he iyohakam taku yuwakanpi kin yute kta, aguyapi tawa kin hee nakaś.

8 Taku wayazanka te qa taku yapotapi kin yute kte śni, qa 


\section{LEWI TOOPE.}

hena on ihduśape kte śni ; Jehowa he miye.

9 Qa taku patan wicawaśi kin he patanpi kta, hecen hena on ihduikcelapi kte śni, qa woahtani hduhapi kte śni; Jehowa wicayuwakan kin he miye.

10 'Tuwe tokeca taku yuwakanpi kin yute kte śni, wawayuśna kici ti, qaiś wohtani opeton kin, he taku yuwakanpi kin yute kte śni.

11 Tuka wawayuśna kin wicanagi wan opeton, mazaska tawa on opetonpi kin he etanhan yute kta; nakun tuwe ti ohnso tonpi kin he, hena aguyapi tawa etanhan yutapi kta.

12 Unkan wawayuśna cunwintku wan wicaśta tokeca he yuze cinhan, taku ikce qupi yuwakanpi kin etanhan yute kte śni.

13 Tuka wawayuśna cunwintku wan wiwazica, qaiś elipe kiyapi kinhan, qa cinca nice ca atkuku ti en hdi kinhan, atkuku talaguyapi etanhan yute kta, wikośka qon iyececa, tuka tuwe tokeca hetanhan yute kte śni.

14 Unkan wicaśta wan taku yuwakanpi onuniyan yute cinhan, he izaptan aokijuye kta, qa taku yuwakanpi kici wawayuśna kin qu kta.

15 Hecen taku Israel cinca yuwakanpi taku Jehowa ektakiya yuwintapi kin hena yuikcekapi lite śni.

16 Tuka taku yuwakanpi kin hena yutapi kinhan, oicazopi woahtani kin he wicakiciyuhapi kta; Jehowa wicayuwakan kin he miye, nakaś.

17 Unkan Jehowa ie ca Mowis heciya,

18 Aaron qa iye cinca, qa Israel cinca owasin hecen ewicakiya wo, Wicaśta tuwe kaśta Israel tiohnaka etanhan, qaiś oyate to- keca etanlian, Israel om ounye cin woahi wan makahi kte cinhan, taku ic̣iconzapi on, qaiś taku ikce qupi kin owasin on, wohulinahyapi wan makahi kte cinhan;

19 Iyokipi yayapi kta heon mdoka zanika, pte etanhan qaiś tahinca etanhan, qaiś tatokadan etanhan kahi kta.

20 Tona ośteka wanjidan yakahi kte śni, hena on iyokipi yayapi kte śni.

21 Unkan wicaśta wan taku iciconze hduecetu kta e wopida wośnapi wan Jehowa kahi kte cinhan, qaiś taku ikceya qu kta, pte wanunyanpi, qaiś tahinca etanhan iyokipi ye kta e he zanike kta, tuktedan ośteke kte śni.

22 Iśta gonga, qaiś huha wan kaśujapi, qaiś lkaśpapi, qaiś ceśpu yukan, qaiś ayaśpuya, qaiś hidihdi, taku hececa Jehowa yakahipi kte śni qa hena etanhan peta wośnapi wan owayuśna akan Jehowa yaqupi kte śni.

23 Tatanka qa tahinca huha iyakicuya, qaiś huha aokpani kin he ikceya yaqu kta, tuka woyakapi wośnapi on iyokipi ye kte śni.

24 Nakun taku kaśujapi, qa kawegapi, qa nahdecapi, qa baśpapi kin Jehowa yakahipi kte śni, qa nitamakocepi ohna taku hececa ecannonpi kte śni.

25 Nakun taku hececa owasin oyate tokeca cinca nape etanhan taku wakanyadapi taaguyapi kin yakahipi kte śni, taku on howinpi hena mahen un nakaś, ośtekapi, niyepi on iyolkipi yapi kte śni.

26 Unkan Jehowa ie ca Mowis heciya,

27 Tatanka qaiś tahinca qaiś tatokadan wanji tonpi ehantanhan śakowin can hunku kici un kta, 
tuka anpetu iśahdogan qa ohakam Jehowa wakiyuśnapi woahi on iyokipi kta.

28 Unkan pte qaiś tawiyedan wan iye cinca kici anpetu wanjidan en yapatapi kte śni.

29 Unkan wopida wośnapi wan Jehowa wayakiduśnapi kinhan, iyokipi yayapi kta e wayakiduśnapi kta.

30 Anpetu kin he en temyayapi $\mathrm{kta}$, ihanhanna hehanyan ongedan oyahdaptapi kte śni ; Jehowa he miye.

31 Qa woahope mitawa yapatanpi kta, qa ecen ecanonpi kta, Jehowa he miye.

32 Qa micaje wakan kin duikcekapi kte śni, tuka Israel cinca wicehna mayawakanpi kta, Jehowa niyuwakanpi kin he miye.

33 Wakanmayadapi kta heon Egupta niakoce etanhan hdinanpe ciyapi, Jehowa he miye.

\section{WICOWOYAKE 23.}

1 Unkan Jehowa ie ca Mowis heciya,

2 Israel cinca kin liecen ewicakiya wo, Jehowa omniciye tawa ieyanyapahapi kta, omniciye wakan ieyanpahapi mitawa kin dena ee.

3 Anpetu śakpe wohtani ecannon kta, tuka anpetu iśakowin kin he anpetu okihpapi wakan, ieyanpahapi wakan, ounyayapi owasin ohna, wohtani takudan econpi kte śni; Jehowa taokihpapi kin hee nakaś.

4 Jehowa ta omniciye ieyanpahapi wakan iyehantu eca ieyanyapahapi kta dena ee.

$5 \mathrm{Wi}$ tokaheya anpetu iake topa en hitayetu otahedan Jehowa toacakśin kin hee.

6 Qa wi kin he anpetu iake zaptan en aguyapi napohyapi śni wohanpi Jehowa tawa, anpetu śakowin aguyapi napohyapi śni yatapi kta.

7 Anpetu tokaheya ieyanpahapi wakan duhapi kta, wayaka wolitani ecannonpi kte śni.

$8 \mathrm{Qa}$ anpetu śakowin peta wośnapi Jehowa yakahipi kta, anpetu iśakowin ieyanpahapi wakan kta, wayaka wohtani takudan ecannonpi kte śni,

9 Unkan Jehowa ie ca Mowis heciya,

10 Israel cinca kin hecen ewicakiya wo, Makoce cicupi kin he ekta yaipi, qa woksapi bayaksapi kinhan, tokaheya bayaksapi etanhan opahte wan wawayuśna ekta hiyuyayapi kta.

11 Unkan iyokipi yayapi kta e Jehowa itokam wopahte kin he koze kta; anpetu okilipapi ihanhanna hehan wawayuśna kin he koze kta.

12 Unkan anpetu wopahte yakozapi kin en, amnos wan zanika, waniyetu wanjidan, wohuhnaliyapi Jehowa yecicagapi kta.

13 Unkan minha tawa aguyapi mdu can wakśica ohnaka nonpa wihdi icicahiyapi, peta wośnapi waśtemna Jehowa iyokipi ye kta, qa woakaśtan kin mini śa wiyatke ohnaka nonpa.

14 Tuwe wakan yadapi kin ekta woahi hiyuyapi śni anpetu kin hehanyan, aguyapi, qa wamnaheza ceunpapi, qa paśdayapi unmana yatapi kte śni; ounyayapi owasin ohna nitoicagepi yeye cin wokage kin he owihanke wanica.

15 Unkan wopahte kosyapi yakahipi anpetu wakan ihanhanna etanhan anpetu wakan śakowin dawapi kta, anpetu wakan ocowasin dawapi kta.

16 Anpetu wakan iśakowin ihanhanna etu dawapi kta, anpetu wikcemna zaptan hehanyan; 


\section{LEWI TOOPE.}

qa minha teca wan Jehowa yakahi kta.

17 Ounyayapi etanhan aguyapi kozapi nonpa, aguyapi mdu can wakśica ohnaka nonpa, napolyapi śpanyanpi, taku tokaheya suton kin Jehowa ekta hiyuyayapi kta.

18 Qa aguyapi kici amnos śakowin zanika waniyetu wanjidun, qa tatankadan pte wanuyanpi cinca wanjidan, qa tamdoka nonpa yakahipi kta, Jehowa liuhnalikiciyapi kta, qa minlia tawapi, qa woakaśtan tawapi, peta wośnapi waśtemna Jehọa iyokipi.

19 Hehan tatokadan mdoka wan woahtani wośnapi on, qa amnos nonpa waniyetu wanjidan wopida wośnapi on, wayakiduśnapi kta.

20 Unkan wawayuśna kin hena koze kta aguyapi tokaheya suton lin ahna, nakun amnos nonpa qon, Jehowa itokam wokosyspi wan, hena Jehowa on wakanpi kta, wawayuśna tawa kta.

21. Unkan anpetu kin he en niyepi on omniciye wakan ieyanpahapi kta, wayaka wohtani takudan ecannonpi kte śni, ounyayapi owasin ohna nitoicagepi yeye cin, he wokage owihanke wanice kta.

22 Unkan makoce nitawapi woksapi kin bayaksapi kinhan, maga nitawapi kahmin owasin bayakse kte śni, qa woksapi nitawa taku okapte cin mnayaye kte śni, wicaśta onśika, qa oyate tokeca on he elipe wicayeciye $\mathrm{kta}$; Jehowa wakan yadapi kin he mive.

23 Unkan Jehowa ie ça Mowis heciya,

24 Israel cinca kin hecen ewicakiya wo, Wi iśakowin anpetu tokaheya kin wookilipe duhapi 180 kta, mazayahotonpi on kiksuyapi, ieyanpahapi wakan,

25 Wayaka wohtani takudan ecannonpi kte śni, qa peta wośnapi wan Jehowa yakahipi kta. 26 Unkan Jehowa ie ca Mowis heciya,

27 Awicakehan wi iśakowin anpetu iwikcemna kin waakahpapi anpetu kin hee, eyanpahapi wakan duhapi kta, qa ninagipi iyokiśin niçiyapi lita, qa peta wośnapi wan Jehowa yakahipi kta.

28 Anpetu kin lie en wohtani takudan ecannonpi kte śni, waakahpapi anpetu kin hee. Jehowa wakan yadapi itokam akahipe niçiyapi lita e hee nakaś.

29 Wicanagi tuwe kaśta anpetu kin he en iyoliśin içiye śni kinhan taoyate etanhan kaśpapi kta. 30 Unkan wicanagi tona anpetu kin he en wohtani econpi wicanagi kin hena owasin taoyatepi etanhan ihang wicawaye kta.

31 Ounyayapi owasin ohna wohtani takudan ecannonpi kte śni, nitoicagepi yeyecin wokage owihanke wanica.

32 Anpetu okilipapi wakan he duhapi kta, qa ninagipi iyokiśin niçiyapi $k t a$, wi anpetu inapcinwanka htayetu etanhan ake litayetu kte cin hehanyan wookilipe nitawapi kin okihpe yayapi kta. 33 Unkan Jehowa ie ça Mowis heciya,

34 Israel cinca kin hecen ewicakiya wo, Wi iśakowin anpetu iake zaptan kin en ohanzi hdepi wohanpi kin hee, anpetu śakowin Jehowa tawa.

35 Anpetu tokaheya ieyanpahapi wakan, he en wayaka wohtani takudan ecannonpi kte śni.

36 Anpetu śakowin peta wośnapi Jehowa yakahipi kta, anpetu iśahdogan eyanpahapi wakan du- 


\section{WICOWOYAKE 24.}

hapi kta, qa peta wośnapi Jehowa yakahipi kta, witaya iheyapi wan hee, wayaka wohtani ecannonpi kte śni.

37 Jehowa taomniciye ieyanyapahapi kta anpetu kin hena ee, eyanpahapi wakan on wohuhnahyapi, qa minha wośnapi, qa woakaśtanpi Jehowa yakahipi kta, anpetu iyohi taku tawa kin iy ececa.

38 Nakun Jehowa taokihpapi, qa nakun taku yaqupi, qa nakun taku niçiconzapi kin, nakun taku ikce yaqupi kin Jehowa yaqupi kin owasin isanpa.

39 A wicakehan wi iśakowin anpetu iakezaptan makoce taku aicage cin mnayayapi linhan, anpetu śakowin Jehowa tawohanpi oyakihanpi $\mathrm{kta}$, anpetu tokaheya wookihpe, qa anpetu iśahdogan wookilipe kta.

40 Unkan anpetu tokaheya can wiciyokipi waskuyeca iyacupi lita, tamar can adetka, qa canhahaka adetka, qa walkpa cohwanjica nakun, qa anpetu śakowin Jehowa wakan yadapi kin itokam widuśkinpi kta.

41 Qa wohanpi Jehowa oyakicihanpi kta, anpetu sakowin ptanyetu otoiyohi nitoicagepi yeye cin, wokage owihanke wanica, wi iśakowin en he woyakihanpi kta.

42 Anpetu śakowin can adetka ohanzi hdepi oyatipi kta, tona Israel tamakoce en tonpi kin hena owasin can adetka ohanzi hdepi ohna tipi kta.

43 Hecen Israel cinca Egupta makoce etanhan hdinanpe wicawaye cehan ohanzi hdepi ohna tjpi wicawaya, nitoicagepi sdonyapi kta; Jehowa wakan yadapi kin he miye.

44 Unkan Mowis Jehowa wokicihanpi toketu Israel cinca owicaliyaka.

\section{WICOWOYAKE 24.}

1 Unkan Jehowa ie ça Mowis heciya ;

2 Petijanjan on can ihdi ecedan bopanpi nicahipi kta, Israel cinca icu wicaśi wo, hecen ide kin ohinniyan wankan ye kta.

3 Woyaatanin cokaya ozanpi itankan, itkokipapi wakeya ohna, htayetu etanhan ihanhanna kta eca Aaron he ideye kta, ohinniyan nitoicagepi yeye cin wokage owihanke wanica.

4 Petijanjan ihupa ecedan akan petijanjan ideye kta, Jehowa itokam ohinniyan.

5 Unkan aguyapi mdu iyacu kta, qa he span yaye kta, aguyapi wikcemna sanpa nonpa, aguyapi otoiyohi ohna can walkśica ohnaka nonpa kta.

6 Unkan hena Jehowa itokam wahna wotapi ecedan alian eyahnake kta, ocankuye nonpa ocankuye iyohi ohna śakpe.

7 Unkan ocankuye akan canśin ska kohdi eyahnake kta, unkan he aguyapi on wokiksuye kta, peta on Jehowa wakiciyuśnapi lita.

8 Anpetu okilipapi otoiyohi Israel cinca etanhan hena Jehowa itokam ehnakapi kta, ohinniyan wicotakuye owihanke wanica.

$9 \mathrm{Qa}$ Aaron qa iye cinca kin he tawapi kta, qa he yutapi kta, oyauke wakan ohna, Jehowa peta wośnapi tawa etanhan, iye on he wakan linca nakaś; wokage owihanke wanica.

10 Unkan Israel winallinca wan cinhintku, qa he Egupta wicaśta wan cinhintku, Israel cinca wicehna inanpa, qa Israel winohinca cinhintku, qa Israel wicaśta wan owanka ohna kicizapi.

11 Qa Israel winohinca cinhintru caje kin atayedan je ca ya181 


\section{LEWI TOOPE.}

s̊ica, unkan Mowis ekta hiyuyapi he bunku Selomit eciyapi; Dibri cunwintku, Dan wicoun etanhan.

12 Unkan kaśka hnakapi Jehowa i kin etanhan owicakiyakapi kte cin hehanyan.

13 Unkan Jehowa ie ca Mowis heciya,

14 Wicaśta yaśice cin he owanka etanhan inanpe ya wo, unkan tona nahonpi kin owasin pa kin nape aputakapi kta, unkan omniciye owasin inyan on ktepi kta.

i5 Unkan Israel cinca kin hecen ewicayakiye kta, wicaśta tuwe kaśta taku iye wakanda yaśice cinhan, iye walitani kin hduha lita.

16 Jehowa caje kin yaśice cinhan tokeśta te yapi kta, omniciye owasin inyan on aohduteyapi kta, wicaśta oyate tokeca, qaiś makoce en tonpi caje kin yaśice cinhan te kta.

17 Unkan tuwe kaśta wicaśta nagi kaśtake cinhan he ktepi kta.

18 Unkan tuwe woteca wan nagi kaśtake cinhan hdajuju kta, nagi on nagi.

19 Unkan wicaśta wan tuwe iye ikiyedan ti kin kiunniye cinhan token econ kin he iyecen ecakiconpi kta.

20 Kawegapi on kawegapi kta, iśta on iśta, hi on hi token wicaśta wan kiunniye cin he iyecen kiunniyanpi kta.

21 Unkan tuwe woteca wan kte kinhan he on hdajuju kta, qa tuwe wicaśta wan kte kin he ktepi kta.

22 Wicaśta oyate tokeca on, qa tuwe makoce en tonpi on, woyaco wanjidan duhapi kta; Jehowa wakan yadapi kin he miye.

23 Unkan Mowis Israel cinca kin ewicakiyaka, unkan wicaśta wayaślce cin he owanka itankan 182 inanpe yapi, qa inyan on ktepi hecen Israel token Jehowa Mowis econ śi kin he iyecen econpi.

\section{WICOWOYAKE 25.}

1 Unkan Jehowa Sina he kin akan ie ca Mowis heciya,

2 Israel cinca kin ekta i qa hecen ewicakiya wo. Makoce wan cicupi kin he ekta yaipi kinhan, makoce kin wookilipapi wan Jehowa on oziiciye kta.

3 Waniyetu śakpe maga nitawa oyaju kta, qa waniyetu śakpe haștanhanka iyuwi nitawa adetka bayaśpe kta, qa taku aicage cin mnayaye kta ;

4 Tuka waniyetu iśakowin kin he makoce tookihpe wakan kta, Jehowa tookilipe, maga nitawa oyaju kte śni, qa hastanhanka iyuwi nitawa adetka kin bayaśpe kte śni.

5 Bayakse ciqon etanhan taku icage cin he bayakse kte śni, qa hastanhanka iyuwi nitawa adetka baśpapi śni hastanhanka aicage cin duśpi k te śni, makoce kin tookilipe omaka kin hee nakaś.

6 Qa waniyetu okihpapi kin he woyute yayapi kta, niye, qa nitahokśidan qa nitawicinyanna, qa 'tuwe tohtani opeyaton, qa tona oyate tokeca nici ounyanpi kin.

7 Qa woteca nitawa, qa wamanica makoce nitawa en unpi taku icage cin owasin woyute yapi kta.

8 Unkan waniyetu okihpapi śakowin yahdawa kta, śakowin akihde waniyetu śakowin, hecen waniyetu okihpapi śakowin, waniyetu wikcemna tom sanpa napcinwanka anpetu iyenakeca kta.

9 Hehan wi iśakowin wi anpetu iwikcemna mazayahotonpi 
hoton yakiye kta, woakahpapi anpetu en, makoce nitawa owancaya mazayahotonpi hoton yayapi kta.

$10 \mathrm{Qa}$ waniyetu iwikcemna zaptan waniyetu kin he duwakanpi kta, qa makoce ohna tona en ounyanpi owasin iye ihduhapi $\mathrm{kta}$, eyanpaha yayapi kta, he Jubele yayapi kta, qa yahdapi kta, otoiyohi tamaga ekta, qa takuwicaye cin ekta.

$11 \mathrm{Qa}$ waniyetu iwikcemna zaptan waniyetu kin he Jubele yayapi kta, woyajupi kte śni, qa taku baksapi qon etanhan icage cin he bayaksapi kte śni, qa hastanhanka iyuwi baśpapi śni taku aicage cin duśpi kte śni.

12 Jubele hee nakaś, wakan yayapi kta, maka kicanyanpi śni, taku aicage cin he yatapi kta.

13 Jubele waniyetu kinhan yahdapi kta, otoiyohi iye tamaga ekta.

14 Unkan tuwe nikiyedan ti kin ekta taku wiyopeyapi iyope yakiye cinhan, qaiś tuwe nikiyedan ti nape etanhan taku opeyaton kinhan, ihnuhan nihunkawanji duhukuye cinhan.

15 Jubele etanhan waniyetu yawapi kin eciyatanhan opeyaton kta, tuwe nikiyedan ti etanhan, qa waniyetu taku aicage kte cin iyenaka eciyatanhan iyopeniciye kta.

16 Waniyetu ota on iyuwin kin duota kta, qa waniyetu tonana on iyuwin kin duoptetu kta, nalraś tona taku ajcage cin yawapi eciyatanhan he iyopeye nicaton.

17 Unkan wicaśta wan tuwe ikiyedan ti kin yakiduśe kte śni, tuka tuwe wakan yada kin koyakipe kta; Jehowa wakan yadapi kin he miye nakaś.

18 Heon wokàge mitawa kin ecen ecanonpi kta, qa mitawoyaco yapatanpi kta, qa ecen ecanonpi kta, hecen okope wanin makoce kin ounyayapi kta.

19 Hecen maka kin waskuyeca tawa niçu kte ca imnahan wayatapi kta, qa okope wanin he ounyayapi $k$ ta.

20 Unkan, Inyun unkojupi kte śni, qa taku icahunkiciyapi mnaunkiyapi kte śni, taku untapi kta hwo, ehapi kinhan.

21 Woyawaśte mitawa cicupi kta, waniyetu iśakpe en, hecen waniyetu yamni on woicahye icaliniciyapi kta.

22 Unkan waniyetu iśahdogan woyajupi kta, qa woicaliye tannina etanhan yatapi kta, waniyetu inapcinwanka kin hehanyan taku aicage cin mnayanpi kin hehanyan tannina yatapi kta.

23 Makoce kin owihanke wanica iyope yapi kte śni, makoce kin he mitawa nakaś, wicaśta ihdakapi, qa mici tipi henicapi heon etanhan.

24 Unkan makoce tawayayapi kin owasin en wopekitonpi yaqupi kta.

25 Nihunkavianji wahpanice ça makoce tawa etanhan iyope kiye cinhan taku wicaya wan opekiciton cin kinhan, taku hunkawanjitku wiyopekiye cin he opekiciton kta.

26 Unkan wicaśta wan tuwedan opekiciton śni kinhan, qa iye nape waśaka on taku iyohiya iy eye cinhan, iye opekiton kta.

27 Qa wiyopekiye ciqon ehantanhan omaka yawa k ta, qa taku iyaye tuwe he opeton qu kte ça maga tawa kin en hdi kta.

28 Tuka taku kicupi kta iyohiya nape kin iyeye śni kinhan, taku iyopekiye cin tuwe he opeton kin nape obna yanke kta, Jubele waniyetu kin hehantu kta; un183 


\section{LEWI TOOPE.}

kan Jubele waniyetu hehan inape kta; unkan maga tarva qon en hdi kta.

29 Unkan wicaśta wan otonwe aconkaśkayapi ohna tipi ounyanpi wan iyope kiye cin he opekiton kinhan iyopekiyapi ehantanhan waniyetu wanjidan mdoketu iyahna anpetu kin hehanyan wopekitonpi kta.

30 Tuka waniyetu mdoketu iyahna ihunniyan hehanyan opekitonpi śni kinhan, tipi otonwe conkaśkayapi ohna tuwe he opeton, qa toicage yeye cin owihanke wanica tawa kta, Jubele en inanpe kte śni.

31 Tuka tipi otonwe cistinna tona ihdukśan conkaśke wanice cin ohna hena owasin makoce maga kin iyecen yawapi $\mathrm{kta}$, hena opekitonpi kta, qa Jubele en inanpe kta.

32 Unkan Lewi otonwe tawapi tipi otonwe tawayapi, Lewi kin tohantu kaśta opekitonpi kta.

33 Unkan tuwe Lewi kin etanhan opeton kinhan, tipi opeton etanhan, qa otonwe tawaye cin etanhan Jubele en inanpe kta, Lewi otonwe tawapi tipi kin hena taku tawayapi ee ; Israel cinca ehna nakaś.

34 Tuka otonwe tawapi ihdukśan tinta maga kin hena wiyopeyapi kte śni, he tawayapi owihanke wanica nakaś.

35 Unkan nihunkawanji nici wahpanica aye ca nape hdugate cinhan, he waśagyakiye kta, he oyate tokeca eśta, en ounye cinhan, hecen nici ni kta.

36 Ihnuhan he etanhan woyahitake qa oicage iyacu kinhan, tuwe wakan yada kin ikoyape kta, hecen nihunkawanji ni kta niyepi kici.

37 Mazaska nitawa woyahtake on yaqu kte śni, qa woyute nitawa oicage cin on yaqu kte śni.

38 Jehowa wakanyadapi Kanan makoce niçupi kta e Egupta makoce etanhan hdinanpe niyanpi kin he miye, hecen taku wakan yadapi kin he miye kta.

39 Nihunkawanji wahpanica, qa niye en wiyopeiciye cinhan, wayaka wohtani on wowidag yakiye kte śni.

40 Wicaśta wohtani opekitonpi iyecece kta. Tuwe makoce ounye cin iyecen nici un kta, Jubele omaka kin hehanyan nici hitani kta.

41 Hehan niye etanhan inanpe kta, qa iye cinca nakun opapi kta, qa takuwicaye cin ekta hde kta, qa atewicaye cin makoce tawapi kin en hde kta.

42 Mitaokiye Egupta etanhan hdinanipe wicawaye cin hena eepi, heon wayaka iyope wicayapi kin he iyecen iyope wicayapi kte śni.

43 Wacinkoya wowidagwicayaye kte śni, tuka tuwe wakan yada kin koyakipe kta.

44 Oyate nihdukśan yakonpi kin hena etanhan hokśidan wicinyanna koya duhe kta, ookiye wica qa winyan hena etanhan opeyaton kta.

45 Nakun wicaśta oyate tokeca niye om ounyanpi kin cincapi kin opewicayaton kta, qa takuwicayapi nitamakoce en tonpi, qa niyepi ehna unpi kin hena etanhan, qa hena tawawicayayapi kta.

$46 \mathrm{Qa}$ nicincapi niyepi iyohakam icagapi kin hena tawawicayapi kta e iwicayacupi kta, owihanke wanin hena wowidag wicayayapi $\mathrm{kta}$, tuka nihunkawanjipi kin Israel cinca kin wacinkoya wowidag wicayeciyapi kte śni. 
47 Unkan tuwe oyate tokeca, qa nici ounye cin wijice cinhan, qa nihunkawanji niciwahpanica wicaśta oyate tokeca nici ounye cin ekta wiyopeiçye cinhan, qaiś oyate tokeca takuwicaye cin wan ekta.

48 Wiyopeyapi iyohakam wopekitonpi kta; iye hunkawanjitku wan opekiton kta.

49 Dekśitku qaiś tahanśitku he opekiton kta, qaiś iye cehpi etanhan tuwe takuye cin he opekiton kta, qaiś iye nape waśake cinhan, opeiciton kta.

50 Unkan tuwe he opeton kici waniyetu yawa kta iyopeiçiye cin ehantanhan, Jubele waniyetu kin iyehantu kta, qa mazaska iyopeiceiye cin etanhan waniyetu yawapi kta, wicaśta wohtani opetonpi kin he iyecen anpetu yawapi kta.

51 Nahanhin waniyetu ota kinhan hena eciyatanhan mazaska on opetonpi qon iyecen kicu kta.

52 Qa waniyetu tonana okaptapi Jubele iyehantu kinhan, waniyetu iyenakeca yawapi eciyatanhan wopekitonpi kicu kta.

53 Tuwe wohtani waniyetu wanjidan opetonpi kin he iyecen, he kici yanke kta, niiśta itokam wacinkoya wowindag ye kte śni.

$54 \mathrm{Qa}$ hena on opekitonpi śni kinhan Jubele waniyetu kinhan inanpé kta, iye qa cinca kin opapi kta.

55 Israel cinca kin ookiye wicamduha nakaś, mita ookiye Egupta makoce etanhan hdinanpewicawaye cin hena eepi; Jehowa Wakan yadapi kin he miye.

WICOWOYAKE 26.

1 Wakan taku śniśni, qa wakagapi pagopi niçicagapi kte śni, qa wakagapi bosdata enicihdepi kte śni, qa inyan śayapi wan itokam patujapi kta e nitamakoce ohna duhapi kte śni, Jehowa wakan yadapi kin he miye nakaś.

2 Okihpapi mitawa kin yapatanpi kta, qa tipi wakan mitawa yakinihanpi kta.

3 Wokage mitawa omayanipi, qa mitawoahope yapatanpi qa ecen ecanonpi kinhan,

4 Iyehantu eca amagaju ciciyapi kta, unkan maka kin taku aicage kta iyececa qu kta, qa waskuyeca can kin tawaskuyeca kin qu kta.

5 Hecen wonapanpi nitawa kin hastanhanka yuśpipi kin iyohi kta, qa-hastanhanka yuśpipi kin wojupi kte cin iyohi kta, qa aguyapi imnahan yahdutapi kta, qa nitamakocepi ohna okope wanin ounyayapi kta.

6 Unkan makoce ohna wookiye waqu kta, hecen makan inonkapi eca yuśinye niyanpi wanice kta, qa makoce nitawa etanhan wamanica śica enawakiye kta, qa ozuye nitamakoce kin omanipi kte śni.

7 Unkan toka niyanpi kin wicayakuwapi kta, unkan nitokam caulipi on licahanpi $k t a$.

8 Unkan niyepi etanhan wicaśta zaptan wicaśta opawinge tawicapapi kta, qa niyepi etanhan opawinge wanjidan kektopawinge wikcemna wicapasipi kta, qa tokaniyanpi nitokam canhpi on hicahanpi kta.

y Unkan acitonwanpi k ta, qa icahiyapi, qa ciyuotapi kta, qa wicotakuye nicipi wahdusuta kta.

10 Hecen tannina śeca kin yatapi kta, qa teca on tannina kin tankan hiyuyayapi kta.

11 Unkan niyepi ehna tipi mitawa ewahde kte, qa minagi hitinihdapi kte śni. 


\section{LEWI TOOPE.}

12 Qa niyepi ehna mawani kta, qa taku wakan yadapi kin he miye kta, qa oyate mitawa kin be niyepi kta;

13 Jehowa wakan yadapi, Egupta makoce etanhan hdinanpe niyanpi kin he miye, qa wowidag niyanpi kte śni, heon wicin icaślke nitawapi kin wapaksa, qa bosdan maniciyapi.

14 Tuka namayahonpi śni, qa mitawoahope kin dena owasin ecen ecanonpi śni kinhan;

15 Qa wokage mitawa ihayaktapi śni kinhan, qa ninagipi woyaco mitawa hitihdapi on mitawoahope owasin ecen ecanonpi śni, hecen wicotakuye mitawa duksapi kinhan,

16 Miś eya hecen ecaciconpi kta, woyuśinye, qa waśinkte, qa tancan kata yuhe niyanpi waśi kta, hena niiśtapi kin sotapi, qa ninagipi kin skan niyanpi kta, hecen wojupi nitawapi taku śni on oyajupi kta, qa toka niyanpi kin he yutapi kta.

17 Qa miite acihnakapi kta, qa toka niyanpi kin nicaśtakapi kta, qa wicaśta śice nidapi kin hena wowidag niyanpi kta, qa tuwedan nipasipi śni eśta nayajicapi kta.

18 Unkan hena on etanhan nayałonpi śni kinhan, sanpa śakowin akihde wayahtanipi on iyopeciyapi kta.

19 Qa nitowaśakapi on walianicidapi kin wakakse kta, qa malipiya nitawapi maza sapa iyececa, qa maka nitawapi kin mazaśa iyececa wakage kta.

20 Qa nitowaśakapi kin. taku śni on sote $k t a$, qa nitamakoce kin takudan aicage kte śni, qa makoce can kin tawaskuyeca kin icahyapi kte śni.

21 Unkan makipajinyan mayanipi, qa namalion tawaten yayapi śni kinhan, ake śakowin akihde cicaśtakapi kta, wayahtanipi kin iyececa.

22 Qa tinta wamanica kin nita. tpe yewaye kta, unkan nicincapi yasotapi kta, qa nitawanunyanpi kin yațapi kta, hecen niyatonanapi kta, qa canku nitawapi kin tuwedan omani kte śni.

23 Unkan hena on miye en nihduecetupi ślli, qa makipajinyan mayanipi kinhan;

24 Hehan miś eya cicipajinpi kta, qa nakun miś wayahtanipi kin on śakowin akihde cicaśtakapi kta.

25 Q a canhipi wan anihiyu waye kta, he wicotakuye mitawa oyapapi śni tokicon kta, hecen otonwe tanka nitawapi en kataiheyayapi kta, unkan niyepi ehna makośice yewaye kta, qa toka niyanpi kin napepi en cicupi kta. 26 Unkan aguyapi sagye cica. ksapi kinhan winohinca wikcemna makacega wanjidan ohna aguyapi nitawapi kin śpanyanpi kta, qa aguyapi nitawapi tkeutapi eciyatanhan nicupi kta, unkan wayatapi kta, tuka inimnapi $k$ te śni.

27 Unkan he on namayahonpi śni, qa mayakipajinpi kinhan,

28 Hehan wocanksiya cicipajinpi kta, qa ake miś eya śakowin akihde wayalitanipi on cicaśtakapi kta.

29 Unkan nicinkśipi celipipi kin yatapi kta, qa nicunkśipi cehpipi kin yatapi kta.

30 Unkan paha tehanwankantuya nitawapi kin wapajuju kta, qa anpetu wi kagapi nitawa wakawanke kta, qa nitancan tapi kin wakagapi nitawapi tapi akan wehnake kta, qa minagi kin wahitenidapi kte śni.

31 Qa otonwe nitawapi hewoskan wakage kta, qa tipi wakan 


\section{WICOWOYAKE 27.}

nitawapi otiwota wakage kta, qa izinyapi waśtemna nitawapi owamna kte śni.

32 Qa miś makoce kin hewoskan*wakage kta, hecen toka niyanpi kin he en ounyanpi kin yuśinyayapi kta.

33 Qa niyepi ikcewicaśta ehna enanakiya iyaye ciyapi lita, qa nihakam isan wahduśdoke kta, qa makoce nitawapi kin hewoskantu kta, qa otonwe nitawapi kin tuwedan oti kte śni.

34 Hecen makoce kin tookihpapi kin iyokipi kta, tona can hewoskan, qa niyepi toka niyanpi tamakocepi en dukanpi hehanyan makoce okihpe kta, qa tookilipapi kin iyokipi kta.

35 Akan ounyayapi qehan okihpapi tawa icunhan okilipe śni, he on anpetu tona hewoskan okihpe kta.

36 Unkan niyepi etanhan tona iyahanpi nicantepi wankapidan wakage kta, tokaniyanpi tamakocepi kin ohna; canwapa hapa hoton nicaliapapi kta, qa canlipi etanhan najicapi iyecen nayajicapi kta, qa nihicahanpi kta, tuwedan nicuwapi śni eśta.

$37 \mathrm{Qa}$ iyahdaskin nihinlipayapi kta, canhipi etanhan najicapi iyecen tuwedan nipasipi śni eśta, qa toka niyanpi itokam najin oyakihipi kte śni.

$38 \mathrm{Qa}$ Ikcewicaśta ehna aniwi hnunipi kta, qa toka niyanpi tamakocepi kin niyasotapi kta.

39 Qa niyepi etanhan tona ihanpi kin hena śicaya ohanyanpi kin en slianpi kta, toka niyanpi tamakocepi ohna, qa nakun hunkake wicayapi śicaya olianyanpi en skanpi kta.

40 Hehan iye śicaya ohanyanpi kin, qa hunkakepi śicaya olanyanpi kin ohdakapi kta, woki- pajin on makipajinpi, qa nakun token makiza manipi;

41 Nakun miś wicakipajinyan mawani, qa toka wicayapi tamakocepi en awicamda, hehan cantepi bakilidayapi śni ihduhukuyapi kinhan, qa śicaya ohanyanpi on śitkihdayapi iyowin kiyapi kinhan;

42 Hehan wicotakuye mitawa Jakob kici weksuye kta, qa.wicotakuye mitawa Izak kici qa wicotakuye mitawa Abereham kici weksuye kta, qa makoce kin weksuye kta.

43 Unkan makoce kin elipeyapi kin, qa tookihpapi on iyuśkin kta, hena en unpi śni icunhan, unkan iye wahtanipi śitkihdayapi kin iyowinkiyapi kta, woyaco mitawa ihaktapi śni, qa nagipi kin wokage mitawa wahtedapi śni kin he on etanhan.

44 Takomni tokawicayapi tamakocepi ohna yakonpi eśta ehpe wicawakiye kte śni, qa ihangwicaye kta, qa wicotakuye wicawecicage cin wahdujuju kta e hitiwicawahda kte śni, Jehowa wakandapi kin he miye.

45 Tuka iyepi on wicotakuye hunkakewicayapi Egupta makoce etanhan wakanmadapi kta e inanpe wicawaye cin he ikcewicaśta wanyakapi hena om wicotakuye weksuye kta, Jehowa he miye.

46 Wokage, qa woyaco, qa woope Jehowa Sina he ekta, Mowis nape eciyatanhan, iye qa Israel cinca otahedan wicaqu kin hena ee.

\section{WICOWOYAKE $2 \%$.}

1 Unkan Jehowa ie ca Mowis heciya ;

2 Israel cinca kin hecen ewicakiya wo; Wicaśta wan tuwe hdu- 


\section{LEWI TOOPE.}

kinukan kta iciconze cinhan, token nagi niyawapi. kin Jehowa qu kta.

3 Unkan hecen niyawapi kta, wica wan waniyetu wikcemna nonpa etanhan wikcemna śakpe aiyahdeya mazaska śeqel wikcemna zaptan niyawapi lita, tipi wakan śeqel iyececa.

4 'Tuka he winyan hecinhan śeqel wikcemna yamni niyawapi lita.

5 Unkan waniyetu zaptan etanhan waniyetu wikcemna nonpa aiyahdeya, wica kinhan śeqel wikcemna nonpa niyawapi kta, qa winyan eqe śeqel wikcemnana.

6 Unkan wi wanjidan etanhan waniyetu zaptan aiyahdeya niyawapi kinhan wica kin mazaska śeqel zaptan, qa winyan eqe mazaska śeqel yamni niyawapi kta.

7 Unkan waniyetu wikcemna śakpe etanhan, qa iwankapatanhan, wica kinhan śeqel wikcemna sanpa zaptan niyawapi kta, qa winyan eqe śeqel wikcemna.

8 Tuka taku niyawapi kin iyohiye śni kinlıan, wawayuśna kin itokam inajin kta, unkan wawayuśna kin he yawa kta, tuwe iciconze taku nape kin iyohiye cin he wawayuśna kin iyukcan lita.

9 Unkan wanunyanpi etanhan woahi Jehowa kahipi kin hena etanhan taku qu kin owasin Jehowa on wakan kta.

$10 \mathrm{He}$ yutokeca kte śni, qa waśte śica on tokiyopeye kte śni, qa nakun śica waśte on, qa wanunyanpi wan wanunyanpi tokeca on tokiyopeye kte cinhan sakim wakan kta.

11 Unkan wanunyanpi śapa etanhan woahi Jehowa kahipi śni hena owasin etanhan kinhan, wanunyanpi kin he wawayuśna kin itokam najin kiye kta.

12 Unkan wawayuśna kin he niciyawa kta, waśte qa śica unman tukte kaśta, token wawayuśna kin he niciyawa kinhan, hecetu kta.

13 Unkan he opekiton kte cinhan, token niciyawapi, qa nakun sanpa izaptan qu kta.

14 Unkan wicaśta wan Jehowa on wakan kta tipi kin hduwakan kte cinhan, wawayuśna kin he waśte qaiś śica iyawa kta, qa token wawayuśna niciyawa kinhan, hecetu kta.

15 Unkan tuwe tipi hduwakan kin he opekiton kte cinhan, token kiciyawapi, qa nakun sanpa izaptan qu kta, hehan hduhe kta.

16 Unkan wicaśta wan maga tawaye cin etanhan onśpa Jehowa on hduwakan kte cinhan, su on wojupi kin eciyatanhan niciyawapi kta, Barle Homer wan woju iyohiye kte cin he mazaska śeqel wikcemna zaptan niciyawapi kta.

17 Unkan Jubele waniyetu etanhan maga hduwakan, token niciyawapi kin hecetu kta.

18 Unkan Jubele iyohakam maga hduwakan kinhan niciyawapi qon etanhan yuaoptetu kta, qa wawayuśna kin waniyetu tona Jubele wanıyetu iyehantu eciyatanhan mazaska kiciyawa kta.

19 Unkan tuwe maga hduwakan kin he opekiton kte cinhan, mazaska niciyawapi qa nakun he kici izaptan qu kta, hecen elkicetupi kta.

20 Unk an maga kin opekiton kte śni, ecen wicaśta tokeca maga kin he opeton kinhan, icimanna opekiton kte śni.

21 Qa maga kin he wakan kta, Jubele kinhan tankan ye kte śni, Jehowa tawa kta, maga yuwa-

188 
kanpi hinca iyececa, wawayuśna kin he tawaye lita.

2.2 Unkan maga wan opetonpi, qa hunkakewicaye cin etanhan maga kin he tawaye śni, he yuwakan kta, qa Jehowa qu kte cinhan ;

23 Wawayuśna niciyawapi kin eciyatanhan. Jubele waniyetu. iyehantu kin taku iyehantu kiciyawa kta, unkan anpetu kin he en token niciyawapi qu kta, he wakan Jehowa tawa.

24 Jubele waniyetu kinhan maga tuwe etanhan opetonpi kin he ekta hdi kta, wicaśta makoce tawaye ciqon he ekta.

25 Taku niciyawapi kin owasin tipi wakan śeqel eciyatanhan, omnica wikcemna nonpa śeqel wanjidan kta.

26 Tokeśta woteca tokaheya wicatonpi kin he Jehowa tawa, heon tokaheya wicatonpi kin he tuwedan yuwakan kte śni, he pte qaiś tahinca unman tukte kaśta Jehowa tawa.

27 Unkan woteca śapa heca opekiton kte cinhan niciyawapi iyecen he izaptan kici qu kta, qa opekiton kte śni kinhan wiyopeyapi kta, niciyawapi kin iyecen.

28 Tokeśta taku yuwảkanpi hinca, taku wicaśta wan Jehowa ekta yuwakan hinca kin, taku tawaye cin owasin etanhan wicaśta, qa wanunyanpi etanhan, qa maga tawaye cin etanhan he wiyopeyapi kte śni, qa opekitonpi kte śni, taku yuwakanpi linca kin he owasin Jehowa tawa, wakan hinca.

29 Wicaśta tona wicayuwakanpi hinca kin hena opewicakitoupi kte śni, tuwe yuwakanpi linca kin he te kta.

30 Unkan maka taku aicage cin iwikcemna kin maka ojupi etanhan, qa can waskuyeca etanhan, he owasin Jehowa tawa, he Jehowa on wakan.

31 Unkan wicaśta wan iwikcemna kin etanhan taku opekiton kte cinhan, iyehantu, qa nakun kici izaptan kin qu kta.

32 Unkan, pte qa tahinca iwikcemna kin owasin tona cansakadan ihukuya iyoptapi kin hena owasin iwikcemna kin Jehowa on wakan kta:

33 Waśte qaiś śica unmanna on kalinige kte śni qa he yutokeca kte śni, qa tokiyopekiya eśta he, qa wanji on tolkiyopekiyapi koya wakan kta, opekiton kte śni.

34 Sina he ohna Israel cinca on Jehowa Mowis woahope qu kin hena ee. 

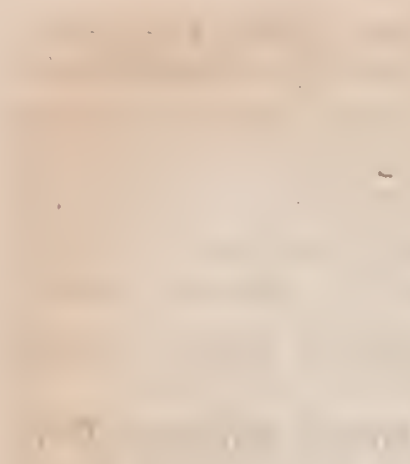

.

$+$

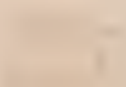

4

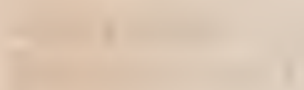

1 .

,

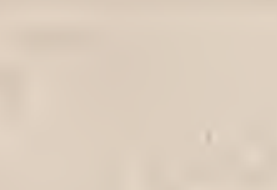

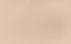

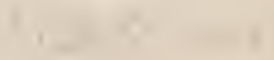

(2) 


\section{Number or
Wratawai (E)}

WICA YA W A PI.

WICOWOYAKE 1.

1 Unkan Egupta makoce etanhan hdinapapi kin waniyetu inonpa wi inonpa anpetu tokaheya en, Sina hopuze cin ohna, itkokipapi wakeya ohna, Jehowa ie, ca Mowis heciya.

2 Israel cinca omniciye ocowasin pa kin wicayawapo, wicowazipi iyecen, ateyapi tipi kin iyecen wicapa kin owasin cajepi yawapi iyecen.

3 Waniyetu wikcemna nonpa etanhan qa aktonpi, Israel ohna wica tona ozuye yapi, niye, qa Aaron wicadawapi kta, obe tawapi kin iyecen,

4 Unkan wicoun otoiyohi etanhan wicaśta wan niyepi om un kta, wicaśta ateyapi tipi itancan heca.

5 Unkan wicaśta niyepi om najinpi kta, wicasta cajepi kin dena eepi, Ruben etanhan Elitzur Sedeur cinhintku.

6 Simeon etanhan Selumiel Tzuriśada cinhintku.

7 Juda etanhan Nahison Aminadab cinhintku.

8 Isakar etanhan Netaneel Tzuar cinhintku.

9 Zubulun etanhan Eliab Helon cinhintku.

10 Josep cinca etanhan, Epraim etanhan, Eliśama Amihud cinhintku, Manaśe etanhan Gamaliel Pedatzur cinhintku.

11 Benjamin etanhan Abidan Gideoni cinhintku.

12 Dan etanhan Ahiezer Amiśada cinhintku.

Num.
13 Aśer etanhan Pagiel Okran cinhintku.

14. Gad etanhan Eleasap Deuel cinhintku.

15 Neptali etanhan Ahira Enan cinhintku.

16 Omniciye etanhan hena wicakicopi, ateyapi wicoun wicaśta yatapi kin, Israel kektopawinge itancan hecapi.

17 Unkan Mowis qa Aaron wicaśta caje wicayatapi qon hena icupi.

$18 \mathrm{Qa}$ wi inonpa anpetu tokaheya en, omniciye owasin yuwitaya hiyu wicayapi, unkan tuwewe etanhan tonpi kin ohdakapi, wicowazipi iyecen, ateyapi tipi on, waniyetu wikcemna nonpa aktonpi caje wicayawapi, wicapa owasin.

$19 \mathrm{Qa}$ Sina liopuze ohna wicayawapi, Jehowa Mowis hecon śi kin he iyecen.

20 Unkan Israel cinhmntku tokapa Ruben cinca kin token wicatonpi wicowazipi iyecen, qa ateyapi tipi kin iyecen caje wicayawapi wicapa kin iyecen, wica waniyetu wilkcemna nonpa aktonpi owasin, tona ozuye yapi owasin ;

21 Ruben wicoun etanhan kektopawinge wikcemna topa sanpa opawinge zaptan wicayawapi.

22 Unkan Simeon cinca etanhan token wicatonpi, wicowazipi iyecen, qa ateyapi tipi kin iyecen, caje wicayawapi wicapa iyecen, wica waniyetu wikcemna nonpa aktonpi owasin, tona ozuye yapi owasin. 
23 Simeon wicoun etanhan kektopawinge wikcemna zaptan sanpa kektopawinge napcinwanka sanpa opawinge yamni wicayawapi.

24 Unkan Gad cinca etanhan token wicatonpi, wicowazipi iyecen, ateyapi tipi kin iyecen, caje vicayawapi wica waniyetu wikcemna nonpa aktonpi, tona ozuye yapi owasin;

25 Gad wicoun etanhan kektopawinge wikcemna topa sanpa kektopawinge zaptan sanpa opawinge śakpe sanpa wikcemna zaptan wicayawapi.

26 Juda cinca etanhan token wicatonpi wicowazipi iyecen, ateyapi tipi kin iyecen, caje wicayawapi waniyetu wikcemna nonpa aktonpi, tona .ozuye yapi owasin;

27 Juda wicoun etanhan kektopawinge wikcemna śakowin sanpa kektopawinge topa sanpa opawinge śakpe wicayawapi.

28 Isakar cinca etanhan token wicatonpi wicowazipi iyecen, ateyapi tipi kin iyecen, waniyetu wikcemna nonpa aktonpi caje wicayawapi, tona ozuye yapi owasin ;

29 Isakar wicoun etanhan kelitopawinge wikcemna zaptan sanpa kektopawinge topa sanpa opawinge topa wicayawapi.

30 Zebulun cinca etanhan token wicatonpi wicowazipi iyecen, ateyapi tipi kin iyecen waniyetu wikcemna nonpa àktonpi caje wicayawapi, tona ozuye yapi owasin;

31 Zebulun wicoun etanhan kektopawinge wilcemna zaptan sanpa kektopawinge śakowin sanpa opawinge topa wicayawaji.

32 Josep cinca etanhan Epraim cinca etanhan token wicatonpi 192 wicowazipi iyecen, ateyapi tipi kin iyecen, waniyetu wikcemna nonpa aktonpi, caje wicayawapi, tona ozuye yapi owasin;

33 Epraim wicoun etanhan kektopawinge wikcemna topa sanpa opawinge zaptan wicayawapi.

34 Manaśe cinca etanhan, token wicatonpi wicowazipi iyecen, ateyapi tipi kin iyecen, waniyetu wikcemna nonpa aktonpi caje wicayawapi, tona ozuye yapi owasin;

35 Manaśe wicoun etanhan kektopawinge wikcemna yamni sanpa kektopawinge nonpa, sanpa opawinge nonpa wicayawapi.

36 Benjamin cinca etanhan token wicatonpi, wicowazipi iyecen, ateyapi tipi kin iyecen, waniyetu wikcemna nonpa aktonpi caje wicayawapi, tona ozuye yapi owasin :

37 Benjamin wicoun etanhan kektopawinge wikcemna yamni sanpa kektopawinge zaptan sanpa opawinge topa wicayawapi.

38 Dan cinca etanhan token wicatonpi, wicowazipi iyecen, ateyapi tipi kin iyecen, waniyetu wikcemna nonpa aktonpi caje wicayawapi, tona ozuye yapi owasin ;

39 Dan'wicoun etanhan kektopawinge wikcemna śakpe sanpa kektopawinge nonpa sanpa opawinge śakowin wicayawapi.

40 Aśer cinca etanhan token wicatonpi wicowazipi iyecen, ateyapi tipi kin iyecen, waniyetu wikcemna nonpa aktonpi caje wicayawapi, tona ozuye yapi owasin ;

41 Aśer wicoun etanhan kektopawinge wikcemna topa sanpa kektopawinge wanjidan sanpa opawinge zaptan wicayawapi.

42 Neptali cinca etanhan token 
wicatonpi wicowazipi iyccen, ateyapi tipi kin iyecen, waniyetu wikcernna nonpa aktonpi caje wicayawapi, tona ozuye yapi owasin ;

43 Neptali wicoun etanhan kektopawinge wikcemna zaptan sanpa kektopawinge yamni sanpa opawinge topa wicayawapi.

44 Mowis qa Aaron qa Israel itancan wikcemna sanpa nonpa ateyapi tipi otoiyohi etanhan wicaśta wanjidan, hena wicayawapi.

45 Hecen Israel cinca kin ateyapi tipi kin iyecen, tona wicayawapi, waniyetu wikcemna nonpa aktonpi, Israel ohna tona ozuye yapi kin owasin ;

46 Tona wicayawapi owasin kektopawinge opawinge śalipe sanpa kektopawinge yamni sanpa opawinge zaptan sanpa wikcemna zaptan eepi.

47 Tuka Lewi kin ateyapi wicoun iyecen hena om wicayawapi śni.

48 Nakaś Jehowa Mowis hecen cciye ciqon;

49 Ihnunhan Lewi wicoun kin wicadawa kin, qa hena oyawa kin Israel cinca kin wicehna aokiju yaye kte śni.

50 Tuka niye Lewi kin wayaatanin tipi kin, qa tawakśica owasin, qa taku ohna un kin owasin yuhe wicayakiye kta. Hena tipi kin he, qa wakśica tawa owasin qinpi kta, qa he kicanyanpi kta, qa tipi kin he ihdukśan etipi kta.

51 Ihdakapi eca Lewi kin tipi kin yujujupi kta, qa etipi eca Lewi kin tipi kin piya iticagapi kta. Unkan tuwe tokcca ikiyedan hi kinhan te yapi kta.

52 Unkan Israel cinca kin otoiyohi wicobe iyecen wicoti tawa, qa wiyokiye tawa ekta ti kta. II.
53 Tuka Lewi kin wayaatanin tipi kin ihdukśan etipi kta, heccn Israel cinca omniciye kin wocanniye awicahinlipaye kte "śni, Lewi kin woyaatanin tipi awanyag wicaśipi kin he iyecen avanyakapi kinhan.

54. Unkan Israel cinca kin token Jehowa Mowis econpi kta eciye cin he iyecen taku owasin econpi.

\section{WICOWOYAKE 2.}

1 Unkan Jehowa ie ca Mowis, qa Aaron hewicakiya.

2 Israel cinca kin otoiyohi wiyokiye tarva ekta, ateyapi tipi wiyokiyedan kin icahda wakeya iticage kta, itkokipapi wakeya ihdukśan owasin wakeya iticagapi kta.

3 Unkan he itokam wi hinape ektakiya Juda' wicoti wiyokihe kin obe tawapi kin iyecen wakeya iticagapi kta. Unkan Naliśon Aminadab cinhintku kin Juda cinca itancan kin hee.

$4 \mathrm{Qa}$ obe tawa kektopawinge wikcemna śakowin sanpa kektopawinge topa sanpa opawinge śakpe wicayawapi.

5 Unkan he icahda Isakar wicoun tipi kta, qa Isakar cinca itancan kin Netanael Tzuar cinhintku kin hee.

$6 \mathrm{Qa}$ obetawa kektopawingc wikcemna zaptan sanpa kektopawinge topa sanpa opawingc topa wicayawapi.

7 Hehan Zebulun wicoun, qa Zebulun cinca itancan kin Eliab Helon cinhintku kin hee.

$8 \mathrm{Qa}$ obe tawa kektopawinge wikcemna zaptan sanpa kektopawinge śakowin sanpa opawinge topa wicayawapi.

9 Juda wicoti wicayawapi owasin kektopawinge opawinge san193 


\section{WICAYAWAPI,}

pa kektopawinge wikcemna śahdogan sanpa kektopawinge śakpe sanpa opawinge topa wicayawapi.

10 Ruben wicoti wiyokiye kin wiyotanhan ektakiya tipi kta, obe tawapi kin iyecen, qa Ruben cinca itancan kin Elitzur Śedeur cinhintku kin hee.

11 Qa obe tawa kektopawinge wikcemna topa sanpa kektopawinge śakpe sånpa opawinge zaptan wicayawapi.

12 Unkan he icahda Simeon wicoun tipi kta, qa Simeon cinca itancan kin Selumiel Tzurisada cinhintku kin hee.

$13 \mathrm{Qa}$ obe tawa kektopawinge wikcemna zaptan sanpa kektopawinge napcinwanka sanpa opawinge yamni wicayawapi.

14. Hehan Gad wicoun, qa Gad cinca itancan kin Eliasap Reuel cinhintku kin hee.

$15 \mathrm{Qa}$ obe tawa kektopawinge wikcemna topa sanpa kektopawinge zaptan sanpa opawinge sakpe sanpa wikcemna zaptan wicayawapi.

16 Ruben om tipi wicayawapi owasin kektopawinge opawinge sanpa kektopawinge wikcemna zaptan sanpa kektopawinge wanjidan, sanpa opawinge topa sanpa wilkcemna zaptan, obe tawapi kin iyecen, qa hena inonpa ihdakapi kta.

17 Hehan itkokipapi wakeya kin ihdake kta, Lewi wicoti om wicoti cokaya, token tipi kin he iyecen ihdakapi kte, otoiyohi oyanke tawa ohna wiyokiyepi om.

18 Epraim wiyokiye obe tawapi kin iyecen wiyohipeyata ektaliya tipi kta, qa Epraim cinca itancan kin Eliśama Amihud cinhintku kin hee.

19 Qa obe tawa kektopawinge 194 wikcemna topa sanpa opawinge zaptan wicayawapi.

$20 \mathrm{Qa}$ he icahda Manaśe wicoun, qa Manaśe cinca itancan kin Gamaliel Pedatzur cinhintku kin hee.

21 Qa obe tawa kektopawinge wikcemna yamni sanpa kektopawinge nonpa sanpa opawinge nonpa wicayawapi.

22 Hehan Benjamin wicoun etipi kta, qa Benjamin cinca itancan kin Abidan Gideoni cinhintku kin hee.

23 Qa obe tawa kektopawinge wikcemna yarmi sanpa kektopawinge zaptan sanpa opawinge tapa wicayawapi.

24. Epraim wicoti om wicayawapi owasin kektopawinge opawinge sanpa kektopawinge śahdogan sanpa opa winge wanjidan, obe tawapi kin iyecen, qa hena iyamni ihdakapi kta.

25 Dan wiyokiye waziyata ektakiya tipi kta obe tawapi kin iyecen, qa Dan cinca itancan kin Ahiezer Amiśada cinhintku kin hee.

26 Qa obe tawa kektopawinge wikcemna śakpe sanpa kektopawinge nonpa sanpa opawinge śakowin wicayawapi.

27 Unkan he icahda tipi kin dena eepi; Aśer wicoun, qa $A$ śer cinca itancan kin Pagiel Okrar cinhintku kin hee.

28 Obe tawa kektopawinge wikcemna topa sanpa kek topawinge wanjidan sanpa opawinge zaptan wicayawapi.

29 Qa Naptali wicoun kin, qa Naptali cinca itancan kin Alira Enan cinhintku kin hee.

30 Qa obe tawa kektopawinge wikcemna zaptan sanpa kektopawinge yamni sanpa opawinge topa wicayawapi.

31 Dan wicoti wicayawapi owa- 


\section{WICOWOYAKE 3.}

sin kektopawinge opawinge sanpa kektopawinge wikcemna zaptan sanpa kektopawinge śakowin sanpa opawinge śakpe hena ehake ihdakapi kta, wiyokiye tawapi kin iyahna.

32 Israel cinca ate wicayapi tipi ecekcen hena wicayawapi, obe tarvapi kin ohnahna, wicoti owancaya denakeca wicayawapi, kektopawinge opawinge śakpe sanpa kektopawinge yamni sanpa opawinge zaptan sanpa wikcemna zaptan.

33 Tuka Lewi kin hena Israel cinca wicehna wicayawapi śni, Jehowa Mowis hecen econ śi nakaeś.

34 Unkan token Jehowa Mowis econ si kin owasin he iyecen Israel cinca kin econpi, hecen wiyokiye tawapi kin icahda iticagapi, qa hecen otoiyohi wicotakuye tawa kin iyecen atewicaya tipi kin om ihdakapi.

\section{WICOWOYAKE 3.}

1 Unkan Aaron, qa Mowis wicoicage kin dee, Sina lie kin ohna Jehowa Mowis kici wohdake cehan.

2 Äaron cinca kin cajepi kin dena eepi, Cinhintku tokapa Nadab, hehan Abihu, Eleazar, qa Itamar.

3 Aaron cinca sda wicayapi cajepi kin hena eepi, hena wośna kagapi kta e nape ojun wicayapi.

4 Tuka Nadab, qa Abihu Jehowa itokam tapi Sina hopuze cin ohna, peta tokeca Jehowa itokam hiyuyapi qehan, qa cinca nicapi. Unkan Eleazar qa Itamar wawayuśnapi ateyapi Aaron itokam.

5 Unkan Jehowa ie ça Mowis heciya,
6 Lewi wicoun kin awicahi wo, qa Aaron wawayuśna kin itokam najin wicayakiye kta, hecen okiyapi kta.

7 Qa iye taku awanyake kte cin, qa omniciye owasin taku awanyakapi kte cin he awanyakapi kta, itkokipapi walkeya itokam, qa tipi toh̆tani kin econpi kta.

8 Qa itkokipapi wakeya wakśica kin owasin awanyakapi kta, nakun Israel cinca kin toawanyake kin owasin, hecen tipi tohtani kin econpi kta.

9 Qa Lewi kin taku qupı wan iyecen Aaron qa iye cinca kin wicayaqu kta, Israel. cinca kin wicehna tanhan hena iye wicakicupi kta.

10 Unkan Aaron qa iye cinca kin hena yuhe wicayaśi kta, unkan wowayuśnapi kin awanyakapi kta, unkan tuwe tokeca ikiyedan hi kinhan teyapi kta.

11 Unkan Jehowa ie ca Mowis heciya.

12 Tho miś Israel cinca wicehna tanhan Lewi kin iwicawacu, lsrael cinca tokapapi, tona winyan tamni nahdoke cin owasin hena eekiya, hecen Lewi kin wicamoluhe kta.

13 Tona tokapa kin owasin mitawa, Egupta makoce ohna tona caske owasin awicawape cin anpetu kin he en Israel wicebna wicaśta, qa woteca tona caske owasin mici wahduwakan, hena mitawa kta, Jehowa he miye.

14 Unkan Sina hopuze cin ohna Jehowa ie ca Mowis heciya.

15 Lewi cinca ateyapi tipi kin iyecen, wicowazipi iyecen, tona wica owasin wicayawa wo, wi wanjidan aktonpi wicadawa kta.

16 Unkan Jehowa i kin eciya195 
tanhan token econ si kin he iyecen Mowis wicayawa.

17 Unkan Lewi cinca cajepi kin dena eepi, Gerśon, qa Qohat, qa Merari.

$18 \mathrm{Qa}$ Gerśon cinca cajepi kin wicowazipi iyecen, dena eepi Libni, qa Simei.

19 Unkan Qohat cinca kin wicowazipi iyecen, Amram qa Itzar, Hebron qa Uziel.

20 Unkan Merari cinca kin wicowazipi iyecen, Malili qa Muśi, Lewi ateyapi tipi wicowazipi kin dena eepi.

21 Gerśnn etanhan Libni wicowazi, qa Śmei wicowazi ; Gerśoni wicowazi hena eepi.

22 Hena wicayawapi tona wica waniyetu wanjidan aktompi owasin kektopawinge śakowin sanpa opawinge zaptan.

23 Gerśoni wicowazi tipi catku tanhan wiyohpeya ektakiya etipi kta.

24 Qa Gerśoni ateyapi tipi itancan kin Eleasap Lael cinhintku kin hee.

$25 \mathrm{Qa}$ itkokipapi wakeya etanhan Gerśoni cinca taku awanyakapi kte cin dee, tipi kin, qa wakeya kin, qa toakahpe kin, qa itkokipapi wakeya tiyopa akahpe cin.

$26 \mathrm{Qa}$ hocoka ozanpi kin, qa locoka tiyopa akalipe cin, he tipi kin qa wahna wośnapi ihdukśan yanka, nakun ikanyanpi kin owasin.

27 Unkan Qohat etanhan Amrami wicowazi, qa Itzar wicowazi, Hebroni wicowazi, qa Uzieli yicowazi; Qohati wicowazi kin hena eepi.

$28 \mathrm{Wi}$ wanjidan aktonpi kin wica owasin, kektopawinge śahdogan sanpa opawinge śakpe, tipi wakan wokicanye kin awanyakapi.
29 Qohat cinca wicowazi kin tipi icahda, wiyotanhan ektakiya etipi kta.

30 Qa Qohati wicowazi ateyapi tipi itancan kin Elitzapan Uziel cinhintku kin hee.

31 Qa hena can wohnaka kin, qa wahna wotapi kin, qa petijanjan ihupa kin, qa wahna wośnapi kin, qa tipi wakan tawakśica kin hena on tohtani econpi kin, qa taku on kicanyanpi owasin, qa tiyopa akahpe kin.

32 Unkan Lewi itancan itancanpi kin Aaron wawayuśna cinhintku kin Eleazar hee kta, iye tipi wakan awanyakapiekin hena awanwicayake kta.

33 Merari etanhan Malili wicowazi kin qa Muśi wicowazi kin; Merari wicowazi kin hena eepi.

34 Wica owasin wi wanjidan aktonpi kektopawinge śakpe sanpa opawinge nonpa.

$35 \mathrm{Qa}$ Merari ateyapi tipi wicowazipi itancan kin Tzuriel Abihael cinhintku kin hee; hena waziyata ekta kiya tipi kta.

36 Unkan Merari cinca kin tipi canmdaska kin, qa can hdakinyan kin, qa can bosdata kin, qa sicu kin, qa tawakśica kin owasin, qa taku on kicanyanpi kin owasin awanyag wicayaśi kta.

37 Nakun hocoka ihdukśan can bosdata kin, qa sicupi kin qa wihutipaspepi kin, qa ikanpi kin owasin.

-38 Unkan Mowis qa Aaron qa iye cinca kin tipi itokam wiyohiyanpa ektakiya, itkokipapi wakeya itokarn wi hinanpa ektakiya etipi kta, Israel cinca kin tipi wakan awanyakapi kin hena awanyakapi kta, qa tuwe tokeca ikiyedan hi kinhan te yapi kta.

39 Lewi kin Jehowa i eciyatanhan Mowis qa Aaron wicayawa- 
pi owasin wicowazipi iyecen, wica wi wanjidan aktonpi wicayawapi owasin kektopawinge wikccmna nonpa sanpa kektopawinge nonpa.

40 Unkan Jchowa Mowis heciya, Israel cinca caskepi owasin wicayawa wo, wi wanjidan aktonpi kin hena, qa cajcpi oyawa icu wo.

41 Qa Jehowa he miye, heon Israel cinca caskepi owasin liee kiya Lewi kin imicicu wo, qa Israel cinca tawanunyanpi caskepi owasin hee kiya Lewi tarvanunyanpi kin;

42 Unkan Mowis Israel cinca caskcpi owasin wicayawa, Jehowa hecon śi kin he iyecen.

43 Unkan caskcpi owasin wi wanjidan aktonpi, cajepi wicayawapi iyecen, kcktopawinge wikcemua nonpa sanpa kektopawinge nonpa sanpa opawinge nonpa sanpa wikcemna śakowin sanpa yamni wicayawapi.

44 Unkan Jehowa ie ça Mowis heciya,

45 Lewi kin iwicacu wo, qa Lewi tawanunyanpi Israel cinca caskepi, qa tawanunyanpi hee kiya, hecen Lewi kin mitawa kta.

$46 \mathrm{Qa}$ Israel cinca caskepi opawinge nonpa sanpa wikcemna śakowin sanpa yamni Lewi kin isanpa hena ope wicakitonpi kta.

47 Hena otoiyohi etanhan pa wicayawapi kin iyecen, śeqel zaptan, tipi wakan seqel iyecen iyacu kta. Seqel wanjidan omnica wikcemna nonpa hec.

$48 \mathrm{Qa}$ wicayawapi iyaye cin mazaska on ope wicatonpi kin he Aaron qa iye cinca kin wicayaqu kta.

49 Unkan Mowis Lewi ope wicakitonpi sanpa iyayapi kin hena etanhan mazaska on opeicitonpi kin he icu.

50 Israel cinca caskepi etanhan mazasia lin icu, śeqel kektopawinge vanjidan sanpa opawinge yamni sanpa wikcemna śakpe sanpa zaptan tipi wakan śeqel iyececa.

51 Qa Mowis mazaska on ope wicakitonpi kin he Aaron qa iye cinca kin wicaqu; Jchowa i kin eciyatanhan Jehowa Mow is cconśi lin he iyecen.

\section{WICOWOYAKE 4.}

1 Unkan Jehowa ie ca Mowis qa Aaron hewicakiya.

2 Lewi cinca wicchna tanhan Qohat cinca kin tonaka wicayawa wo, wicowazipi iyecen, ateyapi tipi kin iyecen.

3 Waniyctu wilicemna yamni aktonpi, qa waniyetu wikcemna zaptan aiyahdeya, tona itkokipapi wakcya wohtani econpi kta e ube cn opapi owasin.

4 Qa Qohat cinca wolitani econpi lita dee, itkokipapi wakeya ohna t.ku wakan linea.

5 Wicoti ihdakapi kte cinhan Aaron qa iye cinca kin en. hipi lita, qa cokaya ozanpi woakahpe kin kun icupi kta, qa he on woyaatanin can wohnake kin aliahpapi kta.

6 Qa he iwankam hoka ha woakalipe wan ehnakapi kta, qa he iwankarn śina wan ocowaśin to ayumdayapi kta, qa tośu kin en iyeyapi kta.

I Qa wahna wotapi itokam hnakapi akan śina to wan ayumdayapi kta, qa he ahan wakśi= ca kin, qa maza tulkina kim, qa wiyatke kin, qa cega etanhan wakanyan kaśtanpi kin chnakapi kta, qa aguyapi ohmmiyan kin he akan yanke kta. 


\section{WICAYAWAPI,}

8 Qa hena akan sina duta wan ayumdayapi kta, qa hoka ha woakahpe wan on akahpapi kta, qa can akiyuhapi kin wiyeya kagapi kta.

9 Qa śina to wan icupi kta, qa petijanjan ihupa kin, qa petijanjan kin, qa petijanjan iyukse kin, qa ohna cahota icupi kin, qa wihdi wakśica on he kicanyanpi owasin akahpapi lita.

$10 \mathrm{Qa}$ he qa tawaśica owasin hoka ha ojuha wan en ohnakapi kta, qa can akiyuhapi wan akan ikoyag yapi kta.

$11 \mathrm{Qa}$ mazaskazi wahna wośnapi akan śina to wan ayumdayapi kta, qa hoka ha woakahpe wan on akalipapi kta, qa can akiyuhapi kin he ikoyag yapi kta.

$12 \mathrm{Qa}$ wakśica kicanyanpi, tipi wakan ohna taku econpi kin hena owasin icupi kta, qa śina to wan akan ehnakapi kta, qa hoka ha ojuha wan en iyeyapi kta, qa can akiyuhapi wan akan ikoyag yapi kta.

13 Qa wahna wośnapi etanhan caliota pakintapi kta, qa akan sind stan wan ayumdayapi kta.

14 Qa he akan taku on kicanyanpi owasin, petaga wakśica kin, qa mazajanjata kin, qa mazamdaska kin, qa waksica kin, taku on wahna wośnapi kicanyanpi owasin ehnakapi kta, qa akan hoka.ha woakahpe wan ayumdayapi kta, qa can akiyuhapi kin ekta ikoyag yapi kta.

13 Unkan tipi ihdakapi eca Aaron qa iye cinca tipi wakan qa taku on kicanyanji kin orvasin akahppapi yuśtanpi kinhan, hena qinpi kta e Qohat cinca kin en hipi kta, tuka taku wakan seca yutanpi kte śni, heconpi kinhan okini tapi kta. Itkokipapi wa- keya ohna Quhat cinca tawaqinpi kin hena eepi.

16 Unkan Eleazar Aaron wawayuśna cinhintku kin wihdi iyoyam yapi kin, qa pejuta izinyapi waśtemna, qa anpetu iyohi minha kin, qa wihdi on sdawicayapi kin hena awanyake kta; tipi wakan ocowasin, qa taku ohna un kin owasin, tipi wakan ohna, qa tawakśica ohna taku un kin awanyake kta.

17 Unkan Jehowa ie ça Mowis qa Aaron hewicakiya,

18 Ihnuhan Lewi kin wicehna tanhan Qohati wicoun wicowazi kin wicayakaśpapi kin.

$19 \mathrm{Qa}$ hecen ecawicayeconpi kta, hecen nipi kta, qa taku wakan, qa taku wakan hinca ikiyedæn hipi eśta țapi kte śni; Aaron qa iye cinca kin en ipi, qa otoiyohi taku ccon, qa taku qin kta okiyakapi kta.

20 Tuka taku tipi wakan ohna akahpapi eca wanyakapi kta e en hipi kte śni, okini țapi kta.

21 Unkan Jehowa ie ca Mowis heciya.

22 Gerśon cinca nakun wicayawa wo, ateyapi tipi kin iyecen, qa wicowazipi kin iyecen ;

23 Waniyetu wikcemna yamni aktonpi etanhan waniyetu wikcemma zaptan aiyahdeya wicadawa kta, obe itkokipapi wakcya ohna wohtani econpi kta e en yapi kin owasin.

24 Gerśoni wicowazi kin wolitani econpi kta, qa taku qinpi kte cin dee.

25 'Tipi ozanpi kin qa itkokipapi wakeya he akalipe cin, qa hoka ha woakahpe kin he iwankam akapa tanhan yanke cin, qa itkokipapi wakeya tiyopa ozanpi kin hena qinpi kta.

26 Nakun hocoka ozanpi lkin, hocoka tipi ihdukśan, qa wahna 


\section{WICOWOYAKE 4.}

wośnapi ihdukśan yanke cin tiyopa ozanpi kin, qa ikan kin qa tawakśica kin owasin, qa taku on hena kicanyanpi kin owasin.

27 Gerśoni cinca taku qinpi, qa taku kicanyanpi kin owasin Aaron qa iye cinca oiepi eciyatanhan econpi kta, qa taku qinpi kta owasin awanyag wicayaśipi kta.

28 Gerśoni cinca wicowazipi itkokipapi wakeya ohna wohtanipi kin hee, qa hena taku awanyakapi kte cin he Itamar Aaron wawayuśna cinhintku nape ihukuya un kta.

29 Merari cinca kin wicowazipi iyecen, ateyapi tipi kin iyccen wicadawa kta ;

30 Waniyctu wikcemna yamni aktonpi waniyetu wilicemna zaptan aiyahdeya, obe itkokipapi wakeya wohtani econpi kta e en upi kin owasin wicadawapi kta. 31 Qa itkokipapi wakcya on wohtanipi kin tawaqinpi awanyakapi kte cin dee; tipi canmdaska kin, qa can hdakinyan kin qa can ipatan kin, qa maza sicu kin.

$32 \mathrm{Qa}$ hocoka ihdukśan maza ipatan kin, qa sicupi kin, qa wihutipaspepi, qa ikanpi kin; nakun taku on hena kicanyanpi, qa yuhapi kin owasin, qa waqinpi awanyakapi kin cajepi on wicayecidawapi kta.

33 Merari cinca wicowazi woltani owasin itkokipapi wakeya ohna htanipi kte cin hee, Itamar Aaron wawayuśna kin cinhintku nape kin ikukuya.

34. Unkan Mowis qa Aaron qa omniciye itancanpikin Qohat cinca wicowazipi iyecen, qa ateyapi tipi kin iyecen wicayawapi,

35 Waniyetu wikcemna yamni aktonpi qa waniyetu wikcemna zaptan aiyahdeya tona itko- kipapi wakeya wohtani econpi kta obe en hipi kin owasin.

36 Kektopawinge nonpa sanpa opawinge sakowin sanpa wikcemna zaptan wicayawapi wicowazipi iyecen qa ateyapi tipi lin iyccen.

37 Qohati wicowazipi etanhan tona itkokipapi wakeya en litanipi owasin wicayawapi kin hena eepi ; hena Mowis qa Aaron wicayawaṕi Jehowa i kin cciyatanhan Mowis nape kin on.

38 Unkan Gerśon cinca kin wicowazipi iyecen qa ateyapi tipi kin iyecen wicayawapi.

39 Waniyetu wikccmna yamni aktonpi qa waniyetu wikcemna zaptan wicatonpi aiyahdeya, tona itkokipapi wakeya en litanipi kta e obe en hipi kin owa sin.

40 Kektopawinge nonpa sanpa opawinge śakpe sanpa wikcemna yamni wicayawapi, wicowazipi iyecen, qa ateyapi tipi kin iyecen.

41 Gerśon cinca wicowazipı etanhan wicaya wapi kin hena eepi, tona itkokipapi wakcya ohna htanipi owasin hena Mowis qa Aaron wicayawapi Jehowa i eciyatanhan.

49 Unkan Merari cinca wicowazi wicayawapi wicowazipi kin iyecen, qa ateyapi tipi kin iyecen.

43 Waniyetu wikcemna yamni aktompi qa waniyetu wilicemna zaptan aiyahdeya, tona itkokipapi wakeya ohna htanipi kta e obe en opapi kin owasin.

44 Kektopawinge yamni sanpa opawinge nonpa, wicowazipi iyecen wicayawapi.

45 Merari cinca wicowazipi etanhan wicayawapi hena Mowis qa Aaron wicayawapi Jeho199 


\section{WICAYAWAPI,}

wa i eciyatanhan Mowis nape lin on.

46 Mowis qa Aaron qa Israel itancanpi kin Lewi kin wicowazipi iyecen, qa ateyapi tipi kin iyecen, wicayawapi owasin,

47 Waniyetu wikcemna yamni aktonpi, qa waniyetu wikcemna zaptan aiyahdeya, tona wohtani econpi kta, qa waqinpi econpi kta e itkokipapi wakeya en hipi kin owasin.

48 Kektopawinge śahdogan sanpa opawinge zaptan sanpa wikcemna śahdog an wicaya wapi.

49 Jehowa i eciyatanhan Mowis wicayawa otoiyohi taku econ kta, qa taku qin kta owicakiyaka, Jehowa Mowis econ śi kin he iyecen.

WICOWOYAKE 5 .

1 Unkan Jehowa ie ca Mowis heciya.

2 Israel cinca kin tona leprosi ececa, qa tona ton kaduze cin, qa tona taku nagi iyaye cin on śape cin, hena owasin wicoti etanhan tokan iyaye wicayapi wicaśi wo.

3 Wica, winyan urman tukte kaśta ye wicayaśipi kta, wicoti, etanhan iyaye wicayayapi kta, hecen wicoti cokaya wati kin he yuśapapi kte śni.

4 Unkan Israel cinca kin ecen econpi, qa hena wicoti itankan iyaye wicayapi, token Jehowa Mowis eciye cin hecen Israel cinca kin econpi.

5 Unkan Jehowa ie ca Mowis heciya,

6 Israel cinca kin hewicakiya wo. Wicaśta qaiś winohinca wicaśta wan en woaktani econ kinhan, qa itonśniyan Jehowa caje yate qa wicanagi kin he hdajuju kta sdotkiye cinhan, 200
7 Woahtani econ qon ohdake kta, qa wokajuju ocowasin hdajuju kta, qa he izaptan aope kiciye ca tuwe en wahtani kin kicu kta.

8 Tuka wicaśta takuye wohdajuju kicupi kta wanice cinhan, wohdajuju kin Jehowa kicupi kta, wawayuśna akahpe kiciye cin he ekta woakahipe tamdoka iyahna.

9 Unkan Israel cinca taku wośnapi etanhan wokosyapi owasin wawayuśna ekta kahipi kin he tawa kta.

10 Qa wicaśta tuwe kaśta taku wośna kin he wawayuśna he kahi kinhan he tawa kta.

11 Unkan Jehowa ie ça Mowis heciya.

12 Israel cinca kin hecen ewicakiya wo. Wicaśta tuwe kasta tawicu ihduhomni; qa iye en natimana awahtani kinhan;

13 Qa wicaśta tokeca wan kici iwanke cinhan, he nahmana econ, qa hihnaku iśta kin wanyake śni, qa ihduśape tuka wayaatanin wanica, hecon icunhan iyeyapi śni nakaś ;

14 Unkan wowinawizi woniya wan en iyopte ca tawicu kin inakiwizi, unkan he ihduśapa; qaiś wowinawizi woniya wan en iyopte ca tawicu inakiwizi, tuka he ihduśape śni kinhan;

15 Wicaśta kin he tawicu kin wawayuśna kin ekta u kiye kta, nakun tawicu toahi kin hiyuye kta, barle mdu can wakśica ohnaka wanjidan, wihdi akaśtan kte śni, qa canśin waśtemna aehnake kte śni, wowinawizi minlia hee nakaś, wokiksuye minha on wicohan śica kiksuyapi kta.

16 Unkan wawayuśna kin he ikiyedan hiyuye ça Jehowa itokam najin kiye kta. 
17 Qa wawayuśna kin mini yuwakanpi maka wakśica wan ohna icu kta, qa tipi wakan owanka etanhan maka mdu nakun wawayuśna icu kta, qa mini kin akada kta.

18 Unkan wawayuśna kin winohinca kin Jehowa itokam najin kiye kta, qa pa yugan iyeye kta, qa nape kin akan wokiksuye minha, wowinawizi minlia kin hee aehnake kta; tuka mini pa taku telika icahye cin he wawayuśna kin nape ohna yuhe kta.

19 Qa wawayuśna kin winohinca kin taku wakan cajeyan kiye kta, qa winohinca heciye kta, Nihduśape śni kinhan, qa nihihna etanhan nihduhomni śni, qa wicaśta tokeca kici inonke śni kinhan, mini pa taku tehika icahye cin de kiunni niye kte śni.

20 Tuka nihihna etanhan nihduhomni, qa nihduśape ça nihihna hee kiya wicaśta tokeca kici inonke cinhan ;

21 Hecen wawayuśna kin winohinca kin taku wakan cajeyan wowicake ohdag śi kta, qa wawayuśna kin winohinca kin heciye kta, Jehowa oyate nitawa wicehna woyaśice nicage kta, Jehowa niceca kin hidi niye ca nitezi po niye cinhan;

22 Qa mini taku tehike icahye cin de niśupe en ye cinhan tezi nipo kta, qa ceca nihdi $k$ ta, eye $\mathrm{kta}$ : Unkan hecetu nunwe amen, winohinca kin eye kta.

23 Unkan wawayuśna kin woyaśice kin he wowapi wan akan owa kta, qa mini pa kin on pajaja kta.

$24 \mathrm{Qa}$ winolinca kin mini pa taku tehika icalye cin he yatke kiye kta: Unkan mini taku telika icahye cin he $\tan$ mahen iyaye ça pa kta.
25 Hehan wawayuśna kin wowinawizi minlia kin winohinca nape etanhan icu kta, qa Jehowa itokam yuwinte kta, qa wahna wośnapi ekta kahi kta.

26 Qa wawayuśna kin minha etanhan nape ohnaka wan wokiksuye on icu kta, qa wahna wośnapi izinye kta, hehan winohinca kin mini yatke kiye kta. 27 Unkan mini yatke kiye cin iyohakam kaketu kta, he ihduśape ca hihnaku nahmana awahtani unkanś, mini taku tehika icaliye cin tan mahen iyaye ça pa kta, heon tezi kin po kta, qa ceca kin hdi kta, unkan winohinca kin he oyate tawa ehna woyaśice kta.

28 Tuka winohinca kin ihduśape śni, qa ska kinhan he kiunniye kte śni. tuka cinca ton kta.

29 Wowinawizi woope kin hee, winohinca wan hihnaku etanhan ihduhomni qa ihduśape cinhan.

30 Nakun wowinawizi woniya wan wicaśta wan en iyaye cinhan, qa tawicu kin inakiwizi, qa Jehowa itokam najin kiye kta; unkan wawayuśna kin woope kin de owasin iyecen ecakicon kta.

31 Hecen wicaśta kin woalitani kiciyawapi kte śni, tuka winohinca iye wahtani kin hduhe kta.

\section{WICOWOYAKE 6.}

1 Unkan Jehowa ie ça Mowis heciya.

2 Israel cinca kin hecen ewicakiya wo: Wicaśta wan qaiś winohinca wan Jehowa ekta ihdukinunkan, Nazari wohdakapi on, Nazari heca kta e iciconze cinhan.

3 Miniśa qa mini wakan etanhan ihdukinunkan kta, minisku201 


\section{WICAYAWAPI,}

mna miniśa etanhan, qa mini wakan etanhan miniskumna yatke kte śni, qa taku kaśta hastanlanka en hpanyanpi kin yatke kte śni, qa haștanhanka do, qaiś puza unmanna yute kte.śni.

4 Tohanyan Nazari heca anpetu owasin hastanhanka iyuwi taku aicage cin ha etanhan qaiś su etanhan kagapi ongedan yute kte śni

5 Tohanyan Nazari iciconze cin icasan wan pa akan ye kte śni, Jehowa on ihdukinunkan ohdake cin anpetu henakeca kin hehanyan paha kin hanska icage kta.

6 Jehowa en Nazari icicu anpetu kin hena icunhan tuwe nagi ye cin en i kte śni.

7 Atkuku on, qaiś hunku on, qaiś sunkaku on, qaiś tawinolitin on, hena tapi on ihduśape kte śni. Tuwe wakan da Nazari kin iye pa akan un nakaś.

8 Tohan Nazari heca hehanyan Jehowa on wakan kta.

9 Unkan tukten yanke cin tuwe ihnuhanna en te cinhan, Nazari pa kin śape kta; unkan anpetu en yuskapi kinhan, pa hdaśda kta, anpetu iśakowin hehan ihdaśda kta.

$10 \mathrm{Qa}$ anpetu iśahdogan tinwakiyedan nonpa, qaiś wakiyedan cinca nonpa wawayuśna kin ekta kahi kta, itkokipapi wakeya tiyopa ekta.

11 Unkan wawayuśna kin wanji woahtani wośnapi kage kta, qa unman wohuhnahyapi kta, hecen wicaśta nagiye cin en wahtani kin akahpe kiciye kta, qa piya pa kin yuwakan kta, anpetu kin he en.

12 Unkan Nazari anpetu kin Jehowa on hdukinunkan kta, nakun wohdajuju on amnos wan waniyetu wanjidan kahi kta, qa 202
Nazari kin hduśape cin on anpetu tokaheya kin yawa kte śni.

13 Unkan Nazari woope kin dee tohan Nazari anpetu henakeca kinhan, anpetu kin he en itkolipapi wakeya tiyopa ekta ihdohi kta.

14 Qa toahi kin Jehowa kalıi kte, woliuhnahyapi on arnnos mdoka wan zanika, waniyetu wanjidan, qa woalitani wośnapi on amnos wiyedan waniyetu wanjidan zanika, qa wopida wośnapi on tamdoka wanjidan zanika.

15 Qa minha tawapi qa woakaśtan tawapi, qa aguyapi napohyapi śni, aguyapi mdu hinca on kagapi, wakiśkotpa ohnaka wan aguyapi hmiyanyan wihdi icicahiyapi, qa aguyapi napohyapi śni wihdi on sdayapi.

16 Unkan wawayuśna kin hena Jehowa itokam kahi kta, qa woahtani wośnapi, qa wohuhnahyapi kin wośna kta.

17 Qa tamdoka kin wopida wośnapi kin Jehowa wakiyuśna kta, aguyapi napohyapi śni wakiśkotpa kin iyahna, qa wawayuśna kin minha kin, qa woakaśtan kin kage kta.

18 Unkan Nazari kin pa kin hdaśda kta, itkokipapi wakeya tiyopa ekta, qa Nazari heca paha kin peta wopida wośnapi ihukuya un kin he akan ehpe kiye kta.

19 Hehan wawayuśna kin tamdoka isto spanyanpi kin icu kta, nakun wakiśkotpa etanhan aguyapi napohyyapi śni wanjidan, qa Nazari nape akan ehnake kta, Nazari heca kaśdapi iyohakam.

$20 \mathrm{Qa}$ wawayuśna kin hena Jehowa itokam yuwinte kta; woyuwintapi wakan hee, wawayuśna tawa kta, maku yuwinta- 
pi kici, qa hiyete kozapi kici. Unkan he iyohakam Nazari kin miniśa yatke kta okihi.

21 Nazari woope kin hee; tuwe Jehowa ekta ihdukinunkan kta oyaka, woahi kahi kte cin taku nape kin iyohiye cin iyahna, token oyake cin he iyecen kahi kta, Nazari woope kin he kici.

22 Unkan Jehowa ie ca Mowis heciya.

23 Aaron qa iye cinca kin hecen ewicakiya wo. Israel cinca kin hecen wicadawaśtepi kta, qa hewicayakiyapi kta.

24 Jehowa niyawaśte, qa nipatan :

25 Jehowa ite kin iyoyam niciya, qa onśinida kta ;

26 Jehowa ite kin anitonwe ca wookiye nicu nunwe.

27 Hecen micaje kin Israel cinca kin awicahnakapi kta, unkan miye wicamdawaśte kta.

\section{WICOWOYAKE $\%$.}

1 Unkan kaketu, Mowis tipi kin ehde yuśtan qehan, qa sdaye ca yuwakan, nakun taku on kicanyanpi owasin; qa wahna wośnapi, qa taku on kicanyanpi owasin, nakun hena sdaye ça yuwakan.

2 Hehan Israel itancan kin ateyapi tipi pa kin hena wakahipi, wicoun itancanpi wicayawapi icunhan en najinpi kin hena eepi.

3 Toahipi kin Jehowa itokam hiyuyapi ; canpahmihma akahpetonpi śakpe, qa tatanka akenonpa, itancan nonpa on canpahmihma wanjidan, qa otoiyohi on tatanka wanjidan, qa hena tipi kin itokam ahipi.

4 Unkan Jehowa ie ca Mowis heciya.
5 Hena icu wo, hecen itkokipapi wakeya wohtani econpi kta e hena unpi kta, qa hena Lewi kin wicayaqu kta, otoiyohi wohtani econ kin iyecen.

6 Unkan Mowis canpahmihma qa tatanka kin hena icu, qa Lewi kin wicaqu.

7 Canpahmihma nonpa, qa tatanka topa Gerśon cinca kin wicaqu, wohtani econpi kin on.

-8 Qa canpahmihma topa, qa tatanka śahdogan Merari cinca kin wicaqu, wohtani econpi kin on ; Aaron wawayuśna kin cinhintku Itamar nape kin ihukuya.

9 Tuka Qohat cinca kin takudan wicaqu śni, hena tipi wakan tawohtanipi hiyetepi akan qinpi nakaś.

10 Unkan wahna wośnapi sdayapi anpetu kin he en, itancanpi kin wahna wośnapi kin yuwakanpi kta e toahipi kin hdoipi, qa itancanpikin toahipi wahna wośnapi itokam hdoipi.

11 Unkan Jehowa Mowis heciya, Wahna wośnapi yuwakanpi kta e itancan otoiyohi anpetu wan tawakiya en toahi kin hdoi kta.

12 Unkan Nahíon Aminadab cinhintku Juda wicoun itancan kin anpetu tokaheya kin toahi kin hdoi.

$13 \mathrm{Qa}$ toahi kin mazaska wakśica wan śeqel opawinge sanpa wikcemna yamni aspeyapi, qa mazaska wakśica on amnimnipi wan śeqel wilscemna śakowin tipi wakan śeqel iyececa, napin aguyapi mdu hinca wihdi icicahiyapi on ojudan minha kin hee.

14. Mazaskazi tukiha wan śeqel,wikcemna wizinyapi on ojudan;

15 Tatanka wanji ptewanunyanpi cinca, tamdoka wanji, a203 
mnos wan waniyetu wanjidan wohuhnahyapi hee;

16 Woahtani wośnapi on tatokadan wanunyanpi mdoka wan;

17 Qa wopida wośnapi on tatanka nonpa, tahincaska mdoka zaptan, tatokadan mdoka zaptan, amnos waniyetu wanjidan zaptan; Nahison Aminadab cinhintku toaki kin hee.

18 Unkan anpetu inonpa kin Natanael Zuhar cinhintku Isakar itancan kin hdoi,

19 Qa toahi kin dee, mazaska wakśica wan śeqel opawinge sanpa wikcemna yamni aspeyapi, qa mazaska wakśica on amnimnipi wan śeqel wikcemna śakowin, tipi wakan śeqel iyececa, napin aguyapi mdu hinca wihdi icicahiyapi on ojudan, minha kin hee ;

20 Mazaskazi tukiha wan śeqel wikcemna wizinyapi on ojudan ;

21 Tatanka wanji ptewanunyanpi cinca, tamdoka wanji, amnos wanji waniyetu wanji, wohuhnahyapi hee ;

22 Tatokadan wanunyanpi mdoka wan woalitani wośnapi on ;

$23 \mathrm{Qa}$ wopida wośnapi on tatanka nonpa, tahincaska mdoka zaptan, tatokadan mdoka zaptan, amnos waniyetu wanjidan zaptan, Natanael Zuhar cinhintku toahi kin hee.

24. Anpetu iyamni Zebulun cinca itancan kin Eliab Helon cinhintku hdoi,

25 Toahi kin mazaska wakśica wan śeqel opawinge sanpa wilkcemna yamni aspeyapi, mazaska wakśica on amnimnipi śeqel wikcemna śakowin, tipi wakan śeqel iyececa, napin aguyapi mdu hinca wihdi icicahiyapi on ojudan, minha kin hee ;

26 Mazaskazi tukiha wan seqel 204 wikcemna wizinyapi on ojudan.

27 Tatanka wanji ptewanunyanpi cinca, tamdoka wanji, amnos waniyetu wanjidan wanji, wohuhnahyapi kin hee ;

28 Tatokadan wanunyanpi mdolka wanji, woalitani wośnapi on;

$29 \mathrm{Qa}$ wopida wośnapi on tatanka nonpa, tahincaska mdoka zaptan, tatokadan mdoka zaptan, amnos waniyètu wanjidan zaptan; Eliab Helon cinhintku toalii kin hee.

30 Anpetu itopa kin Ruben cinca itancan kin Elitzur Śedeur cinhintku hdoi ;

31 Toahi kin mazaska wakśica wanji sequel opawinge sanpa wikcemna yamni aspeyapi, mazaska wakśica on amnimnipi wan śeqel wikcemna śakowin, tipi wakan śeqel iyececa, napin aguyapi mdu hinca wihdi icicahiyapi on ojudan, minha kin hee ;

32 Mazaskazi tukiha wanji śeqel wikcemna wizinyapi on ojudan;

33 Tatanka wanji pte wanunyanpi cinca, tamdoka wanji, amnos wanji waniyetu wanjidan, wohuhnahyapi hee;

34 Tatokadan mdoka wanji woaktani wośnapi on.

35 Qa wopida wośnapi on tatanka nonpa, tahinca ska mdoka zaptan, tatokadan mdoka zaptan, amnos waniyetu wanjidan zaptan, Elitzur Śdeur cinhintku toahi kin hee.

36 Anpetu izaptan kin Simeon cinca itancan kin Śelumiel Tzu riśada cinhintka kin hdoi,

37 'Toahi kin mazaska wakśica wan śeqel opawinge sanpa wikcemna yamni aspeyapi, qa mazaska wakśica on amnimnipi śeqel wikcemna śakowin, tipi wakan śeqel iyececa, napin agu- 
yapi mdu hinca wihdi icicahiyapi on ojudan, minha kin hee.

38 Mazaskazi tukiha wanji śeqel wikcemna wizinyapi on ojudan ;

39 Tatanka wanji pte wanunyanpi cinca, tamdoka wanji, amnos wanji waniyetu wanjidan, wohuhnahyapi kin hee;

40 Tatokadan wanunyanpi mdoka wanji, woalitani wośnapi on : 41 Qa wopida wośnapi on tatanka nonpa, tahincaska mdolka zaptan, tatokadan mdoka zaptan, amnos waniyetu wanjidan zaptan, Selumiel Tzuriśada cinhintku toahi kin hee.

42 Anpetu iśakpe en Gad cinca itancan kin Eleasap Duel cinhintku kin hdoi.

43 Toahi mazaska wakśica wan ścqel opawinge sanpa wikcemna yamni aspeyapi, mazaska wakśica on amnimnipi wanji śeqel wikcemna śakowin, tipi wakan śeqel iyececa, napin aguyapi mdu linca wihdi icicahiyapi ojudan, minlia kin hee.

44 Mazaskazi tukiha wan seqel wikcemna wizinyapi on ojudan ;

45 Tatanka wanji pte wanunyanpi cinca, tamdoka wanji, amnos wanji waniyetu wanjidan, wohuhnahyapi kin hee.

46 Tatokadan mdoka wanji waolitani wośnapi on ;

$47 \mathrm{Qa}$ wopida wośnapi on tatanka nonpa, tahincaska mdoka zaptan, tatokadan mdoka zaptan, amnos waniyetu wanjidan zaptan, Eleasap Duel cinhintku toahi kin hee.

48 Anpetu iśakowin en Epraim cinca itancan kin Eliśama A mihud cinhintru hdoi,

49 Toahi kin mazaska wakśica wan śeqel opawinge sanpa wikcemna yamni aspeyapi, ma- zaska wakśica on amnimnipi wanji śeqel wikcemna śakowin, tipi wakan śeqel iyececa, napin aguyapi mdu hinca wihdi icicahiyapi on ojudan, minha kin hee.

50 Mazaskazi tukiha wanji śeqel wikcemna wizinyapi on ojudan.

51 Tatanka wanji pte wanunyanpi cinca, tamdoka wanji, amnos wanji waniyetu wanjidan, wohulhahyapi kin hee;

52 Tatokadan mdoka wanji woalitani wośnapi on ;

$53 \mathrm{Qa}$ wopida wośnapi on tatanka nonpa, tahinca ska mdoka zaptan, tatokadan mdoka zaptan amnos waniyetu wanjidan zaptan, Eliśama Amihud cinhintku toahi kin hee.

54 Anpetu iśahdogan Manaśe cinca itancan kin Gamaliel Pedatzur cinhintku hdoi,

55 Toahi mazaska waksica wan śeqel opawinge sanpa wikcemna yamni aspeyapi, mazaska waliśica on amnimnipi wan śeqel wikcemna śakowin, tipi wakan śeqel iyececa, napin aguyapi mdu hinca wihdi icicahiyapi on ojudan, minlia kin hee.

56 Mazaskazi tukiha wan śeqel wikcemna wizinyapi on ojudan; 57 Tatanka wanji pte wanunyanpi cinca, tamdoka wanji, amnos wanji waniyetu wanjidan, wohuhnahyapi kin hee.

58 Tatokadan mdoka wanji woahtani wośnapi on;

$59 \mathrm{Qa}$ wopida wośnapi on tatanka nonpa, tamdoka zaptan, tatokadan mdoka zaptan, amnos waniyetu wanjidan zaptan, Gamaliel Pedatzur cinhintku taohi kin hee.

60 Anpetu inapcinwanka en Benjamin cinca itancan kin Abidan Gideoni cinhintku hdoi,

61 Toahi kin mazaska wakśica 


\section{WICAYAWAPI,}

wan śeqel opawinge sanpa wik cemna yamni aspeyapi, mazaska wakśica on amnimnipi wanji śeqel wikcemna śakowin, tipi wakan śeqel iyececa, napin aguyapi mdu hinca wihdi icicahiyaji on ojudan minlia kin hee.

62 Mazaskazi tukiha wan śeqel wikcemna wizinyapí on ojudan;

63 Tatanka wanji pte wanunyanpi cinca, tamdoka wanji, amnos wanji waniyetu wanjidan, woliulinaliyapi kin hee.

64 Tatokadan mdoka wanji woahtani wośnapi on ;

65 Qa wopida wośnapi on tatanka nonpa, tamdoka zaptan, tatokadan mdoka zaptan, amnos waniyetu wanjidan zaptan; Abidan Gideoni cinhintku toahi kin hee.

66 Anpetu iwikcemna en Dan cinca itancan kin Ahiezer Amiśada cinhintku kin hdoi.

67 Toahi kin mazaska wakśica wan śeqel opawinge sanpa wi. licemna yamni aspeyapi, mazaska wakśica on amnimnipi wanji śeqel wikcemna śakowin tipi wakan śeqel iyececa, napin aguyapi mdu hinca wihdi icicahiyapi on ojudan minlia kin hee ;

68 Mazaskazi tukiha wanji śeqel wikcemna wizinyapi on ojudan ;

69 Tatanka wanji pte wanunyanpi cinca, tamdoka wanji, amnos wanji waniyetu wanjidan, wohulinahyapi kin bee;

70 'Tatokadan mdoka wanji. woalitani wośnapi on;

$71 \mathrm{Qa}$ wopida wośnapi on tatanka nonpa, tahinca ska mdoka zaptan, tatokadan mdoka zaptan, amnos waniyetu wanjidan zaptan, Ahiezer Amiśada cinhintku toahi kin hee.

72 Anpetu iakewanji Aśer cin206 ca itancan kin Pagiel Okran cinhintku kin hdoi.

73 'Toahi kin mazaska wakśica wan śeqel opawinge sanpa wikcemna yamni aspeyapi, mazaska wakśica on amnimnipi śeqel wikcemna śakowin tipi wakan śeqel iyececa, napin aguyapi mdu hinca wihdi icicahiyapi on ojudan, minha kin hee.

74 Mazaskazi tukiha wanji śeqel wikcemna wizinyapi on ojudan ;

75 Tatanka wanji pte wanunyanpi cinca, tamdoka wanji, amnos wanji waniyetu wanjidan, wohulinahyapi kin hee;

76 Tatokadan mdoka wanji woahtani wośnapi on;

$77 \mathrm{Qa}$ wopida wośnapi on tatanka nonpa, tamdoka zaptan, tatokadan mdoka zaptan, amnos waniyetu wanjidan zaptan $\mathrm{Pa}$ giel Okran cinhintku toahi kin hee.

78 Anpetu iake nonpa Naptali cinca itancan kin Ahira Enan cinhintku kin hdoi.

79 Toahi kin mazaska wakśica wan śeqel opawinge sanpa wikcemna yamni aspeyapi, mazaska walkśica on amnimnipi vvanji śeqel wikcemna śakowin, tipi wakan śeqel iyececa, napin aguyapi mdu hinca wihdi icicahiyapi on ojudan, minha kin hee.

80 Mazaskazi tukiha wan śeqel wikcemna wizinyapi on ojudan ;

81 Tatanlia wanji pte wanunyanpi cinca, tamdoka wanji, amnos wanji waniyetu wanjidan, wohuhnahyapi kin hee.

82 Tatokadan mdoka wanji woahtani wośnapi on ;

$83 \mathrm{Qa}$ wopida wośnapi on tatanka nonpa, tamdoka zaptan, tatokadan mdoka zaptan, amnos waniyetu wanjidan zaptan, Ahi- 


\section{WICOWOYAKE 8.}

ra Enan cinhintku toahi kin hee.

84 Wahna wośnapi sdayapi qehan Israel itancanpi etanhan yuwakanpi kin dee, mazaska wakśica akenonpa, mazaska waliśica on amnimnipi akenonpa mazaskazi tukiha akenonpa,

85 Wakśica tanka otoiyohi śeqel opawinge sanpa wikcemna yamni, qa wakśica on amnimnipi otoiyohi wikcemna śakowin, wakśica owasin mazaska kin tke utapi kin śeqel kektopawinge nonpa sanpa opawinge topa, tipi wakan śeqel iyececa.

86 Mazaskazi tukiha wikcemna sanpa nonpa wizinyapi on ojudan, otoiyohi śeqel wikcemna, tipi wakan śeqel iyececa, tukiha owasin mazaskazi śeqel opawinge sanpa wikcemna nonpa;

87 Wohuhnahyapi oll tatanka wanunyanpi owasin wikcemna sanpa nonpa, tamdoka wikcemna sanpa nonpa, amnos waniyetu wanjidan wikcemna sanpa nonpa, minha tawapi iyahna, qa woahtani wośnapi on tatokadan mdoka wikcemna sanpa nonpa ;

$88 \mathrm{Qa}$ wopida wośnapi on tatanka wikcemna nonpa sanpa topa, tamdoka wikcemna śakpe, tatokadan mdoka wikcemna śakpe, amnos waniyetu wanjidan wikcemna śakpe ; wahna wośnapi sdayapi iyohakam on yuwakanpi kin hee.

89 Unkan kici wohdake kta e.Mowis itkokipapi wakeya ekta i eca, ho kin nahon, woyaatanin can wohnaka iwankapa tanhan taku eciya, kerubi nonpa otahedan etanhan taku eciya.

\section{WICOWOYAKE 8.}

1 Unkan Jehowa ie ca Mowis heciya,
2 Ie ca Aaron hecen eciya wo, Petijanjan yuwankan yeyaya eca, petijanjan śakowin kin petijanjan ihupa itokapa tanhan iyoyamyapi kta.

3 Unkan Aaron hecen econ petijanjan oide kin petijanjan ihupa itokapa tanhan wankan ye kiya: Jehowa Mowis hecon śi kin he iyecen.

4 Unkan petijanjan ihupa kin kaken kagapi, mazaskazi pagopi, ceca kin, qa adetka kin nakun pagopi: token Jehowa Mowis wanyagkiye ciqon he iyecen petijanjan ihupa kagapi.

5 Unkan Jehowa ie ca Mowi heciya,

6 Lewi kin Israel cinca wicehna tanhan iwicacu, qa wicayuska wo.

7 Qa wicayuskapi kta e hecen ecawicayecon kta, Mini on wicayuskapi kin he akan ehpeya wo; unkan ukapi kin owancaya hdasanpi kta, qa wokoyake hdujajapi kta, hecen skapi kta.

8 Unkan tatankadan pte wanunyanpi cinca wan icupi kta, qa minha tawa aguyapi mdu wihdi icicaliyapi, unkan tatanka inonpa pte wanunyanpi cinca kin iyacu kta woahtani wośnapi on.

9 Qa itkokipapi wakeya itokam Lewi kin ikiyedan awicayahi lkta, qa Israel cinca omniciye owasin yuwitaya hiyu wicayaye lita.

10 Qa Lewi kin Jehowa itokam awicayahi kta; unkan Israel cinca napepi kin Lewi kin awicaputakapi kta.

11 Unkan Aaron Lewi kin wicayuwinte kta, Jehowa itokam, wokosyapi wan Israel cinca etanhan, hecen Jehowa okiyapi kta e wiyeya unpi kta.

12 Unkan Lewi kin napepi ta207 
tanka pa akan awicatputakapi kta, unkan wanji woalitani wośnapi, qa unman wohulinaliyapi Jehowa kicaga wo; hecen Lewi akahpe wicakiciyapi kta.

13 Unkan Aaron itokam, qa iye cinca itokam, Lewi kin najin wicayaye $\mathrm{kta}$, qa wicaduwaśte kta, Jehowa tawokosyapi.

14 Israel cinca wicehna tanhan Lewi kin wicadukinunkan kta, hecen Lewi kin mitawa kta.

15 Unkan he iyohakam Lewi kin itkokipapi wakeya wohtani econpi kta e en upi kta; wicaduska, qa wokosyapi on wicaduwinte cinhan.

16 Hena ocowasin maqupi nakaś, Israel cinca wicehna tanhan winyan owasin cinca toliapa hee kiya, Israel cinca caskepi owasin hee kiya, liena mitawa kta e iwicawacu.

17 Israel cinca ehna wicaśta woteca koya, tona caskepi owasin mitawa, Egupta makoce ohna caskepi owasin wicawakaśtaka, anpetu lin he en hena miciwahduwakan nakaś.

18 Unkan Lewi kin wanna iwicawacu, Israel cinca caskepi owasin hee kiya.

$19 \mathrm{Qa}$ Aaron, qa iye cinca hena yuhapi kta e Israel cinca wicehna tanhan Lewi kin wicawaqu, itkokipapi wakeya ohna Israel cinca tolitanipi econpi kta, qa Israel cinca akalipe wicakiciyapi kta, hecen Israel cinca kin tipi wakan ikiyedan hipi kte śni qa wicehna makośice wanice kta, ikiyedan hipi unkanś makosice yuke kta tuka.

20 Unkan Mowis, qa Aaron, qa Israel cinca omniciye owasin, token Jehowa Mowis Lewi kin ecawicakicon wicaśi kin owasin, he iyecen Israel cinca Lewi kin esawicakiconpi.
21 Unkan Lewi kin ihduskapi, qa wokoyake hdujajapi, unkan Aaron wicayuwinta, Jehowa itokam, wokosyapi wan, qa wicayuska kta e Aaron akahipe wicakiciya.

22 Unkan he iyohakam, Lewi kin itkokipapi wakeya ohna wohtani econpi kta e Aaron itokam, qa iye cinca itokam hipi. Token Jehowa Mowis Lewi kin ecawicakicon śi kin he iyecen ecawicakiconpi.

23 Unkan Jehowa ie ça Mowis heciya.

24 Lewi kin kaken econpi kta, waniyetu wikcemna nonpa sanpa zaptan aktonpi kin hena itkokipapi wakeya en htanipi kin obe en opapi lita.

25 Qa waniyetu wikcemna zaptan aktompi kin hena wohtani obe kin ayuśtanpi kta, qa icimanna en hitanipi kte śni,

26 Tuka sunka wicayapi om taku awanyakapi kin awanyakapi kta e itkokipapi wakeya ohna apeya unpi kta, qa wolitani takudan econpi kte śni, Lewi kin taku awanyakapi kin icahtaka hecen ecawicayecon kta.

\section{WICOWOYAKE 9.}

1 Unkan Israel cinca Egupta makoce etanhan hdinanpapi, waniyetu inonpa wi tokaheya en, Sina hopuza ohna, Jehowa ie ça Mowis heciya.

2 Iyehantu eca Israel cinca woacakśin econpi kta.

3 Wi kin de anpetu iake topa en hitayetu otahedan he yakagapi kta, iyehantu kinhan tokage owasin, qa tawoyaco owasin iyecen he ecanonpi kta.

4 Unkan Mowiś Israel cinca kin woacakśin kagapi kta kewicakiya. 
5 Unkan wi tokaheya anpetu iake topa en, ohtayetu otahedan, Sina hopuza ohna, woacakśin kin kagapi : token Jehowa Mowis econ śi kin owasin hecen Is-. rael cinca kin econpi.

6 Unkan wicaśta wanjigji, wicaśta wan nagi ye cin on śapapi, heon anpetu kin he en woacakśin kagapi okihipi śni, heon anpetu kin he en Mowis itokam, qa Aaron itokam hinajinpi.

7 Qa wicaśta kin hena heciyapi, Unkiyepi wicaśta wan nagi ye cill on unśapapi, tokeca heyatá iyeunyanpi on iyehantu eca, Israel cinca wicehna Jehowa woahi wan unkahipi kte śni he?

8 Unkan Mowis lewicakiya, Kakiya najinpo, Token Jelıowa econ niśipi kte cin he nawahon kta.

9 Unkan Jehowa ie ça Mowis heciya,

10 Israel cinca kin hecen ewicakiya wo, Niyepi ehna, qaiś nitoicagepi yeye cin ehna, wicaśta tuwe kaśta wicaśta wan nagiye cin on śapa, qaiś tehan icimani un eśta, woacakśin kin Jehowa kicage kta,

$11 \mathrm{Wi}$ inonpa anpetu iake topa en ohtayetu otahedan he kage kta, aguyapi napohyapi śni kici, qa peji pa kici he yute kta.

12 Ongedan hanhanna aiyahdeya ohdaptapi kte śni, qa hetanhan huhu wanjidan kaś kawegapi kte śni, woacakśiı wokage owasin iyecen heconpi kta.

13 Unkan wicaśta tuwe ska qa icimani un śni, qa woacakśin kage kapin kinhan, wicanagi kin he iye taoyate etanhan kaśpapi kta, iyehantu Jehowa toahi kin kahi śni, heon wicaśta kin he woahtani kin hduhe $\mathrm{k}$ ta.

14 Unkan wicaśta wan oyate tokeca niyepi om ounye ca woa- cakśin kin Jehowa kicage kte cinhan, woacakśin wokage kin, qa woyaco kin owasin iyecen he kage kta. Wicaśta oyate tokeca on, qa tona makoce en tonpi kin on wokage wanjidan duhapi kta.

15 Unkan tipi kin ehdepi anpetu kin "he en malipiya kin tipi kin akahpa, woyaatanin wakeya kin hee, qa htayetu eca tipi iwankam peta iyececa tanin, ihanlianna hehanyan.

16 Ohinniyan hececa mahpiya kin he akahpe ca hanyen peta iyececa tanin.

17 Unkan tohan mahpiya kin wakeya iwankapa tanhan wankan iyaya, hehan Israel cinca ihdakapi, qa tukten mahpiya kin owanji najin kin, heciya Israel cinca kin etipi.

18 Jehowa i kin eciyatanhan Istael cinca kin ihdakapi, qa Jehowa i kin eciyatanhan tipi ehdepi; tona can mahpiya kin tipi kin iwankam yanka hehanyan ihdakapi śni.

19 Qa mahpiya kin tipi iwankam yanka anpetu ota kinhan, Israel cinca taku Jehowa avacin wicaśi kin he awacinpi, qa ihdakapi śni.

20 Unkan he iyecen mahpiya kin anpetu tonana tipi iwankam yanke cinhan, Jehowa i kin eciyatanhan owanji unpi, qa Jehowa i eciyatanhan ihdakapi.

21 Unkan mahpiya kin hitayetu etanhan ihanhanna hehanyan owanji yanke ça hanhanna yuwankan iyaye cinhan ihdakapi, anposkan qa hanyen unman tukte kaśta mahpiya kin wankan iyaye cinhan ihdakapi.

22 Anpetu nonpa, qaiś wi wanjidan, qaiś sanpa anpetu ota eśta, mahpiya tipi kin iwankam yanke cinhan, hehanyan 


\section{WICAYAWAPI,}

Israel cinca kin wakeya ohna owanji unpi, qa ihdakapi śni ; tuka tohan yuwankan iyaya hehan ihdakapi.

23 Jehowa i kin eciyatanhan wakeya iticagapi, qa Jehowa i eciyatanhan ihdakapi, Jehowa taku awacin wicaśi kin he awacinpi, token Mowis nape on Jehowa econśi kin he iyecen.

\section{WICOWOYAKE 10.}

1 Unkan Jehowa ie ca Mowis heciya,

₹ Mazaska yahotonpi nonpa icicaga wo, pagopi hena yalkage kta, qa omniciye eyanwicayapaha kta, qa oyate ihdag wicayakiye kta e hena duhe kta.

3 Unkan napin on yahotonpi kinhan omnieiye ocowasin niye en yuwitaya hipi kta, itkokipapi wakeya tiyopa ekta.

4. Qa wanjidan on yahotonpi kinhan, itancanpi Israel kektopawinge en tokapapi kin niye en mniciyapi kta,

5 Qa panyanhanhan dahotonpi kinhan, oyate iwiyohiyanpata tipi kin ihdakapi kta.

6 Qa ake panyanhanhan dahotonpi kinhan, oyate itokaga tipi kin ihdakapi kta; ihdakapi kta e panyanharhan yahotonpi kta.

7 Qa oyate kin yuwitaya mniciyapi lite cinhan dahotonpi kta, tuka panyanhanhanpi kte śni.

8 Unkan Aaron cinca wawayuśnapi, kin maza yahotonpi kin yahotonpi kta, nitoicagepi yeye cin wokage kin owihanke wanica duhapi kta.

9 Unkan toka niyanpi niciyuśepi kin nitaritakocepi ohna azu wicayayapi kinhan, mazayahotonpi kin panyanhanhan ho dahotonpi kta; unkan Jehowa wakan ya210 dapi kin itokam niksuyapi kta, qa toka niyanpi etanhan enihdakupi kta.

10 Unkan anpetu widuśkinpi, qa mniniciyapi kin en, qa wi nitawapi anpetu tokaheya en mazayahotonpi kin hoton yayapi kta, wohuhinahyapi nitawapi iwankam, qa wopida wośnapi nitawapi iwankam, qa hena on tuwc wakan yadapi itokam niksuyapi kta, Jehowa wakan yadapi kin he miye.

11 Unkan waniyetu inonpa wi inonpa wi anpetu wikcemna nonpa en, mahpiya kin wayaatanin tipi iwankapa tanhan wankan iyaya.

12 Unkan Israel cinca kin unhdakapi iyecen Sina hopuze kin etanhan ihdakapi; unkan mahpiya kin Paran hopuze kin en iyotanka.

13 Hehan tokaheya Mowis nape eciyatanhan Jehowa ie cin iyecen ihdakapi.

14 Unkan Juda cinca wicoti obe wiyokiye kin tokaheya ihdakapi, qa obe tawa mdetanhunka kin Nahson Aminadab cinhintku kin hee.

15 Unkan Isakar cinca wicoun mdetanhunka lin Netaneel Tzuhar cinhintku kin hee.

16 Unkan Zebulun cinca wicoun mdetanhunka kin Eliyab Helon cinhintku kin hee.

17 Helian tipi wakan kin yujujupi qa Gerśon cinca kin, qa Merari cinca kin tipi kin yuha ihdakapi.

18 Hehan Ruben wicoti obe wiyokiye ihdakapi, qa obe tawa mdetanhunka kin Elitzur Śdeur cinhintku kin hee.

19 Unkan Simeon cinca wicoun mdetanhunka kin śelumiel Tzuriśada cinhintku kin hee.

$20 \mathrm{Qa} \mathrm{Gad}$ cinca wicoun mde- 
tanhunka kin Eleasap Duel cinhintku kin hee.

21 Hehan Qohati kin taku yuwakanpi kin yuha ihdakapi, qa nahanhin ekta kipi śni unmanpi tipi kin ehdepi.

22 Hehan Epraim cinca wicoti wiyokiye kin ihdaka obe tawapi kin iyecen, qa obe tawa mdetanhunka kin Amihud cinhintku Eliśama hee.

23 Unkan Manaśe cinca wicoun obe mdetanhunka kin Gamaliel Pedatzur cinlintku kin hee.

24. Unkan Benjamin cinca wicoun obe mdetanhunka kin Abidan Gideoni cinhintku kin hee.

25 Hehan wicoti owasin hakam Dan cinca wicoti wiyokiye ihdaka, obe tawa kin iyecen, qa obe tawa mdetanhunka kin Ahiezer Amiśada cinhintku kin hee.

26 Unkan Aśer cinca wicoun obe mdetanhunka kin Pagiel Okran cinhintku kin hee.

27 Unkan Naptali cinca wicoun obe mdetanhunka kin Ahira Enan cinhintku kin hee.

28 Israel cinca obepi ihdakapi eca hecen ihdakapi.

29 Unkan Mowis Hobab Raguel cinhintku Midiani kin, Mowis tahanku kin heciya, Unkiyepi oyanke wan Jehowa cajeyate ça he cicupi kta unkekiyapi heciya unkihdakapi, Iho opa wo, kinhan taku waśte ecaunniconpi kta, Jehowa Israel taku waśte icaje wicayate nakaś.

30 Unkan, Mde kte śni, tuka makoce mitawa, qa wicowazi mitawa ekta wahde kta, eciya.

31 Unkan tokin ehpe unyayapi śnin. Niye hopuze kin sdonyaya tukten euntipi kta, hecen iśta unniyanpi kta.

32 Qa unkiyepi om idade cinhan kaketu kta, token Jehowa taku waśte ecaunkiconpi, he iyecen taku waśte ecaunniconp̧ kta,eya.

33 Unkan Jehowa he tawa etanhan ihdakapi, anpetu yainn: canku yeyapi, unkan Jehowa wicotakuye can wohnake kin wicitokam iyaya anpeta yamni canku yapi kin, tukten etipi kta iwanwicakiciyake kta heon etanhan.

34 Qa wicotipi etanhan ihdakapi eca, Jehowa tamahpiya kin anposkan wiciwankan un.

35 Unkan kaketu can wohnaka kin iyaya eca Mowis heya, Jehowa najin ye, qa toka niyanpi kin enanakiya iyayapi kta, qa tona śicenidapi kin nitokapa tanhan najicapi kte.

36 Unkan tipi ehdepi eca, Jehow a hdi ye Israel kektopawinye ota kin ekta, eya.

\section{WICOWOYAKE 11.}

1 Unkan oyate kin Jehowa noge ohna śicaya śicahowayapi Jehowa nahon qehan canniyan, qa Jehowa tapeta aide wicaye ca wicoti ihanke en huhnah wicaya.

2 Unkan oyate kin Mowis hoye kiyapi; unkan Mowis Jehowa cekiya, hecen peta kin anapta.

3 Unkan oyanke kin he Tabera (Wohuhnaga) eya caje yata; hen Jehowa tapeta hulinah wicaya nakaś.

4 Wanna wicota yuwitaya hipi, qa owicapapi kin hena nina wakonpi ; unkan Israel cinca kin nakun ake ceyapi qa heyapi, "Tuwe tado yun unkiyapi kta hwo.

5 Fgupta ohna hogurs imnahan untapi hin he unkiksuyapi; nakun saka yutapi ocaje nunpa, qa pśin ocaje yamni. 
6 Tuka wanna unnagipi śeca nya, takudan wanunyakapi śni, manna kin de lieceedan.

7 Unkan manna kin korianna su kin hiuskokeca, qa wanyakapi kın inyan wicaśake iyececa tanin.

8 Unkan. oyate kin enanakiya iyayapi, qa he mnayanpi, qa wiyukpan ohna yukpanpi, qaiś wibope en bopanpi, qa cega ohna śpanyanpi, qaiś aguyapi hmiyanyanria cahinta ohna śpanyanpi, qa utapi kin aguyapi skuya wilidi icicayapi utapi kin he iyececa.

9 Unkan cu kin hanyen wicoti ahiulipaye çehan manna kin he akan hiyu.

10 Unkan Mowis oyate kin wicowazipi kin ecekcen ceyapi nawicalion, wicaśta otoiyohi tawakeya tiyopa uhna, unkan Jehowa nina śihda nakun Mowis he śicedaka.

11 Unkan Mowis Jehowa heciya, Tokeca nitaokiye śicaya icayecon, qa tokeca nitokam woiyokipi iyewaye śni he? oyate kin de iyuhpa wicaqin mayakiya he?

12 Miye nyate kin de iyuhpa cinca wicawaton he? qaiś miye cinca wicawakaga on, Itpi ohna wicayuha wo, wicaśta wan iye cinhintku azin hduhe cin he iyeccn emayakiya, hecen makoce wan hunkake wicayapi wicayaqu kta yakonze cin heciya awicande kta.

13 Tokiya tanhan tado mduhe ca oyate kin de iyulipa wicawaqu kta he? wanna amaceyapi, qa, Tado unqu po, hecen untapi kta, eyapi

14 Oyate kin de miśnana mduha kta owakihi śni, waqin kta isanpa the nakaś.

15 Hecen ecamiyecon kinhan, 212 tokin te mayayen, woiyokipi nitokam iyewaye cinhan, hecen mitoiyokiśice cin wanwahdake kte śni.

16 Unkan Jehowa Mowis heciya, Oyate hunkayapi etanhan wicaśta wikcemna śakowin mniciyc wicakiya wo, miye ckta, wicaśta oyate kin hunka wicayapi heca sdonwicayaya, qa itancan hecapi, qa itkokipapi wakeya ekta hiyn wicayaye kta; unkan niye $k$ ci hen najinpi kta.

17 Unkan miś kun wau, qa hen nici wowahdake kta, qa woniya niye en un kin etanhan emdaku kta, qa hena en ye waye kta; unkan niśnana oyate kin wicaduha kte śni, hena nici wicay uhapi kta.

18 Unkan oyate kin hewicayakiye kta, Heyake cinhan on ihduwakan po; hecen tado yatapi kta; yaceyapi, qa Tuwe tado yun unkiyapi kta he? Egupta uhna tanyan unyakonpi ehapi kin he Jehowa noge kin iyolipaya, heon Jehowa tado niçupi kta, qa yatapi kta.

19 Anpetu wanjidan yatapi kte śni, qa anpetu nonpa kte śni, qa anpetu zaptan kte śni, qa anpetu wikcemna kte śni, qa anpetu wikemna nonpa kte śni;

20 Tuka wi wanji anpetu owasin hehanyan, nipogepi etanhan inanpe eqa hitiyahdapi kte cin hehanyan; Jehowa niyepi ehna un kin he ilayaktapi śni, qa yaeeyapi qa, Tokeca Egupta etanhan inanunpapi he? ehapi, heon etanhan.

21 Unkan Mowis heya, Oyate ehna waun kin de kektopawinge opawinge śakpe ozuye huiyunpi ; unkan tado wicawaqu lita, qa wi wanji ihunniyan yutapi kta, eha.

24 Tahinca qa pte wicakipa- 
tapi kta, qa hecen ryowicahi kta? qaiśs ininiyowancaya hogan owasin mnawicakiciyapi kta, qa hecen iyowicahi kta he?

-23 Unkan Jehowa Mowis heciya, Jehowa nape kin kaksapi he? Dehan mioie ecetu niciye kta, qaiś ecetu niciye kte śni hecinhari wandake kta.

24. Unkan Mowis tankan inanpe ca Jehowa oie kin oyate kin owicakiyaka, qa oyate hunkawicayapi etanhan wicasta wikcemna śakowin mnawicaya, qa wakeya qon ihdukśan najin wicakiya.

25 Unkan Jehowa mahpiya śapa wan ohna kun hiyu, qa kici wohdake ca woniya iye en un kin hetanhan icu, qa hunkayapi wicaśta wikcemna sakowin qon akan awicahnaka; unkan woniya kin aozi wicaye cehan waayatapi, tuka icimana econpi śni.

26 Tuka wicaśta nompa wicoti ohna owanji yakonpi, wanji Eldad eciyapi, qa unman Medad eciyapi, qa woniya kin iyawicahan, qa hena owicawapi qon en opapi, tuka wakeya qon ekta ipi śni, qa hena wicoti ohna waayatapi.

27 Unkan kośka wan inyanke ca Mowis okiyake ça, Eldad qa Medad wicoti ohna waayatapi ce, eya.

28 Unkan Nun cinlintku Jośuwa; Mowis taokiye, kośka kalinigapi heca wan ayupte ça heya, Itancan Mowis wicakiśica wo.

29 Unkan Mowis heciya. Miye on etanhan winayawizi he? Tokin Jehowa taoyate kin iyulipa wayatapin, qa Jehowa toniya kin owasin wicaqun.

30 Unkan Mowis wicoti kin ekta kihda, iye qa Israel hunkawicayapi.
31 Unkan tateyanpa wan Jehowa eciyatanhan iyaye ca mde kin akotanhan śiyo icu, qa wicoti akan, qa wicoti ihdukśin anoka tanhan, anpetu wan imanipi kin hehanyan ahinhe wicaya, wiciśpa nonpa maka akan.

32 Unkan oyate kin najinpi, anpetu kin he usan, qa hanyetu osan, qa ihanlianna anpetu osan, qa śiyo kin mnayanpi; tuwe aopten mnayan eśta opaha wikcemna muyan, qa hduhapi kta e wicoti ihdukśan hena pceyapi.

33 Unkan conica nalianhin hipi ohna yanka, yasotapi śni itokam Jehowa tocanniye oyate kin aide wicaya, qa Jehowa makośice teliike hinca on oyate kin a wicapa.

34 Unkan oyanke kin he Qiberot Hatawa (Akaskapi kin wicahnakapi,) eya. cajeyatapi ; oyate akaskapi kin hen wicahnakapi nakaś.

35 Unkan Qiberot Hatawa etanhan ihdakapi, Hazerot ekta, qa Hazerot en yakonpi.

\section{WICOWOYAKE 12.}

1 Hehan Miriam qa Aaron Mowis kigepi, Kuśi winohinca yuze cin heon etanhan, Kuśi winohinca wan yuza nakaś.

2 Qa heyapi, Awicakehan Jehowa Mowis iśnana ie kiya he? Unkiś nakun ie unkiyapi śni he? unkan Jehowa he nahon.

3 Wanna wicaśta maka akan hiyeye cin owasin Mowis iśnana wicaśta wahbadan.

4. Unkan ihnuhanna Jehowa Mowis, Aaron, qa Miriam hewicakiya; Upo niyamnipi itkokipapi wakeya ekta, Unkan hena yamni ekta ipi.

5 Unkan Jehowa mahipiya bo213 
sdata ohna kun hiyu, qa itkokipapi wakeya tiyopa ekta yanke ca Aaron qa Miriam wicakipan; unkan napin ekta inanpapi.

6 Unkan heya, Mioie kin nahonpo, Niyepi ehna waayate wan yuke cinhan, iye en Jehowa mihdutanin kta wowanyake en, qa wowihamde en taku ewakiye kta.

7 Tuka mitaokiye Mowis iś hecece śni, wati ocowasin ohna iye wacinye pica.

8 He i i kici wowahdake taninyan, qa hanmdohdake śniyan, qa Jehowa ouncage kin he atonwan, heon tokeca mitaokiye Mowis ayaiapi kin koyakipapi śni he?

$9 \mathrm{Qa}$ Jehowa canniye wicaye ca kihda.

10 Qa mahpiya kin wakeya kin etanhan wankan iyaya; unkan inyun Miriam leprosi ececa, wa iyececa, unkan Aaron Miriam ekta etonwan, unkam leprosi ececa.

11 Hehan Aaron Mowis heciya, Itancan ceciya, tokin woaltani kin de ecinśniyan waunhtanipi kin aunkihnakapi śni miyen.

12 Tokin taku hunku tezi kin etanhan ta inanpe ca cehpi hanke sote cin iyecece kte śnin.

13 Unkan Mowis Jehowa hoyekiye ca heya, Iyotan waśaka ceciciye, wanna okiziya ye.

14 Unkan Jehowa Mowis heciya, Atkuku kin he ite kin atagośa unkanś anpetu śakowin iśtece kte śni he? anpetu śakowin wicoti itankan natakapi kta, qa ohakam en hdi kta.

15 Unkan anpetu śakowin hehanyan Miriam wicoti itankan natakapi, qa Miriam en hdi śni kin hehanyan oyate kin ihdakapi śni.
16 Unkan ohakam oyate kin ihdakapi, Hazerot etanhan, qa Paran lopuza en etipi.

\section{WICOWOYAKE 13.}

1 Unkan Jehowa ie ca Mowis heciya,

2 Kanan makoce Israel cinca wicawaqu kin he iwanniciyakapi kta e wicaśta ye wicaśi wo. Ate wicayapi wicoun otoiyohi etanhan wicaśta wan ye yaśi kta, hena wicehna owasin itancanpi hecapi.

3 Unkan Mowis Jehowa i kin eciyatanhan Paran hopuze kin etanhan ye wicaśi, wicaśta kin hena owasin Israel cinca mdetanhunka hecapi.

4 Qa cajepi kin dena eepi, $\mathrm{Ru}$ ben wicoun etanhan Śsamuha Jakur cinhintku.

5 Simeon wicoun etanhan Ś́apat Hori cinhintku.

6 Juda wicoun etanhan Kaleb Jepune cinhintku.

7 Isakar wicoun etanhan Igal Josep cinhintku.

8 Epraim wicoun etanhan Hośeya Nun cinhintku.

9 Benjamin wicoun etanhan Patti Rapu cinhintku.

10 Zebulun wicoun etanhan Gadiel Sodi cinhintku.

11 Josep wicoun on Manaśe wicoun etanhan Gadi Susi cinhintku.

12 Dan wicoun etanhan Ammiel Gemali cinhintku.

13 Aśer wicoun etanhan Setur Mikael cinhintku.

14 Naptali wicoun etanhan Nahibi Wapsi cinhintku.

15 Gad wicoun etanhan Guel Maki cinhintku.

16 Wicaśta Mowis makoce kin iwanyag ye wicaśi cajepi kin hena eepi. Qa Mowis Hośeya 
Nun cinhintku kin Jehośuwa eya cajeyata.

17 Unkan Mowis Kanan makoce kin iwanyag ye wicaśi, qa hewicakiya, Deciya itokaga en yapo, qa lie kin itan wankanhde yapi. $18 \mathrm{Qa}$ makoce kin he toketu wandakapi kta, nakun oyate en ounyanpi kin, waśakapi, qaiś wankapidan unman tukte hecinhan, nakun tonana, qaiś otapi.

19 Qa makoce ounyanpi kin he waśte qaiś śica, qa otonwe otipi kin hena, wakeya otipi, qaiś conkaśke otipi.

20 Qa makoce kin cepa qaiś tamaheca unman tukte, ohna can yukan qaiś can wanica. Waditakapo, qa maknce waskuyeca kin etanhan icupo, eya. Unkan he ehan hastanhanka toka suton.

21 Unkan iyayapi qa makoce kin iwanyakapi, 'Tzin hopuza etanhan Hamat ektakiya yapi, Rehob ekta hehanyan.

$22 \mathrm{Qa}$ itokaga en ipi, qa Hebron ekta hipi. Unkan Anaq cinca Ahiman, Seśa, qa Talma hen yakonpi. Unkan Tzohan Lgupta ohna kagapi he itokam waniye tu śakowin Hebron kagapi.

23 Unkan Eśkol kaksiza kin en hipi, qa hetanhan adetra wan kaksapi, qa hastanbanka iyage wanjidan, qa cansagye wan alkan akiyuhapi, nakun taspantanka su ota, qa witanśnan etanhan akiyuhapi.

24 Oyanke kin he Eśkol (Iyage) kalssiza eciyapi, Israel cinca kin hastanhanka iyage wan hetanhan kaksapi heon etanhan.

25 Unkan anpetu wikcemna topa hehan makoce iwanyakapi kin etanhan hdipi.

26 Hecen iyayapi qa Mowis ekta, qa Aaron, qa Israel cinca omniciye ocowasin ekta hdipi,
Qadeś, Paran hopuza ohna un kin ekta, qa hośi wicakahdipi, qa makoce waskuyeca wicakipazopi.

27 Qa okiyakapi qa heyapi, Makoce wan ekta ye unyaśipi kin he ekta unkipi, qa awicakehan pteasanpi qa canhanpi tiktica ohna kaduza, qa waskuyeca aicage cin dee.

28 Hececa eśta oyate makoce ounyanpi kin waśakapi, qa otonwe tawapi kin aconkaśkapi, qa nina tankinyanyan. Nakun Anaq cinca kin heciya wanwicunyakapi.

29 Amaleqi makoce itokah ounyanpi, qa Heti kin qa Jebusi kin qa Amori kin He kin en ounyanpi, qa Kanani miniwanca icahda, qa Jordan omdaye kin ounyanpi.

30 Unkan Kaleb Mowis itokam oyate inina yanke wicasi, qa heya, Itanwankanhde unyanpi, qa unhduhapi kta iyececa, ohiunyanpi kta waunśakapi.

31 Tuka wicaśta iye kici iyayapi qon lieyapi, Oyate kin he ekta itanwankanhde unyanpi unkokihipi śni; hena unkiyepi isanpa waśakapi nakaś.

32 Qa makoce iwanyag ipi qon Israel cinca itokam aiapi, qa heyapi, makoce ohna iyaunyuptapi, qa iwanunyakapi kin tona ounyanpi kin wicayasota, qa oyate olina wanunyakapi kin owasin wicaśta hanskaskapi.

33 Qa hen waziya wanwicunyakapi Anaq cinca kin, hena waziya etanhan icagapi qa wicitokam psipsicadan iyeuncecapi.

\section{WICOWOYAKE 14.}

1 Unkan omniciye ocowasin hoyeyapi, qa oyate kin hanyetu kin he ceyapi. 


\section{WICAYAWAPI,}

2 Qa Israel cinca owasin Mowis qa Aaron aiwicahnupi, qa omniciye owasin hena hewicakiyapi, Tokin Egupta makoce ohna untapin, qaiś hopuza kin de ohna unțapin.

3 Qa tokeca Jehowa makoce kin de ekta hiyu unyanpi, hecen canhipi on unhinhpayapi kta, unkitawinpi, qa unkicincapi nakun wayaka unpi kta? Egupta ekta unhdapi kinhan waśte kta naceca.

4 Unkan hekiciyapi, Mdetanhunka wan unkagapi, qa Egupta ekta unhdapi kta.

5 Unkan Mowis qa Aaron makipusdiya hinhpeiciyapi, Israel cinces omniciye owasin wicitokam.

6 Unkan Jośuwa Nun cinhintku, qa Kaleb Jepune cinhintku makoce iwanyakapi etanhanpi wokoyake hdulidelidecapi.

7 Qa Israel cinca omniciye owasin hewicakiyapi, makoce iwanyakapi kta e iyaunyuptapi kin he makoce wan nina waśte hinca.

8 Jehowa iyounkipipi kinhan, makoce kin he ekta ye unkiyapi kta, qa makoce pte asanpi qa canhanpi tiktica kaduze cin he unqupi kta.

9 Takomni ihnuhan yakipajinpi kin, qa makoce oyate kin ihnuhan ikowicayapapi kin aguyapi unkitawapi iyececapi lita, taku wacinyanpi kin he elpewicaya; tuka Jehowa unkicipi un. Ihnuhan ikowicayapapi kin.

10 Unkan omniciye owasin inyan on wicaktepi kta keyapi qehan, Jehowa towitan kin itkokipapi wakeya akan Israel cinca owasin ekta ihdutanin.

11 Unkan Jehowa Mowiś heciya, Tohanyan oyate kin de ihamaktapi kte śni he? qa to216 hanyan wowapetokeca wicehna ecamon on wicamadapi kte śni he?

12 Makośice on awicawape ça ihang wicawaye kta, qa niye etanhan oyate wan tanka, qa hena isanpa waśaka wakage kta.

13 Unkan Mowis Jehowa heciya, Kinhan Egupton he nahonpi kta, hena wicehna tanhan nitowaśake tanka on oyate kin de hiyu wicayaya.

14 Qa makoce kin de ounyanpi kin hewicakiyapi, Niye Jehowa oyate kin de wicehna yaun, qa niye Jehowa iśta kin iśtapi en nihdutanin, malipiya bosdata wiciwankan, qa anposkan mahipiye bosdata ohna wicitokam idade, qa hanyetu eca peta bosdata ohna, he wanna nationpi.

15 Unkan oyate kin de wicaśta wanjidan iyecen te wicayaye cinhan, oyate niye on wotanin nahonpi kin hecen eyapi kta.

I6 Jehowa makoce wakicons owicakiyake ciqon, he ekta hiyu wicaye kta okihi śni, heon hopuza ohna wicakte.

17 Tokin, itancan towaśake kin tanka kten, kehe ciqon he. ivecen.

18 Jehowa tehan silihda śni, tocantekiye tanka; wicolian śica, qa wokipajin kajuju ece, tuka ohinniyan kajuju kte śni, ateyapi wicohan śicapi kin wicoicage iyamni, qa itopa kin hehanyan awicahi ece.

19 Tokin oyate kin de wahtanipi kin wicakicajujun, nitowacantkiye tanka kin iyecen, qa Egupta etanhan dehanyan oyate kin dc wicayecicajuju kin he iyecen.

20 Unkan Jehowa heya, wicawecicajuju, ehe cin he iyecen. 
21 Qa hececa eśta wani kin iyecen Jehowa towitan kin maka owancaya ojudan ye kta.

22 Takomni wicaśta kin tona mitowitan wanyakapi, nakun wowapetokeca ecamon Egupta ohna, qa H்opuza ohna, qa wikcemna akihde iyutan mayanpi, qa maho kin nahonpi śni kin hena owasin,

23 Makoce hunkake wicayapi kin Wakicons owicawakiye cin he wanyakapi lste śni ; ihamaktapi śni dkin hena etanhan wanjidan he wanyakapi kte śni.

24. Tuka mitaokiye Kaleb woniya tokeca yuhe cin heon, qa ohinniyan mihakam u, heon makoce ekta i qon he ekta hiyu waye kta, qa iye cinca kin he tawayapi kta.

25 Wanna Amaleqi qa Kanani kaksiza ohna yakonpi, heon heyakecinhan ihdamnapi, qa Mdeśa ekta-kiya, Hopuza ohna ihdaka po.

26 Unkan Jehowa ie ça Mowis, qa Aaron hewicakiya;

27 Tohanyan omniciye sice kin de imahnupi kin wicawecicajuju kta he? Israel cinca wihnupi imahnupi kin he nawation ce.

28 Hecen ewicakiya wo. Jehowa heya, Wani kin hecen token ehapi nawaron kin he iyecen ecaciconpi kta.

29 Hopuza kin de ohna nitancanpi kin hinhpayapi kta, tona niyawapi, oniwapi qon iyecen waniyetu wikcemna nonpa aktonpi, tona imahnupi owasin etanhan;

30 Ohna ounyayapi kta nape hduwankan iwacu qon awicakehan makoce kin he en yaipi kte śni; tuka Kaleb Jepune cinhintku qa Jośuwa Nun cinhintku kin henana en ipi kta.

31 Nakun nitaśicecapi kin wa- yaka unpi kta kehapi qon, hena ekta awicamde kta, unkan makoce ihayaktapi śni kin he sdonyapi kta.

32 Tuka niyepi nitancanpi kin hopuze kin den hinhipayapi kta.

33 Tuka nicincapi hopuza ohna wiliankiya unpi $k t a$, waniyetu wikcemna topa, qa wawiciliahapi ecanonpi kin hdajujupi kta, nitancanpi kin owasin hopuza ohna sotapi kte cin hehanyan.

34 Tona can makoce iwanyakapi kin anpetu wikcemna topa anpetu otoiyohi on waniyetu wan yawapi kta, waniyetu wikcemua tom, hehanyan wayahtanipi kin yahdajujupi kta, qa mitocanuiye kin sdonyayapi kta.

35 Jehowa miye hepa, omniciye śice cin de miye en amamniciyapi kin awicakehan hecawicawecon kta, hopuza kin de ohna sotapi, qa tapi kta.

36 Unkan wicaśta Mowis makoce iwanyag ye wicaśi, qa makoce śicaya cajeyan hdipi kin heon omniciye ocowasin aihnu wicayapi.

37 Wicaśta makoce śicaya cajeyatapi kin hena makośice on Jehowa itokam tapi.

38 Unkan makoce iwanyag ipi qon hena etanhan Jośuwa Nun cinhintku, qa Kaleb Jepune cinhintku henana nipi.

39 Unkan Mowis wicoie kin hena Israel cinca omniciye owasin owicakiyaka; unkan oyate kin nina liin iyokiśin içiyapi.

40 Unkan hanhanna hin kiktapi qa heyapi, Hopo, oyanke Jehowa cajeyate kin he ekta itanwankanhde unyanpi kte, waunhtanipi nakaś. Qa he paha kin ekta itanwankanhde ipi.

41 Tuka Mowis hewicakiya, Tokeca decen Jehowa oie kin 217 
acayakśinpi he? He ohna tanyan iyahunipi kte śni.

42 Itanwankanhde yapi śni po, Jehowa niyepi ehna un śni heon, hecen toka niyanpi kin wicitokam anipapi kte śni.

43 Amaleqi qa Kanani kin heciya nitokam yakonpi, unkan canhpi on nihinhpayapi kta. Jehowa etanhan nihdamnapi, heon Jehowa nicipi un śni.

44. Unkan lie paha ekta-kiya ihaktapi śniyan itanwankanhde yapi, tuka Jehowa wicotakuye can-wohnaka kin, qa Mowis wicoti cokaya tanhan tokidan ipi śni.

45 Unkan Amaleqi qa Kanani he kin he ounyanpi kin apamahdedan upi qa wicakaśtakapi, qa wicaktepi Horma ekta hehanyan.

\section{WICOWOYAKE 15.}

1 Unkan Jehowa ie ca Mowis heciya.

2 Ic ca Israel cinca kin hecen ewicaliya wo, makoce ounyayapi kta e cicupi kin he ekta yaipi kinhan.

3 Qa peta wośnapi wan Jehowa yecagapi, wohuhnahyapi, qaiś woyakapi hdajujupi wośnapi, qaiś taku ikce qupi on, qaiś mniniciyapi on, woomna Jehowa iyokipi yecagapi kinhan, pte etanhan qaiś tahinca etanhan.

4. Tuwe woahi Jehowa kahi kinhan, minha wan aguyapi mdu can wakśsica ohnaka wan wihdi wiyatke ohnaka wan icicahiyapi ahi kta ;

5 Qa woakaśtan on miniśa -wiyatke ohnaka wan wohulinaliyapi kin, qaiś wośnapi kin ayakaśtan kta e amnos otoiyohi on ;

$6 \mathrm{Qa}$ tamdoka wanjidan on aguyapi mdu can wakśica ohna- ka nonpa, wihdi wiyatke ohnaka wanji sanpa hanke icicahiyapi minha yecage kta.

7 Qa miniśa wiyatke ohnaka wanji sanpa hanke woakaśtan yakahi kta, woomna Jehowa iyokipi.

8 Qa pte wanunyanpi cinca wohuhnahyapi qaiś woyakapi hdajuju wośnapi, qaiś wopida wośnapi Jehowa yecage kte cinhan ;

9 Pte cinca wan on aguyapi mdu can wakśica ohnaka.yamni, wihdi wiyatke ohnaka nonpa icicahiyapi minha kahi kta.

10 Qa woakaśtan on miniśa wiyatke ohnaka nonpa yakahi kta, peta wośnapi woomna Jehowa iyokipi.

11 Hccen econpi kta tatanka otoiyohi on, qa tamdoka otoiyohi on, qa tacincadan on, tahinca ska qaiś tatokadan cincadan on.

12 'Tona waduśnapi kin iyenaka yawapi iyecen otoiyohi on hecen ecanonpi kta.

13 Tona makoce en tonpi kin hena owasin hecen econpi kta, peta wośnapi woomna Jehowa iyokipi kahipi eca.

14 Unkan tuwe oyate tokeca niyepi ehna ihdaka, qaiś niyepi ehna ounye cinhan nitoicagepi yeye cin, qa peta wośnapi waśtemna Jehowa iyokipi kage cinhan token niye ecanonpi kin he iyecen econ kta.

15 Omniciye, wokage wanjidan kta, niyepi on, qa tuwe oyate tokeca niyepi ehna yanke cin on ; wokage owihanke wanica nitoicagepi yeye cin, Jehowa itokam token yaunpi kin he iyecen tuwe oyate tokeca kin un kta.

16 Niyepi on, qa tuwe oyate tokeca niyepi ehna ounye cin on woope wanjidan, qa woyaco wanjidan kta. 
17 Unkan Jehowa ie ca Mowis heciya,

18 Ie ca Israel cinca kin hecen ewicakiya wo, Makoce wan ekta ye ciyapi kin he ekta yaipi kinhan ;

19 Qa makoce taku aicage cin yatapi kinhan, wokosyapi wan Jehowa yaqupi kta.

20 Taku tokaheya boyapanpi kin etanhan aguyapi hmiyanyanna wokosyapi wan yaqupi kta, aguyapi su kapanpi wokosyapi kin he iyecen, he yakozapi kta.

21 Tokaheya boyapanpi etanhan wokosyapi wan Jehowa yaqupi kta, nitoicagepi yeye cin.

22 Unkan ecinśniyan wayahtanipi, qa woahope kin dena Jehowa Mowis eciye cin owasin ecanonpi śni kinhan;

23 Taku Jehowa Mowis nape on econ niśipi owasin, anpetu wan Jehowa Mowis taku econ śi kin hetanhan, qa tokata nitoicagepi yeye cin ohna ecanonpi śni kinhan;

24. Qa omniciye sdotkiyapi śni taku ecinśniyan econpi kinhan, omniciye ocowasin tatanka wan pte wanunyanpi cinca wohuhnahyapi kage kta, woomna waśte Jehowa iyokipi, minha kin iyahna, qa nakun woakaśtan kin woyaco kin iyecen, nakun tatokadan mdoka wan woahtani wośnapi on.

25 Unkan wawayuśna kin Israel omniciye ocowasin akahpe wicakiciye kta, unkan wicakicicajujupi kta, ecinśniyan econpi nakaś, qa toahipi peta wośnapi kin Jehowa kahipi, qa ecinśniyan econpi Jehowa itokam on woahtani wośnapi nakun,

26 Hecen oyate iyuhpa sdonye śni econpi heon Israel cinca, qa tuwe oyate tokeca wicehna ti kin omniciye ocowasin wicakicicajujupi kta.

27 Unkan wicanagi wan ecinśniyan wahtani kinhan, tatokadan wiyedan waniyetu wanjidan wahtani kin on ahi kta.

28 Unkan wawayuśna kin wicanagi ecinśniyan wah̆tani kin akahpe kiciye kta, ecinśniyan wahtani wośnapi Jehowa itokam akahpe kiciye lita on, unkan kicicajujupi kta.

29 Tona ecinśniyan wahtanipi on woope wanjidan duhapi kta Israel cinca makoce tawayapi on, qa tuwe oyate tokeca niyepi ehna ti kin on.

30 Unkan wicanagi tuwe wahan-içidaya taku śica econ kin he makoce tawayapi etanhan, qaiś oyate tokeca etanhan, wicanagi kin he Jehowa kipajin, heon oyate tawa etanhan kaśpapi kta. 31 Jehowa oie kin ihak ta śni, qa tawoahope kin iśtenya, heon wicanagi kin he tolian śica hduha taoyate kin etanhan laśpapi kta.

32 Unkan Israel cinca Hopuza en yakonpi qehan, wicaśta wan anpetu wakan icunhan, cande kin iyeyapi.

$33 \mathrm{Qa}$ he cande iyeyapi kin hena Mowis ekta, qa Aaron ekta, qa omniciye ocowasin ekta kahipi.

34 Qa kaślka hnakapi, token ecakiconpi kta, nahahin owicakiyakapi śni nakaś.

35 Unkan Jehowa Mowis heciya, Wicaśta kin awicakehan te kta, omniciye ocowasin, wicoti itankan, inyan on kininpi kta.

36 Unkan omniciye ocowasin wicoti itankan inanpe yapi, qa inyan on kininpi, Jehowa Mowis econ si kin he iyecen, unkan ta. 37 Unkan Jehowa ie ca Mowis heciya. 


\section{WICAYAWAPI,}

38 Israel cinca owawicakiye ca wicoicag epi yeye cin tawokoyakepi opapun ekta olicilici içicagapi lita, qa opapun ohcilici akan hahonta to wan pahtapi kta, kewicakiya wo.

39 Qa he wowapetogton duhapi kta, qa wandakapi kta, qa Jehowa tawoahope owasin yeksuyapi qa ecanonpi kta, hecen nicantepi opeya, qa miiśtapi opeya yanunipi kte śni, hena opeya wiyacinpi ece qon iyecen.

40 Hecen mitawoahope owasin yeksuyapi qa oyapapi kta, qa tuwe wakanyadapi taoyate wakan yaunpi kta.

41 Jehowa Wakan yadapi kin he miye, qa Wakan mayadapi kta e Egupta makoce etanhan hdinanpe ciyapi, Jehowa Wakanyadapi kin he miye.

\section{WICOWOYAKE 16.}

1 Unkan Qora Itzar cinhintku Qohat cinhintku Lewi cinhintku, qa Datan qa Abiram Eliab cinca kin, qa On Pelet cinhintku Ruben cinca etanhan wohdakapi.

2 Qa Mowis anajinpi, nakun Israel cinca etanhan wicaśta opawinge nonpa sanpa wikcemna zaptan omniciye itancanpi, wicaśta okiniharupi, omniciye ekta wicakicopi hecapi.

3 Qa Mowis Aaron kici amniciyapi, qa hewicakiyapi, Omniciye ocowasin wicaśta otoiyohi owasin wakanpi, qa Jehowa wicacokaya un; heon tokeca Jehowa taomniciye iwankam ye niciyapi, qa niyepi on taku ota yacinpi hie?

4 Unkan Mowis he nation qehan makipusdiya ehpe iciya.

5 Qa Qora, qa okodakiciye tawa owasin hewicalkiya, Hantianna kinhan Jehowa tuwe tawa kin, qa tuwe wakan, qa tuwe iye ikiyedan hiyuye cin hdutanin kta, ya tuwe kahinige cin he iye ekta ikiyedan hiyuye kta.

6 Decen econ po, Qora qa okodakiciye tawa owasin oizinye icupo.

7 Qa hena ohna peta ehnaka po, qa akan wizinyapi akada po. Jehowa itokam heyakecinhan, hehan wicasta tuwe Jehowa kahnige cin hee wakan kta; Lewi cinca niyepi on taku ota yacinpi.

8 Nakun Mowis Qora heciya, Lewi cinca kin namahonpo.

9 Taku Israel wakandapi kin Israel omniciye etanhan niyepi niyukinunkanpi, iye ikiyedan hiyu niyanpi, Jehowa ti wohtani kin ecanonpi kta, qa omniciye itokam nayajinpi qa owicayakiyapi kta, he taku śni yadapi he?

10 Nakun niye qa nihunkawanji Lewi cinca owasin niye kici ikiyedan hiyuniyanpi: unkan nakun wawayuśnapi henicapi k ta e ayakitapi he?

11 Hecen niye qa okodakiciye nitawa owasin Jehowa en amniniciyapi, tuka Aaron taku econ hwo on he iyahnupi.

12 Unkan Mowis tuwe Datan qa Abiram Eliab cinca wicakico ye śi qon, unkan, Ekta unyanpi kte śni eyapi.

13 Hopuza ohna te unyayapi kta, makoce wan pte asanpi qa canhanpi tilktica. ohna kaduze cin etanhan inanpe unyayapi kin he taku śni yada, heon unkiyepi iwankam wicaśta yatapi itancan nicicage kte he?

14 Hiya makoce pte asanpi qa canhanpi tiktica ekta hiyu unyayapi śni qa maga qa hastanhanka iyuwi ojupi tawa un- 
yanpi kta e unyaqupi śni, Wicaśta kin dena iśtapi kin duśdoke kta he? Ekta unyanpi kte śni ce.

15 Unkan Mowis nina canteptanyan, qa Jehowa heciya, Minha tawapi kin ihakta śni ye. Hena etanhan śuktanka śonśonna wanjidan iwacu śni, qa iyepi wanjidan wakimduśe śni.

16 Unkan Mowis Qora heciya, Heyakecinhan niye qa okodakiciye nitawa owasin Jehowa itokam u po, niye qa iyepi qa Aaron.

17 Qa niyepi otoiyohi oizin e ikikcupo, qa hena akan wizinyapi akada po, qa wicaśta opawinge nonpa sanpa wikcemna zaptan oizinyapi ayahipi kta, Jehowa itokam; nakun niye qa Aaron otoiyohi oizinya tawa hduha po.

18 Unkan otoiyohi oizinye ikikcupi, qa peta aehnakapi, qa wizinye akadapi, qa itkokipapi wakeya tiyopa ekta najinpi, nakun Mowis qa Aaron.

19 Unkan Qora okodakiye tawa iyuhpa mnawicaya, itkokipapi wakeya tiyopa ekta; unkan Jehowa towitan kin omnicrye ocowasin en ihdutanin.

20 Unkan Jehowa ie ca Mowis Aaron kici hewicakiya,

21 Omniciye kin de etanhan ihdukinunkanpo, kinhan ihnuhanna wicamdusote kta.

22 Tuka makipusdiya ehpeiciyapi, qa heyapi, Iyotan Waśaka wicacehpi owasin toniyapi wakan nidapi, wicaśta wanjidan wahtani kin heon omniciye iyutipa canniye wicayaye lita he?

23 Unkan Jehowa ie ça Mowis heciya.

24 Ie ça oyate kin Qora qa Datan qa Abiram totipi ildukśan tanhan tokan iyaya po, ewicakiya wo.

25 Unkan Mowis najin qa Datan qa Abiram yakonpi kin ekta i, qa Israel hunkayapi kin ihakam ipi.

26 Qa ie ca oyate kin hewicakiya, Ihopo, wicaśta śice kin dena tawakeyapi etanhan tokan iyaya po; qa ihnuhan taku tawapi kin wanji dutanpi kin. Hecanonpi kinhan okini hena wahtanipi owasin ohna ihang niyanpi kta.

27 Unkan Qora Datan qa Abiram tipi kin ihdukśan tanhan tokan iyayapi; Unkan Datan qa Abiram inanpapi qa tawakeyapi tiyopa ekta najinpi, tawicupi qa cinhintkupi qa cunwintkupi nakun.

28 Unkan Mowis heya, wicohan kin dena owasin ecamon kta Jehowa u maśi, qa miye cinka eciyatanhan śni, détanhan sdonyayapi kta.

29 Wicaśta owasin token tapi kin he iyecen wicaśta kin dena țapi kinhan, qa wicaśta owasin taku akipapi kin dena akipapi linhan Jehowa u maśi śni.

30 Tuka Jehowa taku teca wan kage ca inaka kin i hdukawa, qa nawicapca, nakun taku tawapi kin iyulipa hecen niyake Hades ekta kun yapi kinhan, wicaśta kin dena Jehowa wahtedapi śni sdonyayapi kta.

31 Unkan wicoie kin hena owasin eya hdaśtan qehan, maka kin wicihukuya naptuja ;

32 Qa maka kin i kin hdukawa, qa nawicapca, qa tipi kin nakun, qa Qora wicaśta tawa owasin, nakun taku tawapi kin owasin.

33 Hecen iyepi qa taku tawapi owasin Hades ekta niyake ipi, qa maka kin okahpe wicaya, he221 
cen omniciye etanhan awihnunipi.

34 Unkan Israel wicihdukśan yakonpi kin owasin hena śicahowayapi etanhan najicapi, qa heyapi, Okini maka kin naunpcapi kta.

35 Unkan peta Jehowa etanhan inanpe ca wicaśta opawinge nonpa sanpa wikcemna zaptan wizinyapi ahipi qon hena hulinah wicaya.

36 Unkan Jehowa ie ca Mowis heciya,

37 Eleazar Aaron wawayuśna cinhintku kin heciya wo. $\dot{H} u-$ hnali wicayapi wicehna tanhan oizinyapi icu, qa peta kin kakiya ehpeya wo; hena wakan nakaś.

38 Qa wicaśta iye nagipi kin en walitanipi kin hena oizinyapi tawapi kin kamdayapi kta, qa on wahna wośnapi kin akalipapi kta, hena Jehowa itokam ahipi, heon hena wakan; heccn Israel cinca kin hena wowapetogton yuhapi kta.

39 Unkan Eleazar wawayuśna kin mazaśa oizinyapi kin wicaśta liuhnal wicayapi ahipi gon icu, unkan wahna wośnapi akahpapi kta kamdayapi.

40 Israel cinca kin he wokiksuye yapi, hecen wicaśta tokcca Aaron cinca hetanhan śni wizinyapi izinye kta e Jehowa itokam ikiyedan hi kte śni, qa Qora qa okodakiciye tawa iyecece kte śni ; Jehowa Mowis nape eciyatanhan eciye ciqon he iyecen.

41 Unkan ihanhanna Israel cinca omniciye ocowasin Mowis qa Aaron iwicahnupi qa heyapi, Niyepi Jehowa taoyate te wicayayapi.

42 Unkan omniciye kin Mowis qa Aaron amniciyapi qehan, Itkokipapi wakcya ektakiya etonwanpi; unkan inyun, ma222 lipiya qon he akalipa qa Jehowa towitan kin ilıdutanin.

43 Unkan Nowis qa Aaron itrokipapi wakeya itokam hipi.

44 Unkan Jehowa ie ca Mow is heciya.

45 Oyate kin wicehna tanhan ihdukinunkan po, kinhan ihnuhanna wicamdasote kta. Unkan makipusdiya ilipayapi.

46 Unkan Mowis Aaron heciya, Oizinye ikikcu qa wahna wośnapi akan tanhan peta aonpa wo, qa wizinyapi akada wo, qa oyate ekta, kohanna aya wo, qa akalipe wicakiciya wo. Jehowa etanhan wocanniye iyaya, makośice wanna en wicahi.

47 Unkan Mowis eye cin he iyecen Aaron icu, qa omniciye wicacokaya inyanka; unkan inyun makośice wanna en wicahi. Unkan wizinyapi akada qa akahpc wicakiciya.

48 Unkan tapi qa nipi kin otahedan inajin qehan makośice kin anapta.

49 Wicaśta kektopawinge wikcemna sanpa kektopawinge topa sanpa opawinge śakowin makośica on tapi, Qora oie on țapi qon isanpa.

50 Unkan makośice anapte cin ohakam Aaron Mowis en hdi itkokipapi wakeya tiyopa ekta.

\section{WICOWOYAKE 17.}

1 Unkan Jehowa ie ca Mowis heciya,

2 Israel cinca kin kewicakiya, qa hena etanhan cansakadan icu wo, ateyapi tipi otoiyohi on cansakadan, ateyapi itancanpi owasin on, cansakadan wikccmna sanpa nonpa, wicaśta otoiyohi caje kin cansakadan tawa akan oyawa kta.

3 Qa Lewi cansakadan tawa 
akan Aaron caje kin oyawa kta, ateyapi tipi mdetanhunka iyohi on cansakadan wanjidan $\mathrm{kta}$.

4 Qa hena itkokipapi wakeya ohna, woyaatanin itokam, tukten itkocicipapi eca, hen eyahnake kta.

5 Unkan wicasta tuwe wakalinige cin he cansakadan tawa lin hica kta, hecen Israel cinca imahnupi kin anawapte lita, niyepi inihnupi kin hena.

6 Unkan Mowis Israel cinca kin ecen ewicakiya ; unkan itancanpi owasin cansakadan qupi itancan otoiyohi on cansakadan wanjidan, ateyapi tipi itancan iyohi cansakadan wan, cansakadan akenonpa, qa Aaron cansakadan tawa cansakadan tawapi ehna.

7 Unkan Mowis cansakadan kin hewa ehnake Jehowa itokam itkokipapi wakeya ohna.

8 Unkan ihanliamna Mowis wayaatanin wakeya elkta hi, unkan inyun Aaron cansakadan tawa Lewi ti kin on camni uye ca walica inanpe ye ça hmaska suton.

9 Unkan Mowis cansakadan lkin owasin hiyuya, Jehowa itokapa tanhan Israel cinca owasin ekta, unkan wanyakapi, qa otoiyohi cansakadan ikikcupi.

10 Unkan Jehowa Mowis heciya, Aaron cansakadan tawa woyaatanin itokam ake kihnaka wo, qa he yapatan kta; wowapetokeca wolkipajin cinca kin on, hecen imahnupi ayaśtan wicayakiye kta, qa țapi kte śni.

11 Unkan token Jehowa" econ sil kin he iyecen Mowis econ.

12 Unkan Israel cinca kin iapi qa Mowis heciyapi, unțapi untakunipi śni owasin untakunipi śni.

13 Tona Jehowa ti kin ikiye- dan hipi kin owasin tapi ; unțapi on unsotapi kta he?

\section{WICOWOYAKE 18.}

1 Unkan Jehowa Aaron heciya, niye qa nicinca, qa. niyate tiwahe kin nakun tipi wakan woahtani kin duhapi kta, qa niye qa nicinca kin wawaduśnapi woalitani kin duhapi kta.

2 Nakun nihunkawanjipi kin Lewi wicoun, ateyaya wicoun kin nici hiyuwicaya wo. Hecen onicipapi kta, qa oniciyapi kta; tuka niye qa nicinca kin woyaatanin tiyopa ikiyedan yaunpi kta.

3 Qa wakeya kin ocowasin patanpi kin, qa taku patan wicayaśi kin patanpi kta ; tuka tipi wakan wakśica kin, qa wahna wośnapi kin yutanpi kte śni, hecen tapi kte śni, iyepi; qa niyepi nakun.

4 Unkan onicipapi kta, qa itkokipapi wakeya kin wopatanpi kin patanpi kta, wakeya wolitani kin owasin, tuka tuwe tokeca nicipi ikiyedan hi kte śni.

5 Unkan niyepi tipi wakan awanyakapi kin duhapi kta, nakun wahna wośnapi wopatankin, hecen wocanniye Israel cinca kin icimanna awicahi kte śni.

6 Unkan ito miye nihunkawanji Lewi kin Israel cinca wicehnatanhan iwicawacu, qa taku ikcequpi wan he iyecen hena nicupi, Jehowa on itkokipapi wakeya wolitani kin econpi kta, heon etanhan.

7 Tuka niye qa nicinca om wowayuśnapi kin yakpatanpi kta, taku wahna wośnapi ekta econpi owasin, qa taku cokaya ozanpi mahen econpi, qa wohtani kin ecanonpi kta; taku ikce qupi wan iyecen wowayuśnapi wo223 
htani kin cicupi, qa tuwe tokeca ikiyedan hi kinhan teyapi kta.

8 Unkan Jehowa Áaron heciya, Iho miye wokosyapi patanpi kin cicu, Israel cinca taku yuwakanpi owasin etanhan cicu, wiyutapi nitawa, qa nicinca kin tawapi kta, wokage owihanke wanica.

9 Taku walsan hinca peta etanhan okaptapi kin de niye nitawa kta, woahi tawapi owasin, minha tawapi owasin, qa woahtani wośnapi kin owasin, qa tohdajujupi makahipi kin owasin, wakan hinca hee, niye on, qa nicinca on.

10 Tipi wakan hinca mahen hena yatapi kta, tona wica owasin hetanhan yutapi kta; niye on he wakan kta.

11 De nakun nitawa kta, -wokosyapi, Israel cinca taku yuwintapi owasin etanhan, taku qupi kin, he ciçu, qa nicinkśi, qa nicunkśi nakun opapi kta; wokage owihanke wanica on, oyate ohna tona skapi owasin hetanhan yutapi kta.

12 'Taku iyotan waśte wihdi etanhan, qa miniśa etanhan, qa aguyapi etanhan taku iyotan waśte, taku tokaheya suton Jehowa qupi kin hena ciçu.

13 'Tamakocepi ohna taku tokaheya suton Jehowa kahipi kin nitawa lita; tona skapi yati ohna hetanhan yutapi kta.

14 Israel ohna taku yuwakanpi hinca owasin nitawa kta.

15 Tona caske celipi owasin etanhan Jehowa kahipi, wicaśta qaiś woteca unman tukte kaśta niye nitawa kta. Tuka wicaśta caske kin opekiton wicayakiye kta, nakun wanunyanpi śapa cinca tokapa kin opeton wicayakiye kta. 16 Unkan opekitonpi kta wi hna. wanji tonpi ehentanhan opeyeton kta, dawa kin he iyecen, mazaska śeqel zaptan tipi wakan śeqel iyececa, omnica wikcemua nonpa śeqel wanjidan.

17 Tuka pte tokapa, qa tahincaska tokapa, qa tatokadan tokapa kin opekitonpi kte śni; hena wakanpi, wepi kin wahna wośnapi akan ayakaśtan kta, qa waśinpi kin izinyaye kta, peta wośnapi waśtemna Jehowa iyokipi.

18 Tuka celipipi kin he nitawa kta, maku kosyapi kin, qa hiyete etapa kin he iyecen nitawa kta.

19 Israel cinca taku yuwakanpi kin owasin etanhan, taku Jehowa ekta kozapi wokosyapi owasin ciçu, niye, qa nicinkśi, qa nicunkśi nakun, wokage kin de owihanke wanica miniskuya wicotakuye ohinniyan Jehowa itokam, he niye nitawa kta, qa nakun tona niye etanhan icagapi he tawapi kta.

20 Unkan Jehowa Aaron heciya, Makoce tawapi ohna maga tawayaye kte śni, qa wicehna makoce onśpa duhe kte śni; Israel cinca wicehna wopamni nitawa, qa taku tawa yaye kte cin he miye.

21 Unkan ito Lewi cinca Israel ehna iwikcemna kin owasin tawayapi kta e wicawaqu, wolitani econpi kin on, iyuwin itkokipapi wakeya wohtani kin on.

22 Hecen Israel cinca kin icimana itkokipapi wakeya ekta hipi kte śni, okini woahtani hduhapi kta, qa tapi kta.

23 'I'uka Lewi kin itkokipapi wakeya wolitani kin econpi kta, qa iyepi wahtanipi hduhapi kta, wokage kin de owihanke wanica, nitoicagepi yeye cin maga tawayapi kte śni, Israel cinca wice- 
24. Tuka Israel cinca taku icahyapi etanhan iwikcemna kin Jehowa kahipi wokosyapi kin he Lewi kin wicawaqu, he tawa yapi kta, heon hewicawakiya Israel cinca wicehna maga tawayapi lite śni.

25 Unkan Jehowa ie ça Mowis heciya,

26 Ie ca Lewi kin hecen ewicayakiye $\mathrm{kta}$, Israel cinca etanhan iwikcemna maga heekiya cicupi kin hena iyacupi eca hena etanhan Jehowa tawokosyapi kin yakahipi kta, iwikcemna etanhan iwikcemna kin.

$27 \quad$ Qa woknsyapi nitawapi aguyapi su kapanpi oyanke etanhan icupi he iyecen, qa miniśa hastanhanka yuśkica etanhan he iyecen niciyawapi kta.

28 Hecen niyepi nakun Jehowa tawokosyapi yakahipi kta; iwikcemna Israel cinca nicupi kin owasin etanhan Jehowa wokosyapi kin Aaron wawayuśna kin yaqupi kta,

29 Taku nicupi kin owasin etanhan Jehowa wokosyapi iyohi yakahipi kta, taku iyotan waśte taku yuwakanpi etanhan.

30 Unkan hewicayakiye kta, Taku iyotan waśte yakahipi eca he Lewi wicakiciyawapi kta, maga taku aicage cin, qa hastanhanka iyuwi taku aicage cin he iyecen.

31 Unkan he yatapi kta tukte en yaunpi kta, niyepi qa tona yatipi ohna unpi kta itkokipapi wakeya ohna wolitani ecanonpi on iyuwin nicupi kin hce.

32 Qa he etanhan taku iyotan waśte yakahipi kinhan woahtani yahduhapi kte śni, qa Israel cinca taku, yuwakanpi kin duikcekapi kte śni, qa nițapi kte śni.
WICOWOYAKE 19.

1 Unkan Jehowa ie ca Mowis qa Aaron hewicakiya,

2 Wokage woope Jehowa econ niśipi kin dee, qa heya, Israel cinca kin kewicayakiye kta, Pte he-yuktanna hinśa wan zanika tuktedan ośteke śni, cannapinpi tohinni un kiyapi śni, heca icupi qa nicahipi kta, kehe kta.

3 Qa Eleazar he yaqupi kta, qa wicoti itankan ayapi kta, qa iye itokam patapi kta.

4 Unkan Eleazar wawayuśna kin napsukaza oll we kin etanhan icu kta, qa itkokipapi wakeya tiyopa ektakiya we lin etanhan śakowin akihde ehpeye kta.

5 Unkan iye iśta kin itokam pte-heyuktanna kin liuhnahyapi kta; ha kin, qa tado kin, qa we kin, qa cesdi kin hulinaliyapi kta. 6 Unkan wawayuśna lin hanteśadan can, pejiliota, qa talincaska hin duta icu kta, qa pteheyuktanna lubnage cin akan ehpeye kta.

7 Qa wawayuśna kin wokoyake hdujaja kta, qa celipi minin iyeiçye kta, qa ohakarn wicoti en hi kta, qa litayetu kte cin hehanyan śapc kta.

8 Qa tuwe he hulinaliye cin he wokoyake hdujaja kta, qa cehpi -kin minin niwc kta, qa ilitayetu hehany an śape kta.

9 Unkan wicaśta sape śni wan pte heyuktanna caliota kin mnaye kta, qa wicoti itankan kihnake kta, oyanke wan śape śni en, qa he patanpi kta mini awicamnimni kin on, Israel cinca oyate on woalitani ipakinte cin hee.

10 Unkan tuwe pte-heyuktanna cahota mnaye cin he woko- 
yake hdujaja kta qa htayetu kte cin hehanyan śape lita; qa he Israel cinca on qa tuwe oyate tokeca wicehna ounye cin on en yanke $\mathrm{kta}$, wokage kin de owihanke wanice kta.

11 Tuwe wicaśta nagiye cin cehpi yutan kinhan, anpetu śalowin śape kta.

12 Qa he on ikpakinte kta, anpetu iyamni en, hecen anpetu iśakowin en ska kta, tuka anpe1u iyamni en ikpakinte śni lkinhan, anpetu iśakowin en slia kte śni.

13 Tuwe taku te cin, wicaśta nagiye ca te cin icahtake cinhan, qa ikpakinte śni kinhan, Jehowa toti kin yuśapa, qa wicanagi kin he Israel etanhan kaśpapi kta, wipakintapi mini kin he amnimnipi śni heon hee śapa, toaśape nahanhin askapa.

14 Wicaśta wan wakeya ohna te cin woope kin dee, tona wakeya ohna unpi kin owasin, nakun tona wakeya en hiyupi kin hena owasin anpetu śakowin śapapi kta.

15 Qa wakśica iha ton śni owasin, qa iha akalipapi śni kin hena śape kta.

16 Unkan tuwe wicaśta wan makoskan canhpi on ktepi, qaiś wayazanka ta, qaiś wicahu wan, qaiśs ohna wicahnakapi wan icalitake cinhan anpetu śakowin hehanyan śape kta.

17 Qa tuwe śape cin on wipakintapi kta e pte huhnahyapi qon cahota etanhan icupi lita, qa wakśica wan ohna mini kaduze cin akaśtanpi kta.

18. Unkan wicaśta wan śape śni pejihota icu kta, qa mini kin he en oputkan kta, qa wakeya kin qa wakśica owasin, qa wicanagi hen yakonpi kin owasin awicamnimni kta, nakun tuwe 226 wicahu wan icahtake qaiś tuwe ktepi, qaiś wayazanka țe, qaiś ohnahnakapi wan icaltake cinhan.

19 Unkan wicaśta śape śni kin he tuwe sape cin he amnimni kta, anpetu iyamni en, qa anpetu iśakowin en, .unkan anpetu iśakowin en ihdkusa kta, wokoyake hdujaja kta, qa minin niwe kta, hecen litayetu en ska kta.

$20 \mathrm{Qa}$ wicaśta tuwe śape ca ihduska śni kinhan, wicanagi kin he omniciye etanhan kaśpapi kta, Jehowa tipi wakan tawa kin yuśape cin heon etanhan, wipakintapi mini kin amnimnipi śni heon hee śapa.

21 Unkan woope kin de owihanke wanin yuhapi kta, tuwe wipakintapi mini amnimni kin wokoyake hdujaja kta; qa tuwe wipakintapi mini kin icahtake cinhan ilitayetu hehanyan śape kta.

22 Qa taku kaśta tuwe śapa yutan kinhan śape kta, qa wicanagi tuwe he icahtake cin htayetu kta hehanyan śape kta.

\section{WICOWOYAKE 20.}

1 Unkan wi tokaheya en Israel cinca oyate kin owasin Tzin hopuze cin en hipi, qa oyate kin Qadeś en iyotankapi, qa hen Miriam ta, unkan hen hnakapi.

2 Unkall mini yatkanpi kta wanica, heon oyate Mlowis qa Aaron amniciyapi.

3 Qa oyate kin Mowis kigepi, qa heyapi, Tokin unki hunkawanjipi Jehowa itokam tapi qehan unkiś eya untapin.

4 Qa tokeca unkiye untapi kta e, qa nakun wanunyanpi unkitawapi, hopuze cin de elita Jehowa taomniciye hiyuwicayayapi he? 
5 Qa tokeca Egupta etanhan ilanwankanhde mani unyayapi, qa makoce śice kin den hiyu unyayapi he? tukten wojupi iyecece śni, nakun witanśna hu wanica, qa hastanhanka iyuwi wanica, qa taspantanka su ota wanica, qa mini yatkanpi lita wanica.

6 Unkan Mowis qa Aaron omniciye etanhan upi, qa itkokipapi wakeya tiyopa ekta hipi, qa makipusdiya elipe iciyapi, qa Jehowa towitan kin wanyakapi.

7 Unkan Jehowa ie ca Mowis heciya,

8 Cansakadan qon icu, qa niye qa Aaron nicinye oyate kin mniciye wicayayapi kta, qa wanyakapi icunhan imnija kin eyakiyapi kta, unkan mini tawa wicakicu kta; hecen imnija etanhan mini hinanpe wicayeciye kta, qa oyate kin, qa tawanunyanpi kin yatke wicayakiye kta.

9 Unkan Mowis Jehowa itokapa tanhan cansakadan icu, econ śi kin he iyecen.

10 Unkan Mowis, qa Aaron omniciye kin mniciye wicayapi, imnija itokam, qa hewicakiya. Wawakipajinpi sa anagoptan po. Imnija kin de etanhan mini inanpe unniciyapi kta he?

11 Unkan Mowis nape yuwankan ikikcu, qa cansakadan on imnija kin apa; unkan mini ota inanpa ; unkan oyate kin yatkanpi, tawanunyanpi nakun.

12 Unkan Jehowa ie ca Mowis qa Aaron hewicakiya. Wicamayadapi śni, qa Israel cinca wicitokam mayaduwakanpi śni, heon omniciye kin de makoce wicawaqu kin ekta hiyuwicayayapi kte śni.

13 Meriba (Kigepi) mini kin hee; hen Israel cinca kin Jehowa kigepi, unkan hen ihduwakan.
14 Unkan Qadeś etanhan Mowis Edom wicaśtayatapi ckta wicaśta ye wicaśs, qa heciya. Nisunka Israel hecen eya, Niye woiyokiśice unkakipapi kin owasin sdonyaya.

15 Token hunkake wicunyanpi Egupta ekta ipi, qa Egupta olma unyakonpi anpetu ota, qa token Egupton unkiyuśepi, unkiyepi, qa nakun hunkake wicunyanpi.

16 Unkan Jehowa hoye unkiyapi, unkan ho naunkilionpi, qa ohnihde wan ye si, ga Egupta etanhan hdinarpe unyanpi, unkan iho wanna Qadeś en unyakonpi, otonwe wan makoce nitawa ihanke ekta.

17 Tokin nitamakoce ohna unkiyayapi kten. Maga nitawa ohna, qa hastanhanka iyu i ojupi ohna unyanpi kte śni, qa miniyowe nitawapi etanhan unyatkanpi kte śni, wicaśtayatapi tacanku ohna maunnipi kta, hetanhan etapa tanhan qa catka tanhan unmanna unyanpi kte śni, nitamakoce isanpa unkiyayapi śni kin hehanyan.

18 Unkan Edom heciya. Miyc ohna idade kte śni okini canhipi hduha itkocicipe kta.

19 Unkan Israel cinca kin heciyapi, canku tanka ohna unyanpi kta, qa mini nitawa unyatkanpi kinhan miye qa wanunyanpi mitawa on iyuwin ciçukta. 'Takudan econ śni siha ecedan weçon opta mde kta.

20 Unkan heya, Iyoopta idade kte śni, qa Edom wicota om qa nape waśaka on inanpe ca itkokim $u$.

21 Hecen Edom iye tamakoce ohna Israel ye kta iyoki śni ;

22 Qa Qadeś etanhan ihdakapi, qa Israel cinca omniciye owasin Hor he ekta hipi.

23 Unkan Jehowa ie ca Edom 


\section{WICAYAWAPI,}

makoce ihanke ekta, Hor he ohna Mowis qa Aaron hewicakiya.

24 Aaron iye taoyate ekła okipe yapi kła. wakoce Israel cinca wicawaqu kin he ekta i kte śni, nakas, Kigepi mini kin ekta mioie yakipajinpi heon etanhan.

25 Aaron ya iye cinhintku Eleazar iwicacu, qa Hor he akan hiyuwicaya wo.

$26 \mathrm{Qa}$ Aaron tawolkoyake kin duśdoke kta, qa cinhintku Fleazar he koyag yaye kta, qa hen Aaron te lita, qa okipe yapi kta.

27 Unkan token Jehowa econ śi kin he iyecen Mowis econ, qa Hor he akan ipi kin he oyate owasin wanyakapi.

28 Unkan Mowis Aaron tawokoyake kin kiciyuśdolse ca cinhintku Eleazar he koyag kiya; unkan Aaron hen ta, Hor he paha akan : unkan Mowis qa Eleazar he kin etanhan kun hdicupi.

29 Unkan omniciye owasin Aaron te cin sdonyapi, heon Israel cinca tiyohnaka ocowasin Aaron aceyapi, anpetu wikcemna yamni hehanyan.

\section{WICOWOYAKE 21.}

1 Unkan Arad wicaśtayatapi Kanani kin itokah makoce ounye cin he Israel Atarim canku ohna $u$ nahon qehan azuwicaya, qa etanhan wayaka iwicacu.

2 Unkan Israel wokonze wan Jehowa en iciçonzapi, qa heyapi, Oyate kin de minape en katinyan mayaqu kinhan, otonwe tawapi ocowasin ihang waye kta.

3 Unkan Jehowa Israel ho kin nahon, qa Kanani kin wicaqu : unkan ocowasin ihang wicayapi, otonwe tawapi nakun, heon makoce kin he Horma (Woihangye) eciyapi.
4 Unkan Hor he kin etanban ihdakapi, qa Edom makoce ihdukśan yapi kta, heon Mde śa canku ohna yapi, qa oyate nagipi kin hanyan, canku kin on etanhan.

5 Qa oyate kin Wakantanka aiapi, qa Mowis aiapi, Tokeca Hopuze cin ohna untapi kta e Egupta etanhan hdinanpe unyayapi he? aguyapi wanica, qa mini wanica, qa unnagipi woyute kapojedan kin de hitihda, eyapi.

6 Heon Jehowa wamduśka wahulinahya, oyate kin en hiyu wicaya, unkan wicayahtakapi, hecen Israel oyate etanhan wicota tapi.

7 Hehan oyate kin Mowis elita hipi, qa heyapi, Waunhtanipi, Jehowa unkaiapi, qa niś nakun; Jehowa wamduśka yutokan iyeunkiciciyapi kta e icekiya wo ; unkan Mowis oyate on cewicakiciya.

8 Unkan Jehowa Mowis heciya, Wamduśka wahuhnahya wan icicaga wo, qa can pasdatapi wan akan ikoyag ya wo, unkan tona wicayahtakapi qa he wanyakapi kinhan hena owasin nipi kta.

9 Unkan Mowis mazaśa wamduślka wan kage ca can pasdatapi wan akan ikoyag ya; unkan kaketu wamduśka wicaśta wan yahtake cinhan, mazaśa wamduśka kin atonwan eca ni ece.

10 Unkan Israel cinca kin ihdakapi, qa Obot (Miniiyokaśtan) en etipi.

11 Unkan Obot etanhan ihdakapi, qa Ije-Abarim (Abarim Otiwota) en etipi, he hopuza ohna Moab itokam wihinanpe ektakiya yanka.

12 Unkan hetanhan ihdakapi, qa Zered kaksiza ohna etipi.

13 Hetanhan ihdakapi qa Arnon akasanpa etipi, he hopuza 


\section{WICOWOYAKE 21.}

ohna un, Amori tamakoce ihanke ekta, Amon Moab ihanke kin hee, Moab qa Amori kin otaheden.

14. Heon Jehowa azuye wicaya oyakapi wowapi kin en heyapi, Waheb ekta Supa en, qa Arnon wakpadan ekta.

$15 \mathrm{Qa}$ wakpadan omdaye kin he Ar tipi kin iyohiya, qa Moab makoce ihanke kin icahtaka.

16 Unkan Beer (Miniyowe) ekta hipi, Miniyowe kin he Jehowa ke ca Mowis heciya, Oyate kin yuwitaya u wicakiya wo, kinhan mini wicawaqu kta.

17 Hehan Israel odowan kin de ahiyayapi, Miniyowe inanpa wo. He kahiyayapo.

18 Itancan miniyowe qapi kin hee, oyate akicita yapi kin tasagyepi on, Wicoope lage cin eye cin iyecen. Unkan hopuze cin etanhan Matana en hipi.

19 Unkan Matana etanhan Nahaliel (Kaksiza wakan) en hipi, Nahaliel etanhan Bamot (Pahaota) ekta.

$20 \mathrm{Qa}$ Bamot etanhan Maliomdaye ekta, he Moab tatinta ohna yanka, Pisga paha ihukuya, qa he etanhan hewoskan kin wanyakapi.

21 Hehan Israel Sihon Amori wicaśtayatapi kin ekta ohnihde ye wicaśi, qa heciya,

22 Nitamakoce kin iyopta mde kta ; maga nitawa en unyanpi lete śni, qa hastanhanka iyuwi njupi nitawa ohna unyanpi kte śni ; qa miniyowe nitarva etanhan mini unyatkanpi kte śni; nitamakoce iyoopta unyanpi kin hehanyan, wicaśtayatapi tacanku kin omaunnipi kta.

23 Tuka Silion iye tamakoce Israel opta yapi kta iyowinwicakiye sni, qa Sihon taoyate kin iyuhpa yuwitaya $u$ wicakiye ca
Israel itkokim hiyu, hopuza ektakiya, qa Jahatz (Anatanpi) ekta i, qa Israel azu wicaya.

24 Unkan Israel tacanlipi hi on kaśtakapi, qa tamakoce kin tawa yapi, Aruon etanhan, Jaboq hehanyan, Amon cinca kin elita; Amon cinca tamokoce ihanke kin he aconkaśkayapi, nakaś.

25 Unkan Israel otonwe kin hena owasin icupi, qa Israel Amori totonwepi owasin en iyotankapi, Heśbon qa otonwe cunkśi wicaya owasin.

26 Ḣeśbon Sihon Amori wicaśtayatapi totonwe kin hee.. Iye he itokam Moab wicaśta yatapi kin azuye ca tamakoce Arnon ekta owasin nape kin etanhan icu.

27 Heon dowanpi qa heyapi, Heśbon otonwe elita u po. Silion totonwe kagapi qa aconkaśkaya po.

28 Heśbon etanhan peta wan inanpa, Silion totonwe etanhan ide wan. Ar Moab huhnahya, Arnon paha itancan nakun.

2.9 Moab hehehe, Kemoś taoyate nitakuni śni, cinhintku tona najicapi cunwintku nakun Amori wicaśtayatapi Silion wowidag wicaye kta e wicaqu.

30 Unkkan wicunkutepi, Ḧesbon ihang yapi. Dibon ekta, qa Nopa ekta hewoskan kagapi, he Medeba ekta iyohiya.

31 Hecen Israel Amori tamakoceri kin en iyotankapi.

32 Unkan Mowis wicaśta Jazer ekta tonwe ye wicaśi, unkan totonwe kin icupi, qa Amor hen unpi kin nape wicayapi.

33 Hehan ihdulomnipi, qa Basian canku ohna itanwankanlide yapi ; unkan $\mathrm{Og}$ Baśan wicaśtayatapi kin itkowicakipe kta inanpa, iye qa taoyate owasin Edrei okicize ekta. 
WICAYAW API,

34 Unkan Jehowa Mowis heciya, Ihnutan he ikoyape cir, ninape en he waqu kta, qa taoyate owasin, qa makoce tawa nakun ; unkan token Silhon Amori wicaśtayaui Heśbon en yanke ciqon ecayecoll, he iyecen he ecayecon kta.

35 Unkan kaśtalkapi iye qa cinca kin, qa taoyate owasin, hecen wanjidan niyake okapte śni.

\section{WICOWOYAKE 22.}

1 Unkan Israel cinca kin ihdakapi, qa Moab makomdaya ohna Jordan icahda Jeriko itakasam elipi.

2 Unkan Balaq Tzipor cinhintku taku Israel Amori kin ecawicakicon owasin wanyaka.

3 Unkan Moab nina kokipa, oyáte wicitokam, wicotapi nakaś, qa Israel cinca kin on Moab hanyan. 4 Unkan Moab Midiyani hunkawicayapi kin hewicakiya, Wanna obe kin de taku unkihdukśan un kin owasin sdipapi kta, tatanka wan tinta wato kin sdipa he iyecen. Unkan hehan Moab wicaśtayatapi kin Balaq Zipor cinhintku kin hee.

5 Qa ohnihde Balam Beyor cinhintku ekta ye wicaśi, Petori ekta, he iye tamakoce wakpa kin icahda yanka; kico kta, qa heciya, Tho oyate wan Egupta etanhan inanpa, qa inyun maka ite kin akalipe ca micahda iyotankapi.

6 Heon wanna ceciciya, u qa oyate kin de wicamiciyaśica wo ; wamaśaka eśta hena sanpa waśakavi, okini wicawakaśtaka, qa makoce etanhan nape wicawaya owakihi kta. Tuwe dawaśte kin he yawaśtepi, qa tuwe daśice cin hee yaśicapi e sdon waya nakaś.
7 Unkan Moab hunkayapi, qa Midiyani hunkayapi woayate iyunwin nape ohna yuha iyayapi, qa Balam ekta ipi, qa Balaq oie kin eciyapi.

8 Unkan hewicakiya, Hanyetu kin de den yanka po, qa token Jehowa emakiye cin he iyecen wicoie kin aciyuptapi kta. Unkan Moab itancan kin Balam kici wankapi.

9 Unkan Wakantanka Balam ekta hi, qa heciya, Wicaśta kin dena nici unpi kin tuwepi hwo?

10 Unkan Balam Wakantanka heciya, Balaq Beyor cinhintku, Moab wicaśtayatapi wahomayan,

11 Inyun, oyate wan Egupta etanhan inanpe ca maka ite kin akahipa. Wanna u, qa miciwicayaśica wo, hecen azu wicawaye ca nape wicawaya owakihi kta.

12 Unkan Wakantanka Balam heciya, Ihnuhan om idade cin, ihnuhan oyate kin daśice cin, yawaśtepi nakaś.

13 Unkan Balam hanlianna kikta, qa Balaq ohnihde wicaya, hewicakiya, Nitamakoce ekta hdapo. Niyepi om mde lita Jehowa iyowin makiye śni.

14 Unkan Moab itancan kin najinpi, qa Balaq ekta hdapi, qa heciyapi, Balam unkicipi u kte śni, keya.

15 Unkan Balaq ake wicaśta sanpa wicakinihanpi, qa sanpa ota ye wicaśi.

16 Unkan Balam elita hipi, qa heciyapi, Balaq Tzipor cinhintku kin hecen eya, Ceciciya, ihnuhan ekta mau yakapin kin.

17 Wowitan tanka hinca cicu, qa taku emayakiye cin owasin ecamon kta. U wo, ceciciya, oyate kin de wicamiciyaśica wo. 


\section{WICOWOYAKE 22.}

18 Unkan Balam ayupte ca Balaq taokiye hewicakiya, Balaq iye ti kin mazaska qa mazaskazi ojudan maqu eśta, Taku wakan oie kin kawape ça taku cistinna qaiś taku tanka unmanna econ owakihi kte śni.

19 Heon ceciyapi hanyetu kin de nakun den wankapo, hecen taku Jehowa ake emakiye kta sdon waye kta.

20 Unkan Wakantanka hanyen Balam ekta hi, qa heciya, Wicaśta kin nico hipi hecinhan, najin, qa om ya wo ; tuka wicoie eciciye cin heceedan ecanon lita.

21 Unkan Balam hanhanna kikta, qa śonśonna aqin kiton, qa Moab itancan om iyaya.

32 Unkan iyaye cin heon Wakantanka canniyan, qa kize kta e Jehowa ohnihde kin canku ohna najin. Unkan śonśonna akan iyotanka, qa tahoksiidan nonpa opapi.

23 Unkan śonśonna kin Jehowa ohnihde kin canku ohna najin, qa isan hduśdoka nape ohna wanyaka, heon sonśonna kin canku etanhan ihdutokan, qa tinta omani. Unkan Balam śonśonna kin apa, canku ohna iyaye ye kta heon etanhan.

24 Unkan Jehowa ohnilıde kin hastanhanka iyuwi ojupi ehna canku qapi wan ohna najin anonkatanhan acankaśkayapi.

25 Unkan śonśonna kin Jehowa ohnihde kin wanyake ca conkaślke ipuskica, qa Balam siha kin conlkaślke ekta inatan; unkan ake he apa.

26 Hehan Jehowa ohnihde kin ake ako iyaye ca tukten ocistiyedan ohna inajin: hell canku etanhan tokan iyaye pica śni, etapa tanhan qaiś catka tanhan.

27 Unkan śonśonna kin Jehowa ohnihde kin wanyake ca makipusdi elipe ic̣iye, Balam ihukuya. Unkan Balam canniye ca sagye on śonśonna kin apa.

28 Hehan Jehowá śonśonna i lin yukawa; unkan Balam heciya, Taku ecacicon hwo on etanhan wanna yamni akihde amayapa he?

29 Unkan Balam śonśonna kin heciya, Iyokiśin mayaya heon hecamon, Tokin isan wan minape ohna yukan, unkanś cikte kta tuka.

30. Unkan śonśonna kin Balam heciya, śonśonna nitawa totanhan mayaduhe cin ecen anpe dehanyan akan dotanke śni he? Qa ijehan hecen hecacicon he? Unkan, Hiya, eya.

31 Hehan Jehowa Balam iśta kin yuzamni, unkan Jehowa ohnihde nape kin ohna isan yuśdog hduha, canku onajin wanyake ça makipusdiya ehpe ic̣iya.

32 Unkan Jehowa ohnihde kin heciya, Tokeca wanna yamni akihde sonsonna ayapa he? Niohan mitokam pemni ce, heon miś cicize kta e wahinawapa.

$33 \mathrm{Qa}$ śonśonna wanmayake ça yamni akihde mitokapa tanhan ihdutokan śni unkanś he niwaye kta, qa wanna awicakehan ci kte kta tuka.

34. Unkan Balam Jehowa ohnihde kin heciya, Wawahtani, canku olina amayakipe kta nayajin kin ston waye śni nakaś, qa wanna hecen iyonicipi śni kinhan wahde kta.

35 Unkan Jehowa ohnihde kin Balam heciya. Wicaśta qon om ya wo. Tuka wicoie eciciye cin heceedan ehe kta. Unkan Balam Balaq ohnihde wicaya owicapa.

36 Unkan Balam u kin he Ba231 


\section{WICAYAWAPI,}

laq nahon, qa itkokipe lita e iyaya, Moab otonwe ekta, he Arnon icahda makoce ihanke kin hee.

37 Unkan Balaq Balam heciya, Cico kta e hoye ciciye śni he ? qa tokeca ekta mayahi śni he? Awicakehan nina ciyutan owakihi śni he?

38 Unkan Balam Balaq heciya, Inyun wanna en cihi, taku iwae kta owakihi he? wicoie Jehowa mii en maqu kin heceedan iwae kta.

39 Unkan Balam Balaq kici iyaya, qa Ḣtzot otonwe ékta ipi.

40 Unkan Balaq tatanka, qa tahinca ska wayuśna, qa Balam qa itancan opapi kin tuwe wicakico yeśi.

41. Unkan ihanhanna Balaq Balam icu qa Baal paha kin ekta i, qa hetanhan oyate wicoti ihanke wan wanyaka.

\section{WICOWOYAKE 23.}

1 Unkan Balam Balaq heciya, Wahna wośnapi śakowin den micicaga wo; qa den tatanka sakowin, qa tahin wanunyanpi śakowin wiyeya micicaga wo.

2 Unkan token Balam eye cin hecen econ, qa Balaq qa Balam tatanka wan, qa tamdoka wan wahna wośnapi otoiyohi akan hulinahya.

3 Unkan Balam Balaq heciya, Wohuhnali yaye cin icahda den yanka wo. Miś kakiya mda, okini Jehowa itko makipe u kta, qa wicoie wanyag makiye cin owasin ociciyake kta, eye ca paha śdayehna ekta iyaya.

4. Unkan Wakantanka Balam itkokipa, unkan heciya, Wahna wośnapi śakowin ewahde, qa wahna wośnapi iyohi akan ta232 tanka wan, qa tamdoka wan liuhnah waya.

5 Unkan Jehowa wicoie wan Balam i en yekiye ca heciya, Balaq ekta hde, qa heyakiye kta.

6 Unkan ekta hà, unkan inyun wohulinali ye cin icahda yanka, iye qa Moab itancan owasin.

7 Unkan Balam wicoie wakan kin ohdake ca heya, Balaq Moab wicaśtayatapi kin Aram etanhan, Wiyohiyanpa he kin etanhan u makiye ca heya, Tho Jakob miciyaśica wo, qa kuwa Israel mici canniye ya wo.

8 Iyotan waśake cin he canniye ye śni, miś token canniye waye kta he? Jehowa wicayaśice śni, token wicamdaśice kta he?

9 Inyan paha etanhan wanmdaka, pajodan etanhan aowakasin, Inyun oyate kin de iśnana ounye kta, qa oyate tokeca om wicayawapi kte śni.

10 Jakob maka mdu. sukaza kin, qa Israel ośpaye itopa kin, tuwe yawa okihi he? Tokin wicaśta ecetu tapi kin iyecen minagi te kten qa owihanketa hena iyemacece kten.

11 Unkan Balaq Balam heciya, Taku ecamiyecon hwo? Toka wicawaye cin daśice kta e cico, unkan inyun nina wicadawaśte.

12 Unkan ayupte ca heya, Taku Jehowa mii en ye kiya he patanyan iwae kte śni he?

13 Unkan Balaq heciya, Oyanke tokeca wan ekta mici u wo, qa hetanhan wanwicadake lita, ihanke hinca wan heceedan wandake kta, owasin wanwicadake kte śni, qa hetanhan wicamiciyaśica wo.

14 Qa Tzopim (Iwanyakapi) tinta en Pisga paha ekta hiyuye ca wahna wośnapi śakowin kage ca tatanka, wan, qa tamdoka 


\section{WICOWOYAKE 24.}

wan wahna wośnapi iyohi akan liulinahya.

15 Qa Balaq heciya, Den yanka wo, wohuhnah yaye cin icahda, tuka miś kakiya itkowakipe kta.

16 Unkan Jehowa Balam itkolipe ca wicoie wan i kin en ye kiye ça heciya, Balaq ekta hde ca hecen eya wo.

17 Unkan en hdi, unkan inyun woluhnah ye cin icahda yanka, nakun Moab itancan kin opapi, qa Balaq heciya, Jehowa taku eya he?

18 Unkan wicoie wakan kin ohdake ca heya, Balaq najin qa nahon wo. Tzipor cinhintku kin anoli makiciya wo.

19 Iyotan waśake cin wicaśta heca śni, tohini itonśni, qa Adam cinca śni iyope içiye śni. Iś eye cinhan ecen econ kte śni he ? Ie cinhan hduwicake kte śni he?

20 Inyun iye yawaśte qa yawaśte maśi, unkan mdatokeca owakihi śni.

21 Jakob en wicohan śica atonwe kte śni, qa Israel en woiyokiśice wanyake kte śni, Jehowa wakan dapi kin wica kici un, wicaśta yatapi iyaśapi kin.

22 Iyotan waśake cin Egupta etanhan hdinanpa ya, tinta tatanka iyecen waśaka ce.

23 Jakob en wapiyapi wanica ce, qa Israel en wakan kagapi wanica. Iyotan waśaka Jakob on, qa Israel on, taku econ he? detanhan eyapi kta.

24 Inyun oyate kin mnaja wiyedan iyecen najin hiyaye $\mathrm{kta}$, qa mnaja mdoka wan iyecen yuwankan iye içiye kta. Taku yalitake cin we kin yatke, ca taku yapota yute śni kin hehanyan makan iwanke kte śni.

25 Hehan Balaq Balam heciya,
Ilınuhan wicadaśice cin, qa nakun ihnuhan wicadawaśte kin.

26 Unkan Balam ayupte ca Balaq heciya, Taku Jehowa emakiye cin owasin ecamon lita eciciye śni he?

27 Unkan Balaq Balam heciya, Wanna kuwa, oyanke tokeca ekta unye kta, okini hetanhan miyeciwicadaśice kta e Wakantanka iśta kin en ecetu kta.

28 Qa Balaq Balam iyaye ya, Peyor paha kin elita, hetanhan Jeśimon (Hewoskan) wanyalkapi.

29 Unkan Balam Balaq heciya, Wahna wośnapi śakowin den micicaga wo, qa den tatanka śakowin, qa tamdoka śakowin, wiyeya micicaga wo.

30 Unkan token Balam eye cin he iyecen Balaq econ; unkan wahna wośnapi otoiyohi akan tatanka wan, qa tamdoka wan hulinali ya.

\section{WICOWOYAKE 24.}

1 Unkan Israel wicayawaśte kin he Jehowa iśta kin en waśte Balam wanyaka, heon woayate akin iyaye śni, econ qon iyecen, tuka hopuze cin ektakiya etonwan.

$2 \mathrm{Qa} \mathrm{Balam}$ iśta yuwankan ikikcu, qa Israel wicoun iyecen tipi kin wanwicayaka, unkan Wakantanka toniya kin he akan un.

3 Hehan wicoie wakan ohdake ca heya, Balam Beyor cinhintku heya, wicaśta iśta yuhdokapi kin heya;

4 Tuwe $\mathrm{El}$ oie kin nalion qa Iyotan waśaka towanyake kin wanyaka, he hicahan eśta iśta yuzamni hduhe cin he heya;

5 Israel yatipi kin qa Jakob nitawakeya kin hena waśte hinca. 233 


\section{WICAYAWAPI,}

6 Wakpadan kin iyecen yeye cin, taślkoju wakpa icahda iyecen, hianteśa Jehowa oju kin iyecen, wazi can mini icahda iyececapi.

7 Koka tawa etanhan mini kaduze kta, qa taku iye etanhan icage cin mini ota akan un kta, qa wicaśtayatapi tawa Agag iwankam yanke kta, qa tokiconze kin pajodan kta.

8 Taku wakan waśaka Egupta etanhan inanpe wicaya; Oyate tona tokayapi kin hena temwicaye kta, huhupi kin wicakiyukse kta; tawahinkpe kin ipahdan ihewicaye kta.

9 Patuja mnaja iyecen, qa makan iwanka mnaja wiyedan iyecen, tuwe pahice kta he? Tuwe niyawaśte kin yawaśtepi kta, qa tuwe niyaśice cin yaśicapi kta.

10 Hehan Balaq Balam nina canniye ya, qa nape hdaskape ca Balaq Balam heciya, Toka wicawaye wicadaśice $\mathrm{kta}$ e cico, unkan inyun yamni akihde katinyan wicadawaśte ce.

11 Heon wanna najica ekta hda, wowitan tanka cicu kta epa, tuka inyun niyutanpi kta Jehowa iyoki śni.

12 Unkan Balam Balaq heciya, ohnihde wicayaya mico ye wicayaśi qon hecen ewicawakiye śni he?

13 Balaq iye ti kin mazaska, qa mazaskazi ojudan maqu eśta, Jehowa i kin kawape, qa taku waśte qaiś taku śica econ owakihi śni, tuka taku Jehowa ie cin heceedan iwae kta.

14 Unkan ito wanna oyate mitawa ekta wahde irta, Kuwa wo, taku oyate kin de oyate nitawa ecawicakiconpi $\mathrm{kta}$, tokata anpetu ota iyohakam, he ociciyake kta.
15 Qa Balam wicoie wakan olidake ca heya, Balaq Beyor cinhintku kin heya, wicaśta iśta yuhủokapi kin he heya :

16 Tuwe El oie kin nahon, qa Otancanke tosdonye kin okahniga, Iyotan waśaka towanyake kin wanyaka, makipusdiya hinhpaya eśta iśta yuzamni hduhe cin he heya;

$17 \mathrm{He}$ wanmdake kta tuka dehantu śni, awatonwe kta tuka ikiyedan kte śni, wicanhipi wan Jakob etanhan u kta, mazasagye wan Israel etanhan najin kta, qa Moab ihanke kin owasin kaśtake kta, qa Śset (Owodutaton) cinca kin owasin ihang ye kta.

18 Qa Edom tawaye kta, qa Seyir tokayapi kin he tawaye kta, qa Israel waditagya econ kta.

19 Qa tuwe idake ye kta Jakob etanhan kta, qa tona otonwe etanhan okaptapi ihang wicaye kta.

20 Hehan Amaleq wanyake ca heya, Amaleq oyate tokaheya kin hee, tuka tolsata iś eya awihnuni kta.

21 Hehan Qeni kin wanwicayake ca wicoie wakan ohdake ça heya, yatipi kin sutaya aconkaśkayapi, qa inyan ehna hohpi nicicaga.

22 Hececa eśta Qeni kin tokan awicayapi kta; Tohantu kta he? Aśur wayaka aniye kta.

23 Unkan wicoie wakan kin ohdake ça heya, Hehehe, EI hena econ lin tuwe hehanyan ni kta he?

24. Unkan witawata Kitim huta kin etanhan upi kta, qa Aśur iyokiśin yapi kta, qa Eber iyokiśin yapi kta: unkan iś eya owihanketa awihnuni kta.

25 Hehan Balam najin qa 
WICOWOYAKE 26.

ekta kihda, qa Balaq iś eya elita hda.

\section{WICOWOYAKE 25.}

1 Unkan Israel Śitim en ounyanpi, qa oyate kin Moab cunwintkupi inawicahmanpi on ihduśapapi.

2 Unkan winyan kin hena taku wakandapi wakiyuśnapi ekta oyate wicakicopi, unkan oyate kin yutapi, qa winyan kin taku wakandapi kin ohodapi.

3 Hecen Israel Baal Peyor (Peyor Itancan kin) en ikoyag içiyapi; unkan Jehowa Israel canniye wicaya.

4 Qa Jehowa Mowis heciya, Oyate itancanpi kin owasin wicakico wo, unkan hena Jehowa on anpetu wi itokam otke wicayapi kta, hecen Jehowa Israel nina canniye wicaya anapte kta.

5 Unkan Mowis Israel wayacopi kin hewicakiya, Otoiyohi wicaśta tawa tona Baal Peyor en ikoyag içiyapi kin hena wicaktepo.

6 Unkan Inyun Mowis qa Israel cinca oyate kin itlrokipapi wakeya tiyopa ekta ceyapi icunhan, Israel cinca etanhan wicaśta wan ku, qa Midiyani winohinca wan sunka wicaye cin ekta hiyuya, Mowis qa oyate kin wanyakapi.

7 Unkan Aaron wawayuśna cinhintku Eleazar cinhintku Pinehas he wanyake cehan oyate cokaya tanhan najin iyaye ca nape ohna wahukeza wan icu;

8 Qa Israel wicaśta kin ihakam iyaya, wakeya catkuta ekta, qa napin, cawicapa, wicaśta kin, qa winohinca nakun tezi kin ohna, hecen makośice kin Israel cinca etanhan anapta.

9 Warna makośice on tapi kin hena wicaśta ketkopawinge wikcemna nonpa sanpa kektopawinge topa.

10 Unkan Jehowa ie ca Mowis heciya,

11 Pinehas Eleazar cinhintku Aaron wawayuśna cinhintku lrin, Israel cinca etanhan mitocanniye yutokan, inawawizi iyecen iye wicehna inawizi, qa hecen inawawizi kin en ihang wicawaye śni.

12 Heon hecen eciya wo. Ito wookiye wicotakuye waqu ;

13 Qa wicotakuye wawayuśna wicolian owihanke wanica yuhe kta, iye qa cinkśi wicaye cin iye iyohakam, Wakantanka on inawizi kin Israel cinca akahpe wicaya heon etanhan.

14 Wanna Israel wicaśta ktepi kin Midiyani winobinca kici ktepi kin he Zimri eciyapi, Salu cinhintku, Simeon ateyapi tipi wan en itancan wan hee.

$15 \mathrm{Qa}$ Midiyani winohinca kte. pi kin he Kozbi eciyapi, Tzur cunwintku Midiyani ateyapi tipi wan en itancan wan hee.

16 Unkan Jehowa ie ca Mowis heciya,

17 Midiyani toka wicaye ça wicakaśtaka wo.

18 Iyepi toka niyanpi qa nihnayanpi ecinśniyan ohanyanpi on, Peyor wicoie icahtaka, qa tawinohtinpi Kozbi Midiyani itancan wan cunwintku, Peyor on etanhan, makośice anpetu kin en Irtepi heon etanhan.

\section{WICOWOYAKE 26.}

1 Unkan makośice iyohalram Jehowa ie ca Mowis qa Eleazar Aaron wawayuśna cinhintku kin hewicakiya.

2 Israel cinca oyate kin owasin wicayawa wo, wica waniye235 
tu wikcemna nonpa aktonpi, ateyapi tipi kin iyecen, Israel ohna tona ozuye yapi kin owasin.

3 Unkan Mowis qa Eleazar wawayuśna kin wicoie kin hewicakiyapi, Moab makomdaye ohna, Jordan icahda Jerilio iyotakons.

4. Waniyetu wikcernna nonpa aktonpi wicayawapo, token Jehowa Mowis qa Israel cinca econ wicaśi qon, Egupta etanhan inanpapi qehan, he iyecen.

5 Ruben Israel cinhintku tokapa, Ruben cinca Hanok etanhan Hanoki wicowazi kin, Palu etanhan Palui wicowazi kin,

6 Ḧetzron etanhan Hetzroni wicowazi kin, Karmi etanhan Karmi wicowazi kin.

7 Rubeni wicowazi lin hena eepi, Kektopawinge wikcemua topa sanpa kektopawinge yamni sanpa opawinge śalkowin sanpa wikcemna yamni wicayawapi.

8 Unkan Palu cinhintku Eliab hee.

9 Qa Eliab cinca kin Nemuel, qa Datan qa Abiram. Datan qa Abiram obe wicakicopi kin hena eepi, hena Mowis qa Aaron wicakipajinpi. Qora taomniciye en Jehowa kipajinpi qehan.

10 Qa maka kin i hdukawa, qa nawicapca, qa Qora taobe kin tapi qehan, peta kin wicaśta opawinge nonpa sanpa wikcemula zaptan wicahuhnage cehan; unkan hena wowapetokeca hecapi.

11 Tuka Qora cinca kin tapi śni.

12 Simeon cinca kin wicowazipi kin iyecen, Nemuel etanhan Nemueli wicowazi, Jamin etanhan Jamini wicowazi kin, qa Jakin etanhan Jakini wicowazi kin.

13 Zerah etanhan Zerahi wico236 wazi kin, Śaul etanhan Śauli wicowazi kin.

14 Simeoni wicowazipi kin hena eepi; kektopawinge wikcemna nonpa sanpa kektopawinge nonpa sanpa opa winge nonpa.

15 Gad cinca kin wicowazipi kin iyecen, Tzepon etanhan Tzeponi wicowazi kin, Ḣagi etanhan 亡̇agi wicowazi kin, Śuni etanhan Śuni wicowazi kin,

16 Ozni etanhan Ozni wicowazi kin, Eri etanhan Fri wicowazi kin,

17 Arod etanhan Arodi wicowazi kin, Areli etanhan Areli wicowazi kin.

$18 \mathrm{Gad}$ cinca wicowazipi kin hena eepi wicayawapi iyecen, kektopawinge wikcemna topa sanpa opawinge zaptan.

19 Juda cinca kin Er qa Onan, tuka Er qa Onan Kanan makoce ohna tapi.

20 Unkan Juda cinca kin wicowazipi kin iyecen dena eepi, Śla etanhan Ślani wicowazi kin, Perets etanhan Peretzi wicowazi kin, Zerah etanhan Zerehi wicowazi kin

21 Unlkan Peretz cinca kin Hetzon etanhan Hetzoni wicowazi kin, Hamul etanhan Hamuli wicowazi kin.

22 Juda wicowazipi kin hena eepi wicayawapi iyecen liektopawinge wikcemna śakowin sanpa kektopawinge śakpe sanpa opawinge zaptan wicayawapi.

23 Unkan Isakar cinca kin wicowazipi kin iyecen, Tola etanhan Tolai wicowazi kin, Puwa etanhan Puwani wicowazi yin, 24 Jaśub etanhan Jaśubi wicowazi kin, Śimron etanhan Śtmroni wicowazi kin.

25 Isakar wicowazipi kin hena eepi wicayawapi kin iyecen kektopawinge wikcemna śakpe 
sanpa kektopawinge topa sanpa opawinge yamni, wicayawapi.

26 Zebulun cinca wicowazipi kin iyecen, Sered etanhan Saredi wicowazi kin, Elon etanhan Eloni wicowazi kin, Jahleel etanhan Jahleeli wicowazi kin.

27 Zebulun wicowazipi kin hena eepi kektopawinge wikcemna śakpe sanpa opawinge zaptan wicayawapi.

28 Josep cinca wicowazipi kin iyecen Manaśe qa Epraim.

29. Manaśe cinca Makir etanhan Makiri wicowazi kin, ga Makir Gilead kicitonpi, Gilead etanhan Gileadi wicowazi kin.

30 Gilead cinca kin dena eepi, Jezer Jezeri wicowazi, Heleq etanhan Heleqi wicowazi kin,

31 Qa Asriel Asrieli wicowazi kin ; qa Śekem Śsekemi wicowazi kin,

$32 \mathrm{Qa}$ Śemida Śmidai wicowazi kin, qa Ḣeper etanhan Ḣeperi wicowazi kin.

$33 \mathrm{Qa}$ Tzelopehad Ḣeper cinhintku cinca wica nica cunwintku hecedan, qa Tzelopehad cunwintkn cajepi kin dena eepi Mahla, qa Noya, Hogla Milka, qa Tirtza.

34 Manaśe wicowazipi kin hena eepi kektopawinge wikcemna zaptan sanpa kektoparvinge nonpa, sanpa opawinge śakowin wicayawapi.

35 Epraim cinca kin dena eepi wicowazipi kin iyecen, Sutelah etanhan Śsutelahi wicowazi kin, Beker etanhan Bekiri wicowazi kin, Tahan etanhan Tahani wicowazi kin.

$36 \mathrm{Qg}$ Sutelah cinca kin dena eepi, Enan etanhan Enani wicowazi kin.

37 Epraim cinca wicowazipi kin hena eepi, wicayawapi-kin iyecen kektopawinge wikcemna yamni sanpa kektopawinge nonpa sanpa opawinge zaptan; Josep cinca wicowazipi kin hena eepi.

38 Benjamin cinca wicowazipi kin iyecen, Bela etanhan Belayi wicowazi kin, Aśbel etanhan Aśbeli wicowazi kin, Ahiram etanhan Alirami wicowazi kin.

39 Śupam etanhan Śupami wicowazi kin, $\dot{\mathrm{H} u p a m}$ etanhan $\dot{\mathrm{H}}$ pami wicowazi kin.

40 Qa Bela cinca kin Ard, qa Naaman, Ardi wicowazi kin, Naaman etanhan Naamani wicowazi kin.

41 Benjamin cinca hena eepi wicowazipi kin iyecen, qa kektopawinge wikcemna topa sanpa kektopawinge zaptan sanpa opawinge śakpe wicayawapi.

42 Dan cinca kin wicowazipi kin iyecen dena eepi; Śuham etanhan Śuhami wicowazi kin, Dan wicowazi kin hee wicowazipi kin iyecen.

43 Suhami wicowazi kin owasin wicayawapi kin iyecen kektopawinge wikcemna śakpe sanpa kektopawinge topa sanpa opawinge topa.

44 Aśer cinca wicowazipi lin iyecen, Jimna etanhan Jimnahi wicowazi kin, Jiśui etanhan Jiśuwi wicowazi kin, Beriya etanhan Beriyi wicowazi kin.

45 Beriya cinca etanhan, Heber etanhan Ḧeberi wicowazi kin, Malkiel etanhan Malkicli wicowazi kin.

$46 \mathrm{Qa}$ Aśer cunwintku Sarah eciyapi.

47 Aśer cinca wicowazipi kin hena eepi, wicayawapi kin iyecen kektopawinge wikcemna zaptan sanpa kektopawinge yamni sanpa opawinge topa.

48 Naptali cinca wicowazipi kin iyecen Jahtzel etanhan Jah- 
tzeli wicowazi kin, Guni etanhan Guni wicowazi kin.

49 Jetzer etanhan Jetzeri wicowazi kin, Ślilem etanhan Śilemi wicowazi kin.

50 Naptali wicowazipi kin hena eepi wicowazipi kin iyecen: kektopawinge wikcemna topa sanpa kektopawinge zaptan sanpa opawinge topa wicayawapi.

51 Israel cinca wicayawapi kin hena eepi, kektopawinge opawinge śakpe sanpa kektopawinge wanjidan sanpa opawinge śakowin sanpa wikcemna yamni.

52 Unkan Jehowa ie ça Mowis heciya,

53 Makoce tawayayapi kta e hena wicakicipamnipi kta, cajepi wicayawapi kin iyecen.

54 Wicota kin tankaya tawayapi kta wicayaqu kta, qa tonana on tawayapi cistiyedan wicayecage $\mathrm{kta}$; otoiyohi tona yawapi kin eciyatanhan tawaye kta e qupi kta.

55 Hececa eśta oeconna eciyatanhan makoce pamnipi kta, ateyapi wicoun cajepi eciyatanhan tawayapi kta.

56 Oeconna i kin eciyatanhan makoce tawapi wicakicipamnipi lta, wicota qa tonana otahe dan.

57 Lewi kin wicayawapi kin dena eepi, wicowazipi kin iyecen, Gerśon etanhan Gerśoni wicowazi kin, qa Qohat etanhan Qohati wicowazi kin, qa Merari etanhan Merari wicowazi kin.

58 Lewi wicowazipi kin dena eepi, Libni wicowazi kin, $\dot{\mathrm{H} e}-$ broni wicowazi kin, Malli wicowazi kin Muśi wicowazi kin ; Qorahi wicowazi kin. Unkan Qohat Amram kicitonpi.

59 Unkan Anram tawicu Jokebed eciyapi, Lewi cunwintku,
Egupta ohna he Lewi kicitonpi, unkan he Aaron qa Mowis qa tawinolitinpi Miriam Amram kiciton.

60 Unkan Aaron cinca kin Nadab, qa Abihu, Eleazar qa Itamar.

61 Tuka Nadab qa Abihu peta tokeca Jehowa itokam ahipi qehan țapi.

62 Unkan kektopawinge wikcemna nonpa sanpa kektopawinge yamni wicayawapi, wica wi wanjidan aktonpi owasin. Tuka Israel cinca wicehna makoce tawayapi wicaqupi śni, heon Israel cinca wicehna wicayawapi śni.

63 Mowis qa Eleazar wawayuśna kin hena wicayawapi, Israel cinca wicayawapi qehan, Moab makomdaye ohna Jordan icahda Jerilio iyotakonza.

64 Qa wicehna wicaśta Mowis qa Aaron wawayuśna kin Israel cinca Sina hopuza ohna wicayawapi qon wanjidan ope śni.

65. Hena hopuza ohna tapi kta Jehow a kewicakiya nakaś, hecen hena etanhan wanjidan okapte śni, Caleb Jepune cinhintku, qa Jośuwa Nun cinhintku henana.

\section{WICOWOYAKE $2 \%$.}

1 Helıan Josep cinhintku Manaśe wicowazi kin etanhan Manaśe cinhintku Makir cinhintku Gilead cinhintku Ḧeper cinhintku Tzelopehad cunwintku hipi, qa Tzlopehad cunwintku cajepi kin dena eepi : Mahla qa Noya Hogla Milka qa Tirza.

2 Qa Mowis itokam qa Eleazar wawayuśna itokam, qa omniciye owasin qa itancanpi itokam itkokipapi wakeya tiyopa ekta najinpi qa heyapi ;

3 Ate unyanpi hopuza ohna ta 
tuka omniciye Jehowa amniciyapi kin ope śni, Qora obe kin he; tuka iye wahtani kin on ta, qa cinca wica nica.

4 Tokeca ate unyanpi cinhintku nica heon caje kin wicowazi tawa etanhan kajujupi kta he? Ate sunka wicaye cin wiceIna makoce onśpa unqumiye.

5 Unkan token ecawicakiconpi kta Mowis Jehowa iwanga.

6 Unkan Jehowa ie ca Mowis heciya,

7 Tzelopehad cunwintku kin ecetuya eyapi. Ate yapi tamaga kin hena ek ta iy opta yaye kta.

8 Unkan Israel cinca kin hecen ewicayakiye kta, Wicaśta wan cinca wica nica te cinhan, maga tawa kin cunwintku ekta iyopte yayapi kta.

$9 \mathrm{Qa}$ cunwintku nice cinhan cincu qa sunkaku maga tawa yaqupi kta.

10 Qa hunkawanjitku nice cinhan, atkuku hunkawanjitku kin maga tawa yaqupi kta.

$11 \mathrm{Qa}$ atkuku hunkawanjitku nice cinhan, tuwe wicowazi tawa etanhan iye cehpi takuye linca kin maga tawa yaqupi kta ; unkan iye he tawaye kta. Woyaco kin de Israel cinca owihanke wanin yuhapi kta, Jehowa Mowis econ śi kin he iyecen.

12 Unkan Jehowa Mowis heciya, Abarim he kin de itanwankanhde ye ça makoce Israel cinca kin wicawaqu kin he wanyaka wo.

$13 \mathrm{He}$ wandake kta, hehan niś eya oyate nitawa ekta okipe niyanpi kta, Aaron nicinye okipe yapi kin he iyecen.

14 Tzin hopuza ohna mii kin yakipajinpi oyate kin kigepi qehan, iśtapi en mayaduwakanpi śni, mini ekta, Kigepi mini kin hee, Qadeś ekta, Tzin hopuza ohna.
15 Unkan Mowis ie ça Jehowa heciya,

16 Tokin Jehowa taku wakan wicacehpi toniyapi owasin wicayuhe cin wicaśta wan oyate kin wicayuhe śi kten.

17 He wicitokam inanpe $\mathrm{kta}$, qa wicitokam ku kta, qa inanpe wicaye $\mathrm{kta}$, qa hdi wicaye kta, hecen Jehowa taomniciye kin tahin wanunyanpi waawanyake nica iyececapi kte śni.

18 Unkan Jehowa Mowis heciya, Jośuwa Nun cinhintku icu wo, wicaśta kin he woniya ohna yanka qa nape aputaka wo.

19 Qa Eleazar wawayuśna itokam, qa omniciye ocowasin itokam, najin yakiye kta, qa token econ yaśi kin hena wanyakapi lita.

20 Qa wowitan nitawa etanhan he yaqu kta, hecen Israel cinca omniciye owasin he nahonpi kta.

21 Unkan Eleazar wawayuśna lzin itokam najin kta; unkan he Jehowa ikicíwange kta, iyoyam wicaya woyaco eciyatanhan, Iye i kin eciyatanhan inanpapi kta; qa iye i eciyatanhan hdipi lita, iye qa nakun Israel cinca owasin omniciye ocowasin.

22 Unkan token Jehowa Mowis econ śi he iyecen econ, qa Jośuwa icu qa Eleazar wawayuśna itokam, qa omniciye ocowasin itokam najin kiya.

23 Qa akan nape aputaka, Jehowa Mowis eciye ciqon he iyecen.

\section{WICOW OYAKE 28.}

1 Unkan Jehowa ie ça Mowis. heciya,

2 Israel cinca kin wahokon wicakiye ça hewicakiya wo. Waktaya unpo, iyehantu eca woahi mitawa, woyute mitawa peta 
wośnapi waśtemna wiciyokipi makahipi kta.

3 Qa hewicayakiye kta. Peta wośnapi Jehowa yakahipi kte cin dee, amnos nonpa, napin zanika, waniyetu wanjidan wohulinahyapi ohinniyan anpetu otoiyohi.

4 Amnos wanji hanhanna eca waduśnapi kta, qa amnos inonpa kin olitayetu otahedan ecanonpi lita.

5 Qa mintia on aguyapi mdu can wakśica ohmaka wanjidan, wihdi bopanpi wivatke ohnaka wanjidan icicahiyapi.

6. Wohuhnahyapi ohinniyan Sina he en kagapi, peta wośnapi waśtemna Jehowa iyokipi.

$7 \mathrm{Qa}$ toakaśtan kin amnos wanjidan on wiyatlie ohnaka wanjidan, tipi wakan ohna miniśa lkin Jeliowa okicicaśtan wo.

8 Qa amnos inonpa kin ohtayetu otahedan waduśna kta, hanhanna minha kin qa woakaśtan kin kici yakage lita, peta wośnapi waśtemna Jehowa iyolkipi.

9 Unkan anpetu okihpapi en amnos nonpa waniyetu wanjidan zanika, qa aguyapi mdu can wakśica ohnaka nonpa wihdi icicahiyapi, minha hee, nalsun toakaśtan kin.

10 Anpetu okilipapi wohulinahyapi kin hee, anpetu okilipapi otoiyohi on ohinniyan wohuthahyapi, qa toakaśtan kici.

$11 \mathrm{Qa}$ wi anpetu tokaheya kin en wohuhnahyapi Jehowa yakahipi kta, tatanka pte wanunyanpi cinca nonpa, tamdoka wanjidan, qa amnos śalzowin zanilia.

12 Qa minha kin aguyapi mdu can waksica ohnaka yamni, wihdi icicahiyapi, tatanka otoiyohi on, qa tamdoka on minha aguyapi mdu can wakśica ohnaka nonpa, wihdi icicahiyapi.
13 Qa minha amnos otoiyohi on aguyapi mdu can wakśica ohnaka wanjidan, wihdi icicahiyapi, peta wośnapi, waśtemna Jehowa iyokipi.

14 Qa toakaśtanpi kin miniśa wiyatke ohnaka nonpa, tatanka wan on, qa wiyatke ohnaka wanji sanpa hanke tamdoka kin on, qa wiyatke ohnaka wanjidan amnos otoiyohi on; omaka wi otoiyohi on wi toka tanin eca wohuhnahyapi kin hee,

15 Nakun tatokadan mdoka wan woahtani wośnapi Jehowa wakiyuśnapi kta, ohinniyan wośnapi kici, qa toakaśtan kici. 16 Unkan wi tokaheya anpetu iake topa en woacalsśin Jehowa kicagapi kta.

17 Unkan wi kin he anpetu iake zaptan en Jehowa wokicihanpi kta, Anpetu śakowin aguyapi napohyapi śni yutapi lita.

18 Anpetu tokaheya en wicakicopi wakan, wayaka wohtani takudan econpi lite śni.

19 Qa peta wośnapi wohuhnahyapi wan Jehowa yakahipi kta, tatanka pte wanunyanpi cinca nonpa, tamdoka wanjidan, qa amnos waniyetu wanjidan śakowin, niyepi on zanipike kta.

20 Qa minhapi aguyapi $\mathrm{mdu}$ wihdi icicahiyapi can wakśica olnaka yamni tatanka otoiyohi on, qa tamdoka on can wakśica olnaka nonpa yecagapi kta.

21 Qa amnos śakowin on can wakśica ohnaka śakowin, amnos otoiyohi on can wakśica ohnaka wanjidan, wicayecagapi kta. 22 Qa tatokadan wanjidan woahtani wośnapi akahpe niçiyapi kta.

23 Hena wośnapi yakagapi lita, hanhanna wohuhnahyapi kici, wohuhnahya pi ohinniyan kin hee. 24 Anpetu śakowin kin anpetu 
otoiyohi peta tawote waśtemna Jehowa iyokipi yakagapi kta, wohuhnahyapi ohinniyan toakaśtan kici nakun kagapi kta.

25 Unkan anpetu iśakowin en wicakicopi wakan duhapi kta, wayaka wohtani takudan ecanonpi kte śni.

26 Nakun taku tokaheya suton anpetu en minha teca Jehowa yakahipi eca, anpetu wakan śakowin owihanketa wicakicopi wakan duhapi kta, wayaka wohitani takudan ecanonpi kte śni.

27 Qa wohuhnahyapi waśtemna Jehowa iyokipi yakahipi kta, tatanka pte wanunyanpi cinca nonpa, tamdoka wanjidan, qa amnos waniyetu wanjidan śakowin.

$28 \mathrm{Qa}$ minhapi aguyapi mdu wihdi icicahiyapi can wakśica ohnaka yamni tatanka otoiyohi on, qa can wakśica ohnaka nonpa tamdoka wan on.

29 Qa can wakśica ohnaka śakowin amnos śakowin on, amnos otoiyohi on can wakśica ohnaka wanjidan.

30 Tatokadan mdoka wanjidan akahpe niciyapi kta heon.

31 Wohuhnahyapi ohinniyan, qa taminha kici, hena yakagapi kta, woakaśtanpi kin nakun niyepi on hena zanipike kta.

\section{WICOWOYAKE 29.}

1 Unkan wi iśakowin, wi anpetu tokaheya en wicakicopi wakan duhapi kta, wayaka wohitani takudan ecanonpi kte śni, anpetu mazayahotonpi wiyuśkinyan hotonpi kin duhapi kta. 2 Qa wohuhnahyapi waśtemna Jehowa iyokipi yakagapi kta, tatanka wanji ptewanunyanpi cinca, tamdoka wanji, amnos waniyetu

śakowin zanika.

3. Qa taminhapi kin aguyapi mdu wihdi icicahiyapi, can wakśica ohnaka yamni tatanka kin on, qa ohnaka nonpa tamdoka on,

4. Qa amnos śakowin on otoiyohi on can wakśica ohnaka wanjidan.

5 Qa tatokadan mdoka wanjidan woahtani wośnapi hee akahpe niçiyapi kta.

6 Nakun wi wohulinaliyapi kin taminha kici, qa ohinniyan wohuhnahyapi kin taminha kici, qa toakaśtanpi kin woyaco iyecen, peta wośnapi waśtemna Jehowa iyokipi.

7 Unkan wi jśakowin kin he anpetu iwikcemna en wicakico. pi wakan duhapi kta, qa ninagipi iyokiśin niciyapi kta, he en wayaka wohtani takudan ecanonpi kte śni.

8 Qa wohulinahyapi wan Jehowa yecahipi kta, waśtemna wiciyokipi, tatanka ptewanunyañpi cinca wanji, tamdoka wanji, amnos waniyetu wanjidan śakowin zanika niyepi on.

9 Qa minliapi kin aguyapi mdu wihdi icicahiyapi can wakśica ohnaka yamni tatanka wan on, qa can wakśica ohnaka nonpa tamdoka wanjidan kin on.

10 Qa amnos śakowin on, amnos otoiyohi on can wakśica ohnaka wanjidan.

11 Tatokadan mdoka wanjidan woahtani wośnapi on, nakun akalipe wicakiyapi woahtani wośnapi, qa ohinniyan woliuhnaliyapi kin, qa minha kin, qa toakaśtanpi kin.

12 Unkan wi iśakowin anpetu iake zaptan en wicakicopi wakan duhapi kta, wayaka wohtani takudan ecanonpi kte śni, qa 241 


\section{WICAYAWAPI,}

anpetu śakowin wohanpi Jehowa oyecihanpi kta.

$13 \mathrm{Qa}$ woliulinahyapi wan Jehowa yakahipi kta, peta wośnapi waśtemna wiciyokipi, tatanka ptewanunyanpi cinca wikcemna sanpa yamni, tamdoka nonpa, ammos waniyetu wanjidan aketum zanipike kta.

$14 \mathrm{Qa}$ taminhapi aguyapi mdu wihdi icicahiyapi can wakśica ohnaka yamni tatanka otoiyohi on, tatanka iakeyamni on, qa tamdoka nonpa on, otoiyohi on can wakśica ohnaka nonpa.

$15 \mathrm{Qa}$ amnos aketopa on, amnos otoiyoli on can wakśica ohnaka wanjidan.

16 Qa tatokadan mdoka wanjidan woahtani wośnapi, nakun ohinniyan wohulinatiyapi kin, taminha kin qa toakaśtan kici.

17 Qa anpetu inonpa en tatanka ptewanunyarpi cinca wikcemna sanpa nonpa, tamdoka nonpa, amnos waniyetu wanjidan aletopa zanipika.

18 Qa taminhapi kin, qa toakaśtanpi kin, tatanka on, qa tamdoka on, qa amnos on, woyaco eciyatanhan iyenakecapi iyecen.

19 Qa tatokadan mdoka wanjidan woahtani wośnapi, nakun wohulinahyapi ohinniyan, qa minha tawa, qa toakaśtanpi kin.

20 Qa anpetu iyamni en tatanlia akewanji, tamdoka nonpa, amnos waniyetu wanjidan aketopa zanika.

21 Qa taminhapi, kin qa toakaśtanpi kin, tatanka on, qa tamdoka on, qa amnos on, woyaco eciyatanhan iyenakecapi iyecen.

$22 \mathrm{Qa}$ tatokadan mdoka wanjidan woahtani wośnapi, nakun wohuhnahyapi ohinniyan, qa minlia tawa, qa toakaśtan kin.

$23 \mathrm{Qa}$ anpetu itopa en tatanka wikcemna, tamdoka nonpa, a242 mnos waniyetu wanjidan aketopa zanika.

24 Tatanka on, qa tarndoka on, qa amnos on iyenakecapi, taminhapi, qa toakaśtanpi kin woyaco iyecen.

$25 \mathrm{Qa}$ tatokadan mdoka wanjidan woaktani wośnapi, nakun wohuhnahyapi ohinniyan, taminha kin, qa toakaśtan kici.

26 Qa anpetu izaptan en tatanka napcinwanka, tamdoka nonpa, amnos, waniyetu wanjidan aketopa zanika.

27 Qa tatanka, qa tamdoka, qa amnos iyenakecapi on taminhapi, qa toakaśtanpi, woyaco eciyatanhan.

28 Qa tatokadan mdoka woahtani wośnapi wanjidan, nakun wohuhnahyapi ohinniyan, qa taminha, qa toakaśtan kici.

$29 \mathrm{Qa}$ anpetu iśakpe en, tatan$\mathrm{ka}$ śahdogan, tamdoka nonpa, amnos waniyetu wanjidan aketopa zanika.

30 Qa taminhapi, qa toakaśtanpi kin, tatanka on, qa tamdoka on, qa amnos iyenakecapi on, woyaco eciyatanhan.

$31 \mathrm{Qa}$ tatokadan mdoka woahitani wośnapi wanjidan, nakun woliuhnahyapi ohinniyan kin, tamintia qa toakaśtan kici.

32 Qa anpetu iśakowin en tatanka śakowin, tamdoka nonpa, amnos waniyetu wanjidan aketopa zanika.

33 Qa taminliapi, qa toakaśtanpi kin tatanka iyenaka on, qa tamdoka iyenaka on, qa amnos iyenaka on, woyaco eciyatanhan.

34 Qa tatokadan mdoka woahtani wośnapi wanjidan, nakun wohulinahyapi ohinniyan, taminlia kin qa toakaśtan kici.

35 Anpetu iśahdogan en omniciye wakan duhapi kta, wayaka 
WICOWOYAKE 30 .

wolitani takudan ecanonpi kte śni.

36 Qa wohulinahyapi peta wośnapi waśtemna wiciyokipi Jehowa yakahipi kta, tatanka wanji, tamdoka wanji, amnos waniyetu wanjidan śakowin zani$\mathrm{ka}$.

37 Taminhapi qa toakaśtanpi kin tatanka on, qa tamdoka on, qa amnos iyenakecapi on, woyaco eciyatanhan.

$38 \mathrm{Qa}$ tatokadan mdoka woalitani wośnapi wanjidan, nakun wohuhnahyapi ohinniyan kin, qa taminha kin toakaśtan kici.

39 Taku odakapi kin qa taku ikceya waduśnapi kin, wohulinahyapi nitawapi on, qa minha nitawapi on, qa woakaśtanpi nitawapi on, qa wopida wośnapi nitawapi on, isanpa wohanpi iyehantu eca hena Jehowa yecagapi kta.

40 Unkan token Jehowa Mowis econ si he iyecen Mowis Israel cinca kin owicakiyaka.

\section{WICOWOYAKE 30.}

1 Unkan Mowis Israel cinca wicoun itancanpi kin hewicakiya, Wieoie Jehowa econ niśipi kin dee.

2 Wicaśta wan woyake wan Jehowa okiyake cinhan qaiś taku wakan cajeyate on nagi ihdaśke cinhan, oie kin ihduikceke kte śni, tuka taku i kin eciyatanhan inanpe cin owasin iyecen econ lkta.

3 Unkan winohinca wan woyake wan Jehowa okiyake cinhan, qa nahanhin wikośka atkuliu ti ohna wicaśke on ihdaśke. cinhan,

$4 \mathrm{Qa}$ atkuku woyake kin qa wicaśke on nagi ihdaśke cin he nation, qa atkuku inina yanke cinhan taku oyake cin owasin suta kta, qa wicaśke on nugi kin ihdaśke cin owasin ecetu kta.

5 'I'uka tohan atkuku woyake kin, qa wicaśke on nagi ihduśke cin nation, anpetu kin he en tehinda kinhan, ecetu kte śni, qa atkuku he tehinda kin heon Jehowa kicicajuju kta.

6 Unkan woyake on ihdaśka, qaiś iha on ecinśniyan nagi ihdaśke cehan wicaśta wan he yuhe cinhan ;

$7 \mathrm{Qa}$ hihnaku be nahon, qa anpetu en nahon inina yanke cinhan, woyake kin ecetu kta, qa wicaśke on nagi kin ihdaśke cin suta kta.

8 Tuka hihnaku tohan nahon anpetu kin he en telinda kinhan, woyake kin qa taku iba ecinśniyan nagi kin ihdaśke cin he yujuju kta, unkan Jehowa he kicicajuju kta.

9 Unkan woyake qa taku on wiwazica qaiś winohinca elipekiyapi nagi ihdaśke cin owasin suta kta.

10 Unkan hihnaku ti kin ohna oyake, qaiś taku wakan caje yate ça wicaśke on nagi kin ihdaśka :

$11 \mathrm{Qa}$ hihnaku nahon eśta inina yanke ça tehinda śni kinhan, woyake kin ecetu kta, qa wicaśke on nagi kin ihdaśke cin owasin suta kta.

12 Tuka hihnaku tohan nalion taku iha kin etanhan inanpe cin owasin katinyan yujuju kinhan, woyake kin, qa wicaśke on nagi ihdaśke cin he suta kte śni. hihnaku he yujuju he on Jehowa kicicajuju kta.

13 Woyake owasin; qa nagi iyokiśinye kta e taku wakan caje yate, wicaśke owasin hihnaku yasuta okihi, qa yajuju okihi. 14 Tuka hilinaku anpetu wan243 
ji qa anpetu iyokihe katinyan inina yanka cinhan, taku oyake owasin, qa wicaśke on ihdaśke cin owasin hena yasuta, hena nakon qehan anpetu kin he en inina yanka nakaś.

15 Tuka nahon kin iyohakam hena katinyan yajuju kinhan winyan walitani kin hihnaku yuhe kta.

16 Wokage wicaśta wan, qa tawicu otahedan, qa wicaśta ateyapi qa cunwintku wikośka a kkuku ti ohna yanke cin otahedan, Jehowa Mowis eciye cin hena eepi.

\section{WICOWOYAKE 31.}

1 Unkan Jehowa ie ca Mowis heciya,

2 Israel cinca on Midiyani tokiçon wicaya wo, ohakam oyate nitawa ekta okipe niyanpi lita.

3 Unkan Mowis ie ca oyate kin hewicakiya, Niyepi etanhan ozuye yapi kta e wicaśta wipe kiton wicaya po, unkan Midiyani azu wicayapi kta, hecen Jelowa on Midiyani en watoyeconpi kta.

4 Israel wicoun owasin etanhan wicoun otoiyohi etanhan kektopawinge wanjidan ozuye ye wicayaśipi kta.

5 Unkan Israel kektopawinge etanhan wicoun iyohi etanhan kektopawinge wan, kahnigapi, kektopawinge akenonpa ozuye on wipe kitonpi.

6 Unkan Mowis wicoun iyohi etanhan kektopawinge wan ozuye ye wicaśi nakun Pinehas Wleazar wawayuśna cinhintku kin ozuye yeši, qa taku on tipi wakan kicanyanpi, qa mazayahotonpi on panyanhanhan yahotonpi kin nape kin ohna. 244.
7 Unkan Midiyani kin azu wicryapi, Jehowa Mowis econ śi kin he iyecen, qa tona wica owasin wicaktepi.

$8 \mathrm{Qa}$ wicaktepi iyahna Midiyani wicaśtayatapi kin wicaktepi. Ewi, qa Reqem, qa Tzur, qa Hur, qa Reba Midiyani wicaśtayatapi zaptan, nakun Balam Beyor cinhintku canhipi on ktepi.

9 Unkan Israel cinca kin Midiyani winohinca kin, qa śicecapi kin, wayaka iwicacupi, nakun tawanunyanpi pte talinca ko owasin, qa taku yuhapi kin owasin kipi.

10 Qa otonwe ohna ounyanpi qon owasin, qa taconkaśkepi owasin peta on hulinahyapi.

11 Unkan taku kipi kin owasin icupi, wicaśta woteca koya. $12 \mathrm{Qa}$ wayaka qa taku icupi, qa taku kipi owasin hdohdapi, Mowis ekta, qa Eleazar wawayuśna ekta, qa Israel cinca omniciye ocowasin ekta, wicoti kin ekta; he Moab niakomdaya ohna un, Jordan icahda Jerilio iyotakonza.

13 Unkan Mowis qa Eleazar wawayuśna kin, qa omniciye itancan wicaye cin owasin itkowicakipe kta wicoti itankan inanpapi.

14 Unkan Mowis ozuye mdetanhunkapi kektopawinge itancanpi, qa opawinge itancanpi obe ozuye etanhan hdipi kin canniye wicaya.

15 Qa Mowis hewicakiya, Winyanpi kin owasin ni wicayayapi he?

16 Inyun hena Balam oie eciyatanhan Israel cinca kin Jehowa kipajin wicakiyapi, Peyor wicoie en, heon Jehowa taomniciye en makośice yukan.

17 Heon wanna śiceca ehna 


\section{WICOWOYAKE 31.}

tona wica owasin wicatke po, qa winohinca tona wicaśta kici wankapi on wicaśta sdonyapi owasin wicakte po.

18 Tuka śiceca winyanpi wicaśta sdonyapi śni, qa wica kici wankapi śni hena niyepi on ni wicayapo.

19 Qa niyepi anpetu śakowin wicoti itankan yankapo, qa tona wicanagi wan ktepi owasin, qa tona wicatancan ta icahtakapi owasin anpetu iyamni en, qa anpetu iśakowin en ihduskapo, qa wayaka duhapi kin hena wicayuska po.

$20 \mathrm{Qa}$ heyake owasin, qa taku kaśta taha on kagapi, qa tatokadan hin on kagapi, qa can on kagapi hduska po.

21 Unkan Eleazar wawayuśna kin ozuye wicaśta wokicize etanhan hdipi kin hewicakiya, Woope wokage Jehowa Mowis eciye cin dee.

22 Awicakehan mazaskazi, qa mazaska, qa mazaśa, qa mazasapa, qa mazaskaśdoyapi kin, qa mazasu,

23 Taku kaśta peta ohna opta ya okihi he peta iyopte yayapi kta; nakun wipakintapi mini on pakintapi kta, hecen slia kta, qa taku peta en ye kta okihi śni kin he owasin minin iyopte yayapi kta.

$24 \mathrm{Qa}$ anpetu iśakowin en heyake kin hdujajapo, qa ihduskapo, qa ohakam wicoti en yahipi kta.

25 Unkan Jehowa ie ca Mowis heciya,

26 Ozuye ipi taku icupi kin wicaśta wanunyanpi koya yawa wo, niye qa Eleazar, qa omniciye ateyapi otokapapi kin.

27 Qa taku icupi kin yapamni kta, obe kizapi kta ozuye ipi kin hena hanke wicayaqu kta, qa omniciye ocowasin hanke wicayaqu kta.

28 Qu ozuye obe wicaśta kizapi lkta iyayapi kin hena etanhan Jehowa on onśpa kusyaye kta, opawinge zaptan etanhan wvicanagi wanjidan iyacu kta, wicaśta etanhan, nakun pte etanhan, qa śonśonna etanhan, qa talinca etanhan.

29 Hanke tawapi etanhan Jehowa tawokosyapi kin iyacu kta, qa Eleazar wawayuśna kin yaqu kta.

30 Unkan Israel cinca hanke tawapi etanhan wikcemna zaptan etanhan wanjidan iyacu kta, wicaśta etanhan, qa pte etanhan, qa śonśonna etanhan, qa tahinca etanhan, wanunyanpi owasin etanhan, qa hena Lewi Jehowa toti kin awanyakapi kin wicayaqu kta.

31 Unkan token Jehowa Mowis econ śi hecen Mowis qa Eleazar econpi.

32 Unkan taku icupi ihe cin oyate ozuye ipi taku kipi kin he tahinca kektopawinge opawinge śakpe sanpa kektopawinge wikcemna śakowin, sanpa kektopawinge zaptan.

33 Qa pte kektopawinge wikcemma śakowin sanpa kektopawinge nonpa.

34 Qa śonśonna kektopawinge wikcemna śakpe sanpa kektopawintge wanjidan.

35 Qa wicanagi wicinyanpidan wica kici wankapi eciyatanhan wicaśta sdonyapi śni, winyan wicanagi owasin kektopawinge wikcemna yamni saupa kektopawinge nompa.

36 Qa he hanke obe ozuye ipi wopamni tawapi kin tahinca yawapi kin etanhan kektopawinge opawinge yamni sanpa kektopawinge wikcemna yamni san245 


\section{WICAYAWAPI,}

pa kektopawinge śakowin sanpa opawinge zaptan.

37 Qa tahinca etanhan Jehowa olśpa tawa kin opawinge śakpe sanpa wikcemna śakowin sanpa zaptin.

38 Qa pte kektopawinge wilicemna yamni sanpa kektopawinge śakpe, qa Jehowa onśpa tawa wikcemna śakowin sanpa nonpa.

39 Qa śonśonna kektopawinge wikcemna yamili sanpa opawinge zaptan, qa Jehowa onśpa tawa wikcemna śakpe sanpa wanjidan

40 Qa wicanagi kektopawinge wikcemna sanja kektopawinge śakpe, qa hena etanhan Jehowa onśpa tawa wicanagi wilicemna yamni sanpa nonpa.

41 Unkan Mowis Jchowa onśpa tawa wokosyapi kin Eleazar wawayuśna kin qu, Jehowa Mowis econ si kin he iyecen.

42 Unkan Israel cinca hanke tawapi wicaśta ozuye ipi kin etanhan Mowis icu qon etanhan:

43 Wanna omniciye hanke tawapi kin tahinca etanhan kektopawinge opawinge yamni sanpa kektopawinge wikcemna yamni sanpa kektopawinge śakowin sanpa opawinge zaptan.

$44 \mathrm{Qa}$ pte kektopawinge wikcemna yamni sanpa kektopawinge śakpe.

$45 \mathrm{Qa}$ śonśonna kektopawinge wikcemna yamni sanpa opawinge zaptan.

46 Qa wicanagi kcktopawinge wikcemna sanpa kektopawinge śakpe.

47 Unkan Mowis Israel cinca hanke tawapi kin etanhan taku icu wikcemna zaptan etanhan wanjidan icu, wicaśta etanhan, qa wanunyanpi etanhan, qa he- na Lewi Jehowa toti awanyakapi kin wicaqu, Jehowa Mowis ecoll si kin he iyecen.

48 Unkan ozuye kektopawinge mdetanhunkapi, kek topawinge itancanpi, qa upawinge itancanpi kin Mowis ekta hipi :

49 Qa Mowis heciyapi, Nitaokiye wicaśta unnapepi ihukuya okicize ekta ipi kin hena wicunyawapi, qa unkiyepi etanhan wicaśta wanjidan aokpani.

50 Heon woahi wan Jehowa unkahipi wicaśta otoiyohi mazaskazi oinpi iyeye cin, mazaicicahilia, qa mazahuhu, mazanapcupe, oinpi, qa maza śipto, nagi akahpe unkiciyapi kta Jehowa itokam.

51 Unkan Mowis qa Eleazar taku mazaskazi on kagapi owasin hena etanhan icupi.

52 Mazaskazi wokosyapi Jehowa ekta kosyapi kin he śeqel kektopawinge akeśakpe sanva opawinge sakowin sanpa wikcemna zaptan henakeca, kcktopawinge itancanpi qa opawinge itancanpi" etanhan.

53 Obe wicaśta lin otoiyohi iye on taku ki.

54 Unkan Mowis qa Eleazar wawayuśna kin mazaskazi kin icupi kektopawinge, qa opawinge itancanpi etanlian, qa itkokipapi wakeya en ahipi, Israel cinca on wokiksuye Jehowa itokam.

\section{WICOWOYAKE 32.}

1 Unkan Ruben cinca qa Gad cinca kin wanunyanpi ota yuliapi, ota hinca, qa Jazer makoce kin, qa Gilead makoce kin wanyakapi, qa inyun makoce kin he wallunyanpi makoce heca.

2 Qa Gad cinca qa Ruben cinca upi, qa Mowis qa Eleazar

246 


\section{WICOWOYAKE 32.}

wawayuśna kin, qa omniciye itancanpi kin hewicakiyapi ;

3 Atarot, qa Dibon, qa Jazer qa Nimra, qa Ḣeśbon, qa Elleale, qa Śbebam, qa Nebo, qa Beon.

4 Makoce Jehowa Israel omniciye itokam kaśtake cin, he wanunyanpi on makoce waśte hinca, qa llitaokiye wanunyanpi yuhapi.

5 Niiśta kin en woiyokipi iyeunyanpi kinhan, makoce kin he nitaokiye unqumiye, tawa unyanpi, kta, qa Jordan akasanpa unyanpi kte śni, eyapi.

6 Unkan Mowis Gad cinca, qa Ruben cinca kin hewicakiya, Nihunkawanjipi wokicize ekta yapi, qa niyepi den yakidotanliapi kta he?

7 Tokeca Israel cinca cantepi kin wankadan yakagapi? hecen makoce Jehowa wicaqu kin en iyoptapi kte śni.

8 Niyatepi Qadeś Barneya etanhan makoce iwanyag ye wicawaśi qehan hecen econpi.

9 Hehan Eśkol kalssiza ekta ipi, qa Israel cinca canwankapidan wicakagapi, heon makoce Jehowa wicaqu kin he elita ipi śni.

10 Unkan anpetu kin he en Jehowa canniye ca caje ihdate konze ca heya,

11 Wicaśta waniyetu wikcemna nonpa aktonpi Égupta etanhan itanwankanhde upi kin hena makoce Aberaham qa Izak qa Jakob konza ewicawakiye ciqon he wanyakapi lkte śni, ocowasin omapapi śni nakaś.

12 Kaleb Jepune Qenizi kin, qa Jośuwa Nun cinhintku henana, hena Jehowa iyohakam yuśtanpi nakaś.

13 Unkan Jehowa Israel canniye wicaya, unkan hopuza ohna waniyetu wikcemna topa ihdakapi, wicoicage Jehowa iśta kin en taku śica econpi kin hena sotapi kin hehanyan.

14 Unkan inyun niyatepi heekiya wicaśta wahtanipisa wicoicage nayajinpi, qa Jehowa Israel canniye wicaye cin he sanpa tanka yakagapi kta.

15 Tuka iye etanhan nihdamnapi kinhan, ake hopuza en yanke wicaye lita, qa oyate kin de owasin awihnuni wicayayapi kta.

16 Unkan ikiyedan hipi qa heciyapi, Deciya tahinca wanunyanpi tipi wicunkicagapi kta, qa unkicincapi otonwe wicunkicagapi kta.

17 Qa unkiyepi wipe kiton waditagya Israel cinca wicitokam unyanpi kta, tukten yakonpi kta ek ta hiyuwicunyanpi kin hehanyan, tuka śiceca unkitawapi otonwe aconkaśkayapi ohna iyc)tankapi kta, oyate makoce kin den unpi kin heon etanhan.

18 Israel cinca otoiyohi maga tawa tawaye śni lkin hehanyan untipi en unhdipi kte śni.

19 Qa hena om Jordan akasanpa takudan tawa unyanpi kte śni, Jordan itato wiyohiyanpata ektakiya maga unkitawapi yanke nakaś.

20 Unkan Mowis hewicaliya, Token ehapi hecen ecanonpi kinhan, Jehowa itokam wipe kiton ozuye dapi kinhan,

$21 \mathrm{Qa}$ otoiyohi wipe kiton Jehowa itokam Jordan akasanpa idadapi, iye itokapa tanhan toka wicaye cin nape wicaye cin hehanyan.

22 Qa makoce Jehowa itokam ktepidan, qa ohakam yahdipi kinhan, Jehowa itokam, qa Israel itokam iyaonpepicaśni yaunpi kta, qa Jehowa itokan makoce kin de tawa yayapi kta. 


\section{WICAYAWAPI,}

23 Tuka hecen ecanonpi śni kinhan, inyun Jehowa awayahtanipi, qa wayahtanipi kin iyeniyanpi kta sdonyapo.

24. Siceca nitawapi on otonwe icicagapo, qa tahinca nitawapi tipi wicakicagapo, qa taku niihapi etanhall inanpe cin econpo.

25 Unkan Gad cincà, qa Ruben cinca iapi, qa Mowis leciyapi, Token Itancan unyanpi eye cin ecen econkunpi kta.

26 Unkicincapi unkitawicupi, tahinca unkitawapi, qa wanunyanpi unkitawapi owasin, heciya Gileyad otonwe en yakonpi kta. 27 Tuka nitaokiye wipe kiton obe owasin Jehowa itokam wokicize ekta iyounptapi kta, itancan unyanpi eye cin iyecen.

28 Unkan Mowis hena on Eleazar wawayuśna kin qa Jośuwa Nun cinhintku kin, qa Israel cinca wicoun ateyapi itancanpi kin wahokonwicakiya.

29 Qa Mowis hewicakiya, Gad cinca, qa Ruben cinca wipe kitonpi owasin niyepi om Jehowa itokam wokicize ekta iyoptapi, qa nitokam makoce ktepidan kinhan, Gileyad makoce kin tawayapi kta, e wicayagupi kta.

30 Tuka wipe kiton niyepi om iyoptapi sni kinhan, niyepi ehna Kanan makoce onśpa tawayapi kta.

31 Unkan Gad cinca, qa Ruben cinca ayuptapi, qa heyapi, Taku Jehowa nitaokiye unkekiyapi he econkunpi kta.

32 Unkiyepi wipe kiton Jehowa itokam Kanan makoce ekta iyounptapi kta, hecen Jordan itato makoce kin tawa unyanpi kta.

33 Unkan Mowis Gad cinca, qa Ruben cinca, qa Josep cinhintku Manaśe wicoun hanke, Si248 hon Amori wicaśtayatapi kin makoce idake cin he, qa $\mathrm{Og}$ Baśan wicaśtayapi makoce idake cin, makoce otonwe kin, qa otonwe ihdukśan makoce wanke cin wicaqu.

34 Unkan Gad cinca kin otonwe kagapi Dibon, qa Atarot, qa Aroyer,

35 Qa Aterot-Śopan, qa Jazer, qa Jogbeha.

36 Qa Bet-Nimra, qa Bet-Haran, otonwe conkaśkeyapi, nakun talinca tipi kagapi.

37 Unkan Ruben cinca kin Heśbon, qa Eleale, qa Qireyataim kagapi,

38 Nakun Nebo, qa Bayal Meyon, caje yatokecapi, qua Sibma, qa otonwe kagapi cajepi kin cajehdatapi.

39 Unkan Manaśe cinhintku Makir cinca kin Gileyad ekta ipi, qa he icupi, qa Amori hen tipi kin nape wicayapi.

40 Unkan Mowis Gileyad makoce Manaśe cinhintku Makir cinca kin wicaqu, unkan hen iyotankapi.

41 Unkan Manaśe cinhintku Jair, wicotipi kin ekta i qa hena icu, qa hena Hawot-Jair (Jair totonwe) eya caje yata.

42 Unkan Noba Qenat ekta i qa icu, nakun otonwe ciqadan cunkśi wicaya, qa he Noba eya cajeyata iye caje eciyatanhan.

\section{WICOWOYAKE 33.}

1 Israel cinca obe tawapi iyecen Egupta makoce etanhan inanpapi, Mowis qa Aaron nape kin ihukuya, oihdakapi kin dena eepi.

2 Unkan inanpapi kin ihdakapi iyecen, Jehowa i kin eciyatanhan Mowis owa, qa ihdakapi inanpapi eciyatanhan dena eepi. 
3 Unkan wi tokaheya en wi anpetu iakezaptan en Rameses etanhan ihdakapi, woacakśin ihanhanna, Israel cinca nape yuwankan icupi inanpapi, Egupton owasin wanyakapi.

4 Unkan Egupton tona iyepi etanhan Jehowa wicakaśtaka, caskepi owasin wicahnakapi, nakun taku wakan dapi kin Jelıwa woyaco ecawicakicon.

5 Unkan Israel cinca Rameses etanhan ihdakapi, qa Sukot (ohanzihdepi) ek ta etipi.

6 Unkan Sukot etanhan ihdakapi, qa Etam ekta etipi, he hopuza ihanke ekta yanka.

7 Unkan Etam etanhan ihdakapi qa ihdamnapi Pi-Hahirot ektakiya, he Bayal Tzepon itokam yanka, qa Migdol (Conkaśke) itokam etipi.

8 Unkan Hahirot itokapa tanhan ihdakapi, qa mde cokaya iyoptapi, liopuza ekta, qa Etam hopuza ohna anpetu yamni ihdakapi, qa Mara en etipi.

9 Unkan Mara etanhan ihdakapi, qa Elim en etipi, qa Elim ohna miniyowe akenonpa, qa tamar can wikcemna śakowin, qa hen ticagapi.

10 Unkan Elim etanhan ihdakapi qa Mde śa icahda etipi.

11 Unkan Mde śa etanhanihdakapi qa Sin hopuza ohna etipi.

12 Unkan Sin hopuza etanhan ihdakupi qa Dopqa ohna etipi.

13 Unkan Dopqa etanhan ihdakapi qa Aluś ohna etipi.

14 Unkan Aluś etanhan ihdakapi qa Repidim ohna etipi, qa hen mini yatkanpi kta wanica.

15 Unkan Repidim etanhan ihdakapi qa Sina hopuza ohna etipi.

16 Unkan Sina hopuza etanhan ihdakapi qa Qibrot-Hatawa ohna etipi.
17 Qa Qibrot-Hatawa etanhan ihdakapi qa Hatzerot ohna etipi. 18 Qa Hatzerot etanhan ihdakapi qa Ritma ohna etipi.

19 Unkan Ritma etanhan ihda-. kapi qa Rimmon-Parets ohna etipi.

20 Unkan Rimmon-Parets etanhan ihdakapi qa Libna ohna etipi.

21 Unkan Libna etanhan ihdakapi qa Risa ohna etipi.

22 Unkan Risa etanhan ihdakapi qa Qehelata ohna etipi.

23 Unkan Qehelata etanhan ihdakapi, qa Śaper he kin ekta etipi.

24 Unkan Śaper lie etanhan ihdakapi qa Ḧarada ohna etipi.

25 Unkan Harada etanhan ihdakapi qa Maqhelot ohna etipi. 26 Unkan Maqhelot etanhan ihdakapi qa Tahat ohna etipi.

27 Unkan Tahat etanhan ihdalsapi qa Tarah ohna etipi.

28 Unkan Tarali etanhan ihdakapi qa Mitqa ohna etipi.

29 Unkan Mitqa etanhan ihdakapi qa Ḣámona ohna etipi.

30 Unkan Haśmona etanhan ihdakapi, qa Moserot ohna etipi.

31 Unkan Moserot etanhan ihdakapi qa Bene-Jaaqan olina etipi.

32 Unkan Bene-Jaaqan etanhan ihdakapi qa Hor-Hagidgad (wakinyan ti kin) ek ta etipi.

33 Unkan Hor-Hagidgad etanhan ihdakapi qa Jotbata ohna etipi.

34 Unkan Jotbata etanhan ihdalkapi qa Abrona ohna etipi.

35 Unkan Abrona etanhan ihdakapi qa Etzion-Gaber (wicaśta cankahu) ekta etipi.

36 Unkan Etzion-Gaber etanhan ihdakapi qa Tzin, Qadeś hee, hopuza ohna etipi.

37 Unkan Qadeś etanhan ihda249 
kapi, qa Hor he kin ekta etipi, Edom makoce ihanke ekta.

38 Unkan Jehowa i eciyatanhan Aaron wawayuśna kin Hor he kin akan itanwankanhde i, qa hen ta, Israel cinca Egupta makoce etanhan hdinanpapi waniyetu wikcemna topa wi izaptan anpetu tokaheya en.

39 Qa Aaron waniyetu opawinge sanpa wikcemna nonpa sanpa yamni tonpi ehantanhan Hor he kin en ta.

40 Hehan Arad wicaśtayatapi Kanani kin Kanan makoce itoliaga en ounye cin Israel cinca upi kin nahon.

41. Unkan Hor he kin etanhan ihdakapi qa Tzalmona ohna etipi.

42 Unkan Tzalmona etanhan ihdakapi, qa Punon ohna etipi.

43 Unkan Punon etanhan ihdakapi qa Obot elkta etipi.

44 Unkan Obot etanhan ihdakapi qa Ije-Abarim Moab makoce ihanke ekta etipi.

45 Unkan Ije-Abarim etanhan ihdakapi qa Dibon-Gad ohna etipi.

46 Unkan Dibon-Gad etanhan ihdakapi qa Almon ohna Diblataim ektakiya etipi.

47 Unkan Almon Diblataim ektakiya tanhan ihdakapi qa Abarim he ohna, Nebo itokam etipi.

48 Unkan Abarim he etanhan ihdakapi qa Moab maliomdaye Jordan icahda Jerilio iyotakons etipi.

49 Unkan Jordan icahda Bet Jeśimot etanhan Abel (Woiyokiśica) Śitim ekta Moab makomdaya ohna etipi.

50 Unkan Moab makomdaya ohna Jordan icahda Jeriho iyotakons Jehowa ie ca Mowis heciya,

51 Ie ca Israel cinca kin hewica250 kiya wo, Kanan makoce ekta Jordan iduwegapi kinhan ;

52 Makoce ounyanpi owasin nitokapa tanhan nape wicayayapi kta, qa wakagapi owapi tawapi kin owasin awihnuni yayapi kta, qa wakagapi śdoyapi tawapi kin owasin ihang yayapi kta, qa tawahnawośnapi kin owasin dujujupi kta.

53 Hecen nape wicayayapi kta makoce etanhan, qa ohna yakidotankapi kta, makoce kin he tawayayapi kta e ciçupi nakaś.

54 Unkan oeconna eciyatanhan makoce yapamnipi kta, wicowazipi nitawa iyecen, wicotapi kinhan makoce tankaya wicayaqupi kta, qa tonanapi kinhan makoce aoptetu wicayaqupi kta, otoiyohi taku oeconna eciyatanhan icu kin he tawa ye kta, ate wicayayapi wicowazipi kin iyecen tawa yayapi lita.

55 Tuka makoce ounyanpi kin nitokapa tanhan nape wicayayapi śni kinhan, kaketu kta; tona owicayakaptapi kin hena, wapepeka niiśtapi ohna iyececapi kta, qa taku pepe nicuwipi en, qa makoce ohna yakidotankapi akan toka niyanpi kta.

56 Unkan token ecawicawecon kta kepce cin hecen ecaciconpi kta.

\section{WICOWOYAKE 34.}

\section{Unkan Jehowa ie ca Mowis} heciya,

2 Israel cinca wahokon wicakiye ça hecen ewicayakiye kta, Kanan makoce en yaipi kinhan makoce tawayayapi kta niçupi kta dee, İanan makoce ihanke owasin ekta.

3 Qa itokaga ihanke kin Tzin hopuza etanhan Edom icahda duhapi kta, qa wihinanpe ektakiya miniskuya mde ihanke ito- 


\section{WICOWOYAKE 34.}

kaga etanhan ihanke duhapi kta.

4 Qa makoce nitawa ihanke kin Aqrabim iyakaptapi itokaga tanhan iyaye kta, Tzin elkta i kta; qa Qadeś-Barnea itokaga tanhan iyaye kta Hadar ti ekta, qa Atzmon ekta iyopte kta.

5 Qa Atzmon etanhan ihanke kin yuktanyan ye kta, Egupta wakpa ekta, qa miniowanca ekta ihunni kta.

6 Unkan ihanke wiyohpeyata ekta miniowanca huta kin hee duhapi kta, qa he huta kin wiyohpeyata ektaliiya ihanke duhapi kta.

7 Unkan waziyata ektakiya ihanke kin de duhapi kta, miniowanca etanhan Hor he kin ekta iyecagopi kta.

8 Qa Hor he kin etanhan $\mathrm{Ha}$ mat ocanku ektakiya iyakagopi kta, qa makoce ihanke kin Tzedad ekta iyohi kta.

9 Qa makoce ihanke kin Zipron iyohi lkta, qa-Hatzar-Enan (Miniyowe Otonwe) hehanyan ye kta, waziyata ektakiya ihanke duhapi kta hee.

$10 \mathrm{Qa}$ wiyohiyanpata ektakiya ihanke iyecagopi kta HatzarEnan etanhan Sepain elita.

11 Qa ihanke Sepam etanhan Ribla ekta Ain (Miniyowe) iwiyohiyanpa tanhan qa opapun kin itokaga ye kta, qa Kiniret mde huta kin icahtake Lita wi hinanpe ektakiya:

12 Qa opapun kin Jordan iyokaga ye kta, makoce hecen ihdukśan icagopi duhapi kta hee.

13 Unkan Mowis Israel cinca wahokon wicakiye ca heya, Makoce oeconna eciyatanhan tawayayapi kte cin hee, Jehowa wicoun napcinwanka qa wicoun wan hanke wicaqu maśi hee.
14 Ruben cinca wicoun kin ateyapi tipi kin iyecen, qa Gad cinca wicoun ateyapi tipi kin iyecen wanna icupi qa Manaśe wicoun hanke makoce tawa yapi kte cin he wanna icupi.

15 Wicoun nonpa qa wicoun wan hanke makoce tawayapi kte cin he wanna icupi, Jordan itato Jeriho iwiyohiyanpa tanhan wi hinanpe ektakiya.

16 Unkan Jehowa ie ca Mowis heciya,

17 Wicaśta makoce nicipamnipi kta cajepi kin dena eepi, Eleazar wawayuśna kin, qa Jośuwa, Nun cinhintku.

$18 \mathrm{Qa}$ wicoun otoiyohi etanhan itancan wan makoce pamnipi kta e iyacupi kta.

19 Qa wicaśta cajepi kin dena eepi, Juda wicoun etanhan Kaleb Jepune cinhintku,

20 Qa Simeon cinca wicoun etanhan Śsmuel Amihud cinhintku,

21 Benjamin wicoun etanhan Elidad Kiselon cinhintku,

22 Dan cinca wicoun etauhan itancan kin Buqi Jogeli cinhintku,

23 Josep cinca etanhan, Manaśe cinca wicoun etanhan itancan kin Haniyel Epod cinhintku, 24 Qa Epraim cinca wicoun es tanhan itancan kin Qemuel Siptan cinhintku.

25 Qa Zebulun cinca wicoun etanhan itancan kin, Elitzapan Parenak cinhintku,

$26 \mathrm{Qa}$ Isakar cinca wicoun etanhan itancan kin, Paltiel Azan cinhintku,

27 Qa Aśer cinca wicoun etanhan itancan kin Ahihud Ślomi cinhintku.

28 Qa Naptali cinca wicoun etanhan itancan kin Pedahel Amihud cinhintku, 
29 Hena Jehowa Kanan makoce Israel cinca wicakicipamni wicaśi.

\section{WICOWOYAKE 35.}

1 Unkan Moab makomdaye ohna Jordan icahda Jeriho iyotakons Jehowa ie ca Mowis heciya.

2 Israel cinca wahokon wicakiye ca makoce oeconna eciyatanhan tawayapi kta etanhan Lewi otonwe ohna iyotankapi kta e wicaqupi kta kewicakiya wo ; qa tonwanyanpi kin ihdukśan tinta onśpa wicayaqupi kta ehe kta.

3 Qa otonwe ounyanpi kta e yuhapi kta, qa tinta kin tawotecapi on, qa tawanunyanpi, qa ta$\mathrm{ku}$ yuhapi owasin on yuhapi lita.

4. Qa otonwe tinta kin, otonwe conkaślke anokatanhan ihdukśan wiciśpa kektopawinge iyohi ye kta.

5 Unkan otonwe itankapa tanhan wiyohiyanpata ihanke kin wiciśpa kektopawinge nonpa idutapi kta, qa itokaga ihanke kin wiciśpa kektopawinge nonpa, qa wiyolipeyata ihanke lin wiciśpa kektopawinge nonpa, qa raziyata ihanke kin wiciśpa kektopawinge nonpa, cokaya tonwanyanpi kta otonwe tinta heca yuhapi kta.

6 Unkan otonwe Lewi wicayaqupi śakpe wowinape otonwe heca wicayaqupi kta, on tuwe wicaśta wan kte kinhan ekta inyanke kta, qa nakun otonwe wilicemna topa sanpa nonpa wicayaqupi kta.

$y$ Otonwe Lewi wicayaqupi lita owasin otonwe wikcemna topa sanpa śahdogan, hena qa tinta ihdukśan koya.
8 Qa Israel cinca taku tawayapi etanhan otonwe wicayaqupi kta, otoiyohi otonwe tawa etanhan, Lewi wicaqu $\mathrm{kta}$, makoce oeconna eciyatanhan tawaye cin iyecen, ota etanhan ota yaqupi kta, qa tonana etanhan tonana yaqupi kta.

9 Unkan Jehowa ie ca Mowis heciya,

10 Ie ca Israel cinca kin hecen ewicakiya wo, Kanan makoce ekta Jordan iduwegapi kinhan,

11 Wowinape otonwe niçicagapi kta, qa tuwe wanun wicaśta wanji kte kinhan hena wanji elita inyanke kta e hena duhapi kta.

$12 \mathrm{Qa}$ hena watokicon etanhan wowinape, otonwe duhapi kta; hecen tuwe wicašta wan kte kinhan, yacopi kta e omniciye itokan najin śni kin hehanyan te kte śni.

I3 Otonwe wicayaqupi etanhan wowinape otonwe śakpe duhapi kta.

14 Otonwe yamni Jordan itato yaqupi kta, qa Kanan makoce en otonwe yamni yaqupi kta; wowinape otonwe hecapi kta.

15 Otonwe śakpe kin hena wowinape hecapi kta, Israel cinca on, qa tuwe oyate tokeca, qa niye ehna ounye cin on, tuwe wim canagi wan wanun kaśtake cinhan heciya nape kta.

16 Tuka maza śapa wipe wan on kaśtaka on te cinhan tin kte, qa tinwicakte awicakehan te kta.

17 Unkan inyan on wicaśta țe kta iyececa on apa, unkan ta, tuwe he ape cinhan te kta, tinwicakte heca, tuwe tinwicakte awicakehan te kta.

18 Qaiś can wanji on wicaśta apapi te kta iyececa on ape ca te cinhan tinwicakte lieca, qa 
tuwe tinwicakte heca awicakehan te kta.

19 Tuwe we tokicon kin tinwicakte kin kte kta, tohan he akipc cinhan kte kta.

$20 \mathrm{Qa}$ he sicedaka on taku patan, qaiś elipeye cinhan, qa hecon kte hinca on te cinhan;

21 Qaiś tokaye cin on nape on ape ca on te cinhan, tuwe he ape cin awicakehan te kta, he tinkte; tuwe tokicon kin tohan tinwicakte cin akipe cinhan he kte kta.

22 Tuka he śicedake śni qa ihnunhanna wipe taku kaśta patan, qaiś ehpeye cinhan, qa econ kte hince śni.

23 Qaiś he wanyake śni, qa inyan wan taku kaśta on wicasta te kta iyececa ehpeye ça akan ilhpaye ca on te cinhan, tuka he tolkaye śni, qa akite śni kinhan,

24 Omniciyc kin tuwe ape cin qa we tokiçon kin otahedan yacopi kta, woyaco kin hella eciyatanhan.

$25 \mathrm{Qa}$ omniciye kin tuwe wicakte kin we tokicon nape kin etanhan eyakupi kta, qa omniciye kin wowinape otonwe ekta nape ciqon ake ekta ayapi kta, qa hen yanke kta, wawayuśna tanka wihdi wakan on sdayapi te kte cin hehanyan.

26 Tuka tuwe wicakte wowinape otonwe ekta nape ciqon ihanke itankan inanpe cinhan,

27 Unkan we tokicon kin he wowinape otonwe ihankc itankan iyeye cinhan, qa we tokiçon kin wicakte kin kte kinhan, he on tuwedan we yapi kte śni.

28 Wowinape otonwe tawa en yanke kta iyececa, wawayuśna tanka te kte cin hehanyan, qa wawayuśna tanka te cin iyohakam wicakte kin makoce tawaye cin en hdi kta.
29 Wokage woope kin hena duhapi kta, nitoicagepi yeye cin tonwanyayapi kin owasin ohna. 30 Tuwe wicanagi wan ape cinhan wayaatanin nonpa ipieciyatanhan ktepi kta, tuka wayaatanin wanjidan wicanagi ktepi kta ayuptapi kte śni.

31 Tuwe tinwicakte yacopi nagi kin akahpe kta e woakahpe iyacupi kte śni, tuka awicakehan te kta.

32 Nakun woakahpe iyacupi kte śni on tuwe wowinape otonwe ekta nape cin wawayusna te śni itokam makoce ounye - kita ek ta hde kta.

33 Qa makoce en dukanpi kta duśapapi kte śni, maka kin yuśape cin we kin hee, qa wc makoce ohna papsonpi taku tokeca akahpe kta okihi śni tuwe he papson we tawa on hecedan.

34 Qa makoce ohna ounyayapi kin he duśapapi kte śni, he ohna miye ounwaya, miye Jehowa Israel cinca wicehna ounwaye nakaś.

\section{WICOWOYAKE 36.}

1 Unkan Manaśe cinhintku Makir cinhintku Gileyad cinca wicowazi ateyapi itancanpi Josep cinca wicowazipi etanhan ikiyedan hipi qa Mowis itokam qa Israel cinca ateyapi watokapapi itancanpi itokam iapi.

2 Qa heyapi, Makoce tawayapi- kta e Israel cinca oeconna eciyatanhan Jehowa itancan mitawa wicaqu śi, qa Tzelopeliad maga tawa iye cunwintku Jehowa itancan mitawa wicaqu śi.

3 Unkan Israel cinca wicoun wan cinca etanhan wicaśta wan wicayuze cinhan, maga tawapi ate unyanpi makoce tawapi etanhan yuśpapi kta, qa tuwe wica253 
yuze cin tawicoun tamakoce elita okijuyapi kta, hecen makoce oeconna eciyatanhan tawa unyanpi kin he cistinna kagapi kta.

4 Israel cinca Jubele yuhapi kinhan, winyanpi tamakocepi tuwe wicayuze wicoun tamakoce ekta okijuyapi kta, qa maga tawapi ate unyanpi wicoun tamakoce etanhan yuśpapi kta.

5 Unkan Mowis Israel cinca Jehowa i eciyatanhan wahokon wicakiye ça heya, Josep cinca oiepi kin iyececapi.

6 Wicoie Jehowa Tzelopehad cunwintku econ wicaśi kin dee, heya, Ateyapi wicoun wicowazi etanhan heceedan tuwe wicayuze lkta, tuka hena etanhan tuwe iśtapi en waśte wicayuze kta.

7 Israel cinca makoce oeconna. eciyatanhan tawayapi kin wicoun wanji etanhan wicoun tokeca ekta yutokanpi kte śni ; tuka Israel cinca otoiyohi ate wicaye wicoun tamakoce kin ckta ihdaśke kta.

8 Unkan Israel cinca wicoun 254 etanhan tuwe kasta cunwintke maga tawa ye kta, wicaśta wan ateye wicoun wicowazi etanhan he tawicu kta hecen Israel cinca otoiyohi ate wicaya tamakocepi kin tawaye kta.

9 Qa makoce kin wicoun wanji etanhan wicoun tokeca ekta yutokanpi kte śni, tuka Israel cinca wicoun etanhan wicaśta otoiyohi makoce oeconna etanhan tawaye cin he en ihdaśke lita.

10 Token Jehowa Mowis eciye cin hecen 'Tzelopehad cunwintku econpi,

11 Unkan Malila Tirtza qa Hoglaqa Milka qa Noya Tzelopehad cunwintku ate wicayapi cinca wicayuzapi.

12 Josep cinhintku Manaśe cinca wicowazipi etanhan wicayuzapi qa maga tawapiateyapi wicowazi wicoun en yanka.

13 Wokage qa woyaco Jehowa Mowis nape on Israel cinca econ wicaśi, Moab makomdaya ohna, Jordan icahda, Jeritio iyotakons dena eepi.

WICAYAWAPI IHANKE.

Micotomed by

preservation

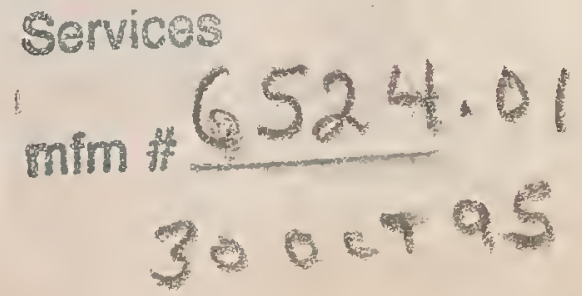




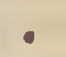

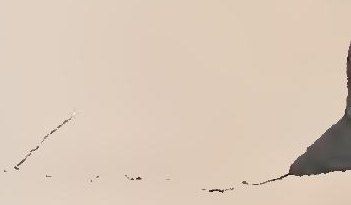





\title{
PSALII WOWAPI.
}

TH $\mathrm{E} \cdot$

\section{BOOK OF PSALMS,}

IN THE

\section{包akata equatuage:}

TRANSLATED FROM THE HEBREW,

\author{
BY S. R, RIGGS, A, M,
}

MISSIONARY OF THE A. B. C. F. M.

NEW YORK:

AMERICAN BIBLE SOCIETY, INSTITUTED IN THE XEAR MDCCCXVI. 


\section{PSALM WOWAPI.}

\section{PSALM 1.}

1 Wicaśta cante waśte hinca;

Tuwe wicaśta śica tawowahokonkiye kin ohna mani śni,

Qa wahtanipi sa tacanku kin en inajin śni,

Qa wauncapi oyanke tawapi kin en iyotanke śni:

2 Tuka Jehowa toope kin wowiyuśkin ya;

Qa tawoahope kin awacin un lkta, anpetu hanyetu koya.

3 Unkan he can wan mini wakpa icahda ojupi, Qa iyehantu eca waskuyeca icaliya ece kin he iyecece kta;

Nakun ape tawa kin śnije kte śni,

Qa taku econ kte cin owasin tanyan yuśtan kta.

4. Wicaśta śicapi kin hececapi śni ;

Tuka hena aguyapi ha tate kahbog iyeye cin he iyececapi.

5 Heon wicaśta śica woyaco kin en najinpi kte śni ;

Qa walitanipi sa wicaśta owotanna tomniciye kin en unpi kte śni.

6 Jehowa wicaśta owotanpidan tacanku kin he sdonya;

Tuka wicaśta śicapi tacanku kin atakuni kte śni.

\section{PSALM 2.}

1 Tokeca Ikcewicaśta kin oqoyapi,

Qa oyatepi takuśni awacinpi kta he.

2 Maka wicaśtayatapi kin odakonkiciyapi, Qa wicaśta itancanpi witaya wohdakapi, Jehowa qa Tuwe Sdakiye cin on.

3 Wicaśke tawapi kin unpapsakapi lkta, Qa hakahmonpi tawapi ehpeunyanpi lita ce.

4 Malipiya kin en iyotanke cin he ilia kta; Itancan kin iwicalialia kta.

5 Hehan tocanniye en wowicakiyake kta; Qa śinhda linca en śitkihda wicaye kta.

6 Tuka Zion Paha Wakan mitawa kin akan, Wicaśtayatapi mitawa sdawakiya ce.

7 Wokonze kin owahdake kta ; Jehowa hemalkiya, Micinkśi kin he niye, Anpe dehan cinca cicaga ce.

8 Makida wo, kinhan Ikcewicaśta tawa wicayaye kta, Qa maka ihanke kin nitawa kta e cicu kta.

9 Maza icapsinte wan on wicayakawihnuni kta, Maka wakśica iyeeen wicayakamdece lita ce. 
10 Heon etanhan wicaśtayatapi, ksapa po;

Maka wayacopi, waonspeiciciya po:

11 Wokokipe yuha Jehowa ohoda po;

Qa wonihinciye on iyaśa po.

12 Cinhintku kin i iputaka po,

Okinni śinhda kta, qa tocanniye kitanna ide kinhan,

Canku kin etanhan nitakunipi kte śni.

Tona iye wacinyanpi owasin cante waśtepi ece.

\section{PSALM 3.}

Dawid tadowan, iye cinhintku Absalom ite kin nakicipa, he ehan.

1 Jehowa, toka mayanpi kin hena ota aya;

Tona takpe maupi kin hena wicota.

2 Wicota minagi heciyapi;

Wakantanka en nikiyapi wanica ce. Sela.

3 Unkan Jehowa, mihdukśan wahacanka kin he niye;

Wowitan mitawa qa pa yuwankan imacu kin.

4 Miho kin on Jehowa hoyewakiya ;

Unkan iye Paha Wakan tawa kin etanhan namahon. Sela.

5 Miye imunke ça miśtinbe;

Ake wekta; Jehowa mayuhe cin heon.

6 Oyate kektopawinge wikcemna kowicawakipe kte śni, Ihdukśan tanhan takpe maupi kin.

7 Jehowa inajin wo;

Wakantanka mitawa nimayan wo;

Tokamayanpi owasin tapon hu kin en awicayapa;

Wicaśta śica hi kin wicayecamdeca.

8 Woehdaku kin he Jehowa tawa;

Woyawaśte nitawa nitaoyate kin en un nunwe. Sela.

\section{PSAM 4.}

David tadcwan. Candowankiyapi on dowan itancan kin.

1 Wakantanka owotanna mitawa,

Houwaye cinhan amayupta wo;

Makakija en mayadutanka;

Onśimalkida, qa wocekiye mitawa nahon wo.

2 Wicaśta cinca, tohanyan mitowitan wowiśtece yayapi kta he ; Tohanyan takuśni waśteyadapi qa woitonśni ayakitapi kta he. Sela.

3 Tuka Jehowa wicaśta wacantkiye cin iye on eiçihde e sdonya po;

Hoyewakiya eca Jehowa namakilion ece kta.

4 Kokipa po, qa wahtanipi śni po,

Nitowinjapi akan nicantepi on awaiçicinpi,

Qa inina yukan po. Sela.

5 Wicoowotanna wośnapi kin wośna po,

Qa Jehowa wacinyan po. 
P $\$$ A $\mathrm{M} 6$.

6 Tuwe taku waśte unkipazopi kta he, wicota eyapi ece: Jehowa, niye nitohnake iyoyanpa kin unkayuhomni po.

7 Wowiyuśkin micante kin en eyahnaka, Aguyapi qa miniśa wicakiciyuotapi kin he isanpa.

8 Wookiye en imunke ça nakun miśtinbe lkta;

Jehowa, niye niśnana wowacinye en ounye mayakiye cin heon.

\section{PSALM 5.}

Dawid tadowan. Cotanka on dowan itancan kin kicagapi.

1 Jehowa mioie kin nahon wo;

Mitawacin kin he iyulcan ye.

2 Mitawocelkiye ho kin he nalion wo,

Wicaśtayatapi mitawa, qa Wakantanka mitawa;

Ceciciye kta heon etanhan.

3 Jehowa, hanhanna eca miho kin nayahon kta ;

Hanhanna eca houciciye ca wankan ewatonwe kta.

4 'Taku Wakan woahtani en iyuśkin kin henica śni ;

Taku śice cin he niciti kte śni.

5 Tona witkotkoke cin niiśta itokam najinpi kte śni :

Tona woahtani econpi kin iyuhpa śice wicayadaka ece.

6 Tona itonpiśni kin hena ihangwicayaye kta;

We wicaśta qa wicahnaye sa kin he Jehowa wahteda kte śni.

7 Tuka miye nitowaonśida ota kin on yati en wau kte;

Kocicipe cin on tipi wakan nitawa etkiya ohowada kta.

8 Jehowa, nitoowotanna on amayan wo, toka mayanpi kin on etanhan ;

Miite itokam canku nitawa owotanna kaga wo.

9 I kin ohna wowicake wanica:

Mahen tanhan wicośice yukan;

Dotepi kin wicawoha yulidokapi kin heca.

Cejipi on wicahnayanpi ece.

10 Wakantanka, kakiświcaya wo ;

Wowahokonkiye tawapi on hinhpayapi nunwe;

Woahtani hduotapi kin on elpewicaya wo ;

Niye nicipajinpi kin heon etanhan.

11 Tuka wacinniyanpi kin owasin wiyuśkinpi kte;

Ohinniyan wiyuśkinyan dowanpi kte;

Niye awanwicayahdake cin heon etanhan:

Nakun tona nicaje waśtedakapi kin hena iniyuśkinpi kte.

12 Wicaśta owotanna kin niye dawaśte kta;

Jehowa, wahacanka iyececa, wowaśte wateśdag yaye kta.

\section{PSALM 6.}

Dawid tadowan. Kuya ahiyayapi en candowankiyapi on dowan itancan kin.

1 Jehowa, nitocanniye en iyopemaye śni ye;

Qa śinyahda en kakiśmaye śni wo.

2 Jehowa, onśimakida ye, wamaśake śni ; 
Jehowa asnimayan ye, mahuhu tehiya kakija.

3 Minagi kin iś eya nina kakija;

Tuka niye Jehowa, tohanyan kta he.

4 Jehowa hdicu ye, minagi ehdaku wo; Nitowaonśida kin on nimayan wo.

5 Wiconte en nilksuyapi kin wanica; Hades ekta tuwe wopida eniciye kta he.

6 Comniwahdazi on waśake śni amayan; Hanyetu osan mitowinja amnitan waya; Iśtamnihanpe amau kin on ipahin spaye wakiya ece.

7 Ceyapi on iśta amatakuni śni ; Tokamayanpi owasin on kan amayan.

8 Taku śica ecanonpi kin owasin mitan tanhan ya po ; Jehowa woceye mitawa ho mamakion kin heon.

9 Jehowa hoyewakiye cin he namalion; Wocekiye mitawa kin he Jehowa icu kta.

10 Tokamayanpi owasin iśtecapi qa nihinciyapi kte ; Ihnuhanna hdicupi qa iśtecapi nunwe.

\section{PSALM 7.}

Dawid tadowan. Kus Benjamite oie kin on Jehowa kahiyaye ciqon.

1 Jehowa Wakantanka mitawa, niye wacinciye; Tehiya makuwapi owasin etanhan nimayan qa emahdaku wo:

2 Okinni mnaja iyecen minagi yaśpaśpa qa yahdehdece kta; Unkan waehdaku wanice kta.

3 Jehowa Wakantanka mitawa, hecen ecamon kinhan; Minape ohna taku sica yuke cinhan;

4 Tuwe wookiye en mici un kin itkom taku śica ecawecon kinhan ;

Han, tuwe taku on etanhan śni śicaya makuwa kin he emdaku ece :

5 Hehan toka kin minagi tapa qa ehdege kte;

Han, mitaniya maka kin en namdu kte;

Qa mitowitan watuśekśeca en ounye kiya nunwe. Sela.

6 Jehowa, canniniye cin en inajin wo ;

Tokamayanpi sinhdapi kin on wankan iyeiçiya wo;

Qa miye on woyaco cajedate cin on ihduhica wo.

7 Unkan oyate mniciyapi kin aohduteniyanpi kta;

Heon etanhan hena on wankan ihdoku wo.

8 Jehowa oyatepi kin wicayaco kta;

Jehowa, mitoowotanna qa mitowaśte en maun kin he iyecen mayaco ye.

9 Tokin wicaśta śica tohanpi śica hena owihanke yen;

Tuka wicaśta owotanna kin he yusuta wo ;

Taku Wakan owotanna wicacante qa wicapakśin iyukcan kin he niye.

10 Wahacanka mitawa kin he Wakantanka etanhan;

Tona cante owotanna niwicaye cin hee. 
11 Wakantanka wayaco owotanna; Qa Wakantanka anpetu iyohi canniyan un :

12 Ihduhomni śni kinhan isan hdube kta; Tinazipe wanna nawinja qa wiyeya ehnaka:

13 Qa wiconte wipe kin elkta epazo; Tunwanhinkpe kin ide kiye kta.

14 Wanyaka wo, woahtani on tinge; Taku śica on ihduśaka, qa woitonśni hiyuya.

15 Woha wan kage ça iye kiça; Qa oqapi iye lkage cin en ohinhpaya.

16 Tolitani iye pa kin en hdi kta; Qa tohan śica iye pesdete ahdilipaye kta.

17 Jehowa toowotanna kin on wakahimdamde kta; Qa Jehowa Tehan wankantu caje kin iwadowan kta.

\section{PSALM 8.}

Dawid tadowan. Gittith on dowan itancan kin.

1 Jehowa Itancan unkitawapi, Maka kin owancaya nicaje wokitanin hinca; Nitowitan mahpiya kin iwankam eyehde.

2 Hokśiyopa qa azin unpi ipi kin etanhan woyatan yakaga; Tona toka niyanpi kin hena on, Toka watokiçon ko wicaduinina kta heon.

3 Mahpiya nitawa ninapsukaza kage cin he wanmdaka eca; Hanyetu wi qa wicanlipi eyahnake cin ;

4. Wicaśta kin taku, hecen ekta ewacanni he; Qa wicaśta cinhintku kin, on en yahi kta he.

5 Kitan ecinyan Taku Wakan kin ihukuya yakaga; Wowitan qa woyuonihan on wateśdag yecaton;

6 Ninape taku kage cin hena idake yakiya; Taku owasin iye siha ihukuya eyahnaka;

7 Tahinca optaye, tatanka ko owasin; Qa nakun tinta woteca kin;

8 Mahpiya okinyanpi, qa miniwanca hogan kin, Taku miniwanca ohna cankuye cin hena.

9 Jehowa Itancan unkitawapi, Maka kin owancaya nicaje wokitanin hinca.

\section{PSALM 9.}

Dawid tadowan. Muth-laben on dowan itancan kin.

1 Micante ocowasin on Jehowa mdatan kta; Taku wowinihan nitawa owasin mdatanin kta.

2 Cante mawaśte qa niye on wimduśkin kta ; Tehan Wankan yaun nicaje ivadowan kta.

3 Tokamayanpi hekta itoheya hdicuyapi kinhan, Nitokam hicahanpi qa hinlipayapi kte.

4 Woakinica qa woyaco mitawa dusuta; 
Oiyotanke kin akan owotanna wayaco idotanke.

5 Ikcewicaśta iyopewicayaya, tona śice cin hena ihangwicayaya; Ohinniyan qa owihanke wanica cajepi yapajuju.

6 Toka woihangye tawapi kin wamna owihanke: Qa totonwepi kin hena dujuju ; Ahna wicakiksuyapi lain he atakuni śni.

7 Tuka Jehowa owihanke wanica ounye kte ; Woyaco on oiyotanke tawa wiyeya ekihde.

8 Unkan wowaśte on maka kin hdaco kta; Woowotanna on oyate kin wicayaco kta.

9 Nakun śicaya wicakuwapi kin hena Jehowa wowinape yapi kta ; Wokakije anpetu kin en he wowinape yapi lita.

10 Unkan tona nicaje sdonyapi hena wacinniyanpi kta; Niye Jehowa, tona anicitapi kin hena ehpewicayaye śni ece.

11 Jehowa Zion en ounye cin he idowan po; Tolian tanka oyate kin en oyaka po.

12 Tohan wicawe akita eca hena kiksuya ece; Tona kakija hoyeyapi kin he akiktonje śni.

13 Jehowa onśimákida wo ;

Tona śicemadapi token kakiśmayanpi kin he wanyaka wo .

Wiconte tiyopa etanhan imacu kin he niye.

14 Hecen Zion cunwintku tatiyopa kin en nitowitan ocowasin omdake kta;

Wiconi nitawa on imduśkin kta.

15 Ikcewicaśta woha wan kagapi kin he ohna ihpayapi ;

Wohmunke wan iye nahmanpi kin he ohna siha icihmunkapi.

16 Woyaco econ kin eciyatanhan Jehowa sdonyapi:

Tuwe śice cin he iye nape ohan kin on hmunkapi ece. Higgaon. Sela.

17 Wicaśta śicapi Hades en hdicuwicayapi kta; Oyate Wakantanka akiktonjapi kin iyuhpa.

18 Wahpanicapi kin ohinniyan akiktonjapi kte śni;

Wicaśta onśika wacinyanpi kin he owihanke wanica ihanke kte śni.

19 Jehowa inajin ye, wicaśta alkantu waśake śni nunwe;

Nitokam Ikcewicaśta kin wicayacopi kte.

20 Jehowa, wakokipe wicakicaga wo;

Hecen Ikcewicaśta wicaśta ikceka hecapi e sdoniçiyapi kta. Sela.

\section{PSALM 10.}

1 Jehowa, tokeca itehan nayajin he; Wokakije anpetu kin en nayakiibe kta he.

2 Wicaśta śica wahaniçida on kakije cin tehiya kuwa ece; Wohnaye awacinpi kin he on icihduzapi nunwe.

3 Wicaśta śica iye nagi taku cantiheye cin on ihdatan; Qa waicucu kinhan ihdawaśte;

Jehowa he wahteda śni tuka. 
4 Wicaśta śica, ite wahaniçida kin on, Takudan akite kte śni, Wakantanka wanica;

Hecen tawacin ece.

5 Tacanku kin ohinniyan suta ;

Nitawoyaco kin iwankam un, iśta kin itehan;

Tokawicaye cin owasin awicapogan kta.

6 Cante mahen heya; Icimana mayutokanpi kte śni;

Wicoicage qa wicoicage hehanyan woiyokiśice wanice kta ce.

7 Woyaśice, woyahnayan qa woyuśice on i kin ojudan;

Ceji ihukuya woyaśkiśkapi qa woạtitani hiyeya.

8 Otonwe en waiyapepi kin hen iyotanke kta;

Woinalibe oyanke kin en wicaśta owotanna wicakte kta; Iye iśta wicaśta onśika iyawicape kta.

9. Mnaja wan waśun ohna yanka, He iyecen, woinalibe oyanke kin ohna waiyape un kta;

Wicaśta onśika yuze kta e iyape k ta;

Wohmunke tawa kin en wicaśta onśika au eca yuza ece.

10 Patuś inajin qa makata iwanka,

Hecen wicaśta waśaka wicayuhe cin on wicaśta onśika hinhpa-

yapi kta.

11 Cante mahen heya; Wakantanka akiktonja;

Ite nakiliman; icimana wanyake kte śni ce.

12 Jehowa, najin ye;

Wakantanka, nape yuwankan ehdaku wo ;

Onśike cin hena akilitonje śni wo.

13 Tokeca wicaśta śica Wakantanka wahteda śni he;

Niye wawinunge kte śni, cante mahen keya he.

14 Niye wandaka; ninape on toyecon kta e

Wicolian śica wocanniye ko ayatonwan ece :

Tuwe onśike cin he wacinniye kta;

Wamdenica kin oyakiya ece.

15 Wahtani sa isto kin kawega wo;

Wicaśta śica tośice cin ayakite kta,

Takudan iyeyaye kte śni hehanyan.

16 Jehowa Wicaśtayatapi ohinniyan qa owihanre wanice kta:

Makoce tawa kin etanhan Ikcewicaśta kin atakunipi śni.

17 Tona onśika taku icekiyapi kin he, Jehowa niye nayahion;

Cantepi dusuta kta; ninoge anohyakiye kta.

18 Wamdenica qa kakiśyapi kin towicayecicon kta;

Hecen wicaśta maka akan icimana wakokipe wicakicage kte śni.

\section{PSALM 11.}

Dawid tadowan. Dowan itancan kin Kicagapi.

1 Jehowa wowinape waye;

Tokeca minagi heyakiyapi he ;

He nitawapi onakipa po, zitkadan iyececa.

2 Wan, wicaśta śicapi tinazipe nakiwinjapi ;

Ikan kin ekta tunwanhinkpe wiyeya ehnakapi ; 
Otpaza en wicaśta cante owotanna wicakutepi kta.

3 Woahde kin hena yujujupi kta:

Wicaśta owotanna taku econ okihi kta he.

4 Jehowa tipi wakan tawa kin ohna yanka;

Jehowa oiyotanke tawa mahpiya kin ekta wanka;

Iye iśta kin wanyaka, iye iśtojuha wicaśta cinca wicayukcan ece.

5 Jehowa wicaśta owotanna kin yukcan kta;

Tuka wicaśta śica qa tona wawicakipi waśtedake cin hena

Iye nagi śicewicadaka ece.

6 Wicaśta śice cin hena wakanhdi awicamagaju kte,

Peta qa inyanzi, tete iyumni wohitike hinca;

Miniy atke ohna wicaqupi kin hee.

7 Jehowa owotanna un, woowotanna waśtedaka ece :

Tona ecetupi kin hena iye ite kin wanyakapi kta.

\section{PSALM 12.}

I'sahdogan on dowankiya itancan kin. Dawid tadowan.

1 Jehowa wanikiya wo; wicaśta wacantkiye cin he owihanke; Wicaśta wicake cin Adam cinca kin etanhan atakunipi śni ce.

2 Otoiyohi iye takodaku kici woitonśni okiciciyapi kta;

Iha śduśduta qa cante nonpa on iapi kta.

3 Jehowa wicaiha śduśduta owasin baśpe kte, Wicaceji watankaicidaya ie cin he nakun.

4 Hena heyapi ; Uncejipi on wounhitikapi kta; Tha unyulanpi; tuwe itancan unyuhapi he.

5 Wicaśta onśika kakijapi kin on, Wicaśta walipanica ceyapi kin on,

Wanna nawajin kta ce, Jehowa eye kta;

Tuwe he cantokpani hinca kinhan, wankan wapa ewahnake kta ce.

6 Jehowa taliu eye cin hena wicoie ecedan;

Mazaska maka oceti ohna yuecedan, Śakowin akihde śdoyapi.

7 Jehowa, niye hena wicayakpatan $k$ ta ;

Wicoicage kin de etanhan ohinniyan eyahdaku kta.

8 Wicaśta cinca wahtepi śni kin wankan ehdepi kinhan,

Wicaśta śica ôwancaya manipi kta.

\section{PSALM 13.}

Dowankiya itancan tawa. Dawid tadowan.

1 Jehowa, tohanyan amiyektonje kta he, ohinniyan kta he; Tohanyan niite namayakilibe kta he.

2 Tohanyan minagi on awacin waun lita, Anpetu otoiyohi cante maśice $k$ ta he ;

Tohanyan tokamaye cin miwankam un kta he.

3 Wanyaka wo, Jehowa Wakantanka mitawa, namahon wo: 


\section{PSA $\mathrm{S} M 15$.}

Iśta iyoyammayan wo, olinni wiconte en miśtinbe kta.

4. Okinni tuwe tokamaye cin, Wanna ohiwaya ce, eye kta; Mahicahe cinhan śicaya malsuwapi kin iyaśapi kta.

5 Tuka miye nitowaonśida wacinwaya; Micante nitowiconi kin on iyaśa kte.

6 Jehowa iwadowan kta, Tanyan ecamicon heon etanhan.

\section{PSALM 14.}

Dowan itancan kin kicagapi. Dawid tawa.

1 Witkotkoke cin he, Taku Wakan wanica ce, cante mahen eya. Hena ihduśicapi, wicohan wałite śni econpi; Tuwedan taku waśte econ śni.

2 Jehowa malipiya eciyatanhan Adam cinca kin ahiwicatonwan; Tuwe wacinksamya ohanyan hecinhan wanyake kta; Tuwe Wakantanka akita hecinhan.

3 Iyuhipa icunonpa iyayapi ; witaya ihduśicapi ; Tiuwedan taku waśte econ śni; han, wanjidan śni.

4. Taku śica econpi kin owasin takudan sdonyapi śni he; Mitaoyate aguyapi iyecen wicayutapi, Qa Jehowa hoyekiyapi śni.

5 Hen wokokipe on kopehdapi; Wakantanka wicoicage owotanna kin en un, heon.

6 Wicaśta onślka tawowacinye lin iśtenyayapi kta ; Jehowa wowinapeye cin heon.

7 Tokin Israel woehdaku tawa kin Zion etanhan $u$ nunwe: Tohan Jehowa taoyate wayaka unpi kin awicahdi kinhan, Jakob iyuśkin kta, Israel pida kta.

\section{PSALM 15.}

\section{Dawid tadowan.}

1 Jehowa, tuwe nitawakeya en ounye kta he; Tuwe paha wakan nitawa kin en ti kta he.

2 Tuwe iyaonpepicaśniyan mani, qa woowotanna econ, Qa cante mahen wowicake ia ece :

3 Truwe iye ceji on waaie śni, Tuwe ikiyedan un kin taku śica ecalkicon śni; Qa takodaku on woyake śica wan icu śni :

4 Tye iśta en wicaśta wahteśni kin wahtedapi śni : Tuka tona Johowa kokipapi kin hena wicayuonihan kta: Iye içiconza on kiunniiciya eśta hdutokeca kte śni :

5 Mazaska tawa ota kamna kta ehnake śni ; Qa wicaśta iyaonpepica śni un kin on wokajuju icu śni ; Tuwe dena econ kin he ohinniyan pahohopi kte śni. 


\section{PSALM 16.}

Dawid tawoinalibe.

1 Wakantanka makpatan wo; Niye wacinciya, heon etanhan.

2 Jehowa heyakiya; Itancan mitawa kin he niye; Mitowaśte kin he niye ekta iyahde śni :

3 Makoce kin en wicaśta wacantkiye cin hena ekta, Qa itancanpi kin, hena en ocowasin wimduśkin.

4. Tona wanji tokeca wolipapi kin hena woiyokiśice ota yuhaji lkta:

We wośnapi kin hena wapapson lkte śni, Qa cajepi kin miiha ohna iwacu kte śni.

5 Jehowa wopamni mitawa qa wiyatke mitawa kin hee; Woailipeye mitawa kin he niye dutanka kta.

6 Taku wiciyokipi kin imakiyutapi :

Han, maga waśte hinca mduha.

7 Jehowa wahokonmakiye $\operatorname{cin}$ he mdatan kta; Nakun hanyetu eca mitapakśin onspemakiya ece.

8 Mitokam Jehowa ohinniyan ewahde; Mietapa ekta un, heon mahohopi kte śni.

9 Heon etanhan micante wiyuśkin, Qa mitowitan ipsica ece :

Han, micelipi wowacinye en wanke kta.

10 Minagi Hades en amayaduśtan kte śni;

Wanji Wakan nitawa kin he wicaliwin wanyagyakiye kte śni.

11 Wiconi canku kin he onspemayakiye kta;

Niite kin he wowiyuśkin ojudan;

Nietapa ekta ohinniyan wiciyokipi.

PSALM $1 \%$.

Dawid tawocekiye.

1 Jehowa, taku owotanna kin nahon ye;

Sicahowamde cin he anohikiya wo;

Wocekiye mitawa namakilion ye,

Iha wicahnaye śni etanhan u kin he.

2 Mayaowotanpidan lin he nitohnake etanhan u kta;

Niiśta taku ocitkonza kin hena wanyake kta.

3 Cante imayaduta, hanyetu en mayahi;

Imayadukcan, takudan iyeyaye kte śni ;

Mii kin mitawacin kakipe kte śni.

4. Wicaśta wicolian tawapi ekta, niiha oie kin on, Waihangye sa olanpi kin iwanmdaka ece.

5 Mioye kin nitacanku yus un ; Siha ewahde kin naśdute śni.

6 Wakantarika houciciya; amayadupte kta heon; Anolimakiya wo, taku epe cin namakition wo. 
7 Tona wacinyaupi niwicayaye nitocantekiye hdutanin wo; Tona najinpi kin etanhan, ninape etapa on.

8 Wiciśta su kin iyecen makpatan wo ; Nihupahu ohanzi ihukuya namayahibe kte;

9 Wicaśta śica ihangmayanpi itepi kin etanhan; Minagi tokayapi aohdutemayanpi kin hena.

10 Waśin tawapi kin en aconkaśkapi ;

Ipi on wahaniçidaya iapi ece.

11 Wanna unkowepi kin en aohduteunyanpi; Iśta on wanyag iheyapi, hdatapi kta heon.

12 Mnaja iyececa, wayapota inahini;

Qa mnaja koślka woinalibe en iyotanke cin iyececa.

13 Jehowa najin wo, itkokim ya wo, patuśkiya wo; Mazasagye nitawa on wicaśta śica etanhan minagi eyaku wo:

14 Jehowa, ninape on, wicaśta kin etanhan;

Wicoicage wicaśta etanhan,

Taku tawapi wiconi kin den un;

Qa taku nahmana yahnake cin on tezi ojuwicayakiye kta: Hena cinca wipipi kta;

Qa taku ohdaptapi kin hokśiyoqopa aihpewicakiyapi kta.

15 Tuka miye woowotanna en niite wanmdake kta;

Tohan ouncage nitawa yuha wekta kinhan, cante mawaśte kta.

\section{PSALM 18.}

Dowankiya itancan kin. Dawid Jehowa taokiye kin he kaga : Jehowa tokayapi owasiu napepi etanhan, qa Sanl nape etanhan eyaku qon, anpetu kin he en odowan oie kin dona Jehowa eciya.

1 Unkan heya;

Jehowa mitowaśake, nina waśte cidake kta.

2 Jehowa imnija mitawa, qa conkaśke mitawa, qa emahdaku ece kin hee:

Wakantanka mitawa, wowinape mitawa, he wacinwaye kta ; Wahacanka mitawa, qa ni makiya he kin, paha tehanwankantu mitawa kin hee.

3 Jehowa yatanpi kte cin he hoyewakiye kta;

Unkan tokamayanpi etanhan nimayanpi kta.

4 Wiconte ikan kin on mayuskiskitapi ;

Qa wicośice wakpa kin mayuśinyaye kta.

5 Hades ikan kin aohdutemayanpi; Wiconte mazahtakiyapi kin mayuzapi.

6 Iyotanhan iyewakiye cin en Jehowa hoyewakiye kta ; Qa Wakantanka mitawa cewakiye kta;

Tipi wakan tawa kin eciyatanhan miho kin nahon kta; Qa wocekiye mitawa iye itokam u kte, iye noge kin en.

7 Hehan maka kin yuhuhuzapi qa cancan;

He oahde kin yuhuhuzapi qa śkanśkan;

Iye sinhda heon etanhan.

8 Canniyan etanhan śota wankan iyaya; 
Qa i kin etanhan peta waliuhnaga;

Heon petaga ideyapi.

9 Nakun mahpiya kin patuślkiye ça kun u;

Qa iye siha ihukuya otpaza.

$10 \mathrm{Qa}$ Kerub wan akan iyotanke ca kinyan iyaya:

Han, tate hupahu kin akan kinyan.

11 Woinahbe tawa otpaza kin he kaga;

Ihdukśan tawokeya kin mini otpaza;

Mahpiya en mahpiya śoka kin hee.

12 Itokam iyoyanpa on mahipiya śoka tawa kin hiyaya;

Wasu qa petaga.

13 Hehan mahpiya ekta Jehowa wakinyan hotonkiya;

Qa Iyotan Wankan kin ho hdatanin:

Wasu qa petaga.

14. Unkan tunwanhinkpe kihiyeya qa napewicaya;

Nakun wakanhdi hiyuye ca yuśinyewicaya.

15 Hehan mini sicu kin wanyakapi; •

Qa maka oahde kin yutaninpi ;

Jehowa niye he iyopeyaya;

Nitocanniye oniya ipogan kin heon.

16 Hehan wankantanhan hiyu qa mayuze kta;

Mini ota etanhan imacu kta.

17 Tokamaye waśake cin etanhan emayaku kta;

Qa tona śicemadapi kin etanhan;

Hena mikapeya waśakapi nakaeś.

18 Tyomakiśica anpetu kin en iyamahpayapi kta; Tuka Jehowa mayusuta kin hee.

19 Unkan makoce tanka en amahi ; Iyokipiwaye cin heon emayaku kta.

20 Mitoowotanna iyecen Jehowa ecamicon kta; Minape ska kin iyecen micicajuju kta.

21 Jehowa tacanku kin hena owape; Qa Taku Wakan mitawa śicaya amduśtan śni.

22 Iye tawoyaco kin owasin mitokam un; Qa toope kin mitantanhan ehpewaye kte śni.

23 Nakun iye itokam ecetu waun ; Qa wawahtani kin etanhan ținsmiçiya.

24. Heon mitoowotanna iyecen Jehowa micicajuju; Iye iśta itokam minape ska kin heon.

25 Tuwe wacantkiye cin he kici wacantkiya nihdutanin kta; Wicaśta owotanna kici oniwotanna kta:

26 Tuwe ecedan un kin he kici ecedan nihdutanin kta; Qa tuwe pemni un kin he kici nihdupemni kta.

27 Niye oyate onśika niwicayaye kte : Tuka iśta wankantupi kin kun hiyuwicayaye kta.

28 Niye petijanjan mitawa ideyaye kta: Jehowa mita Wakantanka otpaza iyoyammiciye kte.

29 Niye on ozuye wan awaimnanke kta; Wakantanka mitawa on conkaśke wan awapsice kta. 
30 Iyotan Waśaka tacanku kin he yuśtanpi ;

Jehowa oie kin he yuecepidan;

Tona wacinyanpi owasin he wahacanka yapi.

31 Hecen Jehowa icunonpa tanhan Taku Wakan kin tukte e he; Qa. Wakantanka unkitawapi icunonpa tanhan tuwe imnija he.

32 Iyotan Waśake cin wowaśake on ipiyag makiton;

Qa mitacanku miciyuśtan.

33 Misiha tatokadan iyececa micaga;

Qa pajodan mitawa akan najin makiya.

34 Minape kicizapi onspemakiya,

Hecen miisto mazaśa itazipa kin yuwinja.

35 Unkan nitowiconi wahacanka kin mayaqu :

Nakun ninape etapa kin najin makiye kta ;

Qa nitowahbadan tanka makage kta:

36 Mihukuya caewahde kin hena mayadutanka kte;

Hecen miiślkahu naśdute kte śni.

37 Tokamayanpi tawicawapa qa ewicawehdege kta ;

Qa bowicasotapi śni hehanyan wahdicu kte śni.

38 Wicawakahuhuge kta, hecen najin okihipi śni;

Misiha ihulkuya ilipayapi kta.

39 Unkan okicize kta wowaśake on ipiyag mayalkiton;

Tona takpe maupi kin hena milıukuya iyewicayaye kta.

40 Nakun tokamayanpi tahupi kin mayaqu;

Qa tona śice madapi kin hena ihangwicawaye kta.

41 Hena howayapi kta, tuka wanikiya wanica:

Jehowa hoyekiyapi kta, tuka awicayupte śni.

42 Hehan wicawakamdumdu kta, tate itokam maka mdu iyececa;

Canku upśija iyecen tankan elipewicawaye kta.

43 Oyate kicizapi kin etanhan emayahdaku kte; Ikcewicaśta kin en itancan emayahnake kte;

Oyate sdonwicawaye śni qon hena wowidagwaye kta.

44 Noge on nalionpi kinhan anamagoptanpi kta;

Wicaśta tokeca cinca kin mayaonihanpi kte.

45 Oyate tokeca cinca kin atakunipi kte śni ;

Qa conkaślke tawapi etanhan cancanyan hiyupi kta.

46 Jehowa ni un; qa Imnija mitawa yawaśtepi nunwe; Qa Wakantanka Wanikiya mitawa kin he yatanpi nunwe.

47 Iyotan Waśaka watokiçonpi maqu ece kin hee; Qa nakun mihukuya oyatepi wicaktedan.

48 Tokamaye cin etanhan emahdaku;

Han, tona takpe maupi kin wiciwankam emayahde kta;

Wicaśta waihangye cin etanhan emayahdaku kta.

49 Heon, Jehowa, Ikcewicaśta ehna ciyatan kte; Qa nicaje iwadowan kta.

50 Wicaśtayatapi tawa kin woehdaku kiciyutanka; Qa tuwe Sdakiye cin he wowaonśida ecakicon ece; Dawid qa iye cinca kin, owihanke wanica. 
PSALM 19.

Dowan itancan kin. Dawid tadowan.

1 Malipiya kin Wakantanka towitan kin oyaka; Qa okotonyan kin iye nape ohan kin yuotanin ece.

2 Anpetu wan anpetu iapi okiciyake kta; Qa hanyetu wan hanyetu wosdonye kiciyuotanin lita.

3 Iapi wanica qa wicoie wanica; Hena ho kin ecaca nahonpi śni.

4 Hena jyutapi ikan kin maka owancaya iyaya; Qa oiepi kin maka ihankc kin elsta.

Hena ehna anpetu wi kin wokeya wan kicaga.

5 Unkan he winohinca yuze kte cin wan Wankan tipi etanhan hdinanpe cin iyececa;

Wicaśta waśaka iyecen kiinyanlra iyuśkin.

6 Mahpiya ihanke kin hetanhan ya ece;

Qa unma ihanke kin ekta ki :

Qa iyokate cin etanhan takudan nałmanpi śni.

7 Jehowa toope lkin yuśtanpi, wicanagi kin yuhomni ; Jehowa tawoyaotanin kin he wowicalre,

Witkotkolse cin wicayuksapa ece.

8 Jehowa taku econ wicaśi kín hena ecetu, Wicacante iyuśkinlkiya;

Jehowa tawoahope kin ecedan, wicaiśta iyoyamya ece.

9 Jehowa kokipapi kin he ska, ohinniyan he kta;

Jehowa tawoyaco kin he wowicake, ocowasin owotanna.

10 Hena mazaskazi isanpa waśtedapi kte;

Han, mazaskazi ecedan ota lin isanpa:

Nakun tulimaga canhanpi isanpa skuya;

Qa tulimaga carihanpi śbu kin.

11 Nakun hena on nitaokiye wahokonkiyapi;

Hena yuhapi kin wolkajuju tanka.

12 Tuwe wahtani ohdahniga olkihi he;

Nahmanpi kin hena micicajuju wo.

13 Nakun woahtani tanka kin etanhan nitaokiye ehdaku wo; Hena wowidagmaye śni nunwe; kinhan omaowotanna kta, Qa woalitani ota iyaonpepica śni waun kta.

14 Jehowa imnija mitawa qa tuwe opemakiton lkin, Mii oie kin qa micante taku awacin kin,

Hena niye iyonicipi nunwe.

\section{PSALM 20.}

Dowan itancan kin on. Dawid tadowan.

1 Wicocante sica anpetu kin en Jehowa nanihon kte; Jakob ta Wakantanka caje kin wankan enihde nunwe.

2 Tipi wakan kin etanhan oniciye kte; Qa Zion etanhan niyuwaśake kta.

3 Nitawośna owasin kiksuye kte;

Qa peta on waduśna kin hena icu lita. Sela. 
4 Nicante yuze cin eciyatanhan niçu kte; Qa nitawacin owasin ecetuniciciye kta.

5 Woehdaku nitawa on unkiyuśkinpi kta; Qa Wakantanka unkitawapi caje kin on Wiyokihedan eunkihdepi kta.

Wocekiye nitawa owasin Jehowa ecetuniciciye kte.

6 Jehowa iye Sdakiye cin he nikiya wanna sdonwaya;

Mahpiya wakan tawa eciyatanhan nakilion kta.

Nape etapa waniye wowaśake kin he on.

7 Hunli canpahmihma qa hunh śuktanka,

Tuka unkiye Jehowa Wakantanka unkitawapi caje on unkiwinlktapi kta.

8 Hena e patujapi qa hinhpayapi:

Tuka unkiye naunjinpi qa bosdan unyakonpi.

9 Jehorva wanikiya wo ;

Hoyeunyanpi anpetu kin en,

Wicaśtayatapi kin naunkihonpi kte.

\section{PSALM 21.}

Dowan itancan kin on. Dawid tadowan.

1 Jehowa, nitowaśake on wicaśtayatapi kin cante waśte kta ; Qa woehdaku nitawa on nina iyuśkin kta.

2 Taku cantokpani kin he yaqu;

Qa iye iha taku da kin he teyahinda śni. Sela.

3 Woyawaśte waśte kin yuha itkoyakipe kta; Mazaskazi wateśdake wan pa akan eyecihnake kta.

4 Wiconi nicida, he yaqu; Anpetu hanska, ohinniyan qa owihanke wanica.

5 Waniyakiye cin on towitan tanka kte; Woyuonihan qa wowaśte akan eyahnake kta.

6 He woyuwaśte owihanke wanica yakage kta;

Niite ctanhan wowiyuśkin on iyuśkinyaliye kta.

7 Wicaśtayatapi kin Jehowa wacinyan un ;

Unkan Iyotan Wankan un tawocantekiye en kahuhuzapi kte śni.

8 Tokaniyanpi owasin ninape iyewicaye kta ;

Tona śicenidapi kin ninape etapa iyewicaye kta.

9 Niite itokam maza śdoyapi oceti ide kin iyecen wicayakage kta;

Jehowa tocanniye on nawicapce kta;

Qa peta kin huhnahwicaye kta.

10 Taku icaliyapi kin maka etanhan ihangyaye kta;

Qa cincapi kin Adam cinca kin etanhan.

11 Taku śica nihiyohi kta cinpi ;

Taku śica konzapi, tuka okihipi kte śni.

12 Heon etanhan wicitokam eyakate cinhan,

Hekta itoheya hdicu wicayaye kta.

13 Jehowa, nitowaśake on nihduwankantuya nunwe,

Unkahiyayapi kta, qa nitowaśake unkidowanpi kta. 


\section{PSALM 22.}

Dowankiya itancan: Anpao talinca kin on. Dawid tadowan.

1 Wakantanka mitawa, Wakantanka mitawa, Tokeea ehpemayaya he:

Waśicahowamde oie kin en tehan omayakiye śni.

2 Wakantanka mitawa, anpetu icunhan hoyewaye kta,

Tuka amayadupte kte śni ;

Nakun hanyetu en, qa inina manke śni.

3 Tuka niye niwakan, Israel yatanpi kin en ounyaye cin.

4 Atewicunyanpi wacinniyanpi qon; Wacinniyanpi unkan ewicayahdaku.

5 Hoyeniciyapi unkan ewicahdakupi ; Wacinniyanpi unkan iśtecapi śni.

6 Tuka miye wamdudan hemaca, qa wicaśta hemaca śni ; Wicaśta ekta wowiśtece mayanpi, qa oyate kin walitemadapi śni.

y Tona wanmayakapi owasin wowihalia mayanpi ; Aikśinmayanpi, apomnamnamakiyapi.

8 Jehowa ihduzeze ce; ehdaku kta; Waśtedake cin heon nikiye kta ce, eyapi.

9 Tuka wicatamni etanhan imacu qon he niye; Ina aze kin akan wowacinye mayaqu.

10 Wicatamni kin etanhan niye en ailipemayanpi; Ina tezi kin hetanhan Wakantanka mitawa he niye.

11 Mitehanyan un śni wo, woiyokiśice kiyedan un; Nakun waokiya wanica, heon etanhan.

12 Tatanka ota aohdutemayanpi ;

Baśan etanhan waśakapi kin ohomni takpe maupi.

13 Mnaja ocinśica qa hoton ece kin, He iyecen aimayukawapi.

14 Mini iyecen mapapsonpi; qa huhu mitawa owasin makap śunpi ;

Micante canśin iyececr, mitezi mahen śdoyapi.

15 Maka cega onśpa iyecen mitowaśakc śpan;

Nakun miceji macaka en askapa,

Qa wiconte maka mdu kin en amayade kta.

16 Sunka ohommi imayayapi ;

Wicaśta śica omniciye kin aohdutemayanpi ;

Minape misilı ko capapi.

17 Huhu mitawa owasin wahdawa;

Ahitonwanpi qa opamalitapi.

18 Wokoyake mitawa kicipamnipi;

Qa mitaśina on kansu kutepi.

19 Tuka Jehowa niye mitehanyan un śni ye;

Mitowaśake he niye, omakiya inahini wo.

20 Mazasagye etanhan minagi ehdaku wo;

Mitawa ecedan kin śunka nape kin etanhan. 


\section{PSALM 23.}

21. Mnaja i kin etanhan nimayan wo:

Unkan tatanka he kin hetanhan amayadupta.

22 Nicaje mihunkawanji owicawakiyake kta ; Omniciye kin colkaya tanhan icidowan kta.

23 Jehowa kokipa yaunpi kin, he idowan po; Jakob cinca owasin, he yuonihan po:

Qa Israel cinca owasin, kokipa po.

24 Tuwe onśika kakije cin he ih̉aha śni qa śicedake śni;

Nakun ite inakilibe śni ; tuka hoyekiya eca nakihon ece.

25 Omniciye tanka kin en niye on wadowan kta;

Tona kokipapi wicitolkam wośna mitawa wahdajuju kta.

26 Wah̉bapidan kin hena wotapi qa imnanpi kta;

Tona Jehowa akitapi kin hena idowanpi kte :

Nicantepi owihanke wanin ni kte.

27 Maka ihanke kin owasin kiksuyapi qa Jehowa ekta ihduhomnipi kta;

Qa Ikcewicaśta en wicowazi owasin nitokam ohodapi kta.

28 Wokiconze kin he Jehowa tawa;

Qa iye Ikcewicaśta kin wowidagwicaya ece.

29 Maka akan tona cepapi owasin wotapi qa ohodapi kta;

Watuśekśeca ekta kun yapi kin iyulipa he itokam patujapi kta:

Qa tuwe iye nagi nikiya okihi śni kin

30 Wicacinca wowidagwicaye kta ;

- Hena Itancan kin wicoicage wicahdawa kta.

31 Upi qa toowotanna kin oyakapi kta;

Oyate wan tonpi kte cin en;

Iye hecon kin heon.

\section{PSALM 23.}

Dawid tadowan.

1 Wonmakiye cin Jehowa hee:

Takudan imakakije kte śni.

2 Peji toto en iwanke maye kta;

Wicoozi mini lain icahda yus amaye kta.

3 Minagi yuecetu kte;

Woowotanna canku kin ohna amaye kta;

Iye caje kin on.

4 Han, wiconte ohanzi kalsiza kin en mawani kinhan,

Taku śica kowakipe kte śni, niye mici yaun heon;

Cansakadan nitawa qa cansagye nitawa, hena cantohnagmaye kta.

5. Tokamayanpi wicitokam -wahna wotapi wan wiyeya miyecihnake kta ;

Wihdi on pa sdamayakiye;

Wiyatke mitawa iyatahde.

6 Awicakehan wani kta anpetu owasin,

Wowaśte wowaonśida ko miyahna un kta:

Qa Jehowa ti kin en ounwaye kta, anpetu hanskaska. 


\section{PSALM 24.}

Dawid tadowan.

1 Maka kin Jehowa tawa, qa woojudan kin; Makowancaya qa tona ounyanpi kin.

2 He iye miniwanca kin akan ehde; Qa wakpa kin iwankam sutaya kaga.

3 Jehowa Paha tawa kin tuwe ekta ye kta he; Qa oyanke wakan tawa ohna tuwe yanke kta he.

4 Tuwe nape kin ska, qa cante kin ecedan; Tuwe takuśniśni ekta nagi yeiçiye śni ; Qa wohnaye on iciconze śni kin.

5 He Jehowa etanhan woyawaśte icu kta; Qa woowotanna, Wakantanka Wanikiya tawa kin etanhan.

6 Wicoicage okide unpi kin he dee: Niite akitapi kin hena Jakob eepi. Sela.

7 Tiyopa kin, pa yuwankan ehdaku po ;

Qa tiyopa ohinniyan un wankan iyeiciya po:

Unkan Wowitan Wicaśtayatapi lin tin hiyu kta.

8 Wowitan Wicaśtayatapi kin he tuwe he: Jehowa waśake ca waditake ;

Jehowa kicizapi en mdetahunka.

9 Tiyopa kin, pa yuwankan ehdaku po ;

Qa tiyopa ohinniyan un wankan iyeiciya po:

Unkan Wowitan Wicaśtayatapi kin tin hiyu kta.

10 Wowitan Wicaśtayatapi kir lie tuwe he:

Jehowa Sabaoth,

Wowitan Wicaśtayatapi kin hee. Sela.

\section{PSALM 25.}

Dawid tawa.

1 Jehowa, niye elita, Minagi wankan wahdomde kta.

2 Wakantanka mitawa, niye wacinciya;

Imaśtece śni nunwe;

Tokamayanpi ohimayanpi śni nunwe.

3 Nakun tona anipepi owasin iśtecapi kte śni :

Takuśni on wahtanipi kin hena iśtecapi kta.

4 Jehowa, nitacanku kin hena makipazo wo;

Niohan kin hena onspemakiya ye.

5 Nitowicake en omani makiye ça onspemakiya wo;

Wakantanka Wanikiya mitawa kin he niye;

Anpetu owasin niye acipe waun.

6 Jehowa, nitowaonśida qa nitocantelkiye kiksuya ye ;

Hena otokahe wanica heon etanhan.

7 Komaśka ehan wawalitani qa taku śica ecamon qon hena kiksuye śni ye ;

Wawacinkta duza tanka eciyatanhan miksuya wo ;

Jehowa, nitowaśte kin heon etanhan. 
8 Jehowa waśte qa owotanna;

Heon canku kin ohna wahtanipi sa onspewicakiye kta.

9 Tona onśihanpi kin woyaco en yus awicaye kta;

Qa onśilianpi kin iye tacanku onspewicakiye kta.

10 Jehowa tolian kin owasin wowaonśida qa wowicake, Tona wicotakuye tawa qa" woyaatanin tawa opapi kin hena en.

11 Jehowa, nicaje kin on etanhan, Wawahtani micicajuju ye, he tanka nakaeś.

12 Wicaśta Jehowa kokipe cin tukte e he; Canku hdahnige kte cin he onspekiye kta.

13 Iye nagi wowaśte en wanke kta; Qa cinca kin makoce kin tawayapi kte.

14 Tona Jehowa kokipapi kin hena kici wohdakapi ece; Unkan wicotakuye tawa kin sdonyewicakiye kta.

15 Miiśta ohinniyan Jehowa ekta; Wohmunke etanhan misiha eyaku kta, heon.

16 Miye ekta ihduhomni qa onśimakida ye ; Miśnana waun qa makakija, heon etanhan.

17 Micante iyotanhan iyekiye cin yutankapi ; Makakije cin etanhan emayaku wo.

18 Iyomakiśica qa wamayazan kin wanyaka wo; Qa wawalitani owasin micicajuju ye.

19 Tokamayanpi kin wanwicayaka wo ; hena otapi ; Qa wośicedake wohitika on śicemadapi ece.

20 Minagi micipatan qa emayaku wo; Imaśtece śni nunwe; wacinciya heon.

21 Woyuecetu qa wicoowotanna mapatanpi kta; Acipe waun kin heon etanhan.

22 Wakantanka, wicokakije owasin etanhan Israel ewicahdaku wo.

\section{PSALM 26.}

Dawid tawa.

1 Jehowa woyaco maqu wo:

Mitoecetu kin ohna mawani, heon:

Nakun Jehowa wacinyan waun;

Mahicahe kte śni.

2 Jehowa mayukcan qa imayutan wo; Mapakśin micante ko yuecedan yc.

3 Nitowaonśida miiśta itokam un; : Qa wowicake nitawa ohna mawani.

4. Wicaśta itonpiśni om imdotanke śni; Qa wanakilimanpi kin en mde kte śni.

5 Śicaya econpi omniciye kin śice wadaka; Qa wahtanipi sa om imdotanke kte śni.

6 Wiyaonpepicaśni ohna nape wahdujaja; Hecen Jehowa, owayuśna nitawa ohomni mde kta.

7 Wopida ho kin on eyanwapaha kta; 


\section{PSALM 2\%.}

Qa niohan wakan owasin omdake kta.

8 Jehowa, yati omyanpi kin he waśte wadaka; Qa nitowitan wokeya oyanke kin he nakun.

9 Wah̆tanipi sa om minagi mnakiye śni wo; Qa mitaniya, we wicaśta lkin om.

10 Hena napepi ohna wicowicaśta śni; Qa nape etapapi wiyopeyapi ojudan.

11 Tuka miye qe mitoecetu ohna mawani kta: Opemakiton qa onśimakida wo.

12 Misiha makomdaya ohna han: Mniciyapi kin en Jehowa mdawaśte kta.

\section{PSALM $2 \%$.}

Dawid tawa.

1 Jehowa iyoyanpa mitawa qa Wanikiya mitawa hee, tuwe kowakipe kta he ;

Jehowa wiconi mitawa wowaśake kin hee, tuwe on kopewahda lita he.

2 Wicaśta śica micehpi yutapi lita e mikiyedan upi qehan, Tolamayanpi qa śicemadapi kin hena eepi hicahanpi qa hinhpayapi.

3 Wicota en ahimawanka eśta, micante kokipe kte śni; Ozuye wan ahimanajin eśta, de wacinyan waun.

4 Taku wanji Jehowa wakida, he awakita ece kta; Anpetu tona wani kin owasin Jehowa ti kin en waun kta ;

Jehowa toiyokipi wanmdake kta, Qa tipi wakan tawa lin ohna wawimunge lite.

5 Anpetu śice cin en tawokeya ohna namahbe kta; Iye tawalkeya onalibe kin en inahbe makiye kta; Imnija wan akan wankan emahnake kte.

6 Unkan nakaha mapa kin toka mihdukśan hiyeya wiciwankam un kte;

Heon iye tawakeya ohna wowiyuśkin wośna kin wawakiyuśna kta;

Wadowan kta, han, Jehowa odowan wakahiyaye kte.

7 Jehowa anagoptan wo, miho on wahowamde;

Hecen onśimakida qa amayupta wo.

8 Miite akita po: Micante heniciya ;

Jehowa, niite awakite kta ce.

9 Niite inamakilibe śni wo;

Canniniyan nitaokiye ehpeye śni ye ;

Omakiye cin he niye; amayuśtan śni wo;

Qa ehpemaye śni, Wakantanka Wanikiya mitawa.

10 Tohan ate qa ina elipemayanpi kinhan,

Hehan Jehowa imacu kte.

11 Jehowa, nitacan'zu kin onspemakiya ye ; Qa ocanku owctanna ohna amayan wo, Tona iwanmayakapi kin hena on. 


\section{P S A L 29.}

12 Tokamayanpi tawacinpi kin en wicamaqu śni wo; Wayaatanin itonpi śni kin itkom mahinajinpi,

Qa tona wośitkihda apoganpi kin.

13 Wiconi makoce kin en, Jehowa towaśte opahta wacinwaye śni kinhan.

14 Jehowa wacinyan wo;

Waditaka wo, kinhan cante niyuwaśake kte :

Han, Jehowa wacinyan wo.

\section{PSALM 28.}

Dawid tawa.

1 Jehowa, niye hoyeciciye lita;

Imnija mitawa, miye on inina yanke śni wo;

Miye on noge nikpe cinhan,

Hehan wicahapi ekta yapi kin iyemacece kta.

2 Houciciya eca wocekiye mitawa ho kin nalion wo;

Nitawakeya wakan ektakiya nape mdugata eca.

3 Wicaśta śica om elipemaye śni wo;

Qa tona woahtani econpi kin om;

Hena wicikiyedan unpi kin om wookiye eyapi;

Tuka cantepi en woyuśica yukan.

4 Taku econpi kin iyecen wicaqu wo;

Qa śicaya ohanyanpi kin iyececa;

Iye nape ohanpi kin on niye wicaqu wo;

'T'aku econpi kin iyecen wicakicu wo.

5 Jehowa tolian kin hena awacinpi kte śni,

Qa iye nape taku kage cin hena,

Heon wicakawanke lkta, qa icaliwicaye kte śni.

6 Jehowa yawaśtepi nunwe;

Wocekiye mitawa ho nalion kin heon.

7 Jehowa mitowaśake qa wahacanka mitawa kin hee:

Micante wacinyan un, unkan omakiyapi:

Heon micante nina iyuśkin lita;

Qa odowan mitawa on mdatan kte.

8 Wowaśake tawapi kin Jehowa hee;

Qa wiconi conkaśke wan iye Sdakiye cin tawa.

9 Nitaoyate niwicakiya wo;

Qa taku nitawa kin hdawaśte wo:

Nakun wonwicaya wo,

Qa owihanke wanica hehanyan wicahduha ye.

\section{PSALM 29.}

Dawid tadowan.

1 Waśakapi cinca kin, Jehowa qu po; Jehowa wowitan wowaśake ko qu po.

2 Iye caje towitan kin Jehowa kicu po; Wokoyake wakan un Jehowa ohoda po. 
3 Jehowa ho kin mini kin akan;

Wowitan Wakantanka wakinyan hotonkiya;

Jehowa mini tanka kin akan un.

4. Jehowa ho kin wowaśake en;

Jehowa ho kin wowitan en un.

5 Jehowa ho kin hanteśa bow eliwega;

Han, Jehowa Lebanon hanteśa kin kawehwega :

6 Qa ptejincadan wan iyecen psipsinwicaya;

Lebanon qa Sirion, tatanka cinca iyececa.

7 Jehowa ho kin peta ide kin yapta ece.

8 Jehowa ho contanka kin yahuhuze kta, Qadeś contanka kin Jehowa yuhuluuze kta.

9 Jehowa ho kin tawiyedan hokśiksuye kiya;

Qa can kin kahna okihi kta:

Unkán iye ti kin ohna owasin, Wowitan, eyapi ece.

10 Jehowa mini tanka kin akan iyotanka;

Han, Jehowa Wicaśtayatapi iyotanka, owihanke wanica.

11 Jehowa iye taoyate wowaśake wicaqu kte:

Jehowa iye taoyate wookiye on wicayawaśte kta.

\section{PSALM 30.}

Odowan. Tipi yuwakanpi kin on dowanpi. Dawid tawa.

1 Jehowa, iyotan ciyawa kta, niye wankan emayahde;

Qa tokamayanpi miye on iyuśkinwicayaye śni heon.

2 Jehowa Wakantanka mitawa, Hoyeciciya, unkan niye asnimayaya.

3 Jehowa, Hades etanhan minagi iyacu ;

Woha ekta yapi kin etanhan nimayaya.

4 Wicaśta wacantkiye tawa, niye Jehowa kahiyaya po;

Qa iye towakan kiksuyapi kin on wopida eya po.

5 Tocanniye kin iśta kakpapi hehanyan;

Toiyokipi kin he wiconi :

H́tayetu eca wicaceya yuke kta,

Tuka hanhanna dowanpi kta.

6 Unkan iniye tanyan waun kin en,

Owihanke wanin mapahohopi kte śni ce, epa.

7 Jehowa, nitoiyokipi kin on paha mitawa wowaśake yakaga;

Niite nayakilibe, unkan mayuśinyaya.

8 Jehowa, niye hoyeciciye kta;

Qa Jehowa wocekiye ewakiye kta.

9 Mawe kin en wokamna yukan he,

Wolia ekta kull mde cinhan:

Maka mdu kin niyatan kta he;

Nitowicake kin oyake kta he.

10 Jehowa, anagoptan qa onśimakida ye;

Jehowa, omalkiye cin he niye nunwe.

11 Waśinwahda kin he wacipi miyecaga;

Heyakeśica mayakiduśdoke ça wowiyuśkin on ipiyag mayakiton. 
12 Heon wowitan inidowan kta, qa inina un kte śni :

Jehowa Wakantanka mitawa, owihanke wanin wopida eciciye kta.

\section{PSALM 31.}

Dowan itancan kin on. Dawid tadowan.

1 Jehowa niye wacinciya:

Owihanke wanin imaśtece śni nunwe :

Nitoowotanna on emahdaku wo.

2 Anolimakiya ye ; emayaku inahni wo:

Wowaśake imnija mitawa kin he niye nunwe;

Qa conkaśke tipi on nimayanpi kte.

3 Imnija mitawa qa conkaśke mitawa kin he niye;

Heon nicaje on napata mayaduze ca yus amayade kta.

4 On hmunkapi nahmana emakionpapi kin etanhan mayadusdute kta ;

Wowaśake mitawa kin he niye, heon etanhan.

5 Mitaniya ninape en owehnake kta;

Jehowa wowicake Wakantanka, niye opemayakiton.

6 Tona takuśni itonśni awacinpi kin hena śicewicawadaka ;

Tuka Jehowa wacinwaya ece. .

7 Nitowaonśida on piwada qa imduślkin kte:

Woiyokiśice mitawa niye wandaka;

Wokakije en minagi sdonyaye ciqon.

8 Nakun toka nape en onamayatake śni;

Makoce tanka en siha emayahde.

- 9 Jehowa, onśimada wo, makakija heon:

Woiyolisíica on iśta maśica amayan;

Minagi qa mitezi nakun.

10 Wani kin woiyokiśica on masote;

Qa waniyetu mitawa comnihdazipi on;

Wawahtani on waśake śni amayan;

Qa mahuhu kin hena silitin.

11 Tokamayanpi owasin wowahteda śni mayanpi,

Nakun mikiyedan unpi kin hena iyotan;

Qa tona sdonmayanpi kin wokokipe mayanpi;

Tona tankan wanmayakapi kin hena namakipapi.

12 Taku ta iyecen cante on amilstonjapi ;

Wakśica tramdecapi wan iyemaceca.

13 Wicota waaiapi kin nawah̆on;

Ihdukśan wokokipe, wiyamaonpapi kin icunhan,

Mitaniya icupi kta akitapi.

14 Tuka Jehowa, niye wacinciya;

Wakantanka mitawa lkin he niye ce, epa.

15 Mitaanpetu ninape ohna un;

Tokamayanpi qa śicaya makuwapi napepi etanhan emahdaku wo.

16 Niite nitaokiye aiyelikiya ye ;

Nitowaonsida on nimayan wo. 
17 Jehowa, imaśtece śni nunwe, hoyeciciye cin heon:

Tona śicapi kin hena iśtecapi nunwe, Tades en inina unpi kte.

18 Woitonśni ihapi kin inina unpi nunwe;

Hena wahaniçidaya qa wawośtehdaya

Ikceya wicaśta owotanna aiapi.

19 Tona konicipapi nitowaśte wicayecihnake cin he tanka hinca; Wicaśta cinca itokam tona wacinniyanpi kin en he wicayakidutanin.

20 Hena nitohnake woinahbe kin en nawicayahbe kta, Wicaśta mniciyapi kin etanhan;

Wicaceji wakinicapi etanhan wokeya wan ohna wicayakpatan kta.

21 Otonwe suta wan ohna wowinihanyan cantemakiya, Heon .Jehowa yawaśtepi nunwe.

22 Tuka miś imanihan qehan, Niiśta itokapatanhan makaśpapi ce, epa:

Hececa eśta cewakiya ho kin namayakihon, Hoyeciciye çehan.

23 Wicaśta wacantkiye tawa kin owasin, Jehowa waśtedaka po: Tona wicakapi kin hena. Jehowa wicakpatan ;

Qa tuwe wahanhaniçidaya alianye cin he iyakiçuya kicicajuju eee.

24 Waśaka po, hecen cante niyuținzapi kte ;

Jehowa wacinyayapi kin owasin.

\section{PSALM 32.}

Wowahokonkiye odowan. Dawid tawa.

1 Tuwe taku śica econ kin kicicajujupi, Qa walitani akahpapi kin he cante waśte:

2 Wicaśta kin he cante waśte, Tuwe Jehowa woalitani kiciyawa śni, Qa taniya en wohnayan wanice cin he.

3 Inina manke cechan mahuhu śeca aya, Anpetu osan waśicahowamde cehan.

4 Anpetu hanyetu ko ninape amahdaskin yanka; Mawe kin mdoketu puze cin iyecen aya. Sela.

5 Wawahtani niye ociciyake kta; Qa taku śica ecamon kin anawakihbe śni : T'aku śica ecamon kin hena Jehowa owakiyake kta ce, epe: Unkan wawalitani śice cin miyecicajuju. Sela.

$6 \mathrm{He}$ on tona wacantkiye cin owasin ceniciyapi kta, Tohan iyeniyanpi kte cinhan: Awicakehan mini ota apaśboka eśta, He hiyohipi kte śni.

7 Woinalibe mitawa kin he niye; Wokakije etanhan emayahdaku kta; Woehdaku odowan on aohdutemayaye kta. Sela.

8 Wahokonciciye ça canku ohna de kte cin he onspeciciye kta ; 
Miiśta opacilita wahokonciciye lita.

9 Ihnuhan śuktanka qa śonśonna wan iyenicecapi kinhan;

Hena takudan okahinigapi śni;

Tiyuwi qa ikan oiyokipi on i pawicahtapi ;

Nikiyedan upi śni heon etanhan.

10 Tona śice cin wokakije ota yuhapi kte:

Tuka tuwe Jehowa wacinye cin he wowaonśida aohduteye kta.

11 Tona owotanna yaunpi, Jehowa on pidapi qa iyuśkin po;

Qa tona cante ecetu yaunpi owasin, wiyuśkinyan iyaśa po.

\section{PSALM 33.}

1 Wicaśta owotanna yaunpi, Jehowa on wiyuślkin po:

Yatanpi kin tona ecetupi iyokipipi ece.

2 Candowankiyapi on Jehowa wopida eciya po;

Candowankiyapi ikan wikcemna on idowan po.

3 Odowan teca wan kahiyaya miye;

Ho tanka on wayupiya yajo po.

4 Jehowa oie kin he ecetu;

Qa ohan kin owasin wowicake.

5 Woowotanna qa wokonze waśtedaka;

Maka kin Jehowa towaonśida on ojudan.

6 Jehowa oie on malipiya kin kagapi ;

Qa iye i taniya on hena obe kin owasin.

7 Miniwanca mini kin kawitaya, paha iyececa;

Ośbe kin mazopiye en ekihnaka.

8 Maka kin ocowasin Jehowa kokipapi kte ;

Maka akan ounyanpi iyulipa itokam inihanpi nunwe.

9 Iye ia, unkan ecetu;

Iye kaken eya, unkan sutaya han.

10 Ikcewicaśta tawacinpi kin Jehowa ecetu śni ya;

Oyate token wakiconzapi kin he yujuju ece.

11 Jehowa tawokonze kin ohinniyan he kta;

Iye cante yuze cin wicoicage qa wicoicage hehanyan.

12 Oyate wan Jehowa Taku Wakan yuhapi kin he cante waśtepi ;

Wicaśta iye tawawicaye kta wicahdalinige cin hena.

13 Jehowa mahpiya eciyatanhan ahitonwan;

Adam cinca kin owasin wanwicayaka.

14. Tukten ounye cin hetanhan etonwan,

Maka akan ounyanpi owasin ekta:

15 Cantepi kin owasin wicakaga;

Wicohian tawapi owasin awacin un.

16 Wicaśtayatapi tuwedan ozuye tanka on niyanpi śni:

Wicaśta waśaka keś wowaśake tanka on ihduśke kte 'śni.

17 Niwicayapi kta on śuk tanka kin wacinyepica śni ;

Qa iye towaśake tanka on waeyaku kte śni.

18 Tho, Jehowa iśta kin tona kokipapi kin hena awicatonwan;

Tona iye towaonśida apepi kin hena. 
19 Wiconțe etanhan nagipi ewicahdaku kta;

Qa wicaakilian en niwicaye kta.

20 Unnagipi Jehowa wacinyanpi;

Ounkiyapi qa wahacanka unkitawapi kin he iye.

21 Tye on uncantepi iyuśkin lite; Iye caje wakan wacinunyanpi heon.

22 Jehowa, nitowaonśida unkiyepi en un nunwe; Unkanipepi kin heon etanhan.

\section{PSALIM 34.}

Dawid tawa. Abimelek itokam togye olianyan; unkan hdesi hehan kihda, he ehan.

1 Anpetu owasin Jehowa mdawaśte kta;

Iye idowanpi kin ohinniyan mii ohna un kta.

2 Jehowa on minagi itan lita:

Tona onśitianpi nahonpi kta qa iyuśkinpi kta.

3 Jehowa mici yatanka miye ;

Qa yuwitaya iye caje kin unyawankantupi kte.

4 Jehowa áwakita, unkan amayupta;

Qa wakowakipe cin owasin etanhan emahdaku.

5 Ekta etonwanpi, unkan iyoyamyapi;

Qa ite naśapi śni nunwe.

6 Onśike cin de howaya, unkan Jehowa nahon, Qa tokakije owasin etanhan ehdaku.

7 Tona kokipapi kin hena wicihdukśan, Jehowa taohnihde kin iwanka,

Qa ewicayaku ece.

8 Uta miye, qa Jehowa waśte e wanyaka po:

Tuwe wacinye cinhan wicaśta lin he cante waśte.

9 Wacantkiya yaunpi tawaniyanpi, Jehowa kokipa po;

Tona kokipapi kin hena takudan icakijapi kte śni.

10 Mnaja cinca wicakiś unpi qa wotektehdapi;

Tuka Jehowa akitapi fin hena taku waśte talkudan icakijapi lite śni.

11 Tho po, micinca, anamagoptan po;

Jehowa tokokipe kin onspeciciyapi kta.

12 Wicaśta wiconi cin, qa taku waśte

Wanyake lita e anpetu waśtedake cin he tuwe he.

13 Taku śica etanhan niceji ehdaku wo;

Qa niiha wohnayan eyapi kte śni.

14 Taku śica elipeya wo, qa taku waśte e econ wo:

Wookiye akite ca kuwa aya wo.

15 Jehowa iśta wicaśta owotanna awicatonwan; Qa howayapi eca anohwicakiya ece.

16 Tona taku śica econpi Jehowa ite kin itkowicakim un; Maka lin etanhan wicakiksuyapi kin icu kte.

1\% Hoyeyapi, unkan Jehowa nation, Qa tokakijepi iyulipa etanhan ewicahdaku. 
18 Jehowa cante kicaksapi kin wicikiyedan un; Qa tona taniya onśike cin hena niwicaya ece.

19 Wicaśta owotanna tokakije hin ota; Tuka owasin etanhan Jehowa eyaku kta.

20 Huhu tawa ovasin awankiciyaka; Hecen wanjidan kaeś kawegapi śni.

21 Wośice tona śice cin hena wicakte lkta; Qa wicaśta owotanna sice wicadakapi hin hena yacopi $\mathrm{kta}$.

22 Jehowa taokiye nagipi kin opewicakiton; Qa tona iye wacinyanpi kin tuwedan yacopi kte śni.

\section{PSALIM 35.}

\section{Dawid tawa.}

1 Jehowa, tona malrigepi kin hena wicakige wo; Tona makizapi kin hena wicakiza wo.

2 Mazamakuakahpe wahacanka ko ikikcu, Qa omakiya inajin wo.

3 Nakun wahukeza ehdaku, Qa tona śicaya makuwapi wicakagi wo ; Wiconi nitawa kin lie miye ce, minagi eciya wo.

4 Minagi odepi kin hena inihanpi qa iśtecapi nunwe; Tehiya amawacinpi kin hena hekta hdicuwicayapi qa iśtenwicayapi nunwe.

5 Aguyapi ha tate itokam hena iyecccapi nunwe: Qa Jehowa taohnihde hena wicakaśtake kte.

6 Tacankupi kin otpaza qa śduśduta nunwe; Qa Jehowa taohnihde lin kuwa awicaye kte.

7 Taku on etanhan śni wohmunke wan miye on nahmana eonpapi ;

Taku on etanhan shi minagi on wohia wan qapi.

8 Sdonye śni kinhan woihangye kin en u nunwe;

Qa wohmunke nalibe cin he iye içihmunka nunwe;

Woihangye kin hee en ohinhpaya nunwe.

9 Hehan minagi Jehowa on iyuślkin kta;

Wiconi tawa kin idowan kta.

10 Mahuhu owasin heyapi kta;

Jehowa, tuwe iyeniceca he;

Tuwe isanpa waśaka etanhan onśike cin eyahdaku;

Han, onśike ca wahpanica kin, tuwe wamakinon kin etanhan.

11 Itonśniyan woyakapi kin hinajinpi;

Taku sdonwaye śni kin hena on imawangapi.

12 Taku waśte on taku śica maqupi;

On minagi kakiśyapi kta.

13 Tuka miye qe, wayazankapi qehan, wakihdaka komdaka; Akihanmiçiya on minagi kakiśwaye :

Unkan wocekiye mitawa mamaku en hdihan.

14. Mitakoda se, misunka hee, he iyecen mawani; Tuwe hunku akiceye cin iyecen waśihda pamahdedan waun. 
15 Tuka nakaha nawaśduta, hehan iyuśkinpi qa witaya mniciyapi ; Waaie sa kin hena miye en mniciyapi, unkan sdonwaye śni:

Wayalidelidecapi qa inina unpi śni.

16 Wota ia śicapi kin hena om;

Hi amahdakinskinzapi.

17 Itancan, tohanyan opahta nanke kta he:

Woihangye tawapi etanhan minagi hdohdi wo;

Waśtewakidake cin, mnaja kin etanhan.

18 Omniciye tanka kin en wopida eciciye kta;

Oyate waśaka om ciyatan kta.

19 Tokamayanpi itonpiśni kin hena miye on iyuśkinpi śni nunwe : Tona taku on etanhan śni śicemadapi kin hena iśta kakpanpi.

20 Hena wookiye iapi kte śni;

Tuka tona makoce kin en, inina unpi kin

Hena wohnayan wicoie wicakicagapi kta.

21 Han, hena nina i makapapi :

Qa heyapi; Hoho, hoho,

Iśta on wanunyakapi ce.

22 Jehowa niye wandaka, inina yanke śni wo;

Itancan, mitehanyan un śni wo.

23 Ihduhica ye, qa mayacopi kte cin on kikta wo;

Makiyuśepi kin on,

Wakantanka mitawa, qa Itancan mitawa.

24 Jehowa Wakantanka mitawa,

Nitoowotanna eciyatanhan mayukcan wo;

Qa hena miye on iyuślkinpi śni nunwe.

25 Cante mahen, Hoho, hecen uncinpi ce, eyapi śni nunwe;

Naunkipcapi ce, eyapi śni llunwe.

26 Tona makakija on iyuśkinpi kin hena iśtecapi,

Qa witaya ite naśapi nunwe;

'Tona miye on ihdatankapi kin hena wowahteda śni,

Qa wowiśtece koyakapi nunwe.

27 Tona mitoowotanna cinpi kin hena iyaśapi,

Qa wiyuślkinpi nunwe;

Han, ohinniyan heyapi kte; Jehowa tanka ce,

Iye taokiye wookiye kicicin kin hee.

28 Unkan miceji nitoöwotanna cajeyate kta;

Niyatanpi kin anpetu osan.

\section{PSALM 36.}

Dowanitancan kin on. Jehowa taokiye wan kaga. Dawid tawa.

1 Micante mahen, wicośice wicaśta śica heciya;

Iye iśta itokam Taku Wakan kokipapi wanica ce.

2 Iye iśta kin en ilidawaśte ;

Tuka wahtani kin iyeyapi qa śicedapi kta.

3 Iye i oie kin hena woitonśni qa wohnayan;

Wacinksamya waśteya econ kta he ayuśtan.

4. Towinja akan woitonśni awacin un kta; 
Canku waśte śni wan ohna iyahe kta;

Taku śica ayuśtan kte śni.

5 Jehowa, nitowaonśida kin he mahpiya elsta un;

Nitowicake malipiya śapa iyahde.

6 Nitoowotanna kin he Iyotan Waśaka ta he kin iyececa:

Wokonze nitawa kin he wośbe tanka:

Jehowa, wicaśta woteca ko ni wicayaya.

7 Wakantanka, nitowaonsilda kin he waśte hinca;

Heon Adam cinca nilipahu ohanzi kin en wacinyanpi kta.

8 Yati wasna kin on imnanpi kte;

Qa wowaśte nitawa walkpa kin etanhan yatke wicayaye kta.

9 Wiconi minilidoka wan nici wanke:

Iyoyanpa nitawa en iyoyanpa wanunyakapi kta.

10 Tona sdonniyanpi kin hena nitowaonśida iyohi wicakiya wo: Qa nitoow otanna, tona cante ecepidan kin:

11 Walianiçidapi siha kin anamatan śni nunwe; Qa ohan śica nape kin napemaye śni nunwe.

12 Hen wicohan śica econpi lin hinhpayapi; Hena makata elipeyapi, qa najin okihipi kte śni.

\section{PSALIM $3 \%$.}

\section{Dawic tawa.}

1 Wicohan śica econpi kin hena on iyokiśiniçiye śni wo; Qa woahtani cconpi kin on winawizi śni wo.

2 Hena ecadan kaśdapi kta, peji iyececa; Qa wato iyecen śnijapi kta.

3 Jehowa wacinyan wo, qa taku waśte econ wo: Makoce lin en ounye ca wowicake akita wo:

4. Nakun Jehowa en iyokipiiciya wo; Kinhan taku iceyakiye cin hena nicu kta.

5 Nitacanku kin Jehowa yuhekiya wo; Nakun wacinyan wo; linhan ecetuye lita:

$6 \mathrm{Qa}$ nitoowotanna iyoyanpa iyecen yutanin kta ; Qa wokonze nitarva kin wiyotanhan iyececa.

7 Jehowa en inina un, qa wacintankaya ape wo: Tuwe canku ohna wapiya un kin he on iyokiśiniçiye śni wo; Tuwe wicotawacin śica hduecetu kin on.

8 Wocanniye ayuśtan qa wośihda elipeya wo; Taku śica ecanon kta e iyokiśiniciye śni wo.

9 Tona taku śica econpi kin hena ihangwicayapi kta ; Tuka Jehowa wacinyanpi kin hena măkoce kin yuhapi kta.

10 Ehake cistiyedan hehan wicaśta śica en un kte śni; Han, tohe kin opalita yaun kta, tuka en un śni.

11 Tuka tona onśiccidapi kin hena makoce kin tawayapi kta; Qa wookiye ota on iyuśkiniçiyapi kta.

12 Wicaśta śica wicaśta owotanna iyape un; Qa hi ahdakinskinza ece.

13 Itancan kin ihaha kta; 
Taanpetu u kta wanyake cin heon.

14 Wicaśta śicapi mazasagye ehdakupi ;

Qa tinazipepi nakiwinjapi ;

Onśika qa wahpanica kin makata elipewicayapi kta ;

Qa tona ohan owotanna kin wicaktepi kta.

15 Mazasagye tawapi kin on iye cante caiçipapi kta;

Qa tinazipepi kin kawegapi kta.

16 Taku cistinna wicaśta owotanna tawa kin he waśte;

Wicaśta śica ota wowijice yuhapi kin isanpa.

17 Wicaśta śica is to kawegapi kta:

Tuka Jehowa wicaśta owotanna najin wicakiya ece.

18 Wicaśta ecetu taanpetu kin hena Jehowa sdonya:

Qa taku yuhapi kin he owihanke wanice kta.

19 Anpetu śica en iśtecapi lite śni;

Qa wicaakilian anpetu kin en imnahan wotapi kta.

20 Tuka wicaśta śice cin hena atakunipi kte śni;

Qa Jehowa tokayapi kin ;

Tahinca cinca śin kin iyecen liuhnagapi ;

Śota en taninpi śni.

21 Wicaśta śica wodota keś wicakicu kte śni :

Tulra wicaśta owotanna wacantkiye ca wawicaqu ece.

22 Tona wicayawaśte kin hena makoce kin tawapi kta:

Qa tona wicayaśice cin hena ihangyapi kta.

23 Jehowa eciyatanhan wicaśta cachde kin yuecetupi;

Qa tacanku kin on iyuśkin kte.

24 Hinlipaye kta, tuka makata elipeyapi kte śni ; Jehowa nape yuze cin heon etanhan.

25 Homakśidan waun, qa wanna wimacahinca;

Tuka wicaśta owotanna kin elipeyapi,

Qa cinca woda unpi wanmdake śni.

26 Anpetu nsan waonśida qa owicaqu ece :

Unkan cinca kin hena woyawaśte.

27 Taku śica ayuśtan qa taku waśte e econ wo ;

Qa owihanke wanin ounyan wo.

28 Jehowa wokonze waśtedaka ece;

Qa wicaśta wacantkiye tawa kin hena elpewicaye kite śni;

Hena ohinniyan wicakpatanpi :

'Tuka wicaśta śica cinca kin hena ihangwicayapi.

29 Wicaśta owotanna makoce kin tawayapi kta;

Qa owihanke wanin en ounyanpi kte.

30 Wicaśta,owotanna i kin woksape ie kta;

Qa ceji wokonze iwohdake kte.

31 Iye ta Wakantanka tawoahope kin eante mahen un;

Caehde tawa kin kaktihanhan kte śnị.

32 Wicaśta śica wicaśta owotanna iwanyaka;

Qa kte kta akita ece.

33 Tuka nape en Jehowa elipeye kte śni ;

Qa aiapi kinhan yaco kte śni.

34. Jchowa ape un wo, qa tacanku patan wo; 


\section{PSALM 38.}

Unkan makoce tawayaye kta e yuwankan inicu kta;

Wicaśta śica ihangyapi kinhan wandake kta.

35 Wicaśta śica wohitika wan wanmdaka;

Qa ihdutanka, can teca icage cin iyececa:

36 'Tuka tanin śni iyaya, unkan inyun en un śni ;

Han owade, tuka iyeyapi śni.

37 Wicaśta ecetu kin he iyukcan, qa owotanna kin wanyaka wo;

Wicaśta kin he owihanke kin woolkiye.

38 Tuka wawakipajinpi kin witaya ihangyapi kte ;

Wahtanipi sa owihanke kin he baśpa iyeyapi.

39 Tuka wicaśta owotanna niwicayapi kin he Jehowa etanhan:

Wokakije anpetu kin en wowaśake tawapi kin hee.

40 Unkan Jehowa owicakiye ça ewicahdaku:

Wicaśta śica etanhan ewicayaku qa niwicaye kta;

Tye wacinyanpi, heon etanhan.

\section{PSALM 38.}

Dawid tadowan. Wokiksuye on.

1 Jehowa, wocanniye nitawa en iyopemaye śni wo;

Qa śinyahda hinca en makapsinpsinte śni wo.

2 Nitunwanhinkpe lkin bomalidokapi ;

Qa ninape sutaya amapaskica.

3 Nitocanniye on micehpi zaniyan un śni ;

Wawahtani on mahulıu en woozi wanica.

4 Wawahtani kin hena pa iwankam imayaya;

Waqin the hinca iyecen on mduśaka ece.

5 Amapapi kin tonyan qa śicamna;

Wimatkotkoke cin heon etanhan.

6 Mahnaśkinyan, nina patuś nawajin;

Anpetu osan waśihda waun.

7 Wowayazan śica wan on pakśin omajudan;

Qa micehipi tuktedan zani śni.

8 Wamaśake śni qa nina makaśuśujapi ;

Cante mayazan heon waśicahowamda ece.

9 Itancan, token cantowakpani owasin nitokam un;

Qa waśicahowamde cin he nanicihmanpi śni.

10 Cante maliniyanyan, waśake śni amayan;

Nakun miiśta iyoyanpa kin hee kaeś iyemacetu śni.

11 Tona waśtemadapi qa mitakuye wamayazan kin en najinpi lite śni ;

Qa mikiyedan unpi qon itehanyan inajinpi.

12 Unkau minagi akitapi kin hena mahmunkapi ;

Qa tona śicaya waun kta cinpi kin hena taku sica eyapi;

Qa anpetu osan wohnayan awacinpi kta.

13 Tuka miye, noge tpa iyececa, nawalion kte śni;

Qa ia olitpani i hdukawa kte śni kin iyemaceca.

14 Hecen wicaśta wanahon śni kin iyecen waun;

Qa tuwe i ohna iyopeyapi wanice cin. 
15 Jehowa niye acipe waun kin heon, Niye waadupte kte, Itancan Wakantanka mitawa.

16 Unkan hepa; Okinni miye on iyuśkinpi kta; Siha nawaśduta ecá miye on ihdatanpi ece.

17 Miye humaśte waun kin heon; Qa iyomakiśice cin ohinniyan mitokam un.

18 Miohan śice cin owahdake kta; Qa wawahtani kin on iyokiśinmiciye kta.

19 Tuka tokamayanpi ni unpi kin hena waśakapi; Qa tona taku on etanhan śni śicemadapi kin hena ota aya.

20 Tona taku waśte on taku śica econpi kin hena, Taku waśte awakite cin on makiyuśepi kta.

21 Jehowa, amayuśtan śni wo:

Wakantanka mitawa, mitehanyan un śni ye.

22 Omakiya inahini wo; Itancan, Wanikiya mitawa.

\section{PSALM 39.}

Jeduthun dowan itancan kin on. Dawid tadowan.

1 Miohan iwanwahdake kta, epa; Hecen miceji on wawahtani kte śni :

Ionatake wan mii on mduhe kta; Wicaśta śica mitokam unpi icunhan.

2 Ia okitpani iyecen inina manka; Taku waśte ee lkaeś ie śni waun;

Qa mitoiyokiśica yuśośapi.

3 Cante mahen makate hinca; Waawacin waun icunhan peta itkon: Hehan miceji on iwaa :

4 Jehowa, omawihanke kin sdonye makiya wo; Qa mitaanpetu kin tohanyan kta hecinhan; Tohan en waun kte śni sdonwakiye kte.

5 Wan, mitaanpetu kin nape hdakinyan yakaga; Qa nitokam wani kin he takuśni iyececa :

Awicakehan wicaśta otoiyohi, sutaya najin eśta, ocowasin takuśni. Sela.

6 Awicakehan wicaśta kin ohanzi wan en omani ; Awicakehan takuśni on nihinciyapi ece: Waeju ece, tuka tuwe hena yuhe kta sdonye śni.

7 Unkan nakaha, Itancan, taku e ape waun he: Niye hincà wacinciya ece.

8 Wawahtani owasin etanhan emayaku wo; Wicaśta witkotkoka wowihahamayanpi kta makage śni wo.

9 Inina waun, i wahdukawa kte śni ; Niye hecanon kin heon etanhan.

10 Amayape cin he imicicu wo; Ninape makaśtake cin on atakuni śni amayan.

11 Iyopeyapi on wicaśta wahtani kin on iyopeyaya; 
Qa wayapote sa iyecen taku iyokipi kin dutakuni śni :

Awicakehan wicaśta otoiyohi takuśni. Sela.

12 Jehowa, wocekiye mitawa nahon wo;

Qa houwaye cin anohkiya ye:

Iśtamuihanpe amau kin on inina yanke śni wo ;

Niye kici wicaśta matokeca,

Unhdaka waun, atewicawaye cin owasin iyececa.

13 Makpatan wo, hecen waśagmiçiye kta,

Imdamde śni qa en waun śni itokam.

\section{PSALM 40.}

Dowan itancan kin on. Dawid tadowan.

1 Apepi on Jehowa ape waun;

Unkan anohmakiye ca hoyewaye cin namakilion.

2 Nakun wicotakuniśni wolia wan etanhan hiyu makiya, upśija linca etanhan;

Qa misiha imnija wan ahan makiye; mioye miciyusuta.

3 Qa odowan teca wan mii kin en ohnaka;

Wakantanka unkitawapi yatanpi kin :

Wicota wanyakapi qa kokipapi kta;

Qa Jehowa wacinyanpi kte.

4 Wicaśta wan cante waśte hinca, Jehowa wowinape yuhe cin hee;

Qa tona wahanhanicidapi kin,

Woitonśni opapi kin hena koya ekta etonwe śni.

5 Jehowa mita Wakantanka taku ota ecanon;

Niolian wakan qa unkiyepi on nitarvacin kin,

Hena owecinhan niciyawapi kte śni ;

Cajemdate ça omdake lita eśta, hena yawapica śni.

6 Wośnapi qa wawicaqupi kin hena iyonicipi śni ;

Noge mayakidulidoka:

Wahuhnahyapi qa woahtani on wośnapi kin yada śni.

7 Hehan hepa; Wanyaka wo, wau ce;

Wowapi yuhmihmapi kin en hecen omiciwapi :

8 Wakantanka mitawa nitawacin ecen ecamon lkta iyomakipi;

Qa nitoope kin micante mahen un.

9 Omniciye tanka kin en woowotanna eyanwapaha:

Wanyaka wo, miiha wakpatan kte śni;

Jehowa niye sdonyaya.

10 Nitoowotanna micante mahen nawalibe śni;

Wacinniyepica qa waniyakiya omdaka;

Nitowaonśida qa nitowicake omniciye tanka kín etanhan nawatibe śni.

11 Jehowa niye,

Nitowaonśida temiyecilida kte śni;

Nitocantekiye qa nitowicake ohinniyan mapatan kte.

12 Taku śica yawapica śni aohdutemayanpi ;

Wawahtani kin hena emihdegapi, 
Qa hecen wawanyaka owakihi śni;

Hena mapa hin isanpa ota;

Qa on cante mawanica.

13 Jehowa, emahdaku iyonicipi nunwe; Jehowa, omakiya inalini ye.

14 Tona minagi ihangya akitapi kin

Hena witaya cante toketu taninśni qa iśtecapi kte ;

Tona śicaya waun kta cinpi kin

Hena hekta hdicupi qa iśtecapi kte.

15 Tona, Hanhan, hanhan, emakiyapi kin

Hena wowiśtece on atakunipi kte śni.

16 Tona anicitapi kin hena owasin cante waśtepi qa iniciyuślinpi kte;

Tona wiconi nitawa waśtedapi kin hena, Jehowa yatankapi nunwe, eyapi kte.

17 Tuka miye onmaśike ça mawahipanica;

Itancan lin amawacin un kta:

Tuwe omakiye ca emahdaku kin he niye;

Wakantanka mitawa yutehan śni ye.

\section{PSALIM 41.}

Dowan itancan kin on. Dawid tadowan.

1 Tuwe wicaśta onśika en ewacin kin he cante waśte; Anpetu śice cin en Jehowa ehdaku kte.

2 Jehowa awanhdake ça nikiye kta;

Maka akan tanyan un kte;

Qa tokayapi tawacin kin en wicayaqu kte śni.

3 Wowayazan ole akan Jehowa yuwaśake kta; Wayazanka icunhan towinja ocowasin piyaya.

4. Miye hepe; Jehowa onśimalkida wo; Minagi asniyan wo; wacicihtani kin heon.

5 Tokamayanpi kin hena taku śica emakiyapi kta; Tohan țe kta qa caje atakuni kte śni he.

6 Qa wanmayag hi kinhan woitonśni oyaka ece kta; Cante mahen woahtani mnakiya;

Tokiya ye kte ça oyake kta.

y Tona śicenıdapi owasin witaya amajijipi kta ;

Hena śicaya cante makiyuzapi kta.

8 Taku śice hinca wan aokaśtanpi ce :

Qa tuwe makata wanke cin he ake kinajin kte śni.

9 Han, wicaśta kici wowakiye, wacinwakiye ciqon, Aguyapi mitawa yute cin he siyete on namahtaka.

10 'Tuka, Jehowa niye, onśimada qa najinmakiya wo; Hecen wicawecicajuju kta.

11 Detanhan waśtemayadaka sdonwakiya;

Tokamaye cin maktedan śni kin heon.

12. Unkan miye, mitoecetu kin en mayadusuta; Qa ohinniyan niite itokam najin mayakiya. 


\section{P S A L M 43.}

13 Jehowa, Israel ta Wakantanka, yawaśtepi nunwe; Otokahe wanica qa owihanke wanica. Amen qa amen.

\section{PSALM 42.}

Dowan itancan kin. Qora cinca wahokonwicakiyapi.

1 Tatokadan mini wakpadan cantokpani,

He iyecen, Wakantanka, minagi cantonikpani ece.

2 Wakantanka on minagi ipuza, Wakantanka ni un kin; Tohan wau qa Wakantanka ite kin en mihdutanin kta he.

3 Iśtamnihanpe amau lin hena woyute waye, anpetu hanyetu koya;

Nita Wakantanka tukten un he, ohinniyan emakiyapi heon.

4 Dena weksuye ça minagi amihdapson kta;

Wicota om imdamde cinhan;

Hena om Wakantanka ti kin ekta wai;

Wowiyuśkin qa woyatan ho kin yuha;

Wicota anpetu wakan opapi kin hena om.

5 Minagi, tokeca pamahdedan yaun,

Qa tokeca miye mahen iyoniciśica he;

Wakantanka wacinyan wo, ake mdatan kte,

Miite omakiye ça mita Wakantanka kin hee.

6 Mahentanhan minagi iyokiśica un;

Heon cilsuye kta, Jordan makoce kin etanhan,

Qa Hermon he, paha cistinna kin hetanhan.

7 Mini tanka nitawa ho kin on, mini śma kin mini śma kipan;

Taja nitawa qa mini ihalia nitawa owasin miwankam iyaya.

8 Anpetu icunhan Jehowa towaonśida kin ekihde kta ;

Qa hanyetu eca iye tadowan kin mici un ;

Wakantanka nimaye cin he cewakiye kte.

9 Wakantanka imnija mitawa hewakiye kta ;

Tokeca amiyektonja he;

Tokeca waśihda imdamda he,

Toka śicaya makuwapi kin on.

10 Tokamayanpi kin iyopemayanpi, mahuhu kaśuśujapi kin iyececa;

Anpetu otoiyohi, Nita Wakantanka toki un he, emakiyapi kinhan.

11 Minagi, tokeca pamahdedan yaun,

Qa tokeca miye mahen iyonicisica he ;

Wakantanka wacinyan wo, ake mdatan kta,

Miite omakiye ça Wakantanka mitawa kin hee. ,

\section{iPSALM 43.}

1 Wakantanka mayaco qa imakiya wo;

Oyate wacantkiye śni kin on;

Wohnayan wicaśta qa wahtani sa etanhan emayahdaku kte.

2 Wakantanka conkaśke mitawa kin he niye ; 
Tokeca ehpemayaya he ;

Tokeca waśihda inıdamde kta he,

Toka śicaya makuwapi kin on.

3 Iyoyanpa nitawa qa wowicake nitawa u kiya wo;

Hena yus amaye kte;

Paha wakan nitawa kin en hi makiye kte;

Qa nakun wokeya nitawa ekta.

4 Hehan Wakantanka owayuśna tawa ekta wau lite;

Taku Wakan mitowiyuślkin tanka ekta.

Unkan candowankiyapi on icidowan lita,

Wakantanka, Wakantanka mitawa.

5 Minagi, tokeca pamahdedan yaun,

Qa tokeca miye mahen iyoniciśica he :

Wakantanka wacinyan wo, ake mdatan kta;

Miite omakiye ça Wakantanka mitawa kin hee.

\section{PSALM 44.}

Dowan itancan kin on. Qora cinca wahokonwicakiyapi.

1 Wakantanka, noge on naunhionpi,

Atewicunyanpi unkokiyakapi,

Iye taanpetu lrin en wicohan ecanon qon,

Wanakaja anpetu lsin en.

2 Niye ninape on Ikcewicaśta hamwicayaya,

Qa iyepi cn owicayakiju;

Oyatepi kin ihangwicayaya,

Qa iye icaliwicayakiya.

3 Mazasagye tawapi on malioce kin icupi śni;

Qa iye isto kin he on eicihdakupi śni ;

Tuka ninape etapa, qa niisto, qa niite iyoyanpa lsin;

Niye cantowicayahnake cin heon.

4 Wakantanka, Wicaśtayatapi mitawa kin he niye; Jakob woehdaku kiconza wo.

5 Niye on tokaunyanpi kin paha iyewicunyanpi kta; Nicaje on tona itkounkipapi kin nawicunpanpi kta.

6 Mitinazipe wacinwakiye kte śni; Qa mazasagye mitawa nimaye kte śni.

7 Tuka niye tokaunyanpi kin etanhan eunyahdakupi ; Qa tona śiceundapi kin iśtenwicayaya.

8 Anpetu osan Wakantanka unkidowanpi; Qa nicaje ohinniyan unyatanpi kta. Sela.

9 Hececa eśta, ehpeunyayapi qa wowiśtece unyakagapi;

Qa ozuye unkitawapi kin om de kte śni.

10 Toka kin itokapatanhan hdicuunyayapi kta: Qa tona siceundapi kin hena wakte hdapi.

11 Tahinea wanunyanpi yutapi kte cin iyececa unyakagapi kta: Qa Ikcewicaśta ehna enanakiya iyeunyayapi.

12 Nitaoyate inyunwin codan wiyopeyakiye kta: Qa iyopeyapi on takudan yakamna śni. 
13 Unkikiyepidan unpi kin en wowiśtece unyakagapi kta;

Unkihdukśan unpi kin en wowilialiaunyanpi qa poptanptanunkiyapi.

14 Ikcewicaśta ekta oweśtepi unyakagapi kta;

Oyatepi en pomnamnaunkiyapi kte.

15 Anpetu osan iśtenmayanpi kin mitokam un;

Qa miite iśtece cin he amahdahpa:

16 'Tuwe wawiyopeye ça wayaśica ho kin on ;

Toka ite qa waihangye cin on etanhan.

17 Dena owasin unkakipapi, tuka unkaniktonjapi śni;

Qa wicotakuye nitawa unhnayanpi śni.

18 Uncantepi helsta hdicu śni ;

Qa unkoyepi nitacanku icunonpa ye śni.

19 Unktehi owanka kin en unyakawehwegapi ;

Qa wiconțe on unkayakahpapi.

20 Wakantarka unkitawapi caje unkakiktonjapi,

Qa taku wakan tokeca ekta nape unhdugatapi kinhan;

21 Wakantanka he ode kte śni he :

Wicacante woinahbe kin hena iye sdonya nakaeś.

22 Niye on anpetu osan unktepi unyakonpi ;

Tahinca wanunyanpi wicaktepi kte cin iyecen unyawapi.

23 Itancan, ihduhica wo, tokeca niśtinma he ;

Kikta wo, hecehin ehpeunyanpi śni po.

24 Tokeca niite nayakihbe $\mathrm{k}$ ta he;

Iyounkiśicapi qa śicaya unkuwapi kin ayektonje kta he.

25 Unnagipi watuśelrśeca ekta patuś yanka;

Untancanpi maka kin ahdaskin wanka.

26 Ounyakiyapi kta on inajin wo;

Qa nitowaonśida on opeunkiton po.

PSALIM 45.

Mnalicalica on dowan itancan kin. Qora cinca wahokonwicakiyapi. Wowaśte odowan.

1 Micante wicoie waśte on ipiga;

Miohan wicaśtayatapi kin owakimdake ;

Wowapi icage wowapi kaga wayupika tawa miceji kin hee.

2 Adam cinca ehna niye iyotan owanyag niwaśte;

Niiha en wowaśte okaśtanpi ;

Heon Wakantanka ohinniyan niyawaśte.

3 Iyotan Wašaka, mazasagye nitawa ceca en iyahdaśka wo;

Nitowitan qa nitoiyokipi ahna:

4. Qa nitowitan hduha śuktanka akan iyoopta ya wo;

Wowicake qa woowotanna onśike cin hena on;

Unkan nape nietapa taku wowinihan onspeniciye kta.

5 Wanhinkpe nitawa pepe hinca;

Nitokam oyatepi hinhpayapi kta;

Tona Wicaśtayatapi tokayapi cantepi kin en.

6 Wakantanka, oiyotanke nitawa ohinniyan qa owihanke wanica; 
Nitokiconze sagye kin he woowotanna sagye.

7 Woowotanna waśteyadaka, qa woahtani śiceyadaka;

Heon Wakantanka, nita Wakantanka kin,

Wowiyuśkin wihdi on sdaniciya,

Kodawicayaye cin isanpa.

8 Nitawokoyake owasin canśin, qa canhaśa, qa pejihuta waśtemna;

Huhu tipi waśte kin etanhan wiyuśkinniyanpi.

9 Wicaśtayatapi cunwintkupi kin winohinca waśteśte nitawa om unpi ;

Wicaśtayatapi tawicu kin nietapa ekta najin,

Mazaskazi Ophir etanhan en.

10 Cunś, anagoptan qa wanyaka qa on anolikiya wo;

Qa nitaoyate niyate ti ko akiktonja wo:

11 Unkan wicaśtayatapi kin nitowaśte waśtedake kte:

He itancan nitawa, hecen itokam patuja wo.

12 Turos cunwintku taku itulianpi wan on niite akite $k$ ta; Oyate kin en wijicapi kin hena.

13 Ti mahen wicaśtayatapi cunwintru kin ocowasin wowitan; Tawolroyake kin mazaskazi kazontapi.

14 Śna ipatapi in wicaśtayatapi kin en ahipi kta;

Wikośkapi takodawicaye ihakam unpi kin hena niye en awicahipi kta.

15 Wowiyuśkin qa wopida yuha en awicahipi kta;

Wicaśtayatapi ti kin timahen hipi lite.

16 Niyatepi eekiya nicinkśipi yuke kta;

Hena maka kin owancaya wicaśta itancan ewicayahde kte.

17 Wicoicage qa wicoicage owasin en nicaje kiksuyewicawaye kta:

Heon oyatepi kin niyatanpi kta, owihanke wanica.

\section{PSALM 46.}

Qora cinca on; dowan itancan kin. Wikośka dowanpi.

1 Wowinape qa wowaśake unkitawapi kin Wakantanka hee; Awicakehan wokakije en waokiye iyeyapi ece.

2 Heon etanhan kopeunhdapi kte śni, maka kin yutoliecapi, Qa he kin miniwanca cante en ayapi eśta.

3 Mini kin hotanin qa śkanśkan kte; Taś tanka on he kin yuhuhuzapi kte. Sela.

4 Wakpa wan-adetka kin Wakantanka totonwe kin iyuśkinye. ktä;

Tipi wakan kin-Tyotan Wankan un ounye cin he.

5 Wakantanka cokaya un, heon pahohopi kte śni ; Anpao hinanpa hehan Wakantanka okiye kta.

6 Ikcewicaśta oqoyapi, wicokiconze kin śkanśkanpi ;

Iye ho hdutanin, maka kin skan kta.

7 Jehowa Sabaoth unkicipi un ;

Jakob ta Wakantanka wowinape unyanpi. Sela. 
$8 \mathrm{U}$ miye, Jehowa tolian kin wanyaka po; Makoce kin en woihangye kaga ece.

9 Maka ihanlse hehanyan ozuye ayuśtan kiya; Itazipa kin kawege kta, qa wahukeza kakse kta ; Canpahmihma kin peta on liuhnaliye kta.

10 Ayuśtan po, qa Wakantanka kin he miye e sdonya po, Ikcewicaśta ehna mayatanpi kte, maka akan mayatankapi kte.

11 Jehowa Sabaoth unkicipi un: Jakob ta Wakantanka wowinape unyanpi. Sela.

\section{PSALM $4 \%$}

Dowan itancan kin on. Odowan; Qora cinca tawapi.

1 Oyate owasin, nape hdaskapa miye; Wowiyuśkin ho kin on Wakantanka iyaśa po.

2 Jehowa Tehan Wankan kin he wowinihan; Wicaśtayatapi tanka, maka kin owancaya.

3 Unkihukun wicaśta kin ewicahnake kta; Qa oyatepi unsihapi ihukuya.

4 Makoce wan unkicicalinigapi kte ; Jakob waśtedake ciqon toiyokipi kin hee. Sela.

5 Iyaśapi yuha Wakantanka wankantkiya ikiyopta; Jehowa mazayahotonpi ho kin hduha.

6 Dowan po, Wakantanka idowan po ; Dowan po, Wicaśtayatapi unkitawapi idowan miye.

7 Wakantanka maka kin owancaya Wicaśtayatapi ; Wowahokonkiye wan on dowan miye.

8 Wakantanka Ikcewicaśta kin wowidagwicaya; Wakantanka oiyotanke wakan kin akan kiyotanka.

9 Oyatepi en itancanpi kin mniciyapi; Abraham ta Wakantanka taoyate kin : Makoce wahacanka kin hena Wakantanka tawa: Iye nina tehanwankan un.

\section{PSALM 48.}

Qora cinca tawapi. Psalm odowan.

1 Jehowa he tanka; qa nina yatanpi kte; Taku Wakan unkitawapi totonwe, Paha wakan tawa kin en.

2 Oyanke waśte hinca, maka ocowasin wowiyuśkin; Zion Paha kin, waziyata opapun ekta; Wicaśtayatapi tanka totonwe kin.

3 Tipi waśteśte tawa kin ohna, Wakantanka wowinape yapi kta sdonyapi.

4. Iho, maka wicaśtayatapi lin mniciyapi; Witaya en hiyayapi :

5 Hena wanyakapi, unkan inihanpi; Kopehdapi qa najica iyayapi.

6 Hen cancan hinhdapi; 
Wayazan hdapi, wiholinca hokśiksuya iyececa.

7 Wiyohiyanpa tanhan tate kin on, Tarśiśs wita wata kin yalkajuju kta.

8 Naunhonpi qon he iyecen wanunyakapi; Jehowa Sabaoth totonwe kin en, Wakantanka unkitawapi totonwe ohna; He Wakantanka ohinniyan hdusuta kte. Sela.

9 Wakantanka, tipi wakan nitawa ohna, Nitocantekiye kin awauncinpi.

10 Wakantanka, nicaje iyececa, Niyatanpi maka ihanke kin hehanyan; Ninape etapa woowotanna ojudan.

11 Zion Paha kin iyuśkin kte ; Juda cunwintkupi cante waśtepi kte ; Woyaco nitawa kin on etanhan.

12 Zion ihdukśan ya miye, ohomni mani po;

Canpasotka kin hena yawa po:

13 Conkaślke tawa kin ekta cante yekiya po ;

Tipi waśteśte kin hena awacin miye;

Hecen wicoicage u kte cin owicayakidakapi kte.

14. Taku Wakan kin de Wakantanka unkitawapi, owihanke wanica:

Wiconte hehanyan yus unkayapi kte.

\section{PSALIM. 49.}

Dowan itancan kin. Odowan Qora cinca tawapi.

1 Oyate owasin, de nalion miye;

Maka akan ounyanpi kin owasin, anagoptan po :

2 Adam cinca, nakun wicaśta cinca,

Wijicapi wahpanicapi kin om.

3 Mii woksape oyake kta;

Qa micante taku awacin kin he wookalinige.

4 Wiyacinpi wan ekta minoge yewaye kta; Candowankiyapi on mitadowan mduotanin kta.

5 Anpetu śica en tokeca liopewahda kta he; Tona amahanpi wahtanipi kin aohdutemayan kinhan.

6 Iye towaśakepi kin wacinkiyapi ; Qa taku ota tonpi kin on ihdatanpi ece:

7 Wicaśta wan iye sunkaku ecaca opekiton kte śni: On wiyopeyapi Wakantanka qu kta okihi śni.

8 Unkan nagipi opekitonpi kin he teliika; Qa owihanke wanica okihipica śni :

9 Hecen hinyahin ohinniyan ni un kta; Qa wicaliwin wanyake kte śni.

10 Wicaśta ksapa tapi kte cin he wanyake kta; Nakun witkotkoka wacintonśni ko atakunipi kte śni ; Qa taku tonpi kin toktokeca aihpewicakiyapi kte.

11 Cante mahen, tipi tawapi owihanke wanice kta, kecinpi; 
Ohna yukanpi kin wicoicage qa wicoicage hehanyan; Makoce tawapi iye cajepi icajeyatapi ece.

12 'Tuka wicaśta woyuonihan en yanke kte śni;

Woteca iyecen kagapi; hena atakunipi śni.

13 Hecen ohanyanpi ; hecen witkotkokapi :

Tuka tona ihakam upi kin hena

Token eyapi qon he waśtedakapi kta. Sela.

14 Optaye wan iyecen Hades ekta awicayapi ;

Wiconte wonwicaye kta;

Qa wicaśta owotanna, anpao lkinhan, wowidagwicayapi kta:

Nakun towaśakepi kin Hades yusote kta;

Iye tipi kin etanhan tawawicaya.

15 Tuka Wakan tanka minagi Hades nape etanhan opemakiton lkta;

Iye emahdaku kta, heon.

16 Wicaśta wan wijica icage cinhan kopehda śni wo;

Tipi tawa wowitan tanka aye cinhan:

17 Tohan te cinhan takudan hduha ye kte śni;

Towitan ihakam kun ye kte śni.

18 Tuka ni un icunhan nagi hdawaśte kta :

Nakun tanyan nicicuwa kinhan niyatanpi kta.

19 Atewicaye wicoicage kin ekta ye kta:

Hena icimana iyoyanpa wanyakapi kte śni.

20 Wicaśta yuonihanpi, tuka okalinige śni kin

He woteca kin iyacinpi, hena atakunipi kte śni.

\section{PSALM 50.}

\section{Asaph tadowan.}

1 Jehowa Wakantanka Iyotan Waśake cin ia, Qa maka kin kipan,

Wihinanpa etanhan wi iyaye cin hehanyan.

2 Zion woiyokipi iyotan lince cin etanhan

Wakantanka iyega.

3 Wakantanka unkitawapi u kta, qa inina un kte śni;

I tokam peta waliulinaliye kta ;

Qa ihdukśan nina icamna kte.

4 Wankan mahpiya kin kipan kta,

Qa nalkun maka kin, iye taoyate wicahdaco kta.

5 Wicaśta cante wicawakiye cin miye en awicau po;

Tona wośnapi on wicotakuye micagapi kin.

6 Unkan mahpiya kin iye toowotanna kin oyake kta; Wakantanka wayaco un kin heon. Sela.

7 Mitaoyate nahon po, kinhan iwae kta; Qa Israel, wahokonciciye kta;

Wakantanka, Wakantanka nitawa kin he miye.

8 Nitawośnapi kin on iyopeciye kte śni ;

Qa peta on waduśnapi kin ohinniyan mitokam un :

9 Yati etanhan tatanka wan iwacu kte śni;

Optaye nitawa etanhan tamdoka kin: 
10 Contanka en wamanica otoiyohi mitawa;

Qa woteca paha kektopawinge akan unpi kin.

11 He ekta. taku kinyan un kin otoiyohi sdonwaya; Qa makomdaye en woteca owasin miciun.

12 Wotektewahda eśta ociciyake kte śni ; Maka kin he mitawa qa taku ohnaka kin.

13 Tatanka conica wate kta he; Qa tamdoka we mdatke kta he.

14 Wakantanka woyatan wakiyuśna wo ; Qa Iyotan Wankan un kin taku iwahoyaya hdajuju wo.

15 Hehan woiyokiśica anpetu kin en, houmakiya wo; Kinhan ecihdaku kta, hecen imayadowan kta ce.

16 Tuka Wakantanka wicaśta śica heciya; Token on mitoope kin odake kta; Qa wicotakuye mitawa tokeca cajedate kta he.

17 Wahokonkiyapi kin he śice yadaka; Qa mioie kin niheyata elipeyaya.

18 Wamanon sa wan wandaka eca takodayaya; Qa wawicihahapi econpi kin owicayapa ece.

19 Nii taku śica en owicayaqu; Qa niceji wohnaye kazonte kta.

20 Nisunka akiia idotanke kta; Nihun cinhintku kin waayape kta.

21 Hena hecen ecanon, tuka inina manke: Niye yaun kin ocowasin iyemaceca kecanni: lyopeciye kta, qa niiśta itokam hena ewahde kta.

22 De awacin po, Wakantanka ayektonjapi kin ; Okinni waehdaku wanin ciyaśpaśpapi kta.

23 Tuwe woyatan wayuśna kinhan, he mayuonihan kta: Qa tuwe tacanku hdiecetu kin

He Wakantanka towiconi kin wakipazo kta.

\section{PSALM 51.}

Dowan itancan kin. Dawid tadowan: Nathan wicasta wokcan en hi qehan, iye Bathśeba en hi qon iyecccil.

1 Wakantanka, nitocantekiye on onśimada ye; Nitowansisida ota on wawahtani micicajuju wo.

2 Taku śica ecamon kin on ocowasin mayujaja ye ; Qa wawahtani kin hena makipakinta wo.

3 Wawahtani kin hena sdonwakiya; Qa taku 'śica ecamon kin ohinniyan mitokam un.

4 Niye niśnana en wacicilitani, Qa niiśta kin en takı śica ecamon; Hecen iyae cin en oniwotanna kte; Wadaco kinhan iyannpepica śni yaun kta.

5 Wanyaka ye, taku śica en matonpi; Qa woahtani en ina mahduha.

6 Wanyaka ye, tanmahen tanhan wowicake yacin ece: 
Qa woinalbe ehta woksape sdonye mayaye kte.

7 Ceyakata on mayakipakinte kta, unkan eciedan waun kta; Mayadujaja kta, kinhan wa isanpa maska kte.

8 Wicocante waśte wowiyuśkin ko nahon mayaye kte ; Hehan huhu yakaśuje cin hena iyuśkin kta.

9 Wawahtani etanhan niite nakihman ye;

Qa taku śica ecamon kin owasin micicajuju wo.

10 Wakantanka, cante ska micaga ye;

Qa miye mahen woniya yuśtanpi miciyuteca wo.

11 Niite etanhan ehpemaye śni ye;

Qa Nitaniya Wakan kin emicihdaku śni wo.

12 Wiconi nitawa wowiyuśkin kin he mieu ye; Qa woniya waśte wan yuhe makiya wo.

13 Hehan śicaya econpi kin nitacanku onspewicawakiye kta; Qa wahtanipi sa niye ekta ihduhomnipi kta.

14 Wakantanka, wicawe etanlaan emahdaku wo ; Wakantanka, Wanikiya mitawa; Miceji nitoowotanna iyuślkinyan idowan kte.

15 Itancan, miiha miyecidukawa k.ta;

Hecen mii woyatan nitawa oyake kte.

16 Wośnapi kin yacin lite śni, he waqu kta tuka;

Peta on wośnapi kin iyonicipi śni.

17 Wośnapi Wakantanka tawa woniya kicaksapi kin hena ee; Wicacante kieaksapi qa kaśuśujapi kin, Wakantanka, śiceyadake kte śni.

18 Nitoiyokipi kin en Zion taku. waśte ecakicon wo : Ierusalem conkaśke kin niye yecage $\mathrm{kta}$.

19 Hehan woowotanna wośnapi kin iyonicipi kta; Peta on wośnapi qa wośnapi ocowasin hulinahyapi : Hehan tatanka owayuśna nitawa adipi kte.

\section{PSALM 52.}

Dowan itancan kin on. Dawid tawowahokonkiye. Doeg Edomi kin en hi qa Saul wokiyaka qa heciya; Dawid Ahimelek ti en hi ce, eye ciqon.

1 Wicaśta waśaka, tokeca taku śica on nihdatan kta he; Iyotan Waśaka towaonśida kin he anpetu osan.

2 Niceji wicohan śica kicanye lita; Wohnayan kaga, putinhin icasan yumanpi iyececa.

3 Taku śica waśteyadaka, taku waśte isanpa; Qa woitonśni, woowotanna oyakapi kin isanpa. Sela.

4. Wicoie waihangye cin owasin waśteyadaka, Wicaceji wicahnayan yaun kin.

5 Heon etanhan Wakantanka owihankeśniyan ihangniye kta; Yutokan iyeniye kta, qa wakeya etanhan inicu kte ; Qa wiconi malroce kin etanhan yujun ehpeniye kta. Sela.

6 Unkan wicaśta owotanna wanyakapi qa kokipapi kta; Qa he on ihapi lsta:

7 Wanyaka po, wicaśta kin he 
Wakantanka wowaśake yuhe kte śni;

Tuka iye towijice icage cin wacinkiye kta ;

Qa tohan śice cin on waśa giçiye kte.

8 Tuka miye Óliwe can teca wan iyemaceca,

Wakantanka ti kin ohna;

Wakantanka tocantekiye wacinwaya,

Owihanke wanica hehanyan.

9 Niye hecanon kin heon ohinniyan ciyatan kta;

Qa nicaje wacinwakiye kta ;

He wicaśta wacantkiya nitawa itokam waśte kin heon.

\section{PSALM 53.}

Dowan itancan kin on. Wowayazan on Dawid tawowahokonkiye.

1 Taku Wakan wanica ce, witkotkoke cin cante mahen eya:

Ihduśicapi qa wicohan śica walite śni econpi ;

Tuwedan taku waśte econ śni.

2 Wakantanka mahpiya eciyatanhan Adam cinca kin ahiwicatonwan ;

Tuwe wacinksamya ohanyan hecinhan,

Tuwe Wakantanka akita hecinhan, he wanyake kta.

3 Iyuhpa nunipi, witaya liwinpi ;

Tuwedan taku. waśte econ śni; han, wanjidan śni.

4 Woahtani econpi kin hena takudan sdonyapi śni he; Aguyapi iyecen mitaoyate wicayutapi ;

Qa Wakantanka hoyekiyapi śni.

5 Wokokipe wanica eśta hen nina wikopapi;

Tuwe en ahiniwanka huhu kin Wakantanka enanakiya elipeya;

Iśtenwicayaya, Wakantanka wahtewicada śni nakaeś.

6 Tokin Israel woehdaku tawa kin Zion etanhan u nunwe:

Tohan Wakantanka taoyate wayaka unpi kin en wicahdi kinhan, Jakob iyuśkin kta, Israel pidapi kte.

\section{PSALM 54.}

Candowankiyapi on dowan itancan kin. Dawid tawowahokonkiye. Ziph oyate upi qa Saul heciyapi; Dawid unkicipi inahbe śni he, eyapi qon he ehan.

1 Wakantanka, nicaje on nimayan wo: Qa nitowaśake on woyaco ecamiyecon kte.

2 Wakantanka, wocekiye mitawa nahon ye; Mii oie kin hena anoĥkiya wo.

3 Wicaśta tokeca en ahimanajinpi heon; Qa teliiya wakuwapi kin minagi akitapi: Iye itokam Wakantanka ehdepi śni. Sela.

4 Wanyaka wo, Wakantanka omakiya ece: Itancan kin tona minagi waśagyapi kin hena om un.

5 Wośice kin tokamayanpi en awicahdi kte: Nitowicake en hena ihangwicaya wo.

6 Taku ituhanpi on waciciyuśna kte; 
Jehowa, nicaje waśte heon mdatan kta.

7 Wokakije owasin etanhan emahdaku;

Qa miiśta tokamayanpi kin opawicahta, heon.

PSALI 55.

Dawid tawa. Candowankiyapi on dowan itancan kin. Wowahokonkiye odowan.

1 Wakantanka, mitawocekiye anohkiya wo;

Qa hoyewaye cin on naiçihbe śni ye..

2 Namalion qa amayupta wo;

Awacin waun qa howamde kta:

3 Toka ho kin on, wicaśta śica śicaya kuwapi kin on ;

Hena woahtani aomakawinliyapi kta;

Qa śinhdapi en tokamayanpi kte.

4 Mitan mahen micante śkanśkan;

Qa wiconțe ikopapi kin amahinhpaya.

5 Kopehdapi qa cancanpi kin mahiyohi;

Qa wonihinciye amakahipa.

6 Unkan hepe; "Tuwe wakiyedan iyecen hupahu wan maqu kta he:

Kinyan imdamde ça ozimiçiye kta.

$7 \mathrm{Ho}$, tehan wanuni kite;

Hewoskan owati kta. Sela.

8 Wowinape onawakipa inawalini kta ;

Tateyanpa hinca etanhan, icamna kin etanhan.

9 Itancan, nawicapca wo, cejipi wicalkiyuśkiśka wo :

Otonwe kin en wicokicize qa wicokicige wanmdaka heon.

10 Anpetu hanyetu ko otonwe conkaśke alran ohomni yapi kte; Qa wicośice wicoiyokiśice ko mahen un.

11 Woalitani cokaya un,

Qa śicaya wicakuwapi wohnayan ko tahocoka kin ayuśtan kte śni.

12 Toka wan iyopemaye kte cin hee śni;

Kinhan inina manke kta tuka:

Tuwe miye on wahaniçida śicemadake cin hee śni ;

He heca unkanś etanhán namicilibe kta tuka.

13 Tuka he niye, wicaśta kici iakinimaskokeca;

Mitakoda qa wacinwaye ciqon hee.

14 Unkiye okonwanjidan wicotawacin skuya unkiçicaga:

Wicota om Wakantanka ti kin en unye ciq̨on.

15 Woihangye awicakipa nunwe;

Niyake Hades ekia kun yapi kte:

Tipi tawapi ohna wicohian śica yukan,

Iye cantepi kin en.

16 Miye qe Wakantanka hoyewakiye kta;

Unkan Jehowa nimaye kte.

17 Ḣtayetu qa hanhanna qa wioytanhan, cewakiye ca hoyewaye kta:

Unkan iye miho kin nahon kte. 
18 Makizapi etanhan, wookiye en, nagi opemalkiton; Wicota om ecamon kin heon etanhan.

19 Iyotan Waśake cin he nawicahion qa awicayupte kte; Qa tuwe wanakajatanhan ounye cin he. Sela.

Tona wicayutokanpi wanin unpi,

Qa Wakantanka kokipapi śni kin hena.

20 Tona om wookiye en un qon hena nape awicahnaka;

Wicotakuye tawa kin he hdaksa.

21 Pteasanpi ihdi iyecen i kin śduśduta;

Tuka cante kin he okicize :

Wihdi isanpa iye oie kin śduśduta;

Tuka hena mazasagye ecedan.

22 Taku niçu kin he Jehowa ailipeya wo;

Unkan iye awanniyake kta:

Wicaśta owotanna wicapahohopi kta tohinni iyowinye kte śni.

23 'Tuka niye, Wakantanka, wicotakuniśni woha en kun iyaye wicayaye kta :

Wicawe wicaśta qa wicahnaye sa kin

Hena anpetu tawapi cokaya iyohipi kte śni.

Hececa tuka miye qe wacinciye kte.

\section{PSALM 56.}

Wicaśta tokeca om wakiyedan inina un kin on, dowan itancan kin kicagapi. Dawid tawoinahbe. Gath elsta Philistine yuzapi qon he ehan.

1 Wakantanka, onśimakida ye, wicaśta i amahdukawa; Anpetu osan watemya kin he kuwa amau ece.

2 Anpetu osan tona iwanmayakapi kin hena i amahdukawapi ; Wahanhaniçidapi ota temmakiyapi kta tuka.

3 Anpetu kin en kopewahda kinhan, Hehan wacinciye kta.

4 Wakantanka en iye oie kin mdatan kte ;

Wakantanka wacinwaya, kopewahda kte śni ;

Wicacehpi taku ecamicon kta okihi he.

5 Anpetu otoiyohi mioie yapemnipi;

Amawacinpi kin owasin śica.

6 Witaya mniciyapi, waiyape yukanpi kta; Mioye kin hena awanyakapi kta;

Minagi iyapepi kin iyececa.

7 Woahtani on ihduśkapi kta he;

Wakantanka, wocanniye en oyatepi kun ehpewicaya wo.

8 Wanuni kin hena dawa ece:

Iśtamnihanpe mitawa ojuha nitawa en ohnaka wo:

Hena nitawowapi en un śni he.

9 Anpetu en hoyewaye cinhan,

Hehan tokamayanpi kin namnipi kta:

He sdonwaya; Wakantanka miciun kin he.

10 Wakantanka en oie kin mdatan kta;

Jehowa en iye oie kin iwadowan kta. 
11 Wakantanka wacinwaya; kopewahda kte śni; Wicaśta taku ecamicon kta okihi he.

12 Wakantanka, taku iwahociye cin hena mduha; Woyatan wośnapi kin cicu kte.

13 Wiconte etanhan minagi edaku; Misiha hinlipaye kte śni :

Wakantanka itokam mawani kta;

Wiconi iyoyanpa kin en.

\section{PSALM 5\%.}

Dowan itancan kin kicagapi. Waihangye śni wo. Dawid tawoinahbe. Makohdoka en Saul ite kin etanhan najice ciqon he ehan.

1 Onśimakida wo, Wakantanka onśimakida wo ; Minagi wacinniyan, heon etanhan; Han, niliupahu ohanzi kin en wowinape awakite kta ; Wośice kin iyoopte kte cin hehanyan.

2 Wakantanka Iyotan Wankan hoyewakiye kta; Iyotan Waśaka tanyan miciyuśtan kin hee.

3 Mahpiya eciyatanhan u wicaśi qa emahdaku kta; Tuwe amapogan kin he iyopeye kta." Sela. Wakantanka iye towaonśida towicake ko hiyuye kta.

4 Minagi mnaja wicehna un;

Ideya unpi kin hena ehna imunke kta;

Wicaśta cincapi hi kin wahukeza qa wanhinkpe;

Qa cejipi kin mazasagye pe hinca.

5 Wakantanka, malipiya kin iwankam niyatanpi nunwe; Nitowitan maka kin ocowasin iwankam.

6 Wohmunke wan misiha on wiyeya ehnakapi ;

Minagi kin patuś ya ;

Mitokam wolia wan qapi ;

He en iye ohinhpayapi. Sela.

7 Micante wiyeya, Walkantanka, micante wiyeya un ;

Wadowan kta qa mdatan kta.

8 Mitowitan, kikta wo, cotanke candowankiyapi ko kikta miye : Miye qe anpao kin mduhice kta.

9 Itancan, oyate kin ehna ciyatan kte;

Oyatepi wicehna icidowan kta.

10 Nitocantekiye tanka kin malipiya iyapațo;

Qa nitowicake kin mah̉piya śapa iyahde.

11 Wakantanka, mahipiya kin iwankam niyatanpi nunwe;

Nitowitan maka kin ocowasin iwankam.

\section{PSALM 58.}

Waihangye sni wo, Dawid tawoinalibe.

1 Wicaśta cincapi, awiçakehan ia oyakitpanipi he; Hecen taku owotanna odakapi lkte śni, Taku ecetu kin dukcanpi kte śni. 
2 Han, cante mahen taku śica ecanonpi kta; Makoce kin en ninapepi wicowicaśtaśni kin tkeuyatapi kte.

3 Wicaśta śicapi wicatamni etanhan nunipi; Itonpiśni, tonpi ehantanhan śicaya econpi.

4 Wamduśka tage śica he iyecen tage śica wicalkiyukan; Sintelida noge kpe cin he iyecen noge ohmus hduza:

5 He wajijipi ho nalion kte śni; Wayupiya wajijipi econ eśta.

6 Wakantanka, hena ipi ohna hi wicakicaksa wo; Jehowa, mnaja kośka hi kin kaśuśuja wo.

7 Mini ohinniyan kaduze cin iyecen skan ayapi nunwe; Tunwanhinkpe nakiwinje kte, kawegapi iyececa.

8 Tusda skan aye cin iyecen tanin kte śni:

Winolinea hinyahinke śni cinca ton kin iyecen, Anpetu wi kin wanyakapi śni.

9 Cega nitawapi wapepeka sdonye śni, Saka qa iś śpan, ecen kahin iyeye kta.

10 Tokiçonpi kin he wicaśta owotanna wanyake cinhan iyuśkin kte :

Wicaśta śica we kin on siha hdujaja kte.

11 Hecen wicaśta wan heye kta; Han, wicaśta owotanna waskuyeca ton;

Han, Taku. Wakan maka akan wayaco yanka ce.

\section{PSALM 59.}

Dowan itancan kin kicagapi. Waihangye śni wo. Dawid tawoinalibe. Saul yewicaśi qehan, qa Dawid ti kin awanyaka, kte kta e heon.

1 Wakantanka mitawa, tokamayanpi kin etanhan makiyuśka wo ; Tona takpe maupi kin on wankan emayahde kta.

2 Tona woahtani econpi kin etanhan makiyuśpa wo; Qa re wicaśta kin etanhan nimayan ye.

3 Wan, minagi iyape yukanpi ;

Waśakapi kin miye on miciyapi ;

Jehowa, wawahtani śni qa taku śica ecamon śni ye.

4 Miohan śice śni eśta anamatanpi qa eicihdepi :

Kikta wo, itkomayakipa qa wandake kte.

5 Heon niye, Jehowa, Wakantanka Sabaoth,

Israel ta Wakantanka, kikta wo,

Oyatepi owasin wanwicadake kte;

Wicaśta śica wahnayanpi kin owasin wicakpatan śni wo.

6 Ḧtayetu kinhan hdicupi kte; śunka iyecen lidopi kte;

Qa otonwe ohomni inyankapi kte.

7 Inyun, ipi kin etanhan hiyuyapi ;

Ihapi kin en mazasagye yukan;

Qa tuwe nahon kta he, eyapi.

8 Tuka Jehowa, niye iwicayahalia kta;

Ikcewicaśta owasin wowihaha wicayaye kte.

9 Towaśake kin on acipe waun kta; 
Conkaśke mitawa Wakantanka hee.

10 Mita Wakantanka iye towaonśida on itkomakipe kta; Tokamayanpi kin Wakantanka opawicalita makiye kta.

11 Itancan, wahacanka unkitawapi, Hena wicakte śni wo, okinni mitaoyate akiktonjapi kte:

Nitowaśake on onuniwicaye ca kun elipewicaya wo.

12 Ihapi oie kin hena ipi woalitani lin hee :

Hecen wahanicidapi kin on wicayuzapi kte;

Woitonśni qa iapi śica eyapi kte cin hena on.

13 Waihangya wo, wocanniye on wicayusota wo;

Qa en unpi kte śni ;

Hecen Jalkob en Wakantanka wicaśtayatapi yanka e sdonyapi kte;

Maka ihanke kin hehanyan. Sela.

14 Unkan htayetu kinhan hdicupi kta;

Sunka iyecen hidopi lste;

Qa otonwe kin ohomni inyankapi kte.

15 Taku yutapi imani unpi kta;

Imnanpi śni eśta hanyetu osan wankapi kta.

16 Tuka miye nitowaśake iwadowan kta;

Qa hanlianna nitowaonśida mdatan kta:

Conkaślke mitawa kin he niye;

Qa iyomakiśica anpetu kin en wowinape mitawa.

17 Wowaśake mitawa, niye icidowan kta:

Wakantanka conkaślke mitawa kin hee,

Wowaonśida Wakantanka mitava.

\section{PSALM 60.}

Dowan itancan kin kicagapi. Woyaotanin Mnahcalica kin on. Dawid tawoinahbe. Waonspekiyapi kta. Aram wakpa nonpa otahedan, qa Aram Zoba ohiwicay: qon, hehan Joab hdica qa Miniskraya kaksiza en, Edom etanhan kektopawinge akenonpa wicakte.

1 Wakantanka, ehpeunyayapi, unyakawehwegapi ;

Canniniyan; tuka ake ounyakiyapi lita.

2 Maka kin duśkanśkan qa naptuśyaya, Naptuptujapi kin yuecetu wo, cancan nakaeś.

3 Nitaoyate taku telika wanyagwicayakiya; Miniśa on cekcegyapi kin yatke unyakiyapi.

4 Tona konicipapi kin wiyokihedan wan wicayaqu, Wowicake on yuwankan iyeyapi kta. Sela.

5 Tona cantewicayakiye cin wicayukcapi kta; Heon ninape etapa on niunkiyapi qa naunlion po.

6 Wakantanka iye towakan kin en ia;

Wakte wahdi kta;

Sekem wapamni kta;

Qa Sukoth kaksiza lin imdute kta.

7 Gilead he mitawa, qa Manassa he mitawa; Ephraim nakun mapa wowaśalie kin; Juda woope kaga mitawa kin hee. 


\section{$P S \mathbb{L} M$ 6.2.}

8 Moab cega oyujaja mitawa kin hee;

Edom en mitahanpe ehpewaye kta;

Philistia, miye on iyaśa wo.

9 Tuwe otonwe aconkaśkapi kin en amaye kta;

Tuwe Edom ekta amai he.

10 Wakantanka, elipeunyayapi qon he niye lrte śni he; Qa Wakantanka, ozuye unkitawapi om de kte śni qon.

11 'Toka kin on etanhan ounkiya miye :

Qa wicaśta alkantu waehdakupi lkin he taku śni.

12 Wakantanka en wowaśake unkiçicagapi kte:

Unkan iye tona tokaunyanpi kin nawicapan kte.

\section{PSALM 61.}

Dowan itancan kin kicagapi. Candowankiyapi Dawid tawa on.

1 Wakantanka, houwaye cin nakion ye;

Wocekiye mitawa awacin un wo.

2 Micante iyokiśica en, maka ihanke kin etanhan houciciye kta: Imnija wan miwankam tehanwankantu kin he en amayai kte.

3 Wowinape mitawa qun he niye; Toka kin itokam, conkaśke suta hinca.

4 Ohinniyan nitawakeya ohna wati kta; Niliupahu ohanzi kin en inawape lita.

5 Wakantanka, niye talku iwahowaye cin he nayahon: Tona nicaje kokipapi taku tawa lkte cin he mayaqu.

6 Wicaśtayatapi taanpetu kin en anpetu yecage kta; Waniyetu tawa kin wicoicage qa wicoicage iyececa.

7 Ohinniyan Wakantanka itokam iyotanke kte; Wowaonśida wowicake ko yuhe yakiye kte; Hena patanpi kta.

8 Heon ohinniyan nicaje iwadowan kta: Anpetu eca taku iwahowaye cin wakajuju ece kta.

\section{PSALM 62.}

Jeduthun eciyatanhan dowan itancan. Dawid tadowan.

1 Wakantanka ecedan en minagi inina yanka;

Iye eciyatanhan nimayanpi.

2 Iye iśnana imnija mitawa qa Wanikiya mitawa;

Conkaślke suta mitawa kin hee;

Nina mapahohopi kite śni.

3 Tohanyan wicaśta wan inihanyayapi lita he;

Niyepi owasin nicamdecapi kte;

Conkaśke takinyan yanka,

Mahinatake yujujupi kin iyececa.

4 Wankan yanke cin etanhan iśnana kun ehpeye wacinpi;

Woitonśni waśtedakapi ;

Ipi kin on wayawaśtepi kta,

Qa tanmahentanhan wayaśicapi lita. Sela. 
5 Wakantanka ecedan en, minagi, inina un wo; Wowacinye mitawa kin he iye eciyatanhan.

6 Iye iśnana imnija mitawa qa Wanikiya mitawa;

Conkaśke mitawa kin hee;

Mapahohopi kte śni.

7 Wiconi mitawa qa wowitan mitawa kin

Wakantanka eciyatanhan;

Imnija suta mitawa qa wowacinye mitawa

Wakantanka en un.

8 Oyate kin, anpetu otoiyohi he wacinyan po;

Itokam nicantepi tpapson po:

-Wowacinye unkitawapi kin Wakantanka hee. Sela.

9 Adam cinca kin takuśni ecedan,

Wicaśta cincapi kin hena woitonśni ;

On tkeutapi kin en wankan yapi ece;

Heñ witaya takupiśni.

10 Wowidagyapi kin wacinyanpi śni po;

Qa wawicalipi on wahanicidapi śni po;

Wowijice icage cinhan cantohnakapi śni po.

11. Wakantanka wancadan ia;

Nonpakiya de nawalion;

Wowaśake he Wakantanka tawa:

12 Nakun Itancan, wocantekiye kin he nitawa;

Tuka otoiyohi iye ohan kin eciyatanhan,

Wicayaqu ece kta.

\section{PSALM 63.}

Dawid tadowan-Juda hewoskan en un qehan.

1 Wakantanka, Taku Wakan mitawa kin he niye; IIanlianna hinca ocide kta;

Niye on minagi ipuza, micelipi cantonikpani ;

Makoce puza en, qa mini codan en mdokiț.

2 Tipi wakan kin ohna wanciyaka qon iyecen, Nitowaśake qa nitowitan kin wanmdake kta.

3 Nitocantelkiye kin wiconi isanpa waśte;

Heon miiha niyatanpi kta.

4 Hecen wani kin en icidowan kta;

Nicaje on nape wahdugate kte.

5 Tacupe qa wasna on iyecen minagi imna kte;

Qa iha wiyuśkin on mii niyatan lrta.

6 Mitowinja akan ciksuye cinhan, Hanyetu osan niye awacicin kta.

7 Omakiye cin he niye;

Unkan niłupahu ohanzi kin en imduskin kta.

o Minagi kiyedan onicihan un;

Ninape etapa sutaya mayuza.

9 Tuka tona minagi ihangya akitapi kin

Hena kun yapi kta, maka cokaya ekta. 
10 Mazasagye nape kin en ehpewicayapi kte; Hena śungidan taku tawa unpi kta.

11 Tuka wicaśtayatapi kin Wakantanka en iyuśkin kta; Tona he cajeyatapi owasin ihdatanpi kta ; Tona itonpiśni kin hena iohmus icupi Ita.

\section{PSALM 64.}

Dowan itancan kin kicagapi. Dawid tadowan.

1 Wakantanka, wocekiye mitawa en miho nation wo; Toka kokipapi kin etanhan wiconi mitawa yapatan kte.

2 Wicaśta śica takodakiciyapi etanhan namayahbe lita; Sicaya econpi oqoyapi kin etanhan.

3 Hena, mazasagye iyecen, ceji hdumanpi ;

Wanhinkpe iyecen, oie pa kin ekatapi;

4 Wicaśta ecetu kin nalimana kutepi kte; Ihnuhanna kutepi. kta, qa kokipapi kte śni.

5. Wicoie śice cin en waśagiçiyapi kta; Nahmana on hmunkapi eonpapi kin he ohdakapi kta; Tuwe wanwicayake kta he, eyapi.

6 Wicohan śica nina akitapi; Wiyeya unkanpi; tanyan yuśtanpi ce:

Unkan malıen wicaśta tawacin qa cante kin temahentu.

7 Tuka Wakantanka ihnuhanna wanhinkpe on wicakute: Hen taopi wicayukan.

8 Hecen naśna wicakiyapi ; iye cejipi kin en wicahdi ; Tona opawicahtapi kin owasin najicapi kta.

9 Heon wicaśta owasin nihinciyapi kta; Qa Wakantanka ohan kin he oyakapi kte; Qa taku econ kin awacinpi kta.

10 Wicaśta owotanna Jehowa en iyuśkin kta, Qa he wacinye kta;

Qa tona cante ecetupi kin owasin witanpi kta.

\section{PSALM 65.}

Dowan itancan kin kicagapi. Psalm odowan. Dawid tawa.

1 Wakantanka, Zion en ainina yatanpi kin he nitawa; Qa taku iwahoyapi kin niçupi kte.

2 Wocekiye nayahon ece kin, Niye ekta wicacehpi owasin u kte.

3 Woahtani wicoie kin iyakapemayanpi ; Taku śica econkupi kin hena niye yakajuju kta.

4 Tuwe yakahnige ca kiyedan ayau kin he cante waśte; Tihocoka nitawa kin en ounye kta;

Yati wowaśte on iunmnanpi kte; Tipi wakan nitawa kin he.

5 Woowotanna en taku wowitonpe on unkayaduptapi kta, Wakantanka Wanikiya unkitawapi; 
Niye maka ihanke kin owasin wacinniyanpi;

Qa tona miniwanca ekta tehan unpi kin.

6 T'owaśake on he kin sutaya ehde;

Wookihi ipiyagkiton kin heon.

7 Miniwanca sa kin yuinina;

Taja sa qa oyate owodutaton kin.

8 Nakun tona ihanke ekta tipi kin

Hena wowape tokeca nitawa kin kokipapi ;

Wiinanpe qa wiiyaya oenape kin hena dowanyakiye kta.

9 Maka kin en yahi qa aminitanyaya:

Waśte hinca yakage kta;

Wakantanka tawakpa kin mini ojudan:

Aguyapi icahwicayeciciye kta;

Wiyeya yakaga nakaeś.

10 Maka yumdupi kin duspaya ;

Cankuyetonton kin boyamdaya;

Magaju on panpanna yakaga;

Taku aicaga ece kin he dawaśte ita.

11 Nitowaśte omaka kin wateśdag yecaton;

Qa nitacanku wasna śbuya.

12 Tinta makoskantu kin śbuya;

Qa pajodan kin wowiyuśkin ipiyagkiton.

13 Tahin optaye kin cincota ogekiton;

Qa omdaye kin aguyapi aihdalipa:

Wowiyuśkin on iyaśapi kta ;

Han, dowanpi kta.

\section{PSALIM 66.}

Dowan itancan kin kicagapi. Woyatan odowan.

1 Maka kin ocowasin,

Wakantanka on iyaśa po.

2 Iye caje wowitan kin idowan miye;

Iye yatanpi wowitan kin qu po.

3 Wakantanka heciya po;

Niolian wowinihan hinca ce;

Nitowaśake tanka on tokaniyanpi kin

Niye en itonpi kte śni :

4. Maka kin ocowasin ohonidapi kta,

Qa inidowanpi kta;

Nicaje kin idowanpi kte. Sela.

5 U miye, Wakantanka ohan kin wanyaka po ;

Wicaśta cinca kin en wowinihanyan ohanyan.

6 Miniwanca kin he puza kaga;

Wakpa kin opta huinyun yapi kta:

Hen iye on unkiyuśkinpi kte.

7 Towaśake on ohinniyan iye wicaśtayatapi ;

Iye iśta Ikcewicaśta kin iwanwicayaka:

Wahanhaniçidapi kin wankan yeiçiyapi kte śni. Sela. 
8 Oyatepi, Wakantanka unkitawapi yawaśte po;

Iye yatanpi ho kin nahon wicakiya miye.

9 Unnagipi wiconi en ehnalka;

$\mathrm{Qa}^{\mathrm{s}}$ unsihapi hicahe kta iyowinkiye śni.

10 Wakantanka, niye iyutanunyayapi ;

Mazaska śdoyapi iyecen unyaduecepidan.

11 On hmunkapi kin en unkayaupi;

Pakśin unkitawapi en wokakije eyahnaka.

$12 \mathrm{~Pa}$ unkitawapi akan wicaśta yanke wicayakiya;

Peta qa mini ehna unhiyupi:

Hehan taku utkanna en inappe unyakiyapi.

13 Peta wośnapi kin yuha yati en hibu kte;

Taku iwahociye.cin hena cicicajuju kta.

14 Miiha hdaotanin qon hena;

Qa makakija en mii taku eye ciqon.

15 Tahinca cepa peta wośnapi waciciyuśna kta, 'Tamdoka wasna kin iyahna:

Tatanka tatokadan kici wamduśna kta. Sela.

16 U miye, tona Wakantanka koyakipapi kin, nahon po;

Unkan taku minagi ecakicon kin he ociciyakapi kte.

17 Mii on hoyewakiya;

Qa woyatan miceji ihukuya un.

18 Micante en woahtani awacanmi kinhan,

Itancan kin nation kte śni.

19 Tuka awicakehan Wakantanka nahon;

Wocekiye mitawa ho kin he anagoptan.

20 Wakantanka yawaśtepi nunwe,

Wocekiye mitawa yutokan iyeye śni,

Qa iye tocantekiye kin emicihdaku śni.

\section{PSALM $6 \%$.}

Candowankiyapi on dowan itancan kin. Woyatan odowan.

1 Wakantanka onśiunkidapi qa unyawaśtepi kte; Qa iye ite on iyounyanpapi nunwe. Sela.

2 Hecen maka akan nitacanku sdonyapi kte; Wiconi nitawa, oyatepi owasin ehna.

3 Wakantanka, oyatepi kin wopida eniciyapi kta; Oyatepi owasin wopida eniciyapi kte.

4 Oyatepi wiyuśkinpi qa iyaśapi kta ; Oyate kin owotanna wicadaco kta heon;

Qa maka akan oyatepi yus awicade kta. Sela.

5 Wakantanka, oyatepi kin wopida eniciyapi kte; Oyatepi iyuhpa wopida eniciyapi kta.

6 Maka kin waskuyeca aicage:

Wakantanka, Wakantanka unkitawapi unyawaśtepi kte:

7 Wakantanka unyuwaśtepi kta;

Qa maka ihanke owasin iye kokipapi kte. 
PSALIM 68.

Dowan itancan kin kicagapi. Woyatan odowan. Dawid tawa.

1 Wakantanka inajin kta;

Tokayapi kin hena enanakiya yapi kta;

Qa tona śicedapi iye itokapatanhan najicapi kta.

2 Sota kalibolia he iyecen wicayakahape kta;

Peta en canśin śdo kin he iyecen,

Wicaśta śica Wakantanka itokam taninpi kte śni.

3 Unkan wicaśta owotanna pidapi kta;

Wakantanka itokam iyaśapi kte ;

Qa wowiyuśkin on iyuśkinpi kte.

4. Wakantanka idowan po, iye caje kin yatan miye;

Tuwe hewoskan śunka wakan akan ye cin he canku kicaga po ; He Jah eciyapi, heon itokam wiyuśkin po.

5 Wamdenica ateyapi, qa wiwazica wayaco tawapi, Wakantanka hee, wowakan oyanke tawa ohna.

6 Tona tanśnana unpi kin hena Wakantanka tipi en owicahnaka ; Tona kaśka yakonpi kin hena wowaśte en hiyu wicakiya ece : Tuka toka kin hena makocc puza en ounyanpi kta.

7 Wakantanka, nitaoyate wicitokam idade ciqon; Hewoskan makoce kin ehna mayani qehan; Sela.

8 Maka kin ślkanśkan;

Han, mahpiya kin śbuyapi, Wakantanka itokam ;

Sina kin de, Wakantanka itokam,

Israel ta Wakantanka kin.

9 Wakantanka, taku ituhanpi kin magajuyaya :

Nitaoyate qa mdokita kin he niye duwaśaka.

10 Optaye nitawa kin hen ounyanpi ;

Wakantanka, nitowaśte on wicaśta onślka taku wicayecamna kta.

11 Itancan kin wicoic kin wicaqu kta:

Tona eyanpahapi kin wicota hinca.

12 Ozuye wicaśtayatapi kin najicapi kta, najicapi kta;

Unkan winohinca moggyanke cin he taku icupi kin pamni kta.

13 Tohan malroce opapun kin en inunkapi kinhan,

Wakiyedan hupahu mazaska apawintapi kin iyenicecapi kta;

Qa śun kin mazaskazi on wiyatpatpa.

14 Hen Iyotan Waśake cin wicaśtayatapi najice wicaye çehan, Zalmon en wapa iyececa.

15 Baśan paha kin he Wakantanka paha tawa;

Baśan paha kin he imnija he kin heca.

16 Paha imnija kin, tokeca aikśinya eyatonwanpi he;

Paha ohna Wakantanka ti kta hdahinge cin hee;

Han, Jehowa ohinniyan en ounye kta.

17 Wakantanka canpahmihma tawa kin

Hena woyawa tanka nonpa, lkektopawingc yuotapi ;

Itancan kin he en owicapa; 
18 Wankan wapa yakiyahda;

Wayaka awicahdapi kin hena ayahdi;

Wicaśta kin elına taku wicaqupi kin iyacu;

Han, tokapi kin ehna;

Jehowa Wakantanka en ounye kte.

19 Anpetu qa anpetu Itancan kin yawaśtepi nunwe:

Waqinunkiyapi, tuka Iyotan Waśake cin niunkiyapi kin hee. Sela.

20 'Taku Wakan kin de wiconi Wakantanka unkitawapi kin hee;

Qa wiconte oenape kin hena Jehowa Itancan tawa.

21 Han, Wakantanka tona tokayapi pa wicakahdehdece kta;

Tuwe woalitani en mani un paha kin.

22 Itancan kin heya;

Baśan etanhan awicawaku kte;

Miniwanca ośbe kin etanhan awicawahdi kta:

23 Hecen nisiha toka we kin en śayapi kta;

Nitaśunke ceji kin he nakun.

24 Wakantanka, iniyopte cin he wanyakapi ;

Mita Wakantanka iyoopte cin;

Wicaśtayatapi mitawa, owanka wakan kin ohna.

25 Tona dowanpi kin hena tokaheya yapi ;

Hektam candowankiyapi kin;

Wicihdukśan wikośka cancega kabupi.

26 Omniciye kin en Wakantanka yawaśte po ;

I tancan kin, Israel minilidoka kin etanhan.

27 Benjamin cistinna kin hen un, ohiwicaya;

Juda itancanpi kin, inyan on wakininpi ;

Zebulon itancanpi; Naphtali itancanpi kin.

28 Nita Wakantanka nitowasake kiconza;

Wakantanka, taku unkoyecihanyanpi kin he yuwaśaka wo.

29 Jerusalem en tipi wakan nitawa kin on,

Wicaśtayatapi kin taku nicahipi kta.

30 Woteca psa ehna unpi kin hena niye iyopewicaya wo;

Wicota waśakapi kin, oyate ptejicadan om;

Mazaska onśpaśpa yuha ahi patujapi kin;

Oyate okicize waśtedapi kin wicayuomdeca wo.

31 Egupta etanhan wicaśta cepa upi kta;

- Kuś Walkantanka ekta nape hdugate kta.

32 Maka wokiconze kin, Wakantanka yatan po ;

Itancan kin idowan miye: Sela.

33 Tuwe malipiya kin akan iyotanka,

Malipiya wanakaja kin;

Inyun, ho hdaotanin, wowaśake ho kin.

34 Wakantanka yawaśaka miye;

Iye towitan Israel akan un,

Qa towaśake kin mahpiya kin ekta.

35 Wakantanka, oyanke wakan nitawa etanhan wowinihan yaun; Israel ta Wakantanka; 
Wowaśake qa wookihi kin oyate wicaqu ece.

Wakantanka yawaśtepi nunwe.

\section{PSALM 69.}

Mnahcalica kịn on dowan itancan kin. Dawid tawa.

1 Wakantarka, nimayan wo;

Mini kin minagi ee kaeś hiyohi, heon.

2 Upśija temahentu mahen imdamde,

Tukten najinpica śni ;

Mini śma kin en wau;

Qa mini kin iwankam imayaya.

3 Waceya on mihduśilitin; dote masaka;

Wakantanka mitawa ape waun kin on iśta mayazan.

4 Tona taku on etanhan śni śicemdapi kin

Hena mapa hin isanpa ota ;

Tona ihangmayanpi kta,

Tokamayanpi itonpiśni kin hena waśakapi ;

Taku iwacu śni kin he wicawecu kta.

5 Wakantanka, wimatkotkoke cin he sdonyaya;

Qa wawahtani kịn hena nanicilimanpi śni.

6 Tona anipepi kin hena miye on iśtecapi śni nunwe ;

Itancan Jehowa Sabaoth:

Tona anicitapi kin hena miye on iśtenwicayapi śni nunwe;

Israel ta Wakantanka kin.

7 Niye on amaiapi waun;

Wowiśtece ite amakalipa.

8 Mihunkawanji ehna togmahdapi waun;

Qa ina cincawicaye cin hena om wicaśta matokeca.

9 Yati cantokpanipi kin he mayutakuni śni;

Qa tona iyopeniyanpi wawiyopeyapi kin he amahinhpaya.

10 Unkan waceya, minagi woteśni un;

Qa he nakun on amaiapi ece.

11 Unkan wakihdaka ogeweton;

Qa heon oweśtepi makagapi.

12 Otonwe tiyopa en iyotankapi kin hena amaiapi ;

Qa tona woyatke suta yatkanpi kin hena dowanpi.

13 Tuka, Jehowa, miye wocekiye eciciya;

Woiyokipi anpetu kin en,

Wakantanka, nitowaonśida untkana on ;

Namahon wo, wiconi nitawa wowicake kin on.

14 Upśija kin etanhan emayaku ye,

Qa mahen imdamde kta iyowinmaye śni ye ;

Tona śicemadapi kin etanhan emayakupi nunwe,

Mini śma kin etanhan.

15 Mini tanka kin aomakahpe śni nunwe;

Qa mini śma kin namapce śni nunwe;

Qa wolia i kin aokibe maye śni nunwe.

16 Jehowa, namahon ye, nitowaonśida waśte kin on; 
Wawacinkta duza tanka ota kin on miye ekta ihduhomni ye.

17 Qa niite nitaokiye nakicihbe śni wo:

Makakija lie on namahon inahini wo.

18 Minagi ikiyedan u wo, he opekiton ye ;

Tokamayanpi kin on etanhan makiyuśka wo.

19 Amaiapi qa iśtenmayanpi qa iyopemayanpi kin, niye sdonyaya;

Tokamayanpi owasin nitokam yakonpi.

20 Waaiapi kin micante kaksa; heon wamayazan;

Unkan onśimadapi kta ape waun, tuka wanica;

Qa cantohnagmayanpi kta, tuka tuwedan iyewaye śni.

21 Nakun wate kta on pizi maqupi ;

Qa imapuza hehan miniskuya yatkemakiyapi.

22 Wicitokam wahna wotapi kin he wohmunke eekiyapi nunwe; Qa tanyan yukanpi kin he mazalitakiyapi kte.

23 Iśta tawapi aotpasyapi nunwe;

Wawanyakapi kte'śni ;

Qa nitepi ohinniyan patuświcakiya wo.

24 Nitocanniye kin awicakaśtan wo ;

Qa wośihda kata nitawa iyahdewicaya nunwe.

25 Hocoka tawapi kin he otiwota ehpeyapi kte;

Qa tawakeyapi ohna tuwedan ti śni nunwe.

26 Tuwe yakaśtake cin he śicaya kuwapi ;

Qa wicayapalidoke iyokiśicapi kin he oyakapi ece.

27 Wahtanipi on woahtani wicaqu wo ;

Qa nitoowotanna kin en upi śni nunwe.

28 Wiconi wowapi kin etanhan wicapajujupi nunwe;

Qa wicaśta owotanna om owicawapi kte śni.

29 Tuka miye iyomakiśice ça makakija ;

Wakantanka, wiconi nitawa wankan emahde kte.

30 Odowan wan on Wakantanka caje kin mdatan kta;

Woyatan dowanpi on indatanka kta.

31 Unkan he Jehowa iyokipi kta,

Tatanka, tatangmdoka he śake ko yuhe cin isanpa.

32 Wicaśta wahbadan he wanyakapi, qa iyuśkinpi kta; Qa Wakantanka ayakitapi kin niye nicantepi ni kte:

33 'Tona onśike cin hena Jehowa nawication;

Qa wicakaśkapi tawa kin hena śice wicadake śni.

34. Mahpiya qa maka kin he yatanpi nunwe;

Miniwanca qa taku obna śkanśkan owasin.

35 Wakantanka Zion nikiye kta;

Qa Juda totonwe kin hena piya kage kta:

Hecen en ounyanpi kta qa tawayapi kta.

36 Nakun iye taokiye cinca kin tawapi kta;

Qa tona iye caje waśtedapi kin hena hen tipi kta.

PSALM \%0.

Dowan itancan kin kicagapi. Dawid tawa. Wokiksuye kta.

1 Wakantanka, emahdaku wo: 
Jehowa, omakiya inahni wo.

2 Tona minagi odepi kin hena token cante yuzapi taninśni qa iśtecapi nunwe ;

Tona śicaya waun kta cinpi kin hena namnipi qa iśtenwicayapi kte.

3 Iśtecapi on namnipi kta;

Tona, Hanhan, hanban, eyapi kin.

4 Tona anicitapi kin owasin iyuśkinpi qa pidapi kte; Qa, Jehowa yatankapi nunwe, ohinniyan eyapi kta;

Tona wiconi nitawa waśtedapi kin hena.

5 Tuka miye makakije ça onmaśika;

Wakantanka en mahi inahini wo;

Tuwe omakiye ca emahdaku kin he niye;

Jehowa yutehan śni ye.

\section{PSALM 71 .}

1 Jehowa niye wacinciya;

Icimana iśtenmayanpi śni nunwe.

2 Nitoowotanna on enayahdaku qa inapemayakiye kta: Anolimakiye ca nimayan wo.

3 Tokin imnija tipi ciciyuhen, ohinniyan en waku kte; Nimakiyapi kta econwicayaśi :

Imnija mitawa qa conkaśke mitawa kin he niye.

4 Mita Wakantanka, wicaśta śica nape etanhan emahdaku wo; Śicaya ohanye ca waliteśni kin napcokaya etanhan.

5 Itancan, wowacinyeciya heon etanhan;

Jehowa, homakśiyopa ehantanhan wacinciya.

6 Wicatamni etauhan niye en imunka; Ina tezi kin etanhan yus amau kin he niye; Niye en ohinniyan mitadowan.

7 Wicota wowinihan mayanpi; Tuka wowinape suta mitawa kin he niye.

8 Niyatanpi kin on mii ojudan kta; Qa, anpetu osan, nitowitan kin on.

9 Wimacahinca kinhan ehpemaye śni ye; Waśake śni amayan en amayuśtan śni wo.

10 Tokamayanpi kin hena amaiapi ; Qa tona minagi iyapepi kin hena witaya wohdakapi;

11 Qa heyapi; Wakantanka ehpeya ce; Kuwa aye ça yuza wo, tuwedan eyaku kte śni.

12 Wakantanka, mitehanyan un śni ye; Mita Wakantanka omakiya inahini wo.

13 'Tona minagi tokayapi kin,

Hena iśtecapi qa atakunipi śni nunwe; Tona śicaya waun kta cinpi kin, Hena waaiapi qa wowiśtece koyakapi nunwe.

14. Tuka miye ohinniyan ape waun kta; Qa niyatanpi kin owasin mdaota kta. 
15 Nitoowotanna kin mii oyake kta; Anpetu osan, wiconi nitawa kin;

Tonakeca sdonwaye śni nakaeś.

16 Jehowa Itancan ohan waśake cin hena en wau kta; Nitoowotanna omdake kta, nitawa ecedan.

17 Wakantanka, homakśiyopa ehantanhan niye onspemayakiya: Unkan dehanyan niohan wakan kin mdaotanin kta.

18 Nakun winiacahinca qa pa maska hehanyan, Wakantanka, amayuśtan śni ye ;

Hecen niisto kin wicoicage kin de owakiyake kta; Nitowaśake, tona u kta owasin en.

19 Nitoowotanna nakun, Wakantanka, tehanwankantu; Taku tanka hinca ecanon ece;

Wakantanka, tuwe iyeniceca he.

20 Niye wokakije ota qa tanka wanyag unyakiyapi:

Yahdicu qa niunyakiyapi kta;

Qa maka ośbe kin etanhan hdicu unyakiyapi kta.

21 Niye mayadutanka kte;

Qa ake cante waśte mayakage kta.

22 Nakun candowankiyapi on ciyatan kta;

Nitowicake on, Wakantanka mitawa:

Israel Taku Wakan tawa, cotanka on ciciyajo kta.

23 Tohan ciciyajo kinhan miiha iyuśkin kta;

Qa minagi opeyakiton kin he nakun.

24 Nakun miceji, anpetu asan, nitoowotanna cajeyate kta:

Tona tanyan waun kte śni cinpi qon,

Hena iśtecapi, hena ite naśapi, heon.

\section{PSALM 72.}

\section{Solomon tawa.}

1 Wakantanka, nitawoyaco wicaśtayatapi kin qu wo; Qa nitoowotanna, wicaśtayatapi cinhintku kin.

2 Woowotanna on nitaoyate wicayukcan kta; Qa onśikapi nitawa kin, woyaco on.

3 Hehan he kin oyate wookiye icahwicakiciye kta; Qa paha kin, woowotanna on.

4 Oyate en tona onśikapi kin on wayaco kta; Wahpanica cinca kin hena niwicaye kta; Qa tehiya wicakuwa kin he kamdemdece kta.

5 Konicipapi kta, tohanyan anpetu wi, Qa hanyetu wi kin yuke kta, Wicoicage qa wicoicage hehanyan.

6 Maga kaśdapi kin amagaju he iyecen hdihe kta ; Magaju maka bospaya kin he iyececa.

7 Iye taanpetu kin en wicaśta owotanna icage kta; Qa wookiye untkanna, hanyetu wi henakeca kta hehanyan.

8 Unkan iye miniwanca etanhan miniwanca ekta wicaśtayatapi kta; 
Qa wakpa kin etanhan maka ihanke kin hehanyan.

9 Iye itokam Ikcewicaśta kin patujapi kta;

Qa tona tolkayapi kin liena maka mdu kin iputakapi kta.

10 Tarśiś wicaśtayatapi qa wita kin

Hena wopida wośna ahiyupi kta;

Śeba qa Seba wicaśtayatapi kin taku itukilianpi kta.

11 Han, wicaśtayatapi owasin iyc itokam makipusdiyapi kta;

Oyatepi owasin wowidagwicaye kta.

12 Wahpanica hoyekiye cin he ehdaku kta;

Qa onśike cin, qa nukun tuwe okiyapi śni.

13 Wicaśta onśika walipanica ko wicakpatan lkta;

Qa wahpanica nagipi kin niwicaye kta.

14 Wohnayan qa wicokicize etanhan nagi opewicakiton $\mathrm{kta}$;

Qa hena wepi kin tehike kta, iye iśta kin en.

15 Unkan ni kta, qa mazaska Śeba etanhan qu kta;

Nakun ohinniyan wocekiye ekiciye kta;

Anpetu osan yawaśte kta.

16 Makoce kin en wamnaheza napohnaka wanjidan eśta, he ipa kin en;

Waskuyeca icage cin Lebanon iyecen kahnahnapi kta:

Unkan otonwe etanhanpi kin maka wato iyecen hica aye lita.

17 Tye caje kin owihanke wanice kta;

Anpetu wi itokam iye caje icage kta :

Unkan iye on wicaśta ihdawaśtepi kta;

Oyate owasin iye yawaśtepi kta.

18 Jehowa Wolrantanka yawaśtepi nunwe;

Israel ta Wakantanka kin;

Iśnana wowinihan wicohan econ ece.

19 Qa iyc cajc wakan lin ohinniyan yawaśtepi nunwe;

Qa iye towakan kin on maka kin ocowasin ojudan kte.

Amen qa amen.

20 Dawid Jesse cinhintku tawocekiye kin henakeca.

\section{PSALM 73.}

Asaph tadowan.

1. Awicakehan Wakantanka Israel wicakiciwaśte;

Tona cante ecepidan kin hena.

2 Tuka miye qe misiha naśdute kta ikeyedan;

Mioye kin kaicucuya hiyeya tuka.

3 Whanhaniçidapi kin inawawizi;

Wicaśta śica wookiye en unpi wanmdake çehan.

4 Hena țapi kinhan wicakaślkapi śni;

Qa wowaśake tawapi kin he cepa.

5 Wicaśta iyotanhan iyekiyapi kin en opapi śni ; Qa wicaśta tokeca om kakijapi śni.

6 Heon etanhan wahanlianiçidapi kin he wanapin kiyapi; Qa wicokicize wokoyake iyecen awicakahpa.

; Cepapi on iśta pajoya unpi; 
Token canteyuzapi ahinanpa ece.

8 Owehanhanpi qa taku śica eyapi ;

Wankantanhan wicokicize cajeyatapi cce.

9 Ipi kin mahpiya iyahdekiyapi ;

Qa cejipi kin maka kin omani un.

10 Heon etanhan iye taoyate dehanyan ahdi;

Qa mini ojudan hdahepapi.

11 Unkan heyapi; Tokiyatanhan Wakantanka sdonye kta he:

Qa Iyotan Wankan kin he wookahinige yuha he.

12 Wanyaka po, dena wicaśta śicapi;

Tuka ohinniyan tanyan unpi,

Sanpa wijica ayapi.

13 Awicakehan ituya micante wahduska;

Qa wiyaonpepicaśni en nape wahdujaja.

14 Nakun anpetu otoiyohi makaśtakapi;

Qa hanhanna eca iyopemayanpi ece.

15 Hecen omdake kta ce, epe cinhan, Iho, nicinca wicoicage kin iyokiśinwicawaye kta.

16 Unkan de okahnih awacanmi qehan, Miiśta kin en tehike :

17 Tipi wakan Wakantanka tawa kin en hibu kinhan, Hehan owiliankepi kin wanmdake kta.

18 Awicakehan canku śduśduta en ewicayahde kta:

Woihangye en ohinhpaye wicayaye.

19 Inyun, ihnuhanna wicotakuniśni en iyolipayapi :

Wanna taninpi śni, wonihinciye on atakunipi śni :

20 Wihamnanpi wan ogungapi elsta be iyececa;

Itancan, yekta kinhan, touncagepi kin śiceyadake kta.

21 Hecen cante śinmiçiya;

Qa mapakśin wakpahdoka:

22 Qa wimatkotkoka qa takudan sdonwaye śni;

Niye en woteca iyemaceca.

23 Tuka miye hinyahin cici waun;

Minape etapa kin yus mayaun.

24 Nitowahokonkiye on yus amayade kta;

Qa iyohakam wowitan iyahdemayaye kta.

25 Mahpiya ekta tuwe mduha he;

Qa maka akan niye kici tuwedan wacin śni.

26 Micehipi qa micante atakuni śni ;

Micante imnija, qa taku ohinniyan mitawa kin Wakantanka hee.

27 Tho, tona nitehanyan unpi kin hena atakunipi kte śni;

Tona ehpeniyan wicin iyayapi kin owasin ihangwicayaya.

28 Tuka miye qe Wakantanka mikiyedan u kin he waśte:

Jehowa Itancan kin wacinwaye :

Niolian owasin omdake kta. 


\section{PSALM 74.}

Waonspekiyapi kta. Asaph tawa.

1 Wakantanka, tokeca ohinniyan waayakta śni he; Tinta nitawa optaye kin en nitocanniye ide kta he.

2 Omniciye nitawa kiksuya wo, Wanakajatanhan opeyaton ;

Taku nitawa cansakadan opeyakiton qon;

Zion Paha kin de en ounyaye cin.

3 Ohinniyan ihangyapi kin ekta caehde nitawa hiyuya wo; Toka taku śica owasin econ, tipi wakan nitawa ohna.

4 Tokaniyanpi nitomniciye kin en hotonpi ece ; Wiyokihedan tawapi kin wiyokihedan ehdepi.

5 Tuwe contanka en onspe yuwankan ikikcu kin He sdonyapi tuka qon.

6 Tuka nakaha ayuco pagopi kin witaya Onspe maziyape ko on kawankapi.

7 Tipi wakan nitawa kin ideyapi ; Nicaje ohna ti qon he makata ehpeyapi.

8 Witaya ihangunyanpi kta ce, cante mahen eyapi ; Makoce kin owancaya Wakantanka tomniciye lkin hulinahyapi.

9 Wowakta wanunhdakapi śni ;

Wicaśta wokcan wanica;

Qa tohanyan kta hecinhan sdonya unkokihipi śni.

10 Wakantanka, tohanyan toka owehanhan kta he; Toka kin ohinniyan nicaje wahiteda kte śni he.

11 Tokeca ninape, han ninape etapa kin, eyahdaku kta he; Nimaku cokaya tanhan ihangwicaya wo.

12 Tuka Wakantanka wanakajatanhan Wicaśtayatapi mitawa ; Makoce cokaya wiconi kaga ece.

13 Niye nitowaśake on miniwanca kin duptuja; Mini akan hogan tankinyanyan pa kin yakaksaksa.

14. Niye unktehi pa kin yakahuhuga; Oyate yutapi kta wicayaqu kta, makoskan unpi kin.

15 Niye miniyowe qa mini tanka kin dukinukan; Wakpa ohinniyan minitan kin dupuza.

16 Anpetu kin nitawa, han, hanyetu kin nitawa; Iyoyanpa qa anpetu wi kin niye yakaga:

17 Niye maka opapun kin owasin eyahnaka; Mdoketu qa waniyetu, hena niye yakaga.

18 De kiksuya wo; toka wan Jehowa yaśica; Qa oyate witkotkoka wan nicaje wowihahaya.

19 Wakiyedan nitawa optaye wotektehdapi kin en wicaqu śni wo; Onśika nitawa optaye kin ohinniyan akiktonje śni wo.

20 Wicotakuye kin he atonwan wo; Maka akan otpaza kin wicakipi ounyanpi kin ojudan.

21 Kiyuśepi kin he iśteca hdicu śni nunwe; 
Wicaśta onśika walipanica ko nicaje yatanpi kta.

22 Wakantanka najin wo; oihdaka wo;

Anpetu osan witkotkoka niyaśice cin he kiksuya wo.

23 Tokaniyanpi ho kin he akiktonje śni wo;

Takpe niupi oqoyapi ohinniyan wankan ye cin.

\section{PSALIM 75.}

Dowan itancan kin kicagapi. Asaph tadowan. Waihangye śni wo. Woahiyaye wan.

1 Wakantanka, wopida unkeniciyapi;

Unkiye wopida unkeyapi ;

Qa nicaje ikiyedan kin he niohan wakan oyakapi.

2 Tohan mniciyapi kta cajemdate cinhan,

Woowotanna on miye wamdaco kta.

3 Maka kin śdo, qa tona akan tipi kin owasin;

Can ipatan tawa kin hena tkeuwata. Sela.

4 'Tona ihdatanpi kin, Ihdatanpi śni po, ewicawakiya ;

Qa wicaśta śica, He hduwankan iyeyapi śni po.

5 He nitawapi hduwankan iyeyapi śni po;

Qa tahu suta hduha iapi śni miye.

6 Wankan yeyapi kin he wiyohiyanpatanhan śni,

Qa wiyohipeyatanhan śni ;

Qa hewoskan makoce kin eciyatanhan śni.

7 Tuka Wakantanka wayaco yanka;

De kun ehpeye kta, qa he wankan iyeye kta.

8 Jehowa nape ohna wiyatke wan;

Qa miniśa ipiga, pejihuta icạhiyapi ;

Unkan hetanhan kaśtan ece;

Ceteta en hiyeye cin henana yaliepapi kta;

Maka akan wicaśta śica owasin yatkanpi lita.

9 'Tuka miye qe ohinniyan womdake kta;

Jakob ta Wakantanka iwadowan kta.

10 Qa wicaśta śica he kin owasin wakaśpe kte:

Tuka wicaśta owotanna he kin wankan icage kta.

\section{PSALM. 76.}

Candowankiyapi on dewan itancan kin kicagapi. Asaph tadowan. Woahiyaye wan,

1 Juda en Wakantanka sdonyaipi;

Israel en iye caje kin tanka linca.

2 Nakun tawokeya Salem en han;

Qa Zion en ounyan ece.

3 Hen itazipe wanhinkpe kin kaweliwega;

Wahacanka qa mazasagye qa okicize kin. Sela.

4 Niye wiyatpa yaun, wakinihan hinca,

$\dot{H}$ e akan wapatapi ece kin isanpa.

5 Tona cante sutapi kin hena taninpi śni;

Woistinma tawa iśtinmapi ;

Qa wicaśta waśakapi tuwedan nape iyekiyapi śni. 
6 Jakob ta Wakantanka, iyopewicayaye cin on, Canpahmihma qa nakun śuktanka iśtinma tapi.

7 Niye niśnana konicipapi kta; Qa tuwe nitokam najin kta he, sinyahda kinhan.

8 Mahpiya eciyatanhan woyaco nahonwicayaya; Maka kin kokipe ca inina yanka;

9 Wakantanka wicayaco najin qehan : Maka akan onsike cin owasin niwicaye kta. Sela.

10 Awicakehan wicaśta śinhdapi kin niyatan kta; Qa śinhdapi ihe cin he ipiyagyeton kta.

11 Jehowa Wakantanka nitawapi taku iwahoyapi qa hdajuju po; Tona ihdukśan yaunpi kin owasin;

Tuwe kokipapi kte cin he taku kahipi kta iyececa.

12 Iye wicaśta itancanpi taniya kin baksa ece:

Maka akan wicaśtayatapi kin kokipapi kta.

\section{PSALM $7 \%$.}

Dowan itancan Jeduthun etanhan. Asaph tadowan.

1 Miho kin Wakantanka elita yewaya, qa cewakiye kta; Miho kin Wakantanka ekta yewaye kta:

Unkan anamagoptan kte.

2 Makakija anpetu kin en Itancan kin awakita; Hanyetu hehan minape yugan un, qa tasaka śni ;

Minagi wakihnapi cin śni.

3 Wakantanka weksuya, qa nihinmiciya; Awacin manka, unkan mitaniya iyokiśica hinca. Sela.

4 Miiśta iśtojuha sutaya yus yaun ; Makaśtakapi, qa ia owakihi śni.

5 Anpetu wanakaja ekta ewacanmi; Wanakaja tanhan waniyetu kin hena.

6 Hanyetu en mitadowan qon he weksuye kta; Micante on awacin waun kta;

Qa mitaniya kin nina wiwanga ece.

7 Ohinniyan Itancan kin warkta kte śni he; Qa ake icimana wacantkiye kte śni he.

8 Towaonśida kin he ohinniyan owihanke he; Wicoicage qa wicoicage hehanyan, oie kin ayuśtan he.

9 Iyotan Waśake cin he waonśida akiktonja; Qa wocanniye on tocantekiye anakipta he. Sela.

10 Unkan hepa; Makakije cin he dee; Iyotan Wankan un nape etapa waniyetu kin.

11 Jah ohian kin mdaotanin kta;

Wanakaja tanhan niohian wakan kin hena weksuye kta.

12 Nakun niolian owasin awacanmi kta; Qa taku ecanon kin hena awacin waun kta.

13 Wakantanka, woowotanna en nitacanku: Wanji waśaka tukte e Wakantanka iyecen tanka he.

14 Iyotan Waśaka wicohian wakan econ kin he niye; 
PSALM 78.

Oyate kin nitowaśake sdonye wicayakjya.

15 Niisto on nitaoyate opewicayakiton;

Jakob qa Joseph cinca kin. Sela.

16 Wakantanka, mini kin wanniyakapi:

Mini kin wanniyakapi ; śkanśkanpi ;

Han, mini śbe cin cancan.

17 Mahpiya śapa kin mini hdaśtanpi;

Malipiya kin hotanin;

Han, nitunwanhinkpe kin kinyan hiyaya.

18 Wakinyan nitawa ho kin tate iyumni en un;

Wakanhdi maka kin iyoyamya;

Maka kin cancan qa śkanśkan.

19 Miniwanca kin en nitacanku;

Qa mini ota ohna cankuyaye;

Hecen nioye kin sdonyapi śni.

20 Optaye wan iyecen nitaoyate yus awicade;

Moses qa Aaron nape kin on.

\section{PSALM $\% 8$.}

Asaph tawa. Waouspekiyapi kta.

1 Mitaoyate, mitoope kin anagoptan po;

Mii oie kin hena anohkiya miye.

2 Wicoie wakan wan on i wahdukawa kta;

Wanakaja tanhan wiyukcanpi lin omdake kta.

3 Hena unkiye naunlionpi qa sdonunyanpi;

Qa ate wicunyanpi unkokiyakapi qon;

4 Hena iye cinca anawicunkihmanpi kte śni,

Wicoicage u kte cin,

Jehowa yatanpi kin owicunkiyakapi kta ;

Qa iye towaśake, qa wicohan wakan econ qon hena.

5 Unkan Jakob en wayuotanin wan ehde;

Qa Israel en woope wan kaga;

Hena hunkake wicunyanpi econ wicaśi,

Iye cincapi owicakiyakapi kta.

6 Hecen wicoicage u kte cin sdonyapi kta;

Wicacinca icagapi kte cin,

Hena najinpi qa iye cinca owicakiyakapi kta.

7 Hecen Wakantanka wacinyanpi kta ;

Qa Iyotan Waśaka ohan kin akiktonjapi kte śni ;

Tuka tawoahope kin opapi kta.

8. Hecen atewicayapi kin iyececapi kte śni;

He wicoicage tahu suta qa wakize sa;

Wicoicage cante hduwiyeye śni;

Qa taniya kin Wakantanka ekta owotanna śni.

9 Ephraim cinca, wanhinkpe wotawe yuhapi kin,

Hena namnipi, kicizapi anpetu kin en.

10 Wakantanka wicotakuye tawa yuhapi śni;

Qa tawoahope eciyatanhan mani wicadapi śni. 
11 Tuka taku econ qon hena akiktonjapi ; Qa iye ohan wakan wanyagwicakiye ciqon.

12 Atewicayapi wicitokam wicohan wakan econ; Fgupta makoce kin en, Zoan tinta kin etu.

13 Miniwanca kin yukinukan qa ohna iyaye wicaya; Qa mini kin maya iyecen najin kiya.

14 Hehan anpetu icunhan malipiya sapa wan on awicaya; Qa hanyetu osan peta iyoyanpa kin on.

15 Hewotahedan makoce kin en imnija kin kaptuja; Qa ośbe tanka etanhan iyecen yatkewicaliya.

16 Hecen imnija wan etanhan mini ihalia kiya; Qa wakpa iyecen mini kin hiyuya.

17 Hececa eśta nakun iye en wahtanipi ; Hewoskan makoce kin en, Iyotan Wankan kin i en hiyeyapi :

18 Qa iye cante en Wakantanka iyutanyanpi ; Wipipi kta on woyute kidapi qehan.

19 Han, Wakantanka aiapi, qa heyapi; Iyotan Waśake cin hewoskan makoce kin en, Wahna wotapi wan wiyeya ehde kta okihi he.

20 Iho, imnija kin apa, unkan mini hiyu; Qa etanhan wakpa wan kaduza:

Nakun aguyapi hiyuya okihi, Qa taoyate kin tado wicaqu kta he.

21. Heon Jehowa nahon qa śinhda:

Unkan Jakob en peta wan ideyapi ;

Qa nakun Israel en wocanniye awicau:

22 Wakantanka wicadapi śni heon etanhan; Qa niwicaye cin he wacinyanpi śni.

23 Unkan wankan mahipiya śapa kin econ śi; Qa mahpiya tiyopa kin hduhdoka;

24 Qa manna yutapi kta e ahinhe wicakiya; Qa mahpiya wamnaheza kin wicaqu.

25 Waśakapi taaguyapi kin wicaśta yutapi; Qa waneya imnahan yuhewicakiya.

26 Malipiya kin ekta wiyohiyanpatanhan tate wan yekiya; Qa iye towaśake on tate itokagatanhan ukiya:

27 Qa maka mdu iyecen conica ahinhewicakiya; Qa taku kinyanpi kin miniwanca wiyaka iyececa.

28 Qa iye tahocoka kin cokaya ahinhpayeya; Tipi kin indukśan hiyeya.

29 Unkan yutapi qa imnanpi hinca ; Taku cinpi qon he wicakahi nakaeś.

30 Hinyanhin taku cinpi qon śicekidapi śni ; Woyute nahanhin i ohnakapi ;

31 Unkan Wakantanka tocanniye kin en wicahi; Qa tona cepapi kin etanhan wicakte; Qa Israel kalinigapi kin patuświcakiya.

32 Hececa eśta nakun wahtanipi; 
Qa wakanyan ohanyan eśta wicadapi śni.

33 Unkan anpetu tawapi kin takuśni en wicakiyusota; Qa waniyetu tawapi, wokakije en.

34 Wicakte qehan, hehan akitapi, Qa hdicupi, qa hanhanna hinca Wakantanka on wiwangapi.

35 Nakun imnija tawapi qon Wakantanka hee e kiksuyapi; Qa Iyotan Waśaka, Iyotan Wankan kin opewicakiton kin hee.

36 Hececa eśta iye ipi on hnayanpi ;

Qa cejipi on oitonpi śni.

37 Qa ekta cante ecetu yuzapi śni; Qa wicotakuye tawa kịn en wicakapi śni.

38 Tuka iye, Waonśida lkin, woah̉tani kajuju, qa ihangwicaye śni ; Nakun ijehan tocanniye anakipta; Qa tośinhda ocowasin hduhice śni.

39 Unkan hena wicacehpi heca liksuya; Tateyanpa wan iyaye ça ake hdi kte śni.

40 Hீewoskan makoce lin ekta i en hiyeyapi ; Qa makoskan jjehan iyokiśinyapi.

41 Nakun namnipi qa Wakantanka iyutanyanpi; Qa Israel Taku Wakan tawapi Wanji aowehanhanpi.

42 Iye nape kin kiksuyapi śni, Anpetu en wokakije etanhan ewicahdaku qon.

43 Egupta en wowakta tawa kin ehnaka; Qa iye ohan wakan kin, Zoan makoce kin en.

44 Nakun wakpa tawapi kin we kaga: Qa mini wakpadan kin yatkan okihipi śni.

45 Tatawamduśka en hiyuya, unkan wicayajipapi; Qa hnaśka, unkan ihangwicayapi.

46 Unkan taku icahyapi kin wamduśkadan wicaqu; Qa wicohtani tawapi kin he psipsicadan qu.

47 Hastanyanka iyuwi yuhapi kin wasu on kte; Qa sakomin can kin, hewanke on.

48 Nakun tawanunyanpi kin wasu en onawicataka; Qa optaye yuhapi kin, peta ide kin en.

49 Iye tocanniye kata en au wicakiya; Wośinhda qa wocanniye qa wonihinciye; Ohnihde śicapi kin hena u wicaśi.

50 Iye tocanniye kin canku wan kiyumdaya; Hena nagipi wiconțe ipiwicada śni; Qa taniyapi makośica en wicaqu.

51 Hecen Egupta en tona tokapa kin owasin awicapa; Ham tawakeyapi ohna towaśakepi tokaheya kin hena.

52 Hehan tahinca optaye iyecen, iye taoyate kin awicahiyu; Qa hewoskan makoce kin en optaye iyecen yus awicaya.

53 Qa tanyan awicaya, unkan wikopapi śni; Tuka tokawicayapi kin hena miniwanca awicakahpa.

54 Hecen opapun wakan tawa kin en awicahi ; Paha kin de en, iye nape etapa ehdaku qon he.

55 Nakun wicitolram oyatepi napewicaya; 
Qa ounyanpi kte cin iyuta wicaqu;

Qa tawakeyapi ohna: Israel wicoun oti wicakiya.

56 Tuka Wakantanka Iyotan Wankan kin

He iyutanyanpi qa śinhdayapi ;

Qa tawoahope kin kiciyuhapi śni.

57 Nakun namnipi, qa hunkake wicayapi iyecen wahnayan ohanyanpi ;

Icunonpa iyayapi, itazipa wahnaye sa iyececa.

58 Qa paha tawapi kin on śinhdayapi ;

Qa wakagapi bagopi kin on nawiziyapi.

59. Wakantanka he nahon, unkan śinhda;

Qa Israel nina śicewicakidake.

60 Hecen Śilo en onnye cin he ayuśtan;

Wakeya wicaśta ehna ti kicage ciqon:

61 Qa iye towaśake kin wayaka ahde kiya;

Qa iye towitan kin toka nape kin en wicaqu.

62 Nakun taoyate mazasagye en onawicataka;

Qa taku tawa hince cin he canniyeya.

63 Wicaśta kahnigapi tawa kin hena peta hulinahwicaya;

Qa wikośkapi tawa kin hena wicayatanpi śni.

64 Wawayuśnapi tawa kin mazasagye on hinhpayapi ;

Qa wiwazicapi kin hena ceyapi śni.

65 Hehan, wicaśta iśtinma se iyecen, Itancan kin kikta;

Wicaśta waśaka miniśa on iyaśa kin iyececa.

66 Qa tokayapi kin lieyata elipewicaya;

Wowiśtece owihanke kte śni wicaqu.

67 Nakun Joseph tawakeya kin akta śni ; Qa Ephraim tawicoun kin kalinige śni.

68 Tuka Juda tawicoun kin he hdaliniga;

Zion Paha kin de waśtedake ciqon.

69 Qa tipi wakan tawa kin tewankantuya kaga;

Maka kin iyecen owihankes̉niyan ekihde.

70 Nakun iye taokiye Dawid kahiniga;

Qa tahinca tipi kin etanhan ikikcu;

71 Tawiye azinpi kin wicihektapatanhan ehdaku;

Jakob iye taoyate kin wonwicaye kta;

Qa Israel iye tawa hince cin.

72 Unkan iye cante ecetu kin on wonwicaya;

Nakun iye nape wayupike cin on yus awicaye kta.

\section{PSALM 79.}

Asaph tadowan.

1 Wakantanka, Ikcewicaśta makoce nitawa kin en hipi;

Tipi wakan nitawa kin he yuśapapi;

Jerusalem paha seksen elipeyapi.

2 Mahpiya okinyanpi kin yutapi kta e

Nitaokiye tancanpi lin wicaqupi;

Wicaśta wacantkiye nitawa cehpi kin maka wamanica wicaqupi. 
PSAIM 80.

3 Jerusalem ihdukśan mini iyecen we papsonpi;

Qa tuwedan wicalie śni.

4 Tona ikiyedan unyakonpi kin hena unkaiapi :

Unkihdukśanpi kin hena pomnamnaunkiyapi qa wowihahaunyanpi.

5 Jehowa, tohanyan kta he;

Ohinniyan canniniye kta he;

Winayawizi kin he peta iyecen ide kta he.

6 Oyatepi sdonniyanpi śni kin

Hena nitocanniye awicakaśtan wo:

Qa wokiconze nicaje hoyekiyapi śni kin hena.

7 Jakob wanna temyapi ;

Qa tamaga kin ihangyapi.

8 Unkitokam unpi wahtanipi qon unkiyepi en kiksuye śni wo; Inalini wo, wacanyakiye cin he itkounkipapi nunwe;

Nina iyotanhan iyeunkiyapi, heon etanhan.

9 Wakantanka niunkiyapi kin, ounkiya po ; .

Nicaje wowitan kin heon etanhan;

Nakun unkiyukea po, qa waunhtanipi kin kajuju wo;

Niye nicaje kin on etanhan.

10 'Taku Wakan tawapi kin toki un he,

Tokeca Ikcewicaśta eyapi kta he :

Nitaokiye we papsonpi tokiconpi kin he,

Unkiśtapi itokam, Ikcewicaśta sdonyapi kte.

11 Kaśka unpi comnihdazipi kin.he nitokam u nunwe ;

Niisto tanka kin eciyatanhan,

Tona tapi kte cin hena niwicaya wo.

12 Itancan, tona ikiyedan unyakonpi,

Wowihaha on iniliahapi kin he,

Iye maku kin ohna, śakowin akihde wicakicu wo.

13 Hecen unkiye, nitaoyate qa tahinca wonwicayaye cin,

Ohinniyan wopida unkeniciyapi kta;

Wicoicage qa wicoicage en niyatanpi kin owicunkiyakapi kta.

\section{PSALM 80.}

Asaph tadowan. Mnalicalica on dowan itancan kin kicagapi. Wayaotaninpi kta.

1 Israel wonwicaye cin, anagoptan ye;

Optaye wan iyecen Joseph yus ade cin;

Kerubim ehna idotanke, iyoyanpa wo.

2 Ephraim qa Benjamin qa Manasse itokam,

Nitowaśake hduhica wo ;

Qa niunkiyapi kta $\mathrm{u}$ wo.

3 Wakantanka, piya kiyotang unkiya po; Qa niite hduwiyakpa, qa niunkiya miye.

4 Jehowa, Wakantanka Sabaoth,

Tohanyan nitaoyate cekiyapi kin izinyaye kta he.

5 Iśtamnihanpe aguyapi yunwicayakiya;

Qa iśtamnihanpe ota hinca yatke wicayakiya. 
6 Oyate ikiyedan unyakonpi kin en woakinica unyakagapi; Qa tokaunyanpi kin iye iyakitedan iliapi.

7 Wakantanka Sabroth, Piya kiyotang unkiya po;

Qa niite hduwiyakpa, qa niunkiya miye.

8 Ifgupta etanhan hastanyanka iyuwi wan ayahdiyaku: Ikcewicaśta kin hieyata elipewicayaye ça he huyahde.

9 Itokam wayakahinta ;

Unkan hutkan icage ca makoce ojudan.

10 Ohanzi kin he paha kin akahpa: Qa adetka, hante śa Wakantanka tawa kin iyececa.

11 Adetka kin miniwanca kin iyahde; Qa hutkan, wakpa kin ekta.

12 Tokeca conkaśke kin dujuju he: Hecen tona en hiyayapi owasin yuksaksapi.

13 Taśkoju etanhan kukuśe mdoka kin yatakuni śni; Qa tinta wamanica kin etanhan yutapi ece.

14 Wakantanka Sabaoth, tokin yahdicun; Mahpiya eciyatanhan ahitonwan, qa wanyaka, Qa hastanyanka iyuwi kin de en hdi wo:

15 Qa nape nietapa huhde qon he hdusuta wo; Qa cinca niye icahyakiye cin he.

16 Peta on hulinaga ; kaksapi ; Niite iyopewicaye cin on atakunipi śni.

17 Nietapa wicaśta kin akan ninape un nunwe; Adam cinhintku niye yahduwaśake cin.

18 Hehan unkiye ehpeunṇiyanpi kte śni:

Niye niunyakiyapi kta, Unkan nicaje hoyeunkiyapi kta.

19 Jehowa, Wakantanka Sabaoth, Piya kiyotang unkiya po ;

Niite hduwiyakpa, qa niunkiya miye.

\section{PSALM 81.}

Asaph tawa. Gittith on dowan itancan kin kicagapi.

1 Wakantanka wowaśake unkitawapi kin Taninyan idowan miye ;

Jakob ta Wakantanka iyuśkinyan kahiyaya po.

2 Odowan ahiyaya po, qa cancega apa po;

Candowankiyapi waśte cotanka kici.

3 Wi teca kin en, mazayahotonpi yajo po ;

Wi mibe cin en, wounhanpi anpetu kin en.

4 Israel en woahope wanji dee; Jakob ta Wakantanka toope kin.

5 Joseph en wayuotannin kin de kaga; Eigupta makoce etanhan hinanpe cin he ehan: Tapi wan owakahinige śni kin he nawahon.

6 Waqinpi kin etanhan tapete eweciyaku; 
Iye nape wakiśkokpa kin ayuśtanpi.

7 Wokakije en yapan, unkan ecihdaku;

Nakun wakinyan hoton oinatibe etanhan aciyupta:

Woakinica mini kin ekta iyutanciya. Sela.

8 Mitaoyate, nahon wo, kinhan wahokonciciye kta;

Israel, anamayagoptan kta hecinhan :

9 Taku wakan tokeca niyepi en un kte śni;

Qa ihnuhan oyate tokeca taku wakan

'Tawa kin en makata ehpeniçiye cinhan.

10 Jehowa Taku Wakan nitawa,

Egupta makoce etanhan uniye cin he miye:

Nina i hdukawa wo, kinhan ojuwaye kta ce.

11 Tuka mitaoyate miho kin anagoptan śni ;

Qa Israel wicamicida śni.

12 Hecen iye cante hdusutapi kin en ehpewicawaya:

Iye tawacinpi kin en omanipi kta.

13 Tokin mitaoyate anamagoptanpi kinhan;

Qa Israel mitacanku ohna manipi kta:

14 Ecadan tokawicayapi kin patuś wicawaye kta; Qa tona śicewicadapi kin minape awicawaknake kta tuka.

15 Jehowa śicedakapi kin hena iye en ihdaśkapi kta: Tuka iye anpetu tawapi kin owihanke wanice kta.

16 Nakun aguyapi conica kin yunkiye kta: Qa imnija etanhan tulimaga canhanpi on imnaciye kta.

\section{PSALM 82.}

\section{Asaph tadowan.}

1 Iyotan Waśaka tomniciye kin en Wakantanka najin; Taku wakan kin wicehna wayaco yanka.

2 Tohanyan ecinśniyan wadacopi kta he; Qa wicaśta śica itepi kin yakinihanpi kta. Sela.

3 Onśika qa wamdenica kin hena wicakiciyukcan po; 'Tuwe kakije ca wahpanica kin owotanna ecawicakicon miye.

4 Onśika qa walipanica kin hdinape wicaya po;

Wicaśta śica nape kin etanhan ehdaku miye.

5 Sdonyapi śni qa okahinili wacinpi śni;

Otpaza en manipi kte hinca:

Maka oahde kin owasin yuhohopi.

6 Taku wakan henicapi ce, miye epa;

Owasin Iyotan Wankan un cinca kin niyepi.

7 'Tuka awicakehan wicaśta iyecen nitapi kta ;

Qa wicaśta itancanpi wanji iyecen nihinhipayapi kte.

8 Wakantanka inajin wo, maka kin hdaco wo: Oyate jyulpa tawawicaye kte cin he niye. 


\section{PSALM 83.}

Psalm odowan. Asaph tawa.

1 Wakantanka, inina yaun śni nunwe: Iyotan Waśaka, inina yanke śni qa owanji yanke śni wo.

2 Iho, tokaniyanpi kin hena hotonpi; Qa śicenidapi kin hena pa yuwankan ikikcupi.

3 Nitaoyate kin śicaya awicaiapi; Qa nawicayakihbe cin hena on wohdakapi ece.

4 Hena heyapi; Upo, oyate kin he ihangwicunyanpi kte; Qa Israel caje kin icimana kiksuyapi lrte śni ce.

5 Cante eciyatanhan witaya awacinpi; Niye on wicotakuye wan hduśtanpi.

6 Edom tawakeyapi, qa Iśmaeli kin; Moab, qa Hagari kin hena:

7 Gebal, qa Ammon, qa Amalel ; Philistia, Turos en ounyanpi lin hena om :

8 Nakun Assur hena owicapa: Hena Lot cinca isto owicaqupi. Sela.

9 Midian en iyecen ecawicakicon wo; Sisera iyececa, Jabin iyececa, Kiśon walspadan en.

10 Endor ekta ihangwicayapi qon: Maga akan tacesdi iyececa icagapi.

11 Alicita tancanpi kin Oreb qa Zeeb iyecen wicakaga wo; Han, wicaśta itancanpi owasin, Zeba qa Zalmunna iyececapi.

12 Hena heyapi; Wakantanka tamaga kin Hena unkiye tawaunyanpi kta ce.

13 Wakantanka mitawa, Hena tate iyumni iyececa wicakaga wo; Tateyanpa itokam aguyapi lia kin iyececa :

14 Peta contanka wan hulinage cin iyececa; Qa ide wan he aide kin iyececa:

15 Hecen tateyanpa nitawa on kuwa awicade kta; Qa tate iyumni nitawa on nihinciye wicayaye lita;

16 Ttepi kin wowiśtece on ojuwicaya wo : Unkan, Jehowa, nicaje wicaśta akitapi kte.

17 Iśtecapi kta, qa nihinciyapi kta, owihanke wanica; Qa ite naśapi kta, qa atakunipi kte śni.

18 Unkan niye Jehowa eniciyapi kin sdonyapi kta; Niśnana Iyotan Wankan yaun, maka kin owancaya.

\section{PSALM 84.}

Gittith on dowan itancan kin kicagapi. Qora cinca tadowanpi.

1 Jehowa Sabaoth, Nitawakeya kin nina wiciyolipi.

2 Jehowa tahocoka kin on, Minagi cantokpani qa nakun niya śni te kta seca: 
Micante qa micehpi Taku Wakan ni un kin hoyekiya ece.

3 Upijate hee kaeś tipi wan iyekiya,

Qa icapśinpśincadan holipi wan,

En cinca elrihnake kta;

Jehowa Sabaoth, owayuśna nitawa kin hena,

Wicaśtayatapi mitawa, qa mita Wakantanka.

4 Yati en ounyanpi kin hena cante waśtepi ;

Hena ohinniyan niyatanpi kta. Sela.

5 Wicaśta tuwe towaśake niye en un kin he cante waśte:

Canku tankapi kin iye cantepi ohna wanka.

6 Iśtamnihanpe Kaksiza ohna yapi kinhan,

He miniyowe kagapi;

Nakun magaju kin woyawaśte on akahipa.

7 Wowaśake etanhan wowaśake ekta yapi kta:

Zion en Wakantanka itokam ihdutanin kta.

8 Jehowa, Wakantanka Sabaoth,

Wocekiye mitawa nahon wo ;

Jakob ta Wakantanka, anagoptan ye. Sela.

9 Wakantanka, wahacanka unkitawapi wanyaka wo;

Qa Sdayakiye ite kin atonwan wo.

10 Nitahocoka ohna anpetu wanjidan keś

Keltopawinge isanpa waśte:

Mita Wakantanka ti tiyopa kin en

Imunke kta wakahiniga:

Qa wicowicaśtaśni wakeya kin ohna wati kte śni.

11 Jehowa Wakantanka anpetu wi qa wahacanka kin heca;

Jehowa wowaonśida wowitan ko wicaqu kta;

Tona ecetuya manipi kin taku waśte ipiwicada kte śni.

12 Jehowa Sabaoth,

Wicaśta wacinniye cin he cante waśte.

\section{PSALM 85.}

Dowan itancan kin kicagapi. Qora cinca tadowanpi.

1 Jehowa, makoce nitawa canteyakiya; Jakob wayaka unpi kin hdicu wicayakiya.

2 Nitaoyate taku śica econpi kin yecicajuju; Wahtanipi kin owasin ayalkahpa. Sela.

3 Śinyahda kin ocowasin anayecipta; Nitocanniye kin ide śni yakiya.

4 Unkiyepi en hdicu wo, Wakantanka. Wanikiya unkitawapi; Qa canniyeunyakiyapi kin anakipta wo.

5 Owihanke wanin canniyeunyayapi kta lie; Nitocanniye wicoicage qa wicoicage ekta iyahdeyaye kta he.

6 Ake yahdicu qa niunyaki yapi kte śni he : Kinhan, nitaokiye iniyuśkinpi kta.

7 Jehowa, nitowaonśida wanyagunkiya po:

Qa nitowiconi kin unyaqupi kte.

8 Jehowa Iyotan Waśake cin he taku eye cin he nawahon kta; 
Iye taoyate qa wicaśta wacantkiye tawa kin wookiye on owicakiye kta :

Tuka ihnuhan wowitkotkoke cin ekta ihdoyapi kinhan.

9 Tona kokipapi kin henana wiconi tawa wicikiyedan un;

Hecen makoce unkitawapi ohna wowitan ounye kta.

10 Wowaonśida qa wowicake itkokicipapi : *

Woowotanna qa wookiye i ikiciputakapi.

11 Wowicake maka kin etanhan icaga;

Qa woowotanna mahpiya eciyatanhan kun ahitonwan.

12 Nakun Jehowa taku waśte kin wicaqu kta;

Qa makoce unkitawapi taku icaliye kta.

13 Woowotanna iye itokam mani kta;

Qa iye oye kin ohna aunhdepi kte.

\section{PSALM 86.}

Dawid tawocekiye.

1 Jehowa, ninoge ukiye ca amayupta wo;

Makakije ça onmaśika, heon.

2 Minagi awanyaka ye, wacantkiye wanji he miye:

Nitaokiye niliya wo, Wakantanka mitawa he niye;

Wacinniye cin heon.

3 Itancan, onśimakida wo ;

Anpetu osan hoyeciciye kta heon.

4 Nitaokiye nagi kin iyuśli inkiya wo;

Itancan, niye ekta minagi wahdomda.

5 Niye, Itancan, niwaśte qa wayakajuju ece;

Qa tona hoyeniciyapi kin owasin,

Wocantekiye utkanna wicayeciyuha.

6 Jehowa, wocekiye mitawa nahon ye ;

Qa cewakiya ho kin he anagoptan wo.

7 Anpetu en makakije cinhan houciciye kta :

Unkan niye amayadupte lita.

8 Itancan, taku wakan ehna tuwedan iyenicece śni;

Qa takudan niohan iyecece śni.

9 Itancan, oyate wicayakage cin owasin,

Hena nitokam upi qa ohodapi kta;

Qa nicaje yaonihanpi kta.

10 Niye nitanka qa wowapetokeca ecanon;

Niśnana Wakannitanka.

11 Jehowa, nitacanku onspemakiya ye;

Nitowicake ohna mawani kta;

Micante nicaje kokipe kta en iyakaśka wo.

12 Itancan, Wakantanka mitawa,

Micante ocowasin on, wopida eciciye kta;

Qa nicaje mdaonihan kta, owihanke wanica.

13 Miye en nitowaonśida kin tanka;

Qa.Hades temahentu kin etanhan minagi edaku.

14 Wakantanka, wahanlianiçidapi kin takpe maupi; 


\section{P S A M 88.}

Qa wicaśta wohitika omniciye kin minagi akitapi;

Qa wicitokam niye enihdepi śni.

15 Tuka Itancan,

Taku Wakan waonśida qa wacantkiya kin he niye,

Wawacinkta yuza tanka,

Qa wowaonśida wowicake ko on winijice.

16 En mahdiyaku qa onśimakida wo;

Nitowaśake kin nitaokiye qu wo;

Qa nitaokiye win cinhintku kin nikiya wo.

17 Wowakta waśte wan makipazo wo;

Hehan tona śicemadapi wanyakapi qa iśtecapi $\mathrm{kta}$ :

Jehowa, niye omayakiye ca wacintonhnag mayaye cin heon.

\section{PSALM $8 \%$.}

Qora cinca tawapi. Psalm odowan.

1 Oahde tawa kin wowakan paha kin en un.

2 Zion tatiyopa kin hena. Jehowa waśtekidaka; Jakob en ounyanpi kin owasin isanpa.

3 Niye en wicoie wakan kin oyakapi, Wakantanka totonwe kin. Sela.

4 Rahab qa Babel sdonmayanpi kin he omdake kta; Iho, Philistia qa Turos Kuś kici ;

De hen teca ni en tonpi ce.

5 Qa Zion on heyapi kta;

Wicaśta kin de qa kae hen tonpi ce:

Unkan iye Iyotan Wankan kin hdusuta kte.

6 Jehowa oyate owicawa kinhan wicayawa kta;

De en teca ni en tonpi ce. Sela.

7 Unkan tona dowanpi, tona yajopi ko;

Minihdoka mitawa owasin niye en un ce, eyapi j.ta.

\section{PSALM 88.}

Psalm odowan. Qora cinca tawapi. Dowan itancan kin hicogapi. Wowayazan kakiśye cin on. Waonspekiyapi lzta. Heman Ezrahi he kaga.

1 Jehowa, Wakantanka Wanikiya mitawa, Nitokam anpetu hanyetu ko hoyewaye.

2 Wocekiye mitawa nitokam u nunwe;

Hoyewaye cin he anolikiya wo.

3 Wokakije minagi imnahan un; Qa mitaniya Hades ikiyedan ayapi.

4 Woha ek ta iyayapi kin opeya mayawapi; Wicaśta wowaśake nice cin he iyemaceca.

5 Tapi kin opeya makiyuśkapi,

Wicaktepi wicahapi en wankapi kin iyececa,

Hena owihanke wanica wicayelssuye śni :

Qa hena ninape etanhan ehpewicayapi.

6 Wolia wan temahentu kin en omayahnaka; 
Otpaza tipi kin en, ośbe kin en.

7 Nitocanniye tkeya iyamahdaskica; Qa taja nitawa owasin on kakiśmayaya. Sela.

8 Tona sdonmayanpi kin hena mitehanyan iyeyaya;

Hena wahitemadapi śni yakaga;

Onamatakapi, qa tankan waku owakihi śni.

9 Wokakije on miiśta atakuniśni ;

Jehowa, anpetu otoiyohi houciciye ;

Minape ciciyugata ece.

10 Tapi kin hena wicolian wakan ecawicayecon $k$ ta he ; Wanagi hdinanpapi qa wopida eniciyapi kta he. Sela.

11 Wicahapi en nitowaonśida oyakapi kta he;

Nitowicake kin wicotakuniśni en.

12 Wicohan wakan nitawa otpaza en sdonyapi kta he;

Qa nitoowotanna, woakiktonje makoce kin en.

13 Tuka miye, Jehowa, hoyeciciya;

Qa hanhanna eca mitawocekiye nitokam u kte.

14 Jehowa, tokeca minagi ayakta kte śni;

Niite anamayakihbe kta he.

15. Homakśiyopa ehantanhan makakije ça mațe kta seca;

Wonihinciye nitawa qin waun;

Token cante mduza tanin śni.

16 Nitocanniye miwankam iyaya;

Wonihinciye nitawa ihangmayanpi :

17 Mini iyececa, anpetu osan, aohdutemayanpi ;

Witaya ohomni imayayapi.

18 Waśtemadake c̣a mitakuye kin mitehanyan iyewicáyaya;

Tona sdonmayanpi qon hena otpaza.

\section{PSALIM 89.}

Waonspekiyapi kta. Ethan Ezrahi kin he tawa.

I Jehowa towaonśida kin ohinniyan iwadowan kta;

Wicoicage qa wicoicage hehanyan,

Mii on nitowicake omdake kta.

2 Wowaonśida ohinniyan icaliyapi lita ce, epe ciqon: Mahpiya kin hena en nitowicake eyehde kta.

3 Wanji wakahinige cin he kici wicotakuye wakaga; Dawid mitaokiye kici iapi weciyasuta:

4 Owihanke wanica hehanyan nicinca sutaya ewahde kta; Qa wicoicage owasin hehanyan oiyotanke nitawa icaliwaye kta. Sela.

5 Unkan, Jehowa, mahpiya kin niohan wakan oyakapi kta; Nakun nitowicake, oyate wakan mniciyapi kin en.

6 Tuwe mahpiya kin ekta Jehowa iyececa he; Waśakapi cinca kin Jehowa iyacinpica he.

7 Oyate wakan onahbeya mniciyapi kin en, Taku Wakan nina kokipapi kta iyececa; Qa tona ihdukśan unpi kin isanpa kinihanpi kte. 
8 Jehowa, Wakantanka Sabaoth, Jah Iyotan Waśaka, tuwe iyeniceca he; Qa nitowicake nihdukśan un kin.

9 Miniwanca wankan ye cin he wowidagyaya;

Taja wankan aya eca niye amdakedan yakaga ece.

10 Wicaktepi iyecen Rahab yakaśuśuja;

Niisto waśaka on tokaniyanpi kin wicaduomdeca.

11 Mahpiya kin he niye nitawa,

Nakun maka kin nitawa;

Makoce owasin qa woojudan kin

Hena niye yakaga.

12 Waziyata qa itokaga, hena niye yakaga;

'Tabor qa Hermon nicaje on iyuśkinpi kta.

13 Isto waśaka wan niyukan;

Ninape waśaka, nape enitapa lkin tehanwankantu.

14. Woowotanna qa woyaco oiyotanke nitawa oyanke kin hee; Wowaonśida qa wowicake niite itokam yapi kta.

15 Oyate wotanin waśte sdonye cin hena cante waśtepi; Jehowa, niite iyoyanpa kin en omanipi kta.

16 Anpetu osan nicaje on iyuśkinpi kta; Qa nitoowotanna on wankan ihdoyapi kta.

17 Iye towaśakepi wowitan kin he niye, - Qa nitoiyokipi kin en he wankan iyeunyeciciyapi kta.

18 Wahacanka unkitawapi kin he Jehowa tawa; Qa wicaśtayatapi unkitawapi kin he Israel Wakandapi kin he tawa.

19 Hehan wanji wacantkiye nitawa woyuotanin en oyakiya, Qa heha; Wanji Waśake cin akan wowaśake ewehnaka; Wanji oyate etanhan kalinigapi kin wankan ewehde:

20 Dawid mitaokiye kin iyewaye;

Wihdi wakan mitawa on sdawakiya:

21 Minape ohinniyan kici un kta;

Nakun miisto yuwaśake kta.

22 Tokapi kin iyokiśinyapi lite śni ; Qa woahitani cincapi kakiśyapi kte śni.

23 Unkan iye itokam tona tokayapi kin wicawakaśuśuje kta ; Qa tona śicedapi kin awicawape kta.

24 Nakun mitowicake qa mitowaonśida kici un kta; Qa micaje on iye he kin wankan icage kta.

25 Qa iye nape kin miniwanca en ewahnake kta; Qa nape etapa kin mini tanka kin en.

26 Iye hoyemakiye kta; Atewaye cin he niye; Mita Wakantanka, qa imnija nimaye cin.

27 Unkan miye he micinca tokapa wakiye kta; Maka wicaśtayatapi kin isanpa wankan un kta.

28 Ohinniyan mitowaonśida weciyuhe kta ; Qa wicotakuye mitawa kici wicake kta.

29 Nakun iye cinca owihanke wanica mdusuta kta; Qa oiyotanke tawa kin mahpiya anpetu kin iyecece kta. 
30 Iye cinca kin mitoope elipeyapi kinhan; Qa mitawoyaco ohna mani wacinpi śni ;

31 Taku omdake cin taku ikceka yawapi ;

Qa mitawoahope opapi śni kinhan:

32 Hehan taku śica econpi kin, icapsinte wan on, Qa walitanipi kin, apapi on iyopewicawaye kta:

33 Tuka mitowaonśida ewecihdaku kte śni ; Qa wowicake mitawa kin en itonśni miçicage kte śni.

34. Wicotakuye mitawa taku ilkceka wakage kte śni; Qa miiha taku enapeye cin mdatokeca kte śni.

35 Taku wanji mitowakan lkin on wakonza ce ; Dawid en itonśni owakihi śni :

36 Iye cinca kin owihanke wanice kta; Qa oiyotanke tawa anpetu wi mitokam iyecece lita.

37 Ohinniyan sutaya yanke kta, hanyetu wi iyececa; Qa mahpiya ekta wayuotanin wicaka. Sela.

38 Hececa eśta niye ehpeyaye ça ayakta śni ;

- Sdayalkiye ciqon he canniyeyaya:

39 Nitaokiye wicotakuye tawa yecalssa; Wateśdake tawa kin makata ehpeyaya:

40 Inatake tawa owasin dujuju; Conkaśke tawa kin woihangye yakaga:

41 Tona canku ohna hiyayapi owasin he ihangyapi ; Tona ikiyedan unpi kin hena wahtedapi śni:

42 Tona śicedakapi nape etapa kin yuwankan iyeyaya; Tona tokayapi owasin iyuśkinwicayaya.

43 Nakun mazasagye tawa kin pe śni yakaga; Qa okicize en kici nayajin śni.

44 Toiyege kin ayuśtanyakiya; Qa oiyotanke tawa kin makata ehpeyaya.

45 Kośka taanpetu kin duptecedan; Wowiśtece on akahipeyeton. Sela.

46 Tohanyan kta; Jehowa, ohinniyan nayakilibe kta he; Peta iyecen nitocanniye ide kta he.

47 Miye ptenyedan wani kin he kiksuya wo; Tokeca Adam cinca owasin ituh yakaga he.

48 Wicaśta tuwe ni un qa wiconte wanyalke kte śni; Hades nape etanhan nagi eihdaku kta he. Sela.

49 Itancan, otolkahe ekta nitowaonśida kin tukte e he; Nitowicake en Dawid wahoyaye ciqon.

50 Itancan, nitaokiye $i$ en hdepi kin he kiksuya wo; Miikpi ohna oyate ota owasin wicamduha:

51 Jehowa, tokaniyanpi i en iyeyapi gon he; On Sdayakiya oye kin i en jyeyapi qon.

52 Jehowa owihanke wanin yawaśtepi nunwe. Amen qa amen. 
PSALM 90.

Wocekiye wan, Moses Wakantanka tawicaḱta tawa.

1 Itancan, niye otiunniyanpi,

Wicoicage qa wicoicage kin en.

2 He kin icage śni he itolkam;

Qa maka maga ko yakage śni he ehan;

Otokahe wanica qa owihanke wanica,

Iyotan Waśaka kin he niye.

3 Maka mdu ee kaeś ekta wiçaśta hdicuyaya;

Qa, Adam cinca hdicu miye, eha ece.

4 Waniyetu kektopawinge, niiśta kin en,

Htanihan wanna henakeca he iyececa;

Qa hanyetu en akicita onajin kin iyececa.

5 Okahibog iyewicayaya;

Woiśtinma wan iyececapi ;

Hanhianna eca, wato iyecen, taninpi śni.

6 Hanhanna eca hica aya, qa hehan tanin śni ;

Hitayetu hehan kaśdapi qa śnija.

7 Nitocanniye kin on unsotapi ;

Qa śinyahda kin on lropeunhdapi ece.

8 Waunhtanipi kin nitokam eyahnaka;

Woanahbe unkitawapi kin niite iyoyanpa kin en.

9 Anpetu unkitawapi owasin nitocanniye en iyaya;

Waniyetu unkitawapi wicotawacin wan iyecen enaunkiyapi.

10 Waniyetu unkitawapi anpetu kin

Hena waniyetu wikcemna śakowin,

Qa wowaśake yuke cinhan, waniyetu wikcemna śahdogan;

Hececa eśta wowaśake kin he wokakije qa wicośice;

Thu uhanna atakuni śni, unkan kinyan unkiyayapi.

11 Nitocanniye wowaśake kin tuwe okaliniga he;

Qa śinyahda kin he konicipapi kin iyececa.

12 Hecen anpetu unkitawapi hdawa onspeunkiya po;

Kinhan woksape ekta cante yeunkiyapi kta.

13 Jehowa hdicu wo; tohanyan kta he :

Qa nitaokiyepi on tawacin hdutokeca wo.

14 Hanhanna eca nitowaonśida on imnaunyan po ;

Kinhan unkiyuśkinpi qa piundapi kta,

Anpetu unkitawapi owasin en.

15 Anpetu en kakiśunyanpi kin he iyecen-wiyuśkinunkiya miye ;

Waniyetu en taku śica wanunyakapi iyececa.

16 Niohan kin nitaokiye wanyagwicaya wo;

Qa iye cinca kin, nitowitan kin.

17 Qa Jehowa Wakantanka unkitawapi

Towaśte kin unkicipi un nunwe;

Qa unnapepi ohan kin unkiciyusuta miye ;

Han, unnapepi ohan kin yuecetu wo. 
PSALM 91.

1 Iyotan Wankan un toinahbe kin en, Tuwe iyotanke cin he, Iyotan Waśaka ohanzi tawa ihukuya iwanke kta.

2 Jehowa hewakiye kta; Wowinape mitawa, qa conkaśke mitawa, Mita Wakantanka, he wacinwaye kta ce.

3 Wahmung ye cin wohmunke tawa kin Etanhan, iye eniyaku kta; Makośica waihangye cin etanhan.

4 Iye sun kin on akahpenicaton kta ; Qa tahupahu ihukuya wowinape iyeyaye kta; Towicake kin wahacanka qa maza makuakalipe.

5 Hanyetu wokokipe kin he koyakipe kte śni; Qa anpetu en wanhinkpe kinyan iyaye cin ;

6 Makośica otpaza en omani un ; Qa wowayazan wiyotanhan eca wayutakuniśni kin.

7 Nicahda kektopawinge hinlipayapi kta; Qa nietapa ekta kek topawinge wilkcemna; 'Tuka niye nikiyedan u kte śni.

8 Niiśta ecedan on ekta eyatonwe kta; Qa wicaśta śica tokiconpi kin wandake kta.

9 Jehowa, wowinape mitawa kin he niye: Iyotan Wankan kin he otipi niçicaga:

10 Heon taku śica nihiyahde kte śni; Qa makośica wan nitawakeya ikiyedan u kte śni.

11 Taohnihde kin niye on wahowicaye kta; Nitacanku owasin en awanniyakapi kta;

12 Napepi ohna niyuhapi kta; Hecen inyan siha ibonito kte śni.

13 Mnaja qa wamduśka si ayahde kta; Mnaja kośka qa sintehda kin amayani kta.

14. Iye cantomahnaka, unkan ewehdaku kta; Micaje sdonye cin on wankan ewehde kta.

15 Hoyemakiye kta, kinhan amdupte kta: Woiyokiśica en kici waun :

Ewehdaku qa mduonihan kta:

16 Anpetu hanskaska on imnawaye kta; Qa wiconi mitarva wanyagwakiye kta.

\section{PSALM 92.}

Anpetu wakan on woyatan odowan.

1 Jehowa yatanpi kin he waśte ; Qa nicaje idowanpi kin, Iyotan Wankan yaun.

2 Hanhanna eca nitowaonśida oyakapi ; Qa nitowicake, hanyetu kin en.

3 Candowankiyapi ikan wikcemna, qa cancega on; 
Cotanke on awacinpi kin he nakun.

4 Jehowa, niohan kin on iyuśkinmayaya ; Ninape olian kin on imduśkin kta.

5 Jehowa, niolian tanka hinca;

Nitawacin kin temahentu hinca.

6 Wicaśta wacintonśni kin he sdonye kte śni; Qa witkotkoke cin he de okalinige kte śni.

17 Wicaśta śica peji iyecen icagapi;

Qa woalitani econpi kin owasin hca ayapi;

Owihanke wanica yutakunipi lite śni heon.

8 Tuka, Jehowa owihanke wanica, Iyotan Wankan kin he niye.

9 Iho, Jehowa, tokaniyanpi kin, Iho, tona tokaniyanpi kin atakunipi kte śni :

Woalitani econpi kin owasin enanakiya iyewicayapi kta.

10 Tuka tinta tatanka he kin iyecen,

He mitawa icaliyaya:

Wihdi to on sdamayanpi.

11 Nakun tokamayanpi kin miiśta wanwicayake kta;

Tona wicohian śica econpi takpe maupi kin

Hena on minoge nalion kta.

12 Wicaśta owotanna kin wazi can iyecen uye kta; Lebanon hante kin iyecen icage lita.

13 Jehowa ti kin en huhdepi ;

Wakantanka unkitawapi tahocoka ohna hcapi kta:

$14 \mathrm{~Pa}$ skapi hehanyan waskuyeca icahyapi kta; Cepapi kta, qa toto unpi kta.

15 Jehowa ecetu kin he oyakapi kta;

He imnija mitawa, qa woowotanua śni takudan ẹ un śni.

PSALM 93.

1 Jehowa wicaśtayatapi, wowitan kohdaka;

Jehowa wowaśake kohdaka, ipiyagkiton ;

Nakun maka kin ehdepi, hecen yuhohopi kte śni.

2 Wanakajatanhan oiyotanke nitawa yusutapi ; Niye otokahe niwanica.

3 Mini tanka kin wankan iyeiçiya; Jehowa, mini tanka kin ho hdawankan iyeya;

Mini tanka kin taja wankan iyeya

4 Mini ota ho kin isanpa;

Miniwanca taja tanlia kin isanpa;

Jehowa wankan un kin he waśaka.

5 Taku odake cin hena wicakapi hinea;

Wowakan yati kin oiyolkipi ;

Jehowa, anpetu hanskaska hehanyan.

$$
\text { PSALM } 94 .
$$

1 Jehowa, watokiçon Wakantanka;

Watokiçon Wakantanka, iyoyanpa wo. 
2 Maka Wayaco yaun, najin wo;

Wahanhaniçidapi kin towicakiçon wo.

3 Jehowa, wicaśta śica tohanyan kta he;

Tohanyan wicaśta śica iwakicipi kta he.

4 Iwaśicunpi, taku śica eyapi ;

Woahtani econpi kin owasin ihdatanpi.

5 Jehowa, nitaoyate wicakapanpi;

Qa taku nitawa kakiśyapi ece.

6 Wiwazica qa tuwe oyate tokeca etanhan tewicayapi; Qa wamdenica kin tin wicaktepi.

7 Tuka heyapi; Jah wanyake kte śni;

Qa Jakob ta Wakantanka awacin kte śni ce.

8 Oyate en wacintonśni yaunpi, awacin po;

Qa winitkotkokapi, tohan wacinksamya niohanpi kta he.

9 Tuwe wicanoge kage cin he wanation kte śni he :

Qa tuwe wicaiśta kage cin he wawanyake kte śni he.

10 Tuwe oyatepi iyopewicaye cin he wicakaśtake kte śni he; Tuwe wicaśta waonspewicakiye cin.

11 Wicaśta tawacinpi kin Jehowa sdonya ece; Hena taku śni.

12 Jah, wicaśta iyopeyaye cin he cante waśte; Qa nitoope eciyatanhan onspeyakiye cin:

13 Anpetu śice cin etanhan ozilkiye yaye kta; Wicaśta śica woha wan wicakicapi kta hehanyan.

14 Jehowa taoyate kin awicayuśtan lite śni ; Qa taku tawa kin ehpekiye kte śni.

15 Tuka woyaco woowotanna en hdi kta; Qa tona cante ecetu kin owasin he pasipi kta.

16 Sicaya econpi kin on tuwe mici najin kta he; Woahtani econpi kin on tuwe micica kta he.

17 Jehowa omakiye śni unkanś, Ecadan minagi woinina en un kta tuka.

18 Misiha naśduta ce, epe cinhan;

Jehowa, nitowaonśida najin makiye kta.

19 Miye mahen mitawacin iyokiśica ota icunhan, Wacintonhnake nitawa minagi iyuśkinkiye kta.

20 Woahtani oiyotanke kin he nicica kta he; Wicohan sica woope iyecen lronze cin he.

21 Wicaśta owotanna nagi kin takpe hipi ; Qa wicawe taku iyaonpepica śni kin he yacopi.

22 Tuka Jehowa conkaśke mitawa kin hee; Qa mita Wakantanka imnija wowinape waye.

23 Unkan iye wahtanipi kin iyahdewicaya; Qa taku śica econpi kiı en ihangwicaye kta; Jehowa Wakantanka unkitawapi ihangwicaye kta.

\section{PSALM 95.}

1 U miye, Jehowa unkahiyayapi kte; Wiconi unkitawapi Imnija kin unkidowanpi kte. 
2 Wopida yuha iye ite kin en unkupi kte; Wowiyuśkin odowan on unkiyaśapi kta.

3 Jeliowa Taku Wakan Tanka; Qa taku wakan owasin iwankan Wicaśtayatapi tanka.

4. Maka ośbe kin hena iye nape ohna hduha; Qa paha ipa kin hena iye tawa.

5 Miniwanca kin he tawa, qa iye kaga; Nakun maka puze cin he iye nape lkaga.

$6 \mathrm{U}$ miye, patuś inaunjinpi qa ohoundapi kte; Jehowa Waicahye unkitawapi kin itokam, Canpeśka makehde unkanpi kte.

y Wakantanka unkitawapi kin he iye; Qa makoce tawa oyate kin unkiyepi; Qa iye nape ohna tahin wanunyanpi kin : Anpetu kin de iye ho kin nayahonpi kta hecinhan;

8 Meribah iyecen cante hdutehipi śni po; Makoskan Massa anpetu kin iyececa.

9 Hunkakewicayayapi imayutanpi qehan ; Imayukcanpi, nakun miohan wanyakapi.

10 Waniyetu wikcemna topa hehanyan wicoicage kin he iyokiśinmayan ;

Unkan hepe; Oyate kin de cante elsta nunipi;

Qa mitacanku sdonyapi śni.

11 Hecen canmaniyan konza kewicawakiya;

Woozi mitawa kin en opapi kte śni.

\section{PSALIM 96.}

1 Jehowa odowan teca wan kahiyaya po; Maka kin ocowasin, Jehowa idowan po.

2 Jehowa kahiyaya po, iye caje yawaśte po ; Anpetu otoiyohi waniwicakiye cin he yaotanin po.

3 Ikcewicaśta kin ehna iye towitan oyaka po; Oyate owasin en iye ohan wakan kin.

4 Jehowa tanka, qa nina yatanpi kte; Taku wakan owasin isanpa kokipapi kta iyececa.

5 Ikcewicaśta taku wakan yuhapi kin owasin taku śni ; Tuka Jehowa mahipiya kin kaga.

6 Iye itokam woyuonihan qa wowitan; Tipi wakan tawa ohna, wowaśake qa wowaśte.

7 Jehowa qu miye, oyate wicowazipi kin, Wowitan qa wowaśake Jehowa qu miye.

8 Iye caje towitan kin Jehowa kicu po; Wopida wośna wan icupi qa tahocoka kin en u po.

9 Wowakan oiyolkipi kin en Jehowa ohoda po; Iye itokam, maka kin ocowasin, inihan miye.

10 Oyatepi ehna, Jehowa Wicaśtayatapi ce, eya po; Nakun maka kin ehdepi, yuhohopi kte śni :

Oyate kin woecetu eciyatanhan wicayaco lkta.

11 Må̉piya kin iyuślkin, qa maka kin pida nunwe; 
Miniwanca qa woojudan kin hotanin nunwe.

12 Maga kin iyuśkin lkte, qa taku ohna un kin owasin; Hehan contanka can owasin wiyuśkinyan dowan kta:

13 Jehowa itokam: He wanna $u$;

Maka kin hdaco u ce:

Woowotanna on wicoicage kin yaco kta;

Qa oyate kin, iye towicake eciyatanhan.

\section{PSALM 9\%:}

1 Jehowa Wicaśtayatapi, maka kin iyuśkin kte;

Wita ota hince cin hena pidapi nunwe.

2 Ihdukśan mahpiya śapa qa otpaza;

Oiyotanke tawa woowotanna qa woyaco akan han.

3 Iye ite kin itokam peta iyaya; Qa ihdukśan tokayapi kin huhnahwicaye kta.

4 Wakanhdi tawa makoce kin iyoyamya; Maka kin wanyaka qa cancan.

5 Jehowa itokam paha kin canśin iyecen śdoyapi ; Maka ocowasin Itancan tawa kin itokam.

6 Malipiya kin hena iye toowotanna yuotaninpi ; Qa oyate owasin iye towitan wanyakapi.

7 Tona okagapi wan okiyapi kin owasin iśtecapi kte ; Tona takuśni on ihdatanpi kin hena: Iye en patuja po, taku wakan owasin.

8 Zion he nation qa iyuśkin; Qa Juda cunwintku wowiyuśkin on iyaśapi : Jehowa, nitawoyaco kin hena on.

9 Niye, Jehowa, maka owancaya Iyotan Wankan yaun; Taku wakan owasin iwankam tehanwankantu.

10 Jehowa waśteyadakapi, taku śica śicedaka po ; Wicaśta wacantkiye tawa nagipi kin awanwicayaka ;

Wicaśta śica napepi etanhan wicayukce kta.

11 Wicaśta owotanna kin iyoyanpa owicakicijupi ; Qa wowiyuśkin, tona cante ecetupi kin on.

12 Owotanna yaunpi kin, Jehowa en iyuśkin po; Qa iye towakan kiksuyapi kin he yatan miye.

\section{- PSALM 98. Odowan.}

1 Odowan teca wan Jehowa kahiyaya po; Wicolian wakan kin heca econ ece: Nape etapa qa isto walkan kin on woohiye içicaga.

2 Jehowa wiconi tawa kin hduotanin; Ikcewicaśta iśta kin en toowotanna hduzamni.

3 Iye towaonśida qa towicake kin kiksuya, Israel tiyohnaka kin on; Wakantanka unkitawapi waniwicakiye cin, 


\section{P S A L M 100.}

Maka ihanke kin owasin wanyakapi.

4 Maka kin ocowasin, Jehowa kahiyaya po ;

Iyaśapi qa wiyuśkinpi qa dowan po.

5 Candowankiyapi on Jehowa kahiyaya po;

Candowankiyapi qa odowan ho kin on.

6 Mazayahotonpi tanka qa cotanka ho kin on, Jehowa Wicaśtayatapi kin itokam iyaśa po.

\% Miniwanca kin sa nunwe, qa woojudan kin; Maka kin, qa tona en ounyanpi nakun.

8 Wakpa kin nape hdaskapapi nunwe;

Qa paha kin witaya iyuśkinpi kte;

9 Jehowa itokam:

He maka kin hdaco u ce;

Woowotanna on wicoicage kin hdaco kta;

Qa oyatepi kin, wokonze eciyatanhan.

\section{PSALM 99.}

1 Jehowa Wicaśtayatapi, oyate kin cancanpi ;

Kerubim otahedan iyotanka, maka kin śkanślkan.

2 Zion en Jehowa tanka;

Qa oyate owasin iwankam tehanwankan yanka.

3 Nicaje tanka qa wowinihan kin he yatanpi kte;

He Wakan hinca.

4 Unkan wicaśtayatapi towaśake kin woyaco waśtedaka;

Niye wicoecetu yahdusuta;

Jakob en woyaco qa woowotanna yakaga.

5 Jehowa Wakantanka unkitawapi yatanka po ; Qa iye siha oahe kin en makata ehpeiciya po: Iye Wakan hinca heon.

6 Moses qa Aaron, wawayuśna tawa kin ehna;

Qa Samuel tona caje hoyekiyapi kin om ;

Hena Jehowa hoyekiyapi, unkan iye awicayupta ece.

7 Malipiya bosdata kin en owawicalkiye:

Woyaotanin tawa kin opapi, qa wokage wicaqu kin.

8 Jehowa Wakantanka unkitawapi, niye awicayadupte;

Iyotan Waśaka, niye wicayecicajuju ;

Tuka wahtanipi kin hena towicayecicon.

9 Jehowa Wakantanka unkitawapi yatanka po;

Qa iye paha wakan tawa kin ohna patuja po;

Jehowa Wakantanka unkitawapi kin he wakan nakaeś.

\section{PSALM 100.}

Odowan wan. Wopida eyapi kta.

1 Maka kin ocowasin, Jehowa iyaśa miye.

2 Wowiyuśkin on Jehowa okiya po;

Itokam dowanwan u miye.

3 Jehowa he Wakantanka e sdonya po: 
Iye unkagapi, qa unkiye unkiçicagapi śni :

Taoyate qa tahinca tinta tawa kin en Hena unkiyepi.

4 Tatiyopa kin en wopida eya u po,

Woyatan yuha iye tahocoka ohna u miye;

Wopida eciya miye ;

Iye caje kin yawaśte po.

5 Jehowa waśte, towaonśida kin owihanke śni ;

Qa towicake kin he wicoicage qa wicoicage hehanyan.

\section{PSALM 101.}

Dawid tadowan.

1 Wowaonśida qa woyaco on wadowan kta ; Jehowa, niye icidowan kta.

2 Canku yuśtanpi wan ohna wacin waksape kta:

Tohan en mayahi kta he:

Cante ncowasin hduha wati ohna mawani kta.

3 Miiśta itokam wicoie śica ewehnake kte śni ;

Taku śica econpi kin hena śice wadaka ;

Miye en amaskape kte śni.

4 Wicacante pemni kin he amayuśtan kta ; Taku śica sdonwakiye kte śni.

5 Tuwe takodaku nalimana aie cin he ihangwaye kta :

Tuwe iśta tanka qa wahianicida kin iyowinwakiye kte śni.

6 Makoce en tona wicakapi kin miiśta awicatonwan; Hena mici unpi kta;

Tuwe canku yuśtanpi omani kin he omakiye kta.

7 Tuwe wohnayan econ kin he wati en ounye kte śni ;

Tuwe itonśni kin he miiśta itokam yanke kte śni.

8 Hanhanna eca, makoce kin en, tona ścapi

Owasin ihangwicawaye kta;

Hecen Jehowa totonwe kin etanhan,

Tona śicaya econpi kin owasin ehpewicawaye kta.

\section{PSALM 102.}

Wocekiye wan. Tuwe iyotanhan iyekiye, kakija qa Jehowa itokam waśicahowaye cin he tawa.

1 Jehowa, wocekiye mitawa nation wo;

Qa houwaye cin he en niu nunwe.

2 Niite anamakihibe śni wo;

Makakija anpetu kin en anohmakiya wo ;

Houwaye anpetu kin en amayupta inahini wo.

3 Mitanpetu kin śota iyaya;

Qa mahuhu can śeca iyecen itkon.

4 Micante peji iyecen śniśyapi qa pus aya: Aguyapi hduta awektonja:

5 Waśicahowamda ho kin on, 
Micelipi kin mahuhu en askapa.

6 Tinta mdega wan iyemaceca;

Hinhankaga otiwota en iyecen waun.

7 Kiktahan waun, qa zitkadan wan Tice ekta iśnana un kin iyemaceca.

8 Anpetu osan tokamayanpi kin iyopemayanpi;

Tona canniyemayanpi kin hena ośtemahdapi ece.

9 Aguyapi iyecen cahota wata;

Qa wamdatke cin he ceyapi kici icahiwaya;

10 Wośinhda qa wocanniye nitawa kin on etanhan:

Yuwankan imayacu qa ehpemayaye cin heon.

11. Mitaanpetu ohanzi takinyan ye cin iyececa;

Qa miye qe peji iyecen maśnija.

12 Tuka niye, Jehowa, owihanke wanin idotanke kta;

Qa wokiksuye nitawa kin he wicoicage qa wicoicage hehanyan.

13 Niye nayajin kta, Zion onśiyakida kta ;

Tohan okiyapi kta wanna iyehantu,

Han, tohan iyehantu hince cinhan:

14 Tohan inyan tawa kin nitaokiye waśtedakapi;

Qa watuśekśeca on iyokipipi kinhan.

15 Unkan Ikcewicaśta Jehowa caje kin kokipapi kta;

Qa nitowitan kin, maka wicaśtayatapi owasin.

16 Jehowa Zion kage cin heon;

Iye towitan en ihdutanin.

17 Onśike tawocekiye ekta ihduhomni;

Qa wocekiye tawapi kin śicedake śni.

18 Wicoicage u kte cin on de owapi kta;

Qa oyate wan icage kte cin he Jah yatanpi kta.

19 Towakan paha kin etanhan eyokasin;

Jehowa malipiya etanhan maka kin ahitonwan;

20 Kaśka unpi comnihdazipi kin nahon kta;

Wiconte cinca kin wicakiyukce kta.

21 Zion en Jehowa caje kin oyakapi kta;

Qa iye yatani kin, Jerusalem en:

22 Oyatepi witaya mniciyapi kin en ;

Qa wokiconze kin, Jehowa ohodapi lkta.

23 Canku ohna mitowaśake kin kun ehpeya;

Mitaanpetu kin yuptecedan.

24 Mita Wakantanka, mitaanpetu cokaya en, emayaku śni wo, epe kta:

Waniyetu nitawa wicoicage wicoicage hehanyan.

25 Otokahe ekta maka kin sutaya eyahde;

Qa ninape taku kage cin mahpiya kin hee.

26 Hena awihnuni kta, tuka niye yani kta;

Qa hena owasin sina iyecen kuke kta;

Wokoyake iyecen hena dutokeca kta;

Unkan yutokecapi kta.

27 Tuka he niye,

Qa waniyetu nitawa owihanke kte śni. 
28 Nitaokiye cinca kin ounyanpi kte; Qa iye cincapi kin nitokam wicayusutapi kte.

\section{PSALM 103.}

Dawid tawa.

1 Minagi, Jehowa yawaśte wo; Qa taku mahen maun kin owasin, iye caje wakan kin.

2 Minagi, Jehowa yawaśte wo; Qa taku waśte econ kin owasin akiktonje śni wo.

3 He wayalitani owasin nicicajuju ; Waniyazan kin iyuhpa asniniyan :

4 Wicotakuniśni etanhan nitaniya opekiton; Wowaonśida qa wocantekiye on wateśdagnicaton.

5 Taku waśte on nitaanpetu ojudan ya ; Hecen koniśka ihduteca, wamdi iyececa.

6 Jehowa woowotanna ece econ, Qa woyaco, tona kakiświcayapi owasin on.

7 Iye tacanku kin Moses sdonyekiya; Qa Israel cinca, iye ohan tanka kin.

8 Jehowa wacantkiye ça waonśida; Tchan canteptanye śni, qa wawacinkta yuza tanka.

9 Ohinniyan wicakige kte śni ; Qa owihanke wanin canniye wicaye kte śni.

10 Waunhtanipi kin iyecen ecaunkiconpi śni ; Qa taku śica unkitawapi iyecen unqupi śni.

11 Mahpiya kin maka iwankam tehanwankantu kin, He iyecen iye towaonśida tona kokipapi kin iwankam waśake hinca.

12 Wiyohiyanpa etanhan wiyolipeyata hehanyan; Thehanyan waunhtanipi kin yutokan ehpeunkiciciyapi.

13 Ateyapi wan iye cinca onśiwicakida kin iyececa, Jehowa tona kokipapi kin onśiwicakida ece.

14 Token unkicagapi kin he sdonya; Maka mdu heuncapi he kiksuya.

15 Wicaśta taanpetu kin ḥena wato iyececa; Tinta wahca kin he iyceen hea aya:

16 Tate oniya wan iwankam iyaya, Unkan en un śni;

Qa tohe kin icimana sdonkiye kte śni.

17 'Tuka Jehowa towaonśida otokahe wanica, Qa owihanke wanice kta, tona kokipapi kin on ; Qa toowotanna kin cincapi sanpa cincapi kin en :

18 Tona wicotakuye tawa kiciyuhapi ; Qa tawoahope econpi kta on kiksuyapi kin hena.

19 Jehowa mahpiya kin en oiyotanke ekihde; Qa tokiconze kin taku owasin wowidagya.

20 Ohnihde wakan tawa, Jehowa yawaśte po; Wowaśake itancanpi, iye oie ecen ecanonpi; 
Iye oie ho kin anayagoptanpi kta.

21 Trobe kin owasin, Jehowa yawaśte po;

Taokiyeniyanpi, iye tawacin ecen ecanonpi.

22 Taku kage cin owasin, Jehowa yawaśte wo;

Tokiconze owanka kin owasin ohna:

Minagi, Jehowa yawaśte wo.

\section{PSALM 104.}

1 Minagi, Jehowa yawaśte ye :

Jehowa, mita Wakantanka, niye nitanka hinca:

Woyuonihan qa wotanka koyahdaka.

2 Śina wan iyecen iyoyanpa hdoin :

Ozanpi wan iyecen mahpiya kin hdúmdaya.

3 Mini tanka on tipi içicaga;

Mahpiya śapa kin canpahmihma ya ;

Tate hupahu kin on icimani ece.

4 Tateyanpa kin hena ohnihde wicakaga;

Peta ide kin taokiye ya ece.

5 Maka kin tohe akan sutaya ehde;

Hecen owihanke wanica yutokanpi kte śni.

6 Miniwanca on wokoyake iyecen ayakahpa; Mini tanka kin paha iwankam hiyeya.

7 Yakiśica hehan najicapi ;

Wakinyan nitawa hoton kin on napapi.

8 Paha iyecen wankan yapi ;

Kaksiza iyecen kun ipi ;

Owanka kin de yecage cin ekta.

9 Oicago wan yakaga, sanpa yapi kte śni ; Ake maka kin apaśbog hiyu kte śni.

10 Kaksiza kin en minihdoka inanpe ya; Hena paha kin otahedan kaduza ece.

11 Tinta wamanica owasin etanhan mini yatkanpi :

Hen śonśonna itu unpi kin ipuza enakiyapi.

12 Iwankam mahpiya akinyanpi kin otipi ;

Can adetka ehna hotonpi ece.

13 Wankan tipi tawa etanhan he kin amagajuya:

Niohan etanhan taku icage cin on maka kin imnahan.

14 Wato kin he woteca icahwicakiciya;

Qa waskuyeca, wicaśta woju kin on ;

Hecen maka kin etanhan aguyapi hinanpe ye kta.

15 Qa miniśa wicaśta cante iyuśkinkiya,

Wihdi isanpa ite kin iyehkiye kta;

Qa aguyapi wicacante yusuta ece kin he.

16 Jehowa can tawa kin imnahan;

Lebanon hante-iye oju kin hena.

17 Hena en zitkadan hohpi kagapi;

Hoka wacantkiye cin he wazi en otipi.

18 He tehanwankantu kin tatokadan tawapi ; 
Qa imnija kin hitunkasan wowinape yapi.

19 Makoncage on hanyeta wi kin kicaga:

Anpetu wi tohan iyaye kte cin he sdonkiya.

20 Otpaza yakłga, unkan ecen hanyetu;

Hehan contanka wamanica owasin hinanpapi.

21 Mnaja cinca taku iyahpayapi kta ahotonpi ;

Qa Wakantanka woyute kidapi ece.

22 Wi hinanpa, hehan witaya hdipi; Qa waśunpi lrin en hdiwankapi ece.

23 Hehan wicaśta wicohtani ekta ya;

Qa litayetu hehanyan wicohan econ ece.

24 Jehowa, niohan kin hena ota hinca;

Woksape on hena owasin yakaga:

Wowijice nitawa on maka kin ojudan.

25 Miniwanca kin de tanka qa ohdakinyan hanska;

Hen taku śkanśkan yukan, qa yawapica śni ;

Taku niyake un cikcistinna tankinyanyan koya.

26 He ohna wita wata kin ohnihdapi :

Unktehi kin de ohna śkate kta yakaga.

27 Hena owasin niye wacinniyanpi :

Tohan iyehantu eca woyute wicayaqu ece kta.

28 Niye wawicayaqu, hena pahipi;

Ninape yahdumdaya, taku waśte on imnanpi ece.

29 Niye niite nayakifibe, hena nihinciyapi :

z... Woniya ewicayeciyaku, hena țapi ;

Qa maka mdu ekta hdapi.

30 Nitaniya yeyaśi, hena icagapi :

Qa makoce ite kin duteca ece.

31 Jehowa towitan kin owihanke wanice kte:

Jehowa iye ohan kin on ihduśkin nunwe.

32 He maka kin en etonwan, unkan cancan:

Paha kin yutan, unkan hena izitapi.

33 Tohanyan wani kin Jehowa iwadowan kta;

Waun kin hehanyan mita Wakantanka wakahimdamde kta.

34. Iye awacin waun kin he skuye kta:

Jehowa en imduśkin kta.

35 Wahtanipi sa maka kin etanhan owihankepi kta;

Qa wicaśta śica icimana en unpi kte śni.

Minagi, Jehowa yawaśte wo.

Jah yatan miye.

PSATM 105.

1 Jehowa wopida eciya po,

Iye caje hoyekiya miye;

Iye ohan tanka oyatepi ehna oyaka po.

2 Iye idowan po, odowan kahiyaya miye;

Iye tawowapetokeca kin owasin awacin po.

3 Iye caje wakan kin itan miye; 
Tona Jehowa akitapi kin hena cante waśtepi lita.

4. Jehowa ode miye, qa iye towaśake kin ;

Ohinniyan ite kin akita miye.

5 Wicohian wakan econ qon hena kiksuya po;

Iye tawowapetokeca, qa woyaco iye i kin etanhan.

6 Abraham iye taokiye etanhan inicagapi;

Jakob cinca wicakahnige ciqon.

7 Jehowa Wakantanka unkitawapi kin he iye;

Tawoyaco kin maka kin owancaya un.

8 Wicotalsuye tawa kin he ohinniyan kiksuya;

Wicoie econ wicaśi qon, wicoicage kektopawinge hehanyan:

9 A.braham kici hduśtan qon he;

Qa Isaak en wokonze eciya:

10 Nakun Jakob he wokage kiciyusuta;

Israel en wicotakuye owihanke wanica.

11 Niye Kanaan makoce kin ciçu lita, Makoce iyutapi nitawapi kta ce, eya.

12 Hinyahin wicayawapi waśakadan; Tonana hinca, qa en unhdaka unpi.

13 Qa oyate lin wicehna ihdaka yapi;

Wokiconze wan etanhan oyate tokeca ekta.

14 Tuwedan kakiświcaya iyowinkiye śni ; Qa iyepi on wicaśtayatapi iyopewicaya :

15 Sdawicawakiye cin hena owicayutanpi śni po; Qa waayate mitawa taku śica ecawicakiconpi śni po.

16 Hehan makoce kin he en wicaakilian wan u śi ; Aguyapi sagye kin owasin kaksa.

17 Wicitokam wicaśta wan ye śi ; Joseph wowidake kta e wiyopeyapi:

18 Wicaślke on siha kiunniyanpi; Iye nagi mazasapa kin en $u$.

19 Oie kin he ecetu hehanyan, Jehowa taku eye ciqon he iyutanye.

20 Wicaśtayatapi kin he ye wicaśi, qa kiyukca; Oyatepi itancan kin hee, qa kiciyuhdoka.

21 Iye ti kin ohna itancan kaga; Qa wowijice tawa kin owasin tawalkiya.

22 Tohan iyokipi kinhan akicita tancan wicakaśke kta; Qa hunkayapi wicayuhe cin ksapa wicakage kta.

23 Hehan Israel Egupta en ahiti; Qa Jakob Ḣam makoce kin en ounyan.

24 Unkan taoyate kin nina wicahduota; Qa tokayapi kin isanpa wicayuwaśake.

25 Hehan cante wicayutokeca, taoyate śice wicadakapi kta; Taokiye wicaye cin hnayan wicakuwapi kta.

26 Moses iye taokiye kin ye śi; Qa Aaron kalinige ciqon he.

27 Iye tawowapetokeca oie kin wicakipazopi : Qa tohan wakan, Ham makoce kin en. 
28 Otpaza ye śi, qa otpaza kaga;

Unkan iye oie kin kizapi śni.

29 Mini tawapi kin he we kaga;

Qa hogan tawapi kin wicakte.

30 Makoce tawapi hnaśka ota icahyya;

Wicaśtayatapi yuhapi wankan tipi kin ohna.

31 Ecen eya, unkan hoponkadan hiyupi;

Qa heya, makoce tawapi owancaya.

32 Magaju kte cin he wasu wicaqu;

Peta ide kin makoce tawapi owancaya.

33 Tastanyanka iyuwi qa suken can tawapi kin hena apa;

Qa tamakocepi ihdukśan can kin kawega.

34 Ecen eya, unkan psipsicadan tanka hiyupi;

Psipsicadan nakun, qa hena yawapica śni :

35 Qa tamakocepi kin en wato owasin yaśdapi ;

Qa tamagapi en taku icage cin temyapi.

36 Nakun tamakocepi en tona tokapapi owasin wicakte;

Iye towaśakepi tokaheya icage cin owasin.

37 Hehan mazaska mazaskazi ko yuha hinapewicaya:

Unkan wicowazi tawa kin en wanjidan hustake śni.

38 Iyayapi qehan Egupta iyuśkin :

Nina kowicakipapi, heon etanhan.

39 Woakalipe kta on mah̉piya śapa hdumdaya;

Qa peta wan, hanyetu en iyojanjan kta.

40 Kidapi, unkan wakiyedan u wicakiya :

Qa mahpiya aguyapi on imnawicaya.

41 Imnija wan kalidoka, unkan mini hiyu ; Hopuza en wakpa wan hiyaya.

42 Tye oie wakan qon he kiksuya heon;

Taokiye Abraham taku eciye ciqon.

43 Qa iye taoyate iyuśkinyan hinanpe wicaya;

Tona wicakalinige cin hena iyakiśapi.

44 Qa makoce Ikcewicaśta tawapi qon he wicaqu :

Unkan oyatepi hitanipi qon he yuhapi.

45 Hecen iye tawoahope kin opapi kta; Qa toope kin hena yuhapi kta.

Jah yatan miye.

\section{PSALM 106.}

1 Jah yatan miye.

Jehowa wopida éciya po ;

Iye waśte, heon etanhan;

Qa towaonśida kin owihanke wanica.

2 Jehowa tohan tanka kin tuwe oyalke kta he;

Iye yatanpi ocowasin tuwe nahonwicaye kta he.

3 Tona wokonze opapi kin hena cante waśtepi ;

Tuwe ohinniyan woowotanna econ kin.

4. Jehowa, nitaoyate cantewicayakiye cin iyecen miksuya ye; 
Nitowiconi kin hiyumakiya wo.

5 Tona yalkahniga wowaśte yuhapi kin wanmdake kta;

Nitaoyate wiyuśkinpi kin on imduśkin kta;

Woaihpeye nitawa kin en wowitan miciye kta.

6 Hunkakewicunyanpi om waunhtanipi;

Pemniyan unkolianyanpi, śicaya econkupi.

7 Egupta en niohan wakan kin

Hunkakewicunyanpi okahnigapi śni ;

Nitowaonśida untkana kin kiksuyapi śni ;

Tuka wawakipajinpi, mde kin ekta, Mde Śa kin en.

8 Heca eśta iye caje kin on niwicakiya;

Towaśake sdonyewicakiye kta e heon.

9 Nakun Mide Śa kin kiśica, unkan pus aya;

Hecen ośbe kin ehna iyaye wicaya, hopuza iyececa.

10 Qa waśicedake nape kin etanhan ewicayaku;

Qa toka nape kin etanhan opewicakiton.

11 Unkan tokawicayapi kin mini awicakañpa;

Etanhan wanjidan eśta okaptapi śni.

12 Hehan oie kin wicadapi;

Iye yatanpi kin ahiyayapi.

13 Koyahanna iye ohan kin akiktonjapi ;

Iye tawowahokonkiye kin akipepi śni.

14 Tuka hewotahedan wocantiheye wan cantiheyapi;

Qa hopuza en Wakantanka iyutanpi.

15 Unkan taku kidapi qon he awicayupta;

Tuka wicotamaheca nagipi en ye śi.

16 Nakun otiwita en Moses inakiwizipi ;

Qa Aaron, Jehowa yuwakan kin he.

17 Hehan maka kin ihduhdoka, qa Dathan napca;

Qa Abiram tona om un ko awicakahipa.

18 Unkan ośpaye kin hena peta wicahulinaga;

Ide wan śicapi kin hena liulnaliwicaya.

19 Horeb en ptejicadan wan kagapi;

Qa taku śdoya kagapi kin itokam patujapi.

20 Hecen iye towitanpi kin hdutokecapi, Tatanka wato yute cin ouncage iyececa.

21 Wakantanka niwicaye ciqon he akiktonjapi;

Egupta en wicolian tanka econ qon:

22 Wowapetokeca Ham makoce kin en;

Mde Śa ekta taku wowinihan.

23 Hehan ihangwicaye kta, keya;

Moses iye kahnige ciqon he itokam,

Otahedan najin śni unkanś;

Tocanniye yutokan iyeye kta, on waihangye kte śni.

24 Qa makoce wiciyokipi kin he aktapi śni;

Iye oie kin wicadapi śni :

25 Tuka tawakeyapi ohna wihnupi ;

Jehowa ho kin he anagoptanpi śni.

26 Hehan hena on nape hdugata; 
Tintoskan ihpaye wicakiye kta:

27 Qa cincapi oyatepi wicehna hinhpaye wicaye kta;

Qa makoce tukeca ekta iyewicaye kta.

28 Unkan Baal Peor en ikoyagiçiyapi ;

Qa țapi taku wakiyuśnapi kin yutapi.

29 Hecen taku śica econpi kin on iyokiśinyapi :

Unkan makośica iyowicahpaya.

30 Hehan Phinehas inajin qa wayaco:

Unkan makośica kin anaptapi.

31 Unkan he woowotanna kiciyawapi ;

Wicoicage qa wicoicage ekta owihanke wanica.

32 Nakun Woakinica mini kin ekta śihdayapi :

Hecen iyepi on Moses taku śica akipa:

33 Iye taniya kin kipajinpi :

Unkan iha on ecinśniyan ia.

34 Oyatepi kin ihangwicayapi śni ;

Jehowa kewicakiye ciqon iyececa.

35 Tuka oyatepi kin om icicahiyapi,

Qa wicohan tawapi onspeiciciyapi :

36 Qa wakagapi tawapi ohodapi ;

Unkan hena e wohmunke yapi kin ee.

37 Han, cinhintkupi qa cunwintkupi kin,

Taku wakan śica wawicakiyuśnapi.

38 Hecen wicawe taku iyaonpepicaśni papsonpi,

Cinhintkupi qa cunwintkupi we kin,

He Kanaan wakagapi kin wawicakiyuśnapi ;

Hecen makoce kin wicawe on yuśapapi.

39 Nakun iye ohanpi kin on ihduśicapi;

Qa śicaya econpi kin on wawicihahapi ic̣icagapi.

40 Unkan Jehowa iye taoyate canniyewicaya; Qa woailipeye tawa kin wahtekida śni.

41 Hehan Ilkeewicaśta napepi kin en wicaqu.

Unkan tona śicewicadapi kin hena wowidake wicayapi :

42 Qa tokawicayapi kin śicaya wicakuwapi :

Hẹcen napepi kin ihukuya patujapi.

43 Iye ijehan wicakiyukca;

Tuka wakiconzapi on kipajinpi ;

Hecen wahtanipi kin on atakunipi śni.

44 'Tuka iyokiśicapi kin he wanyaka,

Śicaho wayapi nation qehan.

45 Qa iyepi on wicotakuye tawa kin he kiksuya;

Qa towaonśida untkanna on tawacin hdutokeca.

16 Qa wayaka wicayuhapi owasin itokam,

Hena onśiwicadapi kta wicaqu.

47 Jehowa Wakantanka unkitawapi, niunkiya po;

Qa Ikcewicaśta etanhan ake unhduwitaya po:

Nicaje wakan kin unyatanpi kta;

Nitowitan kin en unkihdatanpi kta.

48 Jehowa Israel ta Wakantanka kin yawaśtepi nunwe; 
Otokahe wanintanhan qa owihanke wanica.

Unkan wicaśta owasin, Amen, eyapi.

Jah yatan miye.

\section{PSALM $10 \%$.}

1 Jehowa wopida eciya po, iye waśte kin heon; 'Towaonśida kin he owihanke wanica:

2 Tona Jehowa opewicakiton kin hena eyapi kta; Tona toka nape kin etanhan opewicakiton kin.

3 Qa makoce tokeca etanhan wicahduwitaya; Wiyohiyanpa tanhan qa wiyohpeya tanhan, Waziyatanhan qa miniwanca etanhan.

4 Makoskan onunipi, liewoskan canku ohna; Otonwe en ounyanpi kte cin iyeyapi śni.

5 Wotektehdapi, nakun ipuzapi; Tanmahen tanhan nagipi hanyanpi.

6 Hehan iyokiśicapi en Jehowa hoyekiyapi : Unkan wicokakije etanhan ewicayaku;

7 Qa canku owotanna ohna cankuye wicaya; Otonwe en ounyanpi kta he ekta yapi kta.

8 Jehowa wopida eciyapi kte, Iye towaonśida kin on, Qa wowapetokeca Adam cinca ecawicakicon kin hena on.

9 Wicanagi cantokpani kin he imnaya ece; Qa nagi wotektehda kin he taku waśte on ojuya.

10 'Tona otpaza qa wiconțe ohanzi en iyotankapi; Wokakije mazasapa ko on wicakaśkapi :

11 Hena Iyotan Waśaka oie kin kipajinpi ; Qa Iyotan Wankan tawowahokonkiye kin aktapi śni :

12 Hecen wicohtani on cante onśika wicakaga : Hicahanpi qa waokiye wanica.

13 Hehan iyokiśicapi en Jehowa hoyekiyapi : Unkan wicokakije etanhan ewicayaku:

14. Otpaza qa wiconte ohanzi etanhan iwicacu; Qa on wicakaśkapi kin hena yuksa.

15 Jehowa wopida eciyapi kta, towaonśida kin on ; Qa wowapetokeca Adam cinca ecawicakicon kin hera on :

16 Mazaśa tiyopa kin kaptuptuja heon; Qa mazasapa iratake kin kaksaksa.

17 Witkotkopi kin, wahtanipi canku kin on, Qa taku śica econpi kin on, kakiśiçiyapi.

18 Iye nagipi woyute owasin hitihdapi ; Qa wiconte tiyopa kin ikikedan yapi.

19 Hehan iyokiśicapi en Jehowa hoyekiyapi : Unkan wicokakije etanhan ewicayaku:

20 Iye oie kin ukiye ça asniwicaya; Qa wicotakuniśni etanhan hdinanpe wicaya.

21 Jehowa wopida eciyapi kte, towaonśida kin on, 
Qa wowapetokeca Adam cinca ecawicakicon kin hena on:

22 Qa wopida wośnapi wayuśnapi kte ;

Qa dowanpi on iye ohan kin oyakapi kte.

23 Tona miniwanca ekta wita wata ohna yapi,

Qa mini ota kin ohna wicohan econpi kin;

24 Hena Jehowa ohan kin wanyakapi;

Qa ośbe kin en wowapetokeca econ kin.

25 Hehan ecen eya, unkan tate wohitika wan uye;

Qa taja kin wankan icu.

26 Mahpiya ekta wankan yapi ;

Kuya wośbe kin ekta ipi ;

Śicaya unpi kin on nagipi nihinciyapi.

27 Itohomnipi qa kacegya manipi, witkopi iyececa;

Qa wacinksapapi qon iyulipa taninśni iyaya.

28 Hehan iyokiśicapi en Jehowa hoyekiyapi;

Unkan wokakije etanhan hinanpe wicaya.

29 Tate wohitika qon he amdakedan kaga:

Unkan taja tawapi kin inina hiyeya.

30 Hehan owanji yukanpi on pidapi :

Hecen oihunni cinpi qon en awicahi.

31 Jehowa wopida eciyapi kte, towaonśida kin on ; Qa wowapetokeca Adam cinca ecawicakicon kin hena on.

32 Nakun oyate mniciyapi kin en yatankapi kte; Qa hunkayapi omniciye kin èn idowanpi kte.

33 Wakpadan kin hena tintoskan ee kiya;

Qa minilidoka kin liopuza kaga:

34 Waskuyeca makoce wan miniskuya icu kiya; Tona en ounyanpi śicaya econpi kin he on.

35 Tintoskan kin he miniyowe lkiya;

Qa hopuza kin he minihdoka eepi kaga:

36 Qa hen wotektehdapi kin ewicahnaka;

Unkan otonwe en ounyanpi kte cin he kagapi :

37 Qa maga ojupi, qa hastanyanka iyuwi huhdepi ;

Qa woicage waskuyeca icahyapi.

38 Nakun wicayawaśte, hecen nina ihduotapi; Qa tawanuyanpi kin wicayutonna śni.

39 Hena wanistinna ayapi qa patuś unpi tuka qon; Wokakije, wowayazan qa woiyokiśice kin on.

40 Wicaśtayatapi wowah̉teda śni wicakaga; Qa canku codan makoskan onuniwicaya.

41 Tuka wahpanicapi kin wokakije etanhan ewicayaku; Qa optaye iyececa wicowazi wicakaga.

42 Wicaśta ecetupi wanyakapi qa iyuśkinpi kta; Qa woahtani owasin iohmus icu kta.

43 Tona wacinksapapi qa dena a wacinpi kinhan, Hena Jehowa towaonśida kin iyukcanpi kta. 


\section{PSALM 108.}

Psalm odowan. David tawa.

1 Wakantanka, micante wiyeya un;

Wadowan qa mdatan kta, mitowitan kin he on.

2 Cotanka candowankiyapi ko kikta miye;

Miye qe anpao kin mduhice kta.

3 Jehowa, oyatepi ehna ciyatan $\mathrm{kta}$;

Oyate kin en ciyaonihan kta.

4 Malipiya iwankapatanhan nitowaonśida kin tanka;

Qa nitowicake kin mahpiya śapa iyahde.

5 Wakantanka, mahipiya isanpa niyawankantuyapi nunwe;

Qa maka kin ocowasin iwankam nitowitan.

6 Waśtewicayadake cin hena ewicayakupi kta,

Heon ninape etapa on niunkiyapi qa unkayupta po.

7 Wakantanka iye towitan en wohdaka;

Wimduśkin kta; Sekem wapamni kta;

Sukoth kaksiza kin imdute kta ce.

8 Gilead he mitawa, Manassa mitawa;

Ephraim nakun mapa wowaśake kin;

Juda woope kaga mitawa kin hee:

9 Moab woyujaja koka mitawa kin hee;

Fidom akan mitahanpe elpewaye Ita;

Philistia on wimduśkin kta.

10 Tuwe otonwe aconkaśkapi kin en amaye kta he; n Tuwe Edom en amai lita he.

11 Wakantanka ehpeunyanpi qon hee kte śni he; Qa Wakantanka ozuye unkitawapi om ye śni qon.

12 Toka kin etanhan ounkiya miye :

Wicaśta akantu waehdakupi kin he taku śni.

13 Wakantanka eciyatanhan wowaśake unkicagapi kta ; Qa iye tokaunyanpi kin nawicapce kta.

\section{PSALM 109.}

Dowan itancan kin kicagapi. Dawid tadowan.

1 Wakantanka mdatan ece kin, Inina yanke śni ye.

2 Wicai śica qa wicai wahnayan amahdukawapi ;

Wicaceji itonśni on amaiapi.

3 Nakun śicedakapi wicoie on aohdutemayanpi;

Qa taku on etanhan śni makizapi.

4 Wacanwakiye cin on toka mayanpi;

Tuka miye qe wocekiye en waun.

5 Waśte on taku śica iyamaonpapi ;

Qa wośicedake, wocantekiye kin on.

6 Wicaśta śica wan iye awanyagkiya wo; Qa etapa eciyatanhan Satan inajin nunwe. 
7 Tohan yacopi kinhan śica yawapi kte; Qa tawocelkiye kin he woahtani kte.

8 Anpetu tawa kin tonnana kte ; Qa toawanyake kin tuwe tokeca icu kte.

9 Cinca kin wamdenicapi kta ; Qa tawicu kin wiwazice kta.

10 Qa cinca kin onuniyan unpi qa wo dapi nunwe; Qa tihulia kin ehna waakitapi kte.

11. Taku tawa kin owasin icazokiye cin he iyahpaye kte; Qa tohtani kin oyate tolkeca kipi kte.

12 Tuwedan katinyan onśikida kte śni ; Qa wamdenica tawa kin tuwedan cantewicakiye kte śni.

13 Tona etanhan icage cin hena awihnunipi kte ; Wicoicage iyokihe en cajepi pajujupi nunwe.

14. Iye atewicaye walitanipi kin Jehowa kiksuye kte ; Qa hunku taku śica econ kin he kajujupi śni nunwe.

15 Hena ohinniyan Jehowa itokam wanke kte; Hecen kiksuyapi kin maka kin etanhan icu kte.

16 Iye wowaonśida econ kta kiksuye śni heon; Nakun wicaśta onśika qa wahpanica kin śicaya kuwa; Qa wanji cante kaśujapi kin kte kta tuka.

17 Tuka wayaśicapi kin he waśtedaka, hecen akipa; Qa wayawaśtepi kin he waśtedake śni, hecen he itehan iyeyapi.

18 Unkan wokoyake iyecen wayaśicapi kohdaka; Hecen mini iyecen tan mahen hiyu; Qa wihdi iyecen huhu kin en hiyohi.

19 Wokoyake un kin he iyecen kohdake kta; Qa ipiyaka iyececa ohinniyan ipiyagkiton kte.

20 Tona tokamayanpi Jehowa etanhan wokajuju tawapi kin dee; Qa tona minagi taku śica eciyapi kin hena.

21 Tuka niye, Jehowa, Itancan, Nicaje eciyatanhan ecamicon wo ;

Nitowaonśida waśte, lieon makiyuśka wo.

22 Miye makakije c̣a onmaśika;

Qa mahentanhan cante mapahidokapi.

23 Ohanzi wan takinyan ye cin iyecen mataninśni. Qa psipsicadan iyecen kaham iyemayanpi.

24 Akilianpi on hupahu wamaśake śni ; Qa śin manica on micehipi son aya.

25 Nakun miye wowihahamayanpi ; Wanmayakapi, pomnamnamakiyapi.

26 .Tehowa, Wakantanka mitawa, omakiya wo; Nitowaonsida eciyatanhan nimayan wo.

27 Unkan ninape kin he dee sdonyapi kta; Jehowa niye hecanon kin.

28 Hena wayaśicapi kta, tuka niye wadawaśte kta: Hena najinpi qa iśtecapi kta; Tuka nitaokiye iyuśkin kta.

29 Tona tokamayanpi wonihinciye koyakapi kta; 
Qa wowiśtece kin he sina iyecen inpi kta.

30 Mii on Jeliowa nina wopida ewakiye kta;

Qa wicota ehna, mdatan kta.

31 Iye wicaśta onśike cin etapa eciyatanhan najin kta;

Tona nagi yacopi kin etanhan eyaku kta.

PSALM 110.

Dawid tadowan.

1 Jehowa Itancan mitawa kin heciya; Mietapa ekta iyotanka wo, Tona tokaniyanpi siha oahde nitawa wakage kta hehanyan.

2 Nitowaśake icapsinte kin Jehowa Zion etanhan hiyuye kta: Tokaniyanpi kin cokaya wicaśtayatapi yanka wo.

3 Nitowaśake anpetu kin en, nitaoyate waihduśnapi; Wowakan wiciyokipi kin on; Anpao tamni kin etanhan, Koniśka cu kin he yahduha.

4 Jehowa wakonza, qa tawacin hdutokeca kte śni ; Niye wawayuśna owihanke wanica henica kta, Melkizedek touncage kin eciyatanhan.

5 Itancan kin nietapa ekta yanke cin, Iye tocanniye anpetu kin en, Wicaśtayatapi katpa iyewicaya.

6 Ikcewicaśta ehna wayaco kta;

Wicaśta tapi on ojuya ;

Maka ohdakinyan hehanyan pa wicakaliuge.

7 Canku ohna minilidoka kin etanhan yatke kta : Heon etanhan pa yuwankan icu kta.

\section{PSALM 111.}

1 Jah yatan miye.

Micante ocowasin on Jehowa mdatan kta, Wicaśta ecetu takodakiciyapi qa omniciye kin en.

2 Jehowa ohan kin hena tankinyanyan; Tona iyokipipi owasin hena akitapi ece.

3 Iye ohan kin woyuonihan qa wotanka; Qa toowotanna kin owihanke wanica.

4 Iye ohan wakan kin wokiksuye kicaga: Jehowa waonśida qa wacantliya.

5 Tona kokipapi kin woyute wicaqu; Wicotakuye tawa kin ohinniyan kiksuye kta.

6 Iye tohtani wowaśake kin taoyate owicakiyaka; Incewicasta tamakoce kin wicaqu kta.

7 Iye nape taku econ kin hena wowicake qa wokonze: Tawowahokonkiye kin owasin wicakapi :

8 Owihanke wanica hehanyan yusutapi; Wowicake qa woowotanna on econpi. 


\section{P S A L M 113.}

9 Iye taoyate woehdaku wicaqu ;

Wicotakuye tawa kin owihankeśniyan hdusuta:

Iye caje kin wakan qa wokokipe.

10 Woksape otokahe Jehowa kokipapi kin hee;

Tona heconpi kin hena owasin wayupikapi :

Iye yatanpi kin he owihanke wanice kta.

\section{PSALM 112.}

1 Jah yatan miye.

Wicaśta Jehowa kokipe cin he cante waśte;

Tawoahope kin on iyuśkin hinca.

2 Iye cinca maka akan waśakapi kta;

Tona ecetupi wicoicage kin he yawaśtepi kta.

3 Woyuha qa wowijice iye ti kin ohna;-

Qa toowotanna kin owihanke wanice kta.

4. Otpaza en wicaśta ecetu iyoyanpa awicahinanpa ;

Waonśida qa wacantkiya qa owotanna.

5 Wicaśta wacantkiye ça wawicaqu kin he waśte;

He wokonze on tohan kin hduśtan lita.

6 Avicakehan ohinniyan yuhohopi kte śni :

Wicaśta owotanina kin owihankeśniyan kiksuyapi kta.

7 Taku śica nahonpi kin on kopehda kte śni ;

Cante yusutapi, Jehowa wacinyan un.

8 Iye cante kin yusutapi, wakokipe kte śni;

Tokawicaya awicatonwe kte cin hehanyan.

9 Ituwicakihan, wahpanicapi taku wicaqu;

Toowotanna kin owihanke wanice kta :

Iye he kin wowitan on wankan un kta.

10 Wicaśta śice cin he wanyake ça śinhda kta;

Hi hdakinskinze ca skan kta :

Wicaśta śica taku cin kin he awihunni kta.

\section{PSALM 113.}

1 Jah yatan miye.

Yatan po, Jehowa taokiye kin ;

Jehowa caje kin he yatan miye.

2 Jehowa caje kin yawaśtepi nunwe;

Detanhan qa owihanke wanica.

3 Wi hinanpe cin hetanhan, iyaye cin hehanyan, Jehowa caje kin yatanpi nunwe.

4 Jehowa tehan wankan nu, oyate owasin iwankam; Iye towitan mahpiya kin iwankam.

5 Jehowa Wakantanka unkitawspi, Tehanwankantu kiyotanke cin he tuwe iyececa he :

6 Temahentuya etonwe cin hee;

Mahpiya en qa maka kin en.

7 Wahpanica kin watuśekśeca etanhan eyaku; 
Onśike cin tacesdi paha kin etanhan yuwankan eyaku kta;

8 Wicaśta itancanpi kin om iyotanke kiye kta; Iye taoyate itancanpi kin om.

9 Winohinca cinca ton śni ti kin He iyuśkinyan cinca hduha iyotanke kiya. ' Jah yatan miye.

\section{PSALM 114.}

1 Israel Egupta etanhan hinanpe cehan; Jakob ti kin oyate wan śa iápi kin hetanhan;

2 Juda he tipi wakan tawa kin icaga; Tokiconze kin Israel hee.

3 Mini tanka kin he wanyake ça najica; Jordan he nakun namni :

4 Paha kin hena tamdoka iyecen ipsicapi; Qa pajodan kin tacinca kin iyececapi.

5 Mini tanka, tonikeca on nayajica he; Jordan, tokeca nayamni he.

6 Paha kin, tokeca tamdoka iyecen iyapsicapi he; Pajodan, tokeca tacinca iyenicecapi he.

\% Maka kin, Itancan kin itokam, cancan wo; Jakob ta Wakantanka kin itokam.

8 He imnija kin minihdoka kaga; Wanhi inyan kin he miniyowe ee kiya.

\section{PSALM 115.}

1 Unkiyepi śni, Jehowa, unkiyepi śni ; Tuka nicaje wowitan qu wo ;

Nitowa onśida on, nitowicake kin on.

2 Tokeca Ikcewicaśta heyapi kta;

Wanna Taku Wakan tawapi tokiya un he.

3 Tuka Wakantanka unkitawapi malipiya ekta yanka; Token iyokipi kin owasin econ ece.

4. Wakagapi tawapi kin hena mazaska qa mazaskazi; Wicaśta nape taku kage cin hena.

5 Hena i yukanpi, tuka iapi śni ; Iśta yukanpi, tuka wawanyakapi śni :

6 Noge yukanpi, tuka winahonpi śni ; Poge yukanpi, tuka taku omnanpi śni :

7 Nape yukanpi, tuka wiyuzapi śni ; Siha yukanpi, tuka manipi śni; Nakun dote eciyatanhan ho enapeyapi śni.

8 Tona kagapi kin hena iś iyececapi lkta ; Tona hena wacinyanpi kin owasin.

9 Israel, niye Jehowa wacinyan wo; Owicakiye ca wahacanka tawapi kin he iye.

10 Aaron ti kin, Jehowa wacinyan wo; 
Owicakiye ça wahacanka tawapi kin he iye.

11 Jehowa knyakipapi kin, Jehowa wacinyan po;

Owicakiye ca wahacanka tawapi kin he iye.

12 Jehowa unkiye unkiksuyapi; wayawaśte kta; Israel ti kin wicayawaśte kta;

Aaron ti kin wicayawaśte kta.

13 'Tona Jehowa kokipapi kin hena wicayawaśte kta;

Cistinpidan tankinkinyanpi om.

14 Jehowa niyuotapi nunwe;

Niyepi qa nakun nicincapi kin.

15 Jehowa eciyatanhan niyawaśtepi ;

Mahpiya qa maka kage cin hee.

16 Mahpiya mahpiya kin he Jehowa tawa;

Tuka maka kin Adam cinca wicaqu.

17 Tona țapi kin hena Jah yatanpi śni ;

Tona woinina ekta yapi kin owasin nakun.

18 Tuka unkiye Jah unyawaśtepi kta;

Detanhan qa owihanke wanica.

Jah yatan miye.

\section{PSALM 116.}

1 Jehowa waśtewadaka,

Miho qa mita wocekiye nahon kin heon.

2 Iye anohmakiye cin heon, Mitaanpetu kin en, hoyewakiye kta.

3 Wiconte icaśke kin aohdutemayan; Qa Hades wowayazan kin mayuze; Wokakije woiyokiśica ko iyewaye.

4 Hehan Jehowa caje kin hoyewakiye kta: Jehowa, ceciciya ce, minagi ehdaku wo.

5 Jehowa wacantkiye ca owotanna; Qa Wakantanka unkitawapi kin he waonśida.

6 Jehowa ksapapi śni kin hena awanwicayaka: Miye kun ehpemayanpi, unkan nimayan.

7 Minagi, woozi nitawa ekta hdicu wo; Jehowa tanyan ecanicon lkin heon etanhan.

8 Niye wiconte etanhan minagi edaku; Miiśta kin ceyapi etanhan, Misiha hicahanpi etanhan edaku.

9 Jehowa itokam mawani kta, Wiconi makoce kin en.

10 Wicawada, heon iwaa: Nina makakija qon.

11 Nihinmiciye cin en hepa; Wicaśta owasin itonpi śni ce.

12 Jehowa taku ota maqu kin, Taku on wecicajuju kta he.

13 Wiconi miniyatke kin iwacu kta, 
Qa Jehowa caje kin hoyewakiye kta.

14. Jehowa taku iwahowaye cin wecicajuju kta, Iho, iye taoyate owasin wicitokam.

15 Tona wacantkiye tawa kin hena tapi kin He Jehowa iśta kin en tehika.

16 Tokin, Jehowa, nitaokiye miyen; Nitaokiye, nitaokiyewin cinhintku kin miyen: Makaśkapi kin mayakidukca.

17 Wopida wośna wan waciciyuśna kta; Qa Jehowa caje kin hoyewakiye kta:

18 Jehowa taku iwahowaye cin wecicajuju kta, Iye taoyate owasin wicitokam:

19 Jehowa ti hocoka kin en; Jerusalem, niye cokaya. Jah yatan miye,

\section{PSALM $11 \%$.}

1 Oyate owasin, Jehowa yatan miye ; Wicaśta hiyeye cin owasin, he idowan po.

2 Towaonśida kin unkiyepi en tanka hinca; Qa Jehowa towicake kin he owihanke wanica. Jah yatan miye.

\section{PSALM 118.}

1 Jehowa wopida eciya po, iye waśte heon; Towaonsida kin he owihanke wanica.

2 Tokin Israel heyapin ; Iye towaonsida kin he owihanke wanica.

3 'Tokin Aaron ti kin heyapin; Iye towaonśida kin he owihanke wanica.

4. Tokin tona .Jehowa kokipapi kin heyapin; Iye towaonsida kin he owihanke wanica ce.

5 Wokaśke etanhan Jah hoyewakiya; Owanka tanka en Jah amayupta.

6 Jehowa mici un; wakowakipe kte śni; Wicaśta akantu taku ecamicon okihi he.

7 Jehowa mici un, tona omakiyapi kin ehna; Hecen tona śicemadapi kin awicawatonwe kta.

8 Jehowa wacinyanpi kin he waśte, Wicaśta wacinwicayapi kin isanpa.

9 Jehowa wacinyanpi kin he waśte; Wicaśtayatapi wacinwicayapi kin isanpa.

10 Ikcewicaśta owasin aohdutemayanpi ; Tuka Jehowa caje kin on bawicawaspe kta.

11 Aohdutemayanpi, han, aohdutemayanpi ; Tuka Jehowa caje kin on bawicawaśpe kta.

12 'Tulimaga iyececa aohdutemayanpi ; 
Wapepeka ide kin iyececa bowicasnipi ;

Tuka Jehowa caje kin on bawicawaśpe kta.

13 Wacayape, mahinhpaye kta on camayapa;

Tuka Jehowa omakiya.

14 Mitowaśake qa mitadowan kin Jah hee;

Nakun he wanikiya mitawa.

15 Wiconi qa wowiyuśkin ho kin he

Wicaśta owotanna tawakeya kin ohna:

Jehowa nape etapa kin he wowaśake kaga.

16 Jehowa nape etapa hduwankan icu ;

Jehowa nape etapa kin he wowaśake kaga.

17 Mațe kte śni, tuka wani kta;

Qa Jah ohan kin omdake kta.

18 Awicakehan Jah nina iyopemayan;

Tuka wiconte ekta yemaye śni.

19 Woowotanna tiyopa kin makiyulidoka ye ;

Hena ohna hibu kta, Jah wopida ewakiye kta.

20 Jehowa tatiyopa kin he dee;

Ohna wicaśta owotanna tin hiyupi kta.

21 Amayadupta, heon wopida eciciye kta;

Qa.wiconi mitawa kin he niye.

22 Inyan wan ticagapi aktapi śni qon

He oise pa kin icaga ce:

23 He Jehowa eciyatanhan,

Qa unkiśtapi kin en taku wowinihan.

24. Jehowa anpetu kage cin he dee;

En unkiyuśkinpi qa piundapi kta.

25 Ceunniciyapi, Jehowa, dehan niunkiya po;

Ceunniciyapi, Jehowa, dehan wapi unyan po.

26 'Tuwe Jehowa caje on u kin he yawaśtepi :

Jehowa ti kin etanhan unniyawaśtepi.

- 27 Jehowa Iyotan Waśaka qra iyoyanpa unqupi :

Wośnapi kin he hahonta on kaśka po,

Owayuśna he kin en.

28 Iyotan Waśaka mitawa kin he niye,

Heon ciyatan kta;

Wakantanka mitawa, icidowan kta.

29 Jehowa wopida eciya po;

Iye waśte kin heon etanhan;

Towaonśida kin he owihanke wanica.

\section{PSALM 119.}

\section{ALEPH.}

1 Canku ohna ecetupi kin hena cante waśtepi ;

Tona Jehowa toope omanipi kin hena.

2 Tawoyaotanin opapi kin hena cante waśtepi ;

Tona cante ocowasin on akitapi kin hena:

3 Nakun taku śica econpi śni ; 
Tuka iye tacanku kin ohna manipi.

4 Woonspe nitawa yakonza; Awicakehan ounpapi kta e heon.

5 Tokin mitacanku yusutapin, Wokage nitawa owape kta heon.

6 Hehan imaśtece lite śni, Nitawoahope owasin en ewatonwe cinhan.

7 Cante ecetu kin on wopida eciciye kta; Woyaco owotanna nitawa onmaspe kinhan.

8 Wokage nitawa owape lkta; 'Tokin ocowasin ehpemayaye śnin. BETH.

9 Kośka wan taku on tacanku kpakinte kta, Hecen nioie eciyatanhan hduhe kta he.

10 Micante ocowasin on acicita; Nitawoahope etanhan wanuni śni nunwe.

11 Taku ehe cin he cante mahen nawahman; Hecen wacicihtani kte śni.

12 Jehowa, niye niyawaśtepi nunwe; Nitoope kin onspemakiya ye.

13 Nii wayaco kin owasin, Hena miiha on omdaka.

14 Nitawoyaotanin canku kin ohna imduśkin ; Wowijice owasin on iyececa.

15 Wowahokonkiye nitawa awacin waun kta; Qa nitacanku ekta ewatonwe kta.

16 Wokage nitawa en iyuśkin miçiye kta; Nioie kin awektonje kte śni.

GIMEL.

17 Nitaokiye miye wani kta maqu wo ; Kinhan nioie awanmdake kta.

18 Iśta mayukawa wo, kimhan ewatonwe kta ; Nitoope eciyatanhan wicohan wakan.

19 Maka akan unhdaka waun; Nitawoahope kin anamakihbe śni wo.

20 Ohinniyan minagi nitawoyaco

Cantolrpani on kaśuśujapi.

21 Wahanhanicidapi kin hena iyopewicayaya, Wicayaśicapi kin; Hena nitawoahope etanhan nunipi.

22 Waaiapi qa śicedakapi kin yutokan iyemiciciya wo ; Nitawoyaotanin awacanmi kin heon.

23 Wicaśta itancanpi nakun iyotankapi qa amaiapi : Tuka nitaokiye wokage nitawa awacin yanka.

24 Nakun woyaotanin nitawa wowiyuśkin waye; Wicaśta waonspemakiye cin hena ee.

25 Minagi maka mdu en askamic̣iya : 
Nioie eciyatanhan nimayan wo.

26 Mitacanku kin owahdaka;

Unkan amayadupta;

Wokage nitawa onspemakiya wo.

27 Nitawoonspe ocanku kin okahnilmayan ye,

Kinhan niolian wakan kin awacanmi kta.

28 Woiyokiśica on minagi ceya un ;

Nioie eciyatanhan najin makiya wo.

29 Woitonśni canku kin yutokan iyemiciciya wo;

Qa nitoope kin he ionśimakida ye.

30 Wowicake canku kin he wakahniga;

Nitawoyaco kin hena mitokam ewahnaka.

31 Nitawoyaotanin sutaya mduza;

Jehowa, iśtenmaye śni wo.

32 Nitawoahope canku kin ohna waimnanke kta;

Cante mayakidutanka kta, heon etanhan.

HE.

33 Jehowa, wokage nitawa canku ohna amayan wo;

Kinhan, owihanke hehanyan owape kta.

34 Okahilimayan wo, kinhan nitoope awacanmi kta;

Qa cante ocowasin on owape kta.

35 Nitawoahope canku ohna mani mayan wo;

He en wimduskin ece.

36 Nitawoyaotanin ekta cante yemayan ye;

Qa wowijice ekta śni.

37 Takuśni wanyakapi etanhan iș́ta mayuhomni wo :

Nitacanku ohna nimakiya wo.

38 Nitaokiye nioie kin kiciyusuta wo;

He on konicipapi kta.

39 Wowiśtece mitawa kowakipe cin he miciyutokan wo;

Woyaco nitawa kin waśte, heon etanhan.

40 Tho, nitawoonspe kin cantowakpani hinca;

Nitoowotanna kin en nimayan wo.

$$
\text { VAU. }
$$

41 Nakun, Jehowa, nitocantekiye en mau nunwe;

Nitowiconi, nioie kin eciyatanhan.

42 Hecen tuwe taku iyamaonpe cin amdupte kta;

Nioie wacinwaye cin heon etanhan.

43 Nakun mii etanhan wowicake oie kin

Ocowasin ehdaku śni wo;

Woyaco nitawa ape waun kin heon.

44 Hecen nitoope kin ohinniyan ahowape kta;

Owihanke wanica, qa owihanke wanica.

45 Unkan makoce tanka wan omawani kta;

Nitawowahokonkiye awakite cin heon.

46 Nakun wicaśtayatapi wicitokam

Nitawoyaotanin caje mdate kta;

Qa imaśtece kte śni.

47 Nakun nitawoahope en iyuśkin miçiye kta; 
Hena waśtewadake hinca.

48 Nitawoahope waśtewadake cin hena on,

Nape wankan yewaye kta;

Qa wokage nitawa awacin manke kta.

ZAIN.

49 Nioie kin nitaokiye kiciksuya wo;

Wacinye mayakiye cin heon.

50 Makakija en wacintonhnagmaye $\operatorname{cin}$ he dee;

Qa nioie kin he kinimakiya.

51 Wahanicidapi kin hena nina imahahapi;

Tuka nitoope amduśtan śni.

52 Jehowa, nitawoyaco wanakaja kin hena weksuya;

Qa cante waśte miçicaga.

53 Wicaśta śicapi on nina śinwahda;

Nitoope elipeyapi kin heon.

54 Wokage nitawa odowan mitawa kin hee; Ounhdaka tipi mitawa ohna.

55 Jehowa, hanyetu icunhan nicaje weksuya;

Qa nitoope ahowapa ece.

56 Nitawoonspe owape cin he on,

De mduha ece qon.

CETH.

$5 \%$ Jehowa, taku mitawa kin he niye ;

Nioie kin mduhe kta, kepa.

58 Nitocantekiye micante ocowasin on awakita;

Nioie eciyatanhan onśimakida wo.

59 Mitacanku kin awakicanmi ;

Qa nitawoyaotanin ekta misiha hdicu wakiya.

60 Woonspe nitawa mduhe kta e, Heon inawahni qa mdutehan śni.

61 Wicaśta śica ikan tawapi mihdukśan hiyeya ; Tuka nitoope awektonje śni.

62 Ciyatan kta hanyecokaya imdotanke kta, Nitoowotanna woyaco kin hena on.

63 'Tona konicipapi kin owasin kodawicawaya; Qa tona nitawoonspe opapi kin hena.

64. Jehowa, nitownonśida on maka kin ojudan; Wokage nitawa kin onspemakiya wo.

TETH.

65 Jehowa, nitaokiye taku waśte ecayecon,

Nioie kin eciyatanhan.

66 Wiyukcanpi waśte qa wookahnige onspemakiya wo; Nitawoahope wicawada kin heon.

67 Makakije śni he ehan wanuni ;

Tuka nakaha iyae cin he owape.

68 Niye niwaśte qa taku waśte ecanon ece; Wokage nitawa onspemakiya wo.

69 Wahaniçidapi kin hena woitonśni amakagapi ;

Miye qe micante ocowasin on nitawoonspe kin owape kta. 
70 Iye cantepi kin wasna iyecen cepa; Miye qe nitoope on imduśkin.

71 Kakiśmayanpi kin he waśte; He on wokage nitawa onspemiçiciye kta.

72 Nii woope kin he miciwaśte, Mazaska qa mazaskazi kektopawinge isanpa. YOD.

73 Ninape makage ca icahmayan; Onspemakiya wo, kinhan nitawoahope onmaspe kta.

74 Tona konicipapi kin hena wanmayakapi qa wiyuśkinpi kta; Nioie wacinwaye cin heon etanhan.

75 Jehowa, nitawoyaco kin hena woowotanna sdonwaya; Qa wowicake on kakiśmayaya.

76 'Tokin nitowaonśida wacintonhnagmayen:

Nitaokiye taku eyakiye ciqon iyececa.

77 Wacanyakiye cin he mahiyohi kte, hecen wani kta;

Nitoope kin he wowiyuśkinwaye, heon.

78 Wahaniçidapi kin iśtecapi nunwe, Woitonśni on mayapemripi, heon :

Miye qe nitawoonspe awacin manke kta.

79 Tona konicipapi kin hena miye en maupi kte;

Qa tona nitawoyaotanin sdonyapi kin hena.

80 Wokage nitawa en micante zanika un nunwe;

Hecen imaśtece kte śni.

KAPH.

81 Wiconi nitawa on minagi niyaśni iyaya;

Nioie kin on ape waun.

82 Iyae cin on iśta manaka;

Hecen, Tohan cante waśte mayakage kta he, epa.

83 Wojuha wan sota en un kin iyemaceca;

Wokage nitawa kin hena awektonje śni.

84 Nitaokiye taanpetu kin tonakeca he;

Tona śicaya makuwapi kin tohan wicadaco kta he.

85 Wahaniçidapi kin hena wolia miçapi ;

Hena nitoope kin eciyatanhan śni.

86 Nitawoahope kin owasin wowicake;

Woitonśni on śicaya makuwapi; niye omakiya wo.

87 Iśnikaeś makoce kin etanhan ihangmayanpi śni ;

"Tuka nitawoonspe kin amduśtan śni.

88 Nitowaonśida eciyatanhan nimayan wo:

Kinhan ní taku yaotanin kin owape kta.

LAMED.

89 Jehowa, nioie owihanke wanica, Mahipiya kin ekta yusutapi.

90 Wowicake nitawa kin he wicoicage qa wicoicage hehanyan:

Maka kin dusuta, unkan ecen han.

91 Nitawoyaco on anpetu kin dehan hena ecen un;

Hena owasin wowidagyaye cin heon.

92 Nitoope kin he wowiyuśkinwaye śni unkanś, 
Makakije cin en matakuni kte śni tuka.

93 Nitawoonspe kin ohinniyan awektonje kte śni ;

Hena en kinimayakiya nakaeś.

94 'Tawamayaya, nimayan wo;

Nitawoonspe kin awakite cin heon.

95 Wicaśta śica ihangmayan iyapepi ;

Nitawoyaotanin kin hena owakalinige kta.

96 Woecetu kin owasin ihanke kin wanmdaka;

Nitawoahope ohdakinyan tanka hinca. MEM.

97 Nitoope waśtewadake hinca: Anpetu osan he awacin manka.

98 Nitawoahope ksapa makaga, Tokamayanpi kin isanpa;

He ohinniyan mitawa nakaeś.

99 Waonspemakiyapi owasin isanpa, Wacinksamya miohan ;

Nitawoyaotanin kin hena awacin waun.

100 Wicahincapi kin isanpa wowakaliniga;

Nitawoonspe wapatan kin heon.

101 Canku śica owasin etanhan misiha ewehdaku;

Nioie mduhe kta heon etanhan.

102 Nitawoyaco kin icunonpa mde śni;

Niye onspemayakiye cin heon.

103 Micaka en nioie kin skuye hinca;

Tuhmaga canhanpi mii kin ohna he isanpa.

104 Wowahokonkiye nitawa etanhan wookahiniga mduha; Heon woitonśni canku owasin śice wadaka. NUN.

105 Nioie misiha kin en petijanjan hee; Qa mitacanku ohna iyoyanpa kin.

106 Wakonza, nakun ecen ecamon lita; Woyaco owotanna nitawa kin ahowape kta.

107 Miye nina hin makakija; Jehowa, nioie eciyatanhan kinimayan wo.

108 Jehowa ceciciya, mii taku itunicitian kin Hena iyonicipi nunwe;

Qa nitawoyaco kin hena onspemakiya wo.

109 Minagi ohinniyan minape ohna yanka; Tuka nitoope kin he awektonje śni.

110 Wicaśta śica mahmunkapi kta ; Tuka nitawoonspe etanhan wanuni śni.

111 Nitawoyaotanin kin hena ohinniyan mitawa kta; Micante towiyuśkin kin hena ee.

112 Wokage nitawa ecamon kta e cante ekta yewaye; Ohinniyan qa owihanketa he'hanyan.

$$
\text { SAMEK. }
$$

113 Tona cante nonpa kin hena śicewadaka; Tuka nitoope kin he waśtewadaka. 
114 Wowinape mitawa qa wahacanka mitawa kin he niye; Nioie kin he ape manka.

115 Śicaya ecanonpi kin, micuni po:

Kinhan mita Wakantanka tawoahope kin wapatan kta.

116 Wahomayaye cin eciyatanhan najin makiya wo;

Kinhan wani kta;

Qa wacinwaye cin on imaśtece kte śni.

117 Sutaya mayuza wo, kinhan tanyan waun kta;

Qa ohinniyan wokage nitawa en ewatonwe kta.

118 Tona wokage nitawa etanhan nunipi kin

Hena owasin walitewicayada śni ;

Wohnaye tawapi kin he wowiśtece.

119 Ginginca iyececa, maka akan

Wicaśta śica owasin ihangwicayaya;

Heon nitawoyaotanin kin hena waśtewadaka.

120 Kocicipe cin heon micelipi cancan;

Qa nitawoyaco kin hena kowakipa.

AYIN.

121 Woyaco qa woowotanna ccamon ece;

Tehiya makuwapi kin en elipemaye śni wo.

122 Taku waśte on nitaokiye acakśin wo;

Wahaniçidapi kin hena makiyuśepi śni nunwe.

123 Nitowiconi kin on iśta mamdeze śni ;

Qa nakun nitoowotanna oie kin on.

124 Nitocantekiye eciyatanhan nitaokiye ecakicon wo;

Qa wokage nitawa onspemakiya wo.

125 Nitaokiye kin he miye, waokatinilimayan wo;

Qa nitawoyaotanin kin sdony emakiya wo.

126 Jehowa wanna ohanye kta iyececa;

Nitoope kin kicaksapi heon.

127 Heon etanhan nitawoahope waśte wadaka;

Mazaskazi isanpa, han mazaskazi hinca.

128 Heon etanhan nitawoonspe owasin owotanna wadaka;

Woitonśni canku otoiyohi śicewadaka

PE.

129 Nitaw oyaotanin kin hena wowinihan;

Heon etanhan minagi hena ahopa.

130 Nioie yuzamnipi kin he iyoyamya ece;

Tona wacinton śni kin hena wokahinitiwicaya.

131 Mii wahdukawa qa niya śni mața nun seca;

Nitawoahope cantowakpani, heon etanhan.

132 Miye exta ihduhomni qa cantemakiya wo

Tona nicaje waśtedakapi kin iyececa.

133 Nioie kin on mioye kin yasuta wo ;

- Qa taku śica takudan wowidagmaye śni nunwe.

134 Wicaśta wakakiśye cin etanhan opemakiton wo ;

Hecen nitawoonspe kin ahowape kta.

135 Niite kin nitaokiye aojanjanya wo,

Qa wokage nitawa onspemakiya ye. 
136 Miiśta etanhan mini wakpadan kaduza; Nitoope opapi śni kin heon etanhan.

TSADI.

137 Jehowa, niye oniwotanna; Qa nitawoyaco kin hena ecetu.

138 Nitawoyaotanin kin woowotanna eyehde, Qa wowicake hinca.

139 Wacantawahde kin he mahdusota; Tokamayanpi nioie akiktonjapi kin heon.

140 Nioie kin ecedan linca; Heon nitaokiye he waśtedaka.

141 Miye macistinna qa wahitemadapi śni ; Tuka nitawoonspe awektonje śni.

142 Nitoowotanna kin he woowotanna owihanke śni; Qa nitoope kin he wowicake.

143 Wonihinciye qa wokalije mayuzapi; Nitawoahope kin hena wowiyuśkinwaye.

1.44 Nitawoyaotanin kin ohinniyan owotanna; Okalinilimayan wo, kinhan wani kta.

$$
\text { QOPI. }
$$

145 Micante ocowasin on hoyeciciya; Jehowa amayupta wo; Wokage nitawa kin hena owape kta.

146 Houciciya ce, nimayan wo; Kinlan nitawoyaotanin awacanmi kta.

147 Anpao kin en nitokam wau qa houciciya: Nioie kin on ape waun.

148 Miiśta hankiktapi kin kawicapa; Nioie kin awacanmi kta heon.

149 Nitocantekiye eciyatanhan ho namakilion wo; Jehowa, nitawoyaco kin on nimayan wo.

150 Tona taku śica kuwapı kin hena kiyedan unpi; Tuka nitoope kin itehanyan unpi

151 Jeliowa, niye kiyedan yaun; Qa nitawoahope kin owasin wowicake.

152 Wanakajatanhan nitoope kin on sdonwaya; Hena otokahe wanin tanlıan yahduśtan.

$$
\text { REŚ. }
$$

153 Mitokakije wanyake ca emayalsu wo ; Nitoope awektonje śni, heon etanhan.

154 Woakinica mitawa amicinica qa opemakiton wo ; Nioie kin eciyatanhan nimayan wo.

155 Wanikiyapi kin he wicaśta śica itehan un; Wokage nitawa odepi śni kin heon.

156 Jehorva, wocantekiye nitawa kin hena ota; Nitawoyaco kin on kinimakiya wo.

157 Ścaya makuwapi qa tokamayanpi kin hena otapi ; Tuka nitawoyaotanin kin amduśtan śni.

158 Walitanipi sa wanwicamdaka qa wamayazan; 
Hena nioie opapi śni kin heon.

159 Jehowa, nitawoonspe waśtewadake cin he wanyaka ye; Nitocantekiye eciyatanhan nimayan wo.

160 Nioie otokahe kin he wowicake; Qa nitoowotanna woyaco kin owasin owihanke wanica. śIN.

161 Ituh wicaśta itancanpi ścaya makuwapi ; Tuka micante nioie kin kokipa.

162 Taku ehe cin on wimduśkin; Tuwe woyuha tanka iyeye cin iyececa.

163 Woitonśni śicewadake ça walitewada śni ; Nitoope kin he waśtewadaka.

164 Anpetu eca śakowin akihde ciyatan ece; Nitoowotanna woyaco kin hena on.

165 Nitoope waśtedakapi kin hena wookiye ota yuhapi ; Qa takudan ibowicato kte śni.

166 Jehowa, nitowiconi kin ape waun; Qa nitawoahope kin ecamon ece.

167 Minagi nitawoyaotanin kin patan; Qa hena nina waśtewadaka ece.

168 Nitawoonspe qa nitawoyaotanin kin mduha; Miolian owasin nitokam hiyeye cin heon. TAU.

169 Jehowa, waceye cin nitokam ikiyedan u nunwe; Nioic eciyatanhan okalinilimayan wo.

$1 \% 0$ Mitawocekiye nitokam u nunwe; Nioie kin on makiyuśka wo.

171 Miiha woyatan hiyuye kta ; Wokage nitawa onspemayakiye kta heon.

172 Miceji iyae cin ayupta nunwe; Nitawoahope kin owasin owotanna ce.

173 Ninape omakiya un nunwe;

Nitawoonspe kin hena wakahigiga heon.

174 Jehowa, waniyakiye cin he cantowakpani ; Qa nitoope kin he wowiyuśkinwaye.

175 Minagi ni un nunwe, kinhan niyatan kta; Qa nitawoyaco kin omakiya nunwe.

176 'Tahinca wan taninśni iyecen wanuni; Nitaokiye okide wo ; Nitawoahope awektonje śni, heon etanhan.

\section{PSALIM 120.}

\section{Oicimani odowan.}

1 Makakija en Jehowa hoyewakiya : Unkan iye amayupta.

2 Jehowa, woitonśni iha ẹtanhan minagi eyaku wo; Wohnayan ceji kin etanhan.

3 Taku niçu kta he, wohnayan ceji kin ; 
Qa taku sanpa yuheniciye kta he.

4 Wicaśta waśaka tiwanhinkpe pe hinca, Genista petaga koya.

5 Hehehe, miye Meśek en ounwaye; Kedar tawakeyapi kin ohna wati.

6 Tuwe wookiye śicedake cin he Minagi wanna tehan kici ti.

7 Miye wookiye wacin, tuka iwaa eca, Hena e kicizapi kta.

\section{PSALM 121.}

Oicimani odowan.

1 Paha kin ekta miiśta wankan yewaya: Omakiyapi kin he tokiyatanhan une.

2 Omakiyapi kin he Jehowa eciyatanhan, Malipiya maka lio kage cin he.

3 Nisiha naśdute kta iyowinkiye kte śni : Tuwe awanniyake cin he iśtinbe kte śni.

4. Tho, Israel Waawanyake tawa kin He liba kte śni qa iśtinbe kte śni.

5 Waawanyake nitawa kin Jehowa hee; Nietapa ekta ahanziniye cin Jehowa hee.

6 Anpetu eca anpetu wi kin kiunniniye kte śni ; Qa hanyetu wi kin, hanyetu icunhar.

7 Taku śica owasin etanhan Jehowa awanniyake kta ; Ninagi awanyake kta.

8 Tankan de ça tin yahdi eca, Jehowa awanniyake kta, Detanhan qa owilianke wanica.

PSALM 122.

Oicimani odowan. Dawid tawa.

1 Jehowa ti kin ekta unyanpi kta ce, Emakiyapi kin on imduśkin.

2 Jerusalem, nitatiyopa kin en Unsihapi iyahanpi.

3 Otonwe wan icikoyagya he cin He iyececa Jerusalem kagapi.

4. Heciya wicowazipi wankan yapi ece; Jah tawicowazi kin, Israel yaotaninpi kta; Jehowa caje yatanpi kta heon.

5 Hen woyaco oiyotanke kin hdepi; Dawid tiyohnaka oiyotanke tawapi.

6 Jerusalem wookiye kin icekiya po; Tona waśtenidapi kin hena wookiye yuhapi nunwe.

7 Wookiye conkaśke nitawa mahen un nunwe; Qa woozi içiyapi tipi waśteśte nitawa mahen. 
8 Mihunkawanji qa mitakuye on, Wookiye mahen niun nunwe, epe kta.

9 Jehowa Wakantanka unkitawapi ti kin on, Tanyan yaun kta awakite kta.

\section{PSALM 123.}

Oicimani odowan.

1 Mahpiya kin en yakidotanke cin Niye ekta miiśta wankan yewaye.

2 Wan, wicaśta taokiye wicayuhapi napepi kin ekta iśta yeyapi ; Winyan taokiye yuhe cin nape ekta ctonwan ece;

He iyecen unkiśtapi Jehowa Wakantanka unkitawapi ekta etonwanpi, Onśiunkidapi kte cin hehanyan.

3 Onśiunkida po, Jehowa onśiunkida po; Wowahtedaśni on nina iunmnanpi ;

4 Tona owanji yukanpi kin hena Wah̉tedapiśni on unnagipi imnan hinca ; Walianicidapi wowahtedaśni kin he.

\section{PSALM 124. \\ Oicimani odowan. Dawid tawa.}

1 Jehowa unkicipi un śni unkanś; Hecen Israel eyapi kta ;

2 Unkicipi un kin Jehowa ee śni unkanś, Wicaśta akantu anauntanpi qehan;

3 Hehan niyake naunpcapi kta tuka, Wocantahdeunyanpi ide qehan:

4. Hehan mini kin unkiwankam iyaye kta, Wakpa wan unnagipi iwankam iyayc kta;

5 Hehan unnagipi iwankam, Mini ipiga kin iyaye kta tuka.

6 Tye hipi kin on unyahtakapi kta unqupi śni. Heon Jehowa yawaśtepi kte.

7 Unnagipi ihduśke, Zitkadan wan wicaśta wahmunke etanhan he iyececa;

On hmunkapi kin yulidecapi, Unkan unkiye unkiyukcapi.

8 Ounkiyapi kin he Jehowa caje kin en un ; Mahpiya qa maka kage cin he.

\section{PSALM 125.}

\section{Oicimani odowan.}

1 Jehowa wacinyanpi kin hena, Zion Paha kin iyececapi,; Yuhohopi śni, ohinniyan han. 
2 Paha kin Jerusalem ihdukśan hiyeya; Qa Jehowa iye taoyate wicihdukśan un, Detanhan qa owihanke wanica.

3 Wicośice can sagye kin wicaśta owotanna Tamakoce kin akan wanke kte śni; Hecen wicaśta owotanna taku śica Ekta nape yeyapi kte śni.

4 Jehowa, tona waśte taku waśte ecakicon wo; Qa torra cante ecetu kin hena.

5 Tuka tona canku pemni ekta icunom yapi kin, Hena wicohan śica econpi kin om Jehowa iyaye wicaye kta. Israel wookiye kici un nunwe.

\section{PSALM 126.}

Oicimani odowan.

1 Zion hdicu kin en Jehowa ihdohdi qehan, Wihanmnanpi kin iyeuncecapi.

2 Hehan ihapi on i unkojupidan; Qa uncejipi wowiyuślin on: Hehan Ikcewicaśta ehna heyapi; Jehowa taku tanka ecawicakicon ce.

3 Jehowa taku tanka ecaunkiconpi ; On unkiye piundapi.

4 Jehowa, unhdicupi kin en ihdoku wo, Itokali wakpadan kin iyececa.

5 Tona ceya wojupi kin Hena wiyuśkinyan wamnayanpi kta.

6 Ceya ye kta, wojupi opahte yuha ye kta; Wiyuśkinyan ku kta, Aguyapi palitapi hduha ku kta.

\section{PSALM $12 \%$.}

Oicimani odowan. Solomon tawa.

1 Tipi kin Jehowa kage śni kinhan, He en tona kagapi kin ituya śkanpi. Jehowa otonwe kin awanyake śni kinhan, Waawanyake kin ituya kiktahan un.

2 Ituh hanhanna hin yektapi ;

Tehan oziniçiyapi śni;

- Wicohtani woyute yatapi kta. Waśtekidake cin hecen woiśtinma qu ece.

3 Iho, wicacinca Jehowa taku wicaqu kin hee; Wicatamni oicage cin he wokajuju ee.

4 Wanhinkpe wicaśta waśaka nape ohna, Wicakośka cinca kin hena iyececa.

5 Tuwe hena on wanju okikju kinhan, Wicaśta kin he cante waśte hinca: 
Hena iśtecapi kte śni;

Tiyopa kin ohna toka om wohdakapi kta.

\section{PSALIM 128.}

\section{Oicimani odowan.}

1 Jehowa kokipapi kin otoiyohi cante waśte; Iye tacanku ohna manipi kin hena.

2 Ninape ohtani kin he yahdute cinhan, Cante niwaśte hinca, qa tanyan nanke lista.

3 Nitawin hastanyanka iyuwi waicage cin he iyececa, Tipi nitawa mahen ;

Nicinca kin hena Oliwe ojupi kin iyececapi, Wahna wotapi nitawa ihdukśan.

4 Wanyaka wo, wicaśta Jehowa kokipe cin, He hecen yawaśtepi kta.

5 Jehowa Zion etanhan niyawaśte kte; Qa Jerusalem towaśte kin wandake kta, Yani kin anpetu owasin.

6 Nakun nicinca cinca kin wanyahdake kta. Israel wookiye kici un nunwe.

\section{PSALM 199.}

\section{Oicimani odowan.}

1 Komaśka ehantanhan ota kakiśmayanpi ce; Israel eye kta iyececa;

2 Komaśka ehantanhan ota kakiśmayanpi, Tuka maktepidan śni.

3 Mahiyumdupi kin mitapete ayumdupi ; Cankuyetonton hanskaska kagapi,

4 . Tehowa owotanna; Wicaśta śica ikan tawa kin he bapsaka.

5 Tona Zion śicedakapi kin hena Owasin iśtecapi qa hekta hdicuyapi kta.

6 Tipi akan wato icage cin he iyececapi kta; He yujunpi śni itokam śnija ece.

7 He on aguyapi bakse cin nape okikju śni ; Qa tuwe pahite cin he maku en ohnaka śni.

8 Nakun tona en hiyayapi kin heyapi śni ; Jehowa tawoyawaśte en niunpi nunwe; Jehowa caje on unniyawaśtepi ce, eyapi śni.

\section{PSALM 130. \\ Oicimani odowan.}

1 Jehowa, wośbe kin etanhan, houciciya ece.

2 Itancan mihorkin nahon ye ; Wocekiye mitawa ho kin anohkiya wo. 
3 Jah, woahitani cajeyakiyate cinhan, Itancan, tuwe en najin okihi kta he.

4 Tuka wokajuju kin he niye duha;

Hecen konicipapi kta.

5 Jehowa ape waun, minagi ape un;

Qa iye oie kin wacinwaye.

6 Minagi Itancan kin akipe;

Anpao kta ape unpi kin isanpa;

Anpao kta ape unpi kin hena.

7 Israel, Jehowa wacinyan wo;

Jehowa wowaonśida hduha;

Qa wopekitonpi untkanna kici un.

8 Unkan iye Israel opekiton kta,

Wahtani owasin etanhan.

\section{PSALM 131.}

Oicimani odowan. Dawid tawa.

1 Jehowa, micante wahianicida śni,

Qa miiśta wankantuye śni ;

Nakun taku tanka kin en opimiçiye śni,

Qa taku miwankam hiyeye cin.

2 Awicakehan minagi wahduwahbadan,

Qa wahduiniua;

Tuwe hunku azin ehpeye cin iyececa;

Azin enakiya wanji minagi iyececa.

3 Israel, Jehowa wacinyan wo;

Detanhan qá owihanke wanica.

PSALM 132.

\section{Oicimani odowan.}

1 Jehowa, Dawid on kiksuya wo, Tawokakije kin owasin.

2 He Jehowa elrta içiconza; Awicakehan Wanji Waśaka Jakob tawa kin heciya;

3 Wakeya ohna wati kin en wahde kte śni ; Ohehdepi akan manke cin en mde kte śni;

4 Miiśta woiśtinma waqu kte śni; Qa miiśtojuha, owicagunga ;

5 Jehowa owanka wan iyeweciye śni ; Tipi wan Wanji Waśaka Jakob tawa kin on.

6 Iho, Ephrata en he naunlionpi: Can wojupi kin ohna iyeunyanpi ce.

7 Iye ti kin en unkupi kta; Iye siha hde kin en canpeśka makehde inaunjinpi kta.

8 Jehowa najin wo, woozi nitawa kin en; Niye qa nitowaśake can koka kin.

9 Wawayuśnapi nitawa woowotanna koyakapi kte; 
Qa wicaśta wacantkiya nitawa iyaśapi nunwe.

10 Dawid nitaokiye kin on etanhan,

Sdayakiye cin ite kin hduhomni śni wo.

11 Jehowa wowicake eciyatanhan Dawid heciya;

Etanhan namni kte śni ;

Nitancan etanhan icage cin he

Oiyotanke nitawa akan iyotangwakiye kta ce.

12 Nicinca wicotakuye mitawa patanpi kinhan;

Qa mitawoyaotanin onspewicawakiye cin hena;

Hehan nakun iye cinca kin ohinniyan

Oiyotanke kin akan nici iyotankapi kta.

13 Jehowa Zion hdahniga;

Ohna ti kta cin kin heon.

14. Ohinniyan woozi mitawa kin he dee;

Den manke kta, wahdaliniga heon etanhan.

15 Tawoyute woyawaśte on mdawaśte kta;

Walpanicapi tawa kin hena

Aguyapi on imnawicawaye kta.

16 Nakun wawayuśnapi tawa kin

Hena wiconi koyagwicawaye kta;

Qa wicaśta wacantkiya tawa kin

Hena iyaśapi, iyaśapi kta.

17 Hen Dawid he wan icahweciye kta;

Petijanjan wan Sdawakiye cin piweciya.

18 Tona tokawicaye cin wowiśtece koyagwicawaye kta.

Tuka iye akan tawateśdake kin hica kta.

\section{PSALM 133.}

Oicimani odowan. Dawid tawa.

1 Wanyaka wo, hunkawanjinkiciyapi

Nakun witaya tipi kin

He waśte qa wiciyokipi linca.

2 He wihdi waśte kin iyececa, pa akan,

Putinhin ekta kaduza, Aaron putinhin tawa;

Qa taonhdohda okagege kin ekta iyahde.

3 Hermon cu kin iyececa,

Zion paha kin ahinhe cin hee:

Hen Jehowa woyawaśte wan u śi ;

Owihanke wanin wiconi kin hee.

\section{PSALM 134.}

Oicimani odowan.

1 Tho, Jehowa taokiye kin owasin, Jehowa yawaśte miye ;

Hanyetu eca Jehowa ti kin ohna nayajinpi kin.

2 Oyanke wakan kin ekta nape kiyugata po;

Qa Jehowa yawaśte miye. 
3 Jehowa Zion etanhan niyawaśte kta; Tuwe malipiya maka ko kage cin he.

PSALM 135.

Jah yatan miyé.

1 Jehowa caje kin he yatan po;

Jehowa taukiye yaunpi, he idowan po.

2 Tona Jehowa ti kin ohna nayajinpi kin; Wakantanka unkitawapi ti hocoka en yaunpi :

3 Jah yatan miye; Jehowa waśte; Iye caje kin idowan po, he oiyolkipi nakaeś.

4 Jehowa Jakob hdahniga; Israel tawaye kte cin heon.

5 Jehowa tanka hinca he sdonwaya;

Qa Itancan unkitawapi taku wakan owasin isanpa.

6 Jehowa taku cin kin owasin econ ece; Mahpiya kin ekta qa maka akan; Miniwanca qa wośbe owasin en.

7 Maka ihanke etanhan opo wankantkiya iyayeya; Magaju kin wakanhdi kicaga;

Mazopiye tawa etanhan tate kin hiyu kiya.

8 Egupta en tokaheya tonpi kin wicakaśtaka;

Wicaśta qa nakun wanunyanpi kin.

9 Worvapetokeca qa taku wowinihan,

Egupta, niye en ukiye ciqon;

Pharoh qa taokiye kin owasin en.

10 Oyate ota wicakaśtaka, Qa wicaśtayatapi waśakśaka wicakte:

11 Sihon, Amori wicaśtayatapi kin;

Qa Og, Baśan wicaśtayatapi ;

Qa Kanan wokiconze kin owasin:

12 Qa makoce tawapi qon yuhapi kta wicaqu;

Israel iye taoyate yuhapi kta.

13 Jehowa, nicaje kin he owihanke wanica:

Jehowa, niksuyapi kin he

Wicoicage qa wicoicage liehanyan.

14 Jehowa iye taoyate kin hdaco kta;

Qa iye taokiye kin hena on ihduhomni kta.

15 Ikcewicaśta taku wakan tawapi kin

Hena mazaska qa mazaskazi ;

Wicaśta nape on kagapi.

16 I yukanpi, tuka iapi śni;

Iśta yukanpi, tuka wawanyakapi śni ;

17 Noge yukanpi, tuka winationpi śni;

Nakun ipi ohna oniya wanica.

18 Tuwe hena kage cin hena iyececapi;

Nakun tona wacinyanpi kin owasin.

19 Israel tiyohnaka, Jehowa yawaśte po; 
Aaron tiyohnaka, Jehowa yawaśte po.

20 Lewi tiyohnaka, Jehowa yawaśte po; Jehowa koyakipapi kin, Jehowa yawaśte po.

21 Jehowa Zion etarhan yawaśtepi nunwe, Jerusalem en ounye cin. Jah yatan miye.

\section{PSALM 136.}

1 Jehowa waśte, hecen wopida eciya po; Towaonśida kin he owihanke wanica.

2 Taku wakan ta Wakantanka kin wopida eciya po; Towaonśida kin he owihanke wanica.

3 Itancanpi kin en Itancan kin wopida eciya po; Towaonśida kin he owihanke wanica.

4 Tuwe iśnana taku tanka wowinihan econ kin; Towaonśida kin he owihanke wanica.

5 'Iuwe woksape on mahpiya kage cin; Towaonsida kin he owihanke wanica.

6 'Tuwe maka kin mini akan yumdaya; Towaonśida kin he owihanke wanica.

7 Tuwe iyoyanpa tanka kage cin; Towaonsida kin he owihanke wanica.

8 Anpetu wi anpetu en ounye kte cin; rowaonśida kin he owihanke wanica.

9 Hanyetu wi wicanhpi ko hanyetu en ounyanpi kta; Towaonśida kin he owihanke wanica.

10 Tuwe Egupta en tokaheya wicatonpi wicakaśtake ciqon; Towaonśida kin he owihanke wanica.

11 Qa wicacokaya tanhan Israel ewicahdaku; Towaonśida kin he owihanke wanica.

12 Nape waśake ca isto hdugate cin on ; Towaonśida kin he owihanke wanica.

13 Tuwe Mde śa kin yukinukan ehnaka; Towaonśida kin he owihanke wanica.

14 Qa cokaya Israel iyayeye cin; Towaonśida kin he owihanke wanica.

15 Tuka Pharo qa ozuye tarva kin Mde śa kin en elpewicaya; Towaonśida kin he owihanke wanica.

16 Tuwe iye troyate liewotahedan yus awicaye cin; Towaonsida kin he owihanke wanica.

17 Tuwe wicaśtayatapi tanka wicakaśtaka; Towaonśida kin he owihanke wanica.

18 Qa wicaśtayatapi waśaka wicakte; Towaonśida kin he owihanke wanica.

19 Silion, Amori wicaśtayatapi kin; Towaonśida kin he owihanke wanica.

$20 \mathrm{Qa} \mathrm{Og}$, Baśan wicaśtayatapi kin; Towaonśida kin he owihanke wanica. 
21 Qa tamakocepi kin yuhapi kta wicaqu; Towaonśida kin he owihanke wanica.

22 Iye taokiye Israel yuhe kta; Towaonśida kin he owihanke wanica.

23 'Tuwe unwahpanicapi en unkiksuyapi ; Towaonśida kin he owihanke wanica.

24 Qa tokaunyanpi kin etanhan eunhdakupi; Towaonśida kin he owihanke wanica.

25 Tuwe celipi ton owasin woyute wicaqu; Towaonśida kin he owihanke wanica.

26 Mahpiya ta Wakantanka kin wopida eciya po; Towaonśida kin he owihanke wanica.

\section{PSALM $13 \%$.}

1 Babel tawakpa kin icahda, Hen unkiyotankapi, nakun unceyapi, Zion unkiksuyapi qehan.

2 He cokaya cohwanjica kin en, Candowankiyapi unkitawapi otkeunyanpi.

3 Hen wayaka unyuhapi dowanpi oie kin unkidapi; Qa waunkipi kin hena wowiyuśkin dapi ; Zion tadowan etanhan unkicidowan po, eyapi.

4 Token Jehow a tadowan kin, Makoce tokeca en, unkahiyayapi kta he.

5 Jerusalem aciktonje cinhan, Minape etapa kin akiktonja nunwe :

6 Miceji macaka en askapa nunwe, Ciksuye śni kinhan; Mitowiyuśkin tokapa ekta Jerusalem ewahnake śni kinhan.

7 Jehowa, Edom cinca kin ekta, Jerusalem taanpetu kin kiksuya wo: Yujuju po, yujuju po, Taku ahdehe cin hehanyan, eyapi.

8 Babel cunwintku, ihangyapi kte cin, 'Tuwe ecaunyeconpi kin iyecen

Nicicajuju kirhan he carte waśte kta.

9 Tuwe hokśiyopa nitawa iyawicahpaya, Qa inyan en elpewicaye cinhan, he cante waśte kta.

\section{PSALM 138.}

Dawid tawa.

1 Micante ocowasin on wopida eciciye kta; Taku wakan kin wicitokam icidowan kta.

2 Tipi wakan nitawa ekta canpeśka makehde inawajin kta ; Qa nicaje mdatan kta, nitowaonśida qa nitowicake on: Nioie kin nicaje ocowasin isanpa tanka yakaga heon. 
3 Anpetu en hoyewaya, hehan amayadupte; Minagi en wowaśake on waditag mayaya.

4 Jehowa, maka wicaśtayatapi owasin niyatanpi kta; Nii oie kin nahonpi kinhan.

5 Qa Jehowa tacanku ohna dowanpi kta; Jehowa towitan kin he tanka nakaeś.

6 Jehowa tehanwankantu eśta kun unpi kin awiçatonwan; Tuka wahanicidapi kin telıantanhan sdonwicaya.

7 Wokakije cokaya mda eśta nimayaye kta; Tokamayanpi śinhdapi kin ekta nape yahdugate kta; Qa ninape etapa on nimayaye lita.

8 Jehowa taku miciyuśtan kta: Jehowa, nitowaonśida kin he owihanke wanica: Ninape taku kage cin elpeye śni wo.

\section{PSALM 139.}

Dowan itancan kin kicagapi. Dawid tadowan.

1 Jehowa, mayadukcan qa sdonyaya;

2 Imdotanka nawajin ko sdonyaya; Mitawacin tehantanhan oyakaliniga.

3 Omawani qa imunke cin wapetogyaton; Qa miolian kin owasin tanyan idukcan.

4 Miceji en wicoie wanjidan eśta, Iho, Jehowa, niye ocowasin sdonyaya.

5 Milieyata qa mitokam aohdutemayaya, Qa ninape amayahnaka.

6 Wosdonye kin he okahinil owakihi śni; Tehan wankantu, hecen owakihi kte śni.

\% Nitaniya etanhan toki mde kta he; Qa niite etanhan toki nawajin kta he.

8. Wankan mahpiya ekta mde cinhan, hen niye yaun, Qa Hades en owinśwaton kinhan, inyun, niye.

9 Anpao hupahu kin wakamdage kta; Miniwanca ihanke kin en ounwaye kta:

10 Heciya eśta ninape amayan; Qa nape nietapa kin mayuza.

11 Awicalkehan otpaza amahinhipaye kta, epa eśta; Hehan hanyetu kin mihdukśan iyoyanpa.

12 Otpaza hee kaeś aotpasniye śni ; Qa hanyetu kin anpetu iyecen iyojanjan: Otpaza kin he iyoyanpa kin iyececa.

13 Mitapakśin kin niye yakaga; Ina tamni mahen akahpemayaton.

14 Wowinihanyan makagapi heon ciyatan kta; Niohan kin hena wowinihan; Qa he minagi tanyan sdonya.

15 Woinahbe en makagapi qehan, Mahuhu kin ananicitimanpi śni, 
Maka ośbe kin en makazontapi qehan.

16 Wiyapemni waun qon niiśta wanmayaka, Qa mitaanpetu icage kte cin,

Hinahin wanica ehan,

Owasin nitawowapi kin en owapi.

17 Unkan Iyotan Waśaka nitawacin kin hena nina tewahinda; Hena yuwitayapi kin tanka hinca.

18 Hena mdawa kta, tuka wiyaka kin isanpa ota:

Wekta qa hinaliin cici waun.

19 Wakantanka, awicakehan wicaśta śica yakte kta: Heon we wicaśta yaunpi kin, amayuśtan po.

20 Hena taku śica on cajeniyatapi ece:

Tokaniyanpi kin ikcekceya cajeniyatapi.

21 Jehowa, tona śicenidapi kin hena śicewicawada kte sni he; Qa tona nicipajinpi kin hena wahtewicawada kte śni he.

22 Wośicedake wowicake on śicewicawadaka;

Hena tokawicawaya iyececa.

23 Wakantanka, imayukcan qa micante sdonya wo, Imayute qa micanteoze kin sdonya wo.

24 Qa wokakije canku wan en maun kinhan wanyaka wo: Qa canku owihanke wanice cin en amayan wo.

\section{PSALM 140.}

Dowan itancan kin kicagapi. Dawid tadowan.

1 Jehowa, wicaśta śice cin etanhan emayaku wo; Wicaśta wohitika etanhan mayakpatan kta.

2 Hena cante mahen wicolian śica awacinpi ; Anpetu osan wokicize on mniciyapi ece.

3 Ceji hdumanpi, wamduśka iyececa; Ihapi mahen sintehda tage yukan. Sela.

4 Jehowa, wicaśta śica nape etanhan emayaku wo; Wicaśta wawicaki sa etanhan mayakpatan kta; Hena mioye yutelii wacinpi.

5 Wahanicidapi kin hena, On hmunkapi wan emaonpapi, hahonta nakun; Canku icahda wohmunke wan ehnakapi ; Qa mazahtakiyapi emaonpapi. Sela.

6 Wakantanka mitawa kin he niye, Jehowa ewakiya; Jehowa, wocekiye mitawa ho kin nahon wo.

7 Jehowa Itancan, wowaśake wanimakiye cin; Kicizapi anpetu kin en pa akahpemayaton.

8 Jehowa, wicaśta śica taku cin kin ayupte śni wo; Taku awacin kin ecetu śni kiya wo; Wahanicidapi kta. Sela.

9 Tona mihdukśan hiyeye cin

Hena iha taku wayusice cin pa ahdahpapi kta.

10 Petaga aihipewicayapi kta;

Peta en iyewicaye kta, qa mini śma en: 
Hecen ake najinpi kte śni.

11 Wicaśta iwakan kin hena

Makoce kin en yusutapi śni nunwe :

Taku śica wicaśta wawicaki sa ihangya kuwa kta.

12 Jehowa wicaśta onśika woowotanna,

Qa wicaśta wahpanica kin woyaco ecakicon kta sdonwaya.

13 Wicaśta owotanna ecedan nicaje yatanpi kta;

Wicaśta ecetupi kin nitokam iyotankapi kta.

\section{PSALM 141.}

Dawid tadowan.

1. Jehowa, houciciya, en mau inahini ye;

Ceciciya eca maho kin nalion wo.

2 Wocekiye mitawa azintonpi kin nitokam un nunwe;

Minape yugatapi hitayetu wośnapi kin iyececa.

3 Jehowa, mii kin en akicita najin wicaśi wo;

Miiha tiyopa kin awanyaka ye.

4 Wicoie śica wan ekta micante yekiye śni wo ;

Wicaśta woahtani econpi kin om,

Wicośice en htawani kte śni.

Qa woyute waśte yuhapi kin wate kte śni.

5 Wowaonśida on wicaśta owotanna amape;

Qa iyopemayan; he ihepi ee kta,

Mapa tehinda kte śni ;

Tuka nakun iye kakijapi kin en cewakiye kta.

6 Wayaco tawapi kin hena inyan en ehpewicayapi:

Unkan mioie nahonpi, hena skuya nakaeś.

7 Tuwe maka yumdu qa yuksaksa iyececa,

Huhu unkitawapi Hades i kin en kada ehpeyapi.

8 Jehowa Itanean, miiśta kin niye elkta yanke ;

Wowacinye mitawa kin he niye;

Minagi papson śni wo.

9 Mazalitakiyapi emaonpapi kin etanhan imacu wo ;

Qa woalitani econpi wohmunke tawapi etanhan.

10 Wicaśta śica wohmunke iye tawapi en elpeiçiyapi nunwe; Tuka miye qe sam imdamde kta.

\section{PSALM 142.}

Dawid tawoonspe, makolicloka en un qehan. Wocekiye wan.

1 Miho kin on Jehowa hoyewakiya;

Miho kin. on Jehowa cewakiya.

2 Iye itokam taliu awacanmi wakpapson;

I tokam woiyokiśice mitawa owahdaka.

3 Mahentanhan mitaniya ktepidan;

Tuka mitacanku kin niye sdonyaya;

Canku ohna mde cin on wohmunke wan emakionpapi.

4 Etapa tanhan etonwe ca wanyaka wo; 
Unkan tuwedan śdonmaye śni;

Wowinape takudan mduhe śni;

Tuwedan minagi ekta ctonwe śni.

5 Jehowa, niye houciciya:

Wiconi makoce kin en taku mitawa,

Wowinape mitawa kin he niye, epa.

6 Waceye cin he nation wo, nina makakija;

Tona śicaya makuwapi etanhan emayaku wo;

Hena mikapeya waśakapi nakaeś.

7 Wicakaśka tipi kin etanhan minagi icu wo,

Hecen nicaje mdatan kta :

Tanyan ecamiyecon kinhan,

Wicaśta owotanna mihdukśan hiyeye kta.

\section{PSALM 143.}

Dawid tadowan.

1 Jehowa, wocekiye mitawa nahon wo;

Onśiya hoyewaye cin he anolikiya ye :

Nitoowotanna eciyatanhan nitowicake on amayupta wo.

2 Qa nitaokiye kici woyaco akite śni wo;

Niye nitokam ni unpi kin tuwedan owotanna śni.

3 Toka kin he minagi śicaya kuwa;

Wiconi mitawa makata elipeya;

Wanakaś tapi kin iyecen otpaza en ounye makiya.

4 Heon mahentanhan mitaniya aopaskicapi ;

Mahentanhan micante atakuni śni.

5 Anpetu wanakaja kin hena weksuya;

Niohan kin owasin awacanmi ;

Ninape taku kage cin hena awacin waun.

6 Minape ciciyugata;

Maka puze cin iyecen minagi nitokam wanka. Sela.

7 Inalini wo, Jehowa amayupta wo;

Minagi atakuni śni.

Niite inalibe makiye śni wo ;

Okinni wicahapi ekta yapi kin iyemacece kta.

8 Hanhanna eca wocanteliye nitawa nalion mayan wo ;

Niye wacinciya, heon :

Canku ohna mde ktc cin he sdonye mayan wo;

Niye ekta minagi wahdomda, heon etanhan

9 Tokamayanpi kin etanhan emayaku wo;

Jehowa, niye en inawahbe.

10 Nitawacin ecamon kta onspemakiya wo;

Taku Wakan mitawa kin he niye:

Nitaniya kin he waśte;

Makoce omdaya en amayan nunwe.

11 Jehowa, nicaje on etanhan nimayaye kta;

Nitoowotanna on minagi wokakije etanhan inape yaye kta.

12 Nakun nitowaonśida on tokamaye cin ihangyaye kta; 
Qa tona minagi iyokiśinyapi kin owasin atakuniśni iyewicayaye kta;

Nitaokiye kin he miye, heon etanhan.

\section{PSALM 144.}

Dawid tawa.

1 Jehowa, Imnija mitawa, yawaśtepi nunwe;

He minape zuyapi onspekiya;

Qa minapsukaza, kicizapi kin.

2 Wacanmakiye ça conkaśke mitawa,

Wowinape mitawa qa emahdaku ece;

Wahacanka mitawa qa tuwe wacinwaye cin,

Mitaoyate mihukuya hiyuye cin he.

3 Jehowa, wicaśta kin taku he, on sdonyaye kta;

Qa wicaśta cinhintku, unkan en yahi kta he.

4 Wicaśta kin taku śni iyececa;

Taanpetu kin ohanzi wan hiyaye cin iyececa.

5 Jehowa, mahpiya nitawa hduhukuye ça kun u wo;

$\dot{H}$ e kin hena yutan wo, kinhan hena izite kta.

6 Wakanhdi hdutanin qa wicakamdeca wo ;

Wanhinkpe nitawa hiyuye ça yuśinye wicaya wo.

7 Wankantanhan ninape hiyukiya wo;

Makiyuśka qa mini ota etanhan imacu wo;

Toka cinca nape kin etanhan.

8 Hena ipi wohnayan iapi ece;

Qa nape etapa tawapi kin he nape itonśni.

9 Wakantanka, odowan teca wan cicahiyaye kta;

Candowankiyapi ikan wikcemna on icidowan kta.

10 He wicaśtayatapi wanikiyapi wicaqu;

Iye taokiye Dawid isan śice cin etanhan icu ece.

11 Makiyuśka qa toka cinca nape kin etanhan imacu wo;

Hena ipi kin wohnayan iapi ece ;

Qa nape etapa tawapi kin he nape itonśni.

12 Hecen unkicinkśipi kośkapi ehan

Taku ojupi tanka icage cin iyececapi lkta;

Unkicunkśipi kin tipi wakan oise

Inyan ayuco bagopi kin iyececapi kta.

13 Dotopiye unkitawapi kin ocaje owasin on ojudan kta:

Tahinca unkitawapi kin kektopawinge cinca tonpi kta,

Qa kektopawinge yuotapi canku unkitawapi ohna.

14 Tatanka unkitawapi yuśakapi kta;

Yuksapi wanica qa yujujupi wanica ;

Qa canku unkitawapi ohna ceyapi wanice kta.

15 Oyate wan hecen unpi kin he cante waśtepi:

Oyate Taku Wakan tawapi kin

Jehowa hee hecinhan he cante waśtepi. 


\section{PSALM 145.}

Dawid tawa. Woyatan.

1 Wakantanka, Wicaśtayatapi mitawa ciyawankantuya kta; Qa nicaje mdawaśte kta, ohinniyan qa owihanke śni.

2 Anpetu owasin ciyawaśte kta ;

Qa nicaje iwadowan kta,

Ohinniyan qa owihanke śni.

3 Jehowa tanka qa nina yatanpi kta iyececa;

Qa tanka kin he iyukcanpica śni.

4 Wicoicage wicoicage iyahdeya taku ecanon kin yatanpi ; Qa niohan waśaka oyalkapi kta.

5 Wotanka nitawa wowitan oiyokipi kin he, Qa wicoie wowinihan nitawa awacanmi kta.

6 Nakun niolian wakan wokokipe kin he oyakapi ; Unkan miye wotanka nitawa mdaotanin kta.

7 Nitowaśte tanka wokiksuye kin he yatanpi ; Qa nitoowotanna kin on iyaśapi ece.

8 Jehowa waonśida qa wacantkiya; Tehan canteptanye śni qa tocantekiye tanka.

9 Owasin ekta Jehowa waśte ; Qa tocantekiye taku kage cin owasin en un.

10 Jehowa, taku icahyaye cin owasin niyatanpi; Qa wicaśta wacantkiya nitawa kin hena niyawaśtepi kta.

11 Nitokiconze wowitan kin he oyakapi; Qa nitowaśake kin cajeyatapi ece.

12 Tohan tanka kin wicaśta cinca sdonyewicayapi kta; Qa tokiconze wotanka wowitan kin.

13 Nitokiconze kin he wolkiconze owihanke wanica ocowasin; Qa wicaśta niyatapi kin he wicoicage qa wicoicage en.

14 Tona hicahanpi owasin Jehowa najin wicakiya; Qa patujapi kin hena owasin wicayubosdata.

15 Taku iśta yuke cin owasin niye anipepi ; Unkan iyehantu eca woyute wicayaqu ece.

16 Ninape yahdumdaya, Qa taku ni un kin owasin imna wicayaya.

17 Tacanku owasin en Jehowa owotanna; Qa ohian owasin en wacantkiya.

18 Jehowa tona hoyekiyapi kin owasin wicikiyedan un; Tona wowicake en hoyekiyapi kin owasin.

19 Tona iye kokipapi taku cinpi kin ecetuye kta; Qa nakun hoyeyapi kin nation, qa niwicaye kta.

20 Tona waśtedapi kin hena owasin Jehowa wicapatan; Tuka śicapi kin hena owasin ihangwicaye kta.

21 Jehowa yatanpi kin he mii cajeyate kta; Qa wicacehpi owasin iye caje wakan kin he yawaśtepi kta; Ohinniyan qa owihanke wanica. 


\section{PSALIM 146.}

1 Halleluya:

Minagi, Jehowa yatan wo.

2 Tohanyan wani kin hehanyan

Jehowa mdatan kta;

Tohanyan en waun hehanyan

Mita Wakantanka iwadowan kta.

3 Wicaśtayatapi wacinwicayapi śni po;

Adam cinca kin, en wanikiyapi wanica.

4 Taniya kin iyaya, hehan maka ekta hda; Anpetu kin he en tawacin kin atakuni śni.

5 Jakob ta Wakantanka tuwe okiye cin he cante waśte; Ta Wakantanka Jehowa he wacinye cinhan.

6 He mahpiya qa maka kin kaga; Miniwanca qa taku ohnaka kin owasin : He wowicake ohinniyan kpatan.

7 Tona śicaya wicakuwapi kin on woyaco econ ece; Wotektehdapi kin hena aguyapi wicaqu; Kaśka unpi kin hena Jehowa wicayukca.

8 Iśtagongapi kin hena Jehowa iśta wicakiyukawa; Pakopi kin hena Jehowa wicayuowotanna; Wicaśta owotanna kin hena Jehowa waśte wicadaka.

9 Jehowa tona oyate tokeca kin hena awanwicayaka; Wamdenica qa wiwazica kin owicakiya ece :

Tuka wicaśta śica tacanku kin yupemni.

10 Jehowa owihanke wanin wicaśtayatapi un kta; Zion, nita Wakantanka ohinniyan qa ohinniyan kta. Halleluya.

\section{PSALM $14 \%$.}

1 Halleluya.

Wakantanka unkitawapi idowanpi kin he waśte;

He wiciyokipi, heon yatanpi kin he wicimdeza.

2 Jehowa Jerusalem piya kaga;

Israel enanakiya iyayapi kin hena mnawicakiya.

3 Tona cante kicaksapi lin okiziwicaya ;

Qa capapi kin pawicakilita ece.

4 Wicanhpi kin tonakeca eśta hena yawa;

Owasin cajeyata ece.

5 Itancan unkitawapi kin tanka, qa towaśake kin ota; Qa tookahnige kin he yawapica śni.

6 Wahpanicapi kin hena Jehowa najin wicakiya; Sicapi kin hena makata elipewicaya ece.

7. Wopida odowan on Jehowa ayupta po: Candowankiyapi on Wakantanka unkitawapi kahiyaya po.

8 He mahpiya kin mahpiya śapa on akahpa;

Maka kin magaju kicaga; 
Qa he kin peji oicaliya ece.

9 Woteca kin woyute wicaqu;

Kangi cinca nakun, ceyapi kinhan.

10 Suktanka towaśake kin he iyokipi śni;

Wicaśta huha kin hena on iyuśkin śni.

11 Tona kokipapi kin hena Jehowa iyokipi;

Tona iye towaonśida apepi kin hena.

12 Jerisalem, Jehowa yatan wo;

Zion, Wakantanka nitawa idowan wo.

13 Nitatiyopa ihupa kin hena yusuta;

Nicokaya nicinca kin wicayawaśte.

14 Nihdukśan wookiye kaga ece;

Aguyapi śin kin on imnaniyan.

15 Taku eye cin he maka kin ekta ye śi ;

Iye oie kin dus inyanka ece.

16 Wa kin he hiyuya, talinca hin iyececa;

Hewanke kin cahnta iyecen kada ece,

17 Wasu tawa kin onśpaśpa, iyecen elipeya ;

Osni tawa itokam tuwe najinhan najin okihi he.

18 Iye oie kin ye śi, qa hena span kiya;

Tate tawa ukiya eca mini kaduza ece.

19 Iye oie kin Jakob okiyaka;

Tawoahope qa tawoyaco kin, Israel en.

20 Oyate kin owasin hecen ecawicalkicon śni;

Qa tawoyaco kin hena sdonyapi śni.

Halleluya.

\section{PSALM 148.}

1 Halleluya.

Mahipiya kin etanhan Jehowa yatan po;

Tehanwankantu ek ta he yatan miye.

2 Taohnihdewicaye cin owasin, he yatan po;

Wicobe tawa iyulipa, he yatan miye.

3 Anpetu wi qa hanyetu wi, he yatan po;

Iyoyanpa wicanlipi owasin, he yatan miye.

4. Mahipiya mahpiya kin, he yatan po ;

Qa mini, mahpiya iwankam un kin nakun.

5 Hena Jehowa caje kin yatanpi kte ;

Iye eren eya, unkan kagapi, heon etanhan.

6 Qa hena yusuta, owihanke śni hehanyan;

Woope wan wicaqu, unkan he acakśinpi śni.

7 Maka kin etanhan, Jehowa yatan miye;

Hogan tankinyanyan qa ośbe kin owasin.

8 Peta qa wasu, wa qa śota ;

Tateiyumni iye oie econ ece kin :

9 Hie kin hena, qa paha owasin ;

Waskuyeca can, qa hante owasin:

10 Wamanica, qa woteca owasin ;

Watutka, qa taku kinyan un kin: 
11 Maka wicaśtayatapi, qa oyate owasin; Wicaśta itancanpi, qa maka akan wayaco owasin :

12 Kośka, qa nakun wikośka;

Wicahinca qa hokśiyopa om :

13 Hena Jehowa caje kin yatanpi kte; Iye caje kin ecedan yatankapi ece;

Towitan kin maka qa mahpiya kin iwankam.

14 Unkan iye taoyate he wan icaliwicakiciya;

Wicaśta wacantkiya tawa owasin on wowitan kin hee;

Israel cinca kin on, oyate wan iye ikiyedan unpi.

Halleluya.

\section{PSALM 149.}

1 Halleluya.

Jehowa odowan teca wan kahiyaya po ;

Towitan kin, wicaśta wacantkiya mniciyapi kin $\mathrm{en}$.

2 Waicahye cin on Israel iyuśkin nunwe;

Zion cinca Wicaśtayatapi tawapi kin on içidowanpi kte.

3 Wacipi kin on iye caje kin yatanpi nunwe;

Cancega cotanka ko on idowan miye.

4 Jehowa iye taoyate kin on iyuśkin;

Onśiicidapi kin hena wiconi koyagwicaya cce.

5 Wowitan on wicaśta wacantliya iyuśkinpi kte;

Towinjapi akan dowanpi nunwe.

6 Iyotan Waśaka yatanpi kin dotepi ohna un;

Qa napepi ohna mazasagye anog ope kin.

7 Hecen Ikcewicaśta ehna watokiçonpi kta ;

Qa oyatepi kin kakiświcayapi kta.

8 Wicaśtayatapi wicayuhapi mazaicicahilia on wicakaśkapi kta ; Qa tona wicakinicapi kin hena maza icaśke on.

9 Hena en woyaco owapi kin yuśtanpi kta;

Wowitan kin de wicaśta wacantkiya tawa owasin yuhapi. Halleluya.

\section{PSALM 150.}

1 Halleluya.

Tipi wakan tawa ohna Wakantanka yatan po;

Wowaśake mahpiya tawa kin ohna idowan po.

2 Wicolian tanka econ kin on yatan miye;

Iyotan tanka kin iyececa idowan po.

3 Mazayahotonpi on yatan miye;

Cotanka candowankiyapi ko on yatan po

4 Cancega wacipi ko on he yatan miye;

Candowankiyapi cotanka ko on yatan po.

5 Mazasnasna hotanka on yatan po;

Mazasnasna wiyuśkin on he yatan miye.

6 Taku niya un kin owasin Jah yatan po.

Halleluya. 


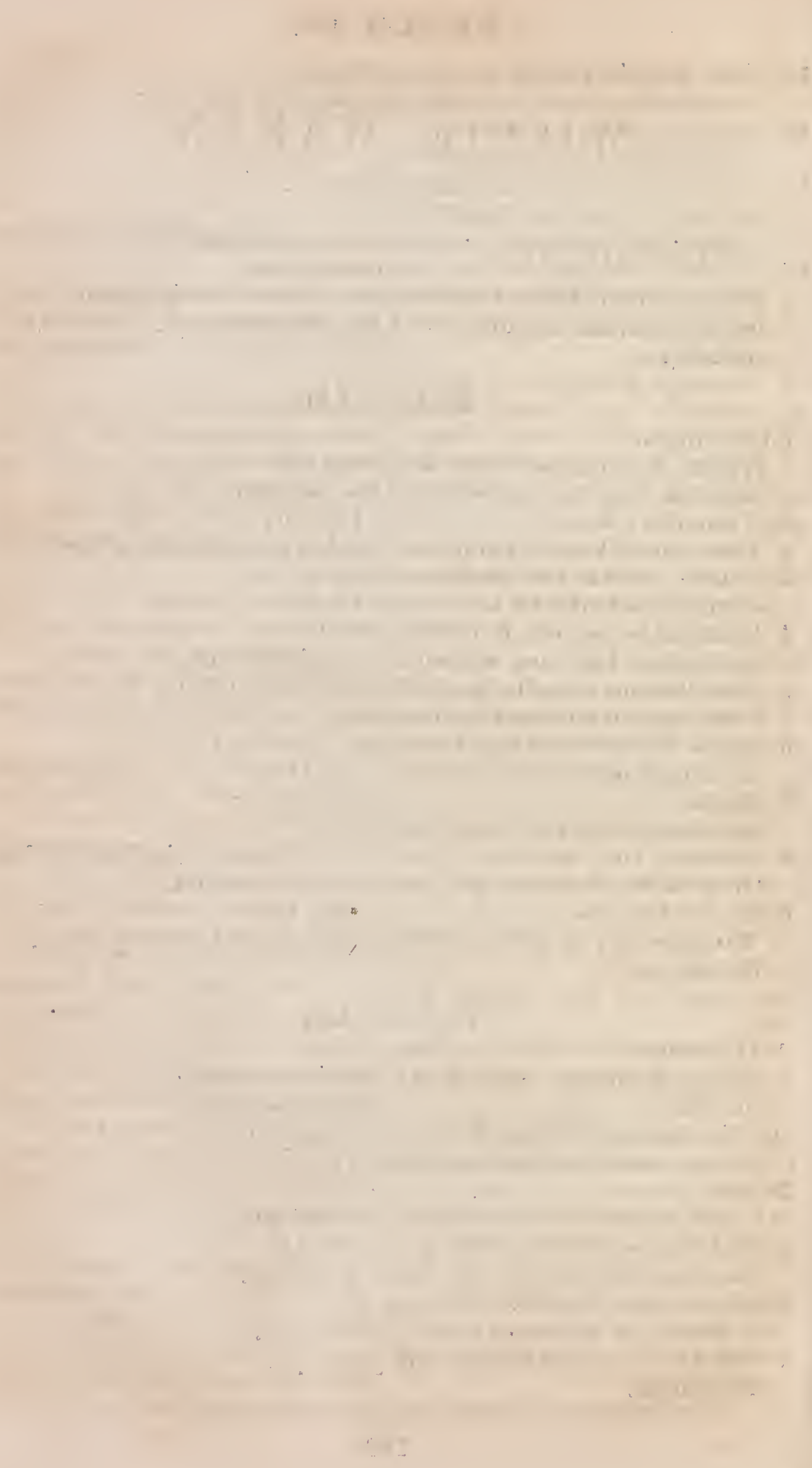




\section{Proverds
lie rube \\ WICOIE WAKAN.}

\section{WICOWOYAKE 1.}

1 Wicoie wakan Israel wicaśta yatapi kin, Dawid cinhintku Salomon he tawa.

2 Woksape woonspe ko sdonye wicakiye kte, wowiyukcan iapi kin okahnili wicakiye kta,

3 Woksape, woonspe, woecetu, woyaco qa wicohan owotanna yuhe wicakiye kta;

4 Tona wacintonśni ksam wicaye kta; kośka kin woksape wowayupike ko wicaqu kta.

5 Wicaśta ksapa nahon kinhan sanpa ksape kta, qa wicaśta wokcan wokonze okahnige kta.

6 Heon wicoie wakan kin okahnigapi kta, wicaśta ksapa oiepi qa taku okahnigapi tehika eyapi kin koya.

7 Jehowa kokipapi kin wosdonye otokahe kin hee, tuka wicaśta witkotkopi woksape qa woonspe ihaktapi śni.

8 Micinkśi, niyate taku onspe niciye cin nahon wo, qa nihun taku ope niśi kin ehpeye śni wo.

9 He wateśdake waśte nipa kin ihdukśan, qa wanapin nitahu kin en un kta.

10 Micinkśi, wahtanipisa niyutitan cinpi eśta ihnuhan wicayada kin.

11 Ito unyanpi kte, wicaśta ktepi kta e nahmana awicunpepi kta, taku śica econpi śni kin liena ituh wicunhmukapi kte;

12 Woha en iyohpayapi ocowasin, qa Hades iyecen ni unpi nawicunpcapi kta;

13 T'aku waśte ota iyeunyanpi

Prov. kta, untipi woyuha ojugjudan unhapi kta:

14 Hena owasin en ounyapapi kta, mazaska opiye okonwanjidan unhapi kta, eniciyapi kinhan :

15 Micinkśi ihnuhan canku kin he en owicayape cin; oye tawapi kin etanhan nisiha kin ehdaku wo.

16 Sihapi kin taku sica oinyankapi, qa wicawe papson inahinipi.

17 Taku hupahu ton hiyeye cin owasin wanyakapi icunhan mazahtakiyapi ituh onpapi.

18 Tuka hena iye we kin hmunkapi, iye nagipi kin on inalimanpi.

19 'Tona śicaya taku kamnapi, qa tuwe tawa ktepi kin liena owasin tacankupi kin hececa.

20 Woksape tankan pan, canku kin ohna hoyeya ece;

21 Otonwe canku ihanke kin ohna, tiyopa icahda mniciyapi ohna ieyanpaha, qa heya,

22 Wacintonpi śni tohanyan wicawitkotkoke cin he waśte yadakapi kta, qa waoncesapi tohanyan wowaonce kin he iyonicipipi kta, qa witkotkopika wosdonye śiceyadakapi kta he?

23 Miye ocihanpi heon ihduecetupo ; mitaniya kin acicaśtanpi kta, mioie kin okahnili ciciyapi kta.

24 Hoyeciciyapi tuka ayaktapi śni, minape kin mdumdaya tuka tuwedan en ewacin śni.

25 Wahokonciciyapi kin owasin ehpeyayapi, qa wakta ciyapi kin iyonicipipi śni. 


\section{WICOIE WAKAN.}

26 Miś eya nihnaśkinyanpi kin icihapi kta, tohan nina koyakipapi kinhan oncicapi kta.

27 Woihangye tohan hiyohi qa koyakipapi kinhan, iyoniciśicapi, wamniomni iyececa wocante śica wicokakije ahna nihiyohipi kinhan :

28 Hehan houmakiyapi kta tuka waamdupte kte śni, hanhanna omadepi eśta iyemayanpi kte śni.

29 Wosdonye śice dakapi, qa Jehowa kokipapi kin kahnigapi śni.

30 Wahokonwicawakiye cin tawațenyapi śni, iyopewicawaye cin owasin wahtedapi śni ;

31 Heon ohanyanpi waskuyeca kin hdutapi kta, qa iye wahokonkiciyapi kin he imna kiciyapi kta.

32 Oziiciyapi kin witkotkopi kin te wicaya, qa iyokipiiçiyapi kin wacintonpi śni kin ihang wicaya ece.

33 Tuka tuwe anamagoptan kin wikope wanin ounye kta, qa taku śica ikope śni owanji un kta ce.

\section{WICOWOYAKE 2.}

1 Micinkśi, tokin mioie kin iyacu qa wahokonciciye cin nalimana niciyehnaken;

2 Ninoge kin woksape anagoptan qa nicante kin wookalinige ekta ye yakiyen.

3 Han wokcan yakipan, qa wookahnige hoyeyakiye kta ;

4 Mazaska iyecen ayakite ca patanyan mazaska wokihnake iyecen oyade cinhan;

5 Hehan Jehowa kokipapi kin oyakahnige kta, qa Wakantanka sdonyapi kin iyeyaye kta.

6 Jehowa woksape wicaqu ece, iye i kin etanhan wosdonye wookahnige koya.
7 Tona owotanna kin hena wiconi awicakicihnaka; tona ecetuya ohanyanpi kin Jehowa wahacanka yapi ;

8 Hecen wicoope canku kin ope wicakiye ça, tona waśte wicakida hena canku awanwicakiciyaka ece.

9 Hehan wicoowotanna, wnyaco, wokonze, oye waśte owasin oyakahnige kta ;

10 Woksape nicante kin en hiyu kinhan, qa wosdonye ninagi kin iyokipi kinhan;

11 Wacintonpi kin awanniciyake kta, wookahinige niyuhe kta.

12 Heon canku śica etanhan enihdaku kta, wicaśta pemniyan ie cin etanhan;

13 Hena canku owotanna ehpeyapi, qa otpaza canku kin omanipi ;

14 Taku śica econpi kta e iyuśkinpi, pemniyan ohanyanpi kin wowinktayapi ;

15 Oye tawapi kin śkośkopa, qa tacankupi kin pemnimni ece.

16 Wihomniwin kin etanhan enihdaku kta, witkowin i skuya etanhan.

17 He tokaheya yuze cin ehpekiye ca, taku wakan tawa tawicoun kin akiktonja.

18 Tiyohnaka kin wiconte ektakiya atakinyan ya, qa oye tawa kin wanna tapi iyahde.

19 Tona en yapi kin hetanhan wanjidan ku śni, qa wiconi canku kin iyahde śni do.

20 Hecen wicaśta waśte tacankupi kin omayani kta, qa wicaśta owotanna tacankupi kin oyape kta.

21 Wicaśta ecetupi kin hena maka kin otipi kta, wicaśta waśte kin hen ounyanpi kita.

22 Tuka wicaśta śica kin maka kin etanhan wicakawankapi 


\section{WICOWOYAKE 3.}

kta, qa tona śicaya ohanyanpi kin hena wicakawihnunipi kta.

\section{WICOWOYAKE 3.}

1 Micinkśi, mitoope kin ihnuhan ayektonje cin, qa mitawoahope kin nicante kin ekta yuha wo.

$2 \mathrm{He}$ wookiye anpetu hanskaska qa wiconi omaka ota aokijuniciye kta.

3 İhnuhan wocantkiye qa wowicake aniyuśtan kin, hena nitahu kin en apahta wo, qa nicantojuha kin akan okiwa wo.

4 Hecen yaksape kta, qa Wakantanka wicaśta ko cante niciye kta.

5 Nicante ocowasin on Jehowa wacinyan wo, qa nitawacin kin ikpatan śni wo ;

6 Canku nitawa kin owasin obna he kiksuya wo, kinhan nioye kin owasin awanniciyake kta.

7 Ihnuhan ksape niçida kin, Jehowa kokipeyahan un qa taku śice cin etanhan ihduhomni wo.

8 He nicelipi zaniyan niniye kta, qa nihuhu acuniciye kta.

9 Woicihde duhe cin on Jehowa ohoda wo, qa taku icahyaye cin otokapa iyahna :

10 Kinhan tipi womnayaye cin woicewinyan on ojudan kta, qa miniśa iyuśkice nitawa kin anapsonpson kta.

11 Cinś Jehowa nicapsinpsinte cin he ihnuhan ihayakta śni; qa iyopeniye cinhan wacin ibośake śni wo.

12 Jehowa tona waśte wicakidake cin hena iyopewicakiya ece, wicaśta wan cinca iyotan kida kin he iyececa.

13 Wicaśta kin woksape iyeye cin, qa wicaśta wookahnige enapeye cin he wopida yuha.
14. He opetonpi kin hee waśte, mazaska opetonpi kin hee śni ce; hetanhan icage cin hee iyotan, qa mazaskazi kapeya.

$15 \mathrm{He}$ inyan tehihika kin he sam iyeya telikika, qa taku iyonicipi iyeye cin owasin hena iyowaja śni.

16 He etapa kin ohna anpetu hanskaska, qa catka kin ohna woyuha wowitan iyahna un.

17 Owe tawa kin owe wiciyokipi, qa tacanku kin owasin wookiye ;

18 Tona kaskita yuzapi kin he can wiconi yanpi, qa tuwe he patan kinhan he wopida ya.

19 Jehowa wolssape on maka kin tinsaya ehnake ca, wookalinige kin on malipiya kin yuśtan.

20 Tosdonye kin on wośbe kin naptujapi, qa malipiya kin $\mathrm{cu}$ kin śbuya.

21 Micinkśi, woksape wicake, wowayupike iyahna awanyaka wo. Ihnuhan hena niiśta kine etanhan nunipi kin;

22 Kinhan hena nitahu en wanapin waśte kta, qa ninagi wiconi yuhe kta.

23 Hecen okope wanin nitacanku kin ohna mayani kta, qa nisiha kin wanahnaye kte śni.

24. Makan inonke cinhan takudan koyakipe kte śni, makan inonka eca tanyan niśtinbe kta ece.

25 Ihnuhanna yuśinye wicaye çin he koyakipe kte śni, qa wicaśta śica wayutakunipiśni wicahiyohi eśta nakun.

26 Nakaes Jehowa wacinyaye kta, qa nisiha icupi kte śni e awanniyake kta ce.

27 Tona onśika ninape taku waśte ecawicakicon okihi kinhan ihnuhan teyahinda kin.

28 Nitawaśi taku yakiduhe cin- 
han, Fkta hda qa ake kuwa wo, heyakecinhan ciçu kta, ihnuhan eyakiye cinhan.

29 Nitakoda takudan ikopeśniyan nikiyedan ti kinhan takudan śica ecakicon śni wo.

30 Wicaśta taku śica ecanicon sni kinhan ihnuhan kici ayakinica kin.

31 Wicaśta wakisa kin icekin śni wo, qa ohan tawa kin wanjidan kahinige śni wo.

32 Jehowa tona pemniyan ohanyanpi kin walite wicada śni, tuka wicaśta ecetupi kin om wohdaka.

33 Jehowa wicaśta śica ti kin yaśica, tuka wicaśta owotanna ounye cin hee yawaśte.

34 Wancapi-en Jehowa waonca, eśta onśiicidapi kin wowaśte wicaqu ece.

35 Tona ksapapi kin hena wowitan ohiyapi kta, tuka tona witkotkopike cin hena wowiśtece en ihduwankantupi.

\section{WICOWOYAKE 4.}

1 Hokśiyopa niyate wahokonniciyapi kin nahonpo, qa wowiyukcan sdonyapi kin cantotpanipo. 2 Woonspe waśte ciçupi ce, mitoope kin ihnuhan ehpeyayapi kin.

3 Ate cincamayan nahanhin mawankàdan, qa miśnana ina icahda waun qehan;

4 Onspemakiya, qa hemakiya; Mioie kin nicante kin yuhe kte, woahope mitawa kin yuha wo, liecen yani kta ce.

5 Woksape kin opeton wo; wokcan kin opeton wo ; akiktonje śni wo, qa mii kin etanhan mioie kin nakicipe śni wo.

6 Ehpeye śni wo, hecen awanniyake kta ; waśte daka wo, hecen nipatan kta.
7 Wicoksape kin he iyotan; wicoksape kin he opeton wo, qa taku mnayaye cin owasin on wowiyukcan kin opeton wo.

8 He iyotan yada kinhan yuwankan inicu kta, kaskita duze cinhan hehan niyuwitan kta.

9 He nipa kin ihduksan wowiciyokipi yuwitaye kta; wateśdake okitanin niçu kta.

10 Cinś, anagoptan wo, qa mioie kin icu wo, hecen omaka wiconi nitawa kin niciyuotapi $\mathrm{kta}$.

11 Wicoksape canku kin ociciyaka, canku owotanna kin ohna omaniciciya.

12 Ohna mayani kinhan oihe yaye cin antinsyapi kte śni, qa yainanka eśta nihicahe kte śni.

13 Woonspe kaskita yuza wo ; ihnuhan aduśtan kin, awanyaka wo heon yani kta ece.

14. Wicaśta ohan śica tacankupi kin ihnuhan ohna idade cin, qa wicaśta pemni kin tacanku kin ohna mayani kte śni.

$15 \mathrm{He}$ ehpeya wo, ihnuhan ohna de cinhan, he etanhan ihduhomni qa tokan iyaya wo.

16 Taku śica econpi śni kinhan iśtimapi śni ce : Tuwedan wankaheyapi śni kinhan wicohba kin wicakipi ece.

17 Wicohan śica aguyapi kin he yutapi; boticapi miniśa kin he yatkanpi ece.

18 Tuka wicaśta ow otanna canku tawapi kin lie iyoyanpa iyega ohinniyan sam wiyatpa aya ecen anpetr wiyotanhan he iyececa.

19 Wicaśta śica tacankupi kin he aotpaza; taku en hicahanpi Ite cin he sdotkiyapi śni.

20 Micinkśi, mioie kin awacin wo, taku eciciye cin anoh kiciya wo.

21 Thnuhan he niiśta kin icunom iyeyakiye cin, nicante kin cokaya yuha wo. 


\section{WICOWOYAKE 5 .}

22 Tona he iyeyapi kin he wiconi tawapi ee, qa celipi kin ocowasin okizi wicaya ce.

23 Taku duhe cin owasin isanpa nicante kin awanhdaka wo, hetanhan wiconi owahinanpe kin hee ce.

24 Wicopemni nii kin. etanhan yutokan wo, qa wicaśtapi śni niiha etanhan hdutehan wo.

25 Niiśta kin nitokam owotanna etonwan kte, niiśtojuha kin nitokapa ecetuya tonwanhanpi kte.

26 Nisiha oye kin awacin wo; hecen canku yaye cin owasin tanyan yahduśtan kta.

27 Nietapa nicatka ihnuhan unmanna eciyatanhan nihduhomni kin, taku sice cin etanhan nisiba kin ehdaku wo.

\section{WICOWOYAKE 5.}

1 Micinkśi, mitoksape kin awacin wo, wookahinige mitawa kin anoh kiciya wo;

2 Hecen woway upike duhe kta, qa nisiha kin wosdonye patan kta.

3 Witkowin iha kin etanhan tuhmaga canhanpi kaduza, qa caka kin wihdi isanpa śduta.

4 Tuka hetanhan taku icage cin peji hota iyecen pa, isan pesto kin iyecen pe hinca.

5 Siha kin apamahdedan wiconțe oiyahdeya, oye kin wakanśica ti kin iyohiya.

6 Wiconi canku kin naśna niciye kta e oye kin kpakśikśan, hecen idukcan kte śni.

7 Hokśiyopa detanhan anamagoptanpo, qa wicoie mii kin etanhan inape cin ihnuhan ehpeyayapi kin.

8 Witkowin kin etanhan canku kin hdutehan wo, qa tatiyopa kin ihnuhan kiyadan de cin.
9 Okini nitowaśake tokan wicayaqu kta, qa omaka nitawa kin wicaśta waonśidapi śni yuhapi kta.

10 Okini toka woyuha nitawa kin on imnapi kta, qa taku on htayani kin witkowin ti kin ohna yanke kta ;

11 Okini oniwihanketa woyute nitawa nicehpi ko sotapi kinhan śicahowade kta;

$12 \mathrm{Qa}$, Hehehe, tokeca woonspe śice wadaka, qa wahokonmakiyapi kin ihawakta śni he.

13 Tona waonspemakiyapi kin nawicawahon śni, qa wahokonmakiyapi ho kin anawagoptan śni ece.

14 Wicota qa omniciye cokaya taku śica owasin en ye miçiye kta tuka, ehe kta.

15 Minilidoka nitawa kin etanhan hdatkan wo, qa wakpadan mini owe nitawa etanhan kaduze cin nakun etanhan.

16 Hecen wakoniya nitawa kin tankaya kaduze kta; otonwe canku kin ohna mini wakpadan kaduze kta;

17 Hena niśnana nitawa kte, tokan tuwedan tawa kte śni.

18 Hecen wakoniya nitawa kin yawaśtepi kta, qa honikśidan qehan winohinca duze ciqon he nihduśkin kte;

19 Tawiyedan waśte dakapi, qa tatokadan wiciyokipi iyecece kta, azinpi kin imna niçiye kte, anpetu owasin, qa waśte yakidake cin ohinniyan wanipi nunwe.

20 Tokeca micinkśi winohinca tokeca wanipi lita, qa witkowin maku kin adoksohan duze kta he?

21 Jehowa wicaśta canku kin owasin wanyake ca tolken olianyanpi kin wicakicitkeuta. 
22 Iye ecinśniyan olianye cin he wicaśta śice cin kihdege kta, qa iye wahtani ikan kin on hmunkapi kte;

23 Witkoya olianye cin ota onuni kta, qa onspekiyapi śni on te kta.

\section{WICOWOYAKE 6 .}

1 Micinkśi, nitakoda wanji taku yecicajuju kta kehe cinhan, qa on wicaśta tokeca nape duza hecinhan;

2 Nii kin etanhan nioie kin he iyanicaśkapi; nii kin on taku ehe cin he niyuzapi ce.

3 Heon micinkśi hecen econ wo, qa ihduśka wo, nitakoda nape kin iyahpade cinhan hunktiya, ihduhukuya qa nitakoda iyopaśtaka wo.

4. Niiśta kin en wicahiba kin qu śni wo, qa niiśtojuha kin on iśtinbe śni wo.

5 Zitkadan tuwe hmunke kta nape kin etanhan ihduśka, qa tatokadan wicanape kin etanhan ihduśke cin he iyecen ihduśka wo.

6 Kuja nanke cin tajuśka kin ekta ya wo, yaksape kta e ohanyanpi kin wanyaka wo.

$7 \mathrm{He}$ itancan nica, akicita nica, qa wicaśta yatapi nica ;

8 Tuka mdoketu eca taku yutapi kihnaka, qa woksapi eca woyute tawa mnayan ece.

9 Tohantu kinhan makan inonke kta? kuja, niśtinbe cin etanhan tohantu yekta kta he?

10 Nihba onge qa niśtinbe onge, qa makan inonke kta e nape pakśijapi onge ;

11. Hecen wowalipanice kin wicaśta icimani wan he iyecen en niu kta, qa wicaśta wipe hduha en nihi kin he iyecen taku owasin inicakije kta.
12 Wicaśta śica wicaśtapi śni i pemni hduha mani;

13 Iśta kin on wiyuta, qa iye siha okiya, qa iye napsukaza on waonspekiya ece;

14 Wacanśinyapi kin cante mahen ohinniyan taku śica kaga, okicize kin he kada elipeya.

15 He etanhan ihnuhanna ihdutakuni kte śni, oliankoya yukukapi kta, qa wokicizi wanice kta.

16 Taku śakpe Jehowa śice daka, qa śakowin nagi kin wahitedaśni.

17 Wiciśta wankantuya, qa wicaceji itonpi śni, qa wicanape tona taku śica econpi śni wewicayapi;

18 Wicacante wicolian śica ewacin, wicasiba taku śica econpi kta e inahniyan inyankapi;

19 Wayaa tanin ecinśniyan woitonśni ipoliya, qa tuwe hunkawanjin kiciyapi iyakitedan kicikigepi oju kin he nakun.

20 Cinś, niyate wahokonniciye cin tanyan yuha wo, qa nihun toope kin elipeye śni wo.

21 Hena ohinniyan nicante kin en palita wo, qa nitahu kin en iyakaśka wo.

22 Hecen mayani eca yus aniye kta ece, niśtima eca awanniyake kta, qa tohan yekta eca oniciye kta ece.

23 Woahope kin he petijanjan qa wicoope kin he iyoyampa, qa iyopewicayapi woonspe kin he wiconi canku kin ee.

24 He winolinca śica etanhan awannihdake kta, winohinca oyate tokeca ceji skuya etanhan.

25 He owanyag waśte eśta nicante kin ekta kon śni wo; qa iśtojuha kin on ihnuhan inicu kin.

26 Witkowin kin wicaśta wan 
WICOWOYAKE 7.

aguyapi ciqadan ekta aye kta, qa wawicihahapi econsa kin wicanagi tehika apasi kta.

27 Wicaśta wan peta ikpihnake cinhan tawokoyake kin lulinage kte śni he?

28 Tuwe petaga amani kinhan siha kin gu kte śni he?

29 He iyecen tuwe wicaśta tokeca tawicu kici iwanke cinhan; tuwe kaśta he yutan kinhan ta'ku śica iyahdeya.

30 Turve wotektehda on woyute manun qa wipiiciciya eśta nina śicedakapi śni ;

31 Tuka iyeyapi kinhan śakowin akihde wicakicu kta; ti kin ohna taku yuke cin owasin eśta wicaqu kta.

32 Tuka tuwe wawicihahapi winohinca wan kici econ kin he cante wanica, tuwe hecon kinhan nagi kin ihang kiya.

33 He apapi kta, qa iśtenyapi kta, qa wowiśtece kin he kajujupi kte śni.

34 Wowinawizi he wicaśta tocanteptanye kin ee, tohan tokiçon kinhan wawitonpe kte śni.

35 Wokajuju tanka eśta ihakta kte śni, taku ota yaqu eśta iyokipi kte śni.

\section{WICOWOYAKE $\%$}

1 Micinkśi, mioie kin yuha wo, qa mitawoahope kin niye eniçilmaka wo.

2 Mitawoahope niiśta su kin iyecen yuha wo, qa mitoope kin, hecen yani kta;

3 Hena ninapsukaza en apahta wo, qa nicante ojuha kin akan owa wo.

4 Wicoksape kin, Nis tanke ciya eciya wo, qa wookalinige, Mitakuye caje yata wo.

5 Hecen witkowin kin etanhan nipatan kta, wihomniwin i skuya kin etanhan.

6 Wati owanyeye kin hetanhan can ipuskica ohna aowakasin qehan ;

7 Wacintonpi śni wicehna ewatonwan, cincapi ehna kośkadan cante nica iyewaya ;

8 Witkowin oise tawa kin ikiyedan mani, canku wan ti kin iyahde kin he ohna ya;

9 Anpetu owihanketa litayetu, hanyetu otpaza he ehan ;

10 Unkan inyun winohinca kin he itkokipa, witkowin wan iyecen ihduze ça cante lin nakihibe.

11 Owanji yanke śni qa wicakihna, siha kin tipi tawa ohna yakonpi śni.

12 Tuktekten tiyopa ekta, tuktekten canku kin ohna, qa oise owasin ikiyedan wicahmonka.

13 He yuze ça i iputake ça waditagya heciya,

14 Wopida wośnapi mduha, nakaha hanmdepi wahduśtan:

15 Heon itkocicipe kta e tankan wahinawape ; niite kin wanciyake lite hinca qa wanna iyeciye.

16 Ohewahde kin śina owinś waya; Egupton wipatapi on apalidan waton;

17 Mitowinja kin mura, canśin qa canhaśa okahtan waya.

18 Ito unhde ca iyokipi kiciya imnaunkiçye kte: Hanyetu osan waśtekicidaya unkiciyuśkin kte ;

19 Wicaśta kin tin yanke śni, tehan icimani iyaya;

20 Mazaska ojutonpi nape ohna icu, wi mibe kin anpetu kin he en hdi kta ce.

21 Wicoie skruya ota on he yahomni, iha skuya on yaptanye ya.

22 Unkan ihnuhanna ihakamya 
mani, tatanka wan tutken patapi kta ekta ye cin he iyecen, qa tahinca hmunkapi kin en inyanke cin he iyecen.

23 Ecen wanhinkpe wan pi kin ipahdan iheyapi; zitkadan wan hmunlrapi en kinyan iyopta, qa on te kta sdotkiye śni he iyecen.

24. Heon micinca anamagoptanpo, qa wicoie mii kin ie cin awacinpo.

25 Ihnuhan nicante kin winohinca kin he oye kin en nihduhomni kinhan, tacanku kin ohna onuniye śni wo.

26 Wicota capapi makata ehpewicaya, wicakte kin hena owasin waśalkapi.

27 'Ti kin he Hades canku iyahde kin hee, wiconte temahentuya ohna iyaye wicaya.

\section{WICOWOYAKE 8.}

1 Awicakehan woksape kin waieyanpaha, qa wookahnige kin hoyeya ece.

2 Paha hinca akan najin, canku icipawega ohna.

3 Tiyopa icahda, otonwe tiyopa en, tukten tiyopa ohna hiyupi ekta wicakipan.

4. Thopo, wicaśta cicipanpi qa Adam cinca kin hoyeciciyapi ce.

5 Tona wacinnitonpi śni kin wowayupike onspeiciciyapo, qa winitkotkopika cante kiksapapo.

6 Anagoptanpo, qa iha wahdukawa ca taku owotanna epe kta.

\%. Micaka kin wowicake eye lita, qa wowicaśta śni miiha lin wahte da śni.

$8 \mathrm{Mii}$ kin etanhan wicoic kin owasin ecetupi, hena en takudan śkope śni qa pemini śni.

9 Wicaśta wiyukcan kin hena owasin mdeza e iyeya, tuwe wo- sdonye akite cinhan hena owotanna e iyeye kta.

10 Mazaska hee śni, tuka waonspeciciyapi kin he icupo, qa mazaskazi hinca hee śni tuka wosdonye kin he kaỉnigapo.

11 Woksape kin he inyan tehihika kin sam iyeya tehika, qa taku kaśta iyowicakipi owasin he iyowaja śni.

12 Woksape he miye, qa iyotan wamdupika; taku wayupika sdonyapi kin hena owasin iyewaya ce.

13 Tona Jehowa kokipapi kin hena wicolian śica śicedakapi; wahaniçidapi, watanka icidapi, śicaya opiiçiyapi, qa wicai pemni kin hena śice wadaka.

14. Wahokonwicakiyapi wiconi ko hena mitawa, wokcan kin he miye, wowaśake he mitawa.

15 Miye eciyatanhan wicaśta yatapi wawidakapi, qa itancanpi taku owotanna wakiconzapi ece.

16 Miye eciyatanhan itancanpi oyate yuhapi, qa tona maka akan wayacopi itancanyan unpi.

17 'Tona waśte madapi kin hena waśte wadaka, qa tona hanhanna hin amakitapi kinhan iyemayanpi kta.

18 Woyuha ota qa wowitan miciyukan, woicihde suta qa wokiconze.

19 Mazaskazi ecedan hin eśta mitawaskuyeca kin he iyotan, qa mazaska kahnigapi eśta taku icahwaye cin hee iyotan waśte. 20 Wicoowotanna canku ohna mani wicawaya, wokiconze oye kin cokaya ohna.

21 Hecen tona waśte madapi kin hena taku hohośni tawayapi kta, qa wopiye tawapi ojudan wicawakiye kta.

22 Jehowa tohan tokaheya mayuhe ciqon, takudan icahye śni he itokam kici waun. 


\section{WICOWOYAKE 9.}

23 Otokahe wanin ehantanhan sdamayanpi, otokahe ekta maka kin itokam.

24 Ośbe wanica hehan matonpi, wakoniya mini ojudan itokam icalimayanpi ;

25 Ḣe imnija kin sutaya ehdepi śni, qa paha kin itokam icañmayanpi ;

26 Maga qa tinta qa maka mdu paha kin kage śni itokam;

27 Mahipiya ehnake cehan en waun; ośbe akan wohmihbe ehde he ehan.

28 Wankantanhan malipiya śoka otke ya; ośbe kin etanhan wakoniya yuśtan he ehan ;

29 Miniwanca kin toope qu, qa mini kin huta kin sanpa ye kte śni konze ${ }^{-c e h a n, ~ m a k a ~ k i n ~ s i c u ~}$ kill yasuta qehan;

30 He ehan ikiyedan manka, kici icalimayanpi, anpetu otoiyohi toiyokipi kin he miye, qa ohinniyan itokam wimduśkin.

31 Wicaśta cincapi om imduśkin, qa maka ounyanpi kin he iyomakipi.

32 He etanhan dehan micinca anamagoptanpo. Tona mitacanku opapi kinhan hena pidaiciyapi.

33 Woonspe kin nalionpo, qa ihduksapapo, ihnuhan he elipeyayapi kin.

34 Wicaśta tuwe anamagoptan anpetu owasin mitatiyopa awanyake ca tiyopa icahda najin kin he wopida.

35 Tuwe iyemaye cin he wiconi iyeya, qa Jehowa en woiyokipi aihdutan.

36 Tuwe miye en walitani kin he nagi kin kiunniyan, tona sice madapike cin hena wiconțe waśte dakapi ece.

WICOWOYAKE 9.

1 Woksape tipi tawa wanna

kage ẹa inyan śakowin oahe kin hdakan :

2 Tado wanna kpata, miniśa kin icicahi, qa wahna wotapi kin ekihde.

3 Wikośka tawa kin ye wicaśi, wicoie wakan otonwe ohna he ipa kin akan pan yanka.

4 Tuwe wacintonśni deci u wo, cante nicapi kin hewicakiya, 5 Iruwapo, aguyapi mitawa etanhan yutapo, qa miniśa wiyeya ewehde kin etanhan yatkanpo.

6 Wicowitkotko kin elipeyapo, hecen yanipi kta; qa wokcan canku lin ohna manipo.

7 Tuwe waonca wahokonkiye cin lie wowiśtece iyahdeiciya, qa tuwe wicaśta śica iyopeye cin he ihduśape kta.

8 Wicaśta waonca ihnuhan iyopeyaye cin, śice nidake kta naceca ; tulka wicaśta ksapa he iyopeya wo, hecen waśte nidake kta.

9 Wicasta ksape cin waonspekiya wo, hecen sanpa ksape kta ; wicaśta owotanna wosdonye qu wo, he icu kta.

10 Woksape otokaheya kin he Jehowa kokipapi kin ee, qa taku wakan sdonyapi kin wokcan itancan kin hee.

11 Miye on anpetu nitawa niciyuotapi kta; nakun wiconi omaka yuhe ciciye kta.

12 Yaksape cinhan nitoksape taku aicage cin he nitawa kta, qa waonyace cinhan niśnana śitkihda niciye kta.

13 Winohinca witkotkoka kin he iwakan, wacintonśni qa takudan sdonye śni.

14 Iye ti tiyopa kin icahda iyotanka, otonwe pajodan ohna canalkanyotankapi akan.

15 Ahiyayapi kin wicakipan, canku katinyan omanipi kin hena; 


\section{WICOIE WAKAN.}

16 Tuwe wacinton śni kinhan, kuwa wo, eciya, qa tona cante nicapi hewicakiya,

17 Mini manonpi kin he skuya ce, qa aguyapi nahmana yutapi kin he wiciyokipi ce, eya.

18 Tuka hen wicanagi yakonpi, tona wicakico Hades osmaka kin ohinhpayapi wicaśta kin sdonye śni.

\section{WICOWOYAKE 10.}

1 Wicoie wakan Salomon tawa. Hokśiyopa ksapa eca atkuku iyuśkin kiya ece; tuka hokśiyopa witkotkoke cin he hunku kin iyokiśinya.

2 Wicohan śica on mazaska mnayanpi kin owicakiyapi śni ; tuka wicoowotanna wiconte ctanhan waehdaku.

3 Jehowa wicaśta owotanna nagi kin akihan ye kte śni, tuka wicaśta śica taku cinpi ehpewicakiciya ecc.

4. Turve nape kuje cinhan ihduwahpanica, tuka wicanape minihcca kin he wijinwicaya ece.

5 Tuwe mdoketu eca wamnayan ece kin he hokśiyopa wayupika, tuka tuwe wamnayanpi icunhan iśtinbe cin he holsíiyopa wawiśtenya ece.

6 Wicaśta owotanna pa kin yawaśtepi, tuka wowiśtece wicaśta śica i liin aohdute ye kta.

7 Wicaśta owotanna kiksuyapi kin yawaśtepi kta, tuka wicaśta śica caje kin kuke kta.

8 Tuwe cante ksape cin he wahokonkiyapi icu kta, tuka tuwe iha witkotkoke cin he wankahe kta.

9 Tuwe wohnaye wanin mani kinhan okope codan mani lkta; tuka tuwe canku kin hdupemni kin he sdonyapi kta.

10 Tuwe iśta kin on wiyute 144 cin he woiyokiśice icaliya, qa tuwe iha witkotkoke cin he licahe kta.

11 Wicaśta owotanna i kin wiconi minilidoka kin hee, tuka wicowohitike kin wicaśta śica ipi kin akahpe kta.

12 Wośicedake kin wicakigepi kaga, tuka wowaśtedake kin he woahtani owasin akahpa.

13 Wicaśta wiyukcan iha kin ohna wolksape iyeyapi kta, tuka wicaśta cante nice cin nite ekta cansakadan kta.

14 Wicaśta ksape cin hena woksape nakilimanpi, tuka tuwe witkotkoka i kin ecadan inihan kta.

15 Wicaśta wawokihika towijice kin conkaśke suta kin hdawa, tuka wowahpanice wicaśta onśika yuśinye wicaya.

16 Wicaśta owotanna kin wiconi iyuwin yapi, tuka wicaśta śica woahtani icaliyapi ece.

17 Tuwe woonspe hduhe cin he canku wiconi iyahde kin he opa; tuka tuwe wahokonkiyapi awakicin śni kinhan nuni.

18 Tuwe iha itonpi śni on wocanniye anakilibe, ca tuwe wowiśtece yaotanin kin iś eya witkotkoka.

19 Tuwe wicoie ota ie cin wahtani, tuka tuwe iha nina hduhc cin he ksapa.

20 Wicaśta olian owotanna cante kin he mazaska kahinigapi, wicaśta śica cante kin he taku śni.

21 Wicaśta owotanna iha kin wicaśta won wicaya ece, tuka wicaśta witkotkopi kin cante nicapi on tapi kta.

22 Jehowa toyawaśte kin wawijinya kin hec, qa iyahna woiyokiśica opeye śni.

23 Taku śica econpi kin wicaśta witkotko imagaga içiyapi, 


\section{WICOWOYAKE 11.}

tuka wicaśta wiyukcan wolksape opa.

24 Wicaśta śica taku ikope cin he akipe kta, qa wicaśta owotanna taku cin lkin he kiçupi kta.

25 Tate iyumni ahiyaya eca wicaśta śica en un kte śni ; tuka wicaśta owotanna oahe kin owihanke wanica.

26 Hastanhanka toto wicahi kiuniyan, qa śota wiciśta kin en he iyecen wicaśta kuja tona yeśipi kin en.

27 Jehowa kokipapi kin he anpetu wicakiciyuota, tuka wicaśta śica omaka tawapi kin wicakiciyuptecedanpi kta.

28 Wicaśta owotanna wowiyuśkin apepi, tuka wicaśta śica taku apepi awihnuni kta.

29 Jehowa tacanku kin wicaśta waśte waśag wicaya ece, tuka wicaśta śica y uśinyewicaya.

30 Wicaśta owotanna ohinniyan yuhohopi kte śni, tuka wicaśta śica maka kin ounyanpi kte śni.

31 Wicaśta owotanna i kin woksape hinanpe ya, tuka wopemni ceji kin baśpapi kta.

32 Wicaśta owotanna iha kin taku wiciyokipi sdonya, tuka wicaśta śica ipi kin wicośkiślze.

\section{WICOWOYAKE 11.}

1 Wohnayan tke utapi kin he Jehowa wahtedaśni, tuka tke utapi ecetu kin he Jehowa toiyokipi ee.

2 Tohan wahanicidapi hi kinkan wowiśtece he nakun u ece, tuka woksape onsilianpi kin wicayukan.

3. Wicaśta waśte towaśtepi kin awicaye kta, qa wicaśta śica topemnipi kin ihang wicaye kta. 4 Wocanniye anpetu ehan woyuha takudan okihi śni, tuka $* 10$ wicoowotanna wiconte etanhan waehdaku.

5 Wicaśta waśte toowotanna kin canku wicakiciyumdaye kte, qa wicaśta śica tohan śicapi kin on hicahanpi kta.

6 Wicaśta ecetupi kin toowotanpi kin wicakiyuśka ece, tuka wicaśta wakonsa tawohnayanpi kin wicahmunka.

7 Tohan wicaśta śica țe cinlian taku cin qon awihnuni kta, qa taku wicaśta ołan śica apepi atakuni śni ece.

8 Wicaśta owotanna woiyokiśice etanhan inapa, qa wicaśta śica wan he en hiyu.

9 Wicaśta wahnayan iye i kin on takodaku ihangya, tuka wicaśta owotanna tosdonyepi kin he ewicahdaku.

10 Wicaśta owotanna tanyan unpi kinhan otonwe kin iyuśkin, qa wicaśta śica awihnunipi eca wiyuśkinyan dowanpi ece.

11 Wicaśta ecetupi yawaśtepi kin he otonwe kin yawankantuya, tuka wicaśta śica ipi-kin he yajuju.

12 'Tuwe takodaku wahtedaśni kin he cante nica, tuka wookalinige wicaśta kin inina yanka.

13 Waaiesa kin taku nahmana oyakapi kin he yaotanin, tuka tona woniya wacinyepica kin hena wicoie anahmanpi ece.

14 Tukten wahokonkiyapi wanice cin hen oyate wankahe kta, tuka wiyukcanpi ota on etanhan wicani.

15 Tuwe wicaśta tokeca taku icazo kicicajuju kta keye cinhan kiunni kta, tuka tuwe he wecicajuju kta eye kapin kin he wacinyepica.

16 Winohinca waśte wowitan yuhe kta, qa wicaśta waśaka woyuha patanpi ece.

17 Wicaśta wacantkiya iye na145 


\section{WICOIE}

gi tanyan ecakicon; tuka tuwe waonśida śni kin he iye celipi kin kiunni.

18 Wicaśta śica wohnaye wolitani econpi; tuka tuwe woowotanna oju kin iyuwin wicaka yuhe kta.

$19 \mathrm{He}$ iyecen wicoowotanna on wicani, qa tuwe wicolian śica opc cinhan teiçiya.

20 Tona cante pemniyan unpi kin hena Jehowa wowahtedaśni, tuka tona tacanku ecetupi kin hena iyokipi yapi.

21 Wicanape tokan nape ekta yekiyapi eśta wicaśta śica śitkihdayapi kta; tuka wicaśta owotanna cincupi kin he inape kta.

22 Winohinca owanyag waśte tuka woksape nice cin he mazaska oinna kukuśe pasu kin ohna kin he iyececa.

23 Wicaśta owotanna taku waśte heceedan cinpi, tuka wicaśta śica wocanniye apepi.

24 Wanji wakadada eśta sanpa mnayan, qa wanji taku owotanna isanpa tehinda eśta ihduwahipanica.

25 Wicanagi wayawaśte kin he cepe kta, qa tuwe wamnimni kinhan amnimnipi kta.

26 Tuwe wamnaheza tehinda kin he oyate kin yaśicapi kta, tuka tuwe he iyopeye cinhan woyawaśte pa akan un kta.

27 Tuwe wowaśte nina akite cinhan woiyokipi iyeye kta, qa tuwe taku śica ode kinhan he iyolii kta.

28 Tuwe towijice kin wacinkiye cinhan hicahe kta, tuka wicaśta owotanna canwapa iyecen tanyan icagapi kta.

29 Tuwe iye ti kin iyokiśinye cinhan tateyanpa ekicihnakapi kta, qa tona witkotkokapi kin hena, tuwe ksape cin wowidag wicaye kta.
30 Wicaśta owotanna taku icaliye cin he can wiconi kin ee, qa tuwe wicanagi ohiwicaye cin he wicaśta ksapa.

31 Wicaśta .owotanna maka akan iyuwin yuhe kta, hecinhan wicaśta śica qa wahtanisa isanpa hececapi kta.

\section{WICOWOYAKE 12.}

1 Tuwe wahokonkiyapi waśte dake cinhan wosdonye waśte daka qa tuwe iyopekiyapi kin śice daka he witkotkoka.

2 Jehowa wicaśta waśte cantekiya, tuka tuwe taku śica awacin kin he yaco kta.

3 Wicaśta wicolian śica eciyatanhan sutaya he kte śni, tuka wicaśta owotanna hutkan tawapi kin hohodan kte śni.

4 Tuwe hihnaku iśtenkiye cin he huhu owasin kuke kiya iyececa, tuka winohinca ksapa wan hihnaku wateśdake tawa kin hee.

5 Wicaśta owotanna cante ozepi kin hena ecetupi, tuka wicaśta śica wahokonkiyapi kin he wohnaye.

6 Tona śica oiepi on wicawe hmunkapi, tuka wicaśta owotanna i tawapi ewicayalkupi kta.

7 Wicaśta śica kawankapi qa en unpi śni, tuka wicaśta owotanna tipi kin sutaya he kta.

8 Wicaśta token ksapa he iyecen yatanpi kta, qa tuwe cante pemni kin woihaktapi śni un kta. 9 'Tuwe ihaktapi śni eśta oukiye wan yuhe cinhan tuwe ihduonihan qa aguyapi codan un kin he isanpa tanyan un ece.

10 Wicaśta owotanna kin tawoteca ni un kin en ewacin cce, tuka wicaśta śica wawacinkta yuzapi kin he wohitika.

11 Tuwe tamaga kicanye cin 
he aguyapi imnaiciye kta, tuka tuwe takuśniśni kuwa kin he cante wanica.

12 Wicaśta śica kin śicapi conliaśke tawapi cin ece, tuka tona owotanna hutkan tawapi kin taku icaliya.

13 Tha itonpi śni kin on śicaya wicahmunkapi, tuka wicaśta owotanna wokakije etanhan inanpe kta.

14 Wicaśta i waskuyeca tawa kin he etanhan taku waśte on imnapi kta, qa taku iye nape kage cin he kicupi kta.

15 Wicaśta witkotko canku tawa kin he owotanna kecin, tuka tuwe wahokonkiyapi anagoptan kin he ksapa.

16 Wicaśta witkotko tocanniye kin ecadan hdutanin, tuka wieaśta wayupika iśtenyapi kin ahdahpa.

17 Tuwe wowicake ie cinhan taku owotanna yaotanin, tuka wayaatanin itonśni kin he wohnaye.

18 Wicaśta wan isan cawicape iyecen ia, tuka tona ksapa cejipi kin he wookizi.

19 Wowicake iha kin he ohinniyan suta kta, tuka ceji itonśni kin he aślkayedan.

20 Tona taku śica awacinpi cantepi kin ohna wohnaye yukan, tuka wookiye wahokonkiyapi kin hena wowiyuśkin.

21 Wicaśta owotanna takudan śica iyahde śni unpi kta, tuka tona śicapi kin hena taku śica ojupidan.

22 Wicaiha itonpiśni kin Jehowa wahtedaśni, tuka tona ecetu ohanyanpi kin hena toiyokipi kin ee.

23 Wicaśta ksapa wosdonye ahdahpa, tuka tona witkotkoka cantepi kin wacintonpi śni ieyanpaha.
24. Wicanape miniheca kin he wawidake kta, tuka wicaśta ku. je cin he wowidake yapi kta.

25 Woiyokiśice wicaśta cante mahen he patuśya, tuka wicoic waśte wan he iyuśkin ya.

26 Wicaśta owotanna kin tawaśitku kin kapeya, tuka wicaśta śica tacankupi kin wicahnayan.

27 Wicaśta kuja taku ihni kin he pasnu śni, tuka wicaśta miniheca woyuha tawa kin he tehika. 28 Wicoowotanna canku kin ohna wiconi, qa oye canku kin ohna wiconte wanica.

\section{WICOWOYAKE 13.}

1 Hokśidan ksape cin he atkuku wahokonkiya nahon, tuka waoncesa iyopekiyapi eśta nahon śni.

2 Wicaśta wan i kin taku icahye cin etanhan taku waśte yute kta, tuka tona pemniyan ohanye cin nagipi kin wicowohitike yutapi kta.

3 Tuwe i kin awanhdake cin he nagi kin kpatan, tuka tuwe iha kin hdugaga woiyokiśica yuhe kta.

4 Wicaśta kuja nagi kin taku cin tuka wanica; tuka tona miniheca nagipi cepapi kta.

5 Wicaśta owotanna wicoie wicake śni śice daka, tuka wicaśta śica honwin qa iśtece kta.

6 Wicoowotanna tona ecetupi kin wicapatan, qa wicolian śica kin wahtanipisa nawicahnaye kta.

7 Wanji ihdawijica tuka takudan yuhe śni, qa wanji ihdawahpanica eśta woyuha ota ton.

8 Wicaśta wan toni kin opekiton kta e towijice kin iyopekiya, tuka wicaśta śica iyopeyapi nation śni. 
9 Wicaśta owotanna toiyoyanpa kin wiyuśkin kiya, tuka wicaśta śica petijanjan tawapi kin bowicakisnipi kta.

10 Walianicidapi kin hecedan wakinicapi kaga, tuka tuwe wahokonkiyapi nation kin he ksapa.

11 Wicahnayanpi kin eciyatanhan woyuha kin he wanistina aye kta, tuka tuwe iye nape kin on kamna kin he hduota kta.

12 Woape kin yutehanpi kin cante kin iyokiśin ya, tuka taku cinpi kin iyohipi kin he wiconi can kin he iyececa.

13 Tuwe wicoie kin ihakta śni kin ihang iciye kta, tuka tuwe woahope kin ikope cin iyuwin yuhe kta.

14 Wicaśta ksapa toope kin he wiconi wakoniya kin heca, on wiconte wihmunke yuiyokapi.

15 Tawacin waśte kin wicoiyokipi icahya, tuka tona wahtedaśniyan ohanyanpi kin canku tawapi kin he tehika.

16 Wicaśta ksapa otoiyohi wosdonye on taku econ, tuka tuwe wacintonśni towitkotkoke cin hduotanin.

17 Wahośiya śica taku śica ohinhpaya ece, tuka wahośiya wicake cin he wookizi.

18 Tuwe woonspe ehpekiye cinhan wowahpanica wowiśtece iyahna icicaga, tuka tuwe iyopekiyapi awacin kin he kinihanpi kta.

19 Taku cinpi kin iyohipi kinhan wicanagi kin iyokipi, tuka wicohan śica ayuśtanpi kin he wicaśta witkotkopi wahtedapi śni.

20 Tuwe wicaśta ksapa om mani kinhan ksape kta, tuka tuwe wicaśta witkotkoke koda wicaye cinhan hee ihang yapi kta.
21 Taku śica wahtanipisa wicapasi, tuka tona owotanna taku waśte wicakicupi kta.

22 Wahtanisa woyula tawa kin wicaśta owotanna wicakicihnakapi, qa wicaśta waśte cinhintku cincapi kin woyuha tawa wicakiye kta.

23 Wicaśta wahpanica malikicanyanpi on woyute ota icaga, tuka owotanna ohanyanpi śni oil apa awihnuni.

24. Tuwe wicapsinte tpatan kin he cinhintku śice kidaka, tuka tuwe he waśte kidake cin he kohanna iyopekiya.

25 Wicaśta owotanna nagi kin imnahan wota ece, tuka wicaśta śica tezipi kin akilianpi kta.

\section{WICOWOYAKE 14.}

1 Winoliinca ksape cin otoiyohi tipi icicaga ece; tuka witkotkoke cin he iye nape kin on ti kin hdujuju.

2 Tuwe iye toecetu kin omani kin he Jehowa kokipa, tuka tuwe canku kin hdupemni kin he ihakta śni.

3 Wicaśta witkotko ipi kin ohna walianiçidapi sagye yukan, tuka tona ksapapi ihapi kin wicahduhapi.

4 Tukten tatanka wanica hen aguyapi tipi takudan ohnaka śni, qa oicage ota kin he tatanka waśake cin he eciyatanhan u ce.

5 Wayaatanin wicake cin he itonśni wanice kta, tuka wayaatanin ecinśniyan kin he woitonśni ipohya.

6 Waoncesa woksape akita eśta he iyeye śni, tuka tuwe wookahnige cin he wosdonye waśakadan iyeya.

7 Wicaśta witkotkoka itokapatanhan ya wo, qa nakun tuwe 
woksape iha yuhe śni sdonyaye cinhan.

8 Wicaśta wayupika toksape kin he tacanku kin iyukcan, tuka wicaśta witkotko towitkotkopike cin he wohnaye.

9 Wicaśta witkotkopi kin woalitani ilialiayapi, tuka tona ecetupi kin ehna wacantkiyapi yukan.

10 Wicacante nagi kin toiyokiśice cin sdotkiya, qa towiyuśkin en tokan icicahiye śni.

11 Wicaśta śica tipi kin kawankapi kta, tuka wicaśta ecetupi tawakeyapi icage kta.

12 Canku wanji ecetu seca wicaśta itokam, tuka he iyohakamya wiconte iyahdeya.

13 Nakun tuktekten ihahapi icunhan cante śicapi, qa worviyuśkin kin he ihanke kin ekta woiyokiśica.

14 Tuwe cante namni kin iye tacanku kin on imna kta, qa wicaśta waśte iye cante oze on ipi kta.

15 Tuwe wacintonśni kin wicoie owasin wicada, tuka wicaśta wayupika caehde kin iwanhdaka.

16 Wicaśta ksapa kokipe ca taku śica etanhan ihduhomni ece, tuka wicaśta witkotkoke cin iyopta iyaye ça waciniçiya.

17 'Tuwe wacinko kinhan witkotkoya ohanyan, qa tuwe taku śica cantiheye cin śice dakapi kta.

18 Tona wacintonpi śni wowitkotko kin tawayapi, tuka wicaśta ksapa wosdonye wateśdag tonpi.

19 Wicaśta śica tona waśtepi wicitokam patujapi, qa tona owotanna tatiyopa itokam.

20 Kodawicaya eśta wicaśta wahpanica kin śicedakapi, tuka wicota wijica wan taku yapi.
21 Tuwe takodaku ihakta śni kin wahtani, tuka tuwe tona onśika onśiwicakida yawaśtep. kta.

22 Tona taku śica kagapi kin hena nunipi, tuka tona taku waśte kagapi wacantkiyapi wowaonśida ahna tawayapi.

23 Taku kicanyanpi kin owasin on taku mnayanpi ece, tuka wicaiha iapi kin wicayuwahpanica heceedan.

24 Wicaśta ksapa woyuha tawapi kin he wateśdag ionpi, tuka tona wacintonpi śni towitkothopike cin he wowitkotko.

25 Wayaatanin wicakc cin he wicanagi ewicayaku, tuka wayaatanin wahnaye cin woitonśni ipoliya.

26 Jehowa kokipapi kin en wowacinye suta yukan, qa tuwe lecon cinca kin hena wowinape yuhapi kta.

27 Jehowa kokipapi kin he wiconi minihidoka kin hee, on wiconte wihmunke kin etanhan ihduśpapi.

280 yate ihduotapi eca wicaśta yatapi kin wowitan yuha ece, tuka oyate kiı wanistina aye cinhan atakuni kte śni e ikopa.

29 Tuwe tehan canteptanye śni kin he wiyukcan tanka, qa tuwe wacinko kin le towitkotkoke cin hdutanin.

30 Cante zanika celipi nikiya kin hee, tuka winawizipi kin he wicahu kin yukuka kin hee.

31 Tuwe wicaśta onśika wan sanpa yuwahipanice cin he tuwe he icahye cin he iśtenya, tuka tuwe lie ohoda kin he wahpanicapi kin cante wicakiya ece.

32 Wicaśta śice cin taku śica en napeyapi kta, tuka wicaśta owotanna ta eśta taku wacinyan un.

33 Woksape kin wicaśta wiyu149 


\section{WICOIE WAKAN.}

kcan cante kin ohna iyozi, qa wicaśta witkotko cokaya kin sdonyapi kta.

34 Wicoowotanna kin he oyate kin wowitan icahwicakiciye cin ee, tuka woahtani kin he oyate Wowiśtece kin ee.

35 Ookiye ksapa kin wicaśta yatapi toiyolsipi kin hee, tuka tuwe wowiśtece kage cin he canniye kta.

\section{WICOWOYAKE 15.}

1 Hbayedan waayupte cin he wowacinko anapta, tuka wicoie tehike cin he wocanteptanyan kaga.

2 Wicaśta ksapa cejipi kin wosdonye yawaśte, tuka wicaśta witkotko ipi kin wowitkotko enapeya.

3 Jehowa iśta kin owancaya wicaśta śica wicaśta waśte ko owasin iyohi wicaya.

4 Wicaceji wawokizi ye cin he can wiconi kin iyececa, tuka wicaceji wayapemni kin wicanagi kin he yawega.

5 Tuwe witkotko kin he atkuku wahokonkiye cin ihakta śni, tuka tuwe iyopeyapi iyowiniciye cin he ksapa.

6 Wicaśta owotanna ti kin ohna woyuha tanka yanka, tuka wicaśta śica taku mnayanpi kin en wośkiśke yukan.

7 Tona ksapa ihapi kin wosdonye kadadapi, tuka tona withotko cantepi kin hecetu śni.

8 Wicaśta śica wośnapi kin he Jehowa wowahteda śni, qa wicaśta ecetupi kin cekiyapi kin he iyokipi.

9 Wicaśta śica tacanku kin he Jhowa walitedaśni, qa tuwe woowotanna ope cin he waśte daka.

10 Tuwe canku elipeye cin wahokonkiyapi kin iyokipi śni, qa tuwe iyopeliyapi śice dake cin he te kta.

11 Jehowa wicațe cin nagipi kin qa tancan atakunipi śni owasin wanyaka, nakun Adam cinca cante ozepi kin owasin.

12 Waoncesa wahokonkiyapi waśte dake śni, qa wicaśta ksapapi kin ekta ye kte śni.

13 Wicacante wiyuśkin kin he itohnake waśte kaga, tuka cante wicaśice cin on wicoksape kin kawanka.

14 Wicacante ksape cin wosdonye akita, tuka witkotko ipi kin wowitkotkoke woyute yapi.

15 Tona iyokiśica anpetu tawapi owasin śicapi, tuka cante waśte kin wohanpi en ohinniyan opapi kin he iyececa.

16 Jehowa wokokipe yapi taku cistina yuhapi eśta hee waśte, tuka woyuha tanka wicośkiśke iyahna hee waśte śni.

17 Wahpe tanka heceedan eśta cante waśteya kiciyus yutapi kin hee waśte, qa tatanka cepa eśta cante śicaya kiciyus yutapi kin hee śica.

18 Wicaśta wacinko kicigepi kaga, qa tuwe tehan canteptanye śni woakinica anapta ece.

19 Wicaśta kujapi tacankupi kin wapepeka mabinatake wán iyececa, tuka wicaśta owotanna tacanku kin he yumdayapi.

20 Hokśidan ksape cin he atkuku wiyuśkin kiya, tuka wicaśta witkotkoke cin he hunku kin ihakta śni.

21 'Tuwe cante nice cin he wowitkotkoke cin wiyuśkinyan, tuka wookaliniga wicaśta ecetuya mani.

22 Wiyukcanpi wanice cinhan cante ozepi kin atakuni śni ece, tuka wiyukcanpi ota on yusutapi kta. 
23 Wicaśta i kin tanyan waayupte cin on iyuśkin kta, qa wicoie iyehantu kin he waśte hinca.

24 Wiconi canku kin wankantuya wicaśta ksapa opa, hecen Hades ihukuya kin yuiyogya.

25 Jehowa wahanicida ti kin yujuju, tuka wiwazica ti kin yusuta ece.

26 Cante ozepi śikśica kin Jehowa wahtedaśni, tuka wicoie wakaśoteśni kin he iyokipi.

27 Tuwe śikśicaya wakamna kin he iye ti kin iyokiśinya ece, tuka tuwe taku qupi kin śice dake cin hee ni kta.

28 Wicaśta owotanna cantepi token waayupte kta awacin, tuka tona śica ipi kin taku śica enapeya ece.

29 Wicaśta śicapi kin Jehowa itehanyan wicaun, tuka wicaśta owotanna cekiyapi eca nawicahon ece.

30 Wicaśta iyoyanpa kin he wicacante iyuśkin, qa taku waśte nahonpi kin wicahuhu cemya ece.

31 Wicanoge wiconi woiyopeye nahon kin he wicaśta ksapa om wanke kta.

32 Tuwe woonspe aktaśni kin he iye nagi kin ehpekiya, qa tuwe woiyopeye nation kin he cante ton.

33 Jehowa kokipapi kin woksape woonspe kin ee, qa onśihan içidapi kin he wowitan itokamya u ece.

\section{WICOWOYAKE 16.}

1 Cante ozepi kin wicaśta etanhan, tuka Jehowa ceji woayupte kin he qu.

2 Wicaśta iye tacanku kin owasin ecedan kecin, tuka woniya wicatkeuta Jehowa hee.
3 Nitolitani kin Jehowa en yakizamni wo, hecen nicante ozepi kin yusutapi kta.

4. Taku Jehowa kage cin owasin akiciyuptapi, qa wicaśta śica anpetu śice cin on nakun.

5 Wicacante wahanicida kin otoiyohi Jehowa wowahtedaśni, nape iciyahdeyapi eśta iyopewicayapi kta ce.

6 Wowaonśida wowicake iyahna on woalitani kajujupi, qa Jehowa kokipapi kin on taku śica etanhan inapapi.

7 Tohan wicaśta wan ohan kin Jehowa iyokipi kinhan tona tokayapi qon takuyapi kta e kaga. 8 Woowotanna kici taku cistina eśta hee waśte, qa woyuha tanka ecetu śni mnayanpi kin he śica.

9 Wicaśta cainte kin tacanku kin hdukcan, tuka cahde tawa kin Jehowa konza.

10 Wokcan wicoie wan wicaśta yatapi iha kin etanhan, wayaco eca i kin nuni kte.śni.

11 Tke utapi ecetu kin he Jehowa etanhan, ojuhadan ohna inyan un kin hena owasin iye kaga.

12 Wicaśta yatapi kin śicaya ohanyanpi kin wowahtedaśni yanpi kte, nakaeś. woowotanna kin oiyotanke kin yusuta.

13 Wiciha owotanna wicaśta yatapi iyowicakipi, qa tuwe ecetuya ie cin he waśte dakapi kta.

14 Wicaśta yatapi tocanniye kin he wiconțe wahośiwicaya iyececa, tuka wicaśta ksapa wan he anapte kta.

15 Wicaśta yatapi itohnake kin iyoyanpa kin ohna wiconi yukan, qa cante wicakiye cin he magaju ehake kin he iyececa.

16 Woksape mnayanpi kin hee iyotan, qa mazaskazi hee śni; 


\section{WICOIE WAKAN.}

qa wokcan iyohiyapi kin he mazaska isanpa cinpi kte.

17 Taku sica yuiyokapi kin he wicaśta ecetupi tacanku yumdayapi kin hee, qa tuwe iye ohan kin awanhdake cin he nagi kin kpatan.

18 Wicotakuniśni itokamya watankaicidapi $u$, qa wicawankahe kte cin itokam woniya wahaniçida ya ece.

19 Onśipika om woniya onśilian icida kin hee waśte, qa wahanicidapi om taku kipi kicipamnipi kin en opapi kin he síca.

20 Tuwe wicoie kin awacin kinhan taku waśte iyeya, qa tuwe Jehowa wacinye cin he yawaśtepi kta.

21 Tuwe cante ksape cinhan wokcan eciyapi kita, qa iha oskuye cin he woonspe icahye kta.

22 Tuwe woksape yuhe cin he wiconi wakoniya wan seca yuha, tıka wicaśta witkotko onspekiyapi kin he wowitkotko.

23 Wicacante ksape cin he $\mathrm{i}$ kin wayupikiya, qa iha kin akan woonspe aokijuya ece.

24 Wicoie wiciyokipi tulimaga canhanpi kin he iyececa, wicanagi woskuye kin, qa wicahuhu okizi kin hee.

25 Canku wanji ecetu seca wicaśta itokam eśta, iyohakamya wiconte canku kin iyahdeya.

26 Tuwe htani kin he iye on litani, i kin iyopaśtaka nakaeś.

27 Wicaśta wicaśtapi śni kin taku śica kicanye ca, iha kin ohna peta ideya seca.

28 Woopcmni wieaśta kin he woakinice icallya, qa wicaśta śin woyag un kin he takodakiciyapi wicayamdece cin hee.

29 Wicaśta wakisa kin takodaku kihnayan, qa canku wan waśte śni kin omanikiya.
30 Woyapemni kin yukcan kta e iśta kin ohmus ikikcu, qa iha kin hdalitaka wicohan śica hduśtan.

31 Wicapa ska woowotanna canku kin ohna iyeyapi kinhan wateśdake owaśtecaka kin hee.

32 Tuwe tehan canteptanye śni kin he wicaśta waśaka kapeya, qa tuwe toniya wowidag kiye cin he wicaśta otonwe icu kin he kapeya.

33 Wicaśta cconpidan kansu kin ojuha ohna iyeyapi, tuka token hinanpe kta Jehowa konza.

\section{WICOWOYAKE 17.}

1 Aguyapi onśpadan wapaye codan eśta wokiyapi en hee waśte, qa tipi wan tado akinicapi ojudan kin hee śica.

2 Ookiye ksape cin he cinca iśtenwicakiye cin he wowidag ye kta, qa sunka kiciyapi om taku tawayapi kta en wopamni yuhe kta.

3 Maka wakśica ohna mazasika iyutapi, qa maza oceti ohna mazaskazi, tuka Jehowa wicacantepi kin iwicayuta.

4 Wicaśta śica wiciha ecinśniyan anagoptan, qa tuwe itonśni kin ceji awihnuniyan kin anoh kiciya.

5 Tuwe wicaśta wahpanica kin iha kin he tuwe he kage cin iśtenya, qa tuwe tokan yuśakapi kin on iyuśkin śitkihdayapi kta. 6 Cinca qa sanpa cinca kin hena wicahinca wateśdag yapi, qa hokśipidan atkukupi kin wowitan wicayapi ece.

7 Iapi tanka kin wicaśta witkotkopika iyewicacecapi śni, qa hc isanpa iha itonpi śni kin wicaśta tokapapi kin iyowiniçiyapi kte śni.

8 'Taku wicaqupi inyan wici- 


\section{WICOWOYAKE 18.}

mdeza iyececa tuwe he yuhe cin iśta kin itokam, taku kaśta ektakiya yuhomnipi taku okihi.

9 Tuwe woahtani akahpeton kin he otakuye akita, qa tuwe he oyakesa he kodakiciyapi tanka yujuju.

10 Iyopeyapi kin wicaśta ksapa nina yaskinya, tuka opawinge apapi keś wicaśta witkotko kin awacin śni.

11 Tuwe wakipajin taku śica hecedan akita, heon wahośi wohitika ekta ye sipi kta.

12 Eeś wicaśta wanji wahanksica cinca owasin kitanin śni akipe cin he tehika cśta, wicaśta witkotkoka towitkotkoke cin ohna he isanpa tẹika.

13 Tuwe taku waśte on taku śica iyowinye cinhan, taku śica iyc ti kin ayuśtan kte śni.

14 Okicigepi otokahe kin he mini okadusyapi kin he iyececa, heon akinicapi kin ihdutaninśni itokam ehpeya wo.

15 Tuwe wicaśta śica yaowotanna, qa tuwe wicaśta owotanna yaśice cin hena napin Jehowa wowalitedaśni.

16 Taku on etanhan woksape opeton kta e witkotkoka nape ohna maza yukan tuka cante wanica?

17 Kodayapi wan ohinniyan takodaku cante kiye kta, qa tehiya unpi eca owicakiye kta ece kin heon sunkayapi wan wicakicitonpi.

18 Wicaśta cante nice cin takodaku itokam icazopi kicicajuju kta keye ça nape yekiya.

19 Tuwe wokipajin waśte dake cin he akinicapi waśte daka, qa tuwe tatiyopa kin hduwankantuya kin he ihang içiye kta e akita.

20 'Tuwe cante pemni kin he taku waśte iyeyc kte śni, qa tu- we ceji kin hdatokeca kin he tạku śica ohinhipaye kta.

21 Tuwe cinca witkotko wan kage cin he woiyokiśin içiya, qa hokśidan wicaśtaśni atkuku kill iyuśkin kte śni.

22. Wicacante wiyuśkinyan tanyan okizi wicaya, q̀a woniya iyokiśice cin he wicahuhu kin naśeca.

23 Taku itpi etanhan qupi kin he wicaśta śica icu, qa woyaco canku kin yapemni.

24 Wokcan wicaśta kin itokamya woksape yukan, tuka wicaśta witkotko iśta kin maka ihankeya kin etonwanpi.

25 Hokśidan witkotko kin he atkuku okiçuninya, qa tuwe ton kin iyokiśinya ece.

26 Wicaśta owotanna iyopewicayapi kin he waśte śni, wicaśta tokapapi kin taku ecetu kin on awicapapi kin he nakun.

27 Tuwe oie kin kpatan kin he wosdonye sdonya, qa woniya inina kin he wookahnige wicaśta.

28 'Tuwe witkotkoka eśta inina un kin hehanyan ksapa kecinpi kta, iohmus un kin he wiyukcan.

\section{WICOWOYAKE 18.}

1 Wicaśta wan iyokipi içiye kta e iśnana iyaye ça taku awacinpi kin owasin on wicakige.

2 Wicaśta witkotko wookalinige iyokipi śni, tuka cante kin toketu ihdutanin kta he iyokipi.

3 Wicaśta śica u kinhan woaktaśni nakun $\mathrm{u}$, qa wicakigepi kin he iwicaśteca opa.

4 Woksape wakoniya kin he wakpa kaduze cin heca, wicaśta i kin etanhan wicoie kin mini śma kin he iyececa.

5 Wayacopi en wicaśta śica wan ihaktapi on wicaśta owo tannæ yapemnipi kin he waśte śni. 


\section{WICOIE WAKAN.}

6 Wicaśta witkotko iha kin akinicapi en ye içiya, qa $\mathrm{i}$ kin apapi kin kipan..

7 Wicaśta witkotko i kin ihdutakuniśni, qa iha kin iye nagi kin kihmunka ece.

8 Tuwe iwakan kin oie kin woyute skuya kin iyececa, wicatezi kin mahentuya iyaya ece.

9 Nakun tuwe tohtani on kuje cinhan tuwe woyuha ihang ye cin he cinye ya.

10 Jehowa caje kin conkaśke suta wan heca, wicaśta owotanna kin hen onapa qa okope wanin yanke.

11 Wicaśta wijica taku yuhe cin he otonwe suta, qa conkaśke tehanwankantuya iyececa kecin.

12 Nawegapi kin itokam wicacante kin wahanicida, qa wowitan itokam onśihan icidapi kin.

13 Tuwe taku nahion śni itokam waayupte $\operatorname{cin}$ he witkotko içiye ca kiunniciya.

14 Wicaśta tancan yazan eśta taniya kin wakiśaka ece, tuka wicaniya naśujapi kinhan tuwe wakiśalke kta okihi he?

15 Wicacante wiyukcan kin he wosdonye ton kta, qa wicaśta lisapa nogepi kin wosdonye akite kta.

16 Taku ituhan kin wicaśta kiciyukan kta, qa wicaśta tanka wicitokam hiyuye kta.

17 Waakinicapi en tuwe tokaheya wohdake .cin he ihdaowotanna, tuka uman hi qa iwange kita.

18 Oeconna kin akinicapi kin anaptc ca wicaśta waśaka wicayukinukan.

19 Sunkakiciyapi akinicapi kin conkaśke tiyopa onatakapi kin iyececa, qa otonwe suta ohiyapi tchika eśta sunkakiciyapi kicipajinpi kin he sanpa tehika.

154
20 .Wicaśta tezi kin taku i kin icaliye cin on imna lita, taku iha kin etanhan inape cin heon imna. kta.

21 Wiconte wiconi ko wicaceji nape kin ohna un, qa tuwe he waśte dake cinhan taku icahye cin he etanhan yute kta.

22 Tuwe tawicu iyeye cinhan taku waśte iyeya, qa Jehowa wicaśta kin he cante kiya.

23 Wicaśta wahpanica wacekiya yanka, tuka tuwe wijice cin he sutaya waayupta.

24 Tuwe wicota kodawicaye cin he kodayapi iyecen ihdutanin lkta, qa wanji wacantkiya sunkayapi wicapatan eśta sam iyeya wicapatan.

\section{WICOWOYAKE 19.}

1 Tuwe iha pemni qa witkotkoka hee śni, tuka tuwe ecetuya rnani walipanica eśta hee waśte.

2 Wicanagi kin wosdonye nice cin he nakun waśte śni, qa tuwe siha kin inahni kin he nuni.

3 Wicaśta towitkotkoke cin canku kin lıdupemni, qa cantc kin Jehowa canniyeya.

4 Wowijice kodayapi ota icahya, tuka tuwe wahpanica kin he takodaku yukinukan.

5 Wayaatanin itonśni kin he iyopeyapi kta, qa tuwe taku ecinkta enapeye cinhan ihduśpe kte śni.

"6 Tuwe nina wo wicaqu kin wicota he ite kin kastostopi kta, qa tuwe ituhansa kin he owasin kodayapi kta.

7 Tuwe onślka eca hunkawanjitku owasin śice dakapi, qa liecen sam iyeya kodawicaya ihdutehanpi, wicoie pasi tuka hena taku śni.

8 Tuwe cante ton kin he nagi 


\section{WICOWOYAKE 20 .}

kin waśte kidaka, tuwe wookalinige patan kin taku waśte iyeye kta.

9 Wayaatanin itonśni kin iyopeyapi kta, qa tuwe taku ecinkta enapeye cinhan awihnuni kta.

10 Wicaśta witkotko taku wiciyokipi yuhe kta he iyecece śni, qa ookiye yuhapi qon wicaśta itancan wowidag wicaye kta e sanpa tehika.

11 Wicaśta toksape kin tocanniye kin kihnaka, qa śicaya ecakiconpi kin iwankam iyaye cin towitan kin hee.

12 Wicaśta yatapi tocanniye kin he mnaja hoton kin iyececa, qa toiyokipi kin cu kin wato akan he iyececa.

13 Hokśidan witkotko atkuku toiyokiśice kin ee, qa winohinca kigesa kin śbuyapi ohinniyan kin heca.

14 Tipi qa woyuha hunkake yapi etanhan tawayapi, tuka tawicu ksape cin he Jehowa etanhan.

15 Wokuje kin he hbapi kaga, qa wicanagi econkapin kin he wotektehda un kta.

16 Tuwe woope kin yuhe cinlian nagi kin hduha, qa tuwe tacanku kin iyakiktaśni kin he te kta.

17 Tuwe wahpanicapi cante wicakiye cin he Jehowa oqu, qa taku waśte kin he iyecen tanyan kicu kta.

18 Nahanhin taku waśte ape oyakihi nicinca iyopekiya wo, qa te yakiye lkta ihnuhan ninagi en inape cin.

19 Tuwe wocanteptanye tanka kin he śitkihdayapi kta, hetanhan edaku eśta ake ecanon lita.

20 Wahokonniciyapi kin awacin wo, hecen iyohakamya yaksape kta.
21 Wicacante ekta taku ota awacinpi, tuka Jehowa taku konze cin he sutaya he kta.

22 Wicaśta toiyokipi towacantkiye kin hee, qa wicaśta wahpanica kin wicaśta itonśni kin kapeya.

23 Jehowa kokipapi kin on wiconi, qa tuwe hecon lin he imnahan yanke kta, taku śica he iyohi kte śni.

24 Wicaśta kuje cin nape kin wakśica ohna yekiya, ake i kin ekta eśta hiyukiye lite śni.

25 Waoncesa nina ayape cinhan tuwe wacintonśni kin he ksape kta, qa wicaśta wokahinige kin he iyopeya wo, wosdonye okahnige kta.

26 Hokśidan waiśtenya qa waihakta śni kin he atkuku kin hdutakuniśni, qa hunku napekiya.

27 Micinkśi woonspe kin wosdonye wicoie kin etanhan nuni wicakiye cin he nahon kta ayuśtan wo.

28 Wayaatanin wicaśta śni kin he woyaco ihaha, qa wicaśta śica ipi kin taku śniśni napcapi.

29 Woyaco wicaśta śica wiyeya wicakicihnakapi, qa wicaśta witkotkopi tapetepi kapsinpsintapi kta.

\section{WICOWOYAKE 20.}

1 Miniśa kin he waihahia qa mini wakan kin he owodutaton wicaya; tuwe unman tukte kaśta on nuni kinhan ksape kte śni.

2 Wicaśta yatapi kin yuśinyewicaye cin he mnaja hoton kin he iyececa, tuwe he camniyeye cinhan iye nagi kin awahtani.

3 Akinicapi anapte cin wicaśta towitan kin ee, tuka wicaśta witkotko otoiyohi wakigepi en yeiçye kta. 


\section{WICOIE WAKAN.}

4 Wicaśta kuja osni kin on maga hdumdu lete śni, he on woksapi icunhan woda kta, tuka wanice kta.

5 Wicaśta taku konze cin he cante mahen mini śma kin iyececa, tuka wicaśta wookahinige kin he tankan hiyuye kta.

6 Wicaśta ota otoiyohi towacantkiye kin ohdake kta, tuka wicaśta wowicake tuwe iyeye kta he?

7. Tuwe ecetuya mani kin he olian owotanna, cinca iyohakam icagapi iś eya tanyan unpi ece.

8 Wicaśta yatapi woyaco oiyotanke akan yanke cin he iśta kin on taku śica owasin bomden iyaye ya.

9 Micante kin mihduecedan, wawahtani kin etanhan mihduska, tuwe eye kta okihi he?

10 Wiyutapi toktokeca, qa tke utapi toktokeca heniyoza Jehowa wahtedaśni.

11 Kośka eśta ohanye cin etanhan ihdutanin, ohan kin ecedan kinhan qa ecetu kinhan.

12 Noge wanalion kin qqa iśta wawanyaka kin heniyoza Jehowa kaga.

13 Ihnuhan woiśtima waśte yadake cin, okini nihduwahpanice kta ; iśta hdukawa hecen aguyapi inimna kta.

14 Śica, śica ce, turve opeton kta eye ca wanna tawa tankan iyaya hehan ihdatan.

15 Mazaskazi yukan, qa tukihasan śa ota eśta, wosdonye wiciha kin he wojuha tehike hinca hee.

16 Tuwe oyate tokeca wicaśta on, wecicajuju kta, eniciye cinhan taśina kin icu wo, qa tuwe wicaśta sdonwicaye śni, wicawecicajuju kta, eye cinhan taku tohanyan kajuju śni duhe kte cin he icu wo.
17 Tokan wicakicicajujupi eyapi kin he aguyapi itonśni, qa iyohakam i kin isbudan oju ye kta.

18 Taku konzapi kin wiyukcanpi kin on yusutapi kta, qa tanyan iyukcanpi kin hehan ozuye kaga wo.

19 Tuwe waaia mani kin he taku nahmana oyakapi kin yaotanin, heon tuwe iha hdakawahan he kici icicahiye śni wo.

20 Tuwe atkuku hunku ko wicayaśice cinhan otpaza cokaya kin petijanjan tawa kin bokicisni kta.

21 Woyuha otokahe ekta inahinina mnayanpi kin he owihanketa yawaśtepi kte śni.

22 Taku śica towecon kta, ihnuhan ehe cin; Jehowa apeya wo, iye niniye kta.

23 Nonpakiya tke utapi kin Jehowa wahteda śni, qa wohnaye aspekiyapi kin he waśte śni.

24 Wicaśta oye kin he Jehowa etanhan, hecen tokiya tanhan wicaśta canku kin hdukcan kta he?

25 Wicaśta tuwe ihnuhanna taku tawa hdawakan kin he hmug içiye, nakun tuwe konze cin iyohakan iwange cinhan.

26 Wicaśta yatapi ksape cin he wicaśta śica wicayamdece ca canpahrnihma wiciwankam hiyuya:

27 Jehowa petijanjan tawa kin wicaśta taniya kin ee, taku wicacante mahentuya un kin owasin iwanyaka.

28 Wocantekiye wowicake ahna wicaśta yatapi kin awanyakapi, qa oiyotanke tawa wocantekiye on hdusuta.

29 Kośka towaśakapi kin iwinktapi kin hee, qa wicahinca kin pa skapi kin wowitan yapi.

30 Bahonpi paskitapi kin he 
taku śica patehan ya, qa apapi kin tezi colkaya iyohi kin he nakun.

\section{WICOWOYAKE 21.}

1 Jehowa wicaśta yatapi cante kin mini wakpadan iyecen nape kin ohna yuhe ca toki cin kin heciya he yuptanyan.

2 Wicaśta iyohi tacanku kin owasin ecetu kecin, tuka Jehowa wicacante owasin aspe kiya.

3 Wośnapi Jehowa iyokipi eśta taku owotanna qa ecctu econpi kin he isanpa iyolipi.

4 Iśta ihduwankantuyapi qa cante kin ihdatankapi kin hena wicaśta śica petijanjan yapi tuka woahtani.

5 Wicaśta miniheca canteozepi on taku mnayanpi ece, tuka tona inalinipi kin owasin on ihdukakijapi ece.

6 Woyuha ota ceji itonśni on mnayanpi kin hena takuśniśni bomdu iyayapi, qa tona heconpi kin hena wiconte akitapi.

7 Wicaśta śica wakipi kin ihang wicaye kta, nakaś woyaco econ tawațenyapi śni.

8 Wicaśta canku pemnimni kin he oyate tokeca, tuka tuwe ecedan kin he tancanku kin ccetu.

9 Tice kahmin otipi kin hee waśte, tuka winohinca wakigesa kici tipi tankaya eśta otipi kin he tehika.

10 Wicaśta śica nagi kin taku śica cin ece, iśta kin takodaku eśta wacantkiya yuze śni.

11 Waoncesa aonpapi kinhan tuwe wacintonśni ksape kta, qa wicaśta ksapa onspe kiyapi kinhan wosdonye icu kta.

12. Wicaśta owotanna kin wicaśta śica toti kin iyukcan : Tuwe wicaśta śica taku śica ohna napewicaya.
13 Tuwe tohan wicaśta onśika hoyekiyapi ca noge kin onakitake cinhan iś eya hoyeye kta, tuka tuwedan ayupte kte śni.

14 Taku nahmana qupi kin he wocanniye anapte, ca itpi kin ohna taku ituhanpi kin he canteptanye tanka.

15 Woyaco econpi kin he tona owotanna iyuśkin wicakiya, tuka śicaya olianyanpi kin wicakahintapi kta.

16 Wicaśta wan wookahinige canku kin etanhan nuni kin he wicaśta tapi yuwitayapi om yanke kta.

17 Tuwe iyokipi iciye kta waśte dake cinhan ihduwahpanica, qa tuwe miniśa wihdi ko waśte dake cin he wijice kte śni.

18 Wicaśta śica tona owotanna ohanyanpi kin wicakicicajujupi kta, qa wicaśta wakisa kin tona ecetupi on.

19 Tinta hewoskantuya ounyanpi kin he waśte, tuka winohinca wakigesa qa wacanniyesa kici tipi kin he śica.

20 Wicaśta ksapa ounye cin olna woyuha wiciyokipi qa wihdi yukan, tuka wicaśta witkotko kin he napca iycya.

2 I Tuwe woowotanna wocantekiye ohna ope cinhan wiconi woowotanna qa wowitan iyeye kta.

22 Wicaśta ksape cin he waśakapi otonwepi kin ohiye ca, taku suta wacinyanpi kin he yuhukuya ece.

23 Tuwe i kin qa ceji kin awanhdake cin he nagi kin taku tehika etanhan kpatan.

24 Tuwe wahanicidaya wocanniye kage cin he wamnaicida, wahaniçida waoncesa eciyapi lita.

25 Wicaśta kuja nape kin hitani kapin, heon wocin kin te kiye. 
26 Anpetu osan wahduhaha kte cin cantotpani, tuka wicaśta owotanna woqu qa tehinda śni.

27 Wicaśta śica wośnapi kin lie wowahtedaśni ece, ecah taku sica awacinyan au kinhan isanpa.

28 Woitonśni wayaatanin kin awihnuni kta, tuka wicaśta token nahon kin yatanin kin he ohinniyan ie kta.

29 Wicaśta śice cin itohnake kin hdusuta, tuka wicaśta owotanna kin canku kin hduecetu.

30 Jehowa itkokim wicoksape wanica, qa wookahnige wanice ca wokcan wanica ce.

31 Kicizapi anpetu kin heon śuktanka kin wiyeya kihnakapi, tuka niwicaye cin Jehowa hee.

\section{WICOWOYAKE 22.}

1 Woyuha ota hee śni, tuka tanyan caje niyatapi kin he kaliniga wo; mazaska mazaskazi kici hee śni, tuka tanyan wacin en niyuzapi kin.

2 Wicaśta wijica qa wahpanica itkokicipapi, Jehowa napin wicakaga.

3 Wicaśta ksape cin taku śice cin he wanyake ca inahma, tuka tona wacintonpi śni kin hena iyoptapi qa kakiświcayapi.

4 Jehowa kokipapi qa onśihan içidapi iyuwin kin he wowitan qa wowijice qa wiconi.

5 Wicaśta pemni tacanku kin ohna wapepeka on hmunkapi ko yukan, tuwe nagi kin awanhdake cinhan hena ihdutehan kta.

6 Otokahe etanhan hokśidan tacanku kin ohna kihiya wo, hecen wicahinca eśta ehpekiye kte śni.

7. Wicaśta wijica wahpanicapi wowidag wicaya, unkan tuwe waodote cin he waoqu kin he okiya ece.

8 Tuwe wicohan śica oju kin he wicotaninśni mnaye $\mathrm{kta}$, qa tocanniye sagye kin sote kta.

9 Iśta wacantkiya kin he yawaśtepi kta, nakaś aguyapi tawa kin onśpa wakanheja wicaqu.

10 Waoncesa napeya wo, hecen wakigepi kin tokan ye kta, nakun waakinicapi kicibapi ko enakiyapi kta.

11 Tuwe wicacante ecedan kin he waśte kidake cin he iha wiciyokipi on wicaśta yatapi kin kodaye kta.

12 Jehowa iśta kin wosdonye patan ece, qa wicaśta wahnayesa oie kin yuptanyeya.

13 Mnaja wan tankan yanka, otonwe canku kin ohna makte kta, wicaśta kuje cin eya.

14 Wihomniwin i kin he woha śbe hinca, tona Jehowa wahte wicadaśni kin hena ohinlipayapi kta.

15 Hoksidan cante kin ohna wopemni opahtapi eśta iyopekiyapi icapsinte kin he yutehan yekiciye kta.

16 Tuwe taku tawa hduota kta e wicaśta onśika wicayuwahpanica, qa tuwe watonka wawicaqu kin he awicakehan wahpanice kta.

17 Nogoptan qa wicaśta ksapa oiepi kin nalion wo, qa nicante kin mitoksape kin awacin kta.

18 Hena maku mahen yapatan kinhan wiciyokipi; niiha kin akan yuecetupi kta.

19 Niś niye Jehowa wacinyaye kta, heon nakaha hena sdonye ciciya.

20 Wahokonkiciyapi qa wosdonye kin taku itancanyan ociciyake sni he?

21 Hecen wowicake wicoie kin 
woecetu kin sdonye ciciye, heon tona ye niśipi kin hena wicoie wicake cin wicayakahdi kta.

22 Wicaśta onśike cin he onśiya un kin on etanhan ihnuhan taku tawa yaki kinhan, qa wicaśta walipanice cin he wayacopi en yaśuje śni wo ;

23 Awicanicapi en Jehowa iwicakiye kta, qa tuwe wawicaki kin he nagi kin ki kta.

24 Ihnuhan wicaśta wacinko wan kodayaye cin, qa wicaśta wacanniyesa kin kici ye śni wo ;

25 Okini ohan tawa kin ccewayakta nace, qa ninagi kin nicihmunkapi nun ce.

26 Tokan on taku wicakicicajujupi kta, icazopi kin wicakicicajujupi kta eyapi kin, ihnuhan owicayape cinhan;

27 Taku on kajujupi oyakihi śni kinhan tokeca owinja nihuhuya tanhan eniciyakupi $k$ ta he?

28 Maga wapetokeca wanakaja nihunkakepi ehdepi qon he dutokan kte śni.

29 Wicaśta wohtani en miniheca wandaka he? wicaśta yatapi wicitokam najin kta ; wicaśta caje wicayatapi śni kin hena wicitokam najin kte śni.

\section{WICOWOYAKE 23.}

1 Wicaśta yatapi wanji kici wayate kta idotanke cinhan, taku nitokam he cin he en ewacin wo.

2 Wayate cin iyakape niye cinhan isan wan dote kin hdakinyan iniyakiye non ce.

3 Woyute tawa wiciyokipi kin he iyotan da śni wo, he woyute kin wicahnayan.

4. Wijinniciye kta e ihdustute śni wo, wookalinige nitawa kin he ahduśtan wo: Wa

5 Taku, iśta kin yeyalkiya he? wanmdi wan mahipiya ektakiya kinyan iyaye cin he iyecen wowijice hupahu içicage ça iyaya ece.

6 Tuwe wohinyesa kin aguyapi tawa kin etanhan yute śni wo, qa woyute skuya tawa kin kon śni wo;

7 Nakaś token nagi mahen ecin kin he iyececa, Wote ca wayatkan wo, eniciya eśta cante kin nici un śni.

8 Onśpa yate cin he hdemyaye kta, qa nioie waśte kin owasin ehpeyakiye kta.

9 Wicaśta witkotkoka noge kin ohna ie śni wo, nioie woksape kin ihakta kte śni.

10 Maga wapetokcea wanakaja kin he dutokan kte śni, qa wamdenica maga tawapi kin an ye śni wo.

11 Nakaś tuwe taku wicaye cin he waśaka, iye hinca taku tawapi kin on nici akinice kta.

12 Woonspe ekta nicante kin hiyukiya wo, qa ninoge kin woksape wicoie kin ekta.

13 Hokśidan iyopekiyapi patan śni wo ; cansakadan on ayapa eśta te kte śni.

14 Cansakadan on ayape sta, hecen nagi kin Hades etanhan edaku kta.

15 Micinkśi nicante kin ksape cinhan micante kin iyuśkin kta, miye hinca;

16 Qa pakśin mitawa nina pida kta, niiha taku ecetu eye cinhan.

17 Ihnuhan nicante walitanisa icewicakin kin, tuka anpetu osan Jehowa kokipeyahan un wo.

18 Nakaś woyuśtan yuke cinhan taku wacinyan ayape kin he nicicaśpe kte śni.

19 Micinkśi niye anagoptan, qa ksapa wo ; qa canku ohna nicante kin hduowotanna wo. 
20 Miniśa yatkanpisa qa iyokipi içiyapi kta e tado napcapisa owicape śni wo.

21 Witkosa qa wotesa kin hena ihduwahpanicapi, qa ogungapi kin hella wokoyake hdelidecahan koyakapi kta.

22 Niyate cinca nicage cin he nation wo, qa nihun wakanka eśta ihnuhan ihayak taśni kin.

23 Wowicake kin opeton wo, qa wiyopekiye śni wo, nakun woksape qa woonspe qa wookahnige.

24 Tuwe olian owotanna kin he atkukı nina iyuśkin kta, qa tuwe hokśidan ksapa wan icahye cinhan he etanhan pida kta;

25 Hecen niyate nihun kici iyuśkinpi kta, qa tuwe niton kin nina pida kta.

26 Cinś nicante kin maquı wo, qa canku mitawa kin he niiśta kin awanyaka nunwe.

27 Witkowin kin he makohdoka temahentuya, qa wihominiwin kin he wolia cistiyedan ec :

28 Awicakehan he wabotica iyecen wawihmunke ca wicaśta ehna wahtanipisa wicayuota.

29 Tuwe, Hehehe, eya hc? tuwe ccya he? Tuwe kicikigepi yuha he? tuwe woiyokiśice yuha lic? tuwe taku on etanhan taninśni apapi he? iśta samyahan un kin he tuwe?

30 Tona miniśa tehan opapi kin, tona miniśa icicahiyapi kin akin yapi kin hena.

31 Miniśa tohan śaiciye, tohan wiyatke ohna iśta kin hdutanin, wiciyokipiya kaduze cin hehan atonwe śni wo.

$32 \mathrm{He}$ iyohakam wamduśka iyccen wayahtake kta, qa sintchida wan iyecen wakiunniye kta.

33 Hehan niiśta kin winohinca tokeca wanwicayake kta, qa ni160 cante kin taku pemnimni kiu enapeye kta;

34 Qa tuwe miniwanca cokaya kin iwanke cin he iyenicece kta, qa tuwe sina watopekiyapi ihupa inkpata wanke cin he iyenicece kta.

35 Makaśtakapi tuka mayazan śni, amapapi tuka weksuye śni, tohan wekta kinhan ake awakite kta, ehe kta.

\section{WICOWOYAKE 24.}

1 Wicaśta śice cin icewicakin śni wo, qa owicayape kta cin śni wo.

2 Cantepi kin waboticapi awacinpi, qa ihapi kin waapatayapi kin ccedan cajeyatapi.

3 Woksapc on tipi kagapi ece, qa wookahnige on sutaya he kta. 4 Wosdonye on wopiye owasin woyuha tehike ça wiciyokipi ojudan yapi kta.

5 Wicaśta ksape cin he waśa$\mathrm{ka}$, qa wosdonye wicaśta kin hc tuwe waśake cin he kapeya.

6 Ozuye yakage cinhan wayupiya econ wo, qa wiyukcanpi ota on ohiyapi ece.

7 Woksape kin he tehanwankantuya un, hecen wicaśta witkotko kin he iyohi śni, otonwe tiyopa kin ohna i kin hdukawa śni. 8 Tuwe taku śica econ wacin kinhan he wicohan sica itancan cciyapi kta.

9 Witkotkoka cante oze kin he woalitani kin ee, qa waopncesa kin he wicaśta wowahitedaśni yapi kta.

10 Iyokiśicapi anpetu kin en cante niwankadan kinhan nitowaśake kin cistinna kta.

11 Tona wiconte en yusdohan awicayapi, qa wicaktepi kta e kaktihar - kin hena ewicayahdaku kta. kinhan. 
12 He sdonunyanpi sni, eha cśta, tuwe wicacante kin iyukcan kin he iyukcan kte śni he? tuwe ninagi kin awanyake cin he sdonye kta, qa wicaśta otoiyohi token olianye cin he iyecen yuhekiye kta.

13 Cinś tulimaga canhanpi kin he waśte heon yuta wo, qa canhunpute kin he nicaka ekta skuye kta;

14 Woksape ninagi kin ekta hecece kta, tohan he iyeyaye cinhan iyuwin yuke kta, qa taku ayape kin heon niçihnaye kte śni he sdonya wo.

15 Wicaśta śica, ihnuhan wicaśta owotanna ounye cin iwandake cin, qa tipi tawa kin awihnuniye śni wo.

16 Wicaśta owotanna śakowin akihde hicahan eśta najin $\mathrm{kta}$, tuka wicohan śice cin he taku śica oilipaye kta.

17 'I'oka niye cin hicahe cinhan ihnuhan iduśkin kin, qa naicipson eśta ihnuhan nicante, kin iyokipi ic̣iye cin ;

18 Okinni Jehowa he wanyake cinhan iyokipi kte śni, qa tocanniye kin he etanhan niye en aniyuhomni nun ce.

19 Wicaśta śicapi iśta ihnuhan iyoniyake cin, qa wicolian śice cin he icekin śni wo;

20 Nakaś wicaśta sica iyuwin yuhe kte śni, qa wicolian śicapi kin hena petijanjan wicakiyusnipi kta.

21 Cinś, Jehowa kokipeyahan un wo, wicaśta yatapi nakun ; qa tona taku tok tokeca akitapi lin hena om opiic̣iye śni wo.

22 Heniyoza tohan kakiś wicayapi kta tuwe sdonya he? woiyokiśicapi ihnuhanna anawicajin kta.

23 Wicaśta ksapa oiepi kin hena nakun eepi: Wayacopi kin en wicite iyekiyapi kin he waśte śni.

24 Tuwe wicaśta śice cin, oniwotanna eciye cin he oyate kin yaśicapi kta; han, owasin wowalitedaśni yapi kta.

25 Tuka tona he iyopeyapi taku wiciyokipi iyeyapi kta, qa tuwe waśte kin wicayawaśte lita.

26 Tuwe wicoie ecetu ayupte cinhan iha kin iputakapi kta.

27 Nitohtani tankan iwanhdaka wo, qa nitamaga kin tanyan hduśtan wo, qu hehan tipi yecage kta.

28 Ihnuhan ituli nitakoda on taku śica odake cin; niiha kin on wicayahnaye kta he?

29 Token ecamiconpi kin he iyecen ecawecon kta, wicaśta otoiyohi" token olianye cin he iyecen owakilianye kta, eye śni wo.

30 Wicaśta kuja wan tamaga kin icahda iyamdupta, qa wicaśta cante nica wan wiyuwi tawa ikiyedan;

31 Unkan inyun hena owancaya haśbe aicage ca itohnake kin osan wapepeka on akalipetonpi, qa inyan on inataktonpi kin he yuwankapi ;

32 Hena awatonwan, qa micante kin elkta ewahnaka ; wanmdake ca waonspemiciciye.

33 Ehake cistiyedån niśtibe, cistiyedan makan inunke kta e nape pakśijapi ;

34 Hecen nitowahpanice kin he wicaśta icimani wan he iyecen en nihi kta, qa wicaśta wipe hduhe cin en u kin iyecen taku owasin inicakije kta.

\section{WICOWOYAKE 25.}

1 Wicoie wakan kin dena nakun Salomon tawa, qa Juda o161 
yate wicaśta yatapi Hizekiya wicaśta tawa kin dena alrta owapi.

2 Wakantanka taku anakilibe kta towitan kin ee, tuka taku tanyan iyulcan kta e wicaśta yatapi kin towitan kin hee.

3 Mahpiya tehanwankantuya kin, qa maka temahentuya kin, qa wicasta yatapi cante kin hena owasin iyukcanpicaśni.

4 Mazaska etanhan gigi kin he yutokan iyeya wo, hecen wakśica wan mazakage cin un lkta e hiyu kta.

5 Wicaśta yatapi itokapa tanhan wicolian śicapi kin hena wicayutokan wo, hecen oiyotanke tawa woowotanna on sutaya he kta.

6 Wicaśta yatapi kin itokam ihdutan śni wo, qa wicaśta tokapapi ohe kin ohna najin śni wo.

7 Nakaś, Wankantuya deciya u wo, eniciyapi kin hee waśte, tuka wicaśta itancan niiśta wanyake cin he itolkam niyuhukuyapi kin hee waśte śni.

8 Ihnuhan inaliniyan woakinice en yeniçiye cin, okini owihanketa tuwe kici ayakinice cin he iśtenniye kta, hehan token ecanon kta he?

9 Tuwe kici ayakinice cin he kiciśnana wohdaka wo, unkan tokan taku nahnaana oniciyake cin he yuotanin śni wo.

10 Okini tuwe nanilion kin he iyopeniye kta, qa iśtenniyanpi lin niciyutokanpi kte śni.

11 Wicoie wan tohan iyececa iapi kin he taspan tanka zizi kin mazaska wakiślsotpa ohna he iyececa.

12 Wawiyopeye ksape cin noge wanahon kin ekta he mazaska oinpi kin, qa mazaskazi wanapin lin he iyececa.
13 Wahośi wacinyepica wan tona ye śipi kin iyowicakipi kin he, mdoketu cokaya wa osni kin iyecen, qa itancan tawa nagi kin ekicetu.

14 Tuwe taku wicaqu kta itonśniyan on ihdatan kin he mahpiya sapa qa tate magaju wanica kin he iyececa.

15 Wacintankapi on wicaśta yatapi kin cinkiyapi, qa ceji skuya kin he wicahulu kin yaksa ece.

16 Tuhmaga canhanpi iyeyaýa he? winipi kta e heceedan yuta wo. Okini iyatahdeniçiye cinhan yahdepe kta.

17 Ihnuhan nisiha kin anpetu owasin nitakoda ti kin en ye yakiye cin; okini ijehan en de cinhan śice nidake kta.

18 Tuwe takodaku oitonśniyan wayaatanin kin he wanhinkpe, qa isan qa canhpi wan iyececa.

19 Wohnaye wicaśta wan, anpetu iyolziśicapi en wacinyanpi kin he hi wan ksahan qa siha pśunwahan kin he iyececa ece.

20 Tuwe cante śice cin he ekta wiyuśkinyan odowan ahiyayapi kin he anpetu sni icunhan heyake yuśdokapi kin, qa wipajaja miniskuya akaśtanpi kin he iyececa.

21 Tuwe śice nidake cin he wotektehda kinhan aguyapi y unkiya wo, qa ipuza kinhan mini yatkekiya wo ;

22 Hecen petaga iye pa kin akan mnayaye kta, unkan Jehowa iyuwin niçu kta.

23 Tate waziya tanhan u kin he magaju kaha iyeya, he iyecen itohnake canksiya wicaceji taku śica nalimana oyake cin.

24 Tice okahmin otipi kin hee waśte, tuka winohinca wakigesa kici tipi tankaya eśta he telika. 
25 Wootanin waśte wan malioce tebantanhan aupi kin he mini sni tuwe ipuza kin ekta he iyececa.

26 Wicaśta owotanna wan wicaśta śica itokam patuje cin he mini owe omanipi kin, qa wakoniya waihang ye cin he iyececa.

27 Tuhmaga canlianpi ota yutapi kin he waśte śni, he iyecen taku tke owasin iyukcanpi kin he tehika.

28 Tuwe toniya hduhe kta okihi śni kin he otonwe wan kawankapi, qa conkaśke wanica he iyececa.

\section{WICOWOYAKE 26.}

1 Mdoketu eca-wa, qa woksapi icunhan magaju, he iyecen tuwe witkotkoka-kinihanpi kin he wiciyokipi śni.

2 Zitkadan kinyanyan, qa icapśinpśincadan anawinyan kin, he iyecen ituli yaśicapi kin he iyowicahi kte śni.

3 Suktanka on wicapsinte, qa śonśonna on iiyuwi kin, qa wicaśta witkotkopi tapetepi kin on cansakadan.

4 Ihnuhan wicaśta witkotko towitkotkoke cin he iyecen adupte cin, okini niś eya he inihdacin kta.

5 Wicaśta witkotko towitkotkoke cin he iyecen ayupta wo ; hecanon śni kinhan okini ksape icida kta.

6 Tuwe taku tanka on wicaśta witkotko wahośi ye cin he siha kin kakse ca iye kiunniiciye.

7 Wicoie wakan wan wicaśta witkotko i kin ohna, tuwe huśte sicogan natitantan kin he iyececa.

8 Tuwe wicaśta witkotko wan wowitan qu kin he inyan teliihi- ka opahte wan inyan paha wan en ehpeye cin he iyececa.

9 Wicoie wakan witkotkoka ipi kin ohna qa wapepela wan wicaśta witko nape kin ohna hena iyececa.

10 Tuwe tanka kin he owasin yuśinye wicaya, wicaśta withotko iyuwin qu, qa tona kapapi nakun iyuwin wicaqu ece.

11 Śunka talsu hdepe cin he ake en hdi kin, he iyecen wicaśta witkotko towitkotkoke cin ake econ ece.

12 Wicaśta wan ksape icida wandaka he? hee śni tuka wicaśta witkotko kin hee ksape kta kecanni kta.

13 Inmutanka canku icahda yukan, otonwe canku kin ohna mnaja wan yanka ce, wicaśta kuje cin cya.

14. 'Tiyopa wan iyuze kin akan ihduhomnimni he iyecen wicaśta kuje cin towinja kin akan ihduptanptan.

15 Wicaśta kuja wakśica ohna nape kin ye kiya, ake i hin ckta hiyukiyc kapin.

16 Wicaśta kuje cin he wicaśta śakowin wayupiya wayuptapi kin hena isanpa ksape icida.

17 Tuwe taku tawa śni akinicapi ahiyaye ça en ye içiye, he tuwe śunka noge kin yuze cin he iyececa.

18 Tuwe magaga iciye kta e petuspe wanhinkpe qa wiconte kaho iyeye cin,

19 He iyecen wicaśta takodaku hnayc ca, tokeśta waślkate śl he? eye cinhan.

20 Can wanice cinhan peta kin sni kta, he iyecen woyakesa wa. nice cinhan kicigepi kin ayuśtanpi ece.

21 Calidi petaga aonpapi, qa can peta akan, he iyecen wica163 


\section{WICOIE WAKAN.}

śta wakigesa woakinice ideya ece.

22 Tuwe iwakan kin oie kin woyute skuya iyececa, qa wicatezi kin mahentuya iyaya ce.

23 Wiciha kata cante wahnaye kici mazaska icicahiyapi kin maka wakśica apawintapi kin he iyececa.

24 Tuwe śice dake cin he iha kin on ihdatokeca, qa iye mahentuya wohnaye kihnaka;

25 Tohan ho kin wiciyokipi kaga he wacinye śni wo, hehan cante mahen taku wahtedapi śni śakowin yukan.

26 Wocanniye kin wohnaye on akalipe, tolian śice cin he omniciye ohna yazamnipi kta.

27 'Tuwe woha wan qe cin he iye ohinhpaye kta, qa tuwe inyan wan paptanye cin he en icicawin hdi kta.

28 Wicaceji itonśni kin he tona wicayaśuje cin śice wicadaka, qa wicai śdute cin wicotakuni śni kaga.

\section{WICOWOYAKE 27.}

1 Ihnuhan heyakecinhan on nihdatan kinhan, anpetu wàn taku ton kta he sdonyaye śni.

2 Nii kin hee kte śrii, tuka tuwe tokeca niyatan nunwe, wicaśta wan oyate tokeca, tuka niiha kin hee kte śni.

3 Inyan wan tke qa wiyaka kin he yuśagwicaya, tuka wicaśta witkotko tocanniye kin heniyoza isanpa tke.

4 Wocanniye he wohitika, qa wocanteptanye kin he minitan wan iyececa, tuka wowinawizi itokam tuwe najin kta okihi he?

5 Nahimana waśte dakapi kin hee sni, tuka taninyan iyopekiyapi kin hee waśte.

6 Kodayapi iyopewicaye cin he wicaka, tuka tuwe sicedake cin i iwicaputaka eśta wicahnayan.

7 Tuwe wipi kin he tuhmaga canhanpi ihakta śni, tuka tuwe wotektehda kin woyute pa eśta owasin skuye daka.

8 Wicaśta oyanke tawa etanhan nuni kin he zitkadan wan hohpi kin etanhan nuni kin he iyececa.

9 Wihdi qa wizinyapi kin wicacante iyuśkin kiya; nakun takodaku nagi kin etanhan wabokonkiye cin oskuya.

10 Nitakoda qa niyate takodaku kin ayuśtan śni wo, qa anpetu winicakije cin hehan nisunka ti kin en ye śni wo; sunkayapi wan tehan yanke cin hee śni, tuka tuwe nikiyedan ti kin hee waśte.

11 Micinkśi ksapa wo, hecen micante kin iyuśkin kta, qa tnwe taku śica emakiye cin itkom taku ewakiye kta.

12 Wicaśta ksape cin taku sica wanyake ca inalima, tuka tona wacintonpi śni kin hena iyoptapi qa kakiświcayapi.

13 Tuwe oyate tokeca wicaśta on wecicajuju kta, eniciye cinhan taśina kin icu wo; qa tuwe wicaśta sdonwicaye śni on wicawecicajuju lrta, eye cinhan taku tohanyan kajuju śni duhe kte cin he icu wo.

14 Tuwe hanhanna hin kikta qa takodaku hotankakiya yawaśte kinhan le yaśicapi kiciyawapi kta.

15 Magaju anpetu icunhan ohinniyan ośbuyapi wan, qa winohinca wakigesa wan hena iakedececa ;

16 Tuwe he anakilibe cin he tate kin anahma he iyececa, qa nape etapa wihdi waśtemua kin he ihdutanin kta. 
17 Mazasapa on mazasapa pekiyapi, qa wicaśta kin takodaku ite kin on pe kiyapi.

18 Tuwe witanśna hu patan kinhan hetanhan waskuyeca yute kta, qa tuwe itancan awanhdake cin he yutanpi kta.

19 Mini ohna ite wan ite kin iyececa, he iyecen wicacante kin wicaśta kin iyececa.

20 Hades qa wiconte kin imnapi śni, he iyecen wiciśta kin imna kte śni.

21. Mazaska maka wakśica on, qa mazaskazi maza oceti on, qa wicaśta kin wicai yatanpi kin on iyutapi.

22 Wicaśta witkotko wan wibope ohna wamnaheza kici wibope ihupa on boyapan eśta, towitkotkoke cin he ayuśtan kte śni.

23 Talinca nitawa lin token taninpi tanyan sdotkiya wo, qa wanuyanpi kin awakicin wo:

24 Nakaś woyuha kin he ohinniyan en un śni, qa wicaśta yatapi wicowazi wanjidan etanhan ohinniyan un kta he?

25 Peji kin inanpa qa wato kin tanin, qa he peji kin he mnayanpi kta.

26 Tahinca ska kin etanhan wokoyake yaton kta, qa tatokadan wanuyanpi on maga wan opeyaton lita;

27 Hecen tatokadan asanpi imna wayate kta, qa tiyohnaka nitawa nakun, qa he on wikośka nitawa nipi kta.

\section{WICOOWOYAKE 28.}

1 Wicaśta śica tuwedan wicakuwa śni eśta najicapi ece, tuka wicaśta owotanna mnaja iyecen waditakapi.

2 Makoce wokipajin kin on wicaśta yatapi ota, tuka wooka- hnige wosdonye wicaśta wan eciyatanhan tehan ecetu kta.

3 Wicaśta wahpanica wan tona onsilka wicayuwahpanice cin he magaju wakawankeya wan on woyute wanica he iyececa.

4 'Tona woope opapi śni kin hena wicaśta śice cin yatanpi, tuka tona woope opapi kin hena wicakigepi ece.

5 Wicaśta śica woyaco okalinigapi śni, tuka Jehowa akitapi kin hena owasin okahnigapi ece.

6 Wicaśta wahpanica toecetu kin omani kin hee waśte, tuka tuwe canku kin hdupemni wijica eśta hee waśte śni.

7 Tuwe woope patan kin he hokśidan ksapa, tuka tuwe wotapisa kodawicaye cin he atkuku iśtenya.

8 Tuwe woyalitake qa ota wicakida on woyuha kin hduota kin he tuwe wahpanicapi onsiwicada kte cin he on he mnaye kta.

9 Tuwe woope nalion kte śni e noge kin hdutokan kin he wocekiye eye cin he wowaliteda śni yapi kta.

10 Tuwe wicaśta ecetu kin canku śica onuni wicakiye cin he woha wan iye qe cin he ohinhpaye kta, tuka tona ecetupi kin hena taku waśte tawayapi kta.

11 Wicaśta watonka kin ksape içida, tuka wicaśta wakahinige cin he iyukcan kta.

12 Wicaśta owotanna iyuśkinpi eca wowitan tanka ece, tuka wicaśta śica wankan ayapi eca wicaśta inahmanpi ece.

13 Tuwe wahtani kin ahdahpe cin he tanyan ihuni kte śni, tuka tuwe ohdake ca ayuśtan kinhan onśidapi kta.

14 Tuwe ohinniyan kokipe cin he wowaśte wicaśta kin heca, 165 


\section{WICOIE WAKAN.}

tuka tuwe cante kin hdusuta kin he taku śica ohinhipaye kta.

15 Wicaśta yatapi sice cin he oyate onsilka akan he mnaja lidoya, qa mato waanatan wan he iyececa.

16 Wicaśta yatapi wookahnige nice cin he tanyate kin nina wicayuwalipanica, tuka tuwe wicakipi śice dake cinhan anpetu tawa kin hduota kta.

17 Tuwe wicaśta we kin on yuśakapi kin he tuwedan yuze kte śni e woha kin ekta inyanka.

18 Tuwe ecetu kiya mani kin he ni kta, tuka tuwe canku ota ohna ihdupemni kin he wanji ohinhpaye kta.

19 Tuwe tamaga kin kicanye cin he woyute imna kta, tuka tuwe tona takuska econpi owicape cin he wowicakakije on imna iciye kta.

20 Wicaśta wicake cin he wowaśte ota, tuka tuwe ihduwijice kta e inahini kin he taku śica akipe kta.

21 Wicite iyekiyapi kin he waśte śni, wicaśta hecon kin he aguyapi onśpadan on walitani kta.

22 Tuwe ihduwijice kta inalini kin he iśta śica, qa heon wowalipanice akipe kta sdotkiye śni.

23 Tuwe ceji skuye cin hee śni, tuka wicaśta iyopeye cin hee iyohakam waśte dapi kta.

24 Tuwe atkuku qa hunku cśta taku tawa icu qa, taku śica ecamon śni, eye cin he waihangyesa dakonya.

25 Tuwe nagi tanka içida kin he kicigepi kaga, tuka tuwe Jehowa wacinye cin he cepa aye kta.

26 Cante kin wacinkiye cin he witkotkoka, tuka tuwe woksape omani kin he ihduśke kta.

\section{6}

27 Tuwe onśipike cin hena cante wicakiye cin he wicakije kte śni, tuka tuwe iśta anakilibe cin he wicota yaśicapi kta.

-28 Wicaśta śica najinpi eca wicaśta inahmanpi ece, tuka hena atakunipi śni eca wicaśta owotanna ota ayapi ece.

\section{WICOWOYAKE 29.}

1 Wicaśta ijehan iyopeyapi eśta tahu kin ihdusuta kin he ihnuhanna kaśujapi kta, qa woasni wanice kta.

2 Tohan wicaśta owotanna ota ayapi eca oyate kin wiyuśkinpi kta, tuka wicaśta śica widakapi eca oyate kin śicahowayapi ece.

3 Tuwe woksape waśte dake cin he atkuku iyuśkinkiye kta, tuka tuwe witkowin om iyokipi içiye cin tawoyuha kin toki ehpekiye kta.

4 Wicaśta yatapi kin woyaco on makoce tawa kin sutaya he kiye kta, tuka tuwe mazaska qupi waśte dake cin he pawanke kta.

5 Tuwe takodaku on ceji kin hduskuye cin he siha kin on mazalitakiyapi wan eonpa.

6 Wicaśta śica wahtani kin ohna mazhitakiyapi wan yukan, tuka wicaśta owotanna wiyuśkin qa dowan kta.

7 Wicaśta owotanna tona onśika wicayacopi kin sdonya, tuka wicaśta śica he sdonye kta okahnige śni.

8 Wicaśta waoncesa otonwe kin ideyapi, tuka wicaśta ksapa wocanniye anaptapi ece.

9 Wicaśta ksapa wan wicaśta witkotko wahokonkiye cinhan, canksiya ia qa iha eśta iyozi wanice kta.

10 We wicaśta kin hena tuwe 
waśte kin śicedakapi, qa wicaśta ecetu nagi kin akitapi.

11 Wicaśta witkotko tawacin kin ocowasin hiyuya, tuka wicaśta ksape cin he tokata ekihnaka.

12 Wicaśta yatapi wicoie itonśni anagoptan kinhan ookiye wicaye cin owasin śicapi lita.

13 Wicaśta wakiyuśe qa wahpanica akicipapi, Jehorva napin iśtapi kin iyoyam wicaya ece.

14 Wicaśta yatapi tona onśika wowicake eciyatanhan wicayaco kinhan oiyotanke tawa kin owihanke wanin sutaya he kta.

15 Hokśidan kiyuślkapi kin he hunku iśtenya, tuka icapsinte iyopekiyapi iyahna he ksapa kaga.

16 Wicaśta śica ihduotapi eca wicohan śica nakun ota kta, tuka wicaśta owotanna hena atakunipi śni kin atonwanpi kta.

17 Nicinkśi kin iyopekiya wo, hecen oziniciyc kte ça ninagi kin iyokipi kta.

18 Tukten Wakantanka oie kin wanice cinhan oyate kin wohitika, tuka woope yuhapi kin he wowaśte oyate.

19 Wowidake wan wicoie on yuecetupi śni, okahniga eśta ayupte kte śni.

20 Wicaśta ia olianko wandake cinhan hee śni, tuka wicaśta witkotko kin hee etanhan taku waśte ayape kta iyececa.

21 Tuwe tawowidake kin hokśiyopa ehantanhan kihna kinhan, owihanketa he cinca ye kta.

22 Wicaśta wacinko kicigepi kin icahya, qa wocanniye wicaśta kin woahtani ota.

23 Wicaśta wamnaicida kin on ihdukuye kta, tuka tuwe woniya kucedan kin he wowitan ton kta.

24. Tuwe wamanusa kici wopamni yuhe cin he nagi kin śice lidaka, yaśicapi kin nahon tuka oyake śni.

25 Wicaśta kokipapi kin he wicahmunka, tuka tuwe Jehowa wacinye cinhan okope wanin un kta.

26 Wicota wicaśta yatapi ite kin akitapi, tuka wicaśta wicayaco kin Jehowa hee.

27 Wicaśta owotanna wicaśta śicc cin he wowahtedapi śni, qa wicaśta śica tuwe ohan owotanna kin he wowahtedapi śni.

\section{WICOWOYAKE 30.}

1 Agur Jake cinhintliu kin wicoie tawa, odowan, wan, Itael eciya, Itacl Yukal ko ewicakiya. 2 Woteca iyemaccea qa wicaśta iyemacece śni, qa wicaśta towiyukcan kin mayuke śni.

3 Awicakehan woksape kin onmaspe śni qa wicaśta wakan wosdonye kin he sdonwaye śni. 4 Tuwc wankantuya Mahpiya ekta i he? qa tuwe hetanhan kun hdi he? Tuwe nape kin ohna tate kin mnayan he? Tuwe mini lzin śina wan ohna opalita he? Tuwe maka ihanke kin owasin ehde he? Token eciyaji qa cinhintku token eciyapi he? sdonyaye cinhan oyaka wo.

5 Taku wakan oie kin owasin peta on yuecepidan; tona he wacinyanpi kin hena wahacanka yapi.

6 Ihnuhan oie tawa kin ekta taku aokijuyaye cinhan, okini iyopeniye $\mathrm{kta}$, qa iyatonśni iyeniyanpi kta.

ry Taku nom cicida, mate śni ecen hena temicihda śni wo ;

8 Wicolian śica qa iapi ecinkta miciyutehan ye, wowahpanice wowijice unmanna maqu śni, woyute wate kta iyececa wonmayan ye; 


\section{WICOIE WAKAN.}

9 Okini wimapi kinhan micihnaye ca, Jehowa he tuwe, epe kta ; qa wimakakije cinhan olini wamawanun, qa iwaton śni eśta taku wakan namalion epe kta.

10 Ihnuhan wowidake wan yuhe cin ekta ayakiia, okini niyaśice kta qa taku śica ayakipe kta.

11 Wicoicage wan atkuku kin yaśice ca hunku kin yawaśte śni.

12 Wicoicage wan wakaśoteśni icidapi, tuka unkce kin wicakiyujajapi śni.

13 Wicoicage wan iśta hduwankanpi hinca, qa iśtojuha wankan ayapi ece.

14 Wicoicage wan hipi kin canhpi kin iyececapi, qa hiskepi kin isan iyececapi; hecen tona onśika maka etanhan temwicayapi, qa tona wahpanica wicaśta ehna unpi kte śni.

15 Tusda cunwintku nonpa, napin, Maqu ye, maqu ye, eyapi. Taku yamni imnapi śni, qa taku topa, Henakeca, eyapi śni ;

16 Hades, qa tamni cinca ton śni, qa maka kin mini imna śni, qa peta, Henakeca, eye śni.

17 Wicaśta wan atkuku ihahia, qa hunku kin nahon kta e ihakłaśni kin he kaksiza kangi kin capapi kta, qa wanmdi cinca kin he yutapi kta.

18 Taku yamni yusingemayan, qa taku topa owakahnige śni ;

19 Wanmdi canku kin mahpiya ohna, wamduśka canku kin imnija akan, wata canku kin mde cokaya, qa wicaśta canku kin wikośka kici.

20 Winolinca wawicihahapi econ canku tawa kin he iyececa, wota qa i kin itpakinte ca, Taka śíca ecamon śni ce, eya.

21 Taku yamni on maka kin śkanśkan qa taku topa on oziic̣iye kta okihi śni.
22 Wowidake on tohan wicaśta yatapi un ece, qa wicaśta witkotko on aguyapi imna wota ece ;

23 Winohinca canniyesa on hihna ton kinhan, qa wayakawin on yuhe ciqon woyuha kin tawaye cinhan.

24 Taku topa maka akan iyotan cistinna tuka ksape hinca;

25 Tajuśka kin oyate waśake śni tuka mdoketu eca woyute kihnakapi ece ;

26 Hitunkasan kin tancan sutapi śni, tuka imnija ohna tipi kicagapi ece;

27 Arebe (psipsicadan ocaje wan) wicaśta yatapi nicapi tuka obe tawapi owasin yuwitaya icimanipi ;

28 Onktomi napepi on yuzapi qa wicaśta yatapi ti kin ohna yakonpi ece.

29 Taku yamni tanyan manipi ece, qa taku topa tanyan yapi ;

30 Mnaja kin he wamanica iyotan waśake ca takudan etanhan ihduhomni śni ;

31 Sunka wakan nite ipiyag tonpi, qa tatokadan mdoka, qa wicaśta yatapi oyate tawa opapi kin he.

32 Witkotkoya nihduwankantuya kinhan, qa taku śica yakonze cinhan, nii kin akan nape kin atputaka wo.

33 Awicakehan pte asanpi kin icicahiyapi kinhan pte asanpi ihdi kin hiyu kta, qa wicapoge yutitanpi kinhan we hiyu kta, qa wocanniye yutitanpi on kicigepi hiyu kta.

\section{WICOWOYAKE 31.}

1 Lemuel wicaśta yatapi wicoie tawa, odowan hunku kin he onspekiye cin dee.

2 Cinś taku hwo? mitamni cin- 


\section{WICOWOYAKE 31.}

ca kin taku eciye kta he? womiciyake ota eciyatanhan citon kin taku hwo?

3 Thnuhan nitowaśake kin he winohinca yaqu kin, qa canku nitawa taku wicaśta yatapi ihang wicaye cin ekta ye yakiye cin.

4 Wicaśta yatapi, Lemuel, wicaśta yatapi miniśa yatkanpi kta he iyecece śni, qa wicaśta yatapi cincapi kin mini wakan cinpi kta he iyecece śni.

5 Okini he yatke cinhan taku konzapi kin akiktonje kta, qa wicaśta onśika tawoyaco kin yaśkiśke kta.

6 Tuwe wanna te kte cin he mini wakan kin qu wo, qa tona cante śicapi kin hena miniśa wicaqu wo.

7 Hecen yatke kta, qa tokakije cin akiktonje kta, qa toiyokiśice cin icimanna kiksuye kte śni.

8 Iapi okitpani kin he on i kin hdukawa wo, qa wamdenica owasin wicayacopi kinhan;

9 I kin hdukawa qa tona onśika qa wicakijapi owasin iwicakiya owotanna wayaco wo.

10 Tuwe winohinca ksapa wanji iyeye kta he? Tukiha tehihika ee kaeś sam iyeya iyawapi.

11 Hihnaku cante kin he wacinkiya, qa woyuha icakije kte śni yan un kta.

12 Tohan ni kin hehanyan taku waśte ecakicon kta, qa taku śica wanice kta.

13 Tahinca hin hahonta on kagapi ko akita, qa nape kin iyokipi ya htani.

14 Wopeton witawata tawapi kin iyececa, tehantanhan woyute tawa kin en ihdoku.

15 Nahanhiin anpa śni kikta, qa tiyohnaka tawa owasin woyute wicaqu, qa wikośka taku econpi kta sdonye wicakiya.

16 Maga wan awacin qa ope- ton, taku nape kin kagapi kin on hastanhanka iyuwi wan huhde.

17 Ipiyaka sutaya iyahdaśka qa hupahu kin hduwaśaka.

18 'Taku wiyopeye cin he waśte e sdonkiye; hanyetu osan petijanjan hde kin kicisni śni.

19 Wipahmu kin ekta nape yekiya qa napsukaza kin iyuhmu kin yuza.

20 Tona onśika kin hena on nape kin hdugata, qa wicaśta oncikpani owicakiye kta e nape kin ye kiya.

21 Wapa keś tiyohnaka tawa kin on ikope śni; nakaś owasin taku icitakihna ece koyakapi.

22 Owinja içicaga; qa miniliuka zibzipedan śinaśa ko koyaka ece.

23 Otonwe tiyopa kin ekta makoce hunkayapi wicehna hihnaku iyotanka eca iyekiyapi ece.

24 Minihuha zibzipedan kage ca wiyopekiye, ipiyaka nakun qa wopeton kin wicaqu.

25 Wowaśake wowitan iyahna ihduze ca tokata anpetu u kte cin iha.

26 I kin hdukawa eca wicoksape ia, qa ceji kin wacantkiya woope yulia.

27 Tiyohnaka tawa olianyanpi kin owasin iwanyaka, qa kujahan aguyapi kin hdute śni.

28 Cinca kin najinpi eca hdawaśtepi; hihnaku nakun qa hdatan.

29 Cunwintkupi ota ksamya olianyanpi, tuka niye owasin kape wicayaya ce, eya.

30 Wowiciyokipi kin he wicahnayan qa wicite waśte kin he ptenyedan, tuka winohinca Jehowa kokipeyahan un kin he yawaśtepi kta.

31 Nape kin taku icahye cin he qupo, qa otonwe tiyopa kin ohna iye ohan kin hena yatanpinunwe. 



\section{EYANPAHA KIN.}

WICOWOYAKE 1.

1 Eyanpaha kin, Dawid cinhintku Jerusalem en Wicaśtayatapi oie kin dena ee.

2 Takuśni, takuśni, Eyanpaha kin eya, takuśni, takuśni, iyuhipa takuśni ce.

3 Anpetu wi ihukuya wicaśta wicolitani econ kin owasin on iyunwin taku yuha he.

4 Wicoicage warji iyaye ca wicoicage wan u ece ; tuka maka kin ohinniyan han.

5 Nakun anpetu wi kin hinanpa, qa anpetu wi kin iyaya, qa totanhan hiyu qon he ekta inalini hda ece.

6 Tate kin itokalikiya iyaye ca waziyatakiya ihduhomni; tate ihduhomnimni, qa totanhan iyaye cin ake en hdi ece.

7. Wakpa iyuhpa miniwanca kin en iyaya; tuka miniwanca kin ojudan śni; wakpa kin totanhan hiyupi qon he ake ekta hdapi ece.

8 Wicoie owasin liunkițapi ojudan; wicaśta oyake kta okihi śni: wiciśta kin he wawanyaka imnan śni, qa wicanoge kin wanahonpi on imnan śni.

9 Taku un qon he u kte cin hee: qa taku econpi ece kin taku econpi kte cin ee : qa anpetu wi kin jhukuya taku teca wanica. Eccle.
10 Taku on, Wanyaka wo, de teca ce, eyepica he : wanna tanina un qon hee, unkiye unkitokapatanhan yanke.

11 Taku hekta un qon hena kiksuyapi śni; qa nakun taku tokata kin tona ohakam unpi kte cin hena kiksuyapi kte śni.

12 Eyanpaha kin he miye, Jerusalem en Wicaśtayatapi manka.

13 Unkan taku mahpiya ihukuya econpi kin owasin woksape on awakite kta, qa imdukcan kta e micante ekta yewaya: wicolitani tehike cin de Wakantanka wicaśta cinca wicaqu, on htanipi kta heon.

14 Anpetu wi kin ihukuya wicolian econpi kin owasin wanmdaka, unkan iho, iyuhpa takuśni qa woniya iyokiśsinyapi kin hee.

15 Taku ślkope cin he yuowotanpidan kta okihipica śni; qa taku iyokpani un kin hena yawapica śni.

16 Micante kici wowahdaka qa hepa; Iho, tanka imacaga qa woksape mnawakiya, tona Jerusalem en mitokam unpi qon owasin isanpa: han, micante woksape qa wosdonye ota wanyaka.

17 Nakun woksape sdonwaye kta micante waqu, wicoksape śni qa wowitkotkoke sdonwaye 
kta: De nakun woniya iyokiśinyapi kin he imdukcan.

18 Woksape ota en woiyokiśice ota yukan ; qa tuwe wosdonye hduota kinhan he wicocanteśica hduota ece.

\section{WICOWOYAKE 2.}

1 Micante en hepa; Iho wo, wowiyuśkin on iciyute kta ce; hecen taku wiciyokipi sdonya wo. Unkan iho, he nakun taku śni.

2 Ihapi kin he, Wowitkotkoka, ewakiya: qa wowiyuśkin, He taku tokon he.

3 Micehpi miniśa on wahdusuta kta micante en awakita, qa micante woksape sdonyewakiye kta; qa wowitkotkoka kin he mduze kta, hecen wicaśta cinca on taku waśte kin tukte c he, anpetu towanakeca nipi kin en, mahpiya ihukuya taku econpi kte cin he wanmdake kia.

4 Taku tanka mic̣icaga; tipi miçicaga; hastanhanka iyuwi ojupi kin hu wehde :

5 Maga qa canwojupi kin micicaga, qa en waskuyeca can ocaje owasin hu wahde:

6 Miniyowe kagapi kin miçicaga, on contanka call icahye cin he spayewaye kta :

7 Wicaśta taokiye qa winyan taokiye opewicawaton, qa wati ohna tonpi wicamduha; nakun tatanka qa tahinca wanunyanpi ota hinca wicawahduha, Jerusalem en mitokam unpi qon owasin isanpa :

8 Nakun mazaska qa mazaskazi mnamiçiye, qa wicaśtayatapi makoce tawapi ko tawokamna kin: wicaśta dowanpi qa winohinca dowanpi heca icahwicawakiya, qa wicaśta cinca tawo- wiyuśkinpi, winohinca qa winohincapi.

9 Hecen matanka, qa tona mi tokam Jerusalem en unpi qon ikapeya taku ota micicaga : nakun woksape mitawa miciun.

10 Qa taku miiśta da kin takudan ipimiçida śni, wowiyuśkin wanjidàn keś micante etanfan wakipatan śni; mitohtani owasin on micante iyuśkin nakaeś : qa mitolitani ocowasin on wokamna mitawa kin hee.

11 Hehan wicohan mirlape econ kin owasin en ewakitonwan, qa wicohtani htawani kta ecamon kin hena; unkan iho, ocowasin takuśni qa woniya iyokisica hee, qa anpetu wi kin ihukuya wokamna wanica.

12 Unkan woksape qa wicoksapeśni qa wowitkotkoke kin iwanmdake kta mihdamna: wicaśta wan wicaśtayatapi ihakam u kin he taku okihi kta he : taku wanna econpi kin heceedan.

13 Hehan woksape en wokamna yukan e wanmdaka, wowitkotkoke kin isanpa, iyoyanpa kin he otpaza isanpa waśte kin he iyececa.

14 Wicaśta ksapa iśta iye pa kin en un; tuka witkotkoke cin he otpaza omani: hececa eśta miye qe taku wanjidan owasin awicakipa ece e wanmdaka.

15 Hehan cante mahen hepa; Witkotkoke cin he taku akipa he iyecen miś eya awakipa; hecen tokiyatanhan miye qe iyotan waksapa he. Heharl cante mahen, De iś eya takuśni ce, epa.

16 Witkotkoke cin iyececa wi- 
caśta ksapa kin ohinniyan kiksuyapi śni nakaeś; taku nakaha un kin hena tokata anpetu kin en owasin akiktonjapi kta, heon etanhan. Qa wicaśta ksape cin iś token te kta he; witkotkoke cin iyececa.

17 Heon etanhan wicani kinśicewakidaka ; anpetu wi kin ihukuya wicohan econpi kin he waciniyowakiśica nakaeś : iyuhpa takuśni qa woniya iyokiśinyapi kin hee.

$18 \mathrm{Han}$, mitohtani anpetu wi ihukuya ecamon kin ocowasin śicewakidake ; wicaśta mihakam u kte cin he ailpewakiye kta, heon etanhan.

$19 \mathrm{Qa}$ he wicaśta ksape kta, qa iś witkotkoka kta tuwe sdonya he. Hececa eśta, mitohtani ecamon kin owasin, qa en anpetu wi ihukuya ksapa mihdutanin kin hena iye tawaye kta. De nakun takuśni.

20 Heon etanhan anpetu wi kin ihukuya wicohtani ecamon kin iyuhpa micante kicunniwakiye kta milidamna.

21 Wicaśta wan tohtani kin woksape en, qa wosdonye en, qa wokonze en un: tuka he wicaśta wan on hitani śni kin he tawa kta ailipekiye kta. He nakun takuśni qa taku śica tanka.

22 Wicaśta ive tohtani owasin, qa cante iyokísica, on anpetu wi ihukuya htani kin etanhan taku hduha he :

23 Taanpetu kin iyuhpa wicocanteśica, qa tokakije kin he woiyokiśica nakaeś : han, hanyetu en iye cante oziiçiye śni. He nakun takuśni.

24 Wicaśta wote kta, qa wayatke kta, qa iye nagi tohtani kin on iyuśkinkiye kta, taku sanpa waśte wanica. De iś eya Wakan- tanka nape kin etanhan wanmidaka.

25 Tuwe wote kta he, qa miye mikapeya tuwe wiyuślkin kta he.

26 Taku iye itokam waśte kin - he Wakantanka wicaśta wicaqu ece, woksape qa wosdonye qa wowiyuśkin : tuka wahtani sa kin he wokakije qu, wamnaye kta qa waeju kta, hecen Wakantanka itokam taku waśte kin he qu kta. He nakun takuśni qa woniya iyokiśica.

\section{WICOWOYAKE 3.}

I Taku iyuhpa iyehantu yukan, qa mahpiya kin ihukuya woawacin owasin tohantu kte cin yukan ece.

2 Wicatonpi iyehantu yukan, Qa wicate kta iyehantu ;

Woju econpi iyehantu;

$\mathrm{Qa}$ wojupi yujunpi kta iyehantu :

3 Wicaktepi iyehantu yukan, Qa okiziyapi iyehantu ;

Wayujujupi iyehantu,

Qa piya kagapi iyehantu yukan :

4 Wicaceya iyehantu yukan, Qa ihapi lita iychantu;

Waśihdapi iyehantu, Qa śkatapi iyehantu yukan:

5 Inyan ehpeyapi iyehantu yukan,

Qa inyan witaya pahipi kta ; Adoksohan wicayuzapi iyehantu yukan,

$\mathrm{Qa}$ poskin wicayuzapi ayustanpi kta.

6 Waakitapi iyehantu yukan, Qa toki ehpeyapi kta iyehantu; Wapatanpi kta iyehantu, $\mathrm{Qa}$ wapaganpi iyehantu $\mathrm{yu}$ kan :

7 Wayuhdehdecapi iyehantu yukan, 
Qa wakagegepi iyehantu yukan ;

Inina yukanpi iyehantu, Qa iapi kta iyehantu yukan:

8 Waśtewicadaka iyehantu yukan,

Qa śicewicadaka iyehantu;

Okicize iy ehantu yukan,

Qa wookiye kagapi kta iyehantu.

9 Wicaśta hitani kin he iye tolitani eciyatanhan iyunwin taku hduha he.

10 Wicohtani en wicaśta cinca hitaniiciyapi kta Wakantanka wicaqu kin he wanmdaka:

11 Taku owasin iyehantu eca owanyag waśte kaga : nakun iye cantepi lin en ohinniyan kte cin he ewicakicilnaka, hecen Wakantanka tohtani kage cin otokaheya tanhan qa owihanke ekta wicasta tuwedan okahnige kte śni.

12 Hena en taku waśte wanica sdonwaya; tuka wiyuśkin kta, qa ni un icunhan taku waśte econ kta :

13 Qa nakun wicaśta otoiyohi wote kita, qa wayatke kta, qa tolitani owasin taku waśte en un kin he on ihduśkin kta ; Wakantanka taku qu kin hee.

14 Taku Wakantanka econ kin owasin owihanke wanice $\mathrm{kta}$ slonwaya; takudan en akagapi kte śni, qa takudan etanhan icupi kte śni : unkan iye itokam wakokipapi kta e Wakantanka hecon ece.

15 Taku hekta un qon he nakaha un, qa taku tokatam u kte cin he wanna hekta un : qa taku hekta un kin he Wakantanka lin ake hdiyohi kiye kta.

16 Unkan nakun anpetu wi ihukuya woyaco oyanke kin wanmdaka, wośice hen yanka; qa 174 woowotanna oyanke kin, wicośice en yanka.

17 Cante mahen hepa; Owotanna kin he qa śice cin Taku Wakan kin wicayaco kta: han, wicotawaein otoiyohi qa wicohan otoiyohi hen iyehantu yukan.

18 Wicaśta cinca kin on cante mahen hepa; Tokin Wakantanka hena iwicayuten, hecen iye qe woteca hecapi e wanihdakapi kta.

19 Wicaśta cinca kin taku akipapi hecinhan, he woteca akipapi ; han, taku wanjidan awicakipa ece : wanji iś token ta hecinhan, unma kin iś iyecen ta : han, owasin woniya okonwanjidan yuhapi: hecen wicaśta kin iś woteca isanpa takudan iciwaśte śni; iyuhpa takuśni nakaeś.

20 Owasin oyanke wanjidan ekta yapi; iyuhpa maka mdu heca, qa iyulipa ake maka mdu icagapi ece.

21 Wicaśta cinca taniya wankan ye cinhan, qa woteca taniya kin iś maka ekta kun ye cinhan, tuwe sdonya he.

22 Heon etanhan wicaśta iye tohan kin on ihduśkin kta taku sanpa. waśte wanica imdukcan; he taku tawa kin ee nakaeś : qa iye iyohakam taku u kte cin he tuwe wanyagkiye kta he.

\section{WICOWOYAKE 4.}

1 Unkan wahdicu qa wokakiśye anpetu wi ihukuya econpi kin hena owasin iwanmdaka: qa iho, kakiśyapi iśtamnihanpe awicau ; qa wicakicanpte yuhapi śni : unkan wakakiświcaye nape ohna wowaśake yukan; tuka wicakicanpte yuhapi śni.

2 Heon etanhan tapi kin, toua wanna tapi kin hena wicadma- 
waśte, nipi, tona nahanhin ni unpi kin isanpa :

3 Han, tuwe tohinni ni un śni kin he hena napin isanpa waśte, wicolian śica anpetu wi ihukuya econpi kin hena wanyake śni nakaeś.

4 Akeś wicolitani owasin iwanmdaka, qa wicolian yuśtanpi otoiyohi, on wicaśta iye takodaku lici nakiciwizipi kin hee. De iś eya takuśni qa woniya iyolkiśica ce.

5 Witkotkoke cin he nape hdukśija qa iye cehpi hduta ece.

6 Napohnaka wanjidan woozi kici hee waśte, nape nonpa ojudan isanpa, wicolitani econpi qa woniya iyokiśica iyahna hecinhan, eya.

7 Hehan wahdiyaku qa anpetu wi ihukuya takuśni kin he wanmdaka.

8 Wanjidan yukan, qa inonpa wanica; han he cinca qa sunkaku takudan hduhe sni; tuka tolitani owasin ayuśtan śni ; nakun iye iśta kin wowijice imna śni; qa, Tuwe on htawani qa minagi taku waśte icaliśwaya he, eye śni. De nakun taku śni; han, wicolitani telika.

9 Nonpa kinhan wanjidan isanpa waśtepi ; wicohtani econpi kin on wokajuju waśte yuhapi nakaeś.

10 Hena hinlipayapi kinhan, unma kin iś takodaku najin kiye kta: tuka, hehehe, tuwe iśnana. un hinhpaye cinhan ; tuwe najinkiye kta yuhe śni nakaeś.

11 Akeś, nonpa sakim wankapi kinhan cozapi ece ; tuka toliyatanhan wanjidan iśnana coza kta he.

12 Unkan wanji kici econ ohiye cinhan, nonpa kici inajinpi kta; qa hahonta yamni kahnonpi kin he yupsakapi tehika.
13 Hokśidan wan wahpanica qa ksapa he waśte, wicaśtayatapi wan wicahinca qa witkotkoka wanna onspekiyapi kta wicada śni kin he isanpa.

$14 \mathrm{He}$ qe wicokaśke tipi kin etanhan wicaśtayatapi kta hiyu; tuka nakun tuwe iye tośkanśkan kin en tonpi kin he walipanica aya.

15 Wicaśta ni unpi owasin anpetu wi kin ihukuya manipi kin hena, hokśidan inonpa kin eekiya inajin kte cin kici iwanwicamdaka.

16 Oyate kin owasin wopteca śni, tona wicitokam unpi qon owasin : nakun tona ohakam upi kin hena he on iyuśkinpi kte śni. Awicakehan he nakun takuśni qa woniya iyokiśice cin hee.

\section{WICOWOYAKE 5.}

1 Wakantanka ti kin ekta da eca nisiha awanhdaka wo, qa witkotkoka tawośna yaqu kte śni e wanalion yanka wo; hena eqe taku śica econpi kecinpi śni. 2 Nii on olianko śni wo, qa nicante Wakantanka itokam taku eya inahini kte śni. Wakantanka malipiya ekta yanka, qa niye maka akan yaun; heon etanhan nioie tonana kta iyececa.

3 Wicolian ota eciyatanhan wihanmnanpi icaga: qa witkotkoka ho kin wicoie ota en un.

4 Tohan Wakantanka ekta ta$\mathrm{ku}$ on niçiconze cinhan, hdajuju dutehan kte śni; iye qe witkotkokapi kin on wiyuślkin śni ; taku on niçiconze cin he hdajuju wo.

5 Niçiconza qa yahdajuju śni kinhan, niçiconze śni unkanś hee waśte kta tuka.

6 Ihnuhan nii kin he nicelipi walitanikiye cinhan; qa Wawa- 
yuśna kin itokam, He woyaśna ce, ebe kte śni : Taku on etanhan Wakantanka niho on sinhda kta, qa ninape taku econ kin ihangye kta he.

7 Wihanmnanpi ota kin en takuśniśni yukan, qa wicoie ota; tuka niye Taku Wakan kin kokipa wo.

8 Wicaśta onśika wicakipi kin he, qa makoce tehan yanke cin en woyaco woowotanna ko yupemnipi kin hena wandake cinhan, he taku kin on yuśinyaye śni wo: tuwe waśaka keś isanpa waśake cin he awacin yanka; qa hera isanpa waśakapi yukan.

9 Nakun maka wokamna kin he wicaśta owasin tawapi ; wicaśtayatapi hee kaeś maga kin wowidagya.

10 Tuwe mazaska waśtedake cin he mazaska imnan kte śni; qa tuwe woyuha ota waśtedake cin he oicage on imnan lkte" śni. He nakun takuśni.

11 Woyuha kin hena ota aye cinhan, tona yutapi kin hena yuotapi; hecen tuwe tawapi lin hena taku iwaśtepi he ; iśta on wanhdakapi heceedan.

12 Tuwe litani iśtinbe cin he skuya, taku tonana qa iś ota yuta eśta : tuka wijice woyuha ota ton kin he iśtinma olkihi śni kiya.

13 Anpetu wi kin ihukuya taku śica tehika wan wanmdaka, woyuha tona tawapi iśicapi kte cin on kihnukapi hee.

14 Tuka woyuha kin hena śicaya waakipapi on atakuni śni ece; unkan cinca wan icalikiye ca takudan nape ohna yanke śni.

15 Iye hunku tamni etanhan u qon he iyecen tancodan hde kta, hiyu qon iyecen ye kta, tohtani etanhan taku nape ohna aye kte cin takudan icu kte śni.
16 Unkan de is eya taku śica tehika, token u qon he iyecehin ake hde kta: hecen tuwe tate htakicini kin wokamna taku yuha he.

17 Nakun taanpetu owasin otpaza en wota, qa wicocante śica ota yuha, qa wayazanka en woiyokiśice.

18 Tho, taku wanmdake cin he dee; taku waśte wan oiyolipi, wote kta qa wayatke kta, qa tohtani anpetu wi ihukuya econ kin owasin, anpetu ni un towanakeca Wakantanka qu kin hehanyan iyuśkin kta; taku tawa kte cin hee nakaeś.

19 Nakun wicaśta otoiyohi Wakantanka woyuha qa wowijice qu kin he etanhan yute kta okihi, qa taku tawa kte cin hduhe kta, qa tolitani on iyuśkin kta: Wakantanka taku qu kin hee.

20 Ota śni qeyaś anpetu ni un kin kiksuye kta; iye cante wiyuśkin kin en Wakantanka toayupte yanka nakaeś.

\section{WICOWOYAKE 6.}

1 Anpetu wi kin ihukuya taku sica wanji yukan wanmdaka, qa he wicaśta ehna tanka lincia:

2 Wicaśta wanji wowijice, woyuha qa woyuonihan Wakantanka qu, hecen iye nagi taku cin kin iyuhpa takudan kiwanice śni, tuka hetanhan hdute kta Wakantanka okihikiye śni, tuka wicaśta tokeca yuta. De iś eya takuśni qa woyazan tehilke.

3 Wicaśta wan opawinge cincaton, qa waniyetu ota ni un, hecen iye tawaniyetu anpetu ota qeyaś, iye nagi taku waśte on imnan śni, qa nakun wicahapi hduhe śni kinhan, okaśkan tonpi wan hee kaeś he isanpa waśte, epa. 
4 He qe opo etanhan hiyu, qa otpaza en iyaya, qa iye caje kin otpaza akahipe kta.

5 Nakun anpetu wi wanyake śni qa sdonye śni, eqe woozi yuha, unma qon isanpa.

6 Han, waniyetu kektopawingc nonpa hehanyan ni qeyaś taku waśte takudan wanyake śni: owasin ounyanpi wanjidan ekta yapi śni he.

7 Wicaśta tohtani ocowasin iye $i$ kin on etanhan, hececa eśta iye nagi kin imnan śni.

8 Wicaśta ksapa wicaśta witkotkoka isanpa taku yuha he: wicaśta onśikc cin ni unpi wicitokam mani sdonye cin he taku yuha he.

9 Wicaśta wawanyake cin he wicanagi on uni kin isanpa waśte. De nakun takuśni qa woniya iyokiśice cin hee.

10 Taku hekta un qon hena wanna cajeyatapi, ' qa he taku kin wicaśta ee sdonyapi : nakun tuwe iye isanpa waśake cin he kici econ kta okihi śni.

11 Hecen wicoie ota takuśniśni yuotapi ece, heon wicaśta hena taku iwaśteka he.

12 Wicaśta wan ni un, takuśni ni un taanpetu iyulipa ohanzi iyecen hiyaye cin he taku waśte yuhe kta tuwe sdonya he: qa anpetu wi kin ihukuya wicaśta wan iyohakam taku u ktc cin tuwe he oliyake kta he.

\section{WICOWOYAKE $\%$}

1 Wicacaje waśte kin he wlhdi waśtemna isanpa waśte, qa aispetu en wicate cin he anpetu en tonpi kin isanpa waśte.

2 Wicaceya tipi ekta yapi kin he wayatkanpi tipi ekta yapi kin isanpa waśte; eqe wicaśta owasin owihankepi kin hee, qa ni un kin he iye cante en ekihinake kta.

3 Waciniyokiśicapi kin he ihapi kin isanpa waśte; wicite iyokiśice cin on wicacante kin yuwaśtepi nakaeś.

4 Wicaśta ksapapi cante kin he wicaceya tipi kin ohna yanka; tuka witkotkokapi cante kin iyaśapi tipi kin ohna yanka.

5 Wicaśta ksapa wawiyopeye cin nahonpi kin he waśte, witkotkokapi dowanpi wicaśta nahọn kte cin he isanpa.

6 Cega wan ihukuya wapepeka ho kin witkotkokapi ihapi kin iyececa. He nakun taku śni.

7 Awicakehan wokakiśy api kin he wicaśta ksapa yuwitkotkoka ; qa taku wicaqupi kin he wicacante kin yuśica ecc.

8 Wicoie ihanke kin he otokaheya kin isanpa waśte; qa woniya waeintanka kin he woniya wahanicida kin isanpa waśte.

9 Ihnuhan nitaniya en canniniyan inayalini kinhan: wocanniye kin he witkotkokapi maku ohna yanka ece.

10 Tokeca anpetu tokaheya qon anpetu kin dena isanpa waśte he, ihnuhan ehe cinhan: woksape eciyatanhan de on winunge śni, heon etanhan.

11 Woksape kin he waśte, woyuha yuhapi kicica; qa tona anpetu wi wanyakapi wokamna tawapi kin hee.

12 Woksape kin he wowinape, qa mazaska he wowinape ; tuka wosdonyc wokamna kin, woksape tona yuhapi wiconi wicaqu ece kin hee.

13 Wakantanka tolian kin awacin wo : taku śkopa kage cin tuwe owotanna kage kta okihi he. 14 Anpetu waśte kin en cante waśtc wo; tuka wośice anpetu kin en waawacin wo: nakun 
dena icitkokim Wakantanka kagra, wicaśta iye iyohakam takudan iyeye kte śni heon etanhan.

15 Anpetu takuśni mitawa kin icunhan dena iyulipa wanndaka: wicaśta owotranna iyc toowotanna en kaśpapi, qa wicaśta śica iye tohan śice cin en ihdutehan ece e yukan.

16 Ihnuhan owotanna hinca yaun, qa ksape hinca niçicage cinhan : tokeca nihdutakuni kte sni he.

17 Ihnuhan śice hinca nicicage ca winitkotkoke cinhan : tokeca nahahin iyenihantu śni nițc lita he.

18 De duze kte cin he waśte; han, nakun detanhan ninape ehdaku śni wo: tuwe Wakantanka kokipe cin he iyuhpa etanhan hdinanpe kta.

19 Woksape he wicaśta ksapa kin yuwaśaka, wicaśta waśaka wikcemna otonwe ohna yukanpi lin isanpa.

20 Awicakehan maka akan wicaśta owotanna, taku waśte ece econ qa wahtani śni kin heca wanica.

21 Nakun wicoie eyapi lin owasin elita cante yeye śni wo; ukinni nitaokiye niyaśica nayalion kta:

22 Niś eya nakun wicaśta tolieca ijehan wicadaśica nicante sdonkiya nakaeś.

23 De ocowasin wolssape on imdute: Waksape kta ce, epa; tuka he mitehan un.

24 Taku itehanyan yanke ca temahentu hince cin he tuwe iyukcan okihi kta he.

25 Miye qa micante mihduhomni, sdonwaye kta, qa owade lita, qa woksape wowiyukcan ko awakite kta, qa wowitkotkoke wośice kin sdonwaye kta, han, wowitkotkoke wotakuśni koya.
26 Unkan winohinca tuwe cante kin mazahtakiyapi qa on hmunkapi, nape kin mazaicaśke lin heca he wiconte isanpa pa binca iyewaye: tuwe Wakantanka itokam waśte kin he etanhan ilıduśpe kta; tuka wahtani sa kin he iye on yuzapi lita.

27 Wanyaka wo, de iyewaya ce, Eyanpaha kin eya; wanjidan wanjidan iyahna wowiyulkcan lin iyeye kta ;

$28 \mathrm{He}$ hinyahin minagi akita, tuka iyewaye śni ce: kektopawinge ehna wicaśta wanjidan iyewaya; tuka dena owasin ehna winohinca wanjidan iyewaye śni.

29 Wanyaka wo, decedan iyewaya, Wakantanka wicaśta owotanna kaga; tuka iye qe wowiyukcan ota alkitapi ece.

\section{WICO.WOYAKE 8 .}

1 Tuwe wicaśta ksapa iyececa he; qa tuwe e wicoie iyukcan sdonya he : wicaśta toksape kin he iye ite kin iyehiliya, qa ite tosuta kin yutokeca kta.

2 Miye wahokonciciya, wicaśtayatapi tawoahope kin patan wo, qa Wakantauka tawokonze oie kin on etanhan.

3 Iye itokapatanhan de kta inahini śni wo; wicoie śica en inajin śni wo; iye qe taku cin kin owasin econ nakaeś.

4 Wicaśtayatapi oie kin tukten yanke cin hen wowaśake yukan; unkan tuwe, Taku ecanon he, eciye kta he.

5 Tuwe woahope lin patan kinhan he taku sica sdonye kte śni : qa wicaśta ksapa cante kin he tohan iyehantu qa wowiyukcan kin sdonye kta.

6 Taku owasin on wiyukcanpi qa tohan iyehantu yukan ece; 
heon etanhan wicaśta wośice tanka kiciyanke :

7 Taku u kte cin he sdonye śni nakaeś: ecin tohantu kte cin tuwe okiyake kta he.

8 Wicaśta tuwedan woniya on waśake śni, iye taniya hduhe kta heon; qa nakun tuwedan wiconte anpetu kin en wowaśake lıduhe śni : qa okicize kin de en tụwedan kiyuśkapi śni: nakun taku śica tona en iyolipayapi kin hena wicayuśpe kte śni.

9 De ocowasin wanmdaka, qa taku anpetu wi ihukuya econpi kin owasin ekta micante ewacin wakiya: tohan wicaśta wanji iś wicaśta tokeca wowidagya on taku śica akipa e iyehantu yukan.

10 Unkan hecen wicaśta śica wicahapi wanmdaka, hena upi qa oyanke wakan kin etanhan iyayapi, unkan otonwe en heconpi kin hen awicakiktonjapi. De nakun takuśni.

11 Taku sica econpi yacopi kin kohanna yuśtanpi śni, heon etanhan wicaśta cinca cantepi kin śicaya econ wacinkiyuzapi hinca.

12 Wahtani sa wan opawinge akihde taku śica econ qa tehan ni eśta, tona Wakantanka kokipapi, tona iye itokam kokipapi lin hena tanyan unpi kta, awicakehan sdonwaya.

13 Tuka tuwe śice cin he taku waśte yuhe kte śni, qa taanpetu hdutehan kte śni, hena ohanzi iyececa; Wakantanka itokam kokipe śni, heon etanhan.

14 Takuśni wan maka akan econpi ; wicaśta owotanna wicaśta śica ohanpi kir eciyatanhan iyecen akipapi ; akeś wicaśta śica kin is wicaśta owotanna olianpi kin eciyatanhan iyecen akipapi. He nakun takuśni ce, epa.
15 Hehan wiyuskinpi kin he mdawaśte, wicaśta anpetu wi ihukuya taku sanpa waśte yuhe śni, wote kta, qa wayatke kta, qa wiyuślin kta: iye tohtani etanhan, anpetu ni un, anpetu wi ihukuya Wakantanka taku qu kin he hduhe kta nakaeś.

16 Unkan woksape sdonwaye kta e micante awacinwakiya, qa maka akan wicohan econpi kin wanmdake kta: nakun tuwe anpetu icunhan qa hanyetu icunhan iśta on woiśtinma wanyake sni yukan:

17 Hehan Wakantanka tohan owasin wanmdaka; anpetu wi ihukuya wicohan econpi kin owasin wicaśta wan iyeye lita okihi śni: wicaśta wan he iyukcan kta htani qeyaś iyeye kte śni; han, nakun wicaśta ksapa he sdonye kta keya eśta, iyeya okili kte śni.

\section{WICOWOYAKE 9.}

1 Dena owasin micante ekta yewaye, qa de ocowasin omdake kta; wicaśta owotanna qa ksapapi, qa wicolian tawapi iyulipa Wakantanka nape ohna yakonpi : taku wicitokam un qon owasin on wicaśta tuwedan wowaśtedake qa wośicedake unmarna sdonye kte śni.

2 Iyulipa akiyecen owasin en awicau; wicaśta owotanna qa wicaśta śica taku wanjidan awicakipa ; tona waśte, qa tona ska qa tona śapapi kin; tuwe wośna qa tuwe wayuśna śni kin : waśte kin he wahtani sa kin iyececa; tuwe Taku wakan cajeyata eciyatanhan taku eye cin he tuwe Taku wakan cajeyata kokipe cin iyececa.

3 Anpetu wi ihukuya taku econpi kin owasin en dee śica, 


\section{EYANPAHA KIN,}

owasin taku wanjidan gkipapi kin hee: han, nakun wicaśta cinca cantepi talsu śica ojugjudan, nakun tohanyan ni unpi wowitkotkoke cantepi kin en un, qa he iyohakam tapi kin ekta iyayapi.

4. Tuwe ni unpi kin iyuhpa ekta kahnigapi kin he wowacinye yuha; sunka ni un kin he mnaja te cin isanpa waśte nakaeś.

5 'Tona nipi kin hena țapi kta sdonkiyapi; tuka țapi kin hena takudan sdonyapi śni, qa nakun sanpa iyunwin yuhapi śni; hena wicakiksuyapi kin hee kaeś akiktonjapi.

6 Nakun wawaśtedakapi qa waśicedakapi qa wawinawizipi qon wanna henakeca; qa hetanhan anpetu wi ihukuya taku econpi kin takudan tawapi śni owihanke wanica.

7 Ya wo, aguyapi nitawa iyuślkinyan hduta wo, qa cante waśteya miniśa hdatkan wo ; nakaha niohan kin Wakantanka iyokipiya.

8 Nitawokoyake kin ohinniyan ska nunwe; qa nipa kin ihepi codan un śni nunwe.

9 Nitawin waśteyakidake cin he kici wiyuśkinyan ni wo, takuśni wiconi nitawa anpetu kin owasin, anpetu wi ihukuya takuśni anpetu niçu kin iyuhpa : wiconi kin de en qa anpetu wi ihukuya wicolian ecanon kin en taku nitawa kte cin hee nakaeś.

10 Ninape taku econ kta iyeye cin he token oyakihi econ wo: Hades ekta de cin heciya wicohan wanica, wicotawacin wanica, wosdonye wanica qa woksape wanica, heon etanhan.

11 Wahdicu qa anpetu wi ihukuya tuwe duzahe cin he oinyanke kin en ohiye śni wanmdaka, qa waśake cin he okicize en. 180 ohiye śni, qa nakun ksapapi kin aguyapi yuhapi śni, qa wicaśta wakahniga kin hena wowijice yuhapi śni, qa nakun wicaśta wayupikapi woiyokipi yuhapi śni; tuka tohantu qa toketu kin hena owasin en awicau ece.

12 Nakun tohantu kte cin wicaśta sdonkiye śni: hogan ho śica wan on wicayuzapi kin iyececa, qa zitkadan on hmunkapi wan on wicayuzapi iyececa; he iyecen wicaśta cinca anpetu sica eri wicayahtakapi, ihnuhanna awicahinhpaye cinhan.

13 Nakun anpetu wi ihukuya woksape kin de wanmdaka, unkan he tanka wadaka:

14 Otonwe wan cistinna qa ohna wicaśta tonana : unkan wicaśtayatapi tanka wan he takpe $u$, qa aonataka qa conkaślke tanka en akaga:

15 Unkan he ohna wicaśta ksapa onśika wan iyeyapi, qa iye toksape kin eciyatanhan otonwe kin nikiya : hececa eśta tuwedan wicaśta onśike cin kiksuye śni.

16 Hehan hepa; Woksape he wowaśake isanpa waśte : hececa eśta wicaśta onśike cin he toksape kin dektayapi qa oie kin anagoptanpi śni.

17 Wicaśta ksapa oiepi kin ainina anagoptanpi, tuwe witkotkokapi ehna itancan hoyeye cin he isanpa.

18 Woksape he okicize wipe kin isanpa waśte : tuka wahtani sa wanjidan taku waśte ota ihangya ce:

\section{WICOWOYAKE 10.}

I Honagidan tapi ihepi kaga tawihdi kin śicamna kiya ece : tuwe woksape qa woyuonihan on yatankapi kin he wowitkotko- 
ke cistinna hduhe cinhan he iyececa.

2 Wicaśta ksapa cante kin iye etapa eciyatanhan yanka, tuka witkotkolka cante kin he catka tanhan yanka.

3 Han, nakun tuwe witkotkoke cin he canku ohna mani eca cante kiwanica, qa iye witkotkoka e owasin owicakiyaka.

4 'Tuwe itancan kin he taniya nitkokim inajin kinhan nitolse ayuśtan śni wo; inina unpi kin he woalitani tanka yuwaśte ece.

5 Anpetu wi ihukuya taku śica wanji wanmdaka, ecinśniyan econpi wan tuwe itancan kin itokapatanhan hiyu :

6 Wowitkotkoke kin wankan wapa ehnakapi, qa wijicapi kin iś kuya iyotankapi :

7. Wowidake unpi kin hena śuktanka akan iyotankapi wanmdaka, qa wicaśtaya tapi makata manipi, wowidake unpi iyececa.

8. Tuwe woha wan qe cinhan en ohinhpaye kta, qa tuwe conkaśke wan yujuju kinhan wamduśka wan yahtake kta.

9 Tuwe inyan qe cinhan hena on kiunniye kta: tuwe can kasdesdece cin he on ksuweiciye kta.

10 Mazasapa kin he pe śni, qa he hdube śni kinlan, nina hin waśagya econ kta: tuka wuksape he yukcankiya okihi.

11 Wamduśka kin ajijipi śni kinhan wicayahtake kta; qa tuwe iwakan kin iś he isanpa waśte śni.

12 Wicaśta ksapa i oie kin hena wowaśte; tuka witkotkoka iha kin iye naicipce kta.

13 Iye i oie tokaheya kin he wowitkotkoke; qa taku eya owihanke kin he iś wowitkotkoke śice hinca.

14 Nakun tuwe witkotkoke cin he oie ota: taku kte cin wicaśta wan sdonye śni; qa iye iyohakam taku u kte cin tuwe okiyake kta okihi he.

15 Witkotkokapi litanipi kir mdokitewicaya ece, ecin token otonwe elita ye kta sdonkiye śni.

16 Hehehe makoce, wicaśtayatapi nitawa hokśiyopa kinhan, ga itancanpi nitawa hanlianna hinca wotapi kinhan.

17 Makoce, niyawaśtepi ce, wicaśtayatapi nitawa wicaśta tanka cinca hecinhan, qa wicasta itancanpi nitawa tohan iyehantu eca wotapi kinhan, waśakapi kta heon qa witkopi kte śni.

18 Wokuje ota on tipi tihuha kin hinhpaya; qa wicanape takudan econ śni kin on tipi kin ohiyu ece.

19 Ihapi kta on aguyapi kagapi, qa miniśa wicani wiyuśkinkiya ece: tuka mazaska taku owasin on waayupte.

20 Nitawacin hee kaeś en wicaśtayatapi kin yaśice śni wo; ga tipi ohna nunke cin mahen tanhan wijice cin yaśice śni wo: mahpiya zitkadan wan wicaho kin aye kta, qa taku hupahu ton kin he wicoie kin oyake kta, heon.

\section{WICOWOYAKE 11.}

1 Aguyapi nilawa mini ite kin akan kada elpeya wo; anpetu ota kinhan ake iyeyakiye kta.

2 Onge śakowin wicaqu wo, qa nakun śahdogan: taku śica maka akan un kta tukte e sdonyaye śni nakaeś.

3 Mahpiya śapa hin mini ojugjudan kinhan maka akan iliducokapi ; qa can wan itokaȟkiya hinhpaya, qa iś waziyatakiya hinhpaya kinhan, tukte en can 


\section{EXANPAHA KIN,}

wankahan hecinhan hen wanke kta.

4 Tuwe tate kin iwanyake cinhan woju kte śni; qa turve mahpiya śapa kin ekta ewacin kinhan aguyapi bakse kte śni.

5 Woniya tacanku kin he, tuwe ihruśaka tamni ohna token huhu icage cin sdonyaye śni, he iyecen Wakantanka taku owasin kage cin he tohan kin sdonyaye śni.

6 Hanhanna eca nitawoju kin olsikju wo, qa litayetu eca ninape ehdaku śni wo : unma tukte icage kta, dee qa lee, qa iś napin akiyecen waśte kta sdonyaye śni.

7 Awicakehan iyoyanpa kin he oiyokipi linca, qa wicaiśta anpetu wi wanyake cin he waśte: - 8 Tuka wicaśta wan waniyetu ota ni, owasin on iyuśkin eśta, otpaza anpetu kin kiksuye kta iyececa; hena iś eya ota kta. Taku u kte cin iyuhpa takuśni.

9 Kośka, honikśiyopa icunhan cante waśte wo; qa koniśka anpetu kin en nicante iyuśkiniciya nunwe; qa nicante tacanku kin ohna mani wo, qa niiśta wawanyake cin en: tuka dena owasin on Wakantanka woyaco en aniu kta, he sdonya wo.

10 Heon etanhan nicante etanhan wicocanteśice hdutokan wo, qa nicehpi etanhan taku śica elipeya wo: hokśiyopa qa kośka unpi kin napin takuśni nakaeś.

\section{WICOWOYAKE 12.}

1 Wanna koniśka anpetu kin cn Wawicahniye cin he kilssuya wo, anpetu śice cin hinyahin ahi śni, qa waniyetu en, Hena ecaca iyomakipi śni ce, ehe kte cin hinyah̉in kiyadan u śni:

2 Anpetu wi, qa iyoyanpa, qa hanyetu wi, qa wicanhipi kin hinyahin aotpasyapi śni, qa mahpiya śapa magaju iyokakam ake hdiyohi icunhan :

3 Anpetu kin en ti awanhdakapi kin cancanpi kta, qa wicaśta waśaka patuś ic̣iyapi kta, qa hi wiyakpan kin hena ayaśtanpi kta, tonana heon, qa owanyeye eciyatanhan eyokasinpi kin aotpasyapi kta;

4 Qa canku ohna tiyopa kin ecen icupi kta, wotpanpi ho kin ayuśtankiyapi kinhan; qa zitkadan hoton kin on inajin kta, qa dowanpi cunwintkupi iyuhpa kun ehpeyapi lita:

5 Nakun taku wankantu kin kokipapi kta, qa canku ohna kopehdapi kta; qa uma kin he aktapi kte śni, qa psipsicadan kin he wośake dapi kta, qa cinpi hin he enakiyapi kta: wicaśta tipi owihanke wanice cin he ek ta hde kta nakaeś, qa tona ccyapi kin hena canku ohna yapi ece :

6 Nahanlin mazaska ikan kin yuśkapi śni, qa mazaskazi wiyatke kin yuptujapi śni, qa wakśica miniyowe kin elsta kamdecapi śni, qa minilidoka en iyuhmilıma kin yuwegapi śni jtokam.

7 Hehan maka mdu kin token un qon maka 'kin ekta hde kta, qa wicaniya kin he Wakantanka qu qon he ekta hde kta.

8 'Takuśni takuśni, Eyanpaha kin eya ; owasin takuśni ce.

9 Unkan Eyanpaha kin he sanpa ksapa nakaeś, heon etanhan nakun oyate kin wosdonye onspewicakiya; han, nina awacin qa wicoie wakan ota owotanna kage kta akita.

10 Wicoie oiyokipi Eyanpaha kin iyeye kta akita: unkan taku owapi kin he owotanna, wowicake oie kin heca. 


\section{WICOWOYAKE 1.}

11 Wicaśta ksapa oie kin hena wicalitake kin iyececa, qa wonwicaye wanjidan etanhan qupi mazaiyokatkuge omniciye itancanpi okatanpi kin iyececa.

12 Nakun, micinkśsi, dena on onspeiciciya wo: wowapi ota kagapi kin he owihanke yuke śni; qa nina wayawapi kin he wicacehpi mdokiteya ece
13 Wicoie ocowasin yuśtanpi kin he naunhonpi kte; Wakantanka kokipa wo, qa iye tawoahope kin ahopa wo: wicaśta ocowasin kin hee.

14 Wakantanka wicolian owasin woyaco on au kta nakaeś, taku nahmanpi iyuhja knya, waśte qa śica unma tukte kaśta.

\section{DOWANPI ODOWAN.}

\section{WICOWOYAKE 1.}

1 Dowanpi Odowan Solomon tawa kin he dee.

2 Iye i oiputake tawa on $\mathrm{i}$ imaputaka nunwe. Nitowaśtedake kin he miniśa isanpa waśte nakaeś.

3 Thepi waśtemna nitawa oomna kin on nicaje wihdi okaśtanpi kin iyececa: heon etanhan witanśna unpi kin waśtenidakapi.

4 Mayutitan ye. Nihakam unkinyankapi kta. Wicaśtayatapi kin he iye ti kin timahen amahi: Piundapi kta qa unkiniyuśkinpi kta; nitowaśtedake kin he miniśa isanpa unkiksuyapi kta : tona ecetu kin hena waśtenidakapi.

5 Jerusalem cunwintkupi, miye masapa tuka owanyag waśte, Kedar tawakeya iyececa, Solomon ozan hdepi tawa kin iyececa.

6 Masapa heon amatonwanpi śni pe, anpetu wi kin he on maśtimaśpan nakaeś : ina cinca canniyemakiyapi ; hastanhanka iyuwi ojupi kin awanyag emahdepi ; hastanhanka iyuwi ojupi mitawa kin awanwahdake śni.
7 Minagi waśtekidake cin niye, ito, omakiyaka ye, tukten wawihanyakiya, tukten wiyotanhan iwanke wicayakiya hecinhan: tokeca miye, nitawaśi taoptaye kin en, wanji nuni kin iyemacece kta he.

8 Winohinca ehna owanyag niwaśte hinca, he sdonyaye śni kinhan, detanhan cankuya ye, optaye owepi ohna, qa wonwicayapi tawakeyapi kin icahda tatokadan cinca nitawa witianwicakiya ye.

9 Wiwaśte mitawa, Pharaoh tacanpahmihma en śung wiye kin he iciyacin.

10 Nitapon oinpi skaskadan on owanyag waśte, nitahu wanapin on.

11 Mazaskazi oinpi unnicagapi kta ye, mazaska taśpudan koya.

12 Wicaśtayatapi kin wahna wotapi kin "en yanka icunhan nard mitawa oomna hiyuya ece.

13 Waśtewakidake cin he canśin waśtemna opalite wan eewakiya, aze mitawa otahedan hanyetu osan wanke kta.

14 Waśtewakidake cin he kopher iyage wan iyececa wada183 


\section{DOWANPI ODOWAN,}

ka, Engedi hastanhanka iyuwi ojupi kin ehna.

15 Iho, wiwaśte mitawa, owanyag niwaśte; han, owanyag niwaśte, niiśta tinwakiyedan iyececa.

16 Tho we, waśtecicidake, niye owanyag niwaśte, han oiyokipi hinca; nakun owanka unkitawa kin he toto.

17 Untipi canludakinyan wanke cin hena hanteśa, wankan obagopi unkitawapi kin hena wazi can.

\section{WICOWOYAKE 2.}

1 Śaron onjinjintka, kaksiza en mnahcahca kin he niye.

2 Mnahcalica wapepeka ehna un kin he iyececa, wiwaśte mitawa kin cunwintkuyapi kin owicapeya un.

3 Taspantanka hu taśloju can lin ehna, he iyecen waśtewakidake cin he cinhintluyapi kin owicapeya un ye. Iye ohanzi tawa kin on imduśkin qa en imdotanka, unkan tawaskuyeca kin micaka en skuye hinca.

4 Miniśa tipi kin en amahi ; unkan tawiyokihedan miwankan un kin he wocantekiye.

5 Hastanhanka on wacintonhnagmayan ye; taspantanka on waśagmayan ye, wowaśtedake on wamayazan heon.

6 Iye catka kin mapa kin ihukuya, qa iye nape etapa kin he poskin mahduza ce.

7 Jerusalem cunwintkupi, tinta tatokadan wiye qa tawiyedan on ceciciyapi ce, waśtewakidake cin yapaninipi ktc śni qa duhicapi kte śni, tohanyan iyokipi hehanyan.

8 Waśtewakidake ho kin! wan, lie kin akan psipsica, paha kin akan psipsin u ye.

9 Waśtewakidake cin he tato- kadan qa tahinca cinca wan iyececa ye: wan, conkaśke iheyata inajin, owanyeye eciyatanhan ahitonwan, ozanpi ohna taniniçiya ye.

10 Waśtewakidake cin he ie ca hemakiya; Wiwaśte mitawa, owanyake waśte mitawa, najin qa kuwa wo.

11 Tho, waniyetu kin wanna henakeca, magaju kin hdapte, wanna sam iyaya;

12 Maka akan wahca kin taninin; dowanpi kte cin wanna hiyohi: qa makoce unkitawapi ohna tin wakiyedan ho kin nahonpi:

13 Suken can kin waskuyeca toto icahya, qa hastanhanka iyuwi hica aye cin hena omnanpi waśte. Wiwaśtc mitawa, owanyag waśte mitawa, najin qa lruwa wo.

14 Tinwakiye dan mitawa, imnija ohdoka ohna, he iyadipi oinahibe kin ohna, tokin niite wanmdalsen, niho nawalion kta; niho kin he oiyokipi, qa niite kin owanyag waśte nakaeś.

15 Tokadan, tokadan cikcistinna hastanhanka iyuwi kiunniyanpi kin hena unkiciyuza miye : hastanhanka iyuwi unkitawapi hastanhanka śtunkaka aicaga nakaeś.

16 Wastewakidake cin he miye mitawa, qa iye tawamayan: mnalicalica kin ehna wawilhankiya ece ye.

17 Anpao hinanpa qa ohanzi kin kinyan iyaye kte cin hehanyan, hdicu ye, waśtecicidake, tatokadan wan iyececa un ye, qa tahinca cinca wan Bether he kin akan iyececa.

\section{WICOWOYAKE 3.}

1 Hanyetu icunhan mitowinja akan tuwe minagi waśtekidake

184 
cin he owakide; owakide tuka iyewaye śni.

2 Wanna nawajin kta, qa otonwe kin en mde kta, canku kin ohna qa ocanku tanka ohna, tuwe minagi waśtekidake cin he owakide kta ce. Owakide tuka iyewakiye śni ye.

3 Waawanyake otonwe ehnalna yapi ece kin hena iyemayanpi. Minagi waśtekidake cin lie wandakapi he, ewicawakiya.

4 Kitan ecinyan hena sanpa imdamda hehan tuwe minagi waśtekidake cin he iyewaya: mduza qa amduśtan kte śni, ecen ina ti kin en awahdi, qa tuwe cinca mayuhe cin ti mahen.

5 Jerusalem cunwintkupi, tinta tatokadan qa tawiyedan kin on ceciciyapi ce, waśtewakidake cin he yapaninipi kte śni qa duhicapi kte śni, tohanyan iyokipi hehanyan.

6 Tuwe śota wankan ye cin iyececa hewoskan tanhan $u$, canśin waśtemna qa wihdi waśtemna wopeton tapejihuta mdu kin owasin koya on aizinyapi kin he tuwe he.

ry Iho we, on akiyuhapi tawa he Solomon tawa; wicaśta waditaka wikcemna śakpe ihdukśan yakonpi, Israel waditakapi kin etanhan.

8 Hena owasin mazasagye hduhapi, kicizapi en wayupikapi : hanyetu en wokokipe on otoiyohi tamazasagye ceca akan yanka.

9 Solomon wicaśtayatapi kin Lebanon can kin etanhan on akiyukapi wan içicaga ye.

10 Bosdan he cin hena mazaska on kaga, onnaptan kin he mazaskazi on, oakalipe kin he minihuha śa stan on, ocokaya kin he wocantekiye en owinśtonpi, Jerusalem cunwintkupi kin wicakicagapi.
11 Zion cunwintkupi, inanpapiqa Šlomon wicaśtayatapi hunku wateśdake teśdag kicaton un kin he wanyaka pe, wakanhduze anpetu kin en, qa iye cante wiyuśkin anpetu kin en.

\section{WICOW OYAKE 4.}

1 Tho wo, wiwaśte mitawa, owanyag niwaśte; iho, owanyag niwaśte; paha nisonpi otahedan niista kin tinwakiyedan iyececa; pa hin nitawa tatokadan optaye wan Gilead paha akan iwankapi kin iyececa.

$2 \mathrm{Hi}$ nitawa optaye wan $\mathrm{yu}$ śdapi wicayujajapi etanhan upi kin iyececa, hena otoiyohi cekpa yuhapi, qa etanhan cinca ton śni wanica.

3 Niiha hahonta duta wan iyececa; iyae cin he wiciyokipi hinca; nawate.nitawa taspantanka su ota onśpa iyececa, paha nisonpi otahedan.

4 Nitahu kin he Dawid taconkaśke kin iyececa, wipe on kagapi, wahacanka kektopawinge en otkeyapi, iyulipa wicaśta waśaka wahacanka tawapi.

5 Aze nom nitawa tacinca nom cekpapi, mnahcahica ehna wihanpi kin iyececa.

6 Anpao kta qa ohanzi kinyan iyaye kta, hehan canśin waśtemna he kin ekta, qa canśin kohdihdi paha kin elkta, mde kte.

7 Wiwaśte mitawa, ocowasin owanyag niwaśte ; tuktedan oniyuhi śni.

8 Lebanon etanhan miciu wo, mitawin, Lebanon etanhan miciu wo: Amana ipa kin etanhan etonwan wo, Śenir qa Hermon paha pajodan kin etanhan, mnaja otipi kin etanhan, inmutanka he kin etanhan.

9 Cante mayaki, mitawin, mi- 
- tankśi ; niiśta wanjidan on, nitahu tawanapin oboyaya wanjidan on cante mayaki.

10 Mitawin, mitankśi, nitowastedake kin he oiyokipi hinca : nitowastedake kin miniśa isanpa waśte : qa ihepi nitawa omnanpi kin he taku waśtemna owasin isanpa.

11 Mitawin, canhanpi tiktica śbu niiha hena ee : niceji ihukuya tulimaga canhanpi pte asanpi ko yukan: qa nitawokoyake omnanpi Lebanon omnanpi kin iyececa.

12 Mitawin, mitankśi canwojupi natakapi kin hee ; minihdoka natakapi, miniyowe askamtonpi kin heca.

13 raku icaliyaye cin taspantanka su ota ojupi kin heca, waskuyeca waśte hinca nakun; canśin oomna waśte nard kicica:

14 Nard qa wiziye kici; sinkpe tawote qa canhaśa, canśin kohdihdi can kin owasin koya; cansin waśtemna qa canśin pa hinca, wizinye waśtemna ocaje owasin koya:

15 Miniyowe canwojupi, minilidoka wakoniya, qa Lebanon etanhan wakpadan kin.

16 Waziyatanhan tate uya, kikta ye; qa itokagatanhan uye cin, $u$ ye: mitawoju kin atateyanpa ye ; hecen oomna waśtemna kin hiyu kta. Tokin waśtewakidake iye canwojupi tawa kin en $\mathrm{ku}$, qa waskuyeca waśte kin hduten.

\section{WICOWOYAKE 5.}

1 Mitawin, mitankśi, canwojupi mitawa kin en hibu: canśin mitawa taku omna waśtenma ko mnawakiya: canhanpi tiktica mitawa tuhmaga canhanpi mitawa kıci wahdute: miniśa milawa pte asanpi iyahna wahdatkan. Mitakuye, wota po ; wayatkan po, han, waśtecicidapi, wiyuśkinyan wayatkan miye.

2 Miśtinbe, tuka micante kiktahan yanke; Waśtewakidake katoto yanka ho kin hee; Mitankśi, wiwaśte mitawa, tinwakiyedan mitawa, wanji taku iyaonpepica śni mitawa, makiyuhidoka wo; mapa kin cu ojudan, qa mapa hin hanyetu ośbuye kin ojudan nakaeś.

3 Onlidohda wahduśdoka, token ake wecun kta he : siha wahdujaja, token ake śamwakiye kta he.

4 Waśtewakidake cin he owanyeye eciyatanhan nape hiyuye, unkan iye on tanmalien miliduśkanśkan.

5 Waśtewakidake wakiyuhdoke kta najin imdamda, unkan inatake iyuze akan minape etanhan canśin śbuya, qa minapsukaza canśin waśtemna śbuya.

6 Waśtewakidake wakiyuhdoka ; tuka waśtewakidake cin he wanna ihdutokan iyaya: ie cehan minagi hiyu tuka: owakide tuka iyewaya owakihi śni: wakipan tuka amayupte śni ye.

7 Waawanyake otonwe ohomni yapi kin hena iyemayanpi, hena amapapi, hena ksuwemayanpi : conkaśke awanyakapi kin hena ite akahpe kin makipi.

8 Jerusalem cunwintkupi, ceciciyapi ce, waśtewakidake cin he iyeyayapi kinhan wowaśtedake on wamayazanka he oyakidakapi kte.

9 Winohinca wicehna owanyag niwaśte hinca, waśteyakidake cin he waśtedakapi tokeca taku on isanpa he; waśteyakidake cin taku on waśtedakapi 
tokeca wan isanpa waśte he, on hecen ceunyakiyari he.

10 Waśtewakidake cin he ska qa samna, kektopawinge wikcemna ehna okitanin hinca.

$11 \mathrm{~Pa}$ kin he mazaskazi ecedan: pa hin kin hena yuhaha qa sapsapa, kangi iyececa :

12 Iśta kin tinwakiyedan mini walspa icahda kin iyececa, pte asanpi ohna yujajapi, ayuco ośtanpi :

13 Iye tapon taku omna waśtemna oicage kin iyececa, wahca waśtemna kin iyececa: iha kin mnahcalica iyececa, canśin waśtemna śbuyapi :

14. Tye nape kin mazaskazi napcupe Tarśiś inyan tehika ośtanpi kin iyececa: tezi kin he huhu wiyatpa inyan toto tehika akahpapi kin iyececa:

15 Iye hu kin inyan skaska ihupa mazaskazi eccedan oyuśtanpi kin en ehdepi iyececa: ite kin Lebanon iyececa, hanteśa kin iyecen oiyokipi hinca.

16 Iye i kin skuye hinca: han, o cowasin waśte hinca. Jerusalem cunwintkupi, waśtewakidake cin he dee, qa mitakuye hinca hee.

\section{WICOWOYAKE 6.}

1 Winohinca ehna owanyag niwaśte linca, waśteyakidake cin he tokiya iyaya he: waśteyakidake cin he tokiya icunonpa iyaya he; niyeci unkodepi kta ce.

2 Waśtewakidake cin he iye canwojupi tawa kin ekta iyaya, taku omna waśtemna oicage ekta: canwojupi kin en wawihanye kta, qa mnahcalica hduspi kta.

3 Waśtewakidake cin he tawamayan, ga wástewakidake cin he miye mitawa : mnahicahica kin ehna wawilianyan ece ye.

4. Wiwaśte mitawa, owanyag niwaśte hinca, Tirzeh iyececa, Jerusalem iyecen oiyokipi hinca, ozuye wiyokihedan yuhapi iyecen wokokipe hinca.

5 Miye etanhan niiśta hduho. mni wo, hena maktepidan: pa hin nitawa tatokadan optaye Gilead en iwankapi kin iyececa.

6 Nihi kin hena tahinca wanunyanpi optaye wicayujajapi etanhan iyayuptapi kin iyececa, henaotoiyohi cekpayuhapi, qa hena etanhan cinca ton śni wanica. 7 Paha sonpi otahedan nawate nitawa taspantanka su ota onśpa iyececa.

8 Wicastayatapi winohinca wikcemna sakpe, qa winohinca wicayuhapi wikcemna śahdogan, qa witanśna unpi yawapica śni.

9 Tinwakiyedan mitawa, wiyaonpica śni mitawa kin he wanjidan, hunku tawa kin he iśnana, tuwe yuhe ciqon hdahinige cin hee. Cunwintkuyapi kin hena wanyakapi qa 'yawaśtepi ; wicastayatapi winohinea qa winolinca wicayuliapi kin hena nakun yaonihanpi.

10 Tuwe anpao iyececa etonwan, hanyetu wi iyecen owanyag waśte, anpetu wi kin iyecen wiyatpa, ozuye wiyokihedan yuhapi iyecen wokokipe hinca, he tuwe he.

11 Hma can wojupi kin ekta kun imdamda, kaksiza en waskuyeca kin wanmdake kta,hastanhanka iyuwi icaga hecinhan, taspantanka su ota camni uye wanmdake kta.

12 Sdonwakiye śni, ihnuhanna minagi Amminadib tacanpahmihma iyececa micicaga.

13 Hdicu wo, hdicu wo, Śsulamith; hdicu wo, hdicu wo, wan187 


\section{DOWANPI ODOWAN,}

unniyakapi kta. Sulamith en taku wandakapi kta he. Ozuye nom owaci kin iyececa ce.

\section{WICOWOYAKE $\%$}

1 Wicaśtayatapi cunwintku, nisiha hanpokiton kin hena owanyag waśte hinca : ceca okihe nitawa wamnuha tuwe wayupika nape on kage cin hena iyececa.

2 Nicekpa kin he wakśica ohmihma miniśa iyujimnana kin hee: nitezi kin he aguyapi su opaha wan mnalicalica ihdukśan hiyeye cin he iyececa.

3 Aze nom nitawa totokadan cinca cekpapi kin iyececa.

4 Nitahu kin he huhu waśte canpasotka kin iyececa: niiśta Heśbon miniyowe kin iyececa, Bath Rabbim tatiyopa kin etu: nipoge Lebanon conkaśke $\mathrm{Da}$ masek ektakiya etoñwe cin he iycceca.

5 Nipa akan niyanke cin he Karmel iyececa, qa nipa bin boyaya kin śa stan iyececa : sonpi kin on Wicaśtayatapi kin kaśkapi ye.

6 Wiwaśte mitawa, owanyag niwaśte, qa wowiyuśkin on oiyokipi hinca yaun.

- Nihanske cin he tamar can kin iyececa, qa aze nitawa iyage kin iyececa.

8 Tamar can kin en mde kta, adetka kin mduze kta ce, epa: unkan hinnakaha aze nitawa hastanhanka iyage imdacin kta, qa nipoge omnanpi kin taspantanka iyecece kta :

9 Qa nicaka kin miniśa waśte iyececa, he waśtewakidake cin en katinyan ya, tona iśtinmapi iha akan iwaśtedan iwicayaya.

10 Waśtewakidake cin he ta188 wamayan, qa iye tocantekiye kin miye en mau ye.

11 Waśtecicidake, kuwa, rnaga kin ekta unye kta, otonwe cistinna kin hena ekta hanyetu unyakon kta.

12 Hanhanna hinca liastanhanka iyuwi ojupi kin ekta unye kta; wiyuwi kin icage cinhan wanunyake kta, jyage śtunkaka wanna tanin hecinhan, taspantanka su ota kin wanna camni uye cinhan: heciya mitocantekiye cicu kta.

13 Wocantekiye waskuyeca kin oomna hiyuya, qa tiyopa unkitawapi ohna waskuyeca waśte ocaje owasin, teca qa tanni, waśtecicidake, hena ecicihnaka ce.

\section{WICOWOYAKE 8.}

1 Mitimdo, ina aze hdazoke ciqon, tokin henicecen; unkanśs canku ohna iyeciciya ehantanhan iiciputake kta: hececa eśta miye wahtemadapi kte śni.

2 Yus aciye kta, ina ti kin en acii $\mathrm{kta}$, he onspemakiye kta: miniśa icicahiyapi taspantanka su ota hanpi mitawa kin he yatheciciye kta tuka ye.

3 Iye nape catka mapa kin ihukuya kta, qa etapa kin poskin mahduze kta tuka.

4 Jerusalem cunwintkupi, ceciciyapi ce, waśtewakidake cin he yapaninipi kte śni, qa duhicapi kte śni, tohanyan iyokipi hehanyan.

5 Tuwe hewoskan tanhan $\mathrm{n}$, waśtekidake cin he hdus un kin he tuwe he. Taspantanka hu kin ihukuya najinciciya; nihun hen niton; tuwe niyuhe cin he hen niton.

6 Askape wan iyecen nicante en, ipuspe wan iyececa niisto akan emahnaka ye : wowaśteda- 


\section{WICOWOYAKE 1.}

ke kin wiconte iyecen waśaka: wowinawizi kin he Hades iyecen suta: he pelinige kin peta pehnige ide hinca heca.

7 Mini ota wowaśtedake kasni kte śni, qa mini tanka kin spayeye kte śni : wicaśta wan iye ti tawahpaya kin iyulipa wowaśtedake on tokiyopekiya eśta, he ocowasin aktapi kte śni.

8 Tankśiunyanpi kin he cistinna qa aze nica. Anpetu en tankśiunyanpi kin dapi kinhan token ecaunkiconpi kta he.

$9 \mathrm{He}$ conkaśke unkans akan mazaska tipi wan unkagapi kta; he tiyopa unkanś hante śa canmdaska on onauntakapi kta tuka do.

10 Miye qe conkaśke hemaca, ga aze mitawa pajodan iyececa. Heon iye iśta kin en wanji wo- cantekiye iyekiye cin hemaceca ye.

11 Baal-Hamon en hastanhanka iyuwi ojupi wan Solomon yuha: wiyuwi ojupi kin he waawanyake owicaqu: waskuyeca on otoiyohi mazaska kektopawinge kahi kta.

12 Wiyuwi ojupi mitawa mitokam wanke cin he mitawa. Solomon, niye kektopawinge duhe kta, qa tona waskuyeca awanyakapi kin hena opawinge nonpa yuhapi kta ye.

13 Canwojupi kin en ounyaye cin, okodakiciye kin hena niho anagoptanpi ece: he iś nalionmakiya wo.

14 Wastecicidake, najica u ye, qa taku omnanpi waste paha kin akan tatokadan wiye qa iś tahinca cinca wan iyececa un ye.

\section{ISAYA TAWOYUKCAN KIN.}

\section{WICOWOYAKE 1.}

1 Isaya Amoz cinhintku towanyake kin, Juda qa Jerusalem on wanyake, Juda wicaśtaytapi Uziya, Jotham, Ahaz, qa Hezekiya taanpetu kin en.

2 Malipiya, nalion wo, qa maka, anohya wo: Jehowa ia ; Cinca icahwicawakiye ça tanka wicawakaga, unkan makipajinpi ce.

3 Tatanka ee tuwe tawa kin sdonkiya, qa sonśonna kin he tuwe yuha dotopiye tawa sdonya; tuka Israel wasdonye śni, mitaoyate waawacin śni.

4 Hehehe, oyate wan wahtani sa, wicasta woah̆tani on hduśakapi. wicoicage-wan śicaya econ- pi, wicacinca wayuśicapi; Jehowa ayustanpi, Israel Wanji Wakan tawa kin he wahtedapi 利i, hektam ihdoyapi.

5 Taku on sanpa anipapi kta he; nakun wawakipajinpi ece ecanonpi kta; wicapa ocowasin wayazanka, qa wicacante kin ocowasin banyeca:

6 Sicu etanhan pa iyahdeya tuktedan zani śni; capapi, kasuśujapi qa ton a wicau; aohduteyapi śni, yuskiskitapi sni qa wihdi on waśte kagapi sni.

7 Makoce nitawapi kin he otiwita, nitotonwepi kin peta on hulinagapi; maga nitawapi wicaśta tolkeca nitokam temyapi, qa hewoskan ehpeyapi, wicaśta tokeca awihnunipi iyececa. 


\section{ISAYA TAWOYUKCAN KIN,}

8 Zion cunwintku kin hastanyanka ojupi wan en ohanzi hdepi lin hc iyecen ehpeyapi, sakayutapi ojupi en wakeya he cin iyececa, otonwe anatanpi kin iyececa.

9 Jehowa ozuyc tawa onajice cistinna wan unkokicicaptapi śni unkanśs Sodom iyeuncecapi kta, qa Gomorrāh iyecen unkicagapi kta tuka.

10 Sodom wicaśtayatapi, Jehowa oic kin nalion po ; Gomorrah oyate, Wakantanka unkitawapi toope kin awacin po.

11 Tokeca taku ota wamayakiduśnapi he, Jchowa eya; tamdoka liulinagapi qa cemyapi wasna kin on imamna hinca; qa tatänka, tacincadan qa tatokadan mdoka we kin iyomakipi śni.

12 Nakun tohan miye en ihdutanin yahipi kinhan, mitahocoka omayanipi kin, tuwe he ninapepi etanhan icu kta he.

13 Detanhan wośnapi takuśni kin heca e aupi śni po ; wizinyapi kin he walitewada śni ; wi teca qa wookilipe omniciye kagapi kin hena mduha owakihi śni ; omniciye wakan kin he hee kaeś woalitani.

14. Wi teca nitawapi qa mniniciyapi ece kin hena minagi ścedaka; hena woyuśake mdawa; yuha mdowakița.

15 Unkan nape yahdugatapi kinhan miiśta anacilimanpi kta; han, wocekiye daotapi kinhan nawahon kte śni ; nape we onijupidan nakaeś.

16 Ihdujaja po, ihduska po; miiśta itokapatanhan taka śica ecanonpi kin elpeya po; sicaya econpi ayuśtan po:

17 Taku waśte econpi onspeiciciya po: wokonze akita po; śicaya wicakuwapi kin hena owotanna ccawicakicon po, wa- mdenica kin hena wicayaco po, wiwazica kin he iciya po.

18 Tho miye, nakaha wounhdakapi kta, Jehowa eya: wayahtanipi kin hena duta eśta wa iyecen ska kta; hena śa stan iyececa eśta tahinca hin kin iyecece lita ce.

19 Hecen yacinpi qa waanayagoptanpi kinhan, makoce taku waśte aicage cin he yatapi kta :

20 Tuka wicayadapi śni-qa wawayakipajinpi kinhan, nazasagye on temniyanpi kta, Jehowa i kin he hecen eya ce.

21 Hehehe, otonwe owotanna qon he witkowinna icaga: wokonze on ojudan, woowotanna ohna ti tuka qon; tuka nakaha tin wicaktepi sa.

22 Mazaska nitawa ginginca icaga; miniśa nitawa mini icahiyapi.

23 Wicaśtayatapi nitawa kin hena wicaśtapiśni qa wamanonpi sa takodawicayapi : otoiyohi taku wicaqupi waśtedaka qa icajuju akitapi ; wamdenica kin hena on wayacopi śni, qa wiwazica tawoakinica kin he en wicahi śni.

24 Heon etanhan Itancan kin, Jehowa ozu ye yuha, Israel Iyotan Waśaka tawa kin he hecen eya; Han, tona makipajinpi kin on iyokipimiçiye kta, qa tokamayanpi kin hena towicawecicon kta ce.

25 Qa minape aciyuhomni kta, qa ginginca nitawa wiśdoye on ciciyuecedan kta, qa maza śdoyapi kin ocowasin elipeciciye ikta.

26 Qa wayaco nitawapi tokaheya ekta iyecen wicamduecetu kta, qa waaiewicakiyapi nitawa otokahe ekta iyececapi lita: hehan iyohakam, otonwe owotan- 
ta, otonwe wacinyepica, eniciyapi kta.

27 Woyaco on Zion opekitonpi lita, qa tona en hdipi kin hena พัoowotanna on.

as Unkan śicaya econpi sa qa wåhtanipi kin witaya ihangwicayapi kta, qa tona Jehowa ayustanpi kin hena yusotapi kta.

29 Qa utuhu can waśteyadakapi qon hena on iśtecapi kta, qa can wojupi yahdahnigapi kin hena on iniśtecapi kta.

30 Utuhu can wan ape śnije cin he iyenicecapi kta, qa can wojupi wan mini wanice cin he iyececa.

31 Qa tuwe waśake cin he hahonta on kagapi kin he iyecece kta, qa iye ohan kin peśnija iyecece kta; unkan napin ptaya ideyapi kta, qa tuwedan kasni kte śni.

\section{WICOWOYAKE 2.}

1 Wicoie Juda qa Jerusalem on, Isaya Amoz cinhintku wanyake cin he dee.

2 Unkan anpetu ehake kin en, Jehowa tipi Paha kin he he ipa kin en chdepi kta, qa paha kin isanpa wankan kagapi kta ; unkan oyate owasin en kawitaya iheye kta.

3 Unkan oyate ota upi qa heyapi kta; U miye, Jehowa Paha tawa kin ekta unyanpi kta, Jaliob ta Wakantanka ti kin ekta; unkan iye tacanku kin onspeunkiyapi kta, kinhan ocanku tawa ohna maunnipi kta: Zion etanhan woope kin inanpe kta, qa Jehowa oie kin Jerusalem etanhan.

4. Unkan iye Ikcewicaśta ehna wayaco kta, qa oyate ota iyopewicaye kta; hecen mazasagye yuhapi kin mahiyumdu kagapi lita, qa wahukeza on isan śkopa kagapi kta: oyate wan oyate tokeca mazasagye apaha kte śni, qa icimana kicizapi onspeiçiciyapi kte śni.

5 Jacob tiyohnaka, u po, Jehowa iyoyanpa tawa kin en maunnipi kta.

6 Heon nitaoyate, Jakob ti kin he, ehpeyakiya; wiyohiyanpata tanhan isanpa ihduotapi, qa hanmdepi, Philistia oyate kin iyececa, qa wicaśta tokeca cinca takodawicayapi, heor etanhan.

7 Nakun makoce tawapi kin mazaska mazaskazi ko ojudan, qa wowijice tawapi kin wopteca sni ; nakun makoce tawapi kin śuktanka on ojudan, qa canpahmihma tawapi kin wopteca sni.

8 Makoce tawapi nakun wakagapi on ojudan; iye nape ohan kin hena ohodapi ece, taku iye napsukaza kagapi kin hena:

9 Hecen wicaśta ikceka patuś inajin, qa wicaśta tanka kin he makata ehpeiçiya ece ; heon wicayecicajuju kte śni.

10 Imnija kin ihukuya ya wo, qa maka mdu kin en inahman wo, Jehowa tokokipe kin itokapa tanhan qa tawootanin wowitan kin heon.

11 Wicaśta wahaniçida iśta kin kun iyeyapi kta, qa wicaśta watankaiçidapi kin he kun ehpeyapi kta; qa anpetu kin he en Jehowa iśnana yatankapi kta.

12 Jehowa ozuye tawa taanpetu kin he tona wahanicida qa watankaicida owasin akan un, qa tona yuwankan iyeiçiya owasin akan; unkan yuhukun ehpeyapi kta :

13 Nakun Lebanon hante sa tehanwankantu qa wankan iyeyapi owasin akan un, qa Bașan utuhu can kin owasin akan ;

14. Qa he tehanwankantu kin 191 


\section{ISAYA TAWOYUKCAN KIN,}

iyuhpa akan, qa paha wankan iyeyapi kin owasin akan yanke ;

15 Qa tipi tehanwankan otoiyohi, qa conkaśke suta ow asin akan;

16 Qa Tarśiś wita wata iyulipa, qa taku owanyag waśte kin owasill akan yanke.

17 Unkan wicaśta wahanicidapi kin kin he kun elipeyapi kta, qa wicasta watankaiçidapi kin he patuśyapi kta; qa anpetu lin he en Jehowa iśnana yatankapi kta.

18 Nakun wakagapi kin hena ocowasin tokan aye kta :

19 Qa imnija ohidoka ohna, qa maka ohdoka kin ohna yapi kta, Jehowa tokokipe kin itokapatanhan, qa tawootanin wowitan kin heon, tohan maka kin wakokipe kicah inajin kinhan.

20 Anpetu kin he en wicaśta mazaska wakagapi qa mazaskazi wakagapi tawa, iye ohoda kta on kagapi kin hena napeilieyatahedan qa hupawakihdakedan kin ekta. elpeyapi kta :

21 Imnija olidoka ohna yapikta, han imnija kahdokapi kin ohna, Jehowa tokokipe kin itokapatanhan, qa tawootanin wowitan kin heon, tohan maka kin wakokipe kicali inajin kinhan.

22 Wicaśta, taniya poge ohna un kin, he ayuśtan po: taku on he taku yawapi kta he.

\section{WICOWOYAKE 3.}

1 Wanyaka, Itancan kin Jehowa ozuye tawa Jerusalem qa Juda etanhan ipatan qa sagye kil icu kta, aguyapi sagye kin ocowasia, qa mini sagye kin ocowasin :

2 Wicaśta waśaka qa wicaśta zuye sa, wayaco qa wicaśta wokcan, tuwe hanmdohdaka qa wicahinca kin ;
3 Wikcemna zaptan en itancan qa tuwe ite kirrihanpi, qa wawiciye kin, qa wabago wayupika, qa tuwe ie ksapa.

4 Unkan kośka wicaśtayatapi wicawaqu kta, qa hokśiyoq $7 a$
iwicadakapi kta.

5 Qa oyate kin otoiyohi kakiśkiciyapi kta, qa otoiyohi ti kiyedan unpi kin nakun; hokśidan kin he wicahinca en ibdatan $\mathrm{kta}$, qa tuwe wahte śni kin he tuwe kinihanpi kin en.

6 Tohan wicaśta wan iye cinyeku, atkuku ti kin etanhan, he hduze ca, Niye wokoyake niciun, itancan unniyuhapi kta, qa woihangye kin de niye ninape en un kta, eye cinhan;

7 Anpetu kin he en nape hdugate heye kta; Wawokiziya hemaca kte śni, wati kin ohna woyute wanica qa wokoyake wanica, heon oyate kin en itancan makagapi śni po.

8 Jerusalem atakuni śni qa ${ }^{\top} u-$ da hinhpaya; iye cejipi qa olianpi kin Jehowa tawowitan iśta kin iyokiśinyapi kin heon etanhan.

9 Iye ite hdutaninpi kin he owicayaka, qa Sodom iyececa iye wahtanipi ohdakapi, nakihmanpi śni. Woiyokiśice iye nagipi en kiciyankapi nunwe, taku ślca ecaiçiconpi nakaeś.

10 Tuwe owotanna kin, Tanyan un kta ce, keciya po; iye ohanpi waskuyeca kin hdutapi ece kta :

11 Hehehe, turís śice cin, tehike kta, iye nape ohan kin iyecen ecakiconpi kta nakaeś.

12 Mitaoyate hokśipidan on wowidagyapi, qa winolinca kin itancan wicayuhapi ece. Mitaoyate, tona wahokonniciyapi kin hena nuniniyanpi, qa canku omayanipi kin he awihnuniyapi. 
WICOWOYAKE 5 .

13 Jehowa wakinin inajin, qa oyate wicahdaco yanka.

14 Jehowa iye taoyate en hunkayapi kin wicahdaco kta, nakun wicaśtayatapi kin. Niye hastanyanka iyuwi ojupi kin temyayapi; wowahpanica taku wicakipi kin he yatipi kin ohna yanke.

15 Tokeca mitaoyate wicayakapanpi qa wahpanica itepi kin wicadumanpi he, Jehowa ozuye Itancan kin eya.

16 Nakun Jehowa heya; Zion cunwintku tankaiçidapi, qa pa hduwankan icu manipi, qa token cincin iśta ycyapi, manipi qa kaicicuya yapi, qa siha on nasnasnapi ;

17 Heon etanhan Itancan kin Zion cunwintku pesdete oyuhiihi on peśda kage kta, qa tancodan unpi kin Jehowa wicayutaniil kta.

$18 \mathrm{Qa}$ anpetu kin he en Itancan kin sicogokaśke wowitan kin ewicakiciyaku kta, qa wateśdake mazaska wanapiu koya;

19 Oinpidan, qa napokaśke, qa ite akahpe kin ;

20 Wapaha qa siha icaśke, qa paipahte, qa wojuha wakan, qa oinpi wakan kin;

21 Napcupe qa poge oinpidan;

22 Wokoyake waśte, qa onlidohida, qa śina, qa mazaśka opiye ;

23 Ihdiyomdasin, qa mahen unpi waśte, qa wateśdake, qa ite alkahpe.

24 Unkan kaketu kte, taku waśtemna on sicamna yuke kta; q̧a ipiyake on telimiso; qa paha sonpi on nasuśda kagapi ; qa śina zipzipedan on wakihdaka ipiyagkitonpi ; owanyag waśte on wicagupi ee yuke kta.

25 Wicaśta nitawapi mazasagye on hinhpayapi kta, qa wicaśta waditaka nitawapi kicizapien.
26 Hecen iye tatiyopa iyokiśica qa ceye kta,"qa iye hinca cokadan makata iyotanke lita.

\section{WICOWOYAKE 4.}

1 Unkan anpetu kin he en winolinca śakowin wicaśta wanjidan en yuzapi qa heyapi kta; Aguyapi unlidutapi kta qa wokoyake kounhdakapi kta, tuka nicaje on cajeunyatapi nunwe, wiyaonpapi eunkiciyakupi kta.

2 Anpetu kin he en Jehowa toicage kin he woyuonihan qa wowitan kta, qa maka waskuycca kin he wowaśte qa woiyokipi $\mathrm{kta}$, Israel tona okaptapi kin hena on.

3 Unkan kaketu kte, Zion en okaptapi qa Jerusalem en mdogyanke cin hena, Owotanna, ewicakiyapi kta, tona wiconi on Jerusalem en owicawapi kin otoiyohi ;

4. Tohan woyaco oniya qa ide oniya kin on, Itancan kin Zion cunwintku tawośape kicicajuju, qa Jerusalem cokayatanhan we kin pakinte cinhan.

5 Unkan Jehowa Zion Pạha oyanke otoiyohi akan, qa tomniciye kin en, anposkan, mahpiya śapa qa śota wan kage kta, qa hanyetu eca, peta ide iyega; wowitan ocowasin iwankam woakahpe kta.

6 Unkan wakeya wan yuke kta, anpetu icunhan waśte kin on ohanzi kta, qa wowinapeyapi $\mathrm{kta}$, qa icamna magaju ko on woinalibe kta.

\section{WICOWOYAKE 5.}

1 Iho, mitakoda wakidowan kta, mitakoda tadowan kin hee, hastanhanka iyuwi ojupi tawa kin on. Mitakoda hastanhanka 


\section{ISAYA TAWOYUKCAN KIN,}

iyuwi ojupi wan yuha, pajodan waśte hinca wan en.

2 Unkan he ihdukśan nakitake, qa etanhan inyan pahi, qa hastanhanka iyuwi hu waśteśte kin en oju, qa cokaya cowahe tehanwankantu wan kaga, qa en iyuśkice wanji nakun kicaga. Hehan hastanhanka aicage kta ape yanka, unkan hastanhanka ikceka aicaga.

3 Hecen dehan, Jerusalem en ounyayapi qa Juda wicaśta, tokin miye qa hastanhanka iyuwi ojupi mitawa imiyeciyukcanpin. 4 Hastanhanka iyuwi ojupi mitawa kin en ecamon kin taku sanpa econpica he. Hecen hastanhanka aicage $k$ ta ape manka, tokeca hastanhanka śica aicaga he.

5 Unkan nakaha, iho, hastanhanka iyuwi ojupi mitawa taku ecawecon kta he ociciyakapi kta: Wapepeka inatake kin emdaku kta, unkan temyapi kta; conkaśke kin he mdujuju kta, hecen amanipi kta :

$6 \mathrm{Qa}$ hewoskan ehpewaye kta; adetka abaśpapi kte śni qa maka akatapi kte śni; tuka wapepeka can pepe ko en icage kta; nakun mahpiya śapa kin wahowaye kta, magaju takudan ahinhe kte śni.

7 Jehowa ozuye tawa hastanhanka iyuwi ojupi tawa kin Israel tiyohnaka kin hee, qa Juda wicaśta wojupi waśteśte tawa kin hena ee: unkan wokonze kta e ape yanka, tuka iho, we papsonpi ; woowotanna on ape un, tuka iho, sicahowayapi.

8 Tona tipi en tipi akagapi, maga en maga ikoyagyapi, hecen oyanke wanica, qa iye iśnana maka cokaya yankapi kta, hena wotehi kiciyankapi nunwe.

9 Minoge en Jehowa ozuye yu- he cin he hecen eya; Awicakehan tipi ota hewotahedan unpi kta tanka qa waśte tuwedan ohna ti kte śni.

$10 \mathrm{Han}$, hastanhanka iyuwi ojupi maka iyutapi wikcemna bath wanji icaliye kta, qa homer wanji ojupi kin he epha wan icahye kta. 11 Wotehi kiciyankapi nunwe, tona mini wakan kuwapi kta hankiktapi, litayetu hehanyan econpi, miniśa wicayuwitko kta ; 12 Qa wohanpi kin en candowankiyapi ikan wikcemnaqa candowankiyapi ikceka, cancega miniśa ko yukan ; tuka Jehowa tolian kin ekta ewacinpi śni, qa iye nape taku econ kin awacinpi śni.

13 Mitaoyate taku yukcanpi śni heon wayaka ayapi, qa wicaśta kinihanpi kin hena akihan tapi, qa wicota tawa ipuzapi on pus aya.

14 Heon etanhan Hades ihdutanka qa nina hin i hdukawa; unkan wowitan tawa, qa wicota tawa, qa owodutaton kagapi, qa tuwe wiyuśkin nakun en yapi kta.

15 Hecen wicaśta ikceka kin makata iyeyapi kta, qa wicaśta tanka kin yuhukun ehpeyapi kta, qa wahaniçidapi iśta kin kun icupi kta :

16 Tuka Jehowa ozuye yuhe cin he woyaco en yatankapi kta, qa Wakantanka wakan kin he woowotanna on yawakanpi kta.

17 Hehan tahine cincapi kin tukten awicayapi hen wihanpi kta, qa makotahedan tona cepapi kin hena wicaśta tokeca temyapi kta.

18 Wotehi kiciyankapi nunwe, tona woahtani tehmiso on wicohan śica hdutitanpi, qa canpahmihma ikan on iyecen walitanipi kin :

194 
19 T'ona heyapi; Ohanko kte, taku econ inalini kte, hecen wanunyakapi kta; qa Israel Taku Wakan tawa kin he ku qa kiyedan $u$ kte, hecen sdonunyanpi kta ce.

20 Tona taku śica waśte eciyapi, qa taku waśte kin he śica ce, cyapi ; tona iyoyanpa otpaza eekiyapi, qa otpaza iyoyanpa eekiyapi; tona taku skuya kin he pa kiyapi qa taku pa skuye kiyapi kin hena wotehi kiciyankapi nunwe.

21 Tona iye iśta kin en ksapapi, qa wayupikeiçidapi kin hena wotehi kiciyankapi nunwe.

22 Tona miniśa yatkan ohitikapi, qa mini wakan icahiya wicaśta waśakapi kin hena wotehi kiciyankapi kta;

23 Tona taku wicaqupi on wicaśta śica wicayaowotaupi, qa wicaśta owotanna toowotanna ekiciyakupi kin hena.

24. Heon etanhan, peta ceji aguyapi hu yasota, qa peji ideyapi hinhipaye cin he iyecen, hena hutkan kin can ponpon iyecece kta, qa wahica tawapi kin maka mdu iyecen wankan ye kta; Jehowa ozuye tawa toope kin ehpeyapi, qa Israel 'Taku Wakan tawa oie kin aktapi śni, heon etanhan.

25 Heon Jehowa tocanniye kin iye taoyate aidervicakiya, qa iye nape awicapaha, qa wicakaśtaka: Hecen paha kin cancan aya, qa wicaśta tapi tacesdi iyecen canku cokaya wankapi. Hececa eśta tocanniye enakiye śni, tuka hinyahin nape hdugan un.

26 Unkan oyate tehanhan unpi kin wiyokihedan wan ewicakihde kta, qa maka ihanke tanhan hena wicakijojo kta; unkan iho, hena inahni dus upi kta.

27 Hena etanhan tuwedan mdo- kite śni, qa tuwedan laktihahan kte śni; iba kte sni qa iśtinbe kte śni; qa nite on ipiyagg kitonpi kin he yukcapi kte śni, qa hanpkan tawapi kin yupsakapi kte śni :

28 Hena tiwanhinkpepi kin pcpe, qa tinazipepi kin owasin ekatapi ; śunka wakan tawapi śakepi kin inyan iyecen yawapi kta, qa canhdeśka tawapi kin tateiyumni wan iyecece kta.

29 Hena hotaniıpi kin mnaja iyececa, mnaja kośka iyecen hotaninpi kta: han, iyaśapi qa wawiyahpayapi kta, qa tanyan kihnakapi kta; unkan tuwedan ehdaku kte śni.

30 Unkan anpetu kin he en miniwanca sa wanke cin iyecen hena. iyawicakiśapi kta; hecen maka kin ekta etonwanpi kinhan, iho, otpaza woiyokiśica, qa mahiya kin ekta iyoyanpa kin he aotpasyapi.

\section{WICOWOYAKE 6.}

1 Waniyetu Uziya wicaśtayatapi ta he elian, I tancan kin oiyotanke tehanwankantu qa wakan ehdepi wan akan iyotanka wanmdaka; unkan tawokoyake kin tipi wakan kin ojuya.

9 Hen icahda seraphim inajinpi ; otoiyohi liupahu śakpe yuhapi; nom on ite ahdalipa, qa nom on siha ahdalipa, qa nom on kinyanpi ece.

3 Unkan wanji wanji tokeca kipan qa heciya; Wakan, wakan, wakan, Jehowa ozuye hduha; towitan kin on maka kin ocowasin ojudan ce.

4 Unkan tuwe pan ho kin on tiyopa taku akan he cin hena śkanśkan, qa tipi kin śota ojudan. 5 Hehan hepa; Hehehe, matakuniśni ce : wicaśta iha śapa he195 


\section{ISAYA TAWOYUKCAN KIN,}

maca, qa oyate wan iha śapapi on wati; miiśta Wicaśtayatapi lin Jehowa ozuye yuhe cin he wanyakapi, heon etanhan.

6 Hehan seraphim wanji kinyan en mahi, qa petaga wan peta oicu on owayuśna kin etanhan icu-qon he nape ohna yuha:

$7 \mathrm{Qa}$ he mii kin icahtagya qa heya; Iho, de niiha kahtaka; qa niohan śice cin eniciyakupi, qa wayalitani kin nicicajujupi ce.

8 Nakun Itancan ho kin heya nawalion; Tuwe yewaśi kta, qa tuwe unkiyepi on ye kta he, eya. Hehan hepa; De miye ce, yeinaśi ye.

9 Unkan heya; Ya wo, qa oyate kin de hewicakiya wo ; Wanahonpi on nayahonpi, tuka oyakahnigapi śni ; qa wanyakapi on wandakapi, tuka sdonyayapi sni ce.

10 Oyate kin de cantepi cemya wo, qa noge kpe wicakaga wo, qa iśtagonge wicakiya wo ; okinni iśta on wanyakapi kta, qa noge on nahonpi kta, qa cante on okahnigapi kta, qa ihduhomnipi kta, kinhan okiziwicawaye kta tuka.

11 Unkan hepa; Itancan, tohanyan kta he. Unkan heya; Otonwe kin ihangyapi kta tuwedan ohna ti śni, qa tipi wicaśta codan, qa makoce kin ocowasin liewoskan ehpeyapi kta;

$12 \mathrm{Qa}$ Jehowa wicaśta kin tehan iyewicaya, qa makoce cokaya yujujupi tanka wanke kta hehanyan.

13 Tuka iwikcemna wanji en un, qa he ake icage kta, qa he woyute yapi kta: wazi can qa utuhu can kin iyececa: kaksapi eśta oicage kin mahen un, he iyecen wicoicage wakan kin he oicage kta ce.

\section{WICOWOYAKE $\%$}

1 Unkan Ahaz, Jotham cinhintku, Uziya cinhintku, Juda wicaśtayatapi taanpetu kin en, Rezin Aram wicaśtayatapi, qa Peka Remaliah cinhintku, Israel wicaśtayatapi lin, hena Jerusalem ekta hipi, azuyapi kta, tuka he ktedan okihipi śni.

2 Hehan, Aram Ephraim takodaya ce, Dawid tiyohnaka kin hecen eya okiyakapi. Unkan iye cante śkanśkan, nakun taoyate cantepi kin, contanka er can tateyanpa on kahuhuzapi iyececa.

3 Unkan Jehowa Isaya heciya; Wanna ekta ya wo, qa Ahaz itkokipa wo, niye qa nicinkśi Sear-Jaśub kici, wankan wapa mde kagapi wakpa iyahde ihanke kin ekta, wayujaja tamaga canku kin ohna :

4 Qa heyakiye kta; Iwanihdake ça inina un wo; kopehda śni qa cante wankadan śni wo; petuspe sonya un sinte kin dena oza on, Rezin Aram kici qa Remaliah cinhintku tocanniye ide kin on :

5 Aram śicaya waniciconza kin heon; nakun Ephrain qa Remaliah cinhintku lin hena heyapi ; 6 Juda ekta unyanpi qa nagiyeunyanpi kta, qa cokaya wicaśtayatapi wan Tabeal cinhintku kin hee, eunhdepi kta ce, eyapi :

7 Heon etanhan Jehowa I tancan kin he hecen eya; He ecen he kte śni, qa ecetu kte śni.

8 Aram pa kin he Damasek, qa Damasek pa kin Rezin hee; nakun waniyetu wikcemna śakpe sanpa zaptan iyotpani Ephraim yuksaksapi kta, hecen oyate kte śni.

9 Nakun Ephraim pa kin Samaria hee; qa Samaria pa kin 
Remaliah cinhintku kin hee: Wicayadapi śni kinhan niyusutapi kte śni.

10 Nakun Jehowa ie ca Ahaz heciya ;

11 Niye on Jehowa Wakantanka nitawa wowakta wan kida wo; kuyatanhan qa iś wankantanhan he kida wo.

12 Tuka Ahaz heya; Wakida li te śni, qa nakun Jehowa iyutanwaye lite śni ce.

13 Unkan heya ; Dawid tiyohnaka, wanna nakon po ; wicaśta akantu ikokiśinyayapi kin le tiku cistimna, tuka nakun mita Wakantanka iyokiśinyayapi kta he.

14 Heon etanhan Itancan kin iye linca wowakta wan nicupi lita: Iho, wikośka wan ihduśalie ca cinca ton; unkan Emmanuel-Wakantanka unkicipi uncya caśkiton kta.

15 Pteasanpi ilddi canhanpi tiktica ko yute kta, taku śica akta śni qa taku waśte kahniga sdonye kta heon.

16 Hokśiyoqopa kin he taku śica akta śni, qa taku waśte kahniga sdonye kta itokam, wicaśtayatapi nom kowicayakipe cin makoce tawapi kin he hewoskan elipeyapi kta.

17 Jehowa, niye en, qa nitaoyate en, qa niyate ti kin en, an. petu tohinni u śni kin heca au kta, anpetu en Fphraim Juda ayuśtan qon hetanhan ; $\Lambda$ śur wicaśtayatapi kin hee kta.

18 Unkan anpetu kin he en kalietu kte, honagidan Egupta wakpa ihanke en un kin he Jehowa kijojo kta, qa tulimaga Aśur maknce en un kin he nakun.

19 Unkan hena upi kta, qa iyu- lipa hewoskan kaksiza lin en ounye kta, qa imnija obdoka kin ohna, qa wapepeka ojupi owa- sin en, qa owihankepi iyulipa akan.

20 Anpetu kin he en, isan pe hinea opetonpi kin, wakpa kin akotanhan lin hena, Aśur wicaśtayatapi kin hee or, Itancan kin pa kasan kta, qa siha kin koya; qa he putinhin nakun eyaku lita.

21 Unkan anpetu kin he en kaketu kte ; wicaśta wan pteheyuktanna wanji qa tawiyedan nom icahwicaye kta:

22 Unkan asanpi ota qupi kin on asanpi ihdi hdute kta; tona makoce cokaya okaptapi kin owasin pteasanpi ihdi canhanpi tiktica ko yutapi ece kta nakaeś

23 Unkan anpetu kin he en kaketu kte, makocajeyate owasin tukten mazaska kektopawinge on hastanhanka iyuwi he kektopawinge yukan tuka qon, hen wapepeka can pepe ko yuke kta. 24 Wanhinkpe itazipa ko hduha en hipi kta; makoce owancaya wapepeka can pepe ko yuke kta heon etanhan.

$25 \mathrm{Qa}$ paha owasin magicamna on qapi qon hen wapepeka can pepe ko kokipapi on ckta de kte śii : tuka lien tatanka awicayapi kta, qa tahinca wanunyanpi amanipi kta.

\section{WICOWOYAKE 8.}

1 Unkan Jehowa hemakiya; Tahasaka tanka wan ikikcu wo, qa wowapi icage wicaśta tawa on, Maher-śalal-haśbaz, (W opate inahnikiya wapata inalini) eya en owa wo.

2 Unkan waayatanin warinycpica iwicawelicu, Uriah wawayuśna lin he qa Zokariah Jeberakiah cinhintku:

3 Qa winolinca wawiyukcan kin he ikiyedan wai ; unlian 


\section{ISAYA TAWOYUKCAN KIN,}

ihduśaka qa cinca ton. Hehan Jehowa hemakiya; Maher-śalalhaśbaz, eya caśkiton wo.

4 Hokśiyopa kin he hinahin, Ate qa ina, eya hoyeya onspe śni itokam, Damasek torvijice qa Samaria tawahpaya kin hena yutokan icupi kta, Aśur wicaśtayatapi ite kin itokam.

5 Unkan ake Jehowa omakiye ca heya ;

6 Oyate kin de Shiloa mini iwahnana iyaye cin wahtedaśni, qa Rezin qa Remaliah cinhintku kin on iyuśkin ;

7 Heon etanhan, iho, Itancan kin wakpa wan mini waśaka qa ota en awicau; unkan kaIrsiza owasin ohna hiyu kta, qa maya apaśbog ye kta :

8 Qa Juda ehna ye kta; apaśbog hiyu qa akam iyaye kta, dote kin hehanyan hiyohi kta: qa tahupahu akamdage kta, Emmanuel, nitamakoce ohdakinyan ojudan kta.

9 Oyate yaunpi, dakonkiciya po; unkan nicamdecapi kta : Makoce tehantu owasin, anagoptan po; ipiyagkiton miye; kinhan nicamdecapi kta; ipiyagkiton po, unkan nicamdecapi kta ce.

10 Woawacin awacin po, unkan yujujupi kta; wicoie wan eya po, unkan ecen he kte śni: Wakantanka unkicipi un nakaeś.

11 Jehowa nape waśaka wan on decen emakiya, qa oyate kin de tacanku kin obna mawani kte śni e onspemakiya qa heya ;

12 Oyate kin de tona, odakota eciyapi kin owasin, odakota eyakyyapi kte śni; qa iye taku kokipapi kin he koyakipapi kte śni, ąa kopeyahdapi kte śni.

13 Jehowa ozuye tawa iye hinca daowotanpi kta; qa iye hinca wowitonpe nitawapi kta, qa iye hinca wokokipe nitawapi ee kta. 14. Unkan iye wowinape kin heca kta: tuka Israel tiyohnaka napin on inyan ibotopi qa imnija on hicahanpi ee kta, Jerusalem en tipi kin hena on wohmunke qa mazahitakiyapi heca kta.

15 Unkan hena wicota hicahahanpi qa hinhpayapi kta, qa wicakamdecapi kta, qa wicahmunkapi kta, qa wicayuzapi kta.

16 Woyaotanin kin pahta qa woope kin apuspa wo, waonspewicawakiye cin hena en.

17 Unkan Jehowa Jakob tiyohnaka on ite anakilibe cin he miye awakipe kta, qa he ekta ewatonwe kta.

18 Wanyaka wo, miye qa micinca Jehowa maqu kin Israel en wowakta qa wowapetokeca unyakonpi, Jehowa ozuye tawa Zion paha en ti kin he eciyatanhan.

19 Unkan tohan, Tona hamdohdag iapi qa tona wahanicidapi jijipi qa wapiya dowanpi kin hena owicade po, eniciyapi kinhan;-oyate wan Taku Wakan tawapi kin akitapi kte śni he; tona nipi on tona tapi kin owicadepi kta he.

20 Woope qa woyaotanin kin akita po; wicoie kin de iyecen iapi śni kinhan, iyoyanpa takudan en wicaun śni heon etanhan. 21 Unkan he ohna iyotanhan iyekiyapi qa wotektehda yapi kta; qa tohan wotehdapi kinhan śinhdaiçiyapi lkta, nakun wicaśtayatapi qa Taku Wakan tawapi wicahdaśicapi kta, qa wankan etonwanpi kta.

22 Nakun maka kin ekta etonwanpi kta, unkan, iho, wonihinciye qa otpaza ; otpaza, woiyokiśica; tuka otpaza kin he yutokan iyeyapi kta. 
WICOWOYAKE 9.

1 Hececa eśta iyokiśinyapi kin en tokaheya otpaza qon iyecece śni, Zebulon makoce qa Naphtali makoce iwaśtedan lkakiśye ciqon he ehan; tuka iyohakam mde kahda, Jordan akasanpatanhan, Galile Ikcewicaśta ounyanpi en nina iyokiśinya.

2 Oyate otpaza en manipi qon hena iyoyanpa tanka wan wanyakapi ; wiconte ohanzi makoce en ounyanpi kin hena iyoyanpa awicahinanpa.

3 Oyate kin duota; wowiyuśkin kin yeciyutanka; woksapi wowiyuśkin kin ohna nitokam wiyuśkinpi ; woyuha kicipamnipi on cante waśtepi kin he iyececa.

4 Can napinpi napin kin he, qa iye tapete cansakadan, qa wowidagye cansagye tawa kin hena duksaksa, Midian anpetu kin en iyececa.

5 Zuye cin otoiyohi kicizapi owodutaton, qa wokoyake we en oputkanpi kin en hanpokihan ece ; qa hulinagapi kta, peta tawote kin hee.

6 Hokśiyoqopa wan unkicitonpi, cinca wan unqupi: unkan wokiconze kin he iye tapete akan yanke kta; qa caje kin, Wawinihan, Wawiciya, Taku Wakan Iyotan Waśaka, Ateyapi Owihanke Wanica, Wookiye Wicaśtayatapi kin eciyapi kta.

y Tokiconze oicage qa wookiye kin he owihanke wanice kta, Dawid oiyotanke tawa akan, qa tokiconze akan, ecen ehde kta, qa woyaco woowotanna ko on hdusuta kta, detanhan qa owihanke wanica: Jehowa ozuye hduha he tomniheca kin he de econ lita.

8 Itancan kin wicoie wan Ja- kob en yeśi, unkan he Israel ahinlipaya.

9 Unkan oyate owasin sdonyapi kta, Ephraim qa Samaria en tipi kin heepi kaeś, hena cante walianicida qa suta kin on heyapi ;

10 Maka śpanyanpi kin hena hinhpayapi, tuka inyan kakanpi on tipi unkagapi kta; sukamin can lin hena kawankapi, tuka hanteśa eeunkiyapi kta ce.

11 Heon etanhan Jehowa tona Rezin śicedakapi kin hena iye itkokim hiyuwicaye kta, qa tokawicaye cin hena wicayuwitaya kta :

12 Aram oyate wiyohiyanpatanhan, qa Philistia oyate wiyohpeyatanhan unpi; qa hena i ocowasin on Israel temyapi kta. Hececa eśta iye tocanniye kin ayuhomnipi śni, tuka hinyahin nape hdugan un.

13 Oyate kin tuwe wicakaśtake cin ekta ihduhomnipi śni, qa Jehowa ozuye yuhe cin he akitapi śni.

14 Heon etanhan, anpetu wanjidan en, Jehowa Israel etanhan pa sinte ko bakse kta, adetka qa psa hu kin nakun.

15 Hunkayapi qa wicaśtayatapi kin he pa kin ee; qa wicaśta wokcan woitonśni oyake cin he sinte kin ee.

16 Tona oyate kin wicayawaśtepi kin hena nuniwicayapi ; qa tona hena on yawaśtepi kin hena napca iyewicayapi.

17 Heon etanhan kośka wicayuhapi kin on Itancan kin wiyuśkin kte śni; qa wamdenica wiwazica ko wicayuhapi kin hena onśi wicada kte śni : otoiyohi wayuśica qa taku śica econpi ; qa wicai otoiyohi witkotkoya ia ece. Hena owasin on iye tocanniye kin etanhan yuhomnipi 
śni, tuka hinyahin nape hdugan un.

18 Wicośice peta iyecen itkon; wapepeka campepe ko huhnage kta, qa contanka otehi kin ohna ide kta, hecen śota wankan ye cin en wankan kahbog yapi kta.

19 Jehowa ozuye yuha tosinlida kin on maka kin aotpasyapi, qa oyate kin peta tawote kin iyecece kta ; tuwedan iye sunkaku kin itonkipe kte śni.

20 Unkan etapa eciyatanhan wicaki kta, tuka wotelktehda kta; qa catkatanhan wote kta, tuka hena imnanpi kte śni: wicaśta otoiyohi iye isto conica hdutapi kta :

2 L Manassa Ephraim, qa Ephraim Manassa; napin witaya Iuda anatanpi. Hececa eśta iye tocanniye kin yuhomnipi śni, tuka hinyahin nape hdugan un.

\section{WICOWOYAKE 10.}

1 Tona woyaco owotanna śni yacopi, qa wowapi kagapi tona wokakiśye owapi kin hena wotehi wicakiciyanka nunwe :

2 Wokonze etanhan wahipanica kin wicayuhomnipi kta, qa mitaoyate onśikapi woowotanna ewicakiciyakupi kta, wiwazica wamawicakinonpi kta, qa wamdenica wawicakipi kta, heon etanhan.

3 Hecen wokakiśye anpetu kin en taku ecanonpi kta he, qa woihangye tehantanhan au kte cinhan, waokiyapi on tuwe ekta nayajicapi kta he; qa nitowitanpi kin tukten eyahnakapi kta he. 4 Miye codan wayaka unpi kin ihukuya canpeśka inakehde inajinpi kta, qa wicaktepi kin wicihukuya hinhpayapi kta. Hececa eśta iye tocanniye kin yuho200 mnipi śni, tuka hinyaliin nape hdugan un.

5 Hehehe, Aśur, mitocanniye icapsinte, qa cansakadan iye napepi ohna wośinhda mitawa kin hee.

$6 \mathrm{He}$ oyate wahnayesa wan takpe yewaśi kta, qa oyate canniyewicawaye cin hena on taku econ waśi kta, wawicaki qa tawahpaya icu kta, qa canku upśija iyecen awicamani kta.

7 Hececa eśta iye hecen le kte śni, qa cante mahen hecen awacin kte śni ; tuka waihangyapi iye cante kin en un, qa oyate tonana śni wicakaśpe kta.

8 He kaken eya; Akicita tancan mitawa kin hena ocowasin wicaśtayatapi śni lie.

9 Kalno he Karliemiś iyecece śni he; Hamath Arpad iyecece śni he ; Samaria Damasek iyecece śni he:

10 Oyate takuśni śni wakandapi, qa wakagapi tawapi, Jerusalem qa Samaria tawa kapapi qon hena minape iyowicahi kin heon,

11 Samaria qa taku wakan tawa token ecawicawecon he iyecen Jerusalem qa taku wakan tawa kin ecawicawecon kte śni he, eya.

12 Heon etanhan kaketu kte, Itancan kin tohan Zion lie kin en, qa Jerusalem en, ocowasin hduśtan kinhan, hehan Aśur wicaśtayatapi cante wahanicida waskuyeca kin he towecicon lita, qa iye iśta wankantu witantan kin.

$13 \mathrm{He}$ kaken eya ; Minape waśake cin on hecamon, qa mitoksape on, wamdupika nakaeś: nakun oyate makoce icagopi kin mdutokan, qa towijicepi wicawaki, qa tuwe waśaka iyececa, en ounyanpi kin hena iwicawadaka: 
14 Qa wahohpi wan iyecen minape oyate towijicepi kin iyeya : qa wanji witka okaptapi pahi kin iyecen malra kin ocowasin mnawaye : unkan takudan hupahu hduśkanśkan śni, qa i hdukawa śni, qa hoton śni ce, eya.

15 Onspe kin he tuwe on wakakan kin iwankam wahaniçida kta he; canibakse kin he tuwe kaśkanśkan kin isanpa watankaicida kta he: cansakadan wan tona he yuwankan yuzapi kin wicitokam ihduśkanśkan he iyececa: cansagye kin, taku can śni iyecen bosdan eihde kte cin he iy ececa.

16 Heon etanhan Itancan kin, ozuye Itancan kin, tona cepapi wicayuhe cin en wicotamaheca ye śi kta, qa iye towitan kin ihukuya ide wan ideye kta, peta ide iyececa.

17 Unkan Israel Toiyoyanpa kin he peta heca kta, qa iye Towakan kin he ide kin ee kta; qa tanpetu wanjidan en wapepeka tawa canpepe koya hulnage ça awihnuni kta :

18 Nakun contanka tawa wowitan, qa iye tamaga waśte kin yusote kta, nagi tancan koya: qa wayazanka wan hanye cin he iyecece kta.

19 Qa contanka tawa can okaptapi kin hena tonana kta, hecen hokśiy opa wan hena ow okihikta.

20 Unkan anpetu kin. he en kaketu kte, tona Israel etanhan okaptapi, qa Jacob ti kin etanhan tona niyanpi kin hena tuwe wicakaśtake cin he icimana wacinyanpi kte śni; tuka Jehowa Israel Towakan kin he awicakehan wacinyanpi kta.

21 Tona okaptapi,. Jakob etanhan owicakaptapi kin hena Taku Wakan Iyotan Waśaka en ihdoupi kta.
22 Israel, nitaoyate miniwanca wiyaka kin iyececa qeyaś, tonana okaptapi kin hena hdipi kta: woihangye konzapi qon he woowotanna on iyatahde kta.

23 Woihangye wan, qa taku konzapi kin he, makoce ocowasin cokaya, Itancan Jehowa ozuye yuhe cin he iś econ kta.

24 Heon etanhan Jehowa ozuye Itancan kir kaken eya; Mitaoyate Zion en ounye cin, Aśur kokipe śni wo: cansakadan on anipe-1rta, qa Egupta ekta econpi qon iyecen niye en cansagye hduwankan icu kta;

25 Tuka ehake cistiyedan hehan wośihda kin henakeca kta, qa mitocanniye tona ihangyapi kin hena en un kta.

26 Unkan Jehowa ozuye yuhe cin he icapsinte wan icalkiciye kta, Midian Oreb imnija ekta wicaktepi qon iyececa; qa cansakadan tawa Mdeśa akan, qa Egupta ekta econ qon he iyecen hduwankan icu kta.

27 Unkan anpetu kin he en kaketu kte, woqin tawa nitapete etanhan eyakupi kta, qa cannapinpi tawa nitahu etanhan ; unkan sdayapi kin on cannapinpi kin yuwegapi kta.

28 Ayath en hi: Migron ekta iyoopte iyaya; Mikmaś en tawahpaya kilinaka;

29 Ire hdoka kin ohna iyayapi; Geba ekta ewankapi : Rama kopehda; Gibeah Saul najica.

30 Gallim cunwintku, ho wankan iyekiya wo: Anathoth onśika, Laśa hehanyan ho hdutanin wo.

31 Madmena najica iyaya : Gebim en ounyanpi kin hena nape kta ihduwiyeyapi.

32 Tuka anpetu kin he Nob en owanji yanke kta; Zion cun201 


\section{ISAYA TAWOYUKCAN KIN,}

wintku he tawa, Jerusalem Paha kin ektakiya nape koze kta.

33 Iho, Itancan Jehowa ozuye yuha wokokipe on adetka kin kaśpe kta: qa tona hanska icagapi kin kaksapi kta, qa wahanicidapi kin hena yuhukun iyeyapi kta. 34 Unkan contanka can kin mazasapa on kawanke kta, qa Lebanon wanji waśaka on hinhpaye kta.

\section{WICOWOYAKE 11.}

1 Unkan Jesse canhute kin etanlıan cansakadan wan hinanpe kta, qa tahutkan etanhan adetka wan icage kta;

2 Qa Jeho:va Taniya kin iye akan yanke $\mathrm{kta}$, woksape qa wookahinige woniya, wowiyukcan qa wowaśake woniya, wosdonye qa Jehowa kokipapi woniya kin he:

3 Unkan he Jehowa kokipapi kin en wakcanyan wayupika kage kta; iye iśta wanyake cin eciyatanhan wayaco kte śni, qa iye noge nakon kin eciyatanhan wiyukcan kte śni :

4 Tuka woowotanna on wicaśta onśika wicayaco kta, qa wolronze on wakinica kta, maka akan wahpanicapi kin hena on etanhan; qa iye $i$ icapsinte on maka kin ape kta, qa iye iha oniya kin on wicaśta śica wicakte kta.

5 Unkan woowotanna kin iye nite ipiyake kin hee kta, qa wowicake ipiyagkiton kta.

6 Nakun sunktokeca kin he tahinca wanunyanpi cinca kin kici ti kta, qa inmuhdeśka kin he tacinca kin kici iwanke kta; qa tatankadan qa mnaja kośka ptejicadan koya witaya unpi kta; unkan hokśidan cistinna wan hena yus awicaye kta.
1 Pte kin nakun qa walianksica kin wihanpi kta; hena cincapi kin witaya makata iwankapi lita; qa mnaja kin he tatanka iyecen aguyapi hu yute kta.

8 Unkan kokśiyoqopa azin kin he sintehda oti kin akan ślkate kta, qa hokśiyopa azin enakiye cin he wamduśka śica ti kin akan nape yekiye kta.

9 He wakan mitawa kin owancaya wakiunniyanpi kte śni qa waihangyapi kte śni : miniwanca mini akahpe cin, he iyecen maka kin Jehowa sdonyapi kin on ojudan kta nakaeś.

10 Unkan anpetu kin he en Jesse hutkan oicage wan yuke kta, he oyate kin en wiyokihedan he kta; iye ekta Ikcewicaśta kin ode upi kta: unkan iye ozikiye tawa kin he wowitan kta.

11 Nakun anpetu kin he en kaketu kte, Itancan kin ake icinonpa nape yeye kta, tona taoyate okaptapi kin opewicakiton kta, tona owicakaptapi kin hena, Aśur etanhan, qa Egupta etanhan, qa Pathros etanhan, qa Kuś e tanhan, qa Elam etanhan, qa Sinar etanhan, qa Hamath etanhan, qa miniwanca wita kin etanhan.

12 Qa Ikcewicaśta wiyokihedan wan ewicakihde kta, qa Israel etanhan heyata iyewicayapi qon hena wicayuwitaya kta, qa Juda etanhan enanakiya iyayapi maka tate ouye topa kin etanhan wicapahi kta.

13 Unkan Ephraim tawowinawizi kin ayuśtan kta, qa Juda tokayapi kin hena wicakaśpapi kta; Ephraim Juda inakiwizi late śni, qa Iuda Ephraim nagiyeye kte śni.

14 Tuka Philistim hinyetepi akan wiyohpeyatakiya kinyanpi 
kta; wiyohiyanpata cinca kin witaya wawicakipi kta; hena Edom qa Moab nape awicahnakapi kta; qa Amon cinca kin anawicagoptanpi kta.

15 Unkan Jehowa Egupta mde ceji kin ocowasin ihangye kta; qa tate waśaka tawa on wakpa kin en nape koza kta, qa adetka śakowin kin en ape kta, qa hanpokiton opta maniwicakiye kta.

16 Unkan iye taoyate okaptapi, Aśur etanhan owicakaptapi kin hena, canku tanka wan yuhapi kta; Israel Egupta makoce etanhan hinanpe cin anpetu qon he iyececa.

\section{WICOWOYAKE 12.}

1 Unkan anpetu kin he en hecen ehe kta; Jehowa, ciyatan kta, canniyemayaya qeyaś nitocanniye yutokan iyeyapi, qa cantohnag mayaya.

2 Iho, Taku Wakan nimakiye cin hee: he wacinwaye kta qa kopewahda kte śni : mitowaśake qa mitadowan kin Jehowa Iyotan Waśake cin hee; nakun iye Wanikiya mitawa kte cin hee.

3 Heon etanhan wowiyuśkin on wanikiyapi miniyowe kin etanhan mini edakupi kta.

4 Nakun anpetu kin he en hecen ehapi kta; Jehowa yatan miye, iye caje kin hoyekiya po, iye olian kin oyate ehna oyaka po, iye caje kin wankan un ce, eya po:

5 Jehowa idowan miye; taku wiciyokipi econ kin heon; maka kin owancaya de sdonyapi ce.

6 Zion en ounye cin hoyeye ça iyaśa wo ; Wanji Wakan Israel tawa nicokaya un kin he tanka hinca, heon etanhan.
WICOWOYAKE 13.

1 Babel tawoyake kin, Isaya Amoz cinhintku wanyake ciqon. 2 IIe tehanwankantu kin akan wiyokihedan wan ehde po, hena hoyewicakiya miye, nape koza po, hecen akicita tancanpi tiyopa tawa kin ohna yapi kta.

3 Wicaśta wakan mitawa wanna econ wicawaśi, nakun mitocanniye on wicaśta waśaka mitawa wicaweco, tona mitawootanin on iyuśkinpi kin hena.

4 Ḣe kin elta wicota hotaninpi, oyate ota iyececa; oyate wokiconze yuwitaya owodutatonyan hotaninpi : kicizapi kta on Jehowa ozuye tawa ozuye kin owicawa.

5 Makoce tehan tanhan upi, mahpiya ihanke kin etanhan, Jehowa qa iye tocanniye wotawe kin hena, makoce ocowasin ihangye kta heon etarhan.

6 Ceya miye; Jehowa taanpetu kin kiyedan ihan; Iyotan Waśake cin etanhan woihangye wan iyecen u kte.

7 Heon etanhan wicanape iyuhpa hinhpayapi kta, qa wicaśta otoiyohi cante skan kta :

8 Qa kokipapi kta; wonihineiye qa woiyokiśice wicayuze kta; wanji cinca ton kte cin iyecen wayazankapi kta: wicaśta iye takodaku kici nihinciyapi kta; iye itepi kin ide ite kin hececapi kta.

9 Tho, Jehowa taanpetu kin u ce, wośinhda qa wocanniye ide kin on wohitika, hecen makoce kin hewoskan kage kta ; qa etanhan walitanipi sa ihangwicaye kta.

10 Mahpiya wicanhipi qa wicanlipi tankapi kin hena iyoyanpa hiyuyapi kte śni; anpetu wi kin hinanpa eca aotpasyapi kta, 203 


\section{ISAYA TAWOYUKCAN KIN,}

qa hanyetu wi kin iś taiyoyanpa iyeliye kte śni.

11 Unkan wośice on ctanhan wicoicage kin śitkihdawaye kta, qa wicaśta śica iye wahtanipi kin on: nakun wicaśta wahanicida towohitike kin he ayuśtanwakiye kta, qa wicaśta wohitika towahanicidapi kin he kun ehpewaye kta.

12 Wicaśta wan mazaskazi isanpa tehika wakage kta; han, wicaśta wan Ophir mazaskazi telike cin isanpa kta.

13 Heon etanhan mahpiya kin mduśkanśkan kta, qa maka kin towanke kin etanhan śkanśkan iyaye kta, Jehowa ozuye yuha tocanniye kin on, qa tośinhda ohitika anpetu kin on.

14 Hecen tatokadan wan niya śni kuwapi, qa tahinca wanunyanpi tuwedan wicayuwitaya śni kin hc iyecece kta; wicaśta otoiyohi iye taoyate ekta hde kte, qa iye tamakoce lin ekta wicaśta otoiyohi najice kta.

15 Tuwe iyeyapi kinhan capapi kta, qa tuwe opeya un kin he mazasagye on ktepi kta.

16 Nakun iye iśta itokam iye cinca kin wicakicicahuhugapi kta; tipi tawapi kin yucokapi kta, qa tawicupi wicakikśanpi kta.

17 Iho, Made oyate kin anawicatan wicawakiye kta, liena e mazaska aktapi kte śni, qa mazaskazi on iyuśkinpi kte śni.

18 Unkan tinazipepi kin kośkapi kin makata elpewicayapi kta, qa wicatammi oicage cin onśidapi kte śni : iye iśtapi wicacinca wicapatanpi kte śni.

19 Hecen Babel wokiconze wiciyokipi, Kasdim wowitan oinpi kin he Wakantanka Sodom qa Gomorrah ihangye ciqon he iyecece kta.
$20 \mathrm{He}$ icimana otipi lite śni, qa wicoicage yeye cin hehanyan tuwedan en ounye kte śni : nakun Arabi wicaśta lin lien ticage kte śni; qa talinca wanunyanpi wonwicayapi kin hen wankapi kte śni.

21 Tuka wamanica kin hena hen wankapi kta; qa wicaśta otipi qon hena hinhankagapi on ojudan kta; qa kangi cinca kin hen tipi kta, qa canotidan kin hena hen wacipi kta.

22 Qa tipi tawapi yujujupi kin ohna wamanica hotonpi kta, ga tipi waśteśte qon ohna unktehi yuke lkta. Unkan he tawaniyetu kin kiyedan ihan, qa taanpetu kin yutehanpi kte śni.

\section{WICOWOYAKE 14.}

1 Jehowa Jakob onśikida kta, qa ehake Israel hdahnige kta, qa iye tamakoce kin ohna ewicahnake kta nakaeś : nakun oyate tokeca kin hena om witayapi kta, qa Jakob ti kin en kokijuyapi kta.

2 Unkan oyate kin iś hena iwicacupi qa tohepi kin en awicahipi kta: qa Israel tiyohnaka kin hcna wicaśta taokiye qa winyan taokiye wicayuhapi kta, Jehowa tamakoce kin eń qa tona iye wayaka wicayuhapi qon hena iś wayaka iwicacupi kta; qa wowidagwicayapi qon hena iś iwicadakapi kta.

3 Unkan anpetu kin he en, nitoiyokiśice etanhan, qa nitokokipe etanhan, qa wowidagyapi tehike en inidakapi qon etanhan Jchowa woozikiye nicu kta:

4 Hehan Babel wicaśtayatapi lkin on wicoie kin de iyacu kta, qa hecen ehe lita; Wawicakiśye cin he ayuśtan, wawicakiśyapi kin he ayuśtanpi ce. 
5 Jehowa wicaśta śica cansag-. ye tawa kin he yulsaksa, wicaśtayatapi tasagye kin.

6 'Tuwe wośinhda on oyate ayuśtan śni awicape ciqon, tuwe wocanniye on Ikcewicaśta i wicadake cin he śicaya kuwapi, qa tuwedan tehinda śni.

7 Maka kin ocowasin ozikiya un, owanji yanke: hena dowarlwan hinhdapi.

8 Han, niye on śinta can iyuśkinpi, Lebanon hante śa kin ; Inunka ehantanhan cankaksa tuwedan unkiyepi en unkupi śni ce, eyapi.

9 Kuyatanhan niye on Hades ihduśkanśkan, yau kin itkonicipe kta: niye on wicaśta tapi kin hena wicayuhica, maka akan mdetahunkapi owasin : oyate wicaśtayatapi owasin iye tohepi etanhan najinwicakiya.

10 Hena iyuhpa waayuptapi qa heniciyapi kta; Niś eya waunśakapi śni iyeccn inicaga he; unyakonpi kin iyeniceca he.

11 Nitowitan kin he Hades ekta kun ahipi, candowankiyapi nitawa ho lin: nibukuya wamdudan owinśyapi, qa woakahpe nitawa wamduśka kin hena ee.

12 Wicanlipi iyega, anpao cinca kir, mahpiya eciyatanhan nihinlipaya ; oyate wicaduwaśake śni qon niye qe makata nicawankapi.

13 Tuka niye nicante en hehe ciqon; Malipiya ekta wankan mde kta ; mitoyanke kin wicanlipi Taku Wakan tawa kin iwankam ewehde kta; nakun omniciye paha kin en waziyata ihanke kin ekta imdotanke kta; 14 Mahpiya śapa wankantu kin isanpa imdamde kta; Iyotan Wankan un kin iyemacece kta ce, eha.
15 Hececa eśta Hades ekta kun elipeniyanpi kta, woha cetete kin ekta.

16 Tona wanniyakapi kin hena opanihtapi kta, iniyukcanpi kta; Wicaśta maka kin yuhubuza, wokiconze kin yuśkanśkan qon he dee se:

17 Makoce kin hewoskan kage ça otonwe en un kin hena ihangya; kaślkawicahnaka tipi wicakiyulidoke śni he dee he, eyapi kta.

18 Oyate wieaśtayatapi kin owasin, han iyulipa, wowitan ohna wankapi, otoiyohi iye ti kin ohna.

19 'Tuka niye qe niliapi śni tankan elipeniyanpi, adetka walite śni wan iyececa, wicaktepi tawokoyakc, mazasagye on capapi, inyan woha kin en elipeyapi kin he iyececa; wicaśta ța wanka amanipi kin iyececa.

20 Hena owicapeya nihapi kte śni ; nitamakoce hewoskan yecage nitaoyate wicayekte nakaeś. Sicaya econpi cinca kin tohinni wicayaotaninpi kte śni.

21 Iye cinca hunkakcwicayapi wahtanipi on wicaktepi wicakicaga miye: hecen inajinpi kte śni, qa maka kin tawayapi kte śni, qa makoce ite kin otonwe on ojuyapi kte śni.

22 Tuka miye qe hena itkom inawajin kta, Jehowa ozuye yuhe cin he hecen eya; qa Babel ctanhan wicacaje, qa wookapte, qa wicacinca, qa wicatakojakpa ko baweciśpe kta, Jehowa eya.

23 Nakun pahin en ounyanpi kta e wakage kta, qa psa mde hecakta : qa wicotakuni śni icahinte kin on wakakinte kta ce, Jehowa ozuye yuhe cin he hecen eya.

24 Jehowa ozuye tawa wakon205 


\section{ISAYA TAWOYUKCAN KIN,}

za on heya; Awicakehan token awacanmi qon he iyecetu kta; qa token wakonze cin he ecetu lita:

25 Makoce mitawa kin ohna Aśur wakamdece kta, qa amawani kta, he mitawa akan: hehan hena etanhan cannapinpi tawa kin tokan iyaye kta, woqin tawa iye tapetepi etanhan tokan ye kta.

26 Wokonze maka kin owancaya wakonzapi kin he dee : qa oyate owasin nape ayukatinpi lin he dee.

27 Jehowa ozuye tawa he wakonza, unkan tuwe ecetuśniye kta he; qa iye nape hdukatin, unkan tuwe he pakam iyeye kta he.

28 Ahaz wicaśtayatapi ta waniyetu kin he en woyake kin he dee.

29 Palestina ocowasin, tuwe anipe icapsinte tawa kawegapi on iyuśkin śni wo; wamduśka oicage etanhan sintehda wan hinanpe kta, qa he cinca kin peta wamduśka kinyan un kin heca kta e heon.

30 Unkan onśikapi cinca tokapapi kin hena-wotapi kta, qa wahpanicapi kin wikopeśniyan wankapi kta: tuka miye wicaakihan on hutkan nitawa wakte kta; qa iye tona onicaptapi kin hena wicakte kta.

31 Tiyopa, śicahowaya wo ; otonwe, hotanin wo; Palestina ocowasin, niye niskan: waziyatanhan śota uye kta, qa tuwedan ozuye tawa etanhan najice kte śni.

32 Hehan oyate wahośi uwicasi kin hena token awicayuptapi kta he. Jehowa Zion sutaya ekihde, hecen iye taoyate onśikapi kin hena he wowinape yapi kta.

\section{WICOWOYAKE 15}

1 Moab tawoyake kin. Hanyetu icunhan Ar-Moab ihangyapi, kaśpapi heon: Hanyetu kin en Kir-Moab ihangyapi, kaśpapi heon etanhan.

2 Dibon tipi, owanka tehanwankantu ekta ceye kta wankan iyayapi: Nebo qa Madeba akan Moab śicahowaye kta; wicapa iyuhpa nasuśdapi, qa putinhin otoiyohi baksapi.

3 Iye tacankupi ohna wakihdaka ipiyagkitonpi kta; iye ti akahpapi akan qa tihocoka ohna otoiyohi śicahowaye kta, ceyaya kun kupi kta.

4. Hecen Heśbon ceye kta qa Elealeh; Jahaz hehanyan hohdataninpi kta: heon etanhan Moab taakicita śicahowayapi kta ; iye nagi tehikekida kta.

5 Moab on micante śicahowaye kta: tawonajice kin Zoar iyahdeyapi, pte wan wanna waniyetu yamni: Luhith ekta wankan yapi kin ohna itanwankanhde ceya yapi kta ; Horonaim canku iyahde ohna woihangye woceye yataninpi kta.

6 Nimrim mini kin liopuza wanke kta; peji kin śnija, wato wanin aya, taku toto wanice hinca.

7 Heon etanhan taku okapta hduhapi, qa taku kihnakapi kin hena coliwanjica kaksiza lkin ekta hdoyapi kta.

8 Waśicahowayapi kin he Moab ihanke kin ohomni iyaya; woceye kin he EgIaim ekta, han, woceye kin he Elim minilidoka ekta iyohi.

9 Dimon mini kin we ojudan kta: Dimon en akihdewaye kta; tuwe Moab etanhan najice cinhan mnaja en awau kta, qa tona makoce en okaptapi kin hena. 


\section{WICOWOYAKE 16.}

1 Makoce wicaśtayatapi kin tacinca wan kaya po, Sela etanban hewoskantu hehanyan, Zion cunwintku Paha tawa kin ekta.

2 Unkan kaketu kte, zitkadan wan, hohpi kiyujujupi kinban, nuni kin he iyecen Moab cunwintkupi Arnon iyuwegapi kin ekta unpi kta.

3 Wiyukcan miye, woowotanna econ po; ohanzi nitawa wiyotanhan colkaya hanyetu iyecen kaga wo; heyata iyewicayapi kiin hena nawicahman wo; tuwe nuni kin he hnaye śni wo.

4 Moab, heyata iyewicayapi mitawa kin hena niye kici ounyewicakiya wo; waihangya ite kin etanhan niye hena awicakahpa yanka wo: waśitkihdaye cin he owihanke, waihangye cin he ayuśtan, waanatanpi kin hena makoce kin etanhan atakunipiśni ce.

5 Unkan wowaonsida on oyanke kin sutaya ehdepi kta; qa iye wowicake on akan iyotanke kta, Dawid tawakeya ohna wayaco yanke, wokonze akite ça woowotanna yuinahni kta.

6 Moab towalianicida kin he naunlionpi, nina wahianicida nakaeś; wotankaicida tawa qa towahanicida qa tośinhda kin hena: tuka woitonśni tawa kin iyecetu kte śni.

7 Heon etanhan Moab Moab on sicahowaye kta, ocowasin waśicahowaye kta: Kirhareś oahde kin on yaceyapi kta, awicakehan kaśtakapi nakaeś.

8 Heśbon tamaga kin śnija aya, qa Sibma hastanhanka iyuwi kin : adetka waśte icage cin hena Ikcewicaśta itancanpi kaksapi ; Yazer hehanyan iyohi, tintoskan onunipi : adetka tawa han- ska icaga mini kin akasanpa iyaya.

9 Heon etanhan Yazer tawoceye on Sibma hastanhanka iyuwi kin awaceye kta: Heśbon, Elealeh kici, iśtamnihanpe mitawa on amagajuciye kta : woceye wan nitawaskuyeca qa nitawojupi kin ahinhpaya heon.

10 Nakun maga waśte kin etanhan wowiyuśkin qa wopida eyakupi : hecen hastanhanka ojupi kin en dowanpi wanice kta, nakun iyaśapi yuke kte śni: wanapanpi kin iyuśkice ohna miniśa takudan naśkicapi kte śni : iyaśapi kin ayuśtan wakiya. 11 Heon etanhan mitezi Moab on, candowankiyapi wan iyecen houye kta, qa mitarmmahentanhan Kirhareś on.

12 Unkan kaketu kte, tohan Moab oyanke tehanwankantu ohna mdokita ihdutanin kinhan, hehan iye tawakeya wakan kin ekta cekiya hi kta; tuka ohiye kte śni.

13 'Tanihan wicoie Jehowa Moab eciye ciqon he dee.

14 Tuka nakaha Jehowa ie ca hecen eya; Waniyetu yamni iyokpani, tuwe" opetonpi tawaniyetu iyececa, hehan Moab towitan kin he takudapi kte śni, wicota tanka hince cin he nakun : unkan wookapte kin he tonana hince kta, ota sni.

\section{WICOWOYAKE $1 \%$.}

1 Damasek tawoyake kin. Tho, otonwe kin etanhan Damasek ihangyapi; hecen otiwita heca kta.

2 Aroer otonwe kin hena ehpeyapi; hen wanunyanpi optaye yuke kta, en wankapi kta, qa tuwedan wakokipe wicakicage lite śni. 
3 Conkaśke kin Ephraim etanhan yutokan iyeyapi kta, qa wokiconze hin Damasek etanhan, qa Aram okaptapi kin etanhan: hena Israel cinca towitan kin iyececapi kta, Jehowa ozuye yuhe cin he hecen eya.

4 Unkan anpetu kin he en Jakob towitan kin waśake śni aye $k t a$, qa iye celipi cepe cin he tamaheca aye kta.

$5 \mathrm{Qa}$ wicasta wamnayan aguyapi hu mnayan, qa isto on wahuwapa pahi ece kin he iyecece kta; nakun Rephaim kaksiza en wahuwapa mnayanpi kin iyecece kita.

6 Tuka hastanhanka yuśnapi en okaptapi kta, Oliwe can kahnapi iyececa, waskuyeca, nonpa yamni adetka wankan oinkpa ekta, topa zaptan adetka waicaliya ece kin en, Jehowa Israel ta Wakantanka hecen eya.

7 Anpetu kin he en, wicaśta wan Waicahye tawa kin ekta ekitonwe kta, qa iye iśta Israel Wanji Wakan tawa kin ihakta lita.

8 Qa owayuśna iye nape kage cin hena ekta ekitonwe kte śni, ya iye napsukaza taku kage cin wanhdake kte śni, wakagapi qa nakun anpetu wi okagapi kin hena.

9 Anpetu kin he en iye totonwe sulssuta kin hena, adetka elipeyapi qa caninkpa tehanwankantu, Israel cinca on okaptapi qon he iyececapi kta: unkan hewoskantu kta.

10 Taku Wakan Wanikiya nitawa kin he ayektonja, qa Imnija nitowaśake kin he yeksuye śni, heon etanhan wojupi waśteśte oyaju lata, qa ehna can tokeca huyahde kta:

11 Anpetu icunhan taku huya208 hde kin he icaliyakiye kta, qa hanhanna eca woyaju kin hcayakiye kta : tuka anpetu tawayapi kte cin en womnayan kin he kinyan iyaye kta, qa woiyokiśice tehike yuke kta.

12 Hehelie, ayate ota wicota kin, miniwanca hoton kin iyecen houyapi: qa oyate owodutatonpi, mini ota ow odutaton iyecen owodutaton kagapi :

13 Mini ota kokijuya iyaye cin iyecen oyate kin owodutatonyan kokijupi kta: tuka iye iyowicaki kte śni, unkan tehan napapi kta, qa he kin akan aguyapi ha tate itokam he iyecen napeyapi kta, qa ohmihma wan tateiyumni itokam he iyececa.

14 Unkan iho, htayetu eca wonihinciye; lianlianna itokam he en un śni. Tona waunkipi taku tawapi, qa tona unyapotapi taku yuhapi kte cin he dee.

\section{WICOWOYAKE 18.}

1 Hehehe, makoce hupahu on ahanziyapi, Kuś tawakpa kin akotanhan wanke :

2 He wahośiyapi miniwanca akan yewicaśi, han, psa wata ohna mini ite kin akan: Wahośiye dusduzahanpi, oyate wan hanskaska qa śicaya kuwapi ekta ya miye, oyate wan otoka. heya tanhan qa dehanyan wohitikapi ; oyate waśake hinca qa wanapan ece, wakpa kin makoce tawapi kicaksaksa :

3 Maka kin en ounyayapi qa makoce akan dukanpi kin owasin wanyaka po, tohan he akan wiyokihedan wan ehde kinhan; qa mazayahotonpi wan yahoton kinhan nahon po.

4 Jehowa kaken emakiya; 0 zimiçiye kta, qa mitowanka en awacin manke kta, wato akan 
amdakedan maśte kin iyececa, womnayan maśte kin en amahipiya cu kin iyecece.

5 Wojupi mnayanpi śni itokam, camni wanna icage ca wahica ohna hastanhanka skuye cin he suton aye kta, hehan isan śkopa on camni kin baśpe kta, qa nakurı adetka kin hena icu qa baksa ehpeye kta.

6 Hena witaya elipeyapi kta, he wakinyanpi qa maka woteca kin hena yuhapi kta: qa wakinyanpi kin hena amdoketupi kta, qa maka woteca iyulipa hena awaniyetupi kta.

7 Hehan woqupi wan Jehowa ozuye yuha liahipi lita, oyate wan hanskaska qa śicaya kuwapi, qa oyate otokaheya tanhan qa dehanyan wohitikapi, oyate waśake hinca qa wanawicapan ece, wakpa kin makoce tawapi kicaksaksa kin hena, Jehowa ozuye yuha caje oyanke kin en, Zion Paha kin ekta.

\section{WICOWOYAKE 19.}

1 Egupta tawoyake kin. Iho, Jchowa mahpiya śapa duzahan wan akan iyotanke ca Egupta en u kta. Unkan iye itokam Cogupta wakagapi tawa kin kena śkanśkanpi lita, qa cokayatanhan Egupta cante skan ikta.

2 Unkan Egupta wicaśta Egupta oyate icitkokim ewicawahde kta; hecen kicizapi kta, wicaśta otoiyohi iye sunkaku kici, qa wicaśta otoiyohi iye taliodaku lici ; otonwe otonwe kici, wokironze wolkiconze kici.

3 Unkan tanmahentanfan Egupta toksape kin atakuni kte śni, qa iye tawacin kin ecetuśniwaye kta: hecen wakagapi kin ekta ode yapi kta, qa tona jijipi, qa $* 14$ tona hanmdepi, qa tona wapiyapi hena awicakitapi kta.

4 Unkan Egupta oyate kin itancan wan ksizeca nape kin en wicawaqu kta; qa wicaśtayatapi wohitika wan wicayuhe kła ce, Itancan Jehowa ozuye yuha he hecen eya.

5 Unkan miniwanca etanhan mini kin wanica aye kta; qa wakpa kin mini oyalie qa puza aye kta.

6 Qa wakpa kin hena sicamna kta; Egupta wakpa kin hena lionke śni qa puza aye lkta; cedi psa ko śnije kta.

\% Wakpicahda tinta kin, wakpa mote ekta, qa taku wakpicahda ojupi kin hena iyulpa śnije kta, kahoya iyeyapi lita, qa en un kte śni.

8 Nakun tona hopsicapi kin hena comnihdazipi kta, qa tona wakpa kin en hoiyupsice elipeyapi kin hen owasin śicahowayapi kta, qa tona ho minin ehnakapi kin hena hanyanpi kta.

9 Nakun tona halionta zipzipedan kagapi qa miniliuha ska kazontapi kin hena iśtecapi kta.

10 Qa tona en itancanpi kin hena kicalsapi kta, tona taku kamnanpi ece kin hena owasin iyokiśicapi lsta.

11 Awicakehan Zoan itancanpi kin witkotkokapi ; wiyukcan ksapapi Pharaoh tawa tawowiyukcan kin he wowitk otlikoke icaga. Wicaśta ksapa cinhintku, wana. kaja wicaśtay atapi cinhintliu kin he miye ce, token Pharaoh eyakiyapi kta he.

12 Hena tokiya unpi he: wicaśta ksapa nitawa toliya unpi he. 'Tokin wanna oniciyak' pin ; qa Jehowa ozuye yuha ken Ligupta on wakonze sdonyapi lite.

13 Zoan itancanpi kin 


\section{ISAYA TAWOYUKCAN KIN,}

witkotkoka icagapi ; Noph itancanpi kin wicahnayanpi ; nakun Egupta hnayaupi, tawicoun mdetahunkapi kin hena.

14. Ne cokaya Jehowa woakinica woniya wan yuicicahi : hecen wicolian owasin en Egupta nuniyapi, wicaś'a witko iye hdepe cin ohna kacegya mani kin he iyececa.

15 Nakun Egupta tohtani wanice kta, taku pa qa sinte, can adetka qa cedi, econ kte cin yuke śni.

16 Anpetu kin he en Egupta winohinca wan iyecece kta; qa nihinciye kta qa cancan kta, Jehowa ozuye yuha nape koze cin on, iye iwankam hduśkanśkan kin heon etanhan.

17 Unkan Juda makoce kin he Egupta ekta wolkokipe ee kta'; tuwe kaśta he cajeyate cinhan nihinciye kta, Jehowa ozuye yuha tawolionze he kiconze cin heon etanhan.

18 Anpetu kin he en Egupta makoce kin ohna otonwe zaptan Kanaan iapi on iapi kta, qa Jehowa ozuye yuha en icicunzapi kta. Wanji, Wicotakuniśni otonwe, eciyapi kta.

19 Anpetu kin he en Egupta makoce cokaya owayuśna wan Jehowa kiciyanke kta, qa opapun ihanke ekta obosdatu wan Jehowa kicihe kta.

20 Qa he wowapetokeca qa woyuotanin Jehowa ozuye yuha tawa kta, Egupta makoce kin en. Tehiya wicakuwapi kin on hena Jehowa hoyekiyapi kta, kinhan he Wanikiya wan, han, wanji waśaka, wicaqu kta, qqa ke ewicahdaku kta.

Unkan Lgupta en Jehowa api.kta, qa anpetu kin he upta wicaśta kin Jehowa api kta, qa wośnapi woqu- pi ko econpi kta; han, Jehowa taku qu ic̣iconzapi kta qa econpi kta.

22 Unkan Jehowa Egupta kaśtake kta; hdaśtake ca okiziye kta. Hehan Jehowa en hdipi kta, unkan iye anohwicakiye kta qa okiziwicaye kta.

23 Anpetu kin he en canku tanka wan Egupta etanhan Aśur iyahde kta; unkan Aśur wicaśta Egupta en hi kta, qa Egupta wicaśta kin iś Aśur elsta i kta, qa Egupta oyate Aśur oyate kici wowidagiciyapi kta

24 Anpetu kin he en Israel wiciyamni kta, Egupta kici qa Aśur kici, hakoce cokaya woyawaśte kta :

$25 \mathrm{He} J$ howa ozuye yuhe cin he heya yawaśte kta; Egupta mitaoyate, qa Aśur minape icahye cin he, qa Israel tawa wicawaye cin hena yawaśtepi nunwe.

\section{WICOWOYAKE 20.}

1 Waniyetu wan Tartan Aśdod ekta hi, Sargon Aśur wicaśtayatapi u śi, qa Aśdod kici kicize ça icu qehan;

$2 \mathrm{He}$ ehan Jehowa oie kin Isaya $\Lambda$ moz cinhintku nape en hi, qa heciya; Ya wo, ka ninite etanhan wakihdaka hduśdoka wo, qa nisiha etanhan nitahanpe hduśdoka wo. Unkan ecen econ, tancodan sicodan ko mani.

3 Unkan Jehowa heya; Isaya mitaokiye Egupta ekta qa Kuś ekta wowapetokeca qa woyuśinyaye on waniyetu yamni tancodan sicodan ko mani kin,

4 He iyecen Aśur wicaśtayatapi kin Egupia oyate wayaka awicaye kta, qa Kuś oyate wayaka awicaye kta, wicahakakta qa wicatokapa, tancokapidan qa 
sicopidan, han, onze taninpi, Egupta iśtece kta heon.

5 Unkan kopehdapi kta, qa IKuś wacinyanpi qa Egupta wo-* witanyapi qon hena on iśtecapi kta.

6 Unkan wita kin de en ounye cin he anpetu kin he en heye kta; Tho, wowacinye unkitawapi kin dececa, tukten ounkiyapi kta on naunpapi, Aśur wicátayatapi nape etanhan eunyakupi kta e heon. Hecen token naunjicapi kta he.

\section{WICOWOYAKE 21.}

1 Miniwanca liewoskantu tawoyake kin. Okaga ekta tate iyumni iyoopte iyaye cin he iyecen makoskan tanban $u$, makoce okokipe kin etanhan.

2 Wowanyake tehke wan omakiyakapi: Wawicaki sa wawicaki ece, qa waihangye cin he waihạgya ece. Elam, itanwankanhde ya wo; Medea, niś aconkaśka wo: comnihdazipi kin owasin ayuśtan wakiya.

3 Heon etanhan nite wowayazan omajudan, winohinca hokśiksuya yazan kin he iyecen wayazan wahda: he nahonpi kin on pamahdedan waun he wanyakapi on mayuśinyaya.

4 Micante nuni, woyuśinyaye mayuśinyeya: hanyetu wimduśkin kin he wokokipe micaga.

5 Wahna wotapi kin wiyeya ehnaka wo, owinja owinśkiton wo, wota wo, wayatkan wo: itancan yaunpi, najin po, wahacanka sdakiya miye.

6 Itancan kin kaken emakiya; ya wo, waawanyake wan ehde wo, taku wanyake cinhan ohdake kta.

"y Unkan canpahmihma wan, śuktanka akan iyotankapi nom, śonśonna tacanpahmihma, camo tacanpahmihma ko wanwicayaka: hehan wanahonpi on anohliya yanka:

8 Qa maja wan iyecen houya; Itancan, owanyake tipi ohna anpetu icunhan uhinniyan nawajin, qa oinajin nitawa uhna hanyetu otoiyohi emahdepi ;

9 Unkan iho, hinnakaha canpahmihma wicasta ohna, śuktanka akan iyotankapi nom upi ce. Hehan waayupte ca heya; Hinhpaya, Babel hinlipaya, qa wakagapi tona wakanda lìn hena iyuhpa makata kamden ehpeya ce.

10 Onapanpi mitawa, qa vonapan mitawa cinca kin: Jehowa ozuye yuha Israel ta Wakantanka taku nahonmaye cin he ociciyakapi.

11 Duma tawoyake kin. Sier etanhan houmakiya; Waawanyake, hanyetu kin toketu he: Waawanyake, hanyetu kin toketu he.

12 Wawanyake kin hecen eya; Wihinanpa kin he u, qa nakun narsyetu kin: wawinungapi k'a hecinhan wawiwanga po: hdicu po, kuwa mije.

13 Woyake Arab akan un kin. Dedanim etanhan icrmayanipi kin, Arab contanka kin en inunkapi kta.

14 Tema makoce en ounyanpi hin hena tuwe ipuza kin itkokim mini kaupi; tuwe najice cin he aguyapi yuha itkokipapi.

15 Hena mazasagye ite kin etanhan najicapi, mazasagye yuwankan icupi ite kin etanhan, qa itazipa nawinjapi etanhan qa kicizapi telike etanban.

16 I tancan kin kaken pmakiya; Waniyetu wanjidan, tuwe wohtani opetonpi tawaniyetu iyececa, hehan Kedar towitan ocowasin tanin kte śni. 
17 Qa itazipa yulapi qon tona okapt spi, Kedar cinca wicaśta waśakśakapi kin hena wicayutonananpidan kta, Jehowa Israel ta Wakantanka kin he hecen eya nakaeś.

\section{WICOWOYAKE 22.}

1 Wowanyake Kaksiza tawoyake kin. Dehan tonikeca, hecen ocowasin tiakalipe kin ekta idada he.

2 Wicośkanśkan ojudan, owodu'aton otonwe, otonwe wiyuśkinyan yaun; tona nicitapi kin hena mazasigye on wicaktepi śnı, qa kicizapi en țąi śni.

3 ltancanpi nitawa owasin witaya najicapi, itazipa yuhapi kin eqe hena wicakaślapi ; tona niye en iyewicayapi kin hena iyulipa witaya wicakaśkapi, tehan najicapi.

4 Heon etanhan hepa; En ematonwanpi śni po, nina waceye kła; mitaoyate cunwintku wakipi kin on cantohnagmayan akitapi śni po.

5 Anpetu kin he woiyokisice qa waanatanpi qa wonihinciye, Jehowa Itancan wicobe tawa kin etanhan, wowanyake kaksiza kin en, conkaśke oqapi qa he kin ekta hoyekiyapi.

6 Unkan Elam wanju hduha u, canpahmihma wicaśta ohnaka, śuktanka akan iyotankapi koya; qa Kir wahacanka hducoka.

7 Urkan kaketu kte, kaksiza kahnigapi nitawa kin hena canpahmihma on ojudan, qa ślktan. ka akan iyotankapi kin hena tiyopa lin en wiyeya eiçihdepi lita.

8 Hehan Juda tawoakahpe kin yuzamni : unk anpetu kin he en contanka tipi wotawe lin ekta eyakitonwan.

9 Nakun Dawid totonwe ohna oyujujupi kin wandakapi, hena ota; qa kuya mini ohnaka mini kin duwitayapi :

10 Qa Jerusalem tipi kin hera dawapi, qa conkaśke piyayapi kta on tipi kin dujnjupi.

11 Nakun conkaśke nonpa otahedan tanina mini ohnaka kin on maka ośkotpa wan yakagapi ; tuka Waicahye cin he ekta eyatonwanpi śni, qa tuwe wanakaja he kage cin he ihayaktapi sni.

12 Unkan anpetu kin he en Jehowa Itancan wicobe tawa kin he ceyapi kta iwicakico, qa waśinhdapi, qa pa yuśdapi, qa wakihdaka ogekitonpi kta:

13 'Tuka iho, wicocante waśte, qa wowiyuśkin, tatanka wicaktepi, qa tahinca wanunyanpi wicakațapi, tado yutapi, qa miniśa yatkanpi : Wauntapi qa waunyatkanpi lita, heyakecinhà unțapi kta ce.

14 Unkan minoge ohna Jehowa wicobe tawa kin he oihdake: Awicakehan woahtani kin de akahpeniciyapi kte śni ecen nitapi kta, Jehowa Itancan wicobe tawa kin he eya ce.

15 Jehowa. Itancan wicobe tawa kin he hecen eya; Hunkatiya, wamnayan kin de ekta ya wo, Shebna tipi awanyake cin hee ekta;

16 Qa heciya wo; Den taku duha, qa tuwe den niciyanka he, hecen den woha wan nihdakan, tuwe wankan woha wan hdakan, imnija ohna tipi wanji ihdakan iyececa.

17 Iho, makata elipeyapi on Jehowa makipusdiya ehpeniye kta, qa woakahpe on anicahpe kia.

18 Taku pahmihma se awicakehan pahmihma iyeniye kta, tapa wan makoce tanka kin en iyececa: hen nite kta, qa hen 
nitowitan canpahmihma kin niyuha ti wowiśtece kin ee kta.

19 Nakun nitoyanke kin etanhan ehpeciye kta, qa nitohe etanhan tuwe kun eniyaku kta.

20 Unkan anpetu lin he en kaketu kte, mitaokiye Eliakim Hilkiah cinhintku kin weco kta:

21 Qa nitawokoyake koyagwakiye kta, ga ipiyake nitawa on waśagwakiye kta, ga wokiconze nitawa iye nape kin ohna ewahnake kta. Unkan Jerusalem en ounyanpi qa Juda tiyohnaka kin hena iye ateyapi kta.

22 Qa Dawid ti tiyopa iyukdoke kin iye hinyete akan ewahnake kta; hecen yuhdoke kta qa tuwedan ecen icu kte śni; qa-iye ecen icu kta qa tuwedan yuhdoke kte śni.

23 Qa he owakatan kta, iyokatkuge owanka wacinyepica kin en iyececa; hecen iye atkuku ti kin en wowitan oyanke heca kta.

24 Unkan atkuku tiyohnaka wowitan ocowasin iye en otkeyapi kta, taku etanhan icage ca inanpe cin, ceganstinna owasin owayujaja wakśsica qa cega mini oyuhapi kin iyulipa.

¿5 Jehowa wicobe tawa kin he hecen eya; Anpetu kin lie en, iyoka thuge owanka wacinyepica en okatanpi qon he yutokan iyeyapi kta, qa kaksapi kta, qa hinhpayc kta: qa taku en otkeyapi qon he kaśpapi kta: Jehowa hecen eya nakaeś.

\section{WICOWOYAKE 23.}

1 Turos tawoyake kin. Tarśiś wita wata tawa, śicahowaya po; he yujujupi nakaeś, hecen tipi wanica, mahen yapi kta wanica: Kittim makoce kin etanhan he wicakiyuotaninpi.
2 Wita en ounyayapi, inina un po; Zidon wopeton tawa mini. wanca akasampa tanhan yapi kin hena ojuniyanpi.

3 Unkan mini tanka kin icahda Sihor tawoju kin, wakpa tawominayan kin he tawokamna kin ee; qa hen Ikcewicaśta wopetonpi ece.

4 Zidon iśteca $W \oplus$, miniwanca kin he ia; miniwanca towaśake kin he hecen eya; Mihduśake śni qa cinca waton śni, qa koślka icahiwicawakiye śni, wikośka nakun icahwakiye śni ce.

5 Egupta oyakapi kin on, he iyecen Turos oyakapi kin on nina wayazanhdapi kta.

6 'Tarśiś ekta akasanpa ya miye ; wita kin en ounyayapi śicahowaya po.

7 Wanji wiyuśkin nitawapi, otokahe ekta icage cin, anpetu wanakaja tanhan, he dee he: ounye kta e iye siha tehan aye kta.

8 Turos wateśdake kiton kin on tuwe hecen wakiconza he; wicaśta wopeton tawa kin hena wicaśtayatapi, qa en wopeton yukanpi kin lıena maka akan wicaśta kinihanpi tuka.

9 Jehowa wicobe tawa kin he hecen wakiconza, wowinkta wowitan ocowasin yuśice kta, maka akan wicaśta kinihanpi owasin walitewicada śni kage kta e heon.

10 'Tarśiś cunwintku, walspa wan iyecen nitamakoce opta ya wo ; detanhan ipiyake wanica.

11 Miniwanca kin ekta nape hdukatin; wokiconze kin liena yuśkanśkan: Jehowa wopeton otonwe kin on econwicaśi, towaśake kin ihangyapi kta.

12 Qa heya; Ake icimana widuśkin kte śni, Zidon cunwintku wikośka kakiśyapi kin; najin 


\section{ISAYA TAWOYUKCAN KIN,}

wo, Kittim ekta akasam ya wo; heciya nakun oziiçiyapi wanin yaun kta.

13 Kasdim makoce kin wanyaka wo; he oyate śni ; Aśur tona tintoskan otipi qon hena he wicakicage; conkaśke kin hena bosdan ehdepi, tipi waśteśte kagapi: tuka iye qe he otiwita kaga.

14 Tarśiśs wita wata, śicahowaya po, nitowaśakepi kin he ihangyapi ce.

15 Unkan anpetu kin he en $\mathrm{ka}$ ketu kte, Turos akiktonjapi kta, waniyetu wikcemna śakowin, wicaśtayatapi wanjidan taanpetu kin hehanyan. Waniyetu wikcemna śakowin iyohakam hohan witkowinna wan iyececa Turos dowan kta.

16 Witkowinna akiktonjapi qon, niye candowankiyapi wan icu qa otonwe kin ohomni ya wo; wayupiya dowàn wo, odowan ota ahiyaya wo; hecen niksuyapi kta.

17 Unkan kaketu kte, waniyetu wikcemna śakowin iyohakam, Jehowa ake Turos ekta etonwe kta; unkan iye wokajuju tawa kin en ihdoku kta, qa maka ite kin owancaya wokiconze maka akan un kin owasin om wawicihahapi econ $k$ ta.

18 Tuka tokamna qa wokajuju tawa kin hena Jehowa ekta wakan kta; hena kihnakapi kte śni qa ejupi kte śni; tokamna kin he tona Jehowa itokam ounyanpi kin imnahan wotapi kta, qa wokoyake waśte hinca heon.

\section{WICOWOYAKE 24.}

1 Tho, Jehowa makoce kin yucoka, qa hewoskan kaga, qa ite yuahdapśinyan ehnaka, qa en ounyanpi kin hena enanakiya iyewicaye kta.
2 Unkan kaketu kte, oyate unpi kin vawayuśna kin iś iyececa; wowidake un kin tuwe yuhe cin iyececa; wikośka un kin tuwe yuhe cin iyececa; wopeton un kin wiyopeye cin iyececa; woqu un kin wodota he iyececa; oicage on nwicaqu kin tuwe oicage kicicajuju kin he iyecen un kta.

3 Makoce kin ocowasin yucokapi, qa ocowasin wakipi kta; wicoie kin de Jehowa ecen eya nakaeś.

4 Makroce kin ceya un, śniś aye ; wicoicage kin hanyan, śniś aya; makoce kin en oyate tehanwankan unpi kin hena hanyanpi.

5 Nakun makoce kin tona en ounyanpi ihukuya yuśapapi ; hena woope kin ahtanipi, wolkonze kin yutokecapi, wicotakuye owihanke śni kin he kicaksapi nakaeś.

6 Heon etanhan woyaśice kin he makoce kin temya iyeya; qa en ounyanpi kin hena śitkihdawicayapi ; heon etanhan makoce en ounyanpi kin wicahuhnagapi, qa wicaśta tonana okaptapi.

7 Miniśa teca kin hanyan ; canwiyuwi kin śniś aya ; tona cante waśtepi ece kin owasin comnihdazipi.

8 Cancega wiyuśkinpi kin ayuśtan, tona wiyuśkinpi iyaśapi kin he owihanke, candowankiyapi towiyuśkin he ayuśtan.

9 Dowanwan miniśa yatkanpi kte śni; mini wakan tona yatkanpi kin he wicakicipa lince kta.

10 Otonwe cokadan un kin he y ujujupi ; tipi otoiyohi natakapi, hecen tuwedan mahen ye kte śrii.

11 Canku ohna miniśa dapi tuka qon; wowiyuśkin ocowasin aotpasyapi, makoce wiyuśkinpi kin he henakeca. 
12 Otonwe kin en woyujuju ecedan, qa tiyopa kin ocowasin kamdecapi.

13 Tohan makoce cokaya oyate kin ehna hecetu'kinhan, oliwe can yuhnapi iyececa, hastanhanka yuśpi yuśtanpi opahipi kin iyecece kta.

14 Hena ho wankan yeyapi kta, Jehowa towitan on iyaśapi kta, miniwanca etanhan nina houyapi kta.

15 Heon etanhan peta kin en Jehow a yatanka po, miniwanca wita kin elsta Jehowa Israel Taku Wakan tawapi kin.

16 Maka ihanke hinca etanhan dowanpi naunhonpi, wicaśta owotanna wicayatanpi kin he. Tuka hepa; Matakuniśni, matakuniśni, hehehe miye; wahnayan econpi kin hena wahnayanpi; han wahnayan econpi kin hena nina wahnayanpi.

17 Maka akan ounyaye cin, wolkokipe, qa wolia, qa wohrnunke kin hena akan niyanka.

18 Unkan kaketıkte, tuwe wokokipe hotanin kin etanhan najice cinhan he wolia ohinhpaye kta, qa tuwe woha cokayatanhan hdicu kinhan wohmunke kin on hmunkapi kta; wankantanhan owanyeye kin hena yuhidokapi, qa maka oahde kin hena śkanśkan nakaeś.

19 Kamdecapi on maka kin kamdecapi, yuksaksapi on maka kin yuksaksapi, maka kin nina śkanśkan.

20 Witkopi iyceeca maka kin kacegya un kta, qa cowahe wan iyecen kahuhuzapi kta: unkan woahtani tawa kin on hduśaka; hecen hinhipaye kta qa ake najin lste śni.

21 Unkan anpetu lin he en kaketu kte, tona wankan unpi optaye wahaniçidapi kin hena Jeho- wa kakiświcaye kta, qa makata wicaśtayatapi maka akan unpi kin hena.

22 Unkan hena wicayuwitayapi kta, wayaka wokaśke tipi ohna wicayuwitayapi kin om, qa conkaśke en onawicatakapi kta, tuka anpetu ota iyohakam en wicahipi kta.

23 Unkan hanyetu wi kin ite naśa kta, qa anpetu wi kin iśtece kta, tohan Jehowa wicobe tawa kin he Zion Paha akan, qa Jerusalem en Wicaśtayapi yanke kta, qa hunkawicayapi tawa wicitokam wowitanyan un kinhan.

\section{WICOWOYAKE 25.}

1 Jehowa Wakantanka mitawa kin he niye, ciyawankantuya kta, nicaje mdatan kta: taku wowinihan ecanon, nitowiyukcan kin hena wanakajatanhan wowacinye qa wowicake.

2 Otonwe kin he woyujuju pahaha yakaga; otonwe aconkaśkapi kin he ihangyapi; oynte tokeca conkaśke tawa kin he otonwe kte śni ; icimana kagapi lite śni.

3 Heon etanhan oyate waśake cin he niyatan kta, oyate wohitikapi totonwe kin konicipe kta.

4 Tona wahpanica wowaśake tawa kin he niye, onśika kakiśyapi kin wowaśake tawa, icamna etanhan wowinape, omaśte kin en ohanzi wan he niye, oyate wohilike taniya kin he conkaślke aicamna kin iyececa qehan.

5 Toka owodutaton hin lie du. hukuya kta, lopuza en maśte kin iyececa; omaśte en mahpiya śapa ohanzi kin he: oyate wohitilxapi tadowanpi kin yuhukuyapi kta.

6 Unkan paha kin de akan Je215 


\section{ISAYA TAWOYUKCAN KIN,}

howa wicobe tawa kin he taku cepa on wohanpi wan oyate owasin wicakicage kta, miniśa koka ohna han oyatke, taku cepa cupe ojudan, miniśa koka etanhan tanyan puskepapi kin

\% Qa paha kin de en ite alalipe oyate owasin akahpapi kin he yatakuni kte śni, qa woakahe kazontapi Ikcewicaśta owasin awicakahpapi kin he nakun.

8 Wiconte ocowasin yatakuni kte śni; qa Jehowa Itancan kin wicite owasin etanhan iśtamnihanpe wicakipakinte lkta; qa iye taoyate tawowiśtece kin maka ocowasin etanhan eyaku kta; Jehowa hecen eya nakaeś.

9 Unkan anpetu kin he en heyapi kta; Iho, Wakantanka unkitawapi kin he dee; he unkapepi, unkan iye niunkiyapi kta: Jehowa dee, he unkapepi, cante unwaśtepi kta qa iye towiconi on unkiyuśkinpi kta.

10 Paha kin de en Jehowa nape kin yanke kta; unkan iye ihukuya Moab ahanpi kta, aguyapi hu tacesdi paha kin en amanipi iyececa.

$11 \mathrm{Qa}$ hena cokaya nape hdugate kta, tuwe niwan niwe kta nape hdugate cin iyececa: tuka iye nape ośkiśkeya ohanye cin on towahanicidapi kin he kun ehpeye kta.

12 Qa conkaśke nitawa ipasotka tehanwankantu kin he yujuju kta, kun elipeye kta, makata ehnake kta, maka mdu kin en.

\section{WICOWOYAKE 26.}

1 Anpetu kin he en Juda makoce kin ohna odowan kin de ahiyayapi kta :

Otonwe suta wan unhapi;

Wanikiyapi kin he conkaśke qa maka qapi kin eekiye kta. 216
2 Tiyopa kin yuhdoka miye,

Hecen oyate owotanna wowicake patanpi kin mahen yapi lita.

3 Wicacante wacinyan un kin he wookiye hinca ohwa duhe lita,

Iye wacinniyan nakaeś.

4 Ohinniyan Jehowa wacinyan miye ;

Wowaśake owihanke wanice cin he Jah Jehowa en un nakaeś.

5 Tona wankantuya tipi kin hena kun iwicacu ;

Otonwe tehanwankantu kin kun elpeye kta,

Kun ehpeye kta, maka kin ekta ;

Maka mdu kin en icu kta.

6 He wicasiha ahe kta;

Onśika sihapi, wahpanica sicuhapi kin.

7 'Tuwe owotanna tacanku kin he owotanna:

Owotanna hinca yaun, wicaśta owotanna tacanku kin dumdaye kta.

8 Han, Jehowa, nitawoyaco canku kin ohna unkanipepi;

Unnagipi cantokpani kin he nicaje qa niksuyapi kin ekta.

9 Hanyetu en minagi on cantocikpani ;

Han, tanmahen tanhan mitaniya on nina acicite kta :

Nitawoyaco kin maka akan un kinhan,

Makoce en ounyanpi kin woowotanna onspeiçiciyapi k ta.

10 Tuwe śica cantekiyapi eśta woowotanna onspeiçiciye śni :

Woecetu makoce kin ohna śicaya ohanyan;

Qa Jehowa tokitanin kin wanyake kte śni.

11 Jehowa, ninape yuwankan 
icupi eśta hena wanyag wacinpi śni :

Tuka wanyakapi kta, qa oyate inawicayakiwizi kin on iśtecapi kta:

Han, tokawicayaya ide kin he huhnahwicaye kta.

12 Jehowa, wookiye unyaqupi kta;

Unkiohanpi owasin niye qe unkoyecilianyanpi nakaeś.

13 Jehowa Wakantanka unkitawapi,

Nicunonpa itancanpi wowidagunyanpi ;

Tuka niye niśnana on nicaje cajeunyatapi kta.

14 Takpe wicayai qa ihangwicayaya;

Qa tokiksuyapi kin ocowasin dutakuni śni :

Heon etanhan țapi, ake kinipi kte śni ;

Nagi iyayapi, hdinanpapi kte śni.

15 Oyate kin duota,

Jehowa; oyate kin duota;

Nihduonihan;

Makoce opapun iyuhpa dutanka.

16 Jehowa, wokakije on en nihipi ;

Kakiświcayaye cin he on

Wocekiye wan hoyeniciyapi.

17 Tuwe ihduśaka wanna ecadan cinca ton lita,

He wayazanhda, hokśiksuya on śicahowaya,

He iyecen, Jehowa, nitokam unyakonpi ce.

18 Unkihduśakapi, waunyazanpi,

Tate hiyuunyanpi seca;

Makoce kin en owicakiyapi econkupi śni ;

Nakun maka ounyanpi kin hena hinhipayapi śni.

19 Tona nicițapi kin hena nipi kta;
Mitancan te cin he kici hdinanpapi kta:

Kikta po, iyaśa miye, tona maka mdu en ounyayapi kin ;

Wato cu kin he cu nitawa;

Qa maka kin wicate cin hiyuye kta.

20 Iho ye, mitaoyate, ti mahen hdicu wo,

Qa tiyopa kin onaiçitag icu wo:

Cistiyedan se anaiçiliman wo ;

Wośinhda kin sam iyaye kta hehanyan.

21 Iho, Jehowa iye tohe etanhan hiyu,

Maka ounyanpi kin wahtanipi on kakiświcaye kta:

Nakun maka kin he we hdutanin kta,

Qa tona wicaktepi tawa kin icimana awicakahpe kte śni.

\section{WICOWOYAKE $2 \%$}

1 Unkan anpetu kin he en, Jehowa, iye tacanhipi tke, tanka qa suta on, Leviathan wanmduśka duzahe cin he kaśtake kta; han, Leviathan wanmduśka pemni kin he; qa unktehi tanka miniyata un kin he kte kta.

2 Anpetu kin he en hastanhanka ojupi wan kahiyaya miye.

3 Jehowa miye he awanwahdaka ; ohinniyan a magajuwaye kta: okinni kiunniyanpi kta, heon hanyetu anpetu ko awanmdake kta.

4. Wośinhda en maun śni: tuwe okicize en wapepeka canpepe ko mitkokim ehnake kta he: hena takpe mde kta, hena ocowasin huhnahwaye kta tuka.

$5 \mathrm{Qa}$ iś mitowaśake en sutaya yuze kta; miye kici wookiye içicage kta: han, wookiye micicaga nunwe. 
6 Anpetu u kte cin en Jakob liutkan icage kta: Israel palia kta qa hea aye kta, qa waskuyeca on maka ite kin ojuyapi kta.

7 Tuwe ape ciqon apapi kin iyecen apa he; qa wicaktepi on iye wicakte qon iyecen ktepi he.

8 Ehpeyapi kin he kici iyuteya ayakinica kta: wiyohiyanpatanhan tate uye anpetu kin en tate wohitika tawa on yutokan iyeya ece.

9 Heon etanhan de on Jakob tohan sice cin kajujupi kta; qa woahtani tawa yutokan iyeye kta oicage ocowasin he dee. Owayuśna inyan kin iyuhpa inyan acetipi kamdemdecapi kin iyecen ehpeye cinhan, wakagapi ga anpetu wi okagapi kin hena bosdan he kte śni.

10 Tuka otonwe aconkaślkapi kin he hewoskan wanke kta : otipi yujujupi qa ehpeyapi, tintoskan iyececa: hen ptejicadan wihan kta, qa hen makan iwanke kta, qa adetka kin temye kta.

11 Tohan adetka śnijapi kinhan yuksapi kta: winohinca kin upi qa hena ideyapi lita: He oyate wiyukcan śni, heon etanhan tuwe icahwicaye cin he onśiwicada kte śni, qa tuwe wicakage cin he cantewicakiye kte śni.

12 Unkan anpetu kin he en wakpa tancan kin etanhan Egupta kaksiza kin hehanyan Jehowa kalina kta; qa, Israel cinca, niyepi wanjigjidan nipahipi kta.

13 Nakun anpetu kin he en mazayahotonpi tanka wan yahotonpi kta; hehan tona Aśur makoce kin en enanakiya unpi, qa Egupta makoce en ehpewicayapi qon hena upi kta, qa Jerusalem ekta, Palıa wakan kin akan, Jehowa ohodapi kta. 218

\section{WICOWOYAKE 28.}

1 Wateśdake tehanwankantu, Ephraim witkopi kin wotehi wicakiciyanka nunwe; qa walica śnije lita iye towaśte wakan kin he, miniśa on ohiyapi kalssiza cepa tawapi oinkpa kin ekta yanke cin he.

2 Iho, wanji wawokihi qa waśaka Itancan kin kici un, wasu hinhe, icamna waihangye cin iyececa, mini tanka nina apaśbogye cin iyecen iye nape on makata ehpeye kta.

3 Ephraim witkopi kin tawateśdake tehanwankantu kin he wicasiha ihukuya napanpi kta:

4. Qa towaśte wakan kin, wahica śnije kta kaksiza cepa oinkpa kin ekta yanke cin he, mdoketu śni suken waskuyeca suton, tur we ekta etonwe wanyake cinhan, nahahin nape ohna un icunhan temya iyeya he iyecece kta.

5 Anpetu kin he en, iye taoyate okaptapi kin on Jehowa wicobe tawa kin he wowakan wapaha qa wowaśte wateśdake kin ee kta;

6 Qa tona wayaco ryotankapi kin hena on woyaco woniya kin heca lita, qa tona otonwe tiyopa kin ohna okicize yuhomnipi kin hena on wowaśake kta.

7 Tuka hena eqe nakun miniśa on nunipi, qa mini wakan on icunonpa unpi; wawayuśna qa wicaśta wokcan kin hena mini wakan on nunipi, hena miniśa on napcapi, mini wakan on icunonpa unpi ; wawanyakapi en nunipi, wiyukcanpi en kaktihanhanpi.

8 Wahna wotapi iyuhpa hdepapi śica on ojugjudan; owanka wanica.

9 Tuwe he wosdonye onspekiye kta he; qa tuwe he woonspe 
okahnihkiye kta he: tona asanpi ayuśtanwicakiyapi, tona aze etanhan ewicayakupi.

10 Woonspe woonspe iyahna, woonspe woonspeiyahna; woope woope iyahna, woope woope iyahna; deciya tonana, heciya tonana ece.

11 Heon etanhan wicaiha yaśkiślkapi qa wicaceji tokeca on oyate kin de om wowahdake kta.

12 Hena hecen ewicakiya; Woozi kin he dee; mdokițapi kin oziwicakiya miye ; qa owanji yankapi kin he dee ce; tuka anagoptan wacinpi śni.

13 Hececa eśta hena en Jehowa oie kin he woonspe woonispe iyahna; woope woope iyahna; deciya tonana, heciya tonana; liecen yapi kta qa itunkam hinhpayapi kta, qa wicakawegapi kta, qa wicahmunkapi kta, qa wicayuzapi kta.

14 Heon etanhan wicaśta wawihaha yaunpi, Jerusalem en oyate kin de iwicayadakapi kin, Jehowa oie kin nalion po.

15 Wiconte kici wicotakuye unkicagapi, qa Hades kici okiciciya unyakonpi; hecen tohan wawiyopeye apaśboka iyopta ye cinhan unkikiyepidan u kte śni ; woitonśni he wowinape unyanpi nakaeś, qa ecinkta eyapi kin en inaunkiẹilimanpi ce, ehapi ;

16 Heon etanhan Jehowa Itancan kin he hecen eya; Iho, Zion en inyan wan oahehde ewahnaka, inyan iyutapi, oise inyan tehika, oahehde hdepi wan; tuwe wicada kin he inalini kte śni.

17 Nakun woope kin en woyaco ewahnake kta, qa woowotanna, on tkeutapi kin en: unkan wasu kin he woitonśni wowinape kin kahin iyeye kta, qa mini kin woinahbe kin apaśboke kta.

18 Hecen wiconte kici wicota- kuye nitawapi kin he yujujupi kta, qa Hades kici oyeciciyapi kin he ecen he kte śni; qa wawiyopeye apaśboke cin he iyoopta iyaye cinhan, hehan anihanpi lita.

19 'Tohan iyaya eca niyuzapi ece kta : hanlianna qa hanlianna iyaye kta, anpetu qa hanyetu: hecen onahonpi kin he okatinigapi kin he wonihinciye ecedan kta.

20 Owinja kin he tuwe anasonson kta iyehantu śni, qa oakahpe kin he cistinna, hecen on iyaiçipemni kte śni nakaeś.

'21 Perazim paha kin ekta Jehowa iyecen inajin kta; Gibeon kaksiza qon en iyecen śinhda kta; hecen iye tolian kin econ kta, iye tohan tokeca kin he; qa iye tohtani hduśtan kta, tohtani tokeca kin he.

22 Heon etanlan wawihahapi śni po, okinni on nicaśkapi kin niciyusutapi kta: woyutakuniśni wan, qa he Itancan Jehowa wicobe tawa kin eciyatanhan nawahon, maka kin owancaya yuśtanpi.

23 Anohya miye, qa miho kin nahon po; anagoptan miye, qa iwae cin nation po.

24 Mahyumdu kin woju kta e on anpetu otoiyoli maga hdumdu he; tamaga hdupanpan qa hduhinta ece kta he.

25 Tohan maga ite kin yumdaya hehan qetsa kada śni he ; komin he oju, qa aguyapi su kin cankuyetonton ohnaka, qa śuktanka tawote kin tukten hdahinige cin en, qa icage kte cin en wayahota kada elipeya ece.

26 Hecen iye Taku Wakan tawa wowiyukcan onspekiya, wokahnilikiya ce.

27 Qetsa kin he maziyapan on kapanpi śni, qa canpahmihma 219 


\section{ISAYA TAWOYUKCAN KIN,}

wan komin ayuhomnipi śni; tuka qetsa kin he cansagye on kapanpi, qa komin he cansakadan on.

28 Aguyapi icahyapi kin he napanpi ece, tuka ohinniyan kapan kte śni, qa canpahmihma tawa kin ayuhmihma kte śni, qa śuktanka akan iyotankapi tawa kin on namdu kte śni.

29 De nakun Jehowa wicobe tawa kin etanhan u ece; iye towiyukcan kin wakan hinca, tohan kin hdutanka ece.

\section{WICOWOYATE 29}

1 Hehehe, Ariel, Ariel otonwe en Dawid tiqon: waniyetu waniyetu en kokijuya po: wośnapi kin ihunnikiyapi kta.

2 Tuka Ariel tehiya wakuwa kta; qa woiyokiśice woceye ko yuke kta: hececa eśta he Ariel iyececa miciyanke kta.

3 'Tuka niye nihdukśan ewati kta, qa ozuye on aonacitake kta, qa niye en conkaśke awakage kta.

4 Hecen kun ehpeniyanpi kta, maka kin etanhan houyaye kta, qa iyae cin he kuya maka mdu etanhan kta, qa niho kin wanagi maka etanhan iyecece kta, qa iyae cin he maka mdu kin etanhan jiji kta.

5 Nakun oyate tokeca ota wicaduhe cin hena watuśekśeca iyececapi kta, qa wicota waśakśakapi kin hena aguyapi ha kahbog iyaye cin iyececapi kta: unkan he ihnuhanna hince kta.

(f) Wakinyan hotonpi, qa maka śkanśkan, qa ho tanka on Jehowa wicobe tawa kin he en nihi kta, magaju qa icamna on, qa peta ide wahuhnahye cin on.

7 Unkan oyate kin owasin wicota hinca, Ariel kici kicizapi, tona iye qa taconkaśke kin kici kicizapi owasin, qa tona iyokisinye cin hena hanyetu en wowanyake ihamnanpi kin iyececapi kta.

8 Tuwe wotektehda wihamna, unkan iho wota, tuka kikta, unkan nagi cokadan, he iyecece kta; qa iś, tuwe ipuza wihamna, unkan iho wayatkan, tuka kikta, unkan iho waśake śni qa nagi ipuza : oyate ota hinca Zion Paha kin en kicizapi kin owasin iyececapi kta.

9 Owanji yukan po, qa nihinciya po ; iyuślkiniçiya po qa iśtagonga po. Hena witkopi, tuka miniśa on etanhan śni ; kacekcegya manipi, tuka mini wakan on etanhan śni.

10 Woiśtinbe hinca oniya wan Jehowa anicaśtanpi, qa iśta niyuotinzapi: wicaśta wokcan qa itancan nitawapi, tona wawanyakapi kin hena wawieakahpa.

11 Hecen wowanyake ocowasin niyepi elrta wowapi oie apuspapi kin iyececa; tuwe wowapi onspe kill he qupi, qa; De yawa wo, ceunniciyapi ce, eyapi : tuka, owakihi śni, he apuspapi ce, eya :

12 Hehan wowapi kin he tuwe wowapi onspe śni kin he qupi, qa; De yawa wo, ceunniciyapi ce, eyapi : tuka, Wowapi onmaspe śni ce, eya.

13 Heon Itancan kin heya; Oyate kin de $i$ on mikiyedan upi, qa ihapi on mayaonihanpi, tuka cantepi mitehanyan iyeiciyapi, qa komakipapi kin he wicaśta tawoonspe kin eciyatanhan onspewicakiyapi ;

14 Heon etanhan iho, oyatokin de ehna wakanyan owahanmde kta, taku wowinihan qa wowapetokeca : tona ksapa wicayuhapi toksapepi kin he atakuni kte 
sni, qa tona wiyulrcanpi tookalnigepi kin analimanpi kta nakaeś.

15 Hehehe, tona towiyukcanpi lin temahentuya Jehowa anakicihman akitapi, qa tohanpi kin otpaza en, qa heyapi, Tuwe wanunyakapi he; qa tuwe sdonunyanpi he.

16 Awicakehan waduptanyanpi kin he maka cega kaga maka tawa kin iyacinpi kta: wicohan wan tuwe econ kin on, He makage śni ce, eye kta he; qa taku kagapi kin he tuwe kage cin on, Takudan okalinige śni ce, eye kta he.

17 Ehake cistiyedan hin hehan Lebanon maga waśte icage kta, qa maga waśte kin he contanka yawapi kte śni he.

18 Qa anpetu kin he en nogetpapi kin wowapi oie kiu hena nalionpi kta, qa iśtagongapi iśtapi kin owotanin śni qa otpaza etanhan wawanyakapi kta.

19 Wicaśta onśiiçidapi kin nakun Jehowa en sanpa wiyuśkiniciyapi kta, qa wicaśta opeya onśikapi kin hena Israel Taku Wakan tawa kin ikiciyuśkinpi kta.

20 Tuwe wohitike cin he atakuni śni, qa wawihlalia kin he ihangyapi, qa toua woahtani iyapepi kin owasin wicakaksapi : 21 Wicoie wanjidan on wicaśta walitani yawapi, qa tuwe tiyopa ohna wayaco kin he hmung wacinpi, qa takuśni on wicaśta owotanna icunonpa iyayeyapi kin hena.

22 Heon etanhan Jehowa Abraham opekiton qon he Jakob tiyohnaka kin hewicakiya; Dehan Jakob iśtece kte śni, qa dehan iye ite nasan kte śni.

23 Tuka tohan iye cinca, minape taku kage cin hena, iye cokaya wanwicahdake cinhan, mica- je yawakanpi kta, qa Jakob Taku Wakan tawa yawakanpi kta, qa Israel ta Wakantanka kokipapi kta.

24 Qa tona tawiyukcanpi en nunipi qon hena nakun wookahnige sdonyapi kta, qa tona wawakipajinpi qon hena woonspe onspepi kta.

\section{WICOWOYAKE 30.}

1 Hehehe, wawakipajinpi cinca, Jehowa eya; hena wokonze hduśtanpi, tuka miye etanhan śni ; qa woyatke wan okaśtanpi, tuka Mitaniya kin eciyatanhan śni, hecen woahtani woahtani en ejupi kta.

2 Hena Egupta ekta apamahde huinyun yapi, tuka mii etanhan wawiwangapi śni; Pharaoh towaśake kin en waśagic̣iyapi kta, qa Egupta ohanzi kin wacinyanpi kta.

3 Heon etanhan Pharaoh towaśake kin he wowiśteceyayapi kta, qa Egupta ohanzi wacinyanpi kin he wonihinciye kta.

4. Iye ta wicaśtayatapi kin Zoan en unpi, qa iye uwicaśi qon hena Hanes en hipi.

5 Hena owasin oyate wan owicakiye kta okihi śni kin he on iśtecapi, waanakikśinpi qa waokiyapi okihipi śni, tuka wowiśtece qa nakun wowilialia ecedan.

6 Itokah woteca tawoyakapi kin. Wonihinciye qa woiyokiśice makoce, etanhan mnaja wiye qa mnaja waśaka, sintelida qa wamduślka kinyan ideya, hena etanhan upi, he ekta towijicepi kin śunka wakan śonśonna amdo akan ayapi kta, qa taku kihnakapi kin camo pśunka kin akan, oyate waokiye kte śni wan ekta. 
7 Egupta wawokiye cin he takuśni, qa takudan okihi śni : heon etanhan decen ewakiye ciqon; Wahianicidapi owanji yankapi ce. 8 Tho wo, wanna wicitokam wahna wowapi wan en he owa wo, qa wowapi en kaga wo, hecen he anpetu u kte cin hehanyan kta, owihanke wanice :

9 Oyate kin de wawakipajin ece, wicacinca itonpi śni, wicacinca Jehowa toope kin anagoptan wacinpi śni hecapi :

10 Hena, Wawanyakapi śni po, wawanyakapi kin ewicakiyapi ; qa wiyukcanpi kin, Taku ecetu unkiciyukcanpi śni po, iapi śdúdutua unkokiyaka po, woitonśni ayata po :

11 Canku lin icunonpa ya po ; ocanku icunonpa wahtani po: Israel Taku Wakan tawa kin he yutokan iyeunkiciciya po, eyapi.

12 Heon etanhan Israel Towakan kin he hecen eya; Oie kin de ayaktapi śni, qa wohnaye woyupemni ko wacinyayapi qa en eniçihnakapi ;

13 Heon etanhan woahtani kin de conkaślke tehanwankantu en okakse wan iyecen he apaha yanka qa hinhpaye kta se, okamdece kin lue iśtakakpapi se ilnuhanna $\mathrm{u}$ kte cin he iyecen niciyankapi kta.

14 Qa he maka cega kaga tawakiśkotpa ocowasin kamdecapi wokamdece kin he iyecen kamdece kta; itonkipe kte śni: hecen okamdece kin he etanhan onśpa oceti etanhan on peta icupi kta, qa on miniyowe etanhan mini eyakupi kta, takudan iyeyapi kte śni.

15 Itancan Jehowa Israel Towakan kin he hecen eya; Hdicupi qa owanji yukanpi kin he on yanipi kta; inina unpi qa wa222 cinyanpi kin on waniśakapi kta tuka: unkan wicayadapi śni.

16 Tuka hehapi ; Hiya, śuktanka akan naunjicapi kta ce; heon etanhan nayajicapi kta: qa, Taku duzahan akan unkiyotankapi kta, ehapi; heon etanhan tona tanipapi kin hena duzahanpi kta.

17 Wanjidan wawiyopeye on kektopawinge wanji najicapi kta, zaptan wawiyopeyapi on nayajicapi kta: hecen niyuhuhuzapi kta, wiyokihedan he akan chdepi qa wapahaśa wan paha ekta he cin iyececa.

18 Heca eśta Jehowa ape un kta, onśinidapi kta; qa heon inajin kta, cantonihnakapi kta e heon: Jehowa Taku Wakan kin he wayaco nakaeś: Tona apepi kin owasin cante waśtepi ece.

19 Oyate kin Zion en Jerusalem ohna ounyanpi kta : iśtamnihanpe aniu yaceye kte śni; tuwe waonśida kin he ho uyaye cin on onśinida kta; he nahon kinhan aniyupte kta.

20 Unkan Itancan kin woiyokiśice aguyapi qa wokakije mini kin niçupi qeyaś, tona waonspeniciyapi kin hena ake icimana naiçilimanpi kte śni, tuka niiśtapi waonspeniciyapi kin wanwicayakapi kte :

21 Qa niheyatanhan ninogepi wicoie wanji nalionpi kta, Cánku kin he dee ce, ohna mani po, eye kta, tohan etapatanhan dapi kta, qa tohan catkatanhan dapi kta hecinhan.

22 Nakun nita mazaska wakagapi oakahpe kin daśapapi kta, qa nita mazaskazi wakagapi okaśtanpi oakahipe kin ; iśnati mahen unpi tawa iyecen hena elipeyaye kta: Tukan yanka wo, eyakiye -kta.

23 Hehan nitawojupi magaju 
kin hiyuye kta, hecen on nitamaga kin oyekju kta; qa maka oicage etanhan aguyapi yuke kta; qa he cepa qa oicah ota $\mathrm{kta}$. Anpetu kin he en nitawoteca tinta tanka en wihanpi kta.

24 Nakun tatanka qa śonśonpidan maga kicanyanpi kin hena woyute skuyeyapi, mazaicu qa icaduge on kadugapi kin he yutapi kta.

25 Unkan he tehanwankan otoiyohi, qa paha pajodan otoiyohi akan wakpadan yuke kta, mini kaduze kta, anpetu kin en tankaya wicaktepi, conkaśke lkin hinlipayapi kinhan.

26 Nakun hanyetu wi iyoyanpa kin he anpetu wi iyoyanpa iyecece kta ; qa anpetu wi iyoyanpa kin he śakowin akihde kta, anpetu sakowin iyoyanpa jyecece kta; anpetu kin en Jehowa iye taoyate okamdece tawa pakifite ça apapi okaśpe kin okiziye cinhan.

27 Iho, Jehowa caje kin tehantanhan $u$, tocanniye ide, qaide kin he tehike : iye iha kin wośinhda on ojudan, qa ceji kin he peta waliulinage cin iyececa.

28 Qa iye taniya wakpa apaśboke wan iyecen tahu cokaya iyahde kta, wicotakuniśni icaduge wan on oyate kin wicakaduge lita: qa nuniwicaye kta e iiyuwi wan oyate ipi kin ohna yanke kta.

29 Odowan wan duhapi kta, hanyetu en omniciye yuwakanpi kin iyececa; qa cante iyuślinpi, trwe cotanka wan hduha Jehowa Paha tawa ekta ye kte cin iyececa, Israel ta Imnija kin elita.

30 Unkan Jehorva towitan ho kin nation wicakiye kta, qa iye isto kun u kin he hdutanin kta, nakun wocanniye wośinhda, qa peta wahuhnage ide kin, icamna qa magaju, qa wasu hinhan.

31 Jehowa ho kin on, Aśur cansagye on wakaśtaka ece qon he kawankapi kta.

32 Unkan cansakadan kahnigapi kin Jehowa akan ehnake kte cin he tukten hiyaye cin otoiyohi, he cancega qa candowankiyapi kicica kta : qa okicize owodutaton on om kicize kta.

33 Tanihantanhan Tophet wiyeya kagapi ; han, wicaśtayatapi kin on yuwiyeyapi ; tanka, temahentu kaga; peta qa can ota ejupi ; Jehowa taniya kin, canśin wakpa wan iyececa, he ideye kta.

\section{WICOWOYAKE 31.}

1 Hehehe, tona owicakiyapi kta on Egupta ekta apamahde iyayapi ; qa śunka wakan wacinwicayapi, qa canpahmihma inapapi, hena otapi heon; qa śuktanka akan iyotankapi, hena waśakapi . hinca nakaeś : tuka Israel Towakan lin he ekta etonwanpi śni, qa Jehowa akitapi śni.

2 Hececa eśta iye nakun lisapa, qa taku śica au kta, qa iye oie kin ehdaku kte śni : tuka wicohan śca econpi tipi kin itkom inajin kta, qa woalitani ecompi waokiyapi kin hena itkowicakipe kta.

3 Nakaeś, Egupta oyate lin hena wicaśta, qa Taku Wakan hecapi śni; qa iye taśunkepi kin hena cehpi qa woniya hecapi śni. Tohan Jehowa nape hdugate cinhan hehan tuwe waokiye cin he kacegya mani kta, qa tuwe okiyapi kin he nakun hinhpaye kta, qa owasin witaya atakunipi kte śni 
ISAYA TAWOYUKCAN KIN,

4 Jehowa hecen emakiye ciqon; Mnaja qa mnaja kośka taliu iyahpaya ahdo, waawanyake wicota takpe upi qeyaś hena hopi on kopehda kte śni, qa hena owodutaton kagapi kin on makata ehpeiciye kte śni; he iyecen Jehowa wicobe tawa kin he Zion he kin on, qa paha tawa kin on kicis kun u kta.

5 Zitkadan waakahpapi ece kin he iyecen Jehowa wicobe tawa kin he Jerusalem ahdahpe kta; ahdahpa qa ehdaku kta ; acakśin qa kpatan kta.

6 Irrael cinca, tuwe itehan icunonpa idadapi kin he elta ihdoku po.

7 Anpetu kin he en wicaśta otoiyohi iye tamazaska wakagapi, qa tamazaskazi wakagapi, niye ninape woahtani on nicicagapi qon hena ehpeyapi kta.

8 Hehan Aśur oyate kin mazasagye on hinhpaye kta, wicaśta tawa śni, qa mazasagye wicaśta akantu tawa śni kin he he temye kta: tuka mazasagye ite kin etanhan nape kta, qa kośka tawa kin hena wayaka umpi kta.

9 Qa iye wokokipe on taconkaśke kin en onajice kta, qa itancan wicayuhe cin hena wiyokihedan kin kokipapi kta ce, Jehowa iye tapeta Zion en un, qa oceti tawa Jerusalem en yanke cin he hecen eya.

\section{WICOWOYAKE 32.}

1 Tho, wicaśtayatapi wan woowotanna on wicaśtayatapi kta, qa itancanpi kin iś woyaco on wakonzapi kta.

2 Hecen wicaśta wan tateyanpa etanhan woinahbe kta, qa.icamna etanhan wowinape iyecece kta; hopuza en mini wakpadan iyececa, makoce ohna wicahtate 224 en imnija tanka ohanzi kin iyececa.

3 Qa tona wanyaka iśtośnijapi kte śni, qa tona wanahonpi nogepi anagoptanpi kta.

4 Nakun tona inalinipi cantepi kin wosdonye okahnigapi kta, qa ia yaśkiśkapi cejipi kin tanyan ia inahnipi kta.

5 Hehan witkotkoke cin icimana wacantkiya eciyapi kte śni, qa watehinda kin he ohanpi eciyapi kte śni.

6 Witkotkoke cin he wowitkothoke ecen ie kta, qa iye cante woahtani kage kta, wicośice econ kta, qa Jehowa on woitonśni oyake kta; tuwe wotektehda un nagi kin he yucoka kta, qa tuwe ipuza tawoyatke kin yuwanice kte.

7 Nakun watehinda taku wowinyunye kin hena śica; he woyuśice waki conza, woitonśni oie kin on wicakiś unpi kin wicayutakuni kte śni, wicaśta onśika ecetuya ia eśta.

8 Tuka wacantkiye cin he taku wocantekiye kin konza ece ; qa wocantekiye on sutaya najin kta.

9 Winohinca wahanicidapi inajin miye; kopehda śni cunwintkupi, maho kin nation po, iwae cin anagoptan po.

10 Waniyetu anpetu ota ahna nihinniciyapi kta, kopehda śni yaunpi; hastanhanka oicage ylke kte śni, mnayanpi kin he u kte śni.

11 Wahaniçida yaunpi, cancan po ; kopehda śni yaunpi, nihinciya po, ihduśdoka po, qa tancokapidan po, qa nite en ipiyagkiton po.

12 Maku en aiçipapi kta, omdaye waśte hinca on, qa hastanhanka iyuwi waicahye cin on.

13 Mitaoyate tamakoce kin en wapepeka can pepe ko uye kta; 
han, wowiyuśkin tipi iyulipa akan, otonwe wiyuśkin kin en.

14 'Tipi tehanwankantu, kin ay uśtanpi, oyate ota tonwanyanpi kin he ehpeyapi; paha qa conkáske kin hena ohinniyan makoĥdoke kta, śonśonna itu unpi on wiyuśkinpi, wanunyanpi en wilianpi kta:

15 Ecen Woniya kin wankantanhan unkakaśtanpi kta, qa tintoskan kin he maga waśte kta, qa maga waśte kin he contanka yawapi kta.

16 Hehan wokonze tintoskan ounye kta, qa woowotanna maga waśte kin ohna yanke kta.

17 Unkan woorwotanna ohan kin wookiye hee kta, qa woowotanna yuśtanpi kin he oziiçiyapi qa wowacinye owihanke wanice kta.

18 Qa mitanyate wookiye owanka wan en ounyanpi kta, qa tipi wacinyepica ohna, qa wikope śni ozikiyapi owanke kin en.

19 Unkan wasu contanka kin ahinhe kta, qa otonwe kin omdaye wan en yuhukun elipeyapi kta.

20 Mini owasin icahda woyajupi cante niwaśtepi, tatanka qa śonśonna silıa kin heciya yeyaśipi.

\section{WICOWOYAKE 33.}

1 Hehehe, niye wawicayaki tuka wanicipi śni ; qa wawicayahnayan tuka niye nibnayanpi śni : Tohan wawicaki arluśtan kinhan hehan wanicipi kta; tohan wawicahnayan enayakiye cinhan hehan niye nihnayanpi kita.

2 Jehowa onśiunkida miye ; unkanipepi. Hanlianna eca hena isto niyuhapi nunwe; nakun wokakije anpetu kin en waniunkiyapi kin he niye kte.
3 Owodutaton hoyeye cin on oyate kin najicapi ; wankan nihdohda on oyate kir enanakiya iyayapi.

4 Unkan wayakipi kin he mnayanpi kta, psipsicadan mnaiçiyapi kin hee kta; psipsica tanka inyankapi kin he iyecen ainyankapi kta.

5 Jehowa wankan hdoyapi; wankan ounyan nakaeś : wokonze qa woowotanna on Zion ojuya.

6 Qa iye nitaanpetu woyusuta kin hee, wanikiyapi wowaśake, woksape qa wosdonye kin liena eepi kta: "wokihnaka tawa Jehowa kokipapi kin hee.

7 Tho, mdetahunkapi wicayuhapi kin tankan śicahowayapi kta: wookiye on yewicaśipi qon hena nina ceyapi kta.

8 Canku tanka kin hena hewotahedan ehpeyapi ; oicimani wanica. Wicotakuye kin he kicaksa, otonwe kin wahtewicada śni, wicaśta kin ihawicakta śni.

9 Maka kin lianyan, ceya: Lebanon iśteca, śeca aya: Saron tintoskan iyececa: qa Baśan Karmel kici kahnapi.

10 Wanna nawajin kta ce, Jehowa eya: wanna wankan mahdoyapi kta ; wanna mihduwantu kta ce.

11 Peji śeca on ihduniśakapi kta, aguyapi hu yatonpi kta; nitaniyapi kin petaiyecen huhnaliniyanpi kta.

12 Qa oyate kin inyan acetipi kin hecapi kta; wapepeka kahin iyeyapi iyecen peta en huhnagapi kta.

13 Tehan yaunpi, taku ecamon kin he nalion miye; qa kiyedan yaunpi, mitowaśake kin he sdonya po.

14 Zion en wahtanipi kin hena kopehdapi ; wicacancan wayuśa225 


\section{ISAY 1 TAWOYUKCAN KIN,}

papi kin wicayuza. Unkiyepi etanhan tuwe peta wahulinage cin en ounye kta he ; unkiyepi etanhan tuwe ide owihanke wanice cin en ounye kta he.

15 Tuwe owotanna mani, qa ecetuya ia ece; tuwe wohnayc wokamna śicedaka, on opetonpi lite cin yuza nape hdatata, weyapi nahon kte śni on noge okihmuze, qa taku śica wanyake kte śni on iśtohmuza icu ;

16 He wankan owanka kin en ounye kta; imnija conkaśke kin wowinapeye kta; aguyapi qupi ecc kta ; mini tawa kin he ohinniyan kta.

17 Niiśta wicaśtayatapi iye toiyokipi kin en wanyakapi kta ; makoce tehantu hince cin lie wanyakapi kta.

18 Nicante wonihinciye qon he kiksuye kta. Wowapi kage cin he tokiya un he ; tuwe waaspeya ece kin he tokiya un he; tuwe conliaślie yawa ece qon he tokiya un he.

19 Oyate wan ite wawinihan kin heca wandakc ktc śni; oyate wan iapi nayalion kta isanpa teliika; ceji yaśkiśka, taku kapi tanin śni kin heca.

20 Zion otonwe ohna mniunkiciyapi ece lin he wanyaka wo: Jerusalem woozi ounyanpi kin he niiśta wanyakapi kta ; wakeya kahipapi kte śni, canhutipaspe tawa icimana yujun icupi lite śni, qa taikan kin wanjidan yuksapi kte śni.

21 Tuka hen Jehowa waśake hince cin he wakpa qa wakpadan ohdakinyan tankapi kin eeunkiyapi kta; wamnaheca wata takudan he ohna ye kte śni, nakun wita wata waśaka en hiyaye ktc śni.

22 Wayaco unkitawapi kin Jehowa hce; woope kaga unkitawapi 226
Jehowa hee; Jehowa he Wicaśtayatapi unkitawapi ; iye niunyanpi kta.

23 Ikan nitawa yukcapi, can ihupa kin en sutaya iyakaśkapi kte śni: sina on watopapi kin hena yumdayapi kte śni. Helfan wawicakipi tanka wan kicipamnipi kta : taku icupi kin tona huśte iyahpayapi.

24 'Tuwe en ounye cin, Wamayazanka ce, eye kte śni ; oyate en tipi kin hena wahtanipi wicakicicajujupi nakaeś.

\section{WICOWOYAKE'?4.}

1 Ikcewicaśta, nayahonpi kta e kiyadan u miye, qa oyate yaunpi, anagoptan po: maka kin nalion kte, qa toojudan kin ; maka kin tona etanhan icagapi kin hena koya.

2 Jehowa tocanniye kin Ikcewicaśta owasin akan yanke, qa ozuye tawapi owasin wośinhda awicaun : Hena iye wicakiconza, wicaktepi kta e wicaqu.

3 Unkan wicaktepi tawapi kin hena tankan ehpeyapi kta, qa tancan țapi kin etanhan śicamna au kta, qa hena we kin on he kin hpan kta.

4 Qa mahpiya taobe kin iyulipa skan kta, qa malipiya kin hena tahasaka wan iyecen opeharipi kta, qa taobepi owasin hinhpaye kta, hastanhanka iyuwi ape hinhpaya, qa suken can etanhan ape śnije cin iyececa.

5 Han, mazasagye mitawa mahpiya kin en imnan: iho, woyaco on Edom en ahiyu kta, qa oyate wicamdaśice cin en.

6 Jehowa tamazasagye kin he we on ojudan, waśin on cepa, tahin wanunyanpi cinca qa tatokadan we on, tamdoka tapakśin sin on: Bozra en wośna wan Jeho- 
wa kicagapi nakaeś, qa wokte tanka wan Edom makoce kin en.

7 Unkan tinta tatanka hena om apamahde upi kta, qa tatanlia tatang mdoka kin om : unkan iye tamakoce kin we on imnan kta, qa maka mdu kin waśin on cepe kta.

8 Jehowa towicakiçon anpetu kin dee, Zion toakinica on wokajuju waniyetu kin hee nakaeś.

9 Hecen tawakpa kin hena canśin icage kta, qa maka mdu kin inyanzizi kta, qa makoce $\mathrm{kin}$ canśin ide eceh icage kta.

10 Hanyetu qa anpetu he kasnipi kte sni ; etanhan śota kin he owihanke wanin wankantkiya ye kta; wicoicage qa wicoicage hehanyan hewoskan wanke kta; tuwedan he opta ye kte śni, owihanke wanica.

11 Tuka mdega qa pahin he tawayapi kta; nakun hoka qa kangi hen unpi kta; qa toketu tanin śni ikan kin he ayutitanpi $\mathrm{kta}$, qa woihangye inyan kin.

12 Wicaśta tankapi kin wicaś. tayatapi kta e iwicakicopi kta, tuka wanica; qa akicita tancanpi tawa owasin sotapi kta.

13 Qa tipi waśteśte tawa kin en wapepeka uye lita, haśbe canpepe ko conkaśke tawa kin ohna: qa canotidan kin hena he tipi yapi kta, hinhankaga cinca tahocolia kin liee kta.

14 Qa tintoskan tanhan woteca kin wita etanhan wamanica kin itkowicalkipapi kta, qa hohnogeca kin he taliodaku kipan kta; nakun wanagi kin he hen yanke kta, qa woozi makoce wan iyeiçiye kta.

15 Hen wanhinkpe wamduśka kin waśun icicage kta, qa witka kada kta, qa man kta, qa taohanzi kin en mnawicaye kta; nakun heciya kangi tanka kin mniciya- pi kta, otoiyohi iye takodaku kici.

16 Jehowa tawowapi kin ohna akita po, qa wanyaka po; dena wanjidan en un śni wanice kta, otoiyohi iye takodaku kicica kta. Mii kin he de econwicaśi, qa iye taniya kin he hena mnawicaya nak:eś.

17 Iś eya hena makoce wicakipamni, qa iye nape ikan on de iwicakiciyute: hena ohinniyan tawayapi kta, wicoicage qa wicoicage hehanyan hen ounyanpi kta.

\section{WICOWOYAKE 35.}

1 Dena on tintoskan qa makoce puze cin iyuśkin kta, qa hopuza. kin he wiyuśkin kta qa onjinjintka iyecen hica kta:

2 Hcalica kta qa iyuśkin kta, han, wowiyuśkin qa dowanpi kin on. Lebanon towitan kin qupi kta, Karmel qa Śaron toiyokipi kin: Hena Jehowa towitan wanyakapi kta, Wakantanka unkitawapi toiyokipi kin.

3 Wicanape suta śni kin hena yuwaśaka po, wicahupahu waśake śni kin hena wicayusuta miye. 4 Tona cante inalinipi kin hena hewicakiya po; Ihdutinza po; kopehdapi śni po: Taku Wakan nitawapi wanhdaka po: watokiconpi u kta, Wakantanka tawokajuju kin he ; iye hinca u qa niniyanpi kta ce.

5 Hehan iśtagongapi kin iśta wicakiyukawapi kta, qa noge tpapi kin hena noge wicakiyuhidokapi kta.

6 Hehan tuwe huśte kin he taindoka wan iyecen ipsice kta, qa ia okitpani ceji kin dowan kta: hewoskan mini bohdog hiyu nakaeś, qa wakpa hopuza kin en.

7 Qa makoce aśpanśpan kin he mde icage ktạ, qa maka puze 


\section{ISAYA TAWOYUKCAN KIN,}

cin he minithdoke kta: Canotidan ounyanpi tukten wankapi fin hen cedi psa ko yuke kta.

8 Unkan canku tanka wan hen wanke kta, qa ocanku kta; qa he Woowotanna canku eciyapi kta : tuwe sape cin he ornani kte śni : tuka he iye tawapi lita: oicimanipi kin hena witkotkopi qeyaś nunipi kte śni.

9 Hen mnaja wanice kta, qa wamanica wayapote cin he ohna ye kte śni, hen iyayapi kte śni : tuka tona opewicakitonpi kin hena omanipi kta :

10 Qa tona Jehowa opewicakiton qoi hena hdicupi kta, qa iye pa akan dowanpi qa wowiyuśkin owihanke śni hduha Zion en hipi kta: wowiyuśkin wopida ko hduhapi kta, qa woiyokiśice comnihdazipi ko najice kta.

\section{WICOWOYAKE 36.}

1 Unkan Hezekiya waniyetu aketopa wicaśtayatapi he en, Senakerib Aśur wicaśtayatapi kin Juda otonwe aconkaśliapi kin owasin takpe wicau qa iwicacu. (2) Qa Aśur wicaśtayatapi kin Lakiś etanhan Rabśaka, ozuye t:ınka wan kici, Hczekiya Jerusalem en un kin he ekta ye śi. Unkan wankan miniyowe wakpa liagapi kin icahda hinajin, wayujaja tamaga canku tanka kin he oinra.

3 Hehan Eliakim Hilkiya cinhintku, tipi awanyake cin he, qa Śbona wowapi kaga ece, qa Joali Asaph cinbintku, owicawa cce kin, hena ekta hinanpapi.

4 Unkan Rabśaka hewicakiya; Ito, Hezekiya heciya po; Wicaśtayatapi tanka, Aśur wicaśtayatapi kin he heren eya ; Wowacinye kin de wacinyaye cin he tukte e he.
5 Kicizapi on wowiyukcan qa wowaśake, hena c wicoie takuśni ecedan cajemdate. Iho, tu. we e wacinyaya qa on mayaki: pajin he.

6 Iho, cedi yuksapi sagye kin de, Egupta he wacinyaya; he wicaśta wan apatan kinhan nape en iyaye ca pahdoke kta. Pharaoh Egupta wicaśtayatapi kin hececa tona. wacinyanpi kin owasin en.

7 Tuka, Jehowa Wakantanka unkitawapi kin he wacinunyanpi ce, emayakiye cinhan ; tuwe pahata tipi kagapi tawa, qa owayuśna tawa Hezekiya tokan ehpeye ca Juda Jerusalcm kici, Owayuśna kin de itokam ohoyadapi kta ce, ewicakiya, he dee śni he.

8 Heon nakaha ceciciya ce, mayuhe cin, Aśur wicaśtayatapi kin, kici ekiciya wo, kinhan śuktanka kektopawinge nonpa ciçu kta, wicaśta akan iyotangwicakiya oyakihi kinhan:

9 Hecen token mayuha taokiye itancan cistinna wanjidan qeyaś ite kin napeyakiye kta, qa canpahmihma śuktanka akan iyotankapi ko Egupta iwacinyaye kta he.

10 Qa nakaha Jehowa codan makoce kin de ihangya wau he. Makoce kin de takpe ya wo, qa ihangya wo, Jehowa emakiya ce.

11 Hehan Eliakim qa Śebna qa Joah Rabśaka heciyapi; Tokin nitaokiye Aram iapi on unkokiyapin; he unkokahnigapi: qa Juda iapi on unkokiyapi śni po, conkaśke akan oyate yukanpi kin nahonpi kta.

12 Tuka Rabśaka heya; Mayuhe cin he niyuhapi qa niyepi wicoie kin dena eciciyapi kta e umaśi he ; qa wicaśta conkaśke 


\section{WICOWOYAKE 37.}

akan iyotankapi, iyc cesdi hdutapi kta qa iye deje cin niyepi om hdatkanpi kte cin hena ekta umaśi śni he.

13 Hehan Rabśaka najin qa Juda iapi en hotankakiya hoyeye co heya; Wicaśtayatapi tanka, Ásur wicaśtayatapi, oie kin nalion po.

14 Wicaśtayatapi kin he heya ce; Hezekiya nihnayanpi śni nunwe ; iye enihdakupi kta okihi śni.

15 Nakun ihnuhan Hezekiya Jehowa wacinye niyanpi kinhan : Awicakehan Jehowa eunhdakupi kta ; otonwe kin de Aśur wicaśtayatapi nape kin en qupi lite śni ce, eya qeyaś :

16 Hezekiya anagoptanpi sni po: Aśur wicaśtayatapi kin he kaken eya; Wookiyc micaga po, qa tankan en mau po; kinhan wicaśta otoiyohi iye hastanhanka iyuwi etanhan, qa snken can tawa kin etanhan hdute kta, qa otoiyohi miniyowe nitawapi etanhan yảhdatkanpi kta :

17 Ecen wau qa makoce wan nitamakocepi kin iyececa, wamnaheza miniśa ko malsoce, aguyapi qa hastanhanka iyuwi ojupi makoce kin ekta aciyapi kta cc.

18 Jehowa cunhdakupi kta ce, Hezekiya eye cin on ihnuhan awacinniyanpi kinhan. Oyate tokeca taku wakan yuhapi kin iyc tamakoce Aśur wicaśtayatapi nape kin etanhan elidaku he.

19 Hamat qa Arpad taku wakan tawapi lin hena tokiya un he: Sepharvaim taku wakan tawa kin tokiya un he : qa Samaria minape etanhan eyakupi he.

20 Makoce kin hena owasin taku wakan tawapi kin tukte wanji iye tamakoce minape etanhan ehdaku he; hccen Jehowa Jeru- salem minape etanhan ehdaku kta, eya.

21 Tuka inina yukanpi qa oie wanjidan ayuptapi śni : wicaśtayatapi kin hecen econ wicaśi, qa, Takudan ayuptapi śni po, eye ciqon.

22. Hehan Eliakim Hilkiya cinhintku tipi awanyake cin he, qa Sebna wowapi kage cin, qa Joah Asaph cinhintku owicawa ece kin, hena wokoyake hdupotapi, Hezekiya en hdipi qa Rabśaka oie kin okiyakapi.

\section{WICOWOYAKE $3 \%$.}

1 Unkan Hezekiya he nation qehan, tawokoyake hdulidece, ca wakihdaka ogekiton, qa Jehowa ti kin en tin iyaya:

2 Qa Eliakim tipi awanyake cin he, qa Śbna wowapi knge cin, qa wawayuśna itancanpi kin hena wakihdaka ogekitonpi Isaya Amoz cinhintku wicaśta wokcan kin ekta ye wicaśi.

3 Unkan hena heciyapi " Hezeliya hecen eya ce; Anpetu kin de woiyokśice qá wośitkihda qa woiyopeye anpetu kin hee: hokśiyoqopa hiyupi kta wanna en hipi, tuka hiyuwicayapi kta e wowaśake yuke śni.

4 Okinni Jehowa Wakantanka nitawa Rabśaka oie lin nalion kta, he iye yuhe cin Aśur wicaśtayatapi kin Taku Wakan ni un kin yaśice kta e uśi, qa wicoie Jehowa Wakantanka nitawa nalion kin hena iyopeyc kta : heon etanhan tona okaptapi en unpi kin hena on worekiye hdawankan ewicayeciye kta.

5 Unkan Hezekiya wicaśtayatapi taokiye kin Isaya en hipi.

6 Unkan Isaya hewicakiya; Niyuhapi kin hecen eyakıyapi kta ; Jehowa kaken eya ce; Wi229 


\section{ISAYA TAWOYUKCAN KIN,}

coie nayahon kin on $A$ śur wicaśtay atapi taokiye wicaye cin mayaśicapi kin hena kokipe śni wo.

\% Tho, worriya wan en yewaśi kta, nakun woyake wan nation lita, qa iye tamakoce kin ekta hde kta: qa iye tamalioce kin en mazasagye on hinlipaye waye lita ce.

8 Hecen Rabśaka hdicu qa Aśur wicaśtayatapi Libna kici kiciza en hdi: Lakiś etanhan kihda lie nahon tuka qon.

9 Unkan iye Tirhaka Kunś wiraśtayatapi on heyapi nahon; Nicize kta e wanna hiyu ce. $\mathrm{Qa}$ he nahon qehan Hezelkiya ekta wahośi yewicaśi qa hoya;

10 Hezekiya Judi wicaśtayatapi kin oyakiyapi qa kaken eyakiyapi kta; Ihnuhan Taku Wakan nitawa wacinyakiye cin he nihnayan kinhan, on hehe cin, Jerusalem Aśu wicaśtayatapi nape kin en qupi kte śni ce.

11 Iho, Aśur wicaśtayatapi kin makoce owasin token ecawicakiconpi.kin he niye nayahon, hena ocowasin ihangyapi; unlian niye enihdakupi $\mathrm{k}$ ta he.

12 Ikcewicaśta oyate tona atewicaye cin ihangyapi qon hena taku wakan tawapi ewicahdakupi he: Gozan, qa Haran, qa Rezep, qa Eden cincapi Telasar en unpi kin.

13 Hamat wicaśtayatapi kin tokiya un he, qa Arpad wicaśtayatapi, qa Sepharvaim otonwe, Hena qa Ivah en wicaśtayatapi lin tokiya un he.

14 Unkan wahośiyapi napepi etanhan Hezekiya wowapi kin he icu qa yawa. Hehan Hezekiya Jehowa ti kin ekta ye ca he Jehowa itokam yumdaya.

15 Qa Hezekiya Jehowa cckiye ça heya;

16 Jehowa wicobe tawa, Israel 230
Taku Wakan tawa, Kerubim oyati, maka akan wokiconze owasin en niye niśnana Wakannitanka; niye mahpiya qa maka kin yakaga :

17 Jehowa anohye ca nahon wo; Jehowa niiśta hdumdaye ca wanyaka wo; qa Senakerib oie kin, on Wakantanka ni un yaśice kta e u wicaśi kin, hena owasin nahon wo.

18 Jehowa, awicakehan Aśur wicaśtayatapi kin maka ounyanpi owasin qa malioce tawapi kin yutakunipi śni ;

19 Qa taku wakan tawapi kin peta en qupi ; hena taku wakan śni nakaeś, tuka wicaśta nape taku kagapi kin heca, can qa inyan, heon etanhan hena ihangyapi.

20 Heon nakaha Jehowa Wakantanka unkitawapi, he nape etanhan niunkiya po; hecen maka wokiconze kin owasin Jehowa he niye, niye niśnana e sdonyapi kta.

21 Hehan Isaya Amoz cinhintku Hezelsiya ekta yewicaśi qa heciya; Jehowa Israel ta Wakantanka kin hecen cya; Senakerib Aśur wicaśtayatapi kin on wocekiye emayakiye cin heon etanhan,

22 Jehowa oie wan eciye cin he dee: Wikoślka Zion cunwintku kin he wahitenidaśni, nina inihaha: Jerusalęm cunwintku kin niye on pomnamna ce.

23 Wawiyopeyaye ça daśice cin he tuwe he: tuwe on ho hdawankan iyeyaye ça nüśta yahduwankan he : Israel Towakan kin hee.

24 Nitaokiye napepi on Itancan kin iyopeyaya, qa heha; Canpahmilima ota mitawa on he paha kin elita hibu, Lebanon opapun kin ekta ; qa hante śa tankinyan- 
yan qa wazi can kahnigapi kin hena wakawanke kta, qa wankan ihanke kin ekta wai lsta, qa maga waśteśte contanka kin en.

25 Miye maka waqe ca mini mdatkan; qa misiha sicu kin on otonwe aconkaśkapi wakpadan kin owasin oyahewaya ce, eha.

26 Wanakaja taku ecamon kin he nayalion śni he; ehanna anpetu kin en he wakaga: Dehan nakaha ecetuwakiye, niye otonwe aconkaśkapi kin wicotakuniśni on ihangyaye kta:

27 Heon en ounyanpi kin hena nape ptecedan, nihinciyapi qa wacinhnunipi; hena maga wato kin iyececapi, peji toto, tipi alahpapi kin ekta wato, qa taku icage śni itokam śnija he iyececapi. 28 Tuka tukten inunke cin he miye sdonwaya, qa tankan idada, qa tin hidu ece, qa canniyemayaye cin nakun:

29 Canniyemayaye ca nihdatanka kin hena noge en hiyomahpaya, heon etanhan maziyukśan mitawa nipoge en iyewaye kta, qa iiyuwi mitawa niiha en, qa canku ohna hidu qon he ohna hdicu ciciye kta ce.

30 Unlkan de wowakta yaye kta ; Omaka kin de taku iye cinka icage cin etanhan yatapi kta ; qa ornaka inonpa taku he etanhan icage cin hena: tuka mdoketu iyamni hehan woju po, qa wamnayan po, qa hastanhanka iyuwi huhde po, qa waskuyeca etanhan hduta po. 31 Unkan tona najicapi, Jùda tiyohnaka etanhan okaptapi kin, hena ake kuyatanhan hutkan icagapi kta, qa wankantanhan waskuyeca icaliyapi kta.

32 Jerusalem etanban wookapte hiyu kta, qa Zion etanhan niiciyapi yuke kta: Jehowa wicobe tawa kin he tocantekiye kin hecon kita ce.
33 Heon etanhan Jehowa Aśur wicaśtayatapi kin on kaken eya; Otonwe kin de en hi kte śni, qa wanhinkpe wanji en hiyuye kte śni, qa wahacanka hduha itokam u lte śni, qa ihdukśan maka qe kte śni :

34 Canku ohna hiyu qon he ohna ake hde kta, qa otonwe kin de en u kte śni, Jehowa eya.

35 Miye on etanhan qa mitaokiye Dawid on etanhan otonwe kin de niwaye kta e awahdalipe kta ce.

36 Hehan Jehowa tawahośiye wan iyaye ca Aśur oyate wankapi kin en kektopawinge opawinge qa kektopawinge wikcemna śahdogan sanpa kektopawinge zaptar wicakaśtaka. Unkan hanhianna hin kiktapi hehan, inyun, hena owasin ta wankapi.

37 Hecen Senakerib Aśur wicaśtayatapi kin ihdake ca kihde ça hdicu qa Ninewa elrta eti.

38 Qa iye taku wakan tawa Nisrok ti kin ohna cekiya icunhan iye cinhintku Adramelek qa Śarezer mazasagye on hdaśtalrapi ; qa Ararat makoce kin en niiçiyapi. Unkan cinhintlku Esar-hadon iye eekiya wicaśtayatapi yanka.

\section{WICOWOYAKE 38.}

1 Anpetu kin hena en Hezekiya te kta wayazanka. Unkan Isaya wicaśta wokcan Amoz cinhintku kin he en hi qa heciya; Jehowa hecen eya ce; Yati kin hduwiyeya wo; nițe kta qqa yani kte sni, heon etar.han.

2 Hehan Hezekiya tiyonaptan ektakiya ite hduhomni qa Jehowa cekiya,

3 Qa heya; Hehehe, Jehowa ceciciya ce, wowicake en qa cante ecetu hduha nitokam ma231 


\section{ISAYA TAWOYUKCAN KIN,}

wani, qa niiśta kin en taku waśte ecamon ece kin he dehan kiksuya ye, eya. Unkan Hezekiyu woceye tanka on ceya.

4. Hehan Jehowa oie kin Isaya en hi qa heya;

5 Ya wo qa Hezekiya heciya wo; Jehowa Dawid ateyaya ta Wakantanka kin he hecen eya ce; Nitawocelriye kin he nawahon, iśtamnihanpe nitawa wanmdaka; iho, nitaanpetu kin en waniyetu akezaptan ewaju kta.

6 Qa Aśur wicaśtayatapi nape kin etanhan ecihdaku lita, niye qa otonwe kin de; qa otonwe kin de awahdahpe kta.

7 Qi Jehowa taku cajeyata wicohan kin de econ kta e Jehowa etanhan decen wowakta yaye kta:

8 Tho, iyadipi ohanzi kin hdicuwakiye kta, Ahaz iyadipi tawa en anpetu wi kici iyayc cin, he iyadipi wikcemna hekta hdicu kta, eya. Unkan anpetu wi kin iyadipi wikcemna hdicu, iyadipi kin hena wanna iyaya nakaeś.

9 Hezckiya Juda wicaśtayatapi kin wayazanke ca wayazanke etanhan kini qon. he ehan tadowan kin he dee :

10 Miye hepe ciqon;

Mitaanpetu kaksapi en Hades tiyopa ekta mde kta;

Mitawaniyetu okaptapi $\mathrm{kin}$ hena makipi.

11 Jah wanmdake kte śni ce, epa,

Jah wicani makoce kin ohna:

Icimana wicaśta wanmdake kte śni,

Nakun maka akan ounyanpi kin hena.

12 Mitoicage kin he ihdag iyaya, Qa tahinca awanyaka tawakeya iyecen cmiciyakupi :

Wakazonta wan iyecen wani kin wahduksa: 232
Halionta etanhan iye mayukse kta :

Anpetu etanhan hanyetu elita owihanke mayaye kta.

13 Hanhanna hehanyan mitokam ewahde ;

Mnaja iyecen mahuhu iyulipa yuwehwege lita;

Anpetu etanhan hanyetu elita owihanke mayaye lkta.

14 Icapśinpśincadan ohomni kinyan he iyecen wahotonton;

Tinwakiyedan iyecen waceya :

Wankan etonwanpi on iśta matke :

Woiyokiśice miciun, Jchowa anamakikśin wo.

15 Token cpe kta he;

Iye omakiye ça nakun iśnana he hduśtan;

Minagi iyokiśica on waniyetu mitawa owasin iwaśtedan mde kta.

16 Itancan, dena on wicani ece ;

Qa dena owasin on mitaniya ni un :

Hecen asnimayaye ça nimayaye kta.

17 Iho, wookiye on wicocante śice hinca imicicaga ;

Qa minagi canteyakiya on wicotakuniśni woha etanhan edaku :

Wawahtani owasin nilieyata ehpeyaya nakaeś.

18 Hades niyatan kte śni, wiconțe inidowan kte śni;

Tona wolia kin ekta iyayapi hena nitowicake apepi kte śni.

$19 \mathrm{Ni}$ un kin, ni un kin he niyatan kta,

Anpetu kin de ecamon iyececa ;

Ateyapi kin iye cinca nitowicake kin sdonycwicakiye kta.

20 Jehowa nimaye cin liee: 
Hecen candowankiyapi on mitadowan unkahiyayapi kta, Anpetu unnipi kin owasin,

Jehowa ti kin ohna.

21 Isaya heye ciqon; Suken waskuyeca kapanpi icupi kta qa śiyaka ahnakapi kte, kinhan asni kta ce.

22 Nakun Hezekiya, Jehowa ti ekta mde kta e wowakta tukte e lie, eye ciqon.

\section{WICOWOYAKE 39.}

1 He ehan Merodak Baladan, Baladan cinhintku Babel wicaśtayatapi kin he wowapi qa taku qu kin iyahna Hezekiya kaye wicaśi : he wayazanka qa akisni nalion kin heon.

2 Unkan Hezekiya hena on iyuśkin, qa tipi ohna wakihnake cin he wanyagwicakiya, mazaska, qa mazaskazi, qa taku waśtemna, qa wihdi waśte, qa tawotawe tipi kin ocowasin, taku mazopiye tawa kin en iyeyapi kin iyulipa koya; taku iye ti kin ohna qa tośkanśkan ocowasin en Hezekiya wanyag wicakiye śni wanica.

3 Hehan Isaya wicaśta wokcan Hezekiya wicaśtayatapi kin en hi qa heciya; Wicaśta kin dena taku eyapi he; qa tokiyatanhan hipi he. Unkan Hezekiya heya; Makoce wan tehan tanhan $\mathrm{Ba}$ bel hee etanhan en mahipi.

4 Hehan heya; Yati ohna taku wạnyakapi he. Unkan Hezekiya ayupta ; Wati ohna taku owasin wanyakapi; taku wehnake cin taku wanyagwicawakiye śni wanica, eya.

5 Unkan Isaya Hezekiya heciya; Jehowa wicobe tawa he oie kin nalion wo:

6 Iho, anpetu kin u kta, hehan taku yati ohna yanke cin iyuhpa, qa niyatepi taku kihnakapi anpetu kin dehanyan, hena owasin Babel ekta ayapi kta ; takudan okaptapi kte śni ce, Jehowa eya.

7 Qa nicinca niye etanhan inanpapi kta, tona cincawicayaye cin etanhan awicayapi kta; qa hena Babel wicaśtayatapi ti kin ohna wowidake unpi kta ce.

8 Hehan Hezekiya Isaya heciya; Jehowa oie odake cin he waśte. Nakun, Wookiye qa wowicake mitaanpetu kin en un kta ce, eya.

\section{WICOWOYAKE 40.}

1 Wacintonhnagya po, mitaoyate wacintonhnagya miye, nita Wakantanka eya.

2 Jerusalem cante kin okiya po qa hoyekiya po, toozuye kin yuśtanpi, taku śica econ qon hena kicicajujupi; iye wahtani owasin on Jehowa nape kin etanhan nonpa akihde kicupi nakaeś.

3 Wicaho wan hewoskan hoyeya; Jehowa tacanku kin yuwiyeya po; hewoskantu Wakantanka unkitawapi canku tanka wan owotanna kicaga miye.

4 Kaksiza otoiyohi wankan kagapi kta, qa lie paha ko otoiyohi kun icupi kta; qa hośkiśki kin makomdaya kta, qa tahtaga kin he yumdayapi kta:

5 Qa Jehowa towitan kin yutaninpi kta, qa he wicacelipi owasin yuwitaya wanyake kta; Jehowa i kin he hecen eya nakaeś. 6 Wicaho kin he, Hoyeya wo, eya. Unkan, Taku eyanwapaha kta he, eya. Wacacehipi iyuhipa wato, qa towaśte kin ocowasin maga wahica kin iyececa :

7 Wato kin śnija, wahca kin san aya; Jehowa taniya kin atateyanpa nakaeś: awicakehan oyate kin wato heca. 


\section{ISAYA TAWOYUKCAN KIN,}

8 Wato kin śnija, wahica kin san aya: tuka Taku Wakan unkitawapi oie kin he ohinniyan un kta.

9 Zion, wotanin waśte eyanyapaha kin, paha tehanwankantu ekta ihdoya wo ; Jerusalem, wotanin waśte eyanyapaha kin, waśagya niho hdawankan iyeya wo: Hdawankan iyeya kokipe śni wo; Taku Wakan nitawa wanhdaka miye, Juda otonwe kin ewicakiya wo.

10 Tho, Jehowa Itancan kin wowaśake hduha u kta, qa iye is to lkin he wawidagye kta: Iho, iyopeyapi hduha un, qa tokajuju kin iye itokam yanka.

11 Wonwicaya wan iyececa iye optaye tawa kin wonwicakiye kta ; isto on tacincadan mnawicakiye kta, qa maku ohna wicahduhe kta: tona azinwicakiye cin hena iwaśtedan awicaye kta.

12 Tuwe mini kin nape cokaya ohna iyuta he, qa napapaśdecapi on mahpiya kin iyuta : qa maka mdu kin wiyutapi wan en onakitaka, qa he kin hena on aspeyetonpi en, qa paha kin on tkeutapi on aspeyeton kin he tuwe he.

13 Tuwe Jehowa Taniya kin wahokonkiya he, qa iś wicaśta wiyukcan tawa; hecen waonspekiye kta he.

14. Tuwe he kici wohdaka, qa wokahnilikiya, qa woyaco canku en onspekiya, qa wosdonye onspekiya, qa wookahnige canku kin wanyagkiya he.

15 Tho, Ikcewicaśta kin cega etanhan śbuyapi wan iyececapi qa on aspeyetonpi akan watuśekśeca iyecen yawapi : iho, wita kin hena taku śni seiyecen yuwankan icu kta.

16 Qa Lebanon ideyapi kta henakeca śni, qa wamanica tawa 234 kin hena woliulnage wośnapi on henakeca śni.

17 Iye itokam Ikcewicaśta iyuhpa takupi śni; qa hena taku wanica qa taku śni hdawa ece.

18 Hecen tuwe e Wakantanka idacinpi kta he; qa wiyacinpi kin tukte e he iyececa yadapi kta he.

19 Wakagapi wan wabago kin kaga, qa mazazikage cin he mazaskazi on akahpa, qa mazaska icicahiha kicaga.

20 Tuwe wośna iwahipanica kin he can wan ponpon kte śni hdahnige kta; wabago wayupika wan akite kta; wakagapi bagopi wan yuhohopi kte śni kin heca ehde kta.

21 Sdonyayapi sni he; nayahonpi śni he ; otokaheya tanhan he oniciyakapi śni he; maka kagapi kin ehantanhan he oyakahnigapi śni he;

22 He maka mihbe akan iyotanke cin hee, unkan tona ounyanpi kin hena psipsicadan iyececapi: he ozanpi wan iyecen mahpiya kin hdumdaya, qa hena wakeya otipi kta iyecen hdutitan :

$23 \mathrm{He}$ iye wicaśta itancanpi kin wicayutakuni śni; maka akan wayaco yukanpi kin hena takuśni iyececa wicakaga ce.

24. Han, hena huhdepi śni ; han, hena ojupi śni ; han, hena tancanpi maka en hutkan icagapi śni : tuka iye hena awicatateyanpa, unkan śnijapi kta, qa tateiyumni kin hena kahbog iyewicaye kta, aguyapi ha iyececa.

25 Hecen tuwe e imayadacinpi kta qa iyemacece kta he, 'Tuwe Wakan kin he hecen eya.

26 Niiśtapi wankan yeya po, qa wanyaka po, tuwe dena kaga he, wicayawa iyecen obepi kin dena hinanpe wicaye cin he tu- 
we he : tookihi tanka kin on hena owasin cajeyata; wowaśake on waśake hinca he on wanjidan okapte śni.

27 Mitacanku Jehowa anakicihimanpi, qa Taku Wakan mitawa mitawoyaco kin acakśin ce, tokeca Jakob, hecen ehe kta he, qa Israel, tokeca hecen iyaa he.

28 Sdonyaye śni he; nayahon śni he ; Taku Wakan Owihanke wanica, Jehowa, maka ihanke kage cin he hanye kte śni, qa mdokite kte śni : tookahnige kin he iyukcanpica śni.

29 'Tona mdokitapi kin hena wookihi wicaqu, qa tona takudan okihipi śni kin hena wowaśake wicakicaga ece.

30 Kośka kin heepi kaeś mdokițapi qa hanyanpi kta, qa wicakahinigapi kin hena waśakapi śni, waśakapi kte śni :

31 Tuka tona Jehowa apepi kin hena sanpa ihduwaśakapi kta, wamdi iyecen hupahu on wankan yapi kta ; hena iyankapi kta, tuka mdokițapi kte śni; manipi kta, tuka hustakapi kte śni.

\section{WICOWOYAKE 41.}

1 Wita, mitokam inina yukan po ; kinhan oyate kin ihduwaśakapi kte; hena kiyedan upi kte; hehan iapi kte; woyaco oiyotanke kin en witaya kiyedan unkupi kta.

2 Tuwe wiyohiyanpatanhan wicaśta owotanna kin yulica he, iye siha ekta kico, qa itokam Ikcewicaśta kin qu, qa wicaśtayatapi wowidagyekiya he; watuśekśeca iyecen iye tamazasagye kin en, peji okahbogyapi kin iyecen iye tinazipe kin en wicaqu.

3 Hena kuwa awicaya, tanyan iyopta, iye siha canku wan ohna ye śni kin he ohna.

4 'Tuwe he kage ça hduśtan, otokaheya tanhan wicoicage kin cajewicayata he : Jehowa miye, tokaheya qa ehake un kin he miye ce.

5 Wita kin hena wanyakapi qa kokipapi ; maka ihanke kin hena cancanpi; kiyedan hiyupi qa hipi.

6 Wicaśta otoiyohi iye takodaku kin okiciyapi kta, qa otoiyohi iye sunkaku, Waśaka wo, ekiciyapi kta.

7 Hecen wabago waśdoya kin waśagye ; tuwe maziyape on kaśduśdute cin he maziyapapi ape cin he waśagya; qa mazaśdoyapi kin he waśte ce, eya: Unkan iyokatkuge on kasutapi, hecen yuhohopi kte śni.

8 Tuka Israel mitaokiye, Jakob wahdahnige cin, mitakoda Abraham cinca kin he niye :

9 Maka ihanke kin etanhan ecihdaku, qa hunnaptan tanhan cico qa heciciya; Mitaokiye kin he niye ; cihdahnige ca elipeciye śni ce.

10 Wakokipe śni wo, cici waun nakaeś; nihinciye śni wo, Wakantanka nitawa kin he miye : ciyuwaśaka ; han, ociciya : han, mitoowotanna nape etapa kin on ciyuza.

11 Iho, tona canniyeniyanpi kin hena owasin iśtecapi qa nihinciyapi kta; hena takuśni iyececapi kta; qa wicaśta nicigepi kin hena atakunipi kte śni.

12 Wicaśta nicigepi qon hena awicayakite kta tuka iyewicayaye kte śni ; wicaśta nicizapi kin hena taku śni qa taku sotapi kin iyececapi kta.

13 Jehowa Taku Wakan nitawa, nape nietapa yus un kin he miye nakaeś: Kokipe śni wo ; 235 


\section{ISAYA TAWOYUKCAN KIN,}

ociciya ce, tuwe eniciye cin he miye.

14 Jakob wamdudan, wakokipe śni wo, qa Israel wicaśta kin; miye ociciye kta ce, Jehowa, qa tuwe openiciton, Israel Towakan kin he hecen eya.

15 Iho, mazicapan pe hinca teca hi yulan heca cicage kta; lie kin hena yalkapan qa yakandu kta, qa paha kin hena aguyapi ha iyececa yakage lita.

- 16 Hena yakaduge lita, unkan tate kin hena yuwankan iwicacu kta, qa tateiyumni kin hena kahoya ehpewicaye kta: Tuka niye Jehowa on iduśkin kta, Israel Towakan kin on nihdatan kta.

17 Onśikapi qa wahipanicapi kin hena mini odepi, unkan wanica, ipuzapi on ceji sakapi ; hehan Jehowa miye nawicawalion kta, Israel 'Taku Wakan tawa lin miye hena elpewicawaye kte śni.

18 Hopuza en wakpa mduhdoke kta, qa kaksiza cokaya miniyowe kta: tintoskan kin he mimilidoka qa makoce puze cin he miniyowe wakage kta.

19 Ḣante śa, sitta can, qa hedas can, qa wilhdi can hena tintoskan owaju kta ; wazisaka can utulıu can, qa wazi can hewoskan makoce kin en witaya huwahde kta.

20 Hecen wanyakapi kta, qa sdonyapi kta, qa iyukcanpi kta, qa witaya okahnigapi $\mathrm{kta}$; Jehowa nape kin he de econ qa Israel Towakan kin he kaga.

21 Woakinica nitawapi kin aku po, Jehowa eya: Wicoie waśaka nitawapi hduha kiyedan u po, Jakob ta Wicaśtayatapi kin eya. 22 Hena aupi kta, qa taku u kte cin wanyagunyanpi kte; taku otokahe ekta un qon hena 236 yutaninpi kta, hena taku hecinhan, hecen hena awauncinpi kta, qa hena owihanke kin sdonunyanpi kta; qa iś taku u kte cin hena unkokiyakapi kte.

23 'Taku tokata u kte cin hena pazo po, hecen taku wakan henicapi sdonunyanpi kta: han, taku waśte qa iś taku śica econ po, kinliar ekta euntonwanpi kta qa wanunyakapi kta.

24 Tho, takuśni enitanhanpi, qa niohanpi kin taku śni; tuwe nicalinigapi kin he wowalite śni.

25 Waziyatanhan tuwe mdulica, unkan he u kta ; wihinanpatanhan micaje hoyemakiye kta; wicaśtayatapi kin en wicau kta, upśija iyecera, qa wakśica kaga wan maka nacoco kin iyececa.

26 Tuwe otokaheya tanhan oyaka he; hecen sdonunyanpi kta; qa itokamtu, hecen, Owotanna ce, unkeyapi kta: Han, tuwedan wayuotanin śni; han, tuwedan woyake śni; han, tuwedan nioiepi nahon śni.

27 Tokaheya kin he Zion heciye kta; Wanyaka, wanwicayaka wo: unkan tuwe wotanin waśte au kin he Jerusalem waqu lita.

28 Unkan ewatonwan, tuka wicaśta wanica; han, hena ekta, tuka wiyukcan wanica; qa hena wiwicamunga, tuka oie wanjidan amayuptapi śni.

29 Iho, hena iyulipa takupi śni, iye olianpi kin taku śni; wakagapi śdoyapi yuhapi kin hena tate qa toketu taninśni.

\section{WICOWOYAKE 42.}

1 Tho, mitaokiye sutaya wahduze; wahdahinige cin minagi iyuśkin kin hee; he Mitaniya awahnaka; iye wokonze 
Ikcewicaśta kin wicakaye kiye kta.

2 Pan kte śni, qa hdawankan iyeye kte śni, qa canku ohna ho hdatanin kte śni.

3 Cedi kaśnjapi kin he yukse kte śni, qa hahonta kitanna ide kin he bosni kte śni: wowicake ekta wokonze au kta.

4 lye hanye kte śni qa wacin ibośake kte śni, ecen wokonze maka kin en ekihde kta: qa wita kin hena iye toope kin apepi kta.

5 Jehowa Wakantanka, mahipiya kin kage ca hdumdaya ; tuwe maka kin kamdaya qa taku etanhan uye cin; tuwe oyate dkan unpi wiconi wicaqu, qa tona amanipi woniya wicaqu ece kin he hecen eya:

6 Jehowa mije woowotanna en cico, nakun nape cihduze $k$ ta, qa cipatan kta, qa oyate wicotakuyc tawapi kta, qa lkcewicaśta ekta iyoyanpa cicage kta.

7 Iśta tonwanpi śni kin wicadumdaye kta, wicakaśkapi kin wicokaśke etanhan hiyuwicayaye kta, tona otpaza en iyotankapi okaślke tipi kin etanhan.

8 Jehowa he miye; micaje kin hee: unkan mitowitan kin he tuwe tokeca waqu kte śni, qa mitoyatan kin taku bagopi kin waqu kte śui.

9 Tho, taku tokaheya qon hena yuecetupi, nakun taku teca omdaka ece; hena icage śni itokam nahonciyapi kta.

10 Odowan teca wan Jehowa kahiyaya po, iye toyatan maka ihanke kin etanhan, tona miniwanca ekta idadapi qa taku on ojudan; wita kin hena, en ounyanpi kin hena koya :

11 Tintoskan qa en tonwanyanpi ho wankan yeyapi kte, Kedar en tonwanyanpi kin hena : imni- ja ounyanpi kin hena dowanpi kte, he ipa kin etanhan iyaśapi nunwe.

12 Wowitan Jehowa qupi nunwe, qa iye toyatan kin he wita kin ekta oyakapi kte.

13 Wicaśta waśaka iyecen Jehowa hinanpe kta: wicaśta zuye cin iyecen wowinawizi hduhice kta: iyaśa kta, han, ho hdatanin kta; tokawicaye cin on ihduwaśake kta.

14 Wanakajatanhan inina manka; ie śni waun ; tinsmiçiya ce ; tuka nakaha, winohinca hokśiksuya iyecen waśicahowamde kta ; wamdapote kta qa wancahna wanawapce kta.

15 He qa paha kin hena hewoskan wakage kta, qa wato en un kin iyulipa mduśnije kta ; qa wakpa kin hena iliewita wakage kta; qa mdedan kin hena mdupuze kta :

16 Qa canku wan sdonyapi śni kin he olma iśtagongapidkin maniwicawaye kta.; ocanku sdonyapi śni kin ohna yus awicawau kta: wicitokam otpaza kin he iyoyanpa kta, qa taku śkośkopa owotanna wakage kta. Dena taku kin ecawicawecon kta, qa awicamduśtan kte śni.

17 Tona wakagapi bagopi wacinyanpi, tona wakagapi śdoyapi kin, Taka wakan unkitawapi kin he niyepi ce, eyapi kin hena nina iśtecapi kta, hena hekta hdicuwicayapi kta.

18 Noge nitpapi, nalion po ; qa iśtanigongapi, etonwan po, hecen wandakapi kta ce.

19 Tuwe mitaokiye iyecen iśtagonga he; qa tuwe wahosilya mitawa yewaśi kte cin iyecen nogetpa he: tuwe koda iyecen iśtagonga, qa tuwe Jehowa taohiye iyecen iśta wanica he.

20 ' 'aku ota wandaka, tuka 237 


\section{ISAYA TAWOYUKCAN KIN,}

awacanni kte śni: noge yuldokapi, tuka wanahon kte śni.

21 Jehowa iye toowotanna on iyuśkin hinca; woope kin he hdutanka qa hduonihan kta.

22 Tuka oyate kin de wicakipi qa waboticapi; hena owasin makohidoka ohna wicayuzapi, qa wicokaśke tipi ohna nawicalimanpi : hena wayaka awicayapi, qa tuwedan ewicahdaku śni ; wawicakipi qa tuwedan, Wicakicu wo, eye śni.

23 Niyepi etanhan tuwe de awacin kta he; tuwe anohkiye ca tokata anagoptan kta he.

24 Jakob wakipi kta wicaqu, qa Israel wamanonpi en hiyuwicaye cin he tuwe he: Jehowa waunkicihtanipi kin hee śni he. Iye tacanku kin ohna mani wacinpi śni, qa toope kin anagoptanpi śni nakaeś.

25 Heon etanhan iye tocanniye wohitika qa wicokicize wowaśake kin he akaśtan : unkan he ohomni aidekiya, tuka sdonye śni ; qa iye guya tuka cante ekta yus wacin śni.

\section{WICOWOYAKE 43.}

1 Tuka wanna Jakob, tuwe nicage cin he, qa Israel, tuwe icaliniye, Jehowa hee hecen eya; Wakokipe śni wo, opeciciton nakaeś; nicaje on cico, tawaciya.

2 Mini kin ehna idada kinhan cici waun kta; qa wakpa kin opta, he akam iniyaye kte śni; peta ehna mayani kinhan nigu kte śni, nakun ide kin ainide kte śni.

3 Jehowa Wakantanka nitawa kin he miye nakaeś, Israel Towakan, Wanikiya nitawa; opeciciton kta on Egupta waqu, niye on Kuś qa Seba iyopewaya.
4 Miiśta en nitelikika heon niyuonihanpi, nakun miye waśte. cidaka; heon etanhan niye on wicaśta wicawaqu kta, qa oyate ninagi on.

5 Wakokipe śni wo, miye cici waun nakaeś; wicoicage nitawa wiyohiyanpatanhan awaku kta, qa wiyolipeyatanhan mnaciye kta :

6 Waziyata kin hewakiye kta ; Hiyuya wo; qa itokaga kin; $\mathrm{Pa}$ tan śni wo; tehantanhan micinkśi awicau wo, qa micunkśi kin maka ihanke kin etanhan:

7 Tona micaje on caśtonpi qa mitowitan on wakaga owasin ; miye wakaga nakaeś, han icahiwaya.

8 Oyate iśtagongapi kin hena hiyuwicaya wo, qa tona iśta yukanpi; qa nogetpapi, qa tona noge yukanpi :

9 Ikcewicaśta owasin witaya upi kta, qa oyate kin mniciyapi kta; hena etanhan tuwe he de oyake kta, qa taku otokahe ekta un qon unkipazopi kta he: wayaotanin wicahdoupi kte, hecen ihdaowotanpi kta; qa iś nahonpi qa, He wowicake ce, eyapi lite.

10 Wayaotanin mitawa kin he niyepi, qa mitaokiye wahdahinige ciqon, Jehowa eya; liecen sdonyayapi qa wicamayadapi kta, qa he miye e oyakahnigapi kta : mitokam taku wakan takudan kagapi śni, qa mihakam wanicc kta.

11 Jehowa he miś miye: qa micunonpatanhan Wanikiya wanica.

12 Miye womdake ca waniwakiya qa wamdaotanin, wanji tokeca niyepi ehna un śni qehan; heon etarhan wayaotanin mitawa kin niyepi, Wakantanka he miye e, Jehowa eya. 


\section{WICOWOYAKE 44.}

13 Han, anpetu kin he itokam he miye; qa tuwedan minape etanhan waeyaku ukihi śni : miye taku ecamon kta, qa tuwe anapte lita he.

14 Jehowa Wanikiya nitawapi, Israel Towakan kin he hecen eya; Niyepi on Babel ekta yewicawaśi, qa wonajice tawa iyuhpa kun ehpewicawaya, nakun Kasdim oyate wita wata ohna iyaśapi kin hena.

15 Jehowa 'Taku Wakan nitawapi, Israel Icahwicaye cin, Wicaśtayatapi nitawapi kin he miye ce.

16 Tuwe miniwanca ohna canku wan kage ça mini tanka en ocanku wan ;

17 Tuwe canpahmihma qa śuktanka, ozuye qa wowaśake hiyuye cin, Jehowa hee hecen eya; Witaya iwankapi kta, ake inajinpi lste śni : hena atakunipi śni, hahonta ide kin iyecen kasnipi ce.

18 Taku tolkaheya qon hena kiksuyapi śni po; qa taku tanina un kin hena awacinpi śni po.

19 Iho, taku teca wan ecamon; wanna icage kta; he sdonyayapi kte śni he. Han, tintoskan canku wan wakage kta, hewotahedan wakpa wanke kta.

20 'Tinta wamanica kin, unktehi qa hinhankaga cinca hena mayuonihanpi kta; tintaoskan mini hiyuwaye, liewotahedan wakpa wicawaqu, mitaoyate wahdahnige ciqon mini yatkewicawakiye kta heon.

21 Oyate kin de miye miçicaga: hena mitoyatan yaotaninpi kta.

22 Tuka Jakob, hoyemayakiye śni; Israel, miye on mdoyakite ciqon:

23 Wohuhnage nitawa tahinca cinca mayakahi sni; qa nitawośna on mayaduonihan śni. Wo- śnapi on wowidagciye śni, qa wowizite on iyotanhan iyeciciye śni.

24 Mazaska on sinkpe tawote takudan opemiyeciton śni, qa nitawośna cepa on imnamayaye śni : tuka wayalitani on wowidag mayaya, taku sica ecanon kin on iyotanhan iy emayakiya.

25 Miye, miye hinca on, tuwe taku śica nitawa nicicajuju kin he miye; qa wayalitani kin hena weksuye kte śni.

26 Kiksuyemayan ye ; unkiciyukcan kta: nihdaowotanna kta e on wohdaka wo.

27 Niyate tokaheya qon he walitani, qa tona waonspeniciyapi kin hena makipajinpi.

28 Heon etanhan tipi wakan itancanpi kin wicamdaśica, qa Jakob woyaśice en iyewaya, qa Israel woiyopeye ekta.

\section{WICOWOYAKE 44.}

1 Tuka nakaha Jakob mitaokiye, nakion wo; qa Israel wahdahnige cin :

2 Jehowa hecen eya; Tuwe nicage ca wicatamni etanhan icahniye cin he oniciye kta: Jakob mitaokiye, wakokipe śni wo; qa niye Jeśurun wahdalinige cin.

3 Tuwe ipuza kin he mini àwakaśtan kta, qa mini tanka maka puza kin akan : nicinca kin Mitaniya awakaśtan kta, qa mitoyawaśte cincawicayaye cin akan:

4 Hecen peji ehna icagapi kta, wakpicahda coliwanjica lin iyececa.

5 De, Jehowa tawamayan ce, eye kta; qa he Jakob caje eya caje ihdate kta ; qa wanji iś, Jehowa tawa ce, iye nape en okiwa kta, qa Israel caje eya caje ihdate kta.

6 Jehowa Israel ta Wicaśtaya239 
tapi, qa tuwe opekiton qon, Jehowa wicobe tawa kin he hecen eya; Tokaheya lin he miye, qa ehake un kin he miye; qa micunonpatanhan Taku Wakan wanica ce.

7 Qa tuwe, miye iyececa eyanpaha kta, qa he oyake kta, qa wiyeya emicihnake kta, wanakaja oyate ewicawahde lin iyececa he: hecen taku tona u, qa taku u kte cin hena owicakiyakapi kta.

8 Cancanpi śni qa wakokipapi śni po : hetanban miye nahonciyapi śni qa omdake śni he; wayaotanin mitawa kin he niyepi nakaeś: Micunonpatanhan Taku Wakan yukan he. Hiya, Imnija wanica, takudan sdonwaye śni.

9 Tona wakagapi bagopi kagapi kin hena owasin takupi śni ; taku waśtedakapi kin hena takudan okihi kte śni : nakun iye ihdaotaninpi; hena wawanyakapi kte śni, qa wasdonyapi kte śni; hecen iśtecapi kta tuka.

10 Tuwe taku wakan wan kaga, qa wakagapi wan takudan okihi śni kin heca śdoya he.

11 Iho, tawaśitku kin iyuhpa iśtecapi kta: qa tona lagapi kin hena wicaśta etanhanpi : hena owasin yuwitayapi kta, hena najinpi kte; hena wakokipapi kta, hena witaya iśtecapi kta.

12 Maza kage cin he onspe wan kaga, qa petaga ehna htani, qa nakun maziyape on kicanyan qa iye isto waśake cin on yuśtan kta : han, iye hinca wotektehda, unkan towaśake wanica; mini yatke śni, unkan hunkița.

13 Cankajipe cin he caniyute wan on iyuta ; icago wan on icagokta ; canicahdoke on kaśpekta ; qa mazicago wan on kago kta; qa hehan wicaśta ouncage iyece- ca kage kta, wicaśta towaśte lin iyececa; hecen tipi kin ohna he kta.

14 Hante śa hdaksa, nakun uskuyeca qa utuku can icu, he contanka can kin ehna kiyuwaśake kta ; wazi can wan oju, unkan magaju kin icahye kta.

15 Unkan he wicaśta wan peta en aonpe kta; qa hetanhan iye icu lsta, qa on cosiciyc kta ; han, iceti qa aguyapi śpanyan; han, on taku wakan wan kage ca ohoda; etanhan wakagapi bagopi wan kage ca itokam makata ehpeiciya.

16 Hanke etanhan peta en huhnaga; hanke on tado yuta; wośpanye ceonpa qa imnan; han, cosiçiya, ya, Áha, macoza, peta wanmdaka ce, eya:

17 Qa taku okaptapi kin he taku wakan kaga, wakagapi bagopi iye tawa kin hee; en makata elipeiciya, qa ohoda, qa celiya, qa, Emahdaku wo, taku walkan mitawa kin he niye nakaeś, eya. 18 Hena sdonyapi śni, qa okahnigapi śni; iye hena iśta aohdutewicaya, hecen wanyakapi kte śni, cantepi nakun, hécen okałnigapi kte śni.

19 Heon tuwedan cante on awacin śni, nakun wosdonye wanica, qa wookahnige twanica, on heye kta tuka; Hanke peta en huhnahwaye; han, nakun he petaga on aguyapi spanwaye; on tado cowaqin qa wata: unkan taku okaptapi kin he etanhan wowahtedaśni wan wakage kta he; cankaga wan en makata ehpemiciye kta he.

20 Caliota yuta; cante hnayanpi wan he icunonpa iyayeya, heon iye nagi eihdaku kte śni, qa, Minape etapa ohna woitonśni yuke śni he, eye kte śni.

21. Dena kiksuya wo, Jakob qa 
Israel; mitaokiye kin he niye nakaeś: miye cicaga, mitaokiye kin he niye: Israel, miye aciktonje kte sni.

22 Taku śica ecanon kin, malipiya soka iyececa, wăkajuju; qa wayahtani kin mahpiya sapa iyececa: Miye en ihdoku wo, upeciciton kin heon ctanhan.

23 Malipiya, dowan miye, Jehowa hecon nakaeś: maka ihukuya kin, iyaśa po: paha kin niye, contanka qa ohna can otoiyohi, kahiyaya po: Jehowa Jakob opekiton nakaeś, qa Israel en wowitan içicaga.

24 Jehowa openiciton qa wicatamni etanhan icaliniye cin he hecen cya; Jehowa taku owasin kaga, iśnana mahpiya kin halumdaya qon he miye: maka lin miśnana wahdumdaya :

25 Tona itonpi śni wowakta tawapi kin yuecetu śni, qa wapiye sa kin wicayuhnaślinye kta ; wicaśta ksapa helata itoheya hdicawicaya, qa tookahinigepi kin yuwitkotkole kta:

26 Iye taokiye oie kin yuecetu, qa wahosiya tawa tawowalokionkiye kin ecetukiciciyc kta; tuwe, Ounniyanpi kta cc, Jerusalem eciya; qa .Juda otonwe kin, Ake nicagapi kta, qa makoskantuya wanke cin ake piwayc kta ce, eye cin he miyc.

27 Tuwe mini śbe cin heciya : Puza wo, qa walipa nitawa oyaliewaye kta cc :

28 'Tuwe Kuros heciya; Wonwicaye mitawa hee, hecen mitoiyokipi ocowasin econ kta; qa Jcrusalem heciye kta; Nicagapi kte; qa tipi wakan kin, Ohehdepi nitawa ehnakapi kta ce, eciyc lita.

WICOWOYAKE 45.

1 Jehowa Kuros sdakiye cin * 16 hee, itokam Ikcewicaśta kin awicahanpi lita e nape etapa sutaya mduza; qa wicaśtayatapi ipiyake wicawakiyuśke kta, hecen he itokam tiyopa itakihna kin yuhdoliapi lita; qa tiyopa kin hena ecen icupi kte śni, hecen eciya ;

2 Nitokam miye mile kta, qa taku ślkopa lin he owotanna wakage kta: mazaśa tiyopa kin mduwehwege kta, qa maza sapa inatake kin wakaksaksa kta.

3. Qa otpaza en womnaye kin cicu kta, qa wowijice onalibe en nahmanpi kin hena; hecen Johowa miye, tuwc nicaje cajeyate cin, Israel 'Taku Wakan tawa kin he miye c sdonyaye kta.

4 Jakob mitaokiye qa Israel wahdalinige cin heon nicaje on cico kta: sdonmayaye śni eśta caściton kta.

5 Jehowa he miye, qa tokeca wanica, micunonpatanhan Taku Wakan wanica: miye ipiyagciton kta, sdonmayaye śni eśta.

6 Hecen tona wihinanpatanhan qa wiyohpeyatanhan kin hena micunonpatanhan wanica e sdonyapi kta. Jehowa he miye, qa toleca wanica.

7 Iyoyanpa icaliwaya, qa otpaza wakaga; wookiye mduśtan, qa wośice wakagga; Jehowa miye dena owasin ecamon ece.

8 Mahpiya kin wankantanhan śbuya miye, qa malipiya śoka kin hena woowotanna hinheya nunwe ; maka kin ihdulidoke kte, hecen wowanikiye icaliyapi kte, qa woowotanna kici icaga nunwe: Jehowa miye hena wakaga ce.

9 Hehehe, tuwc Waicalye tawa kin kipajin hecinhan; wakśica onśpa maka wakśica onśpa kicica. Upśija kin he tuwe kage cin, Taku ecanon he, eciye 241 


\section{ISAYA TAWOYUKCAN KIN,}

kta he: qa nitolitani, Nape wanica ce, eye kta he.

10 Hehehe, tuwe atruku heciya; Taku icahyaya he; qa winohinca kin, Taku yaton he, eciyc cinhan.

11 Jehowa Israel Towakan qa Waicaliye tawa kin he hecen eya; Micinca on taku tokata $u$ kte cin hena imawanga po, qa minape taku kage cin on econ mayaśipi ktr.

12 Miyc maka kin wakaga, qa wicaśta aicaliwaye. Miye minape kin mahpiya kin yumdayapi, qa obe tawa kin iyuhpa mdusuta.

13 Woowotanna on iye icaliwaya, qa tacanku owasin mduowotanna kta: iye mitotonwe kin kage kta, qa wayaka mitawa hdicuwicklkiye kta, on opetonpi qa wiyopeyapi wanica, Jehowa wicobc tawa kin he hecen eya.

14 Jehowa hecen eya; Egupta tohtani, qa Iruś tokamna, nakun Sabea wicaśta tanka tokamna kin niye en u kta, qa hena nitawa kta; nitokam upi lita; mazaicicahilia ikoyag ihdoupi kta, qa nitokam makata ehpeiciyapi kta ; hena ccniciyapi kta; $\Lambda$ wicakehan Taku Wakan kin nici un, qa wanji tokeca yuke śni, taku wakan wanica ce, eyapi kta.

15 Israel ta Wakantanka, Wanikiye cin he, awicakchan Taku Wakan anaicilibe cin he niye.

16 Hena owasin witaya iśtecapi qa nakun nihinciyapi; wakagapi kagapi kin hena witaya wonihinciyc elita iyayapi.

17 Jehowa en wowanikiye owihanke wanica on Israel nikiyapi lita : niye iniśtecapi kte śni, qa nihinniciyapi kte śni, owihanke wanica.

18 Jehowa mahpiya kin kaga; Wakantanka iye hinca maka kin 242 icahye ca kaga; he hdusuta, taku on etanhan śni kagc śni, ohna tipi kta e icaliye cin he hecen eya; Jehowa he miye, qa tokeca wanica.

19 Woinahibe en, maka otpaza awanke cin ohna iwae śni: Jakob cinca kin, Taku śni on miite akita po, ewicawakiye śni : Jehowa woowotanna ohdake cin he miye, taku ecetu kin hena omdaka ece.

20 Mniciyapi qa u po; Ilkcewicaśta etanhan nayajicapi kin witaya kiyedan ihdou po. Tona wakagapi bagopi can kin ekihdepi qa taku wakan wanikiya okihi śni celkiyapi kin hena takudan sdonyapi śni.

21 Woyaka po qa kiyedan ihdou po: han witaya ikiciyukcanpi kte. Wanakaja tanhan de tuwe oyaka he ; hetanhan tuwe yaotanin he: Jehowa miye śni he ; qa micunonpatanhan taku wakan wanica; Taku Wakan owotanna qa Wanikiya; micunonpa tanhan wanica.

22 Maka ihanke kin owasin ahimatonwan po, kinhan yanipi kta: Wakantankn he miye, qa tokeca wanica nakaeś.

23 Miye hinca on wakonza, woowotanna on wicoie kin mii ctanhan enapeya, unkan hdicu kte śni: Miye en wicahupahu otoiyohi patuje kta, qa wicaceji otoiyohi wohdake lita:

24 Awicakehan Jehowa en woowotanna qa wowicake mduha ce, eye kta : iye en ahi kta; qa tona canniyeyapi kin owasin iśtecapi kta.

25 Israel cinca kin owasin Jehowa cn ihdaowotanpi qa itanpi kta.

WICOWOYAKE 46.

1 Bel canpeśka makehde ina- 
jin, Nebo patuś un: wakagapi tawapi kin hena woteca qa wanunyanpi kin akan hiyeya: waqimpi nitawapi kin iakan ehnakapi; mdokite cin he yuśaka.

¿ Patuś unpi, witaya canpeśka makehde yukanpi; taku qinpi kin anakikśin okihipi śni, tuka iyepi kaeś wayaka iyayapi.

3 Jakob tiyohnaka qa Israel tipi etanhan tona olsaptapi, wicatezi etanhan wicayuhapi, wicatamni etanhan wicaqinpi owasin niye anamagoptan po :

4 Wicahinea elıan yaipi kta hehanyan he miye; qa pa skapi helanyan ciçinpi kta; miye wakaga nakun mduhe kta; miye hinca wawaqin kta, qa ecihdakupi kta.

5 Hecen turve e imayadacinpi kta he, qa iyececa mayakagapi kía he, qa kici iwanmayadakapi kta, hecen akiyeuncecapi kta.

6 Mazaska opiye etanhan mazaskazi hiyuyapi, qa onaspeyapi kin on mazaska theutapi ; mazaskazi kage cin wan opetonpi kta ; unkan he taku wakan wan kage kta: en makata elipeiciyapi kta, han ohodapi kta:

7 Hinyete akan ehnakapi kta, qinpi kta, qa tohe kin en ehdepi lita; unkan en lie kta; tohe kin etanhan tokan ye kte śni : han, tuwe hoyekiya eśta ayupta okihi kte śni, qa tokakije etanhan eyaku kte śni.

8 De kiksuya po, qa wicaśta yaunpi hdutanin po: ake cante en yuza miye, wayahtanipi sa.

9 Taku wanakajatanhan un kin hena kilksuya po; lyotan Waśalie cin he miye, qa tokeea wanica; Taku Wakan kin he miye, qa tuwedan iyemacece śni.

10 Otokaheya tan̆han owihanke kin omdaka, qa wanakajatanhan taku nahanhin econpi śni kin; Mitowiyulscan kin ecen he kta, qa mitoiyokipi kin iyulipa mduśtan kta ce, epa:

11 Wihinanpatanhan zitkadan wayapote sa ivan weco, qa makoce tehan wanke cin etanhan mitowiyukcan wicaśta kin : Han, miye ecen epa, nakun ecetuwakiye kta; wakonza, nakun ecen ecamon lata ce.

12 Tona cante nisutapi, woowotanna itehan yaunpi kin, anamagoptan po:

13 Mitoowotanna kin he kiyedail awaku; he itehan yanke kte śni, qa wowaniliye mitawa kin he lianhi kte śni. Tuka Israel mitowitan kin on Zion en wowaniliye ewehnake kta

\section{WICOWOYAKE $4 \%$.}

1 Babel eunwintku witanśna, kun u qa watuśekśeca en iyotanka wo: makata iyotanka wo: Kasdim eunwintku, oiyotanke waśte wanica ; detanhan icimana wankadan qa suta śni eniciyapi kte śni.

2 Inyan wiyutpan kin icu qa wotpanpi kaga wo: ite akahpe nitawa eyaku wo, nitośle hduśdoka wo, sican hducoka wo, wakpa kin copa wo.

3 Tanconikadan śdayehna un kta; han, nitowiśtece kin wanyakapi kıa: watokiçonpi ccamon kta, qa wicaśta kici oweciye kte śni.

4 Opeunkitonpi kin he Jehowa wicobe tawa eciyapi, Israel Towakan kin hee.

5 Kasdim cunwintku, inina iyotanka wo, qa otpaza elsta ya wo; detanhan icimana, Wokiconze wicaśtayatapi winohinca, eniciyapi kte śni.

6 Mitaoyate eanniyewicawakiya, taku mitawa kin he mduśape 243 


\section{ISAYA TAWOYUKCAN KIN,}

ca ninape en wicawaqu. Ecaca onśiwicayada śni; wicahincapi kin hena cannapinpi nitawa tkeya awicayahnaka.

7 Qa heha; Ohinniyan wicaśtaYatapi wimanohinca kta ce. Hecen dena en nicante cyahnake śni, cwihanke kte cin he yeksuYe śni.

8 Heon etanhan de nahon wo, oiyokipiiciya nanke cin, oziiçiya ilotanke, nicante en hehe cin; Miye qa micunonpatanhan tuwedan śni, wiwazica imdotanke lite śni, qa wicacinca taninpi śui kin he sdonwaye kte śni cc, eha.

9 Tuka dena oza ihnuhanna, anpetu wanjidan en aniu kta, wicacinca taninpi śni, qa wiwazica unpi kin: hena ocowasin cn ni u kta, wakan yakaga ota qeyaś; nina hỉn wapiyapi ecanon eśta.

10 Niolian śice cin he wacinyakiya; Tuwedan wanmayake śni cc, eha. Nitoksape qa nitookahnige kin he nihnayan: hecen nicante en heha; Miyc, qa micunonpatanhan tuwedan śni ce.

11 Heon etanhan taku śica en niu kta; tokiyatanhan hinanpa sdonyaye kte śni ; qa wośice anihinlipaye kta; yutokan iyeyaye lita ryakihikte śni; qa woihangye sulonyayc kte śni kin he ihnuLanna en niu kta:

12 Wanna wapiyapi nitawa, qa wakan yakaga ota hinca, inicaga ehantanhan ehna yaśkan kin hena hduha inajin wo ; okin. ni iwaśte yaun oyakihi kta, okinni wakokipc wicayecage kta.

13 Wowiyukcan nitawa ota on mdoyakita. Iho, wanna tona ma. lipiya iyutapi kin, tona wicanlipi wanyakapi, tona wi kin on waayatapi ece kin hena inajinpi, qa taku en aniupi kte cin etanhan eniyakupi nunwe.

\section{4}

14 Tho, hena aguyapi hu iyececapi ; peta hulinahwicaye kta ; ide nape kin etanhan iye nagi cihdakupi kte śni: petaga on cosiçiyapi, qa peta itokam iyotankapi kte cin wanice kta.

15 Tona inicaga chantanhan om htayani, wicaśta wopeton nitawa kin hena hecen nicuwapi kta; otoiyohi makoce tawa kin ekta hde kta; tuwedan niniye kte śni.

\section{WICOWOYAKE 48.}

1 Jakob tiyohnaka, de nation po, tona Israel caje on cajeniyatapi, qa Juda mini kin etanhan inayapapi, tona Jehowa cajc on wakonzapi qa Israel ta Wakantanka cajeyatapi ece, tuka wowicake qa woowotanna eciyatanhan śni.

2 Hena otonwc wakan kin icajeiciyatapi, qa Taku Wakan Isracl tawa kin he wacinyanpi nakacś: He Jehowa wicube tawa eciyapi.

3 Otokaheya tanhan taku tokaheya qon hena omdaka; unkan hena mii etanhan enape, qa hena mdaotanin : ilhnuhanna ccamon, unkan hena ecetu.

4 Nitehika e sdonwaya, qa nitahu maza ikan kin heca, qa niite mazaśa, nakaeś :

5 Heon etanhan otokahcya tanhan hena ociciyaka; hinyahin ecctu śni chan ciciyaotanin : okinni, Wakagapi mitawa dena econ; qa wakagapi bagopi mitawa, qa wakagapi śdoyapi mitawa kin he hena yuśtan ce, ehe lita nace.

6 Wanna nayahon, dena owasin wanyaka wo; unkan niye orlakapi kte śni he. Detanhan taku teca wanyagciciya, taku nahmanpi qon, unkan hena sdonyaye śni. 
7 Hena nakaha kagapi, qa otokaheya tanhan śni; anpetu kin de itokam hena nayahon śni; okinni, Tho, hena sdonwaya ce, ehe kta tuka qon.

8 Han, nayahon śni; han, slonyaye śni ; han, nakun itolam noge niyulidokapi śni. Wahnayan oyaliande kta, qa wicatamni etanhan wahtani sa eniciyapi kin he sdonwaya.

9 Micaje on etanhan mitocanniye wahdapte kta, qa mayatanpi kta e niye on anamiçipte kta, hecen cicakse kte śni.

10 Iho, ciyuecedan, tuka mazaska kicica śni ; wokakije oceti kin en cihdah̉niga.

11 Miye on etanhan, miye hinca on etanhan ecamon lita: tokeca micaje yuaśapapi kta he: qa mitowitan kin he tuwe tokeca waqu kte śni.

12 Jakob anamagoptan wo, qa Israel weco qon; He miye, Tokaheya qa nakun Ehake un kin he miye.

13 Nakun minape maka oahe cin ehnaka; qa minape etapa mahpiya lin hdumdaya. Hena wicawakipan eca witaya inajinpi ece.

14 Niyepi owasin mniciyapi qa nahon po: hena etanhan wanji tukte dena oyaka he. Jehowa he waśtedaka: iye toiyokipi kin Babel en econ kta, ca iye nape Kasdim akan yanke kta.

15 Miye, miye iwae; han, miye he weco; miye awahi; un: kan iye tacanku kin tanyan ihurnikiye kta.

16 Mikiyedan u po, de nalion miye: otokaheya tanhan oina. libe en iwae śni; totanhan un kin hen miś waun. Unkan nakaha Jehowa Itancan kin u masi, qa iye Taniya kin.

17 Jehowa openiciton kin, Is- rael Towakan kin he hecen eya; Jehowa Taku Wakan nitawa, token oyakihi lrta onspeniciye, canku opeya de kte cin be ohna aniye cin he miye.

18 Pokin mitawoahope kin hena anayagoptan unkanś; hehan wookiye nitawa kin wakpa.wan iyeccce kta, qa nitoowotanna miniwanca taja kin iyecece $\mathrm{kta}$ tuka.

19 Nakun nicinca wiyaka kin iyecece kta, qa nitezi etanban icage cin hena casmu iyececapi kta: iye caje lin kaksapi kte śni, qa mitokapatanhan ilangyapi kte śni tuka.

20 Babel etanhan hdinanpa po, Tíasdim etanhan najica po; dowanpi ho kin on eyanpala miye, de oyaka po, maka ihanke kin hehanyan yaotanin miye; Jehowa iye taokiye Jakob he opekiton ce, eya po.

21 Unkan hena hewotahedan makoce kill ohna awicaye ciqon hehan ipuzapi śni: imnija kin etanhan mini hiyuwicakiciciya; nakun imnija kin kahdoka, unlsan mini bomdu hiyu.

22 Tona śicapi kin hena wookiye nicapi ce, Jehowa eya:

\section{WICOWOYAKE 49.}

1 Wita kin, niye namahon po; qa oyate tehan yaunpi, anohiciya po: Wicatamni etanhan Jehowa makipan, ina tezi etanhan cajemayata.

2 Qa mii kin he mazasagye pe hinca iyececa kaga; iye nape ohanzi kin en namahbe, qa wanhinkpe pabezapi makaga; iye wanju tawa kin onamahinan;

3 Qa hemakiya; Israel, mitaokiye kin he niye, niye on miliduwitan kta ce.

4 Hehan miye hepe; Takuśni $24 \xi$ 


\section{ISAYA TAWOYUKCAN KIN,}

nin htawani; etanhan takudan icage śni, qa takuśni on mitowaśake wabdusota; Tuka woyaco mitawa kin he Jehowa kici un, qa mitubtani kin mita Wakankanka kici un.

5 Unkan nakaha Jehowa wicatamui etanhan taokiye maye kta makaga, Jakob iye en awaku kta, he kaken eya; Israel mnayanpi śni qeyaś Jehowa iśta kin en wowitanyan waun kta, qa mita Wakantanka wowaśake mitawa kin hee kta.

6 Nakun hecen eya; Mitaokiye he niye, Jakob towicoun nain wicayakiye kta, qa Israel okaptapi kin wicaduecetu kte cin he taku tanka śni : nakun 1kcewicaśta lin iyoyanpa yaun kta ciç, heon maka ihanke kin heharyan wowanikiye mitawa kin he niye kta.

7 Jehowa Israel opehiton, qa Towakan kin he, tuwe nina sicedakapi, tuwe Ikcewicaśta walitedapi śni, wicaśtayatapi tawowidake kin he heciya; Wicaśtayatapi wanyakapi kta, qa wicaśta itancanpi kin inajinpi kta, nakun patujapi kta. Jehowa wacinyepica, Israel Towakan kin on; unkan iye nicahnigapi kta.

8 Jehowa kaken eya; Tohan oiyokiji kinhan nacihon, q̨a wowanikiye anpetu kin en ociciya: Unkan awanciyake kta, qa oyate lin en wicotakuye wan ecihde kta, maka kin yusutapi kta, qa ounyanpi hewoskantu kin tawa wicayaye kta:

G Hecen wicakaśkapi kin, Tankan u po, ewicayakiye kta: tona ópaza en unpi kin hena, Irdutanin po, ewicayakiye kta. Canku kin ohna wotapi kta, qa paha ślayehna owasin akan ta wilianpi yuke kta.

10 Hena e wotektehdapi lite $+x^{2} 46$ śni, qa ipuzapi kte śni ; qa oma śte qa anpetu wi kin aśpanwicayapi kte śni: 'Tuwe onśiwicakida kin he yus awicaye kta nakaeś, qa miniyowe kin en awicai kta.

11 Qa he mitawa owasin canku wakage kta, qa ocanku tanka mitawa kin hena wankan yeyapi kta.

12 Iho, dena tehantanhan hipi kta; qa iho, dena waziyatanban qa wiyohpeyatanhan; qa dena Sinim makoce kin etanhan.

13 Mahpiya, dowan miye; qa maka, wiyuśkinyan un wo; nakun paha kin, dowan hinhda miye ; Jehowa iye taoyate cantohnagwicaya nakaeś, qa kakiświcaye cin hena onśiwicakida kta.

14 Tuka Zion heya; Jehowa amayuśtan, qa Itancan mitawa amiktorija ce.

15 Winohinca hokśiyoqopa azir kin he akiktonje kta, hecen iye tamni cinca kin onśikida kte śni he: Han, hena wakiktonjapi kta nace ; tuka miye qe aciktonje kte śni.

16 Iho, minape cokaya en bacigo ; conkaśke nitawa ohinniyan mitokam wanke.

17 Nicinca inahipi : tona niyujujupi qa niyutakunipi śni qon hena nitantanhan napapi kta.

18 Ohomni iśta yuwankan ikikeu qa wanyaka wo: dena owasin ihduwitayapi; hena en niupi. Miye wani, Jehowa eya, hecen dera iyuhpa koyahdake kta, oinpi wan iyececa, ģa winohinra wan hihnaton kte cin iyecen ipiyahdake kita.

19 'Tintoskan nitawa, qa hewoskan makoce nitawa, qa makoce en niyutakunipi śni qon he dehan wicota en ounyanpi kin on onikan kte śni, qa nanipcapi qon hena itehan unpi kta. 
20 Nakun cinca nicitaninpi śni qon hena ninoge en heyapi lita; Makoce ocikan śni, makiyukan wo, hecen en ounwaye kta ce.

21 Hehan nicante en hehe kta; Dena tuwe icahmiciya he, cinca makitanin śni, qa cinca codan wayaka waun qaunhdaka waun; unkan dena tuwe icaliwicaya he. Iho, miśnana oinakaptapi ; dena tokiya unpi tuka he.

22 Jehowa Itancan kin he hecen eya: Iho, Ikcewicaśta ekta nape wahdugate kta, qa oyate kin en wiyokihedan wan ewehde kta : unkan maku ohna nicinkśi awicaupi kta, qa nicunkśipi kin hinyete akan wicaqinpi kta.

$23 \mathrm{Qa}$ ateyayapi woniçupi kte cin wicaśtayatapi eepi kta, qa winohinca tanka tawapi kin hena azinniciyapi kta : hena niye en makipusdiya patuś inajinpi kta, qa nisiha watuśekśeca kin asdipapi kta: unkan Jehowa he miye sdonyaye kta ; tona wacinmayanpi kin hena iśtecapi kte śni nakaeś.

24 Wakipi kin he wicaśta waśaka etanhan icupi kta he, qa owotanna wayaka un kin lie ehdakupi kta he.

25 Tuka Jehowa hecen eya; Wicaśta waśaka wayaka wicayuhapi kin liena ewicayakupi kta, qa wakipi kin wicaśta wohitika ewicakicihdakupi kta ce. 'Tuwe nicize cin he kici wecize kta nakaeś, qa nicinca niwicawaye kita.

26 Qa tona kakiśniyanpi kin hena iye cehpi hdunwicawakiye kta, qa iye we kin on witkopi kta, miniśa teca on iyececa ; unkan Jehowa Wanikiva nitawa, qa tuwe openiciton, Wanji Waśaka Jakob tawa kin he miye e wicacelipi owasin sdonyapi kta.

\section{WICOWOYAKE 50.}

1 Jehowa hecen eya; Kicipaganpi wowapi on nibun elipewa. kiye cin tukte e he: qa icaro. makiyapi kin tukte wanji en wiyopeciciyapi he. Iho, wayahtanipi on wiyopeniciyapi, qa taku śica ecanonpi kin on etanhan inayayapi kin kpaganpi.

2 Heon etanban wahi qehan tokeca wicaśta wanica he: wapan qehan tokeca tuwedan amayupte śni he. Minape ptecedan, ptecedan, liecen wopekiton oki hi śni he; qa waehdaku owakihi śni he. Iho, wawiyopewaye on miniwanca kin mdapuze kta ; wakpa kin hena hewotahedan wakage kta; hogan tawapi śicamna kta, mini wanica nakaeś, qa ipuza on țapi kta.

3 Mahpiya kin otpaza koyagwaye kta, qa wakihdaka woakalipe wecage lita ce.

4 Jehowa Itancan kin waonspekiyapi ceji kin maqu, hecen wicoie wanji on tuwe mdokita iwakiy a onmaspe lita : hanhanna, hanlianna wayulice kta, minoge wicaśta waonspe iyecen wanalion kta e yulice kta.

5 Jehowa Itancan kin noge makiyulidoka; unkan wawakipajinyan waun śni, qa helkta wahdicu śni.

6 Mitapete waapapi kin wicawaqu, qa hin yujunpi kin iyoha mitawa wicawaqu : miite wowiśtenyapi qa atagośapi kin etanhan nawakilibe śni.

7 Tuka Jehowa Itancan kin omakiye kta, heon etanhan mayuśinyaye kte śni: heon etanhan miite wanhi iyecen wahduza, hecen imaśtece kte śni sdonwakiya.

8 Tuwe mayaowotanna kin he ilkiyedan un: tuwe amakinica 247 


\section{ISAYA TAWOYUKCAN KIN,}

kta he; witaya naunjinpi kte: tuwe makipajin kta he ; he mikiyedan u nunwe.

9 Iho, Jehowa Itancan kin omakiye kta ; tuwe mayaco kta he: iho, hena owasin wokoyake iyecen kuke kta; wayapote sa kin hena wicayapotapi kta.

10 Niyepi etanhan tuwe Jehowa kokipe, tuwe e iye taokiye ho kin anagoptan, tuwe otpaza omani qa iyoyanpa codan un he ; he Jchowa caje kin wacinye kta, qa ta Wakantanka en eicihde nunwe.

11 Iho, niycpi owasin ceyatipi, qa peśnija on aohduteniciyapi; peta nitawa iyoyanpa kin en mani po, qa peśnija ideyayapi kin en. Minape etanhan de nitawapi kta; woiyokiśice en inunkapi kta.

\section{WICOWOYAKK 51.}

1 Tona woowotanna yapasipi, Jehowa ayakitapi, niye anamagoptan po : imnija etanhan nicakanpi kin he ekta ekitonwan po, qa maka olidoka etanhan niçapi qon he ekta:

2 Abraham ateyayapi kin he ekta etonwan miye, qa Sara niyuhapi kin he ekta: iye ecedan weco, qa mdawaśte qa mduota ce.

3 Jehowa Zion wacintonhnagya; makoce puza tawa ocowasin wacintonhnagya nakaeś : qa tintoskan tawa kin he Eden iyececa kage kta, qa hopuza tawa Jehowa tawoju kin iyececa: wicocante waśte qa wiyuśkinpi hen un kta, wopida eyapi qa dowanpi ho kin hena.

4 Mitaoyate, anamagoptan po : qa oyate mi tawa, anohmakiya po: woope kin mitantanhan inanpe kta, qa mitawoyaco oyate kin eı iyoyampa ewehde kta nakaeś.
5 Mitoowotanna kin he kiyedan un; wowanikiye mitawa hinanpa; qa miisto oyate kin wicayaco kta: wita kin hera amapepi kta, qa miisto kin he wacinyanpi kta.

6 Mahpiya kin ekta iśta hduwankan iyeya po, qa kuya maka kin ckta ctonwar po. Mahpiya kin śota iyecen tanin śni iyaya, qa maka kin wokoyake iyecen kuka aye kta; qa tona en ounyanpi kin iś eya iyecen tapi kta; tuka wowanikiyc mitawa kin he owihanke wanice kta, qa mitoowotanna kin he ihangyapi kte śni.

7 Tona woowotanna sdonyayapi, oyate mitoope nicantepi en un kin, anamagoptan po : wicaśta wawiyopeyapi kin he kokipapi śni po, qa wayaśicapi kin hena on nihinciyapi śni po.

8 Hena wokoyake iyececa wayapote sa temwicaye kta, qa talinca hin iyececa wamduśkadan hena wicayapotapi kta: tuka mitoowotanna kin he owihanke wanice kta, qa wowanikiye mitawa kin he wicoicage qa wicoicage hchanyan.

9 Ihduhica, ihduhica wo; Jchowa isto, ihduwaśaka wo; wanakaja anpetu kin en iyececa, kikta wo, wanakaja wicoicage kin en iyececa. Rahab kaksaksa qa unktehi pahdoke ciqon he niye śni he :

10 Miniwanca, mini tanka kin yupuze ciqon he niye śni he; tuwe miniwanca ośbe kin en canku kaga, hecen tona opewicakitonpi kin ohna yapi kta.

11 Heon ctanhan Jehowa opewicakiton kin hena hdicupi kta, qa dowanwan Zion ekta kupi kta; unkan wowiyuśkin owihanke śni kin he iye pa akan yanke kta : wicocante waśte qa wowi- 
yuśkin icupi kta; woiyokiśice qa comnihdazipi kin heua najice kta.

12 Miye, han tuwe wacintonlinagniyanpi kin he miye: turve niye he, hecen wicaśta te kte cin koyakipe kta he, qa wicaśta cinhintku peji iyececa kagapi kte cin he.

13 Qa Jehowa icahniye cin, mahpiya kin hdumdaya qa maka oahehde kin ehde kill he ayektonja; qa wawakipajin tocanniye kin on anpetu otoiyohi ohinniyan kopeyahda, waihangya okihi kta iyececa. Unkan wawakipajin tocanniye kin tukte e he.

14 Patuś un kin he kiyuśkapi kta inahini, qa hecen woha kin ohna te kte śni, qa aguyapi kiwanice kte śni.

15 Tuka Jehowa he miye, Taku Wakan nitawa, miniwanca yuśkanśkan, unkan taś tawa hoton; Jehowa wicobe tawa eciyapi.

16 Unkan mioie kin hena nii en owahnaka, qa minape ohanzi kin ohna akalipecicaton; hecen mahpiya kin ti wecage kta, qa maka oahehde kin ewehde kta, qa, Mitaoyate kin niye, Zion ewakiye kta heon.

17 Ihduhica wo, ihduhica wo, Jerusalem, Jehowa nape etanhan iye tocanniye miniyatke dahepe ciqon, inajin wo: witkopi miniyatke cetete hehanyan paskicapi kin dahepa.

18 Cinca wicaton kin owasin etanhan tuwedan yus aye śni; qa cinca icahwicakiye cin iyuhipa etanhan wanjidan napata hduze śni.

19 Taku kin dena oza en niupi ; tuwe aniceye lita he: wicotakuni śni qa woihangye, qa wicaakilian, qa mazasagye : tokiyatanhan wacintonhnagciye kta he.

20 Nicinca kin hena hustakapi; canku otoiyohi ipa kin en wankapi, tatokadan wan wohrmunke en un kin iyececa. Jehowa tocanniye kin on ojupidan, Taku Wakan nitawa towiyopekiye kin on.

21 Heon etanhan, kakiśniyanpi qa winitko yaun tuka miniśa on etanhan śni, ceciciya, de nation wo ;

22 Jehowa Itancall nitawa qa Wakantankta nitawa, tuwe taoyate iwicakiye cin he hecen eya; Iho, witkopi miniyatke kin ninape etanhan emdaku, mitocanniye miniyatke hminyanna kin hee; ake icimana datke kte śni.

23 Tuka tona kakiśniyanpi napepi kin en wicawaqu kta ; tona ninagi heciyapi; Patuja wo, kinhan akan unkiyayapi kta ce: unkan iwankam iyayapi kin hena en nitancan maka iyecen enicihnaka, qa canku kin iyececa.

\section{WICOWOYAKE 52.}

1 Kikta wo, kikta wo; Zion, nitowaśake kohdaka wo: Jerusalem otonwe wakan kin, wokoyake waśteśte kin kohdaka wo ; detanhan cehpi bakihdayapi śni qa aśapapi kin icimana en niupi kte śni.

2 Maka mdu kin etanhan ihdatata wo; Jerusalem, najin, iyotanka wo; nitahu iyakaśkapi kin hduśdoka wo, Zion cunwintku wayaka yaun kin.

3 Jehowa hecen eya; Taku sni on wiyopeniciyapi; unkan mazaska codan openicitonpi kta. 4 Jehowa Itancan kin he hecen eya; Ehanna mitaoyate Egupta en ounyanpi kta e ekta 249 


\section{ISAYA TAWOYUKCAN KIN,}

apamahde iyayapi; unkan taku on etanhan śni Aśur oyate kin kakiświcayapi.

5 Unkan nakaha den taku mici un he, hecen mitaoyate taku śni on ewicayakupi he, Jehowa eya ; tona iwicadakapi kin hena iyaśapi, hecen ohinniyan anpetu osan micaje yaśicapi ce, Jehowa eya.

6 Heon etanhan mitaoyate micaje sdonyapi kta: heon etanhan anpetu kin he en he miye hepa e sdonyapi kta; iho, he miye.

$7 \dot{\mathrm{H} e}$ kin akan tuwe wotanin waśte au, tuwe wookiye eyanpaha, he siha oiyokipi hinca; tuwe wowaśte wotanin au, tuwe wowanikiye eyanpalia; tuwe, Wakantanka nitawa he Wicaśtaya tapi ce, Zion eciye cin.

8 Waawanhdake nitawa hopi kin; ho wankan yeyapi ; witaya dowanpi kta; iśta iśta itkokim wankiciyakapi kta nakaeś, tohan Jehowa Zion ake en hdi kinhan.

9 Jerusalem otiwita kin, wiyuśkin hinhda po, witaya dowan iniye; Jehowa iye taoyate wacintonhnagwicaya, Jerusalem opekiton nakaeś.

10 Oyate owasin iśta kin itokam Jehowa iye isto wakan kin hduzamni : unkan maka ihanke kin iyuhpa Wakantanka unkitawapi tawowanikiye kin wanyakapi kta.

11 Hanta po, hanta po, hetanhan tankan ya po, taku śapa yutanpi śni po ; cokaya tanhan tankan u po; Jehowa tawakśica yaqinpi kin niye ihduska miye.

12 Hetanhan inaliniyan inayapapi kte śni, qa najica dapi kte śni: Jehowa nitokapatanhan ye kta, qa Israel ta Wakantanka kin he hektapatanhan mnaniyanpi kta.

13 Iho, mitaokiye wacinksam250 ya ohanye kta, ihduwankantu kta, qa yuwankan iyeyapi kta, qa tehan wankan un kta.

14 Token niye on wicota nina yuśinyayapi : iye tookitanin kin nina kaśicapi, wicaśta isanpa, qa itohnake kin Adam cinca isanpa.

$15 \mathrm{He}$ iyecen oyate ota awicamnimni kta: iye on wicaśtayatapi iohmus icupi kta : taku owicakiyakapi śni kin he wanyakapi kta; qa taku nahonpi śni qon he awacinpi kta nakaeś.

\section{WICOWOYAKE 53.}

1 Wounyakapi kin he tuwe wicada he; qa Jehowa isto kin, tuwe ekta yutaninpi he.

2 Unkan iye itokam camni wankadan iyecen uye kta; qa maka puza etanhan hutkan wan iyececa: wookitanin codan un, qa owanyag waśte śni ; hecen wanunyakapi kinhan waśteundakapi kta e owanyake waśte wanica.

3 Walitedapi śni qa wicaśta on elipeyapi; wicaśta cante śica qa woiyokiśice sdonkiya; qa tuwe ite anaunkicilimanpi kin iyececa ; wahtedapi śni, unkan unkiye awauncinpi śni.

4 Awicakehan waunyazanpi kin hena iye yuha, qa wicocante sica unkitawapi kin hena iye qin: tuka kaśtakapi, Wakantanka eciyatanhan apapi qa kakiśyapi e canunkinpi.

5 Tuka waunhtanipi kin hena on iye palidokapi, taku śica econkupi kin on kaśujapi : wookiye unkitawapi on wokakiśye iye akan yanke; qa iye apapi kin eciyatanhan woasni unhapi.

6 Unkiye owasin tahinca wanunyanpi iyecen unnumipi ; otoiyohi iye tacanku kin ekta unkihduhomnipi; unkan Jehowa un- 


\section{WICOWOYAKE 54.}

kiye owasin taku śca econkupi kin hena iye en yuwitaya ehnaka.

7 Nagiyeyapi qa iye hinca kakiśyapi eśta ihdukawa kte śni ; tahinca cinca iyecen ktepi kta en ahipi, qa tahinca wanunyanpi hin yuśdapi, wicitokam inina yanke cin he iyecen iye i hdukawa kte śni.

8 Kaśkapi qa yacopi etanhan eyakupi; unkan toicage kin tuwe oyake kta he: wicani makoce kin etanhan kaśpapi nakaeś; mitaoyate taku sica econpi kin on, iyepi on kaśtakapi.

9 Unkan wicaśta śicapi om woha qupi, tuka te ceehan wijicapi kin owicapeya wanka: ecin wośice takudan econ śni, qa iye i ohna wohnaye wanica nakaeś. 10 Tuka kaśuje kta Jehowa iyokipi ; wanna iyokiśnya: Tohan iyonagi woahtani wośna yakage cinhan, hehan cinca wanhdake kta, anpetu hduhanska kta, qa Jehowa toiyokipi kin iye nape ohna ecetu kta.

11 Iye nagi hitani kin etanhan wanhdake kta, imnan kta: Iye sdonyapi kin on mitaokiye owotanna wicota wicayuowotanna kta ; qa wahtanipi kin iye wicakicicin kta.

12 Heon etanhan wicaśta tanka nm wecipamni kta; qa iye wakipi kin wicaśta waśaka om okini kta ; wiconte hehanyan iye nagi kpapson nakaeś. Unkan śicaya econpi sa om yawapi; qa wicota wahtanipi kin hena iye wicakiciçin; hecen śicaya econpi sa kin hena iwicakiye kta.

\section{WICOWOYAKE 54.}

1 Cinca okitpani, cinca yaton sni, dowan wo: kokśinyeksuye śni, dowan hinhda qa iyaśa wo: tuwe iśnana ehpeyapi kin he cinca ota nakaeś, winohinca hihnaton cinca kin isanpa, Jehowa eya.

2 Nitawakeya owanka kin hdutanka wo, qa yati ozanpi kin yutitanpi nunwe; kicunni śni wo, ikan hduhanska qa hutipaspe hdusuta wo.

3 Etapatanhan qa catkatanhan nihduota kta; qa nicinca Ikcewicaśta kin tawawicayapi kta, qa otonwe otiwita elipeyapi kin en ounyanpi lita.

4. Wakokipe śni wo, iniśtece kte śni nakaeś; nakun wiśtece śni wo, iśtenniyanpi kte sni nakaeś: tokaheya inicaga wowiśtece kin he ayektonje kta, qa wiwazica yaun iyopeyapi qon he icimana yeksuye kte śni.

5 Wawicahniye cin he nihihna ee nakaeś; Jehowa wicobe tawa eciyapi ; qa tuwe openiciton Israel Towakan kin hee, Taku Wakan maka ocowasin tawa eciyapi kta.

6 Winohinca wan ehpeyapi qa nagi iyokiśica, qa aniktapi śni qehan wikośka yuzapi kin iyececa Jehowa nico ce, Wakantanka nitawa eya.

7 Iśta kakpapi se hehanyan ehpeciya ; tuka wowaonśida tanka on cikpahi kta.

8 Wośinhda hiyuyapi kin en iśta kakpapi se hehanyan miite anacilibe;"tuka wowaśte owihanke wanica on onśicida kta ce, Jehowa openiciton kin he hecen eya.

9 Miye ekta Nowa mini kin he de iyececa: Nowa mini kin icimana ake maka akahpe ye kte śni, keya wakonze ciqon, he iyecen nakun icimana canniyeciye kte śni, qa iyopeciye kte śni, keya wakonza.

10 H́e kin hena tokan aye kta, 251 
qa paha kin yutokan iyeyapi kta; tuka mitowaśte tokan iniciyaye kte śni, qa wookiye mitawa wicotakuye kin he yutokan iyeyapi kte śni, Jehowa canteniciyc cin he hecen eya.

11 Kakiśyapi, kahuhuzapi, wacintonhnagyapi śni, iho, miye hinca inyan nitawa waśeśa on ewahnake kta, qa oahehde nitawa inyan tehihika on.

12 Qa ipasotka nitawa inyan wiyatpatpa on wakage kta, qa nitatiyopa peśniśnija inyan kin on, qa nihdukśan owancaya inyan wiciyokipi kin on.

13 Unkan nicinca owasin Jehowa waonspewicakiye kta; qa nicinca wookiye tanka yuhapi kta.

14 Woowotanna on niyusutapi kta: wicokakiśyapi kin itehan un wo; koyakipe kte śni nakaeś: qa wonihinciye kin, he nikiyedan u kte śni.

15 Tho, mniciyapi, mniciyapi $\mathrm{kta}$, tuka wapahaśa mitawa ekta śni : tona niyc itkokim mniciye cin hena niye on hinhpaye kta.

16 Tho, mazakaga peta en petaga apogan, qa iye tolitani on ikicanye wan hiyuye cin he miye hinca icahwaya: qa nakun waihangye ihangye kte cin he miye wakaga ce.

17 Ikicanye otoiyohi niye nicis kagapi kte cin he takudan okihi kte śni; qa wicaceji niye en wayaco inajin kte cinhan otoiyohi niye daco kta. Jehowa taokiye taku yuhapi kte cin lie dec, qa iye toowotanpidan kin he miye eciyatanhan, Jehowa eya.

\section{WICOWOYAKE 55.}

1 Ho, ipuzapi kin owasin, mini kin en u po; qa turve mazaska codan un eśta; kuwa miye, wo252 petonpi qa wota po; han, u miye, mazaska codan qa iyunwin codan miniśa qa pteasanpi opeton po.

2 Tokeca aguyapi śni on mazaska yahdusotapi kta he; qa tokeca taku imnawicaye śni kin on litayanipi he: Anagoptanyan anamagoptan po, qa taku waśte kin he yuta po; kinhan ninagipi waśin on iyuśkiniçiyapi kta.

3 Anohmakiyapı qa en mau po ; waanagoptan miye, kinhan ninagipi ni kta; unkan wicotakuye owihanke wanica wan cicicagapi kta, Dawid towaonśida wowicake kin hee.

4 Iho, iye wayaotanin kta e oyate kin wicawaqu, oyate kin en wicaśtayatapi qa waonspekiya.

5 Iho, oyate wan sdonyaye śni qon he yeco kta; qa oyate sdonniyanpi śni kin hena niye en inyang upi kta, Jehowa nita Wakantanka kin on, qa Israel Towakan kin on etanhan; iye niyuwakan nakaeś.

6 Jehowa iyeyepica icunhan akita miye, ikiyedan un kin icunhan hoyekiya po :

7 Tuwe sice cin he tacanku ahduśtan kte, qa wicaśta wahtani sa iye tawacin elpekiye kta; qa Jchowa en ilidoku kta, kinhan onśikida kta; qa Wakantanka unkitawapi kin ekta, iye wokajuju hduota kta nakaeś.

8 Niye nitawacinpi kin mitawacin kin ee śni, qa mitacanku kin he nitacankupi kin ee śni, Jehowa eya.

9 Mahpiya kin maka kin isanpa tehanwankantu, he iyecen nitacanku nitacankupi kin isanpa tehanwankan, qa mitawacin nita wacinpi kin isanpa nakaeś.

10 Magaju kin he kun u, qa wa 
kin malipiya eciyatanhan, qa ake ekta hnihde śni, tuka maka kin imnakiye ça waicahyekiye ça camni uyekiya, hecen tuwe woju kin ojupi qu kta, qa wayute cin he woyute qu kta ;

11 He mioie mii etanhan inanpe cin iyecece kta : miye en cokadan ihdoku kte śni; tuka taku iyomakipi kin he yuśtan kta, qa taku on yewaśi kin he ecetukiye k ta.

12 Niye wowiyuśkin yuha inayapapi kta, qa wookiye hduha yus aniyanpi kta : nitokapatanhan he qa paha kin dowan hinhdapi kta, qa maga can kin iyulipa nape hdaskapapi kta.

13 Canpepe. lkin eekiya wazi can uye kta, qa wapepeka eekiya hanteśa uye kta: unkan he Jehowa en wocajeyatapi ee kta, qa wowakta owihanke wanica kaśpapi kte śni kin heca kta.

\section{WICOWOYAKE 56.}

1 Jehowa hecen eya; Woyaco patan po, qa woowotanna econ miye: wowanikiye mitawa kin he kiyedan u nakaeś, qa mitoowotanna kin he yuotaninpi kta:

2 Wicaśta tuwe de econ kte, ca wicaśta cinhintku de sutaya yus un kte cinhan; tuwe anpetu okihipapi yuśape śni kpatan, qa iye nape taku śica ccoll śni awanihdake cinhan, he cante waśte.

3 Unkan wicaśta tokeca cinhintku Jehowa en askamiçiye cin he ie ça, Jehowa iye taoyate kin etanhan ocowasin heyata iyemayan ce, ihnuhan eye cinhan; nakun, Wanyaka -wo, can śeca hemaca ce, ihnuhan yunokos eye cinhan.

4 Jehowa hecen eya; Yunokos tona anpetu okihpapi mitawa patanpi, qa taku iyomakipi kahnigapi, qa wicotakuye mitawa yus unpi kinhan;

5 Hena wati ohna qa conkaśke mitawa mahen owanka wicawaqu kta, qa wicacaje cinhintkuyapi qa cunwintkuyapi isanpa waśte ; wicacaje owihanke wanica icimana kaśpupi kte śni waqu kta.

6 Nakun wicaśta tokeca cinca Jehowa en askamiçiyapi, ohodapi kta, qa Jehowa caje kin waśtedapi kta, iye wowidagwicaye kta, wicaśta otoiyohi anpetu ohilipapi yuśape śni patan, qa wicotakuye mitawa sutaya yus un kinhan ;

7 Hena eepi kaeś paha wakan mitawa kin ekta awicawau kta, qa wocekiye tipi mitawa ohna, wiyuśkin wicawaye kta : wohulinage wayuśnapi qa wośna tawapi kin owayuśna mitawa akan oiyokipi kta; wati kin he oyate owasin on wocekiye tipi eciyapi kta nakaeś.

8 Iehowa Itancan Israel enanakiya iyewicayapi qon mniciye wicakiye cin he hecen eya; Tona iye en mniciyapi kin isanpa nakun en mniciye wicawakiye kita ce.

9 Tinta wamanica owasin, niye watemya u miye; contanka wamanica kin iyuhpa.

10 Waawanyake tawa kin hena owasin iśtagongapi, wasdonye śni unpi, owasin śunka inina unpi, wapa okihipi śni ; wihaninanpi, iwankapi, iśtinma waśtedakapi.

11 Han, hena śunka wotapi sa, imnanpi sdonkiyapi śni, qa hena iś wonwicaya okahnih okihipi śni ; hena owasin iye tacankupi ekta ekiktonwanpi, otoiyohi iye oyanke tawa kin etanhan tawokamna kta ekta. 


\section{ISAYA TAWOYUKCAN KIN.}

12 Kuwa miye, miniśa iwacu kta, unkan mini wakan on wipiunliciyapi kta; qa heyakecinhan anpetu kin de iyecece kta, tanka, wopteca śni, iyotan linca kta ce, eyapi.

\section{WICOWOYAKE $5 \%$}

1 Wicaśta owotanna taninśni iyaya, tuka tuwedan cante ek ta yeye śni : qa wicaśta waonśida ewicayakupi, tuka wicaśta owotanna taku śica etanhan eyakupi tuwedan kecin śni.

2 Wookiye en ye kta ; towankapi kin akan ozikiyapi kta; tuwe ecetuya mani kin.

3 Tuka wapiye sa cinhintkupi, deciya kiyedan u po, wiinalimanpi qa wawiciliahapi econ sa cinca kin.

4. Tuwe on iyahahapi he : tuwe on i yahdukawapi he, ceji hiyuyayapi he : wokipajin cinca, woitonśni ojupi kin henicapi śni he:

5 Uskuyeca can ehna can teca otoiyohi ihukuya iyokipiiciya yaunpi, hokśiyopapi kin kaksiza olna wicayaktepi, imnija yuptuptujapi kiu ihukuya:

6 Kaksiza en inyan śduśduta ehna taku oyakini qon yanke; hena, hena e taku tawayaye cin ee: nakun hena en woyatke wośna yahdaśtan, wośna wayahduśna. Hena on iyokipimiçiye kta he.

7 Paha pajodan qa tehanwankantu akan nitohe eyehde; han, heciya wahduśna idada.

8 Nakun tiyopa can qa tiyopa kin ilieyatanhan wokiksuye eyehde; miye icunonpatanhan nihduzamni, qa wankan. idada; ohehdepi nitawa yahdutanka, qa hena om wicotakuye niçicaga; iye ohehdepi tawapi kin waśteyadaka, owanka yahdutanka.

\section{4}

9 Wihdi on wicaśtayatapi kin anihduwiyeya, qa wihdi waśtemna yahduota; qa makoce te. han ekta wahośi yewicayaśi, qa Hades e qeś ekta nihduhukuya. 10 Canku hanska nitawa on mdokițe niçiya; tuka, Wacinyepica śni ce, ehe śni ; ninape towiconi kin he iyeyakiya; heon etanhan waśake śni niçida śni.

11 Unkan tuwe e on kopeyahda qa koyakipa he, hecen iyatonśni, qa miyeksuye śni, qa en cante duze śni he : wanakajatanhan kaeś inina manke śni he : hecen komayakipe śni,

12 Miye nitoowotanna omdake kta, qa niolian nakun; tuka hena iniwaśte kte śni.

13 Hoyeyaye cinhan hehan nitomniciye kin hena enihdakupi nunwe: tuka tate kin he hena i.yuhpa kahbog iyewicaye kta, takuśni iwicacu kta. Tuka tuwe wacinmaye cinhan makoce kin yuhe kta, qa paha wakan mitawa kin he tawaye kta:

14 Qa heye kta; Pahaya ehnaka po, pahaya ehnaka po, canku wiyeya laga miye, can ibotopi kin mitaoyate ewicakiciyaku po. 15 Wanji tehanwankantu qa wankan un, otokahe wanica en ounyan, qa tuwe Wakan eciyapi kin he hecen eya; Tehanwankantu qa owanka wakan kin hen wati kta, nakun tuwe taniya kasujapi qa onśike cin he kici ; tona onśikapi taniyapi niwicawaye kta, qa cante kasujapi kin hena kiniwicawakiye kta heon.

16 Owihanke wanica hehanyan wawakize kte śni, qa ohinniyan śinwahda kte śni : mitokam woniya kin lianye kta nakaeś, wicataniya wakage cin nakun.

17 Tokamna śica woahtani kin on sinwahda qa wakaśtaka : namiçihbe qa sinwahda; heca eśta 
iye cante yuze canku kin ohna pemniyan ikiyopta.

18 Tacanku kin wanmdaka, unkan okiziwaye kta; nakun yus amde. kta, qa iye akiceyapi qon hena kuya om wacintonhnagwaye kta.

19 Wicaiha oicage cin he miye wakaga; Wookiye, wookiye, tuwe itehan un qa tuwe ikiyedan un kin kici, Jehowa ekiciya: unkan miye okiziwaye kta cc.

20 'Tuka wicaśta' śicapi kin hena miniwanca taś ton kin iyececapi, he owanji yanka okihi śni, mini tawa kin upśija qa maka hiyuya ece.

21 Wicaśta śicapi kin hena wookiye nicapi ce, Jehowa eya.

\section{WICOWOYAKE 58.}

1 Dote eciyatanhan pan wo, ihnuhan inina nanke cinhan, maza yahotonpi wan iyecen niho wankan yeya wo; qa mitaoyate taku śica econpi, qa Jakob tiyohnaka iye wahtanipi kin owieakiciyaka wo.

¿ Tuka anpetu otoiyohi omadepi, qa mitacanku sdonya iyokipipi, oyate wan woowotanna econ ece, qa Taku Wakan tawapi tawoahope ehpeye sni kin he iyececa: Woowotanna wokonze kin hena makidapi, Taku Wakan ikiyedan upi iyolkipipi.

3 Tokeca akihan unkiciyapi tuka wandake kte sni he: nagi iyokiśinunkiçiyapi tuka sdonye wacanni kte śni he, eyapi. Wanyaka wo, anpetu en akilianniciyapi hehan iyokipi niçiyapi kta, qa wi cohtani ecaniconpi kin ocowasin wicayakidapi ece.

4 Wanyaka wo, wicokicige qa woakinica on akihanniçiyapi, qa wośice nape on waayapapi. kta heon: Anpetu kin de iyececa nihopi wankantanhan nahonwicayayapi kta e akihanniçiyapi kte śni.

5 Akihaniçiyapi wakahnige kte cin he de iyecece kta he : Anpetu wan en wicaśta jyokiśiniçiye lita: hintkan hu iyecen pamahdedan un kta, qa wakihdaka cahota ko owinśkiton kta he. He akilianiciyapi qa anpetu Jehowa iyckipi kta, eya cajedate kta he.

6 Akihaniçiyapi wakahinige kte cin he dee śni he ; wicośice icaśke kin yukcapi kta, canwanapin wiçin kin kiyuśkapi kta, tona kakiśyapi kin hena wicakiyukcapi kta, qa can napinpi owasin duksapi kta :

7 Wotektehdapi kin aguyapi nitawa wicayakipamni kte cin hee śni he ; qa tona onśika kakiśyapi kin hena yati en awicayaku kta ; tuwe tancodan un wandake cinhan wakahpeyecaton $\mathrm{kta}$, qa wicacehpi nitawa kin etanhan nanicilibe kte śni.

8. Hehan iyoyanpa nitawa anpao iyecen inanpe kta, qa. woasni nitawa kohanna hiyu kta; nakun nitoowotanna nitokam ye kta, qa Jehowa towitan kin he hekpatanhan awanniyake kta.

9 Hehan niye hoyeyaye kta, unkan Jehowa waayupte kta; niye yapan kta, unkan iye, Iho, miye ce, eye kta. Nicokayatanhan canwanapin kin ehpeyakiye cinhan, napsukaza pazopi qa ecinkta eyapi kin hena:

10 Qa wotektehdapi kin ekta ninagi hiyuyaye ça wicanagi kakiśyapi kin he imnayaye cimhan, hehan iyoyanpa nitawa kin otpaza en limanpe kta, qa owotaninśni nitawa kin he wiyotanhan iyecece kta.

11 Unkan Jehowa ohinniyan yus aniye kta, qa iwicapuza en 


\section{ISAYA TAWOYUKCAN KIN,}

ninagi imnaye kta, qa nihuhu kin cemniciye kta: hecen niye maga wan mini imna lin he iyenicece kta, qa minilidoka wan mini wicahnaye kte śli kin he iyececa.

12 Unkan niye eciyatanhan otiwita wanakaja kin hena piya kicagapi kta; wicoicage qa wicoicage oahehdepi kin ake yakage kta; qa, Conkaśke kawankapi piya kage cin, Canku otipi kta yuecetu kin, eniciyapi kta.

13 Anpetu okilipapi kin etanhan siha eyahdaku, anpetu wakan mitawa en nitoiyokipi kin ecen ecanon kte śni ; qa anpetu okihpapi kin he woiyokipi, Jehowa towakan kin he wowitan, keya cajedate cinhan; qa nitacanku ohna oyahande śni, nitoiyokipi oyakide śni, qa niye nioie oyahdake śni, on he duonihan kinhan :

14 Hehan Jehowa en iyokipiniçiye kta; unkan malsa paha kin akan iyotanke ciciye kta, qa Jakob niyate taku tawa qon etanhan wonciye kta ce, Jehowa i kin he hecen eya nakaeś.

\section{WICOWOYAKE 59.}

1 Tho, Jehowa nape niwicaya okiki kte śni e yuptecedan śni; qa iye noge wanahon okihi kte śni e kpe śni :

2 Tuka taku śica ecanonpi kin hena niyepi qa 'Taku Wakan nitawapi yukinukan iyeniyanpi ; qa wayalitanipi kin hena iye ite anahbeniciyapi, hecen nalion kte śni.

3 Ninapepi we on aśapapi nakaeś, qa ninapsukazapi śicaya econpi kin on : niihapi woitonśni iapi, nicejipi wośice ajijipi ece.

4 Tuwedan woowotanna kipan 256 śni, qa tuwedan wowicale eciyatanhan wayaco śni: hena taKuśni wacinyanpi, qa woitonśni ohdakapi ; wicohian sica on ihduśakapi qa woahtani hiyuyapi.

5 Wamduślka itka kin manpi, qa unktomi tahokata kazontapi : tuwe witka tawapi yute cinhan te kta, qa wanji pakpipi kinhan sintehda wan liyu kta.

6 Taku kazontapi kin hena wokoyake kte śni, qa iye ohanpi kin on ihdahpapi kte śni : iye olianpi kin wośice wicohan kin heca, qa iye napepi ohna wakipi ohanyanpi yukan.

7 Iye siha taku śica ekta inyankapi, qa tona taku iyaonpepica śni unpi we kin he papson inahnipi; iye tawacinpi śicaya econpi wicotawacin kin heca; woihangye qa wokakse iye tacankupi ohna yanka.

8 Wookiye canku kin sdony api śni; qa manipi kin en wokonze wanica : ocanku śkośkopa içicagapi ; tuwe ohna ye cinhan woolíye sdonye kte ośni.

9 Heon etanhan wokonze unkitehanpi yanka, qa woowotanna unkihdegapi kte śni : iyoyanpa unkapepi, tuka iho, otpaza ; iyowotanin unkapepi, tuka ow otanin śni unkomanipi.

10 Iśtagongapi iyececa conkaśke yutantan unkakitapi, iśta codan iyecen unyutantanpi : wiyotanhan siha ibountopi, hanyetu en iyececa; maga waśteśte ohna wicaśta tapi iyeuncecapi.

11 Unkiye iyuhpa wahanksica iyececa unhidopi,.qa tinwakiyedan ceya iyecen unceyapi: wokonze unkapepi, tuka wanica; wowanikiye unkapepi, tuka unkitehanpi yanka.

12 Taku śica econkupi kin hena nitokam yuotapi, qa waunhtanipi kin hena unkoyakapi 
ece : taku śica econkupi kin hena unkicipi hiyeya, qa waunlitanipi kin hena sdonunkiyapi :

13 Śicaya econpi, qa Jehowa en itonpi sni, qa Taku Wakan unkitawapi ayuśtanpi; wokakiśyapi qa okicize cajeyatapi, woitonśni oie awacinpi qa cante eciyatanhan eyapi kin hena on.

14 Hecen woyaco hek ta itoheya iyeyapi, qa woowotanna itehan inajin; hocoka ohna wowicake hinhpaya, qa woecetu kin en u kta okihi śni.

$15 \mathrm{Han}$, wowicake wanica aya; qa tuwe taku śica ayuśtan kin he wakipi ece : Unkan he Jehowa wanyake ca woyaco wanice cin he śicedaka.

16 Qa wicaśta wanica he wanyaka, qa wawiciya wanice cin heon yuśinyaya: heon etanhan iye isto wowanikiye hdamna; qa iye toowotanna kin he ihdusuta.

17 Woowotanna kin he maku akahpe iyecen kicun: qa wowanikiye wateśdalie wapaha kiya; qa watokiçonpi wokoyake kin hena kohdaka, qa wowinawizi śna iyecen hdowin nakaeś.

18 Iye ohanyanpi kin eciyatanhan wicakicu lita, tona kipajinpi kin, wocanniye ; tokayapi kin hena wotokicon wicakicu kta; wita kin hena wicohan on towicakicicon kta.

19 Heon wiyohpeyatanhan Jehowa caje kin kokipapi kta, qa iye towitan wihinanpatanhan. Wakiyuśe kin wakpa tanka iyecen hiyu kinhan, Jebowa Taniya kin itkokim wiyolkihedan ekihde kta.

20 Hehan Wopekiton kin Zion en u kta, qa Jakob etanhan tona śicaya econpi ayuśtanpi kin hena en, Jehowa eya.

21 Unkan miye, wicotakuye * 17 mitawa wicakici un kin he dee, Jehowa eya: Mitaniya en niun, qa mioie nii en owehnake cin hena nii ayuśtan kte śni, qa nicinca ipi kin, qa nicinca sanpa cincapi ipi kin awicaynśtan kte śni, detanban qa owihanke wanica, Jehowa eya.

\section{WICOWOYAKE 60.}

1 Najin wo, iyojanjan wo; iyoyanpa nitawa wanna $u$, qa Jehowa towitan kin anihinanpa.

2 Iho, otpaza maka kin akahpe kta, qa otpaza soka oyate kin awicakahpe kta; tuka Jehowa anihinanpe kta, qa iye towitan kin anitanin kta.

3 Qa Ikcewicaśta kin iyoyanpa nitawa en manipi kta, qa wicaśtayatapi kin hena yahinanpa ojanjan kin en.

4 Ihdukśan iśta hduwankan iyeye ca wanyaka wo ; hena owasin witaya mniciyapi, niye en niupi ; nicinkśipi tehantanhan upi kta, qa nicunkśipi adoksohan wicayuhapi kta.

5 Hehan wandake ca widuśkin kta; qa nicante cancan qa tanka aye kta: miniwanca wicota kin niye en yuhomnipi kta, Ikcewicaśta towijicepi kin niye en niu kta.

6 Camo ota hinca anicahpapi kta, Midian qa Epha etanhan camo kośka kin hena: Śeba etanhan hena owasin upi kta; mazaskazi qa canśin waśtemna kin ahipi kta, qa Jehowa yatanpi wotanin waśte oyakapi kta.

7 Kedar optaye kin iyuhpa niye en mniciyapi kta, Nebayoth tamdoka kin hena iwicadake kta; hena oiyokipiya owayuśna mitawa adipi kta, unkan mitowi$\tan$ tipi kin he wahduwitan kta. 8 Mahpiya śapa iyececa, qa tin257 


\section{ISAYA TAWOYUKCAN KIN,}

wakiyedan iyecen iye tipi ekta kinyan upi kin dena tuwepi he.

9 Awicakehan wita kin hena amapepi kta; qa Tarśiś tawitawata tokaheya, tehantanhan nicinkśi awicaupi kta, mazaska qa mazaskazi tawapi koya, Jehowa Taku Wakan nitawa caje kin ekta, qa Israel Towakan kin ekta, he iye niyuwitan nakaeś.

10 Unkan wicaśta tokeca cincapi conkaśke nitawa kagapi kta, qa wicaśtayatapi tawapi kin hena oniciyapi kta. Sinwahda qehan cicaśtaka, tuka canmawaśte kin en onśicida nakaeś.

11 Unkan nitatiyopa ohinniyan yulidog he lita, hena anpetu hanyetu koya ecen iyeyapi kte śni ; hecen Ikcewicaśta tawowijice kin niye ekta aupi kta, qa wicaśtayatapi tawapi kin hena upi kta.

12 Oyate qa wicośkanśkan tukte wanji oniciye kte śni kinhan he atakuni kte śni; han, Ikcewicaśta kin hena ocowasin ihangyapi kta.

13 Lebanon towitan kin he en niukta ; wazisaka can, wazi can qa hanteśa kicica, tipi wakan nitawa owanka kin yuwaśte kta; unkan tukten siha wahde kin he wahduwitan kta ce.

14 Hehan tona kakiśniyanpi qon hena cinca patuś en niupi kta; qa tona walitenidapi śni qon owasin siha yahde kin en makata patujapi kta; qa, Jehowa Totonwe, Zion Israel 'Towakan tawa, eniciyapi kta.

15 Niye eh̉peniyanpi qa śicenidapi, hecen tuwedan opta ye śni ; heon etanhan wowaśte owihanke wanica, wicoicage yeye cin hehanyan wowiyuskkin cicage kta.

16 Nakun Ikcewicaśta asanpi tawa dazoke lita, qa wicaśtaya258 tapi aze ayazin kta: qa Jehowa Wanikiya nitawa qa Wopekiton nitawa, Wanji Waśaka Jakob tawa kin he miye e sdonyaye kta.

17 Mazaśa on mazaskazi eekiya awau kta, qa mazasapa on mazaska eekiya awau kta, qa can on mazaśa, qa inyan on mazasapa kta: Nakun akicita tancan nitawa wookiye wicawakage kta, qa wicaśtayatapi nitawa woowotanna kta.

18 Wawicakipi kin he nitamakoce ohna icimana nahonpi kte śni, woihangye qa woyutakuniśni nitaopapun mahentanhan; tuka conkaśke nitawa, Wowanikiye, qa nitatiyopa kin, Woyatan, eya caśyakiton kta.

19 Hetanhan anpetu icunhan anpetu wi kin iyoyanpa nitawa lite śni, qa iyojanjan kta on hanyetu wi kin iyoyamniye kte śni; tuka niye en iyoyanpa owihanke wanica Jehowa ee kta, qa nita Wakantanka wowitan nitawa ee kta.

20 Anpetu wi nitawa ake icimana isinyan iyaye late śni, qa hanyetu wi nitawa icimana eihdaku kte śni; iyoyanpa owihanke wanica nitawa Jehowa ee kta, qa anpetu en yaceye cin hena henakeca kta.

21 Nakun nitaoyate iyulipa owotanna kta : makoce kin ohinniyan tawapi kta, adetka huwahde, minape taku kage cin heepi, hecen mayuwitanpi kta.

22 Wanji cistinna kin he kektopawinge icage kta, qa tonana kin he oyate wasake kta. Tohan iyehantu kinhan Jehowa miye he mduinalini kta ce.

WICOWOYAKE 61.

1 Jehowa Itancan Taniya kin 
akan mayanka; Jehowa sdamakiya, wotanin waśte wicaśta onśihanpi owicawakiyake kta heon etanhan; tona cante kaśujapi wicamduskiskite kta e yemaśi; wayaka unpi wicayukcapi kta owicawakiyake kta, qa kaśka unpi wicokaśke tipi wicakiyuhidokapi kta :

2 Jehowa tawaniyetu oiyokipi kin omdake kta, qa Wakantanka unkitawapi anpetu watokiconpi tawa kin; tona ceyapi owasin wacintonhnagwicawaye kta:

3 Tona Zion en ceyapi kin hena wakoyagwicayapi kta, cahota eckiya wateśdake wicaqu kta, wicaceya eckiya wowiyuśkin wihdi, cante wankadan e ekiya woyatan wokoyake kin wicaqu kta: heon etanhan, IVoowotanna uskuyeca can, Jehowa tawoju, ewicakiyapi kta, hecen iye yuwitanpi kta:

4. Unkan otiwita kin hena piya kagapi kta, tanina yujujupi kin hena bosdan ehdepi kta, qa otonwe otiwita wanke cin hena piyapi kta, wicoicage qa wicoicage liew oskantuya wanke ciqon.

5 Hehan oyate tokeca etanhan najinpi qa nitawanunyanpi wonwicayapi kta, qa wicaśta tokeca cinca kin hena maga niciyumdupi qa hastanhanka iyuwi nicipakintapi kta.

6 Tuka niyepi, Wawayuśnapi Jehowa tawa, eniciyapi kta: Wakantanka unkitawapi taokiye, eniciyapi kta. Ikcewicaśta tawowijicepi kin yatapi kta, qa towitanpi kin on nihdatanpi kta.

7 Nitowiśtccepi kin on nonpa akihde kta, qa wonihinciye on taku tawapi kin on wiyuśkinpi kta; heon etanhan tawapi ohna nonpa akihde yuhapi kta; wowiyuśkin owihanke wanica wicakiciun kta.
8 Jehowa miye, woyaco waśtewadaka; waliulinage wośnapi kta on wawicakipi kin śicewada$\mathrm{ka}$; qa iye olianyanpi iyecen wicawaqu kta, wowicake eciyatanhan, qa wicotakuye owihanke wanica wan wicawecage kta.

9 Hehan iye oicage tawa. Ikcewicaśta ehna sdonwicayapi kta, qa etanhan icagapi kte cin oyate kin wicehna: Tona wanwicayakapi owasin, oicage Jehowa yuwaśte lsin hena eepi e sdonwicayapi kta.

10 Miye qe iyuśkinyan Jehowa on imduśkin kta, minagi mita Wakantanka on pida kta: iye wowanikiye wokoyake koyagmayan nakaeś, woowotanna śina kin amakalipa; wicaśta tawicu ton kta wateśdake hduwaśte, qa winohinca hihna ton kta oinpi kicun kin iyececa.

1 i Maka kin camni ukiye ca maga kin taku en ojupi icahkiye cin he iyecen Jehowa Itancan kin woowotanna qa woyatan oyate owasin wicitokam icahye kta.

\section{WICOWOYAKE 62.}

1 Zion on etanhan inina manke kte śni ; qa Jerusalem on etanhan oziwakjye kte śni ; toowotanna kin woiyege iyececa inanpe kta, qa towanikiye kin petijanjan ide kin iyecece kta hehanyan.

2 Unkan Ikcewicaśta kin nitoowotanna wanyakapi kta, qa wicaśtayatapi owasin nitowitan kin. Qa wicacaje teca, Jehowa i cajeyate kte cin he icajeniyatapi kta.

3 Nakun Jehowa nape ohna wowitan wateśdake kin he niye kta, qa wicaśtayatapi tawapaha Wakantanka nitawa nape kin ohna. 
4 Hetanhan icimana, (Azubah) Hhpeyapi, eniciyapi kte śni; qa nitamakoce icimana, (Śemamah) Hewoskan, eciyapi kte śni; tuka, (Hephzibah) Mitowiyuśkinen-un, eniciyapi kta, qa makoce nitawa, (Beulah) Wakanyuzapi, eciyapi kta: Jehowa iniyuśkin, qa nitamakoce kin he wakan yuzapi kta nakaeś.

5 Kośka wan wikośka wan wakan yuze cin he iyecen nicinlsśipi wakan nihduzapi kta; qa wicaśta winohinca yuze cin on iyuśkin kin he iyecen nita Wakantanka iniyuśkin kta.

6 Jerusalem, conkaśke nitawa akan waawanyake ewicawahnaka, anpetu owasin qa hanyetu owasin ohinniyan inina unpi kte śni : tona Jehowa kiksuyeyayapi, ihnulaan inina dukanpi kinhan ;

7 Qa woozikiye qupi śni po, Jerusalem hdusuta qa maka kin ohna woyatan kage kte cin hehanyan.

8 Jehowa iye nape etapa kin on, qa isto waśake cin on iciconza ; Awicakehan icimana wamnaheza nitawa tokaniyanpi yutapi kta wicawaqu kte śni; qa oyate tokeca cincapi kin minis̉a nitawa on litayanipi kin he yatkanpi kte śni.

9 Tuka tona mnakiyapi kin he hdutapi kta, qa Jehowa yatanpi kta ; qa tona wayuśpipi kin henà mitowakan hocoka kin ohna hdatkanpi kta.

10 Ohna ya po, tiyopa kin ohna ya po; oyate tacanku kin wiyeya kaga po: wankan ehnaka po, canku wankantu kaga po; etanhan inyan pahi po ; wiyokihedan wan oyate kin wankan ewicakicihde po.

11 Tho, Jehowa maka ihanke hehanyan nahonwicaya; Zion cunwintku kin heciya po; Iho, wowanikiye nitawa kin u ce; iho, iny unwin hduha un, qa woecon tawa kin heitokam yanke.

12 Unkan hena, Oyate wakan, Jehowa opewicakiton, ewicakiyapi kta: qa niye, Akitapi, otonwe ehpeyapi śni, eniciyapi kta.

\section{WICOWOYAKE 63.}

1 Edom etanhan, Bozra etanhan sina duta in $u$ kin he tuwe he; wokoyake waśte kohdaka, iye towaśake tanka on icimani un kin he tuwe he. Woowotanna eciyatanhan ia, wanikiya waśake cin he miye ce.

2 Tokeca nitawokoyake kin duta, qa nitaśina turve miniśa iyuśkice naśkice cin iyececa he.

3 Miniśa iyuśkice cin miśnana nawaśkica; qa oyate kiı etanhan tuwedan micica śni: lieon mitocanniye on nawicawaśkice $\mathrm{kta}$, qa mitośinhda on nawicawapan kta; qa iye hanpi kin mitaśina amnimnipi $k t a$, qa mitawokoyake ocowasin wahdustan kta.

4 Watokiçonpi anpetu kin he micante en un, qa opewicawakiton tawaniyetu kin he wanna hiyohi nakaeś.

5 Unkan ekta ewatonwe, tuka waokiya wanica; qa tuwedan wayusuta śni heon mayuśinyaya; heon etanhan miye miisto wowanikiye hdamna, qa mitośinhda kin he mahdusuta.

6 Hecen mitocanniye on oyate kin nawicawapan kta, qa mitośinhda on witkowicawakiye kta, qa iye hanpi kin he makata ehpewaye kta.

7 Jehowa wawacinkta yuza tanka kin he mdatan kta, Jehowa yatanpi kin, Jehowa taku icantounhnakapi kin owasin eci- 
yatanhan, qa wowaśte tanka Israel tiyohnaka icantowicahnaka, iye towaonśida eciyatanhan qa iye wawacinkta yuza tanka ota kin eciyatanhan.

8 Unkan iye heya; Awicakehan hena mitaoyate, wicacinca taku ecinkta eyapi kte śni ce. Hecen Wanikiya tawapi kin he iye.

9 Toiyokiśicapi iyuhipa en iye iyokiśica; qa iye ite Taohnihde kin he niwicakiya: iye towaśtedake qa tocantekiye kin on opewicakiton, qa wicaqin, qa wanakaja anpetu kin owasin iye wicahduha un.

10 Tuka hena eqe wawakipajinpi qa iye Taniya Wakan kin iyokiśinyapi; heon etanhan iye tokawicaye kta ihduhomni, iye hinca wicakiza.

11 Hehan wanakaja anpetu kin hena kiksuya, Moses qa iye taoyate: Tuwe mini tanka etanhan awicau qon he tukten un he, obe tawa wonwicaye ciqon : 'Tuwe iye Taniya Wakan mahen ehnake ciqon he tukten un he.

12 Tuwe Moses nape etapa on yus awicaya, iye isto wakan kin on wicitokam mini kin wicakiyukinukan, caje owihanke wanica wan icicage $\mathrm{kta}$.

13 Tuwe mini śbe kin ehna maniwicakiya, suktanka wan hopuza en un kin iyececa, hecen kaktihanhanpi kte śni.

14. Woteca wan kaksiza ekta iyaye cin iyecen Jehowa Taniya kin oziyekiya; he iyecen niye nitaoyate yus awicada, wowitan caje nicicage kta heon.

15 Malipiya kin eciyatanhan kun ahitonwan wo, qa nitoowotanna qa nitowitan tipi kin etanhan wanyaka ye : nitowinawizi qa nitowaśake kin tukte e he; nitowaonśida qa miye en nito- cantekiye kin hena anakiptapi he.

16 Awicakehan niye ateunniyanpi, Abraham sdonunkiyapi śni qa Israel iyeunkiyapi śni qeyaś: niye, Jehowa, Ateunniyanpi, Wopekiton unkitawapi; nicaje kin he otokahe wanica.

17 Jehowa, tokeca nitacanku etanhan nuniunyakiyapi he ; nitokokipe icunonpa cante unyadusutapi he. Nitaokiyepi on, wicoun tawawirayaye cin on etanhan hdicu ye.

18 Oyate wakan nitawa ptenyedan tawakiyapi : tokaunyanpi kin hena tipi wakan nitawa naśapapi.

19 Unkiye wanakajatanhan unyakonpi : hena e tohinni wowidagwicayaye śni: hena nicaje icajewicayatapi śni.

\section{WICOWOYAKE 64 .}

1 Tokin mahipiya kin yahduhdecen, kun hidu kta; hecen nitohnake on he kin skan aye kta. 2 Peta canliaka ideya, peta mini ipiliya ece kin iyececa; tona tokaniyanpi nicaje sdonyewicakiye kta, nitohnake on Ikcewicaśta cancanpi kta heon.

3 Taku wowinihan ecanon qehan, hena unkapepi śni; kun hidu, nitohnake on lie kin skan aya.

4 Wakantanka, nicunonpatanhan, tuwe wacinyan un taku kicage kte cin, otokaheyatanhan nahonpi śni, wicanoge kin en iyohpaye śni, wicaiśta wanyake śni.

5 Tuwe wiyuśkin qa woowotanna econ ece kin he itkoyakipa; nitacanku ohna niksuyapi kin hena: Iho, niye śinyahda; unkan waunhtanipi: dena en ohinniyan, tuka niunyanpi kta. 
6 Tuka unkiye owasin taku śapa iyeuncecapi, qa woowotanna unkitawapi iyulipa wokoyake awepi kin iyececa ; qa iyulipa canwapa iyecen śniś unkayapi; qa taku śica econkupi kin hena, tate iyececa, yutokan unkayapi kta.

7 Hececa eśta tuwedan nicaje loyekiye śni, tưwedan en niyuze kta ilıduhica śni; niye niite ahaunyecilimanpi nakaeś, qa taku śica econkupi kin on awihnuniunyakiyapi.

8 Tuka nakaha Jehowa, Ateunyanpi kin he niye; maka coco kin he unkiyepi, qa cega kaga unkitawapi kin he niye; qa ninape taku kage cin he unkiyepi iyulipa.

9 Jehowa nina śinhda śni wo, qa ohinniyan. woahtani kiksuye śni ye: iho, wanyaka ye, ceunniciyapi, unkiye owasin nitaoyate unyakonpi.

10 Otonwe wakan nitawa kin hena makoskantu; Zion hewoskantu; Jerusalem otiwita.

11 Tipi wakan qa owanyag waśte unkitawapi, ohna atewicunyanpi niyatanpi qon he peta on huhnaga: qa taku oiyokipi unkitawapi iyuhpa ihangyapi.

12 Jehowa, dena on ananicipte kta he; inina nanke ca nina kakiśunyayapi kta he.

\section{WICOWOYAKE 65.}

1 Tona wimawangapi śni qon hena amakitapi ; tona omadepi śni qon hena iyemayanpi: Ikcewicaśta micaje on icajeyatapi śni qon; Wanmayaka ye, wanmayaka ye, ewicawakiya.

2 Oyate wan wawakipajin, canku waśte śni ohna iye tawacinpi eciyatanhan mani kin he anpetu. osan nape wicawakiyugate: 262
3 Oyate wan miite itokam ohinniyan sinhdamayanpi, can wojupi ehna wawayuśnapi, qa maka omdoton ohna wizinyapi ece.

4 Hena wicaśta hnakapi ehna yukanipi, qa woinalibe kin en iwankapi; kukuśe śin yutapi, qa cega ohna tado aśapa hanpi hduhapi.

5 Hena heyapi; Ako inajin wo ; mikiyedan u śni wo; niye isanpa miye mawakan nakaeś. Dena mapoge ohna sota heca, peta wan anpetu osan itkon.

6 Iho, mitokam wowapi; Inina manke kte śni, tuka watolicon ecamon kta; han, iye maku ohna wicawecu kta;

7 Wayahtanipi qa niyatepi wahtanipi yuwitaya: hena he kin akan wizinyapi, qa paha kin akan iśtenmayanpi: heon etanhan iye ohanyanpi qon hena maku ohna iwicaweciyute kta ce, Jehowa eya.

8 Jehowa kaken eya; Miniśa teca kin hastanhanka iyage kin en un, qa, Ihangye śni wo, woyawaśte wan en un ce, eyapi kin, he iyececa mitaokiye on ecamon $k t a$, hecen iyulpa ihangwicawaye kte śni.

y Unkan Jakob etanhan oicage wan awau kta, qa Juda etanhan tuwe paha mitawa tawaye kita wan: hecen wicawahdahnige cin hena tawayapi kta; qa mitaokiye kin hena he en ounyanpi kita.

10 Unkan Śaron tahinca optaye otipi kin heca kita, qa Akor kakśiza kin he wanunyanpi owanka kin hee kta, mitaoyate amakitapi kin hena on.

11 Tuka Jehowa ayuśtanpi, paha wakan mitawa akiktonjapi, Gad (wicanlipi tanka) wahna wotapi wan wiyeya ekicihnakapi, qa Meni, (anpao wicanlipi) 
woyatke wan okicaśtanpi kin hena niyepi.

12 Heon etanhan mazasagye ekta ciyawapi kta, qa niyepi owasin wicaktepi en yapatujapi kta : cicipanpi tulka amayaduptapi śni, iwaa tuka nayahonpi śni, tuka miiśta itokam taku śica ecanonpi, qa taku iyomakipi śni kin he yakahnigapi;

13 Heon etanhan Jehowa Itancan kin hecen eya; Iho, mitaokiye wotapi kta, tuka niye wotekteyahdapi kta; iho, mitaokiye wayatkanpi kta, tuka niye inipuzapi kta; iho, mitaokiye wiyuśkinpi kta, tuka niye winistecapi kta :

14. Iho, mitaoliye cante waśte on dowanpi kta, tuka niye cante śicapi on yaceyapi kta, qa woniya kaksapi on yaśicahowadapi kta.

15 Qa nicajepi woyaśice icajeyatapi kin he wicawahdalinige cin aihpewicayayapi kta : qa Jehowa Itancan kin nikte lita, qa iye taokiye kin wicacaje tokeca eya icaśkiton kta.

16 Hecen tuwe maka akan ihdawaśte kinhan Taku Wakan wicake cin on ihdawaśte kta; qa tuwe maka akan taku eya iciconze cinhan, Taku Wakan wicake cin on konze kta; tanina woiyokiśica kin hena akiktonjapi qa miiśta etanhan anahmanpi kin heon etanhan.

17 Iho, malipiya teca qa maka teca wakaga; unkan tanina qon hena kiksuyapi kte śni, qa wicacante kin en u kte śni.

18 Tuka niyepi, taku wakage cin on ohinniyan pidapi qa wiyuśkin po ; iho, Jerusalem he wiyuśkinpi, qa taoyate kin wowiyuśkin wakaga heon etanhan.

19 Hecen Jerusalem on imdu- śkin kta, qa mitaoyate on cante mawaśte kta: unkan he ohna wicaceya ho kin icimana nationpi kte śni, qa śicahowayapi ho kin nakun.

20 Hetanhan anpetu hokśiyoqopa wan icimana yuke kte śni, qa wicalinca anpetu imna śni wanice kta: hokśiyoqopa kin eqe waniyetu opawinge cinca hehan te kta nakaeś: tuka walitanisa kin he waniyetu opawinge kinhan yaśicapi kta.

21 Unkan tipi kagapi kta qa ohna tipi kta; qa hastanhanka hu hdepi kta qa etanhan waskuyeca hdutapi kta:

22 Tipi kagapi kinhan turve tokeca ohna ti kte śni; wojupi kinhan tuwe tokeca yute kte śni : mitaoyate taanpetu kin can anpetu kin iyecece kta ; qa tona wicawahdahnige cin hena iye nape ohanyanpi kin tehan hdutapi kta.

23 Takuśni on litanipi kte śni, qa woiyokiśica on cinca tonpi kte śni : tona Jehowa wicayawaśte cinca kin hena eepi, qa tona hena etanhan icagapi nakur.

24. Unkan kaketu kte, hena hoyeyapi śni itokam waamdupte kta, qa iahan unpi icunhan nawahon kta.

25 Śunktokeca qa tahinca cinca kin akiptan wihanpi kta; qa mnaja kin he tatanka iyececa peji yute kta; qa wamduśka tawoyute maka mdu kin hee kta. Paha Wakan mitawa owancaya wayuśicapi kte śni, qa waihangyapi kte śni, Jehowa eya.

\section{WICOW OYAKE 66.}

1 Jehowa hecen eya; Mahpiya kin he mitoiyotanke kin ee, qa maka kin misiha oahde kin hee. Tipi miyecagapi kte cin tukte 
e he; qa woozikiye mitawa kin tukten yanka he.

2 Dena owasin minape kaga, qa dena owasin ecen un, Jehowa eya: tuka de ekta ewatonwe kta, tuwe onśike ca taniya kaśujapi, qa mioie on cancan kin he.

3 Tuwe tatanka kikte kin he wicaśta kaśtaka iyececa; tuwe tahinca cinca wayuśna kin śunka tahu kakse cin iyececa; tuwe minha wan wayuśna kin he kukuśe we iyececa ; tuwe wizinyapi wokiksuye wośna kin he wakagapi wan yawaśte kin iyececa. Han, hena e canku hdalinigapi, qa taku śapa tawapi on nagi ihduśkinpi.

4 Miś eya wicoiyoliśica wicawecicahnige kta, qa taku kokipapi kin hena en awicawau kta. Wicawakipan qehan tuwedan waayupte śni ; iwae cehan nahonpi śni; tuka miiśta itokam taku śica econpi, qa taku iyomakipi śni kin hena hdahinigapi kin heon etanhan.

5 Tona iye oie on nicancanpi kin, Jehowa oie kin nalion miye : Nihunkawanjipi śicenidapi, qa micaje on etanhan tankan ehpeniyanpi qon hena, Jehowa yuwitanpi nunwe, eyapi : tuka widuśkinpi kta on ihdutanin kta, qa hena eqe iśtecapi kta.

6 Oqoyapi ho wan otonwe kin etanhan; tipi wakan kin etanhan wicaho wan; Jehowa tona tokayapi towicakicicon ho kin hee.

7 Hoksiksuye sni itokam hiyuya, hinyahin wayazanhda śni itokam hokśidan wan ton.

8 'Taku he iyececa tuwe nation he; tuwe taku hececa wanyaka he. Maka kin anpetu wanjidan en waicaliye kta he; oyate wan wancahna wicatonpi kta he: 264
Tuka Zion hokśiksuya hehantudan cinca hiyuwicaya.

9 Ihdulidece wakiye kta qa hiyuwakiye kłe śni he, Jehowa eya: hiyuwakiya eśta onawatake kta he, nita Wakantanka eya.

10 Jerusalem kici wiyuśkin po, qa tona waśte yadakapi owasin kici cante waśte miye ; wowiyuśkin on kici wiyuśkin po, tona ayakiceyapi kin iyuhpa.

11 Hecen ayazinpi kta qa tocantohnake aze on winipipi kta; hecen dazokapi kta, qa iye towitan aze ojudan kin etanhan wiyuśkinniçiyapi kta.

12 Jehowa kaken eya; Iho, wakpa iyecen wookiye waqu kta, qa Ikcewicaśta towitanpi kin mini kaduze cin iyececa. Hehan ayazinpi kta, maku ohna niyuhapi kta, qa śiyoto akan niyuhnahnapi kta.

13 Tuwe hurku cantohnagye cin he iyecen cantohnagciyapi kta ; qa Jerusalem en wacintohnagniyanpi kta.

14 Tohan wandakapi kinhan nicantepi iyuśkin kta, qa nihuhupi kin wato iyecen icage kta. Unkan Jehowa nape kin he iye taokiye sdonyapi kta, qa tokawicaye cin en iye tośinhda kin tanin kta.

15 Iho, Jehowa peta yuha u kta, qa canpahmihma tawa kin tate iyumni iyececa; hecen tocanniye kin wośinhda kici, qa toiyopeye kin peta ide kici wicaqu kta.

16 Peta on qa iye tamazasagye on Jehowa wicacehpi iyuhpa om wohdake kta: unkan Jehowa wicakte kin hena ota kta.

17 Tona ihduwakanpi qa can wojupi ehna ihduskapi, cokata wanji ihakam, kukuśsésin, qa taku aśapa, hitunkadan ko yutapi 
kin hena yuwitaya awihnunipi kta, Jehowa eya.

18 Unkan kaketu kte, iye ohanpi qa tawacinpi kin hena on etanhan Ikcewicaśta qa wicaceji iyuhpa mduwitaya kta: unkan hena upi qa mitowitan kin wanyakapi kta.

19 Unkan hena wicehna wowakta wan ewahnake kta, qa tona etanhan najicapi kin hena Ikcewicaśta kin ekta yewicawaśi kta, Tarśiś, Pul qa Lud, hena itazipa ekatapi ; Tubal qa Javan, wita tehan unpi kin, tona omayakapi nahonpi śni qa mitowitan wanyakapi śni. Unkan hena iś Ikcewicaśta ehna mitowitan yaotaninpi kta.

$20 \mathrm{Qa}$ hena nihunkawanjipi owasin awicaupi kta, Jehowa wakiyuśnapi wan, Ikcewicaśta o wasin etanhan, śuktanka akan, qa canpahmihma ohna, qa tatokadan akan, qa śonśonna akan, qa camo cincapi akan, Jerusalem
Paha Wakan mitawa ekta, Israel cinca wośna wan wakśica ska ohna Jehowa ti kin en ahipi kin he iyececa, Jehowa eya.

21 Unkan miś eya hena etanhan wawayuśnapi qa Lewi wicaśta hecapi kta e iwicawacu kta ce, Jehowa eya.

22 Malipiya teca qa maka teca wakage kte cin hena mitokam wanke kta, he iyecen niye nicincapi qa nicajepi unpi kta ce, Jehowa eya.

23 Unkan kaketu kte, wi teca wan etanhan witeca ekta, qa wookilipe etanhan wookilipe ekta hehanyan, wicacehpi owasin mitokam waohoda au kta, Jehowa eya.

24. Qa hena yapi kta, qa wicaśta wamicihtanipi tancan tapi kin wanwicayakapi kta. Wamduśka tawapi kin te kte śni, qa peta tawapi kin kasnipi kte śni: tuka wicacehpi owasin hena wowaliteda śni yawapi kta.

\section{Microformaci by}

\section{Preservation}

Services

min $6524, d$

30 otT 95 





WICOWAZI OWICAWAPI.

WAKANKICIYUZAPI. 
WICOWAZI OWICAWAPI.

WICATONPI。 


\section{WICOWAZI OWICAWAPI.}

WICATONPI. 


\section{WICOWAZI OWICAWAPI.}

\section{WICATIE CIN.}






\section{A KOTA}

\section{WOW API WAKÄN KIN.}

\section{THE NEW TESTAMENT,}

IN THE

DAKOTA LANGUAGE:

TRANSLATED FRON THE ORIGINAL GREEK,

BY STEPHEN R. RIGGS,A. M.

MISSIONARY OF THE A. B. C. F. M.

NEW YORK:

AMERICAN BIBLE SOCIETY,

INSTITUTED IN THE YEAR MDCCCXVI. 


\section{WICOTAKUYE TECA KIN.}

\begin{tabular}{|c|c|}
\hline Wicowroyake. & Wicomoyake. \\
\hline theos .................. 28 & I. Timotheos......... \\
\hline Larkos ... & II. Timotheos. . \\
\hline .... 24 & Titos ......... \\
\hline .......21 & Philemon... \\
\hline inyanpi kin ............. 28 & Hebrew... \\
\hline oma.................... 16 & Jakob \\
\hline Korinth ............... 16 & I. Inyan... \\
\hline II. Korinth ............. 13 & II. In \\
\hline Galatia................ & I. Jol \\
\hline Ephesos $\ldots \ldots \ldots \ldots \ldots \ldots \ldots \ldots$ & II. Johannes ... \\
\hline Philippi.................. 4 & III. Jollannes ... \\
\hline 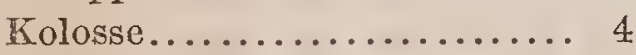 & ........ \\
\hline essalonike ............ 5 & Wayuotanin ........... \\
\hline aessalonike.... & \\
\hline
\end{tabular}




\section{MATTHEOS.}

Jesus eya caśyaton $\mathrm{kta}$; iye taoyate kin wahtanipi kin etanhan niwicaye kta, heon etanhan.

22 Unkan dena owasin hecen conpi, Itancan oie, wicaśta wokcan oyake ciqon he yuecetupi kta; he hecen eya;

23 Iho, witanśna un wan ihduśake ca cinca ton kta; unkan limmanuel eya caśtonpi kta; Wakantanka unkicipi un, eyapi kin he kapi.

24 Unkan Joseph iśtinma etanhan kikta, hehan Itancan taohnihde token econśi qon he ecen econ, qa tawicu hduza.

25 Tuka cinca tokapa cinca ton śni hehanyan sdonye śni; unkan Jesus eya caśkitorr.

\section{WICOWOYAKE 2.}

1 Unkan Herod wicaśtayatapi taanpetu kin he ehan, Bethlehem Juda makoce kin en Jesus tonpi, hehan iho, wiyohiyanpatanhan wicaśta ksapa Jerusalem en hipi qa heyapi ;

2 Tuwe Juda oyate kin en wicaśtayatapi kta tompi kin he tukten un he; wicanhpi tawa kin he wiyohiyanpata wanunyakapi qa ohoundapi kta on unhipi ce.

3 Herod wicaśtayatapi kin he nahon, unkan nina yuśinyaya, Jerusalem ocowasin nakun.

4 Unkan wośnapi kagapi owasin qa oyate kin en wowapi kagapi kin hena wicayuwitaya, hehan Messiya tukten tonpi kta hecinhan, he iwicawanga.

5 Unkan heciyapi; Juda makoce kin en Bethlchem otonwe kin hetu ce ; wicaśta wokcan kin en kaken owapi ;

6 Qa niś Bethlehem, Juda makoce kin en, Juda itancanpi kin ehna iyotan nicistinna śni ; enitanhan wicaśtayatapi wan u kta, he Israel mitaoyate kin wonwicaye kta.

7 Hehan Herod wicaśta ksapapi qon nalimana wicakico, qa wicanhpi kin tohanhan tanin ece, he atayedan iwicawanga.

8 Hehan Bethlehem ekta ye wicaśi qa heya; Ya po, qa hokśiyoqopa kin nina ode po; qa iyeyayapi kinhan hośimakahdi po; miś eya wau qa ohowada kta, eya.

9 Wicaśtayatapi ia nalionpi hehan tankan iyayapi; unkan iho, wicanhipi wiyohiyanpata wanyakapi qon he wicitokam iyaye ca hokśiyoqopa wanke cin he iwankam inajin.

10 Unkan wicanhpi kin wanyakapi qehan wowiyuśkin tanka on iyuślkinpi.

11 Unkan ti mahen ipi hehan hokśiyoqopa kin wanyakapi, hunku Mary nakun, qa itokam makata ehpeiçiyapi qa ohodapi : unkan wopiye hdulidokapi hehan taku qupi, mazaskazi, qa canšin pejihuta waśtemna koya.

12 Unkan wowihanmde on Wakantanka wahowicaya, Herod ekta hdapi kte śni, hehan canku tokeca ohna iye tamakoce ekta hdapi.

13 Hena wanna kihdapi hehan, iho, Itancan taohnihde wan wowihanmde on Joseph en ihdutanin qa heya; Najin qa hokśiyoqopa hunku kici iwicacu qa Egupta ekta najica wo, qa tohan ociciyake kte cin hehanyan hen un wo: Herod hokśíyoqopa kin kte wacin on etanhan ode lita ce.

14 Hecen hanyen najin hiyaye, ca hokśiyoqopa hunku kici ewicahdaku, qa Egupta ckta iyaya : $15 \mathrm{Qa}$ hen un, Herod te cin hehanyan: Hecen Itancan wicaśta wokcan on taku eye ciqon 
WICOWOYAKE 3.

he yuecetupi kta; Egupta etanhan micinkśi weco ce.

16 Wicaśta ksapapi qon Herod hnayanpi wanhdake cehan nina śihda ; qa akicita ekta yewicaśi, qa Bethlehem qa makoce ihdukśan koya en, hokśiyopa owasin wicakte, tona waniyetu nonpa qa ihukuya, wicaśta ksapapi wiwicawange ciqon he iyacinyan.

17 Unkan hehan Jeremiya wicaśta wokcan oie qon yuecetupi, he kaken eya;

18 Rama en wicaho wan nahonpi, içidowanpi, wicaceya qa waśihdapi tanka; Racel cinca awicakiceya qa wacintonknakapi kta cin śni, cinca en unpi śni nakaeś.

19 Tuka Herod wanna ta hehan, iho, Itancan taolinilide wan Egupta elita Joseph en taniniciya ;

£,0 Qa heya; Najin qa hokśiyoqopa hunku kici ewicahdaku, qa Israel makoce tawa kin ekta hda wo; tona hokśiyoqopa ta cinpi qon hena wanna tapi.

21 Unkan najin hiyaye ca' hokśiyoqopa hunku kici ewicahdaku qa Israel tamakoce kin en hdi.

22 Qa Arcelaos, Juda makoce kin en, atkuku Herod tohe kin en wicaśtayatapi yanka, he nahon liehan ekta hde kta kokipa; tuka wowihanmde on Wakantankâ wahoye cin on, Galile makoce kin ekta iyaya:

23 Qa otonwe wan Nazare th eciyapi kin he en hi qa hen yanka; hecen, Nazareth wicaśta eciyapi kta ce, wicaśta wokcan eye ciqon he yuecetupi.

\section{WICOWOYAKE 3.}

1 Unkan anpetu kin hena en, Johannes baptem wicaqu ece kin he Juda tamakoce hewoskan ekta woyaka hi;

2 Qa heya; Ihduecetu po, malipiya wokiconze kin wanna kiyedan u ce.

3 Isaya wicaśta wokcan cajeyate ciqon he dee, kaken eya; Wicaho liewoskan en hotanin; Itancan tacanku kin wiyeya ehnaka po, ocanku tawa kin owotanna kaga po.

4 Unkan Johannes eqe tawokoyake taku wan kamel eciyapi he hin kin on kagapi, qa tehmiso wan ipiyagkiton; unkan tawoyute kin psipsicadan qa tulimaga canhanpi kin hee.

5 Hehan Jerusalem qa Juda qa Jordan ihdukśan tanhan owasin en hipi :

6 Unkan Jordan en baptem wicaqu, woalitani ohdakapi.

7 Unkan Pharisee qa Saddukee wicota baptem wicaqu kin en hipi wanwicayaka, hehan hewicakiya; Wamduśka wicoicage kin, wocanniye u kte cin nayecipapi kta tuwe e wahokonniciyapi he.

8 Heon etanhan ihduecetupi kta iyececa waskuyeca icahya po :

9 Qa, Abraham ateunyanpi ce, eyeciyapi kta awacinpi śni po; miye heciciyapi, Inyan kin dena etanhan Wakantanka Abraham cinca kicage kta okihi.

10 .Unkan wanna onspe kin can hute kin en ehnakapi; heon etanhan can otoiyohi waskuyeca waśte aicage śni kin he kawankapi qa petan ehpeyapi ece.

11 Miye nihduecetupi kta on mini on baptem ciçupi; tuka wanji iyotan waśaka mihakam $\mathrm{u}$, he tahanpe kin weciyuhe kta iyemicihantu śni ; he qe Woniya Wakan qa peta on baptem niçupi kta. 


\section{MATTHEOS.}

12 Icaduge nape ohna hdulia, owanka tanyan hdahinte kta, qa aguyapi su kin dotopiye ohna mnakiye kta, tuka ha kin peta kasnipica śni on hulinahye kta.

13 Hehan Jesus Galile etanhan $u$, qa Jordan ekta Johannes en hi, baptem qupi kta e heon.

14 Tuka Johannes tehinda qa heya; Baptem mayaqu kta iyemaceca, qa niś en mayahi he.

15 Tuka Jesus ayupte ca heciya; Dehan iyowinyan wo ; decen wicoowotanna ocowasin unśtan kta iyececa. Hehan iyowinkiya.

16 Unkan Jesus baptem qupi qehan ecahankeya mini kin etanhan itawankanhde hda ; unkan iho, mahpiya kin ayuzamnipi, qa Wakantanka Taniya kin wakiyedan kahya kuntkiya $u$, qa en hiyahan, wanyaka.

17 Unkan iho, malipiya eciyatanlian wicaho wan heya; Micinkśi waśte wakidake cin he dee, on iyomakipi hinca ce.

\section{WICOWOYAKE 4.}

1 Hehan Jesus woniya on hewoskan makoce kin ekta ayapi, Wakanśica iyutanye kta e heon.

2 Unkan wanna anpetu wikcenina topa qa hanyetu wikcemna topa wote śni un, hehan wotektehda

3 Unkan wawiyutanyan kin en hi qa heciya; Wakantanka Cinhintku kin he niye hecinhan, inyan kin dena aguyapi icage kta keya wo.

4 Tuka iye ayupte ça heya; Aguyapi ecedan on wicaśta ni kte śni, tuka wicoie otoiyohi Wakantanka i eciyatanhan enanpe cin hena on, hecen wowapi kagapi qon.

5 Hehan Wakanśica otonive wakan kin ekta aya, qa tipi wakan oinkpa wan alian ehde;

6 Qa heciya; Wakantanka Cinhintku kin he niye hecinhan, hetanhan kun ehpeiciya wo; kaken wowapi en kagapi ce; Taohnihde kin niye on econ wicaśi kta, hecen nape ohna niyuhapi kta, inyan wan en siha ibonițo kte śni.

7 Tuka Jesus heciya; Akeś wowapi en kagapi; Itancan Wakantanka nitawa kin he iyutanyaye kte śni.

8 Hehan ake Wakanśica paha wan tehanwankantuya kin he ekta aya, qa maka akan wokiconze kin owasin wowitan ko kipazo ;

9 Qa heciya; Makata ehpeniciye ca ohomayada kinhan, dena owasin cicu kta ce.

10 Hehan Jesus heciya; Satan miheyata ya wo; Itancan Wakantanka nitawa kin he ohoyada $\mathrm{kta}$, qa iśnana wowidagniye kta ce, eya owapi tuka qon.

11 Hehan Wakanśica ayuśtan; unkan iho, mahpiya ohnihde kin en hipi qa okiyapi.

12 Unkan Johannes kaśkapi he nation hehan Jesus Galile ekta iyaya :

13 Qa Nazareth ehpeya iyaye ca Kapernaum en hiyanka, he mde kin icahda, Zabulon qa Nephtalim opapun kin hetu.

14 Hecen Isaya oie qon yuecetupi kta;

15 Zabulon makoce qa Nephtalim makoce, mde kahda ocanku kin, Jordan akasanpa tanhan, Galile Ikcewicaśta ounyanpi kin :

16 Oyate otpaza en iyotanke ciqon he iyoyanpa tanka wanyakapi, qa tona wiconte makoce qa ohanzi kin en iyotankapi kin hena iyoyanpa awicahinanpa ce. 17 Unkan hetanhan Jesus wo- 


\section{WICOWOYAKE 5.}

yaka aye ça heya ece ; Ihduecetu po, mahpiya wokiconze kin wanna kiyedan u ce.

18 Unkan Galile mde kin icahda mani icunhan, wicaśta nom sunkakiciyapi wanwicayaka, Simon, Inyan eciyapi, qa sunkaku Andrew eciyapi, ho wan mde kin en iyeyapi ; hena hopsicapi kin hecapi :

19 Unkan hewicakiya; Miyahna u po, kinhan wicaśta wicadupsicapi ece e kta cicagapi kta ce.

20 Unkan ecahankeya ho kin ehpeyapi qa ihakam iyayapi.

21 Unkan hetanhan iyoopta ye ca, nakun nom sunkakiciyapi wanwicayaka, Jakob Zebede cinhintku qa sunkaku Johannes, wata kin ohna atkukupi Zebede kici unpi qa lio piyapi ; unkan hena wicakico.

22 Iś eya wata kin atkukupi ko oliankoya ehpeyapi.qa ihakam iyayapi.

23 Unkan Jesus Galile owancaya ryaya, omniciye tipi yuhapi kin ohna waonspewicakiye ca, wokiconze wotanin waśte kin oyaka ece, qa oyate kin en wowayazan owasin qa wicocante śica owasin asniyan ece.

24. Unkan ohan kin Suriya owancaya oyakapi; hecen tona śicaya yukanpi, qa tona wowayazan ocaje owasin on wayazanpi, qa tehiya unpi, qa tona taku wakan śica yuhapi, qa tatapike cin hena koya owasin en awicahipi ; unkan asniwicaya.

25 Hecen oyate ota ihakam yapi ece, Galile etanhan, qa Dekapolis qa Jerusalem qa Juda etanhan, qa Jordan akasanpa tanhan.

\section{WICOWOYAKE 5.}

1 Unkan wicota wanwicayaka hehan paha wan ekta iyaya; qa hen iyotanka hehan waonspewicakiye cin en hipi.

2 Unkan i hdukawa qa waonspewicakiye ca heya ;

3 . Tona cante mahen onśiicidapi kin hena wicayawaśtepi ce ; mahpiya wokiconze kin he tawapi ece.

4 Tona ceyapi kin hena wicayawaśtepi ; hena wicakihnapi kta.

5 Tona onśihanpi kin hena wicayawaśtepi; maka kin de tawapi kta.

6 Tona woowotanna on wotektehdapi qa ipuzapi kin hena wicayawaśtepi ce; hona imnanpi kta.

7 Tona waonśidapi kin hena wicayawaśtepi ; hena onśiwicadapi kta.

8 Tona cante en ecepidan kin hena wicayawaśtepi ; hena Wakantanka wanyakapi kta.

9 Tona wookiye kagapi kin hena wicayawaśtepi ; hena Wakantanka cinca ewicakiyapi kta.

10 Tona woowotanna econpi kin on śicaya kuwapi kin hena wicayawaśtepi mahpiya wokiconze kin he tawapi ece.

11 Tohan aniiapi qa śicaya nicuwapi, qa miye on itonśniyan taku śica owasin eniciyapi kinhan, niyawaśtepi ece.

12 Wiyuśkinpi qa nina cante waśte po, mahpiya ekta ocinyopeniyanpi tanka yanka; wicaśta wokcan nitokam unpi qon hena iś eya hecen wicakuwapi qon.

13 Maka miniskuya kin he niyepi. Tuka miniskuya kin he skuye śni kinhan taku e on skuyeyapi kta he; hetanhan takudan okihi kte śni, tankan elipeyapi kta qa wicaśta amanipi kta.

14. Oyate iyoyanpa kin he niyepi. Otonwe wan paha akan kagapi kin he anahbepica śni ece.

15 Nakun petijanjan ideyapi qa 
koka ohdateya iyeyapi śni, tuka petijanjan ihupa wan en ośtanpi, hecen tipi kin'ohna owasin iyoyamwicaya ece

$16 \mathrm{He}$ iyecen wicaśta itokam iyoyanpa nitawa iyojanjan kta, hecen wicohan waśte nitawa wanyakapi kta, qa mahipiya ekta ateyayapi yanke cin he yuonihanpi kta.

17 Woope qa wicaśta wokcan kin mdutakuni kte śni on wahi kecinpi śni po, ihangyapi on -wahi śni tuka yuecetupi kta on.

18 Awicakehan heciciyapi ; Mahipiya qa maka kin atakuniśni eśta, oowa wanjidan qa oehnake wanjidan woope kin etanhan icupi kte śni, owasin ecetu kta.

19 Heon etanhan, tuwe woahope kin dena wanji cistinna ahope śni, qa hecen wicaśta onspewicakiye cinhan, he mahpiya wokiconze kin en iyotan cistinna yawapi kta; tuka tuwe ecen econ qa iyecen waonspewicakiye cinhan, he mahpiya wokiconze kin en tanka yawapi kta.

20 Miye heciciyapi ce; Wicoowotanna nitawapi kin, wowapi kagapi qa Pharisee tawapi kin isanpa tanka śni kinhan, mahiya wokiconze kin en ecaca oyapapi kte śni.

21 Tuwedan yakte kte śni, hunkawicayapi kin eyapi keyapi nayalionpi; qa tuwe tin wicakte kinhan he yacopi kta ce.

22 'Tuka miś heciciyapi; Tuwe taku on etanhan śni sunkaku śicekidake cinhan he yacopi kta; qa tuwe sunkaku, Wahteśni, eciye cinhan he omniciye tanka kin en ayapi kta; qa tuwe, Winitko tkoka, eciye cinhan, he Wakanśica tapeta kin en iyohpeyapi kta iyececa.

23 Heon etanhan wośnapi nitawa owayuśna kin ekta ayahi kin- han, qa hen nisunka taku iyanionpa he yeksuya hecinhan ;

24 Ito wośnapi nitawa owayuśna kin itokam ehnake ca ekta ya wo, tokaheya nisunka kici okiciciye ça hehan ku qa wahduśna wo.

25 Tuwe taku en aniu hecinhan he kohanna kici okiciyuwaśte wo, canku ohna kici yaun kin icunhan; okinni wayaco ckta aniye kta, qa wayaco kin iś akicita en aniye kta, qa hecen kaśka nihnakapi kta :

26 Awicakehan heciciya; Tohanyan mazaśadan ehake yahdajuju śni kinhan hetanhan tankan yaku lite śni.

27 Wawicihahapi ecanon kte śni, hunkawicayapi qon eyapi keyapi nayahonpi.

28 Tuka miś heciciyapi ; 'Tuwe winohinca wan en etonwe ca cantiheye cinhan he wanna cante ekta kici wawiciliahapi econ. 29 Niiśta etapa eciyatanhan kin he wahtaniniye cinhan hduśdoke ca elipeya wo; niiśta wanji atakuniśni kinhan he waśte kta, qa tancan ocowasin Wakanśica ti kin en elipeniyanpi kte śni.

30 Qa ninape etapa kin walitaniniye cinhan hdakse ca ehpeya wo ; nihuha wanjidan atakuniśni kinhan he waśte kta, qa tancan ocowasin Wakanśica ti kin en iyohpeniyanpi kte śni.

31 Tuwe tawicu elipekiye kta hecinhan kpaganpi wowapi wan qu kta, keyapi qon.

32 Tuka miś heciciyapi; 'Tuwe tawicu elipckiya, wawicihahapi econ kin on etanhan śni, he wawiciliahiapi econkiya ece; qa tuwe winohinca ehpeyapi kin he yuze cinhan he nakun wawicihahapi econ ece.

33 Akeś hunkawicayapi qon heyapi keyapi nayahonpi; Itonśni 
nicicage kte śni, tuka taku konza Itancan lkin eyakiye cinhan hena yahduecetu kta ce.

34 'Tuka miś heciciyapi; 'Taku cajedate ca he namalion ce, eye śni wo; mahpiya kin he hecen cajedate kte śni, he Wakantanka oiyotanke tawa kin hee.

35 Qa nakun maka kin de, he Wakantanka siha oahe kin ee; qa nakun Jerusalem hecen cajedate kte śni, Wicaśtayatapi tanka totonwe kin hee.

$36 \mathrm{Qa}$ nakun nipa kin he caje yahdate kte śni, paha wanjidan kaeś ska qa iś sapa yakage kta oyakihi śni, heon etanhan.

3\%" Tuka taku ehe cin, Han, han; Hiya, hiya, ecedan kta; taku dena sanpa kinhan he taku śica etanhan icage kta.

38 Iśta wanji iśta wanjidan on etanhan, qa hi wanji lii wanjidan on etanhan, keyapi nayahonpi.

39 'Tuka miś heciciyapi; Taku śica tokiconpi śni po; tuka tuwe tapon etapa eciyatanhan anipe cinhan unma eciyatanhan kipazo po.

$40 \mathrm{Qa}$ tuwe woope on niyaco qa onhdolida nici kta cin kinhan, nitaśina nakun tehinda śni wo.

$41 \mathrm{Qa}$ tuwe wiyutapi hanska wanji hehanyan kici yeniye k ta hecinhan, nom kici ya wo.

42 'Tuwe taku nicida kinhan qu wo; qa tuwe wonicidota hecinhan etanhan ihdamna śni wo.

43 Tuwe nikiyedan un kin he waśteyadake kta, qa tuwe tokaniye cin he śiceyadake kta, eyapi qon nayalionpi.

44 Tuka miś heciciyapi ; 'Tona tokaniyanpi kin hena cante wicakiya po; qa tona niyaśicapi kin liena wicayawaśte po; tona śicenidapi kin hena taku waśte ecawicakicon po; qa tona taku i en iyeniyanpi qa tehiya nicuwapi kin hena wocekiye ewicakiciya po.

45 Hecen Ateyayapi mahpiya ekta un kin he cincaniyanpi yaunpi kta; he qe anpetu wi tawa kin wicaśta śica wicaśta waśte ko en awicahinanpeya ece, qa wicaśta owotanna owotanna śni ko awicamagajuya ece. 46 Tona waśtenidapi kin hena waśte wicayadapi kinhan wokajuju taku dubapi he; wamnayanpi kin hena eepi kaeś heconpi śni he.

$47 \mathrm{Qa}$ sunka wicayayapi kin henana napewicaduzapi kinhan, taku econpi ece sanpa tukte e ecanonpi he; wamnayanpi kin hena eepi kaeś heconpi śni he.

48 Heon etanhan Niyatepi mahipiya ekta un kin he owotanna hinca he iyecen niś eya owotanna hinca un po.

\section{WICOWOYAKE 6.}

1 Wicaśta itokam ihnuhan taku ituyahanpi kinhan, wanniyakapi kta heon; hecen ecanonpi kinhan Niyatepi mahpiya ekta un kin he takudan nicupi kte śni.

\& Heon etanhan tohan taku wicayaqu kta hecinhan nitokam cotanka yajowicayakiye kte śni, wanaicihmanpi kin orniciye tipi qa canku ohna econpi ece kin iyececa, wicaśta on wicayatanpi kte cin heon; awicakehan heciciyapi; Hena wokajuju hduhapi ece.

3 Tuka niye taku wicayaqu eca ninape etapa taku econ kin he nicatka sdonye kte śni.

4 Hecen wawicayaqu kin he nahmana un kta; qa Niyatepi taku nahmana un kin wanyalka ece kin he iye taninyan nicicajuju kta. 


\section{MATTHEOS.}

5 Qa tohan ceyakiya eca wanaicilimanpi kin iyenicece kte śni ; hena eqe omniciye tipi qa canku oise kin en najin cekiyapi waśtedakapi ece, wicaśta wanwicayakapi kta e heon; awicakehan heciciyapi; Hena wokajuju hduhapi ece.

6 Tuka niye qe ceyakiye cinhan, tipi cistinna nitawa kin en timahen yakiyahde. ca tiyopa ecen iyacu kinhan, hehan $\mathrm{Ni}$ yate nahmana un kin he cekiya wo, qa Niyate nahmana wawanyaka ece kin he taninyan nicicajuju kta.

7 Ceyakiyapi kinhan akihdehde oie kicunpi śni po, Ikcewicaśta econpi kin iyececa; hena e iapi ota unpi kin on nawicahon kta kecinpi.

8 Niye hena iyenicecapi kte śni ; hinahin yakidapi śni itokam; hena taku kin yacinpi, Niyatepi sdonya ece.

- 9 Heon etanhan kaken cekiya po; Ate unyanpi mahipiya kin en; Nicaje wakandapi kte;

10 Nitokiconze u kte; Nitawacin ecer econpi nunwe, mahpiya kin en iyececa, nakun maka akan:

11 Anpetu kin de anpetu woyute unqu miye:

12 Qa waunhtanipi kin unkicicajuju po, tona waunkicilitanipi wicunkicicajujupi kin he iyececa :

13 Qa taku wawiyutanyan un kin en unkayapi śni po, tuka taku sice cin etanhan eunhdaku po: Wokiconze kin he niye nitawa, qa wowaśake kin, qa wowitan kin, owihanke wanica. Amen.

14 Niye wicaśta wahtanipi wicayecicajujupi kinhan, Niyatepi malipiya ekta un kin he nakun niye nicicajujupi kta.
15 'Tuka niye wicaśta wahtanipi kin wicayecicajujupi śni ehantanhan, nakun Niyatepi wayahtanipi kin nicicajujupi kte śni.

16 Tohan wote śni yaunpi kinhan wanaicilimanpi kin iyecen ite śnkiyapi śni po; hena eqe ite hduśicapi ece, hecen wicaśta ekta wote śni unpi e ihdutaninpi kta; awicakehan heciciyapi, Hena wokajuju hduhapi ece.

17 'Tuka niye qe wote śni yaun kinhan pa sdakiye ça ite hdujaja wo:

18 Hecen wicaśta en wote śni yaun nihdutanin kte śni, tuka Niyate nahmana un kin he en nihdutanin kta; qa Niyate nahimana wawanyake cin he taninyan nicicajuju kta.

19 Woyuha maka akan ekihnakapi śni po ; hen wamduśkadan qa gigi kin wayutakuniśni ece, qa wamanon sa wayujujupi qa wamanonpi ece.

20 Tuka woyuha mahpiya kin ekta ekihnaka po; heciya wamduśkadan qa gigi kin wayuśice cin wanica, qa wamanon sa wayujujupi śni qa wamanonpi śni.

21 Tukten woyuha niciyankapi hecinhan, nicantepi nakun hen un kta.

22 Wiciśta kin he wicatancan iyoyanpa kin hee; heon etanhan niiśta tokeca śni kinhan nitancan ocowasin iyoyanpa kta:

23 Tuka niisśta śice cinhan nitancan kin ocowasin otpaze kta. Heon iyoyanpa en niun kin he otpaza hecinhan, otpaza kin he tanka hinca.

24 "Tuwedan wicaśta itancan nom anawicagoptan kta okihi śni; wanji śice .kidake kta, qa unma kin iś waśte kidake kta : wanji en iyaihdaśke kta, qa unma lin iś en etonwe kte śni; 
Wakantanka qa mammon napin anagoptan oyakihipi kte śni.

25 Heon etanhan heciciyapi ; Yanipi kin on nagiyeiçiyapi śni po, taku yatapi kta, qa taku datkanpi kta; qa nakun nitancanpi on taku kodakapi kta: Wiconi lin he woyute isanpa śni he; qa wicatancan kin iś wokoyake isanpa yawapi śni he.

26 Malipiya okinyanpi kin hena wanwicayaka po, hena wojupi śni, qa woksapi śni, qa mazopiye en omnayanpi śni, tuka Niyatepi malipiya ekta un kin he hena wo wicaqu ece; unkan niye hena isanpa niyawapi śni he.

27 Niyepi etanhan wanji tukte e he awacannipi on wiciśpa wanjidan kaeś sanpa nihduhanskapi oyakihipi kta he.

28 Qa tokeca wokoyake on iyokiśinniciyapi he; tinta mnalicalica kin wanyaka po, icage cin; htani śni qa nakun wapahmun śni ;

29 Tuka heciciyapi; Solomon wowitan tanka hduha un qon lıee kaeś de wranji iyececa wokoyake kicun śni.

30 Unkan tinta peji, dehan icage ca heyakecinhan oceti en elipeyapi ece kin, he Wakantanka hecen koyagya hecinhan, niș niye wacinyan nicistinpidan, sanpa wakoyagniyanpi kte śni he.

31 Heon etanhan, Taku untapi, qa taku unyatkanpi, qa taku kounyakapi kta he, eya wacin iyokiśicapi śni po.

32 Traku kin hena owasin Ikcewicaśta akitapi ece; qa Niyatepi mahpiya ekta un lin he dena takuowasin yacinpi esdonya ece.

33 Tuka Wakantanka tokiconze kin he tokaheya akita po, qa toowotanna nakun; kinhan dena taku owasin koya niçupi kta.

34 Heon etanhan heyakecinhan on iyokiśiniçiyapi śni po ; heyakecinhan he taku tawa kin on iyokiśica ece e kta; anpetu eca taku sica tawa kin yukan ece e kta.

\section{WICOWOYÄKE $\%$}

1 Wayacopi śni po, hecen niyacopi kte śni.

2 Woyaco tukte e on wadacopi hecinhan he on niś eya niyacopi kta; qa wiyutapi tukte e on widutapi eca hee on winiciyutapi ece e kta.

3 Qa nisunka iśta kin en takuśni yanka e tokeca wandaka he ; qa cankaga wan niiśta ohna yanke cin he wanyahdake śni.

$4 \mathrm{Qa}$ iś token nisunka, Ito niiśta kin etanhan takuśni kin icicicu kta, eyakiye kta he; qa iho, cankaga wan niiśta ohna yanka ce.

5 Wanaiciliman, tokaheya cankaga iśta ohna niyanke cin he hduśdoka wo; qa hehan takuśni nisunka iśta ohna yanke cin he iyacu kta tanyan wandake kta.

6 Taku tona wakan kin hena śunka wicaqupi śni po, qa inyan teliika nitawapi kin kukuśe wicitokam ehpeyapi śni po; okinni siha on amanipi kta, qa akeś hdicupi qa niyahdecapi kta.

7 Da po, kinhan niçupi kta; ode po, kinhan iyeyayapi kta; en katoto po, kinhan niciyulidokapi kta.

8 Tuwe da eca icu ece; qa tuwe ode eca iyeya ece; qa tuwe tiyopa katoto eca he kiciyuhidokapi ece.

9 Unkan enitanhanpi wicaśta wanji cinhintku aguyapi kida kirhan, inyan wanji qu kta he.

10 Qa hogan kida kinhan wamduśka wan qu kta he.

11 Heon etanhan niye niśicapi 


\section{MATTHEOS.}

eśta, taku waśte nicinca wicayaqupi kta onnispepi ehantanhan, Niyatepi malipiya ekta un kin he, sam iyeya tona kidapi kin, taku waśteśte wicaqu ece e kta.

12 Heon etanhan taku tona wicaśta ecaniconpi kta yacinpi kinhan, hena owasin ecawicakicon po; woope qa wicaśta wokcanpi kin iho hee.

13 'Tiyopa ciqadan kin he ohna ya po; Tiyopa kin tanka qa canlzu nakun tanka wicotakuniśni iyahde, qa ohna iyayapi kin wicota hinca.

14 Tuka tiyopa kin ciqadan qa canku nakun ocistiyedan wiconi iyahde; qa wicaśta tonana iyeyapi ece.

15 Wicaśta wokcan itonpiśni kin hena kowicakipa po; hena tahinca wanunyanpi iyecen koyag en nihipi, tuka mahen śunktokeca way apota ece kin hecapi.

16 Waskuyeca tawapi kin on sdonwicayayapi kta.. Wicaśta wapepeka etanhan hastanhanka yuśpipi he, qa toka hu kin etanhan suken waskuyeca icupi he.

17 He iyecen can waśte owasin waskuyeca waśte icahya ece; tuka can śice cin waskuyeca śica aicaga ece.

18 Can waśte kin he waskuyeca śica icahye kta ohihi śni; qa nakun can śice cin he washuyeca waśte icaliye kta ohihi śni.

19 Can otoiyohi waskuyeca waśte aicage śni kin he kawankapi qa petan elipeyapi ece.

20 Heon etanhan waskuyeca tawapi on sdonwicayayapi kta.

21 Tona, Itancan, Itancan, emakiyapi kin owasin mahpiya wokiconze kin en yapi kte śni ; tuka tuwe Atewaya malipiya ekta un kin tawacin kin ecen econ kinhan hee en un kta.
22 Anpetu kin he en wicota hemakiyapi kta; Itancan, Itancan, nicaje on wiunyukcanpi śni he; qa nicaje on taku wakan śica napewicunyanpi; qa nicaje on wicohan tanka ota econkupi ce.

23 Tuka hehan hewicawakiye kta; Tohinni sdonciyapi śni, wicohan śica ecanonpi kin mitantanhan iyaya po.

24 Heon etanhan tuwe mioie kin dena nation, qa ecen econ kinhan, he wicaśta ksapa e imdacin kta, he qe imnija wan akan ti kicaga.

25 Unkan nina magaju qa minitan aya; nakun tateyanpa hinca, qa tipi kin he en iheya, tuka hinlipaye śni; inyan akan kagapi nakaeś.

$26 \mathrm{Qa}$ tuwe mioie kin dena nahon, tuka ecen econ śni kinhan, he wicaśta witkotkoka iyececa, he wiyaka akan tipi kicaga.

27 Unkan nina magaju qa mini tanka icaga, nakun tateyanpa hinca, qa tipi kin he en iheya; unkan hinlipaya; qa ihangyapi kin he tanka.

28 Unkan Jesus wicoie kin dena ihunnikiya, hehan wicota wahokonwicakiye cin on yuśinyayapi.

29 Wanji wookihi yuhe cin iyecen wahokonwicakiya, qa wowapi kagapi econpi kin iyecece śni.

\section{WICOWOYAKE 8.}

1 Unkan paha kin etanhan kun ku qehan, wicota hinca ihakam unpi.

2 Unkan iho, wicaśta wan lepros ececa he en hi qa canpeśka makehde inajin qa heciya; Itancan, yacin kinhan, asnimayaye kta oyakihi. 
3 Unkan Jesus nape ekta yekiye ca yutan, qa heciya; Wacin; hecen asni wo. Unkan ecehnana wowayazan kin ayuśtan.

4. Unkan Jesus heciya; Ihunhan tuwe oyakidake cinhan; tuka elxta ye ca wośnapi kage cin he en ihdutanin wo, qa taku Moses econniśi qon he wayuśna wo, sdonyapi kta e heon etanhan.

5 Unkan Jesus Kapernaum en hi qehan, akicita tancan wan en u qa cekiya ;

6 Qa heya; Itancan, hokśidan mitawa tiyata wanka tataka, nina yazan.

7 Unkan Jesus heciya; Wau qa aśniwaye kta.

8 Hehan akicita opawinge wicayuhe cin he ayupte ca heya; Itancan, wati en timahen yau kta iyemacece śni; tuka oie kin ecedan eya wo, kinhan hokśidan mitawa asni kta:

9 Miś eya wicaśta itancan hemaca, qa akicita wicamduha; unkan wanji, Ya wo, ewakiya eca, ya ece ; qa wanji tokeca, U wo, ewakiya eca, u ece ; qa mitaokiye, Kaken econ wo, ewakiya eca, ecen econ ece.

10 Unkan Jesus he nahon qehan yuśinyaya, qa ihakam unpi kin hewicakiya; Awicakehan heciciyapi, Israel hee kaeś en wacinyanpi hinsko tanka iyewayc śni ce.

11 Nakun heciciyapi; Wiyohiyanpa tanhan qa wiyohpeya tanhan wicota upi kta, qa Abraham qa Isaak qa Jakob om iyotankapi kta, mahpiya wokiconze kin en :

12 Tuka wokiconze cincapi kin hena tankan iyewicayapi kta, otpaze hinca ekta; heciya wicaceya hi hdakinskinzapi ko yuke kta.

13 Unkan Jesus akicita opa- tate iyumni wan tanka; hecen winge wicayuhe cin heciya; Hda wo, wacinyaye cin iyecen ecaniconpi nunwe. Unkan wihiyayedan oape kin he en hokśidan tawa kin asniyanpi.

14 Unkan Jesus Inyan ti kin en tin iyaya, hehan kunku kin makata wanka wanyaka, tancan kata.

15 Unkan nape kin yutan, hehan tancan kate cin ayuśtan; unkan najin qa wowicakihan.

16 Wanna htayetu hehan wakan sica wicayuhnaśkinyan ota en awicahipi ; unkan oie kin on wakan śica napewicaya; qa tona wayazankapi kin owasin asniwicaya.

17 Hecen Isaya wicaśta wokcan taku eye ciqon he yuecetupi kta; he kaken eya; Iye tchiya unyakonpi kin eunkiciyakupi qa waunyazanpi kin yutokan iyeunkiciciyapi ece.

18 Unkan Jesus wicota hinca ihdukśan yukanpi kin hena wanwicayaka hehan akasanpa yapi kta keya.

19 Unkan wowapi kaga wan en hi qa heciya; Waonspeliya, tokiya da eca cihakam waun ece e kta.

20 Unkan Jesus heciya; Śungidan waśun tonpi, qa mahpiya okinyanpi kin holipi yuhapi ece ; tuka Wicaśta Cinhintku kin tukten pa ipahinye kta yuhe śni.

21 Hehan waonspewicakiye cin tokeca wanji heciya; Itancan, tokaheya wahde ca ate wehe kta e iyowinmakiya ye.

22 Tuka Jesus heciya; Mihakam un wo ; ito tapi kin hena tona kitapi kin wicakihapi kta.

23 Unkan wata wan en opa, kam upi.

24. Unkan iho, mini kin ekta 13 hehan waonspewicakiye cin iha- 


\section{MAT'THEOS.}

wata kin taja on akahpapi; tuka iye iśtinma yanka.

25 Unkan waonspewicakiye cin en hipi, qa yuhicapi qa heciyapi ; Itancan, niunkiya po, unțapi kta ce.

26 Unkan hewicakiya; Cistinna wacinyayapi kin, tolreca koyakipapi he. Hehan inajin qa tate mini ko kiśica; unkan amdakedan tanka.

27 Tuka wicaśta kin hena yuśinyayapi qa keyapi; De taku wicaśta lxa hwo, hecen tate mini ko anagoptan ece.

28 Unkan akasanpa i, Gadarene makoce tawapi kin en, hehan wicaśta nom wakan śica wicayuhnaśkinye cin hena itkokipapi, wicaśta hnakapi kin etanhan upi, wohitikapi hinca, hecen tuwedan canku kin he ohna ye kta okihi śni.

29 Unkan iho, heya howayapi; Jesus Wakantanka Cinhintku, taku unkiyotahedan un he; hinahinke śni tuka itokam kakiśunyayapi kta on yahi he.

30 Unkan yukanpi kin itehan kukuśe optaye wan wo wicaqupi ece.

31 Hecen wakan śice cin hena cekiyapi qa heyapi ; Napeunyayapi kinhan kukuśe optaye kin en unkiyayapi kta iyowinunkiya miye.

32 Unkan; Ya po, ewicakiya. Hecen tankata hinanpapi hehan kukuśe optaye kin en iwicayayapi. Unkan iho, kukuśe optaye lin iyulipa maya hinca apamahde inyang naśdog iyayapi, mde kin en, qa minin țapi.

33 Unkan tona wo wicaqupi qon hena najicapi; qa otonwe en kipi, qa taku owasin oyakapi, wicaśta wakan śica wicayulnaśkinyanpi qon, taku ecawicakiconpi kin hena koya.
34 Unkan iho, otonwe kin ocowasin Jesus itkokim upi, qa wanyakapi qehan, makoce tawapi kin etanhan hde kta icekiyapi.

\section{WICOWOYAKE 9.}

1 Hehan wata kin en okipe ca akasanpa kihde ca iye totonwe kin en ki.

2 Unkan iho, wicaśta tatake cin wan owinja akan wanka $c$ he en ahipi. Unkan Jesus wacinyanpi kin wanyake cehan tatake cin heciya; Cinś, cante waśte wo; wayalitani kin nicicajujupi ce.

3 Unkan iho, wowapi kagapi kin wanjikji iyakitedan hekiciyapi; Wicaśta kin de śicaya ia.

4 Unkan Jesus canteyuzapi kin wanyaka hehan heya; Tokeca cante mahen taku śica awacannipi he.

5 Wayahtani kin nicicajujupi ce, eyapi; qa iś; Najin qa mani wo, eyapi kin, unma tukte waśakadan he.

6 Tuka Wicaśta Cinhintku kin woahtani maka akan kajuja kta okihi e he sdonyayapi kta; hehan tatake cin heciya; Najin wo, nitowinja ehdaku qa tiyata hda wo.

7 Unkan najin hiyaye ça iye ti ekta hda.

8 Tuka wicota he wanyakapi hehan yuśinyayapi, qa Wakantanka wicaśta wookihi hinsko tanka wicaqu kin he yatanpi.

9 Unkan Jesus hetanhan iyoopta iyaya hehan wicaśta wan Mattheos eciyapi, mazaska mnayanpi kin en iyotanka he wanyaka; qa heciya; Miyahna u wo. Unkan najin hiyaye ca ihakam iyaya.

10 Unkan ti mahen wota iyo- 
tanka yanka hehan, iho, mazaska mnayanpi walitanipi sa ko ota en hipi, qa Jesus kici iyotankapi, waonspewicakiye cin om.

11 Unkan Pharisee kin iś he wanyakapi hehan waonspewicakiye cin hewicaliyapi ; Tokeca Waonspekiye nitawapi mazaska mnayanpi walitanipi sa ko om wota he.

12 Unkan Jesus he nation qehan hewicakiya; Tona zanipi kin hena pejihutawicaśta cinpi śni, tuka wayazankapi kin.

13 Tuka ya po, qa de taku kapi hecinhan sdonya po; Wowaonsida iyomakipi qa wośnapi wacin śni. Wicaśta owotanna wicakico wahi śni, tuka wahtanipi sa iyopeiçiyapi kta on wahi ce.

14 Hehan Johannes waonspewicakiye cin en hipi qa heciyapi; Tokeca unkiye qa nakun Pharisec kin ijehan akilianunkiciyapi, tuka waonspewicayakiye cin akihaniçiyapi śni.

15 Unkan Jesus hewicakiya; Wakankiciyuza cincapi kin tohanyan tawicu ton kte cin om un kinhan, hehanyan token ecin iyokiśicapi kta he ; tuka anpetu u kta tawicu ton kte cin ewicakiciyakupi kta, unkan hehan akihaniciyapi kta.

16 Tuwedan śina teca onśpa wolroyake tanika en akihdag ton śni ; kinhan onśpa teca qon he mnawahe kta qa sanpa tanka lidecahan aya ece.

17 Nakun miniśa teca ojuha tanika en okaśtanpi śni ece; kinhan ojuha kin napopa, qa miniśa kin skepa, qa ojuha kin ihangyapi ece. Tuka miniśa teca ojuha teca en okaśtanpi ece, hecen napin tanyan yuhapi.

18 Hena hecen ewicakiya icunhan, iho, wicaśta itancan wan en u qa canpeśka makehde ina- jin qa hcciya; Micunkśi nakaha ța, tuka en yau qa nape on ayaputake cinhan kini kta.

19 Unkan Jesus najin qa kici hda, nakun waonspewicakiye cin.

20 Unkan iho, winolinca wan waniyetu ake nonpa wayazanla, we ece iheya, he idazatanhan en u qa taśina opapun kin yutan:

21 Taśina kin hee kaeś mdutan kinhan amasni kta ce, cante mahen eye ca hecon.

22 Tuka Jesus ihdamna, qa wanyake çehan heya; Cunś, cante waśte wo ; wacinyaye cin he asniniyan ce. Unkan wihiyayedan oape kin he en winohinca kin asni.

23 Unkan Jesus wicaśta itancan qon ti kin en i, qa tona cotanka yajopi, oyate ceyapi ko wanwicayaka;

24 Hehan hewicakiya; Alro iyaya po; wicinyanna kin te śni, tuka iśtinma ce. Unkan ihahapi.

25 Tuka oyate tankan hdicuwicayapi, hehan ti mahen i, qa nape on yuza; unkan wicinyanna kin inajin.

26 Unkan he makoce kin owancaya oyakapi.

27 Unkan Jesus hetanhan iyoopte iyaya, hehan wicaśta nom iśtagongapi kin hena ihakam yapi, howayapi qa heyapi; Dawid Cinhintku onśiunkida miye. 28 Unkan ti mahen iyaya, hehan wicaśta iśtagongapi kin en hipi : Unkan Jesus hewicakiya; Hecen ecamon lita owakihi wicayadapi he. Unkan, Han, Itancan, eyapi.

29 Hehan iśta en wicayutan qa heya; Wacinyayapi kin iyecen ecaniconpi nunwe.

30 Hecen iśta hdukawapi. Unkan Jesus waśagya wahokonwi- 
cakiye ca heya; Ito tuwedan sdonye kite śni

31 Tuka tankan hdapi hehan makoce kin he owancaya nina oyakapi.

32 Unkan hena iyayapi hehan iho, wicaśta wan ia okitpani, wakan śica yuhnaśkinyan, he en ahipi.

33 Unkan wakan sice cin napeyapi hehan ia okitpani qon ia; unkan oyate kin inihanpi qa heyapi; Israel en tohinni decen wanyakapi śni tuka qon.

34 'Tuka Pharisee kin heyapi; Wakanśica itancan kin on taku wakan śica napewicaya ece.

35 Unkan Jesus otonwe kin owasin, otonwe ciqadan ko iyaza un, omniciye tipi yuhapi kin ohnahna waonspekiye ça wokiconze wotanin waste kin he oyaka ece; qa wayazankapi owasin, oyate kin en tehiya unpi ko asniwicaya ece.

36 Tuka wicota wanwicayaka hehan onśiwicakida; nina mdokițapi qa enanakiya iyayapi ece, tahinca wanunyanpi tuwedan wicayuhe śni iyececa.

37 Hehan waonspewicakiye cin hewicakiya; Awicakehan woksapi-kin he tanka, tuka litanipi kte cin hena tonana:

38 Heon etanhan woksapi en Itancan kin iye tamaga kin en wicaśta hitanipi kte cin hena hiyuwicaye kta icekiya po.

\section{WICOWOYAKE 10.}

1 Unkan waonspewicakiya akenonpapi kin hena wicakico qehan, taku wakan śica on wowaśake wicaqu, napewicayapi kta, qa nakun wowayazan śicaya unpi ko, owasin asniwicayapi kta. 2 Unkan wahośiyapi akenonpapi kin kakenken ewicakiyapi; tokaheya Simnn he nakun Inyan eciyapi, qa Andrew iye sunkaku; Jakob Zebede cinhintku qa Johannes iye sunkaku ;

3 Philip qa Bartholomew; Thomas qa Mattheos wamnaye ciqon; Jakob Alpheus cinhintku qa Lebbeus he nakun 'Thaddeus eciyapi ;

4 Simon Kananite kin, qa Judas Iskariot wiyopeye kte cin hee.

5 Dena akenonpapi kin Jesus yewicaśi, wahokonwicakiye ca heya; Ikcewicaśta tacanku wan ohna yapi śni po, qa Samaria oyate otonwe tawapi kin en yapi śni po:

6 Tuka Israel ti kin etanhan tahinca wanunyanpi taninpi śni kin hena ekta ya po.

7 Qa dapi eca yantanin po, qa; Mahpiya wokiconze kin wanna kiyedan un ce, eya po.

8 Tona wayazankapi kin asniwicaya po, tona lepros ececapi kin ska wicakaga po, tapi kin hena kiniwicaya po, qa taku wakan śica napewicaya po ; iyunwin codan iyacupi, iyunwin codan wicaqu po.

9 Ipiyake nitawapi ohna mazaskazi, qa mazaska, qa mazaśa unmana yuhapi śni po.

$10 \mathrm{Qa}$ icimayanipi kin on wojuhadan, qa onhdohida nonpa, qa hanpa, qa cansagye, yuhapi śni po; tuwe hitani kin he woyute qupi ece e kta iyececa.

11 Qa otonwe tanka otonwe ciqadan ko, wanji tukte en yaipi kinhan, tuwe iyececa hecinhan iwicawanga po, qa he kici yukan po, tohanyan etanhan yahdapi śni kinhan.

12 Qa tipi wanji en yaipi kinhan, Wookiye en un nunwe, eya po.

13 Qa tipi kin he iyececa hecinhan woukiye nitawapi akan 
un kta ; tuka iyecece śni kinhan wookiye niye en nihdipi kta.

14 Qa tukten iyowinniciyapi śni qa nioiepi kin nalionpi śni lkinhan, tipi kin he, qa otonwe kin he, etanhan tankan yahdapi kinhan, wasuśekśeca siha onitkapapi kin he natata po.

15 Awicakehan heciciyapi ; Woyaco anpetu kin en otonwe kin he tehilya un kta, Sodom qa Gomorra makoce kin isanpa.

16 Iho, tahinca wanunyanpi śunktokeca wicehna iyecen yeciśipi ; heon etanhan wamduśka iyecen ksapa po, qa wakiyedan iyecen watibapidan po.

17 Tuka wicaśta iwanwicayaka po; woyaco omniciye kin en aniyanpi $\mathrm{kta}$, qa omniciye tipi yuhapi kin ohna nicapsinpsintapi kta :

$18 \mathrm{Qa}$ wicaśta itancanpi wicaśtayatapi ko, en aniyanpi kta, miye on etanhan, hecen iyepi qa Ikcewicaśta ko wicayakiyutaninpi kta.

19. Unkan tohan hecen wicaniçupi kinhan, taku, qa token ehapi kte cin, heon iyokiśiniçiyapi śni po; wihiyayedan oape kin he en token ehapi kta he nicupi kta ce.

20 Ie kte cin he niyepi śni, tuka Niyatepi Taniya en niunpi kin he iye ie kta.

21 Hunkawanjinkiciyapi kin iś unma ktepi kta e wicaqu kta ; qa wicaśta wan iye cinhintku kin nakun; qa wicacinca kin iś hunkakewicayapi itkowicakipapi qa wicakiktepi kta.

22 Qa micaje on owasin sicenidapi kta ; tuka tuwe owihanke kin hehanyan sutaya un kinhan he ni kta.

23 Tuka tohan otonwe kin de en śicaya nicuwapi kinhan, wanji tokeca ekta najica po; awica. kehan heciciyapi, Israel otonwe tawapi kin iyaza yaunpi kte śni ecen Wicaśta Cinhintku kin hi kta.

24. Waonspekiyapi kin he waonspekiya tawa kakipe śni; qa tuwe wowidake un kin he yuhe cin isanpa tanka śni ece.

25 Tuka waonspekiyapi kin waonspekiya tawa kin -iyececa, qa wowidake un kin yuhe cin iyececa hecinhan hecetu kta. Tipi tawa kin he Beelzebub eciyapi hecinhan, tiyohnake tawa kin iś nakun hewicakiyapi kte śni he.

26 Heon etanhan kowicakipapi śni po ; taku akalipapi qa yuzamnipi kte śni wanica, qa taku anahmanpi qa sdonyapi kte śni wanica.

27 Taku otpaza en ociciyakapi kin he anpa en daotaninpi kta; qa taku noge on nayalionpi kin he tipi akalipe kin etanhan eyanpaha odakapi kta.

28 Unkan tona wicatancan ecedan ktepi kta okihipi, qa wicanagi kte okihipi śni kin hena kowicakipapi śni po ; tuka tuwe wicanagi wicatancan ko Wakanśica ti kin en ihangye kte cin hee e kokipa po.

29 Zitkadan nom mazaśa wanjidan iyopeyapi śni he; qa etanhan wanjidan kaeś Niyatepi codan makata ilipaye kte śni.

30 Tuka paha nitawapi kin hee kaeś owasin yawapi ece.

31 Heon etanhan kopehdapi śni po ; zitkadan ota isanpa niyawapi ece.

32 Heon etanhan tuwe kaśta wicaśta wicitokam miye omayake cinhan, he Ate malipiya en un kin itokam omdake kta.

33 Tuka tuwe wicasua wicito17 
kam miye elipemaye cinhan, he miye Atewaye mahpiya en un kin itokarn ehpewaye kta.

34 Wookiye maka akan wicawaqu kta on wahi kecinpi śni po; wookiye wicawaqu kta wahi śni tuka mazasgye.

35 Wicaśta wan atkuku śicekidake wakiye kta wahi, qa cunwintku iye hunku kin, qa takośku" iye kunku kin śicekidake kta heon.

$36 \mathrm{Qa}$ wicaśta tona tokayapi kin iye ti en unpi kin hena eepi kta.

37 Tuwe miye isanpa atkuku qa iś hunku kin waśtekidake cinhan, he mici un kta iyecece śni ; qa tuwe miye isanpa cinhintku qa iś cunwintku kin waśtelkidake cinhan, he mici un kta iyecece śni :

$38 \mathrm{Qa}$ tuwe cansusbeca tawa kin ehdaku śni qa miyahna u śni kinhan, he mici un kta iyecece śni.

39 Tuwe wiconi tawa kpatan kinhan, he toki ehpekiye kta; qa tuwe miye on etanhan wiconi tawa kpagan kinhan, he kpatan kte cin ee.

40 Tuwe iyowinniciyapi kin he iyowinmakiya; qa tuwe iyowinmakiye cin he tuwe umaśi kin he iyowinkiya ecc.

41 Tuwe wicaśta wokcan, wicaśta wokcan caje on iyowinkiye cinhan, he wicaśta wokcan iyunwin tawa yuhe kta; qa tuwe wicaśta owotanna, wicaśta owotanna caje on iyowinkiye cinhan, he wicaśta owotanna iyunwin tawa kin.icu kta.

42 Qa tuwe waonspekiyapi caje on, dena cikcistinpidan wanji wiyatke ohna mini sni ecedan yatkekiye cinhan, awicakehan hecicivani, he iyunwin tawa toki elipékiye kte śni.

\section{WICOWOYAKE 11.}

1 Unkan Jesus waonspewicakiye akenonpapi kin taku econwicaśi ayaśtan, hehan hetanhan iyaya, otonwe tawapi kin ohnahna woyake kta qa waonspekiye kta.

2 Unkan Johannes kaślka hnakapi kin en Messiya ohan kin nahon qehan, iye waonspewicakiye cin nom ekta yewicaśi ;

3 Qa heciya; Tuwe u kte ciqon he niye he, qa iś tuwe tokeca wanunyakapi kta he.

4 Unkan Jesus waayupte ca hewicakiya; Hda po, qa taku tona nayahonpi qa wandakapi kin hena Johannes okiyaka po:

5 Iśtagongapi kin tonwanpi ece, luuśtepi kin manipi ece, tona lepros ececapi kin ska wicakagapi ece, tapi kin kinipi ece, qa tona onśikapi kin hena wotanin waśte owicakiyakapi ece.

6 Qa tuwe miye on naśdute śni kinhan, he yawaśtepi ce, eya.

7 Hena wanna kihdapi hehan Jesus Johannes on oyate kin wowicakiyaka; Taku wandakapi kta on hewoskan yaipi he; cedi wan tateyanpa kahuhuza:

8 Tuka taku e wanyag yaipi he ; wicáśta wan wokoyake panpanna koyake cin he; iho tona taku panpanna koyakapi kin hena wicaśtayatapi tipi ohna yakonpi ece.

9 Tuka taku e wanyag yaipi he; wicaśta wokcan kin heca he; han, eciciyapi, qa wicaśta wokcan isanpa.

10 Wowapi en kagapi qon, Iho, niite itokam wahośiya mitawa yewaśi, he nitokam canku nitawa nicage kta ce, iho hee.

11 Awicakehan heciciyapi ; Tona winohinea eciyatanhan 
wicatonpi kin wanji Johannes baptem wicaqu ece kin sanpa tanka icage śni; tulka tuwe mahpiya wolkiconze kin en iyotan cistïnna kin he sanpa tanka.

12 Nakun Johannes baptem wicaqu taanpetu kin hetanhan dehanyan mahpiya wokiconze kin nina akitapi, qa nina akite cin hena iyahpayapi ece.

13 Johannes hehanyan woope qa wicaśta wokcanpi owasin woyalkapi :

14. Qa niś wicada wacannipi hecinhan Elias u lite ciqon he dee.

15 Tuwe noge winalion yukan hecinhan he ito nalion kta.

16 Tulka ito wicoicage lin de taku e imdacin kta he. Hokśipidan tihocoka en iyotankapi kin hena e iyececa, takodakupi 'wicakioanpi,

17 Q unnicrifajopi tuka niye wayacipi śni ; "waśinunhdapi tula niye yaceyapi śni.

18 Johannes wote śni qa wayathe śni hi ; unkan, Taku wakan śica yuha ce, eyapi.

19 Wicaśta Cinhintlku kin wota qa wayatkan hi ; unkan heyapi ; Iho, wicaśta wan wote sa qa miniśa yatkan ece, wamnayanpi qa walitanipi sa takodawicaya cce. Tuka woksape iye cinca kin on owotanna oyakapi ece.

20 Hehan otonwe kin tona en iye olian tanka cconpi qon hena iyopewicaya, iyopeiçiyapi śni lieon.

21 Hehehe Korazin, hehehe Bethsada; wicolian tanka niyepi en econpi kin, hena Turos qa Sidon en econpi unkanś, wanakaja heyake śica cahota ko en iyopeiciyapi lita tuka.

22 Heon etanhan heciciyapi; Woyaco anpetu kin en, tehiya yaunpi kta, qa Turos Sidon kici aopten unpi kta.

23 Qa Kapernaum niye, mahpiya ekta wankan aniyanpi, tuka wicahapi elita ehipeniyanpi kta; wicolian tanka niye en cconpi kin heina Sodom en econpi unkanś, anpetu kin dehanyan ecen un kta tuka.

24 Heon etanhan heciciyapi; Woyaco anpetu kin en tehiya yaun kta, qa Sodom makoce kin ahecece kta.

25 Anpetu kin hena en Jesus waayupte ca heya; Ate, mahpiya maka ko Itancan kin, ciyatan, dena taku kin tona ksapapi qa wasdonyapi kin anawicayakihbe ca holśsiyoqopapi owicayalidaka ece.

26 Han, Ate hecctu, heccn iyonicipi nakaeś.

27 Ate taku owasin maqu; unkan tuwedan Cinhintkuyapi kin sdonye śni, A teyapi un kin hecedan sdonkiya; nakun tuwedan Ateyapi kin sdonye śni, tuka Cinhintku hin hecedan; qa tuwe Cinhintku kin sdonyeliye cin he nakun.

28 Tona htayanipi qa tkeya wayaqinpi kin owasin en mau po, kinhan ozikiyapi cicupi kta.

29 Can napinpi mitawa kin he icu po, qa miye eciyatanhan onspeiçiciya po, cante ekta onśimicida qa wahbayedan waun; hecen ninagipi en woozikiye duhapi kta.

30 Can napinpi mitawa kin he tchike śni, qa waqin mitawa kin he kapojedan.

\section{WICOWOYAKE 12.}

1 Anpetu kin hena en, anpetu wakan icunhan, Jesus aguyapi ojupi kin ehna iyaya; unkan waonspewicakiye cin wotekte- 
lidapi, qa aguyapi yuksa icupi qa yutapi.

2 Unkan Pharisce kin he wanyakapi hehan heciyapi; Wanyaka wo, waonspewicayakiyc cin, anpetu wakan icunhan taku econpi kte śni ece kin, hee econpi.

3 Unkan iye hewicakiya; Dawid taku econ qon he tohinni dawapi śni he; iye qa tona om un kin wotektelydapi qehan;

4 Tipi wakan kin en i, qa aguyapi taninyan chnakapi kin yuta, he iye qa tona om un kin yutapi kte śni, tuka wośnapi kagapi kin ecedan yutapi kta tuka.

5 Qa woope kin en nakun dawapi śni, wośnapi kagapi kin tipi wakan ohna anpetu wakan ahopapi śni eśta iyaonpcpica śni unpi.

6 Tuka heciciyapi; Tuwe tipi wakan kin isanpa tanka wan den un.

7 Wowaonśida wacin qa wośnapi wacin-śni ce, eyapi qon he idukcanpi unkanś, tona wahtanipi śni kin hena wicadacopi kte śni tuka.

$8 \mathrm{Qa}$ Wicaśta Cinhintku kin he anpetu wakan Itancan kin he nakun ce.

9 Hchan hetanhan iyaye ça omnigiyc tipi tawapi kin eu i.

10 Unkan iho, wicaśta wan nape śnija hen un; unkan wiwangapi qa heciyapi; Anpetu wakan icunhan waasniyapi kta iyececa he; he iyaonpapi kta e heyapi.

11 Unkan hewicakiya; Enitanhanpi wicaśta wanji tahinca wanunyanpi wanjidan yuhe cinhan, he anpetu wakan icunhan wolia cn iyolipaya hecinhan, tancan en yuze ça ehdaku kte śni he.
12 Wicaśta kin he taliinca wanunyanpi isanpa ece. Hcon etanhan anpetu wakan icunhan taku waśte econpi kta iyececa.

13 Hehan wicaśta qon heciya; Nape hdukatin wo; hecen yukatin, unkan unma kin iyecen asni icaga.

14. Unkan Pharisee kin hetanhan tankan hdapi, qa token kicepi kte cin he akiiapi.

15 Tuka Jesus he sdonye ça hetanhan tokan iyaya; unkan wicota linca ilakam unpi, qa iye hena owasin asniwicaya :

16 Qa nina oyake śni wicaśi.

17 Hccen Isaya wicaśta wokcan kin taku eye ciqon yuecetupi kta; he kaken-eya;

18 Tho, mitaholśidan wahrahinige ciqon; waśte wakidake cin on minagi iyuśkin ece; mitaniya kin waqu kta, horen Ikcewicaśta kin wora owicakiyake kta.

19 Wicakize kte śni, qa pan kte śni, qa ho kin tuwedan canku ohna nahon kte śni :

20 Cedi kaśuśujapi kin he yukse kte śni, qa hahonta ide lite cin he kasni kte śni, woyaco he woohiye ckta au śni kinhan :

21 Qa iye cajc kin Ikcewicaśta wacinyanpi kta.

22 Hehan wanji wakan śica yuhnaśkinyan he en ahipi, iśtagonge ca ia okitpani; unkan asniyan, hecen iśtagongc ca ia okitpani kin he ia qa nakun tonwan.

23 Unkan wicaśta ota kin owasin yuśinyayapi qa heyapi; Dawid cinhintku kin he dee śni he. 24 Tuka Pharisee kin iś he nahonpi qehan heyapi; Wicaśta kin de wakan śica napewicaye cin he Beelzcbub wakan śica itancan kin on hecon ece.

25 Unkan Jesus token cante- 
yuzapi kin he sdonye ça hewicakiya; Wokiconze wan iye iyakitedan kiciza eca hinhpaya ece; nakun otonwe qa tipi otoiyohi iye iyakitedan kicizapi ehantanhan ecen he kte śni.

$26 \mathrm{Qa}$ Satan Satan napeye cinhan wanna iye iciciza; hecen token tokiconze kin sutaya he kta he.

27 Qa miye Beelzebub on wakan śica napewicawaya ehantanhan, nicincapi kin iś taku on napewicayapi he; heon etanhan hena niyacopi kta.

28 Tuka miye Wakantanka Taniya on wakan sica napewicawaya hecinhan, Wakantanka tokiconze kin wanna en nihipi.

29 Wicaśta waśaka ti lrin en, tuwe tin iyaye ca taku tawa kin ihangye kta token okihi kta he; tokaheya wicaśta waśake cin kaśke cinhan hehan tipi kin ihangye kta.

30 Tuwe mici un śni kinhan he makipajin ece; qa tuwe micipahi śni kinhan he yutakuni śni ece.

31 Heon etanhan heciciyapi; Woahtani owasin iapi śica ko wicaśta wicakicicajujupi kta; tuka Woniya Wakan aiapi kin he wicaśta wicakicicajujupi kte śni.

$32 \mathrm{Qa}$ tuwe Wicaśta Cinhintku kin taku sica eciye cinhan, he kicicajujupi kta; tuka tuwe Woniya Wakan kin taku śica eciye einhan, he kicicajujupi kte śni, wiconi kin de en, qa nakun wiconi u kte cin en.

33 Can kin waśte kaga po, kinhan waskuyeca waśte kta; qa iś can kin śica kaga po, kinhan waskuyeca śice kta; can kin waskuyeca tawa kin on sdonyapi ece.

34 Wamduśka wicoicage, ni- śicapi hecen token taku waśte ehapi kta oyakihipi he; wicacante ojudan kin he eciyatanhan wicai kin ia ece.

35 Wicaśta waśte kin iye cante waśte ojudan kin eciyatanhan taku waśte hiyuya ece; qa nakun wicaśta śice cin iye cante śica ojudan kin etanhan taku śica hiyuya ece:

36 Tuka wicoie waśte śni otoiyohi wicaśta eyapi kin hena on woyaco anpetu kin en ohdakapi ece e kta, he miye eciciyapi.

37 Nioie kin on niyaowotanpi kta, qa nakun nioie kin on niyacopi kta.

38 Hehan wowapi kagapi wanjikji qa Pharisee kin ayuptapi qa heciyapi ; Waonspekiya, wowapetokeca wan wanyagunyakiyapi kta uncinpi.

39 Tuka iye wayupte ca hewicakiya; Wicoicage śice ca wawiciliahapi econ sa kin he wowapetokeca da, tuka wowapetokeca wanjidan qupi kte śni, Jonas wicaśta wokcan wowapetokeca tawa kin heceedan kta.

40 Anpetu yamni qa hanyetr yamni hehanyan Jonas hogan tanka tezi kin ohna un qon, he iyecen anpetu yamni qa hanyetu yamni Wicaśta Cinhintku kin maka cante kin en un kta.

41 Woyaco anpetu kin en Ninewe wicaśta kin inajinpi qa wicoicage kin de yacopi kta; hena e Jonas woyake cin on iyopeiciyapi tuka; unkan iho, Jonas isanpa tanka wanji den un.

42 Itokali wicaśtayatapi winohinca qon he woyaco kin en inajin kta, qa wicoícage kin de yaco kta; eqe Solomon tolssape nahon kta on maka ihanke kin etanhan u tuka; unkan iho, 


\section{MATTHEOS.}

wanji Solomon isanpa tanka den un.

43 Woniya śape cin wicaśta kin etanhan tankata hinanpa, hehan makoce puza en omani, ozikiyapi akita, tuka takudan iyeye śni;

44 Hehan heya; Tipi mitawa etanhan wahinape ciqon he ake ekta wahde kta. Unkan ekta ki, qa tuwedan ohna yanke śni, kahintapi qa ayuco yuśtanpi, hecen iyeya.

45 Hehan iyaye ca woniya tokeca śakowin iye isanpa śicapi, hena iwicacu qa om mahen iyaya, qa ohna yukanpi ; unkan wicaśta kin he hetanhan tehiya un, tokaheya un qon isanpa. Wicoicage śice cin de hecece kta.

46 Unkan oyate kin hecen wowicakiyaka icunhan, iho, iye hunku qa hunkawanjitku kin tankan hinajinpi qa okiyapi kta cinpi.

47 Unkan wanji heciya; Iho, nihun qa nihunkawanji tankan najinpi qa oniciyapi kta cinpi.

48 Tuka iye waayupte ca tuwe heye cin he heciya; Tuwe inawaya he, qa mihunkawanji kin hena tuwepi he.

49 Unkan waonspewicakiye cin ektakiya nape hdugate ca heya ; Iho, mihun qa mihunkawanji dena eepi ce.

50 Tuwe kaśta Ate mah̉piya ekta un tawacin kin ecen econ kinhan he mihunkawanji qa mitawinolitin qa mihun ee.

\section{WICOWOYAKE 13.}

1 Unkan anpetu kin hena en Jesus tipi kin etanhan tankata iyaye ca mde kin icahda iyotanka.

2 Unkan wicota hinca en mniciyapi, "hecen iyotanke kta e wata wan en opa, qa oyate kin iyuhpa hutata najinpi.

3 Unkan wiyacinpi on taku ota owicakiyaka, qa heya; Iho, wicaśta woju wan woju iyaya:

4 Unkan woju icunhan, apa canku icahda hinhpaya; hecen wahupakoza en hipi qa temyapi. 5 Qa apa tukten inyan ota ehna ihpaya, hen maka tonana; unkan ohankoya hinanpa, maka tonana nakaeś.

6 Tuka wi kata aya hehan owasin gu; qa hutkan nica heon etanhan śnija.

7 Unkan apa iś wapepeka ehna ihpaya; unkan wapepeka tanka icage ca hecen kte.

8 Tuka apa maka waśte en ihpaya qa etanhan ota icaga, apa opawinge, apa wikcemna śakpe, apa wikcemna yamni.

9 Tuwe noge wination kta yukan hecinhan, ito nahon kta.

10 Unkan waonspewicakiye cin en hipi qa heciyapi; Tokeca wiyacinpi on owawicayakiya he.

11 Unkan iye awicayupte ca heya; Mahipiya wokiconze woanahbe kin niye oyakahnigapi kta nicupi, tuka hena hecen wicaqupi śni, heon etanhan.

12 Heon tuwe taku yuhe cinhan nakun qupi kta, hecen iyakicuya yuhe kta ; tuka tuwe takudan ton śni, tona yuhe cin hee kaeś kipi ece e kta.

13 Heon etanhan wiyacinpi on wowicawakiyaka ece ; hecen tonwanpi keś wanyakapi śni, qa winahonpi keś nahonpi śni, qa nalsun okahinigapi śni.

$14 \mathrm{Qa}$ hecen Isaya wicaśta wokcan taku eye ciqon iyepi on yuecetupi; Winahonpi on nayahonpi kta, tuka oyakahinigapi kte śni ; qa tonwanhan wandakapi kta, tuka idukcanpi kte śni ce. 
15 Oyate kin de cantepi km cepa aya, qa nogepi kin kitan se winalionpi, qa ištohmuzapi; hecece śni unkanś, iśta on wanyakapi kta, qa noge on nalionpi kta, qa cante on iyukcanpi kta, qa ihduhomnipi kta, kinhan asniwicawaye kta tuka.

16 Tuka niiśtapi kin wowaśte, on wandakapi ece; qa ninogepi kin wowaśte, on nayalionpi ece.

17 Awicakehan heciciyapi; Wicaśta wokcan ota, qa wicaśta owotanna, taku wandakapi ece kin hena wanyakapi lita cinpi, tuka wanyakapi śni; qa taku nayalionpi ece kin, hena nalionpi kta cinpi, tuka nahonpi śni.

18 Heon etanhan wicaśta woju qon wiyacinpi kin he ito nahion po.

19 Wicaśta otoiyohi wolkiconze oie kin nahon keś, okahnige śni kinhan, he tuwe śice cin en hi, qa taku cante en ojupi qon eyalku ece; iho; canku icahda ojupi icu qon hee.

20 Unkan inyan ota en ojupi icu qon he dee; tuwe wicoie kin nation qa ohankoya iyuśkinyan icu ;

21 Tuka iye en hutkan wanica, hecen ecadan enakiya; tohan wicoie kin on wokakije qa śicaya kuwapi akipa hecinhan, hinnakaha ayuśtan iyaya ece.

22 Unkan wapepeka ehna ojupi icu qon, tuwe wicoie kin nahon kin hee; tuka wiconi kin de awacinpi qa woyuha ota wohnaye kin hena wicoie kin kte ece, hecen waskuyeca takudan aicage śni.

23 Tuka maka waśte en ojupi icu qon, he tuwe wicoie kin nahon qa okahniga; qa hecen waskuyeca icahya ece; apa opawinge, qa apa wikcemna śakpe, qa apa iś wikcemna yamni icaga ece.

24 Wiyacinpi tokeca wan owicakiyake ca heya; Malipiya wokicouze kín wicaśta wan iyc tamaga en su waśteśte oju kin he iyececa.

25 Tukawiciśtinma hiyeya icunhan, toka wan en hi, qa aguyapi ehna wayahota oju, qa kihda.

26 Unkan ape kin wanna uye ca wahuwapa icage kta, hehan vivayahota iś nakun tanin.

27 Unkan tipi tawa wowidakewicaye cin en hipi qa heciyapi ; Itancan, nitamaga en su waśteśte oyaju śni he; hecen wayahota kin tokiyatanhan yuha he.

28 Unkan iye hewicakiya; Wicaśta wan toka kin heca e hecon. Unkan wowidakewicaye cin hena heciyapi; Unyanpi qa unpahipi kta yacin he.

29 Tuka iye heya; Hiyá; okinni wayahota kin yapahipi kinhan, nakun aguyapi koya dujunpi kta. 30 Ito woksapi kta hehanyan napin icicahiya icage kta. Unkan wolssapi anpetu kin he en, wayuksapi kin hewicawakiye kta; Tolraheya wayahota pahipi qa hulinahyapi kta on pahta po; tuka aguyapi kin he dotopiye mitawa en omnayan po.

31 Wiyacinpi tokeca wan owicakiyake ca heya; Mahpiya wokiconze kin sinape su wanji iyececa, he wicaśta wan icu qa iye tamaga kin en oju.

$32 \mathrm{He}$ su kin owasin iyotan cistinna; tuka icaga liehan wato kin owasin iśnana tanka; qa can iyececa icaga, hecen malipiya okinyanpi kin en upi, qa adetka kin en ahiyahan ece.

33 Wiyacinpi tolkeca wan owicakiyaka; Mahpiya wokiconze kin on napoliyapi kin iyececa, 


\section{MATTHEOS.}

he winohinca wan icu qa wotpanpi wiyutapi yamni en inahman, hecen ocowasin napohyapi.

34 Dena taku owasin Jesus wiyacinpi on oyate kin owicakiyaka, qa wiyacinpi codan takudan owicakiyake śni.

35 Hecen wicaśta wokcan taku eye ciqon yuecetupi, he hecen eya; Wiyacinpi on i wahdukawa kta; taku maka kagapi ehantanhan anahmanpi kin he omdalke kta ce.

36 Hehan Jesus oyate kin hdewicaśi, qa tipi wan en tin iyaya. Unkan waonspewicakiye cin en hipi qa heciyapi; Maga en wayahota wiyacinpi kin he unkokiyaka miye.

37 Unkan iye awicayupte ca hewicakiya; 'Tuwe su waśteśte oju qon he Wicaśta Cinhintku kin hee.

38 Maga kin he maka ocowasin ee; su waśteśte qon he wokiconze cinca kin hena eepi; wayahota kin he tuwe śica cinca kin hena eepi :

39 Toka hena oju qon Wakansica hee. Woksapi kin he wiconi kin de owihanke kin hee; qa wayuksapi kte cin mahpiya ohnihde kin hena eepi.

40 Heon etanhan, wayahota pahipi qa peta en huhnagapi qon he wiconi kin de owihanke kin ekta hecece kta.

41 Wicaśta Cinhintku kin tona ohnihdewicaye cin hena yewicaśi kta; unkan tokiconze kin etanhan tona wah̉tanipi qa taku śica econpi kin owasin pahipi kta :

42 Qa peta mde kin en ehpewicayapi kta; hen wicaceya hi hdakinskinzapi ko yuke kta.

43 Hehan wicaśta owotanna kin anpetu wi kin iyecen iyega- pi kta, atkukupi tokiconze kin en. Tuwe noge winahon kta yukan hecinhan, ito nahon kta.

44 Akeś mahpiya wokiconze kin he mazaska, maga wan en nahmanpi kin he iyececa, he wicaśta wan iyeya, unkan anahbe ça, wiyuśkinyàn iyaye ca, taku yuhe cin owasin wiyopekiye ca, maga kin he opeton.

45 Akeś mahipiya wolkiconze kin wicaśta wopeton inyan tehika akite ciqon he iyececa ye : $46 \mathrm{He}$ inyan tehika wanjidan, ota iyopeyapi kin, he iyeya, unkan iyaye ca, taku tawa kin owasin wiyopekiye ca, he opeton.

47 Akeś mah̉piya wokiconze kin he ho wan, mde kin en iyohpeyapi, qa ocaje owasin etanhan manaye cin, he iyececa ;

$48 \mathrm{He}$ wanna ojudun hehan hutata ahdipi, en iyotankapi, qa waśteśte kin hena cega ohna mnakiyapi, tuka śikśice cin hena ehpeyapi.

49 Maka owihanke kinhan he iyecece kta; hehan mahpiya ohnihde kin upi kta, qa tona śice cin owotanna unpi kin wiciyopeyapi kin hena iwicacupi kta ; $50 \mathrm{Qa}$ peta mde kin en iyohpewicayapi kta. Heciya wicaceya hi hdakinslkinzapi ko yulke kta.

51 Jesus hewicakiya; Dena owasin oyakahinigapi he. Han, Itancan, eciyapi.

52 Hehan hewicakiya; Heon etanhan wowapi kaga otoiyohi, mahpiya wokiconze on onspekiyapi kin, he wicaśta wan tipi yuthe ca, mazopiye tawa kin etanhan taku teca tannika ko ikikcu ece kin he iyececa.

53 Unkan Jesus wiyacinpi kin dena ihunnikiya, hehan hetanhan tankan iyaya: 


\section{WICOWOYAKE 14.}

54 Qa iye tamakoce kin en ki qehan, omniciye tipi yuhapi kin ohna waonspewicakiya; hecen yuśinyayapi qa heyapi; Woksape kin de, qa wicohan tanka, wicaśta kin de tokiyatanhạn yuha he.

55 Cankajipa cinhintku kin he dee śni he; hunku he Mary eciyapi, qa sunkawicaye cin hena Jakob qa Joses qa Simon qa Judas:

$56 \mathrm{Qa}$ tawinolitin hena owasin unkiyopeyapi unpi śni he ; hecen tokiyatanhan dena taku kin owasin yuha he.

57 Unkan canniyeyapi. Tuka Jesus hewicakiya; Wicaśta wokcan yuonihanpi ece, tuka iye tamakoce qa iye ti kin ohna yuonihanpi śni.

58 Unkan wicolian tanka ota hen econ śni, wicadapi śni heon etanhan.

\section{WICOWOYAKE 14.}

1 Unkan anpetu kin hena en Herod wicaśtayatapi kin Jesus oyakapi kin he nahon;

2 Qa tahokśidan kin hewicakiya; Johannes baptem wicaqu ece kin he dee ; tapi kin etanhan kini, heon etanhan wicohan tanka econ ece.

3 Herod Johannes yuze ca kaśka, qa conkaśke en ehnaka, Herodias iye sunkaku Philip tawicu qon heon.

4 Duhe kta iyecece śni ce, Johamnes eciye ciqon.

5 Unkan heon kte kta cin, tuka oyate kin kowicakipa; hena e wicaśta wokcan heca kecinpi.

6 Tuka Herod anpetu en tonpi qon he ake hiyohipi, hehan. Herodias cunwintku kin wicacokaya waci, qa Herod iyokipiya.

7 Heon etanhan, taku wakan cajeyate ca, taku kida kta hecinhan, awicakehan qu kta, keciya.

8 Unkan he itokam hunku onspekiya, heon heya; Wanna Johannes baptem wicaqu ece qon he pa kin wakśica wan ohna maqu ye.

9 Unkan wicaśtayatapi kin iyokiśice hinca, tuka wakanyan wacajeyate cin heon, qa tona om wota iyotanke cin hena on, he qu wicaśi.

10 Hecen ekta ye wicaśi, qa conkaśke mahen Johannes pa baksa.

11 Unkan pa kin he wakśica ohna akupi, qa wikośka kin qupi ; unkan iye iyoopta hunku qu. 12 Unkan waonspewicakiye cin hena en hipi, qa tancan kin eyakupi qa hapi; hehan yapi qa Jesus okiyakapi.

13 Unkan Jesus he nahon qehan, hetanhan watom iyaye ça, iśnana lewoskan makoce wan ekta i. Unkan oyate kin he nalionpi qehan, otonwe etanhanhan huinyun ihakam yapi.

14 Unkan huta ekta ihunni qehan, wicota wanwicayaka; qa onśiwicakida, qa tona teliiya unpi kin hena asniwicaya.

15 Unkan wanna htayetu hehan waonspewicakiye cin en hipi qa heyapi; Makoce kin de liewotahedan, qa wanna litayetu, heon oyate kin hde wicaśi wo, hecen otonwe ciqadan kin en yapi kta, qa taku yutapi opeicitonpi kta.

16 Tuka Jesus hewicakiya; Hdapi kta iyecece śni; ito niye taku yutapi wicaqu po.

1\% Unkan heciyapi ; Aguyapi kagapi zaptan qa hogan nonpa, henana den unhapi ce.

18 Unkan heya; Hena malkau po. 


\section{MATTHEOS.}

19 Unkan oyate kin wato en iyotanke wicaśi, qa aguyapi zaptan qa hogan nonpa nakun icu, qa malipiya ekta etonwan, hehan yawaśte, qa yuśpaśpa qa waonspewicakiye cin wicaqu, unkan wannspewicakiye cin iś oyate kin wicaqupi.

20 Unkan owasin wotapi qa imnanpi ; unkan oyaptapi etanhan pahipi, makanopiye akenonpa ojudan.

21 Unkan tona wotapi kin hena wicaśta kektopawinge zaptan ecetu, nakun winohinica śiceca koya.

22 Hehan ecahankeya Jesus waonspewiyakiye cin wata wan en ope wicaśi, iye itokam akasanpa yapi kta, oyate kin hde wicaśi icunhan.

23 Unkan wanna oyate kin hde wicaśi, hehan iśnana paha wan ekta cekiya iyaya. Wanna otpaza tuka iśnana ekta yanka.

24 Tuka wata kin wanna mde kin cokaya un, taja on iyotanhan iyekiyapi, tatohekiya yapi nakaeś.

25 Unkan hanyetu en itopa kiktapi hehan Jesus mini kin amani en wicaya.

26 Unkan waonspewicakiye cin mini kin amani wanyakapi qehan, yuśinyayapi qa heyapi ; $\mathrm{He}$ wanagi; qa kokipapi on śicahowayapi.

27 'Tuka Jesus kohanna owicakiye ca heya; Cante waśte po; de milye ce; kopehdapi śni po.

28 Unkan Inyan ayupte ca heya; Itancan he niye hecinhan, ekta mini kin amani u maśi wo.

29 Unkan; U wo, eciya. Hecen Inyan wata kin etanhan kun iyaye ca Jesus ekta ye kta mini kin amani.
30 Tuka wohitiya tateyanpa wanyaka, hehan kokipa. Qa spaye kta iyaya, hehan śicahowaya, qa heya; Itancan, nimayan wo.

31 Unkan Jesus ecahankeya nape ekta yekiye ca yuwankan icu, qa heciya ; Cistinna wacinyaya, tokeca wacetunyahda he.

32 Unkan wata kin en opapi hehan amdakedan icu.

33 Hehan wata en unpi kin en hipi, makata ehpeiciyapi qa heciyapi ; Awicakehan Wakantanka Cinhintku kin he niye.

34 Unkan akasanpa ihunnipi hehan Geneseret makoce kin en hipi.

35 Unkan wicaśta makoce kin he en unpi kin hena iyekiyapi, qa ihdukśan wanke cin owancaya hośi wicakayapi. Unkan tona tehiya unpi kin owasin en awicahipi ;

$36 \mathrm{Qa}$ cekiyapi, taśina opapun heceedan kaeś yutanpi kta heon; unkan tona yutanpi kin owasin tanyan asnipi.

\section{WICOWOYAKE 15.}

1 Hehan Jerusalem etanhan wowapi kagapi qa Pharisee kin Jesus en hipi qa heyapi;

2 Tokeca waonspewicayakiye cin hunkawicayapi woope oyakapi qon acakśinpi he; qa aguyapi yutapi kte cinhan nape hdujajapi śni.

3 Unkan iye waayupte ca hewicakiya; Qa niś nakun tokeca woope odakapi kin on Wakantanka toope lin acayakśinpi he. 4. Wakantanka heya econ wicaśi ; Niyate nihun kici wicahduonihan wo; nakun; Tuwe atkuku hunku kici wicayaśice cinhan wiconte on te kta ce.

5 Tuka niye hehapi; Tuwe 
atkuku qa iś hunku kin heciye kta iyececa; Taku on ociciye lite cin he wanna wośnapi ce; qa hecen atliuku hunku kici wicahduonihan śni ece

6 Hecen woope odakapi kin on Wakantanka toope kin dutakunipiśni.

7 Wananicilimanpi, niyepi on Isaya tanyan woyake ça heye ciqon ;

8 Oyate kin de $\mathrm{i}$ on mikiyedan upi, qa iha on mayaonihanpi ; tuka cantepi kin hena mitehan unpi ece.

9 Wicaśta wicoope kagapi kin liena woope keya onspewicakiyapi, qa on mayuonihanpi kte cin he takudan okihi śni ece.

10 Hehan oyate kin wicakico qa hewicakiya; Nahonpi qa okaliniga po:

11 Taku wicai kin ohna iyaye cin he wicaśta kin yuśape śni ; tuka taku wicai kin etanhan hiyu kin he wicaśta yuśape cin 'ee.

12 Hehan waonspewicakiye cin en hipi qa hec̀iyapi ; Wicoie kin de Pharisee kin nahonpi qa śihdapi, he sdonyàya he.

13 Unkan waayupte ca heya; Taku ojupi kin, tona Ate malipiya elkta un kin oju śni kinhan, hena owasin yujunpi kta.

14 Awicayuśtan po; iśtagongapi iśtagongapi awicayapi kin hena hecapi. Unkan tuwe iśtagonga istagonga wan yus aye cin . han, napin makolidoka en ohinhpayapi kta.

15 Hehan Inyan ayupte ca heciya; Wiyacinpi kin de unkokiyaka miye.

16 Unkan Jesus heya; Niśs nakun hinahin oyakahnigapi śni he.

17 Taku wicai lkin ohna iyaye cin he wicatezi kin ekta $i$ ece, qa hehan hetanhan tankan ehpe- yapi ece; he hinahin oyakahnigapi śni he

18 Tuka taku wicai kin etanhan hiyu kin hena wicacante lin eciyatanhan u ece; qa wicaśta yuśape cin hena ee.

19 Wicacante kin eciyatanhan wicotawacin śica hiyu ece ; tinwicaktepi, wawicilahapi, wiinahmanpi, wamanonpi, woitonśni qa wicayaśicapi kin.

20 Wicaśta yuśape cin dena ee; tuka nape hdujaja śni wotapi kin he wicaśta kin yuśape śni.

21 Hehan Jesus hetanhan tokan iyaye ca, 'Turos qa Sidon makoce ihdukśan wanke cin he ekta i.

22 Unkan iho, Kanan winohinca wan makoce kin hetanhan hiyu, qa hoyekiye ca heya ; Itancan, Dawid Cinhintku, onśimada ye, micunkśi wakan śica yuhnaśkinyan, śicaya un.

23 Tuka iye oie wanjidan ayupte śni. Unkan waonspewicakiye cin en hipi, cekiyapi qa heyapi; Hdeśi wo, unkihakam sicahowaya ece.

24 Tuka iye waayupte ca heya; Israel tiyohnaka tahinca wanunyanpi nunnipi kin henana ekta umaśipi ce.

25 Hehan winolinea qon en hi, makata elipeiciye ça heciya ; Itancan, omakiya we.

26 Tuka iye ayupte ca; Hokśiyopa aguyapi tawapi kin icupi qa śunka wicaqupi kin he hecetu śni ce, eya.

27 Unkan iye; Han, Itancan; tuka śunka kin iye wicayuhapi wahna wotapi tawapi kin etanhan ośnaśna hinhpaye cin hena etanhan yutapi ece, eya.

28 Hehan Jesus ayupte ça heciya; Winohinca, wacinyaye cin he tanka; token yacin kin he enicicetu nunwe. Unkan wihi- 


\section{MATTHEOS.}

yayedan oape kin he hetanhan cunwintku kin asni.

29 Unkan Jesus hetanhan iyaye ca Galile mde kin ikiyedan u; qa paha wan ekta iyaye ca akan iyotanka.

30 Unkan wicaśta ota en hipi, tona huśtepi, iśtagongapi, ia okitpanipi, hu bawicaksapi, qa toktokeca ota en awicahipi, qa Jesus siha kin en ewicahnakapi; unkan asniwicaya.

31 Hecen wicota yuśinyayapi, ia okitpanipi qon hena iapi, hu bawicaksapi qon hena tokecapi śni, huśtepi qon hena manipi, qa iśtagongapi qon hena tonwanpi wanwicayakapi, heon etanhan; qa Israel Taku Wakan tawapi kin he yatanpi.

32 Hehan Jesus waonspewicalkiye cin wicakico qa hewicakiya; Oyate kin onśiwicawakida, wanna yamni can taku yute śni mici unpi ; qa wote śni hdewiçawaśi lita tawațenwaye śni, okinni tahepi ihpayapi kta.

33 Unkan waonspewicakiye cin heciyapi; Makoce liewotahedan kin de en tokiyatanhan aguyapi iyenakeca unhapi qa oyate hinsko tanka imnawicunyanpi kta he.

34 Unkan Jesus hewicakiya; Aguyapi kragapi tona duhapi he. Unkan; Śakowin qa hoganstinna tonana nakun, eyapi.

35 Unkan oyate kin makata iyotanke wicaśsi ;

$36 \mathrm{Qa}$ aguyapi śakowin icu, hogan lkin hena nakun, qa yawaśte, hehan kiyuśpaśpa qa waonspewicakiye cin wicaqu; unkan waonspewicakiye cin oyate kin wicaqupi.

37 Unkan owasin yutapi qa imnanpi; qa oyaptapi kin hena pahipi, makanopiye sakowin ojudan.
38 Wotapi kin hena wica ece kektopawinge topa, nakun winohinca śiceca koya.

39 Hehan oyate kin hdewicaśi, qa wata wan en ope ca Magdala makoce kin en hi.

\section{WICOWOYAKE 16.}

1 Unkan Pharisee qa Sadukee etanhan en hipi, qa mahpiya eciyatanhan wowapetokeca wan wicakiyutanin śipi, iyutanyanpi kta.

2 Tuka iye waayupte cea hewicakiya; Tohan htayetu eca, Mahipiya śaśa heon owaśtecake kta ce, ehapi ece.

$3 \mathrm{Qa}$ hanhanna eca, Ecin ośicecake kta, mahpiya śaśa qa śapśapa, heon etanhan. Wananicilimanpi, mahpiya ite kin he y ukcan onnispepi, tokeca anpetu kin dena wowapetogton oyakahnigapi śni he.

4. Wicoicage śice ça wawicihahapi econ sa kin he wowapetokeca wan da; tuka wowapetokeca wanjidan qupi kte śni; Jonas wicaśta wokcan tawowapetokeca kin heceedan. Unkan elipewicaya iyaya.

5 Unkan waonspewicakiye cin akasanpa ipi, qa aguyapi icu akiktonjapi.

6 Hehan Jesus hewicakiya; Pharisee qa Sadukee on napolyapi tawapi kin he iwanyakapi qa kokipa po.

7 Unkan iye iyakitedan akinicapi qa heyapi; Aguyapi unkicupi śni, heon etanhan, heya ce.

8 Tuka Jesus lie sdonye ca hewicakiya ; Cistinna wacinyayapi kin, tokeca niye iyakitedan ayakinicapi he, aguyapi iyacupi śni heon.

9 Hinahin oyakahnigapi śni, wicaśta kektopawinge zaptan 
ça aguyapi zaptanna, qa makanopiye ojudan tonakeca yapahipi he yeksuyapi śni he.

10 Nakun wicaśta kektopawinge topa qa aguyapi śakowin, qa makanopiye ojudan tonakeca iyacupi he.

11 Tokeca oyakahnigapi śni he, Pharisee qa Sadukee on napohyapi tawapi kin he kokipe ciśipi, he aguyapi on heciciyapi śni.

12 Hehan aguyapi on napoliyapi kin he kokipe wicaśi śni, tuka Pharisee qa Sadukee woonspe tawapi kin hee kokipapi kta, he okahnigapi.

13 Jesus Kesarca Philippi makoce kin en i qehan waonspewicakiye cin wiwicawange ca heya; Wicaśta Cinhintku kin miye, matuwe kin, wicaśta keyapi he.

14 Unkan heyapi ; Apa Johannes baptem wicaqu qon; qa apa iśs Elias; qa tolktokeca ; Jeramias, qa iś wicaśta wolscan wanji.

15 Qa niś matuwe kehapi he, ewicakiya.

16 Unkan Simon Inyan ayupte ca; Messiya Wakantanka ni un Cinhintku kin lie niye, eya

17 Unkan Jesus ayupte ça heciya; Simon Jona cinhintku niyawaśtepi ce; wicacehpi qa wicawe kin he oniciyake śni, tuka Ate mahpiya elta un kin he oniciyaka

18 Qa miś nakun heciciya; Inyan he niye, qa imnija kin de akan okodakiciye mitawa wakage kta; qa wakanśica tatiyopa kin he yujuju kte śni.

19 Unkan malipiya wokiconze iyuhdoke kin he ciçu kta; hecen taku maka akan yakaśke cinhan he mahpiya ekta kaśkapi kta; qa taku maka akan yakiduśke cinhan he mahpiya ekta kiyuśkapi kta.

20 Hehan cin Jesus Messiya kin he iye, he tuwedan okiyake śni wicaśi. 21 Hetanhan Jesus waonspewicakiye cin wowicakiyaka, Jerusalem ekta ye kta iyececa, qa wicaliincapi, wośnapi kagapi, wowapi kagapi lko on taku ota kakije kta, qa lktepi kta, qa anpetu iciyami hehan ake kini kta, hena owicakiyaka.

22 Hehan Inyan Jesus icu qa iyopeyc ca heya; Itancan, hccetu śni ; dena ayakipe lite śni.

23 Tuka iye ihduhomni qa Inyan heciya; Satan mihektapa tanhan ya wo, tokamayaya; Wakantanka taku tawa kin awacanni śni, tuka wicaśta taku tawapi kin.

- 24 Hehan Jesus waonspewicakiye cin hewicakiya; 'Tuwe miyahna u kta cin kinhan, waipiiçida lita, qa cansusbeca ehdaku qa mihakam un kta.

25 'Tuwe kaśta wiconi tawa kpatan kinhan, he toki ehpekiye kta; qa tuwe miye on wiconi tawa kin kpagan kinhan, he iyeye kta.

26 Wicaśta wan maka kin ocowasin kamna, qa nagi kin toki ehpeye cinhan he taku okihi kta he; qa wicaśta nagi kin taku iyopekiye kta he.

27 Wicaśta Cinhintku kin iye Atkuku towitan hduha taohnihdewicaye cin om u kta; liehan wicaśta otoiyohi iye ohanpi kin eciyatanhan wicaqu kta.

28 A wicakehan lieciciyapi; Tona den najinpi kin etanhan wanjikji wiconte utapi kte śni, eccn Wicaśta Cinhintku kin iye tokiconze kin en u wanyakapi kta.

\section{WICOWOYAKE 17.}

1 Unkan iyohakam anpetu sakpe hehan Jesus Inyan qa 29 


\section{MATTHEOS.}

Jakob, qa iye sunkaku Johannes, hena iwicacu, qa paha wan tehanwankantu ekta awicaya;

$2 \mathrm{Qa}$ wicitokam yutokecapi; ite kin anpetu wi kin iyecen iyega, qa tawokoyake kin iyoyanpa iyecen ska.

3 Unkan iho, Moses qa Elias en ihdutaninpi qa kici wohdakapi.

4 Hehan Inyan ayupte ca Jesus heciya; Itancan, den unyakonpi kin he waśte; yacin kinhan, den wakeya yamni ti unkagapi kta, wanji unnicagapi kta, qa Moses Elias kici wanjikji wicunkicagapi kta.

5 Iahan icunhan iho, iyoyanpa mahpiya wan aowicahanzi, qa iho, mahplya kin eciyatanhan wicaho wan heya; Micinkśi waśtewakidake ça on iyomakipi hince cin he dee ce; anagoptan po

6 Unkan waonspewicakiye cin he nahonpi hehan makipusdiya ehpeiçiyapi, qa nina kokipapi.

7 Tuka Jesus en wicahi, qa wicayutan qa heya; Inajin po, qa kokipapi śni po.

8 Unkan iśta yuwankan ikikcupi hehan, tuwedan wanyakapi śni, Jesus ecedan.

9 Unkan pâha kin etanhan kun hdicupi, hehan Jesus wahokonwicakiye ca heya; Wowanyake kin he tuwedan olxiyakapi śni po, Wicaśta Cinhintku kin wiconte etanhan piya ikicage śni hehanyan.

10 Unkan waonspewicakiye cin wiwangapi qa heciyapi: Wowapi kagapi kin, Elias tokaheya hi kta ce, eyapi kin, tokeca heyapi hwo.

11 Unkan Jesus waayupte ca hewicakiya; Awicakehan Elias tokaheya hi kta, qa taku owasin yuecetu kta.
12 Tuka heciciyapi; Elias wanna hi tuka iyekiyapi śni, qa token cinpi qon ecakiconpi. He iyececa Wicaśta Cinhintku kin iyepi eciyatanhan kakije kta.

13 Hehan waonspewicakiye cin okahnigapi, Johannes baptem wicaqu qon e he ke ca heon hewicakiya.

14 Unkan wicota en hdipi qehan, wicaśta wan en hi qa canpeśka makehde inajin,

15 Qa heya; Itancan, micinkśi onśimicida ye, hanyetu wi ihnaśkinyan qa śicaya un; otakiya peta en ilipaye ca otakiya mini en iyolipaya ece :

16 Qa waonspewicayakiye cin wicawakahi, tuka asniyanpi kta okihipi śni.

17 Hehan Jesus ayupte ca heya; Wicoicage wacinye śni qa wacin tehilke, tohanyan cicipi waun kta be ; tohanyan iyowinciciyapi kta he; den makau po. 18 Unkan Jesus iyopeya, qa hecen wakan śice cin tankata hinanpa; unkan wihiyayedan oape kin hetanhan hokśidan kin asniyanpi.

19 Hehan waonspewicakiye cin Jesus iśnana yanke cin en hipi qa heyapi ; Tokeca unkiye lie napeunyanpi kta unkolihipi sni he.

20 Unkan Jesus hewicakiya; Wacinyayapi śni heon; awicakehan heciciyapi ; Sinape su wanjidan hinskokeca wacinyayapi unkanś, paha kin de; De-tanhan tokan iyaya wo, eyakiyapi kta; unkan tokan iyaye kta, qa taku oyakitpanipi wanice kta.

21 Tuka taku decece cin he cekiyapi qa wote śni unpi ecedan on tankan hinanpa ece.

22 Unkan Galile en unpi icunhan Jesus hewicakiya; Wicaśta 
Cinhintku kin wanna ccadan wicaśta napepi kin en wicaqupi lkta.

23 Unkan ktepi kta, qa anpetu iciyamni kinhan ake piya ikicage kta ce, eya. Unkan nina iyokiśicapi.

24 Hehan Kapernaum en hipi qehan, tona mazaska mnayanpi ece kin Inyan en hipi qa heyapi ; Waonspeniciyapi kin he mazaska mnayanpi en iyohpeye kta he.

25 Unkan, Han, eya. Hehan tin hdicu qehan Jesus tokaheya ie ca heciya ; Simon token idukcan he; maka akan wicaśtayatapi kin tokiyatanhan mazaska mayanpi he; iye cincapi etanhan, qa iś wicaśta tokecapi kin etanhan.

26 Wicaśta tokecapi etanhan, Inyan eya. Unkan Jesus heciya; Hehan cincapi kin wicakaśkapi śni ece:

27 Tuka śihda wicunyanpi kte śni, e heon niś mde kin ekta ye ca hinśkijupi wan minin iyeya wo, qa hogan tokaheya hiyu kin lie icu wo; he i kin dukawa kinhan ohna kangi kagapi wan iyeyaye kta; he icu qa miye qa niye on wicaqu wo.

\section{WICOWOYAKE 18.}

1 Unkan hehan waonspewicakiye cin Jesus en hipi qa heciyapi ; Mahpiya wokiconze kin cn tuwe iyotan tanka he.

2 Unkan Jesus hokśíyoqopa wan kico qa wicacokaya ehnaka;

3 Qa heya; Awicakehan heciciyapi ; Nihduhomnipi śni, qa hokśiyoqopa iyenicecapi śni kinhan, mahpiya wokiconze kin en yaipi kte śni.

4 Tuwe ihduhukuye ça hokśiyoqopa kin de iyecen canteyuze cinhan, mahpiya wokiconze kin en iyotan tanka kin hee.

5 Qa tuwe hokśiyoqopa dececa wan, micaje on, iyowinkiye cinhan, he miye iyowinmakiya ece. $6 \mathrm{Qa}$ tuwe hokśiyoqopa kin dena, wäcinmayanpi kin, wanji wahtanikiye cinhan, he inyan wiyutpan wan tahu en iyakaśkapi, qa iye miniwanca ośbe kin en iyolipeyapi unkanś waśte kta tuka.

7 Wahtaniwicayapi kin on oyate kin wotehi yuhapi kta; eya wahitanikiyapi yuke kta iyececa; tuka wicaśta tuwe wahtaniwicaya hecinhan, he wotelii yuhe kta.

8. Hcon etanhan, ninape qa nisiha walitaniniyanpi hecinhan, hena hdaksc ca ehpeya wo; huha kaksapi wiconi en yai kta hee waśte, qa nape nonpa qa siha nonpa hduha peta owihanke wanica en iyohpeniyanpi kte cin he śica.

9 Qa niiśta wahtaniniye cinhan, hduśdole ca ehpeya wo; iśta wanjidan hiduha wiconi en yai lite cin he waśte, qa iśta napin hduha peta owihanke wanica ell iyolipeniyanpi kte cin he sica.

10 Ihnuhan dena cikcistinpidan wanjidan eśta śiceyadake cinhan ; heciciyapi ce ; Mahpiya ekta ohnihdewicayapi tawapi kin hena Atc malipiya ekta un kin ite ohinniyan wanyakapi ece.

11 Wicaśta Cinhintku kin taku atakuniśni qon he niye lkta on hi.

12 Heccn token idukcanpi he. Wicaśta wan tahinca wanunyanpi opawinge wicayuhe, ca etanhan wanjidan kitaninśni hecinhan, wikcemna napcinwanka sanpa napcinwanka hena owanjidan ewicahnake ca he kin ekta iyaye ca wanji taninśni kin he okide śni he. 


\section{MATTHEOS.}

13 Qa iyeye cinhan, awicalsehan heciciyapi ; hee on iyuśkin, qa wikcemna napcinwanka sanpa napcinwanka toliya iyayapi śni kin hena on iyuśkin śni ece.

14 He iyececa, dena cikcistinpidan wanjidan eśta toki ehpeyapi kta, Niyatepi mahpiya ekta un kin hecen tawacin śni.

15 Tuka nisunka wanicilitani kinhan, ekta ye ca iye niye kiciśnana he okiciyaka ye; nanilion kinhan nisunka ake yahduhe kta.

16 Tuka ananigoptan śni kinhan, hehan wanji qa iś nonpa om de kta; hecen waayatanin nonpa, qa iś yamni ipi kin on wicoie owasin yusutapi kta.

17 Tuka hena nakun anawicagoptan śni kinhan, hehan okodakiciye kin owicakiyaka wo; unkan okodakiciye kin anawicagoptan śni kinhan, hehan lkcewicaśta qa wamnayanpi kin iyecen yahdawa kta.

18 Awicakehan heciciyapi; Taku maka akan yakaślkapi kinhan, he malipiya ekta kaśkapi kta; qa taku maka akan yakiduśkapi kinhan, he malipiya ekta kiyuśkapi lita.

19 Akeś heciciyapi; Tuwe nom maka akan taku wanjidan yadapi kta e wayeconzapi kinhan, Ate mahpiya ekta un kin he ecen ecawicakicon kta.

20 Tukten nom yamni, micaje on mniciyapi kinhan, hen wiciyopeya waun ece.

21 Hehan Inyan en hi qa heciya; Itancan, misunka tona wamicihtani qa wecicajuju kta he; śakowin liehanyan he.

22 Jesus heciya; Sakowin hehanyan kta, eciciye śni, tuka wikcemna śakowin ece śakowin. 23 Heon etanhan mahpiya wokiconze kin wicaśtayatapi wan iyececa, he wowidake wicayuhe cin wicakiciyawa kta:

24 Unkan wicakiciyawa icunhan wanji - mazaska tkeutapi tanka kektopawinge wikcemna iyohiye śni kin he ahipi.

25 Unkan on kajuju kta takudan yuhe śni, hecen yuhe cin he tancan wiyopeyapi kta, qa tawicu, qa cinca, qa taku yuhe cin owasin, qa kajujupi kta, keya.

26 Heon wowidake un kin he en makipusdiya elipeiciya, cekiye ca lieya; Itancan, amakipe ye, owasin cicicajuju kta.

27 Hehan yuhe cin he wowidake tawa kin onśikida, kiyuśka qa ikicazo qon kicicajuju.

28 Tuka wowidake un kin he tankan iyaye ca om idakapi kin wanji, kaśpapidan opawinge ikicazo kin he iyeya, qa iyahpaye ca poskin yuze ça heciya ; Tona imiyecazo kin micicajuju wo.

29 Hehan kici idakapi un kin he siha en ehpeiçiya, cekiye ca heya; Amakipe ye, lkinhan owasin cicicajuju kta.

30 Unkan wicada śni; tuka iyaye ça conkaślie mahen kaśkahnaka, oicazo hdajuju lita hehanyan.

31 Unkan kici wowidake unpi kiu hena taku econpi kin wanyakapi qehan, nina iyokiśicapi : heon en hipi qa wicayuhe cin taku econpi kin owasin okiyakapi.

32 Hehan yuhe cin he akc kico qa heciya; Wowidake niśice hinca, icazopi hinsko tanka qon he cicicajuju, cemayakiye cin heon.

33 Hecen miye onsicida kin he iyecen kici wowidake yaun kin iś eya onśiyakida lkta iyecece śni he.

34 Unkan yuhe cin śihda qa kakiśyapi kte cin en wicaqu, 
tona iyohiye śni qon ocowasin hdajuju kta hehanyan.

$35 \mathrm{He}$ iyecen, Ate mahpiya ekta un kin ecaniconpi kta, otoiyohi can e eciyatanhan nisunkapi tona wanicihtanipi kin wicayecicajujupi śni kinhan.

\section{WICOWOYAKE 19.}

1 Unkan Jesus wicoie kin hena heya ayaśtan, hehan Galile makoce kin etanhan iyaye ca Juda makoce, Jordan wakpa akasanpa tanhan he en hi :

2 Qa wicaśta ota hinca ihakam unpi; unkan hen asniwicaya.

3 Pharisee kin iś nakun en hipi, qa iyutanyanpi kta heciyapi ; Taku owasin on wicaśta tawicu elipekiye kta iyececa he.

4 Unkan waayupte ca hewicakiya; Tuwe otokahe ekta wicakage cin he wica winyan ahna wicakaga, he tohinni dawapi śni he :

5 Qa heya; Heon etanhan, wicaśta atkuku hunku ko ehpewicaye kta, qa tawicu kin en iyaihdaśke kta; qa henaoza cehpi wanjidan kta.

6 Heon, hetanhan nonpapi śni, tuka wicacehipi wanjidan ece. Heon, taku Wakantanka yuokonwanjidan kin he ihnuhan wicaśta kiyuśpe cinhan.

7 Hehan heciyapi ; Itancan, Moses kpaganpi wowapi wan qu kta, qa ehpeye kta, tokeca heconwicaśi hwo.

8 Unkan hewicakiya; Nicantepi suta kin heon winohinca elipewicayayapi kta, Moses iyowinniciyapi; tuka otokaheya tanhan hecetu śni..

9 Qa miś heciciyapi ce; Tuwe, wawicihahapi econ kin on etanhan śni, tawicu ehpekiye ça wanji tokeca yuze cinhan, he wawicihahapi econ ece; qa tuwe winyan chpeyapi kin yuze cinhan, he nakun wawicilialiapi econ ece.

10 Unkan waonspewicakiye cin heciyapi; Wicaśta winohinca kici hecetu kinhan, tawicu tonpi kin he waśte śni.

11 Tuka hewicakiya; Wicoie kin he owasin okihipi śni, tona wicaqupi kih henana okihipi.

12 Yunokos heca yukan; hunh hunku tezi kin etanhan heca icagapi; qa hunh wicaśta on heca wicakagapi ; qa hunh mahpiya wokiconze kin on heca içicagapi. Tuwe he icu okihi kimhan he icu kta.

13 Hehan hokśiyoqopa en awicahipi, nape awicaputake ca cekiye kta e heon; unkan waonspewicakiye cin tehindapi.

14 Tuka Jesus heya; Hokśiyoqopa en mabipi kta iyowinwicakiya po, qa tehindapi śni po ; malipiya wokiconze kin he hetanhanpi ece.

15 Unkan nape awicaputake ca hetanhan tokan iyaya.

16 Unkan iho, wanji en hi qa heciya; Waonspekiye waśte, taku waśte ecamon kta, on wiconi owihanke wanica mduhe kta, tukte e he.

17 Unkan heciya; Tokeca waśte emayakiya he; tuwedan waśte śni, wanji heceedan waśte, Wakartanka hee. Tuka wiconi en yai kta yacin kinhan woahope kin hena ahopa wo.

18 Hehan heciya; Tukte e he. Unkan Jesus heya; Dena, Tinwicayakte kte śni; Wawiciha-" hapi ecanon kte śni; Wamayanon kte śni; Woitonśni odake kte śni ;

19 Niyate nihun kici wicahduonihan wo; qa, Niye inihda- 
cin nikiyedan un kin waśteyadalie kta.

20 Kośka kin he heciya ; Imacaga ehantanhan dena owasin ecen ecamon ece ; taku hinahin iyotpani waun he.

21 Jesus heciya; Owotanna yaun kta yacin kinhan, hde ca taku nitawa wiyopeye ca wahipanicapi kin wicaqu wo; kinlan mahpiya ekta woyuha duhe kta; hehan ku qa nihakam un wo.

22 Tuka koślka kin he wicoie kin de nation qehan iyokiśica kihda: taku ota ton kin heon etanhan.

23 Unkan Jesus waonspewicakiye cin hewicakiya; Awicakehan heciciyapi; Tuwe wijica un kin he kitan se mahpiya wokiconze kin en i kta.

$24 \mathrm{Qa}$ ake heciciyapi ; Kamel wan tahinśpaciqadan ohdoka ohna iyaye kte cin he telike śni, qa wicaśta wijica Wakantanka tokiconze kin en ye kte cin he tehika.

25 Waonspewicakiye cin he nahonpi, hehan nina yuśinyayapi qa heyapi; Tuwe hehan ni okihi kta he.

26 Unkan Jesus ekta etonwe ça hewicalriya: Wicaśta ekta he okihipica śni ; tuka Wakantanka ekta taku owasin okihipica ece.

27 Hehan Inyan ayupte ca heciya; Tho, taku owasin ehpeunyanpi qa nihakam unyakonpi ; hecen taku unhapi kta he

28 Unkan Jesus hewicakiya; Awicakehan heciciyapi; Niyepi -tona teca ni en wicatonpi kin de en omayapapi kin, tohan Wicaśta Cinhintku kin wowitan oiyotanke tawa kin en kiyotanke cinhan, niś eya oiyotanke akenompa akan idotankapi kta, qa
Israel wicoun akenonpa wicadacopi kta ce.

29 Qa tuwe kaśta, tipi, qa hun. kawanjitku, qa lawinohtin, qa atkuku, qa hunku, qa tawicu, qa cinca, qa maga, micaje on etanhan elipeye cinhan, he opawinge akihde hduhe kta, qa nakun owihanke wanin wiconi innaiciye kta.

30 Tuka tokaheya unpi kin wicota ehake unpi kta; qa ehake unpi kin hena tokaheyapi kta.

\section{WICOWOYAKE 20.}

1. Malipiya wokiconze kin he wicaśta wan tipi yuhe cin he iyececa, he hanlianna linca hastanhanka iyuwi ojupi tawa kicanyanpi kte cin wicaśta opeton iyaya.

2 Unkan wicaśta anpe'u eca kaśpapidan wanjidan on opewicaton, hehan hastanhanka iyuwi ojupi tawa kin en yewicaśi.

3 Unkan wihiyayedan oape yamni, hehan ake tankan iyaye ça toktokeca tihocoka en taku tukon śni najinpi wanwicayaka; 4 Qa hewicakiya; Ní́s eya hastanhanka iyuwi ojupi kin en ya po; kinhan token owolanna ciçupi kta ce. Unkan en iyayарi.

5 Ake oape iśakpe ya inapcinwanka hehanhan tankan iyaye ça ake iyecen econ.

6 Hehan uape iakewanji ecetu, ake tankan iyaye ca toktokeca taki tokon śni najirpi wanwicayaka, qa hewicakiya; Tokeca anpetu osan taku tokon śli nayajiupi: he.

7 Unkan, Tuwedan opeuntonpi śni ce, eciyapi Hehan hewicakiya; Niś eya hastanhanka iyuwi ojupi kin en ya po, kinhan taku owotanna jyacupi kta. 
8 Wanna hitayetu hehan, hastanhanka iyuwi ojupi yuhe cin iye taokiye itancan lin heciya; Wicaśta htanipi kin wicakico qa wokajuju wicaqu wo, ehake hipi kin hena tokaheya, ecen tokaheya hipi qon wicayeciyuśtan kta ce.

9 Unkan tona oape wanjidan htanipi kin hena hipi, qa otoiyohi kaśpapidan wanji icupi.

10 Tokaheyapi kin hena en hipi qehan, sanpa yuhapi kta kecinpi, tuka iś eya otoiyohi kaśpapidan wanji icupi.

11 He icupi hehan tipi tawa kin bapi ;

12 Qa heyapi; Ehake hipi kin dena oape wanjidan htanipi, tuka unkiye anpetu osan, wi kata eśa a htaunnipi, iyeuncecapi wicayakaga ece.

13 Unkan iye waayupte ça wanji heciya; Koda, taku śica ecacicon śni ; kaśpapidan wanjidan on htamiyecini kta kemayakiye śni he.

14 Taku nitawa kin ehdaku qa hda wo; ehake hi kin de niye ciçu kin iyecen waqu ece e kta:

15 Miye mitawa kin token wacin kin ecen ecamon kta"iyecece šni he ; miye mawaśte kin heon etanhan iśta niśica he.

16 Hecen ehake unpi kin hena tokaheyapi kta; qa tokaheya unpi kin hena ehake unpi kta; wicota wicakicopi, tuka tonana wicakahinigapi ece.

17 Unkan Jesus Jerusalem ekta itanwankanhde ya, lehan waonspewicakiya akenonpa kin canku kin icunonpa iśnana awicaya, qa hewicakiya;

18 Tho, Jerusalem ekta itanwankanhde unyanpi, qa Wicaśta Cinhintku kin wośnapi kagapi wowapi kagapi lo nape en wi- caqupi kta; unkan te kta on yacopi kta,

19 Qu ihahapi kta, kapsinpsintapi kta, qa icipaweh okatanpi kta on Ikcewicaśta iyoopta wicaqupi k!a; unkan anpetu iciyamni hehan kini kta ce.

20 Hehan Zebedee cinca hunkupi kin en hi, cinhintku napin om, cekiye ca taku wanji kida.

21 Unkan iye heciya; Takn yacin he. Hehan heciya; Micinissi kin denaoza wokiconze nitawa en, unma nietapa ekta iyotanke kta, qa unma iś canitka en iyotanke kta, keya ye.

22 Tuka Jesus wayypte ca heya; Taku yadapi kin sdonyakiyapi śni. Wiyatke etanhan mulatke kte cin he etanhan yatkan oyakihipi he; qa baptem maqupi kte ci I he niś eya niçupi kta oyakihipi he. Unkan, Unkokihipi ce, eciyapi.

23 Hehan hewicakiya; Awicakehan wiyatke mitawa kin etanhan datkanpi kta, qa baptem maqupi kte cin heon niś eya baptem niçupi kta; tuka ematapa qa camatka tanhan iyotankapi wicaqupi kte cin he miye mitawa śni; tuka Ate tona wiyeya wicakiciyuśtan kinhan hena tawapi kta.

24 Unkan wikcemnapi kin hena he nahompi qehan sunkakiciyapi nom on sibdapi.

25 Tuka Jesus wicakico qa heya; Ikcewicaśta ekta tona itancanpi kin hena oyate wicayuhapi, qa wicaśta tankinyanyanpi kin oyate wowidakewicayapi ece, he sdonyayapi.

26 Tuka niyepi ekta hecece kte śni ; tuka wanji tukte tanka yaunpi kta yacinpi kinhan, he ookiye duhapi kta:

$27 \mathrm{Qa}$ tuwe niyepi en tokapa 


\section{MATTHEOS.}

un kta hecinhan, wowidake nitawapi kin hee kta.

28 He iyececa, Wicaśta Cinhintku wowidake wicayube kta on hi śni, tuka idakapi kta, qa wiconi tawa wokajuju kta wicota on wicaqu kta heon hi.

29 Unkan Jeriko etanhan iyooptapi, hehan wicota ihakam unpi. 30 Unkan iho, wicaśta iśtagonga nonpa canku icahda iyotankapi, Jesus en hiyaya nahonpi, hehan howayapi qa heyapi; Itancan, Dawid Cinhintku onśiunkida miye.

31 Unkan wicota kin tehindapi qa inina yanke wicaśipi ; tuka iś heban heon iyotan huwayapi qa heyapi; Onśiunkida po, Itancan, Dawid Cinhintku.

32 Uṇkan Jesus owanji najin qa wicakico qa heya; Taku ecaciconpi kta yacinpi he.

33 Itancan iśta unhdukawapi kta ye, eciyapi.

34 Hehan Jesus onśiwicakida qa iśta wicayutan; unkan ecahankeya iśta on tonwanpi qa ihakam unpi.

\section{WICOWOYAKE 21.}

1 Unkan Jerusalem ikiyedan yapi, qa Bethpage ehan ipi, Oliwe paha kin etu, hehan Jesus waonspewicakiye cin nom yewicaśi ;

2 Qa hewicakiya; Dukanpi kin otonwe iyotakuns wanke cin he ekta ya po; kinhan kohanna śonśonna wan cinca iyahna han iyeyayapi kta ; kiyuśkapi qa makaku po.

3 Qa tuwe taku eniciyapi kinhan, Itancan kin hena cin ce, eya po ; kinhan kohanna awicau niśipi kta.

4 Dena owasin econpi, wicaśta wokcan oie qon yuecetupi kta, he kaken eya;
5 Zion cunwintku kin heciya po; Tho, Wicaśtayatapi nitawa en niu, walibadan qa śonśonna wan akan iyotanka, śukcinca wan, śonśouna cinca kin akan $u$ ce.

6 Unkan waonspewicakiye cin yapi, qa Jesus econ wicaśi kin ecen econpi ;

7 Qa śonśonna cinca iyahna awicahipi, qa iye laśina akan ehpeyapi, qa hena iakan Jesus ehnakiapi.

8 Unkan wicota canku ohna śina elipeyapi; qa apa canwapa. kaksapi qa canku ohnahna owinśyapi.

9 Qa wicota tokata yapi, qa tona ihakam upi kin, howayapi qa heyapi; Dawid Cinhintku woohiye yuha nunwe. Tuwe Itancan caje on u kin yawaśtepi nunwe: Mahpiya ekta woohiye yuha nunwe.

10 Unkan Jerusalem en hi qehan otonwe kin ocowasin śkanśkanpi, qa heyapi; De tuwe he. 11 Unkan wicota kin heyapi; Jesus wicaśta wokcan Galile en Nazareth otonwe kin etanhan he dee.

12 Unkan Jesus tipi wakan Wakantanka tawa kin en i, qa tipi wakan ohna wiyopeyapi ece, wopetonpi ko, hena tankan iyewicaya; qa mazaska tokiyopeyapi ahna econpi kin hena yuahdapśinyan ehpeya, tona wakiyedan wiyopeyapi oiyotanke tawapi koya ;

13 Qa hewicakiya; Tipi mitawa kin he wocekiye tipi eciyapi kta, eya wowapi en kagapi; tuka niye wamanon tipi yakagapi.

14 Hehan iśtagongapi huśtepi ko tipi wakan kin en hipi; unkan okiziwicaya.

15 Unkan wośnapi kagapi, wo- 
wapi kagapi ko taku wowapetokeca econ kin hena wanyakapi, qa hokśiyoqopapi tipi wakan kin ohna, Dawid Cinhintku kin woohiye yuha nunwe, eya howayapi, hehan nina sihdapi :

16 Qa heciyapi; Dena taku eyapi kin nayalion he. Unkan Jesus hewicakiya ; Han ; Hokśiyoqopa, tona azinpi ko ipi kin etanhan wowitan duśtan ce, eyapi kin he tohinni dawapi śni he.

17 Hehan awicayuśtan, qa otonwe kin etanhan tankan iyaye ca Bethani ekta i, qa hen wanka.

18 Unkan hanlianna hinca otonwe kin ekta icicawin hda, unkan wotektehda;

19 Qa suken can wanji canku ohna han wanyake cehan, he en i, tuka takudan iyeye śni, ape eceedan aicaga; unkan heya; Icimana waskuyeca ainicage śai nunwe. Unkan suken can kin kohanna śnija aya.

20 Unkan waonspewicakiye cin he wanyakapi qehan yuśinyayapi qa heyapi; Ehanqon suken can kin kohanna śnija aya.

21 Jesus waayupte ca hewicakiya; Awicakehan heciciyapi ; Wowacinye duhapi qa wacetunyalıdapi śni kinhan, taku suken can kin de ecakiconpi kin heceedan ecanonpi kte śni, tuka paha kin de, Tokan iyaye ca miniwanca kin en iyohpeniyanpi nunwe, eyakiyapi kinhan, ecetu kta.

22 Qa taku tona wocekiye eya yadapi, qa wicayadapi kinhan, hena owasin duhapi ece e kta.

23 Unkan tipi wakan kin en hi, qa waonspewicakiya icunhan, wośnapi kagapi, qa oyate kin en wicaśta tankapi ko, en hipi qa heciyapi; Wicookihi tukte e on dena ecanon he; qa tuwe wicookihi kin de niçu he.
24 Unkan Jesus waayupte ca hewicakiya; Miś eya taku waiji iciwangapi kta; he omayakidakapi kinhan, hehan wicookihi kin tukte on dena ecamon kin ociciyakapi kta.

25 Johannes baptem wicaqu qon he tokiyatanhan he; mahipiya eciyatanhan qa iś wicaśta eciyatanhan. Unkan he iyakitedan aiapi qa hekiciyapi; He mahpiya eciyatanhan, unkeyapi kinhan ; Tokeca he wicayadapi śni he, eye kta:

26 Tuka, He wieaśta eciyatanhan, unkeyapi kinhan, oyate kin kowicunkipapi ; owasin Johannes wicaśta wokcan heca kecinpi.

27 Unkan ayuptapi qa Jesus heciyapi; Sdonunyanpi śni ye. Unkan iś hehan lewicakiya; Miś nakun wicookihi tukte on dena ecamon kin ociciyakapi kte śni.

28 'Tuka token idukcanpi he: Wicaśta wan cinhintku nonpa; unkan tokapa kin en hi qa heciya ; Cinś, anpetu kin de hastanhanka iyuwi ojupi mitawa kin en htani ya wo.

29 Unkan ayupte ça heya; Ecamon kte śni ye. Tuka iyohakam iyopeiciye ça iyaya.

30 Unkan hakakta kin en hi, qa iyecen eciya; Unkan iye ay upte ca; Itancan, mde kta ce, eya. liuka ye śni.

31 Denaoza unina tukte atkukupi tawacin kin ecen econpi he. Unkan, Tokapa kin, eciyapi. Hehan Jesus hewicakiya; Awicakehan heciciyapi; Wamnayanpi qa wa wicilithapi econpi sa niyepi nitokam Wakantanka tokiconze kin en yapi kta.

32 Johannes wicoowotanna canku kin ohna en niupi. tuka wicayadapi śni; tuka warnna- 


\section{MATTHEOS.}

yanpi qa wawiciliahapi econpi sa, hena wicadapi; he wandakapi eśta nakun iyohakam wicayadapi kta e on nihduhomnipi śni.

33 Wiyacinpi wan tokeca nalon po; Wicaśta wan tipi yuhe cin lie hastanhanka iyuwi ojupi wan oju, qa ihdukśan natake ca ohna iyuśkice wan qe ca nakun cowahe wan en kaga; qa wicaśta kicanyanpi lkta owicaqu, hehan icimani iyaya.

34. Unkan wanna waskuyeca suton kta, hehan wowidake wicayuhe cin wicaśta kicanyanpi kin ekıa yewicaśi waskuyeca etanhan icupi kte cin heon.

35 Unkan wicaśta kicanyanpi kin wowidake unpi kin hena wicayuzapi ; wanji apapi, wanji ktepi, qa wanji inyan on kininpi. 36 A kcś wowidake toktokeca ota ekta yewicaśi; unkan iś iyecen wicakuwapi.

37 Hehan ehake iye cinhintku kin ekta yeśi, qa heya; Micinliśi yuonihanpi kta

38 Tuka maga kicanyanpi kin cinhintku wanyakapi, hehan hekiciyapi ; Tawa kte cin he dee; iho po, unktepi kta, hehan taku yuhe cin he unkicupi kta.

39 Hecen yuzapi qa hastanlanka iyuwi ojupi kin etanhan tankan elipeyapi qa ktepi.

40 Hecen tohan hastanhanka iyuwi ojupi tawa kin hi kinhan kicanyanpi kin hena token ecawicakicun lita he.

41 Unkan heciyapi; Wicaśta śikśice cin hena tehiya ihangwicaye kta, qa kicanyanpi toktokeca tohan iychantu eca waskuyeca kicupi ece e kta hena hastanhanka iyuwi ojupi kin owicaqu kta.

42 Hehan Jesus hewicakiya; Inyan wan tipi kagapi kin ehpe- yapi qon he oise pa kin icaga; he Itancan kin eciyatanhan hecetu, ca unkiś api en taku wowapetokeca kin hee, eyapi kin he tohinni wowapi wakan kin en dawapi śni he.

43 Heon etanhan heciciyapi; Wakantanka tokiconze kin eniciyakupi kta, qa oyate wan waskuyeca icahyapi kte cin.he wicaqupi kta.

44 Unkan tuwe inyan kin de ahinhpaye cinhan kamdell ehpeyapi kta; qa tuwe he akan ihpaye cinhan tanyan kamdu kta.

45 IJnkan wośnapi kagapi hena, qa Pharisee ko wiyacinpi tawa kin nahonpi qehan, iye wicaka e heya sdonyapi.

$46 \mathrm{Qa}$ heon yuze wacinpi, tuka oyate kin kowicakipapi ; owasin wicaśta wokcan heca kecinpi.

\section{WICOWOYAKE 22.}

1 Unkan Jesus waayupte ca ake wiyacinpi on wowicakiyake ca heya ;

2 Wicaśta wan wicaśtayatapi heca cinhintku kin wakankiciyuzapi kicage cin he mahpiya wokiconze kin iyececa.

3 Unkan wowidake wicayuhe cin hena yewicaśi, tona wakankiciyuzapi en wicakicopi qon hena uwicaśipi kta; tuka upi kta wicadapi śni.

4 Akeś wowidake toktokeca ekta yewicaśi qa hewicakiya; Tona wicakicopi qon hena hewicakiya po; Wotapi mitawa wiyeya yanka ; tatanka mitawa ptejicadan ko wanna ktepi, qa taku owasin wiyeya hiyeya; iho po, wakankiciyuzapi kin en $u$ po.

5 Tuka iha hapi qa toki iyayapi ; wanji tamaga ekta iyaye ca, wanji is mazopiye tawa ekta iyaya. 
$6 \mathrm{Qa}$ hunh wicakico ipi kin hena wicayuzapi, qa śicaya wicakuwapi qa wicaktepi.

7 Wicaśtay itapi kin he nahon qehan nina śibda; qa taakicita elita yewicaśi, qa tinwicaktepi kin hena ihangwicaye ca totonwepi kin buhnahya.

8 Hehan wowidake tawa kin hewicakiya; Wakankiciyuzapi kin wiyeya yanka, tuka tona en wicakicopi qon hena opapi kta iyececapi śni.

9 Heon etanhan canku ojate kin elktakta ya po, qa tona iyewicayayapi kin wakankiciyuzapi kin en uwicaśi po.

10 Hecen wowidake unpi kin hena canku ojate kin ektakta iyayapi, qa tona iyewicayapi, śica qa waśte ko, en awicaupi; hecen wakankiciyuzapi wicaśta ota en opapi.

II Unkan wicaśtayatapi kin tona wotapi kte cin hena wanwicayaka tin hiyu, qa iho, wicaśta wanji wakankiciyuzapi wokoyake kin kiçun śni hen wanyaka :

12 Qa heciya; Koda, tokeca wakankiciyuzapi wokoyake kicun śni en hidotanka he. Unkan tákudan eye śni.

13 Hehan wicaśtayatapi kin taokiyewicaye cin hewicakiya; Siha nape ko pahtapi qa detanhan aya po, qa tankan otpaza kin en elipeya po; heciya wicaceya qa hi hdakinskinzapi yuke kta.

14 'Wicota wicakicopi tuka tonana wicakahnigapi ece.

15 Hehan Pharisee kin iś yapi, q? token iapi on yuśkiśkapi kta aiapi.

$16 \mathrm{Qa}$ iye waonspewicakiyapi kin Herodian hena om ekta yewicaśipi qa heyapi; Waonspekiye, iewicayaka sdonunyanpi, qa awicakehan Wakantanka ta- canku kin onspeunyakiyapi ece, qa tuwedan koyakipe śni; wicaśta akautu ekta eyatonwe śni nakneś.

17 Heon token idukcan he; unkokiyaka po; Kesar mazaska qupi ece kin he owotanna he.

18 Unkan Jesus taku śica en wicaun kin he sdonya qa heya; Wananiẹihmanpi, tokeca iyutanmayayapi he :

19 Mazaska mnayanpi ece kin he makipazo po, eya. Unkan kaśpapidan wanji kahipi.

20 Hehan hewicakiya; Wicite qa wowapi kagapi kin de tuwe tawa he.

21 Unkan, Kesar tawa ce, eyapi. Hehan hewicakiya ; Heon etanhan Kesar taku tawa kin kicu po, qa Wakantanka taku tawa kin kicu po.

2.2 He nahonpi hehan yuśinyayapi qa ehpeya hdicupi.

23 Unkan anpetu kin he en Sadukee kin en hipi, hena piya wicoicage kin wanica ce, eyapi ece, qa wiwangapi ;

24 Qa heciyapi; Waonspekiye, Wicaśta wan cinca yuhe śni te cinhan, tawieu qon he iye sunkaku yuze kta, qa cincu cinca icahkiciciye kta, Moses keye ciqon.

25. Ünkan unkiyepi en wicaśta śakowin sunkakiciyapi; unkan tokapa kin he winohinea wan yuza tuka ța; qa cinca nica heon iye iyokihe cin he tawicu ailipekiya.

$26 \mathrm{Qa}$ icinonpa iyecen econ, qa iyamni, qa ecen śskowinpi kin.

27 Hehan ehake winohinca qon ta.

28 Hecen piya wicoicage kin en, hena śakowinpi kin wanji tukte winohinca kin he hduhapi kta he ; owasin yuzapi.

29) Jesus waayupte ça hewicakiya ; Yanunipi, wowapi wakan 
qa Wakantanka towaśake kin sdonyayapi śni.

30 Piya wicoicage kin en winohinca wicayuzapi śni, qa hihnatonpi śni, tuka mahpiya ekta Wakantanka ohnihdewicaye cin iyececapi ece.

31 Tuka wicate cin piya icagapi kin on Wakantanka taku eniciyapi kin he dawapi śni he; he kaken eya;

32 Abraham Wakantanka tawa, Isaak Wakantanka tawa, qa Jakob Wakantanka tawa kin he miye : Wakantanka wicaśta țapi kin tawapi śni, tuka ni unpi Wakantanka tawapi ece.

33 Unkan oyate kin hena nahonpi hehan taku onspewicakiye cin on yuśinyayapi.

34. Tuka Pharisee kin hena, Jesus Sadukee kin inina yanke wicakiya, he nalionpi hehan en mniciyapi :

$35 \mathrm{Qa}$ etanhan wanji woope aiekiyapi kin heca wiwange ca iyutanyan heciya;

36 Waonspekiye, woope kin en woăhope kin tukte iyotan tanka he.

37 Unkan Jesus heciya; Itancan Wakantanka nitawa kin he nicante ocowasin on, qa ninagi ocowasin on, qa nitoksape ocowasin on waśteyakidake kta.

38 Woahope tokaheya qa tanka kin hee.

39 Qa inonpa kin he iyececa; Nikiyedan un kin niye inihdacin waśteyadake kta ce.

40 Woahope kin denaoza woope ocowasin wicaśta wokcan ko en ikoyaka ece.

41 Pharisee kin iś en mniciyapi icunhan Jesus wiwicawange ca heya;

42 Messiya on token idukcanpi he ; tuwe cinhintku he. Unkan, Dawid Cinhintku ce, eciyapi.
43 Hehan hewicakiya; Hecen token Dawid Woniya Wakan kin on Itancan, eciya he; qa heya;

44 Itancan kin Itancan mitawa heciya; Mietapa ekta iyotanka wo, tona tokaniyanpi kin siha oahe nitawa wicawakage kta, hehanyan.

45 Hecen Dawid, Itancan, eciya hecinhan, token ecin cinhintkuye kta he.

46 Unkan tuwedan oie wanjidan ayupte kta okihi śni; qa anpetu kin hetanhan tuwedan ake wi wanga tawatenye śni ece.

\section{WICOWOYAKE 23.}

1 Hehan Jesus oyatc kin, iye waonspewicakiye cin om wowicakiyaka :

2 Qa hewicakiya; Wowapi kagapi Pharisee ko Moses oiyotanke tawa kin en iyotankapi ece.

3 Heon etanhan taku tona openiśipi kin hena owasin opapi qa econ po; tuka iye ohanpi kin ohna econpi śni po; taku eyapi, tuka iyecen econpi śni ece.

4 Waqinpi tketke qa qinpi telika lieca oheyunpi, qa wicaśta tapete akan ehnakapi ece, tuka iye qe śaśte on yutanpi kta tawațenyapi śni.

5 Qa wicolian econpi kin owasin wicaśta wanwicayakapi kta e heon econpi ece. Wateśdake tanka içicagapi, qa śina opapun hdutankapi ece.

6 Wotapi en oyanke waśteśte cinpi, qa omniciye tipi kin ohna wankan iyotankapi kte cin he waśtedakapi ece :

7 Qa tihocoka kin en wicayuwintapi, qa, Itancan, Itancan, wicaśta ewicakiyapi kta cinpi.

8 Tuka niye, Itancan, eniciya- 
pi kte śni; wanji mdetahunka duhapi, Messiya hee; qa niyepi owasin hunkawanjinyeciyapi.

$9 \mathrm{Qa}$ nakun maka akan tuwedan, Ate, eciyapi śni po; Wanji mahpiya ekta un kin he Ateyayapi.

$10 \mathrm{Qa}$ nakun, Waonspekiye, eniciyapi kte śni; Wanji Waonspekiye duhapi, Messiya hee.

11 Qa tukte wanji iniyotanpi kta hecinhan, he wowidake nitawapi ee kta.

12 Tuwe yuwankan iyeiciye kta hecinhan, he yuhukun ehpeyapi kta; qa tuwe ihduhukuya hecinhan, he yuwankan iyeyapi kta.

13 Tuka wowapi kagapi qa Pharisee kin, wananiçihmanpi, wotehi niciyankapi nunwe; wiwazica tipi tawapi ihangyayapi ece, qa wanniyakapi kta on wocekiye hanska ehapi ece; heon etanhan woyaco tanka iyahdeniciyapi kta.

-14 Wowapi kagapi qa Pharisee kin, wananiçihmanpi, wotelii niciyankapi nunwe; mahpiya wokiconze kin wicaśta anawicayeciptapi, niye en dapi kte śni, qa tona en yapi kta cinpi kin teyahindapi ece.

15 Wowapi kagapi qa Pharisee kin, wananiçihmanpi, wotehi niciyankapi nunwe; miniwanca maka ko aokibeyayapi, wicaśta wanjidan duhomnipi kta ; qa ecetu kinhan, niye isanpa nonpa akihde. Wakanśica cinca yakagapi ece.

16 Tokakeya mani iśtagongapi kin, wotehi niciyankapi nunwe; hehapi ece; Tuwe tipi wakan kin on wakonze cinhan he taku śni; tuka tuwe tipi wakan kin en mazaskazi yuke cin, he on wakonze cinhan, he walitani ece.
17 Winitkotkokapi qa iśtanigongapi; mazaskazi, qa iś tipi wakan mazaskazi wakan kage cin unma tukte iyotan he.

18 Nakun, Tuwe owayuśna kin on wakonze cinhan he taku śni; tuka tuwe woślıa owayuśna akan un kin he on wakonze cinhan, he wahtani ece.

19 Winitkotkokapi qa iśtanigongapi kin ; wośnapi qa iś owayuśna wośnapi kin wakan kage cin unma tukte tanka he.

20 Heon etanhan tuwe owayuśna kin on wakonze cinhan, he owayuśna qa taku akan un kin koya on wakonza ece.

21 Qa tuwe tipi wakan kin on wakonze cinhan, he tipi wakan, qa nakun tuwe ohna ti ece kin, hee on wakonza ece.

22 Qa tuwe mahpiya kin on wakonze cinhan, he Wakantanka oiyotanke tawa, qa nakun tuwe akan iyotanke cin on wakonza ece.

23 Wowapi kagapi qa Pharisee kin, wananicihmanpi, ,w otehi niciyankapi lunwe; ceyakata wahpe skuya, wacanga ko, iwikcemna kin waduśnapi ece; qa woope kin en taku tanka, woowotanna, wowaonśida qa wowacinye, hena ayaktapi śni ece; dena e ecanonpi kta, qa unma nakun econ śni yaunpi kte śni tuka.

24 Tokaheya mani iśtagongapi kin, hoponkadan puskem edakupi, qa kamel heca napca iyeyayapi ece.

25 Wowapi kagaapi qa Pharisee kin, wananiçihmanpi, wotehi niciyankapi nunwe; wakśica wiyatke ko akantanhan duskapi ece, tuka mahen wamanonpi ca wicotawacin śica on ojugjudan. 26 Pharisee iśtagonge, tokaheya wiyatke wakśica ko ma41 


\section{MATTHEOS.}

hentanhan ska kaga wo, kinhan akantanhan he nakun ska kta.

27 Wowapi kagapi qa Pharisee kin, wananicihmanpi, wotehi niciyankapi nunwe; ohna wicaliapi sanyanpi henicecapi; hena akantanhan owanyag waśte, tuka mahentanhan wicaśta huhu qa taku śica owasin on ojugjudan ece.

$28 \mathrm{He}$ iyecen niyepi wicaśta ekta akantanhan owotanna yaunpi seececa, tuka mahentanhan wanaicilimanpi qa woowotanna śni on onijupidan.

29 Wowapi kagapi qa Pharisee kin, wananiçilimanpi, wotehi niciyankapi nunwe; wicaśta wokcan ohna wicahapi kin en tipi wicayecagapi, qa wicaśta owotanna ohna wicahnakapi kin hena sanyayapi ece :

30 Qa hehapi ; Atewicunyanpi ni unpi he anpetu kin en unyakonpi unkanś, wicaśta wokcan wicaktepi qon en ounpapi kte śni tuka ce.

31 Hecen tona wicaśta wok can wicaktepi qon cincapi kin hena niyepi e onihdakapi ece.

32 Qa nakun niye niyatepi wicohan tawapi kin hena wicayeciyuśtanpi lkta.

33 Wamduśka henicapi, sintehida wicoicage, token wakanśica ti iwicayacopi kin nayecipapi kta he.

34 Heon etanhan iho, wicaśta wokcan, wicaśta ksapa, wowapi kagapi hena koya, niyepi ekta yewicawaśi ; unkan etanhan apa wicayaktepi kta, qa icipaweh owicayakatanpi kta ; qa apa omniciye tipi nitawapi kin ohnahna wicayakapsinpsintapi kta; qa otonwe iyasyaza śicaya wicayakuwapi ece e kta.

35 Hecen maka akan wicaśta owotanna wicaktepi hena we kin ocowasin niyepi en aniupi kta; Abel owotanna we kin hetanhan, Zakarias, Barakias cinhintku, tipi wakan qa owayuśna kin iyotahedan yaktepi qon he we kin hehanyan.

36 Awicakehan heciciyapi; Dena owasin wicoicage kin de en awicau kta.

37 Jerusalem, Jerusalem, wicaśta wokcan wicayakte sa, qa tona niye ekta yewicaśipi qon hena inyan on wicayakinin ece qon, wahupakoza wan iye cinca hupahu ihukuya wicatpahi ece kin, he iyecen wanna ota nicinca wicawapahi kta wacin, tuka niye wicayadapi śni ece.

38 Iho, yatipi kin wanna otiwota elipeniciyapi :

39 Qa heciciyapi; Detanhan tohanyan, Tuwe Itancan caje yuha $u$ kin he yawaśtepi nunwe, ehapi śni, hehanyan wanmayadakapi kte śni.

\section{WICOWOYAKE 24.}

1 Unkan Jesus tipi wakan kin etanhan tankan inanpe ca tokan iyaya; unkan waonspewicakiye cin en hipi qa tipi wakan token kagapi kin he wanyag śipi. 2 Unkan Jesus hewicnkiya; Dena owasin wandakapi śni he; awicakehan heciciyapi; Dena inyan wanji inyan itakihna wanke kte śni, owasin yujujupi lita.

3 Unkan Oliwe paha kin akan iyotankehan yanka, hehan waonspe wicakiye cin iśnana on hipi qa heciyapi ; Dena tohan ecetu kta, qa niye yahi kte ça maka ihanke kta, taku on sdonyapi kta, he ito unkokiyaka miye.

4 Unkan Jesus waayupte ca hewicakiya; Ihnuhan tuwe nihnayanpi kinhan.

5 Wicota micaje on upi kta qa, 
Messiya he miye ce, eyapi kta; qa wicaśta ota wicahnayanpi kta.

6 Unkan wicokicize qa kicizapi oyakapi kin nayahonpi kta; ihnuhan nihinniciyapi kinhan; hena owasin hecetu kte, tuka owihanke kin hinahinke śni.

7 Oyate wam oyate azuwicaye kta, qa wokiconze wokiconze anatan kta; qa tuktekten wicaakihan qa makośikśica qa maka ślkanśkan yuke kta.

8 Dena owasin wicoiyokiśice tokaheya kin hee.

9 Hehan wicokakije en yeniyanpi kta qa niktepi kta; qa micaje on oyate owasin śicenidapi kta.

10 Unkan hehan wicaśta ota śihdapi kta, qa otoiyohi kicihnayanpi kta qa śicekicidapi kta.

$11 \mathrm{Qa}$ wicaśta wokcan itonśni ota icagapi kta, qa wicota wicahnayanpi kta.

12 Hehan wicohan sica ota icage kta, heon wicota waśtedake ciqon he sni aye kta.

13 Tuka tuwe owihanke hehanyan najin kinhan he ni kta.

14 Unkan wokiconze wotanin waśte kin de maka kin owancaya oyakapi kta, oyate owasin en wotanin un kta; qa hehan owihanke kta.

15 'Johan wośice woihangye kin, Daniel wicaśta wokcan cajeyata qon he tipi walsan kin en han wandakapi kinhan; tuwe yawa kinhan okahniga nunwe ;

16 Hehan tona Juda makoce kin en unpi kin he kin ekta napapi kta.

17 Qa tuwe tipi akan un kinhan kun ku, qa taku ti mahen tanhan ehdaku kte śni.

$18 \mathrm{Qa}$ nakun tuwe magata un kinhan, hdicu qa wokoyake ikikcu kte śni.
19 Unkan tona anpetu kin hena en ihduśakapi, qa hokśiyoqopa azinwicakiyapi kin hena wotehi yuhapi kta.

20 Tuka waniyetu, qa anpetu wakan icunhan, nayajicapi kte śni e ito cekiya po.

21 Hehan wokakije tanka kte, maka kagapi lkin ehantanhan dehanyan taku hecece śni, qa nakun tohinni tokata ekta hecece kre śni.

22 Qa anpetu kin hena yuptecedan śni kinhan, wicaśta tuwedan ni kte śni ; tuka wicakahnigapi kin hena on anpetu kin hena yuptecedan kta.

23 Hehan tuwe, Messiya den un ce, qa, kakiya un ce, eniciyapi eśta, wicadapi śni po.

24 Messiya itonśni, qa wicaśta wokcan itonśui kin heca icagapi kta, qa wowanyake tanka wi cohan tanka ko econpi kta, hecen okihipica unkanś wicakahinigapi kin hena eepi kaeś wicahnay anpi kta tuka.

25 tho, itokam hena ociciyakapi.

26 Heon etanhan; Iho, hewotahedan un ce, eniciyapi eśta, ekta yapi śni po; qa, Iho nahmana un ce, eniciyapi eśta, wicadapi śni po.

27 Wakanhdi wiyohiyanpa tanhan $u$, qa wiyolipeyata hehanyan tanin hinhda ece, Wicaśta Cinhintku kin hi kte cin he iyecece kta.

28 Tukten taku ta wanka eca, hen wamdi kiwitayapi ece e kta. 29 Unkan wokakije anpetu kin hena ahankeya, anpetu wi kin aotpaza kta, qa hanyetu wi kin iyoyanpa kte śni, qa wicanlipi mahpiya eciyatanhan hinhpaye kta, qa mahpiya wowaśake kin yuhuhuzapi kta.

30 Unkan hehan mahpiya en, 43 
Wicaśta Cinhintku kin wowakta tawa kin tanin kta. Hehan maka akan oyate owasin nihinciyapi kta, qa Wicaśta Cinhintku kin wowaśake qa wowitan taiska hduha, mahpiya śapa akan u wanyakapi kta.

31 Unkan mazayahotonpi tanka hotanin kin on ohnihdewicaye cin yewicaśi kta, qa hena tate uya topa eciyatanhan, tona wicakahnige ciqon hena wicapahi kta, nılipiya ihanke kin etanhan unma ihanke kin ekta hehanyan.

32 Wanna suken can kin etanhan wiyacinpi wan onspeiciciya po. Can intpa hinahin suta śni, qa wanna ape uya, hehan mdolietu kin wanna ikiyedan sdonyayapi ece.

33 He iyecen dena owasin ecetu wandakapi kinhan, wanna ikiyedan, tiyopa kin ehantu e'sdonya po.

34 Awicakehan heciciyapi; Wicoicage kin de ihanke kte śni, ecen dena owasin ecetu kta.

35 Mahpiya maka ko ihanke kta, tuka mioie kin owihanke kte śni.

36 Tuka anpetu kin he, qa wihiyayedan oape kin he tuwedan sdonye śni; mahpiya ohnihde kin hena sdonyapi śni ; Ate ecedan sdonya.

37 Nowa taanpetu qon hena, Wicaśta Cinhintku kin hi kte cin iyecece kta.

38 Minitan qon isanpa anpetu kin hena en, wotapi qa wayatkanpi, winohinca wicayuzapi qa wicaśta hihnawicayapi ece, Nowa wata kin en iyaye cin anpetu kin hehanyan;

$39 \mathrm{Qa}$ iyukcanpi śni, ecen minitan aye ca iyulipa ihang wicaya : iho, Wicaśta Cinhintku kin u kte cin he iyecece kta.

40 Hehan wicaśta nom magata unpi kta; wanji icupi kta, qa unnia kin iś okaptapi kta.

41 Winohinca nom wiyutpan on wotpan unpi ktá; wanji icupi kta, qa unma kin okaptapi kta.

42 Heon etanhan waktaya un po, wihiyayedan oape tukte en Itancan nitawapi u kta sdonyayapi śni.

43 Tuka de sdonya po; wicaśta tipi tawa kin wamanon sa tohan hi kta sdonya unkanś, kikta un qa tipi tawa yulidokapi kta iyowinwicakiye kte śni tuka.

44 Heon etanhan niś eya wiyeya un po ; Wicaśta Cinhintku kin wihiyayedan oape tukte wanji en hi kta, he sdonyayapi śni.

45 Tuwe wowidake owotanna qa wayupika, itancan tawa tipi awanyagkiya, tohan iyehantu eca wo wicaqu ece e kta.

46 Wowidake kin hecen econ ece, itancan tawa en hdi qa wanhdake cinhan, he yawaśtepi ece.

47 Awicakehan heciciyapi; Taku yuhe cin owasin awanyagkiye kta.

48 T'uka wowidake sice cin he cante mahen, Itancan mitawa tehan hdi śni ce, eye cinhan;

$49 \mathrm{Qa}$ tona om wowidake un kin hena wicakaśtake ça, witkopi om wote ca wayatke cinhan; 50 Anpetu wan waktaya un śni, qa wihiyayedan oape wanji iye kecin śni, he en wowidake un kin yuhe cin hdi kta;

51 Qa cokaya kicakse kta, qa wanaicihmanpi kin om taku yuhekiye kta ; heciya wicaceya qa hi hdakinskinzapi yuke kta.

\section{WICOWOYAKE 25.}

1 Hehan malpiya wokiconze kin witanśna unpi wikcemna iyacinpi kta; petijanjan ihupa 
ikikcupi qa tawicu ton kte cin he itkokim iyayapi.

2 Unkan zaptan ksapapi, qa zaptan witkotkokapi.

3 Witkotkokapi kin hena petijanjan ihupa icupi, tuka wihdi takudan yuhapi śni.

4 Tuka ksapapi kin hena cega ohna wihdi hduhapi, petijanjan ihupa iyabna.

5 Unkan tawicu ton kte cin he u śni; hehanyan iyulipa libapi qa iśtinmapi.

6 Unkan hanyecokaya hehan wicaho wan tanin, Iho, tawicu ton kte cin he u ce; itkokim ya"po.

7 Hehan witanśna unpi kin hena owasin iyotankapi, qa petijanjan kin piyapi.

8 Unkan witkotkokapi kin hena ksapapi kin hewicakiyapi; Wihdi nitawapi etanhan ongege unqu miye, petijanjan unkicisnipi kta.

9 Tuka ksapapi kin waayuptapi qa hewicakiyapi ; Hiya, okinni unkiye niyepi nakun iyounhipi kte śni ; tuka tona wopetonpi ece kin ekta ya pe, qa opeiceiton miye.

10 Unkan he opeton iyayapi, hehan tawicu ton kte cin he hi; unkan tona wiyeya unpi kin hena wakankiciyuzapi kte cin en kici iyayapi; hehan tiyopa kin ecen icupi.

11 Iyohakam witanśna unpi unmapi kin en hipi, qa heyapi; Itancan, Itancan, tiyopa unkiciyuhioka miye.

12 T'uka iye waayupte ca; Sdonciy api śni, awicakehan eciciyapi ce, eya.

13 Heon etanhan kiktahan un po, anpetu qa wihiyayedan oape kin tukte wanji en, Wicaśta Cinhintku kin hi kte cin he sdonyayapi śni.
$14 \mathrm{He}$ iyecen wicaśta wan makoce tokeca ekta ye kta, hehan wowidake wicayuhe cin wicakico, qa woyuha tawa kin wicakipamni.

15 Unkan wanji mazaska tkeutapi tanka zaptan qu, qa wanji nom, qa wanji iś wanjidan qu; otoiyohi token okihi kta hecen wicaqu; hehan icimani iyaya.

16 Unkan tuwe tkeutapi tanka zaptan icu qon he iyaye, ca on wopeton ece econ, qa nakun tkeutapi tanka zaptan kamna.

17 Qa tuwe nom icu qon iś eya iyecen nom nakun kamna.

18 Tuka tuwe wanjidan icu qon he iyaye ca, maka qe ca, yuhe cin mazaska tawa kin inalima.

19 Unkan wanna tehan hehan wowidake unpi kin dena itancan tawapi kin hdi, qa wicakiciyawa.

20 Unkan tuwe tkeutapi tanka zaptan icu qon he zaptan toktokeca koya ahi qa heya; Itancan, tkeutapi tanka zaptan mayayu; iho, nakun tkeutapi tanka zaptan hena awakamna, eya

21 Unkan yuhe cin heciya; Han, wowidake waśte qa wacinyepica; taku tonana on wacinyepica yaun, taku ota yuheciciye kta; niyuha towiyuśkin kin en opa wo.

22 Hehan tuwe tkeutapi tanka nom icu qon he hi, qa heya; Itancan, tkeutapi tanka nom mayaqu; iho, nakun tkeutapi tanka nom hena awakamna.

23 Itancan tawa kin heciya; Han, wowidake waśte qa wacinyepica; taku tonana on wacinyepica yaun, ito taku ota yuheciciye kta ; niyuha towiyuślkin kin en opa wo.

24 Hehan tuwe tkeutapi tanka wanjidan icu qon he hi, qa heya; 


\section{MATTHEOS.}

Itancan, wicaśta nitehika sdonwaya, tukten woyaju śni eśta etanhan wamnayaya ece, qa tukten taku elipeyaye śni kin hetanhan wayapahi ece.

$25 \mathrm{Qa}$ kowakipa, heon imdamde ca mazaska tkeutapi tanka nitawa maka kin en nawahbe; iho, nitawa kin yahduha.

26 Itancan tawa kin wayupte ca heciya; Wowidake niśice ca nikuja; tukten wowaju śni kin hetanhar wamnawaya ece, qa tukten taku wakada śni hetanhan wawapahi ece e sdonyaya;

27 Heon etanlan mazaska mitawa wicaśta wopetonpi kin yuhewicayakiye kta iyececa tuka; hecen wahdi, qa mitawa oicage ko ewehdaku kta tuka.

28 Heon tkeutapi tanka kin ekiciyaku po, qa tuwe tkeutapi tanka wikcemna yuhe cin he ito qu po.

29 Tuwe taku ota yuhe cin he nakun qupi ece e kta, hecen iyakicuya yuhe kta; tuka tuwe takudan yuhe śni kin hetanhan taku ton kin hee kaeś ekiviyakupi ece e kta.

30 Qa wowidake wakamna śni kin he tankan otpaza kin en elipeya po; heciya wicaceya qa hi hdakinskinzapi yuke kta.

31 Tohan Wicaśta Cinhintku kin wowitan hduha, ohnihdewicaya wakan kin owasin om u kinhan, hehan wowitan niyotanke tawa kin en kiyotanke lita:

32 Qa itokam oyate kin iyuhpa mniciyapi kta; unkan wicayukinukan kta, waawanhdake wan tahinca wanunyanpi, tatokadan etanhan wicapahi ece kin he iyececa.

$33 \mathrm{Qa}$ tahinca wanunyanpi kin liena iye etapa eciyátanhan, qa tatokadan kin iye catka eciyatanhan ewicahnake kta.
34 Hehan Wicaśtayatapi kin tona etapa eclyatanhan unpi lin hena hewicakiye kta; $U$ po, Ate eciyatanban niyawaśt kin, wokiconze maka kagapi.kin ehantanhan wiyeya niciyankapi kin he ito hduha po.

35 Wotektewahda, unkan wo mayaqupi imapuza, unkan wayatkemayakiyapi ; wicaśta matokeca waun, unkan tin hiyumaya yapi :

36 Tancomakadan, unkan waamayakahpapi ; wamayazanka, unkan onśimayadapi ; makaśkapi, unkan wanyag mayahipi ece.

37 Hehan wicasta owotanna kin hena ayuptapi qa heciyapi kta; Itancan, tohinni wotekteyahda wanunniyalsapi, qa wo unniçupi he; qa inipuza qa wayatkeunniciyapi he :

38 'Tohinni wicaśta tokeca yaun wanunniyakapi, qa tin hiyuunniyanpi he; qa tancodan yaun, qa wokoyake unnicupi he:

39 Tohinni waniyazanka qa kaśka nihnakapi yaun wanunniyakapi, qa en unnihipi he.

40 Unkan Wicaśtayatapi kin waayupte ca hewicakiye kta; Awicakehan heciciyapi; Mihunkawanji kin dena cikcistinpidan hecawicayeconpi kin he miye ecamiyeconpi ece.

41 Hehan tona catka kin eciyatanhan unpi kin hewicakiye kta ; Niyaśicapi kin, mitantauhan iy?ya po, peta owihanke wanica ekta, wakanśica iye taokiyewicaye cin koya on kagapi qon.

42 Wotektewahda, tuka wo mayaqupi śni ; imapuza, tuka wayatkemayakiyapi śni :

43 Wicaśta matokeca waun, tuka lin hiyumayayapi śni ; tancodan waun, tuka wokoyake mayaqupi śni; wamayazanka qa 
kaśka mahnakapi waun, tuka onśimayadapi śni.

44 Hehan ayuptapi qa heciyapi kta; Itancan, tohinni wotekteyahda, qa inipuza, qa wicaśta tokeca-yaun, qa tanconikadan, qa waniyazanka, qa kaśka nihnakapi yaun wanunniyakapi, qa ounniciyapi śni he.

45 Hehan awicayupte ca heye kta; Awicakehan heciciyapi ; Dena cilcistinpidan hecawicayeconpi śni kin he miye ecamiyeconpi śni ece.

46 Unkan dena owihanke wanin wokakije kin en yapi kta; tuka wicaśta owotanna kin hena owihanke wanin wiconi kin en yapi kta.

\section{WICOWOYAKE 26.}

1 Unkan Jesus wicoie kin dena owasin ihunnikiya, hehan waonspewicakiye cin hewicakiya;

2 Anpetu nom iyohakam woacakśin kin hehantu kta sdonyayapi ; hehan Wicaśta Cinhintku kin icipaweh okatanpi kta e wicaqupi kta.

3 Hehan wośnapi kagapi, wowapi kagapi qa oyate kin en wicaśta tankapi kin hena koya mniciyapi, wośnapi kagapi itancan, Kaiaphas eciyapi, he ti kin en.

4 Qa Jesus token nahmana icupi qa ktepi kta he airpi.

5 Tuka wotapi anpetu kin hena icunhan śni, okinni oyate kin owodutaton kta, eyapi.

6 Unkan Jesus Bethani en Simon lepros ecece ciqun he ti kin ohna yanka.

7 Hehan winohinca wan inyan opiye wan wihdi waśtemna tehika ojudan he yuha en hi, qa iye iyotankehan yanka icunhan, pa kin en okaśtan.

8 Tuka waonspewicakiye cin hena he wanyakapi qehan sihdapi, qa heyapi; 'T'okeca de awihnuniyapi he.

9 Wihdi waśtemna kin de ota iyopeyapi kta, qa wahpanica wicaqupi kta iyececa tuka ce.

10 Jesus he okalinige ca hewicakiya: Tokeca winohinca kin de iyokiśinyayapi he; wicolian waśte wan ecamicon.

11 Wahpanicapi kin ohinniyan wicaduhapi, tuka miye ohinniyan mayaduhapi śni.

12 Wihdi waśtemna. kin de mitancan amakaśtan kin, mahnakapi kte cin on etanhan hecon.

13 Awicakehan heciciyapi; Maka kin owancaya wootanin waśte kin de tukten oyakapi hecinhan, winohinca kin de taku econ kin he wowitan on oyakapi ece e kta.

14 Hehan akenonpapi kin etanhan wanji, Judas Iskariot eciyapi, he wośnapi kagapi kin ekta wicai ;

15 Qa heya; He cicupi kinhan taku mayaqupi kta he. Unkan mazaska wikcemna yamni qupi kta, keciyapi.

16 Unkan hetanhan token hnayan wicaqu kta e he akita.

17 Aguyapi on napohyapi codan anpetu tokaheya, hehan waonspewicakiye cin Jesus en hipi, qa heciyapi ; Tukten woacakśin kin yate kta wiyeya unnicihnakapi kta yacin he.

18 Unkan heya; Otonwe kin en ya po, qa wicaśta kae heciya po; Wanna iyemicihantu, woacakśin kin yati en waonspewicawakiye cin om ecamon kta ce, W aunspekiye cin heya ce.

19 Unkan waonspewicakiye cin Jesus econ wicaśi kin ecen econpi, qa woacakśin kin wiyeya ehnakapi. 


\section{MATTHEOS.}

20 Wanna litayetu hehan akenonpapi kin om en iyotanka.

21 Qa wotapi icunhan heya; Niyepi etanhan wanji wiyopemayayapi kta, awicakehan eciciyapi ce.

22 Unkan iyokiśicapi hinca, qa otoiyohi heciyapi; Itancan, he miye he.

23 Unkan waayupte ca heya; Tuwe wakśica ohna kici nape yewaye cinhan he wiyopemaye kte cin ee.

24 Wowapi en kagapi qon he iyecen, II icaśta Cinhintku kin ye kta; tuka wicaśta kin Wicaśta Cinhintku kin wiyopeye cinhan he wotehi kiciyanke kta; wicaśta kin he tohinni tonpi śni unkanś waśte kta tuka.

25 Unkan Judas wiyopeye kte cin he ayupte ca; Itancan, he miye he, eya. Unkan, Niye heha ce, eciya.

26 Unkan wotapi icunhan Jesus aguyapi icu, qa yawaśte, qa yuśpaśpa, qa waonspewicakiye cin wicaqu, qa heya; Icupi qa yuta po; mitancan kin he dee.

27 Qa wiyatke hin ehdaku, qa wopida eye ca wicaqu, qa heya ; Owasin etanhan yatkan po.

28 Mawe kin he dee, wicotakuye teca kin en, wicota on papsonpi, woahtani kajuju kta heon.

29 Tuka heciciyapi, Detanhan hastanhanka iyuwi waskuyeca kin etanhan icimana mdatke kte śni, Ate tokiconze kin en anpetu wan teca unyatkanpi kte cin hehanyan.

30 Unkan odowan wan ahiyayapi hehan tankan iyayapi, Oliwe paha kin he elsta.

31 Hehan Jesus hewicakiya; Hanyetu kin de niyepi owasin elipemayayapi kta. Kaken wowapi en kagapi; Waawanlıdake 48 kin he awape kta, unkan hetanhan tahinca wanunyanpi optaye kin enanakiya iyayapi kta.

32 Tuka ake wakini kinhan Galile ekta nitokam mole kta.

33 Hehan Inyan ayupte ça heciya; Owasin ehpeniyanpi eśta, miye tohinni ehpeciye late śni.

34 Jesus heciya; Hanyetu kin de anpaohotonna hoton śni itokam yamni anamayakihbe kta, awicakehan eciciya.

35 Inyan heciya; Nici mața eśta anacilibe kte śni. Waonspewicakiye cin iyuhpa nakun iyecen eyapi.

36 Hehan Jesus waonspewicakiye cin om can wojupi wan Gethsemene eciyapi kin he en i, qa hewicakiya; Den iyotanka po, ito kalkiya cekiya mde kta.

37 Unkan Inyan qa Zebedee cinhintku kin napin iwicacu, qa iyokiśica hinhda qa nina yuśaka. 38 Hehan hewicakiya; Minagi iyokiśice linca, mațe kta iyececa; den kiktalıan mici yukan po. 39 Unkan hetanhan kitan ecinyan iyoopte iyaye ca makata ehpeiçya, cekiye ça heya; A te, okihipica kinhan, wiyatke lin de tokan imiciyaye kta ; tuka miye wacin kin hee śni, niye token yacin kin hee ecetu kte.

40 Unkan waonspewicakiye cin iśtinma liyeya en wicabdi, qa Inyan heciya; Tokeca wihiyayedan oape wanji kiktahan mici yaunpi kta oyakihipi śni he. 41 Kiktahan unpi qa cekiya po, okinni taku wawiyutanyan un kin en idadapi nun ce. Wicanagi kin he wicada, tuka wicacehipi kin waśake śni.

42 Ake inonpa ekta iyaye ca cekiye ca heya; Ate, wiyatke kin de mdatke śni tokan imiciyaye kta okihipica śni kinhan, nitawacin ecen econpi nunwe. 
43 Hehan ake en wicahdi, qa iśtinma hiyeya wanwicahdaka ; iśta h̉bapi hinca nakaeś.

44 Unkan ehpewicaye ca ake iyaye ca iyamni cekiya, oie un qon hena ake ohna ecen eya.

45 Hehan waonspewicakiye cin en wicahdi qa hewicakiya; Wanna niśtinmapi qa oziniciyapi he ; iho, wanna iyehaniu, qa Wicaśta Cinhintku kin wahtanipi sa napepi kin en wicaqupi.

46 Najin po, unyanpi kta; iho, tuwe wiyopemaye cin he kiyedan u ce.

47 Unkan iahan icunhan, iho, Judas, akenonpapi kin wanji ee, he hi, qa wicota wośnapi kagapi qa oyate kin en wicaśta tankinyanyanpi etanhan, mazasagye cansagye ko yuha kici hipi.

48 Unkan tuwe wiyopeye cin he iwaktawicaye ca heya; Tuwe i iwaputake cinhan hee kta; sutaya yuza po.

49 Unkan katinyan Jesus en hi qa, Iho. Itancan, eye ca i iputaka. 50 Unkan Jesus heciya; Kicuwa, taku on yahi he. Hehan en hipi qa Jesus nape ahnakapi qa yuzapi.

51 Unkan, iho, Jesus kici unpi wanji nape hdukatin, qa mazasagye ehdaku, qa on, wośnapi kagapi itancan taokiye wanji ape ça noge kaśpa.

52 Hehan Jesus heciya; Mazasagye nitawa ojuha en iyekiya wo; tona mazasagye ehdakupi kin owasin mazasagye kin on tapi kta.

53 Nakaha Ate cewakiya owakihi śni kecanni he; qa hinnakaha mahpiya ohnihde wicobe akenonpa sanpa maqu kta tuka.

54 Tuka hecetu kinhan, wowapi en kagapi, he decetu kta eyapi qon he token yuecetupi lkta he.
55 Wihiyayedan oape kin he en Jesus oyate kin hewicakiya ; Tokeca wamanon sa iyecen, mazasagye cansagye ko hduha takpe nayahipi he ; anpetu oloiyohi tipi wakan kin ohna waonspeciciyapi manka, tuka mayaduzapi śni.

56 Tuka dena owasin ecoupi, wicaśta wokcan taku owapi qon he yuecetupi kta heon. Hehan waonspewicakiye cin owasin ehpeyapi qa najicapi.

57 Unkan tona Jesus yuzapi kin hena Kaiaphas wośnapi kagapi itancan kin ekta ayapi, wowapi kagapi wicahincapi ko mniciyapi kin en.

58 Tuka Inyan itehan ihakam $u$, wośnapi kagapi itancan ti kin hehanyan, qa mahen ye ca ookiyewicayapi kin om iyotanka, owihanke kte cin he wanyake kta.

59 Unkan wośnapi kagapi qa wicahincapi kin, qa omniciye ocowasin, woitonśni wootanin oyakapi Jesus on akitapi, ktepi kte cin heon:

60 Tuka takudan iyeyapi śni; woitonśni woyakapi ota hipi, tuka takudan iyeyapi śni. Unhanketa woitonśni oyakapi nom hipi;

61 Qa heyapi; Wicaśta kin de, Tipi wakan Wakantanka tawa kin he mdujuju, qa anpetu yamni kinhan ake piya wakage kta owakihi ce, eya ce.

62 Hehan wośnapi kagapi itancan kin najin hiyaye ca heciya; Dena taku iyanionpapi kin takudan adupte śni he.

63 Tuka Jesus inina yanka. Unkan wośnapi kagapi itancan kin heciya; Wakantanka ni un kin on wacajeciciyate, Messiya Wakantanka Cinhintku kin he niye hecinhan, unkokiyaka miye. 
64 Unkan Jesus heciya; Niye ecen eha; tuka nakun heciciyapi ; Tokata ekta Wicaśta Cinhintku kin wowaśake etapa eciyatanhan iyotanka qa mahpiya śapa akan u wandakapi kta.

65 Hehan wośnapi kagapi itancan kin śina hduhdehdece ca heya; Taku śica eya; taku on sanpa woyakapi unkakitapi kta he; iho, wanna śicaya ie cin he nayalionpi :

66 'l'oken idukcanpi he. Unkan ayuptapi qa, He te lita iyececa ce, eyapi.

67 Hehan ite atagośapi qa nape on apapi; qa hunli kapsinpsintapi ;

$68 \mathrm{Qa}$ heyapi ; Messiya he niye, tuwe anipe cin he ito unkokiyalka po.

69 Inyan tankan tipi kin ohna iyotankehan yanka; unkan wikuśka wan en hi qa heciya; Qa niś nakun Jesus Galile etanhan kin he kici yaun quil.

70 Tuka iye owasin wicitokam anakilibe ca heya; Taku ehe cin he sdonwaye śni.

71 Unkan ohanzi hdepi kin ekta tankan iyaya, hehan wanji tokeca wanyake ca tona en unpi kin hewicakiya; De nakun Jesus Nazareth etanhan kin he kici un qon.

72 Unkan akeś anakilibe ca, Taku wakan namahon, wicaśta kin de sdonwaye śni ce, eya.

73 Hehan ake iecadan tona en najinpi kin hena hipi, qa Inyan heciyapi ; Awicakehan ewicatanhan yaun; iyae cin he oniyaka ece.

74 Hehan iapi śica ota eye ca, Taku wakan namuhon, wicaśta kin he ecaca sdonwaye śni ce, eya. Unkan ecahankeya anpaohotonna kin hoton.

75 Hehan Inyan, Jesus taku 50 eciye ciqon, Anpaohotonna hoton śni kin itokam yamni anamayakilibe kta ce, eye cin he kiksuya. Unkan tankan iyaye ca nina ceya.

\section{WICOWOYAKE 27.}

1 Unkan hanhanna hiuca wośnapi kagapi kin owasin qa oyate kin en wicahincapi kin hena koya, Jesus token ktepi kta e he aiapi.

2 Unkan kaśkapi hehan ayapi, qa Pontios Pilate wicaśta itancan kin he kaipi.

3 Unkan Judas wiyopeye cin he wanna yacopi wanyaka, hehan iyopeiciye ca, mazaska wikcemna yamni kin, wośnapi kagapi wicahincapi ko ake wicakahi ;

4 Qa heya; Wawahtani, wicawe taku iyaonpepica śni kin wiyopewaya ce. Unkan heyapi; He etanhan unkitokecapi sni; he niś niye.

5 Unkall mazaska kin tipi wakan kin en ehpeye ca kihda; qa hetanhan iyaye ca poskin icikte.

6 Unkan wośnapi kagapi kin mazaska kin hena icupi, qa heyapi ; Mazaska opiye kin en unkohnakapi kta iyecece śni, wicawe iyopeyapi kin dena ee, heon etanhan.

7 Hecen wiyukcanpi qa hena on wakśica lage cin tamaga he opetonpi ; ohna wicaśta tokeca wicahapi kta.

8 Heon etanhan maga kin he, Wicawe maga, eciyapi, anpetu kin dehanyan.

9 Hehan Jeramiya wicaśta wokcan taku eye ciqon yuecetupi ; he kaken eya; Unkan mazaska wikcemna yamni, tuwe iyopeyapi qon, Israel cincapi kin hecen iyacinpi qon, hena icupi, 


\section{WICOWOYAKE 27.}

10 Qa walkśica kage cin tamaga kin iyopeyapi, Itancan kin econmaśi iyececa.

11 Unkan Jesus wicaśta itancan kin itokam inajin. Qa wicaśta itancan kin wiwange ca heciya; Juda wicaśtayatapi kin he niye he. Unkan; Niś heha ce, Jesus eya.

12 Unkan wośnapi kagapi wicahincapi ko taku iyaonpapi, tuka takudan ayupte śni.

13 Hehan Pilate heciya; Dena taku iyanionpapi kin nayation sini he.

14 Tuka oie wanjidan eśta ayupte śni, hecen wicaśta itallcan kin nina yuśinyaya.

15 Wotapi kin de iyehantu eca wicaśta itancan kin wicakaśkapi wanji kiyuśka ece, tukte wanji oyate kin kicapi.

16 Unkan he ehan wanji tanyan śdonyapi, Barabbas eciyapi, he kaśka hnakapi.

17 Hecen en mniciyapi hehan Pilate hewicakiya; Unma tukte ciciyuśkapi kta yacinpi he, Barabbas qa iś Je=us nakun Messiya eciyapi.

18 Winawizipi on he iye en kahipi e sdonya.

19 Unkan woyaco oiyotanke kin akan kiyotanka hehan tawicu kin tuwe en uśi, qa heciya; Wicaśta owotanna kin he takudan ecakicon śni we, on anpetu kin de wiwahamna en nina mayuśinyaya ce.

20 Tuka wcrónapi kagapi wicahincapi ko oyate kin wahokonwicakiyapi, Barabbas kicapi lita, qa Jesus ihangyapi kta.

21 Hehan wicaśta itancan kin ake wiwicawange ca hewicakiya; Unma tukte ciciyuśkapi kta yacinpi he. Unkan, Barabbas, eyapi.

22 Pilate hewicakiya; Jesus nakun Messiya eciyapi kin he token ecamon kta he. Unkan owasin; Icipaweh okatanpi kta, eciyapi.

23 Unkan wicaśta itancan kin heya; Tokeca, taku śica econ kin tukte e he. 'l'uka nina panpi qa; Icipaweli okatanpi kta, eyapi.

24 Hecen Pilate takudan okihi śni, qa nina owodutat on icaga he wanyaka, hehan mini icu, qa wicota wicitokam nape hdujaja, qa heya; Wicaśta owotanna kin de we kin miye cn amaupi kte sini; niye ito wanyaka po.

25 Hehan oyate kin iyuhipa ayuptapi qa lieyapi; He we lin unkiyepi qa unkicincapi koya en unkaupi nunwe.

26 Unkan hehan Barabbas wicakiciyuślia; qa Jesus kapsin$p$ inta hehan icipaweh okatunpi kta e wicaqu.

27 Hehan wicaśta itancan taakicita kin Jesus ti mahen ayapi, qa wicobe ocowasin en mniciyapi.

28 Qa iye tawokoyake kiyuśdokapi, qa śina duta wan inkiyapi.

29) Qa wapepeka kazontapi on wateśdake kagapi qa teśdagkicatonpi, qa cedi wan nape etapa kin on yuskiyapi; qa itokam canpeślza makehde inajinpi, qa ihahapi, qa heyapi; Iho, Juda Wieaśtayatapi kin.

30 Qa atagośıpi, qa cedi kin icupi qa pa en apapi.

31 Unkan hecen ihahapi hehan śina kin ekiciyakupi qa iye tawoknyake qon kiçunkiyapi, qa icipareh okatanpi kta ayapi.

32 Tankan ayapi hehan wicaśta wan Kurene etanhan, Simon eciyapi, he wanyakapi; unkan he cansusbeca kin kicicinkivapi.

33 Unkan makoce wan Grolgo- 
tha eciyapi, he wicapahu makoce eyapi kin he kapi, he elkta ipi;

34 Hehan miniśi pizi icuhiyapi kin hetanhan yatkekiyapi; tuka uta hehan yatke kta wicada śni.

35 Unkan hen icipaweh okatanpi, oeconna econpi qa tawokoyake kicipamnipi. Hecen wicaśta wokcan oie qon yuecetupi; Mitawokoyake kicipamnipi qa onlidolida mitawa on oceonna econpi ce.

36 Unkan hen iyotankapi qa awanyakapi :

37 Qa wiyaonpapi kin de owapi qa pa iwankam ehnakapi ; JESUS, JUDA WICAŚTAYAT'API KIN HE DEE.

38 Nakun wicaśta wamanon sa nom kici icipaweh owicakatanipi, unma etapa eciyatanhan, qa unma iś catka tanhan econpi.

39 Unkan tona en hiyayapi kin hena aiapi, qa apomnamnapi ;

40 Q aheyapi; Tipi wakan ihangyaye ca anpetu yamni ake piya yakage kte ciqon, niiçiya wo. Wakantanka Cinhintku kin he niye hecinhan, cansusbeca kin etanhan kun ku wo.

4 L He iyececa, wośnapi kagapi kin ihthapi, qa wowapi lkagapi wicahincupi ko om heyapi ;

42 Wicaśta tokeca niwicaya ece, iye nuiciye kta okihi śni; Israel wicastayataji tawapi heciuhan, iho, wanna cansusbeca kin etanhan kun ku kta, kinhan wicaundapi kta.

43 Wakantanka wacinkiye cigon; tawa kta hecinhan wanna ehdaku kta; Wakantanka Cinhintku kin he miye ce, eye ciqon.

44 Nakun wicaśta wamanon sa, kici icipaweh owicakatanpi kin hena iś eya iyecen i en hiyeyapi

45 Unkan wihiyayedan oape śakpe hetanhan napcinwanka ape cin hehanyan makoce kin owancaya otpaza.

46 Unkan napcinwanka apa hehantu ecetu, Jesus howaye ca hotankakiya heya; Eli, Eli, lama sabakthani; Wakantanka mitawa, Walkantanka mitawa, tokeca ehpemayaya he, eyapi kin hee.

47 Unkan en najinpi kin he nahonpi qehan, apa heyapi; Elias kipan ce.

48 Unkan wanji etanhan inyang ye ça mini iyuhepe wan icu, qa miniskuya en oputkan, cedi wan icape ca yatkekiya.

49 Unmapi kin heyapi; Hinnahanka, Elias nikiya hi kta hecinhan ito wanunyakapi kta.

50 Tuka Jesus ake hotankakiya hotanin qehan, taniya kin hiyuya.

51 Unkan iho, tipi wakan cokaya ozanpi kin he okiyuhdecapi, wankantanhan qa kun ihanke kin hehanyan; nakun maka kin śkanśkan qa inyan namdecapi :

5: Qa wicahapi kin yuhdokapi, qa wicaśta waśte iśtinmapi kin hena ota hiyupi ;

53 Qa wicahnakapi kin etanhan hninanpapi, qa otonwe wakan lin en hipi, qa wicota en taniniciyapi.

54 Unkan akicita tancan qa tona om Jesus awanyake cin hena maka kin śkanśkan qa taku econpi ko wanyakapi qehan, nina yuśinyayapi qa, A wicakehan Wakantanka Cinhintku kin he dee tuka ce, eyapi.

55 Unkan winohinca ota hen unpi qa itehanyan wanyakapi; tona Galile etanhan upi qa okiyapi ece qon :

56 Mary Magdalene he wanji ee, qa Mary Jakob qa Joses hunku, qa Zebede cinca hunkupi kin. 
57 Wanna hitayetu hehan Arimathea etanhan wicaśta wijica wan en hi, Joseph eciyapi, he nakun Jesus waonspewicakiye cin wanji ee :

$58 \mathrm{He}$ Pilate en i, qa Jesus tancan kin kida. Hehan Pilate tancall kin he quwicaśi.

59 Unkan Joseph tancan kin he icu qehan, miniliuha ska wan opazunta;

60 Qa ohna hnakapi tawa teca, imnija kin en kahdoke cin, he en ohnaka; qa inyan wan tanka ohna hnakapi i kin en eonpa, hehan kihda.

61 Unkan ohna hnakapi kin iyotakons, Mary Magdalene qa Mary unma qon iyotarkehan yukanpi.

62 Unkan anpetu en ihduwiyeyapi ece kin he ihanhanna, hehan wośnapi kagapi qa Pharisee kin hena Pilate en hipi,

$63 \mathrm{Qa}$ heyapi ; Itancan, wicahnaye sa kin de ni un qehan, Anpetu yamni hehan wakini kta ce, eya keyapi, he unkiksuyapi.

64. Heon etanhan wicahnakapi kin natag wicaśi wo, anpetu yamni hehanyan; okinni waonspewicakiye cin hena en hipi qa tancan kin manon akiyahdapi kta, qa hehan, Wicate cin etanhan kini ce, oyate kin ewicakiyapi kta; hecen ehake wicahnayanpi kin he tokaheya kin isanpa tanka kta.

65 Pilate hewicakiya; Akicita wicaduhapi ; hdapi qa token oyakihipi sutaya nataka po.

66 Hecen kihdapi qa wicahnakapi kin sutaya natakapi ; inyan kin he apuspapi qa akicita en najinwicakiyapi.

WICOWOYAKE 28.

1 Anpetu wakan owihanke kin ekta, wanna anpetu tokaheya ayanpa kta hehan, Mary Magdalene qa Mary unma kin, wicahnakari kin wanyag hiji :

2 Unkan iho, maka ślkanśkan wan tanka; mahpiya eciyatanhan Itancan taohnihde wan kun $u$, en hi qa tiyopa kin etanhan inyan kin yuptanye ça akan iyotanka.

$3 \mathrm{He}$ ite kin wakanhdi kin iyececa, ga tawokoyake kin wa iyecen ska:

4 Unkan he kokipapi on awanyakapi kin nina cancanpi qa wicaśta tapi kin iyecen ayapi.

5 Unkan malipiya ohnilide kin he waayupte ca winolinca kin hewicakiya; Niye kokipapi śni po; Jesus icipaweli ukatanpi qon he oyadepi sdonwaya :

6 Den un śni; wanna ihduekicetu, heye ciqon iyececa : Kuwa po, Itancan kin wanke ciqon he wanyaka miye.

7 Qa kohanna hdapi qa, Wicate cin etanhan kini ce, waonspewicakiye cin ewicakiya po ; unkan iho, Galile ekta nitokam iyaya ce, hen wandakapi kta; iho, wanna ociciyakapi.

8 . Unkan wokokipe wowiyuśkin tanka ko yuha koyahanna kihdapi; waonspewicakiye cin hośi wicakakipi kta inyang hdapi. 9 Unkan waonspewicakiye cin hośi wicakahdapi icunhan, iho, Jesus itkowicakipa qa heya; Wiyuśkin po. Unkan en hipi qa siha en yuzapi qa ohodapi.

10 Hehan hewicakiya; Kopehdapi śni po; mihunkawanji owicakiyag ya po, Galile ekta yapi kta; heciya wanmayakapi kta ce.

11 Unkan hena hdapi icunhan, akicita awanyakapi qon etanhan wanjikji otonwe kin en hipi, qa wośnapi kagapi kin taku econpi kin owasin owicakiyakapi. 


\section{MARKOS.}

12 Unkan hena witaya mniciyapi, wicahincapi ko om, qa he akiiapi, hehan akicita mazaska ota wicaqupi ;

13 Qa heyapi; Unkiśtinmapi icunhan waonspewicakiye cin hanyen hipi qa manon icupi ce, eya po.

14 Unkan he wicaśta itancan kin nahon kinhan, unkiye ceunkiyapi kta, qa anaunnicikśinpi kta.

15 Hecen mazaska kin icupi, qa token onspewicakiyapi kin iyecen econpi ; heon wicoie kin he Juda oyate kin en oyakapi ece, anpetu kin dehanyan.

16 Hehan waonspewicakiye cin akewanjipi, hena Galile ekta iya- yapi, paha wan Jesus cajeyate ciqon hetu.

17 Unkan wanyakapi hehan ohodapi ; tuka apa cetunhdapi.

18 Unkan Jesus en wicahi, owawicakiye ca heya; Mahpiya ekta qa maka akan wowaśake ocowasin maqupi :

19 Heon etanhan ya po, oyate kin owasin waonspewicakiya po, qa Ateyapi, Cinhintkuyai, qa Wuniya Wakan kin caje on baptem wicaqu po ;

20 Qa taku tona econciśipi kin owasin yuhapi kta e onspewicakiya po. Unka: iho, anpetu owasin cicipi waun, maka owihanke kin hehanyan. Amen.

\section{A R K $0 \mathrm{~S}$}

\section{WOTANIN WASTE TAWA.}

\section{WICOWOYAKE 1.}

1 Jesus Messiya Wakantanka Cinhintku kin Wotaniı Waśte tawa tokaheya kin he dee.

2 Wicaśta wokcanpi kin en owapi qon iyececa; Iho, niite kin itokam wahośi mitawa yewaśi $\mathrm{k}$ ta, nitokam canku wiyeya enicihnake kta.

3 Wicaho wan hewotahedan hotanin kin hee, Itancan tacanku kin wiyeya ekicihnaka wo, ocanku owotanna kicaga wo.

4 Johannes hewotahedan baptem wicaqu, qa woahtani kajujupi kta, on iyopeiciyapi baptem wicaqupi kin he oyaka ece.

5 Unkan Juda makoce kin owancaya tanhan, qa nakun Jerusalem etanhan ekta yapi; unkan woahtani ohdaka, Jordan wakpa kin en, iye owasin baptem wicaqu.

6 Unkan Johannes kamel hin kin koyake ca telimiso wan ipiyagkiton; qa psipsicadan tulimaga canhanpi ahna yuta ece: 7 Qa woyake ca heya; Mihakam wanji mikapeya waśake cin he $u$ ce, he patuś nawajin qa hankpan weciyuśdoke kta iyemicihantu śni.

8 Miś miye awicakehan mini on baptem ciçupi ; tuka iye qe Woniya Wakan kin on baptem niçupi kta ce.

9 Unkan anpetu kin hena icunhan, Galile en Nazareth etanhan Jesus u, qa Jordan en Johannes baptem qu.

10 Unkan mini kin etanhan itanwankanhde ku qehan, ecahankeya mahipiya kin yuzamnipi qa Woniya Wakan kin wakiye- 


\section{WICOWOYAKE 1.}

dan kahya kuntkiya u qa en hiyahan, he wanyaka.

11 Unkan mahpiya eciyatanhan wicaho wan tanin, Micinkśi wastewakidake ca tanyan iyomakipi kin he niye ce.

12 Hehan ecahankeya Woniya kin hewoskan makoce kin ekta kaham aya.

13 Unkan anpetu wikcemna topa liewotahedan makoce kin en uil; qa hen Satan iyutanyan; wamanica kin om un, unkan mahpiya ohnihde kin okiyapi ece.

14 Unkan Johannes kaśka hnakapi qehan, Jesus Galile en hi, qa Wakantanka tokiconze wotanin waśte kin oyaka:

15 Qa heya; Wanna iyehantu, Wakantanka tokiconze kin kiyedan un; iyopeiçiya po, qa wotanin waśte lin wicada po.

16 Unkan Galile mde kin icahda omani, hehan Simon wanyaka, qa iye sunkaku Andrew nakun, mde kin en ho wan iyohpeyapi; hena hopsicapi kin hecapi nakaeś.

17 Unkan Jesus hewicakiya; Miyahna u po, kinhan wicaśta wicadupsicapi lita cicagapi kta ce.

18 Unkan ecahankeya ho kin ehpekiyapi, qa ihakam iyayapi.

19 Unkan lietanhan kitan ecinyan iyoopta ye ça, Jakob Zebede cinhintku, nakun sunkaku Johannes eciyapi, wanwicayaka; hena wata ohna ho kin piyapi.

20 Unkan ecahankeya wicakico; unkan atkukupi Zebede, qa tona wowidake wicayuhe cin hena om, wata kin en ehpewicayapi, qa ihakam iyayapi.

21 Hehan Kapernaum en yapi; unkan anpetu wakan icunhan omniciye tipi kin katinyan en ye cá, woyaka.

$22^{\circ}$ Unkan taku oyake cin on yuśinyayapi ; wicaśta itancanyan waonspewicalkiya, qa wowapi kagapi kin iyecece śni.

23 Unkan omniciye tipi lkin he ohna wicaśta wan woniya śica yuha; unkan he howaya,

24 Qa heya; Unkayuśtan po; Jesus Nazareth etanhan, taku ecaunniconpi he ; ihangunyayapi kta yahi he; nituwe kin sdonwaya, Taku Wakan Wanji Wakantanka tawa kin he niye.

25 Hehan Jesus iyopeye ça heciya; Inina yanke ca, tankata hninanpa wo.

26 Uukan woniya śice cin he wicaśta kin yuhdece ca, hotankakiya hotanin, hehan tankan hinanpa.

27 Unkan "owasin inihanpi qa heya ikiciwangapi; De taku he; de woonspe teca kin tukte e he; woniya śice cin itancanyan owawicakiya, unkan anagoptanpi ece.

28 Unkan hetanhan Galile makoce ihdulían owancaya ohan kin oyakapi.

29 Unkan omniciye tipi kin etanhan hdicupi, hehan katinyan Simon qa Andrew tipi tawapi kin en yapi, Jakob qa Johannes nakun opapi.

30 Tuka Simon tawicu hunku kin he tancan kata on wayazanka yanka; unkan he okiyakapi.

31 Hehan en hi, qa napata yuze ca najinkiya; unkan ecahankeya tancan kata ayuśtan, qa hehan owicakiya.

32 Wanna htayetu, wi iyaya hehan, wayazankapi kin owasin yanke cin ell awicahipi, tona wakanśica wicayulınaśkinyan. hena koya.

33 Hecen otonwe kin ocowasin tiyopa kin en kata iheya.

34 Unkan wowayazan toktokeca on wayazanpi kin ota asni- 


\section{MARKOS.}

wicaya, qa taku wakan sica ota nakun tankan hinanpe wicakiya; qa wakan śice cin hena ia iyowinwicakiye śni, hena iyekiyapi nakaeśs.

35 Unkan ihanhianna, hinahin anpa itehan, hehan inajin, qa tankan inanpe ca, liewotahedan makoce wan ekta ye ça, hen wocekiye eya.

36 Unkan Simon tona om un kin hena koya ihakam iyayapi.

37 Qa iyeyapi hehan heciyapi ; Owasin anicitapi ce.

38 Unkan hewicakiya; Otonwe ikiyedan kin hena en unyanpi kta; hen nakun womdake kta; heon etanhan wahi ce, eya.

39 Unkan Galile makoce kin owancaya, omniciye tipi kin ohna woyake ca, wakan śica hinape wicakiya ece.

40 Unkan wicaśta wan lepros eqeca, he en hi, qa cekiya, canpeśka makehde inajin qa heciya ; Yacin kinhan ska mayakage kta oyakihi.

41 Unkan Jesus onśikida, nape ekta yekiye ca, yutan qa heciya; Wacin, ska un wo.

42 Unkan heye cin hecehnana, lepros ececa qon he ayuśtan, qa ska icaga.

43 Hehan tanyan wahokonkiye ca hetanhan hdeśi;

44 Qa heciya; Ihnuhan tuwe taku eyakiye cinhan; tuka hde ca, wośna kage cin he en hdutanin wo, qa niyuskapi $\mathrm{kta}$ on taku Moses econ wicaśi qon hena wayuśna wo, hecen iyepi ekta woyutanin kta.

45 Tuka tankan hde ca, nina ohdake ca, owancaya yaotanin, hecen hetanhan Jesus otonwe kin en taninyan ye kta okihi sni, tuka tankan hewotahedan un ece: unkan owancaya tanhan yanke cin en hipi ece.

\section{WICOWOYAKE 2.}

1 Anpetu tonana iyohakam hehan ake Kapernaum en i; unkan ti mahèn yanka he oyakapi.

2 Unkan hecehnana wicota en mniciyapi, hecen tuktedan en hipi kte śni, tiyopa kin itankan tanhan ocikan śni : unkan wicoie kin owicakiyaka.

3 Unkan hehan en hipi, wicaśta wan tatake cin wicaśta tom akiyuhapi, he en aupi.

4 Unkan wicota kin on tokadan ikiyedan upi śni, hehan tipi akahpe kin yuhdokapi ; he yujujupi hehan tatake cin owinja akan yanka ecehna en yuhukun iyeyapi.

5 Unkan wacinyanpi kin he Jesus wanyake cehan, țațalie cin heciya; Cinś wayalitani kin nicicajujupi ce.

6 Tuka wowapi kagapi wanjikji hen iyotankehan unpi kin hena cante mahen hecinpi;

7 Tokeca wicaśta kin de śicaya ia he : Tuwe woalitani kajuju kta okihi he, Wakantanka ecedan.

8 Unkan hena hecekcen awacinpi kin, Jesus iye tawacin ekta sdonya, hehan hewicakiya ; Tokeca hena hecekcen awacannipi he.

9 Wayahtani nicicajujupi, qa iś, Najin, owinja ehdaku qa mani wo, tatake cin eciyapi kta, unma tukte waśakadan he.

10 Tuka Wicaśta Cinhintku kin he maka akan woahtani kajuju kta okihi, he sdonyayapi kta; hehan tatake cin he heciya;

11 Najin, qa owinja ehdaku, qa yati ekta hda wo, eciciya ce, eya.

12 Unlan hecehnana najin hiyaye ca, owinja ehdaku, qa owa sin wicitokam tankan kihda; he 


\section{WICOWOYAKE 2.}

cen owasin inihanpi, Wakantanka yatanpi qa heyapi; Tohinni taku dececa wanunyakapi śni.

13 Hehan ake tankan mde kahda iyaya; unkan wicota en mniciyapi; unkan waonspewicakiya.

14 En hiyaya icunhan, Lewi, Alpheus cinhintku, mazaska mnayanpi ece kin en iyotanka, he wanyake ca; Miyahna u wo, eciya. Unkan najin hiyaye ça ihakam ya.

15 Unkan he ti kin ohna Jesus wota iyotanka, hehan mazaska mnayanpi wahtanipi sa ko ota Jesus kici iyotankapi, waonspewicakiye cin hena koya: wicota qa ihakam unpi ece.

16 Unkan wowapi lragapi Pharisee ko, wamnayanpi qa walitanipi sa om wota yanka wanyakapi, hehan waonspewicakiye cin hewicakiyapi; Tokeca e wamnayanpi wahtanipi sa ko

- om wota qa wayatkan he.

17 Jesus he nalion, hehan hewicakiya; Tona zanipi kin hena pejihutawicaśta kin cinpi śni, tuka wayazankapi kin hena e cinpi: wicaśta owotanna kin heca on wahi sni, tuka walitanipi sa iyopeiçiyapi kta e wicakico wahi ce.

18 Johannes waonspewicakiye cin hena, qa Pharisee tawapi kin akitianiçiyapi ece; unkan en hipi qa heciyapi; Tokeca e Johannes waonspewicakiye cin hena, qa nakun Pharisee tawapi kin, akihaniciyapi he, tuka waonspewicayakiye cin hena akilianiciyapi śni.

19 Unkan Jesus hewicakiya; Wakankiciyuzapi cinca kin bena tohanyan tarvicu ton kte cin om un kin hehanyan akihaniciyapi kta okihipi he. Tohanyan tawicu ton kte cin yuhapi kinhan, hehanyan akihaniçiyapi kte śni.

20 Tuka anpetu kin u kta, tohan tawicu ton kte cin he ewicakiciyakupi kta, unkan anpetu kin hena en akilianiçiyapi kta.

21 Tuwedan śina teca onśpa onlidohida tanike cin en akihdagton śni; hecon kinhan onśpa teca en akagegepi kin he tanike cin etanhan namna kta, hecen oyulidece kin sanpa tanka aya.

22 Nakun tuwedan miniśa teca ojuha tanike $\operatorname{cin}$ en okaśtan śni; hecon kinhan miniśa teca kin he ojuha tanike cin kahideca ece, hecen miniśa skepe ça ojuha kin ihangyapi; tuka miniśa teca kin ojuha teca kin en okaśtanpi kta.

23 Unkan anpetu wakan icunhan wojupi kin ehna iyaya; qa waonspewicakiye cin, yapi icunhall, wahuwapa yuksapi.

24 Hehan Pharisee kin heciyapi; Wanyaka wo, anpetu waKan icunhan, taku econpi kta iyeccee śni tokeca econpi he.

25 Unkan hewicakiya; Dawid qa tona om un qon, wotektehdapi qa cinpi qehan, token econpi qon he dawapi śni he.

26 Abiathar wośnapi kaga itancan taanpetu kin en, Wakantanka ti kin en ye ca, wayutanin aguyapi kin he yute ca, om un kin hena nakun wicaqu ; he wośnapi kagapi iśnana yutapi kta tuka.

27 Unkan hehan hewicakiya; Anpetu wakan kin he wicaśta on kagapi, qa wicaśta kin iś anpetu wakán kin on kagapi śni :

28 Heon etanhan Wicaśta Cinhintku kin anpetu wakan kin en Itancan un. 
WICOWOYAKE 3.

1 Hehan ake omnicige tipi kin en iyaya ; unkan wicaśta wanji nape śnija hen yanka.

2 Unkan anpetu wakan icunhan asniye kta hecinhan he iawanyakapi; taku iyaonpapi kta e heon.

3 Unkan wicaśta nape śnije cin he heciya; Cokata inajin wo.

4 Hehan hewicakiya; Anpetu wakan icunhan taku waśte econpi kta iyececa, qa iś taku śica econpi kta, unma tukte he; waniyanpi kta qa iś wațeyapi kta he. Tuka inina hiyeya.

5 Unkan icanksiya awicatonwan, cante tehipike ein on iyokiśica, hehan wicaśta kin heciya; Nape hdukatin wo. Unkan hduowotanna; hecen nape kin ocowasin yuecetupi, unma kin iyececa.

6 Hehan Pharisee kin hena iyayapi, qa ecahankeya Herodian kin om wohdakapi, token he ihangyapi kta e heon.

7 'Tuka Jesus mde kin ekta hdicu, waonspewicakiye cin om ; qa Galile etanhan qa Juda makoce kin etanhan wicota hinca ihakam yapi.

8 Nakun Jerusalem etanhan, qa Idumia, qa Jordan akasanpa tanhan; qa Turos qa Sidon ihdukśan tanhan wicota, taku wicohan tanka econ nahonpi, hehan en hipi.

9 Unkan wicota aotinsyapi kta e heon, wata cistinna wan yuha waonspewicakiye cin akipepi kta, kewicakiya.

10 Wicaśta ota asniwicaya; heon tona tancan śilitinpi kin hena yutanpi kta e yúotinzapi.

11 Qa tona woniya śapa yuhapi kin hena wanyakapi, hehan itokam makata ilipayapi qa he- ya howayapi, Wakantanka Cinhintku kin he riye ye.

12 Unkan oyakapi kte śni nina kewicakiya.

13 Unkan hetanhan paha wan ekta ye ça, tona cin kin hena wicakico; unkan en hipi.

14 Unkan akenonpa wicakaliniga, ohinniyan kici unpi kta, qa woyaka yewicaśi lita;

15 Qa wayazankapi kin asniwicayapi kta, qa wakan śica napewicayapi kta, wowaśake yuhapi kta.

16 Unkan Simon he Inyan eya caśton;

17 Qa Jakob, Zebede cinhintku, qa Johannes Jakob sunkaku ; unkan hena Boanerges ewicakiya, Wakinyan cincapi he kapi :

18 Qa Andrew, qa Philip, qa Bartholomew, qa Mattheos, qa 'Thomas, qa Jakob Alpheus cinhintku, qa Thaddeus, qa Simon Kananite ;

19 Qa Judas Iskariot, wiyopeye cin hee. Unkan tipi wan ti mahen iyayapi.

20 Hehan ake wicota en mniciyapi, hecen aguyapi yuta okihipi śni.

21 Unkan iye takuwicaye cin he nahonpi qehan, yuzapi lita e ekta iyayapi; qa, Wanna wacinhnuni ce, eyapi.

22 Nakun wowapi kagapi Jerusalem etanhan hipi kin hena heyapi; He Beelzebub yuha, qa wakanśica itancan kin on wakan śca napewicaya ece.

23 Unkan iye hena wicakico qa wiyaeinyan hewicakiya; Token Satan Satan napeya okihi kta he.

24 Qa wokiconze wan iye iyakitedan kicize cinhan, wokiconze kin he ecen he kte śni.

25 Qa tipi wanji iye iyakite- 
dan kicize cinhan, tipi kin he ecen he kte śni.

26 Nakun Satan inajin, qa iye iyakitedan kicize ca ihdunonpa kinhan, he ecen un kta okihi śni, tuka wanna owihanke kta.

27 Tuwedan wicaśta waśaka ti kin en ye ça, tawalipaya kin ihangye kte śni; wicaśta waśake cin he tokaheya kaśke cinhan, hehan tipi tawa kin ihangye kta. 28. Awicakehan heciciyapi ; Walitanipi owasin wicaśta cincapi kin wicakicicajujupi kta, qa iapi śica on wayaśicapi ece kin hena nakun;

29 Tuka tuwe Woniya Wakan kin yaśice cinhan, he icimana kicicajujupi kte śni; tuka woyaco owihanke wanica kicihe kta :

30 Woniya śapa wan yuha ce, eyapi kin heon etanhan heya.

31 Unkan hehan iye hunkawanjitkupi qa hunku kin hena en hipi, tankan hinajinpi, wahoyapi qa kicopi.

32 He icunhan wicota ihdukśan iyotankapi; unkan heciyapi; İho, tankan nihun qa nihunkawanjipi anicitapi ce.

33 Unkan heya awicayupta; Inawaye cin he tuwe, qa mihunkawanji tuwepi he

34 Unkan tona okśan iyotankapi kin hena ekta ewicatonwe ca heya; Ina qa mihunkawanji, wanwicayaka po.

35 Tuwe Wakantanka tawacin kin ecen econ kinhan, he mihunkawanji ee, mitawinohtin qa inawaye cin nakun.

\section{WICOWOYAKE 4.}

1 Hehan ake mde kin icahda waonspewicakiya; unkan wicota hinca en mniciyapi, hecen wata wan en ope ca, mini ekta iyotanka; unkan oyate kin owasin mole icahda maka akan unpi. 2 Unkan wiyacinpi on taku ota onspewicakiya ; qa waonspewicakiya icunhan hewicakiya ;

3 Nalion po: Tho, wicaśta woju wan woju iyaya.

4 Unkan woju icunhan, apa canku icahda hinhpaya; unkan zitkatanka en ahiyahe ça temyapi.

$5 \mathrm{Qa}$ apa inyan ehna hinhpaya, hen maka tonana; unkan maka akantuyedan heon oliankoya hinanpa.

6 Tuka wi hinanpa hehan gu; qa hutkan nice cin heon śniś aya.

7 Unkan apa wapepeka hu kin ehna hinhpaya; unkan wapepeka kin hu uye ça aoținsya, hecen waskuyeca takudan icage śni.

8 Unkan apa maka waśte kin en hinhpaya, he waskuyeca aicaga; hinanpe ca icaga, qa suton, hunli wikcemna yamni, hunh wikcemna śakpe, qa hunh opawinge akihde aicaga ece.

9 Hehan hewicakiya; Tuwe noge wination yukan hecinhan, ito nahon kta.

10 Unkan iśnana un hehan, akenonpapi qa tona om indukśan unpi kin hena wiyacinpi kin he on iwangapi.

11 Unkan hewicakiya; Wakantanka tokiconze woanahbe kill hena niye sdonyayapi kta e niçupi; tuka tankan unpi kin hena taku owasin wiyacinpi kin on econpi.

12 Hecen wawanyakapi on wanyakapi kta, tuka iyukcanpi kte śni; qa winahonpi on nahonpi kta tuka okahnigapi kte śni; hecece śni kinhan okinni wicayuhomnipi kta, qa woahtani wicakicicajujupi kta. 
13 Unkan hewicakiya; Wiyacinpi kin de sdonyayapi śni he, hecen token ecin wiyacinpi owasin oyakahnigapi kta he.

14 Tuwe woju kin he wicoie kin oju ece.

15 rona canliu icahda qon, tukten wicoie kin ojupi, tuka he nahonpi hehan, ecahankeya $\mathrm{Wa}$ kanśica en hi, qa wicoie cantepi kin en ojupi qon he icu ece, iho, hena eepi.

$16 \mathrm{Qa}$ he iyececa, inyan ota en ojupi qon, tona wicoie kin nahonpi eca, kohanna iyuśkinyan icupi ece kin hena eepi ;

17 Tuka iyepi mahen hutkan nicapi heon aśkayedan icaga; tohan wicoie kin on śicaya wicakuwapi qa sitkihdawicayapi icaga, hehan hinnahanka sihdapi.

18 Unkan tona wapepeka hu ehna ojupi qon, tona wicoie kin nahonpi ;

19 Unkan maka kin de awacinpi, qa wijicapi on wicahnayanpi, qa taku tokeca cantiheyapi kin hena en u, ga wicoie kin yuoținza, hecen wasuton śni, hena eepi.

20 Unkan maka waśte kin en ojupi qon, tona wicose kin nahonpi eca icupi ece, qa waskuyeca icahyapi, wikcemna yamni, wikcemna śakpe, qa opawinge akihde, iho, hena eepi.

21 Unkan hewicakiya; Petijanjan koka wan ihukuya, qa iś ohehdepi ihukuya iyeyapi kta, qa petijanjan ihupa en ośtanpi kte śni, aupi he.

22 Taku akahpetonpi qa yutaninpi kte śni wanica; qa taku anahmanpi qa iyoyanpa en aupi kte śni wanica ce.

23 Tuwe noge wination yukan hecinhan he nalon kta.

24 Unkan hewicakiya; Taku nayahonpi kin awacin po. Wiyutapi kin tukte on widutapi ece kin, he on ake winiciyutapi kta, qa tona anagoptan dukanpi kin sanpa niçupi ece e kta.

25 Tuwe wayuhe cin he sanpa taku qupi kta; qa tuwe takudan yuhe śni, taku yuhe cin hee kaeś kipi kta.

26 Unkan heya; Wakantanka tokiconze kin he wicaśta wan taku su kin maka kin en iyohpeya, he iyececa ;

$27 \mathrm{Qa}$ iśtinma, qa hanyetu anpetu ko inajin; unkan su kin he hinanpe ça icage kta, tuka token icaga he sdonye śni.

28 Maka kin he iye cinka waicaliya ece, ape kin he tokaheya, hehan wahuwapa, qa hehan wahuwapa en tanyan wasuton ece.

29 Unkan wanna suton hehan kohanna isan śkopa kin en iyekiya, wanna kaśdapi kta iyehan u nakaeś.

30 Hehan heya; Taku e Wakantanka tokiconze kin iyececa unyawapi kta he; qa wiyacinpi kin tukte e on unkiyacinpi kta he.

31 Sinape su wan iyececa, he maka kin en elipeyapi, hehan taku su maka en un kin owasin he iyotan cistinua;

32 Tuka maka kin en ojupi, hehan uye ca wato owasin sanpa tanka icaga; adetka tankinyanyan aicaliya, hecen mahpiya okinyanpi kin ohanzi ihukuya manpi ece.

33 Unkan wiyacinpi hecekcen ota on wicoie kin owicakiyaka, token nahon okihipi kin he iyececa.

34 Qa wiyacinpi codan takudan owicakiyake śni. Unkan iśnana yukanpi, hehan taku owasin waonspewicakiye cin hdaheya owicakiyaka ece. 
35 Unkan anpetu kin he en, wanna htayetu hehan, hewicakiya; Akasanpa unyanpi kta.

36 Unkan wicota kin wanna hdewicaśipi, hehan ecehna wata kin en ohnakapi : qa nakun wata cikcistinna toktokeca opapi.

37 Unkan tate tanka wan uya; qa taja wata kin apaspa, hecen wanna mini ojudan.

38 Tuka iye wahektapa tanhan ipahin wan akan iśtinma wanka. Unkan yuhicapi qa heciyapi; Waonspekiye, unkowihankepi lita he etanhan initokeca śni he.

39 Unkan iyotanka, tate kin kiśice ça mde kin heciya ; Owanji yanka wo. Unkan tateyanpa ayuśtan qa amdakedan tanka.

40 Hehan hewicalkiya; Tokeca kopeyahdapi he ; toleca wicayadapi śni he.

41 Unkan nina kolkipapi, qa iyakitedan hekiciyapi; De taku wicaśta hwo, tate kin hee kaeś, mde ko anagoptanpi ece.

\section{WICOWOYAKE 5.}

1 Unkan mde kin akasanpa ihunnipi, hehan Gadarene makoce kin en ipi.

2 Unkan wata kin etanhan inanpa, hehan ecahankeya wicaśta wan woniya śica yuha wicahnakapi etanhan itkokim hiyu:

3 He wicahnakapi kin en un ece; qa tuwedan kaśla okihi śni, mazaicicahilia hee kaeś on.

4. Wanna ota kaśkapi, siha on wicakaśkapi mazaicicahilia ko on; tuka mazaicicahilia kin hena yuweliwega ece, qa siha on wicakraśkapi kin yuksaksa ece; qa tuwedan wahbadan kaga okihi śni.

5 Ohinniyan, hanyetu anpetu koya, wicahnakapi kin ehna, qa he kin ekta śicahowaya un, qa inyan on kiihdalssa ece.
6 Tuka Jesus tehan tanhan wanyaka, hehan ekta inyang ye ca ohoda.

7 Qa lotankakiya hotanin qa heya; Jesus Wakantanka 'Tehanwankantu Cinhintku kin, taku'e unkiyotahedan un he; Taku Wakan cajeyan ceciciya, kakiśmayaye kte śni.

8 Wanna, Woniya śapa, wicaśta kin etanhan hinanpa wo, eciya nakaeś.

y Unkan, Token eniciyapi he, eya iwanga. Unkan heciya; Wicota, emakiyapi ce, wiuncotapi nakaeś.

10 Unkan makoce kin he etanhan hde wicaśi kte śni, nina cekiya.

11 Imnija he kin hen ikiyedan kukuśe optaye wan tanka wo wicaqupi.

12 Hecen wakan śicapi kin cekiyapi qa heyapi; Kukuśe kin ekta ye unśi po, mahen wicunyanpi kta ce.

13 Unkan hecehnana Jesus iyowinwicakiya. Hecen woniya śapapi kin tankan hinanpapi qa kukuśe kin mahen iwicayayapi. Unkan optaye kin ecehna maya hince cin en naśdog eyaye ca minin iyayapi; owancaya kektopawinge nonpa ecetu, qa minin țapi.

14 Unkan tona kukuśe wo wicaqupi qon hena najicapi, qa otonwe kin en makoce ibdukśan koya he oyakapi. Unkan taku econpi kin he wanyag ipi.

15 Qa Jesus en hipi, qa wicaśta wakan sica yuhnaśkinyan, qa wicota yuhe ciqon, wanna ogekiton qa tawacin ecetu iyotankehan yanka he wanyakapi; unkan yuśinyayapi.

$16 \cdot$ Unkan tona wanyakapi qon bena, wicaśta wakan śica yuhnaśkinyanpi, kukuśe kin hena ko 


\section{MARKOS.}

token ecawicakiconpi qon hena owicakiyakapi.

17 Hehan makoce tawapi kin etanhan hde kta e icekiyapi.

18 Unkan wata kin en okipa hehan tuwe wakan śica yuhe ciqon he cekiya, kici un kta heon.

19 Tuka Jesus iyowinkiyc śni, qa heciya; Takuwicayaye cin ekta hda wo, qa taku tona Itancan lin ecanicon qa onśinida, hena owicakiyaka wo.

20 Heccen kihde ca Dekapolis en taku tona Jesus ecakicon kin he ohdaka ece. Unkan owasin yuśinyayapi.

21 Unkan Jesus ake watom akasanpa ki qehan, oyate ota en mniciyapi ; qa mde kin ikiyedan un.

22 Hehan iho, omniciye tipi itancanpi wanji, Jairus eciyapi, he en hi; qa wanyaka hehan siha kin en makata ehpeiciya,

$23 \mathrm{Qa}$ nilla cekiye ca heya: Micunkśi cistinna wanna te kta wanka; en u qa nape on aputake ca asniya ye; hecen ni kta ce.

24 Unkan kici iyaya; qa wicaśta ota ihakam yapi qa aoṭinsyapi.

25 Unkan winohinca wan waniyetu ake nom hehanyan we ece iheya ;

$26 \mathrm{Qa}$ pejihutawicaśta ota on tehiya kuwapi, qa taku owasin on hdusota, qa etanhan waśte icage śni, tuka sanpa śica aya ;

$27 \mathrm{He}$ Jesus oyakapi nahon qehan, lieyata tanhan wicota ehna hiyu, qa tawokoyake yutan.

28 Tawokoyake hee kaeś mdutan kinhan, amasni kta ce, eye ciqon.

29 Unkan hecchnana we iheye ciqon anapta; qa tancan mahen he wayazan kin on wanna akisni sdonkiya.
30 Unkan hecehnana woasni etanhan hiyu kin Jesus atayedan sdonkiya, wicota ehna ihduhomni qa heya; Milawokoyake tuwe yutan he.

31 Unkan waonspewicakiye cin heciyapi; Wicaśta ota aotinsniyanpi he wandaka, qa, Tuwe mayutan he, eha he.

32 Unkan tuwe hecon kin he wanyake kta e ohomni awicatonwan.

33 Tuka winohinca lkin taku ecakiconpi kin sdonkiya, hecer kokipeyahan cancanyan en hi, qa itokam makata elipeiciye ca owasin awicakehan okiyaka.

34 Unkan heciya; Cunś, wacinyaye cin he asniniyan; wookiye hda wo, qa waniyazan qon okizi un wo.

35 Unkan iahan icunhan omniciye tipi itancan ti kin eciyatanhan upi qa heyapi; Nicunkśi wanna ta ; tokeca sanpa Waonspekiye cin nagiyeyaya he.

36 Tuka Jesus taku eyapi nahon qehan, ilecehnana omniciye tipi itancan kin heciya; Kokipe śni wo, wicada wo ecedan.

37 Unkan tuwedan kici ye kta iyowinkiye śni, Inyan, qa Jaknob, qa Johannes Jakob sunkaku, henananpidan.

38 Unkan omniciye tipi itancan ti kin he en hi, qa owodututon kin he wanyaka, tona ceyapi qa nihinciyapi kin hena koya.

39 Unkan en i qehan hewicakiya; Tokeca owodutaton yakagapi qa yaceyapi he; wicinyanna km te śni, tuka iśtinma ce.

40 Unkan nina ihapi. Tuka owasin tankan iyewicaya, hehan wicinyanna atkuku qa hunku: qa tona on un kin hena iwicacu, qa wicinyanna wanke cin en i ;

$41 \mathrm{Qa}$ wicinyanna kin nape yuze ca heciya; Talitha kumi, 
Wicinyanna najin wo, eciciya, eyapi kin he kapi.

4.2 Unkan ecahankeya wicinyanna kin najin qa mani; wanna waniyetu akenonpa. Unkan nina hin yuśinyayapi.

43 Hehan nina oyake śni wicaśi; qa taku yutapi qupi kta, liewicakiya.

\section{WICOWOYAKE 6.}

1 Unkan hetanhan iyoopta iyaye ca iye tamakoce kin ekta ki ; qa waonspewicakiye cin hena opapi.

2 Unkan wanna anpetu wakan hehar omniciye tipi kin ohna waonspewicakiya. Qa wicota nahonpi qehan yuśinyayapi qa heyapi; Tokiyatanhan dena yuha he; qa taku woksape qupi he; qa token iye nape on wicolian tanka dececa econpi he.

3 Cankajipe cin, Mary cinhintku, qa Jakob, qa Joses, qa Judas, qa Simon cincu kin he dee śni he; qa tawinolitin kil hena owasin den unkicipi unpi śni he. Unkan he on canniyeyapi.

4 Tuka Jesus hewicakiya; Wicaśta wokcan iye tamakoce en, qa iye takuwicaye cin ehna, qa iye ti kin ohna, ecedan yunnihanpi śni ece.

5 Unkan heciya wicohan tanka takudan econ okihi śni; tuka wayazankapi tonana napé on awicaputake ça asniwicaya.

6 Unkan wicadapi śni kin heon yuśinyaya. Qa otonwe ihdukśan wanke cin ektakta waonspewicakiya iyaya.

7 Unkan akenonpapi kin wicakico, qa nom nom yewicaśi ; qa woniya śapa on wowaśake wicaqu ;

$8 \mathrm{Qa}$ wahokonwicakiya, icimani yapi kte cin on takudan icupi kte śni, cansagye ecedan; wojuha, qa waneya, qa mazaśa opiye ohna hduhapi kte śni.

9 Tuka hanpokihanpi kta; qa onlidolida norn kiçunpi kte śni.

10 Unkan hewicakiya; Tukten tipi wanji mahen yaipi kinhan, tohanyan hetanhan iyoopta dapi śni, hehanyan hen yukan po.

11 Qa tona iyowinniciyapi śni qa ananigoptanpi śni kinhan, tohan hetanhan dapi kinhan, owicadakapi kta e heon watuśekśeca siha etanhan yahdatatapi kta. Awicakehan heciciyapi ; Woyaco anpetu kin en, otonwe kir he kakije kte cin Sodom qa Gomorrah ahecece kta.

12 Unkan iyayapi, qa wicaśta ihduecetupi kta e woyakapi.

13 Qa taku wakan śica ota napewicayapi, qa wayazankapi kin ota wihdi on sdawicayapi qa asniwicayapi.

14 Unkan Herod wicaśtayatapi kin hena nalion; caje kin tankaya oyakapi nakaeś ; hehan heya ; Johannes baptem wicaqu qon he tapi kin etanhan kini, heon etanhan wicohan tanka écon ece.

15 Tuka toktokeca heyapi; Elias hee. Qa toktokeca iś; He wicaśta wokcan, qa iś wicaśta wokcan wanji iyececa ce, eyapi.

16 Tuka Herod nahon qehan heya; Johannes pa bawakse ciqon hee, wiconțe etanhan kini ce.

17 Herod atayedan yewicaśi, qa Johannes yuze ca háḱka hnaka, Herodias, iye sunkaku Philip tawicu qon heon etanhan; he yuza nakaeś.

18 Unkan Johannes; Nisunka tawicu duze cin he hecetu śni ce, Herod eciya.

19 Heon etanhan Herodias can- 
niyekiye ça kte kta cin tulka okihi śni.

20 Herod Johannes kolkipa, wicaśta ow otanna qa waśte sdonya, heon itonpa; qa oie nahon eca taku oła ecen econ, qa iy uślkinyan nalion ece.

2 L Tuka anpetu iyehantu qehan, Herod anpetu en tonpi qon he ehan, wicaśta itancanpi wicay uhe ca, akicita tancan tankapi, qa Galile en wicaśta tokaheya kin hena, htayetu wotapi wan wicakicaga.

22 Unkan Herodias cunwintku kin en hi, qa waci, qa Herod tona om un kin hena koya iyokipiwicaya, hehan wicaśta yatapi kin heciya; Taku yacin kinhan makida wo, hecen cicu kta.

23 Nakun taku wakan cajeyan heciya; Taku mayakida kinhan ciç -kta, mitokiconze hanke hehanyan.

24 Unkan tankan iyaye ca hunku heciya; Taku wakida kta he. Unkan iś, Johannes baptem wicaqu ece pa kin, eya.

25 Heban ecahankeya inaliniyan wicaśtayatapi ekta tin hiyu qa heya kida; Johannes baptem wicaqu pa kin, wakśica obna kohanna mayaqu kta wacin ce, eya.

26 Unkan wicaśtayatapi kin nina iyokiśica, tuka taku wakan cajeyan heye cin heon, qa tona om wota iyotanke cin hena on, he ipida kta tawatenye śni.

27 Unkan hecehnana wicaśtayatapi kin wicakte wan ekta. yeśi, qa pa kin aku śi: Unkan he ekta iyaye ça, wicakaśka tipi kin ohna pa baksa;

28 Qa wakśica wan ohna pa kin ahdi, qa wikośka kin qu; unkan wikośka kin iś iyoopta hunku kin qu.

29 Unkan iye waonspewica- lkiye cin hena, he nahonpi qehan, en hipi qa tancan ehdalsupi, qa ohna hnakapi wan en hnakapi.

30 Unkan tona yewicaśi qon hena Jesus en hdipi, qa taku owasin okiyakapi, taku econpi qa taku oyakapi ko.

31 Hehan hewicakiya; Niye ito icunonpa hewotahedan ekta u po, qa cistiyedan oziiçiya po. Wicota upi qa hdapi ece, qa hecen wotapi kta hee kaeś okihipi śni.

32 Unkan heon liewoskan makoce wan ekta iśnana watom iyayapi.

33 Tuka yapi kin wicaśta ota wanyakapi, qa he iye iyekiyapi ; qa otonwe owasin etanhan ekta inyang iyayapi, qa kawicapapi, qa en i kte cin hen mniciyapi.

34 Unkan Jesus ekta ihunni, hehan wicota wanwicayaka, qa onśiwicakida, tahinca wanunyanpi tuwedan wicayuhe śni kin iyececapi heon, qa hecen taku ota onspewicakiya.

35 Unkan wanna wi kucedan hehan, waonspewicakiye cin en hipi qa heciyapi; De hewotahedan makoce qa anpetu kin wanna owihanke :

36 Hde wicaśi wo, hecen makoce ihdukśan, qa otonwe kin ektakta yapi, qa woyute opeiçitonpi kta; taku yutapi takudan yuhapi śni.

37 'Tuka iye waayupte ca hewicakiya; Niś ito wo wicaqu po. Unkan heciyapi; Unyanpi qa kaśpapi opawinge nonpa aguyapi iyopeunyanpi, qa wo wicunqupi kta he.

38 Aguyapi śpanyanpi kin tona duhapi he; ito wanyaka po, ewicakiya. Unkan sdonyapi hehan; Zaptan qa hogan nonpa, eyapi.

39 Unkan owasin wato kin 
akan optayetonton iyotangkiye wicaśi.

40 Unkan optayeton iyotankapi, opawingege qa wikcemna zaptanptan.

41 Unkan aguyapi zaptan qa hogan nonpa hena icu, hehan mahpiya ekta etonwe ca yawaśte, qa aguyapi yuśpaśpa, qa waonspewicakiye cin wicaqu, oyate kin wicaqupi kta heon; nakun hogan nonpa kin hena owasin wicakipamni.

42 Unkan iyuhpa wotapi qa imnanpi.

43 Qa oyaptapi kin etanhan makanopiye akenonpa ojudan pahipi, nakun hogan kin etanhan.

44 Unkan aguyapi yutapi kin hena kektopawinge zaptan ecetu.

45 Hehan ecahankeya waonspewicakiye cin wata kin en okipe wicaśi, qa tokaheya akasanpa Bethseda ekta yapi kta, kewicakiya; icunhan iye wicota kin hde wicaśi kta.

46 Unkan hena hde wicaśi, hehan paha wan ekta wocekiye eya iyaya.

47 Wanna hitayetu hehan, wata kin mde kin cokaya ún, qa iye iśnana maka akan un.

48 Unkan watopapi iyotanhan iyekiyapi wanwicayaka ; tatohekiya yapi nakaeś. Unkan hanyetu en awanyakapi itopa hehan, mini amani ekta wicaya.

49 Tuka mde kin amani wanyakapi qehan, he wanagi kecinpi, qa śicahowayapi.

50 Owasin wanyakapi qa yuśinyayapi. Unkan hecehnana owicakiye ca hewicakiya; Cante waśte po, he miye, kokipapi śni po.

51 Unkan ekta wicaya, wata kin en; hehan tate kirı ayuśtan.
Hecen nina yuśinyayapi qa iliniyanyanpi.

52 Aguyapi econ qon he awacinpi śni; cantepi kin aotpasyapi nakaeś.

53 Unkan akasanpatanhan ipi, hehan Gennesaret makoce kin en yapi, qa hutata ihunnipi.

54 Unkan wata kin etanhan inanpapi, hehan hecehnana iyekiyapi ;

55 Qa makoce kin he owancaya inyang yapi, qa tukten un nahonpi kinhan, wicaśta wayazankapi kin owinja akan wicayuhapi, qa en awicayapi.

56 Unkan otonwe ciqadan, qa otonwe tanka, qa ihdukśan makoce wanke cin, tukte en ya eca, canku ohna wayazankapi kin ahiwicahnakapi qa cekiyapi ; tawokoyake opapun hee kaeś yutanpi kta ; unkan tona yutanpi kin hena asnipi ece.

\section{WICOWOYAKE 7.}

1 Hehan Pharisee qa wowapi kagapi wanjikji, Jerusalem etanhan upi qa en ahimniciya;

2 Qa iye waonspewicakiye cin wanjikji nape śapa, nape hdujajapi śni he kapi, aguyapi yutapi wanyakapi qehan wicabapi.

3 Pharisee kin Juda wicaśta ko owasin tanyan nape hdujajapi śni kinhan wotapi śni ece, qa wicalincapi tawoahope kin opapi.

4 Qa mazopiye etanhan hiyupi eca ihdujajapi śni ehantanhan wotapi śni. Unkan taku hececa ota opapi kta icupi, miniyatke, qa can koka, qa cega, ohehdepi ko yujajapi ece.

5 Hehan Pharisee kin wowapi kagapi ko wiwangapi; Tokeca waonspewicayakiye cin wicahincapi tawoahope kin ahopapi 
śni he, tuka nape hdujaja śni aguyapi yutapi ece.

6 Unkan waayupte ça hewicakiya; Wananiciitimanpi, niyepi on Isaya tanyan woyake ciqon, hecen wowapi en kagapi ; Oyate kin de ihapi kin on mayaonihanpi, tuka cantepi kin mitehanyan un ece.

7 Tuka wicaśta akantu tawoahope kin hena woonspe eekiyapi, qa oyakapi ece kin heon ohomadapi kin be taku śni.

8 Wakantanka tawoahope kin ehpeyayapi, qa wicaśta akantu toope kin duhapi, cega qa wakśica yujajapi kin; qa taku hececa ota ecanonpi ece.

9 Unkan hewicakiya; Wicaśta akantu toope duhapi kta e lieon Wakantanka tawoahope kin elpeyayapi, he tanyan ecanonpi he.

10 Moses hecen eya; Niyate nihun kici wicahduonihan wo. Nakun; 'Tuwe atkuku, qa iś hunku kin hdaśice cinhan, he wiconte on te kta ce.

11 Tuka niye hehapi ; Wicaśta wan atkuku, qa iś hunku; He wohduze, tokan wicaqupi he kapi, hecen on ociciye kte sni, eciye cinhan he hecetu ce.

12 Hecen atkuku qa hunku taku sanpa owicakiye kta iyoyakipi śni ;

13 Qa niye woope yakagapi qa wicayaqupi kin on Wakantanka tawoahope kin dutakunipi śni : qa taku hececa ota ecanonpi ece.

14 Unkan oyate kin orvasin wicakico, hehan hewicakiya; Orvasin anamagoptan po, qa okaliniga po.

15 Wicaśta tankan tanhan taku mahen iyaye cin takudan yuśica okihi śni; tuka tona mahen tanhan hinape cin wicaśta yuśice cin hena ee.
16 Tuwe noge wination yukan hecinhan he nahon kta.

17 Unkan wicota wicehna tanhan ti mahen iyaye cechan, waonspewicakiye cin wiyacinpi kin he on wiwangapi.

18 Unkan hewicakiya; Niś eya oyakahinigapi śni he. Taku kaśta tankan tanhan wicaśta mahen iyaye cin he yuśape kta okihi śni e idukcanpi śni he.

19 Hena cante kin en i śni, tuka tezi mahen, qa hehan tankan ehpeyapi, hecen woyute owasin yuecetupi.

20 Unkan heya; Taku wicaśta kin etanhan hinanpe cin he wicaśta kin yuśapa ece.

21 Mahentanhan, wicacante kin etanhan wicotawacin śica hiyu; wawicihahiapi, wiinahmanpi, tinwicaktepi,

22 Wamanonpi, waicucupikte, wośice, wohnaye, wowiliaha, iśtaśica, wayaśicapi, wahaniçidapi, witkotkokapi ;

23 Dena taku śice cin owasin mahentanhan hiyu, qa wicaśta yuśape cin hena ee.

24 Unkan hetanhan najin hiyaye ca, Turos qá Sidon makoce opapun kin en i, qa tipi wanji timahen iyaya; unkan tuwedan sdonye kte śni cin, tuka analimanpi kta okihipi śni.

25 Winohinca wan cunwintku cistinna woniya śapa yuhe cin he nation qehan, en hi qa siha kin en makata ehpeiciya.

$26 \mathrm{He}$ Hellenes winohinca kin heca, Surophenikiya oyate kin etanhan; unkan taku wakan śica cunwintku kin etanhan hinanpe śi kta e icekiya.

27 Tuka Jesus heciya; Wicacinca kin liena tokaheya wotapi kta; wicacinca aguyapi tawapi kin icupi, qa sunka wicaqupi kta he hecetu śni. 
28 Unkan iye wayyupte ca heciya; Han, Itancan; tuka wahna wotapi kin ihukuya hokśiyopa yuśnapi kin śunka yutapi ece.

29 Unkan heciya; Wicoie kin de on ekta hda wo, taku wakan śica nicunkśi etanhan hinanpa ce. 30 Unkan iye ti kin ekta ki, hehan taku wakan śica tankan iyaye ca, cunwintku ohehdepi kin akan wanka e wanhdaka.

31 Unkan ake Turos makoce kin ehpeye ca, Sidon opta, Dekapolis makoce kin cokaya omani, qa Galile mde kin ekta ki.

32 Unkan wicaśta wan noge tpa qa ia olkitpani he en ahipi, qa nape ahnake kta icekiyapi.

33 Unkan wicota etanhan iśnana icunonpa aya, qa nape noge ohna iyeye ça, tagośa qa on ceji yutan :

34. Hehan malipiya ekta etonwe ca comnihdazi qa heciya; Ephatha, yuhdokapi nunwe, eyapi kin he kapi.

35 Unkan ecahankeya noge yuhdokapi, qa ceji ikan kin yukcapi, qa hdaheya ia.

36 Hehan he tuwedan okiyake śni wicaśi ; tuka taku ewicakiye cin on etanhan sanpa nina oyakapi ece.

37 Qa nina yuśinyayapi qa heyapi ; Taku owasin tanyan econ ece; noge tpapi kin winahonwicakiye ca, nakun ia okitpani iewicakiya ece.

\section{WICOWOYAKE 8.}

1 Unkan anpetu kin hena en nina wicota hinca, qa takudan yutapi śni, hehan waonspewicakiye cin wicakico qa hewicakiyã ;

2 Wicota kin onśiwicawakida, wanna yamni can mici unpi, qa taku yutapi takudan yuhapi śni.
$3 \mathrm{Qa}$ wote śni hde wicawas̉i kinhan, okinni tahepi ilipayapi kta; hunh tehan tanhan hipi nakaeś.

4 Unkan waonspewicakiye cin ayuptapi; Makoce hewotahedan kin den, tokiya tanhan dena aguyapi imnahan yute wicakiyapi kta he.

5 Unkan, Aguyapi kagapi tona duhapi he, eya iwicawanga. Unkan iś, Sakowin, eyapi.

6 Unkan oyate kin maka akan iyotanke wicaśi ; qa hehan aguyapi śakowin qon icu, yawaśte qa yuśpaśpa qa waonspewicakiye cin wicaqu, iyoopta wicaqupi kta; unkan iś oyate kin wicalkipamnipi.

7 Nakun hogan tonana yuhapi ; hena yawaśte qa wicaqupi kta, keya.

8 Hecen wotapi qa imnanpi; unkan aguyapi oyaptapi kin etanhan makanopiye śakowin pahipi.

9 Tona wotapi kin hena kektopawinge topa ecetu; hehan hde wicaśi.

10 Hehan ecahankeya wata wan en opa, waonspewicakiye cin om, qa Dalmanutha makoce kin ekta $i$.

11 Unkan Pharisee kin en hipi, qa iyutanyan wiwangapi, qa mahiya eciyatanhan wowapetokeca kida.

12 Unkan iye cante ekta comnihdazi qa heya; Tokeca wicoicage kin de wowapetokeca alkita he. Awicakehan heciciyapi ; Wicoicage kin de wowapetokeca takudan qupi kte śni.

13 Hehan ehpewicaye ca wata kin en okipe ca akasanpa hda.

14. Unkan aguyapi icupi kta akiktonjapi, qa wata ohna aguyapi wanjidan sanpa yuhapi śni. 15 Hehan wahokonwicakiye ça 67 


\section{MARKOS.}

heya; Wanyaka po, qa on napohyapi Pharisee tawapi qa Herod tawa kin he kokipa po.

16 Hecen iye iyakitedan hdokinicapi qa heyapi; Aguyapi unhapi śni, heon etanhan heya ce.

17 Jesus he sdonya hehan hewicakiya; Tokeca aguyapi duhapi śni on ayakinicapi he. Hinahin oyakahinigapi śni qa widukcanpi śni he. Hinahin cante aotpasniyanpi he.

18 Iśta niyukanpi wandakapi śni, qa noge niyukanpi eśta nayahompi śni, qa yeksuyapi śni he.

19 Aguyapi zaptan wicaśta kektopawinge zaptan wicawakipamni, hehan oyaptapi kin makanopiye ohnaka tona yapahipi he. Akenonpa, eyapi.

20 Hehan śakowin qa kektopawinge topa, hehan oyaptapi makanopiye ohnaka tona iyacupi he. Unkan, Sakowin, eyapi.

21 Hehan hewicakiya; Tokeca oyakah̉nigapi śni he.

.22 Unkan Bethseda en hi. Hehan iśtagonga wan en ahipi, qa yutan kta e icekiyapi.

23 Unkan iśtagonge cin napata yuze ca otonwe kin tankan aya; qa iśta kin en atagośa hehan nape kin akan ehnaka, qa taku wany aka hecinhan he iwanga.

24 Unkan wankan etonwe ca heya; Wicaśta wanwicamdaka, mani unpi, can kin iyececa.

25 Hehan ake nape kin iśta akan ehnaka, qa ake wankan etonwekiya; unkan hehan yuecetupi, qa taku owasin tanyan wanyaka.

26 Hehan iye ti ekta hde sí, qa heya; Otonwe kin en ye śni wo, qa otonwe kin en tuwedan okiyake śni wo.

27 Unkan Jesus hetanhan Ke- sareya Philippi otonwe kin hena ekta iyaya, waonspewicakiye cin om. Unkan tahepi waonspewicakiye cin heya iwicawanga; Matuwe, wicaśta keyapi he. 28 Unkan ayuptapi ; Johannes baptem wicaqu kin; qa wanjikji; Elias; qa wanjikji ; Wicaśta wokcan wanji he niye, eyapi.

29 Unkan hewicakiya; Qa niye matuwe kehapi he. Unkan Inyan waayupte ça heciya; Messiya he niye.

30 Unlan he tuwedan okiyake śni wicaśi.

31 Hehan wowicakiyaka, Wicaśta Cinhintku kin taku ota kakije kta; wicahincapi qa wośnapi kagapi, wowapi kagapi ko lieyata iyeyapi kta, qa ktepi kta; qa ampetu yamni iyohakam kini kta.

32 Unkan hena taninyan ohdaka. Hehan Inyan icu qa iyokiśni.

33 Tuka ihduhomni qa waonspewicakiye cin awicatonwan, hehan Inyan iyopeye ca heya; Satan milieyata ya wo; taku Wakantanka tawa kin awacanni śni, tuka wicaśta akantu taku tawa kin.

34 Unkan oyate kin waonspewicakiye cin om wicakico qehan, hewicakiya; Tuwe kaśta omape kta hecinhan, waipiiçida kta, qa cansusbeca kiçin kta, qa mihakam u kta.

35 Tuwe wiconi tawa tehinda kinhan he toki elipekiye kta; tuka tuwe miye on qa wotanin waśte kin on wiconi toki elipekiye cinhan, he qe wiconi hduhe kta.

36 Qa wicaśta wan maka lin de ocowasin ohiya eśta, qa iye nagi kin toki ehpekiye cinhan, taku on iwaśte kta he. 
37 Qa taku e wicaśta nagi kin tokiyopekiye kta he.

38. Heon etanhan tuwe, wicoicage wiinahman qa wahtani sa kin de en, miye qa mioie kin on iśtece cinhan, tohan Wicaśta Cinhintku kin iye Atkuku towitan hduha, ohnihde wakan kin om u kinhan, wicaśta kin he on iśtece $\mathrm{kta}$ ce.

\section{WICOWOYAKE 9.}

1 Hehan hewicakiya; AwicaKehan heciciyapi; den najinpi etanhan wanjikji wiconte utapi śni itokam, Wakantanka tokiconze wowaśake on hi wanyakapi kta.

2 Unkan anpetu sakpe iyohakam Jesus, Inyan, qa Jakob, qa Johannes iwicacu, qa icunonpa paha wan tehanwankantu kin ekta awicaya ; qa wicitokam yutokecapi :

3 Tawokoyake kin wiyatpatpa, ska hinca, wa iyececa; maka akan wayuska kin tuwedan iyecen ska kaga okihi śni.

4. Unkan hen Elias qa Moses en ihdutaninpi; qa hena Jesus kici wohdag yukanpi.

5 Hehan Inyan waayupte ca Jesus heciya; Itancan, den unyakonpi he waśte; ito wakeya yamni ti unkicagapi kta; wanji niye. on, qa wanji Moses, qa wanji Elias unkicagapi kta.

6 Taku eye kta sdonkiye śni; nina yuśinyayapi nakaeś.

7 Unkan mahpiya wan aohanziwicaya; qa malipiya kin eøiyatanhan wicaho wan tanin qa heya; Micinkśi waśtewakidake cin he dee, he anagoptan po.

8 Hehan ecahankeya ihdukśan etonwanpi qehan, wicaśta tuwedan wanyakapi śni, Jesus iśnana kici unpi.
9 Unkan paha kin etanhan apamahde kupi icunhan wahokonwicakiya, taku wanyakapi kin tuwedan okiyakapi kte śni, Wicaśta Cinhintku kin tapi etanhan kini śni hehanyan.

10 Unkan wicoie kin he awacinpi, qa tapi etanhan kini eyapi kin he taku kapi hecinhan he ikiciwangapi.

11 Unkan heya iwangapi ; Tokeca e Elias tokaheya hi kta ce, wowapi kagapi kin eyapi he.

12 Unkan awicayupte ca heya; Awicakehan Elias tokaheya hi kta, qa taku owasin yuecetu kta ; qa Wicaśta Cinhintku kin token taku ota kakije kta, qa heyata iyeyapi kta e wowapi en kagapi qon, he nakun owicakiyaka.

13 Tuka heciciyapi ce; Awicakehan Elias wanna hi; unkan token cinpi kin ecen ecakiconpi, wowapi en kagapi qon iyececa.

14 Unkan waonspewicakiye cin en hdi qehan, wicihdukśan wicota hinca wanwicayaka; qa wowapi kagapi kin wiwicawangapi.

15 Unkan oyate kin iyulipa wanyakapi qehan nina yuśinyayapi, qa ekta inyang yapi qa okiyapi.

16 Unkan wowapi kagapi kin, Taku e on wiwicanungapi he, eya iwicawanga.

17 Unkan wicota etanhan wanji ayupta; Waonspekiye, micinkśi woniya ie śni kin heca yuhe cin he cicahi.

$18 \mathrm{Qa}$ takten eyaku eca yuhidehdeca ece; hecen i minitaga au, qa hi hdakinskinza, qa hanyan ece; unkan waonspewicayakiye cin hena tankan ehpeyapi kta kewicawakiya, tuka okitpanipi.

19 Unkan waayupte ca hewicakiya; Wicoicage wicada śni, 69 


\section{MARKOS.}

tohanyan cicipi waun kta; tohanyan iyowinciciyapi kta he. En makau po.

20 Hehan en kahipi. Unkan wanyag iheye cin hecehna woniya kin yuhidehdeca; qa makata ehpeye ça, i minitaga au paptanptan iyeiçiya wanka.

21 Unkan atkuku kin heya iwanga; Tohantanhan hececa he. Unkan iś; Hokśiyoqopa ehantanhan, eya.

$22 \mathrm{Qa}$ ota petan ehpeye „ca, minin elipeya, ihangye kta e heon ; tuka niś taku oyakihi kinhan, onśiunkida po qa ounkiya po.

23 Jesus heciya; Wicada oyakihi kinhan; tuwe wicada kin he on taku owasin okihipica ce.

24. Unkan hecehnana hokśidan atkuku kin hotanin qa ceya heya; Itancan wicawada; wicawada śni kin he niye omakiya wo.

25 Jesus oyate kin en inyang hiyupi wanwicayaka, hehan woniya śapa iyopeye ça heciya; Woniya ia okitpani qa noge tpa, tankan hinanpe ca, icimana en ye śni wo, he miye e econ ciśi ce, eya.

26 Unkan hotanin qa nina yuhdehdeca, qa tankata hinanpa; unkan wicaśta ța iyececa, hecen, Wanna ta ce, wicota eyapi.

27 Tuka Jesus napata yuze ca yuwankan icu; unkan najin hiyaya.

28 Unkan tin hiyu qehan, waonspewicakiye cin nahmana iwangapi; Tokeca unkiye tankan elipeya unkokihipi śni he.

29 Unkan hewicakiya; Ocaje kin de wote śni cekiyapi kin ecedan on tankan hiyu kta okihi ece. 30 Hehan hetanhan yapi qa Galile iyoopta iyayapi ; qa tuwedan sdonye kte śni cin.

$31 \mathrm{He}$ icunhan waonspewica- kiye cin wowicakiyaka qa; Wicaśta Cinhintku kin wicaśta napepi kin en wicaqupi, unkan ktepi kta; qa ktepi iyohakam anpetu iciyamni hehan kini kta ce, ewicakiya.

32 Tuka wicoie kin he okahinigapi śni, qa on iwangapi kta kokipapi.

33 Unkan Kapernaum en hi; qa ti mahen yanka hehan wiwicawanga; Canku ohna taku e on ayakinicapi he.

34 'T'uka inina yukanpi ; canku ohna wanji tukte iyotan tankapi kta e heon hdokinicapi.

35 Unkan iyotanke ca akenonpapi kin wicakico qa hewicakiya; Wicaśta wan tokaheya un kta cin kinhan, hee ehake un kta, qa owasin wowidagyapi kta. 36 Hehan hokśiyoqopa wan icu, qa wicacokaya ehnaka; qa nape ohna eyaku, hehan hewicakiya; 37 Tuwe hokśiyoqopa dececa wanji, micaje on tin hiyuye cinhan, miye tin hiyumayan ece; qa tuwe tin hiyumaye cin he miye śni, tuka tuwe u maşi kin he tin hiyuya ece.

38 Hehan Johannes ayupte ca; Waonspekiye, wicaśta wan nicaje on taku wakan śica tankan elipeya wanunyakapi, he unkihakam u śni ; unkan unkihekam u śni kin on etanhan teunhindapi ce, eya.

39 Tuka Jesus heya; Tehindapi śni po; wicaśta tuwe nicaje on wicohian tanka econ kinhan, he ituya mayaśice kta okihi śni.

40 Tuwe unkizapi śni kinhan, he unkicipi un ece.

41 Tutve micaje on miniyatke ohna mini datkanpi lkta niçupi kinhan, Messiya niyuhapi kin heon, he wokajuju toki ehpekiye kte śni, awicakehan eciciyapi. 
42 Qa tuwe dena cikcistinpidan wacinmayanpi kin wanji wahtanikiye cinhan, tahu en inyan wiyutpan. wan iyakaśkapi qa miniwanca kin en ehpeyapi unkanś, he waśte kta tuka.

43 Qa ninape wahtaniniye cinhan hdaksa wo ; nape ksa wiconi kin en yai kinhan hee waśte kta, qa nape napin hduha Wakanśica ti, peta icimana kasnipi kte śni kin en iyohpeniyanpi kte śni.

44 Hen wamduśka tawapi kin te kte śni, qa peta kin kasnipi śni ece.

45 Nakun nisiha walitaniniye cinhan hdaksa wo ; huniśte wiconi kin en yai kinhan, he waśte lkta, qa siha nom hduha Wakanśica ti peta icimana kasnipi kte śni.kin en iyohpeniyanpi kte śni.

46 Hen wamduśka tawapi kin te kte śni, qa peta kin kasnipi śni ece.

47 Nakun niiśta wahtaniniye cinhan hduśdoka wo; iśta wanjidan hdulia Wakantanka tokíconze kin en de cinhan, hee waśte kta, qa iśta napin hduha Wakanśica ti peta kin en iyohpeniyanpi kte śni.

48 Hen wamduśka tawapi kin te kte śni, qa peta kin kasnipi śni-ece.

49 Wicaśta otoiyohi peta on skuyeyapi kta, qa wośnapi otoiyohi miniskuya on skuyeyapi kta.

50 Miniskuya kin he waśte; tuka miniskuya kin skuye śni aye cinhan taku e on skuyeyapi kta he. Miniskuya ihduha po, ya wookiye kiciyuha po.

\section{WICOW'OYAKE 10.}

1 Hetanhan najin hiyaye ca, Jordan akasanpatanhan, Juda makoce kin en hi: unkan ake oyate kin en witaya kata iheya; qa econ ece qon he iyecen ake wowicakiyaka.

2 Unkan Pharisee kin en hipi qa wiwangapi, Wicaśta tawicu ehpeye kta iyececa hecinhan, he iyutanyanpi.

3 Unkan waayupte ca hewicakiya; Moses token econ niśipi he.

4. Unkan; Moses kpaganpi wowapi kage ca ehpeye kta iyowinyan ce, eyapi.

5 Unkan Jesus waayupte ca hewicakiya; Cante nitehipi kin heon Moses woope kin he nicagapi.

6 Tuka wicoicage otokaheya tanlan Wakantanka hena wica winyan ahna wicakaga :

$\gamma$ Heon etanhan wicaśta wan atkuku hunku ko ehpewicaye kta, qa tawicu en iyaihdaśke kta:

8 Qa henaoza wicacehipi wanjidan kta ce. Hecen hetanhan nonpapi śni, tuka wicacehpi wanjidan.

9 Heon taku Wakantanka yuokonwanjidan lin he ihnuhan tuwe yujuju kinhan.

10 Unkan ti mahen yukanpi, hehan waonspewicakiye cin he akta wiwangapi.

11 Unkan hewicakiya; Tuwe winnhinca ehpekiye ca wanji tokeca yuze cinhan, he wawicihahapi ecakicon ece.

$12 \mathrm{Qa}$ winohinca wan hihnaku ehpeye ca wanji tokeca hihnaye cinhan, he nakun wawicihahapi econ ece.

13 Hehan hokśiyoqopa en awicahipi, wicayutan kta heon; tuka waonspewicaliye cin tona awicaupi kin iyowicakipiśni.

14 Tuka Jesus he wanyaka, hehan nina iyokiśice ca hewica- 


\section{MARKOS.}

kiya; Hokśiyoqopapi en maupi kta iyowinwicakiya po, qa telindapi śni po; tona dececapi kin Wakantanka tokiconze lkin eepi ce.

15 Awicakehan heciciyapi; Tuwe Wakantanka tokiconze kin hokśiyoqopa iyecen icu śni kinhan he mahen ye lite śni.

16 Hehan poskin wicayuza, qa nape awicaputake ça wicayawaśte.

17 Unkan canku kin ohna iyaye çehan, wanji inyang $u$, qa en canpeśka makehde inajin, qa wiwanga; Waonspekiye waśte, wiconi owihanke wanice cin he ohimiciye kta e on taku ecamon kta he.

18 Unkan Jesus heciya; Tokeca waśte emayakiya he ; tuwedan waśte śni; Wakantanka iśnana.

19 Woahope kin sdonyaya; Wawicihahapi econ śni wo, Tinwicakte śni wo, Wamanon śni wo, Woitonśni oyake śni wo, Wawicaki śni wo, Niyate nihun kici wicahduonihan wo.

20 Unkan waayupte ca heciya; Waonspekiye, dena owasin homakśiyopa ehantanhan ecamon ece.

21 Hehan Jesus atonwe cehan waśtekidake ca heciya; Taku wanji ninica; hde ca taku duhe cin wiyopeya, qa wahpanicapi kin wicaqu wo, kinhan mahpiya ekta wowijice duhe kta; hehan $\mathrm{ku}$, can susbeca kicin, qa mihakam u wo.

22 Unkan oie kin he on iyokiśica, qa cante śica hda; taku ota yuha nakaeś.

23 Unkan Jesus ihdukśan etonwe ca waonspewicakiye cin hewicakiya; Tona watonpike cin hena Wakantanka tokiconze kin en yapi kte cin he tehika ce.
24 Unkan oie kin on waonspewicakiye cin yuśinyayapi. Tuka Jesus ake waayupte ca hewicakiya; Hokśiyoqopa, tona wowijice wacinyanpi kin hena Wakantanka tokiconze kin en yapi kte cin he tehike hinca.

25 Wicaśta wijica Wakantanka tokiconze kin en ye kta he teliika, qa kamel wan tahinśpaciqadan ohdoka ohna ye kte cin he aoptetu.

26 Unkan heon nina yuśinyayapi qa heliciyapi; Hehan tuwe ni kta he.

27 Jesus awicatonwe ca heya; Wicaśta elita he okihipica śni, tuka Wakantanka ekta hecece śni; Wakantanka ekta taku owasin okihipica ece.

28 Hehan Inyan heciya; Tho, unkiye taku owasin elpeunyanpi, qa nihakam unyakonpi.

29 Jesus wayyute ca heya; Awicakehan heciciyapi, tuwe, miye qa wotanin waśte kin on, tipi qa hunkawanjitku, qa tawinohtin, qa atkuku, qa hunku, qa tawicu, qa cinca, qa makoce ehpeye cinhan ;

30 Nakaha wiconi kin de en opawinge akihde icu kta; tipi, qa hunkawanjitikupi, qa tawinolitin, qa hunkupi, qa cincapi, qa makoce ko, wośitkihdapi iyahna; qa wiconi u kte cin he en wiconi owihanke wanica yuhe kta.

31 Tuka wicota tokaheya kin hena ehake unpi kta, qa ehake unpi kin hena tokaheyapi kta.

32 Hehan canku ohna Jerusalem ekta yapi; unkan Jesus wicitokam ya; qa iyepi qeś ihakam upi, inihanpi qa kopehdapi. Unkan akeś akenonpapi kin iwicacu, qa taku akipe kte cin hena kohan owicakiyaka :

33 Iho, Jerusalem ekta itan- 
wankanhde unyanpi; qa Wicaśta Cinhintku kin wośnapi kagapi, wowapi kagapi ko napepi kin en wicaqupi kta; unkan hena te yacopi kta, qa Ircewicaśta kin iyoopta wicaqupi $\mathrm{kta}$ :

34 He qe ihahapi kta qa kapsinpsintapi kta, qa atagośapi kta, qa ktepi kta; unkan anpetu iciyamni kinhan ake kini kta.

35. Hehan Jakob qa Johannes, Zebede cinhintkupi, en hipi qa heciyapi ; Waonspekiye, taku uncinpi kin he ecen ecaunyeconpi kta uncinpi.

36 Unkan, Taku e ecaciconpi ktå yacinpi he; ewicakiya.

37 Unkan heciyapi ; Nitowitan kin en, wanji nietapa qa unma nicatka tanhan unkiyotankapi kta e unqu po.

38 Tuka Jesus hewicaliya; Taku yadapi kin sdonyakiyapi sni. Wiyatke etanhan mdatke kte cin he niś yatkan oyakihipi he; qa baptem miye maqupi kte cin he on niś eya niẹupi kta oyakihipi he.

39 Unkan, Unkokihipi ce, eciyapi. Unkan Jesus hewicakiya; Awicakehan wiyatke etanlan mdatke cin niś etanhan datkanpi kta ; qa baptem maqupi kin he iś nakun niçupi kta;

40 Tuka mietapa qa micatka tanhan iyotankapi kin wicaqupi kte cin he miye mitawa śni; tuka tona wiyeya wicakicihnakapi kta.

41 Unkan wikcemna kin he nahonpi qehan, Jakob qa Johannes nina canniyerwicayapi.

42 Tuka Jesus wicakico qa hewicakiya; Oyate kin ekta tona itancan unpi kin wowidagwicayapi ece; qa tona tanka unpi kin hena oyate wicayuhapi ece.

43 Tuka niyepi ekta hecece kte śni ; tuka tuwe iyotan tanka yaunpi kta hecinhan, he ookiye duhapi kta :

$44 \mathrm{Qa}$ tuwe tokaheya yaunpi kta hecinhan, he owasin wowidagyapi kta.

45 Wicaśta Cinhintku kin hee kaeś waokiyapi kta hi śni, tuka wáowicakiye kta, qa wi cota opewicalitonpi kta on wiconi tawa ehpeye kta on hi.

46 Unkan Jeriko en hipi; qa Jeritro etanhan oyate ota qa waonspewicakiye cin om hdinanpa, hehan Bartimeus, Timeus cinhintku, iśtagonge ciqun he canku icahda wada iyotanka.

47 Unkan he Jesus Nazareth etanhan hee nahion qehan, hotanin qa heya; Jesus, Dawid Cinhintku, onśimalsida wo.

48 Unkan wicota iyokipiśni, inina un kta; tuka iye iyotan hotankakiya heya; Dawid Cinhintku, onśimakida wo.

49 Unkan Jesus owanji najin qa kicopi kta, keya ; hecen iśtagonge cin kicopi qa heciyapi; Cante waśte wo, najin wo, wanna nico ce.

50 Unkan iye śina ehpeya najin hiyaye ca Jesus en hi.

51 Unkan Jesus wayupte ca heciya; Taku ecacicon kta yacin he. Iśtagonge cin; Itancan, watonwe kta, eciya.

52 Unkan Jesus heciya; Hda wo, wacinyaye cin he asniniyan ce. Unkan hecehnana tonwe ca canku ohna Jesus ihakam ya.

\section{WICOWOYAKE 11.}

1 Unkan wanna Jerusalem ikiyedan hipi, Bethphage qa Bethani hetu, Oliwe Paha kin en, hehan waonspewicakiye cin nom ye wicaśi ;

2 Qa hewicakiya; Otonwe iyo73 
takons yanke cin en ya po; unkan wanna en yaipi kinhan, hehan sukcinca wan, tohinni wicaśta akan iyotanke śni, he iyeyayapi kta; yukcapi qa aku po.

3 Qa tuwe, Taku on hecanonpi he, eniciyapi kinhan, Itancan kin cin ce, eya po. Unkan hecehnana au niśipi kta.

4 Unkan ekta iyayapi, qa canku nonpa ipaweh wanka, tiyopa wan en śukcinca kin kaśka najin iyeyapi ; hecen kiyukcapi.

5 Unkan hen najinpi kin wanjikji, Tokeca śukcinca kin dukcapi he, ewicakiyapi.

6 Unkan Jesus econ wicaśi qon he ohna ewicakiyapi: hecen iyowinwicakiyapi.

7 Hehan śukcinca kin Jesus kahipi ; qa tawokoyake akan ehnakapi ; unkan iye akan iyotanka.

8 Unkan wicota canku ohna tawokoyake kin owinśyapi; qa apa can kin etanhan adetka kaksapi, qa canku ohna ehpeyapi.

9 Unkan tona tokaheyapi qa tona ihakam upi kin hena hotaninpi qa heyapi ; Osanna, tuwe Itancan caje on u kinhan, he yawaśtepi ce.

10 Dawid ateunyanpi tokiconze Itancan caje kin on $u$ kin he yawaśtepi: Osanna iyotan tehan wankantu.

11 Unkan Jesus Jerusalem en ye ca, tipi wakan kin en iyaya; qa wanna taku owasin ekta etonwe ca, litayetu aya, hehan akenonpapi kin om Bethani ekta kihda.

12 Unkan ihanhanna Bethani etanhan kupi, hehan wotektehda.

13 Qa itehan suken can wan ape yuke cin. he wanyaka, he en hi, okinni en waskuyeca iyeye kta; tuka en hi qehan takudan iyeye śni, ape ecedan; suken icage kta hinalinke śni.
14 Unkan Jesus wayupte ca heciya; Detanhan tuwedan waskuyeca enitanhan yute kte śni, owihanke wanica. Unkan waonspewicakiye cin he nationpi.

15 Hehan Jerusalem en hipi ; unkan Jesus tipi wakan kin en i, qa tona tipi wakan kin ohna wiyopeyapi qa wopetonpi kin hena tankan iyewicaya, qa mazaska tokiyopeyapi ahna econpi kin ahdapśinyan ehpeya, qa wakiyedan iyopeyapi can akan iyotankapi liena koya:

16 Qa tipi wakan kin ohna tưwe cega yuhe kta iyowinwicakiye śni.

17 Unkan wowicakiyake ca hewicakiya; Wati kin he oyate owasin on wocekiye tipi eciyapi kta ce, eya wowapi en kagapi śni he; tuka niye wamanon sa otipi kin yakagapi ce.

18 Wośnapi lragapi qa wowapi kagapi kin he nahonpi, unkan token ihangyapi kta akitapi; kokipapi, wicaśta owasin tawoonspe kin on yuśinyayapi nakaeś.

19 Unkan wanna hitayetu hehan otonwe kin etanhan tankan iyaya.

20 Unkan ihanhanna en hiyayapi hehan suken can kin wanna hute hehanyan śnija e wanyakapi.

21 Unkan Inyan he kiksuye ca heciya; Itancan, wanyaka wo, suken can daśice ciqon he wanna śnija.

22 Unkan Jesus waayupte ca hewicakiya; Wakantanka wacinyanpi kin he yuha po.

23 Awicakehan heciciyapi ; Tuwe paha kin de, Tokan iyaye ça miniwanca kin en iyohpaya wo, eciye ca, cante kin en cetunhda śni, qa taku tona eye cin hena hecetu kta e wicada 
kinlıan; he taku eye cin hena ecen yuhe kta.

24 Heon etanhan heciciyapi; Ceyakiyapi eca taku tona yacinpi kin hena iyacupi e wicada po, kinhan hena duhapi kta.

25 Unkan cekiya nayajinpi kinhan, tuwe taku on yeksuyapi kinhan kicicajuju po; hecen Ateyayapi mahpiya ekta yanke cin he wayahtanipi kin nicicajujupi kta.

26 Qa niś wayecicajujupi śni kinhan, nakun Ateyayapi mahipiya ekta yanke cin wayahtanipi kin nicicajujupi kte śni.

27 Unkan ake Jerusalem en hdipi; qa tipi wakan kin ohna mani un qehan, wośnapi kagapi wowapi kagapi wicahincapi koya en hipi ;

$28 \mathrm{Qa}$ heciyapi ; Taku wowaśake on dena taku kin ecanon he; qa tuwe dena econ niśi, qa hecen ecanon he.

29 Unkan Jesus waayupte ca hewicakiya ; Miś eya taku wanji iciwangapi kta, amayupta po, kinhan wowaśake tukte e on dena ecamon kin ociciyakapi kta.

30 Johannes baptem wicaqu qon wicohan kin he mahpiya eciyatanhan, qa iś wicaśta eciyatanhan, unma tukte he ; amayupta po.

31 Unkan hdokinicapi qa hekiciyapi ; He mahpiya eciyatanhan, unkeyapi kinhan, hehan; Tokeca wicayadapi śni he, eye kta.

32 Tuka, He wicaśta akantu eciyatanhan, unkeyapi kinhan, oyate kin kowicunkipapi; wicaśta owasin Johannes wicaśta wokcall kin heca kecinpi.

33 Unkan waayuptapi qa Jesus heciyapi; Sdonunyanpi śni. Unkan Jesus waayupte ça hewicakiya; Miś eya wowaśake tukte e on dena ecamon kin he nakun ociciyakapi kte śni.

\section{WICOWOYAKE 12.}

1 Hehan wiyacinpi on wowicakiyaka; Wicaśta wan hastanhanka iyuwi wojupi wan oju, qa ohomni natake ca, miniśa iyuśkice wan en qe ca, cowahe tehan wankantu wan lkaga; qa kicanyanpi kte cin owicaqu, qa makoce wan tokeca ekta icimani iyaya.

2 Qa wanna iyehantu qehan taokiye wan kicanyanpi kin ekta ye śi, kicanyanpi kin etanlıan hastanhanka ojupi kin waskuyeca icu kte cin heoll etanhan.

3 Unkan he yuzapi, qa apapi, qa cokadan hde śipi.

4 Unkan ake taokiye wanji tokeca ekta ye śi; tuka e qe inyan on kininpi, qa pa kahdecapi, qa tehiya kuwapi hde śipi.

5 Unkan ake wanji tokeca ekta ye śi; qa he qe ktepi: unkan nakun wicota, hunh awicapapi qa hunhi wicaktepi.

6 Hehan cinhintku wanjidan waśtekidake cin he hinahin hduha; unkan he ehake ekta ye śi, qa; Micinkśi yuonihanpi kta ce, eya.

7 Tuka kicanyanpi kin hekiciyapi; Tawa kte cin he dee; iho po, unktepi kta; hecen he unkitawapi kta ce.

8 Unkan hecen yuzapi qa ktepi, qa wojupi kin etanhan tankan elipeyapi.

9 Heon hastanhanka ojupi yuhe cin he taku econ kta he. En hi qa kicanyanpi kin hena ihangwicaye kta, qa hastanhanka ojupi kin wicaśta tokeca owicaqu kta.

10 Wowapi kin de dawapi śni he; Ticagapi inyan wan ehpe- 
yapi qon he oise en pa icaga ce.

11 He Itancan kin econ, qa unkiśtapi kin en taku wowinihan.

12 Hehan yuze wacinpi, tuka oyate kin kowicakipapi ; wiyacinpi kin de iye wicaka e heya sdonyapi. Unkan ehpeya kihdapi.

13 Hehan Pharisee qa Herodian etanhan wanjikji elsta ye wicaśipi ; taku eye cin on yuzapi kta.

14 Unkan hena en hipi qehan heciyapi; Waonspekiye, iewicayaka qa tuwedan koyakipe śni sdonunyanpi; wicaśta tancan ekta eyatonwe śni ece, tuka awicakehan Wakantanka tacanku kin odaka ece; Kesar wamnayanpi qupi kta iyececa he.

15 Unqupi kta, qa iś unqupi kte śni he. Tuka iye wohnaye hduhapi sdonye ça hewicakiya ; Tokeca iyutanmayayapi he. Kaśpapidan wanji makau po, wanmdake kta ce.

16 Unkan kahipi. Hehan hewicakiya; Wicite qa wowapi kin de tuwe tawa he. Unkan, Kesar tawa ce, eciyapi.

17 Hehan Jesus waayupte ça; Kesar taku tawa kin kicu po; qa Wakantanka taku tawa kin kicu po, ewicakiya. Unkan heon inihanpi.

18 Hehan Saddukee kin, piya iwicacage kte cin wanica keyapi, hena etanhan en hipi; qa wiwangapi qa heyapi ;

19 Waonspekiye, Moses wowapi unkicagapi, wicaśta wan tawicu ehpeya țe ça, cinca nice cinhan, sunkaku winohinca kin he hduze kta, qa cincu qon cinca kicage kta ce.

20 Hunkawanjinkiciyapi śakowin ; unkan tokapa kin winohinca wan yuze ca, cinca codan ța.
21 Hehan iyolkihe kin he hduze ca nakun ta, qa cinca nica; unkan iyamni kin iś nakun.

22 Unkan ecen śakowinpi kin hduzapi, qa cinca nicapi : ehake winohinca kin iś eya ța.

23 Hecen piya iwicacage cin en inajinpi kinhan, wanji tukte tawicuyapi kta he: śakowin hduzapi tuka.

24 Unkan Jesus waayupte ca hewicakiya; Wowapi wakan kin sdonyayapi śni, qa nakun Wakantanka towaśake kin, heon yanunipi.

25 Wiconte etanhan piya wicoicage kinhan tawicu tonpi śni qa hihnaku yuhapi śni; tuka mahpiya ekta ohnihde unpi kin iyececapi.

26 Unkan tona tapi kin hena piya icagapi kia, on Moses tawowapi kin en dawapi śni he; can opamna etanhan Wakantanka okiye ca heya; Abraham Wakantanka tawa, qa Isaak Walkantanka tawa, qa Jakob. Wakantanka tawa kin he miye ce.

27 Wicaśta tapi kin Wakantanka tawapi śni, tuka ni unpi kin Wakantanka tawapi: heon etanhan śogya yanunipi.

28 Kehan wowapi kagapi wanji en hi, qa akinicapi kin nahon, qa tanyan awicayupta iyukcan heon etanhan, Woahope tokaheya kin tukte e he, eya iwanga.

29 Unkan Jesus ayupta; Israel nahon po, Jehowa Wakantanka unkitawapi kin he Itancan wanjidan, woahope owasin tokapa kin hee ce.

30 Unkan Jehowa Wakantanka nitawa kin he nicante ocowasin on, qa ninagi ocowasin on, qa nitawacin ocowasin on, qa nitowaśake ocowasin on, 
waśteyakidake kta ce; woahope tokaheya kin hee.

31 Qa inonpa kin he he iyececa; Nikiyedan un kin he niye inihdacin waśteyadake kta. Woahope dena isanpa tanka wanica.

32 Unkan wowapi kage cin he heciya; Han, Waonspekiya, wowicake ecen eha, Taku wakan wanjidan, qa icunonpa tanhan wanica.

33 Qa wicacante ocowasin on, qa wicotawacin ocowasin on, qa wicanagi ocowasin on, qa wicowaśake ocowasin on waśtedakapi, qa wicikiyedan unpi kin wiciye ihdacin waśtewicadapi kin, peta on wośnapi qa ikce wośnapi owasin, he isanpa tanka ece.

34 Unkan he wacinksamya ayupta Jesus wanyaka, hehan heciya; Wakantanka tokiconze kin itehan yaun śni. Unkan hetanhan tuwedan sanpa wiwanga tawatenye śni.

35 Hehan Jesus tipi wakan kin ohna wowicakiyaka icunhan waayupte ca heya; Wowapi kagapi kin Messiya he Dawid Cinhintku ce, tokeca heyapi he.

36 Dawid iye qe Woniya Wakan on heya; Itancan kin Itancan mitawa heciya; Mietapa ekta iyotanka wo, tona tokaniyanpi kin hena siha oahe nitawa kin wakage kta hehanyan.

37 Heon etanhan Dawid iye qe Itancan eciya; hecen token cincaya he. Unkan oyate ikceka kin wiyuśkinyan.nahionpi.

38 Unkan wowicakiyaka icunhan hewicakiya; Wowapi kagapi kin kowicakipa po, hena wokoyake hanskaska kicun waśtedakapi, qa wopeton tipi kin ohna owicakiyapi kta nakun :

39 Qa omniciye tipi kin en oi- yotanke tokapapi, qa wotapi kin en oyanke waśteśte kin:

40 Hena e wiwazica tipi kin temyapi, qa ihdutaninpi on wocekiye hanska eyapi ece : hena woyaco iyotan tanka yuhapi kta.

41. Unkan Jesus mazaska opiye kin iyotakons iyotanka, qa token oyate kin mazaska opiye kin en, mazaska ehpeyapi kin he wanyaka. Unkan wicota wijicapi kin hena odota en ehpeyapi.

42 Unkan wiwazica wahpanica wan en hi, qa mazaśa onśpadan nom en elipeya, he mazaśa wanjidan yawapi.

43 Unkan waonspewicakiye cin hena wicakico qa hewicakiya; Dena owasin mazaska opiye kin en elipeyapi, tuka wiwazica wahpanica kin de owasin isanpa en ehpeya, awicakehan eciciyapi.

44. Dena owasin taku ota tompi kin eciyatanhan en ehpeyapi ece; tuka iye qe nice cin etanhan taku yuhe cin ocowasin en elpeya, on ni kte cin ocowasin.

\section{WICOWOYAKE 13.}

1 Unkan tipi wakan kin etanhan kinanpa hehan waonspewicakiye cin wanji heciya; Waonspekiya, inyan tankinyanyan kin dena, qa tipi kagapi kin dena wanyaka wo.

2 Unkan Jesus waayupte ca heciya; Tipi tankinyanyan kỉn dena owasin wandaka he. Inyan wanji inyan itakihna wanke kte śni, owasin jujuwahe lita.

3 Unkan Oliwe Paha kin akan iyotanka, tipi wakan kin iyotakons, hehan Inyan qa Jakob qa Johannes qa Andrew nahmana wiwangapi ;

4 Tohan hena hecetu kta he, qa dena owasin iyecetu kta e 
wowapetokeca tukte e hecinhan, unkokiyaka po.

5 Unkan Jesus waayupte ca hewicakiya; Iwanihdaka po, okinni tuwe nihnayanpi kta.

6 Wicota micaje on hipi qa ; He miye ce, eyapi kta, qa wicaśta ota wicahnayanpi kta.

7 Unkan tohan wicokicize qa kicizapi kta oyalkapi kin nayahonpi kinhan, nihinciyapi śni po: hena hecetu kta, tuka owihanke kin hinahinke śni.

8 Oyate wan oyate tokeca on riajin kta, qa wokiconze wan wokiconze tokeca on ; qa tuktekten maka śkanśkan yuke kta, qa wicaakilian qa wicokicize yuke kta. Dena wicoiyokiśice tokaheya kin ec.

9 Tuka iwanihdaka po ; omniciye tanka kin en aniyanpi kta; qa omniciye tipi kin ohna nicaśtakapi kta; qa miye on wicaśta itancanpi wicaśtayatapi ko en aniipi kta, owicayakapi kta heon.

10 Tuka tokaheya wotanin waśte kin he oyate owasin owicakiyakapi kta.

11 Tuka tohan aniyanpi qa wicanicupi kinhan, itokam taku ehapi kta awacinpi śni po, qa on cante śicapi śni po; tuka wihiyayedan oape kin he en taku niçupi liin he ohna eya po ; eye kte cin he niyepi śni, tuka Woniya Wakan kin hee kta.

12 Hunkawanjinkiciyapi wiconțe en kiciçupi kta, qa wicaśta wan iye cinca nakun; qa wicacinca inajinpi kta qa hunkakewicayapi kin tewicayapi kta.

13 Unkan miye on owasin śicenidapi kta; tuka tuwe owihanke kin hehanyan ope cinhan he ni kta.

14 Tuka tohan wośice woihangye, Daniel wicaśta wokcan cajeyate ciqon, tukten najin kta iyecece śni, hen wandakapi kinhan, tuwe yawa kinhan okalinige kta, hehan tona Juda makoce kin en unpi kin he kin ekta najicapi kta:

15 Qa tuwe tipi akan yanke cin he kun $\mathrm{ku}$, qa timahen tanhan takudan ehdaku kte śni :

16 Qa nakun tuwe magata un kinhan, wokoyake ehdaku kta on hdicu kte śni.

17 Tuka anpetu kin hena en tona ihduśakapi qa hokśiyoqopa azinwicakiyapi kin hena tebiya unpi kta.

18 Unkan waniyetu icunhan nayapapi kte śni e icekiya po.

19 Anpetu kin hena en wicokakije yuke kta, tokaheya Wakantanka taku kage cin ehantanhan, qa dehanyan takudan iyecece śni, qa nakun tokata ekta iyecece kte śni.

20 Unkan Itancan kin anpetu kin hena yupteccdan śni kinhan, wicacehpi takudan ni kte śni; tuka wicakahnigapi tona wicakahnige cin hena on anpetu kin hena yuptecedan.

21 Unkan hehan tuwe; Iho, Messiya hen yanka ce, qa iś; Kan yanka ce, eniciyapi eśta, wicadapi śni po.

22. Messiya itonpiśni qa wicaśta wokcan itonpiśni kin heca icagapi kta, qa wowapetokeca taku wowinihan ko econpi kta, okihipica unkanś wicakahnigapi kin hena eepi kaeś wicahnayanpi kta tuka.

23 Tulka iwanihdaka po; iho, itokam hena ociciyakapi.

24. Tuka anpetu kin hena en, wicokakije lkin iyohakam, anpetu wi kin aotpasyapi kta, qa hanyetu wi kin iyoyanpa kte śni ;

25 Qa mahpiya wicanhpi kin hinhpaye kta, qa mahpiya ekta wowaśake kin yuhuhuzapi kta. 
26 Unkan hehan Wicaśta Cinhintku kin wowaśake tanka wowitan ko hduha malipiya śapa akan u wandakapi kta.

27 Unkan hehan ohnihdewicaye cin hena ye wicaśi kta, qa tate ouye topa kin etanhan, maka ihanke kin etanhan qa mahpiya ihanke kin hehanyan, wicakahinige ciqon hena witaya wicakpahi kta.

28 Suken can kin etanhan wiyacinpi kin de okahniga po: Can intpa kin hinaliin wankadan, qa etanlian ape icage cinhan, hehan mdoketu wanna ikiyedan sdonyayapi ece.

$29 \mathrm{He}$ iyececa, niś tolian dena owasin iyecetu wandakapi kinhan, wanna ikiyedan, tiyopa kin etu, e sdonya po.

30 Awicakehan heciciyapi, wicoicage kin de tokan iyaye śni, ecen dena owasin econpi kta.

31 Malipiya maka ko tokan iyaye kta, tuka mioie kin hena tokan iyaye kte śni.

32 Tuka anpetu kin he, qa wihiyayedan oape kin he tuwedan sdonye śni, mahipiya ohnihde kin heepi kaeś, qa nakun Cinhintkuyapi kin ; tuka A teyapi kin ecedan sdonya.

33 Iwanihdaka po, wakta unpi qa cekiya po; tohan iyehantu kte cin sdonyayapi śni nakaeś.

34 Wicaśta wan icimani ye kte cin, tipi ehpeye ca taokiyewicaye cin taku econ wicaśi, otoiyohi wicohan wicaqu, qa tiawanyake cin he awanyake sí, he iyececa.

35 Heon etanhan waktaya un po ; tipi ohna itancan kin tohan hdi kta sdonyayapi śni, htayetu, qa hanye cokaya, qa anpaohotonna hoton, qa iś hankanna kinhan.

36 Okinni ihnuhanna hdi kinhan niśtinmapi iyeniyanpi kta.
37 Unkan taku eciciyapi kin he owasin ewicawakiya ece, Waktaya un po.

\section{WICOWOYAKE 14.}

1 Anpetu nonpa iyohakam he. han woacakśin qa aguyapi napohyapi śni: Unkan wośnapi kagapi wowapi kagapi ko token hnayan yuzapi kta akitapi, ktepi kta e heon.

2 Tuka heyapi; Wośnapi anpetu kin hehantu kte śni; okinni oyate kin owodutaton kta.

3 Unkan Bethani etu, Simon lepros ececa qon he ti kin en wota iyotanka, hehan winohinca wan inyan opiye wan wihdi waśtemna tehike hinca he yuha hi, qa opiye kin yuhdoke ca iye pa kin akaśtan.

4 Unkan hen wanjikji cante mahen iyokipipi śni qa heyapi; Tokeca wihdi waśtemna kin hecen yutakunipi śni he.

5 Wihdi waśtemna kin de kaśpapi opawinge yamni sanpa iyopeyapi qa wahpanica wicaqupi kta tuka. Hecen winolinca qon icaptapi.

6 Unkan Jesus heya; Ayuśtan po; tokeca nagiyeyayapi he. Wicohan waśte wan ecamicon.

7 Wahpanicapi kin ohinniyan wicayahduhapi, qa tohan yacinpi kinhan tanyan ecawicayeconpi kta oyakihipi; tuka miye ohinniyan mayaduhapi śni.

8 Taku okihi kin ecen econ; mitancan hapi kte cin on itokam sdaye kta on hi.

9 Awicakehan heciciyapi; Maka kin owancaya tukten wotanin waśte kin de oyakapi kinhan, winohinca kin de nakaha taku econ kin he oyakapi lita, wokiksuye kta e heon.

10 Unkan Judas Iskariot, ake- 
nonpapi kin wanji ee, wośnapi kagapi kin elkta ya, he hnayan wicaqu kta heon.

11 Unkan he nahonpi qehan wiyuśkinpi, qa mazaska qupi kta keciyapi. Unkan token tanyan hnayan wicaqu kta he akita.

12 Unkan aguyapi napohye śni anpetu tokaheya en wośna ktepi ece kin hehantu, waonspewicakiye cin heciyapi ; Tukten woacakśin wośnapi yate kta wiyeya eunhnakapi kta yacin he.

13 Unkan waonspewicakiye cin nom ye wicaśi, qa hewicakiya; Otonwe kin en ya po, kinhan wicaśta wan mini ohna wakiśkotpa wan qin itkonicipapi kta, he ihakam ya po.

14 Qa tukte en tin kihde cinhan, tipi tawa kin heciya po; Waonspekiye cin heya ce; Wankan tipi kin tukten waonspewicawakiye cin om wośnapi kin wate lita tukte e he.

15 Unkan wankan tipi wan tanka, tanyan yuśtanpi qa wiyeya yanka, nicipazopi kta; hen wiyeya eunkicihnaka po.

16 Unkan waonspewicakiye cin iyayapi, qa otonwe kin en ipi, qa token ewicakiye cin hecen iyeyapi ; unkan wośnapi kin wiyeya ehnakapi.

17 Unkan wanna litayetu hehan akenonpapi kin om en hi.

18 Unkan wota iyotankapi icunhan Jesus heya; Awicakehan heciciyapi; Mici wayatapi kin wanji wiyopemayayapi kta.

19 Hehan iyokiśica hinhdapi, qa otoiyohi heciyapi; He miye he; qa ake wanji, $\mathrm{He}$ miye he, eyapi.

20 Unkan hewicakiya; Akenonpapi kin etanhan wanji wakśica ohna mici oputkan lkin hee ce. 21 Wicaśta Cinhintku kin awi- eyapi. cakehan token wowapi en kagapi qon he iyecen iyoopta ya, tuka wicaśta kin tuwe Wicaśta Cinhintku kin wiyopeye cinhan, he wotehi yuhe kta; wicaśta kin he tohinni tonpi śni unkanś waśte kta tuka.

22 Unkan wotapi icunhan Iesus aguyapi icu, yawaśte, qa kiyuśpa, qa wicaqu, qa heya; Icupi qa yuta po; mitancan kin he dee.

23 Nakun wiyatke kin icu; qa yawaśte hehan wicaqu. Unkan owasin etanhan yatkanpi.

24 Unkan hewicakiya; Wicotakuye teca kin en, mawe kin he dee, wicota on papsonpi.

25 Awicakehan heciciyapi; Detanhan wiyuwi waskuyeca etanhan icimana moatke kte śni, anpetu wan en Wakantanka tokiconze kin en teca mdatke kte cin hehanyan.

26 Uukan odowan wan ahiyayapi hehan Oliwe Paha kin ekta iyayapi.

27 Hehan Jesus hewicakiva; Hanyetu kin de miye on iyuhpa nihicahanpi kta; kaken wowapi en kagapi qon, Waawanhdake kin he awape lita, hecen talinca wanunyanpi kin mdecahanpi kta ce.

28 Tuka ake wakini iyohakam, Galile elsta nitokam imdamde kta.

29 Tuka Inyan heciya; Owasin hicahanpi eśta, miye qe ecamon kte śni.

30 Unkan Jesus heciya; Awicakehan heciciya; Anpetu kin de, hanyetu kin de en, anpaohntonna nonpa hoton śni, ecen yamni akihde anamayakilibe kta.

31 Hehan iyotan nina kitan hinca; Cicimața eśta anacicilibe kte śni. Iyulipa nakun iyecen 
32 Unkan wojupi wan en ipi, Gethsemane eciyapi; hehan waonspewicakiye cin hewica"kiya; Den iyotanka po, icunhan cewakiye kta.

33 Unkan Inyan qa Jakob qa Johannes hena iwicacu, hehan inihan qa nina iyokiśica hinhda;

34. Qa hewicakiya; Minagi nina iyokiśice linca, mațe kta iyececa; den yukanpi qa waktaya un po.

35 Unkan hetanhan kitan ecinyan iyoopta ye ca, makata ihpaye ca cekiya, okihipica kinhan, wihiyayedan oape kin he tokan iyaye kta.

36 Unkan heya; Abba, Ate, niye ekta taku owasin okihipica ; wiyatke kin de emiciyaku wo; tuka miś taku wacin kin hee kte śni, taku yacin kin he hecetu kta.

37 Hehan en hdi, qa iśtinmapi iyewicaya, unkan Inyan heciya; Simon niśtinma he; wihiyayedan oape wanjidan kiktahan yaun kta oyakihi śni he.

$38^{\circ}$ Kiktahan unpi qa cekiya po; okinni taku wawiyutanyan un kin en yaipi nun ce; wicanagi kin he awicakehan wicada; tuka wicacehpi kin waśake śni.

39 Unkan ake iyaye ça rekiya, qa wicoie un qon hena ake ecen eya.

40 Unkan ake en hdi qehan, ake iśtinmapi iyewicaya, iśta hbapi hinca, unkan token ayuptapi nun tanin śni.

41 Unkan iciyamni en hdi, qa hewicakiya; Iśtinma po qa oziiciya po; wanna henakeca, wihiyayedan oape kin wanna iyehantu; wanyaka po, Wicaśta Cinhintku kin wahtanipi sa napepi kin en hnayan wicaqupi.

42 Najin po, unyanpi kta; iho, tuwe mahnaye cin he wanna kiyedan u ce.
43 Unkan ecahankeya, iahan icunhan, Judas hi, akenonpapi kin wanji ee, qa kici wicota hinca, mazasagye can oțoza ko yuhapi, wośnapi kagapi, wowapi kagapi, qa wicahincapi kin etanhan upi.

44 Unkan tuwe hnayan wicaqu kte cin he iwaktawicaye ca; Tuwe i iwaputake cinhan hee kta; yuzapi qa tanyan akiyahda po, eya.

45 Unkan en hi qehan, ecahankeya en ye ca, Itancan, Itancan, eciye ca, i itputaka.

46 Hehan nape ahnakapi qa yuzapi.

47 Unkan wanji en najinpi kin mazasagye ehdaku, qa wośnapi kagapi itancan taokiye wanji ape ca noge baśpa.

48 Unkan Jesus waayupte ca hewicakiya; Wamanon sa takpe hipi iyececa, mazasagyo can otoza ko yuha mayaduzapi kta on yahipi he.

49 Anpetu eca tipi wakan kin ohna ocipapi qa waonspekiya waun, tuka mayaduzapi śni ; tuka wowapi wakan kin yuecetupi kta.

50 Hehan owasin elipeya najicapi.

51 Unkan kośka wan ihakam ya, tancodan un tuka miniliuha ska tancan iyakipemni. Unkan kośkapi kin hena yuzapi :

52 Hehan minihuha ska kin he elipeya, qa tancodan kihda.

53 Unkan Jesus wośna kagapi itancan kin ekta ayapi; qa hen wośnapi kagapi, wicalincapi, wowapi kagapi ko owasin mniciyapi.

54 Unkan Inyan itehan ihakam u, qa wośna kagapi itancan ti kin en hi; qa ookiyewicayapi kin om iyotanke ca peta kin en cosiçiya. 


\section{MARKOS.}

55 Unkan wośnapi kagapi qa omniciye ocowasin tuwe Jesus on taku oyakapi kta odepi, ktepi kta e heon, tuka takudan iyeyapi śni.

56 Wicota itonśniyan woyakapi, tuka taku oyakapi kin ocitkonze śni.

$5 \%$ Hehan wanjikji inajinpi, qa itonśniyan taku iyaonpapi, qa heyapi ;

58 Unkiye, Tipi wakan nape on kagapi kin de ihangwaye kta, qa anpetu yamni hehan wanji tokeca nape codan kagapi kin he wakage kta ce, eya naunlionpi ce.

59 Tuka iś eya taku oyakapi kin ocitkonze śni.

60 Hehan wośna kagapi itancan kin wicacokaya inajin, qa Jesus iwange ca; Dena taku iyanionpapi kin takudan adupte śni he, eya.

61 Tuka iye inina un qa takudan ayupte śni. Ake wośna kagapi itancan kin wiwange ca heciya; Messiya, Iyotan Waśte Cinhintku kin he niye he.

62 Unkan Jesus heya; He miye; qa. Wicaśta Cinhintku lkin wowaśake etapa eciyatanhan iy otanke ca, mahipiya śapa akan u wandakapi kta ce.

63 Hehan wośna kagapi itancan kin wokoyake hdupote ça heya ; Taku on woyakapi sanpa uncinpi kta he.

64. Wayaśice cin he niye nayahonpi : token idukcanpi he. Unkan owasin, țe kta iyececa, yacopi.

65 Hehan wanjikji atagośapi qa ite ogekicitonpi, qa apapi qa; Wokcan wo, eciyapi. Unkan ookiye unpi kin iś eya nape on apapi.

66 Unkan Inyan ihukuya tipi kin ohna yanka; hehan wośna lragapi itancan winyan wowidagwicaye cin wanji en hi ;

$67 \mathrm{Qa}$ Inyan petiśkan yanka wanyake cehan en etonwe ca heya; Qa niś eya Jesus Nazareth kici yaun qon.

68 Tuka anakihbe ça heya; Taku ehe cin he sdonwaye śni, qa nakun owakahinige śni ce. Hehan tankan ohanzi hdepi kin ekta iyaya: unkan anpaohotonna wan hoton.

69 Unkan ake wikośka wan wanyake ca, tona en najinpi kin hewicakiya; De iś nakun hetanhan.

70 Unkan iś ake anakiliman. Hehan kitan ecinyan iyohakam tona en najinpi kin ake Inyan heciyapi; Awicakehan niś henitanhan; Galile wicaśta henica; qa iyae cin he iyececa ce.

71 Tuka iye wayaśice ça taku wakan cajeyata; Wicaśta kin de cajedatapi kin ecaca sdonwaye śni ce, eya.

72 Hehan inonpa anpaohotonna kin hoton. Unkan hehan Inyan Jesus taku eciye ciqon; Anpaohotonna nonpa hoton śni, yamni akihde anamayalihibe $\mathrm{kta}$ ce, wicoie kin he kiksuya. Unkan he awakicin hehan ceya.

\section{WIC.OWOYAKE 15.}

1 Unkan wanna anpao, hehan wośnapi kagapi qa wicahincapi, wowapi kagapi ko omniciye ocowasin om he aiapi, qa Jesus kaśkapi, qa ekta ayapi qa Pilate qupi.

2 Unkan Pilate wiwanga; Juda oyate Wicaśtayatapi kin he niye he. Unkan Jesus ayupte ca, Niye ecen eha ce, eya.

3 Unkan wośnapi kagapi kin taku ota iyaonpapi ; tuka takudan ayupte śni. 
4 Unkan Pilate ake wiwange ca; Takudan adupte śni he; iho, taku ota iyanionpapi ce, eya.

5 Tuka Jesus takudan ayupte śni ; hecen Pilate yuśinyaya.

6 Wośnapi kin de ekta wicakaśkapi wanji wicakiciyukca ece, wanji tukte cinpi kinhan.

7 Unkan wanji Barabbas eciyapi, he tona om inajin qon kaśkapi yanka, he inajinpi qehan nakun tinwicakte.

8 Unkan oyate kin, token econ ece qon he iyecen ecawicakicon kta, panyehan keciyapi.

9 Tuka Pilate awicayupte ca; Juda. Wicaśtayatapi kin he ciciyukcapi kta yacinpi he.

10 Wośnapi kagapi kin winawizipi on kahipi e sdonya.

11 Truka wośnapi kagapi kin oyate kin wahokonwicakiyapi, Barabbas he wicakiyukca kta e heon.

12 Unkan Pilate ake awicayupte ça; Hehan tuwe Juda Wicaśtayatapi eyakiyapi kin he token ecamon kta yacinpi he, ewicakiya.

13 Unkan ake panyehan heyapi ; Icipaweli okatan wo.

14 Hehan Pilate hewicakiya; Tokeca, taku śica econ kin tukte e he. Unkan hehan iyotan nina; Icipaweli okatan wo, panyehan eyapi.

15 Unkan Pilate oyate kin iyokipiwicaye kta cin, heon Barabbas wicaliciyukca, qa Jesus kapsinpsinta hehan icipaweli okatanpi kta wicaqu.

16 Unkan akicita kin tipi wan tanka, Pretorium eciyapi, he ekta ayapi ; qa wicobe ocowasin wicakicopi.

17 Unkan śina duta wan inkiyapi, qa wapaha wan wapepeka on kazontapi qa unkiyapi.
18 Qa hehan okiyapi, Iho, Juda Wicaśtayatapi kin.

19 Qa cedi tanka wan on pa kin en apapi, qa atagośapi, qa canpéśka makehde inajinpi, qa ohodapi.

20 Unkan hecen ihahapi, hehan śina duta kin ikicicupi, qa iye tawokoyake qon ake kicunkiyapi. Hehan icipaweli okatanpi kta e ekta ayapi.

21 Unkan Simon, Kurene wicaśta, en hiyaya, he Alexander qa Ruphus atkuku kin, makoce kin etanhan u kin he can susbeca tawa kin kiciçinkiyapi.

22 Unkan Golgotha owanka kin en ahipi, he, Wicapahu makoce, eyapi kin he kapi.

23 Unkan miniśa pejihuta icahiyapi kin he yatke kta e qupi ; tuka wicada śni.

24 Unkan wanna icipaweli okatanpi, hehan tawokoyake qon kicipamnipi, qa on oeconna econpi, wicaśta otoiyohi taku icupi kta. 25 Unkan wanna wihiyayedan oape yamni, hehan icipaweh okatanpi.

26 Unkan taku iyaonpapi wowapi iwankam owapi kin, JUDA WICAŚSTAYATAPI KIN, hee.

27 Unkan kici wamanon sa nom icipaweh owicakatanpi, wanji etapa eciyatanhan, qa unma catka eciyatanhan.

28 Hecen wowapi kagapi qon yuecetupi ; Qa wahtanipi sa om yawapi ce, eya.

29 Unkan tona en hiyayapi kin hena yaśicapi, poptanptanpi qa heyapi; Iho, tipi wakan kin ihangyaye kta, qa anpetu yamni ake piya yakage kta:

30 Ito, niiçiya wo, qa cansusbeca kin etanhan kun ku wo.

31 Nakun wośnapi kagapi kin ihahapi, qa wowapi kagapi om iye iyakitedan hekiciyapi; Wi- 
caśta tokeca niwicaya ece, tuka iye atayedan niiçiye $\mathrm{kta}$ okihi śni.

32 Messiya Israel Wicaśtayatapi kin, ito cansusbeca kin etanhan kun hdicu kta, hecen wanunyakapi qa wicaundapi kta. Unkan tona kici icipaweh owicakatanpi qon iś eya ihahapi.

33 Unkan wihiyayedan oape iśakpe, hehan maka kin owancaya otpaza, oape inapcinwanka hehanyan.

34 Qa wihiyayedan oape inapcinwanka, hehan Jesus hotankakiya panyehan; Eloi, Eloi, lama sabakthani, eya: Wakantanka mitawa, Wakantanka mitawa, tokeca ehpemayaya he, eyapi kin he kapi.

35 Unkan en najinpi wanjikji he nahonpi qehan heyapi; Iho, Elias kipan.

36 Unkan wanji inyang ye ca, mini iyuhepe wan miniskuya en oputkan, qa can icam yatkekiye kta, qa heya; Hinnakahan po, Elias kun eyaku kta on hi kinhan wanunyakapi kta.

37 Unkan Jesus hotankakiya hotanin, qa taniya hiyuya.

38 Unkan tipi wakan cokaya ozanpi qon he owangya okinalidece ca, wankan tanhan qa kun ihanke kin aiyahdeya.

39 Unkan akicita opawinge wicayuhe cin iyotakons najin, he hecen pan qa taniya hiyuya wanyaka, hehan heya; Awicakehan wicaśta kin de Wakantanka Cinhintku kin.hee.

40 Nakun winohinca itehan tanhan wanyag yukanpi; Mary Magdalene, qa Mary, Jakob cistinna qa Joses hunku kin, qa Salome, hena opapi.

41 Galile en un qon he ehan ihakam unpi qa okiyapi ece kin hena eepi, qa nakun winohinca toktokeca

kici Jerusalem

en hipi qon.

42 Unkan wanna hitayetu, Ihduwiyeyapi kta anpetu, anpetu wakan kin itokam anpetu kin hee, heon,

43 Joseph, Arimathea etanhan, woope aiekiyapi owotanna, qa nakun Wakantanka tokiconze kin ohinniyan ape un kin he $\mathrm{u}$, qa waditagya Pilate en ye ça, Jesus tancan kin kida.

44. Unkan wanna ta hecinhan Pilate yuśinyaya; heon akicita opawinge wicayuhe cin he kico, qa wanna wanakaja tanhan ța hecinhan he iwanga.

45 Unkan akicita opawinge wicayuhe cin he ecen okiyaka, hehan tancan kin Joseph qu.

46 Unkan he minitiuha ska waśte opeton, qa tancan Fin kun eyaku, qa minihuha ska kin iyapemni, qa wicaliapi ohdoka wan imnija en kagapi, he en ohnaka, ga inyan tanka wan wicahapi ohdoka i kin en eonpa.

47 Unkan Mary Magdalene qa Mary Joses hunku kin hena tukten hnakapi kin he wanyakapi.

\section{WICOWOYAKE 16.}

1 Unkan anpetu wakan kin wanna owihanke, hehan Mary Magdalene, qa Mary Jakob hunku, qa Salome hena tancan kin sdayapi kta on pejihuta waśtemna aupi : 2 Unkan anpetu tokaheya hanhanna hinca, wicahapi ohdoka kin en hipi, wanna wi hinanpa hehantu.

3 Unkan hekiciyapi ; Tuwe wicahapi ohdoka i kin etanhan inyan kin yutokan iyeunkiciciyapi kta he.

4 Unkan ekta etonwanpi, hehan inyun, inyan kin wanna yutokan iyeyapi; he tanka hinca. 
5 Unkan wicahapi ohdoka kin mahen ipi, hehan kośka wan etapa eciyatanhan iyotanka, wokoyake ska hanska koyaka, he wanyakapi ; unkan yuśinyayapi.

6 Tuka iye hewicakiya; Ihnuhan niyuśinyayapi kinhan; Jesus Nazareth etanhan, icipaweh okatanpi qon he oyadepi; wanna ihduekicetu; den wanke śni; tukten hnakapi qoin he wanyaka po.

7 Tuka niśs hda po, qa waonspewicakiye cin, qa Inyan nakun, nitokam Galile ekta ye kta, heciya wanyahdakapi kta, eniciyapi qon iyececa, he owicakiyaka po.

8 Unkan hecen koyahanna hdicupi, qa wicahapi ohdoka kin etanhan najica kihdapi; nina cancanpi qa yuśinyayapi; qa tuwedan takudan eciyapi śni, inihanpi nakaeś.

9 Unkan anpetu wakan anpetu tokaheya hanhinna hinca, Jesus ihduekicetu hehan, Mary Magdalene, etanhan taku wakan śica śakowin ehpekiciye ciqon, he tolaheya en taniniçiya.

10 Hehan iye hde ça, tona om un qon iyokiśicapi qa ceya yukanpi kin owicakiyaka.

11 Unkan hena, wanna kini, qa he wanyaka keya ohdaka nahonpi eśta, wicadapi śni.

12 Hehan iyohakam etanhan wicaśta nom makoce ekta ici- mani yapi, hehan icunhan en taniniciya.

13 Hena unmapi kin hośi wicakakipi, tuka nakun wicadapi śni. 14 He iyohakam akewanjipi wota iyotankapi icunhan en taniniciya, qa wacetunhdapi qa cante sutapi on iyopewicaya ; kini iyohakam tona wanyakapi kin heepi kaeś wicawicadapi śni heon.

15 Unkan hewicakiya; Maka kin owancaya ya po, qa wicaśta otoiyohi wotanin waśte kin owicakiyaka po.

16 Tuwe wicada qa baptem qupi kinhan he ni kta; qa tuwe wicada śni kinhan he yacopi kta.

$17 \mathrm{Qa}$ tona wicadapi kin hena wowapetokeca dena yuhapi kta ; Micaje kin on taku wakan śica tankan ehpeyapi kta; wicaceji tokeca on iapi kta ;

18 Sintelida yuwankan icupi kta; qa pejihuta sica yatkanpi eśta etanhan itokecapi kte śni ; wayazankapi kin nape awicahnakapi kta, kinhan asnipi kta ece.

19 Hecen Itancan kin owicaliya, hehan malipiya ekta eyakupi, qa Wakantanka etapa eciyatanhan kiyotanka.

20 Unkan hena iyayapi, qa owancaya woyakapi; qa Itancan kin wicohan tanka econwicakiye cin on wicoie kin yuecetu ece. Amen. 


\section{U K A S}

\section{WOTANIN WAŚTE TAWA.}

\section{WICOWOYAKE 1.}

1 Taku tona cetunhdaśniyan wicaundapi kin hena wicota hdaheya oyag utapi ;

2 Tona otokahe ehantanhan iśta on wanyakapi qa wicoie oyakapi ece kin hena token unkokiyakapi qon he iyececa :

3 Heon etanhan miś eya, taku kin hena owasin otokahe liinca ehantanhan tanyan owakahinge cin on, Taku Wakan waśtedaka iyotan waśte, hena hdaheya wowapi cicage kta iyomakipi.

4 Hecen taku onspeniciyapi qon hena wicakapi e tanyan sdonyaye kta.

5 Herod Juda wicaśtayatapi taanpetu kin en wawayuśna wan Zacariyas eciyapi, Abia optaye kin hetanhan; qa tawicu kin iś Aaron cunwintkupi kin etanhan; unkan he Elisabeth eciyapi.

6 Hena sakim Wakantanka itokam owotanpidan, qa Itancan tawoahope qa wicoope tawa kin owasin omanipi, iyaonpepica śni unpi.

7 Unkan Elisabeth hokśin yuhe śni un, qa hecen cinca nicapi ; unkan sakim wanna kanpi.

8 Unkan iye optaye kin ohna wośna wicolian kin Wakantanka itokam econ eca,

9 Tipi wakan Itancan tawa kin timahen iyaya eca, waazintonpi econ śipi ece, wośna wicohan econpi kin eciyatanhan.

10 Unkan waazintonpi icunhan, oyate kin owasin itankan cekiya hiyeya.

11 Unkan Itancan taohnihde wan en taniniçiya, ya wahna azintonpi kin etapa tanhan hinajin.

12 Unkan Zacariyas he wanyake cehan, wacin iyokiśice ca wakolkipe hinca.

13 Tuka ohnihde wakan kin heciya; Wakokipe śni wo, Zacariyas; wocekiye nitawa kin nahonpi; qa nitawin Elisabeth hokśidan wan niciyuhe kta, unkan he Johannes eya caśyakiton kta.

$14 \mathrm{Qa}$ he on wowiyuśkin wopida ko duhe kta, qa tonpi kin on wicota iyuśkinpi kta.

$15 \mathrm{Qa}$ iye Itancan kin itokam tanka kta ; qa miniśa miniwakan ko takudan yatke kte śni; qa hunku ikpi kin etanhan Woniya Wakan kin on ojudan kta.

16 Unkan iye Israel cinca kin wicota Itancan Wakantanka tawapi kin ekta wicayahomni kta. 17 Qa Elias toksape qa towaśake kin yuha itokam ye kta, qa atewicayapi cincawicayapi kin kici cante wicayuokonwanjidan, qa wanahonpi śni kin hena owotanna unpi wicoksape kin ekta wicayuhomni kta; qa oyate wan Itancan on ihduśtanpi kin wiyeya kicihnake kta e heon etanhan.

18 Unkan Zacariyas ohnihde wakan kin heciya; Wanna wimacahinca, qa mitawin wanna kan; hecen taku on de sdonwaye kta he. 
19 Unkan ohnihde wakan kin waayupte ca heciya; Gabriel, Wakantanka itokam najin kin he miye; qa ociciye ça, wotanin waśte kin de sdonyeciciye kta e u maśipi.

20 Unkan iho, mioie kin tohan iyehantu kinhan yuecetupi kte cin he wicayada śni kin he on, tohanyan yuecetupi śni kin hehanyan, ie śni, qa ia okitpani yaun kta ce.

21 Unkan oyate kin Zacariyas ape hiyeye, ca tehan tipi wakan kin ohna yanke cin on etanhan inihanpi.

22 Unkan tankan hdicu qehan, owicakiye kte cin okitpani : unkan namwicakicawin, qa ie śni un; on etanhan tipi wakan kin ohna taku wakan wanji wanyaka e iyukcanpi.

23 Unkan wicohan econ ece kin anpetu kin wanna ihunnikiya, hehan iye ti kin ekta kihda.

24 Unkan anpetu kin hena iyohakam tawicu Elisabeth hokśin itpihnaka; unkan wi zaptan hehanyan inahbe, ça heya;

25 Anpetu kin en, Itancan kin wicaśta kin taku i en mahdepi qon he emiciyaku kta on ahimatonwe cechan, decen ecamicon ce.

26 Unkan wi iśakpe kin en, Gabriel ohnihde wakan kin Wakantanka etanhan u śipi ; Galile ekta, otonwe wan Nazareth eciyapi kin hetu;

27 Wikośka wan wicaśta wan Joseph eciyapi, Dawid wicowazi tawa kin etanhan, yuze kte cin he ekta; unkan wikośka kin Mary eciyapi.

28 Unkan ohnihde wakan kin he en hi qa heciya; Wiyuśkin wo, waśteya yaun kin; Itancan kin niciun, winolinca owicapeya niye niyawaśtepi ce.
29 Unkan he wanyake cehan, oie kin on inihan, qa okiyapi kin de taku kta hecinhan he awacin un.

30 Unkan ohnihde wakan kin heciya; Mary, wikope śni wo; Wakantanka ekta wowaśte iyeyaya.

31 Qa iho, hokśin itpiyahnake ca hokśidan wan yaton lkta, qa J̇esus eya caśyaliton kta.

32 Iye tanka kta, qa Iyotan Wankantu kin Cinhintku kin eciyapi kta; qa atkuku Dawid tohe kin Wakantanka Itancan kin hduhekiye kta.

33 Qa Jakob ti kin he ohinniyan idake kta; qa wokiconze tawa kin okiciwihanke kte śni ce, eya.

34 Hehan Mary ohnihde wakan kin heciya; Wicaśta sdonye śni waun kin heon, de token on iyecetu kta he.

35 Unkan ohnihde wakan kin waayupte ca heciya; Woniya Wakan kin en niu kta, qa Iyotan Wankantu towaśake lkin aohanziniye kta, qa hecen taku wakan wan yaton kte cin he Wakantanka Cinhintku kin eeiyapi kta.

36 Unkan inyun, Elisabeth icepanśiyaye cin, he wakanka tuka iś eya wanna hokśin itpihnaka ce, qa hokśin yuhe sni eciyapi qon, he wanna wi kin de iśalipe.

37 Wakantanka ekta taku okihipica śni wanica ce.

38 Unkan Mary heya; Itancan ookiyemaye cin wanmayaka we; nioie kin iyecen ecamiconpi nunwe. Hehan ohnihde kin elpeya kihda.

39 Unkan anpetu kin hena en Mary inajin, qa koyahanna hośki makoce kin, Juda otonwe wan ekta iyaya : 


\section{LUKAS.}

40 Qa Zacariyas ti kin en tin iyaye, ca Elisabeth okiya.

41 Unkan Elisabeth Mary ia nahon qehan, itpi mahen hokśiyoqopa kin śkehanhan; hehan Elisabeth Woniya Wakan kin ojudan :

42 Qa hotankakiya hotanin, qa heya; Winohinca opeya niye niyawaśtepi, qa niitpi kin etanhan icage kte cin he yawaśtepi ce.

43 Qa Itancan mitawa hunku kin ekta mahi kte cin de token kapi he.

44 Inyun, iyae cin noge iyohpemaye cehan, hecehnana miitpi mahen hokśiyoqopa kin wowiyuśkin on śkehanhan.

45 Unkan tuwe wicada qon he yawaśtepi: Itancan kin taku eciye ciqon hena ecen yuśtanpi kta ce, eya.

46 Unkan Mary heya; Minagi kin Itancan kin yatan;

47 Qa mitaniya kin he Wakantanka Wanikiya mitawa kin on iyuśkin ece.

48 Taokiye kuya un kin ekta ahimatonwan on etanhan inyun, detanhan wicoicage kin owasin yawaśtepi emakiyapi kta ce.

49 Tuwe waśalie cin he taku hinca ecamicon; unkan caje kin wakan ce.

50 Tona kokipapi kin hena wicoicage qa wicoicage kin onśiwicakida ece.

51 Iye isto kin.on wowaśake hdutanin; witantanpi kin hena taku cante mahen wacinyuzapi kin en wicayuomdeca.

52 Waśakśakapi kin oyanke etanhan kun hdicuwicaye ca, kuya unpi qon hena wankan wapa awicaya.

53 Wotektehdapi kin hena taku waśteśte on imnahanwicaye, ça wijicapi kin iś cokakađan tokan yewicaśi.

54 Iye towaonśida kiksuye cin on taokiyeye cin Israel he iciya ece.

55 Hunkakewicunyanpi owicakiye ciqon iyececa, Abraham iye cinca kin om, owihanke wanica.

56 Unkan wi yamni ecetu hehanyan Mary Elisabeth kici un, qa hehan iye ti ekta kihda.

57 Elisabeth cinca ton lite cin wanna iyehantu; unkan hokśidan wan ton.

58 Unkan ikiyedan unpi kin hena, içepanwicaye cin koya, Itancan kin nina onśikida nahompi; hecen kici iyuśkinpi.

59 Unkan anpetu iśahdogan qehari, hokśiyoqopa kin bahdayapi kta on hipi; unkan atkuku Zacariyas caje kin he icajekiyapi.

60 Ünkan hunku kin waayupte ca heya, Hiya ; tuka Johannes eciyapi kta.

61 Unkan heciyapi; Takuwicayaye cin tuwedan hecen eciyapi śni ce.

62 Unkan atkuku kin iś token eya cajeyate kta hecinhan, he iyuteya iwangapi.

63 Unkan iś wowapi akan kagapi wan da, qa; Johannes he caje kin ee ce, eya owa. Unkan owasin inihanpi.

64 Unkan hecehnana i kin yukawapi, qa-ceji kin kiyuśkapi; unkan ie, ca Wakantanka yatan.

65 Unkan okśan tipi kin owasin wikopapi: unkan iapi kin dena owasin 'Juda hosski kin owancaya yaotaninpi.

66 Unkan toni nahonpi kin owasin cante mahen awacin unpi, qa heyapi; De taliu hokśiyoqopa knta he. Unkan Wakantanka nape kin he kici un. 


\section{WICOWOYAKE 2.}

67 Hehan atkuku Zacariyas Woniya Wakan ojudan, qa wokcan qa heya;

68 Itancan Wakantanka Israel tawa kin he taoyate kin wanna wicahiyohi, qa opewicakiton, on etanhan yawaśtepi nunwe:

69 Taokiye Dawid ti kin en wanikiyapi he wan yuwankan eunkicihdepi ce :

70 Maka kin tokaheya icage cin ehantanhan, wicaśta wolcan wakan tawa ipi kin eciyatanhan, oie kiton kin hena iyecetu :

71 Hecen tokaunyanpi qa unkiyuśepi kin hena napepi kin etanhan niunkiyapi kta;

72 Wowaonśida hunkakewicunyanpi cajewicakiyate ciqon, he ecen econ kta, qa wokiyapi wakall tawa kin he kiksuye kte ;

73 Abraham ateunyanpi kin he taku wakanyan eciye ciqon he hee kta ce ;

74 Decen ionśiunkidapi kta, tona tokaunyanpi nape kin etanhan unkiyuśpapi kinhan, wolkokipe codan ohoundapi kta,

75 Iye itokam, wicoowotanna qa wicoecetu kin en, tohanyan unnipi kin hehanyan.

76 Qa niye, hokśiyoqopa, Wicaśta wokcan Iyotan Wankantu tawa kin eniciyapi kta; Itancan tacanku kin wiyeya yecage kta on ite kin itokam de kta;

77 Taoyate woahtani wicakicicajujupi kin eciyatanhan wanikiyapi sdonyapi kte cin he wicayaqu kta ;

78 Wakantanka unkitawapi canteunkiyapi kin heon etanhan; qa heon wankan tanhan anpao kin hiyounhipi ce ;

79 Hecen otpaza qa wiconte ohanzi kin ehna iyotankapi kin hena iyoyamwicaye kta; qa unsihapi kin wookiye canku kin ohna unkayapi kta ce, eya.
80 Unkan hokśiyopa kin icage, ca tawacin kin waśaka aya ; unkan Israel en taniniçiye kte cin hehanyan, makoskan en ece un.

\section{WICOWOYAKE 2.}

1 Unkan anpetu kin hena en, Kesar Agustus, maka kin owancaya wicaśta owicawapi kta, wakiconza.

2 Kurenios Suria en wicaśtayatapi yanke cin he ehan tokaheya owica wapi kin de yuśtanpi.

3 Unkan owasin toketken tonwanyanpi qon owicawapi kta e elktakta kipi.

4 Unkan Joseph, Dawid ti qa wicowazi tawa kin hetanhan, heon iś eya, Galile en Nazareth otonwe kin etanhan ye ca, Bethlehem Dawid totonwe kin ekta ki ;

5 Mary tawicuye kte cin he kici owapi kta; he wanna cinca ton kta ikiyedan.

6 Unlaan hen yukanpi kin icunhan, anpetu en cinca ton kte cin wanna iychantu.

7 Hecen cinhintku tokapa kin ton, qa śina on hduskite ça, wanunyanpi en wotapi wan en ohnaka; timahen owicakan śni heon etanhan.

8 Unkan makoce kin he en tahincaska awanwicayakapi kin heca hanyen optaye tawapi kin awanwicahdakapi.

9 Unkan iho, Itancan taohnihde wan en wicahi, qa Itancan tawookitanin kin ohomni iyojanjanwicaya; unkan nina wikopapi.

10 Unkan ohnihde wakan kin hewicakiya; Wikopapi śni po; iho, wopida tanka wootanin waśte oyate kin owancaya ecen ye kte cin, he eca hośi cicahipi ce.

11 Anpe dehan Dawid totonwe 
kin en Wanikiya wan nicitonpi, Messiya Itancan kin he hee.

12 Qa on sdonyayapi kte cin he dee; Hokśiyoqopa kin śina on yuskiskitapi qa wanunyanpi en wotapi wan ohna wanka iyeyayapi kta ce.

13 Unkan ihnuhanna mahipiya ekta unpi kin wicota ohnihde wakan kin kici hiyeya, Wakantanka yatanpi qa heyapi ;

14 Iyotan wankantu ekta Wakantanka wowitan yuha nunwe, maka akan wookiye qa wicaśta ekta wicokiciyuwaśte.

15 Unkan mahpiya ohnihde kin hena elipeya wicakiyahdapi qehan, wicaśta tahincaska awanwicayakapi kin hekiciyapi ; Wanna Bethlehem ekta unyanpi, qa taku hiyohi Itancan unkokiyakapi kin de wanunyakapi kta ce.

16 Hecen koyahanna upi, qa Mary Joseph kici, qa hokśíyoqopa kin wanunyanpi en wotapi wan ohna wanka iyeyapi.

17 Unkan wanyakapi qehan, hokśiyoqopa kin on iapi nahonpi qon, he owancaya yaotaninpi.

18 Unkan tahincaska awanwicayakapi taku yaotaninpi kin he tona nahionpi kin owasin yuśinyayapi.

19 Tuka Mary wicoie kin dena owasin cante mahen okihnake ca awakicin un.

20 Unkan tahincaska awanwicayakapi kin hena kihdapi, qa taku nahonpi qa wanyakapi kin on Wakantanka yaonihanpi qa yatanpi ; taku owicakiyakapi qon he iyececa.

21 Unkan wanna anpetu iśahdogan, hokśsyoqopa bahdayapi kta hehan JEsus eciyapi ; nahalin itpihnakapi śni he ehan malipiya ohnihde kin hecen caśton.
22 Unkan Moses woope kage cin iyecen, anpetu en ihdukaśote kte śni hena wanna henakeca, hehan Itancan kin qupi kta e Jerusalem ekta hdoupi.

23 Itancan woope tawa kin en owapi qon iyececa ; Tona tokapapi, wica eca, otoiyohi Itancan kin ekta wakan eciyapi kta ce.

24 Qa nakun wawayuśnapi kta, token Itancan toope kin en eyapi qon he iyececa; Wakiyedan tawanjidan, qa iś wakiyedan cinca nonpa ce.

25 Unkan iho, Jerusalem en wicaśta wan Simon eciyapi; he wicaśta owotanna qa cekiya ece kin heca, ya Israel Wokicanpte kin ape un; unkan Woniya Wakan kin kici un.

26 Unkan he țe śni itokam Itancan Messiya tawa kin he wanyake kta, Woniya Wakan kin okiyaka.

27 He Woniya kin on tipi wakan kin en tin hiyu ; unkan hunkakewicaye cin hokśiyoqopa Jesus, wicoope kin eciyatanhan ecakiconpi kta e en hdoupi ;

28 Hehan adoksohan yuze, ca Wakantanka yawaśte, qa heya; 29 Mayuha, nitaokiye kin wanna wookiye yuha iyayeyaye kta; 30 Wowanikiye nitawa kin he miiśta kin on wanmdaka ce ;

31 Oyate kin owasin wicitokam wiyeya yehnake cin he;

32 Ojanjan wan oyate kin a0janjanwicaye kte cin, qa nitayate Israel wookitanin kin, he hee ce, eya.

33 Unkan taku esiyapi kin hena on hunku qa Joseph yuśinyayapi.

34 Unkan Simon wicayawaśte, qa hunku Mary heciya; Inyun, Israel en wicota hicahanpi, qa ake inajinpi kte cin on hokśiyopa kin de ehdepi, qa nakun 
wowapetokeca ienhdepi kte cin ee kta;

35 Qa nakun mazasagye wan on nitaniya kin canipapi kte; qa hecen wicacanteoze ota yuotaninpi kta ce, eya.

36 Unkan waayate wanji Anna eciyapi, Phanuel cunwintku, qa Aser wicowazi kin etanhan; he wanna wakanka hinca, qa hihnaton kin etanhan waniyetu śakowin hehanyan hihnaku kici un :

$37 \mathrm{Qa}$ he wanna wiwazica, waniyetu wikcemna śahdogan sanpa topa ecetu, qa tipi wakan kin en ece un, qa wote śni unpi wocekiye eya ahna on ohoda ece.

38 Unkan he hehantudan hin en $\mathrm{u}$, qa iś eya Itancan kin yatan, qa Jerusalem en tona opewicakitonpi kin ape unpi kin hena owasin Jesus cajewicakiyata.

39 Unkan Itancan wicoope tawa kin owasin ecekcen yuśtanpi qehan, Galile en iye otonwe tawapi Nazareth he ekta kihdapi.

40 Unkan hokśiyopa kin icage, ça woniya kin en waśaka aye, ça wicoksape kin on ojudan; unkan Wakantanka towaśte kin he kici un.

41 Woacakśin wotapi eca, hunkakewicaye cin waniyetu towanakeca Jerusalem ekta ipi ece.

42 Unkan Jesus wanna waniyetu akenonpa, hehan wotapi wicohan kin iyececa, Jerusalem ekta ipi.

43 Uukan anpetu kin yuśtanpi qehan, hdicupi, tuka hokśiyopa Jesus hekta Jerusalem en yanka; tuka hunku Joseph kici he sdonyapi śni.

44 Tuka ptaya hdapi kin en opa kecinpi, hecen anpetu wan- jidan iyooptapi, qa takuwicayapi kici sdonkiciyapi ko wicehna okidepi.

45 Qa iyeyapi śni, hehan Jerusalem ekta ake icicawin akin ipi.

46 Unkan yamni can, hehan tipi wakan 'kin en waonspekiyapi kin wicacokam waanawicagoptan, qa wiwicawanli yanka iyeliyapi.

47 Unkan wacin ksape cin, qa waayupte cin tona nahonpi qon owasin inihanpi.

48 Unkan wanhdakapi qehan yuśinyayapi; qa hunku kin heciya ; Cinś, tokeca decen unkoyakihandapi he ; iho we, niyate kici wacin iyokiśinya ocide ce.

49 Unkan hewicakiya; Tokeca omayadepi he: Atewaye cin wicolian tawa kin he ito owecipe kta kecannipi śni he.

50 Unkan taku ewicakiye cin he okalinigapi śni.

51 Hecen om hde, ça Nazareth ekta ki, qa anawicakigoptan un. Unkarl hunku kin wicoie kin dena owasin cante mahen yuha un.

52 Unkan Jesus sanpa ksape ça hanska icaga; qa Wakantanka wicaśta ko ekta waśtedakapi.

\section{WICOWOYAKE 3.}

1 Unkan Tiberius Kesar wicaśtayatapi un waniyetu iakezaptan, Pontius Pilate Juda en itancan, Herod Galile en itancan, qa iye sunkaku Philip Itureya qa Trakonitos en itancan, qa Lusanius Abilene en itancan ;

2 Qa Annas Kaiaphas kici wośna kaga itancanpi qon he ehan, hewotahedan ekta, Johannes Zacariyas cinhintku kin Wakantanka oie kin hiyohi. 
3 Unkan he Jordan kahda makoce kin owancaya en $u$, qa woahtani kajujupi kta on iyopeiçiyapi baptem wicaqupi kin he oyaka:

4. Wicaśta wokcan Isaya oie wowapi kin en owapi qon he iyececa; Wicaho wan tuwe makotahedan ekta heya niyan; Itancan tacanku kin wiyeya kicaga po, ocanku tawa kin liciyuowotan po:

5 Kaksiza owasin ojuyapi, qa heyaka paha ko owasin kahukun iyeyapi kta, qa pakśikśan kin hena yuowotanpi, qa ocanku tahtage cin hena yumdayapi kta ce ;

6 Qa wicacehpi kin owasin Wakantanka wowanikiye kin wanyakapi kta ce.

7 Unkan wicota baptem wicaqu kte cin' en ahi kin hena hewicakiya; Wamduśka wicoicage kin, tuwe wocanniye u kte cin etanhan nape niśipi he.

8 Hecen iyopeiciyapi iyececa waskuyeca icaliya po, qa, Abraham ateunyanpi ce, cante mahen ecinpi śni po; miye heciciyapi; Wakantanka inyan kin dena etanlıan Abraham cinca kicage kta okihi.

9 Unkan iś eya onspe kin wanna can hute kin en ehnakapi; heon etanhan can kin tona waskuyeca waśte aicage śni kin hena owasin kawankapi qa petan ehpeyapi ece.

10 Unkan oyate kin, Hehan unkiye token econkupi kta he, eya iwangapi.

11 Waayupte ca hewicakiya; Tuwe onhdohda nonpa yuhe cinhan, he tuwe nice cin unma qu kta; qa tuwe woyute ton kinhan, iś eya ecen econ kta ce.

12 Hehan wamnayanpi kin iś nakun baptem wicaqu kta on en hipi qa heciyapi; Waonspekiye, unkiye token econkupi kta he.

13 Unkan hewicakiya; Wakiconzapi kin isanpa kicapi śni po, eya.

14 Unkan akicita kin iś eya wiwangapi qa heciyapi; Qa unkiś token econkupi kta he. Unkan hewicakiya; Wicaśta Kin tuwedan wakokipe kicagapi śni, qa tuwedan oitonśniyan taku iyaonpapi śni po ; qa wokajuju niẹpi kin he on cante waśte po.

15 Unkan oyate kin owasin wakta hiyeya, qa wicaśta owa. sin Johannes on awacinpi, he Messiya kin hee, qa iś hee śni hecinhan;

16 Hehan Johannes waayupte ca owasin hewicalkiya; Awicakehan mini on baptem cicupi; tuka wanji mikapeya waśaka u, he iye tahanpe hankpan kin weciyuśke kta iyemacece śni; he qe Woniya Wakan peta ko on baptem nicupi kta.

17 He iye wicaduge nape ohna hduhe, ca owanka tawa kin tanyan hduska kta; qa aguyapi su kin he tpahi, qa canwohriaka kin en okihnake kta; tuka ha kin e peta kasnipica śni wan on hulinahye kta ce, eya.

18 Unkan wahokonwicakiye cin en taku toktokeca nakun ota oyate kin owicakiyaka.

19 Unkan Herod wicaśta itan. can kin, iye sunkaku tawicu Herodias hee on, qa taku śica Herod econ kin owasin on iyopeya; 20 Hecen taku owasin sanpa akicage ca, wicakaśka tipi kin en Johannes kaśka hnaka.

21 Unkan oyate kin owasin baptem wicaqupi, ca Jesus iś eya baptem qupi, qa cekiya un rehan, mahpiya kin yuzamripi;

$22 \mathrm{Qa}$ Woniya Wakan kin wakiyedan kaliya tantonyan fun $u$, 
qa en iyahan; unkan mahpiya eciyatanhan wicaho wan hiyu, qa heya; Micinkśi waśtewakidake cin he niye; niye on iyomakipi hinca.

23 Unkan Jesus iye wanna waniyetu wikcemna yamni kta, Joseph cinhintku kin hee kecinpi, he Heli etanhan,

24 He Matthat etanhan, he Lewi etanhan, he Melki etanhan, he Janna etanlian, he Joseph etanhan,

25 He Mattathias etanhan, he Amos etanhan, he Naum etanhan, he Esli etanhan, he Nagge etanhan,

$26 \mathrm{He}$ Maath etanhan, he Mattathias etanhan, he Seme etanhan, he Joseph etanhan, he Juda etanhan,

27 He Joanna etanhan, he Resa etanhan, he Zorobabel etanhan, he Salathiel etanhan, he Neri etanhan,

28 He Melki etanhan, he Addi etanhan, he Kosam etanhan, he Elmodam etanhan, he Er etanhan,

$29 \mathrm{He}$ Jose etanhan, he Eliezer etanhan, he Jorim etanhan, he Matthat etanhan, he Lewi etanhan,

30 He Simeon etanhan, he Juda etanhan, he Joseph etanhan, he Jonan etanhan, he Eliakim etanhan,

31 He Melea etanhan, he Menan etanhan, he Mattatha etanhan, he Nathan etanhan, he Dawid etanhan,

32 He Jesse etanhan, he Obed etanhan, he Booz etanhan, he Salmon etanhan, he Naasson etanhan,

33 He Aminadab etanhan, he Aram etanhan, he Esrom etanhan, he Phares etanhan, he Juda etanhan,
34 He Jakob etanhan, he Isaak etanhan, he Abraham etanhan, he Thara etanhan, he Nacor etanhan,

35 He Seruk etanhan, he Ragu etanhan, he Phalek etanhan, he Eber etanhan, he Sala etanhan, $36 \mathrm{He}$ Kenan etanhan, he Arphaksad etanhan, he Sem etanhan, he Nowa etanhan, he Lamek etanhan,

37 He Mathusala etanhan, he Enok etanhan, he Jared etanhan, he Maleleel etanhan, he Kanan etanhan,

$38 \mathrm{He}$ Enos etanhan, he Seth etanhan, he Adam etanhan, he Wakantanka etanhan.

\section{WICOWOYAKE 4.}

1 Unkan Jesus Woniya Wakan kin ojudan, Jordan eciyatanhan hdi; qa Woniya kin makotahedan ekta iyayeya ;

2 Anpetu wilkcemna topa hehanyan Wakanśica wawiyutanyan. Unkan anpetu kin hena en takudan yute śni ; tuka hena ihunniyan hehan wotektehda.

3 Hchan Wakanśica heciya; Wakantanka Cinhintku kin he niye hecinhan, inyan kin de aguyapi icage kta, keya wo.

4 Unkan Jesus waayupte, ca heciya ; Aguyapi ecedan on wicani kte śni ; tuka Wakantanka oie kin owasin on wicani kta ce; eya owapi ce.

5 Hehan Wakanśica paha wan tehanwankantu ekta aye ça, wancake maka wokiconze kin owasin kipazo.

6 Unkan Wakanśica heciya; Wicowaśake kin de wicookitanin ko owasin cicu kta; hena owasin yuhemakiyapi, qa tuwe wacin kinhan waqu kta.

7 Heon etanhan niś mitokam 93 
makata elipeniciye cinhan, owasin nitawa kta ce.

8 Unkan Jesus waayupte ca heciya ; Satan, mitapetepa tanhan ya wo: Itancan Wakantanka nitawa kin, he iye itokam makata ehpeniciye kta, qa iye iśnana ahoyape kta ce, eya owapi ce.

9 Hehan Jerusalem ekta ahi, qa tipi wakan inkpa kin akan ehde, qa heciya; Wakantanka Cinhintku kin he niye hecinhan, hetanhan kun ehpeiciya wo.

10 Kaken wowapi en kagapi qon; Ohnihdewicaye cin hena niye on econ wicaśi, niyuhapi kta;

11 Qa napepi kin ohna yuwankan niyuzapi kta; qa hecen tukten inyan kin en siha ibonito kte śni, eya owapi ce, eya.

12 Unkan Jesus waayupte ca heciya; Itancan Wakantanka nitawa kin he iyutanyaye kte śni ce, eya owapi qon.

13 Unkan Wakanśica wowiyutanye kin owasin yuśtan qehan, tona can ehpeya kihda.

14 Hehan Jesus Woniya towaśake kin yuha Galile en hdi ; unkan ihdukśan makoce wanke cin owancaya he oyakapi.

15 Unkan omniciye tipi tawapi kin ohnahna waonspekiya ece, hecen owasin yuonihanpi.

16 Unkarı Nazareth en icahyapi qon he ekta hdi; qa econ ece kin he iyecen, anpetu wakan icunhan, omniciye tipi kin en tin iyaye ca, wowapi yawa kta inajin.

17 Unkan Isaya wicaśta wokcan tawowapi kin qupi. Unkan wowapi kin yumdaye cehan, decen owapi kin iyeya;

18 Itancan Woniya tawa kin he akan maun; heon wahpanicapi kin wotanin waśte kin owi- cawakiyake kta e sdamakiya; tona cante psakawahanpi kin asniwicawaye kta on u -maśi ; wayaka unpi kin wicakiyuśkapi kta, qa iśtagongapi kin ake tonwanpi kte cin omdake kta ; tona kahdehdecapi kin hena wicawakiyuśke kta;

19 Qa Itancan omaka oiyokipi tawa kin omdake kta ce.

20 Unkan wowapi kin ecen eyaku, qa ookiye kin he kicu. qa iyotanka. Unkan omniciye tipi kin ohna hiyeye cin owasin opahtapi.

21 Unkan hehan hewicakiya; Anpetu kin de ninogepi kin en wowapi kin de yuecetupi ce.

22 Unkan owasin yaotaninpi, qa wowaonśida oie iye i kin etanhan inanpe cin on yuśinyayapi qa heyapi ; Joseph cinhintku kin he dee śni he.

23 Unkan hewicakiya; Awicakehan iapi kin de ecen emayakiyapi kta; Niye pejihuta wicaśta kin, asniiçiya wo: Kapernaum ekta token ecanon naunhonpi kin, makoce nitawa kin den rakun ecen econ wo.

24 Unkan heya; Awicakehan heciciyapi; Wicaśta wokcan tuwedan iye tamakoce kin en iyowinkiyapi śni ece.

25 Tuka awicakehan heciciyapi ; Elias taanpetu kin en, waniyetu yamni sanpa wi śake hehanyan malipiya kin natakapi; qa makoce kin owancaya wicaakilian hince ciqon he ehan, Israel en wiwazica ota.

26 Tuka hena wanjidan Elias ekta ye śipi śni; Sidon en Sarepta otonwe kin en, winohinca wiwazica wan heceedan ekta ye sipi.

27 Nakun Elisha wicaśta wokcan ni un qehan, lepros ececa wicota Israel en unpi, tuka hena 
wanjidan yuskapi śni, Naeman Suriya wicaśta kin heceedan ce. 28 Unkan omniciye tipi ohna hiyeye ciqon hena nalionpi qehan, owasin śihdapi hinca ;

29 Qa najin hiyayapi qa otonwe kin itankan iyayeyapi, qa paha akan otonwe hdepi kin he matete ekta ayapi, qa kun elpeyapi kta. 30 Tuka iye wicacokaya tanhan iyoopta iyaye ça kihda.

$31 \mathrm{Qa}$ Galile en Kapernaum otonwe kin he ekta hi, qa anpetu wakan eca waonspewicakiya ece.

32 Unkan waonspewicakiye cin on inihanpi ; oie kin he wowaśake kicica nakaeś.

33 Unkan omniciye tipi kin ohna wicaśta wan wakan śica śapa wonliya kin yuha; qa he hotankakiya hoyeya;

34 Qa heya; Hanta wo, Jesus Nazareth, niye qa unkiye taku unkicicapi he; ihangunyayapi kta yahi he; Wakantanka Taku Wakan Wanji tawa kin he niye e sdonciya ce.

35 Unkan Jesus kiśice ca heciya; Inina un, qa wicaśta kin etanhan tankan hinapa wo, eya. Unkan wakanśice cin wicaśta qon wicacokam ehpeye çehan, etanhan tankan hinapa; tuka ksuweye śni.

36 Unkan owasin inihanpi, qa iyakitedan hekiciyapi; De taku oie kin; wicookihi wicowaśake ko on woniya śape cin econ wicaśi eca, tankan hinanpapi ece.

37 Unkan ihdukśan makoce wanke cin owancaya he oyakapi.

38 Unkan omniciye tipi kin etanhan hdicu, qa Simon ti kin en tin iyaya. Unkan Simon kunku kin he tancan wicakata ececa linca; hecen on cekiyapi.
39 Unkan en inajin, qa tancan kata kin he kiśica; unkan asni hinhda: qa hecehnana najin hiyaye ça, owicakiya.

40 Unkan wi iyaya hehan, woyazan ocaje ota on wicayazan tona wicayuhapi kin hena en awicahipi ; unkan otoiyohi nape awicahnake ca asniwicaya.

41 Qa wakan śica iś eya ota wicota etanhan hinanpapi, qa heya niyanpi ; Wakantanka Cinhintku Messiya kin he niye ce. Unkan iye wicakiśice ca, Messiya hee sdonyapi kin lie, eyapi kta iyowicaki śni.

42 Unkan wanna anpao hinape cehan tokan iyaye, ca makotahedan ekta ya; unkan oyate kin odepi, qa yanke cin ekta ahi, qa ehpeya iwicayaye kte cin iyanaptapi.

43 Unkan hewicakiya; Wakantanka tokiconze kin otonwe toktokeca nakun ohna omdake kta; heon etanhan u maśipi ce. 44 Hecen Galile omniciye tipi kin liena ohnahna woyaka ece.

\section{WICOWOYAKE 5.}

1 Unkan oyate kin Wakantanka oie kin nahonpi kta e aohduteyapi qehan, Geneseret mde kin icahda inajin.

$2 \mathrm{Qa}$ mde kin en wata nonpa wanka wanyaka; tuka hokuwapi kin iś wanna tokan iyayapi, qa ho kin hdujajapi.

3 Unkarl wata unma Simon tawa kin he en ope cea, kitanna canan wapa iyayeye kta cin. Hehan en iyotanke ça, wata kin etanhan oyate kin waonspewicakiya.

4 Unkan wanna ayaśtan qehan Simon heciya; Mini śbe kin ekta iyaye ça ho kin minin iyohpeya wo. 
5 Unkan Simon waayupte, ca heciya; Waonspekiye cin, hanyetu osan htaunnipi, qa takudan unktepi śni; tuka nioie kin on ho kin iyohpewaye kta ce.

6 Unkan heconpi qehan, hogan utkanna yuzapi ; unkan ho lkin papsakapi.

7 Unkan tawaśitkupi wata unma en opapi qon hena namwicakicawinpi, en upi qa owicakiyapi kta. Unkan en lipi qa wata napin ojukiyapi, hecen iyujimnana hiyeya.

8 Unkan Simon Inyan he wanyake cehan, Jesus hupàhu kin en makata ehpeiciye ca heya; Itancan, elipeya imayaya wo, wicaśta walitani sa hemaca ce.

9 He iye qa tona om un qon hena hogan yuzapi kin on etanhan owasin inihanpi.

10 Unkan Jalrob qa Johannes Zebede cinhintku kin hena oza, Simon tawaśitkuwicaya, iś eya inihanpi. Unkan Jesus Simon heciya; Wikope śni wo, detanhan wicaśta kin wicaduze kta ce.

11 Unkan wata qon hutata ahdihunipi qehan, owasin ehpekiyapi, qa iyahna yapi.

12 Unkan otonwe wan en un qehan, iho, wicaśta wan lepros ececa hinca, he Jesus wanyake cehan, ite makipuskin elipeiciye ca, cekiye ca heya; Itancan, yacin kinhan mayaduska kta oyakihi.

13 Unkan nape yekiye, ca yutan, qa heya; Wacin ce: ska un wo, eya. Unkan wancake lepros ececa qon asni hinhda.

14 Unkan tuwedan okiyalre śni śi; tuka wawayuśna kin ekta ye ca wanyagiciye kta, qa wayuskapi kta on token Moses econ wicaśi qon he ecen econ kta, keya; hecen wowicakiyake kta.
15 Tuka heon iyotan tankaya wicohan kin he oyakapi ; hecen wicota en mniciyapi, nahonpi kta, qa wayazankapi wicayuhapi kin asniwicaye lite cin heon.

16 Hehan icunonpa makotahedan wan ekta ye ca wocekiye eya.

17 Unkan anpetu wan waonspewicakiya icunhan, Pharisee qa wicoope onspekiye cin hena, Galile otonwe kin owasin, qa Juda qa Jerusalem etanhan, en ahi kin hena en iyotankapi; unkan Itancan towaśake kin asniwicaye kta e en un.

18 Unkan iho, wicaśta wan tatake cin he owinja ohna wicaśta yuha upi: qa timahen ayapi qa itokam ehnakapi kta akitapi.

19 Tuka wicota kin on token en aipi kte śni wanyakapi qehan, tipi akan inajinpi, qa akahpe yuhidokapi eciya tanhan cokaya Jesus itokam kun hiyuyapi.

20 Unkan wacinyanpi kin he wanyake cehan, heciya; Wicaśta, wayahtani kin nicicajujupi ce.

21 Unkan wowapi kagapi qa Pharisee kin iś waakinicapi qa heyapi; De tuwe e taku sica decen eya he. T'uwe woahtani kajuju kta okihi he; Wakantanka ecedan okihi.

22 Tuka tawacinpi kin Jesus iwicayukcan, hehan waayupte ca hewicakiya; Taku e cante mahen ayakinicapi he :

23 Wayahtani kin nicicajujupi ce; qa iś, Najin qa mani wo, eyapi kte cin unma tukte waśakadan he.

24 Tuka Wicaśta Cinhintku kin he iś maka akan woahtani kajuju kta olihi sdonyayapi kta ; hehan tatake cin he heciya; Najin qa nitowinja ehdaku qa 
yati kin ekta lida wo, eciciya ce, eya.

25 Unkan wancake wicitokam najin hiyaye, ça akan wanke ciqon he ehdaku, qa iye ti kin ekta hde, ca Wakantanka yatan.

26 Unkan owasin inihanpi, qa Wakantanka yatanpi ; qa wikopapi hince, ca heyapi ; Wicolian nakaka toktokeca wanunyakapi ce, eyapi.

27 Unkan taku kin dena iyohakam hetanhan ye, ca wamnayanpi kin heca wanji Lewi eciyapi, wamnayanpi oyanke kin en iyotankehan yanka e wanyaka; qa, Miyahna u wo, eciya.

28 Unkan taku owasin ehpeye ça, najin hiyaye ça, ihakam iyaya.

29 Unkan Lewi iye ti kin en wohanpi tanka kicaga; unkan wamnayanpi kin iś wicota, qa toktokeca nakun en opeya wotapi kta iyotankapi.

30 Tuka wowapi kagapi wicayuhapi qa Pharisee kin hena waonspewicakiye cin wicakigepi, qa heyapi ; Tokeca e wamnayanpi qa walitanipi sa om wayatapi qa wadatkanpi ece he.

31 Unkan Jesus waayupte ca hewicakiya; Tona zanipi kin hena pejihutawicaśta cinpi śni, tuka wayazanpi kin.

32 Owotanpidan kin hena iyopeiçiyapi kta e wicakico wahi śni, tuka walitanipi sa.

33 Unkan heciyapi; Tokeca e Johannes waonspewicakiye cin hena ijehan wote śni unpi qa wocekiye eyapi he, qa Pharisee tawapi kin iś eya; tuka nitawa kin wotapi qa wayatkanpi ece.

34 Unkan hewicakiya; Hihnaton ti kin cinca kin hena tawicu ton kin he iś owicapeya un kin icunhan wote śni wicayakiyapi kta oyakihipi he.
35 Tuka anpetu kin u kta, en tawicu ton kin he ewicakiciyakupi kta; hehan anpetu kin hena en wote śni unpi kta.

36 Unkan wiyacinpi wan owicakiyaka: Wicaśta tuwedan śina teca onśpa śina tanike cin en akihdagye śni ece; heconpi kinhan teca qon iś yuhdeca ece, qa onśpa teca etanhan kin he tanike cin kicica śni ece.

37 Qa nakun wicaśta tuwedan miniśa teca ojuha tanike cirı en okaśtan śni ece; heconpi kinhan miniśa kin skepe kta, qa ojuha kin atakuni kte śni.

38 Tuka miniśa teca kin he ito ojuha teca en ohnakapi kta; qa sakim yutehanpi ece.

39 Iś eya wicaśta tuwedan miniśa tanika wanna yatke ça, hehan hinnakaha teca cin śni; Tanike cin eeś waśte ce, eya ece.

\section{WICOWOYAKE 6.}

1 Unkan anpetu wakan inonpa tokaheya, hehan wojupi kin- ehna iyaya; unkan waonspervicakiye cin iś wahuwapa yuksapi, napopabagapi, qa yutapi.

2 Hehan Pharisee kin wanjikji hewicakiyapi ; Tokeca e anpetu wakan kin en taku iyowinwicakiyapi śni kin he eca ecen ecanonpi he.

3 Unkan Jesus awicayupte ca heya; Dawid token econ qon hee kaeś tohinni dawapi śni he; iye qa tona om un qon wotektehdapi qehan.

4 Wakantanka tipi tawa kin en tin iyaye, ca aguyapi taninyan ehnakapi, qa wawayuśnapi kin iye iśnana hetanhan yutapi kte cin iyowinwicakiyapi qon, he icu, qa yute, ca om unpi qon hena iś eya hetanhan wicaqu.

5 Unkan hewicakiya; Wicaśta 
Cinhintku kin iś nakun anpetu wakan kin Itancan ce.

6 Unkan anpetu wakan tokeca wan omniciye tipi kin en tiı iyaye, ça waonspekiya: unkan wicaśta wan nape etapa kin śnija e en yanka.

7 Unkan wowapi kagapi qa Pharisee kin hena anpetu wakan icunhan asniye kta hecinhan he iwanyalkapi, taku iyaonpapi kta akitapi.

8 Tuka iye tawacinpi kin sdonwicaya, qa wicaśta nape śnije cin he heciya; Najin qa cokaya inajin wo. Unkan najin hiyaye ca en inajin.

9 Unkan Jesus hewicakiya; Miśs ito taku wanji iciwangapi kta; Anpetu wakan icunhan taku waśte, qa iś taku śica econpi kta he; wanikiyapi, qa iś wateyapi kta, unma tukte hecetu he, eya.

10 Unkan itanihdukśan owasin awicatonwe cehan, wicaśta qon heciya; Nape kin hdukatin wo. Unkan ecen econ; qa nape kin unma kin iyecen iyekicetu.

11 Unkan śihdapi hince, ça iye iyakitedan Jesus token ecakiconpi kta hecinhan he iwohdaliapi.

12 Unkan anpetu kin hena en Jesus paha wan ekta wocekiye eya $\mathrm{i}$; qa hanyetu osan Wakantanka cekiya un.

13 Unkan wanna anpa qehan, waonspewicakiye cin wicakico, qa etanhan akenonpa wicahdahnige ça, Wahośiyapi, ewicakiya.

14 Simon he Inyan nakun eya caśton, qa he sunkaku kin Andrew, Jakob qa Johannes, Philip qa Bartholomew ;

15 Mattheos qa Thomas, Jakob Alpheus cinhintku, qa Simon nakun Zelotes eciyapi ;
16 Qa Judas Jakob sunkaku, qa Judas Iskariot, nakun wiyopeye kte cin hee.

17 Hehan hena om kun hdicu. qa makomdaya en hdinajin; unkan waonspewicakiye optaye kin, qa Juda owancaya qa Jerusalem, qa mde kahdaya Turos qa Sidon, henakiya etanhan wicota hinca, hena ia nahonpi, qa tona wayazankapi kin asniwicayapi kta e en ahi :

18 Nakun tona woniya śapa on kakijapi kin hena; ükan asniwicayapi.

19 Unkan wicota kin iyulipa yutanpi kta akitapi; etanhan wowaśake hiyu qa owasin asniwicaya ece kin heon etanhan.

20 Unkan waonspewicakiye cin awicatonwe, ca heya; Niwahpanicapi kin niś niyawaśtepi ce ; Wakantanka tokiconze kin he niye nitawapi.

21 Delian wotekteyahdapi kin niś niyawaśtepi ce ; imnahanniyanpi kta. Dehan yaceyapi kin uis niyawaśtepi ce ; iyahapi kta. 22 Tohan wicaśta śicenidapi, qa yuiyog iyayeniyanpi, qa iennihdepi, qa Wicaśta Cinhintku kin on nicajepi kin taku śica iyecen tankan ehpeyapi kinhan, niyawaśtepi ece.

23 Anpetu kin he en wiyuślkinpi, qa wopida on śkehanhian po; iho, mahpiya ekta wokajuju tanka duhapi; atewicayapi kin iś eya iyecen wicaśta wokcanpi wicakuwapi qon.

24 Tulka winijicapi kin niś woteli niciyankapi ece; wicocante waśte kin he wanna yahduhapi.

25 Winipipi kin niś wotehi niciyankapi ece; wotekteyahdapi kta. Dehan iyahapi kin niś wotehi niciyankapi ece; waśinyahdapi qa yaceyapi kta. 
26 Tohan wicaśta owasin tanyan eniciyapi kinban, wotehi niciyankapi ece; atewicayapi kin hecen wicaśta wokcan itonśni wicakuwapi qọn.

27 Tuka nation dukanpi kin heciciyapi ce, Tokaniyanpi kin hena cantewicaliya po, qa śicenidapi kin hena tanyan ecawicakicon po;

28 Niyaśicapi kin wicayawaśte po, qa wawicanksiya nicuwapi kin hena wocekiye ewicakiciya po.

29 Qa tuwe iyolia sani kin en anipe cinhan unma eciyatanhan kipazo wo; qa tuwe śina inicicu kinhan, onhidolida kin nakun tehinda śni wo.

30 Wicaśta otoiyohi taku nicida kin qu wo; qa tuwe woyuha nici kinhan, ake kica śni wo.

31 Qa wicaśta kin token ecaniconpi yacinpi kin, he ivecen ecawicakicon po.

32 Tona waśtenidapi kin henana waśtewicayadapi kinhan, wopida tukte e duhapi kta he: wahtanipi sa kin eepi kaeś tona cantewicakiyapi kin iś itkom cantewicakiyapi ece.

33 Qa tona tanyan ecaniconpi kin henana tanyan ecawicayeconpi kinhan, wopida tukte e duhapi kta he; walitanipi sa kin iś iyecen econpi ece.

$34 \mathrm{Qa}$ ake niçupi kta kecannipi kin henana owicayaqupi hecinhan, wopida tukte e duhapi kta he; wahtanipi sa kin iś wahtanipi sa owicaqupi ece, iyacinyan wicakicupi kta heon.

35 Tuka niś tona tokaniyanpi kin cantewicakiya po, taku waśte econpi qa owicaqu po, ake taku niçupi kta kecinpi śni po ; kinhan wokajuju tanka duhapi kta, qa Iyotan Wankantu kin he cinca yaunpi kta: he iye tona pida śni qa śicapi kin cantewicakiya ece.

36 Hecen Niyatepi kin iś token waonśida kin niś eya he iyecen waonśida po.

37 Wayacopi śni po, kinhan niyacopi kte śni : owawicakiyepi śni po, kinhan owaniciyepi kte śni : wicakicicajuju po, kinhan nicicajujupi kta:

38 Wicaqu po, kinhan niçupi kta ; wiyutapi waśte, paotinzapi, paipuskicapi qa apaśboka, hecen wicaśta kin maku kin en nicupi kta. Wiyutapi on widutapi kin hee on winiciyutapi ece e kta.

39 Unkan wiyacinpi wan owicakiyaka: Iśtagonge cin iśtagonga napata yus aya okihi he : napin ośkokpa kin en iyolipayapi kte śni he.

40 Waonspekiyapi kin he waonspekiye cin itokapa śni ; tuka tuwe yuśtanpi kin otoiyohi waonspekiye tawa kin iyecece kta.

41 Qa tokeca nisunka iśta kin ohna sukaza yanke cin he wandaka; tuka niye niiśta kin ohna cankaga wanke cin he wanyahdake śni he.

42 Qa, Misunka, sukaza iśta ohna niyanke cin he ito ciciyuśdoke kta ce, token nisunka eyakiye kta he, qa icunhan niye niiśta kin ohna cankaga wanke cin he wanyahdake śni. Wanayakilibe cin, niś tokaheya cankaga iśta ohna niwanke cin he ito tokan elipeya wo; qa hehan hinakaha nisunka iśta kin ohna sukaza yanke cin he yeciyuśdoke kta mdesya wandake kta ce.

43 Can wanji waśte kin waskuyeca śica icahye śni; qa nakun ean wanji śice cin he waskuyeca waśte icaliye śni.

44 Can kin otoiyohi waskuyeca tawa kin on sdonyapi ece: wicaśta kin taspanhu kin etanhan 
suken pahipi śni, qa iś eya tokahu kin etanhan hastanhanka pahipi śni.

45 Wicaśta waśte kin he cante woyuha waśte kin etanhan taku waśte icahya ece; qa wicaśta śice cin he cante woyuha śice cin etanhan taku śice icaliya ece: wicacante taku iyakicuya ohnaka kin he etanhan i kin eciyatanhan enapeya ece, eya.

46 Unkan tokeca, Itancan, Itancan, emayakiyapi ece, qa taku epc cin ecen ecanonpi śni he. 47 Tuwe ekta mahi, qa mioie nahon, qa ecen econ kin he tuwe kicica hecinhan he ito ociciyakapi kta.

48 Wicaśta wan tipi kaga; unkan temahentu maka qe, ca oahehde kin imnija wan akan ehde: unkan minitan aye cehan, wakpa kin tipi kin he en nina kaduza, tuka kahuhuze kta okitpani, imnija akan ehdepi nakaeś, he iyececa.

49 Tuka tuwe nahon tuka ecen econ śni kinhan, wicaśta wan oahehde codan maka akan tipi kage cin he iyeccea; unkan he en wakpa kin nina kaduza, qa hccehnana hinhpaya; qa tipi kin he woatakuniśni tanka.

\section{WICOWOYAKE $\%$}

1 Unkan oyate nahon hiyeye cin en iapi kin dena owasin wanna ecen yaśtan qehan, Kapernaum en $\dot{i}$.

2 Unkan akicita opawinge wicayuhe cin wanji taokiye wan tehinda kin he wayazanke ca wanna te kta.

3 Unkan he wanna Jesus on nahon qehan, Juda hunkayapi kin hena ekta ye wicaśi, u kta qa taokiye kin asniye kte cin he icekiya.
4 Unkan hena Jesus ekta hipi qehan, awicakelian cekiyapi, qa, Hecakiconpi kte cin wicasta kin he iyececa ce;

5 Unkoyatepi kin cantewicakiye ça, omniciye tipi wanji wanna unkicagapi ce, eyapi.

6 Unkan Jesus hena om hda. Unkan wanna tipi lin itehan śni qchan, akicita opawinge wicayuhe ciqon he iś kodawicaye cin hena ekta yewicaśi, qa heciya; Itancan kin, nagiyeiciye śni wo, wati kin en tin yahidu kte cin iyemacece śni ce :

7 Heon etanhan miye ekta ciu kta nakun iycecea micida śni : tuka oie kin ecen eyà wo, kinhan mitaokiye kin asni kta.

8 Miś eya wicaśta wicookihi ihukuya hnakapi kin hemaca, qa mihukuya akicita wicamduha; qa wicaśta kin de, Ya wo, ewakiya eca, ya ece; qa wanji tokeca, U wo, ewakiya eca, u ece; qa mitaokiye kin, Decon wo, ewakiya eca, ecen econ ece, eya.

9 Unkan Jesus hena nahon qehan, on yuśinyaye ca, ihduhomni, qa oyate ihakam unpi kin hewicakiya; Israel hee kaeś en wowacinye hinsko tanka kin iyewaye śni, eciciyapi ce.

10 Unkan ye wicaśipi qon icicawin tiyata kipi qehan, ookiye wayazanke cin he wanna asni iyeyapi.

11 Unkan ianpetu qehan otonwe wan Nain eciyapi kin en i; unkan waonspewicakiye cin etanhan ota qa oyate ota hena kici yapi.

12 Unkan otonwe tiyopa kin he ikiyedan wanna ehan i qehan, inyun, wicaśta wan ta tankan aupi; hunku kin wiwazica heca, qa cinhintku hecedan. Unkan otonwe kin etanhan wicota opapi. 


\section{WICOWOYAKE $\%$.}

13 Unkan he Itancan kin wanyake cehan, onśida qa heciya; Ceye śni wo.

14. Unkan canakiyuhapi kin en u, qa yutan. Unkan akiyuhapi quon iś owanji najinpi. Unkan heya; Kośka, bosdan yanka wo, eciciya ce, eya.

15 Unkan te ciqon iyotanke, ca ia. Unkan hunku kin kicu.

16 Unkan owasin nina wikopapi, qa Wakantanka yatanpi qa heyapi; Waayate wanji tanka unkiyopeyapi icaga ce, qa Wakantanka taoyate kin wanna wicahiyohi ce, eyapi.

17 Unkan Juda makoce qa okśan makoce wanke cin owancaya he yaotaninpi.

18 Unkan Johannes waonspewicakiye cin dena taku kin owasin okiyakapi.

19 Hehan Johannes waonspewicakiye cin nom wicakico, qa Jesus ekta ye wicaśi, qa heya; Tuwe u kte ciqon he niye, qa iś wanji tokeca unkapepi kta he.

20 Unkan wicaśta kin hena wanna ekta hipi qehan, heyapi; Johannes baptem wicaqu ece qon he niye ekta unkuśipi, qa, ruwe u kte ciqon he niye he, qa iś tuwe tokeca unkapepi kta lie, eya ce.

21 Unkan hehantudan hinca wayazankapi, qa makośica ececapi, qa woniya śikśica ececapi, hena wicota asniwicaya; qa iśtagongapi kin heca ota tonwewicaya.

22 Hehan Jesus waayupte ca hewicakiya; Hda po, qa taku wandakapi qa nayahonpi kin hena Johannes okiyaka po: Iśtagongapi kin liena tonwanpi, qa huśtepi kin iś manipi, qa lepros ececapi kin iś wicayuskapi, qa nogetpapi kin iś wanahonpi, qa țapi kin iś kiniwicakiyapi, qa walipanicapi kin iś wootanin waśte kin he owicakiyakapi.

23 Qa tuwe miye on śinhda śni kin he yawaśtepi ce.

24 Unkan Johannes wahośiyapi tawa kin wanna kihdapi, hehan Johannes on oyate kin hewicakiya; Taku e makotahedan elsta wanyag yaipi he. Cedi hu wan tate kahuhuze cin heca he.

25 Tuka taku e wanyag yaipi he. Wieaśta wan wokoyake panpanna koyake cin heca he. Iho, tona waśteya wakoyakapi, qa tanyan nipi kin hena wicaśtayatapi tipi kin en unpi ece.

26 Tuka taku e wanyag yaipi he. Wicaśta wokcan lie. Han, eciciyapi, qa wanji wicaśta wokcan isanpa tanka.

27 Tho, wahośiya mitawa kin he niite itokam ye waśi, nitokam canku wiyeya nicicage kta ce, eya owapi qon he dee.

28 Qa miye heciciyapi; Winohinca eciyatanhan wicatonpi kin wicaśta wokcan tuwedan Johannes baptem wicaqu qon isanpa tanka śni: tuka Wakantanka tokiconze kin en tuwe iyotan cistinna kin he he kapa ce.

29 Unkan oyate kin qa wamnayanpi kin tona nahonpi owasin Wakantanka yaowotanpi, Johannes baptem wicaqu kin en opapi.

30 Tuka Pharisee kin; wicoope aiapi kin om, Wakantanka wahokonwicakiye cin en iyapatoiçiyapi, Johannes baptem wicaqu śni nakaeś.

31 Unkan Itancan kin heya; Wicaśta wicoicage kin de taku e imdacin kta he ; qa taku iyececapi he.

32 Siceca iciyopekiciyapi kin en iyotankapi, qa hoyekiciyapi qa heyapi; Unniciyajopi, tuka wayacipi sni ; waśinunnicihdapi, 
tuka yaceyapi śni ce, eyapi kin he iyececapi ce.

33 Johannes baptem wicaqu qon he aguyapi yute sni, qa miniśa yatke śni $u$; unkan, He taku wakan śica yuha ce, ehapi.

34 Wieaśta Cinhintku kin he wote ca wayatke hi ; unkan hehapi; Wan, wicasta wan wote sa, qa miniśa yatke sa, wamnayanpi qa wahtanipi sa takodawicaya ece.

35 Tuka Woksape iye cinca kin owasin on yaowotanpi ece.

36 Unkan Pharisee wanji kici wote kta e cinkiya. Hecen Pharisee ti kin en tin iyaye ça, wote lita iyotanka.

37 Unkan iho, otonwe kin en winohinca wahtani sa kin heca, he, Pharisee ti kin en wota iyotanka sdonye çehan, inyan opiye ihepi ohna yuha en hi ;

38 Qa tapetepa tanhan siha kin en ceya najin, qa iśta minihanpe kin on siha kiciyujaja, qa paha kin on kicipakinta ca, siha kin ikiciputake, ça ihepi qon on sdakicieiya.

39 Unkan Pharisee kico qon he wanyake cehan, cante mahen awacin qa heya; Wicaśta kin de wicaśta wokcan unkanś, yutan kin he tuwe qa taku winolinca kin sdonye kta tuka; he wahtani sa kin heca.

10 Unkan Jesus waayupte ca heciya; Simon taku eciciye kia mduha ce. Unkan iś, Waonspekiya, katinyan eya wo, eya.

41 Wicaśta wan wicaśta nonpa oicazo wicaqu : unma kin kaśpapi opawinge zaptan iyohiye śni, qa unma kin iś wikcemna zaptan.

42 Unkan taku on hdajujupi kte cin yuhapi śni qehan, wancake sakim wicakicicajuju ce. Hecen unma tukte iyotan waśte- dake kta hecinhan, he omakiyaka wo.

43 Simon waayupte ca; Tuwe ota kicicajuju hee nace epca ce, eya. Unkan, Tanyan idukcan ce, eciya.

44 Hehan winohinca qon ekta ihduhomni, qa Simon heciya; Winohinca kin de wandaka he: Yati kin en wahi, unkan mini on siha wahdujaja kta mayaqu śni ; tuka iye kin iśta miniharpe kin on siha mayujaja, qa paha kin on pakinta.

45 I imayaputake śni; tuka winolinca kin de wahi kin ehantanhan siha imaputaka ayaśtan sni.

$46 \mathrm{~Pa}$ kin wihdi on sdamayrye śni ; tuka winohinca kin de ihepi on siha sdamayan ce.

47 Hecen on heciciya ce, Wahtani kin hena ota eśta kicieajujupi ; he nina waśtedaka nakaeś: tuka tuwe waniqadan kicicajujupi kin he iwaśtedan waśtedaka ece.

48 Hehan winyan qon heciya; Niohan sice cin hena nicieajujupi ce.

49 Unkan tona kici wota iyotankapi kin hena helian cante mahen heyapi ; De turve e woahtani nakun kajuju he.

50 Unkan winolinca yon heciya; Wacinyaye cin he niniyan ce; wookiye on hda wo.

\section{WICOWOYAKE 8.}

1 Unkan he iyohakam, otonwe tankinkinyan otonwe cikciqadan ko, owasin iyaza un, qa wootanin waśte Wakantanka tokiconze ahna yaotanin. Unkan akenonpapi kin kici unpi :

2 Qa winohinca wanjikji taku wakan śica yuhapi qa wayazankapi, tuka asniwicayapi, Mary 
he Magdalene eciyapi, he etanhan wakan śica śakowin hinanpapi qon hee;

3 Qa Joanna, Cuza Herod waawanyagkiye ciqon lie tarvicu, qa Susanna, qa nakun toktokeca uta, hena woyuha yuhapi kin on okiyapi ece.

4 Unkan wicota mniciyapi, qa otonwe otoiyohi etanhan en ahi qehan, wiyacinpi on wowicakiyaka:

5 Woju heca wan taku su kin oju iyaya. Woju, unkan apa canku icahda hinhpaya; unkan naaținzapi, qa mahipiya okinyanpi kin temyapi.

6 Unkan apa imnija wan ahinhpaya; unkan uye cehan, spaye śni on wancake śnija.

7 Unkan apa wapepeka ehnahna hinhpaya; unkan wapepeka opeya uye, ca aotinsya.

8 Unkan apa iś maka waśte ahinlipaya; unkan uye, ça opawingege suton, eya. Unkan hena hecekcen eye çehan, heya niyan; Tuwe noge winahon yukan hecinhan, ito nalion kta ce, eya.

9 Unkan waonspewicakiye cin wiwangapi, qa heciyapi ; Wiyacinpi kin de toketu he.

10 Unkan heya; Niyepi Wakantanka tokiconze woanahibe kin hena oyakalinigapi kta nicupi ; tuka tokan kin iś wiyacinpi ece : hecen etonwanpi keś wanyakapi kte śni, qa wanahionpi keś okahnigapi kte śni ce.

11 Wiyacinpi kin he dee: Su kin he Wakantanka oie kin ee.

12 Canku icahda qon he tona nahonpi kin heepi; hehan wakan śice cin en hi, qa wicoie kin cantepi kin etanhan eyaku ece, awacinpi qa niwicayapi kte śni heon.

13 Tona wicoie nahonpi eca iyuśkinyan icupi ece kin hena imnija akan un qon hena ee; hena hutkan nicapi, qa cistiyedan wicadapi, tuka taku iyutanwicayapi hehan ayuśtanpi ece.

14. Qa tona wanna nahonpi, qa hehan tokiya yapi, qa wiconi kin de woawacin kin, qa wowijice, qa wowiyuśkin kin, hena on aotinzapi, qa hecen tanyan sutonpi śni kin hena wapepeka ehna hinhpaye ciqon eepi ce.

15 Tuka tona wanna wicoie nahonpi eca, cante waśte qa owotanna mahen yuha unpi, qa wacintankaya sutonpi kin hena maka waśte akan un qon hena eepi ce, eya.

16 Wicaśta kin tuwedan petijanjan wan ideye, ça rvakśica wan iyahdapśinyan elipeye śni, qa nakun owinja wan ohdateya ehnake śni ece; tuka petijanjan ihupa akan ehde ece: hecen tona en ipi kinhan, ijanjan kin wanyakapi kta.

17 'Taku anahmanpi qa yuotaninpi kte śni wanica; qa nakun taku yutaninśniyan hnakapi qa yutaninyan eyakupi kte śni wanica ce.

18 Heon token nayahonpi hecinhan itonpa po; tuwe wayuhe cin he nakun qupi kta; qa tuwe wayuhe śni kin he taku yuha kecin kin hee ikicicupi kta ce, eya.

19 Hehan hunku qa hunkawanjitku kin hena en hipi, qa wicota kin on ikiyedan upi kta okihipi śni.

20 Unkan okiyakapi qa heyapi; Nihun qa nihunkawanji tankan najinpi, qa wannihdake kta cinpi ee.

21 Unkan waayupte, ca hewicakiya; Wakantanka oie nahonpi, qa ecen econpi kin dena ina qa mihunkawanji eepi ce, eya. 
22 Unkan anpetu kin hena wanji en, waonspewicakiye cin om wata wan en opa; qa hewicakiya; Mde kin akasanpa unyanpi kta ce. Hecen canan eyaya.

23 Tuka śina watopeliyapi icunhan iye iśtinma: unkan tate iyumni wan mde kin en $u$; qa mini ojudan yakonpi, hecen tapi kta kokipapi.

24 Hehan en hipi qa yuhicapi qu heyapi; Itancan, Itancan, untakunipi śni. Unkan najin hiyaye ca, tate qa mini taja ko kisica: hecen ayuśtan, qa amdakedan tanka.

25 Unkan hewicakiya; Wacinyayapi kin tukte e he. Unkan iś wilkopapi, inihanpi qa hekiciyapi ; De taku wicaśta he; tate mini kici ee kaeś okiya eca, anagoptan ece.

26 Unkan Galile itakasanpa Gadarene tamakocepi kin en ipi.

27 Unkan lien ihe cehan, wicaśta wannakaja tanhan wakanśica yuhe, ca wokoyake koyake śni, qa tuktedan tipi oti śni, tuka wicaśta hnakapi kin en ece un kin, heca wan otonwe kin eciyatanhan itkokipa.

$28 \mathrm{He}$ Jesus wanyake cehan, hoyeye ca itokam makata ehpeiciye ca hotankakiya heya; Jesus Wakantanka Iyotan Wankantu Cinhintku kin, token unyakon kta he. Ceciciya ce, Kakiśmaye śni wo.

29 Taku wakan śape cin wicaśta kin etanhan hinanpe śi heon etanhan. He ijehan yuza ece; unkan mazaicicahilia wicaśke ko on kaśka hnakapi; tuka wicaśke kin yupsapsake ca, wakanśice makotahedan ekta iyayeya ece.

30 Unkan Jesus wiwange ca heya; Token eniciyapi he. 104
Unkan, Wicota, eya : taku wakan śica ota tan mahen jpi kin heon etanhan.

31 Unkan ośbe kin ekta ye wicaśi kte śni, keya icekiyapi.

32 Unkan he kin en kukuśe ota optaye wan won unpi ; hecen en iwicayayapi kte cin he iyowinwicakiye kta keya cekiyapi ; unkan iyowinwicakiya.

33 Hecen wakanśice cin wicaśta kin etanhan tankan hinapapi, qa kukuśe kin en iwicayayapi; unkan optaye maya hinca wan en apamahde nina hin naśdog iyayapi, qa mde kin en iyolipeiciyapi, qa minin tapi.

34 Unkan taku econpi kin he kukuśe wo wicaqupi qon wanyakapi qehan, nakipapi, qa otonwe qa makoce kin ekta ohdag kipi.

35 Unkan taku ecompi qon he wanyag ai, qa Jesus ekta ahi, qa wicaśta wakanśica etanhan hinapapi qon he ogeton, qa tawacin ekicetu, qa Jesus siha kin en iyotankahan yanka wanyakapi ; unkan wikopapi.

36 Tona wanyakapi qon iś eya wicaśta wakan śica yuhe ciqon token asniyanpi lin he owicakiyakapi.

37 Hehan ihdukśan Gadarene tamakocepi kin owancaya taku econpi qon he nalionpi qchan, owasin nina wikopapi on etanhan cekiyapi, qa tokan yeśipi. Unkan wata kin en okipe, ca icicawin kihda.

38 Unkan wicaśta etanhan wakan śice cin hinanpapi qon he kici un kta keya cekiya. Tuka Jesus hde śi, qa heya;

39 Niye yati kin ekta hde, ca Wakantanka taku hinca ecanicon kin he yaotanin wo, eya. Unkan kihde, ca Jesus talru linca ecakicon qon he otonwe owancaya ohdaka. 
40 Unkan Jesus hdi qehan, oyate kin iyowinkiyapi ; owasin ape hiyeya nakaeś.

41 Unkan iho, wicaśta wan Jairus eciyapi kin he en $u$, omniciye tipi itancan kin wanji hee; unkan he Jesus siha kin en makata ehpeiciye ca, iye ti kin ekta u kta icekiya.

42 He cunwintku wanjidan, wanna waniyetu akenonpa ecetu ; unkan iś wanna țe kte wanka. Tuka ekta ye cin hepiya oyate kin aohduteyapi.

43 Unkan winohinca wan waniyetu akenonpa hehanyan we au, qa taku yuha owasin pejihutawicaśta on wanna hdusota ; tuka hecen tuwedan asniye kta okihi śni ;

44 Hee tapetepa tanhan $u$, qa tawokoyake opapun kin yutan: unkan hecehnana we au qon he anapta.

45 Unkan Jesus heya; Tuwe mayutan he. Unkan owasin anakihmanpi qehan, Inyan qa tona kici unpi qon hena heyapi; Waonspekiye cin, wicota kin aohduteniyanpi, qa aoținsniyanpi ; heca eśta, Tuwe mayutan he, eha ce.

46 Unkan Jesus heya; Tuwe mayutan; wowaśake ematanhan hiyu kin he sdonwakiya ce.

47 Unkan winolinea qon wanna inahbe śni e wanhdake cehan, cancanyan $u$, qa itokam makata ehpeiciye, ca oyate kin owasin wicitokam, taku on yutan, qa token wancake asniyanpi kin he okiyaka.

48 Unkan heciya; Cunś, cante waśte wo; wacinyaye cin he asniniyan ce: wookiye on hda wo.

49 Unkan Jesus iahan icunhan, omniciye tipi idaka ti kin eciyatanhan tuwe u, qa heciya; Wa- onspekiye cin nagiyeye śni wo; nicunkśi wanna ta ce, eya.

50 Tuka Jesus he nalion qehan, waayupte ca heciya; Wikope śni wo; awicakehan wicada wo, kinhan niyanpi kta ce.

51 Unkan tipi kin en tin hiyu qehan, tuwedan tin ye lita iyowinkiye śni; Inyan, qa Jakob, qa Johannes, qa wikoślka atkuku hunku kici henana en ipi.

52 Unkan owasin aceyapi qa waśinhda enapeyapi ece. Tuka heya ; Ceyapi śni po ; te śni, tuka iśtinma ce.

53 Unkan wanna ța sdonyapi kin heon wowiliayapi.

54 Unkan owasin tankan iyayewicaye, ça napata yuze, ca kipan qa heya; Wikoślka najin wo, eya.

55 Unkan nagi kin kiyohpaya, qa hecehnana najin hiyaya: hehan wo qu wicaśi.

56 Unkan hunkakeku kin inihanpi : tuka taku econ kin he tuwedan okiyake śni wicaśi.

\section{WICOWOYAKE 9 .}

1 Unkan waonspewicakiya akenonpapi kin hena wicakico, qa wowaśake wookihi ko, wakanśica owasin on, qa wowayazan asniyanpi kta hecen wicaqu.

2 Qa Wakantanka tokiconze kin oyakapi kta, qa wayazankapi kin asniwicayapi $k$ ta ye wicaśi.

3 Qa hewicakiya; Icimayanipi kte cin on cansagye, qa wojuhadan, qa waneya, qa mazaska takudan eyakupi śni po; qa nakun onlidohda nom nom yuhapi śni po. 4 Qa tukte tipi wan en yaipi kinhan, hen ece yukan po, qa hetanhan iyoopta ya po.

5 Qa tuwe kaśta iyowinniciyapi śni kinhan, otonwe kin he inayapapi kinhan, nisihapi watuse- 


\section{LUKAS.}

kśeca onistakapi kin hee kaeś katata po, he owicayalke kta ce. 6 Unkan iyayapi, qa otonwe iyaza yapi, qa wootanin waśte oyalapi, qa owancaya waasniyanpi.

7 Unkan taku econ kin owasin, Herod wicaśtayatapi kin nalion; qa hehan token cante yuza nun tanin śni ; apa Johannes wiconte kin etanhan kini keyapi kin heon etanhan:

8 Qa apa iś, Elias hi ce, eyapi ; qa apa tokeca iś, Wannakaja waayatapi qon hena etanhan wanji kini ce, eyapi.

9 Unkan Herod heya, Johannes pa bawaksa ce : tuka de tuwe e on taku decekceca nawahon he. Unkan wanyake kta cin.

10 Unkan wahośiyapi kin hena wanna hdipi qehan, taku token econpi kin owasin okiyakapi. Unkan ewicahdaku qa nahmanna icunonpa makotahedan wan ekta i, he otonwe wan Bethsaida eciyapi kin ahankeya.

11 Unkan oyate kin sdonyapi qehan, ihakam eyaya: unkan iyowinwicakiye ça, Wakantanka tokiconze kin he iwowicakiyake ca, tona asniyanpi cinpi kin hena asniwicaya.

12 Unkan wanna litayetu aye çehan, akenonpapi kin upi, qa heciyapi, Wicota kin tokan yewicaśi wo; hecen otonwe qa ihdukśan makoce wanke cin hena ektakta yapi, qa taku yutapi oiçidepi, qa iwankapi kta ce; den makotahedan unyakonpi heon etanhan.

13 Tuka hewicakiya; Niye wo wicaqu po. Unkan heyapi ; Aguyapi zaptan qa hogan nonpa henana unhapi ; oyate denakeca woyute opewicakiciton unyanpi śni kinhan.

14. Wicaśta kektopawinge zaptan ecetupi. Unkan waonspe106 wicakiye cin hewicakiya; Wikcenina zaptanptan ptaptaya iyotang wicakiya po, eya.

15 Unkan ecen econpi, qa owasin iyotang wicakiyapi.

16 Hehan aguyapi zaptan qa hogan nonpa qon hena icu; qa malipiya ekta etonwan, qa yawaśte, qa yuśpaśpa, qa waonspewicakiye cin wicaqu; qa iye oyate kin wicitokam ewicakicihnakapi kta.

17 Unkan wotapi qa owasin wipipi ; unkan onśpaśpa iwicakiciyaye cin hena pahipi, makanopiye akenonpa.

18 Unkan iśnana wocekiye eya un qehan, waonśpewicakiye cin hena kici unpi: unkan wiwicawange ca heya; Miye matuwe oyate kin keyapi he.

19 Unkan wayuptapi qa, Johannes baptem wicaqu qon; qa apa iś, Elias ; qa apa tokeca iś ; Wanakaja waayatapi qon hena etanhan wanji kini ce, eyapi ce, eyapi.

20 Unkan hewicakiya; Tuka niye matuwe kehapi he. Unkan Inyan waayupte ça heya; Wakantanka etanhan Messiya kin.

21 Unkan nina owicakiye, ça wicaśta kin tuwedan okiyake śni wicaśi ;

22 Qa heya; Wicaśta Cinhintku kin taku otakiya on ito iyotanhan iyekiye kta, qa wicaśta tokapapi, qa wawayuśnapi itancanpi, qa wowapi kagapi kin hena heyata iyeyapi, qa teyapi kta ce: unkan anpetu iyamni kinlian kini kta ce, eya.

23 Unkan owasin hewicakiya; Tuwe miyahna u wacin kinhan, waipiiçida, qa anpetu otoiyohi cansusbeca kin kiçin, qa miyahna u kta.

24 Tuwe kaśta wiconi kin hduhe wacin kinhan, togelipekiye 
kta ce; tuka tuwe kaśta miye on wiconi kin togehpelkiye cinhan, hee hduhe kta ce.

25 Wicaśta togelipeiciya, qa iś tokan ehpeyapi hecinhan, maka kin ocowasin ohiiciciya eśta, taku ecin on tanyan un kta he.

26 Tuwe miye qa mioie kin on iśtece cinhan, hee on Wicaśta Cinhintku kin iśtece kta, tohan iye towitan, qa Atkuku tawa qa taohnihde wakan tawapi kin hduha u kinhan.

27 Tuka awicakehan heciciyapi ; Den najiupi kin apa Wakantanka tokiconze kin tohanyan wanyakapi śni kin helıanyan țapi kte śni.

28 Unkan wicoie kin dena iyohakam anpetu śahdogan ecetu, hehan Inyan, qa Johannes, qa Jakob, hena ewicayaku, qa cekiye kta paha wan ekta i.

29 Unkan cekiya icunhan ite kin tokeca hinhda, qa tawokoyalke kin ska qa wiyatpatpa.

30 Unkan iho, wicaśta nonpa lrici wohdakapi, Moses qa Elias hena eepi.

31 Hena wolkitaninyan hinajinpi, qa iye iyaye kte cin, Jerusalem ekta hduśtan kte cin he iwohdakapi.

32 Tuka Inyan qa om un kin hena nina iśtinmapi; unkan kiktapi qehan wookitanin tawa kin he wanyakapi, qa nakun wicaśta nonpa kici najinpi kin hena.

33 Unkan hena ehpeya kihdapi icunhan, Inyan Jesus heciya; Itancan, den unkanpi kte cin he waśte; ito wakeya yamni unkagapi kta, wanji niye, qa wanji Moses, qa wanji Elias, hecen unnicicagapi kta; taku eye cin he sdonkiye śni.

34 Hena hecen eya icunhan, mahpiya śoka wan $u$, qa aohanziwicaya. Unkan mahpiya śoka kin en hiyayapi hehan nina wikopapi.

35 Unkan mahpiya qon etanhan wicaho wan hinape, ca heya ; Micinkśi waśte wakidake cin he dee ce : he anagoptan po.

36 Unkan wicaho qon yaśtian qehan, Jesus iśnana en najin. Unkan iye inina unpi, qa taku wanyakapi qon anpetu kin hena en wicaśta kin tuwedan olkiyakapi śni.

37 Unkan ianpetu qehan, paha qon eciyatanhan wanna en hdipi qehan, oyate ota itkokipapi.

38 Unkan iho, wicota kin etanhan wicaśta wanji hoyekiye ça heya; Waonspekiye cin, ceciciya ce, micinkśi kin atonwan wo; micinca hecedan :

39 Unkan iho, woniya wan yuza eca, ihnuhana śicahowaya ece; qa yuhdehdeca, qa hecen minitaga apaśdiya, qa onśiya kuwa, qa kitan se ayuśtan ece.

40 Unkan waonspewicayakiye cin cewicawakiye, ça napeye wicawaśi ; tuka okitpanipi, eya.

41 Unkan Jesus waayupte ça heya; Wicoicage wicada śni qa tawacin tehika, tohanyan cicipi waun kta, qa iyowinciciyapi kta he : nicinkśi kin den au wo.

42 Unkan u kin hepiya, wakanśice cin makata ehpeye, ca yuhdehdeca. Unkan Jesus woniya śape cin kiśice ca, hokśidan kin asniye ça, atkuku kin kicu.

43 Unkan Wakantanka towaśake hince cin on owasin inihanpi. Tuka Jesus taku token econ kin on owasin inihanpi icunhan, waonspewicakiye cin hewicaliya ;

44 Wicoie kin dena ninogepi kin en iyohpayapi kte; Wicaśta Cinhintku kin wicaśta napepi kin en wicaqupi kta. 
45 Tika iapi kin he okahnigapi śni, qa anawicalilimanpi, hecen iyukcanpi śni: unkan wicoie kin on iwangapi kte cin itonpapi.

46 Unkan hehan wanji tukte iyotan tankapi kte cin he hdokinicapi.

47 Unkan cante ekta hdokinicapi kin he Jesus wanyake cehan, hokśiyoqopa wan icu qa iye iyolihe ehde;

$48 \mathrm{Qa}$ hewicakiya; 'Tuwe kaśta holśsiyopa kin de micaje on eyaku kinhan, he miye e emayaku; qa tuwc kaśta miye emayaku kinhan, he umaśi kin hee eyaku ece; hecen niyepi owaśin wanji tukte nicistinpidan kinhan, hee tanka kta ce, eya.

49 Unkan Johanncs waayupte ca heya; Itancan, tuwe nicaje on taku wakan śica napewicaya wanunyakapi, qa den ounpapi śni kin on etanhan teunhindapi.

50 Unkan Jesus heciya; Tehindapi śni po: tuwe unkipajinpi śni kin he unkicicapi ece.

51 Unkan anpetu en wankan eyakupi kte cin wanna kiyedan aya, hehan Jerusalem ekta ye kta e ite kin hdusuta;

52 Qa wahośiyapi kin heca tokata ye wicaśi. Unkan iyayapi, qa taku wiyeya kicihnakapi kta e Samariya wicaśta tonwanyanpi kin wanji en ipi.

53 Unkan Jerusalom ekta ye kta sece cin on etanhan iyowinkiyapi śni.

54. Unkan waonspewicakiye cin, Jakob qa Johannes he wanyakapi qehan, heyapi; Itancan, Elias econ qon he iyecen unkiye, malipiya eciyatanhan peta kun $u$, qa hiuhnahwicaye unśipi kta yacin he.

55 Tuka ihduhomni qa wica- liśice, ca heya; Tawacin toniketupi kin sdonyaliyapi śni ce.

56 Wicaśta Cinhintru kin wicaśta tewicaye kta on hi śni, tuka niwicaye kta heon hi ce, cya. Unkan otonwe wan tokeca ekta iyayapi.

57 Unkan canku ohna yapi icunhan, tuwe heciya; Itancan kin, tokiya kaśta de cinhan ciyahna mde kta ce, eya.

58 Unkan Jesus heciya; Śungidan kin waśun tonpi, qa malipiya okinyanpi kin holipi yuhapi, tuka Wicaśta Cinhintku kin iye pa kin tukten iwanke kte cin ee kaeś hduhe śni ce.

59 Unkan wanji tokeca heciya; - Miyahna u wo. Tuka heya; Itancan, tokaheya ate wehe mde kte cin he iyowinmakiya ye.

60 Jesus heciya; Tona tapi kin ta wicayuhapi kin hena ito wicakiliapi kta; tuka niye qe ye ca Wakantanka tokiconze kin he oyaka wo.

61 Unkan wanji tokeca nakun heya; Itancan, ciyahna waun kta; tuka ito tokaheya wahde, ca wati kin ohna un kin tanyan wehmake kte cin he iyowinmakiya wo.

62 Unkan Jesus heciya; Wicaśta kin tuwe mahiyumdu wanna yuze, ca hehan hinakaha hakikta kinhan, he Wakantanka tokiconze kin en ope kta iyecece śni ce.

\section{WICOWOYAKE' 10.}

1 Unkan taku kin hena iyohakam, Itancan kin toktokeca wikcemna śakowin cajewicayate, ca iye otonwe tona ekta ye kte cin, qa tokiya kaśta ek ta ye kte cin, hena kohan cktakta nomnom yewicaśi.

$2 \mathrm{Qa}$ hecen ewicakiya; Awicakehan woksapi kin tanka, tuka 108 
litanipi kin e tonana ce; he on ctanhan woksapi Itancan kin, he iye litanipi kin heca woksapi lin en yewicaśi kte cin he icekiya po.

3 Ya po; iho, tahincaska śunktokeca ehna unpi he iyecen ye ciśipi.

4 Mazaska opiye, qa wojuhadan, qa hanpa hena hduhapi śni po: qa tahepi wicaśta tuwedan okiyapi śni po.

5 Qa tukten tipi wan en tin idadapi kinhan, tokaheya, Wookiye tipi kin den un nunwe, eya po.

6 Unkan wookiye cinhintku kin en un kinhan wookiye nitawapi kin en iyahe kta: tuka hecece śni kinhan, ake en nihdipi kta.

ry Qa tipi kin he en ece un po, taku nicupi kin yuta qa yatkan dukanpi kta; tuwe htani kin he wokajuju kicupi ece e kta: 'Tipi iyaza yapi śni po.

$8 \mathrm{Qa}$ tukten otonwe wanji en yaipi, qa iyowinniciyapi kinhan, taku nitokam enicihnakapi kin he ece yuta po.

9 Qa wayazanka en unpi kin hena asniwicaya po, qa, Wakantanka tokiconze kin he kiyedan nihiyohipi ce, ewicakiya po.

10 Qa tukte otonwe wanji en yaipi qa iyowinniciyapi śni kinhan, otonwe kin he canku kin en hdicupi qa lieya po;

11 Nitotonwepi watuśekśeca ountkapapi kin hee kaeś itkomı unnicicatatapi ce; tuka Wakantanka tokiconze kin he kiyedan nihiyohipi kin sdonya po.

12 Tuka heciciyapi ce, Anpetu kin he en otonwe kin he tehiya un kte cin he Sodom aoptenya un kta ce, eya.

13 Korazin wotehi niciyanka ce ; Bethsaida wotehi niciyanka ce; niyepi kin en wicohan tanka econpi kin hena Turos qa Sidon en econpi unkanś, wanakaja wahinske ojuha cahota ahna en iyotanka iyopeiçiyapi kta tuka.

14 Tuka wayacopi kin ekta niye tehiya yaunpi kte cin he Turos Sidon kici aoptenya unpi kta.

15 Qa niye Kapernaum, yuwankan mahpiya ekta aniyanpi kin, kuya walkanśica ti kin ekta iyaye niyanpi kta ce, eya.

16 Tuwe ananigoptanpi kin, he miye e anamagoptan; qa tuwe walitenidapi śni kin, he miye e wahtemada śni; qa miś tuwe walitemada śni kin, he umaśi kin hee wahteda śni ece, eya.

17 Unkan wikcemna śakowinpi qon wopidaya hdipi, qa heyapi ; Itancan, nicaje kin eciyatanhan wakanśicapi kin iyepi kaeś anaungoptanpi ece.

18 Unkan hewicakiya; Mahpiya kin eciyatanhan wakanhdi iyecen Satan hinhpaya wanmdaka ce.

19 Iho, wowaśake ciçupi, hecen wamduśka sintehda ko ayahanpi kta, qa toka token waśakapi eśta isanpa yaunpi kta; hecen takudan ksuweniyanpi kte śni.

20 Tuka wakan śice cin hena ananigoptanpi kin heon wiyuśkinpi śni po ; tuka mahpiya ekta caje oniwapi kin hee on wiyuśkin po, eya.

21 Unkan he ehan Jesus cante mahen iyuśkin, qa heya; Ate, mahpiya maka ahna on Itancan, niye taku kin dena ksapapi, qa wayupikapi kin hena anawicayakihbe, ça hokśiyoqopa kin heca sdonyewicayakiye cin, he pidamayalkiya ce; han, Ate; niye hecen iyonicipi kin heon etanhan.

22 A tewaye cin he taku owasin yuhemakiya; qa Cinhintkuyapi 
kin he tuwe kin tuwedan sdonye śni, Ateyapi kin heceyedan; qa Ateyapi kin iś tuwe kin, Cinhintkuyapi kin iye qa tona owicakiyake kte cin henana sdonyapi ece.

23 Unkan waonspewicakiye cin ekta ihduhomni, qa nalimana heya; Taku wandakapi kin hena wiciśta tona wanyakapi kin hena yawaśtepi ece.

$24 \mathrm{Qa}$ heciciyapi; Waayatapi kin wicaśtayatapi ko ota taku wandakapi kin hena wanyag cinpi, tuka wanyakapi śni; qa taku nayahonpi kin he nation cinpi, tuka nahonpi śni, eya.

25 Unkan inyun, wicoope aiapi kin heca wanji najin hiyaye, ca wawiyutanye, ca heciya; Waonspekiye cin, token ecamon qa owihanke wanin wiconi ohimiciye kta he.

26 Unkan heciya; Wicoope kin en token owapi he; token dawa ece he.

27 Unkan waayupte, ca heya; Itancan Wakantanka nitawa kin he nicante kin ocowasin, qa ninagi ocowasin, qa nitowaśake ocowasin, qa nitawacin ocowasin on waśteyadake kta; qa niye inihdacin kin he iyecen nikiyedan un kin waśteyadake kta ce.

28 Unkan heciya; Tanyan waadupta; hecen econ wo, kinhan yani kta ce.

29 Tuka iye ihdaowotanna wacin, qa Jesus heciya; Unkan mikiyedan un kin he tuwe he.

30 Unkan Jesus ayupte ça heya; Wicaśta wan Jerusalem etanhan Jeriko ekta iyaya; qa wamanonpi sa awicakipa, hena tawokoyake kin kipi, qa apapi, qa țe icakiś elipeya kihdapi.

31 Unkan ehantudan wawayuśna wanji canku kin he ohna hiyaya; qa he wanyake cehan iyoopta iyaya.

32 Unkan Lewite heca wanji iś eya ehan i qchan, atonwe ca iyoopta iyaya.

33 Unkan Samariya wicaśta wanji icimani un, he wanke cin en hi; qa wanyake cehan onśida ;

34 Qa en i, qa kaśujapi kin pahite ca wihdi miniśa ko en okaśtan; hehan iye taśunke kin akan yankekiye ça, owote tipi wan en ahi, qa awanyaka.

35 Unkan ihanhanna iyoopta ye kta, hehan kaśpapidan nom chdaku, qa tipi tawa kin qu, qa heciya; Tanyan yuha wo, qa dena sanpa tona on dusote cinhan, tohan waku kinhan cicu kta ce.

36 Iho, hena yamnipi kin, wamanonpi sa awicakipe ciqon wanji tukte ikiyidan un idukcan he.

37 Unkan, Wanji onśida qon hee ce, eya. Hehan Jesus heciya; Ye ça, iyecen econ wo.

38 Unkan hetanhan iyoopta iyayapi, hehan otonwe wan en i; unkan winohinca wan Martha eciyapi, he iye ti kin en kico.

39 Unkan he tankaku kin, Mary eciyapi kin he Jesus siha kin en iyotanke ça, oie kin nation ece.

40 Tuka Martha wicolian ota on token cante yuza tanin śni; heon en hi qa heya; Itancan, mitanka ehpemaye ca miśnana taku ota ecamon ece kin niś ekta ewacanni śni he; heon etanhan omalkiye kta keciya ye.

41 Tuka Jesus waayupte ca heciya; Martha, Martha, taku ota yakicanda wacanni, qa on nagiyeniçiya ece :

42 Tuka taku wanjidan cinpi- 
ca; unkan Mary taku waśte kin he kahiniga, he icimana kipi kte śni ce.

\section{WICOWOYAKE 11.}

1 Unkan tukten cekiya un, qa ayaśtan qehan waonspewicakiye cin wanji heciya; Itancan, wocekiye eya onspeunkiya po, Johannes waonspewicakiye cin onspewicakiye ciqon he iyececa.

2 Unkan hewicalkiya; Ceyakiyapi kinhan kaken eya po; Ateunyanpi mahpiya ekta nanke cin, nicaje wakandapi nunwe: Nitokiconze kin u kte: Mahpiya ekta nitawacin econpi kin he iyecen maka akan econpi nunwe.

3 Anpetu otoiyohi anpetu woyute unqu po.

4 Qa waunhtanipi kin unkicicajuju miye, unkiś tona śicaya ecaunkiconpi wicunkicicajujupi kin he iyececa. Qa taku wawiyutanyan un kin en unkayapi śni po; tuka taku śice cin etanhan eunhdaku po.

5 Unkan hewicakiya; Niyepi kin wanji tuwe kodayayapi, qa hanye cokaya ekta yaipi, qa heyakiyapi ; Koda, aguyapi yamni omaqu wo:

6 Mitakoda wanji icimani hi, tuka taku itokam ewahnake kta takudan mduhe śni ce.

7 Hehan iye timahen tanhan waayupte, ca heye kta; Nagiyemaye śni wo; tiyopa wanna natakapi, qa micinca om mahen imunka; hecen nawajin qa ciçu kta owakitpani ce.

8 Heciciyapi; He lrodaya on etanhan najin qa qu kte śni qeyaś, nina kitan kin heon etanhan, najin qa tona cin kinhan qu kta ce.

9 Nakun heciciyapi; Da kinhan niçupi kta; akita kinhan iyeyapi kta; katoto po, kinhan niciyulidokapi ece e kta. 10 Tuwe da eca he icu ece; qa tuwe akita eca he iycya ece; qa tuwe katoto eca he kiciyulidokapi ece e kta.

11 Cinca niyukanpi kin, wanji tukte cinhintku aguyapi kida kinhan, inyan wan qu kta he; qa iś hogan wan da lkinhan, hogan on wamduśka wan qu kta he.

12 Qa iś witka wanji da kinhan sirıtehda wan qu $\mathrm{k}$ ta he.

13 Hecen niś niśicapi kin, nicincapi taku waśteśte wicayaqupi kte cin he onnispepi ehantanhan, iś iyotan mahpiya Ateyayapi kin tona Woniya Wakan kidapi kinhan wicaqu kta ce, eya.

14 Hehan wakan śica wanji tankan iyayeya, unkan ia okitpani. Unkan wakan śice cin wanna tankan hinape cehan, ia okitpani qon hehan ia; hecen oyate kin inihanpi.

15 Tuka apa heyapi; Beelzebub wakan śica itancan kin he eciyatanhan wakan śica napewicaya ece.

16 Qa apa toktokeca iś iyutanyanpi kta on mahpiya eciyatanhan wowapetokeca kidapi.

17 'Juka tawacinpi kin sdonwicaye ca hewicakiya; Wokiconze kin tona iye iyakitedan kicipajinpi kin hena atakuni śni ece; qa tipi wanji iye içipajin kinhan he jujuwahan ece.

18 Qa Satan iś iye içipajin kinhan tokiconze kin he token ecin ecen he kta he. Beelzebub eciyatanhan wakan śica napewicawaya ece, kehapi kin heon etanhan.

19 Unkan miye Beelzebub esi, yatanhan wakan śica napew.cawaya ehantanhan, niye nicica- 
pi kin hena tuwe eciyatanhan napewicayapi he; hecen iyepi kin niciyukcanpi kta.

20 Tuka miye Wakantanka napsukaza on wakan śica napewicawaya ehantanhan, Wakantanka tokiconze kin awicakehan nihiyohipi ce.

21 Wicaśta wan waśake ca wipe ton ti awanhdake cinhan, hecen woyuha tawa kin tanyan hiyeya ece.

22 Tuka wanji ikapeya waśake cin he takpe hi, qa ktedan kinhan, tawipe wacinkiye ciqon he owasin ekiciyaku, hehan taku tawa qon pamni ece.

23 Tuwe micica śni kin he makipajin ; qa tuwc miciwapahi śni kin he wayumdeca ece.

24 Woniya śapa kin wicaśta wan etanhan hinanpa eca makocc puze cin en ece un, qa oziiçiye kta akita; tuka takudan iyeye śni kinhan, hehan heya; Wati etanhan wahinawape ciqon he ake ekta wahde kta ce.

25 Unkan en hdi eca, wanna kahintapi qa ayuco yuśtanpi iyekiya ece.

26 Hecen tokiya ye ca, woniya toktokeca iye kin isanpa wicaśtapiśni śakowin ewicayaku, qa hena en yapi, qa otipi : hecen wicaśta kin he otokahe kin isanpa oehake kin he śica ece.

27 Unkan hena hecekcen eya icunhan, wicota kin etanhan winohinca wan ho wankan yeye ça heciya; Wicitpi etanhan inicage cin he, qa aze dazoke cin hena yawaśtepi ce.

28 Tuka iye heya; Han, tuka tona Wakantanka oie kin nahonpi, qa ccen econpi kin hena iś iyotan wicayawaśtepi ce.

29 Unkan oyate kin wanna en hiuniciyapi qehan, hewicaliya; Wroicage kin de śica; wowa- petokeca akitapi, tuka wowapetokeca takudan wicaqupi kte śni; Jonas waayate ciqon wowapetokeca tawa kin hcceedan.

30 Ninewe oyate kin Jonas wowapetokeca yuhapi qon, he iyecen wicoicage kin de Wicaśta Cinhintku kin wowapetokeca yuhapi Irta.

31 Woyaco kin ekta itokaga wicaśtayatapi winohinca qon he wicoicage kin de itkom nawicajin, qa wicayaco kta; he iye maka owihanke tanhan Solomon toksape kin nahon hi; unkan iho, wanji Solomon isanpa tanka den un ce.

32 Woyaco kin ekta Ninewe wicaśta kin hena wicoicage kin de on inajinpi kta, qa yacopi kta; he iye Jonas wowicakiyake cin on iyopeiciyapi ; unkan inyun, wanji Jonas isanpa tanka den un ce.

33 Tuwedan petijanjan wan ideya eca nahmana ehde śni, qa iś ohna wiyutapi kin iyahdapśinyan hnake śni ; tuka petijanjan ihupa wan akan ehde ece; qa hecen tona en hipi kinhan ojanjan kin wanyakapi lkte.

34 Wicatancan iyoyanpa kin wiciśta kin hee: heon etanhan niiśta kin hecetu kinhan, nitancan kin iś eya iyoyanpa ojudan ece; tuka niiśta kin he śica eca, nitancan kin iś eya ocowasin otpaza ojudan ece.

35 Hecen iyoyanpa en niun kin he otpaze kte śni e wakta wo.

36 Heon nitancan kin ocowasin iyoyanpa ojudan, qa tuktedan otpaze śni ehantanhan, petijanjan wan yehya ide iyoyamniyan kin he iyecen ocowasin iyoyanpa ojudan kta ce.

37 Unkan heya icunhan Pharisee wan kico: hecen en is qa. wote lita iyotanka. 
38 Unkan wote śni itokam ihdujaja śni kin he Pharisee qon wanyake cehan, inihan.

39 Unkan Itancan kin heciya; Niye Pharisee lkin, miniyatkan wakśica ko akapatanhan duskapi ece; tuka mahen tanhan wawicakipi wicolian śica ko onijupidan ece.

40 Winitkotkopike cin, akapatanhan un kin he tuwe kaga hecinhan, he iś mahen un kin nakun kage śni he.

41 Tuka taku duhapi kin etanhanhan wicaqu po; kinhan taku owasin niciskapi kta ece.

42 Tuka Pharisee kin niyepi wotehi niciyankapi ece; ceyakata, wacanga, wahpe waśtemna ko owasin etanhan iwikcemnamna wicayaqupi ece ; tuka wicolian owotanna Wakantanka waśtedakapi ko, hena acayakśinpi ece; dena e iś ecen ecanompi kta, qa unma qon oyakaptapi kte śni tuka.

43 Pharisee kin niyepi woteli niciyankapi ece; omniciye tipi kin en oyanke waśteśte kin hena waśteyadakapi ece, nakun wopeton tipi kin ekta yaonihanyan oniciyapi kin.

44 Wowapi kagapi qa Pharisee, wanaicilimanpi kin, wotehi niciyankapi ece; wicaśta hnakapi tanin śni hiyeye ca wicaśta kin sdonye śni amanipi kin henicecapi ece.

45 Unkan hehan wicoope aiapi kin heca wanji waayupte, ca heciya; Waonspekiye cin, hecen eha eca, unkiś eya ienunyahdepi ece.

46 Unkan heya; Wicoope aiapi kin niś eya wotehi niciyankapi ece; waqinpi qinpi telika wicaśta qinwicayakiyapi ece qa niyepi kin napsukaza wanjidan on waqinpi kin dutanpi kte śni.
47 Wotehi niciyankapi ece; wicaśta wokcan wicahnakapi kin hena niye yakagapi, qa niyatepi kin hena wicaktepi qon.

48 Atewicayayapi ohanyanpi qon iyowinwicayakiyapi e awicakehan nihduotaninpi; he iye hena wicaktepi e wicakapi, qa niye wicaśta hnakapi kin wicayecagapi ece.

49 Heon iś eya Wakantanka toksape kin heya; Waayatapi qa wahośiyapi heca ekta yewicawaśi kta ee ; unkan apa wicaktepi, qa onśiya wicakuwapi kta;

50 Hecen maka kagapi kin ehantanhan wicaśta wokcanpi we papsonpi kin he owasin wicoicage kin de iwicawangapi kta ;

51 Abel we kin hetanhan, qa Zacariyas tipi wakan wahna wośnapi kin okitahedan awihnuni qon he we kin iyahdeya: Awicakehan heciciyapi, He wicoicage kin de etanhan iwicawangapi kta ce.

52 Niye wicoope aiapi kin wotehi niciyankapi ece; niś wosdonye iyulidoka yutokan edakupi, qa niye ohna idadapi śni, qa tona ohna iyayapi kte cin hena anawicayaptapi ece.

53 Unkan hena hecekcen ewicakiya icunhan, wowapi kagapi qa Pharisee kin taku ota eyekiyapi kta e nina hin nagiyeyapi :

51. Taku iyaonpapi kta akitapi, heon taku i kin etanhan inanpe kta on hinunkapi kta iyapepi.

\section{WICOWOYAKE 12.}

1 Unkan wicota hinca en ahimniciya, hecen akicihanpi ece, hehan waonspewicakiye cin hewicakiya; Tokaheya Pharisee kin on napoliyapi tawapi kin he 
itonpa po, he wanaiçihmanpi kin hee ce.

2 Taku akalipetonpi qa yuzamnipi kte śni wanica; qa nakun taku anabmanpi qa yuotaninpi kte śni wanica ce.

3 Hecen otpaza ehna taku token ehapi kin he anposkantuya nahonpi kta; qa onahbe tipi kin ohna wicanoge kin en token ehapi kin he tipi akan ieyanpahapi kta.

4. Qa kodamayayapi kin, niś lieciciyapi; Wicatancan ktepi qa sanpa takudan okihipi śni kin hena kowicakipapi śni po.

5 Tuka tuwe koyakipapi kte cin he ito kanyehan ociciyakapi kte: Tuwe wicakte, qa hehan wakanśica tipi kin en ehpewicaya okihi kin he eca kokipa po; han, Hee kokipa po, eciciyapi ce.

6 Zitkadan zaptan mazaśadan nonpa iyopewicayapi śni he: heca eśta Wakantanka hena wanjidan kaeś akiktonje śni.

7 T'uka niyepi kin paha ee kaeś owasin niyawapi ece. Heon etanhan wikopapi śni po; zitkadan ota isanpa niyawapi ece.

8 Qa nakun heciciyapi ce; Tuwe kaśta wicaśta kin wicitokam miye mayaotanin kinhan, Wicaśta Cinhintku kin Wakantanka taohnihde kin hena itokam he yaotanin kta.

9 Tuka tuwe wicaśta kin wicitokam amakta śni kinhan, Wakantanka taohnihde kin hena itolkam, he aktapi kte śni.

10 Qa tuwe kaśta Wicaśta Cinhintku kin itkom wicoie wanji eciye cinhan, kicicajujupi kte; tuka tuwe Woniya Wakan waośtehda kinhan, kicicajujupi kte śni.

11 Tohan omniciye tipi kin en anihipi kinhan, qa wicaśtayatapi wowaśake ko ekta, taku qa token aduptapi kta, qa taku ehapi kte cin awacinpi śni po.

12 Woniya Wakan kin he iye iyehantudan hin taku ehapi kta iyececa he onspeniciyapi lita ce.

13 Unkan wicota kin etanhan wanji heeiya; Waonspekiye cin, misunka woyuha hanke micu kta, keciya wo.

14 Unkan heriya; Wicaśta kin, niwankam wiyukcan qa wapamni kte cin tuwe makaga he.

15 Unkan hewicakiya; Iwanihdaka po, qa wakonpi kin kokipa po; wicaśta nipi kin he taku iyakicuya tonpi kin he en un śni ece.

16 Hehan wiyacinpi wan owicakiyake ca heya; Wicaśta wijica wan tamaga kin taku ota icahya.

17 Unkan cante mahen he awacin qa heya; Token ecamon kta he; waskuyeca mitawa kin tukten wehnake kte cin mduhe śni ce.

18 Hehan heya; Kaken ecamon lkta; wopiye wahdujuju kta, qa tankinkinyar wakage lita ; qa hena ohna talsu imicicaga woyuha mitawa ko owasin ewaju kta.

19 Qa minagi kin hewakiye kta; Minagi, taku ota yehnake cin he waniyetu ota oiyakide Ista ce; ito oziiciye, ca wote, ca wayatke, ca wiyuśkin wo, cya.

20 Tuka Wakantanka heciya; Winitkotkoke cin, hanyetu kin de ninagi kin inicicupi kta; hecen wayehnake cin hena hehan tuwe tawa kta he.

21 'Tuwe woyuha kihnalka, tuka Wakantanka elkta wijice śni kin he he kicica ce, eya.

22 Unkan waonspewicakiye cin hewicakiya; Hecen on hecici- 
yapi ce, Yanipi kin on taku yatapi kte cin, qa iś tancan lkin taku kodakapi kte cin, hena on nagiyeiçiyapi śni po.

23 Wicani kin he woyute kin isanpa ece; qa wicatancan kin iś wokoyake isanpa ece.

24 Kangi kin hena iwicayukcan po; he iye wojupi śni qa woksapi śni, qa dotopiye wolia ko nicapi : unkan Wakantanka hena wo wicaqu ece. Wahupakoza kin isanpa niyawapi śni he.

25 Qa niyepi wanji awacannipi kin on wiciśpa wanjidan kaeś nihduhanskapi oyakihipi he.

26 Hecen taku iyotan cistinna kin he oyakihipi śni ehantanhan, unma kin on tokeca ecin nagiyeniçiyapi he.

27 Mnalicalica kin he token icaga ece kin he iyukcan po; litani śni qa nakun wakahmun śni; tuka heciciyapi ce, Solomon token olitanin eśta, dena wanji iyecen wakoyake śni.

28 Hecen Wakantanka peji anpedehan magata hiyeya, qa heyakecinlian petan ehpeyapi kin hee kaeś wokoyake qu ehantanhan, kitanna wicayadapi kin, niś iyotan wokoyake niçupi kte śni he.

29 Qa taku yatapi qa datkanpi kte cin he ihnihdapi śni po; qa nakun wacețundaya wacin yuzapi śni po.

30 Maka oyate kin hena owasin hecen ihnihdapi ece; qa niye taku kin hena yacinpi kin Ateyayapi kin sdonya ece.

31 Tuka Wakantanka tokiconze kin hee tokaheya ihnihda po, kinhan dena owasin koya niçupi kta.

32 Optaye cistinna kin, wikopapi śni po; wokiconze kiı hee niçupi kta e Ateyayapi kin iyokipi ece.
33 Taku yatonpi kin wiyopekiya po, qa wicaqu po.' Wojuha kukaka aye kte śni, malipiya ekta woyuha owihanke kte śni hena icicaga po; heciya wamanon sa kiyedan u śni, qa wamduśkadan wayapote śni ece.

34 Woyuha tukten niciyankapi hecinhan, iś eya nicantepi kin en un ece e kta.

35 Ipiyag kiton yaunpi kta, qa petijanjan nitawapi ideyahan un kta;

36 Qa niś wicaśta wicayuhe cin kiciyuzapi kin eciyatanhan. hdi kta ape hiyeye cin hena iyecen un po; tohan hdi qa tiyopa hdatoto kinhan wancake kiciyuhidokapi kta.

37 Wicayuhe cin hdi kinhan, ookiye kin tona wakta unpi iyewicakiye cin, hena wicayawaśtepi ; awicakehan heciciyapi ce, Wicayuhe cin iye ipiyagkiton, qa ookiye kin hena wote kta iyotangwicakiye, ca u, qa owicakiye kta ce.

38 Hecen inonpa waktapi kin en, qa iś iyamni waktapi kin iyehan hdi, qa ecen iyewicaye cinhan, ookiye kin hena wicayawaśtepi ece.

39 Qa de sdonya po, tipi tawa kin wamanon sa tohan hi kta sdonya unkanś, wakta un qa tipi kin kiciyulidokapi kte cin anapte kta tuka.

40 Heon etanhan niśs eya wiyeya un po; Wicaśta Cinhintku kin tohan awacannipi śni kinhan hehan u kta ce.

41 Unkan Inyan hehan heciya; Itancan, wiyacinpi kin de unkiśnana unkoyakidakapi, qa iś owasin wicayaka he.

42 Unkan Itancan kin heya; Ookiye wanji waawanyagkiyapi kin he wayupike, ca okiksape cinhan, yuhe cin tiwahe kin yu115 


\section{LUKAS.}

hekiye kta, qa hecen iyehanyan wo wicaqu ece e kte cin he tuwe he.

43 Itancan tawa kin $\mathrm{ku}$, qa trokiye wanji hecen econ yanka wanhdake cinhan, hee yawaśtepi ece.

44 Awicakehan heciciyapi ; 'Taku token tawaye cin he owasin yuhekiye kta ce.

4.5 Tuka ookiye kin he cante mahen, Itancan kin tehan hdi kte śni ecin; qa wicaookiye qa winyanookiye kin hena awicape, ça wote, ça wayatke, ça witkoiciye cinhan,

46 Ookiye kin he anpetu wan yuhe cin hdi ape śni, qa sdonkiye śni hehan hdi, qa kicakse, ca wacetunhdapi kin hena owicapeya ounyekiye kta.

$47 \mathrm{Qa}$ ookiye wanji yuhe cin tawacin kin sølonya, tuka wiyeya icihnake śni, qa tawacin kin okicipe śni kinhan, he ota apapi kta. 48 Tuka tuwe sdonye śni kinhan, he iś apapi kte cin iyecen olianyan eśta tonana apapi kta ce. Tuwe taku ota qupi kinhan, hee taku ota iwangapi kta; qa wicaśta kin tuwe taku ota yuhekiyapi ca, he iyotan ota kicapi ece e kta.

49 Maka akan peta iyayewaye kta wahi; qa token wacanmi he, wanna ideyapi eśta.

50 'Tuka baptem wanji on baptem maqupi kta; qa he tohanyan yuśtanpi śni hehanyan aotinsmayanpi ce.

51 Maka akan wookiye wicaqu wahi kecannipi he. Hiya, tuka wicayuomdecapi kin iś ee kta, eciciyapi ce.

52 Detanhan tipi wanjidan ohna zaptan yukanpi kta, qa yuomdecapi kta, yamni nonpa om, qa nonpa yamni om kicipajinpi kta.
53 Wicaśta wan iye cinhintku kin kipajin kta, qa cinhintku kin iś atkuku kin; hunku kin cunwintku kin kipajin kta, qa cunwintku kin iś hunku kin; nakun kunku kin takośku kin, qa takośku kin iś kunku kin kipajin ece e kta, eya.

54 Unkan nakun oyate kin hewicakiya; Wiyolipeyata tanhan malipiya wanji wankan u wandakapi eca, wancake, Magaju kta ce, ehapi : unkan ecetu ece.

55 Qa itokaga tate uya eca, Odidita kta ce, ehapi: unkan ecen hiyohi ece.

56 Wanaicilimanpi kin, niś mahpiya ite kin maka ko iyukcan oyakihipi ; tuka tokeca e nakaha kin idukcanpi śni he.

57 Han, niye cinka tokeca e taku owotanna kin idukcanpi śni he.

58 Tohan tuwe icazoniciye cin kici wicoope awanyake cin ekta de cinhan, tahepi niyuśkapi kte cin akite hinca wo: okinni he wayaco kin ekta aniye kta, qa wayaco kin iś akicita kin ekta niçu kta, qa hecen kaślka nihmakapi kta.

59 Mazaśadan ehake hince cin he tohanyan yecu śni hehanyar hetanhar yahdicu kte śni, eciciya ce, eya.

\section{WICOWOYAKE 13.}

1 Unkan hehan en opapi kin wanjikji, Pilate token Galile wicaśta heca we kin iye wośnapi tawapi kin kici icicahiye ciqon, he okiyakapi.

2 Unkan Jesus waayupte ca hewicakiya; Galile wicaśta kín decen ecawicaonpi kin heon etanhan Galile wicaśta owasin isanpa hena wahtanipi sa kecannipi he. 
3 Hiya; tuka niye nihduecetupi śni ehantanhan, niś eya owasin iyecen nitakunipi kte śni, eciciyapi ce.

$4 \mathrm{Qa}$ iś Siloam conkaśke akeśahdogan awicahinhpaye, ça wicakte qon, wicaśta owasin Jerusalem en unpi qon henana iyotan wicohan śicapi, kecannipi he.

5 Hiya; tuka niye nihduecetupi śni ehantanhan, niś eya owasin iyecen nitakunipi kte śni, e ciciyapi ce, eya.

6 Unkan wiyacinpi kin de nakun owicakiyaka; Wicaśta wan hastanhanka iyuwi maga tawa kin en suken can wanji oju ; unkan en waskuyeca ode hi, tuka takudan iyeye śni.

7 Hehan hastanhanka iyuwi maga kicanye cin he heciya; Can kin de ekta omaka yamni wanna waskuyeca ode wahi, tuka ongedan iyewaye śni ece; kawanka wo; tokeca ecin maka yuśagya han he.

8 Unkan waayupte, ca heciya; Itancan kin, omaka kin de nakun ayuśtan wo, ecen ohomni maka waqe, ça tacesdi en ewahnake kta;

9 Okinni waskuyeca icaliye kta; tuka wanice cinhan, hinnakaha hehan yahdawanke kta ce.

10 Unkan anpetu wakan icunhan omniciye tipi wan ohna waonspewicakiya yanka:

11 Unkan iho, winohinca wan waniyetu ake śahdogan hehanyan woniya waśake śni kin yuha, tancan kin wanna pako icu, qa tokadan ihduowotanna śni.

12 Jesus he wanyaka, unkan kico qa heciya; Winohinca waniyazanke cin etanhan niciyuśkapi ce.

13 Hehan nape kin ahnaka, un- kan wancake owotanna icu, qa Wakantanka yaonihan.

14. Unkan anpetu wakan icunhan Jesus waasniyan kin on etanhan omniciye tipi idake cin he canteptanyan; hecen waayupte, ca oyate kin hewicakiya; Wicaśta kin litanipi kte cin anpetu śakpe yukan ce; hecen anpetu kin hena en u po, qa asniniyanpi kta ce; tuka anpetu wakan icunhan upi śni po, eya.

15 Unkan Itancan kin waayupte ca heciya; Wanaicihbe cin, niyepi otoiyohi, anpetu wakan, tatanka qa iś śonśonna nitawapi kin yakiduśkapi qa miniyatkekiya adapi śni he.

16 Unkan winohinca kin de Abraham cunwintkuwicaye cin wanji ee, Satan iho, waniyetu ake śahdogan hehanyan kaśka hnaka, he iś anpetu wakan icunhan kiyuśkapi kte śni iyececa he.

17 Unkan hena hecen eye cehan, kipajinpi qon owasin iśtecapi ; unkan wokitaninyan taku econ qon hena owasin on oyate owasin iyuśkinpi.

18 Hehan heya; Wakantanka tokiconze kin he taku iyececa he; qa taku e imdacin kta he.

19 Sinape sukaza wanji iyececa, he wicaśta wan icu, qa maga tawa kin en iyohpeya; unkan icage, ca can tanka aya; unkan mahpiya okinyanpi kin adetka kin en iyahanpi ece.

20 Unkan ake heya; Wakantanka tokiconze kin he taku e imdacin kta he.

21 On napohyapi kin he iyece$\mathrm{ca}$, he winohinca wan icu, qa wotpanpi wiyutapi yamni en analiman, hecen ocowasin napogan ce.

22 Unkan otonwe qa otonwe ciqadan iyaza ye ca, waonspekiya; qa Jerusalem etkiya ya. 
23 Unkan tuwe heciya; Itancan, niwicayapi kte cin hena tonananpidan he. Unkan hewicakiya ;

24 Tiyopa cistiyedan kin he ohna dapi kta nina akita po: wicota ohna yapi akitapi kta, tuka okihipi kte śni, eciciyapi ce.

25 Tipi tawa kin tohan wanna najin hiyaye, ca tiyopa kin ecen ikikcu, qa itankan nayajinpi, qa tiyopa yakatotopi, qa ; Itancan, Itancan, unkiciyulidoka miye, ehapi; qa iye waayupte ca, Tonitanhanpi kin sdonwaye śni ce, eniciyapi kta;

26 Hehan niś hehapi kta; Nanke cin en wauntapi, qa waunyatkanpi ece; qa niś ocanku unkitawapi kin ohnahna waonspeyakiye ciqon.

27 Tulka iye heye kta; Tonitanhanpi kin sdonwaye śni, eciciyapi ce; woowotanna śni ecanonpi ece kin owasin mitantanhan iyaya po.

28 Abraham, qa Isaak, qa Jakob, qa wicaśta wokcan owasin Wakantanka tokiconze kin en opapi tohan wandakapi, qa niyepi kin tankan iyayeniyanpi kinhan, wicaceya hihdakokokapi ko yuke lita.

29 Wiyohiyanpata, wiyolipeyata, waziyata, itokaga, henakiya eciyatanhan au, qa Wakantanka tokiconze kin en iyotankapi kta ce.

30 Unkan iho, ehake yakonpi kin hena tokaheyapi kta; qa tokaheyapi kin hena iś ehake yakonpi lita ce, eya.

31 Unkan anpetu kin he en Pharisee kin wanjikji ekta hipi qa heciyapi; Detanhan ye ca tokan iyaya wo; Herod nikte kta cin.

32 Unkan hewicakiya; Yapi 118 qa sungidan kin he heciya po ; Iho, anpetu lin de qa nakun heyakecinhan, wakan śica napewicawaye ca, wayazankapi kin asniwicawaye kta, qa anpetu iciyamni kinhan mayuśtanpi kta ce.

33 Takomni anpetu kin de, qa heyakecinhan, nakun iyokihe kin he en mawani kta iyececa; ecaeś Jerusalem itankan wicaśta wokcan wanji te kte pica śni.

34 Jerusalem, Jerusalem, wicaśta wokcan kin hena wicayakte, qa niye ekta u wicaśipi qon hena inyan on wicayakininpi ece; eca ijehan anpachotonna wan hupahu ohdateya cinca wicatpahi ece kin he iyecen niye nicinca kin wicacicipahi kta, tuka yacinpi śni.

35 Tho, yatipi kin otiwota ehpeniciyapi : qa, Tohanyan tuwe Itancan caje on u kin he yawaśtepi nunwe, ehapi śni hehanyan wanmayadakapi kte śni, awic:akehan eciciyapi ce.

\section{WICOWOYAKE 14.}

1 Unkan Pharisee itanean wanji ti kin en anpetu wakan icunhan wote kta tin iyaya; unkan Pharisee kin awanyag kuwapi.

2 Unkan iho, wicaśta wan dropsi ececa lin he itokam yanka.

3 Hehan Jesus waayupte ça, wicoope aiapi kin, qa Pharisee kin hena hewicakiya; Anpetu wakan icunhan waasniyanpi kte cin iyowinwicakiyapi he.

4 Tuka inina unpi. Hehan wicaśta qon eyaku, qa asniye, ça hdeśi ;

5 Qa waayupte, ca hewicakiya : Niyepi kin wanji tatanka qa iś śonśonna yatonpi, qa anpetu wakan icunhan makolidola wan 
ohinlipaye cinhan, wancake hdicuiyakiyapi śni naceca he.

6 Unkan hena on ayuptapi kta okitpanipi.

ny Unkan wicakicopi kin wiyacinpi wan owicakiyaka, oyanke tokapa ece kahnigapi kin he wanyake cehan; qa hewicaliya ;

8 Tuwe tohan kiciyuzapi kin ekta nicopi kinhan, oyanke tokapa kin en iyotanke śni wo; okinni nikapeya kinihanpi wanji kicopi kta;

9 Kinhan, sakim nicopi kin he $\mathrm{u}$, qa, Wicaśta kin de kiyukan wo, eniciye cinhan, hehan hinakaha wowistenya oyanke hakakta kin hee ekta idotanke lita ce.

10 Tuka nicopi kinhan ye, ça oyanke hakakta kin en iyotanka wo; hecen nico kin he tohan u kinhan, Koda, wankan wapa ya wo, eniciye kta; hecen om wota idotanke cin hena niyuonihanpi kta.

11 Tuwe kaśta ilıduwankantu kinhan, hee yuhukun iyayeyapi kta ce; qa tuwe ihduhukuye cinhan, hee yuwankantuya ayapi ece e kta.

12 Unkan hehan kico qon he heciya; Tohan wiyotanhan wotapi, qa iś hitayetu wotapi yakage cinhan, kodawicayaye, ça nihunkawanji, qa takuwicayaye cin, qa nikiyedan wijinya unpi kin, hena wicakico śni wo; iś ake okinni nicopi, qa hecen nicicajujupi kta.

13 'Tuka wotapi wan yakage cinhan, wahpanicapi, qa huha ksawahanpi, qa huśtepi, qa iśtagongapi kin, hena wicakico wo;

14 Kinhan niyawaśtepi kta ce; hena nicicajujupi kta okitpanipi nakaeś, owotanpidan kinipi kte cin ekta nicicajujupi kta ce, eya.

15 Unkan opeya wota yankapi kin etanhan wanji hena hecen eya nation qehan, heciya; Wakankanka tokiconze kin en tuwe aguyapi yute kta hecinhan hee yawaśtepi ce.

16 Unkan hehan heciya; Wicaśta wan htayetu wotapi wan tanka kage, ca wicota wicakico:

$17 \mathrm{Qa}$ wotapi kta wanna iyehantu, hehan taokiye wan wicakicopi qon hewicakiye kta ye śi U po, wanná taku owasin wiyeya hiyeya ce.

18 Unkan owasin okonwanjidan ihdatokanpi. Tokaheya kin he heciya; Makoce onśpa opewaton, qa he wanhdag mde kta iyececa; ceciciya ce, micunni wo.

19 Unkan"wanji tokeca heya; Tatanka tazaptan opewaton, qa iwicahdute mde kta; ceciciya ce, micunni wo.

20 Unkan wanji tokeca heya; Winohinca wan mduza, hecen wau owakitpani ce.

21 Unkan hecen ookiye qon he ku, qa heyapi kin yuhe cin ecen okiyaka. Unkan hinakaha tipi tawa kin sihda, qa taokiye qon heciya; Koyahan wo, qa otonwe canku kin ekta ye, ca wahpanicapi kin, huha ksawahanpi, huśtepi, iśtagongapi kin, hena den awicau wo, eya.

22 Unkan ookiye qon heya; Itancan kin, ehe cin he wanna ecen econpi, tuka nahahin okan ce, eya.

23 Unkan Itancan kin ookiye qon heciya; Ocanku hince cin, qa mahinatake ektakta ye, ca uwicakiya wo, hecen wati hin ojudan kta.

24 Heciciya ce; Wicakicopi qon wanjidan kaeś wowahe cin etanhan onśpadan yutapi kte śni ce, eya.

25 Unkan wicota hinca kici 119 
yapi; hehan ihduhomini qa hewicakiya ;

26 Tuwe ekta mahi, qa atkuliu, hunku, tawicu, cinca, sunka$\mathrm{ku}$, tawinohtin kin, hena, qa iye ni kin heeś koya śicekida śni ehantanhan, waonspewicawakiye cin wanji ee kta okitpani ce.

27 Qa tuwe kaśta cansusbeca kin lzicin, qa miyahna u śni ehantanhan, waonspewicawakiye cin wanji ee kta okitpani ce.

28 Niyepi kin wanji conkaśke yakagapi kta kecannipi ehantanhan, ito tokaheya idotankapi, qa yahduśtanpi kte cin iyena yatonpi hecinhan, he iyahdukcanpi śni he.

29 Hecanonpi śni kinhan, okinni oahehde kin he wanna eyahnakapi, qa yahduśtanpi kte cin oyakitpanipi kinhan, tona wanyakapi kinhan owasin inihapi, qa heyapi kta;

30 Wicaśta kin de taku kage kta, tuka hduśtan kta okihi śni ce.

31 Qa nakun wicaśtayatapi wan wicaśtayatapi tokeca azuye kte cinhan, ito tokaheya iyotanke, ca iye kektopawinge wikcemna wicayuhe cin hena on, tuwe kektopawinge wikcemna nonpa yuha takpe u kin he itkokipa okihi kta hecinhan, he ihdukcan śni naceca he.

32 Qa iś, unma kin hinahin itehan un, tuwe ekta ye śi, qa wokiyapi kida naceca.

33 Tho, he iyecen niyepi kin tuwe taku yuhe cin owasin tpagan śni ehantanhan, waonspewicarvakiye cin wanji ee kta okitpani ce.

34 Miniskuya he waśte; tuka miniskuya woskuye kin en un śni ehantanhan, taku ecin on skuya kagapi kta he.

35 Maga qa watuśekśeca paha kin unmana on waśte śni ; tuka tankan elipeyapi ece. Tuwe noge winahon yukan hecinhan, nalion kta ce.

\section{WICOWOYAKE 15.}

1 Unkan. hehan wamnayanpi qa wahtanipi sa, hena owasin -nahonpi kta e on en ahi.

2 Unkan Pharisee kin wowapi kagapi ko ikapapi qa heyapi; Wicaśta kin de walitanipi sa iyowinwicakiye ca om wota eee. 3 Hehan wiyacinpi kin de owicakiyake ça heya;

4 Niyepi kin etanhan wicaśta wanji talinca wanunyanpi opawinge wicayuhe ça, wanjidan kitanin śni lkinhan, wikcemna napcinwanka sanpa napcinwanka kin hena makotahedan en awicayuśtan, qa tanin śni qon he tohanyan iyeye śni hehanyan okide śni naceca he.

5 Qa tohan iyeye cinhan, wiyuśkinyan hiyete alkan akionpa ece.

6 Qa wanna tiyata ki eca, kodawicaye ca, ikiyedan tipi kin hena witaya wicakico, qa hewicakiya ; Miciiyuśkin po, tahinca wanunyanpi wan makitanin śni qon he wanna iyewakiya ce.

7 Heciciyapi ce; he iyececa, wahtanipi sa wanjidan iyopeiciya eca, mahpiya ekta wiyuśkinpi ece e kta, wicaśta owotana qa woahtani on iyopeiçiyapi kta iyecccapi śni wikcemna napcinwanka sanpa napcinwanka on iyecen wiyuśkinpi kte śni.

8 Qa nakun winohinca tuwe kaśpapidan wikcemna yuhe, ca wanji togehpekiye cinhan, hecen petijanjan ideye, ça tipi kin kahinte, ça tohan iyekiye śni kin hehanyan okide śni naceca he, 
9 Qa tohan wanna iyeye cinhan, kodawicaye cin, qa ikiyedan tipi kin hena witaya wicakico qa hewicakiya; Miciiyuśkin pe, kaśpapidan toki elpewakiye ciqon he wanna iyewakiya ce.

$10 \mathrm{He}$ iyececa wahtanipi sa wanjidan iyopeiciya eca, on Wakantanka taohnihde kin wicitokam wiyuśkinpi ece, eciciyapi ce.

11 Unkan heya; Wicaśta wan cinhintku nonpa.

12 Unkan hakakta kin he atkuku kin heciya; Ate, woyuha mitawa kte cin he micu wo. Unkan woyuha kin akipam wicaqu.

13 Unkan iyohakam anpetu tonana hehan cinhintku hakakta gon he taku owasin witaya tpahi; qa itehanyan makoce wan ekta icimani iyaya: qa hen śicaya ohanyanpi kin on taku yuhe cin hdutakuni śni.

14 Unkan wanna owasin hdusote cehan, makoce kin he en wicaakithan tanka icaga; hehan iye wicakiś un.

$15 \mathrm{Qa}$ makoce kin hen unpi kin wanji ekta ye ca kici yanka : unkan he kukuśe wo wicaqu kta e maga kin ekta ye śi.

16 Unkan omnica kukuśe yutapi kin hee etanhan wipiiciye wacin; tuka tuwedan takudan qu śni.

17 Unkan iciksuye cehan heya ; Ookiye opetonpi heca aguyapi iyakiçya yuhapi kin tona ate wicayuha hwo;-qa miye e wotektehdapi on atakuni śni amayan ce.

18 Ito nawajin qa ate ekta wahde kta, qa hewakiye kta; Ate, mahpiya kin en, qa niye nakun nitokam wawahtani :

19 Qa detanhan cincamayaye kta iyemacece śni ; wicaśth opewicayaton kin he iś wanji iyececa makaga wo, epe kta ce.

20 Unkan najin hiyaye ca atkuku kin ekta hda. Tuka nahahin itehan atkuku wanhdake ca onśikida, qa ekta inyang ye ça, poskin hduze ca i itputaka.

21 Unkan cinhintku kin heciya; A te, mahpiya kin en qa niye nitokam wawahtani ; qa detanhan cincamayaye kta iyemacece śni ce.

22 Tuka atkuku taokiye kin hewicakiya; Śina iyotan waśte kin he aupi qa inkiya po; qa mazanapcupe wan nape kin en iyekiya po, qa hanpa ohekiya po: 23 Qa ptejicadan cemyapi kin he den aupi qa kte po; wauntapi qa unkiyuśkinpi kta ce :

24 Micinkśi kin de ța, tuka kini; tanin śni, tuka iyeyapi ce, eya. Unkan hinnakaha wiyuśkinpi.

25 Tuka cinhintku tokapa qon he magata un; unkan ti kiyedan ku hehan dowanpi qa wacipi nahon.

26 Unkan ookiye wanji kipan, qa token he kapi hecinhan he iwanga.

27 Unkan heciya; Nisunka hdi; unkan ni un, qa zaniyan hdi kin on etanhan niyate ptejicadan cemyapi qon he kikte ce, eya.

28 Unkan hecen sihda, qa tin kihde wacin śni: hehan atkuku kin tankan hiyu, qa cekiya.

29 Unkan waayupte ça atkulku kin heciya; Wanyaka wo, wanna waniyetu ota ociciye ça tohinni nioie kawape śni ; hececa eśta kodawicawaye cin om wimduśkin kta e tacincadan wan tohinni mayaqu śni.

30 Tuka nicinkśi witkowinpi kin om woyuha nitawa kin tem- 
niciyê cin de hdi ca, wancake ptejicadan cemyapi kin he yecicața ce, eya.

$3 i$ Unkan heciya; Cinś, ohinniyan mici yaun, qa taku mitawa kin he iyulpa nitawa.

32 Nisunka kin de ta, unkan kini; tanin śni, unkan iyeyapi kin, on etanhan ito cante unwaśtepi, qa unkiy uśkinpi kte cin he hecetu ce.

\section{WICOWOYAKE 16.}

1 Hehan waonspewicakiye cin hewicakiya; Wicaśta wijica wanji waawanyake wan yuha; unkan he yuhe cin woyuha tawa yutakuni śni ce, keya okiyakapi.

2 Hehan kico qa heciya; Taku eniciyapi kin de toketu he; waawandake cin ito ohdaka wo; wanna waawandake cin aduśtan kta ce.

3 Unkan waawanyake cin cante mahen heya; Token ecamon kta he; waawanmdake cin he mayuhe cin imicicu; maliqapi kin owakitpani, qa timatayapi kin he imaśteca.

4 Taku ecamon kte cin he sdonwakiya, heon tohan waawanmdake cin he etanhan heyata iyemayanpi kinhan, tipi kin en tin hiyumayanpi kta ce.

5 Unkan hecen yuhe cin tona ikicazopi qon owasin wicakico, qa tokaheya kin he heciya; Mayuhe cin tona nahahin yecu śni he.

6 Unkan, Wihdi iyutapi opawinge ce, eya. Unkan heciya; Koyahanna wowapi nitawa kin ehdaku, qa iyotanke, ça wilkcemna zaptan e okiwa wo.

7 Unkan wanji tokeca hehan heciya; Qa niś tona nahahiin yecu śni he. Unkan, Aguyapisu iyutapi opawinge ce, eya. Unkan heciya; Wowapi nitawa kin ehdaku, qa wikcemna śahdogan e okiwa wo.

8 Unkan yuhe cin waawanyake owotanna śni kin yaonihan, okiksamya econ kin heon etanhan: maka kin de cincapi kin i wicacage cin en iyoyanpa cinca. kin isanpa ksapapi ece.

9 Unkan miś heciciyapi; Woyuha owotanna.śni kin on tawaśitku ic̣icaga po; hecen tohan niye oniçihipi śni kinhan, iye tipi owihanke kte śni en tin hiyuniciyapi kta ce.

10 Tuwe taku iyotan cistinna on wicaka hecinhan, hee taku ota nakun on wicaka ece; qa tuwe taku iyotan cistimna kin on owotanna śni hecinhan, hee taku ota kin nakun on owotanna śni ece.

11 Hecen woyuha owotanna śni kin he on wicayakapi śni ehantanhan, wicake cin he tuwe ecin yuheniciyapi kta he.

12 Qa wicaśta tokeca taku tawa kin on wicayakapi śni ehantanhan, niye nitawapi kin hee tuwe ecin nicupi kta he.

13 Ookiye kin tuwedan yuhe cin nonpa en wowidagiciye kta okihi śni; wanji śicedake kta, qa unma iś waśtedake kta; qa iś wanji en iyaihdaśke kta, qa unma kin iś ekta ewacin kte śni. Wakantanka qa mammon napin en wowidagniciyapi kta oyakihipi śni ce.

14 Unkan Pharisee kin wakonpi kin, hena owasin nahompi ; qa wowihayapi.

15 Unkan hewicakiya; Wicaśta kin wicitokam ihdaowotanpidan kin he niyepi ; tuka Wakantanka nicante ozepi kin he sdonniyanpi ce : ecaeś wicaśta ekta taku takudapi hince cin hee $\mathrm{Wa}$ kantanka wah̉teda śni ece. 


\section{WICOWOYAKE 17.}

16 Woope qa wicaśta wokcan kin hena Johannes hehanyan; tuka hetanhan Wakantanka tokiconze kin oyakapi ece, qa owasin en opapi kte hinca.

17 Mahpiya maka ko tokan iyaye kte cin he aoptetu, qa woope oowa wanjidan kaeś yuecetupi kte śni kin hee tehika ce.

18 Tuwe tawicu ehpekiye ca wanji tokeca yuze cinhan, hee wawicihahapi econ ece : qa hihnaku ehpeye ciqon he tuwe yuze cinhan he wawicihahapi econ ece.

19 Wicaśta wanji wijica, he wokoyake stan qa minihuha zimzipedan ece koyaka, qa anpetu otoiyohi wiyuśkinyan un.

20 Nakun wicaśta wahpanica wan Lazaros eciyapi, he tatiyopa kin en ahionpapi, tancan owancaya lidihdi ;

21 Qa wicaśta wijica wahna wotapi tawa kin etanhan ośnaśna hinhpaye cin hena yunkiyapi kta cin. Tuka śunka kin en ahi, qa ohdilidi kin sdipapi.

22 Unkan wahpanica kin he ta; qa mahpiya ohnihde kin Abraham maku kin ekta ayapi. Wicaśta wijica qon iś eya ța, qa liapi.

23 Unkan wakanśica tipi kin en kakiś yanke cehan, wankan etonwe, ca tehan tanhan Abraham wanyaka; qa maku kin olna Lazaros yanka.

24 Unkan heya niyan; Ate Abraham, onśimakida qa Lazaros u śi wo, napsukaza inkpa kin mini en oputkan qa ceji makasni kta; oide kin de ohna makalija ce.

25 Tuka Abraham heya; Cinś, yani qon he ehan taku waśteśte yahduha, qa Lazaros, taku śikśica yuha, he kiksuya wo; tuka nakaha iye kicanptapi qa niye kakiśniyanpi.

26 Qa nakun unkiyotahedan kaksiza wan tanka yanka; hecen tona deciya tanhan niye ekta ye wacinpi keś okitpanipi ece; qa nakun heciya tanhan den ekta unhipi kta okitpanipi ece.

27 Hehan heya; Heon ate, ceciciya ce, ate ti kin ekta ye yaśi kta;

28 Misunka zaptan, hena wowicakiyake kta; iś eya okinni wokakije kin de en upi nun ce.

29 Abraham heciya; Moses qa wicaśta wokcan kin hena yuhapi; hena iś anawicagoptanpi eśta śni.

30 Tuka heya; Hiya, ate Abraham: tuka tapi kin eciyatanhan wanji ekta wicai unkanś, ihduecetupi lita tuka.

31 Unkan heciya; Moses qa wicaśta wokcan kin hena anawicagoptanpi śni ehantanhan, tapi kin eciyatanhan wanji kini qeyaś, nakun wicadapi kte śni ce, eya.

\section{WICOWOYAKE 17.}

1 Hehan waonspewicakiye cin hewicakiya; Wahtaniyapi kin u kte śni pica śni; tuka tuwe eciyatanhan hena $u$ kinlan, hee wotehi kiciyanka ece.

2 Dena cikcistinpidan kin tuwe wanji wahtaniye cinhan he inyan wiyukpan wanji tahu kin en napinkiyapi, qa miniyowanca kin oiyohlpeyapi unkanś, eeś waśte kta tuka.

3 Iwanihdaka po. Nisunka śicaya ecanicon kinhan kiśica wo, qa iye iyopeiciye cinhan, kicicajuju wo.

4 Qa śakowin akihde anpetu wanjidan śicaya ecanicon, qa śakowin akihde anpetu wanji123 


\section{LUKAS.}

dan niye ekta ihduhomni, qa, Iyopemiciya ce, eniciye cinhan, hecen yecicajuju kta ce, eya.

5 Unkan wahośiyapi kin Itancan kin heciyapi ; Wacinunyanpi kin unkiciyusanpa miye.

6 Unkan Itancan kin heya; Sinape sukaza wan iyecen wacinyayapi kinhan, sukamin can kin de, Hute ihdamna qa miniwanca kin en huicihde wo, eyakiyapi kta, kinhan ananigoptanpi kta tuka.

7 'Tuka niyepi kin wanji tukte taokiye wan yuha, mahyumdu qa iś wanunyanpi wilianwicakiya un, qa tokeśta maga kin eciyatanhan hdi kinhan, Tho wo, ye ca wota iyotanka wo, eciye lkta he.

$8 \mathrm{Qa}$ iś kaken, Woyute wiyeya micihnake ca, ipiyagkiton qa omakiya wo; qa miś tohan wawate ca wamdatke cinhan, iyohakam niś ito wayate ca wadathe kta ce, eciye kte śni he.

9 Hecen ookiye kin he taku econ śipi kin ecen econ kin, on etanhan wopida eciye kta he. Hepce śni ce.

10 Niś eya iyecen taku token econ niśipi kin, owasin ecen duśtanpi kinhan, heya po; Ookiye wakamna śni kin heuncapi; taku econkupi kta iyecece cin heceedan econkupi ece.

11 Unkan Jerusalem ekta ye cehan, Samaria qa Galile cokaya iyoopta iyaya.

12 Qa otonwe wan en i qehan, wicaśta wikcemna lepros ececapi ithokipapi, qa itehan hinajinpi :

13 Qa ho wankan iyeyapi qa heyapi; Jesus, Itancan, onśiunlida miye.

14 Unkan wanwicayake cehan, hewicakiya; Wawayuśnapi kin ekta wicaya po, qa wan- yagiciya po. Unkan ekta yapi icunhan akisnipi.

15 Unkan wanji wanna asni sdotkiye cehan, ihdamna, qa hotankakiya Wakantanka yaonihan ;

16 Qa siha kin en ite makipuskin ehpeiciye ca, wopida eciya : unkan he Samaria wicaśta kin heca.

17 Unkan Jesus waayupte ça heya; Wikcemna asniwicayapi śni he: tuka napcinwanka kin hena tokiya unpi he.

18 Wakantanka yaonihan kta hdipi kin tuwedan iyeyapi śni, oyate tokeca etanhan kin de iśnana ce.

19 Hehan heciya; Najin qa hda wo; wicayada kin he asniniyan ce.

20 Unkan Pharisee kin iś Wakantanka tokiconze kin he tohan hiyohi kta hecinhan, he iwangapi qehan, waayupte ca lewicakiya; Wakantanka tokiconze kin he wowanyake on hi kte śni.

21 Nakun, Tho, dee ce, qa iho, kae ce, eyapi kte śni : Wanyaka po, Wakantanka tokiconze kin he maken niunpi ece.

22 Hehan waonspewicakiye cin hewicakiya; Anpetu wan hiyohi kta, Wicaśta Cinhintku kin anpetu tawa kin wanji wandakapi kta yacinpi kta, tuka wandakapi kte śni.

$23 \mathrm{Qa}$, Iho dee, qa iho kae ce, eniciyapi kta; tuka wiciyahna yapi śni po, qa owicapapi śni po. 24. Wakanhdi mahpiya ihukuya tokiyatanhan wiyatpa $u$, qa mahpiya ihukuya unma ekta iyahde ece kin he Wicaśta Cinhintku anpetu tawa kin en iyecece kta.

25 Tuka, ito tokaheya taku ota on iyotanhan iyekiye kta, qa

124 
wicoicage kin de on heyata iyeyapi kta ce.

26 Unkan Nowa taanpetu qon en token unpi qon, Wicaśta Cinhintku taanpetu kin en he iyecece kta.

27 Nowa wàta kin en okipe ciqon anpetu kin hehanyan wotapi, qa wayatkanpi, tawicutonpi, qa hihnatonpi ece; unkan minitan $u$, qa owasin owihankewicaya.

28 Qa nakun Lot anpetu tawa qon cn iś eya iyececa, wotapi, qa wayatkanpi, wopetonpi qa wiyopeyapi, wojupi qa ticagapi ece :

29 Tuka Lot Sodom etanhan tankan hinape ciqon anpctu kin hee hinca en malipiya kin eciyatanhan peta, mazakan noge iyuwintapi kin ahna hinhe, ca owasin owihankewicaya.

30 Wicaśta Cinhintku kin yuotaninpi kte cin he iyecece kta ce.

31 Anpetu kin he en tuwe tipi akan yanke ca tawahpaya timahen hiyeye cinhan, kun ku qa ehdaku kte śni; qa tuwe magata un kinhan, iś eya taku hekta un kin ekta ihdamna kte śni.

32 Lot tawicu qon he kilssuya po.

33 Tuwe wiconi tawa kpatan cin kinhan, he toki elipekiye kta; qa tuwe wiconi tawa toki elipekiye cinhan, hee hduhe kta.

34 Heciciyapi ce; Hanyetu lin he en wicaśta nom owinja wanjidan akan wankapi kta; wanji icupi kta, qa unma kin iś chpeyapi kta.

35 Winyan nonpa akiptan wotpan unpi kta; wanji icupi kta, qa unma kin iś ehpeyapi kta.

36 Wicaśta nom magata unpi kta; wanji icupi kta, qa unma kin iś ehipeyapi knta ce.
37 Unkan waayuptapi qa heciyapi; Itancan, tukte etu he. Unkan hewicalkiya; Tukten wicatancan wanka eca, hen wamdi kin kawitaya imniciyapi ece e kta.

\section{WICOWOYAKE 18.}

1 Unkan wicaśta kin ohinniyan wocekiye eyapi kta, qa iyakicunnipi kte śni e on wiyacinpi wan owicakiyaka;

2. Qa heya; Otonwe wan en wiyukcan wanji Wakantanka kokipe śni, qa nakun wicaśta wicakinihan śni.

3 Unkan otonwe kin he en wiwazica wan, he wiyukcan kin ekta hi qa, Makipajin kin he tomicicon ye, eya.

4 Unkan tokaheya econ wacin śni; tuka ocim cante mahen hcya; Wakantanka kowakipe śni, qa nakun wicaśta kin wicawakinihan śni qeyaś;

5 Wiwazica kin de nagiyemaye cin on etanhan ito towecicon kta okinni ohinni hecetu u kinhan wacin iyolkiśinmaye kta naceca ce.

6 Unkan Itancan kin heya; Wiyukcan owotanna śni ie cin he nahon po.

7 Unkan Wakantanka tona wicakalinige, ca anpetu hanyetu ko cekiyapi kin, hena tehan towicakiciçon śni qeyaś, ocim econ kte śni he.

8 Ihnuhanna towicakicicon kta, eciciyapi ce. 'Tuka Wicaśta Cinhintku kin he tohan hi kinhan, maka akan wacinyanpi iyeye lita he.

9 Unkan apa iye ohan owotanna içidapi, qa tokan kin wahtewicadapi śni kin, hena wiyacinpi kin de owicakiyaka.

10 Wicaśta nonpa tipi wakan kin ekta cekiya ipi ; unna kin 
Pharisee heca, qa unma iś wamnayanpi kin heca.

11 Unkan Pharisee kin iśnana najin, qa kaken wocekiye eya; Wakantanka, wicaśta tokeca iyemacece śni, wawicakipi sa, olian owotanpidan śni, wawicihahapi econpi sa, qa wamnaye cin dee kaeś iyemacece śni heon wopida eciciya.

12 Anpetu wakan olritahedan nonpa akihanmiçiye ca taku mduhe cin owasin etanhan iwikcemnamna kin wicawaqu ece.

13 Unkan wamnaye cin iś itehanyan inajin, qa iśta ee kaeś wankan malipiya kin ekta ikikeu śni ; tuka maku kin ohna aicipe, ca heya; Wakantanka onśimakida wo, wawahtani sa.

14 Wicaśta kin de yuowotanpidan tiyata kihda, qa unma qon hee śni, eciciyapi ce : Qa tuwe ihduwankantuya kinhan, he yuhukun iyeyapi kta; qa tuwe ihduhukuye cinhan, hee yuwankan ayapi lita ce.

15 Unkan hokśiyocyopa kin nakun wicayutan kta e en awicahipi tuka waonspewicakiye cin he wanyakapi qehan, wicakiśicapi.

16 - Tuka Jesus wicakico, qa heya; Hokśiyoqopa lkin ekta mahipi kte cin iyowinwicakiya po, qa tehindapi śni po ; Wakantanka tokiconze kin he hececapi etanhan ece.

17 Wowicake eciyatanhan heciciyapi; 'Tuwe hokśiyoqopa iyeceea Wakantanka tokiconze kin icu śni kinhan, he tokadạ en ope kte śni ce.

18 Unkan wicaśta ihdawa wanji wiwange ca heya; Waonspekiye waśte, token ecamon, qa owihanke wanin wiconi ohimiciye kta he.

19) Unkan Jesus heciya ; Toke- ca e waśte emayakiya he; tu. wedan waśte śni; Wakantanka hecedan waśte.

20 Woahope kin hena onnispe; Wawicihahapi ecanon kte śni; Tin wicayakte kte śni ; Wamayanon kte śni; Woitonśniyan wodake kte śni; Niyate nihun kici wicahduonihan wo.

21 Unkan heya; Macistina ehantanhan hena owasin ahowapa ce.

22 Unkan Jesus hena nalion qehan heciya; Nahanhin taku wanji iyotpani yaun; taku yaton kin owasin wiyopekiye ca wahpanicapi kin wicakipamni wo, kinhan mahpiya ekta woyuha duhe kta ce; hehan u qa mihakam un wo.

23 Unkan he nahon qehan nina iyokiśica; wijice hinca nakaeś. 24 Unkan nina wacin iyokiśice cin he Jesus wanyake cehan, heya; Wowijice tonpi kin hena Wakantanka tokiconze kin en yapi kte cin he tehike linca.

25 Wicasta wijica wan Wakantanka tokiconze kin en ye kte cin he tehika, qa kamel heca wanji tahinśpaciqadan ohdoka kin ohna ye kte cin hee kaeś aoptetu ce.

26 Unkan tona he nahonpi kin hena heyapi; Hehan tuwe ni lita he.

27 Unkan heya; Wicaśta kin ckta taku econpica śni kin, he Wakantanka ekta econpica ece. 28 Unkan Inyan heya; Tho, unkiś taku owasin untpaganpi, qa niyahna unyakonpi ce.

29 Unkan hewicalsiya; Awicakehan heciciyapi ce, Wicaśta kin tuwe ti kin, qa hunkakewicaye cin, qa liunkawanjitku, qa tawicu, qa cinca, hena wanji Wakantanka tokiconze kin on lipagan kinhan,

30 Hce dehan nakaha taku 
sanpa ota yuhe kta, qa wiconi u kte cin he en wiconi owihanke wanica yuhe kta ce.

31 Unkan akenonpapi kin ewicayaku qa hewicaliya; Iho, Jerusalem ekta unyanpi, qa wicaśta wokcan Wicaśta Cinhintku kin on token owapi qon owasin yuecetupi kta.

32 Oyate tokeca napepi kin en wicaqupi kta; hecen iśtenyapi, qa wawicanksiya kuwapi, qa atagośapi kta ;

33 Qa kapsinpsintapi qa teyapi kte; unkan anpetu iyamni kinhan kini kta ce, eya.

34. Unkan ecaca okahnigapi śni, qa oie kin de anawicakiȟmanpi, qa taku oyakapi kin sdonyapi śni.

35 Unkan Jeriko ikiyedan wanna u qehan, wicaśta wan iśtagonge canku icahda wada yanka.

36 Unkan wicota ahiyaye cin he nation qehan, he token kapi hecinhan he iwicawanga.

37 Unkan, Jesus Nazareth etanhan kin he hiyaya ce, eya okiyakapi.

38 Unkan hotankakiya heya; Jesus Dawid Cinhintku kin onśimakida wo.

39 Unkan tokahanpi kin hena iyolripi śni, inina yanlze lita ; tuFa heon ifotan, hotankakiya heya; Jesus Dawid Cinhintku kin onśimakida wo.

40 Ulkan Jesus inajin qqa aupi kta kewicalkiya. Unkan wanna liyedan u qehan, wiwange ça,

41 Token ecacicon kta yacin lie, eya. Unkan iye, Itancan, watonwe kta, eya.

42 Unlian Jesus heciya; Tonwan wo, wacinyaye cin he niniyan ce.

43 Unkan wancake tonwe ca iyahna hiyu, qa Wakantanka yaonihan: unkan oyate kin he wanyakapi qehan, owasin Wakantanka yatanpi.

\section{WICOWOYAKE 19.}

1 Unkan Jeriko en i, qa iyoopte iyaya.

2 Unkan iho, wicaśta wan Zakeus eciyapi, wamnayanpi itancan kin wanji hee, qa wijica.

3 Unkan Jesus tuwe kin hecinhan he wanyag alkita ; tuka tancan ptecedan, hecen wicota lin on tokadan okihi śni.

4 Unkan toliata inyang ye ca sukamin can wanji adi, wanyake kta e heon, hẹ hiyaye Ixta nakaeś.

5 Unkan Jesus wanna ehan i qehan, wankan etonwe ca wanyaka, qa heciya, Zakeus koyahanna kun hdicu wo, anpetu lisin de yati kin en ito manke kta ce.

6 Unkan koyahanna kun ku, qa iyuśkinyan iyowinkiya.

7 Unkan he wanyakapi qehan owasin ikapapi qa heyapi, Wicaśta wan wahtani sa he kici wote kta iyaya ce.

8 Unkan Zakeus najinhan najin qa Itancan kin heciya; Itancan wanyaka wo, woyuha mitawa kin hanke wahpanicapi kin wicawaqu ece; qa tuwe oitonśniyan taku iwecicu kinhan, topa akihdo wecu kta ce.

9 Unkan Jesus heciya: Anpetu kin de wanikiyapi tipi kin de hiyohi; Abraham cincaye cin wanji hee nakreś.

10 Wicaśta Cinhintku kin taliu a takuni śni qon, he akite ça niye kta e heon hi ce, eya.

11 Unkan hena nalionpi qehan nakun wiyacinpi wan owicalkiyaka; wanna Jerusalem ikiycdan un, qa Wakantanka toki- 
conze lin ecadan u kta kecinpi kin heon etanhan:

12 Qa heya; Wicaśta ihdawa wan makoce itehan wan ekta iyaya, wokiconze icu qa ku kta.

13 Unkan taokiye wikcemna wicayuhe cin, hena wicakico, qa mazaska tkeutapi wikcemna wicaqu, qa hewicakiya; Tohanyan wahdi śni hehanyan un po.

14 Tuka taoyate kin walitedapi śni, qa tuwe iyahna yeśipi, qa heyapi; Wicaśta kin de unkidakapi kta uncinpi śni ce.

15 Unkan wokiconze kin icu qa hdi qehan, taokiye mazaska wicaqu qon hena wicakicopi kta keya; wopetonpi kin on tona wanna hdamnanpi hecinhan, he sdonye kta.

16 Unkan tokaheya kin he $u$, qa heya; Itancan kin, mazaska nitawa qon, he wanna mazaska wikcemna kamna ce.

17 Unkan heciya; Han, ookiye niwaśte; taku tonana on wacinyepica yaun, heon etanhan ito otonwe wikcemna idaka wo.

18 Unkan iyokihe kin he 11 , qa heya; Itancan kin, mazaska nitawa qon, he wanna mazaska zaptan kamna ce.

19 Unkan iś eya heciya; Niś otonwe zaptan idaka wo.

20 Unkan wanji tokeca $u$, qa licya; Itancan kin, wanyalka wo, mazaska nitawa wanapin ohna yuha waun, he dee:

21 Wicaśta niśake cin lieon kocicipa ce; taku eyahnake śni kin, he ece edaku; qa woyaju śni kin he ece duksa ce, eya.

22 Unkan heciya: Nii kin eciyatanhan ciyaco kta, ookiye winicaśtaśni; wicaśta maśake cin he sdonyaya; taku ewehnake śni kin he ece emdaku, qa wowaju śni kin hee mduksa ece.

23 Hecen tokeca mazaska mi- tawa kin he iciyopekiciyapi kin en ade śni he; kinhan wahdi kin ekta mitawa kin oicage ahna weca kta tuka ce.

24. Hehan hen najinpi kin hena hewicakiya; Mazaska kin he ikicicupi qa tuwe mazaska wikcemna yuhe cin hee qu po.

25 Unkan heciyapi; Itancan kin, he wanna mazaska wikcemna yuha ce.

26 Tona taku yuhapi kin hena owasin nakun wicaqupi cce e kta; qa tuwe taku yuhe śni kinhan, taku yuhe cin hee kaeś ekiciyakupi kta ece, eciciyapi ce.

27 Tuka tokamayanpi iwicawadake kta cinpi śni qon hena den awicaupi qa mitokam wicakte po, eya ce.

28 Unkan hena hecen eye çehan, tokata ye ca Jerusalem ekta itawankanhde iyaya.

$29 \mathrm{Qa}$ Bethphage qa Bethani, paha wan Oliwe eciyapi kin, he wanna ikiyedan ehan i qehan, waonspewicakiye cin etanhan nonpa ye wicaśi ;

30 Qa heya; Otonwe iyotakons wanke cin he ekta ya po; qa tokaheya en yaipi kinhan śukcincadan wan iyeyayapi kta, wicaśta tuwedan tohinni akan iyotanke śni; he kiyuśkapi qa aku po.

31 Qa, Tokeca yakiduśkapi he, tuwe eya iniwangapi kinhan, niś hehan, Itancan kin cin ce, eyaliziyapi kta.

32 Unkan ye wicaśi qon hen? iyayapi, qa token ewicakiye cin ecen iyeyapi.

33 Unkan śukcincadan kiyuśkapi icunhan, tuwe tawapi kin hena hewicakiyapi ; Tokeca yakiduślkapi he.

34 Unkan, Itancan kin cin ce, eyapi. 
35 Unkan Jesus kahdipi; qa wokoyake tawapi kin śukcinca kin aihpeyapi, qa Jesus akan yankekiyapi.

36 Qa ye cin tahepi canku ohna wokoyake kin hdumdayapi.

37 Unkan ikiyedan Oliwe paha kin apamahde iyayapi kin hehan wanna i qehan, waonspewicakiya wicota owasin, wicowasake wicolian wanyakapi qon owasin on etanhan hinakaha iyuśkinpi, qa hotankakiya Wakantanka yatanpi,

38 Qa heyapi ; Wicaśtayatapi tuwe Itancan caje kin on u kin he yawaśtepi ce; mahpiya ekta wookiye, qa iyotan wankantu ekta wowitan un nunwe.

39 Unkan Pharisee wicota en opapi qon apa heciyapi; Waonspekiye cin, waonspewicayakiye cin wicakiśica wo.

40 Unkan waayupte ca hewicakiya; Dena inina unpi kinhan, inyan kin wancake howaye kta ce, eciciyapi ce.

41 Unkan wanna ikiyedan $u$, qa otonwe wanyake çehan, aceye, ca heya;

42 Niye kin anpetu nitawa kin de en taku on wookiye duhe kte cin sdonyakiya unkanś: tuka wanna icimana iśta on wanyahdake kte śni.

43 Anpetu kin hiyonihipi kta, tona tokaniyanpi kin nihdukśan maka qapi, qa okśan niyankapi kta, qa ohomni ohinniyan anapte nanijinpi kta:

$44 \mathrm{Qa}$ makata elipeniyanpi kta, nicinca en niunpi kin hena koya; qa inyan nonpa icitakihna wanke śni ehpeniyanpi kta; anpetu en taku waśte nihiyohipi kin he sdonyakiye śni heon etanhan.

45 Unkan tipi wakan kin en tin iyaye, ca tona ohna wiyope- yapi, qa wopetonpi qon, hena tankan ehpewicaya ;

46 Qa hewicakiya; Tipi mitawa kin he wocekiye tipi, eya owapi qon; tuka niye owamanonpi kin heca yakagapi ce.

47 Unkan anpetu iyohi tipi wakan kin ohna waonspekiya. Tuka wawayuśnapi itancanpi, qa wowapi kagapi, qa oyate kin en wicaśta ihdawapi kin, hena teyapi kta akitapi ;

48 Tuka token econpi kta takudan iyeyapi śni; oyate kin owasin oie kin nahonpi kta cinpi ece.

\section{WICOWOYAKE 20.}

1 Unkan anpetu kin hena wanji en tipi wakan kin ohna oyate kin waonspewicakiye, ca wootanin waśte kin he oyake cehan, wawayuśnapi itancanpi, qa wowapi kagåapi, qa wicahincapi kin, hena ekta ahi,

2 Qa okiyapi, qa heciyapi; Tukte wicookihi on dena decen ecanon he; qa wicookihi kin de tuwe e niçu hecinhan, unkokiyaka po.

3 Unkan waayupte ca hewicakiya ; Miś eya taku wanji iciwangapi kta; he amayupta po:

4 Johannes baptem wicaqu qon he malipiya eciyatanhan, qa iś wicaśta kin eciyatanhan, unma tukte he.

5 Unkan iye iyakitedan iapi, qa heyapi ; Mahipiya kin eciyatanhan ce, unkeyapi kinhan, Tokeca wicayadapi śni he, eye kta : 6 Tuka, Wicaśta kin eciyatanhan, unkeyapi kinhan, oyate kin inyan on unkininpi kta; Johannes wicaśta wokcan kin heca kecinpi.

\% Unkan ayuptapi, qa tokiyatanhan un kin sdonyapi śni, keyapi. 
'8 Unkan Jesus hewicakiya; Miś eya wicookihi tukte on dena ecamon kin nakun ociciyakapi kte śni ce.

9 Hehan wiyacinpi kin de oyate kin owicakiyaka: Wicaśta wan hastanhanka iyuwi maga wan oju, qa malikicanyanpi kin heca owicaqu, qa makoce itehan wan ekta tehan yanka.

10 Unkan wanna iyehantu qehan, taokiye wanji hastanhanka iyuwi maga waskuyeca kin etanhan qupi kta e, malikicanyanpi qon ekta yeśi ; tuka malikicanyanpi qon kaśtakapi, qa cokadan hdeśipi.

11 Unkan ake taokiye wanji tokeca yeśi ; unkan iś eya kaśtakapi, qa śikśicaya kuwapi, qa cokadan hdeśipi.

12 Unkan akeś iyamni wanji yeśi ; unkan iś eya taopi, qa tankan elipeyapi.

13 Hehan hastanhanka iyuwi maga tawa kin heya; Token ecamon kta he. Micinkśi waśtewalkidake cin, hee yewaśi lita ce; okinni he wanyakapi kinhan yuonihanpi kta.

14 Tuka mahikicanyanpi kin he wanyakapi qehan, iye iyakitedan iapi, qa heyapi ; Tawaye kte cin he dee ce; iho po, unktepi kta; hecen unkiye tawaunyanpi kta ce.

15 Hecen hastanhanka iyuwi maga itankan ehpeyapi, qa ktepi. Heon etanhan hastanhanka iyuwi maga tawa kin token ecawicakicon kta he.

16 Ito ku kta, qa mahikicanyanpi kin hena owihankewicaye, ca hastanhanka iyuwi maga kin he tokan wicaqui kta ce, eya. Unkan he nahonpi qehan, Hecetu śni ce, eyapi.

17 Unkan awicatonwe cehan heya; Hecen wowapi en kagapi 130 qon he token kapi he; Tipi kagapi kin inyan lieyata iyeyapi qon hee wanna oise en itancan icaga ce :

18 Qa tuwe inyan kin he ahinhpaye cinhan kaliuliugapi kta; qa iś inyan kin tuwe ahinlipaye cinhan, hee pamdu kta ce.

19 Unkan wawayuśnapi itancanpi, qa wowapi kagapi kin, hena he ehan iyahpayapi kta cinpi ; tuka oyate kin kowicakipapi; wiyacinpi oyake cin he iye wicaka e iyukcanpi.

20 Unkan awanyag kuwapi, qa wanjikji iye owotanna konzapi kin hena iwanyakapi kta e ekta ye wicaśipi ; hecen oie kin iyalipayapi, qa wicaśta itancan towaśake qa tawacin kin en qupi kta.

21 Unkan hena wiwangapi, qa heyapi; Waonspekiye cin, niye tanyan iyae, ca waonspeyakiya ece; qa tuwedan en eyatonwe śni; tuka Wakantanka ocanku tawa kin he awicakehan onspewicayakiya e sdonunniyanpi ce: 22 Iho, Kesar wamnayanpi kin unqupi, qa iś unqupi kte śni, unma tukte hecetu he.

23 Unkan wayupiya hnaye wacinpi kin he iwicayukcan, qa hewicakiya ; Tokeca iyutanmayayapi he.

24 Kaśpapidan wanji makipazo po. Akan owapi qa wakagapi kin de tuwe tawa he. Unkan ayuptapi qa; Kesar tawa ce, eyapi.

25 Unkan hewicakiya; Heon Kesar taku tawa kin hena Kesar kicu po; qa Wakantanka taku tawa kin Wakantanka kicu po.

26 Unkan oyate kin wicitokam oie kin iyahpayapi kte cin okitpanipi, qa waayupte cin he on inihanpi, qa inina hiyeya.

27 Hehan Sadukee ake wica- 
kini kta wanica keyapi kin hena wanjikji en hipi ; wiwangapi,

28 Qa heyapi; Waonspekiye cin, Moses kaken wowapi unkicagapi; Tuwe cincu tawicu ton qa cinca nica ecen te cinhan, iye sunkaku tawicu qon hduze ça cincu cinca icahikiciciye kta ce.

29 Unkan hecen hunkawanjinkiciyapi śakowin: unkan tokapa kin tawicu ton, qa cinca nica ecen ta.

30 Unkan iyokihe qon he hehan hduza; tuka cinca nica ecen ta.

31 U̇nkan iyamni qon hduza; qa śakowinpi qon owasin hecen hduzapi, qa ecen țapi ; unkan cinca nicapi.

32 Unkan ehake winolinca iś eya ta.

33 Iho, hecen wicakini kte cin ekta śakowin hduzapi qon, hena tukte wanji tawapi kta he, eyapi. 34 Unkan Jesus waayupte, ça hewicakiya; Maka kin de cinca kin tawicutonpi, qa hihnatonpi :

35 Tuka tona makoce kin he en opapi kte cin, qa wicate cin etanhan kinipi kta iyececa wicadakapi -kin hena tawicu tonpi śni, qa hihnatonpi śni ece.

36 Qa nakun ake tapi kta okihipi śni; tuka hena mahpiya ohnihde kin om akiyececapi ece, qa wicakini kin he hena cinca kin on etanhan Wakantanka cincawicaya ece.

37 Unkan wicate cin hena kiniwicakiyapi kta, Moses hee kaeś can yuliaka ekta niciyaotaninpi ; Abraham Wakantanka tawa, qa Isaak Wakantanka tawa, qa Jakob Wakantanka tawa, Itancan kin eciye ciqon he ehan.

38 Tona tapi kin hena Wakantanka tawapi śni, tuka ni unpi kin hena : owasin iye ekta ni unpi ece.
39 Unkan wowapi kagapi kin wanjikśi hehan heciyapi; Waonspekiye cin, tanyan eha ce.

$40 \mathrm{He}$ iyohakam tuwedan sanpa wiwanga tawațenye śni.

41 Unkan hewicakiya; Token on Messiya Dawid Cinhintku kin eciyapi he.

42 Odowan wowapi kin en Dawid iye hinca heya; Itancan kin Itancan mitawa kin heciya; Mietapa kin ekta iyotanka wo,

43 Ecen tokaniyanpi kin hena nisiha oahe wicawakage kta ce. 44 Hecen Dawid Itancan eciya hecinhan, token on cinhintku kta he.

45 Unkan hehan oyate owasin nahon hiyeye cin en, waonspewicakiye cin hewicakiya;

46 Wowapi kagapi kin itonwicapa po; he iye sina hanskaska in omanipi, qa iciyopekiciyapi kin en owicakiyapi, qa omniciye tipi kin oyanke tokapa, qa wotapi kin en ohehdepi tokapa kin, hena hecelicen cinpi :

$47 \mathrm{Qa}$ wiwazica tipi tawapi kin temyapi ; qa ihdutaninpi kta on wocekiye hanska eyapi ece; hena iś iyotan woyaco tanka iyahdeiçiyapi kta ce.

\section{WICOWOYAKE 21.}

1 Unkan wankan etonwe, ça wicaśta wijicapi kin womnaye opiye kin en taku wicaqupi kin ehpeya ayapi wanyaka.

2 Unkan wiwazica wanji wahpanica kin iś mazaśadan nonpa en elipeya e wanyaka.

3 Unkan heya; Awicakehan heciciyapi, Wiwazica wahpanica kin de iśnana ota en ehpeya ce.

4 Dena owasin taku iwicakiciyaye cin hee Wakantanka taku qupi kin en elipekiyapi ece; 131 
tuka iye taku ton śni, etanhan. taku on ni kte cin ocowasin en ehpeya ce.

5 Unkan apa iś tipi wakan kin inyan waśteśte, qa taku wicaqupi kin on ayucopi kin, he cajeyatapi qehan, heya;

6 Anpetu hiyohi kta, en taku wandakapi kin dena inyan nonpa icitakihna wanke śni ehpeyapi kta ce.

7 Hehan wiwangapi, qa heyapi; Waonspekiye cin, tohan kinhan hena hecetu kta he; qa hena hecetu kte cin wanna iyehantu kinhan, taku on sdonyapi kta he.

8 Unkan heya; Ihnuhan nihnayanpi kinhan; wicota micaje kin on upi qa, He miye ce, qa wanna ecadan iyehantu kta ce, eyapi kta: tuka wicihakam yapi śni po.

9 Tuka okicize owodutatonpi ko cajeyatapi nayahonpi kinhan, nihinciyapi śni po ; ito tokaheya hena hecen hiyohi kta iyececa, tuka owihanke kin hinyahinke kte śni ce.

10 Hehan hewicakiya; Oyate wan oyate tokeca azuye kta, qa wokiconze wan wokiconze tokeca on inajin kta :

11 Qa otakiya maka kin nina śkanśkan, qa wicaakihan, qa makośikśice, qa wowanyake wokokipe, qa mahpiya kin eciyatanhan wowapetoktokeca, hena hecekcen yuke kta ce.

12 Tuka taku kin dena owasin itokaptu, micaje kin on etanhan iyanihpayapi, qa śicaya nicuwapi, qa omniciye tipi, kaśka wicahnakapi tipi ko en aniyanpi, qa wicaśtayatapi wicaśta iwicadakapi kin ko wicitokam aniyanpi kta ce :

13 Unkan hecen on oniyakapi ece e kta.
14. Hecen icantekicun po; token waaduptapi kte cin he kanyehan awacinpi śni po:

15 Wicai wicoksape ahna cicupi kta, hecen tokaniyanpi kin tuwedan taku iennihdepi kte śni, qa iyakapeniyanpi kte śni.

16 Tuka hunkake wicayayapi, qa hunkawanjin wicayayapi, qa takuwicayayapi, qa koda wicayayapi kin, hena e wiyopeniyanpi kta ; qa apa teniyanpi kagapi kta ce :

17 Qa micaje kin on etanhan owasin niciyuśepi kta.

18 Tuka paha wanjidan kaeś awinitukapi kte śni ce.

19 Wacinnitankapi kin on nicantepi kin hduha po.

20 Jerusalem ihdukśan ozuye yankapi wandakapi kinhan, ihangyapi kte cin he wanna kiyadan e sdonya po.

21 Hehan tona Juda makoce kin en unpi kinhan, he kin ekta najicapi kta; qa tona cokaya en unpi kinhan tankan inapapi kta; qa okśán makoce wanke cin tona en yakonpi kinhan, ekta hdicupi kte śni.

22 Tokiconpi anpetu kin hena ee; hecen taku token owapi qon hena owasin ecen yuśtanpi kta ce.

23 Tuka anpetu kin hena en tona ihduśakapi, qa tona hokśin azinwicakiyapi kin hena wotehi wicakiciyanke kta; maká kin akan wokakije tanka kta, qa wocanniye oyate kin de en awicaun kta.

24 Qa mazasagye ope kin on wankahanpi kta; qa oyate kin owasin ektakta wayaká awicahdapi kta; qa Ikcewicaśta kin Jerusalem naatinzapi kta, ecen Ikcewicasta kin taku iyewicakicihantu kta ce.

25 Anpetu wi, qa hanyetu wi, 


\section{WICOWOYAKE 22.}

qa wicanlipi kin hena ekta wowapetokeca yuke kta; qa maka akan oyate kin yazanhdapi kta, qa wicotoketu tanin śni ; qa miniwanca otaja ko sa wanke kta.

26 Wicaśta kin kopa, qa maka akan taku hiyohi kte cin he ape hiyeye cin, hena on cante toki wicayanka tanin kte śni ece; mahpiya wowaśake kin hena huhuzahe kta heon.

27 Hehan Wicaśta Cinhintku kin mahpiya śapa wan akan, wowaśake qa wookitanin tanka hduha u wandakapi kta.

28 Unkan dena decen aye cinhan, hehan wankan etonwanpi, qa pa yuwankan icu po; openicitonpi kin he wanna kiyadan u ce, eya.

29 Unkan wiyacinpi wan owicakiyaka: Suken can, qa can kin owasin, hena wanyaka po ;

30. Canwapa paha eca, mdoketu wanna ikiyedan, niye cinka wandakapi, qa sdonyayapi ece.

31 Iho he iyececa, taku kin dena iyecetu wandakapi kinhan, Wakantanka tokiconze kin he wanna ikiyedan un e sdonya po. 32 Awicakehan heciciyapi, Wicoicage kin de tokan iyaye śni, ecen dena owasin iyecetu kta ce.

33 Mahpiya kin maka ahna tokan iyaye kta, tuka mioie kin e tokan iyaye kte śni ce.

34 Unkan itonicipa po ; okinni tukten wotapi, qa witkopi, qa wiconi kin de awacinpi kin, hena on nicantepi kin niyuśakapi kta naceca; qa hecen sdonyakiyapi śni ecen anpetu kin he nihiyohipi kte.

35 Maka ite kin akan yakonpi kin owasin wohmuke wan iyecen wicahiyohi kta ce.

36 Heon ohinniyan wakta po, qa cekiya po; -hecen taku hiyohi kte cin dena owasin icunom ida- dapi, qa Wicaśta Cinhintku kin itokam nayajinpi kta iyececa niyawapi kta ce.

37 Unkan anpetu icunhan tipi wakan kin ohna waonspekiya un; qa hanyetu hehan tankan hdicu, qa paha wan Oliwe eciyapi kin he en yanka.

38 Unkan hanhanna hinca oyate kin owasin oie nahonpi kta e tipi wakan kin en ahi.

\section{WICOWOYAKE 22.}

1 Unkan aguyapi napoliyapi śni yutapi woacakśin eciyapi kin, he wanna ecadan iyehantu kte.

2 Unkan wawayuśnapi itancanpi, qa wowapi kagapi kin, hena token econpi, qa teyapi kte cin he akitapi ; tuka oyate kin kowicakipapi.

3 Unkan akenonpapi kin etanhan, wanji Judas Iskariot eciyapi kin he Satan tancan mahen iyaya.

4 Unkan he elkta ye ca, wawayuśna itancanpi, qa akicita itancanpi kin hena om wohdaka, token econ qa wicaqu kte cin heon.

5 Unkan iyuśkinpi, qa mazaska qupi kta keciyapi.

6 Hecen wicada, qa wicota kin en unpi śni icunhan wicaqu kta akita.

7 Unkan aguyapi napohyapi śni anpetu en woacakśin ktepi kte cin wanna hehantu.

8 Hehan Inyan qa Johannes ye wicaśi qa heya; Ya po, woacakśin untapi kte cin he wiyeya unkicihnaka po.

9 Unkan heciyapi ; Tukten wiyeya unhnakapi kta yacin he.

10 Unkan hewicaliya; Otonwe kin en yaipi kinhan, wicaśta wan wakiśkokpa mini ohnaka yuha itkonicipapi kta; iho, iye 


\section{LUKAS.}

tukten tin kihde cinhan, iyahna ya po.

11 Qa tipi tawa kin heciya po; Waonspekiye cin heniciya ce; Wotapi tipi ohna woacakśin kin he waonspewicawakiye cin om wate kte cin lie tukte e he.

12 Kinhan, wankan tipi wan tankaya, qa ayucopi e nicipazopi kta; hen wiyeya hnaka po, eya.

13 Unkan iyayapi, qa token ewicakiye ciqon ecen iyeyapi; qa woacakśin wiyeya hnakapi.

14 Unkan wanna iyehantu qehan, en iyotanka, qa wahośiyapi akenonpapi kin nakun.

15 Unkan hewicakiya; Makakije kte cin he itokamya woacakśin lin de untapi kte cin nina wacin qon.

16 Qa heciciyapi ce; Tohan Wakantanka tokiconze kin ekta yuecetupi śni kin, hehanyan detanhan ake wate kte śni ce, eya.

17 Unkan miniyatkan kin he icu, qa wopida eye ca heya; De icupi qa kicipamni po:

18 Qa heciciyapi ce; Wakantanka tokiconze kin he tohan hiyohi śni kin, hehanyan hastanhanka iyuwi waskuyeca kin etanhan mdatke kte śni ce, eya.

19 Unkan aguyapi kin icu, qa wopida eye, ça yuśpaśpa, qa wicaqu, qa heya ; Mitancan niyepi on qupi kin he dee ce; decen miksuya econ po.

20 Unkan miniyatkan kin iś eya wotapi iyohakamya wicaqu, qa heya; Mawe niyepi kin on papsonpi kin, en okodakiciye teca kin, miniyatkan kin he dee ce.

21 Tuka iho, wiyopemaye kte cin he nape kin wahna wotapi kin akan miciun.

22 Unkan Wicaśta Cinhintku kin token kiconzapi qon ecen ye kta e hecetu; tuka tuwe he wiyopeya hecinhan he wicaśta kin wotelii kiciyanka ce.

23 Unkan hinakaha iye iyalkitedan tukte wanji hecon kte cin he ikiciwanl yeyapi.

24 Unkan tukte wanji iyotan yawapi kte cin he nakun hdokinicapi.

25 Unkan hewicakiya; Oyate kin wicaśtayatapi wicayuhapi kin hena iś iwicadakapi, qa tona wicookihiya owicakihanyanpi kin hena ohanwaśtepi, ewicakiyapi ece.

26 Tuka niś henicecapi kte śni; tuka niyepi kin ekta tukte wanji tokapa kinhan, hee hakakta kin iyecece kta ce.

27 Wota yanke cin, qa wayutankiyapi kin, hena unma tulite iyotan tanka he. Tuwe wota yanke cin hee śni he 'Tuka miś wayutankiyapi kin he iyecen cicipi waun ce.

28 Wawiyutanmayanpi kin en kici maunpi kin he niyepi :

29 Heon Atewaye cin he wokiconze wan maqu kin he iyecen ciçupi :

$30 \mathrm{Qa}$ hecen wokiconze mitawa kin en, wahna wotapi mitawa. kin ekta wayatapi, qa wadatkanpi kta; qa wicaśtayatapi oyanke kin heca akan idotankapi kta, qa Israel wicoun akenonpapi kin wicadacopi kta ce.

31 Hehan Itancan kin heya; Simon, Simon, iho, Satan niyuhe ca, aguyapi su iyecen niyucan kta e cin tuka ce.

32 Tuka wacinyaye cin he iyotpani kte śni e wocekiye eciciya; qa tohan niś niyuhomnipi kinhan, nihunkawanji wicahduwaśaka wo, eya.

33 Unkan heciya; Itancan, wicokaśke wiconte ko en nici mde kta wiyeya waun ce. 


\section{WICOWOYAKE 22.}

34 Unkan heya; Simon, anpetu kin de anpaohotonna hoton śni, ecen yamni akihde sdonmayaye cin he anayakihbe kta, eciciya ce.

35 Unkan hewicakiya; Mazaska ojuha, qa wojuhadan, qa hanpa, hena codan yeciśipi qon he ehan, taku wanjidan kaeś iniciyotpanipi he. Unkan, Takudan śni ce, eyapi.

36 Hehan hewieakiya; Tuka nakaha tuwe mazaska ojuha ton kinhan, ehdaku kta; qa wojuhadan nakun: qa tuwe mazasagye nice cinhan, wokoyake tawa kin wiyopekiye, ça wanji opeton kta.

37 Taku wowapi en kagapi qon, Wicohan śica econpi kin om yawapi ce, eyapi kin he miye en yuecetupi kta, eciciyapi : Unkan dena miye on ecen econpi kta ce.

38 Unkan heciyapi; Itancan, wanyaka wo, den mazasagye nonpa ce. Unkan, Henakeca ce, ewicakiya.

39 Unkan tankan hdicu, qa Oliwe paha kin ekta i ece qon, he ake ekta ya ; unkan waonspewicakiye cin iś iyahna yapi.

40 Unkan wanna ehan i qehan, hewicakiya; Wowiyutanye kin en idadapi kte śni e cekiya po.

41 Hehan wicicunonpa inyan ekta ehpeyapi kin hehanyan ecen $i$, qa canpeśka makehde inajin qa cekiya,

42 Qa heya; Ate hecen nitawacin kinhan, miniyatkan kin de tokan imiciyaye kta; tuka miye mitawacin kin hee śni, niye nitawacin kin hee ecen econpi nunwe.

43 Hehan mahpiya eciyatanhan ohnihde wan en hi qa yuwaśaka.

44 Unkan nina hin yazanhda qehan, iyotan wokitanyan cekiya; qa temni kin he we ośbuye tankinkinyan makata hinlipaya se ececa.

45 Unkan cekiye cin hetanhan wanna najin hiyaye, ça waonspewicakiye cin en wicahdi qehan, wacin iyokiśicapi kin on iśtinmapi iyewicaya.

$46 \mathrm{Qa}$ hewicakiya; Tokeca niśtinmapi he: najinpi qa cekiya po; hecen wowiyutanye kin en yaipi kte śni.

4.7 Hena hecen eya icunhan, iho, wicota, qa akenonpapi kin etanhan wanji Judas eciyapi kin, he wicitokam $u$, qa Jesus i iputake kta e ikiyedan hiyu.

48 Tuka Jesus heciya; Judas, Wicaśta Cinhintku kin i iputakapi wan on wicayaqu he.

49 Unkan kici unpi qon hena, taku toketu kte cin wanna wanyakapi qehan heciyapi; Itancan, mazasagye on waaunpapi kta he.

50 Unkan wanji wawayuśna itancan taokiye wanji ape, ca etapa kin eciyatanhan noge kín kaśpa.

51 Unkan Jesus waayupte ca, Hehanyan iyowinmakiya wo, eye ça noge yutan, qa asniyan.

52 Hehan Jesus wawayuśna itancanpi, qa tipi wakan kin en itancanpi, qa tokapapi en au qon, hena hewicakiya; Mazasagye can ko yuha wamanon sa wan tatpe yapi se iyecen yahipi he.

53 Anpetu iyohi tipi wakan kin ohna opeya ciunpi qon ehan, tuwedan nape kin itkom hiyumayakiyapi śni ; tuka dehan nakaha kin he niye nitawapi, qa otpaza wowaśake kin, eya.

54 Hehan yuzapi qa ayapi, qa wawayuśna itancan ti kin ekta aipi. Unkan Inyan itehan ihakam $u$. 
55 Unkan itankan tipi kin en cokaya cetipi, qa ptaya iyotankapi qehan, Inyan iś eya wicehna iyotanka.

56 Tuka petkahda iyotankehan yanke cin, hehan wikośka wan wanyake ca, opahte ca heya; Wicaśta kin de iś eya he kici un tuka ce, eya.

57 Unkan anakilibe, ca, Winohinca, he tuwe kin sdonwaye śni ce, eya.

58 Unkan iecadan wanji tokeca wanyake ca heya; Niś eya henitanhan ce. Unkan Inyan heya; Wicaśta, hematanhan śni ce.

59 Unkan iyohakam wihiyayedan oape wanji ecetu, hehan wanji.tokeca yaatinze ca heya; Galile wicaśta kin de heca, on etanhan awicakehan iś "eya he kici un tuka ce.

60 Unkan Inyan heya; Wicaśta, token ehe cin he sdonwaye śni ce. Unkan hecehnana, iahan icunhan, anpaohotonna kin hoton.

61 Unkan Itancan kin ihduhomni, qa Inyan atonwan. Hehan Itancan kin, Anpaohotonna hoton śni kin itokam yamni akihde anamayakilibe kta ce, eye ciqon, oie kin he Inyan kiksuya.

62 Hehan Inyan tankan kihde ca nina hin ceya.

63 Unkan wicaśta Jesus yus unpi kin hena ihahapi qa apapi.

$64 \mathrm{Qa}$ ite kin akahpetonpi hehan, ite kin ohna apapi, qa heya iwangapi; Tuwe anipa hecinhan ito-iyukcan wo.

$65 \mathrm{Qa}$ taku toktokeca ota itkom waośtehdaya eciyapi.

66 Unkan wanna anpao qehan, oyate kin en tona tokapapi, qa wawayuśna itancanpi, qa wowapi kagapi kin, hena mniciyapi, qa omniciye kin en aupi ;
67 Qa heyapi ; Messiya he niye hecinhan unkokiyaka po. Un. kan hewicakiya; Ociciyakapi eśta wicayadapi kte śni.

$68 \mathrm{Qa}$ wiciwangapi eśta amayaduptapi kte śni, qa amayaduśtanpi kte śni.

69 De iyohakam Wicaśta Cinhintku kin Wakantanka towaśake etapa kin eciyatanhan iyotanke kta ce, eya.

70 Unkan o cen Wakantanka Cinhintku kin he niye he. Unkan, He miye kehapi ce, ewicakiya.

i1 Unkan heyapi; Iye i kin eciyatanhan unkiyepi hinca wanna naunhonpi ce; hecen tokeca ecin sanpa wayaotaninpi unkodepi kta he.

\section{WICOWOYAKE 23.}

1 Unkan wicota kin owasin najin hiyayapi, qa Pilate kiayapi.

2 Hehan hinnakaha wiyaonpapi, qa heyapi; De oyate kin owicayuśice, ca Kesar wamnayanpi qupi kin he tehinda iyeunyanpi; qa iye Messiya Wicaśtayatapi kin heca keiçiya ce, eyapi.

3 Unkan Pilate wiwange ca heya; Juda Wicaśtayatapi kín he niye he. Unkan ayupte, ca, Niye ecen eha ce, eya.

4 Hehan Pilate wawayuśna itancanpi qa oyate kin hena hewicakiya; Wicaśta kin de on taku śica iyewaye śni ce.

5 Unkan hecen iyotan wohitipike ca heyapi; Oyate kin owodutatonwicaya ece, qa Galile etanhan Juda makoce kin owancaya dehan oiyahdeya waonspekiya ece.

6 Unkan Pilate Galile eyapi nalion qehan, wicaśta kin he 
Galile wicaśta kin heca hecinhan he iwicawanga.

7 Unkan Herod taoyate kin hetanhan e Pilate sdonya, hehan Herod ekta iyayeya; he qe wanna Jerusalem en un.

8 Herod Jesus wanyake cehan nina iyuśkin; taku ota on nahon kin heon wanakajatanhan wanyaka cin; qa wowapetokeca wanji econ wanyake kta kecin.

9 Hehan iapi ota un wiwanga; tuka takudan ayupte śni.

10 Unkan wawayuśna itancanpi, qa wowapi kagapi kin, hena inajinpi, qa wohitiya wiyaonpapi.

11 Unkan Herod ozuye wicaśta wicayuhe cin om takuda śni, qa ihahia kuwa, qa śina wan ayucopi in kiye, ca ake Pilate ekta iyayeya.

12 Unkan anpetu kin he en Herod Pilate kici kiciyuwaśtepi ; he itokamya tokakiciyapi.

13 Unkan Pilate wawayuśna itancanpi, qa itancanwicakiyapi, qa oyate kin hena witaya wicakico qehan, hewicakiya;

14. Wicaśta kin de oyate kin owicayuśice cin wan iyecen mayakahipi; unkan iho nitokam iwanmdaka, tuka taku iyayaonpapi kin hena on, taku śica wicaśta kin de en takudan iyewaye śni.

15 Qa nakun Herod hee kaeś, ekta aye ciśipi; unkan iho, te kte cin iyecece cin takudan ecakiconpi śni.

16 Hecen wakapsinpsinte, ça wakimduśke kta ce, eya.

17 Wotapi kin he icumhan wicaśta wanji wicakiciyuśke kta e heon.

18 Unkan owasin okonwanjidan heya niyanpi; De tokan aya wo; qa Barabbas hee unkiciyuśka miye.

19 He qe otonwe kin en woki- pajin kage, ca tinwicakte on etanhan kaśka wicahnakapi tipi kin en ohnakapi.

20 Pilate Jesú kiyuśke wacin kin heon ake iyecen ewicakiya.

21 Tuka howayapi qa heyapi ; Icipaweh okatan wo, icipaweh okatan wo.

22 Unkan iyamni hewicakiya; Tokeca, tukte ecin taku śica econ lie. On etanhan te kte cin iyececa takudan iyewaye śni; hecen wakapsinpsinte, ça wakimduśke kta ce, eya.

23 Unkan hotankakiya kitanpi, icipaweli okatanpi kta e he kapi. Unkan iye hopi kin, wawayuśna itancanpi kin om iyakapeyapi.

24 Hecen token cinpi kin he iyecen Pilate kiconza.

$25 \mathrm{Qa}$ tuwe cinpi kin wokipajin kage, ca tinwicakte qon, hee wicakiciyuśke; tuka iye tawacinpi kin en Jesus. wicaqu.

26 Unkan tokan yus ayapi qehan, Kurene wicaśta wan Simon eciyapi makoce kin eciyatanhan u kin he yuzapi, qa cansusbeca kin he Jesus ihakam qin kta e aihpeyapi.

27 Unkan oyate kin wicota linca winohinca ko iyahna yapi, qa waśihda a ceyapi.

28 Tuka Jesus ekta ihduhomni qa hewicakiya; Jerusalem cunwintkupi kin, miye amaceyapi śni po ; tuka niyepi qa nicincapi kin aiçiceya po.

29 Iho, anpetu hiyohi kte cin hena en heyapi kta; Hokśin yuhapi śni, qa wicitpi tohinni cincaton śni, qa aze tohinni azinpi śni kin hena yawaśtepi ce. 30 Hehan hinnakaha he kin, Unkahinhpaya po, qa paha kin, Unkahpa po, eciyapi ece e kta. 31 Qa can teca kin en decen 
econpi kinhan, can śeca kin en token econpi kta he, eya.

32 Unkan wicaśta tokeca nonpa, ohan śicapi kin, hena wicaktepi kta e kici awicayapi.

33 Unkan tukten Wicapahu eciyapi kin wanna ehan ipi qelan, hen icipaweh okatanpi, qa nakun wicolian śica econpi qon hena, unma iye etapa eciyatanlian, qa unma kin iś catka tanhan.

34 Hehan Jesus heya; Ate, wicakicicajuju wo ; taku tokonpi kin sdonkiyapi śni ce. Unkan tawokoyake kin kicipamnipi, qa oeconna econpi.

35 Unkan oyate kin opalita najin hiyeya: Qa itancanwicakiyapi kin iś eya hena om ihahapi qa heyapi; Wicaśta tokeca niwicaya ece qon; iye atayedan niiçiya eśta śni, Messiya Wakantanka hdahinige cin hee hecinhan.

36 Unkan akicita kin iś eya ihahapi, en au, qa miniskuya kipazopi ;

37 Qa heyapi ; Juda wicaśtayatapi kin he niye hecinhan, niiçiya wo.

38 Unkan iwankam wowapi kin de, Helenes qa Roma qa Hebrew iapi henakiya en owapi ; JUDA WICAŚTAYATAPI KIN HE DEE CE.

39 Unkan ohan śicapi en owicakatanpi qon wanji śicaya owakiye ca heya; Messiya he niye hecinhan niiçiya wo, qa unkiś nakun.

40 Tuka unma kin waayupte ca kiśice ça heya; Wakantanka koyakipe śni he; wicayacopi okonwanjidan kici en yaun :

$41 \mathrm{Qa}$ unkiye kin en he owotanna; taku econku eciyatanhan unkakipa ece; tuka iye qe taku śica takudan econ śni ce.

42 Unkan hehan Jesus heciya;
Itancan, wokiconze nitawa kin en yaku kinhan, miksuya wo.

43 Unkan Jesus heciya; Anpetu kin de en wowaśte makoce kin en mici yaun kta ce, awicakehan eciciya.

44. Wanna wihiyayedan oape śakpe hehantu; unkan makoce kin owancaya aotpaza, naṕcinwanka apa hehanyan.

$45 \mathrm{Qa}$ anpetu wi kin otpaza icu, qa tipi wakan kin en minihuha ozanzan hiyeye cin he cokaya okinalideca.

46 Unkan Jesus hotankakiya hoyekiye cehan, hehan heya; Ate, ninape kin en minagi kin owehnaka ce. Unkan hecen eye cehan ta iyaya.

47 Unkan akicita opawinge wicayuhe cin he token econpi qon he wanyake cehan, Wakantanka yaonihan, qa heya; Awicakehan wicaśta owotanna he de heca ce. 48 Unkan oyate kin witayahe wanyag ahi qon, hena owasin token econpi qon liena wanyakapi qehan, maku kin ohnahna aiçipapi, qa kihdapi.

49 Unkan tona sdonyapi qon hena owasin itehan inajinpi, qa winohinca Galile etanhan ihakam upi qon hena taku kin dena wanyakapi.

50 Unkan iho, wicaśta wan Joseph eciyapi, wahokonwicakiya ece kin heca, wicaśta waśte qa owotanna;

51 He iye kin wakiconzapi qa olianpi qon wicawicada śni : Arimathea, Juda totonwepi kin wanji hee etanhan; iś eya Wakantanka tokiconze kin he ape un.

52 He Pilate ekta ye ça Jesus tancan kin kida.

53 Unkan kun eyaku, qa minihuha ska opazunte, ca imnija kalidokapi wicaśta hnakapi wan 
en ohnaka; he itokam tuwedan en ohnakapi śni.

54 Unkan anpetu en ihduwiyeyapi ece kin he hee, qa anpetu wakan kin wanna ikiyedan.

55 Unkan winohinca Galile etanhan kici upi qon hena ihakam iyayapi, ohna hnakapi kin he, qa tancan kin token hnakapi kin hena wanyakapi.

56 Qa hetanhan kihdapi, qa pejihuta waśtemma ihepi ko wiyeya ehnakapi. Hehan woahope kin eciyatanhan anpetu wakan kin okilipapi.

\section{WICOWOYAKE 24.}

1 Unkan anpetu wakan en anpetu tokaheya, hanhanna linca, hehan wicaśta hnakapi kin ekta, pejihuta waśtemna wiyeya ehnakapi qon hena yuha en upi; qa toktokeca nakun owicapapi.

2 Unkan wicaśta hnakapi kin etanhan inyan kin wanna tokan iyeyapi e wanyakapi :

3 Qa mahen ipi, tuka Jesus Itallcan tancan kin he iyeyapi śni.

4 Unkan token iyukcanpi nun tanin śni qehan, iho, wicaśta nonpa wokoyake wiyatpatpa unpi icahda nawicajinpi.

5 Unkan kokipapi, qa ite kin makipuskin ehpeiciyapi qehan, hewicakiyapi; Tolkeca e tapi kin wicehna ni un lkin ayakitapi he. 6 Den un śni ; tuka kini ce. Nahanliin Galile en un qon ehan token eniciyapi qon he kiksuya po:

7 Wicaśta Cinhintku kin wicaśta śica napepi kin en wicaqupi kta, qa icipaweh okatanpi kta; tuka anpetu iyamni kinhan kini kta ce, eya ce.

8 Unkan hecen eye ciqon he kiksuyapi.

9 Unkan wicaśta hnakapi kin etanhan kihdapi, qa akewanjipidan unmapi ko hena owasin owicakiyakapi.

10 Unkan wahośiyapi kin hena hecen owicakiyakapi qon, Mary Magdalene, qa Joanna, qa Mary Jakob hunku kin, qa toktokeca om unpi qon hena eepi.

11 Unkan oiepi kin ikcekceya hitunkakanpi kin iyececa dakapi, qa wicawicadapi śni.

12 Hehan Inyan najin hiyaye ça wicaśta hnakapi kin ekta inyang iyaya; qa en eyokasin qehan minibuha wokoyake kin iśnana ehnakapi wanyaka; hehan hdicu qa cante mahen taku econpi kin on inihanyan awacin un.

13 Unkan iho, anpetu kin he en, iyepi kin etanhan wicaśta nonpa otonwe wan Emmaus eciyapi kin he ekta yapi, Jerusalcm etanhan maka iyutapi wikcemna śakpe ecetu.

14 Unkan taku hiyohi kin dena owasin on wohdakapi.

15 Unkan wohdakapi, qa iyukcan awacinpi icunhan, Jesus iye hinca kiyedan $u$, qa om wicaya.

16 Tuka iśta on iyekiyapi kte śni wicayapi.

17 Unkan hewicakiya; Wicoie kin dena tukte e he, mayanipi icunhan woyahdakapi qa iyokiśin yaunpi kin.

18 Unkan unma Kleophas eciyapi kin he waayupte ca heciya ; Jerusalem en yaun śni ece, dehan en taku liyohi kin dena sdonyaye śni he.

19 Unkan hewicakiya; Hena taku he. Unkan heciyapi; Jesus Nazareth etanhan kin on, he wicaśta wokcan, wicohan wicoic ko on waśaka, Wakantanka qa oyate kin owasin wicitokam.

$20 \mathrm{He}$ wawayuśna itancanpi qa 


\section{LUKAS.}

itancanwicunkiyapi kin hena te kta kiconzapi qa icipaweh okatanpi.

21 Tuka Israel opewicakiton kte cin hee unkiśs unkecinpi tuka; unkan heconpi kin ehantanhan wanna anpetu kin de iyamni.

22 Nakun unkanpi kin etanhan winohinca wanjikji hanhanna linca wicaśta hnakapi kin ekta ipi, qa inihanunyanpi :

23 Iye tancan kin iyeyapi śni qehan, hdicupi qa ohnihde wakan kin heca wanwicayakapi, qa hena he ni un keyapi. ce, eya unkokiyakapi.

24 Hehan om unkanpi kin wanjikji wicaśta hnakapi lkin ekta ipi, qa winolinea oyakapi qon iyececa hinca iyeyapi : tuka iye kin wanyakapi śni ce.

25 Unkan hewicakiya; Witkotkokapi qa wicaśta wokcan taku oyakapi qon hena owasin wicada cante hunkapi śni kin:

26 Messiya dena decekcen ito kakije ca wowitan tawa kin en hde kte cin he hecetu śni he.

27 Unkan hecen Moses e tokahcya, qa hehan wicaśta wokcan owasin, taku iye kin on wowapi en kagapi qon hena owasin owicakiyaka.

28 Unkan otonwe ekta yapi qon he wanna ikiyedan ehan ipi qehan, iye sanpa ye kta se ececa.

29 Tuka iyakapeyapi, qa heyapi : Unkicipi un wo, wanna htayetu aya, qa anpetu kin wamna owihanke kta. Unkan om un kta e ti mahen iyaya.

30 Unkan om wota yanke cehan, aguyapi eyaku, qa yawaśte, qa yuśpaśpa, qa wicaqu.

31 Hehan iśta kin wicalkiciyukawapi, qa iyekiyapi : unkan atanin śni iyaya. 32 Unkan hekiciyapi; Canku iyececa ce; eya. kin ohna wounkiyakapi qa wo: wapi wakan kin unkiciyuzamnipi qon he ehan cante unkata śni he. 33 Unkan wihiyayedan oape kin he en najin hiyayapi, qa Jerusalem ekta hdapi, qa akewanji tona om unpi ko hena witaya yulk anpi iyewicayapi ;

34 Qa heyapi; Ehanqon Itancan kin kini, qa Simon en tanin. iciya ce.

35 Hehan iye canku kin ohna tokenketu, qa aguyapi yuśpaśpa kin on iyekiyapi qon, hena he: cekcen owicakiyakapi.

36 Unkan hena hecen eyapi icunhan, Jesus iye hinca wica cokaya hinajin qa hewicakiya; Wookiye nicipi un nunwe.

37 Unkan inihanpi, qa kokipapi, qa wanagi wanyakapi kecinpi.

38 Unkan hewicakiya; Tokeca e iyoniciśicapi he; qa tokeca cante mahen taku cetunyahdapi he.

39 Minape misiha ko, de miye hinca e wanmayaka po ; mayutanpi qa iyukcan po; celipi huhı waton wandakapi kin wanagi kin iyecen ton śni ece.

40 Unkan hecen eye cehan, nape siba ko wicakipazo.

41 Unkan wowiyuśkin on wicadapi śni, qa hinyahin inihanpi, hehan hewicakiya; Den taku yutapi duhapi lie.

42 Unkan hogan pasnonpi onśpa tulimaga canhanpi ahna qupi.

43 Unkan icu, qa wicitokam yuta.

44 Qa hewicaliya; Nahahin om ciunpi qon ehan, wicoie ociciyakapi qon he dee ce; Moses wicoope tawa, qa wicaśta wokean, qa odowan kin, henakiya en miye on taku token owapi qon owasin ecen yuśtanpi kta 


\section{WICOWOYAKE 1.}

45 Unkan hehan tawacinpi kin wicakiciyuzamni, qa hecen wowapi kill okahnigapi kte.

46 Unkan hewicakiya; Hecen owapi, qa Messiya hecen kakije ca, anpetu iyamni kinhan ake kini kte ciqon, he hecetu :

47 Qa hecen iye caje kin on ihduecetupi qa woahitani kajujupi kin, oyate kin owasin en oyakapi kta, Jerusalem en tokaheya kta.

48 Qa taku kin dena oyalkapi kte cin he niyepi ce.

49 Unkan iho, Ate taku iwahowicaye ciqon he niyepi kin en uwakiye kta; tuka tohanyan wankantanhan wowaśake yuhe- niciyapi śni kin hehanyan Jerusalem otonwe kin en ece yukan po.

50 Hehan tankan awicaya, Bethani hehanyan; unkan nape wicakiyuwinta qa wicayawaśte.

51 Unkan wicayawaśte icunhan, yutokan ewicakiciyakupi, qa wankan mahpiya kin ekta ayapi.

52 Unkan iye ohodapi, qa wowiyuśkin tanka yuha Jerusalem ekta kipi :

53 Qa ohinniyan tipi wakan kin ohna ece yukanpi, Wakantanka yatanpi qa yawaśtepi ece. Amen.

\section{JOHANNES}

\section{WOTANIN WAŚTE TAWA.}

\section{WICOWOYAKE 1.}

1 Otokahe ekta Wicoie kin hee; Wicoie kin he Wakantanka kici un, qa Wicoie kin he Wakantanka kin ee.

2 He otokahe ekta Wakantanka kici un.

3 Iye eciyatanhan taku owasin kagapi; qa taku kin tokan tanhan takudan kagapi śni.

4 Iye kin en wiconi; qa wiconi kin he wicaśta iyoyamwicaye cin hee.

5 Iyoyanpa kin hee otpaze cin en omdesya un, tuka otpaze cin he iyowinkiye śni.

6 Wicaśta wan Wakantanka eciyatanhan u śipi, he Johannes eciyapi.

7 He wayaotanin hi, Iyoyanpa kin oyake kta; hecen iye eciyatanhan owasin wicadapi kta.
8 Iyoyanpa kin he iye śni, tuka iyoyanpa kin he yaotanin kta e u śipi.

9 Iyoyanpa wicakapi lince cin hee, wicaśta otoiyohi wicoicage kin en u iyoyamye cin hee.

$10 \mathrm{He}$ wicoicage kin en un, qa iye wicoicage kin kaga, tuka wicoicage kin sdonyapi śni.

11 Tona tawa kin hena ekta wicahi, tuka tawawicaye cin hena iyowinkiyapi śni.

12 Tuka tona iyowinkiyapi kin hena Wakantanka cinca wicaya yakonpi kta e okihi wicaya, tona iye caje wicadapi kin hena.

13 Hena wicawe eciyatanhan śni, qa wicacelipi tawacin kin eciyatanhan śni, qa nakun wicaśta tawacin kin eciyatanhan śni, tuka Wakantanka eciyatanhan wicatonpi.

14 Unkan Wicoie kin he wica. 
cehpi kagapi, qa unkiyepi kin en ounyan; qa wootanin tawa kin wanunyakapi, wootanin kin he Atkuku eciyatanhan iśnana icaga tawa kin he iyececa; wowaonśida wowicake ko ojudan.

15 Johannes he yaotanin, qa heya niyan; Tuwe mihakain u kte cin he mitokam yawapi ce, epe'ciqon he dee; he mitokam un nakaeś.

16 Unkan woojudan tawa kin etanhan unkiye owasin unkicupi, qa he wowaśte wicowaśte lin on.

17 Woope kin he Moses wicaqu ; tuka wicowaśte wowicake ko hena Jesus Messiya eciyatanhan $\mathrm{u}$.

18 Tuwedan tohinni Wakantanka wanyake śni; tuka Cinhintku iśnana icaga Atkuku maku ohna un kin he sdonye unkiyapi.

19 Unkan Johannes woyake ciqon he dee; Jerusalem etanhan Juda oyate Wośnapi kagapi Lewi wicaśta ko ekta ye wicaśipi qa, Tuwe niye he, eciyapi.

20 Tuka Johannes ohdake ca anakilibe śni, tuka ohdaka; Messiya kin he miye śni ce.

21 Unkan wiwangapi; Nituwe he; Eliyas he niye he. Unkan, He miye śni ce, eya. Wicaśta wokcan kin he niye he, eciyapi. Tuka, Hiya, eya.

22 Hehan, Ecin tuwe niye he, eciyapi : tona deciya unkuśipi hecinhan hośi wicunkakipi kta e heunniciyapi ; Token onihdaka he.

23 Unkan heya; Jehowa tacanku kin owotanna kicaga po, tuwe hewoskan eyanpaha un kin he miye ce, Isaya wicaśta wokcan eye ciqon iyececa.

24. Unkan tona en u wicaśipi qon hena Pharisee etanhanpi.

142
25 Unkan wiwangapi qa heciyapi; Hecen tokeca e baptem wicayaqu he; Messiya he niye śni, qa Eliyas he niye śni, qa wicaśta wokcan kin he niye śni hecinhan.

26 Johannes awicayupte ca heya ; Miye mini on baptem wicawaqu ece : tuka wanji nayajinpi kin cokaya najin tuka sdonyayapi śni ;

27 Tuwe mihektam u kte ciqon, tuka mitokam yawapi kin hee; tahanpe kin wakiyuśdoke kta eśta owakihi śni ce.

28 Hena taku kin Bethabara, Jordan akasanpatanhan, econpi, he en Johannes baptem wicaqu.

29 Anpetu iyokihe hehan Jesus etkiya u kin Johannes wanyake ca heya; Tacinca Wakantanka tawa, oyate wahtanipi kin tokan elipeye kte cin he wanyaka po.

30 Tuwe on hepe ciqon hedee; Wicaśta wan mihakam u kte cin he mitokam yawapi; he mitokam un nakaeś.

31 Qa miś eya he sdonwaye śni; tuka Israel oyate kin ekta yuotaninpi kta e beon mini on baptem wicawaqu wahi ce.

32 Unkan Johannes wayaotanin qa heya; Woniya kin he wakiyedan kaliya mahpiya cciyatanhan kuntkiya u wanmdaka, unkan he en hiyanka.

33 Unkan he tuwe kin sdonwaye śni, tuka mini on baptem wicaqu maśi qon he omakiyaka; Tohan malipiya kin eciyatanhan Woniya kin kuntkiya ye ca, tuwe iyahde wandake cinhan he iye Woniya Wakan on baptem wicaqu kta.

34 He wanmdaka e on mdaotanin; Wakantanka Cinhintku kin hee. 


\section{WICOWOYAKE 2.}

35 Ake ihanhanna hehan Johannes waonspewicakiye cin nom om en najin :

36 Qa Jesus omani wanyake cehan heya; Iho, Tacinca Wakantanka tawa kin he dee ce.

37 Unkan waonspewicakiya nom ie cin he nahonpi, qa Jesus ihakam yapi.

38 Hehan Jesus ihdamna, qa ihakam upi wanwicayake ca hewicakiya; Taku ayakitapi he. Unkan, Itancan, Waonspekiye eyapi kin he kapi, tukten yati he, eciyapi.

39 Upi qa wanyaka po, ewicakiya. Unkan kici hdapi, qa tukte en ounye cin he wanyakapi, qa anpetu ihunniyan kici yukanpi : wanna oape wikcemna hehantu.

40 Nom Johannes ia nalionpi qa Jesus ihakam yapi qon, Andrew Simon Inyan sunkaku kin he unma ee.

$41 \mathrm{He}$ cincu Simon tokaheya iyeye ca heciya; Messiya, he Sdayapi eyapi kin, iyeunyanpi ce :

42 Qa Jesus ekta ai. Unkan Jesus wanyake cehan heya ; Simon Jona cinhintku kin he niye: Kephas eniciyapi kta, he Inyan eyapi kin hee.

43 Ihanhanna kin hehan Jesus Galile heciya ya, unkan Philip wanyake ca heciya; Mihakam u wo.

44. Philip Bethsada hetanhan, nakun Andrew Inyan kici he otonwe tawapi.

45 Philip Nathanael akipa, qa heciya; Moses woope en owa qon, qa wicaśta wokcanpi kin nakun, he wanna iyeunyanpi, Jesus Nazareth etanhan, Joseph cinhintku kin hee.

46 Unkan Nathanael heciya; Nazareth etanhan taku waśte $u$ okihi kta he. U qa wanyaka wo, Philip eciya.

47 Jesus Nathanael en $u$ wanyake ca he on heya; Wanyaka po, Israel wicaśta łinca wan he dee, wicahnaye wanin un ce.

48 Nathanael heciya; Tokiyatanhan sdonmayaya he. Jesus ayupte ca heciya; Philip nico qon he itokam can ihukuya nayajin qon hen wanciyaka.

49 Nathanael ayupte ca heciya; Itancan, Wakantanka Cinhintku, Israel oyate Wicaśtayatapi kin he niye.

50 Unkan Jesus ayupte ca heciya ; Can ihukuya nayajin wanciyaka epa, unkan heon wicayada he. Dena taku isanpa tanka wandake lita ce.

51 Unkan heciya; Wowicake, wowicake eciyatanhan heciciyapi, Tokata mahpiya kin yuzamnipi, qa Wakantanka taokiye kin wankantkiya yapi, qa ake kuntkiya upi, qa Wicaśta Cinhintku kin en himniciyapi wandakapi kta ce.

\section{WICOWOYAKE 2.}

1 Anpetu iciyamni kin hehan Galile makoce en otonwe wan Kana eciyapi hen kiciyuzapi omniciye kagapi ; unkan Jesus hunku kin en opa.

2 Jesus nakun waonspewicakiye cin on kiciyuzapi kin en kicopi.

3 Unkan miniśa wanna henakeca hehan, Jesus hunku kin heciya; Miniśa takudan yuhapi śni ye.

4 Unkan Jesus heciya; Winohinca hetanhan iwamayatokiya he; nahanhin iyemicihantu śni ce.

5 Unkan hunku kin wayutan wicakiyapi, He tokehin eniciya- 


\section{JOHANNES.}

pi owasin ecen ecom, ewicakiya.

6 Inyan wakiśkokpa śakpe en laan, ohna Juda oyate ihdujajapi kta e hdepi; otoiyohi wiyutapi nom yamni kipi.

7 Wakiśkokpa kin mini ojudan okaśtan po, ewicakiya. Unkan ojugjudan okaśtanpi.

8 Hehan, Onge etanhan icupi qa omniciye itancan kin he kaya po, Jesus ewicakiya. Unkan kayapi.

9 Unkan omniciye kin en itancan kin onge yatkan uta, he mini tuka miniśa icahyapi, qa he tokiyatanhan kin sdonye śni, tuka wayutan wicakiyapi mini akupi qon hena sdonyapi, unkan itancan kin tawicu ton qon kico ;

10 Qa heciya, Wicaśta eca miniśa waśte tokaheya yatke wicakiya ece; qa tohan wicaśta wipipi hehan taku aoptetu kin; tuka niye miniśa waśte linca dehanyan yehnaka ce, eya. 11 Galile makoce kin hen, Kana otonwe he en, Jesus toka taku wapetokeca econ, qa towitan hdutanin; unkan hecen waonspewicakiye cin wicadapi.

$12 \mathrm{He}$ iyohakam Kapernaum ekta apamahde iyaya, iye qa hunku qa sunkaku qa waonspewicakiye cin hena koya om; unkan anpetu tonana hen yalronpi.

13 Unkan Juda oyate woacakśin wośnapi tawapi kin wanna ikiyedan; heon Jesus Jerusalem ekta itanwankanhde iyaya.

14 Unkan tipi wakan kin en wicaśta kin eya, tatanka, tahinca, wakiyedan ko wiyopewicakiyapi, qa maza ska tokiyopekiciyapi kill en iyotankapi e wanwicayaka ;

15 Qa icapsinte cikciqadan yu144. gaga wan kage ceehan, tipi wakan kin etanhan owasin tankan iyewicaya, tahinca tatanka ko; qa mazaska tokiyopekiciyapi qon iś kada iyeya, qa can wahna wotapi kin he nakun paahdapśinyan elipeya.

$16 \mathrm{Qa}$ wakiyedan wiyopeyapi kin iś, Dena owasin tankan iyeya po, qa, Ate ti kin de wopeton tipi kagapi śni po, ewicakiya.

17 Unkan waonspewicakiye cin wowapi en kagapi qon he kiksuyapi, Yati owakiye cin he mayapota ce.

18 Hehan Juda oyate ayuptapi qa heciyapi; Tokiya tanhan he oyakihi qa hecanon he.

19 Unkan Jesus waayupte ca, Tipi kin de ihangya po, qa anpetu iciyamni kinhan ake ekicetuwaye kta, ewicakiya.

20 Waniyetu wikcemna topa qa sam sakpe hehanyan tipi kin de kagapi qa yuśtanpi qon, niye nakaha anpetu iyamnina ekicetu yaye kta he, Juda oyate kin eciyapi.

21 Tuka tipi eye cin he iye tancan kin he ka e heon etanhan heya.

22 Heon wiconte etanhan ihduekicetu, hehan hecen ewicakiye ciqon he waonspewicakiye cin kiksuyapi; unkan wowapi wakan qa Jesus taku ewicakiye cin hena koya wicadapi.

23 Unkan Jerusalem en woacakśin wośnapi kin icunhan, taku wapetokeca econ kin hena wicota wanyakapi qehan iye caje kin on wicadapi.

24 Tuka Jesus owasin sdonwicaye cin lieon etanhan wacinwicaye śni.

25 Qa wicaśta on tuwedan wayaotanin kta cin śni ; iye ataye-dan wicaśta taku en un kin sdonya, heon etanhan. 
WICOWOYAKE 3.

1 Wicaśta wan Pharisee etanhan Nikodemos eciyapi, Juda oyate itancanpi kin lie wanji ee; 2 Hanyetu wan Jesus yanke cin en i qa heciya; Itancan, waonspekiye Wakantanka eciyatanhan u kin henica sdonunniyanpi; taku wapetokeca ecanon ece kin tuwedan hecen okihi śni, Wakantanka kici un śni linhan.

3 Unkan Jesus ayupte ca heciya; Wowicake, wowicake eciyatanhan heciciya, Wicaśta kin wankantanhan teca nien tonpi śni kinhan Wakantanka tokiconze kin wanyaka okihi kte śni.

4 Wicaśta kin kan elantanhan token ecin teca ni en tonpi kta he. Ake hunku tezi kin mahen kihde ca ake teca ni en tonpi lita he token okihi kta he, Nikodemos eciya.

5 Unkan Jesus ayupte; Wowicake, wowicake eciyatanhan heciciya, Wicaśta kin mini qa Woniya kin on tonpi śni kinhan Wakantanlia tokiconze kin en tin iyaya okihi kte śni ce, eya.

6 Tuwe wicacelipi on tonpi kin he wicacehpi, qa tuwe Woniya kin eciyatanhan tonpi kin he woniya.

7 Wankantanhan nitonpi kta ce, eciciye cin heon ihnuhan niyuśinyaye cin.

8 Tateyanpa kin tokiyatanhan cin eca eciyatanhan ipogan ece, qa oqo kin he nayahon, tuku tokiyatanhan $u$, qa tokiya ye cin he sdonyaye śni. Wicaśta Woniya kin eciyatanhan tonpi kin owasin hecetu.

9 Nikodemos ayupte ça heciya; Hena token okihipica he.

10 Jesus ayupte ca heciya; Israel en waonspekiye cin heni- ca, tuka dena oyakahnige śni he.

11 Wowicake, wowicake eciyatanhan heciciya; Taku sdonunyanpi kin hena unkeyapi ece, qa taku wanunyakapi kin dena unkoyataninpi, tuka wounyataninpi kin ayaktapi śni.

12 Taku maka kin detanhan ociciyakapi kin wicayadapi śni ehantanhan, mahpiya kin ekta taku ociciyakapi kinhan hee token wicayadapi kta he.

13 Nahahin tuwedan mahpiya kin ekta i śni, tuwe mahpiya kin eciyatanhan kuntkiya $\mathrm{u}$, qa hi kin he Wicaśta Cinhintku kin hee, nahahin nalsun mahipiya kim ekta yanka.

14 Nakun hewoskan wanmduśka Moses wankan ehde qon he iyecen Wicaśta Cinhintku kin wankan elıdepi kta.

15 Heon tuwe awacin kinhan owihanke lite śni, qa owihanke wanin wiconi yuhe kta.

16 Wakantanka oyate kin cantewicakiya, heon Cinhintku iśnana icage cin wicaqu, qa tuwe awacin kinhan owihanke kte śni, tuka owihanke wanin wiconi yuhe kta.

17 Wakantanka Cinhintku kin wicoicage kin en u śi kin, heon oyate kin wicayaco kta e hecon śni ; tuka iye on oyate kin nipi kta e hecon.

18 Tuwe he wacinye cinhan he yacopi kte śni; tulka tuwe wacinye śni kinhan he wanna. yacopi ; Wakantanka Cinhintku iśnana icage cin he caje kin wacinye śni kin heon etanhan.

19 Wicayacopi kin he kaketu; Oyate kin iyoyanpa en wicahiyohi, tuka wicaśta kin otpaza e waśtedakapi, qa iyoyanpa e cinpi śni, wicohan śicapi kin heon.

20 Tuwe taku síica econ kin he 145 


\section{JOHANNES.}

iyoyanpa śice daka, qa iyoyanpa ikiyedan u śni, ohan iyopeyapi kte cin heon.

21 Tuka tuwe wowicake eciyatanhan ohanye cin he iyoyanpa kin en $u$, ohan yuotaninpi kta, Wakantanka eciyatanhan ohanye cin heon.

22 Hena iyohakam Jesus waonspewicakiye cin om Juda makoce kin en i; qa en om un, qa baptem wicaqu.

23 Unkan he icunhan Johannes iś eya Enon Salim ikiyedan baptem wicaqu, hen mini ota kin he etanhan. Unkan baptem wicaqupi kta e etkiya aye.

24 Hehanyan hinyahin Johannes kaśkapi śni.

25 Hehan taku hdokinicapi, Johannes waonspewicakiye cin wanjikji qa Juda wicaśta om, wicayujajapi kin he icahtagya.

26 Unkan Johannes ekta ipi qa heciyapi; Itancan, Jordan wakpa akasanpa tanhan tuwe kici yaun qa daotanin qon he, iho, wanna baptem wicaqu, unkan owasin en ece iyoptapi.

27 Unkan Johannes waayupte ça heya; Wicaśta kin mahpiya eciyatanhan taku qupi śni ehantanhan takudan iye cinka icu okihi kte śni ece.

28 Messiya kin he miye śni, tuka itokam u maśipi ce, epe ciqon he niye sdonyayapi.

29 Tuwe tawicu ton kin he hihna yanpi kin hee, tuka hihna yanpi takodaku kin en taninyan anagoptan najin, qa hihna yanpi ho kin nahon kin heon iyuśkin ece. Heon etanhan wowiyuśkin mitawa kin wanna yuecetupi ce.

30 Iye kin nina sanpa icage kta, tuka miye yuhukun amaye kta.

31 Tuwe wankan tanhan hi kin 146 he taku owasin iwankam ounye. Tuwe maka etanhan hi kin he maka etanhan, qa maka kin iyecenya oie kiton. Tuwe malipiya kin eciyatanhan hi kin he taku owasin on iyotan.

32 Qa taku wanyake ca taku nahon kin he owasin yaotanin ece ; tuka taku yaotanin kin tuwedan wicada śni.

33 Taku yaotanin kin he tuwe wicada kinhan Wakantanka wicake cin he yutanin ece.

34 Wakantanka tuwe u śi kin he Wakantanka oie oyaka; Wakantanka Taniya kin iyuta qu śni, ocowasin qu.

35 Atkuku kin Cinhintku waśtekidake ça, taku owasin iye nape kin en qu :

36 Hecen tuwe Cinhintkuyapi kin wacinye cinhan owihanke wanin wiconi kin wanna yuha; tuka tuwe wacinye śni kinhan, he wiconi wanyake kte śni, tuka Wakantanka tocanniye kin wanna akan un.

\section{WICOWOYAKE 4}

1 Jesus waonspewicakiya ota içicage ça baptem wicaqu, Johannes econ qon sam iyeya, Pharisee kin hecen nahonpi, he Itancan kin sdonya.

2 Tuka Jesus iye atayedan baptem wicaqu śni, waonspewicakiye cin hena heconpi.

3 Hehan Juda makoce kin ayuśtan, qa Galile heciya ake ekta hda.

4 Qa Samariya he en acankuye kta iyececa.

5 Hecen otonwe wan Sukar eciyapi Samariya makoce en, Joseph en ounye kta e Jakob atkuku qu qon he ikiyedan, he ekta hi.

6 Jakob miniyowe tawa kin he 
etu; Jesus mdokite hda, qa miniyowe kin he ahankeya iyotanka. Anpetu kin he en wiyotanhe cin hehantu.

\%. Samariya oyate etanhan winohinca wan mini huwe hi ; unkan Jesus, Mini maqu wo, eciya.

$8 \mathrm{He}$ icunhan waonspewicakiye cin otonwe kin ekta taku yutapi opeton iyayapi.

9 Winohinca Samariya oyate etanhan qon hecen eya, Juda wicaśta kin henica, Samariya oyate kin hematanhan, he tokeca e mini mayakida he. Juda oyate Samariya oyate kici takudan ikicitukapi wanice ciqon.

10 Jesus ayupte ca heciya; Wakantanka taku wicaqu kin he, qa, Mini maqu wo, eniciye cin he tuwe kin sdonyaya unkanś, niye yakida kta, kinhan mini wiconi kin niçu kta tuka ce.

11 Itancan, mini kin de temahentuya hiyeya qa takudan on edaku kte śni; tokiya tanhan mini wiconi kin duha he, winohinca kin eciya.

12 Jakob ateunyanpi miniyowe kin de unqupi, qa etanhan iye mini yatkan, qa cinca qa tawanunyanpi ko, he hecon tuka niye iniyotan he.

13 Unkan Jesus ayupte ca heciya; Tuwe mini kin de etanhan yatke cin nakun ipuza kta;

14 Qa tuwe mini waqu kte cin etanhan yatke cin icimana ipuza kte śni. Tuka mini waqu kte cin he owihanke wanin wiconi iyahdeya kaduze kta, eya.

15 Unkan winohinca kin heciya; Itancan, mini kin he etanhan maqu ye, hecen imapuza kte śni, qa nakun icimana mini kin de huwe wahi kte śni, eya.

16 Unkan, Nihihna kico ya wo, qa den kici hi wo, Jesus eciya.

17 Tuka winolinca kin waayupte ça heciya; Mihihna kin wanica ce. Nilihna wanica kehe cin he hecetu e ecen eha ce;

18 Nihihna zaptan, tuka nakaha hihnayaye cin de nitawa śni; hecen ehe cin he wicayaka ce, Jesus eciya.

19 Hehan winolinca kin heciya; Itancan, wicaśta wolkcan kin henica imdukcan;

20 Hunkake wicunyanpi paha kin de en ohodapi ece; tuka niye, Jerusalem he en wicaśta ohodapi ece e kta, kehapi ce.

21 Unkan Jesus heciya; Winolinca, wicamada wo, anpetu wan u ce, paha lin de en, qa Jerusalem nakun en, Ateyapi kin ohoyadapi kte śni.

22 Niye taku ohoyadapi kin sdonyayapi śni; unkiye taku ohoundapi kin sdonunyanpi ece. Juda oyate kin eciyatanhan wanikiyapi kin u ce.

23 Tuka anpetu $u$ kin he wanna hiyohi, tona wowicake eciyatanhan ohodapi kin hena woksape wowicake eciyatanhan Ateyapi kin ohodapi kta. Ateyapi kin he tona hecen ohodapi kte cin akita ece.

24 Woksape kin he Wakantanka kin ee; qa tona ohoda kinhan hena woksape wowicake eciyatanhan ohoda kta.

25 Hehan winolinca kin heciya; Messiya Sdayapi eyapi kin hi kta, tohan hi kinhan taku kin owasin unkokiyakapi kta e sdonwaya.

26 He miye e ociciya ce, Jesus eciya.

$27 \mathrm{He}$ icunhan waonspewicakiye cin en hdipi, qa winolinca kin kici okiciciya un kin heon yuśinyayapi. 'I'uka wanjidan; 
Taku ayakita he; qa, Tokeca kici iyaa he, eyapi śni.

28 Hehan winohinca kin wakiśkolrpa ehpeya kihde ca, otonwe kin ekta ki, qa wicaśta kin hewicakiya;

29 Wicaśta wan taku ecamon qon owasin omiciyake cin he ito wanyag $u$ miye. He Messiya kin ee śni he.

30 Hehan otonwe kin etanhan enanpa, qa yanke cin en ai.

31 He icunhan waonspewicakiye cin cekiyapi qa, Itancan, wota wo, eciyapi.

32 Tuka iye hewicakiya; Taku yutapi sdonyayapi śni kin heca wate kta e mduha ce.

33 Heon, Tuwe taku yutapi kahi he, wonspewicakiye cin ekiciyapi.

34 Tuka Jesus hewicakiya; Tuwe $u$ maśi kin he tawacin kin ecen ecamon kta, qa iye tohan kin weciyuśtan kte cin he taku wate cin ee.

35 Whake wi topa kinhan taku mnaunyanpi kta ce, ehapi. Tuka miś kaken eciciyapi; Wankan etonwanpi qa maga kin wanyaka po, wanna owasin ska qa mnayanpi kin wanna iyehantu.

36 Qa tuwe taku mnaye cin he wokajuju icu ece, qa owihanke wanin wiconi waskuyeca rnnayan ece; hecen on tona woju, qa tona mnaye cin napin wiyuśkinpi kta.

37 Wanji woju qa wanji mnayan, eyapi qon dee e wicakapi.

38 Niye htayanipi śni tuka wamnayanpi he econ ciśipi. Wicaśta tokeca htanipi unkan he hitanipi kin en niye oyapapi.

39 Winohinca kin, Token ecamon qon owasin omiciyaka ce, eye ciqon heon wicaśta Samariya oyate otonwe kin he en ota Messiya hee kin wicadapi.
40 Heon Samariya oyate Jesus en hipi qa celkiyapi, iye tipi kin en un śipi. Unkan nonpa can hen un.

41 Unkan ia nalionpi kin hehan wicota wicadapi.

$42 \mathrm{Qa}$, Taku odake cin he etanhan wicaundapi kin hee śni, unkiś eya naunhionpi, Messiya hee qa oyate wanikiye kte cin hee e sdonunyanpi, winohinca kin eciyapi.

43 Anpetu nonpa iyohakam hehan hetanhan ye ça Galile heciya hda.

44 Jesus iye atayedan hdaotanin, wicaśta wokcan kin heca iye tamakoce kin en yuonihanpi śni ece.

45 Unkan Galile ekta ki, hehan Galile wicaśta kin iyowinkiyapi, Jerusalem ekta wośnapi kin icunhan taku econ qon owasin wanyakapi kin heon etanhan; iś eya wośnapi kin ekta ipi nakaeś.

46 Unkan Galile en Kana otonwe kin, mini ikceka miniśa icaliye ciqon he ake en hdi. Unkan Kapernaum ekta wicaśta itancan wanji cinhintku wayazanka:

47 He Jesus Juda makoce kin hetanhan Galile en hi, nahon kin heon ekta ye ca cekiya, cinhintku kin asniye kta e ekta u kta; wanna te kta ikiyedan.

48 Hehan Jesus heciya; 'Taku wowanyake wowapetokeca ko wandakapi śni keś wicayadapi kte śni ce.

49 Unkan wicaśta itancan kin heciya; Itancan, micinkśi țe śni itokam kun u wo.

50 Tuka Jesus heciya; Hunktiya wo, nicinkśi ni ce. Unkan wicaśta kin Jesus ie cin wicada, qa ekta hda.

51 Tiyatakiya hda, unkan wi- 


\section{WICOWOYAKE 5.}

caśta wowidake wicayuhe cin awicakipa; unkan, Nicinkśi kin ni ce, eya okiciyakapi.

52 Wi kin tohan yanka hehan asni aya he, eya iwicawanga. Unkan, Ḣtanihan wiyotanhan kitanna inaśdoka, hetanhan tancan kata ayuśtan ce, eyapi.

53 Unkan atkuku kin tohan Jesus, Nicinkśi ni ce, eciye ciqon hehantu e kiksuya. Unkan iye qa tiyohnaka tawa koya wicadapi.

54 Jesus Juda makoce etanhan Galile icicawin en hdi, wowapetokeca econ kin icinonpa kin he dee

\section{WICOWOYAKE 5.}

1 Helian iyohakam Juda oyate omniciye wan kagapi he icunhan Jesus Jerusalem he en $i$.

2 Jerusalem en tahinca wanunyanpi tiyopa kin he en ikiyadan, mini kiwitayapi, Hebrew iapi Bethesda eciyapi, zaptankiya aohanziya hdepi.

3 Hena ohna wicaśta wayazanka ota, huśte, iśta gonga, tataka ko ota en makan hiyeya, mini śkanśkan ape hiyeya.

4 Tohan iyehantu eca, malipiya ohnihde wan mini kin en lii qa śkanśkanya ece. Tolkiya yazan kin owasin tohan mini kin he śkanśkan eca tuwe tokaheya en iyohpeiçiya eca asni ece.

5 Wicaśta wan he en wanka, waniyetu wikcemna yamni sanpa śahdogan hehanyan wayazanka.

6 Wicaśta kin he makata wanka, wanakaja tanhan wayazanka Jesus wanyaka, qa, Anisni kta yacin he, eciya.

7 Wicaśta wayazanke cin heciya; Itancan, mini kin śkanśkan keś tuwedan en iyolipemayeśni; en mde kta keś tuwe tokeca mitokam en iyeiciya ece.

8 Najin, owinja ehdaku qa mani wo, Jesus eciya.

9 Unkan ecahankeya woicaśta kin akiśni, qa owinja kin ehdaku, qa mani. Unkan anpetu. kin he anpetu wakan.

10 Heon etanhan Juda oyate kin wicaśta asniyanpi qon heciyapi ; Nakaha anpetu wakan; owinja yeçin kin he hecetu śni ce.

11 Tuwe asnimaye cin he, Owinja ehdaku qa mani wo, emakiya ce, ewicakiya.

12 Hehan, Wicaśta kin, Owinja ehdaku qa mani wo, eniciye cin he tuwe he, eya iwangapi.

13 Tuka wicaśta asniyanpi kin he tuwe kin sdonye śni : he icunhan wicota hen unpi hecen Jesus icunonpa iyaya.

14. Hehan iyohakam tipi wakan en wicaśta kin he Jesus wanyaka, unkan, Wanyaka ye, wanna anisni ; icimana wahtani sni wo ; okinni ake taku wanji śica sanpa tanka iyanihde kta ce, eciya.

15 Unkan wicaśta kin he kihde ça, Tuwe asniye ciqon he Jesus ee, Juda oyate owicakiyaka.

16 Heon etanhan Juda oyate Jesus kuwa ayapi, qa anpetu wakan kin icunhan hena econ kin heon teyapi kta e akitapi.

17 Unkan, Ate nahahin litani he etanhan miś eya litawani, Jesus ewicakiya.

18 Unkan Juda oyate hehan iyotan Jesus teyapi kta sanpa akitapi, anpetu wakan botica, qa nakun Wakantanka atayedan Ateya keiciye cin, Wakantanka kici akiyecen ihdawa kin, heon etanhan.

19 Unkan Jesus waayupte ca 


\section{JOHANNES.}

hewicakiya ; Wowicake, wowicake eciyatanhan heciciyapi, Cinhintkuyapi kin takudan iye cinka econ kta okihi śni ; Ateye cin taku econ wanyaka eca, hehan Cinhintku kin iś eya iyecen econ ece.

20 Ateyapi kin Cinhintku waśte kidake cin heon taku econ kin hena owasin onspekiya; qa nakun eeś wicohan dena sanpa tanka onspelkiye kta, hecen ininihanpi kta.

21 Ateyapi kin tuwe ta keś niye ca wiconi wicaqu ece; he iyecen Cinhintku kin iś tona cin kinhan wiconi wicaqu ece.

22 Nakun Ateyapi kin tuwedan yaco śni, tuka woyaco owasin Cinhintku kin qu.

23 Heon etanhan owasin Ateyapi kin token ohodapi hecinhan he iyecen Cinhintku kin ohodapi kta. Tuwe Cinhintku kin ohoda śni kin he Atkuku u śi kin he nakun ohoda śni.

24 Wowicake, wowicake eciyatanhan heciciyapi, Tuwe mioie kin anagoptan, qa u maśi kin he awacin kinhan, owihanke wanin wiconi yuhe kta; qa woyaco en i kte śni, wanna wiconte etanhan wiconi ekta e i.

25 Wowicake, wowicake eciyatanhan heciciyapi, Anpetu wan u qon wanna hi, wicaśta ta tona Wakantanka Cinhintku ho kin nahon kta; qa tona nahon kinhan hena ni kta.

26 Ateyapi kin iye cinka wiconi hduha, he etanhan Cinhintku kin iś iye cinka wiconi hduhe kta e qu.

$27 \mathrm{Qa}$ nakun wowaśake woyaco kin he nakun Cinhintku qu, Wicaśta Cinhintku kin e heon etanhan.

28 Ihnuhan hena ininihanpi kin do ; anpetu wan hi kta ce, wicaśta wicahapi kin owasin iye ho kin nahonpi kta;

$29 \mathrm{Qa}$ hninanpapi kta, tona waśte econpi kin hena wiconi woekicetu ekta; qa tona taku śica econpi kin hena woyaco woekicetu ekta hiyupi kta.

30 Miye cinka taku ecamon owakitpani; taku mdaco kin hena taku nawalion kin heciya tanhan; qa taku mdaco kin hena hecetu. Miye mitawacin he owakide śni, tuwe ù maśi kin he tawacin owade.

31 Miye mihdaotanin kinhan, taku mdaotanin kin he wowicake śni.

32 Wanji tokeca mayaotanin ece; unkan wayaotanin iye mayaotanin kin he wowicake e sdonwakiya.

33 Johannes ekta ye wicayaśipi ; unkan taku yaotanin kin wowicake kin hee.

34 Tuka wicaśta akantu wayaotaninpi kin iwacu śni; tuka dena on yanipi kta e heciciyapi. 35 He peta ijanjan iyege ca okitanin; unkan iyoyanpa tawa kin on kohanna iyuśkin wacannipi.

36 Tuka Johannes sam iyeya tanka wayaotanin mduha; wicohan ecamon kta Ate maqu qon hena e ecamon ece; Ate umaśi e yuotanin kin hena ee.

37 Qa Ateyapi umaśi qon he nakun mahduotanin: He tohinni ho kin nayahonpi śni, qa itohnake wandakapi śni:

$38 \mathrm{Qa}$ oie kin en niunpi śni, tuwe u śi kin he wicayadapi śni, heon etanhan.

39 Wowapi wakan kin en akita po; heciyatanhan wiconi owihanke wanica duhapi kecannipi ; unkan omayake cin hena ee. 40 Tuka owihanke wanin wi- 


\section{WICOWOYAKE 6.}

coni duhapi kta e on miye en mayaupi yacinpi śni.

41 Wicaśta ekta mitawootanin kin awecite śni.

42 Tuka Wakantanka waśtedakapi kin he en niunpi śni e sdonciyapi.

43 Ate cajeyan wahi, tuka iyowinmayakiyapi śni; tuwe iye caje atayedan hduha hi kin hee iyowinyakiyapi kta.

44 Niye iyakitedan nitawootaninpi kin ayecitapi, qa Wakantanka eciyatanhan wootanin kin ayakitapi śni, he token on wicayadapi kta he.

45 Ate en aciyapi kta e kecanmakinpi śni po; Moses he wacinyayapi qon he iye taku iyanionpapi ce.

46 Moses wicayadapi unkanś miś eya wicamayadapi kta tuka. He miye on wowapi kaga.

47 Tuka iye wowapi kage cin hena wicayadapi śni kinhan, ecin token mioie kin wicayadapi kta he.

\section{WICOWOYAKE 6.}

1 Hena iyohakam Jesus Galile mde kin, he nakun Tiberias mde kin ee, he akasanpa iyrya.

2 Unkan oyate ota ihakam yapi, wicaśta wayazanpika en wapetogya ohanyan wanyakapi qon heon etanhan.

3 Unkan Jesus paha wan ekta iyaya, qa waonspewicakiye cin om akan iyotanka.

4 Unkan woacakśin, Juda oyate wośnapi tawapi wan, he wanna ikiyedan ihan.

5 Jesus iśta yuwankan ikikcu, qa wicota iye yanke cin en au wanwicayake cehan, Philip he"ciya; Aguyapi dena yutapi kte cin tokiyatanhan opeuntonpi kta he.

6 He yukcan kta on etanhan heciya; token econ kte cin he sdonkiya tuka.

7 Hehan Philip ayupte; Kaśpapidan opawinge nonpa aguyapi iyopeyapi, qa otoiyohi cikcistinna icupi eśta, nakun iyowicahi kte śni ce, eya.

8 Hehan waonspewicakiye cin wanji, Simon Inyan sunkaku, Andrew eciyapi, he nakun heciya;

9 Psin ska aguyapi zaptan qa hogan nom hena den hokśidan wan yuha: tuka wicota qa henana on token hanpica he.

10 Unkan, Wicaśta kin iyotanke wicaśi po, Jesus eya. He en peji ota. Wicaśta kin hena iyotankapi, unkan kektopawinge zaptan iyecetu.

11 Hehan Jesus aguyapi kin hena icu qa yawakan, qa waonspewicakiye cin wicaqu ; unkan waonspewicakiye cin iyotang hiyeye cin wicakipamnipi ; qa nakun hogan tohanyan cinpi kin.

12 Unkan imnanpi hehan waonspewicakiye cin hewicakiya, Oyaptapi kin hena pahi po, hecen takudan atakuni kte śni.

13 Unkan psin ska aguyapi oyaptapi qon he etanhan makanopiye ohnaka ake nonpa pahipi ; wotapi etanhan iwicakiciyaye cin hena ee.

14 Taku wapetogya Jesus econ kin oyate kin wanyakapi, qa heon heyapi, Wicaśta wokcan itancan oyate en hi kta keyapi qon he wanna dee.

15 Oyate kin Jesus icupi, qa wicaśtayatapi kah wacinpi e sdonkiya, qa paha kin icunonpa akan iśnana iyaya.

16 Unkan wanna hitayetu hehan waonspewicakiye cin mde kin ektakiya yapi ;

17 Qa wata wan en opapi qa 


\section{JOHANNES.}

mde kin he opta yapi, Kapernaum heciya i wacinpi. Unkan otpaza tuka nahanhin Jesus ekta wicahi śni.

18 Unkan tateyanpa hinca, on etanhan mde kin nina taja aya.

19 Unkan wiyutapi wikcemna nonpa sam zaptan, qa iś wikcemna yamni hehanyan watopapi, liehan wata kin ikiyedan Jesus mini kin amani u wanyakapi ; unkan yuśinyayapi.

20 Tuka, He miye ce, ihnuhan koyakipapi kin do, ewicakiya.

21 Hehan iyokipiya wata kin en iyowinkiyapi ; unkan tokiya yapi qon, wata kin kohanna he en ihunni.

22 Ihanlianna hehan oyate mde kin icahda yukanpi kin, hen wata wanica, wanji waonspewicakiye cin en opapi qon heceyedan, qa Jesus waonspewicakiye cin om wata kin en ope śni, tula waonspewicakiye cin iśnana iyayapi, he wanyakapi :

23 Tuka Jesus aguyapi yawakan qa yutapi qon he en ikiyadan Tiberias eciyatanhan wata toktokeca en hipi.

24 Oyate kin Jesus waonspewicakiye cin om he en yukanpi śni wanyakapi, unkan oyate kin wata kin hena en opapi qa mde kin opta yapi, Jesus Kapernaum heci un kin he akin yapi.

25 Unkan mde kin akasanpa hen iyeyapi qehan, heciyapi; Itancan tohinni den yahi he.

26 Jesus awicayupte ca heya ; Wowicake, wowicake eciyatanhan heciciyapi, Omayadepi kin he taku wapetokeca wandakapi kin heon hecanonpi śni, aguyapi imnahan yatapi qon e heon.

$2 \%$ Taku yutapi on wicatakuni s̉ni heon litanipi śni po ; tuka taku yutapi owihanke wanin wiconi hehanyan kte cin hee on litani po, he Wicaśta Cinhintku kin nicupi kta; Wakantanka A teyapi kin wanna he askamton.

28 Hehan heciyapi ; Token econkupi $\mathrm{kta}$, on Wakantanka tolian kin unkolianyanpi kta he.

29 Jesus waayupte ça hewicakiya; Wakantanka wicolian tawa kin he dee, tuwe u śi kin he wicayadapi kta.

30 Heon etanhan heciyapi; Taku wapetokeca ecanon kin tukte e heon, wanunyakapi qa wicaunnidapi kta ; taku yakaga he.

31 Hunkake wicunyanpi kin eya wanna liewoskan manna yutapi qon; Malipiya eciyatanhan aguyapi yutapi kta e wicaqu ce, eya owapi qon he iyececa.

32 Unkan Jesus hewicakiya; Wowicake, wowicake eciyatanhan heciciyapi; Moses aguyapi kin he malipiya kin eciyatanhan niçupi śni; tuka Atewaye cin he aguyapi wowicake kin malipiya eciyatanhan nicupi.

33 Aguyapi Wakantanka tawa kin he tuwe mahpiya eciyatanhan kuntkiya $u$, qa oyate wiconi wicaqu kin hee.

34 Unkan, Fca Itancan, aguyapi kin he ohinniyan unqu po, eciyapi.

35 Tuka Jesus hewicakiya ; Aguyapi wiconi kin he miye ce. Tuwe en mau kinhan wotektehda śni un kta; qa tuwe wicamada kinhan ipuza śni un kta.

36 Eya wanna wanmayadakapi, tuka wicamayadapi śni e, eciciyapi qon.

37 Ate tona maqu kin owasin miye en mau kta; qa tuwe en mau kinhan tankan iyewaye kte śni.

38 Mahipiya eciyatanhan wahi kin, he mitawacin owape kte śni, tuwe u maśi kin he tawacin ow ecipe lita. 


\section{WICOWOYAKE 6.}

39 Atewaya u maśi liin he tawacin kin he dee, tona maqu kin wanjidan toki elipewaye śni, ecen anpetu ihankeya kin piya icahwaye lita.

40 Tuwe u maśi kin he tawacin kin he dee, tona Cinhintlruyapi kin he wanyake ca wicada kin hena owihanke wanin wiconi yuhe kta, qa anpetu ihankeya kin he en piya icaliwaye kta.

41 Heon Juda oyate kin ajijipi; Aguyapi mahpiya eciyatanhan kun $u$ lkin he miye ce, eye ciqon heon etanhan.

42 Qa heyapi; Jesus Joseph cinhintku qon he dee śni he, atkuku hunku lro sdonwicunyanpi ce : hecen token, Mahpiya eciyatanhan wahi ce, eya okihi he.

43 Heon Jesus waayupte ca hewicakiya; Niye iyakitedan waajijipi śni po.

44. Tuwedan iye cinka en mau kte śni, Ate u maśi qon tuwedan en au śni kinhan, qa anpetu ihankeya kin en piya icahwaye lita.

45 Unkan owasin Wakantanka eciyatanhan onspewicakiyapi kta ce, wicaśta wokcan kin en eya owapi qon. Heon tona Ate waonspewicakiye ça anagoptanpi kin hena owasin en maupi kita.

46 Tuwedan Ateyapi kin tohinni wanyake śni, tuwe Wakantanka etanhan u kin hecedan Atkuku wanhdaka.

47 Wowicake, wowicake eciyatanhan heciciyapi, 'Tuwe miye wicamada kinhan, he owihanke wanin wiconi yuhe $k$ ta.

48 Aguyapi wiconi he miye.

49 Nihunkakepi kin hewoskan manna yutapi, tuka tapi.

50 Mahpiya eciyatanhan aguyapi hi kin he dee, tuwe etanhan yute cinhan te kte śni.
51 Aguyapi ni un kin he miye, mahpiya eciyatanhan wahi ; tuwe etanhan yute cinhan owihanke wanin ni lita. Aguyapi wicawaqu kte cin he micelipi kin ee, he oyate wicani kta e wicawaqu ce, eya.

52 Wicaśta kin de iye cehpi etanhan unqupi qa untapi kta heye cin, he token okihi kta e heya he, Juda oyate iyakitedan eya hdokinicapi.

53 Hehan Jesus hewicakiya; Wowicake, wowicake eciyatanhan heciciyapi; Wicaśta Cinhinthu kin cehpi etanhan yatapi śni, qa we kin datkanpi śni kinhan wiconi takudan en niunpi kte śni.

54. Tuwe micehpi etanhan yute ca mawe yatke cinhan owihanke wanin wiconi yuha ece, qa anpetu ihankeya kin en piya icahwaye kta.

55 Micehpi kin he woyute otancanke, qa mawe kin he woyatke otancanke.

56 Tuwe micehpi yute ça mawe yatke cin he mici un, qa miś kici waun.

57 A te u maśi kin he ni un, qa Ate on ni waun, he etanhan tuwe mayute cinhan miye on ni un lita.

58 Aguyapi malipiya eciyatanhan hi kin he dee. Nihunkakepi manna yutapi qon de iyecece śni, he yutapi tuka tapi. Tuwe aguyapi kin de etanhan yute cinhan owihanke wanin ni kta ce, eya.

59 Kapernaum en omniciye tipi liin he ohna waonspekiya icunhan hena hecen eya.

60 Heon etanhan waonspewicakiye cin ota he nahonpi qehan, Wicoie kin de tehika ce, tuwe e nahon okihi lita he, eyapi.

61 Unkan Jesus waonspewica153 
kiye cin hena heya ajijipi kin, iye atayedan sdonye ça hewicakiya; Tokeca de iyanipațopi he.

62 Wicaśta Cinhintku kin wankan un qon ake ekta kihde ca, wandakapi kinhan, tokeca kta he.

63 Woniya kin he wiconi kaga ece, wicacelipi kin takudan okihi śni. Wicoie eciciyapi kin hena woniya qa wiconi kin hee.

64 Tuka wanjikji he wicayadapi śni. Otokahe ekta tanhan tona wicadapi kte śni, qa nakun tuwe wiȳopeye kte cin hena Jesus sdonva.

65 Unkan heya; He etanhan heciciyapi qon, Tuwedan iye cinka en mau okihi kte śni, tuwe Atewaye cin he qu kinhan heceyedan okihi kta.

66 Hecetu, unkan heon waonspewicakiye cin ota ayuśtanpi, qa icimana ihakam yapi śni.

67. Hehan Jesus akenonpapi kin, Niś eya yahdapi kta he, ewicakiya.

68 Unkan Simon Inyan ayupte; Itancan, tuwe ekta unyanpi kta he; owihanke wanin wiconi wicoie duhe cin;

69 Qa Messiya Wakantanka ni un Cinhintku kin he niye e wicaundapi qa sdonunyanpi ye, eya.

70 Unkan Jesus awicayupte; Akeninonpapi kin miye cicalinigapi śni he ; tuka wanji wakanśica yaunpi ce, eya.

71 Simon cinhintku Judas Iskariyot he ke ça hecen eya. Ake nonpapi kin he etanhan tuka he wiyopeye kte cin ee.

\section{WICOWOYAKE 7.}

1 Hena iyohakam Jesus Galile en un; Juda oyate te yapi kta 154 akitapi heon Juda makoce en un wacin śni.

2 Juda oyate woacakśin wośnapi ece kin he wanna kiyedan aya.

3 Heon etanhan iye sunkawicaye cin heciyapi ; Detanhan ya wo, qa Juda makoce kin en un wo, hecen waonspewicayakiye cin niohan ecanon ece kin hena wanyakapi kta.

4 Tuwe ihdutanin cin kinhan, taku nalimana econ śni ece. Dena ecanon kta hecinhan, iho oyate kin en ihdutanin wo.

5 Sunkawicaye cin iyepi nakun wicadapi śni.

6 Heon Jesus hewicakiya; Miye anpetu kin hinyanhin iyemicihantu śni ; tuka niye qe anpetu kin ohnniyan wiyeya niciyankapi.

7 Oyate kin śicenidapi okihipi śni, tuka miye e śicemadapi ; wicohan tawapi kin hena śica e mdaotanin ece kin heon etanhan.

8 Niye qe wośnapi kte cin ekta ya po. Miye qe nahahin anpetu iyemicihantu śni, heon wośnapi kin he ekta mde kte śni ce.

9 Hena hewicakiye ca hehan Galile en un.

10 Tuka sunkaku iyayapi kin iyohakam hehan, iś wośnapi kte cin ekta ya, taninyan ye śni, tuka nalimana ya.

11 Wośnapi kin icunhan Juda oyate odepi qa, Tokiya un he, eyapi.

12 Qa oyate kin iapi ota on aiapi. Apa, He wicaśta waśte ce, eyapi: qa toktokeca iś, Hiya, oyate kin wicahnayan ce, eyapi.

13 Juda oyate kowicakipapi, heon etanhan tuwedan taku a taninyan eye śni.

14. Wośnapi kin cokaya hdoipi, unkan Jesus tipi wakan ohna inajin, qa wowicakiyaka. 


\section{WICOWOYAKE 7.}

15 Unkan Juda oyate kin inihanpi qa heyapi; Wicaśta kin de tokiya tanhan wowapi kin onspe he; tohinni onspekiyapi śni qon.

16 Unkan Jesus awicayupte ca heya; Wociciyakapi kin de miye etanhan śni, tuwe u maśi kin he iye etanhan.

17 Tuwe iye tawacin ecen econ wacin kinhan, woyakapi kin de Wakantanka eciyatanhan hecinhan, qa iś miye cinka hena epa hecinhan, sdonye kta.

18 Tuwe iye en oie iciye cinhan he iye towitan okide ece; tuka tuwe iś, tuwe uśi kin he towitan okicide kinhan, he iye wowicake, qa wicowicaśtaśni takudan en un śni.

19 Moses woope kin niçupi śni he; tuka tuwedan woope kin ecen ecanonpi śni; taku on țemayayapi kta e ayakitapi he.

20 Oyate kin ayuptapi qa heyapi ; Wakan śica niyuhnaśkinyan; tuwe e teniyan akita he.

21 Hehan Jesus waayupte ca hewicakiya; Wicohan wanji ecamon, unkan owasin niyuśinyayapi.

22 Moses bahdayapi wicohan kin nicupi; he Moses etanhan śni, tuka hunkakewicayapi kin etanhan; unkan anpetu wakan eca wicaśta wan bayecihdayapi ece.

23 Hecen anpetu wakan eca wicaśta wan bakicihdayapi hecinhan, on Moses woope kage ciqon yujujupi kte śni; wicaśta wan anpetu wakan en ocowasin okiziwaye cin heon cantamayahdepi he.

24 Wiyacinyan wayacopi śni po, wokonze e eciyatanhan wayaco po.

25 Hehan Jerusalem etanhan wanjikji heyapi; Tuwe teyan akitapi kin he dee sni he.
26 Unkan iho, kagi śni ia tuka tuwedan taku eciye śni. Wicaśta itancanpi kin awicakehan Messiya kin he dee e sdonyapi he.

27 Tuka wicaśta kin de tokiyatanhan u kin sdonunyanpi; tohan Messiya hi kinhan, tokiyatanhan u kin tuwedan sdonye kte śni, eyapi.

28 Hehan Jesus tipi wakan kin ohna waonspekiya icunhan hotankakiya heya; Tuwe miye kin sdonmayayapi, qa tokiya tanhan wau kin lie nakun: qa miye cinka wahi śni; tuka tuwe u maśi kin he wicaka, eqe sdonyayapi śni.

29 Tuka miye he sdonwakiya, he iye etanhan wahi, qa u maśi kin hee.

30 Unkan heon tinsa yuze wacinpi, tuka nahahin iyehantu śni kin heon tuwedan nape on yutanpi śni.

31 Unkan oyate kin etanhan wicota he iye kin wicadapi qa heyapi ; Tohan Messiya hi kinhan, de taku wapetogya econ kin, he sam iyeya hecon kta he, eyapi.

32 Oyate kin iyakitedan hekiciyapi he Pharisee kin nahonpi ; unkan wośna kagapi Pharisee ko, yuzapi kta e on akicita ekta ye wicaśipi.

33 Hehan Jesus hewicakiya; Ehake tokata cistiyedan ocipapi waun kta, helian tuwe u maśi kinhan heciya wahde kta.

34 Omayadepi kta, tuka iyemayayapi kte śni ; qa tokiya waun kin ekta yaupi kta eśta, oyakitpanipi kta.

35 Hehan Juda oyate kin iyakitedan hekiciyapi; Tokiya ye kta he, qa iyeunyanpi unkolkihipi kte śni; Helenes oyate kin ehna enanakiya iyayapi kin ekta 


\section{JOHANNES.}

ye ca, Helenes wowicakiyake kta he.

36 Omayadepi kta tuka iyemayayapi kte śni, qa tokiya waun kin ekta yaupi kta eśta, oyakitpanipi kta ce, eye cin wicoie kin he toketu he, eyapi.

37 Wośnapi anpetu ehake qa tanka kin en, Jesus najin qa hotankakiya heya; 'Tuwe ipuza kinhan en mau qa mini yatkekta.

38 Tuwe wicamada kinhan, wowapi wakan eye cin iyececa, iye tanmahen tanhan wiconi mini wakpadan kaduze kta ce.

39 Tuka Woniya, tona iye wacinyanpi kin icupi kte cin hee on hecen eya: he ehan nahahiin Woniya Wakan kin wicaqupi śni, nahahin Jesus wowitan ehan i śni.

40 Oyate kin etanhan wicota wicoie kin de nahonpi qehan, Awicakehan wicaśta wokcan kin he dee ce, eyapi.

41 Toktokeca iś, Messiya kin he dee ce, eyapi. 'Tuka apa iś, Messiya Galile etanhan u kta he, eyapi.

42 Dawid wicoicage tawa kin etanhan Messiya hinanpe kta, qa Bethlehem oton we Dawid en un qon hetanhan u kta ce, wowapi wakan eye śni he.

43 Hetanhan oyate kin akipam wacinkiyuzapi.

$44 \mathrm{Qa}$ hunh yuze wacinpi, tuka tuwedan nape on yutan śni.

45 Hehan akicita kin, wośna lkagapi Pharisee ko ekta icicawin wicakipi; unkan, Tokeca e ayahipi śni he, ewicakiyapi.

46 Akicita kin awicayuptapi ; Wicaśta kin tuwedan tohinni wicaśta kin de iyecen oie ye śni ce, eyapi.

47 Hehan Pharisee kin iś awicayuptapi, Niś nakun nihnayanpi he.
48 Wicaśta itancanpi kin wan: jikji qa Pharisee ko he wicadapi he.

49 Tuka oyate kin de, woope yukcanpi śni kin hena wahtepi śni ce, eyapi.

50 Nikodemos hanyetu ehan Jesus yanke cin en i qon, he itancanpi kin wanji ee, hewicakiya ;

51 Woope unkitawapi kin, wicaśta token eye cin nalion śni, qa taku econ kin atayedan sdonye śni itokam, yaco he.

52 Unkan ayuptapi qa heciyapi; Niś Galile henitanhan he: Wawiwange ca wanyaka wo; wicaśta wokcan tuwedan Galile etanhan u śni ce.

$53 \cdot$ Hehan otoiyohi iye tipi kin ekta kihdapi.

\section{WICOWOYAKE 8.}

1 Jesus Oliwe Paha kin ekta i. 2 Qa ihanlianna anpao hehan tipi wakan kin en ki, unkan oyate kin owasin en ahi ; unkan en iyotanke ça wowicakiyaka.

3 Unkan wośna kagapi Pharisee ko winolinca wan nahma ihduśaka e en kahipi, qa cokata ehdepi ;

4 Qa heciyapi, Waonspeliye, winohinca kin de wawicihahiapi econ wanka icunhan iyeyapi.

5 Unkan Moses, woope kin en, tona hececa kin inyan on wicakininpi kta, unkekiciyapi qon: tuka niś token ehe kta he.

6 Taku iyaonpapi kte cin he akitapi e heon etanhan he iyutanyan heciyapi. Tuka Jesus patuś inajin, qa nape on maka kin en owa.

7 Unkan kitanyan wiwangapi, hehan Jesus owotanna inajin, qa hewicakiya; Wanji tukte woalitani wanin yaunpi hecinhan, 
he tokaheya inyan on kinin kte.

8 Hehan ake patuś inajin qa ake maka kin en owa.

$9 \mathrm{He}$ nahonpi, unkan iye tawacin on ihdacopi, heon wanjikjidan tankata kinanpapi, tokaheya wicaśta tanka unpi, qa ecen hakaktapi koya owasin; qa Jesus iśnana un elipeyapi, winohinca cokata hdepi qon he koya.

10 Unkan Jesus ihduowotanna, qa tuwedan wanyake śni, winohinca ecedan ecen najin, unkan heciya, Winohinca, wiyanionpapi qon owasin toki iyayapi he; tuwedan niyaco śni he.

ll Han, Itancan, tuwedan śni, eya. Miś eya ciyaco śni, hunktiya wo, tokata yeye cin icimana walitani śni wo, Jésus eciya.

12 Hehan ake Jesus owicakiye ca heya; Oyate iyoyamwicaye cin he miye. Tuwe mihakam u kin he otpaza ehna mani kte śni, iyoyanpa wiconi hee tawa kta.

13 Hehan Pharisee kin heciyapi; Niye atayedan nihdaotanin, heon wadaotanin kin he wowicake śni.

14 Jesus waayupte ca hewicakiya; Miye atayedan mihdaotanin qeyaś mihdaotanin kin he wowicake; tokiya tanhan wahi kin he sdonwakiya, qa nakun tokiya mde kte cin he; qa niyepi kin tokiya tanhan wau, qa tokiya mde cin hena sdonyayapi śni.

15 Wicacehpi eciyatanhan wadacopi ; tuka miye qe tuwedan mdaco śni.

16 Tuka nakun wamdaco kinhan, wamdaco kin he wowicake kta ; miśnana waun śni, tuka Ateyapi u maśi kin he miciun ece.

17 Wicaśta nom waayataninpi kin he wowicake ce, woope duhapi kin en owapi qon.

18 Miye atayedan mihdaotanin, qa Ate u maśi kin he nakun mayaotanin.

19 Unkan, Niyate tukte e he, eciyapi. Jesus iś awicayupte, Sdonmayayapi śni, qa nakun Atewaye cin sdonyayapi sni: Miye kin sdonmayayapi unkanś, Ate iś nakun sdonyayapi kta tuka.

20 Tipi wakan kin en mazaska opiye he cin he en, Jesus waonspewicakiya icunhan hena hewieakiya. Heca tuka tuwedan Jesus otan wacinpi śni, anpetu kin nahahin kicihi śni heon.

21 Hehan Jesus ake hewicakiya; Wahde kta, qa omayadepi ecen woalitani ehna nițapi kta; qa tokiya wahde cin heciya dapi kta eśta oyakitpanipi kta.

22 Unkan Juda oyate kin heyapi; Iye içikte kta he. Tokiya mde cin heciya dapi oyakitpanipi kta ce, eye cin.

23 Hehan iye hewicakiya; Niye kuya tanhan yaunpi; qa miye e wankan tanhan waun; maka kin detanlıan yampi, tuka miye maka kin dematanhan śni.

$24 \mathrm{He}$ etanhan wayahtanipi kin en nițapi kta ce, eciciyapi. He miye kin wicayadapi śni kinhan wayalitanipi kin ehna nițapi kta ce.

25 Unkan, Tuwe niye he, eciyapi. 'Tokag ehan eciciyapi qon he dee, Jesus ewicakiya.

26 Taku ota eciciyapi, qa ciyacopi kta e mduha; tuka tuwe u maśi kin he wowicake; qa taku emakiye cin owasin oyate kin owicawakiyaka ce.

27 Iye Atkuku kin on hena hewicakiye cin he okalinigapi śni.

28 Hehan Jesus hewicakiya; 
Tohan Wicaśta Cinhintku kin wankan eyahdepi kinhan, hehan he miye kin śdonyayapi kta, qa miye cinka takudan ecamon śni; tuka Ate taku onspemakiye cin hena ecedan ecen omdaka ece.

29 Tuwe $u$ maśi kin he mici un; Ate miśnana amahduśtan śni; taku iyokipi ecen ecamon ece, heon etanhan, eya.

30 Hewicakiya icunhan wicota wanna wicadapi.

31 Heon Juda oyate kin etanhan tona Jesus wicadapi kin hena hewicakiya; Mioiẹ kin ohinniyan oyapapi kinhan, awicakshan waonspewicawakiye cin he niyepi kta;

32 Kinhan wowicake kin sdonyayapi kta; qa wowicake kin he niciyuśkapi kta ce.

33 Hehan ayuptapi; Abraham towicoun kin unketanhanpi, qa tohinni tuwedan wowidag unyanpi śni; hecen, Niciyuśkapi kta ce, ehe cin he token heha he.

34 Jesus awicayupte; Wowicake, wowicake on heciciyapi, Tuwe woahtani econ kin he woahtani en wowidake un ece.

$35 \mathrm{Qa}$ wowidake un kin he ohinniyan tipi kin ohna un śni ece; tuka Cinhintku kin liee ohinniyan timahen ounyan.

36 Heon Cinhintkuyapi kin niciyuśkapi hecinhan, awicakehan tawaniçiyapi kita.

37 Abraham towicoun kin enitanhanpi sdonwaya; tuka țe mayayapi yacinpi, mioie kin en niunpi śni, heon etanhan.

38 Taku Ate ekta wamdake cin hena omdaka ece ; qa niś niyate ekta taku wandakapi kin hee ecen ecanonpi ece.

39 Hehan ayuptapi qa heciyapi ; Abraham he ateunyanpi kin ee. Unkan Jesus hewicakiya;
Abraham cinca kin he niyepi unkanś, Abraham ohanye cin he ecen ecanonpi kta tuka.

40 'Iuka nakaha temayan ayakitapi, wicaśta wan wowicake Wakantanka eciyatanhan nawahon kin he ociciyakapi kin he miye tuka: Abraham e qe hecen econ śni qon.

41 Niyatepi ohan. kin hee ecen ecanonpi ece, eya. Hehan heciyapi ; Tuwe cinca tanin śni kin heuncapi śni ; ate unyanpi wanjidan unhapi, Wakantanka kin hee.

42 Heon Jesus hewicakiya; Wakantanka he ateyayapi unkanś waśte mayadakapi kta tuka; miye kin Wakantanka kin hetanhan wahinape ca wau; qa miye cinka wahi śni, iye kin u maśi.

43 He tokeca e iwae cin oyakahnigapi śni he; mioie kin anagoptan wacannipi śni kin heon etanhan.

44 Wakanśica ateyayapi kin henitanhanpi, qa niyatepi tawacin kin ecen ecanonpi yacinpi ece. He otokahe etanhan tinwicakte kin heca, qa wowicake kin en najin śni, wowicake takudan en un śni nakaeś. 'Tohan woitonśni oyaka eca, he iye cinka ohdaka ece ; iye kin woitonśni sa, qa woitonśni atkuku kin hee.

45 Tuka miye wowicake eciciyapi kin, heon etanhan wicamayadapi śni.

46 Niyepi kin wanji tukte he wawahtani e duwicakapi he. Qa wowicake epe cinhan, tokeca e wicamayadapi.śni he.

47 Tuwe Wakantanka etanhan kin he Wakantanka oie anagoptan. Niye Wakantanka anayagoptanpi śni ece, heon etanhan henitanhanpi śni. 
48 Hehan Juda oyate kin ayuptapi qa heciyapi; Samariya oyate henitanhan, qa Wakanśica niyuhnaśkinyan ce, unkeniciyapi qon he wicaunkapi ce.

49 Jesus ayupte; Wakanśica mayuhnaślkinye śni; tuka Ate wahduwitan, qa niye wowiśtenmayayapi.

50 Miye mitowitan awecite śni ; tuka tuwe waakite ça wayaco yukan ce.

51 Wowicake, wowicake eciyatanhan heciciyapi, Tuwe mioie kin de yuhe cinhan, tohinni wiconte wanyake kte śni, eya.

52 Hehan Juda oyate kin heciyapi ; Wakanśica niyuhnaśkinyan e dehan iyotan sdonunyanpi. Abraham hee kaeś ța, qa wokcanpi kin hena nakun țapi ; tuka niye, Tuwe mioie yuhe cinhan tohinni wiconțe ute kte śni ce, eha ce.

53 Abraham ateunyanpi kin hee kacś te cin, niye e iniyotan he. Wokcanpi kin nakun heepi kaeś tapi ; qa niye nituwe e niçicaga he, eyapi.

54 Unkan Jesus waayupte; Miye cinka mihduonihan kinhan, mitowitan kin he taku kte śni; tuka Atewaye cin he mahduonihan ece; unkan he Wakantanka nitawapi, kehapi.

55 Heca eśta tohinni sdonyayapi śni; tuka miye e sdonwakiya. Qa he sdonwaye śni ce, epe cinhan, miś eya niyepi iyecen iwaton kte śni ; tuka miye sdonwaya, heon etanhan oie kin tanyan weciyuha.

56 Abraham ateyayapi qon mitaanpetu kin wanyake kta cin hinca; unkan he wanyake ca iyuśkin ce, eya.

57 Hehan Juda oyate kin heciyapi ; Nahahin waniyetu wikce- mna nizaptan śni, tuka Abraham wandaka he.

58 Jesus hewicakiya; Wowicake, wowicake eciyatanhan heciciyapi; Abraham un qon he itokam wanna waun ce, eya.

59 Hececa unkan inyan icupi, qa on kininpi kta ; tuka Jesus naicilibe ca, tipi wakan etanhan wicehna tanhan tankata inanpe ca, etoopteya iyaya.

\section{WICOWOYAKE 9.}

1 Jesus ye cin tahepi, wicaśta wan toka tonpi ehantanhan tonwe śni un e he wanyaka.

2 Unkan waonspewicakiye cin wiwangapi qa heyapi; Itancan, tuwe wahtani he, wicaśta kin de atkuku, hunku, iyeka, wanji tukte wahtani he, on iśta gonga tonpi he.

3 Jesus waayupte; $\mathrm{He}$ iye, qa atkuku, hunku, wanji walitanipi on hecece śni; tuka Wakantanka olian kin e he ayutaninpi kta e heon.

4 Anpetu icunhan, tuwe u maśi wicohan tawa kin ecen ecamon kta iyececa; hanyetu kin he hi kta, he en tuwedan wicohan econ okihi kte śni.

5 Tohanyan wicaśta opeya waun kin, oyate iyoyamwicawaya ece, eya.

6 Hena heye ça hehan makata tagośa, qa tage on upśija kage ca, iśtagonge cin iśta kin en upśija ikiciun ;

7 Qa heciya; Siloam miniyowe, yeśipi eyapi kin he kapi, he ekta ye ca en ihdujaja wo. Unkan ekta i, qa on ihdujaja qa hdi, unkan wawanyaka.

8 Heon itan anog tipi, qa tona wicaśta iśtagonge cin wanyakapi ece qon hena, Tuwe timata iyotang un qon he dee se, eyapi. 
9 Wanjikji, He dee ce, eyapi: qa toktokeça iś, He de iyececa ce : tuka iye kin, $\mathrm{He}$ miye ce, eya.

10 Unkan, Tokiya tanhan de on niiśta kin dumdaya he, eciyapi.

11 Unkan iye wayupte ca heya; Wicaśta wan Jesus eciyapi kin he upśija kage ça, iśta anokatanhan imiciun, qa, Siloam mininwe kin ekta ye ca, mini on ihdujaja wo, emakiya qehan, ekta wai, qa mihdujaja, unkan watonwan, eya.

12 Unkan, Wicaśta kin he tukte e he, eciyapi. Sdonwaye śni ce, eya.

13 Wicaśta iśtagonge ciqon he Pharisee kin en wicakaipi.

14 Unkan anpetu wakan icunhan, Jesus upśija kage ca on wicaśta kin he iśta yukawakiya.

15 Hehan Pharisee kin iś ake, token on iśta yukawapi hecinhan he iwangapi. Unkan, Upsija e iśta kin en imaun qa mihdujaja, unkan watonwan, ewicakiya.

16 Unkan Pharisee kin wanjikji ; Wicaśta lin he anpetu wakan awacin śni, heon etanhan Wakantanka lietanhan śni, eyapi. Unkan apa, Wicaśta wan walitani sa hecinhan, wowapetokeca dececa token econ okihi kta he, eyapi. Hecen on akipam wacinhnakapi.

17 Ake wicaśta iśtagonge ciqon heriyapi ; Qa niś token eyakiya he, iśta tonweniye cin. Unkan, He vicaśta wolkcan kin heca ce, cya.

18 Wicaśta kin he iśta gonga tuka ihnuhanna, tonwe cin he Juda oyate kin wicadapi śni, lieon tuwe iśta tonweycyapi kin atkuku hunku ko wicakicopi ;

19 Qa wiwicawangapi qa heyapi; De nicincapi qa tonwe śni tonpi kehapi he; qa tokiyatanhan nakaha tonwan he.

20 Atkuku hunku ko waayuptapi qa heyapi ; De unkicincapi tonwe śni tonpi he sdonunkiyapi ;

21 Tuka nakaha tokiya tanhan on tonwe cin de sdonunyanpi śni. Qa iśta yukawa kin de naluun sdonunyanpi śni; iś eya hokśike śni kin, iye iwanga po, toketu kin he ecen eye kta ce.

22 Juda oyate kowicakipapi, qa heon etanhan atkuku hunku kici hecen eyapi. Tuwe, Messiya kin hee, taninyan eye cinhan, he omniciye tipi, kin etanhan tankan iyeyapi kta, Juda oyate kin wanna wakiconzapi.

$23 \mathrm{He}$ etanhan, Iye eś hokśike śni kin, iye atayedan winungapi śni, atkuku hunku kici eyapi.

24. Hehan ake icinonpa wicaśta iśtagonge ciqon he wiwangapi qa heciyapi; Wakantanka woyatan qu wo; wicaśta kin he wahtani sa sdonunyanpi ce.

25 Iye waxyupte ca heya; $\mathrm{He}$ wahtani sa hecinlian sdonwaye śni ; tuka taku wanji sdonwaya; iśtamagonga tuka nakaha watonwe cin he.

26 Unkan, Taku tokanicon qa on iśta kin niyukawa he, ake eciyapi.

27 Unkan awicayupte; Wanna eya keś ociciyakapi, tuka nayahonpi śni; ake icinonpa nayahonpi yacinpi he; niś eya waonspewicakiye cin hen ope nihdawapi kta he, eya.

28 Hehan iwalitedapi 'śni qa heyapi; Waonspewicakiye cin he wanji niye; tuka Moses waonspewicakiye cin he unkiyepi.

29 Wakantanka Moses he okiya sdonunyanpi, tuka wicaśta kin de tokiyatanhan u kin sdonunyanpi śni ce. 
30 Unkan wicaśta kin he waayupte ça hewicakiya; Taliu wowinihan kin he dee; naka iśta mayumdaya, tuka tokiyatanhan u kin sdonyayapi śni.

31 Walitanipi sa kin hena Wakantanka anawicagoptan śni ece, tuka tuwe Wakantanka yuonihan, qa tawacin okicipe cin, hee Wakantanka anakigoptan e sdonunyanpi.

32 Otokaheya tanhan tuwe wicaśta tonwe śni tonpi iśta kiyukawa oyakapi kin tuwedan nalion śni ce.

33 Wicaśta kin de Wakantanka eciyatanhan śni unkanś takudan okihi kte śni ce, eya.

34 Unkan waayuptapi qa, Woalitani ocowasin ehna nitonpi, tuka waonspeunyakiyapi kta kecanni he, eciyapi; qa tankan iyeyapi.

35 Tankarr iyeyapi kin he Jesus nahon; qa akipa, unkan, Wakantanka Cinhintku kin wicayada he, eciya.

36 Iye waayupte ca, He tuwe he, Itancan, wicawada kta ce, eya.

37 Wandake ca he iye e oniciya, Jesus eya.

38 Unkan, Itancan wicawada, eyaya itokam canpeśka makehde inajin.

39 Unkan Jesus heya; Oyate lin de ekta wicawahi, wicaśta tona tonwe śni kin hena tonwanpi kta, qa tona tonwe cin hena iśtagongapi kta, he woyaco kin hduha wahi ce.

40 Unkan Pharisee kicicapi kin hena nahonpi, unkan, Unkiś eya iśtaungongapi he, eciyapi.

41 Iśtanigongapi unkanś woalitani wanin yaunpi kta; tuka nakaha, Untonwanpi ce, ehapi ; heon woalitani niyukanpi, Jesus ewicaliya.

\section{WICOWOYAKE 10.}

1 Wowicake, wowicake eciyatanhan heciciyapi; Tuwe tahinca tipi tiyopa ohna tin iyaye śni, tiyopa icunonpa tanhan ti iyolipaye cin he wabotice ca wamanon kin heca.

2 Tuka tuwe e tiyopa eciyatanhan tin iyaye cin hee talinca tawa wicaye cin ee.

3 Qa iye tiyopa awanyake cin tiyopa kiciyugan ece, unkan tahinca kin he ho kin nahonpi ece; tahinca tawa kin otoiyohi cajeyan wicakico, qa tankan wicahdoya ece.

4 Unkan talinca tawa kin tankan awicau eca, wicitokam ya ece; unkan tahinca kin iś ihakam yapi ece, ho kin iyekiyapi nakaeś.

5 Qa tuwe tokeca kinhan he ihakam yapi kte śni, tuka nakicipapi kta; tuwe tokeca ho kin iyekiyapi śni, heon etanhan.

6 Wiyacinpi kin de Jesus ecen ewicakiya; tuka taku owicakiyake cin hena okalinigapi śni.

7 Hehan ake Jesus hewicakiya; Wowicake, wowicake on heciciyapi, Tahinca tipi tiyopa kin lie miye ce.

8 Tona mitokam hipi kin hena owasin waboticapi qa wamanonpi kin hecapi ; tuka tahinca kin anàwicagoptanpi śni.

9 Tiyopa kin he miye; tuwe miye eciyatanhan ti iyolipaye cinhan ni kta; qa mahen ye ca tankata inanpa eca wato tanyan iyeye kta.

10 Wabotice cin he wamanonpi, tin wicaktepi, waihangyapi henakiya on hi ece; tuka wiconi yuhapi kta, qa takudan icakije śni unpi kta e heon miye wahi.

11 Waawanhdake waśte kin he miye. Waawanhdake waśte kin 


\section{JOHANNES.}

he tahinca kin on wiconi tawa ehpekiya.

12 Tuka tuwe odotapi un, qa waawanhdake kin ee śni, qa talinca tawa śni kin he śunktokeca en $u$ wanyaka eca, tahinca kin ehpewicaye ca najica ece; unkan śunk tokeca iyawicahpaye ça ihangwicaya ece.

13 Odotapi un kin he wodotapi un, qa tahinca kin ihawicakta śni, heon etanhan napa ece.

14 Waawanhdake waśte kin he miye, qa tahinca mitawa kin hena sdonwicawakiya, qa mitawa kin iś nakun sdonmakiyapi ece.

15 Ate token sdonmakiye cin he iyecen miś Ate sdonwakiya; qa talinca kin hena on wiconi mitawa kin ehpewakiya.

16 Qa tahinca toktokeca nakun wicamduha, tahinca tipi kin den un śni : hena nakun awicawahi kta, hecen miho kin nahonpi kta; hetanhan tahinca tipi wanjidan kta, qa waawanhdakc wanjidan kta.

17 Wiconi mitawa elipewakiye ca, ake ewehdaku kte cin heon A te waśte makidaka.

18. Tuwcdan he maki kte śni; qa miye cinka he wicawaqu; he amduśtan kte cin owakihi, qa ewehdaku kte cin nakun owakihi; Ate hecen econ maśi ce, eya.

19 Akeś wicoie kin dena on Juda oyate kin akipam tawacinpi.

20 De taku wakan śica yuha, qa he ihnaśkinyan, tokeca e nayahonpi he, wicota eyapi.

21 Tuka apa iś, Tuwe wakan śica yuhe cin decen oieye śni ece; taku wakan śice cin he iśta gongapi iśta wicakiyukawa okihi kta he, eyapi.

22 Wanna Jerusalem en yuwo- hduze tonpi omniciye kagapi ece qon hehantu; unkan waniyetu.

23 Unkan icunhan tipi wakan ohna, Solomon ohanzi hdepi tawa qon he en Jesus omani.

24 Hehan Juda oyate itan ihdukśan himniciyapi qa heciyapi ; Tohanyan wacin akipam yusunyakiyapi he; Messiya kin he niye hecinhan taninyan rnkokiyaka po.

25 Jesus awicayupta; Wanna ociciyakapi tuka wicamayadapi śni qon; Ate caje kin eciyatanhan wicohan ecamon kin hena omayakc cin ee.

26 Tahinca tawa wicawaye cin he oyapapi śni, he etanhan wicamayadapi śni, eciciyapi qon he iyececa.

27 Tahinca mitawa kin miho kin nahonpi, qa hena sdonwicawakiya, qa mihakamı unpi.

28 Qa owihanke wanin wiconi wicawaqu; qa icimana owihankepi kte śni, qa minape kin etanhan tuwedan wicayuśpe kte śni. 29 Ate hena maqu kin he taku owasin sam iyeya tanka; qa Ate nape kin etanhan tuwedan wicayuśpa okihi śni.

30 Miye, qa Atewaye cin unwanjipidan, eya.

31 Hehan Juda oyate ake inyan icupi, on kininpi kta e heconpi.

32 Unkan Jesus awicayupta; Ate eciyatanhan wicohan waśte ota cicipazopi; hena tukte wanji on nakaha inyan on mayakininpi kta he, eya.

33 Juda oyate ayuptapi qa heyapi ; Wicohan waśte on unnicininpi ktc śni, tuka iapi śica on, qa winicaśta tuka Wakantanka nicicage cin heon etanhan. 34 Jesus awicayupte; Taku wakan kin henicapi, eciciyapi ce, he woope nitawapi kin en hecen kagapi śni he. 
35 Hecen tona Wakantanka oie kin en wicahiyohi kin hena taku wakanpi ewicakiya hecinhan, qa wowapi wakan kin he yujujupica śni ;

36 Hecen tuwe Ateyapi kin hduwakan qa makata u śi kin he, Wakantanka Cinhintku lin he miye ce, epe ciqon, heon, 'Taku śica eha ce, token eyakiyapi kta he.

37 A te olian kin hena ecen ecamon śni kinhan, wicamadapi śni po.

38 Tuka hecen ecamon kinhan, miye wicamayadapi śni eśta, wicolian kin hena wicada po; hecen Atewaye cin micica, qa miye he wecica, heciyatanhan sdonyayapi qa wicayadapi kta ce, eya.

39 Heon etanhan nakun ake tinsa yuze wacinpi ; tuka napepi kin etanhan ihduśpa.

40 Qa ake hetanhan Jordan wakpa kin he en, Johannes baptem wicaqu ece qon he en i, qa en ounyan.

41 Unkan wicota en hipi qa heyapi; Johannes takudan wapetogya ecen econ śni, tuka Johannes wicaśta kin de etanhan tona oyake cin owasin wowicake.

42 Unkan hen wicota wicadapi.

\section{WICOWOYAKE 11.}

1 Unkan wicaśta wan wayazanka, Lazarus eciyapi, Bethani hetanhan, Mary qa cunku Martha totonwepi kin hee.

2 Mary wihdi waśtemna on Itancan kin sdaye ca, paha kin on siha kin kipakinta, Lazarus he hunkawanjitku wayazanke cin hee.

3 Unkan tawinohtinpi kin, Itancan, wicaśta wan waśteyadake ciqon he wayazanka, eya hośi kaipi.

4 Jesus nahon unkan hecen eya, Wowayazan kin he wiconte ekta śni, tuka he Wakantanka towitan kin ee, qa heon Wakantanka Cinhintku kin yuwitanpi kta.

5 Martha iye tankaku, Lazarus kici, Jesus waśte wicadaka.

6 Wayazanke cin nahon, tuka anpetu nonpa ekta ye śni yanka. 7 Hehan Jesus waonspewicakiye cin liewicakiya; Juda makoce kin ekta unhdapi kta.

8 Unkan waonspewicakiye cin heciyapi ; Itancan, nahanbin aśkatudan Juda oyate kin inyan on nicininpi akitapi, qa ake ekta hde wacanni he.

9 Jesus waayupte; Anpetu wanjidan keś, wihiyayedan oape akenonpa śni he; tuwe anpetu icunhan mani kinhan naśna śni ece; maka akan iyoyanpa kin he wanyaka heon.

10 Tuka tuwe hanyen mani kinhan hee naśna kta, iyoyanpa takudan en un śni kin heon.

11 Hena hecen eya; qa hehan iyohakam hewicakiya; Lazarus koda unyanpi qon iśtinma, ekta mde ca mduliice kta ce.

12 Hehan waonspewicaliye cin, Itancan, iśtinma hecinlan he hecetu kta ce, eyapi.

13 He wanna ta e Jesus heya; tuka iśtinmapi on oziiçiya e heya kecinpi.

14 Unkan hehan Jesus taninyan hewicakiya; Lazarus wanna ta ce :

15 Qa he icunhan hen waun śni kin heon niyepi en wimduśkin, hecen on wicayadapi kta. Ho po, ekta unyanpi kta.

16 Hehan Thomas Didumos eciyapi kin he waonspewicakiye unmapi kin hewicakiya; Ekta unyanpi, qa kici unțapi kta ce. 
17. Unkan Jesus en u qehan, liapi etanhan wanna topa can he sdonya.

18 Jerusalem Bethani ikiyedan, wiyutapi akezaptan ecetu hehanyan.

19 Unkan Martha Mary'kici yukanpi en Juda oyate wicota magagaye wicaipi, hunkawanjitkupi kin heon.

20 Jesus en $u$ Martha nation, unkan itkokim ya; tuka Mary e timahen iyotanka yanka.

21 Hehan Martha Jesus heciya; Itancan, den yaun unkanś, mihunkawanji te kte śni tuka;

22 Tuka detanhan taku Wakantanka yakida kinhan, Wakantanka nicu kta e sdonwaya ce.

23 Nihunkawanji ekicetu kta, Jesus eciya.

24 Eya woekicetu anpetu ihankeya kin hehan ekicetu kta e sdonwaya, Martha eya.

25 Unkan Jesus heciya; Woekicetu qa wiconi kin he miye, tuwe wicamada kinhan toke hin ța eśta ni kta.

26 Qa tuwe ni un, qa wicamada kinhan icimana țe kte śni. He wicayada he.

27 Han, Itancan; Messiya Wakantanka Cinhintku makata hi kte ciqon he niye e wicawada ce, eciya.

28 Unkan hena hecen eya, hehan hde ca, tawinohtin Mary analibeyahan kico, qa heciya; Itancan kin den un, qa nico ce.

29 He nalion iheya, unkan oliankoya najin hiyaye ça ekta ya.

30 Nahanhin Jesus otonwe kin ehan i śni, tuka Martlıa itkokipe ciqon he en un.

31 Hehan Juda oyate en yukanpi, qa magagayapi, he icunhan Mary ohankoya najin hiyaye cin wanyakapi qehan, ihakam iya- yapi qa, Wicaśta hnakapi ekta ceye kta e ekta ya, eyapi.

32 Jesus najin kin Mary ehan i, qa wanyake cehan, ihukuya canpeśka makehde ehpeiçiye ça, Itancan, den yaun unkanś, mihunkawanji țe kte śni tuka, eciya.

33 Mary ceye cin he Jesus wanyake ca, Juda wicaśta kici ipi kin hena nakun ceyapi; unkan iye atay edan comnihdazi, qa iliniyanyan.

34 Qa tokiya eyahnakapi he, eya. Unkan, Itancan, ekta u qa wanyaka ye, eciyapi.

35 Jesus ceya.

36 Hehan Juda oyate kin heyapi; Wanyaka po, ehan qon waśte dake hinca ce.

37 Qa wicaśta wanjikji, Wicaśta iśta aohduta ee kaeś yukawa, wicaśta kin de țe śni unkiya okihi kta naceca ce, eyapi.

38 Ake Jesus iye atayedan comnihdazi, qa wicaśta hnakapi kin en ya. He imnija olidoka ohna hnakapi, qa inyan wan tanka aokatinzapi.

39 Inyan kin tokan iyeya po, Jesus eya. Wicaśta te cin tawinohtin Martha heciya, Itancan, wanna topa can yanka e śicamna.

40 Wicayada kinhan, Wakantanka towitan wandake kta ce, eciciye śni he, Jesus eciya.

41 Hehan inyan kin tokan iyeyapi ; wicaśta ța wanke cin etanhan. Unkan Jesus iśta yuwankan ikikcu, qa heya ; Ate ciyawaśte, namayahon kin heon;

42 Qa ohinniyan namayahon kin he sdonwakiya; tuka wicaśta hnakapi kin de en mitan okśan najinpi kin de on, he niye u mayaśi kin wicadapi kta e on hepe.

43 Hecen eye ca hehan, Lazarus nunke cin hetanhan tankata 
hninanpa wo, awitukadan hoyekiya qa eya.

44 Unkan wicaśta te cin tankata hninanpa, siha nape ko pahtapi, qa ite opemni tonpi kin hena ecehna. Kiyuśkapi qa ayuśtan po, iyaye kta, Jesus ewicakiya.

4.5 Hehan Juda wicaśta Mary kici ipi qon, hena Jesus taku econ kin he wanyakapi, unkan wicota wicadapi.

46 Tuka wanjikji Pharisee kin ekta wicakipi, qa Jesus taku econ kin owicakiyakapi.

47 Hehan wośna kagapi Pharisee ko mniciyapi qa, Token econkupi he ; wicaśta kin de taku wapetokeca ota econ kin, eyapi.

48 Iyowinunkiyapi kinhan wicaśta owasin wicadapi kta ; kinhan Roma oyate kin den upi kta, qa otonwe kin de unkasotapi, qa nakun unkitaoyatepi kin hena ko.

49 Hehan wanji Kaiaphas eciyapi waniyetu kin he en wośna kagapi itancan kin hee hewicakiya; Takudan oyakalinigapi śni.

50 Qa nakun awacannipi śni, wicaśta wanjidan oyate on te cinhan, hecen on oyate kin wicațe kte śni, he unkiyepi kin on hecetu kta ce, eya.

51 Iye etanhan on hecen eye śni, tuka omaka kin he en wośna kagapi itancan kin heon, Jesus oyate kin on te kte cin he iyukcanyan heya.

52 Qa oyate kin heceedan śni, tuka Wakantanka cinca omdecahe cin hena owasin wanjidankiya kiwitaye kta.

53 Hehan anpetu kin hetanhan witaya akiiapi, țeyapi kte cin heon.

54 Heon etanhan Jesus Juda oyate kin wicehna taninyan omani śni, tuka makoce wan liewoskan ikiyedan wanke, otonwe wan Ephraim eciyapi kin he ekta ya, qa waonspewicakiye cin om he en un.

55 Unkan woacakśin Juda oyate wośnapi kagapi wanna ikiyedan aya; unkan wanna kayehan oyate ota Jerusalem en kata iheya aya, woacakśin kin itokam piiçiyapi kta.

$5 \dot{6}$ Hehan Jesus akitapi, qa tipi wakan kin ohna najinpi icunhan iyakitedan hekiciyapi ; Token idukcanpi he; wośnapi kin en u kte śni he.

57 Wośna kagapi tankapi qa Pharisee ko wanna wakiconzapi, tuwe Jesus tukten yanka sdonye cinhan, he oyake kta, hecen yuzapi kta.

\section{WICOWOYAKE 12.}

1 Woacakśin kin itokam sakpe can, hehan Jesus Bethani en $i$, Lazarus wicaśta ța, tuka ekicetuyapi en un qon otonwe kin hee.

2 Unkan hen litayetu wotapi wan kicagapi, qa Martha e wapamni ; tuka kici iyotang wotapi kin Lazarus wanji hee.

3 Hehan Mary wihdi waśtemna qa taku ota iyopeyapi, tke utapi wanjidan, he icu qa on, Jesus siha kin sdakiye ca, iye paha kin on siha kipakinta; unkan ecamna kin tipi kin ojudan.

4 Hehan Simon cinhintku, Judas Iskariot eciyapi, waonspewicakiye cin wanji hee, wiyopeye kte cin hee hecen eya;

5 He tokeca wihdi kin he kaśpapidan opawinge yamni iyopeyapi, qa wahpanicapi kin wicaqupi śni he.

$6 \mathrm{He}$ wamanon heca, qa mazaska opiye yuhe ça, ohna mazaska omnayan, heon hecen eya; wo- 
JOHANNES.

wahpanica kin incantowicahnake cin heon heye śni.

7 Hehan Jesus heya; Nagiyeye śni wo; anpetu wan mahnakapi kte cin he en amayuhe cin hee ce.

8 Tokeśta wowalipanica ohinni wicaduhapi kta; tuka miye e ohinniyan mayaduhapi kte śni, eya.

$9 \mathrm{He}$ icunhan hen un kin Juda oyate wicota sdonyapi; unkan Jesus ecedan on en hipi śni, tuka Lazarus, wiconte etanhan najinkiye ciqon, he wanyakapi kta on etanhan.

10 Unkan wośna kagapi tankapi Lazarus nakun teyapi kta akitapi :

11 Hee nakun on Juda oyate ota hduheyapapi, qa Jesus wicadapi.

12 Ihanlianna wicaśta ota wośnapi kin en hipi kin hena, Jesus Jerusalem etkiya $u$ nahonpi hehan,

13 Canwapa tanka etanhan cankika icupi, qa itkokim yapi, qa panyanhan heyapi ; Hosanna, tuwe Israel Wicaśtayatapi, Jehowa caje kin on u kinhan, he yawaśtepi nunwe.

14 Unkan Jesus śonśonna cinca wan iyeya, hehan akan iyotanka; liecen wowapi kagapi qon;

15 Zion cunwintku ihnuhan koyakipe cin; iho, Wicaśtayatapi nitawa śunka śonśona cinca wan akan yanka u ce.

16 Dena taku kin tokaheya waonspewicakiye cin okahinigapi śni ; tuka Jesus yutanpi hehan hena hecen wowapi en kagapi qon, qa nakun hena ecen ecakiconpi kin, hehan kiksuyapi.

17 Heon oyate kicicapi kin, Lazarus hnakapi tuka kipan, qa wiconte etanhan ekicetuye ciqon, hena he yaotaninpi.
$18 \mathrm{Qa}$ heon oyate kin itkokipapi, taku wapetokeca tanka kin de econ nahoupi kin heon.

19 Unkan heon etanhan Pharisee kin iyakitedan hekiciyapi; Wanyaka po, takudan oyakihipi śni; iho, wicaśta owasin he ihakam iyayapi.

20 Helenes oyate kin etanhan wanjikji wośnapi kin icunhan en ohodapi kta e ekta itanwankanhde upi ;

21 Hena eepi Philip, Galile makoce Bethsada otonwe kin etanhan, he en ipi, qa, Itancan, Jesus wanunyakapi uncinpi ce, eciyapi.

22 Philip ta, qa Andrew okiyaka; qa hehan Philip Andrew kici Jesus okiyalkapi.

23 Unkan Jesus awicayupte ca heya; Anpetu wan en Wicaśta Cinhintku kin yutanpi kte cin wanna hiyohi.

24 Wowicake, wowicake eciyatanhan heciciyapi; Aguyapi su kin maka ekta elipeyapi, te śni kinhan, iśnana un kta; tuka te cinhan ota icaliye kta.

25 Tuwe wiconi kin de waśte kida kinhan, toki ehpeye kta; qa tuwe maka kin den wiconi śice kida kinhan, hee owihanke wanin wiconi ekta akpatan kta:

26 Tuwe omakiye kta cin lkinhan mihakam un kta; qa tukten waun hecinhan mitaokiye kin iś nakun hen un kta; nakun tuwe omakiye kta hecinhan, Ate wicaśta kin he yuonihan kta.

27 Wanna minagi kin nagiyeyapi, hecen token epe kta he. A te, anpetu kin de etanhan ema-hdaku wo. Tuka heon anpetu kin de en wahi ce.

28 Ate nicaje kin hdutan wo. Unkan mahpiya kin eciyatanhan wicaho wan u qa; Wanna eya 
micaje kin wahdutan, nakun wahdutan kta, eya.

29 Unkan wicaśta en najinpi kin he nahonpi qa, He wakinyan hoton, eyapi. Malipiya ohnihde heca e okiya ce, apa eyapi.

30 Unkan Jesus waayupte ça heya; Wicaho kin de miye make sni, he niye nicapi.

31 Wanna oyate kin wicayacopi ; qa wanna maka akan itancan kin tankan elpeyapi.

32 Qa tohan maka kin detanhan wankan emahdepi kinhan, wicaśta kin owasin miye en iwieawacu kta, eya.

33 Wiconte kin tukte on te kte cin he ka e hecen eya.

34 Oyate kin ayuptapi; Woope kin eciyatanhan, Messiya owihanke wanin un kta ce, eyapi naunlionpi ; hecen, Wicaśta Cinhintku kin wankan ehdepi kta ce, token on hecen eha he; Wicaśta Cinhintku kin he tuwe he.

35 Hehan Jesus hewicakiya; Iyoyanpa kin ehake cistiyedan nicipi un; iyoyanpa duhapi kin icunhan en omani po; otpaze cin iyapaśtag niyanpi nun ce; tuwe otpaza icunhan omani kin he tokiya ye cin sdonye śni.

36 Iyoyanpa duhapi kin en iyoyanpa kin he wicada po, hecen on iyoyanpa cinca yaunpi kta ce. Jesus hena hecen eye ça, hehan iyaye ca inahbe wicakiya.

37 Taku wapetokeca ota econ, qa wanyagwicaya, tuka wicadapi śni.

38 Hecen Isaya wicaśta wolican taku eye ciqon wanna iyecetu; Itancan, tuwe wounyakapi kin wicada he; qa tuwe en Jehowa hupahu kin yutaninpi he. $39 \mathrm{Qa}$ heon etanhan wicadapi lkta okihipi śni; heon Isaya ake hecen eya ;

40 Iśta gonge wicaye ça, cante kin ekta nakun wacinton śni wicaya; hecen iśta on wiwanyalkapi kte śni, qa cante on takudan yukcanpi kte śni, qa ihduecetupi lkte śni, qa hecen wokiziwicawaye śni.

41 Towitan kin he Isaya wanyake ciqon he ehan hena hecen ekiciya.

42 Hececa tuka itancanpi etanhan wicota wicadapi; tuka Pharisee kin hena on etanhan yaotaninpi śni, omniciye tipi kin etanhan tankan iyewicayapi kta ikopapi heon.

43 Wicaśta akantu yaonihanpi kin he iyotan dapi, qa Wakantanka wicayaonihan kin he iś iyotan dapi śni.

44 Hehan Jesus panyanhăn heya; Tuwe wicamada kinhan, he miye wicamada śni, tuka tuwe u maśi kin hee wicada ece. $45 \mathrm{Qa}$ tuwe wanmayake cin he nakun tuwe u maśi kin he wanyaka.

46 Miye maka akan iyoyanpa wahi; hecen tuwe wicamada kinhan he otpaza en omani kte śni.

47 Qa tuwe mioie kin nahon, qa wicada śni eśta mdaco śni ; wicaśta wicayacopi kin heon wahi śni, tuka waniyapi lin hee on wahi.

48 Tuwe amáktaśni, qa mioie kin icu śni kinhan he tuwe yaco kte cin hduha; wicoie omdake cin anpetu ihankeya kin en yaco kte cin hee.

$49 \mathrm{He}$ miye cinka hecen oiewaye śni ; tuka Ate u maśi kin he iye econ maśi, taku epe kte ca taku omdake kte cin.

$50 \mathrm{Qa}$ toope kin he owihanke wanin wiconi e sdonwaya. Token epe cin hena, Ate hena hecen emakiya, heon etanhan hecen epa ece, eya. 


\section{WICOWOYAKF 13.}

1 Anpetu en wośnapi ece kin itokam, anpetu qon wanna hiyohi, he en maka kin detanhan iyaye kta, Atkuku ektakiya hde kta, Jesus sdonkiya, tona maka akan tawa kin waśtewicadake cin hena owihanketa waśtewicakida.

2 Wotapi ayaśtanpi hehantu; Judas Iskariot Simon cinhintku kin hee, Jesus wiyopeye kte cin, Wakanśica wanna hecen cante ozekiya.

3 Hehan Jesus Atkuku taku owasin tawa kiye ciqon he sdonkiya un, Wakantanka eciyatanhan hi, qa ake Wakantanka ekta hde kta.

4 Hecen wotapi kin etanhan najin, qa śina ekihnaka; qa napipakinte wan icu, qa ipiyaka.

5 Qa hehan mini wakśica en okaśtan, qa waonspewicalkiye cin siha wicakiyujaja, qa napipakinte wan ipiyake ciqon heon siha kin wicakipakinta.

6 Simon Inyan ehan ahi; unkan, Itancan, niye siha mayakidujaja kta he, eciya.

7 Unkan Jesus ayupte ca, Taku ecamon kin de nakaha sdonyaye śni, tuka ihakamya sdonyaye kta, eciya.

8 Icimana siha mayakidujaja kte śni, ake Inyan eciya. Hehan Jesus ayupte; Ciciyujaja śni kinhan takudan on miyecica kte śni, eya.

9 Hehan Simon Inyan heciya; Itancan, siha ecedan śni, tuka minape, mapa koya.

10 Unkan Jesus heciya; Tuwe wanna ihdujaja kin he takudan sanpa cin śni, siha ecedan yujajapi kta, eya wanna ocowasin ska; unkan niye niskapi, tuka iyulipapi śni.
11 Tuwe wiyopeye kte cin he sdonya, heon etauhan, owasin niskapi śni ce, eya.

12 Unkan siha wicakiyujaja yuśtan kin hehan śina ehdaku qa hdowin qa iyotanke ça, Taku tokaciconpi kin de sdonyayapi he, ewicakiya.

13 Waonspekiya qa Itancan, emayakiyapi ece; he tanyan ehapi ; he miye nakaeś.

14 Heon etanhan miś miye Waonspekiya qa Itancar mayaduhapi kin, siha ciciyujajapi kinlian, niś eya otoiyohi siha yeciyujajapi kta iyececa.

15 Waonspeciciyapi kin he niś he iyecen ecanonpi kta e hecaciconpi.

16 Ookiye unpi kin he Itancan kin iyakapa śni; qa tuwe u śipi kin he tuwe u si hecinhan, he iś iye kapa śni, wowicake, wowicake eciyatanhan eciciyapi.

17 Taku kin dena sdonyayapi hecinhan, ecen ecanonpi kinhan waśteya yaunpi kta.

18 Taku epe cin de niye iyulipa heciciyapi śni ; tona wicawakahnige cin hena sdonwicawakiya ; tuka wowapi en kagapi qon yuecetu kta; Tuwe kici aguyapi wata ece kin he miye on siha yuwankan ehdaku ce.

19 Taku kin dena ecetu śni itokam ociciyakapi; hecen tohan iyecetu kinhan, he miye e wicayadapi kta.

20 Tuwe tokiya yewakiye cin tuwe iyowinkiye cinhan, he miye e iyowinmaye; qa tuwe iyowinmaye cinhan, he tuwe u maśi kin he iyowinkiya, wowicake, wowicake eciyatanhan eciciyapi.

21 Jesus hena hecen eye ca hehan toksape kin ekta iyahdeiciya, hdaotanin qa heya; De dukanpi kin etanhan wanji wi- 
yopemayayapi kta, wowicake, wowicake on eciciyapi ce.

22 Hehan waonspewicakiye cin opakicihta yukanpi, wanji tukte e on heye cin sdonyapi śni.

23 Unkan Jesus waonspewicakiye cin wanji nina waśte kidake ciqon, he eca maku kin en makan iwanka.

24. Heon Simon Inyan he wikiyuta, qa wanji tukte he on heye cin he iwange śi.

25 Hehai he Jesus maku kin en iwanke ca, Itancan, he tuwe he, eya.

26 Jesus ayupte; Wanji tukte hpanyanpi onśpa waqu kinhan hee kta, eya. Hecen onśpa hpanye ça, Judas Iskariot eciyapi Simon cinhintku he qu.

27 Unkan Judas hpanyanpi kin icu, hehan Satan mahen iyaya. Hecen Jesus heciya; Taku ecanon kte cin kohanna econ wo.

28 Wahna wotapi kin ihdukśan yukanpi kin, taku on heye cin tuwedan okahnigapi śni.

29 Judas mazaska opiye yuhe cin heon, Jesus wośnapi kta on taku cinpi kin he opeton śi; qa iś, wahpanicapi kin taku wicaqu śi, apa kecinpi.

30 Unkan hpanyanpi qon he icu qehan, hecehnana tankan iyaya; wanna hanyetu hehantu.

$31 \mathrm{He}$ tankan iyaya, hchan Jesus heya; Wanna Wicaśta Cinhintku kin yutanpi, qa nakun iye eciyatanhan Wakantanka iś yu tanpi.

32 Qa iye eciyatanhan Wakantanka yutanpi hecinhan, hehan Wakantanka iś iye kin on hdutan kta, nakun kohanna hdutan kta.

33 Hokśiyoqopa ehake ptenyedan ocipapi kta. Amayakitapi kta, qa Juda oyate ewicawakiye ciqon; Tolkiya mde cin ekta yaipi kta oyakihipi śni ce; he iyecen nakaha eciciyapi.
34. Woahope teca wan ciçupi, otoiyohi waśteyecidapi kta ; waśtecicidapi kin he iyecen waśtcyecidapi kta.

35 Heon waonspeciciyapi yaunpi kin wicaśta owasin sdonyapi kta, otoiyohi waśteyecidapi kinhan.

36 Itancan, tokiya de $\mathrm{kta}$ he, Simon Inyan eciya. Jesus ayupte; Tokiya mde cin ekta de kta eśta nakaha oyakihi kte śni, tuka ihakamya mihektam yau kta, eya.

37 Unkan Inyan heciya; Itancan, tokeca nakaha cihakam waun kta owakihi śni he; niye on wiconi mduhe cin ehpewaye kta, eya.

38 Jesus ayupte; Miye on wiconi nitawa ehpeyaye kta he. Wowicake, wowicakc on heciciya; Anpaohotonna kin hoton śni itokam yamni akihde sdonmayaye śni, kehe kta ce, eya.

\section{WICOWOYAKE 14.}

1 Ihnuhan cante niśicapi kinhan; Wakantanka wicayadapi, qa miśs nakun wicamayadapi.

2 Ate ti kin en ounyanpi kte cin ota yanka; he hecece śni unkanś ociciyakapi kta tuka; ekta wahde ca oyanke kin ciciyuśtanpi kta.

3 Ekta mde ca oyanke ciciyuśtanpi kinhan, hehan ake wau qa acihdapi kta; hecen miś waun kin he en niś nakun yaunpi kta. 4 Unkan tokiya mde cin he sdonyayapi, qa canku kin he nakun sdonyayapi.

5 Itancan, tokiya de cin sdonunyanpi śni, tokiya tanhan canku kin he sdonunyanpi kta he, Thomas eciya.

6 Unkan Jesus heciya; Canku, qa wowicake, wiconi ko he mi169 
ye; tuwedan Ateyapi kin en u śni, miye eciyatanhan ecedan en u ece.

'7 Sdonmayayapi unkanś nakun Atewaye cin sdonyayapi kta tuka; qa detanhan sdonyayapi qa wandakapi ce.

8 Itancan, Ateyaye cin wanyag unyan po, hecen iunmnanpi Irta, Philip eciya.

9 Hehan Jesus heciya; Philip, wanna wanakajatanhan cicipi waun, tuka nahanhin sdonmayaye śni he; tuwe wanmayake cin he Ateyapi kin wanyaka; hecen tokeca, Ateyapi kin wanyag unyan miye, eha he.

10 Atewaye cin he wecica qa Atewaye cin he micica kin he wicayada śni he. Wicoie eciciyapi kin dena miye cinka eciciyapi śni; Atewaye micica kin he wicolian kin hena iye econ.

11 Atewaye cin he wecica qa Atewaye cin he micica he wicamada po; qa iś wicohan kin hena e on wicamada po.

12 Wowicake, wowicake on heciciyapi; Tuwe wicamada kin he wicohan ecamon kin hena iś iyecen econ kta, nakun eeś sanpa tanka econ kta; Ate ekta wahde kte cin heon etanhan.

13 Qa micaje kin on taku yadapi kinhan, ecen ecamon kta; Ateyapi kin Cinhintku kin on yutanpi kta e heon.

14 Taku wanji micaje on yadapi kinhan, ecen ecamon kta.

15 Waśte mayadapika hecinhan, mitawoahope kin tanyan yuha po.

16 Hehan Ate cewakiye kta, unkan wicakicanpte wan tokeca nicupi kta, he owihanke wanin nicipi un kta.

17 Wowicake Woniya kin hee, oyate kin he icu okihipi śni, tohinni wanyakapi śni qa sdonya- pi śni; tuka niye he sdonyayapi, he nicipi un qa en niunpi kta. 18 Wandenica elipeciyapi kte śni-; ake cihdiyohipi kta.

I9 Wanna ptenyedan kinhan oyate wanmayakapi kte śni, tuka niye wanmayadakapi kta; wani waun, qa niś yani yaunpi kte cin heon.

20 Anpetu kin he en, Atewaye cin he wecica, qa niś miyecicapi, qa miś ocicipapi kin he sdonyayapi kta.

21 Tuwe mitawoahope yuhe ca, liena ope cinhan he waśtemadake cin ee; qa tuwe waśtemadake cinhan, Atewaye cin he waśtedake kta, qa miśs nakun waśtewadake kta, qa en mihdutanin kta ce.

22 Hehan Judas, Iskariot hee śni, he heciya ; He tolieca Itancan, unkiśnana sdonniye unyayapi kta he, qa oyate kin sdonniyanpi kte śni.

23 Jesus waayupte ca heciya; Tuwe waśtemadake cinhan mioie kin yuhe kta; qa Atewaye cin waśtedake kta, qa tukten un kinhan en unhipi kta, qa ti kin en kici unyakonpi kta.

24 Tuwe waśtemadake śni kin he mioie lin yuhe śni. Wicoie nayahonpi kin de mitawa śni, Atewaya umaśi kin he iye tawa. 25 Ocipapi kin en taku kin hena heciciyapi.

26 Tuka Wicakicanpte, Woniya Wakan kin hee, Atewaye cin micaje on uśi kte cin he, taku owasin onspeniciyapi kta, qa ta$\mathrm{ku}$ eciciyapi kin owasin kiksuyeniciyapi kta.

27 Wokiyapi aihpeciyapi; wookiye mitawa ciçupi: wicaśta akantu kicicupi he iyecen cicupi śni. Ihnuhan cante niśicapi qa kopehda yaunpi kinhan.

28 Wahde ca ake en ciupi kta 
eciciyapi kin he wanna nayahonpi. Waśte mayadapi kinhan iduśkinpi kta; Atewaye cin ekta wahde kta epe cin he; Atewaye cin he iye mikapeya tanka.

29 Qa nakaha hena heciciyapi, taku kin itokam; tohan he hiyohi kinhan hehan wicayadapi kta. 30. Detanhan taku ota eciciyapi kte śni; maka akan itancan kin wanna $u$, tuka miye kin en takudan tawa śni.

31 Tuka Atewaye cin waśtewakida; qa Ate taku emakiye cin owasin ecen ecamon ece, he oyate kin sdonyapi lita. Najin po, detanhan icunonpa unyanpi kta.

\section{WICOWOYAKE 15.}

1 Hastanhanka iyuwi wowicake kin he miye, qa Atewaye cin he kicanye cin ee.

2 Adetka tona miye on wasliuyeca icaliye śni kin hena yupśun ; qa adetka tona waskuyeca icahye cin hena pakinta, hecen on waskuyeca ota icahye kta.

3 Eya wanna niskapi, wicoie ociciyakapi kin heon.

4 Mici un po, hecen miś cicipi waun kta. Hastanhanka iyuwi adetka wiyuwi kin en ikoyake śni kinhan waskuyeca icahya okihi śni ece; he iyecen miyeci yaunpi śni ehantanhan, niś eya oyakihipi kte śni.

5 Hastanhanka iyuwi kin he miye, adetka kin he niyepi. Tuwe mici un qa miś kici waun kin he waskuyeca ota icaliya; miye kin en icunonpa yaunpi kinhan takudan oyakihipi kte śni.

6 Tuwe mici un śni kinhan adetka iyecen tankan ehpeyapi, hecen śnija aya; unkan hena wicaśta pahipi qa petan elipeyapi, hecen huhnaga ece.

7 Miyeci yaunpi qa mioie kin he nicipi un kinhan, taku tona yacinpi kin hena yadapi kta, unkan ecen ecaniconpi lita.

8 Waskuyeca ota icaliyayapi kinhan Atewaye cin nina yutanpi kta; qa heciya tanhan waonspeciciyapi yaunpi kta.

9 Atewaye cin token waśte makida kin he iyecen waśte cicidapi; mitowaśte kin he kici un po.

10 Mitawoahope kin duhapi kinhan, initowaśte kin he kici yaumpi kta; Ate tawoahope mduhe cin heon towaśte kin kici waun kin he iyececa.

11 Taku eciciyapi kin dena on mitowiyuśkin kin nicipi un kta e, qa nitowiyuśkinpi kin he ecetu niciciyapi lita.

12 Mitawoahope kin he dee, waśtecicidapi kin he iyecen waśteyecidapi kta.

13 Tuwe takuwicaye cin on wiconi elipekiye cinhan, he wowaśte lkin tuwedan kapeye śni.

14 Taku econ ciśipi kin owasin ecen ecanonpi kinhan takuciyapi yaunpi.

15 Ookiye wicayapi wanna eciciyapi śni; ookiye yapi un kin he Itancan ye cin taku econ keś sdonye śni. Tuka takuciyapi eciciyapi; Atewaye cin tona nationmaye cin owasin sdonye ciciyapi kin heon.

16 Niye he mayakahnigapi śni, tuka miye e cicahnigapi qa ciyusutapi; hecen tokiya dapi qa waskuyeca icahyayapi kta; qa waskuyeca nitawapi kin ohinniyan un lita; hecen micaje on Atewaye cin taku yakidapi kinhan nicupi kta ce.

17 Otoiyohi waśte kicida po, he iwahokonciciyapi ce.

18 Oyate kin śice nidapi eśta, he itokam miye śice madapike ciqon he sdonyayapi.

\section{a

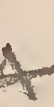




\section{JOHANNES.}

19 Oyate kin enitanhanpi unkanś oyate kin taku iye tawapi waśte kidapi kta; tuka oyate kin henitanhanpi śni, miye e oyate kin etanhan cicahnigapi kin heon oyate kin śice nidapi.

20 Wicoie wan eciciyapi qon he yeksuyapi; Ookiye yapi un kin he Itancan yuhe cin kakipe "ẹa iyotan tanka śni. Śicaya makuwapi hecinhan, niś oyate kin śicaya nicuwapi kta; qa mioie yuhapi hecinhan, niś nioie kin niciyuhapi kta.

21 Tuka nicaje kin on liena owasin ecen ecaniconpi kta; tuwe u maśi kin he sdonyapi śni heon etanhan.

22 Wahi śni qa owicawakiye śni kinhan, woahtani nicapi kta tuka; tuka detanhan woahtani woakahpe nicapi.

23 Tuwe śice madake $\operatorname{cin}$ he nakun Atewaye cin śice daka.

24 Wicohan tuwedan hecen econ śni iyecen ecawicawecon sni unkanś, woahitani nicapi kta tuka; tuka wanna Atewaye cin kici wanmayakapi, qa napin śice undakapi.

25 Tuka woope tawapi kin en wicoie kagapi qon he wanna iyecetu; 'Takudan on etanhan śni śice madakapi ce.

26 Tuka Wicakicanpte, Atewaye cin etanhan uwaśi kte cin he, Wowicake Woniya kin hee, tohan hi kinhan, he iye Atewaye cin etanhan u kta, qa omayake kte cin hee.

27 Qa niś nakun omayadakapi kta, toka ehantanhan miyahna yaunpi qon heon.

\section{WICOWOYAKE 16.}

1 Wayahtanipi kte śni e heon dena taku kin ociciyakapi.

2 Omniciye tipi kin etanhan 172 tankan iyeniyanpi kta; nakun anpetu wan u kta, hehan tuwe kaśta niktepi kinhan, he Wakantanka iyokipiya kecin kta.

3 Atewaye cin sdonyapi śni, qa miś nakun sdonmayanpi śni, he etanhan henakiya hecen ecanionpi kta.

4 'Tuka dena ociciyakapi, he . cen tohan iyehantu kinhan, dena wanna eciciyapi kin he yeksuyapi kta. Qa dena otokahe ekta eciciyapi śni, hinyahin cicipi waun kin heon etanhan.

5 Tuka wanna tuwe umaśi kin he ekta wahde kta; qa wanjidan, Tokiya de kta he, emayakiyapi śni.

6 Tuka taku kin dena ociciyakapi, heon nicantepi kin ekta iyoniyakapi.

7 Tuka wowicake ece ociciyakapi; wahde cinhan heon tanyan yaunpi kta, qa wahde śni kinhan Wicakicanpte kin en nihipi kte śni; tuka wahde cinhan niyepi ekta uwaśi kta.

8 Unkan he tohan hi kinahan, woahtani, woowotanna qa woyaco kin on oyate kin sdonyewicaye kta:

9 Woahtani kin, wicamadapi śni kin heon:

10 Woowotanna kin, Atewaye cin ekta wahde kta, qa icimana wanmayadakapi kte śni, heon :

11 Woyaco kin, oyate kin de itancan un kin he wanna yacopi, heon etanhan.

12 Nakun taku ota eciciyapi kta mduha, tuka hena e qe nahanhin yuha oyakihipi śni.

13 Tuka tohan Wowicake Woniya kin he iye hi kinhan, wowicake ocowasin en aniyanpi lita ; iye atayedan on ie kte śni, tuka taku nahon kin hena oyake kta, qa taku tokata ekta hena oniciyakapi kta. 
14 Mayutan kte cin hee, taku mitawa kin hena icu, qa oniciyakapi kta.

15 Taku Ate tawa kin hena owasin mitawa; he etanhan, Tona mitawa kin hena icu, qa oniciyakapi kta ce, eciciyapi.

16 Wanna aśkayedan hehan wanmayadakapi kte śni ; hchan akeś aśkadan ake wanmayadakapi kta, Ate ekta wahde kte cin hcon.

17 Hehan waonspewicakiye cin iyakitedan hckiciyapi; Unkckiciyapi kin he taku hwo; Wanna aślkayedan wanmayadakapi kte śni, qa hehan aśkadan ake wanmayadakapi kta; qa, Ate ekta wahde kte cin heon.

18 Hcon heyapi; Aśkayedan eye cin he taku he; taku eye cin sdonunyanpi śni ce.

19 Wiwangapi cinpi kin he Jesus sdonya, unkan heon hewicakiya; Wanna aśkayedan wanmayadakapi kte śni, qa hen ikiyedan wanmayadakapi kta, epe cin he, he token kapi hwo, otoiyohi eyeciyapi he.

20 Wowicake, wowicake on heciciyapi; Nihinniçiyapi qa yaceyapi kta, tuka oyate kin iyuślkinpi kta ; iyoniciśicapi kta, tuka iyoniciśicapi kin he wowiyuśkin wan en un kta.

21 Winolinca cinca ton-kta cca yazan hda, wanna iyekicihantu kin hcon; tuka tohan hokśiyogopa ton eca, yazan hda qon he kiksuye śni, wicaśta oyate kin den tonpi eca wowiyuśkin ye cin heon.

22 He iyecen nakaha iyokiśin dukanpi, tuka ake wanciyakapi kta, kinhan nicantepi kin wiyuśkin kta, qa widuśkinpi kte cin he tuwedan nicipi kte śni.

23 Unkan anpetu kin hc en takudan imayanungapi $k$ te śni.
Wowicakc, wowicake on heciciyapi; Taku micaje on Atewaye cin yakidapi kinhan niçupi kta.

24 Nahahin dehanyan micaje on takudan yadapi śni. Da po, iyacupi kta, hecen wowiyuśkin ojudanniciyapi kta.

25 Hena taku iyacinyan heciciyapi ; tuka anpetu wan u kta, hehan wiyacinyan takudan cciciyapi kte śni, tuka taninyan Ateyapi kin on ociciyakapi kta. 26 Tohan iyehantu kinhan micaje on taku yadapi kta, tuka Atcwaye cin wocekiye eciciyapi kta, epe śni.

27 Ate iye atayedan waśte nidapika, waśte mayadapike ca Wakantanka eciyatanhan wahi kin he wicayadapi kin heon.

28 Atcwaye cin eciyatanhan wau, qa oyate kin de en wicawahi Ake oyate kin ehpewicawaye kta qa Ate ekta wahde kta.

29 Wanna wiyacinyan iyac śni, ataninyan iyaa ce, waonspewicakiye cin eciyapi.

30 Taku owasin sdonyaye ca, tuwe winiwange kta iyecece śni yaun e wanna sdonunyanpi ; heon etanhan Wakantanka eciyatanhan yahi kin he wicaundapi, eyapi.

31 Jesus awicayupte; Wanna wicayadapi he.

32 Anpetu wan u kta qa wanna hiyohi, enanakiya otoiyohi yahdapi kta, qa miśnana ehpemayayapi kta; tuka nakun miśnana waun śni, Atewaye cin he mici un.

33 Wookiye amayaduhapi kta heon etanhan hena heciciyapi. Oyate kin de en wokakije duhapi kta; tuka wiyuśkin po, miye oyate kin ohiwicawaya ce. 


\section{WICOWOYAKE 17.}

1 Jesus hena hecen eye ca hehan iśta yuwankan icu, qa malipiya kin ekta etonwan qa, Ate wanna anpetu kin hiyohi, Nicinkśi kin hdutan wo, hecen on Nicinkśi kin nihdutan kta ce.

2 Wicacehpi owasin on wowaśake yaqu qon, hecen tona yaqu kin owasin owihanke wanin wiconi wicaqu kta.

3 Unkan owihanke wanin wiconi kin he dee, wowicake Wakantanka niye e sdonniyanpi kta, qa Jesus Messiya uyaśi qon.

4 Maka kin akan ciyutan; qa wicokicanye mayaqu qon wanna owasin wahduśtan.

5 Unkan nakaha, Ate niye kin on miye mahdutan wo, wicoicage wanice cin he itokam wowitan niyeci hduha waun qon heon.

6 Oyate kin den wicaśta tona mayaqu kin hena en nicaje modaotanin; hena nitawa, tuka hena mayaqu; unkan hena nioie qon niciyuhapi.

7 Taku mayaqu kin owasin he niye etanhan $u$, wanna sdonyapi.

8 Nioie mayaqu qon he wanna wicawaqu; unkan icupi, qa niye eciyatanhan wahi kin he tanyan sdonyapi, qa niye e umayaśi kin he wicadapi.

9 Hena on wocekiye ewicaweciya, maka akan wicaśta kin hena on wocekiye eciciye śni, tuka tona mayaqu kin hena on wocekiye eciciye, hena nitaw a kin he etanhan.

10 Taku mitawa kin hena owasin nitawa, qa niś taku nitawa lzin hena mitawa, qa hena on wowitan waun.

11 Wanna oyate kin dematanhan śni, tuka iye e oyate kin en opeya ni unpi, qa miye wanna nitankiyadan waku kta. Ate wakan, nicaje kin on dena mayaqu qon hduha wo, hecen unwanjidan qon iś iyecen wanjidan hta.

12. Oyate kin den om waun kin he icunhan nicaje kin on wicawahduha. Mayaqu qon hena e wicawahduha, qa owasin elipekiyapi śni, wicaśta wayutakuni śni cinhintku kin hecedan ehpekiyapi, hecen wowapi en un qon wanna iyecetu.

13 Wanna nitankiyadan waku kta, oyate kin den opeya waun kin en ehake hecen epa, hecen on mitowiyuśkin kin iyepi en wicalriyuśtanpi kta.

14 Nioie wicawaqu, qa oyate kin śice wicadapika, oyate kin hetanhanpi śni heon etanhan hececa, oyate dematanhan śni he iyecen.

15 Oyate kin detanhan ewicayahdaku kta e heon woceliye eciciye śni, tuka taku śice cin he en anawicayecipte kta e heon.

16 Oyate kin de ematanhan śni lkin he iyecen iś oyate kin de etanhanpi śni.

17 Wowicake nitawa kin be on wicayuwakan wo; nioie kin he wowicake.

18 Oyate kin deci u mayaśi qon, he iyecen iś oyate kin ecen ye wicawaśi.

19 Qa iyepi on miye mibduwakan, hecen on wowicake eciyatanhan wicayuwakanpi kta.

20 Qa denana on wocelkiye ewicaweciye śni, nakun tona wicoie oyakapi eciyatanhan wicamadapi kin hena koya.

21 Hecen on owasin wanjipidan kta, niye kin iyecen, Ate, miyecica, qa miś cicica; qa hecen iś unkicicapi kta; qa oyate kin niye u mayaśi kin he wicadapi kta. 
22 Wowitan mayaqu qon he wanna wicawaqu; hecen on unwanjidan kin iyecen wanjipidan lita.

23 Miś wicakici waun, qa niś mici yaun, hecen wanjidan en wicayuśtanpi kta; qa hecen niye umayaśi kin he oyate kin sdonyapi kta; qa waśtemayakida kin he iyecen waśtewicawakida ece.

24 Ate, tona mayaqu kin hena, tokiya waun kin hen mici unpi kta wacin; hecen wowitan mayaqu qon he wanyakapi lita, wicoicage kin de itokam waśtemayakida kin heon etanhan.

25 Ate owotanna, oyate kin sdonniyanpi śni ye, tuka miye sclonciye ciqon, qa dena iś niye e u mayaśi kin he sdonyapi.

26 Unkan nicaje kin he sdonye wicawakiya qa nakun sdonye wicawakiye kta; hecen wawacinkta duza on waśtemayakida qon he dena en un kta, qa miś nakun owicawape kta.

\section{WICOWOYAKE 18.}

1 Jesus hena hecen eya, hehan waonspewicakiye cin om Kedron wakpadan kin akasanpa iyaya, hen can wojupi wan, unkan he en $i$, waonspewicakiye cin om.

2 Unkan Judas wiyopeye cin he iś nakun makoce kin he sdonya, Jesus hen waonspewicakiye cin om emniciya ece qon heon.

3 Heon Judas akicita wicoun wan icu, qa akicita tancan ko, wośna kagapi qa Pharisee kin etanhan, om wipe yuhapi, qa petijanjan can itkonya ko yuha en yapi.

4 Jesus taku hiyahde kte cin owasin sdonkiya, itkowicakipe ca, Tuwe e oyadepi he, ewicakiya.
5 Jesus Nazareth etanhan, eya ayuptapi. Unkan Jesus, He miye ce, eya. Unkan Judas he wiyopeye ciqon ee en opa.

6 Unkan Jesus, He miye ce, ewicakiya hehan icicawin kihdapi, qa makata ilipayapi.

7 Heon etanhan ake wiwicawanga; Tuwe ayakitapi he, eya. Unkan, Jesus Nazareth etanhan, eyapi.

8 Jesus waayupte; He miye ce, eciciyapi qon; hecen miye e omayadepi hecinhan dena ito kihdapi kta.

9 Wicoie wan eye ciqon he iyecetu kta, Tona mayaqu kin wanjidan elipewakiye śni ce.

10 Unkan Inyan mazasagye wan yuhe cin ehdaku, qa on wośna kagapi itancan ookiye wicaya wan noge etapa eciyatanhan ape ca kaśpa iyeya, ookiye unna kin he Malkos eciyapi.

11 Hehan Jesus Inyan heciya; Mazasagye nitawa ojuha en iyekiya wo. Wiyatke Ate maqu kin he mdatke kte sni he.

12 Juda oyate akicifa tancan, akicita eyanpaha, akicita ikcedan ko Jesus yuzapi, qa pahtapi.

13 Qa tokaheya Annas kaipi; Kaiaphas omaka kin he en wośna kagapi itancan kin he tunkanku kin hee.

14 Wicaśta wanjidan oyate kin on te kte cin he hecetu kta ce, Juda oyate iwahokonwicakiye ciqon Kaiaphas ee.

15 Unkan Simon Inyan Jesus ihakam ya, nakun waonspewicakiye cin tokeca wan; unkan waonspewicakiye cin tokeca liin he wośna kagapi itancan kin sdonya qon, heon hee wośna kagapi itancan tihocoka kin en Jesus kici iyaya.

16 Tuka Inyan e tankan tiyopa kin en najin. Hehan waon- 
spewicakiye cin unma, wośna kagapi itancan sdonya qon, he tankan hiyu, qa tiyopa awanyake cin he okiye ça, Inyan tin hiyuya.

17 Hehan wikośka tiyopa awanyake $\operatorname{cin}$ he Inyan heciya; Wicaśta kin de waonspewicakiye cin wanji niye he. Unkan, Hemaca śni ce, eya.

18 Akicita eyanpaha wowidag wicayapi ko hen najinpi, sni hdapi qa cetipi; unkan he en petiśkanpi : unkan Inyan iś owicapeya inajin, qa petiśkan.

19 Hehan wośna kagapi itancan kin Jesus wiwanga, waonspewicakiye cin heon, qa iye woonspe tawa kin he nakun.

20 Jesus ayupte; Oyate kin en taninyan womdaka ece; omniciye tipi etu, qa tipi wakan ko en, Juda oyate mniciyapi ece kin en ohinniyan waonspekiya waun, qa takudan anahbeyahan epe śni ce.

21 Taku on wimayanunga he; token ewicawakiye cin tona nahonpi kin hena e wiwicawanga wo, token epe cin he sdonyapi ce.

22 Hena hecen eya, unkan akicita eyanpaha wan en najin kin he Jesus apa, qa heya; Wośna kagapi itancan kin hecen adupta he.

23 Unkan Jesus ayupte ; Sicaya iwaa hecinhan taku śica epe cin he yaotanin wo; tuka tanyan epa hecinhan, tokeca amayapa he.

24 Annas Kaiaphas wośna kagapi itancan liin palitapi ecen ekta aye wicakiye ciqon.

$25 \mathrm{He}$ icunhan Simon Inyan petiśkan najin. Heon heciyapi; Waonspewicakiye cin he wanji niye he. 'Tuka anakilibe ça, Hemaca śni ce, eya.
26 Wośna kagapi itancan kin wowidake tawa wan, Inyan noge kaśpe ciqon he takuya, Wojupi kin en kici yaun wanciyake śni he, eciya.

27 Tuka ake Inyan anakiliman; unkan he icunhan anpa ohotonna wan hoton.

28 Hehan Kaiaphas etanhan woyaco tipi kin en Jesus aipi; unkan wanna anpa. Ihduśapapi kte śni e heon woyaco tipi kin en ipi śni, wośnapi kin hdutapi kta e heon.

29 Unkan Pilate tankata hinanpe ca, en wicau qa, Wicaśta kin de taku e iyayaonpapi he, ewicakiya.

30 Waayuptapi qa heciyapi : Wicaśta kin dc ohan śice śni unkanś unnicahipi kte śni tuka, eyapi.

31 Hehan Pilate hewicaliya; Niye ito eyaku po, qa wayeconzapi kin eciyatanhan yaco po. Hehan, Wicaśta teyapi unkiye unkokihipi śni ye, Juda oyate eciyapi.

32 Hecen Jesus oie qon ecetu $\mathrm{kta}$, token wiconțe on te kta eye ciqon he.

33 Hehan woyaco tipi timahen Pilate kihde ca, Jesus en au wicakiyc ça, Juda oyate wicaśtayatapi qon he niye he, eciya.

34 Jesus ayupta; He niye cinka heha he; qa iś tuwe tokeca miye on hecenl oniciyaka he.

35 Pilate ayupte; Juda wicaśta hemaca he ; nitaoyatepi kin hena, qa wośna kagapi tankapi kin he iye miye en anihipi. 'Token oyalianda he.

36 Jesus ayupte; Wokiconze mitawa kin he maka kin detanhan śni; wokiconze mitawa kin maka kin detanhan unkanś, mitaokiye kin kis unpi kta, qa Juda oyate kin makaśkapi kte śni 
trka. Tuka nakaha wokiconze mitawa kin detanhan śni.

37 Hehan Pilate heciya; Wicaśtayatapi kin henica he. Unkan Jesus ayupte; Wicaśtayatapi hemaca e ecen eha; hemaca kta e on matonpi, qa heon makata wahi, wowicake mdaotanin kta e heon. Tuwe wowicake eciyatanhan un kinhan he miho kin nalion ece.

38 Wowicake kin he taku he, Pilate eciya. Hena hecen oie ya, unkan Pilate tankata inanpe ca, Juda oyate en wicaye cá, Wicaśta kin he taku śica econ takudan iyewaye śni, ewicakiya. 39 Tuka wicohan duhapi kin heciya tanhan, woacakśin anpetu ehan unkipi eca, tuwe kaśka hnakapi wanji wakiyuśka ece qon. Juda oyate wicaśtayatapi kin de wakiyuśka yacinpi he, eya.

40. Unkan akc owasin panyehan howayapi qa heyapi; Wicaśta kin dee kte śni, tuka Barabbas e hee kta. Unkan Barabbas e qe wawicaki sa kin hcca.

\section{WICOWOYAKE 19.}

1 Unkan hehan Pilate Jesus icu qa kapsinpsinta.

2 Unkan akicita wapepeka wapaha wan kazontapi qa wapaha kiyapi, qa śina to wan in kiyapi. 3 Qa, iho, Juda Wicaśtayatapi kin, eciyapi, qa nape on apapi.

4 Pilate ake tankan inanpe ca, Iho, wicaśta kin dee e tankan cicaupi, hecen on taku śica takudan en iyewaye śni e sdonyayapi kta, cwicakiya.

5 Hehan Jesus wapepeka wapaha kin un, qa sina to kin in, tankata inanpa. Unkan, Wanyaka po, wicaśta kin dee ce, ewicakiya.
6 Unkan wośna kagapi tankapi qa akicita tancanpi kin, he wanyag iheyapi hehan, panyehan heyapi, Icipawel okatan wo, icipaw eli okatan wo. Niśnana icupi qa icipaweh okatan po, miye ge taku śica econ takudan iyewaye śni, Pilate ewicakiya.

7 Wowalkiconze wan unhapi, wowakiconze unhapi kin eciyatanhan te kta iyececa, Wakantanka Cinhintku keiciye cin heon etanhan, Juda oyate kin eya ayuptapi.

8 Unkan Pilate iapi kin he nahon qehan, iyotan inihan;

9 Qa woyaco tipi kin timahen kihde ca, Nitokiya tanhan he, Jesus cciya. Tuka Jesus ayupte śni.

10 Hehan Pilate heciya; Amayadupte śni he. Iciyaweh onicatanpi owakihi ; qa niciyuśkapi nakun owakihi, he sdonyaye śni he.

11 Hehan Jcsus ayupte; Wankan tanhan taku kin de niçupi śni unkanś takudan on omayakihi kte śni tuka; heon tuwe niye en amahi kin he iye woahitani tanka.

12 Hetanhan Pilate kiyuśkapi akita. Tuka, Wicaśta kin de yakiduśke cinhan Kesar takuyaye śni ; tuwe wicaśtayatapi ic̣icàge cinhan Kesar i en hiyeya ece, Juda oyate kin panyehan eyapi.

13 Pilate iapi kin he nahon, unkan Jesus tankan yuha inanpa, qa oiyotanke tawa kin he ohna kiyotanka, inyan mdaya omanipi kin en, he Hebrew iapi kin ctanhan Gabbatha eciyapi.

14 Woacakśin itokam anpetu piiciyapi ece kin hetu, qa wiyotanhe cin hehantu; Wicaśtayatapi nitawapi lin wanyaka po, Pilate Juda oyate kin ewicakiya. 15 Tuka, Icunonpa, icunonpa $1 \% 7$ 
iyeye ca icipaweh okatan wo, panyehan eyapi. Wicaśtayatapi nitawapi kin de icipawch owakatan kta he, Pilate ewicakiya. Unkan wośna kagapi tankapi kin ayuptapi ; Kesar ecedan wicaśtayatapi unhapi ce.

16 Hehan icipaweh okatanpi kta e wicaqu. Unkan Jesus icupi qa tokan ayapi.

17 Unkan Jesus can susbeca kicin, qa makoce wan Wicapahu eciyapi, he Hebrew iapi on Golgotha eciyapi, he ekta aya.

18 Hen icipaweh okatanpi, qa wicaśta tok tokeca nom itan anokatanhan ewicahdepi, qa Jesus okitahedan.

19 Unkan Pilate wowapi wan kage ca, can susbeca inkpa kin en ehnaka; JESUS NAZARETH ETANHAN, JUDA OYATE WICAŚTAYATAPI KIN.

20 Wowapi kin de Juda wicaśta ota yawapi; makoce kin Jesus en icipaweh okatanpi kin he otonwe kin ikiyedan, qa he Hebrew, qa Helenes, qa Roma iapi henakiya wicoie kin on owapi.

21 Hehan Juda oyate etanhan wośna kagapi tankapi kin Pilate heciyapi; Juda oyate Wicaśtayatapi kin, eya owa śni wo ; tuka, Juda oyate Wicaśtayatapi kin he miye ce, eiciye cin, hee e ecen owa wo.

22 Tuka Pilate waayupte; Taku owawa kin he wanna owawa ce, eya.

23 Akicita Jesus icipaweli okatanpi, hehan tawokoyake icupi, qa wopamni tom kagapi, akicita otoiyohi kicipamnipi; qa nakun taśina icupi ; śina kin he okagege wanica, wankan tanhan kun ihankeya kin hehanyan okagege codan kagapi :
24. Heon etanhan iye iyakitedan hekiciyapi ; Śina kin he unyuhdccapi kte śni, econkupidan qa yeunkiyapi kta, hecen tuwe ohiye cinhan yuhe kta. Hecen wowapi en kagapi qon he iyecetu; Mitawokoyake kicipamnipi, qa mitaśina kin he oeconna en yekiyapi ce. Unkan akicita kin hena hecen econpi.

25 Unkan Jesus hunku, qa tankaku kicica, Mary Kleopas tawicu, qa Mary Magdalene, hena can susbeca kin icahda najinpi.

26 Jesus hunku wanhdaka, qa en icunonpa ikiyadan waonspewicakiye cin wanji iyotan kidake ciqon wanhdaka, unkan, Winohinca, nicinkśi kin hee ce, hunku kin eciya.

27 Qa waonspekiyapi kin he iś, Wanyaka wo, nihun kin hee ce, eciya. Unkan waonspekiyapi kin he anpetu kin hetanhan iye ti kin ekta aki.

28 Wanna taku kin owasin ecetu Jesus wanyaka, qa wowapi en kagapi qon iyecetu kta e heon, Imapuza ce, eya.

29 Unkan mini śkumna wakśica ojudan en icunhan han; mini śkumna icupi, qa nakun mini iyuhepe wan en oputkanpi, qa peji hota hu wan icam yeyapi, qa. i kin en qupi.

30 Unkan Jesus mini śkumna icu qehan, Wanna yuśtanpi ce, eya; hehan pa makata iyeye ça taniya kin hiyuya.

31 Hehan Juda oyate kin Pilate cekiyapi, hu kawegapi qa etanlıan icupi kta; hecen anpetu wakan icunhan, tancan kin can susbeca kin akan un kte śni, anpetu en piiciyapi kin wanna" hehantu, qa anpetu wakan kin he anpetu tanka, heon.

32 Unkan akicita en hipi, qa tokaheya kin hu kawegapi, qa 
nakun unma kici icipaweh okatanki qon.

33 Hehan Jesus ehan ipi, unkan wanna ța wanyakapi, heon hu kin kawegapi śni.

34 Tuka akicita wanji wahukeza wan on cuwi kin en capa; unkan we mini ko au.

35 Tuwe wanyake ca yaotanin kin he wayaotanin kin he wowicake. Wowicake eye cin he sdonkiya, qa hecen on wicayadapi kta.

36 Hena hecetu, unkan wicowoyake wowapi en kagapi qon iyecetu; $\mathrm{Hu}$ kin wanjidan kawegapi kte śni ce.

37 Qa nakun wỏwapi icinonpa hecen eya; Tuwe pahidokapi kin he wanyakapi kta.

38 Hehan ihakam Joseph Arimathea etanhan Pilate cekiya, Jesus tancan kin icu kta e heon; unkan iś eya Jesus waonspewicakiye cin wanji ee, tuka analibeya, Juda oyate kowicakipe cin heon; unkan Pilate ayupta. He en u, qa Jesus tancan kin hdokiyahda.

39 Unkan Nikodemos, tuwe tokaheya hanyen Jesus en hi qon hee, nakun en hi, qa pejihuta waśtemna nom icicahiyapi, tkeutapi opawinge ecetu, yuha hi.

40 Hehan Jesus tancan lin icupi, qa minihuha ska iyapemnipi, pejihuta waśtemna iyahna, Juda oyate wicaśta hnakapi ece kin he iyececa.

41 Unkan icipaweh okatanpi qon he en ikiyadan wojupi wan han, wojupi he cin he en ohna wicaśta hnakapi wan iyuśtan yanka, tuwedan he ohna oonpapi śni.

42 Juda oyate piiciyapi anpetu kin he icunhan, obna wicaśta hnakapi en ikiyadan yanke cin heon Jesus tancan kin en ohna kapi.

\section{WICOWOYAKE 20.}

1 Anpetu wakan kin en, anpetu tokaheya hanlianna hinea, nahanhin otpaza, hehan Mary Magdalene hnakapi qon ekta i, qa inyan kin hnakapi etanhan icunonpa iyeyapi e wanyaka.

2 Hehan inyang hde ça Simon Inyan hee, qa waonspewicakiye cin unma Jesus waśtekidake ciqon, hena en hi qa hewicakiya; Itancan kin hnakapi kin etanhan eyakupi, qa tukten hnakapi kin sdonunyanpi śni ye.

3 Unkan Inyan waonspewicakiye cin unma qon kici tankan inanpapi, qa en hnakapi qon ekta yapi.

4 Napin inyang yapi ; qa waonspewicaliye cin unma qon hee duzahe ca Inyan kapa, qa iye tokaheya ehan i;

5 Patuś inajin, qa hnakapi kin aokasin, miniliuha ecedan wanyaka, tuka mahen iyaye śni.

6 Hehan iś Simon Inyan iyohi, unkan katinyan mahen iyaya, miniliuha ecedan makata yanka. wanyaka.

7 Minihuha pa akan ehnakapi kin he, minihuha umanpi qon en ope śni, he iś aiyog pehan yanka.

8 Hehan waonspewicakiye cin unma tokaheya hi qon he iś hnakapi kin mahen iyaya, qa iś eya wanyake ca wicada.

9 He wiconte etanhan ekicetu kta, eyapi, wowapi en kagapi qon he hinyahin tanyan sdonyapi śni.

10 Hehan waonspewicakiye cin hena iye tipi ekta icicawin hdapi.

11 Tuka hnakapi kin itankan Mary e ecen najin, qa ceya. 
Unkan ceyaya patuś inajin, qa hnakapi kin en aokasin.

12 Qa mahpiya ohnihde nom wokoyake ska ece koyakapi kin hena wanwicayaka, Jesus tancan wanke ciqon itan anokatanhan, unma pa wanke ciqon eciyatanhan, qa unma is siha qon eciyatanhan yukanpi.

13 Unkan, Winohinca, taku on yaceya he, eciyapi. Unkan iś, Itancan mitawa tancan kin icupi, qa toki ehnakapi kin sdonwaye śni ce, ewicakiya.

14. Heye ca hehan ihdamna unkan Jesus en najin wanyaka, tuka Jesus hee kin sdonye sni.

15 Unkan Jesus heciya, Winohinca taku on yaceya he; tuwe ayakita he. Unkan iś he wakicanye hee kecin, qa lieon, Itancan, he niye iyacu hecinhan tokiya eyahnake cin omakiyaka ye, kinhan ewehdaku kta ce, eciya.

16 Unkan Jesus heciya, Måry. Unkan iye ihdamna, qa, Rąbboni, eciya; he Waonspekiye eyapi.

17 Unkan Jesus heciya; Ihnuhan omayadutan kin; nahanhin Ate ekta wahde śni ce. Tuka mihunkawanji ekta wicaye ca hewicakiya wo, Ate ekta wahde kta, he ate yayapi kin ee, he Wakantanka mitawa kin ee, qa niś he Wakantanka nitawapi kin ee ce, eya.

18 Hehan Mary Magdalene ku qa, Itancan kin wanhdake ca hena hecen eciye cin, waonspewicakiye cin owicakiyaka.

19 Anpetu kin he anpetu tokaheya kin ee, ibtayetu kin he en waonspewicakiye cin, Juda oyate kowicakipa, qa aonaiçitag yukanpi, en Jesus wicacokaya inajin, qa, Wookiye nicipi un kte, ewicakiya.
20 Hecen eye ca hehan nape cuwi ko wicakipazo. Waonspewicakiye cin Itancan kin wanyakapi, unkan wiyuśkinpi tanka.

21 Wookiye nicipi un nunwe, ake Jesus ewicakiya. Ate $u$ maśi kin he iyecen miś ye ciśipi. 22 Hecen eye ca hehan awicaniya qa, Woniya Wakan icu po, ewicakiya.

23 Tona woahtani wicayecicajujupi kinhan, hena wicakicicajujupi kta ; qa tona wicayakipaganpi śni kinhan, hena wicakipaganpi kte śni.

24 Ake nonpapi kin etanhan Thomas Didumos eciyapi he Jesus hi kin he icunhan en ope śni. 25 Itancan kin wanunyakapi, waonspewicakiye cin unmapi kin eciyapi. 'Tuka iye kin hewicakiya; Maza on nape en okatanpi qon he wanmdake śni, qa napsukaza maza okatanpi kin en omdutan śni, qa euwi nakun en nape yewaye śni kinhan, wicawada kte śni ce, eya.

26 Unkan anpetu śahdogan iyohakam, hehan ake waonspewicakiye cin mahen yukanpi, qa Thomas owicapeya un. Hehan Jesus en hi, tiyopa onatag han, qa wicacokaya inajin, qa, Wookiye nicipi un nunwe, ewicakiya.

27 Hehan 'l'homas heciya; Ninapsukaza uye ca minape kin wanyaka wo; qa ninape kin uye qa micuwi kin en oputkan wo, qa wacetunhda śni wicada wo.

28 Unkan Thomas ayupte ca heciya; Itancan mitawa, qa Wakantanka mitawa.

29 Jesus heciya ; Thomas, wanmayadake cin heon wicayada; tuka tona wanmayakapi śni eśta wicadapi kinhan, hena wicayawaśtepi ce.

30 Unkan waonspewicakiye cin wanyakapi en taku tokeca ota 
wapetokeca Jesus econ, tuka hena eqe wowapi kin de en kagapi śni.

31 Tuka dena e owapi, heon Jesus he Messiya Wakantanka Cinhintku kin hee e wicayadapi kta; qa wicayadapi kin on, iye caje kin eciyatanhan, wiconi duhapi kta.

\section{WICOWOYAKE 21.}

1 Hena iyohakam Jesus ake waonspewicakiye cin en ihdutanin, Tiberias mde kin etu; unkan ihdutanin kin he kaketu :

2 Simon Inyan, qa Thomas Didumos eciyapi, qa Nathanael, Kana Galile makoce kin hetanhan, qa Zebedee cinhintku napin, qa waonspewicakiye cin tokeca nonpa, hena witayapi etu.

3 Ho wakuwa kta ce, Simon Inyan ewicakiya. Niyahna unyanpi kta ce, eciyapi. Hecehna yapi, qa wata wan en opapi, tuka hanyetu kin he takudan ktepi śni.

4 Ihanlianna qehan wicihuta elita Jesus najin; tuka waonspewicakiye cin Jesus hee kin sdonyapi śni.

5 Unkan Jesus hewicakiya, Hokśíyopa, taku yutapi duhapi he. Unkan, Hiya, eyapi.

6 Unkan hewicakiya; Ho kin he wata etapa kin eciyatanhan ehpeya po, etanhan iyeyayapi kta ce. Unkan ecen iyohpeyapi, tuka ehaeś hogan kin ota kin heon ehdakupi okitpanipi.

7 Hehan waonspekiyapi wan Jesus waśtedake ciqon he Inyan heciya; Itancan kin hee ce. Simon Inyan, Itancan kin hee, eya nahon qehan, tancodan un kin heon onlidolida kiçun qa minin ehpeiçiya.

8 Unkan waonspewicakiye cin unmapi kin. wata cistinna wan ohna upi, huta kin icannan yakonpi, wiciśpa opawinge nom hehanyan, qa ho kin hogan ojudan akupi.

9 Wanna hutata kihunnipi, hehan petaga itkon wanyakapi, qa hogan aguyapi ko akan yanka.

10 Unkan Jesus hewicakiya, Hogan nakaha iyacupi kin etanhan aku po.

11 Simon Inyan ekta ye ca ho kin heyata hiyuya, hogan tankinyanyan ece opawinge qa sanpa wikcemna zaptan qa sam yamni ; hogan kin ota ho kin ohnaka tuka iyulidece śni.

12 Upi qa de yuta po, Jesus ewicakiya. Unkan waonspewicakiye cin etanhan, 'Tuwe niye he, eyapi kta tuka tuwedan tawațenye śni; Itancan hee kin he sdonyapi kin he etanhan.

13 Hehan Jesus en ye ca aguyapi icu, qa wicaqu, hogan nakun iyecen econ.

14 Piya ekicetu kin etanhan, de iyamni Jesus waonspewicakiye cin en ihdutanin.

15 Unkan wotapi ayaśtanpi, hehan Jesus Simon Inyan heciya; Simon Jona cinhintku, dena eqe sam iyeya waśte mayadaka he. Han, Itancan, waśte cidaka e sdonyakiye ciqon, eya. 'Tahincadan mitarva kin taku yutapi wicaqu wo, eciya.

16 Ake icinonpa, Simon Jona cinhintku waśte mayadaka he, eciya. Han, Itancan, waśte cidaka e sdonyakiya, eya. Tahinca mitawa kin wo wicaqu wo, eciya.

17 Ake iciyamni, Simon Jona cinhintku waśte mayadaka he, eciya. Tamni akihde, Waśte mayadaka he, eya iwange cin heon Inyan iyokiśica; unkan heciya, Itancan, taku owasin sdonyaya; waśtecidake cin he 181 


\section{OHANYANPI QON.}

sdonyaya. Hehan Jesus, Tahinca mitawa kin wo wicaqu wo, eciya.

18 Wowicake, wowicake on heciciya; Koniśka qehan niye cinka ipiyag yeton qa tokiya yacin eca ekta da ece, tuka tohan winicahinca kinhan wicaśta tokeca wan ekta nape yeyakiye kta, kinhan ipiyag nicaton qa tokiya yacin śni eśta ekta aniye kta ce, eya.

19 Wiconte tukte e on Wakantanka yutan kta e he kiciyaotanin kta heon hecen eya. Hecen eye cin hehan, Miyahna u wo, eciya.

20 Hehan Inyan hakikta, qa waonspekiyapi wan Jesus iyotan kidake ciqon hee wicihektam u e wanyaka, hitayetu wotapi icunhan maku kin ipahin wanke ca, Itancan, tuwe e wiyopeniye lita he, eye ciqon hee.
21 Inyan he wanyaka unkan Jesus heciya, Itancan, de iś taku e akipe kta he.

22 Ake tohan wahi kte cin hehanyan decen un kta wacin eśta, he etanhan initokeca he; niye miyahna u wo, Jesus eciya.

23 Heon etanhan wicoic kin de hunkawanjinkiciyapi kin wicehna iyaya; waonspekiyapi kin de țe kte śni, eyapi kin. Tuka Jesus, He te kte śni ce, eciye śni ; tuka, Wahi kte cin hehanyan decen un kta, wacin eśta, he etanhan initokeca he.

24 Taku kin hena waonspekiyapi kin he yaotanin qa wowapi en kaga; qa taku yaotanin kin he wowicake kin e sdonunyanpi. 25 Nakun taku ota Jesus econ, tuka nakun hena owasin otoiyohi kagapi eśta, oyate hiyeye cin wowapi kagapi kte cin he yuhapi kta epce śni. Amen.

\section{YEWICAŚIPI KIN OHANYANPI QON.}

\section{WICOWOYAKE 1.}

1 Theophile, wicoie tokaheya wakage cin he en Jesus tolkaheya taku econ qa waonspckiye cin omdaka;

2 Tona wicakalinige ca yewicaśi qon hena Woniya Wakan kin eciyatanhan wahokonwicakiye cin iyohakam, anpetu wan en wankan eyakupi kin hehanyan.

3 Iye kakije cin iyohakam hena eqe en ni un taniniciya, woyuotamin ota on, anpetu wikcemna topa hehanyan wanyagiciya, qa Wakantanka tokiconze taku tawa kin cajehdata ece ;
4 Qa mniciyapi kin en owicapeya un qehan, Jerusalem etanhan ye śni wicaśi, qa Ateyapi kin wahowicaye ciqon he apepi kta; He miye eciyatanhan nayahonpi ce ;

5 Awicakehan Johannes mini on baptem wicaqu, tuka tokata anpetu tonana ihan, hehan Woniya Wakan on baptem niçupi lita ce, eya.

6 Unkan hena witaya yukanpi hehan, Itancan, wanna dehantu Israel oyate wokiconze kin piya wicayaqu kta he, eya iwangapi. 7 Unkan hewicakiya; Tohanyan qa tohantu kin, Ate iye to- 
waśake kin en eliihnake cin hena sdonyayapi ktu iyecece śni ;

8 'Tuka Woniya Wakan kin en nihipi kinhan, wowaśake duhapi kta; hehan Jerusalem en, qa Juda makoce kin owancaya, qa $\mathrm{Sa-}$ maria qa maka ihanke kin hehanyan mayadaotaninpi kta ce.

9 Unkan hena hecen eye ca, wanhdakapi icunhan, yuwankan icupi ; qa wanyakapi kin etanhan mahpiya śapa wan eyaku.

10 Unkan he wankan iyaye cin, mahpiya ekta etonwan yukanpi kin he icunhan, iho, wicaśta nom ska ihduzapi en wicikiyadan najinpi ;

11 Qa, Galile wicaśta, tokeca e malipiya ekta etonwan nayajinpi he. Jesus e qe niyepi etanhan malipiya ekta eyakupi kin, he mahpiya ekta wankan iyaya wandakapi kin he iyecen ake $u$ kta, ewicakiyapi.

12 Hehan Oliwe paha eciyapi kin hetanhan Jerusalem ekta icicawin hdapi ; he Jerusalem ikiyedan, anpetu wakan oicimani wanji ecetu.

13 En hdipi qa wankan tipi wanji tin iyayapi qa hen iyukanpi, Inyan, qa Jakob, qa Johannes, qa Andrew, Philip, qa Thomas, Bartholomew, qa Mattheos, Jakob Alpheus cinhintku, qa Simon Zelotes, qa Judas Jakob sunkaku :

14. Hena owasin tawacin wanjidan wocekiye eyapi qa kida yukanpi, winolinca kin hena om, qa Mary Jesus hunkú, qa iye hunkawanjinwicaye cin hena kroya.

15 Unkan anpetu kin hena en Inyan ookiye unpi kin wicacokaya najin, (wicacaje owasin opawinge sanpa wikcemna nom wic ayawapi ;)

16 Qa heya; Wicaśta hunka- wanjinciyapi, Judas tona Jesus iyahpayapi qon en mdetahunka kin he on, ehanna Woniya Wakan, Dawid i kin eciyatanhan, ie ciqon, wowapi en kagapi kin, he ecetuyapi kta hunśte.

$17 \mathrm{He}$ iye unkiyopeyapi yawapi, qa wicohtani kin de en opa tuka.

18 Unkan wicaśta kin lıe woahtani iyunwin kin maga wan iyopekiye ca, ehna makata ilipaya, unkan tancan kin kamdas ilipaye ca, śupe kin owasin tankan hiyu. 19 'Tona Jerusalem en yakonpi sa owasin he sdonyapi ; qa heor. maga kin he iye iapi kin eciyatanhan, Akeldama eciyapi, he We maga, eyapi.

20 Odowan wowapi kin en kaken kagapi; He tukten ti qon hewoskan yanke ca, tuwedan ohna ti śni nunwe; qa, Awanhdakapi wicolian yuhe ciqon he tuwe tokeca icu kta.

21 Heon etanhan wicaśta kin dena ohinniyan om unyakonpi, Jesus Itancan kin unkopapi, ti mahen i, qa tankan ye cin,

22 Tokaheya Johannes baptem wicaqu qon hetanhan, anpetu kin ell unkiyepi etanhan wankan eyakupi kin hehanyan, hena etanhan, piya ikicage cin, wanji unkiyopeyapi oyaka ece e kta, eya.

23 Unkan nom najinwicakiyapi, Joseph Barsabas eciyapi, he, Owotanna, eya caśtonpi, qa Matthias.

24. Hehan cekiyapi qa heyapi ; Itancan niye wicacante owasin sdonya nanka, wicaśta kin dena unma tukte yakahnige cin he yutanin wo ;

25 Ookiye wicolian qa yewicaśipi kin de en ope kta, Judas iye oyanke tawa kin ekta hde kta e etanhan hinhpaye cin he. 


\section{OHANYANPI QON.}

26 Hehan woohiye elipeliyapi, unkan woohiye kin Matthias ahinhpaya; qa hecen yewicaśipi akewanjipidan kin owicapeya yawapi.

\section{WICOWOYAKE 2.}

1 Anpetu iwikcemna zaptan kin wanna iyehantu, unkan owasin tawacin wanjidan witaya yukanpi.

2 Helian ihnuhanna, tate wan waśagya uye cin he iyecen, oqo wan mahpiya kin eciyatanhan hiyu, qa tipi ohna yukanpi kin ocowasin ojudan.

3 Unkan wicaceji, peta se ececa, en wicatanin, qa otoiyohi kicipamnipi kin en awicahan.

4 Unkan owasin Woniya Wakan kin on ojupidan qa, wicaceji toktokeca on iapi, Woniya Wakan ie wicakiye cin he iyecen.

$5 \mathrm{He}$ ehan wicaśta owotanna Juda wicaśta kin hecapi, mahipiya ihukuya oyate hiyeye cin etanhan Jerusalem en tipi.

6 Wicaho kin de otanin, unkan wicota en mniciyapi qa inihanpi, otoiyohi iye iapi tawapi kin on, hena ia yukanpi he nakitionpi kin heon.

7 Owasin inihanpi qa yusinyayapi qa otoiyohi hekiciyapi; Tona ia yukanpi kin hena owasin Galile wicaśta kin hecapi śni he.

8 Token on otoiyohi iapi kin yuha untonpi kin hena ia nawicunhonpi heg.

9 Parthe qa Mede qa Elamite, qa Mesopotamia en tipi qon, Juda makoce kin en, qa Kappadokia en, Pontos qa Asia en ;

10 Phrugia qa Pamphulia en, Egupta qa Libue makoce kin en, Kurene ihdukśan, Roma oyate kin etanhan, Juda wicaśta, qa en opewicakiyapi qon,

11 Krete qa Arabe kin heun184 capi, Wakantanka taku tanka econ kain, unkiye unkiapi kin eciyatanhan, hena ia oyakapi kin naunhonpi ce, eyapi.

12 Owasin inihanpi, qa yuśinyayapi, qa otoiyohi hekiciyapi; De taku kapi hwo.

13 Qa wanjikśi ihaliapi qa; Dena miniśa skuya on imnanpi ce, eyapi.

14 Tuka Inyan ake wanjipidan kin om najin, ho yuwankan icu, qa hewicakiya; Juda wicaśta, tona Jerusalem en dukanpi ko, owasin he de sdonyapi, qa mioie kin anagoptan po.

15 Niye hecannipi kin hecetu śni, wanna anpetu kin en yamni ape cin hehantu, heon wicaśta kin dena witkopi śni.

16 Tuka Joel wicaśta wokcan oyake ciqon he dee.

17 Unkan anpetu ihankeya kin en, (Wakantanka eya,) mitaniya kin wicacelipi owasin awicawakaśtan kta; unkan nicinkśipi nicunkśipi ko wokcanpi kta, qa kośka nitawapi kin wowanyalke kin wanyakapi kta, qa wicahinca nitawapi kin wowihamde ihamnanpi kta ce.

18 Anpetu kin hena en wicaśta taokiye, qa winyan taokiye mitawa kin, hena en mitaniya awicawakaśtan kta, qa hena e wokcanpi kta.

19 Unkan wankan mahpiya kin ekta taku on yuśinyayapi wicawaqu kta, qa kuya maka kin akan wowapetokeca kin, we qa peta qa śotoju.

20 Itancan taanpetu tanka wowinihan kin he hi śni itokam, anpetu wi kin otpaza icu kta, qa hanyetu wi kin iś we icage kta.

21 Unkan hehan tuwe Itancan caje kin hoyekiye cinhan he nikiyapi kta ce. 
22 Israel wicaśta, wicoie kin de nalion po; Jesus Nazareth etanhan qon, wicaśta wan niyepi kin ekta, Wakantanka hdutanin qor, wicowaśake, taku on inihanpi, qa wowapetokeca niyepi cokaya Wakantanka econ kiye ciqon hena e niye sdonyayapi.

23 He qe Wakantanka atayedan wakiconze ca, iye osdonye kin eciyatanhan qupi qon, he iyacupi, qa napc śikśica on icipaweh oyakatanpi, qa yaktepi.

24 Hee Wakantanka ekicetu ye ca, wiconțe icaśke kin kiyuśka, en yuhapi kta okihipica śni nakaeś.

25 Dawid he ke ca, kaken eya; Ohinniyan mitokam Jehowa wanmdaka, mietapa kin ekta yanke, heon pahoho śni waun kta.

26 Heon micante kin iyuśkin, qa miceji kin ipsica; qa micehipi kin woape en wanke kta.

27 Minagi kin he hades ekta ehpemayakiye śni, qa Wanji Wakan nitawa kin he wicaliwin wanyake kta e on yaqu kte śni.

28 Wiconi canku kin sdonye mayaye kta; qa niite kin etanhan wowiyuśkin on imnamayaye kta.

29 Wicaśta hunka wanjinciyapi, Dawid hunkakeyapi un kin he eciyatanhan taninyan ociciyakapi kta; he te ça hapi, qa anpetu kin delianyan ohna hnakapi kin unkicipi wanka.

30 He wicaśta wokcan kin heca, qa Wakantanka wokonze on konza, wicacehpi eciyatanhan iye tancan aicage kte cin he etanhan Messiya, iye oiyotanke kin akan iyotanke kta e icahye kta he sdonya.

31 Hecen tokaheya tanhan he wanyake ça Messiya ekicetuya- pi kin he cajeyata, nagi kin hades ekta ehpekiyapi kte śni, qa celipi kin wicahwin wanyake kte śni.

32 Jesus iye qe Wakantanka ekicetuye cin he unkiyepi owasin unyaotaninpi ece.

33 He Wakantanka etapa kin ekta yuwankan icupi, qa Atkuku kin Woniya Wakan iwahoye ciqon he icu, heon nakaha taku wandakapi qa nayalionpi kin he akaśtan.

34 Dawid e qe mahpiya ekta wankan iyaye śni, tuka kaken eya; Jehowa Itancan mitawa kin kaken eciya, Mietapa kin eciyatanhan iyotanka wo,

35 Tona toka niye cin hena nisiha oahe kin ewahnake kte cin hehanyan.

36 Heon etanhan, Israel- tiyohnaka owasin, de tanyan sdonya wo, Jesus icipaweli oyakatanpi qon, hee Wakantanka Itancan qa Messiya lkaga ce.

37 He nahonpi unkan cante kin ekta iyoyakapi, qa Inyan qa yewicaśipi unmapi kin om, Wicaśta hunkawanjin unniyanpi token econkupi kta he, ewicakiyapi.

38 Hehan Inyan hewicakiya; Ihduecetu po, qa woahtani nicicajujupi kta e on Jesus Messiya caje kin on baptem niçupi kta; kinhan Woniya Wakan itunicilianpi kin he iyacupi kta.

39 Wahoyapi kin de niye nitawapi, qa nicincapi, qa tona itehanyan yukanpi kin owasin, tona Itancan unkita Wakantankapi kin wicakico kte cin hena tawapi ce, eya.

$40 \mathrm{Qa}$ wicoie tokeca ota on wicakiyatanin, qa wahokonwicakiye ça heya; Wicoicage śice cin de etanhan niicciya po.

41 Unkan tona oie kin iyuśkin185 


\section{OHANYANPI QON.}

yan icupi kin hena baptem wicaqupi; hecen anpetu kin he en wicanagi kektopawinge yamni en opapi.

$42 \mathrm{Qa}$ yewicaśipi kin taku onspewicakiyapi kin en katinyan opapi ece, qa om taku yuhapi, aguyapi kiyuśpapi qa wocekiye eyapi kin en ece unpi.

43 Unkan wicaśta owasin nina yuśinyayapi; unkan yewicaśipi kin taku wakanyan econpi qa wowapetokeca ota econpi ece.

44 Unkan tona wicadapi kin hena owasin witaya yukanpi, qa taku owasin ptaya yuhapi.

$45 \mathrm{Qa}$ tona maga qa woyuha yuhapi kin hena e wiyopekiyapi, qa otoiyohi cinpi kin iyecen owasin wicakipamnipi.

$46 \mathrm{Qa}$ anpetu otoiyohi, tawacin wanjidan, tipi wakan kin ohna yukanpi, qa tipi iyaza aguyapi kiy uśpapi, wowiyuśkin cante okonwanjidan ko yuha taku yutapi icupi.

47 Wakantanka yatanpi, qa oyate kin owasin en wowaśte yuhapi. Unkan Itancan kin tona nipi kte cin heca, anpetu otoiyohi, okodakiciye kin en ope wicakiya.

\section{WICOWOYAKE 3.}

1 Unkan hehan Inyan qa Johannes kici, tipi wakan kin ekta kaitawankanhde yapi, napcinwanka ape cin, wocekiye eyapi ece kin hehantu.

2 Unkan wicaśta wan tonpi kin ehantanhan huśte un kin he yuha ahipi, anpetu otoiyohi tipi wakan tiyopa wan, Waśte eciyapi kin he en ehdepi, tona tipi wakan tin yapi kte cin hena taku wicakida kte cin heon.

3 Hee Inyan Johannes kici tipi wakan kin timahen yapi kta 186 wanwicayake ca, taku wicakida.

4 Unkan Inyan opalita, qa Johannes kici, qa, Ekta unkatonwan po, eya.

5 Taku okini kta kecin qa hecen anawicagoptan yanka.

6 Hehan Inyan; Mazaska mazaskazi ko talkudan mduhe śni, tuka taku mduhe cin cicukta, Jesus Messiya Nazareth etanhan, he caje on najin, qa mani wo, eciya.

7 Qa nape etapa kin en yuze ca najin kiya; unkan ihnuhanna siha iśkahu ko waśag hinhda.

8 Hehan iye kin psin iyaye ca najin, qa mani, qa om tipi wakan kin timahen iyaya, mani, qa psipsica, qa Wakantanka idowan.

$9 \mathrm{He}$ mani qa Wakantanka idowan kin oyate kin iyuhipa wanyakapi ;

10 Qa wicaśta tipi wakan, tiyopa Waśte eciyapi, kin en hiyotanke ca taku da ece qon he dee sdonyapi ; qa taku ecakiconpi kin on etanhan nina inihanpi, qa yuśinyayapi.

11 Unkan, wicaśta huśte qon wanna okizi kin he Inyan Johannes kici wicayus najin, hena en oyate owasin kiwitaya inyang iheyapi, qa nina yuśinyayapi, omanipi Solomon tawa kin hetu.

12 Unkan Inyan he wanyake cehan, oyate kin hewicakiya; Israel wicaśta, tokeca he on niyuśinyayapi, qa tokeca opaunhtapi nayajinpi he; unkiye wowaśake qa wowaśte unhduhapi kin heon wicaśta kin de mani unyanpi kecanunyakinpi seca.

13 Abraham, qa Isaak, qa Jakob Wakantanka tawapi ; hunkake wicunyanpi Wakantanka tawapi kin he Cinhintku Jesus wanna hdutan; he niyepi e wi- 
cayaqupi, qa Pilate itokam yakipajinpi, he iye kiyuśke kte hinca tuka.

14 Tuka Wanji Wakan qa Owotanna un kin he niye yakipajinpi, qa wicakte sa wan kiyuśkapi yacinpi.

$15 \mathrm{Qa}$ Wiconi Itancan kin he yaktepi; he qe Wakantanka wicate cin etanhan najinkiye cin unlriye unkoyakapi ece.

16 Qa iye caje kin, caje wicadapi kin heon, wicaśta kin de wandakapi qa sdonyayapi kin he yuwaśaka ; han, iye wacinyanpi kin he wicaśta kin de tanyan okiziya, niyepi owasin en.

17 Unkan nakaha mihunkawanji, iyukcan śni hecanonpi, qa wicaśta itańcan wicaduhapi kin hena nakun, he sdonwaya.

18 Tuka Wakantanka Messiya token kakije kte cin, wicaśta wokcan kin owasin i kin eciyatanhan, oyake ciqon hena he en hduecetu.

19 Heon ihduecetupi, qa ihduhomni po; hecen wayahtanipi kin nicicajujupi kta ce; tohan anpetu yuecetupi lain Itancan itohnake kin eciyatanhan u kinhan :

20 Qa Jesus Messiya oniciyakapi qon he uśi kta.

$21 \mathrm{He}$ qe malipiya kin elsta eyakupi kta, taku owasin ekicetuyapi kta anpetu kin hehanyan, Wakantanka maka icage cin hetanhan wicaśta wokcan wakan wicahduhe cin owasin i kin eciyatanhan oyake ciqon hee.

22 Moses iye qe hunkakeyapi kin hewicakiya; Nihunkawanjipi kin etanhan wicaśta wokcan wanji, miye iyemaceca, Jehowa Wakantanka nitawapi kin icaliniciciyapi kta; he taku eniciyapi kte cin owasin anayagoptanpi kta.
$23 \mathrm{Qa}$ wicanagi otoiyohi, wicaśta wokcan kin he anagoptan śni kinhan, he oyate kin etanhan ihangyapi kta ce.

24 Unkan wicaśta wolican owasin, Samuel hetanhan, tona iyohakam woyakapi qon anpetu kin dena cajeyatapi.

25 Wicaśta wokcan cincapi kin he niyepi, qa wicoun Wakantanka hunkake wicunyanpi wicakicage ciqon, he qe Abraham heciya; Unlan nicinca liin on etanhan wicowazi maka akan un kin owasin yawaśtepi lita ce. 26 Wakantanka iye Cinhintku Jesus najinkiye ca niyawaśtepi kta e on niyepi tokaheya en u śi, otoiyohi wayahitanipi kin etanhan ihduhomni niyanpi kta e heon.

\section{WICOWOYAKE 4.}

1 Unkan hena hecen oyate kin ewicakiyapi kin icunhan, wośna kagapi, qa tipi wakan en itancan kin, Saddukee ko en wicahipi ;

2 Oyate kin waonspewicakiyapi, qa Jesus eciyatanhan wiconte etanhan woekicetu kin he oyakapi kin heon śihdapi ;

3 Qa nape on iyawicalipayapi, qa wicakaślka tipi kin en iyewicayapi, ihanhanna kte cin hehanyan; wanna htayetu nakaeś. 4 Tuka wicoie nahonpi qon hena wicota wicadapi; unkan wicaśta kektopawinge zaptan ecetu.

5 Unkan iharhanna hehan, wicaśta itancanpi, hunkawicayapi kin, wowapi kagapi ko ;

6 Qa Annas wośna kagapi itancan, qa Kaiaphas, qa Johannes, qa Aleksandros, qa tona wośna kagapi itancan takuwicaye cin hena koya Jerusalem en mniciyapi.

7. Unkan wicacokaya ewica187 
hdepi, qa wiwicawangapi ; Taku wowaśake, qa tuwe caje kin on hecanonpi he, eyapi.

8 Hehan Inyan Woniya Wakan on ojudan, hecen ewieakiya; Oyate en wicaśta itancanpi, qa Israel hunkawicayapi kin;

9 Anpetu kin de wicaśta huśte qon tanyan ecakiconpi, qa token on okizi kin, he on wiunyanungapi hecinhan;

10 Niyepi owasin, qa Israel oyate kin owasin de sdonya po, Jesus Messiya Nazareth etanlian, icipaweh oyakatanpi qon, Wakantanka wicate cin etanhan ekicetuye cin, he caje on wicaśta kin de nitokam okizi najin.

11 Inyan kin de ticagapi yaunpi kin ayaktapi śni, tuka he oise en itancan kin ee.

12 Qa tuwe tokeca eciyatanhan ranikiyapi kin yuke śni; qa wicacaje tokeca mahpiya kin ihukuya wicaśta wicaqupi, on unnipi kte cin wanica ce.

13 Unkan Inyan qa Johannes waditakapi kin he wanyakapi, qa taku ota onspeiciciyapi śni qa wicaśta ikceka kin hecapi e sdonyapi qehan, nina yuśinyayapi, qa hena Jesus kici unpi qon he iwicay ukcanpi.

14 Qa wicaśta okiziyapi qon he wiciyopeya najin wanyakapi; unkan taku awicayuptapi kte cin takudan yuhapi śni.

15 Tuka omniciye kin etanhan tankan ye wicaśipi, hehan he akiiapi ;

16 Qa iyakitedan hekiciyapi; Wicaśta kin dena token ecawicunkiconpi kta hwo; wowapetokeca tanka kin de econpi kin he tona Jerusalem en yakonpi hin owasin sdonyapi, qa anaunlimanpi kta unkokihipi śni.

17 Tuka he oyate kin en ana188 ptapi kta e heon nina wakokipe wicunkicagapi kta, qa caje kin de on tohinni wicaśta wowicakiyakapi kte śni, unkeyapi kta ce. 18 Unkan hehan wicakieopi, qa Jesus caje kin on takudan ie śni, qa waonspekiye śni wicaśipi.

19 Tuka Inyan Johannes kici awicayuptapi qa heyapi; Wakantanka itokam, niyepi anaunnigoptanpi kta, qa iś Wakantanka anaungoptanpi kta, unma tukte owotanna kin, niś iyukean po.

20 Taku sdonunyanpi qa naunhonpi qon hena oyake śni unyakonpi kta e unkokihipi śni.

21 Hehan ake wakokipe wicakicagapi tamwicakiyapi, qa hde wicaśipi, taku on taku iyawicaonpapi kta takudan iyeyapi sni, oyate kin on etanhan; taku econpi kin heon owasin Wakantanka yatanpi.

22 Wicaśta wookizi wowapetokeca kin he en econpi qon he wanna waniyetu wikcemra topa sam iyeya.

23 Unkan kihdewicayapi, hehan iye takodawicayapi kin ekta hdapi, qa wośna kagapi, hunkawicayapi kin ko, taku token ewicakiyapi qon owasin ohdakapi.

24 Unkan hena nahonpi qehan, witaya Wakantanka hoyekiyapi qa heyapi ; Itancan Wakantanka he niye, mahpiya, maka, miniwanca qa taku ohnaka ko owasin yakaga.

25 Dawid nitaukiye kin i kin eciyatanhan kaken ehe cin; Tokeca e Ikcewicaśta kin oqoyapi, qa oyate kin taku ecinśniyan yulcanpi he.

26 Maka wicaśtayatapi kin najin hiyaye ça, wicaśta itancanpi kin witaya mniciyapi, Itancan 


\section{WICOWOYAKE 5.}

kin en, qa Sdakiye ciqon he nakun.

27 Awicakehan Jesus Nicinkśi wakan sdayakiye ciqon he en, Herod, qa Pontiyos Pilate, Ikcewicaśta, Israel oyate ko mniciyapi ;

28 Ehanna taku, ninape nitawacin ko on, econ yakonze ciqon, he ecen econpi kta heon etanhan.

29 Unkan nakaha, Itancan, token wakokipe unkicagapi kta tamunkiyapi kin he wanyaka wo; qa nitaokiye kin nioie nina waditagya oyakapi kta e wicaqu wo ;

30 Wawokiziya on ninape kin yahdugate cin heon; qa Jesus Nicinkśi wakan caje kin on taku wapetokeca, qa on yuśinyayapi kte cin hena econpi kta.

31 Unkan wocekiye eyapi, hehan tukten witaya yukanpi kin

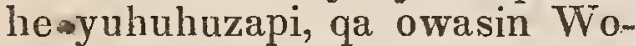
niya Wakan kin on ojupidan, qa Wakantanka oie kin waditagy a oyakapi.

32 Unkan wicota wicadapi kin hena wicacante wanjidan wicanagi wanjidan ko yuhapi; qa taku tonpi kin tuwedan, De mitawa ce, eye śni ; tuka taku owasin witaya yuhapi.

33 Unkan yewicaśipi kin hena wowaśake tanka on Jesus Itancan piya ikicage cin he oyakapi ; unkan wowaśte tanka owasin en un.

34 Unkan hena tuwedan takudan icakije śni unpi : tuka tona maga, qa tipi yuhapi qon hena wiyopekiyapi, qa taku wiyopeyapi kin on taku icupi kin he ahipi,

35 Qa yewicaśipi kin siha hdepi kin en ehnakapi: umkan wicaśta otoiyohi taku token cinpi kin hecen pamni wicaqupi ece.
36 Unkan Joses, he yewicaśipi kin Barnabas, eya cajeyatapi qon, Wicakicanpte cinhintku kin, eyapi kin he kapi, Lewi wicowazi qa Kupros makoce kin etanhan;

37 Hee makoce onśpa hduhe cin wiyopekiye ca mazaska kin hdohi, qa yewicaśipi silha hdepi kin en ehnaka.

\section{WICOWOYAKE 5.}

1 Tuka wicaśta wan Ananias eciyapi, tawicu Sapphira kici, maga wan wiyopekiya;

2 Qa mazaska apa anakihbe, tawicu kin iś nakun sdotkiya; unkan apa ahi, qa yewicaśipi kin sihapi kin en ehnaka.

3 Tuka Inyan heciya; Ananias, tokeca e Woniya Wakan kin oiyaton kte śni e Satan nicante kin en ocowasin iyohpaya, qa maga iyopeyapi kin apa anayakiliman he;

4. Nahahin ecen un qon he ehan yahduhe śni he; qa wiyopekiyapi kin he ehan nakun nitawa śni he. Tokeca nicante ekta hecen econ wacani he. Wicaśta akantu kin hee śni, tuka Wakantanka en iyatonśni ce.

5 Unkan Ananias hena oie kin nalion qehan, hinhpaye ca ta iyaya. Unkan tona hena taki kin nahonpi kin owasin nina wikopapi.

6 Unkan kośka kin najinpi, qa opazuntapi, qa tankan ayapi, qa hiapi.

$7 \mathrm{He}$ iyohakam wihiyayedan oape yarnni, hehan tawicu kin taku econpi qon sdonye śni ecen tin hiyu.

8 Unkan Inyan heciya; Kana on maga iyopeyakiy api he, omakiyaka wo. Unkan iś; Han, kana on, eya. 


\section{OḦANYANPI QON.}

9 Unkan Inyan; Token on sakim Itancan Taniya kin iyutanyayapi kta ecen wacinduzapi he. Wanyaka wo, tona nihihna liapi kin hena sihapi kin tiyopa kin en najinpi, qa niye nakun tankan aniyanpi kta, eciya.

10 Hehan iś eya hecehnana iye siha kin ekta hinhpaye ca ta iyaya. Unkan kośkapi qon en hiyupi, qa wanna ța e wanyakapi, hehan is eya tankan ayapi, qa hihnaku kin kici hapi.

11 Unkan okodakiciye kin ocowasin, qa tona hena taku kin nahonpi owasin nina wikopapi.

12 Unkan yewicaśipi kin napepi kin on oyate kin en wowinihan wicohan qa wowapetokeca ota econpi. Unkan owasin wicacante wanjidan yuha Solomon omanipi tawa kin ohna yukanpi.

13 Qa wicaśta tokeca tuwedan en owicapa okihi śni, tuka oyate kin wicakinihanpi.

14 Qa tona wicadapi kin sanpa ota Itancan kin en opapi, wica winyan ko wicota.

15 Hecen tona wicayazan kin hena tankan awicaupi, qa canku otoiyohi ohna, ohehdepi qa owinja ko akan ahiwicahnakapi, Inyan hen hiyaye cinhan hunh ohanzi wicaye kte cin heon.

16 Unkan, otonwe itan ihdukśan kin hena etanhan wicota Jerusalem en hipi, qa tona wayazanpi, tona taku wakan śica wicayuhnaśkinyanpi ko en awicahipi ; unkan owasin asnipi.

17 Hehan wośna kagapi itancan kin najin hiyaye ca, tona om un kin, Saddukee wicowazi kin etanhanpi, hena winawizipi on ojupidan ;

18 Qa nape on yewicaśipi kin iyawicahpayapi, qa wicakaśka tipi ikceka kin ohna ewicahnakapi.

19 Tuka Itancan taohnihde ye cin he hahyen wicakaśka tipi tiyopa kin yuzamni, qa tankan awicau qa heya;

20 Hunktiya po, tipi wakan kin ohna inajinpi qa wiconi kin de wicoie kin owasin oyate kin owicakiyaka po.

21 He nahonpi, unkan hanhanna hin tipi wakan kin timahen yapi, qa wowicakiyakapi. Hehan wośna kagapi itancan, qa tona om un kin hena witaya iheyapi, qa omniciye tanka kin mniciye wicaśi, Israel cinca hunkawicayapi kin owasin koya; unkan wicakaśka tipi kin ekta wicahuweye wicaśipi.

22 Tuka akicita kin wicakaśka tipi kin en hipi, qa iyewicayapi śni, hehan hośi hdapi ;

23 Qa heyapi ; Awicakehan wicakaśka tipi kin sutaya anatakapi ecen iyeunyanpi, qa tiawanyag wicakiyapi kin tiyopa kin itokam najinpi ; tuka unyuhdokapi qehan, tuwedan en wanunyakapi śni.

24 Unkan wośna kagapi itancan, qa tipi wakan en itancan kin, wośna kagapi kin hena koya, hena wicoie kin nahonpi qehan, he toketu kta hecinhan iyukcanpi śni.

25 Hehan wanji en hi qa; Iho, wicaśta wicakaśka tipi kin en ewicayahnakapi qon hena tipi wakan kin ohna inajinpi qa oyate kin waonspewicakiyapi ce, eya owicakiyaka.

26 Hehan akicita tancan kin akicita ko ekta yapi qa iwaśtedan awicaupi, oyate kin inyan on wicakininpi kta e kokipapi lieon etanhan.

27 En awicahipi qa omniciye kin wicacokaya najin wicakiya- 
pi ; unkan wośna kagapi itancan kin wiwicawanga,

$28 \mathrm{Qa}$ heya; Wicacaje kin de on wowicayalkidakapi kte śni, nina unkeniciyapi śni he; unkan iho, woonspe nitawapi kin on Jerusalem ojudan yayapi, qa wicaśta kin de we kin en unkayaupi kta wacannipi.

29 Hehan Inyan, yewicaśipi kin. om, awicayupte ça heya; Wicaśta akantu hee śni, tuka Wakantanka hee e anaungoptanpi kta iyececa.

30 Jesus yaktepi qa can akan otkeyayapi qon he atewicunyanpi ta Wakantankapi kin hduecetu :

31 Hee kin Wakantanka wankan ehdaku, iye nape etapa kin ekta Itancan qa Wanikiya un kta, Israel ihduecetupi qa woahtani kajujupi kin wicaqu kta e heon.

32 Unkan unkiye, iye on, wicoie kin hena unkoyakapi ece, qa Woniya Wakan tona Wakantanka anagoptanpi wicaqu kin, he iś eya hena yuotanin ece.

33 He nahonpi qehan canteptanyanpi, qa tewicayapi hta e akiiapi.

34 Hehan Pharisee wan Gamaliel eciyapi, woope waonspekiya, qa oyate kin en okinihan, he omniciye kin en najin, qa yewi caśipi kin cistiyedan tankan aye wicaśi :

35 Qa hehan hewicakiya; Israel wicaśta, wicaśta kin dena taku ecawicakicon wacannipi kin he itonpa po.

36 Anpetu kin dena itokam Thudas icaga, he taku wicaśta keiçiya ; unkan wicaśta opawinge topa ecetu ihakam yapi ; tuka he iye ktepi, qa tona wicadapi qon owasin enanakiya iyewicayapi qa takuni śni aya.
$37 \mathrm{Qa}$ he iyohakam Judas Galile etanhan, owicawapi anpetu kin he ehan inajin, qa oyate ota ihakam awicaya; he nakun ihangyapi, qa tona wicadapi qon owasin enanakiya eyaya.

38 Heon nakaha heciciyapi ; Wicaśta kin dena wiciheyata najinpi, qa awicayuśtan po ; wokonze qa wicohan kin de wicaśta akantu kin etanhan hecinhan atakuni kte śni ;

39 Tuka Wakantanka eciyatanhan un kinahan, yujuju oyakihipi śni ; okinni Wakantanka kici yeciza dukanpi kta naceca, eya. 40 Unkan he anagoptanpi, qa yewicaśipi kin wicakipanpi, qa wicakapsinpsintapi qehan, Jesus caje kin on woyake śni wicaśipi, qa wicakiyuśkapi.

41 Unkan iye omniciye kin etanhan hdapi, qa Jesus caje kin on wowiśtece en awicaupi iyececa wicayawapi kin heon wiyuśkinpi.

$42 \mathrm{Qa}$ anpetu otoiyohi tipi wakan kin ohna, qa tipi iyaza wahokonwicakiyapi, qa Jesus Messiya oyakapi ayaśtanpi śni.

\section{WICOWOYAKE 6.}

1 Unkan anpetu kin hena en, waonspewicakiyapi kin wanna wicota aya qehan, Helenes Hebrew oyate kin wicabapi, anpetu otoiyohi wapamnipi kin en wiwazica wicayuhapi kin hena awicatonwanpi śni kin heon etanhan.

2 Hehan akenonpapi kin waonspewicakiyapi kin owasin wicakicopi qa hewicakiyapi ; Wakantanka oie kin unkayuśtanpi, qa. wahna wotapi wiyeya eunhnakapi kte cin hecetu śni.

3 Heon hunkawanjin unniyanpi enitanhanpi wicaśta owotanna 191 


\section{OȞANYANPI QON.}

yawapi, qa Woniya Wakan wicoksape ko on ojupidan śakowin wicakaliniga po; wicohan kin de yuhe wicunkiyapi kta.

4 Tuka unkiye qe cekiyapi qa wicoie oyakapi kin en ohinniyan ye unkiciyapi kta ce.

5 Unkan wicoie kin he oyate kin owasin iyokipipi; qa Stephanos, wicaśta wan wacinyanpi Woniya Wakan ko ojudan, kin he kahnigapi, qa Philip, qa Procoros, qa Nikanor, qa Timon, qa Parmenas, qa Nikolas Antiyoka ekta en opekiyapi qon.

6 Hena yewicaśipi kin wicacokaya ewicahdepi; unkan wocekiye eyapi, hehan nape on awicaputakapi.

7 Unkan Wakantanka oie kin icage ca Jerusalem en waonspewicakiyapi kin nina ota aya; qa wośna kagapi kin ota nakun wacinyanpi kin en opapi.

8 Unkan Stephanos wowacinye wowaśake ko on ojudan, taku on yuśinyayapi, qa wapetokeca tanka econ, oyate kin en.

9 Unkan wicaśta wanjikji omniciye tipi wan, Wicakiyuśkapi tawapi eciyapi kin hetanhan, qa Kurene qa Aleksandre wicaśta kin, qa Kilikia qa Asia etanhanpi, hena en inajinpi qa Stephanos kici hdokinicapi.

10 Tuka Woksape Woniya ko on ie cin he okihipi śni.

11 Hehan wicaśta opewicatonpi, unkan hens e, Wicaśta kin de Moses qa Wakantanka nakun wicoie śica ewicakiya, naunhonpi ce, eyapi.

12 Hecen oyate kin, qa hunkawicayapi, wowapi kagapi ko iyowicapaśtakapi ; unkan anatan. hipi, qa yuzapi, qa omniciye kin en ahipi.

13 Qa oitonśni waayatanin kin heca najin wicakiyapi; hena iś heyapi ; Wicaśta kin de tipi wakan kin de wicoope ko on taku śica eye cin ayaśtan śni.

14 Jesus Nazare th etanhan qon he tipi kin de ihangye ca, wicohan Moses unqupi qon hena yutokeca kta ce, eya naunhonpi ce.

15 Unkan omniciye kin en iyotankapi kin owasin opalita yukanpi, qa ite kin he mahpiya ohnihde wan ite kin iyececa wanyakapi.

\section{WICOWOYAKE $\%$.}

1 Hehan wośna kagapi itancan kin heya; Hena hecetu he.

2 Unkan iye hehan heya; Wicaśta hunkawanjinciyapi, ateciyapi ko anamagoptan po. Wowitan Wakantanka kin, Abraham ateunyanpi Mesopotamia en ounye ciqon, Carran en ahiti kin he itokam, en ikpazo;

3 Qa heciya; Nita makoce qa nitakuye kin etanhan ya wo, qa makoce wan cicipazo kte cin he ekta u wo, eciya.

4 Hehan Kaldeya makoce kin etanhan' hiyu qa Carran en ahi ti. Hen atkuku kin ta, unkan hetanhan makoce kin en nakaha oununyanpi kin den ahi ti.

5 Unkan tawa kte cin siha oahe kin hinskokeca qu śni, tu$\mathrm{ka}$ he yuhe kta qa iyohakam cinca kin yuhapi kta e qu kta iwahoya; nahahin cinca wanica. 6 Unkan Wakantanka kaken eya; He cinca kin makoce tokeca en unhdaka unpi kta; unkan hen wowidake wicayuhapi, qa waniyetu opawinge topa hehanyan śicaya wicakuwapi kta.

7 Qa oyate kin hena wowidag wicayuhapi kte cin he wicamdaco kta; qa he iyohakam etanhan hiyupi kta qa makoce kin 


\section{WICOWOYAKE 7.}

den ohomadapi kta ce, Wakantanka eya.

8 Hehan bakihdayapi wicoope kin he qu. Unkan hecen Isaak cincaya, qa anpetu iśahdogan hehan bakilidaya. Isaak Jakob cincaya; qa Jakob hunkakewicayapi akenonpapi qon cincawicaya.

9 Unkan hunkakewicayapi qon Joseph inakiwizipi, qa Egupta ekta wiyopekiyapi. Tuka Wakantanka kici un;

10 Qa wokakije kin owasin etanhan ehdaku, qa Pharo Egupta wicaśtayatapi kin itokam wowaonśida woksape ko qu; unkan Egupta en wicaśta itancan kaga, qa iye ti kin ocowasin en.

11 Hehan Egupta makoce Kanan ko owancaya wicaakikan, qa nina wicakakija; unkan hunkake wicunyanpi kin taku yutapi iyeyapi śni.

12 Tuka hẹan Egupta heciya wamnaheza yukan, Jakob nahon, unkan hunkake wicunyanpi kin tokaheya ekta ye wicaśi.

13 Unkan inonpa hehan, Joseph hunkawanjinwicaye cin sdonkiyewicakiye, ca hehan Joseph otakuye kin Pharo en oyakapi.

14 Hehan Joseph atkuku kin wahoye ca kico, qa takuwicaye cin owasin nakun, wicanagi wikcemna śakowin sanpa zaptan.

15 Hecen Jakob Egupta ekta apamahde iyaye ca hen ta, iye qa nakun hunkake wicunyanpi kin :

16 Unkan Sucem ekta awicayapi, qa wicahnakapi wan, Abraharn Sucem atkuku Emmor he cinca kin, mazaska on, opewicakicaton qon he en wicahnakapi.

17 Tuka anpetu wahoyapi, Wakantanka Abraham konza iwa- hoye ciqon he wanna kiyadan $u$, hehan oyate kin icage ca Egupta en ihduotapi.

18 Hehan wicaśtayatapi tokeca wan Joseph sdonye śni kin he icaga.

19 Unkan he qe takuwicunyanpi kin wicahnaye ça, hunkake wicunyanpi kin śicaya ecawicakicon, hecen hokśiyoqopa kin nipi kte śni e tankan ehpewicakiyapi qon.

$20 \mathrm{He}$ ehan Moses tonpi, he qe hokśiyopa owanyag waśte liinca; unkan wi yamni hehanyan atkuku ti kin ohna icahkiyapi.

21 Qa tankan elipeyapi, hehan Pharo cunwintku kin eyaku, qa iye cinca kin hecen icaliya.

22 Unkan Moses Egupta oyate wicoksape kin owasin onspekiyapi, qa wicoie, wicohan ko on waśaka.

23-Unkan wanna waniyetu wikcemna topa, hehan hunkawanjitku Israel cinca kin wanwicahdag ye kta wacinyuza.

$24 \mathrm{Qa}$ wanji śicaya ecakiconpi kin he wanhdake cehan, okiye ca śicaya ecakiconpi qon he tokiciçon, qa Egupta wicaśta kin kaśtaka.

25 Hunkawanjitku kin, iye nape kin on Wakantanka wiconi wicaqu kte cin he iyukcanpi kta kecin, tuka okahinigapi śni.

26 Thanhanna hehan ake kicizapi kin en taniniçiye ca wookiye wicakicage kta wacin qa heya; Wicaśta hunkawanjin yeciyapi, tokeca śicaya ecayeciconpi he.

27 Tuka tuwe takodaku kin śicaya ecakicon kin he paha iyeye ca, Tuwe unkiyepi en wicaśta itancan qa wayaco nicaga he. 28 Egupta wicaśta litanihan yakte qon he iyecen mayakte kta he, eya. 


\section{OHANYANPI QON.}

29 Wicoie kin de on Moses nape ca Madiyan makoce kin en liewotahedan un, qa hen hokśidan nom cinca wicaya.

30 Unkan wanna waniyetu wikcemna topa ihunniyan, hehan hewoskan makoce kin en Sina Paha kin hetn, can yuliaha wan ohna peta ide kin en, Itancan taohnihde wan taniniciya.

31 Unkan Moses he wanyake cehan, wowanyake kin on yuśinyaya; qa tanyan wanyake kta e on kiyedan ya, hehan Itancan ho kin en tanin;

32 Qa, Nihunkake Wakantanka tawapi kin he miye; Abraham ta Wakantanka, Isaak ta Wakantanka, qa Jakob ta Wakantanka kin, eya. Unkan Moses cancan qa ekta etonwan okihi śni.

33 Unkan Itancan kin heciya; Nitahanpe kin hduśdoka wo, tukten nayajin kin he maka wakan ce.

34 Ekta ewatonwe ca, Egupta en mitaoyate token kakijapi kin he wanmdake ca comnihdazipi kin nawahon, qa wicawakiyuśke kta e on kun hibu; unkan nakaha u wo, Egupta ekta ye ciśi kta.

35 Moses aktapi śni qa; Tuwe wicaśta itancan qa wayaco nicaga he, eciyapi qon he Wakantanka wicaśta itancan wanikiya ko kage ça ye śi, mahpiya ohnihde can yuliaha kin en taniniçiye cin he nape kin on.

36 Unkan hee tankan awicau, qa taku on yuśinyayapi wapetokeca ko, Egupta makoce kin en, Mde śa ekta, qa hewoskan makoce kin etu, waniyetu wikcemna topa hehanyan econ.

37 Moses Israel cinca kin; Hunkawanjin wicayayapi etanhan wicaśta wokcan wan miye iyemaceca, Itancan Wankantanka nitawapi kin icahniciciyapi kta, he anayagoptanpi kta ce, ewicakiye ciqon he dee.

$38 \mathrm{Qa}$ hewoskan makoce kin ekta okodakiciye kin kici un qon hee, Sina paha kin etu, mahpiya ohnihde kin okiye ca nakun hunkake wicunyanpi kin; qa he wiconi oie kin unqupi kta e icu.

39 Unkan hee kin hunkake wicunyanpi kin anagoptonpi cinpi śni, tuka lieyata iyeyapi, qa cantepi kin en Egupta ekta hdapi.

40 Qa Aaron heciyapi; Taku wakan unkitokam yapi kte cin unkicaga : po ; Moses Egupta makoce kin etanhan unkaupi qon he tokiya iyaya sdonunyanpi śni ce.

41 Unkan anpetu kin hena en ptejicadan wan kagapi, qa wakagapi kin he taku wakiyuśnapi, qa iye nape kagapi kin on wiyuśkinpi.

42 Hehan Wakantanka ihduhomni, qa mahpiya ohnaka kin ohodapi kta e awicayuśtan; wicaśta wokcan tawowapi en kagapi kin he iyececa; Israel ti kin woteca ktepi wośnapi ko, hewoskan makoce kin en, waniyetu wikcemna topa hehanyan mayaqupi he.

43 Unkan ito Molok tawakeya kin, qa Remphan taku wakan yadapi wicanhpi tawa kin he iyacupi ; hena wakagapi kin itokam canpeśka makehde inayajinpi kta e yakagapi : Unkan ito Babulon akotanhan aciyapi kta ce.

44 Hunkake wicunyanpi kin hewoskan makoce kin en waaya. tanin wakeya kin yuhapi ; tuwe Moses okiye ca, token wanyake cin he iyecen kage kta keciye ciqon, hee yuśtan qon iyececa.

194 


\section{WICOWOYAKE 8.}

45 He hunkake wicunyanpi kin icupi, qa Joshua kici Ikecwicaśta tamalkoce kin en ahipi, hena e Wakantanka hunkake wicunyanpi itepi kin etanhan heyata iyaye wicaya, Dawid tanpetu kin hehanyan ;

$46 \mathrm{He}$ Wakantanka itokam wowaonśida iyeye ca, Jakob ta Wakantanka kin tipi wan iyekiciciye kta cin.

47 Tuka Solomon tipi wan kicaga.

48 Hececa tuka, Iyotan Wankan un kin tipi wicanape on kagapi kin ohna ti śni; wicaśta wokcan hecen eye ciqon;

49 Mahpiya kin he oiyotanke mitawa kin ee, qa maka kin he misiha oahe kin hee; ecin tukte tipi miyecagapi $\mathrm{k}$ ta he, qa tukten ozimiciye kta he.

50 Minape kin taku henana owasin kage śni he, Itancan kin eya ce.

51 Tahu nisutapi, qa cante noge ko banicilidayapi śni kin, ohinniyan Woniya Wakan kin yakipajinpi, nihunkakepi econpi qon he niye iyenicecapi.

52 Wicaśta wokcan kin tukte wåjji e nihunkakepi kin śicaya kuwapi śni he. Wanji Owotanna un kin he u kte cin tona oyakapi qon hena wicaktepi; qa he qe liiś niyepi wanna iyopeyayapi qa yaktepi.

53 Mahpiya ohnihde ahipi kin he eciyatanhan woope kin iyacupi, tuka tanyan yeciyuhapi śni ce, eya.

54 Unkan hena taku kin nalionpi hehan, cante kin ekta nina iyoyakapi, qa hi ahdakokokapi.

55 Tuka iye Woniya Wakan kin ojudan mahpiya ekta opahta yanke ca, Wakantanka towitan kin, qa Jesus Wakantanka etapa kin eciyatanhan najin e he wanyaka ;

$56 \mathrm{Qa}$ heya; Wanyaka po, mahpiya kin yuzamnipi, qa Wicaśta Cinhintku kin Wakantanka etapa kin eciyatanhan najin e wanmdaka ce, eya.

57 Hehan hotankakiya hotaninpi, qa nogepi kin ohmus hduzapi, qa tawacin wanjidan on iyahpayapi ;

58 Qa otonwe kin tankan ehpeyapi, qa inyan on kininpi. Unkan waayataninpi kin wokoyake kin kośka wan, Salos eciyapi, he siha kin en ekihnakapi.

59 Unkan Stephanos inyan on kininpi, he icunhan cekiye ca heya, Jesus Itancan, minagi kin ehdaku wo.

60 Hehan canpeśka makehde inajin qa hotankakiya hoyelkiya; Itancan woahtani kin de iyepi kin en awicau śni wo. Hens hecen eye ça hehan iśtinma iyaya.

\section{WICOWOYAKE 8.}

1 Unkan he te cin Salos iyowinkiya. Unkan anpetu kin he en okodakiciye Jerusalem en un kin sicaya wicakuwapi tanka. Qa owasin Juda makoce qa Samaria ekta enanakiya iyewicayapi, yewicaśipi kin henana yapi śni.

2 Unkan wicaśta awacin unpi kin heca Stephanos yuha ayapi, qa nina akiceyapi.

3 Tuka Salos okodakiciye kin yutakuni śni wicakuwa, tipi iyaza tin iyaye ca, wica winyan ko wicayuze ca, wicakaśka tipi kin en iyewicaya.

4 Heon etanhan tona enanaki. ya iyayapi qon hena owancaya iyayapi, qa wicoie kin oyakapi ece.

5 Hehan Philip Samaria oton- 


\section{OHANYANPI"QON.}

we kin ekta apamahde iyaye ca, Messiya owicakiyaka.

6 Unkan oyate kin Philip taku wapetokeca econ kin nalionpi qa wanyakapi qehan, taku tona oyalie cin cante wanjidan on anagoptanpi.

7 Taku wakan śica hotankakiya hotaninyan tona wicayuhnaśkinyanpi kin etanhan inanpapi, qa tona tatapike cin huśtepi ko ota okizipi.

8 Unkan otonwe kin he en wiyuśkinpi tanka.

9 Unkan wicaśta wan Simon eciyapi, otonwe kin he en wanakaja tanhan wapiyapi econ, qa Samaria oyate kin yuśinyewicaye ca, iye wicaśta tanka keiciya.

10 He owasin anagoptanpi, cistinpidan tankinkinyan ko, qa; Wakantanka towaśake tanka kin he dee ce, eyapi.

11 Wanakaja tanhan wapiyapi econ kin on yuśinyewicaye cin heon he anagoptanpi.

12 Tuka Wakantanka tokiconze kin, qa Jesus Messiya caje kin eciyatanhan taku Philip oyake cin hena wicadapi qehan baptem wicaqupi, wica winyan ko.

13 Qa Simon iś eya wicada, qa baptem qupi; hehan Philip kici un, qa wowakta taku wapetokeca tanka ko wanyake cin heon yuśinyaya.

14. Unkan Jerusalem ekta yewicaśipi kin, Samaria Wakantanka oie kin icupi kin he nalionpi qehan, Inyan qa Johannes kici ekta ye wicaśipi.

15 Hena e ekta apamahde hipi, qa Woniya Wakan icupi kta heon wocekiye ewicakiciyapi.

$16 \mathrm{He}$ nahahin tuwedan ahinhe śni, Jesus Itancan caje kin on baptem wicaqupi kin hecedan.
17 Hehan nape on awicaputakapi, unkan Woniya Wakan kin icupi.

18 Unkan yewicaśipi kin nape on awicaputakapi kin he eciyatanhan Woniya Wakan wicaqupi kin he Simon wanyaka, hehan mazaska wicakahi;

19 Qa heya; Tuwe nape on awaputake cinhan he Woniya Wakan icu lita e wowaśake kin he maqu po.

20 Tuka Inyan heciya; Mazaska nitawa kin niye kici atakuni śni nunwe. Taku Wakantanka wicaqu ece kin he mazaska on opetonpi kta kecanni kin heon etanhan.

21 Wicoie kin de en oyape śni qa takudan nitawa śni ; Wakantanka itokamya nicante kin owotanna śni.

22 Heon etanhan taku śice cin de on iyopeiciye ça, Wakantanka cekiya wo, nicante taku awacin kin nicicajujupi nun ce.

23 Nahanhin pizi pa hinca, qa woahtani icaśke kin en yaun e wanmdaka ce.

24 Hehan Simon ayupte ca heya; Taku ehapi kin takudán mahiyahde kte śni heon niś ito miye on Itancan kin cekiya po.

25 Unkan wanna yaotaninpi, qa Itancan oie kin oyakapi qehan, Jerusalem ekta icicawin hdapi, qa Samaria otonwe ota kin en wotanin waśte kin oyakapi.

26 Unkan Itancan taohnihde ye cin Philip okiye ca heciya; Najin qa itokahkiya, canku wan Jerusalem hetanhan iyaye ca Gaza en iyohpaye cin he en i wo, he hewoskan kin ee.

27 Hehan iye najin, qa iyaya. Unkan iho, Ethiopa wicaśta wan yunokos heca, Ethiopa wicaśta- 


\section{WICOWOYAKE 9.}

yatapi winohinca, Kandake eciyapi, he ihukuya wicaśta tanka, qa woyuha tawa kin owasin kiciyuha un kin, he Jerusalem ekta cekiye kta e i.

$28 \mathrm{Qa}$ he wanna hde ca canpahmihma ohna kiyotanke ca, Isaya wicaśta wokcan kin he yawa yanka.

29 Unkan Woniya Wakan kin Philip heciya; Ekta ye ca canpahmihma kin de kici ya wo.

30 Hehan Philip ekta inyang ye ca Isaya wicaśta wokcan kin yawa yanke cin he nalion qa; Taku dawa kin he oyakaliniga he, eciya.

31 Unkan; Tuwedan onspemakiye śni ecin token owakihi kta he, eye ca, Philip en u qa kici iy o tanke kta, keya.

32 Wowapi yawa yanke cin iapi kin he dee; Tahinca wan ktepi kte cin en aipi, qa tacincadan hin wicayuśda kin itokam inina yanke cin, he iyecen iś iye i hdukawa śni.

33 Wicawahpanica en un qehan, yacopi kin he ekiciyakupi ; qa wicoicage tawa kin tuwe oyake kta he; wiconi tawa kin maka kin etanhan ayapi ce.

34 Hehan yunokos kin Philip ayupte ca heciya; Ceciciya ce, tuwe on wicaśta wokcan kin hena hecen cya he, iye içica qa iś wicaśta tokeca ka.

35 Hehan Philip i hdukawa qa wowapi kin he etanhan ie ca Jesus e okiyaka.

36 Unkan canku kin ohna hdapi, qa mini yuke cin wan iyohipi. Hehan yunokos kin ; Mini wanyaka wo, baptem maqupi kta e taku tehinda kta he, eya.

37 Unkan Philip, Nicante kin ocowasin on wicayada kinhan enicicetu kta, eciya. Hehan iś ayupte ca; Jesus Messiya he
Wakantanka Cinhintlku kin ee wicawada ce, eya.

38 Hehan canpahmihma kin owanji yanke śi; qa mini kin ekta liun iyayapi, Philip qa yunokos kin; unkan baptem qu.

39 Unkan mini kin etanhan hdicupi qehan, Itancan Taniya kin Philip eyaku, ga yunokos kin ake wanyake śni; unkan canku kin ohna iyuślkinyan hda. 40 Tuka Philip Azotos ekta iyekiyapi; qa otonwe kin owasin opta ye ca wotanin waśte kin oyaka, ecén Kesareya ekta iyohi.

\section{WICOWOYAKE 9.}

1 Unkan Salos waaiapi tin wicaktepi ko Itancan waonspewicakiye cin awicapogan, wośna kagapi itancan kin en i;

2 Qa Damaskos en omniciye tipi kin ekta wowapi da; hecen canku kin de ohna yakonpi kin tona iyewicaye kte cin, wica winyan ko, Jerusalem ekta kaśka awicahdi kta e heon.

3 Unkan icimani ye cin $\mathrm{Da}$ maskos ikiyedan ya; unkan ihnuhanna ojanjan wan mahpiya eciyatanhan ihdukśan iyoyanpa. 4 Hehan makata hinlipaye cehan, wicaho wan nahon, Salos, Salos, tokeca śicaya mayakuwa he, eciya.

5 Unkan iś; Itancan nituwe he, eya. Hehan Itancan kin iś ; Jesus śicaya yakuwa kin he miye ; can pesto kin nayahtake cin he tehika ce, eya.

6 He cancan qa yuśinyaye ca ; Itancan taku ecamon yacin he, eya. Unkan Itancan kin; $\mathrm{Na}-$ jin qa otonwe kin ekta ya wo, token ecanon kte cin oniciyakapi kta ce, eciya.

7 Unkan wicaśta om icimani kin yuśinyaya najinpi, wicaho 


\section{OHANYANPI QON.}

kin nalionpi tuka tuwedan wanyaliapi śni.

8 Unkan Salos maka kin etanhan najin qa iśta kamdaya hehan, tuwedan wanyake śni; unkan nape on yus ayapi, qa Damaskos ekta aipi.

9 Unkan anpetu yamni wawanyake śni un; qa takudan yute śni qa yatke śni.

10 Unkan Damaskos en waonspekiyapi wan, Ananias eciyapi, he wowihanmde en I tancan kin; Ananias, eciya. Unkan iś ; Itancan, de miye ce, eya.

11 Hehan Itancan kin heciya; Najin qa canku wan Owotanna eciyapi kin he ekta ye ca Judas ti kin en Salos eciyapi, Tarsos etanhan he akita wo; wanna cekiya un ;

12 Qa wowihanmde wan en wicaśta wan Ananias eciyapi tin hiyu, qa tonwe kta e on nape on aputake cin he wanyaka ce.

13 Hehan Ananias ayupta; Itancan, wicota etanhan wicaśta kin he Jerusalein ekta, tona owotanna wicaduhe cin taku śica ecawicakicon kin hena nawahon;

14 Qa deciya nakun wośna kagapi kin eciya tanhan wowaśake yuha un, tona nicaje hoyekiyapi kin hena owasin wicakaśke kta ce, eya.

15 Tuka Itancan kin iś heciya; Hunktiya wo, wakśica wakahinige cin he wanji ee, micaje kin Ilcewicaśta, qa wicaśtayatapi kin Israel cinca ko itokam yuha un kta:

$16 \mathrm{Qa}$ micaje kin on taku token kakije kte cin he wakipazo kta ce, eya.

17 Hehan Ananias ye ça, tipi kin timahen iyaye ca, nape on aputake ca heya; Salos mihunkawanji, yatonwe ça Woniya
Wakan on onijudan kta e Itancan kin u maśi ; Jesus canku ohna yau kin en taniniçiye ciqon hee.

18 Unkan ihnuhanna taku hoceśpu kin iyececa iśta kin etanhan hinhpaya; unkan ecahankeya tonwe ca najin, qa baptem qupi.

19 Unkan taku yutapi icu qehan, waśaka aya. Unkan Salos anpetu tonana waonspewicakiyapi kin, Damaskos en yakonpi, hena om un.

$20 \mathrm{Qa}$ kohanna omniciye tipi kin ohna Messiya he Wakantanka Cinhintku kin ee ce, eya oyaka.

21 Unkan tona nahonpi lin owasin yuśinyayapi qa heyapi; Jerusalem ekta tona wicacaje kin de loyekiyapi kin sicaya wicakuwa, qa nakaha wicakaśke ca, wośna kagapi kin ekta awicaye kta e on den hi kin he dee śni he.

22 Tuka Salos sanpa waśaka icage ca Juda wicaśta Damaskos ekta yakonpi kin hena inina yanke wicakiye ca, $\mathrm{He}$ Messiya kin ee e yawicaka.

23 Wanna anpetu ota hehan Juda oyate kin he țe yapi kta e akiiapi.

24 Tuka hecen konzapi kin he Salos sdonya. Unkan ktepi kta e anpetu hanyetu ko otonwe tiyopa kin awanhdakapi.

25 Hehan waonspewicakiyapi kin hanyen icupi, qa makanopiye wan ohna otonwe conkaślke kin etanhan kun iyeyapi.

26 Unkan Salos Jerusalem en hdi qehan, waonspewicakiyapi kin om un kta cin; tuka owasin kokipapi, qa wanna waonspekiyapi kin heca e wicadapi śni.

27 'Tuka Barnabas he icu, qa yewicaśipi kin en ahi, qa token 
tapehi Itancan kin wanyake ça, he okiye ca, token Damaskos ekta Jesus caje kin on waditagya oyake cin hena owicakiyaka.

28 Unkan Jerusalem elkta om un, qa timahen u qa tankan ya ece; qa Jesus Itancan kin on waśagya woyaka.

29 Qa Helenes oyate kin om hdokinica. Tuka hena eqe he ktepi kta akitapi.

30 Hunkaw anjin kiciyapi kin he sdonyapi, unkan Kesareya ekta aipi, qa Tarsos heciya hde śipi.

31 Hehan okodakiciye kin Juda makoce kin owancaya, qa Galile, qa Samaria ekta wookiye yuhapi, qa icagapi ; qa Itancan kin kokipeyahan manipi, qa Woniya Wakan wicakicanpte cin en unpi, qa hecen ihduotapi.

32 Unkan Inyan makoce kin owasin opta iyaye çehan, Ludda ekta wicaśta owotanna yukanpi kin hena en wicahiyohi.

33 Qa wicaśta wan Eneas eciyapi hen wanyaka; he waniyetu śahdogan makan wanka, țațaka ececa.

34 Unkan Inyan; Eneas Jesus Messiya asniniyan, najin qa owinja hdatata wo, eciya. Unkan ihnuhanna najin hiyaya.

35 Unkān tona Ludda qa Saron en yakonpi kin owasin he wanyakapi, qa Itancan kin ekta ihduhomnipi.

36 Unkan Joppa ekta waonspekiyapi wan Tabitha eciyapi, he Tatokadan eyapi kin he kapi; winohinca kin he olian waśte qa taku wacantkiya econ kin ojudan :

37 Unkan he anpetu kin hena en wayazanka, qa ta iyaya. He yujajapi qehan, wankan tipi wan ekta ehnakapi.

38 Unkan Ludda Joppa ikiyedan, qa Inyan hen un kin waon- spewicakiyapi kin nahonpi qon, heon wicaśta nom ekta ye wicaśipi, qa ecadan en wicau kta e iwahoyapi.

39 Hehan Inyan najin qa hena om hda. Ekta i, unkan wankan tipi kin en aipi, unkan wiwazica kin owasin en najinpi, qa ohde wokoyake ko, 'Tabitha ni un qehan, kage ciqon hena pazo yankapi, qa ceyapi ece.

40 Tuka Inyan hena owasin tankan iyewicaye ça, canpeśka makehde inajin qa wocekiye eya, hehan tancan wanke cin ekta ihdarnna qa; Tabitha, najin wo, eya. Unkan iśta kamdaye ca Inyan wanyake cehan iyotanka.

41 Unkan nape yekiye ca najinkiya; qa wicaśta owotanna wiwazica ko wicakico qehan, he ni un e wicakipazo.

42 Unkan Joppa owancaya he otanin, qa wicota Itancan kin wacinyanpi.

43 Unkan Inyan anpetu ota Joppa en, wicaśta wakpanyan wan Simon eciyapi, he kici yanka.

\section{WICOWOYAKE 10.}

1 Unkan Kesareya en wicaśta wan Kornelios eciyapi, akicita opawinge obe wanjidan, Italike eciyapi, kin he en itancan kin hee ;

2 Wicaśta wakinihan, qa Wakantanka kokipeyahan un, tiyohnaka kici ; qa oyate kin nina cantewicakiya, qa ohinniyan Wakantanka cekiya un ece.

$3 \mathrm{He}$ qe anpetu wan en, napcinwanka apa hehantu, wowanyake wan en, Wakantanka taohnihde wan en hi, qa, Kornelios, eciye cin he taninyan wanyaka. 4 Unkan he opalita yanke ca inihan qa; Itancan taku he, eya. 


\section{OHANYANPI QON.}

Unkan iś ito heciya; Wocekiye nitawa qa wacantkiya oyahande cin he Wakantanka itokam kiksuyapi.

5 Heon nakaha Joppa ekta wicaśta ye wicaśi, qa Simon Inyan eciyapi kin he kico wo.

6 Wicaśta wakpanyan wan Simon eciyapi ti kin miniwanca kahida han, he kici yanka. He token ecanon kte cin oniciyake lita ce.

1 Unkan mahpiya ohnihde Kornelios okiye cin he wanna kihda, hehan tiawanyag wicakiye cin nom, qa akicita waśte ohinniyan tankiyadan wicahduhe cin wanjidan, hena wicakico;

8 Qa hena taku kin owasin owicakiyake cehan, Joppa ekta ye wicaśi.

9 Unkan icimanipi, qa ihanhanna wanna otonwe kin ikiyadan, hehan Inyan tipi kin akan wocekiye eya iyaya, wanna wiyotanhe cin hehantu.

10 Nina wotektehda, qa wote kta cin, tuka taku yutapi wiyeya ehnakapi kin he icunhan wokiksuye śni wan ahinhpaya.

11 Unkan mahpiya kin yuzamnipi e wanyaka, qa heciyatanhan taku wan miniliuha ska tanka iyececa, oise topa kin iciyakaśkapi he kutkiya u, qa maka kin ahe kiyapi.

$12 \mathrm{He}$ ohna taku maka akan wahutopa ocaje owasin, qa woteca, qa watutka, qa malpiya okinyanpi kin.

13 Unkan wicaho wan tanin qa; Inyan, najin, qa kte, qa yuta wo, eciya.

14 Tuka iś; Itancan, hecetu śni, tohinni taku ikceka qa aśape cin wate śni ce, eya.

15 Unkan ake icinonpa wicaho kin tanin qa; Taku Wakantan200 ka pakinte cin he ikceka eciye śni wo, eya.

16 Yamni akihde hecen econpi, qa hehan taku kin he malipiya kin ekta akiyahdapi.

17 Unkan Inyan wowanyake wanyake cin he taku kin iyukcan śni un, he icunhan, ito wicaśta Kornelios u wicaśi qon hena Simon ti kin akitapi, qa wanna tiyopa kin itokam en hinajinpi ;

18 Qa panpi qa; Simon Inyan eciyapi den yanka he, eya iwicarvangapi.

19 Wowanyake kin he Inyan awakicin un kin he icunhan, Woniya Wakan kin heciya; Wanyaka wo, wicaśta yamni onidepi.

20 Heon najin qa kun hde ca, takudan cețunhda śni om ya wo, hena u wicawaśi ce, eya.

21 Hehan Inyan wicaśta Kornelios u wicaśi qon hena elta kun wicai, qa; Tuwe ayakitapi kin he miye ce; taku on yahipi he, ewicakiya.

22 Unkan iś heyapi; Kornelios akicita opawinge wicayuhe cin, wicaśta owotanna, qa Wakantanka kokipeyahan un he Juda oyate kin iyulpa oyakapi, hee mahpiya ohnihde wakan wan okiye ça, tuwe nihuwe u wicaśi, qa iye ti kin ohna nioie kin nalion kta, keya ce, eyapi.

23 Hehan tin u wicaśi qa won wicaya. Unkan ihanhanna Inyan hena om hda, unkan Joppa etanhan hunkawanjinkiciyapi kin wanjikji opapi.

24 Unkan ihanhanna qehan Kesareya iyohipi. Unkan Kornelios tona takuwicaye ça koda wicaye cin hena wicakico, qa om ape yanka.

25 Unkan Inyan en $u$, hehan Kornelios itkokim ye ça, siha 
kin en makata ehpeiciye ca cekiya.

26 Tuka Inyan iś najin kiye ça; Najin wo, miś eya wimacaśta ce, eya.

27 Qa kici wohdaka ecen tipi kin timahen i, qa wicota mniciyapi kin wanwicayaka.

28 Hehan hewicakiya; Tuwe Juda wicaśta kin heca e oyate tokeca etanhan wan kici un, qa en hi kin he hecetu śni e sdonyayapi, tuka tuwedan wicaśta ikceka qa aśape cin ewakiye kte śni he Wakantanka sdonye makiya.

29 Heon etanhan mahuwe ipi kin ecen wacețuhda śni hibu, heon taku on miyecopi hecinhan he iciwangapi, eya.

30 Unkan Kornelios heya; Wanna ehantanhan anpetu topa he ehan, qa nakaha oape kin dehantu, wote śni waun, qa napcinwanka ape cin hehan wati kin en cekiya waun; unkan wicaśta wan wokoyake wiyatpa koyake cin he en mahinajin ;

$31 \mathrm{Qa}$, Kornelios wocekiye nitawa kin he nahonpi, qa taku wacantkiya ecanon kin he Wakantanka itokam kiksuyapi ce.

32 Heon Joppa ekta tuwe ye śi, qa Simon Inyan eciyapi kin he kico wo ; he wicaśta wakpanyan wan, Simon eciyapi, ti kin miniwanca kahda he cin he ohna yanka. He hi kinhan taku eniciye kta ce, eya.

33 Heon kohanna wahociya; qa yahi kin he tanyan ecanon. Heon nakaha unkiyepi kin owasin Wakantanka itolkam unkanpi, taku Wakantanka eye niśi kin owasin naunhonpi kta ce

34 Hehan Inyan i hdukawa qa heya; Awicalkehan Wakantanla wicaśta itohnake kin iwanyake śni e imdukcan.
35 Tuka oyate otoiyohi en, tuwe kokipeyahan un, qa owotanna ohanye cin he waśtedaka ece.

36 Jesus Messiya kin, owasin en Itancan kin hee, he eciyatanhan wicoie Israel cinca kin wookiye iwahowicayapi qon.

37 Wicoie kin he sdonyayapi, Juda makoce kin owancaya oyakapi, Galile etanhan, Johannes baptem oyake cin hehan tanhan. 38 Jesus Nazareth etanhan kin he Wakantanka sdakiya, Woniya Wakan wowaśake ko on; qa he qe taku waśte econ iyaya ece, qa tolla taku wakan śica wicayuhnaśkinyan kin hena okiziwicaya ece, Wakantanka kici un kin heon.

39 Qa taku Juda oyate tamakoce, qa Jerusalem ekta econ qon owasin unyaotaninpi ece. He iye kin ktepi, qa can wan akan otkeyapi qon.

40 Tuka anpetu iciyamni qehan Wakantanka piya icahkiye ca, taninyan wicalkipazo.

41 Oyate kin iyuhpa śni, tuka tona otokaheya ekta Wakantanka wicakalinige ciqon hena en, waayataninpi kin unkiyepi, wiconte etanban piya ikicage cin iyohakam kici wauntapi qa waunyatkanpi ece.

42 Unkan oyate kin wahokonwicakiye unśipi qa nipi tapi ko en wayaco Wakantanka kage cin he iye ce, eya oyake unśipi. $43 \mathrm{He}$ wicaśta wokcan kin owasin oyakapi ; iye caje kin eciyatanhan tuwe wicada kinhan woahtani wokajuju kin yuhe kta ce.

44 Wicoie kin dena Inyan ecen eye cin he icunhan, tona oie kin nahonpi owasin Woniya Wakan kin awicahinhpaya.

45 Unkan bakikidayapi kin etan201 
hanpi tona wicadapi qon, tona Inyan kici hipi kin hena yuśinyayapi, Woniya Wakan wicaqupi ece lkin he Ikcewicaśta iyepi kaeś awicakaśtanpi kin heon etanhan.

46 Wicaceji tokeca on iapi, qa Wakantanka yatanpi kin he nahonpi. Hehan Inyan waayupte ça ;

47 Tuwe mini tehinda okihi, qa hena baptem wicaqupi kte śni he; hena eqe unkiyepi kin iyecen Woniya Wakan kin icupi ce, eya.

48 Hehan Itancan caje kin on baptem wicaqu wicaśi. Hehan anpetu ota en yanke kta e icekiyapi.

\section{WICOWOYAKE 11.}

1 Unkan yewicaśipi kin hunkawanjinkiciyapi ko, Juda makoce kin en yakonpi qon, Ikcewicaśta Wakantanka oie icupi kin he nahonpi.

2 Unkan Inyan Jerusalem en hdi kin, hehan tona bakilidayapi kin etanhanpi qon he kici akinicapi ;

3 Qa heyapi, Wicaśta bakihidayapi śni kin heca en wicayai, qa om wayata ce.

4 Hehan Inyan wohdake ca owasin ecekcen owicakiyake ça heya;

5 Joppa otonwe kin en, wocekiye eya waun, unkan wokiksuye śni wan en taku wowanyake wanmdaka, taku wan miniliuha ska tanka iyececa, oise topa kin iciyakaśkapi mahpiya kin eciyatanhan kun aupi, qa miye ekta mahiyohi.

6 He opahta manke ca awacin waun, unkan maka akan taku wahutopa, qa wamanica, qa watutka, qa malipiya okinyanpi kin wanmdaka.
7 Unkan wicaho wan; Inyan najin, qa kte, qa yuta wo, emakiye cin he nawalion.

8 Tuka miś; Itancan hecetu śni, taku ikceka qa aśape cin tohinni mii kin ohna iyaye śni, epa.

9 Tuka mahipiya kin eciyatanhan wicaho kin ake amayupta; Taku Wakantanka pakinte cin he ikcela eciye śni wo, eya.

10 Yamni akihde hecen econpi, qa hehan mahpiya kin ekta owasin hdokiyahdapi.

11 Unkan ito wicaśta yamni, Kesareya etanhan miye ekta mahiyu śipi qon, hena wanna tipi ohna waun kin itokam hinajinpi.

12 Hehan Woniya Wakan kin iś; Takudan cețunwicahda śni hena om yahde kta ce, emakiya. Unkan hunkawanjinkiciyapi kin dena śakpe om imdamde ca wicaśta qon he ti kin en unkipi.

13 Hehan iye, token mahpiya ohnibde wan iye ti kin en hinajin wanyake ciqon he unkokiyakapi; hee kin kaken eciya; Joppa ekta wicaśta ye wicaśi, qa Simon Inyan eciyapi kin he u śi wo.

14 He taku eniciye kte cin on yani kta, qa nakun tiyohnaka nitawa kin owasin, eya ce, eya.

15 Unkan miś tokaheya iwac cin, hehan Woniya Wakan kin awicahinhpaya, tokag ehan unkiyepi kin en hi qon iyececa.

16 Hehan Itancan oie qon weksuya; Awicakehan Johannes mini on baptem wicaqu, tuka niyepi kin Woniya Wakan on baptem niçupi kta ce, eye ciqon he.

17 Hecen unkiye Jesus Messiya Itancan kin wicaundapi, Wakantanka taku unqupi qon he iyecen wicaqu kinhan, miś 
matuwe he, Wakantanka anawapte kta owakihi he.

18 Hena oie kin nahonpi unkan inina yukanpi qa Wakantanka yatanpi qa heyapi ; Awicakehan Ikcewicaśta on nipi kta e ihduecetupi kin Wakantanka wicaqu ce.

19 Unkan Stephanos te cin on sicaya wicakuwapi qon, heon enanakiya iyewicayapi kin hena Phenike, qa Kupros, qa Antiyoka ekta ipi, qa wicoie kin Juda oyate kin ecedan owicakiyakapi.

20 Unkan hena wanjiljji $\mathrm{Ku}$ pros qa Kurene wicaśta kin heca, hena Antiyoka en hipi qehan, Helenes wicaśta kin wowicakiyakapi, qa Jesus Itancan kin oyakapi.

21 Unkan Itancan nape kin hena om un, qa wicota wicadapi, qa Itancan kin ekta ihduhomnipi.

22 Hena taku kin okodakiciye Jerusalem en un kin nogepi Kin en otanin, hehan Barnabas Antiyoka ekta i kta e ye śipi.

$23 \mathrm{He}$ ekta i, uniran Wakantanka towaonśida kin wanyake cehan iyuśkin, qa cante kin ocowasin on Itancan kin opapi kta e owasin iwahokonwicakiya.

24 He wicaśta waśte, qa Woniya Wakan wacinyanpi ko on ojudan. Unkan wicota Itancan kin opapi.

25 Hehan Barnabas Salos akite kta e Tarsos ekta iyaya. He iyeya, unkan Antiyoka en ahdi.

26 Qa waniyetu mdoketu ahna, tipi wakan kin en mniciyapi, qa oyate ota waonspewicakiyapi ; unkan Antiyoka elita, waonspewicakiyapi kin tokaheya Kristian ewicalkiyapi:

27 Unkan anpetu kin hena en, wicaśta wokcan Jerusalem etanhan Antiyoka ekta hipi.
28 Hena wanji, Agabos eciyapi kin he inajin, qa maka kin owancaya wicaakihan wan tanka "u kta e Woniya Wakan kin eciyatanhan oyaka. Unkan he Kladios Kesar taanpetu kin en ecetu.

29 Unkan hehan waonspewicakiyapi kin otoiyohi, token okihipi kin, hecen hunkawanjitkupi Juda makoce kin en yakonpi kin, wawokiyapi kin wicaqupi kta e konzaji.

30 Hecen econpi, qa Barnabas Salos kici napepi kin on hunka wicayapi kin wicakai wicaśipi.

\section{WICOWOYAKE 12.}

1 Unkan hehan Herod wicaśtayatapi kin okodakiciye kin wicakiyuśe kta e nape yekiya ;

$2 \mathrm{Qa}$ Jakob Johannes cincu qon he mazasagye on kte.

3 Qa he Juda oyate kin iyokipipi e wanyake cehan, Inyan nakun yuze kta aya: aguyapi on napoliyapi codan anpetu kin he ehan.

4 Unkan he iyahpaye cehan, wicakaśka tipi kin en ehnake ca akicita ake śakpe awanyag wicaśi, wośnapi kin enakiyapi kinhan oyate kin elsta tankan au kta kecin.

5 Hecen Inyan wicakaśka tipi kin ohna awanyakapi; unkan on okodakiciye kin Wakantanka nina cekiyapi.

6 Unkan Herod wanna tankan au kta hehan, hanyetu kin he en Inyan akicita nom, unma itan anokatanhan iśtinma wanke ca, maza icicahilia nom on iyaliaślkapi, qa tiawanyag kiyapi kin hena tiyopa kin itokam wicakaśka tipi kin awanyakapi.

7 Unkan inyun, Itancan taohnihdeye cin en hi, qa ojanjan wan 203 


\section{OHANYANPI QON.}

wicakaśka tipi kin en iyoyanpa ; upkan Inyan cuwi kin en ape ca najin kiye ca heya; Kohanna najin wo. Únkan maza icicahiha kin nape kin etanhan hinhpaya.

8 Hehan mahpiya ohnihde kin; Ipihdag kiton qa hanpokihan wo, eciya. Unkan iś ecen econ. Hehan; Śina hdowin qa miyahna u wo, eciya.

9 Hehan tankan ya: unkan iś eya ihakam ye ca, mahpiya ohnihde taku ecakicon kin he wicakapi kin sdonye śni ; tuka wowanyake wan wanyaka kecin.

10 Unkan wicakaśta tipi tokaheya qa iyokihe kin etanhan hinanpapi, hehan maza tiyopa kin en iyohipi, he otonwe kin ekta iyahde, he iye cinka wicakiyuhdog iyaya. Unkan tankan yapi, qa canku wanjidan ihunnipi, hehan mahpiya ohnihde kin ehpeya kihda.

11 Unkan Inyan kilssuya hinhda qa heya; Nakaha awicakehan Itancan kin ohnihde tawa kin u śi, qa Herod nape kin, Juda oyate apepi ko, owasin etanhan emahdaku e sdonwakiya ce.

12 Unkan he awakicin qehan, Johannes Markos eciyapi kin, he hunku Mary ti kin ekta i, hen wicota mniciyapi, qa cekiya yukanpi.

13 Unkan Inyan tipi tiyopa lkin kabubu yanke cin he icunhan, wicinyanna wan Rhoda eciyapi, he ekta wanahon $\mathrm{i}$ :

14 Qa Inyan ho kin iyekiye cehan, wowiyuśkin on tiyopa yuhidoke śni, tuka inyang hdicu qa, Inyan tiyopa kin itokam najin e oyaka.

15 Unkan, Niyuhnaślkinyanpi ce, eciyapi. Tuka iś hecen eya kitan. Hehan, Mahpiya ohnihde tawa kin hee ce, eyapi.
16 Tuka Inyan katinyan tiyopa kin kabubu yanka. Unkan yulidokapi qa wanyakapi hehan yuśinyayapi.

17 Tuka inina yukanpi kta e namwicakicawin, qa token on Itancan kin wicakaśka tipi kin etanhan tankan hiyu kiye cin he owicakiyaka. Qa heya, Jakob qa hunkawanjinkiciyapi kin hena oie kin owicakiyaka po. $\mathrm{He}$ han tankan kinape ca tokan iyaya.

18 Unkan anpa kamdeze cin hehan Inyan toki iyaye cin he akicita kin nina aoqoyapi.

19 Unkan Herod akita, tuka iyeye śni hehan, tiawanyag wicakiyapi kin hena wiwicawange ca, wicaktepi kta e konza. Qa hehan Juda makoce kin etanhan apamahde iyaye ça Kesareya en oullyan.

20 Unkan Herod Turos qa Sidon oyatepi kin wicakize wacin ; tuka hena e wicocante wanjidan en hipi, qa Blastos wicaśtayatapi ohehdepi tipi tawa kin awanyake cin he yuwaśtepi qehan, wookiye dapi; iye tamakocepi kin wicaśtayatapi tawa kin on icaliyapi kin heon etanhan.

21 Unkan anpetu wan cajeyatapi qon he en Herod wicaśtayatapi ihduze ça, woyaco oiyotanke kin akan kiyotanke ca, wowicakiyaka.

22 Ilehan oyate kin hotankakiya hotaninpi qa; He wicaho śni, Wakantanka ho kin hee ce, eyapi.

23 Unkan ihnuhanna Itancan taohnihde ye cin he kaśtaka, Wakantanka wowitan qu śni kin heon etanhan. Unkan wamduśkadan yutapi qa hecen ța iyaya.

24. Tuka Wakantanka oie kin icage ca tankaya aya. 
25 Unkan Barnabas Salos kici wawokiyapi qon hduśtanpi qehan, Jerusalem etanhan hdicupi, qa Johannes Markos eciyapi qon he yuha hdapi.

\section{WICOWOYAKE 13.}

1 Unkan Antiyoka ekta okodakiciye kin en wicaśta wokcan waonspekiya ko wanjikji yukan; Barnabas, qa Simeon he nakun Niger eciyapi, qa Lukios Kurene etanhan, qa Manaen, Herod wicaśta ihdawa qon kici icage cin hee, qa Salos.

2 Hehan Itancan kin cekiyapi qa wote śni unpi kin icunhan, Woniya Wakan kin heya ; Barnabas Salos kici wokicanye iwicaweco kin heon ewicamicihde po, eya.

3 Unkan hehan takudan yute śni wocekiye eyapi, qa nape awicaputakapi, hecen ye wicaśipi.

4 Hena Woniya Wakan tokiya ye wicaśi, unkan Selukiya elkta iyohipi, qa hetanhan Kupros elitakiya watom yapi.

5 Qa Salamis en iyukanpi hehan, omniciye tipi Juda oyate tawapi kin ohna Wakantanka oie kin oyakapi, qa Johannes he ookiye yuhapi.

6 Unkan wita kin opta yapi, qa Paphos ekta iyohipi qehan, wicaśta wapiyapi econ kin, wicaśta wokcan itonśni, Juda wicaśta kin heca wan, Barjesus eciyapi he wanyakapi :

7 He Sergios Palos, wicaśta ksapa, makoce kin en itancan yanke cin he kici un. He qe Barnabas qa Salos kici wicalico, qa Wakantanka oie kin nalion cin.

8 'Tuka Elumas wapiye cin, caje kin hecen kapi, he wicakipajin, qa wicaśta itancan kin wacinyanpi kin etanhan yuhomni kta e akita.

9 Hehan Salos, iye nakun $\mathrm{Pa}-$ los eciyapi, Woniya Wakan on ojudan he opalita najin,

10 Qa lieya; 'Taku wohnaye owasin on ojudan, qa wicohan śica owasin, Wakan śica cinca niye ca, taku owotanna owasin toka yaye cin, Itancan tacanku owotanna kin kaśeyaye cin he aduśtan kte śni he.

11 Hecen dehan inyun, Itancan nape kin iyahdeniyan; unkan anpetu ota iśta nigonge ca anpetu wi kin wandake kte śni, eciya. Unkan ihnuhanna opo qa otpaza wan ahinhpaya; qa tuwe nape yus aye kta e akin iyaya.

12 Hehan taku econpi kin wicaśta itancan kin wanyake cehan wicada, qa Itancan tawoonspe kin on yuśinyaya.

13 Unkan Palos tona om un kin hena Paphos etanhan pacanan iyeyapi, qa Pampuliya ekta Perge en iyohipi. Hehan Johannes awicayuśtan qa Jerusalem ekta icicawin hda.

14 Hehan Perge etanhan inanpapi, qa Antiyoka Pisidiya en, he ekta ipi, qa anpetu wakan hehan omniciye tipi kin en tin iyayapi, qa iyotankapi.

15 Unkan woope, qa wicaśta wokcan tawowapi kin, hena yawapi enakiyapi qehan, omniciye tipi kin en wicaśta itancan wicakiyapi kin iś wicakicopi qa, Wicaśta hunkawanjin unniyanpi wicakicanpte oie wanji duhapi kinhan oyate kin owicakiyaka po, ewicakiyapi.

16 Hehan Palos najin, qa namwicakicawin qa heya, Israel wicaśta, qa tona Wakantanka kokipapi kin anagoptan po.

17 Israel oyate kin de Wakan205 


\section{OHANYANPI QON.}

tanka tawapi kin he hunkake wicunyanpi kin wicakahnige ca, oyate kin Egupta makoce kin en yakonpi kin he icunhan yuwankan iwicacu, qa isto hduwankan iyeye cin on tankan awicau.

$18 \mathrm{Qa}$ waniyetu wikcemna topa hehanyan hewoskan makoce kin en won wicakiya.

19 Qa oyate śakowinpi Kanan makoce kin en, ihang wicaye cehan, makoce kin woohiye eciyatanhan kicipamni wicakiya.

$20 \mathrm{Qa}$ he iyohakam, wicaśta wayaco kin heca wicaqu, waniyetu opawinge topa sanpa wikcemna zaptan hehanyan, ecen Samuel wicaśta wokcan qon ica$g a$.

21 Unkan hehan wicaśtayatapi wan cinpi ; qa Wakantanka Saul, Kiś cinhintku, Benjamin wicoun kin hetanhan, wicaśta kin he wicaqu, waniyetu wikcemna topa hehanyan.

22 Qa he lieyata iyeye cehan, Dawid icaliwicakiciciye ca wicaśtayatapi kaga; he oyake ca heya, Dawid Jesse cinhintku lín he iyewakiya, wicaśta waśte wakidake cin, he mitawacin kin ocowasin ecen econ kta ce.

23 Wakantanka token eye ciqon he eciyatanhan, wicaśta kin de cinca kin etanhan, Jesus Wanikiya kin, Israel oyate kin icahwicakiciciya.

24 He ihdutanin śni qon itokam, Johannes baptem on ihduecetupi kte cin he Israel oyate kin iyuhpa en eyanpaha owicakiyaka.

25 Unkan Johannes oinyanke kin ihunniye kte cin, hehan hecen eya, Miś matuwe kecannipi he: He miye śni; tuka iho, wanji mihektam $u$ kin he tahanpe siha kin etanhan kiyuśka owakihi śni ce.
26 Wicaśta hunkawanjin ciyapi kin, Abraham cinca wicaye cin, qa tona Wakantanka kokipapi kin, niś niyepi wiconi oie kin de oniciyakapi.

27 Tona Jerusalem en yakonpi, wicaśta itancan wicayuhapi ko, he iyekiyapi śni, qa wicaśta wokcan oiepi, anpetu wakan eca yawapi ece kin he nakun; hecen he yacopi kin on hena ecetuyapi.

$28 \mathrm{Qa}$ taku on te kte cin takudan iyeyapi śni qeyaś, he ktepi kta e Pilate icekiyapi.

$29 \mathrm{Qa}$ on taku wowapi en kagapi qon owasin ecetuyapi qehan, can kin etanhan eyakupi, qa ohna hnakapi wan en ohnakapi.

30 Tuka te cin etanhan Wakantanka najin kiya.

31 Unkan tona Galile etanhan Jerusalem ekta om hi qon hena anpetu ota wanhdakapi, qa hèna eepi dehan oyate kin wicakiyaotaninpi ece.

32 Unkan unkiye wotanin waśte unkoniciyakapi, hunkake wicunyanpi taku iwahowicaye ciqon,

33 He cincawicayapi unkiyepi kin en, Wakantanka ecetukiya, Jesus piya icahkiye cin hee on ; hecen odowan icinonpa kin en kaken owapi qon, Micinkśi kin he niye, anpe dehan cinca cicaga ce.

34 Qa wicate cin etanhan najin kiye ca, wicahwin kin ekta i kte śni, heon kaken eya, Dawid towaonśida wicakapi kin he ciçupi kta ce.

35 Heon etanhan wanji tokeca en hecen eya; Wanji Owotanna nitawa kin he wicahwin wanyake kta e iyowinyakiye kte śni.

36 Dawid wicoicage tawa kin 
tanyan owicakicilian, Wakantanka tawacin kin eciyatanhan, hehan iśtinma iyaye ca, hunkake wicaye cin om hnakapi, qa wicaliwin wanyaka.

37 Tuka tuwe Wakantanka najin kiye cin he wicahwin wanyake śni.

38 Heon wicaśta hunkawanjin ciyapi, wicaśta kin de eciyatanhan, woahtani kajujupi kin oniciyakapi e sdonya po.

39 Qa taku owasin, Moses tawoahope kin niyuowotanpidan kta e okitpani kin hena ee kaeś etanhan, tuwe wicada kinhan iye on owotanna kagapi kta.

40 Heon itonicipa po, okinni taku wicaśta wokcan kin en cajeyatapi qon he en nihiyahdepi kta :

41 Tona ayaktapi śni kin, wawanyakapi qa inihanpi qa yutakuni śni iyeiçiya po ; anpetu nitawapi kin en wicohan wanji ecamon kta, qa wicolian kin he tuwe oniciyakapi eśta wicayadapi kte śni ce.

42 Unkan Juda wicaśta kin omniciye tipi kin etanhan tankan hdicupi, hehan wicoie kin hena anpetu wakan hepiya, owicakiyakapi kta e icewicakiyapi.

43 Unkan omniciye kin wanna enakiyapi, hehan Juda wicaśta ga oyate tokeca etanhan' he en opapi qon, cekiya unpi kin hena wicota Palos qa Barnabas kici owicapapi. Unkan wowicakiyakapi, qa Wakantanka towaonśida kin en unpi kta e iwahokonwicakiyapi.

44. Unkan ake anpetu wakan, hehan Wakantanka oie nalionpi kta e otonwe kin iyulipa se en kiwitaya iheyapi.

45 Tuka Juda wicaśta kin hena wicota kin wanyakapi qehan, winawizipi on ojupidan, qa $\mathrm{Pa}$ - los taku eye cin he alinicapi qa aiapi.

46 Hehan Palos Barnabas kici waditake içicagapi qa heyapi, Wakantanka oie kin niyepi tokaheya oniciyakapi kta e hecetu; tuka he ayaktapi śni, qa owihanke wanin wiconi kin iyenihantupi śni nihdukcanpi kin heon, iho, Ikcewicaśta kin ekta unkihdamnanpi.

47 Hecen Itancan kin iwahounyanpi ; Ikcewicaśta iyoyanpa kin ecihnaka, maka ihanke kin hehanyan niwicayakiye kte cin heon.

48 Unkan Ikcewicaśta kin, hena oie kin nahonpi qehan, nina iyuśkinpi, qa Itancan oie kin yatanpi. Unkan tona owihanke wanin wiconi yuhapi kta e wicakalinigapi qon hena wicadapi.

49 Unkan Itancan oie kin makoce kin he owancaya oyakapi.

50 Tuka Juda wicaśta kin iś, winohinca okinihan cekiya unpi kin hena iyowicapaśtakapi, qa otonwe kin en wicaśta itancanpi kin nakun, hecen Palos Barnabas kici śicaya wicalkuwapi, qa makoce kin he etanhan hdicu wicayapi.

51 Tuka iś iyepi kin, hena en, siha watuśekśeca askape cin he hdata tapi, qa Ikonium ekta ipi.

52 Unkan waonspewicakiyapi kin wowiyuśkin Woniyạ Wakan ko on ojupidan.

\section{WICOWOYAKE 14.}

1 Unkan Ikonium ekta, salim omniciye tipi, Juda oyate tawapi kin he en timahen yapi, qa iyecen iapi, liecen Juda wicaśta Helenes ko ota wicadapi.

2 Tuka Juda wicaśta tona wicadapi śni kin hena Ikcewicaśta kin iyowicapaśtakapi, qa hun207 


\section{OHANYANPI QON.}

kawanjin kiciyapi kin śicaya wacinwicakiyus wicakiyapi.

3 Unkan tehan en yukanpi, qa Itancan kin on waditagya iapi, he qe wowaonsida oie kin hdaotanin, qa wowapetokeca wakanyan econpi ko econ wicakiya.

4 Tuka otonwe kin oyate kin akipam iyeyapi; apa Juda wicaśta kin om tipidan, qa apa iś yewicaśipi kin hena owicapapi.

5 Unkan Ikeewicaśta, qa Juda oyate kin, tona itancan wicayuhapi kin hena koya, hena anawicatanpi, śicaya wicakuwapi qa inyan on wicakininpi kta ;

6 He iye sdonkiyapi, qa Lustra qa Derbe, Lukaoniya en otonwe kin, makoce ihdukśan ko en onapapi.

7 Qa hen wotanin waśte kin oyakapi.

8 Unkan Lustra en wicaśta wan siha on okitpani iyotankehan yanka, tonpi kin ehantanhan huśte, qa tohinni mani śni.

9 He Palos ie cin nalion; unkan he opalita yanke ca, wacinyanpi on ni kte cin yuha e wanyaka,

10 Hehan hotankakiya, Nisiha on bosdan najin wo, eya. Unkan iś psin iyaye ca mani.

11 Unkan Palos taku econ kin he oyate kin wanyakapi qehan ho yuwankan icupi qa Lukaoniya iapi kin eciyatanhan, Taku wakan kin wicaśta ihduza unkiyepi en kun unhiyupi ce, eyapi.

12 Qa Barnabas Jupiter eya caśtonpi ; qa Palos iś, Hermes eciyapi, iapi on itancan kin hee, heon etanhan.

13 Hehan Jupiter otonwe itokam yanke cin he wośna kagapi tawa kin he tatanka wateśdake ko, tiyopa kin en ahi, qa oyate kin om wośna kta tuka.
14 He yewicaśipi kin, Barnabas Palos kici, nalonpi qehan, wokoyake aihdupotapi, qa oyate kin ekta inyang yapi, hotaninpi,

15 Qa heyapi, Wicaśta kin, tokeca e hecanonpi he; niye yaunpi kin jyecen, wicaśta akantu kin heuncapi, qa hena takuśniśni kin aduśtanpi qa Wakantanka ni un kin ekta nihduhomnipi kta e iwahokonunniciyapi; he malipiya, maka, qa miniwanca, taku ohnaka ko, owasin kage cin hee.

$16 \mathrm{He}$ wicoicage hekpata un kin en, oyate kin owasin canku tawapi kin ohna manipi kta e iyowinwicakiya.

17 Hececa eśta tanyan econ, qa mahpiya eciyatanhan magaju wicaqu, makoncage taku ota icahye cin hena ko, qa woyute wowiyuśkin ko on cante imnaunyanpi qon, he eciyatanhan ohinniyan ihdutanin ce.

18 Unkan wicoie kin hena on wawicakiyuśnapi kte cin oyate kin kitan se ayuśtan wicayapi.

19 Hehan Antiyoka qa Ikonium etanhan Juda wicaśta wanjikji en hipi qa oyate kin iyowicapaśtakapi ; hecen Palos inyan on kininpi qa otonwe kin tankan yusdohan ayapi, wanna ța kecinpi.

20 Tuka waonspewicakiyapi kin ihdukśan hinajin yukanpi kin icunhan, iye najin hiyaye ca otonwe kin en i. Unkan ihanhianna qehan Barnabas kici Derbe ekta iyaya.

21 Unkan otonwe kin he en wotanin waśte kin oyakapi, qa wicota waonspewicakiyapi qehan, hehan Lustra, qa Ikonium, qa Antiyoka ek ta icicawin hdapi. $22 \mathrm{Qa}$ wannspewicakiyapi nagipi kin waśag wicakiyapi, qa wacinyanpi kin en kitanyan unpi 
kta e iwahokonwicakiyapi, qa wokakije ota iyoopteya Wakantanka tokiconze kin en unkipi kta ce.

23 Unkan okodakiciye otoiyohi en hunka wicayapi kin wicakagapi, qa wote śni wocekiye eyapi qehan, Itancan wicadapi qon he awanwicahdake kta $\mathrm{e}$ icekiyapi.

24 Unkan Pisidia opta yapi hehan Pampulia en kiyohipi.

$25 \mathrm{Qa}$ Perge en wicoie kin oyakapi qehan, Atalia ekta apamahde iyayapi.

26 Qa hetanhan Antiyoka ekta watom hdapi, wicohtani wanna hduśtanpi kin heon, Wakantanka towaonśida kin yuhapi kta e icekiyapi qon he hetu.

$2 \%$ Hen hdipi qa okodakiciye kin mniciye wicaśipi, qa Wakantanka token econ wicakiye cin hena owasin owicakiyakapi, qa wacinyanpi tiyopa wan Ikcewicaśta kin wicakiyuhdoke cin he nakun.

28 Unkan hen waonspewicakiyapi kin om tehan owanji yukanpi.

\section{WICOWOYAKE 15.}

1 Unkan wicaśta wanjikji Juda makoee kin etanhan apamahde yapi, qa hunkawanjinkiciyapi kin wahokonwicakiyapi qa, Moses toope kin eciyatanhan banicilidayapi śni kinhan yanipi kte śni ce, eyapi.

2 Unkan Palos Barnabas kici hena wicakigepi, qa nina awicakinicapi, hehan wiwicawangapi kin he on, Palos Barnabas kici, qa iyepi kin etanhan wicaśta tokeca wanjikji ko, yewicaśipi kin, qa hunkawicayapi kin, Jerusalem ekta yakonpi kin, hena en wicayapi kta e konzapi.

3 Unkan okodakiciye kin hena hecen yewicakiyapi qehan, Phenike, qa Samaria opta yapi, qa Ikcewicaśta ihduhomnipi kin he oyakapi; qa hunkawanjin kiciyapi kin owasin en wowiyuśkin tanka kagapi.

4 Unkan Jerusalem ekta ipi qehan, okodakiciye kin he, qa yewicaśipi kin, hunkawicayapi kin hena ko, tin hiyu wicakiyapi ; hehan Wakantanka token econ wicakiye cin owasin ohdakapi.

5 Tuka Pharisee yuomdecapi kin etanhan wanjikji wicadapi qon hena inajinpi qa heyapi, Bawicakilidayapi qa Moses toope kin ope wicaśipi kta iyececa ce, eyapi ce.

6 Unkan wicoie kin de akiiapi kta e on yewicaśipi kin, hunkawicayapi ko witaya mniciyapi.

7 Qa nina wikiciwangapi hehan, Inyan inajin qa hewicakiya, Wicaśta hunkawanjinciyapi kin, ehanna unketanhanpi kin Wakantanka miye makahinige ca, mii kin eciyatanhan Ikcewicaśta kin wotanin waśte oie kin nahonpi, qa wicadapi kta e konza sdonyayapi.

$8 \mathrm{Qa}$ Wakantanka wicacante kin sdonye cin he wicayaotanin, qa Woniya Wakan unkiye unqupi qon, he iyecen wicaqu.

9 Qa takudan ocitkonze śni unkagapi, iye cantepi kin nakun wicadapi kin on wicakipakinta.

10 Heon nakaha tokeca e Wakantanka iyutanyayapi, qa can napinpi wan, hunkake wicunyanpi qa unkiyepi kin yuha unkokihipi śni kin he waonspewicakiyapi tahupi kin en eyahnakapi kta he.

11 Tuka Jesus Messiya Itancan kin towaonśida kin on, iś iyepi kin wiconi yuhapi kte cin, he iyecen unkiś unhapi kta e wicaundapi ce. 


\section{OHANYANPI QON.}

12 Hehan omniciye kin owasin inina yukanpi, qa Barnabas Palos kici, wowapetokeca wakanyan econpi ko, Wakantanka Ikcewicaśta kin ekta econ wicakiye cin hena ecen ohdakapi kin nahonpi.

13 Unkan wanna ayaśtanpi, hehan Jakob ayupte ça heya; Wicaśta hunkawanjin ciyapi, miye namahon po.

14 Otokahe ekta token Wakantanka Ikcewicaśta kin etanhan oyate wanji, iye caje kin on, iwicacu kta e ekta wicai qon he Simon oyaka.

15 Qa lie kici wicaśta wokcan oiepi qon okonwanjidan, kaken wowapi en kagapi ;

16 He iyohakam mihdamna, qa Dawid tawakeya jujuwabe cin he piya wakage kta; qa mdecahe cin hena ake wakage ca ecen he wakiye kta.

$17 \mathrm{Qa}$ hecen wicaśta okaptapi kin, Ikcewicaśta owasin ko, tona micaje icajewicayatapi kin hena Jehowa akitapi kta ce, Itancan kin hena owasin econ kin he heya ce.

18 Otokaheya tanhan Wakantanka taku icaliye cin owasin sdonkiya.

19 Heon etanhan miś kaken imdukcan, Ikcewicaśta kin tona Wakantanka ekta ihduhomnipi kin hena nagiyewicunyanpi kte śni.

20 Tuka wakagapi on aśapapi kin, qa wiinahmanpi kin, qa we, qa taku weye śni yuțapi kin, hena itehan yeiciyapi kta e wowapi wicunkicagapi kta.

21 Wanakaja tanhan tona Moses oyakapi kin hena otonwe otoiyohi kin en yukanpi, qa anpetu okilipapi eca, omniciye tipi kin ohna he yawapi ece, eya.

22 Hehan iś iyepi kin etanhan 210 wicaśta kahinigapi kin heca, Jndas Barsabas eciyapi, qa Silas, hunkawanjin kiciyapi kin en wicaśta okinihan, hena Palos qa Barnabas kici, Antiyoka ekta ye wicaśipi kta e, yewicaśipi kin, hunka wicayapi kin, okodakiciye kin owasin ko iyokipipi.

23 Qa heciya ayapi kta e kaken wowapi kagapi ; Yewicaśipi kin, qa hunka wicayapi, hunkawanjin kiciyapi kin hena ko, hunkawanjin kiciyapi. Jkcewicaśta etanhan, Antiyoka, qa Suria, qa Kilikia en yakonpi kin, Wookiye yukan po.

24 Unkiyepi etanhan wanjikji iyayapi, qa wicoie on nagiyeniyanpi, qa ninagipi kin nipahohopi qa, Baniçilidayapi, qa wicoope kin oyapapi lkta ce, eniciyapi ce, naunhonpi; unkiye tohinni heye wicunśipi śni tuka:

25 Heon etanhan tawacin wanjidan hduha unwitayapi qehan, wicaśta kahnigapi kin heca e niyepi kin ekta ye wicunśipi kta e iyounkipipi, Barnabas qa Palos waśtewicunkidakapi kin hena om :

26 Hena wicaśta kin wiconi elipekiyapi seca, Jesus Messiya Itancan unkiyapi kin heon etanhan.

27 Hecen Judas qa Silas ye wicunśipi, hena e wicoie on atayedan hecen oniciyakapi kta.

28 Woniya Wakan kici hecen iyounkipipi, waqinpi tokeca unkanihnakapi kte śni, tuka dena taku owotanna kin hecehnana;

29 Taku wakagapi wakiyuśnapi, qa we, qa taku we ye śni yutapi, qa wiinalimanpi kin hena itehan yeniciyapi kta. Hena itehanyan yaunpi kinhan tanyan ecanonpi kta. Tanyan yaunpi nunwe.

30 Hecen hena ye wicasipi qe- 
han, Antiyoka ekta ipi, qa oyate kin kawitaya iheyapi, hehan wowapi kin he wicaqupi.

31 He yawapi, unkan wahokonwicakiyapi kin on nina iyuśkinpi.

32 Unkan Judas qa Silas wicaśta wokcan kin hecapi, heon hunkawanjin kiciyapi kin oie ota on wahokonwicakiyapi, qa wicayasutapi.

33 Wanna anpetu ota en yukanpi, hehan hunkawanjin kiciyapi kin hena yewicaśipi kin ekta wookiye hde wicaśipi.

34 Tuka Silas en un kta e iyokipi.

35 Palos qa Barnabas Antiyoka en unpi, wicaśta tokeca ota ko, oyate kin waonspewicakiyapi, qa Itancan oie kin oyakapi.

36 Unkan iyohakam anpetu tonana, hehan Palos Barnabas heciya, Tukten Itancan oie kin unkoyake ciqon, ake otonwe otoiyohi ekta unye ca, hunkawanjin wicunye cin wanwicunhdake ca, token yakonpi kin sdonunye kta ce, eya.

37 Unkan Barnabas he iś, Johannes Markos eciyapi, yuha yapi kta cin.

38 Tuka he Pampulia ekta elipewicaye ca wicohtani kin en owicape śni qon, heon etanhan Palos he yuha yapi kte cin iyokipi śni.

39) Unkan he on hdokinicapi qa hecen otoiyohi ehpekiciyapi : unkan Barnabas Markos yuha Kupros elkta watom iyaya.

40 Tuka Palos Silas kalinige ca, Wakantanka towaonśida kin kici un kta e hunkawanjinkiciyapi kin icekiyapi qehan, hehan iyaya ;

41 Qa Suria qa Kilikia opta iyaye ça, okodakiciye kin suta wicakaga.
WICOWOYAKE 16.

1 Hehan Derbe qa Lustra ekta iyohi ; unkan ilıo, waonspekiyapi kin wanji hen un, Timotheos eciyapi, Juda winohinca wicake cin he cinhintku, tuka atkuku kin e Helenes oyate kin etanhan.

2 Hunkawanjin kiciyapi Lustra, qa Ikonium en yukanpi kin hena, he waśte e oyakapi.

3 Hetanhan Palos kici ya cin, qa Juda wicaśta hen unpi kin hena on, he icu qa bakicilidaya; atkuku kin Helenes heca e owasin sdonyapi.

4 Unkan otonwe iyaza yapi, qa yewicaśipi kin, kunka wicayapi ko, Jerusalem en yukanpi kin woahope kagapi qon hena tanyan yuhe wicaśipi.

5 Hecen okodakiciye kin wacinyanpi kin en wicayusutapi, qa anpetu otoiyohi nina yuota aya.

6 Unkan Phrugia qa Galatia makoce kin opta iyayapi, qa Asia en wicoie kin oyakapi kte sin Woniya Wakan kin tehinda qehan ;

7 Hehan Musia ekta ipi, qa hetanhan Bithunia ekta ye wacinpi, tuka Woniya kin iyowicaki śni.

8 Unkan Musia opta iyayapi qehan, Troas en iyohipi.

9 Unkan lianyen Palos wowanyake wan wanyaka; Wicaśta wan Makedonia etanhan taninyan inajin, qa cekiýe ca, $\mathrm{Ma}$ kedonia en $u$, qa ounkiya po, eya.

10 Wowanyake kin de wanyake cin heon hinnakaha Makedonia heciya unyanpi kta e uncinpi, Itancan kin hena wotanin waśte kin owicunkiyakapi kta cin heon unkicopi e unhdukcanpi. 


\section{OHANYANPI QON.}

11 Hecen Troas etanhan pacanan iyeunyanpi, qa Samotrake ekta owotanna unyanpi, qa hetanhan ihanhanna Neapolis en unkipi.

12 Qa hetanhan Philippi en unkipi, otonwe teca qa Makedonia makoce onśpa kin he en otonwe tokapa kin hee. Qa anpetu tonana otonwe kin he en unkanpi.

13 Unkan anpetu wakan hehan, otonwe kin etanhan inaunpapi, qa wakpa wan icahda wocekiye eyapi ece qon he en unkipi; hen unkiyotankapi, qa winolinca ece en mniciyapi kin hena owicunkiyapi.

14. Unkan winohinca wan, Ludia eciyapi, Thuatira otonwe kin etanhan, wokoyake duta ece wiyopeye cin heca, Wakantanka kokipeyahan un he nation yanka: unkan cantc kin Itancan kin yuzamni, on Palos oie kin anagoptan.

15 Unkan tiyohnaka kin om baptem qupi qehan, hehan ceunkiyapi qa, Itancan kin on wicawaka kecanmayakinpi hecinhan, wati kin en tin upi, qa ohna yukan pe, eye ca ecen econ unkiyapi.

16 Hetanhan wocekiye eya unyanpi eca, wicinyanna wan itkounkipapi ece, he taku wakan woyaka ece kin yuhe ca, wakanyan wohdag un kin heon tawayapi kin taku ota wicakicamna.

$17 \mathrm{He}$ Palos unkiyepi ko unkihakam $\mathrm{u}$, qa; Wicaśta kin dena Wakantanka tehan wankantuya un kin taokiye wicaye cin, wiconi canku kin unkokiyakapi kin hena eepi ce, eya hoyekiya.

$18 \mathrm{Qa}$ anpetu ota hecen econ. Tuka Palos nagiyeye cin heon hakikta qa, Jesus Messiya caje on winohinca kin de etanhan 212 tankata inanpe ciśi, taku wakan kin eciya. Unkan wihiyayedan oape kin he en tankan hiyu.

19 Unkan winolinea kin de yuhapi kin heepi, taku kamnanpi kte cin he akitakunipi śni kin he wanyakapi e etanhan, Palas Silas kici wicayuzapi, qa tipi hocoka kin ekta wicaśta tokahe wicakiyapi kin en awicayapi :

20 Qa wicaśta itancan wicakiyapi kin en wicakaipi, qa heyapi, Wicaśta kin dena Juda wicaśta kin hecapi, otonwe unkitawapi kin nina nagiyeyapi ;

21 Qa wicohan onspewicakiyapi kin hena e, Roma wicaśta heuncapi kin, unkicupi qa unkopapi kta e iyecece śni.

22 Unkan oyate kin owasin witaya takpe wicaupi; qa wicaśta itancan wicakiyapi kin wokoyake awicayupotapi, qa wicakapsinpsinte wicaśipi.

23 Unkan ota awicapapi, hehan wicakaśka tipi kin cn iyewicayapi, qa tipi awanyake cin sutaya wicayuhe śipi.

24 He hecen econ śipi, unkan wicakaśka tipi temahentuya kin he en onawicataka, qa siha kin can on kaśkapi ece kin en sutaya kaśka wicahnaka.

25 Unkan hanye cokaya hehan, Palos Silas kici Wakantanka cekiyapi, qa yatan dowanpi ; unkan wicaśta wicakaśkapi kin he nahonpi.

26 Unkan ihnuhanna maka kin nina huhuzahan, qa tipi ahe cin hena yuhuhuzapi, qa tiyopa lin owasin kazamni iyaye ça, on pawicalitapi kin owasin mdecahan hinhda.

27 Hececa, unkan wicakaśka tipi awanyag kiyapi kin kikta, qa tiyopa kin yuzamni han wanyaka, unkan wicaśta wicakaśkapi kin owasin kihdapi kecin, he- 
on mazasagye ehdaku ga on icikte kta ;

28 Tuka Palos nina hoyekiye ca, Kiuniiçiye śni wo, owasin den unkanpi ce, eya.

29 Hehan petijanjan wan da, qa tin iyaye ca, cancan Palos Silas kici siha hdepi kin en elipeiciya;

30 Qa tankan awicaye ca, Itancanpi wani kta e token ecamon kta he, ewicakiya.

31 Unkan, Jesus Messiya Itancan kin wacinyan wo, hecen nicinca om yani kta ce, eciyapi.

32 Qa Itancan oie kin okiyakapi, tona ti kin en yukanpi kin hena owasin koya.

33 Unkan lianyetu kin wihiyayedan oape kin he en, iwicacu, qa oape kin wicakiyujaja, qa hehan baptem qupi, tiyohnaka owasin ko.

34 Qa iye ti kin en awicaki qa won wicaya; qa tiyohnaka kin om wicada qa iyuśkin.

35. Unkan wanna anpa qehan, itancan wicakiyapi kin akicita eyanpaha kin ekta ye wicaśipi, qa, Wicaśta kin hena wicakiyuśka wo, eyapi.

36 Unkan wicakaśka tipi awanyake cin, hena oie kin Palos okiyake ca, Itancan wicakiyapi kin niciyuśkapi kta keya hośi hipi, heon etanhan tankan kinanpapi, qa wookiye hda po, eya.

37 Tuka Palos hewicakiya, Roma wicaśta heuncapi, tuka unyacopi śni ecen taninyan unkapsinpsintapi, qa onatag eunhnakapi, qa hinnakaha anahbeya hde unśipi he : hiya, iye atayedan upi, qa tankan unkaupi kta, eya.

38 Hehan akicita eyanpaha kin itancan wicakiyapi kin dena owicakiyakapi, unkan Roma wicaśta kin hecapi e nahonpi kin heon inihanpi.

39 Qa iye atayedan ekta ipi, qa cewicakiyapi, qa tankan awicaupi, qa otonwe kin etanhan hdapi kta icewicakiyapi.

40 Unkan wicakaśka tipi kin etanhan tankata inanpapi,qa Ludia ti kin en ipi ; qa hunkawanjin kiciyapi lkin wanwicahdakapi, hehan wicakihnapi, qa etanhan tokan iyayapi.

\section{WICOWOYAKE 17.}

1 Unkan Amphipolis qa Apolonia opta yapi kin, hehan Thesalonike en ipi; hen Juda oyate omniciye tipi kin wanji yukan.

2 Unkan Palos token econ ece kin ake ecen econ, qa timahen i, qa anpetu okilipapi yamni, wowapi wakan kin eciyatanhan, oyate kin wowicakiyaka.

3 Ie ca Messiya te kte ca, wiconte kin etanhan najin kta $e$ hecetu e yaotanin, qa, Jesus ociciyakapi kin he Messiya kin ee ce, eya.

4 Unkan hunh wicadapi, qa Palos Silas kici owicapapi, Helenes wicaśta owotanna ota, qa winohinca tokapapi kin wanistinpidan śni.

5 Tuka Juda oyate wicadapi śni kin hena woliinyanpi, qa wopeton tipi hocoka kin etanhan wicaśta śica wanjikji iwicacupi, qa kiwitaya iheyapi, qa otonwe kin ocowasin oqoyeyapi, hehan Jason ti kin takpe ipi, qa oyate kin ekta tankan biju wicayapi kta e akitapi.

6 Qa iyewicayapi śni qehan, Jason hunkawanjin kiciyapi wanjikji om otonwe kin en wicaśta itancanpi kin ekta awicaipi, qa hotankakiya hotaninpi, Tona ma213 


\section{OḦANYANPI QON.}

ka kin yuahdapśinyan ehpeyapi qon hena den hipi :

7 Qa Jason hena tin u wicaśi. Unkan hena owasin Kesar toope kin opapi śni, qa, Wicaśtaya tapi tokeca wan Jesus hee ce, eya oyakapi ece, eyapi.

8 Unkan otonwe en wicaśta itancanpi kin oyate ko, hena nahonpi kin inihan wicayapi.

9 Unkan Jason qa unmapi kin taku iwakta wicayapi, hehan awicayuśtanpi.

10 Hehan hunkawanjin kiciyapi kin Palos Silas kici hanyen Berea ekta awicayapi. Hena ekta ipi, qa Juda oyate omniciye tipi yuhapi kin he en timahen yapi.

11 Hena iś Thesalonike oyate kin sam iyeya wacin ksapapi, wicole kin tawacin inahini on icupi, qa hena hecetu e sdonyapi kta, heon anpetu iyohi wowapi wakan kin en akitapi ece.

12 Heon etanhan wicota wicadapi, Helenes winohinca okinihan, qa nakun wicaśta ota.

13 Tuka Berea ekta Palos Wakantanka oie kin oyake cin he, Juda wicaśta Thesalonike en un kin hena sdonyapi qehan, ekta ipi, qa wicota kin wicayaśkiśkapi.

14. Unkan hehan hunkawanjinkiciyapi kin Palos miniwanca kin ekta ye kta se tokan ye śipi ; tuka Silas qa Timotheos hen owanji yukanpi.

15 Unkan tona Palos ayapi qon hena $\Lambda$ thenas elkta aipi ; qa Silas Timotheos kici kohanna en upi kta e iwahowicaye cin he hośi wicakahdapi.

16 Unkan hena Athenas ekta upi kta e Palos awicape yanke cin, he icunhan, otonwe kin wakagapi ojudan e wanyake ca on wacin iyokiśica hinhda.
17 Qa heon omniciye tipi kin ohna Juda oyate, qa tona owicapapi kin hena om wohdaka, qa nakun anpetu otoiyohi wopeton tipi hocoka kin ekta tona itkowicakipe cin hena om.

18 Unkan hehan Epikureos qa Stoikos oyate kin etanhan, wicaśta woksape cinpi wanjikji Palos itkokipapi qa, I wakan kin de token eye kta he, hunhi eyapi ; qa hunh iś, He taku wakan tokeca ehde sececa ce, eyapi; Jesus qa wiconte etanhan woekicetu kin hena owicakiyaka, heon etanhan.

19 He icupi, qa Areyos paha kin ekta aipi qa, Woonspe teca odake cin he taku kin sdonunyanpi kta uncinpi:

20 Taku wotanin tokeca noge on nahion unyayapi kin hena token kapi hecinhan, sdonunyanpi kta ce, eciyapi.

21 Athenas oyate, qa wicaśta tokeca en yukanpi kin owasin, taku tokeca econpi śni, taku nakaha otanin kin oyakapi, qa nahonpi kin heceyedan.

22 Hehan Palos Areyos paha kin cokaya inajin, qa heya ; Athenas wicaśta kin, taku wakan kin nina awacannipi e, taku owasin on imdukcan.

23 Naka taku wakan yadapi kin en awahimdamda, unkan iho, wahna wośnapi wan, Taku wakan sdonyapi śni un kin, akan owapi kin he iyewaye. He iyukcanśniyan ohoyadapi kin hee e ociciyakapi.

24 Taku Wakan kin tuwe maka taku ohnaka ko owasin kage ca, mahpiya maka ko Itancan kin hee, heon tipi wakan wicanape on kagapi kin ohna ti śni ;

25 Qa iye kin owasin wiconi, qa woniya, qa taku owasin ko wicaqu ece kin, he taku icakije 
cin iyecen, wicaśta nape kin on okiyapi śni ece.

$26 \mathrm{Qa}$ wicawe wanjidan on, wicaśta oyate hiyeye cin owasin, maka kin owancaya etipi kta e wicakaga; qa tohanhan nipi, qa tuktekten tipi kte cin hena otokahe tanhan wicakiciyuśtan.

27 Heon Itancan kin akitapi kta iyececa, okinni yutantan akitapi kinhan iyeyapi kta, otoiyohi unkitehanpi śni un tuka ce.

28 Iye atayedan on unnipi, qa unśkanśkanpi, qa en unyakonpi ece : enitanhanpi wicaśta odowan kagapi ece kin hena wanjikji heyapi qon, Unkiś eya he cinca unyakonpi ce.

29 Hecen Wakantanka cinca unyakonpi kin, mazaskazi, qa mazaska, qa inyan, wicaśta wayupike wacinksape bagopi kin, Taku Wakan kin iyacinpi kta iyececa unkecinpi kte śni.

$30 \mathrm{Qa}$ wookahnige śni omaka kin hena, Wakantanka ekta etonwe śni ; tuka wanna wicaśta owasin owancaya ihduecetu wicaśi.

$31 \mathrm{Qa}$ anpetu wan en wicaśta kin woowotanna on wicayaco kta e konza, wicaśta kalinige ciqon he on hecon kta, qa wiconte kin etanhan najin kiye cin heon he owasin sdonye wicaya ce, eya.

32 Unkan wicate cin piya icahyapi kin he nationpi qehan, hunh ihahapi ; qa apa iś, He on ake naunnihonpi kta ce, eyapi.

33 Hecen Palos wicacokaya tanhan tokan iyaya.

34 Tuka wicaśta wanjikji opapi, qa wicadapi; Dionusius Areyos paha omniciye kin en ope ciqon he wanji ee, qa winohinca wan Damaris eciyapi, qa nakun tok.tokecapi.

\section{WICOWOYAKE 18.}

1 Hena iyohakam Palos Athenas ayuśtan, qa Korinth ekta i :

2 Qa hen Juda wicaśta wan iyeya, Akula eciyapi, Pontos en tonpi, qa aśkatudan Italia etanhan hiyu, tawicu Priskila kici; Kladios Juda wicaśta kin owasin Roma ayuśtan wicaśi kin heon etanhan. Unkan hena en wicahi ;

$3 \mathrm{Qa}$ kici wicohtani okonwanjidan, heon om un, qa om hitani, wakeya kagapi kin hecapi.

$4 \mathrm{Qa}$ anpetu wakan eca omniciye tipi kin ohna wowicakiyaka ece, qa Juda oyate Helenes ko cinwicakiya.

5 Unkan Silas qa Timotheos Makedonia etanhan hiyupi qa en hipi qehan, Palos nagi kin ekta iyopaśtag içiye ça, Jesus he Messiya kin ee ce, Juda oyate kin owicakiyaka.

6 Unkan hena e wakipajinpi, qa śicaya iapi kin, hehan Palos wokoyake aihdatata, qa hewicakiya, Niwe kin nipapi kin akan niunpi nunwe, miye mabapica śni waun, detanhan Ikcewicaśta kin ekta mde kta ce.

7 Unkan hetanhan tankan ye ca, wicaśta wan Wakantanka ohoda, Justos eciyapi he ti kin omniciye tipi kin ikiyadan han; unkan tipi kin he en tin iyaya.

8 Unkan Krispos, omniciye tipi kin en itancan kin, he Itancan kin wacinyan, tiyohnaka owasin om; qa Korinth oyate kin wicota nahonpi qehan wicadapi, unkan baptem wicaqupi.

9 Unkan hehan hanyen wowihamde en, Itancan kin Palos heciya, Ihnuhan taku koyakipe cin, tuka nina ie ça, inina yanke śni wo.

10 Miye cicica, qa tuwedan 215 


\section{OHANYANPI QON.}

kiuniniye kta e iyanilipaye kte śni ; otonwe kin de ohna oyate ota wicamduha ce, eya.

11 Unkan hen wi ake śahdogan hehanyan iyotanke ca, Wakantanka oie kin onspewicakiya.

12 Unkan Galiyo Akeya makoce kin en itancan un kin, hehan Juda oyate witaya Palos takpe ipi, qa woyaco oiyotanke kin en ahipi ;

13 Qa, Woahope kin eciyatanhan śni Wakantanka cekiyapi kta e wicaśta kin onspewicakiye cin he dee ce, eyapi.

14 Unkan Palos i hdukawa kta, hehan Galiyo Juda oyate kin hewicakiya; Juda wicaśta kin, de taku śica econpi, qa iś wicaśtaśniyan olianyanpi kin hee unkanś, nacilionpi kta iyececa tuka :

15 Tuka wicoie, qa wicacaje, qa woope nitawapi on wiwangapi kin heca hecinhan, niś ito ekta etonwan po; taku hececa on wimdukcan kte śni ce.

16 Hehan woyaco oiyotanke kin etanhan kaliam iyewicaya.

17 Unkan Helenes wicaśta kin owasin Sosthenes, omniciye tipi kin en itancan kin he, icupi, qa woyaco oiyotanke kin itokam apapi. Tuka Galiyo hena taku kin ekta ewacin śni yanka.

18 Unkan he iyohakam Palos anpetu ota hen yanke cehan, hunkawanjin kiciyapi kin awicayuśtan, qa Suria ektakiya watom iyaya, Priskila Akula kici opapi. Kenkreya ekta paha kin bakiśda, hecen içiconza kin heon.

19 Hehan Ephesos ekta iyohi, qa hen elipewicaya; tuka iye kin omniciye tipi kin en tin iyaye ca, Juda oyate kin om wohdaka.

20 Unkan en tehan owanji yanke kta e cinpi eśta wicada śni :
21 Tuka napewicayuze ca, Jerusalem ekta wośnapi u kte cin he en owape kta, tuka Wakantanka iyokipi kimhan, ake en cihdipi kta ce, eya. Hehan Ephesos etanhan watom iyaya.

22 Qa Kesareya en huta ekta ihunni, qa lien ihe cea okodakiciye kin napewicayuze cehan, Antiyoka ekta apamahde iyaya.

23 Qa anpetu ota hen un kin, hehan Galatia qa Phrugia makoce kin owancaya ye ça, waonspewicakiyapi kin owasin waśag wicakiya.

24 Unkan Juda wicaśta wan, Apolos eciyapi, Aleksandrea en tonpi, wicaśta wayapike ca wowapi wakan on waśaka, he Ephesos ekta hi.

25 Wicaśta kin he Itancan tacanku kin onspe, qa nagi kata, heon ninana ie ca, Itancan taku tawa kin onspewicakiya, Johannes baptem tawa kin hecedan sdonya tuka.

26 Hee omniciye tipi kin ohna waditagya ia; unkan Akula qa Priskila nalionpi qehan, he icupi, qa Wakantanka tacanku kin sanpa tanyan okiyakapi.

27 Unkan Alkeya ekta ye wacin, hehan hunkawanjin kiciyapi kin heciya wowapi wicakicagapi, qa he iyowinkiyapi kta e iwahokonwicakiyapi. He heciya $i$, qa tona wowaonśida kin on wicadapi qon hena nina owicakiya ;

28 Qa Juda oyate kin omniciye en nina awacin wicaye ca, wowapi wakan kin eciyatanhan Jesus he Messiya kin ee e yatanin.

\section{WICOWOYAKE 19.}

1 Unkan Apolos Korinth en un kin icunhan, Palos iheyata makoce kin opta ye ca, Ephesos 
ekta iyohi, qa hen waonspewicakiyapi wanjikji iyewicaya :

2 Qa hewicakiya, Wicayadapi ehantanhan Woniya Wakan kin iyacupi he. Unkan iś, Woniya Wakan yukan e nahahin naunhonpi śni ce, eyapi.

3 Unkan, Taku on baptem nicupi he, ewicalkiya. Unkan, Johannes baptem tawa kin hee ce, eyapi.

4 Unkan Palos, Johannes ihduecetupi kte cin on baptern wicaqu, qa oyate kin tuwe u kte cin he wicadapi kta, kewicakiya, he Jesus Messiya kin ee ce, eya.

5 He nahonpi, unkan Jesus Itancan caje kin on baptem wicaqupi.

6 Hehan Palos nape on awicaputake ca, Woniya Wakan en wicahi, unkan ceji tokeca on iapi qa wokcanpi.

7 Hena owasin wica ece wikcemna ake nonpa.

8 Unkan omniciye tipi kin tin iyaye ça, wi yamni waditagya ie ca wohdaka, qa Wakantanka tokiconze taku tawa kin onspewicakiya.

9 Tuka wanjikji suta içicagapi qa wicadapi śni kin hena, wicota kin en, canku kin de on sicaya eyapi qehan, hena awicayuśtan, qa waonspewicakiyapi kin aiyog iyewicaye ca, anpetu otoiyohi, tuwe Turannos eciyapi, he oyawa tipi yuhe cin ohna woyaka ece.

10 Waniyetu nonpa hehanyan hecen econ, unkan tona Asia en yakonpi kin hena owasin Jesus Itancan oie kin nahonpi, Juda Helenes wicaśta ko.

11 Unkan Palos nape kin on Wakantanka wowapetokeca wakan kin econ ece.

12 Hecen iye tancan kin etan- han, napipakinte qa makuakahpe kin wayazanpi kin en akipi; unkan wowayazan kin ayuśtan iyaye ca, taku wakan śicapi kin tankan inanpapi.

13 Unkan wanjikji, Juda wicaśta wapiyapi kin heca, tona taku wakan śica yuhapi kin hena en, Jesus Itancan kin cajeyata utapi, qa; Jesus Palos oyalke cin he cajeyan naunlion po, eyapi.

14 Qa Juda wicaśta wośna kagapi wanji, Skewa eciyapi, he cinhintku śakowin kin hena heconpi.

15 Tuka woniya śice cin he waayupte ca, Jesus sdonwaye ca, Palos nakun sdonwaya, tuka niś nituwepi he, eya.

16 Unkan wicaśta taku wakan sica yuhe cin he iyawicahpaye ca, ohiwicaya, hecen tancodan kiunniyanpi ecen tipi kin etanhan tankan najicapi.

17 Unkan tona Ephesos en yukanpi kin, Juda qa Helenes oyate kin owasin he sdonyapi ; qa wokokipe owasin awicahinhpaya, unkan Jesus Itancan caje kin yatanpi.

18 Unkan tona wicadapi kin etanhan wicota en ahi, qa wohdakapi, wicolian qon ohdakapi;

19 Qa tona wapiyapi econpi kin hena wicota wowapi tawapi kin en ahipi, qa owasin wicitokam huhnahyapi ; hena iyopeyapi kin hdawapi, qa mazaska kektopawinge wikcemna zaptan iyawapi.

20 Hecen Itancan oie kin nina icage ça ohiya.

21 Unkan hena taku kin wanna enakiyapi, hehan Palos woniya eciyatanhan konza, Makedonia qa Akeya opta ye cinhan, hehan Jerusalem ekta ye kta, qa, Heciya wai kinhan hehan Roma wanmdake kta ce, eya. 


\section{OHANYANPI QON.}

$22 \mathrm{Qa}$ ookiye wicayuhe cin nom, Timotheos qa Erastos, hena Makedonia ekta ye wicaśi, tuka iye anpetu tonana Asia en owanjidan yanka.

23 Unkan he icunhan canku kin de on nina owodutaton.

24 'Tuwe Demetrios eciyapi, mazaska kage cin heca, he mazaska kokadan Diana kicaga ece, qa tona woecon kin he econpi kin hena taku ota wicakicamna.

25 Hena eepi, qa tona opeya htanipi kin hena ko wicakico qa hewicakiya, Wicaśta kin, wicohitani kin de on woyuha untonpi e sdonyakiyapi.

26 Qa nakun Ephesos ecedan śni, tuka Asia owancaya, $\mathrm{Pa}$ los kin de oyate ota wahokonwicakiye ça, wicayuhomni qa, Taku wakan nape on kagapi kin hena wakan śni ce, eya owicakiyaka e sdonyayapi qa nayahonpi.

27 Qa hecen taku on waunkamnanpi ece kin he wahtedapi kte śni seca, qa hecedan śni, llakun Diana taku wakan tanka tipi wakan tawa kin takudapi lkte śni, qa wowitan hduhe cin yutakuni kte śni, he qe Asia owancaya, qa oyate kin iyuhpa ohodapi tuka ce, eya.

28 Unkan hena nahonpi qehan, nina canteptanyanpi qa, Diana Ephesos oyate tawapi kin he tanka ce, eyaya hotaninpi.

29 Unkan otonwe kin owancaya nina owodutaton; qa Gayos qa Aristarkos kici, Makedonia wicaśta kin, Palos kici hipi qon hena iwicacupi, qa tawacin wanjidan owanyake tipi kin en inyang yapi.

30 Unkan Palos oyate kin en wicai lita, tuka waonspewicakiyapi kin.ıyokipi śni :

218
31 Qa Asia en wicaśta tokapapi wanjikji, iye takodawicaye cin hena wahoyapi, qa owanyake tipi kin timahen iyohpaye kte śni e icekiyapi.

32 Heon hunh taku eya hotaninpi, qa hunh iś togye eyapi ; omniciye kin nina oqoyapi; qa tokeca e on mniciyapi kin ota sdonyapi śni.

33 Hehan Aleksander wicota kin etanhan ahiyupi, qa Juda wicaśta kin he cokata iyaye yapi. Unkan Aleksander namwicakicawin, qa oyate kin wowicakiyake kta.

34 Tuka he Juda wicaśta kin heca e sdonyapi, unkan wihiyayedan oape nonpa hehanyan, owasin ho wanjidan hotankakiya hotaninpi, Diana Epliesos oyate tawapi kin he tanka ce, eyaya yukanpi.

35 Unkan wowapi kage cin he oyate kin wicayuinina qehan, hecen eya, Ephesọ wicaśta kin, Ephesos otonwe kin de Diana taku wakan tanka he tipi wakan tawa kin awanyag un, qa wakagapi Jupiter etanhan hinhpaye ciqon he nakun, he tuwe wicaśta kin he sdonye śni he.

36 Dena taku kin cețunhdapica śni, heon inina dukanpi, qa takudan inahniyan ecanonpi kte śni tuka.

37 Wicaśta kin dena tipi wakan kin etanhan takudan manonpi śni, qa taku wakan nitawapi kin yaśicapi śni, tuka den awicayahipi.

38 Demetrios qa tona om hitani kin hena tuwe en taku aupi cinpi kinhan, woyaco anpetu yukan, qa nakun wicaśta wayacopi kin ; otoiyohi wiyakicionpapi kta.

39 Qa niś taku tokeca on winungapi hecinhan, omniciye wi- 
coope kin eciyatanhan un kin he en yacopi kta.

40 Anpetu kin de owodutaton kin on wiunwangapi kta naceca ; omniciye kin de on etanhan taku unkeyapi kte cin takudan unhapi śni ce, eya.

41 Unkan hena hecen eye cehan, hehan omniciye kin hide wicaśi.

\section{WICOWOYAKE 20.}

1 Owodutaton kin he henakeca, hehan Palos waonspewicakiyapi kin wicakico, qa i iwicaputaka, qa Makedonia ekta ye kta e hetanhan iyaya.

2 Unkan makoce kin hena opta iyaye ca, oie ota on iwaliolronwicakiye cehan, Helenes makoce kin en iyohi :

3 Qa wi yamni hen owanji yanka. Unkan Suria ekta watom ye kta, hehan Juda wicaśta kin nahmana iyapepi kin heon Makedonia opta icicawin hde kta e iciconza.

4 Unkan Sopater Berea etanhan, qa Thesalonike etanhan Aristarkos Sekundos kici, qa Gayos Derbe etanhan, qa Timotheos, qa Asia wicaśta, Tucikos qa Trophimos, hena Ásia ekta Palos kici yapi.

5 Hena e tokata yapi, qa Troas en unkapepi.

6 Unkan aguyapi on napohyapi codan anpetu kin iyohakam, Philippi etanhan watounpapi, qa anpetu zaptan hehan Troas en wicunkihdegapi, qa hen anpetu śakowin unkokilipapi.

7 Unkan anpetu tokaheya kin he en waonspewicakiyapi kin aguyapi kiyuśpa imniciyapi, hehan Palos wowicakiyaka, hanhanna hetanhan hde kta, hecen hanye cokaya hehanyan wahokonwicakiya yanka.
8 Unkan wankan tipi ohna mniciya yulkanpi kin he en petijanjan ota hdepi.

9 Unkan kośka wan, Yutikos eciyapi kin he owanyeye kin en iyotankehan yanke ça, iśtinbe finca: unkan Palos tehan woyake cin icunhan, iśtinma iwanke ca, owanka iciyamni kin hetanhan hinhpaye ça ța ecen eyakrupi.

10 Hehan Palos kun ye ca akan elpeiciye ca, poskin yuze ca, Ihnuhan yaceyapi kin, taniya kin nahahin mahen un ce, eya.

11 Qa ake wankan ye ca, aguyapi kiyuśpe ca, yute cạ, tehan ia yanke cin, anpa kamdeze cin hehanyan, hehan hetanhan kihda.

12 Unkan kośka qon ni ikikcupi qa nina pidapi.

13 Unkan unkiye tokaheya wita wata kin ekta unyanpi, qa Așos ekta watom unyanpi, heciyatanhan Palos en ope unśipi kta unkecinpi ; iye qe hehanyan mani cin, qa heon hecon unśipi.

14 Unkan Asos ekta eunkihdegapi qehan, hehan en ope unkiyapi, qa Mitulene en unhipi.

15 Unkan hetanhan iy oopta watom unyanpi, qa ihanlianna qehan Kios en iyotakons unyanpi ; qa ake ihanlianna qehan Samos iyounhipi, qa "Trogulios en owanji unkanpi; qa ake ihanlianna qehan Miletos ekta unkipi.

16 Palos Ephesos en icunom watom iyaye wacin, Asia en okilipe kte śni, qa okihi kinhan anpetu iwikcemna zaptan kin he en Jerusalem en un kta cin, qa heon inahini.

17 Unkan Miletos etanhan ye wicaśi, qa Ephesos en okodakiciye hunkawicayapi kin wicakico. 


\section{OHANYANPI QON.}

18 Unkan hena en hipi, hehan hewicakiya; Asia en wahi qon anpetu tokaheya hehantanhan, token ohinniyan cicipi waun kin sdonyayapi.

19 Tawacin ocowasin wahbayedan waun, Itancan kin ohowada, iśtamnihanpe ota, qa taku wawiyutanyan un kin, Juda oyate nahmana iyamapepi kin on mahiyahde kin hena koya.

$20 \mathrm{Qa}$ taku on tanyan yaunpi kte cin takudan ipicidapi śni, tuka omniciye kin en, qa tipi iyaza wociciyakapi qa waonspeciciyapi ece.

21. Wakantanka ekta ihduecetupi, qa Jesus Messiya Itancan unkiyapi wacinyanpi kin hena, Juda Helenes oyate ko en mdaotanin ece.

22 Unkan nakaha iho, Woniya kin on makaśkapi ecen Jerusalem ekta mda, qa heciya taku mahiyahde kte cin sdonwakiye śni.

23 Tuka otonwe otoiyohi en Woniya Wakan mayaotanin qa, On kaśkapi qa wokakije miciun, keya ce.

24. Tuka hena takuwada śni, qa wiconi mitawa kin tehike wakida śni, iyuśkinyan ihunniyan waimnanke kta, qa wicohan Jesus Itancan maqu kin, Wakantanka towaonśida wotanin waśte omdake kte cin hee tanyan mduśtan kinhan.

25 Qa nakaha iho, niyepi kin Wakantanka tokiconze kin ociciyag ciipi qon owasin ake miite kin wanmayadakapi kte śni e sdonwakiya.

26 Heon anpetu kin de waayatanin ciciyapi, wicaśta owasin we kin on aśape śni waun.

27 Wakantanka tawacin kin ocowasin ociciyakapi qa takudan anawalibe śni.
28 Heon tanyan piiciyapi, qa optaye owasin en, Woniya Wakan waawanhdake nicagapi kin hena koya, qa Wakantanka taokodakiciye kin, iye we kin on opekiton qon he awanhdaka po. 29 Miye indamde cinhan, hehan sunktokeca ocin śicapi kin niyepi en niipi kta, qa tahinca kin itonwicapapi kte śni he sdonwaya.

30 Qa niyepi enitanhanpi wicaśta icagapi kta, qa iś iyepi ihakam waonspewicakiyapi kin u wicakiyapi kta e nina yaśkiśkapi kta.

31 Heon kiktahan un po, qa waniyetu yamni, anpetu hanyetu ko, iśtamnihanpe amau, qa otoiyohi wahokonciciyapi amdaśtan śni, he kiksuya po.

32 Hunkawanjinciyapi, nakaha Wakantanka iś iye, qa towaonsida oie kin en aciupi; he icahniyanpi, qa tona ska wicakagapi kin owasin om taku yuhe niciyapi kta e okihi.

33 Tuwe mazaska, qa mazaskazi, qa wokoyake tawa kin wakon śni.

34 Han, minape kin dena on taku wacin kin mihdamna, qa tona om waun kin hena. wicawecamna qon he niye sdonyayapi.

35 Hena owasin en waonspeciciyapi, hecen litayanipi, qa tona waśake śni un kin owicayakiyapi kta, qa Jesus Itancan oie qon yeksuyapi kta, Wicaqupi kin hee wowaśte, qa icupi kin hee aoptetu, eye ciqon he.

36 Hena hecen eya, unkan hehan canpeśka makehde inajin, qa owasin om cekiya.

37 Unkan owasin nina ceyapi, qa Palos poskin yuzapi, qa i iputakapi.

38 Miite kin ake tohinni wanmayadakapi kte śni eye ciquon, oie 
kin de on iyotan nina iyokiśicapi. Unkan wita wata kin ekta kici yapi.

\section{WICOWOYAKE 21.}

1 Unkan hena ehpewicunyanpi, qa pacanan iyeunyanpi, hehan Kos ekta owotanna unkupi, qa ihantianna hehan Rodos ekta, qa hetanhan Patara en iyounhipi.

2 Qa hen wita wata wan Phenike ekta watom ye kte cin iyeunyanpi, qa he en unkopapi, qa hetanhan unyanpi.

3 Qa Kupros taninyan wanunyakapi, hehan catka tanhan elipeya unhiyupi, qa Suria ekta watom unkupi, qa Turos en unhdihunipi, hen wata taku ohnaka kin wahetajupi kta.

4 Unkan waonspewicakiyapi kin iyewicunyanpi, qa hell anpetu śakowin unkokihpapi; hena e qe'Woniya Wakan eciyatanhan Palos Jerusalem ekta ye kte śni, keciyapi.

5 Tuka anpetu kin hena wanna unkihunnipi, hehan hetanhan inaunpapi, qa hena iśs tawicupi śiceca ko owasin opapi, otonwe itankata hehanyan, qa huta kin ekta canpeśka makehde inaunjinpi, qa ptaya ceunkiyapi.

6 Unkan hehan nape unkiciyuzapi, qa unkiś wata kin en unkopapi, tuka iyepi kin ekta hdapi.

7 Unkan Turos etanhan watom unkupi kin he ihunni unkiyapi, hehan Ptolemais ekta unhipi; qa hunkawanjin kiciyapi kin nape wicunyuzapi, qa anpetu wanjidan oin unkanpi.

8 Unkan ihanhanna qehan, tona Palos kici unyakonpi kin, hetanhan inaunpapi, qa Kesarea en iyounhipi ; qa Philip wotanin waśte oyake cin he ti kin en unyanpi, śakowinpi qon he wanji ee, qa lie kici unyakonpi.
9 Unkan he cunwintku topa witanśna unpi, hena wokcanpi ece.

10 Unkan hen ampetu ota owanji unkanpi kin icunhan, wicaśta wokcan wan Agabos eciyapi, Juda makoce kin hetanhan apamahde hi.

$11 \mathrm{Qa}$ en unhiyupi qehan, $\mathrm{Pa}$ los ipiyaka tawa kin icu, qa on siha nape ko iyaihdaśke ca, Woniya Wakan kin heya ce, Wicaśta tuwe ipiyaka kin de tawa hecinhan, he Juda wicaśta, Jerusalem ekta, hecen kaśkapi kta, qa Ikcewicaśta kin nape kin en wicaqupi kta ce, eya.

12 Unkan he naunhonpi qehan, tona en tipi kin hena om, ceunkiyapi, Jerusalem ekta ye kte śni. 13 Tuka Palos, Token ecanonpi qa yaceyapi, qa hecen cante mayadutakunipi śni he, Jesus Itancan caje kin on Jerusalem ekta makaśkapi, qa nakun mațe kta e wiyeya manka ce, eya.

14. Unkan wicada śni qehan, unkayaśtanpi qa, Itancan tawacin kin ecen econpi nunwe, unkeyapi.

15 Unkan anpetu kin hena iyohakam owasin piunkiçiyapi, qa Jerusalem ekta itawankanhde unyanpi.

16 Nakun Kesarea etanhan waonspewicakiyapi kin wanjikji unkopapi, qa wanji Mnason eciyapi kin he aupi, he Kupros etanhan, wanakaja waonspekiyapi kin heca, he ti kin en unyakonpi kta.

17 Unkan Jerusalem ekta iyounhipi, hehan hunkawanjinkiciyapi kin iyuśkinyan tin unkicopi.

18 Unkan ihanhanna kin hehan, Palos kici Jakob ekta unyanpi; unkan hunkawicayapi kin owasin en yukanpi. 


\section{OHANYANPI QON.}

19 Hena nape wicayuze ca, kan kin tin iyaya; taku qupi hehan Ikcewicaśta kin ekta iye woyake cin on token Wakantanka econ qon owasin ecekcen owicakiyaka.

20 Unkan hena nahonpi qehan, Itancan kin idowanpi qa Palos hcciyapi, Cinye, Juda rvicaśta kektopawinge ota wicadapi, qa owasin wicoope kin sutaya awakicinpi kin he wandaka.

21 Qa niye, Juda oyate Ikcewicaśta ehna yakonpi kin hena owasin Moses toope kin ahduśtanpi kta e onspewicayakiye ca, cinca bawicakicilidayapi kte śni, qa wicohan kin hena eciyatanhan manipi kte śni, kcha keyapi e nalionpi.

22 Heon toketu kta he. Awicakehan wicota kin kawitaya iheyapi kte. Wanna yahi kin he nahonpi.

23 Hecen token unkeniciyapi kin de ecen econ wo. Wicaśta tom token ic̣iconzapi kin hena om unyakonpi.

24 Hena owicape ca, om ihduska, qa mazaska on owicakiya wo, hecen pahi kin hduśdapi kta. Unkan hecen token oniyakapi nahonpi qon hena taku śni, qa niś eya owotanna mayani, qa wicoope kin opeya yaun kin he owasin sdonyapi kta.

25 'Tuka Ikcewicaśta, tona wicadapi kin hena wowapi wicunkicagapi, qa wicohan kin dena takudan opapi kte śni unkeyapi qon, tuka taku wakagapi wakiyuśnapi, qa we, qa taku we ye śni yuțapi kin, qa wiinahmanpi kin hena en ihdoipi kte śni ce, hecedan unkeyapi.

26 Hehan Palos wicaśta kin hena owicape ca, ihanhanna kin hehan om ihduska, qa ihduskapi anpetu kin wanna henakeca kte cin he yuotanin kta e tipi wawan otoiyohi on wośnapi kta hehantu.

27 Unkan anpetu sakowin qon wanna henakeca kte hehan Ju. da wicaśta, Asia etanhan upi qon hena tipi wakan kin en un wanyakapi qehan, oyate kin owasin iyowicapaśtakapi qa $P a$ los iyahpayapi ;

$28 \mathrm{Qa}$ hotaninpi, Israel wicaśta kin, ounkiya po ; wicaśta kin de oyate kin en, qa wicoope kin, qa nakun tipi kin de aie ca, owancaya hecen onspewicaliiya; qa nakun Helenes wicaśta kin tipi walkan kin en awicahi, qa hecen tipi iyotan waśte lin de aśamya ce, eyapi.

$29 \mathrm{He}$ itokam Trophimos, Ephesos wicaśta kin heca, e otonwe kin en kici un qon he wanyakapi, unkan he Palos tipi wakan kin en ahi, kecinpi.

30 Unkan otonwe kin ocowasin nina oqoyapi, oyate kin kawitaya iheyapi, qa Palos icupi, qa tipi wakan kin etauhan tankan yusdohan ayapi, unkan ecahankeya tiyopa kin ecen iyeyapi.

31. Wanna ktepi kta e akitapi, hehan akicita tancan wicobe yuhe cin he, Jerusalem owancaya owodutaton ce, eya okiyakapi kin he nation;

32 Qa kohanna akicita, akicita tancan ko iwicacu, qa inyang ye ca ekta wicai. Unkan akicita tancan akicita ko wanwicayakapi qehan, Palos apapi kin ayuśtanpi.

33 Hehan akicita tancan kin en yc ca, icu, qa maza icicalilia nom on kaśke wicaśi; qa he tuwe kin, qa token econ kin he, eya iwicawanga.

34 Tuka oyate kin hunh taku eya hotaninpi, qa hunli iś togye eyapi. Unkan owodutaton kin 


\section{WICOWOYAKE 22.}

on wowicake kin sdonya okitpani, heon conkaśke kin mahen aye wicaśi.

35 Unkan can iyadipi kin en hdipi, hehan akicita kin yuha ayapi, oyate kin aoțins upi kin heon etanhan.

36 Oyate kin iyulipa ihakam yapi qa, Yutokan aya po, eya hotaninpi.

37 Unkan conkaśke kin mahen ayapi kta, hehan Palos akicita tancan kin heciya, Taku eciciye kta tuka ce, iyowinmayakiye kta he. Unkan iś, Helenes iapi kin onnispe he, eya.

38 Egupta wicaśta anpetu kin dena itokam icage ca, wicaśta wabotica kin hecapi kektopawinge topa ihakam hewoskan makoce kin ekta awicaye ciqon he niye śni he.

39 Unkan Palos, Juda wicaśta Kilikia en, Tarsos otonwe kin hetanhan waun, otonwe śice śni en imacaga. Ceciciya ce, oyate kin taku ewicawakiye kta, iyowinmakiya wo, eya.

40 Hecen iyowinkiya; unkan Palos can iyadipi kin akan inajin, qa oyate kin namwicakicawin. Unkan inina yukanpi qehan, Hebrew iapi kin on ie ca, hewicakiya;

\section{- WICOWOYAKE 22.}

1 Wicaśta hunkawanjinciyapi qa hunkakeciyapi kin, nakaha niyepi en wowahdake cin namahon po.

2 Unkan Hebrew iapi un wowicakiyake cin he nahonpi qehan, iyotan inina yukanpi.

3 Unkan hecen eya; Juda wicaśta kin hemaca, Kilikia ekta Tarsos en matonpi, qa otonwe kin de en icahmayanpi, qa Gamaliel siha kin ekta, hunkake wicayapi wicoope yuhapi kin tanyan onspemakiyapi, qa Wakantanka on nina inawahni, niyepi kin owasin nakaha yaunpi kin iyececa.

4 Qa canku kin de wiconte aiyahdeya śicaya wakuwa, wicawakaśke ca; wicakaśka tipi kin en iyewicawaya, wica winyan ko.

5 Hecen wośna kagapi itancar, qa hunka wicayapi kin owasin mayaotaninpi ece. Hena eepi hunkawanjinkiciyapi kin wowapi wicakicagapi qa maqupi qon, lsecen Damaskos ekta imdamde, heciya tona yukanpi kin hena wicakaśka Jerusalem ekta wicakaśtakapi kta e awicawau kta, heon etanhan.

6 Ohnihde waun, qa Damaskos wanna ikiyadan, unkan wiyotanhe cin hehan, iho, ihnuhanna mahpiya kin eciyatanhan iyoyanpa tanka wan aohdute mayan.

7 Hehan makata mahinhpaye ca, wicaho wan nawahon, Salos, Śalos, tokeca e śicaya mayakuwa he, emakiya.

8 Unkan miś amdupte ca, Itancan nituwe he, epa. Hehan iś, Jesus Nazareth etanhan śicaya yakuwa kin he miye ce, emakiya. 9 Unkan tona om waun kin ojanjan kin wanyakapi, qa nina inihanpi, tuka wicaho omakiye cin he nahonpi śni.

10 Hehan miś, Itancan token ecamon kta he, epa. Unkan Itancan kin, Najin, qa Damaskos ekta ya wo, heciya token ecanon kte cin owasin oniciyakapi kta ce, emakiya.

11 Unkan iyoyanpa wiyatpa kin on wawanmdake śni, hecen tona om waun kin hena minape kin yus amayanpi, qa Damaskos en wai. 


\section{OHANYANPI QON.}

12 Qa wicaśta wan, Ananias eciyapi, wicoope kin eciyatanhan owotanna, Juda oyate hen yakonpi kin owasin he oyakapi ;

$13 \mathrm{He}^{-}$en mahi, qa najin, qa hemakiya, Salos, misunka tonwan wo. Unkan wihiyayedan oape kin he en ekta ewatonwan.

14 Hehan iye heya; Hunkake wicunyanpi Wakantanka tawapi kin nicahniga, iye tawacin kin sdonyaye kta, qa Wanji Owotanna kin wandake ca, he i kin eciyatanhan ho kin nayalion kta;

$15 \mathrm{Qa}$ iye on taku wandake ca, nayalion kin wicasta owasin owicayakidake kta heon.

16 Unkan nakaha taku e ayape he. Najin wo, baptem niçupi kta, Itancan caje kin hoyeyakiye kta, hecen wayahtani kin yalipakinte kta ce, eya.

17 Unkan ake-Jerusalem ekta icicawin wahdi, qa tipi wakan kin ohna cekiya waun, hehan woakiktonje wan mahiyahde;

18 Qa Itancan kin wanmdaka; he kaken emakiya; Inahni, qa kohanna Jerusalem etanhan inanpa wo, mayadaotanin kin he wicadapi kte śni ce, eya.

19 Unkan hepa; Itancan, tona wacinniyanpi kin hena wicawakaśka, qa omniciye tipi kin ohna wica wakaśtaka ece qon he sdonyapi :

20 Qa Stephanos waayatanin nitawa kin we papsonpi qehan, lien nawajin, qa he iyowinwicawakiye ça, tona ktepi taśina awanwicaweciyaka qon.

21 Hehan, Hunktiya wo, detanhan tehan ye ciśi, Ikcewicaśta kin ekta, emakiya ce.

22 Unkan wicoie kin de hehanyan nation yukanpi, hehan nina hotaninpi qa, Wicaśta kin de ma224 ka kin etanhan aya po, ni kta e iyecece śni ce, eyapi.

23 Unkan hotankakiya hotaninpi, qa śina ehpekiyapi, qa watuśekśeca wankan yeyapi kin;

24 Hehan akicita tancan kin, conkaślze mahen aupi, qa kapsinpsintapi kin on wiwangapi kta, keya; hecen tokeca on oyate kin nina aoqoyapi kin he sdonye kta.

25 Unkan tehimiso on kaśkapi icunhan, Palos akicita tancan wan en najin kin heciya, Roma wicaśta kin heca, qa nahahin yacopi śni, tuka yakapsinpsintapi kta oyakihipi he.

26 Akicita tancan kin he nahon, unkan akicita tancan tanka kin hośi hde ca heya; Taku ecanon kte cin he itonpa wo, wicaśta kin he Roma kin heca.

27 Hehan akicita tancan tanka kin en hi, qa heciya, Roma wicaśta kin henica he, omakiyaka wo. Unkan, Han, eya.

28 Unkan akicita tancan qon heya, Ihduhapi kin de ota iyopewakiya, eya. 'Tuka miś hecen matonpi ce, Palos eya.

29 Heon kohanna tona Palos wiwangapi kte cin hena ayuśtan iyayapi, qa akicita tancan tanka kin nina kopehda; he kaśka tuka Roma wicaśta kin heca.e sdonye cin he etanhan.

30 Unkan ihankanna qehan, Juda oyate taku iyaonpapi hecinhan, he atayedan sdonye kta, heon icaśke kin etanhan kiyuślke ça, wośna kagapi, hunkawicayapi ko owasin mniciye wicaśi, qa hehan en ahi, qa wicacokaya ehide.

WICOWOYAKE 23.

1 Unkan Palos omniciye kin opawicahta yanke ca, Wicaśta 


\section{WICOWOYAKE 23.}

hunkawanjin ciyapi, Wakantanka itokam mitawacin ocowasin waśte, anpetu kin dehan wani, eya.

2 Unkan Ananias wośna kaggapi itancan kin, tona ikiyadan najinpi kin Palos i kin ohna ape wicaśi.

3 Hehan Palos heciva, Conkaśke skayapi kin, Wakantanka anipe kta ce; wicoope kin eciyatanhan mayadaco kta e yahidotanka tuka wicoope kaśeya amape wicayaśi.

4. Unkan tona en najinpi kin, Wakantanka wawayuśna itancan tawa kin he śicaya owayakiya he, eyapi.

5 Unkan Palos, Hunkawanjinciyapi, he wawayuśna itancan kin ee sdonwaye śni, eya; Wowapi en kaken kagapi, Nitaoyate kin en wicaśta itancan kin daśice kte śni ce.

6 Tuka apa Saddukee, qa apa iś Pharisee kin hecapi e Palos iyukcan qehan, hehan omniciye kin en hotankakiya heya; Pharisee kin hemaca, qa nakun Pharisee cinhintku kin, qa wicate cin piya ikicagapi kte cin ape waun kin heon etanhan nakaha mayacopi ce.

7 Unkan hecen eye çehan, Pharisee Saddukec kin om hdokinicapi, qa omniciye kin aksipam iyeyapi.

8 Piya ikicagapi kin wanica, qa mahpiya ohnihde, wanagi ko wanica ce, Saddukee kin eyapi ece; tuka Pharisee kin, Napin yukan ce, eyapi ece.

9. Hecen nina owodutaton, qa wowapi kagapi Pharisee etanhanpi kin hena inajinpi, qa wicalkigèi, qa heyapi, Wicaśta kin de en aku śica takudan iyeunyanpi śki: tuka wanagi, qa malipiya ohnir.le wan taku okiyake cin he hecetu kinhan, Wakantanka kici unkicizapi kte śni. 10 Nina hdokinicapi, unkan Palos yuśpaśpa icupi kta e akicita tancan tanka kin kokipe ca, heon akicita kin kun ye wicaśi, hena wicacokaya tanhan icupi, qa conkaśke kin mahen akupi kta.

11 Unkan he ilitayetu, hehan Itancan kin en hinajin qa, Palos cante waśte wo, Jerusalem en mayadaotanin kin he iyecen $\mathrm{Ro-}$ ma ekta mayadaotanin kta ce, eciya.

12 Unkan anpa kamdeze cin, hehan Juda wicaśta wanjikji okodakiciye wan kagapi, qa witaya içiconzapi, takudan yutapi kte śni, qa takudan yatkanpi kte śni, Palos ktepi śni kin hehanyan lkta, keyapi.

13 Wakiconzapi kin hena wicaśta wikcemna topa aktonpi.

14 Unkan hena wawayuśna tankapi, qa hunka wicayapi ko en wicayapi, qa heyapi; Palos unktepi śni kin hehanyan, takudan untapi kte śni e wokonze on unkiconzapi.

15 Heon etanhan hanhanna, niye omniciye kin owasin om akicita tancan tanka kin he au yaśipi kta, taku sanpa atayedan inungapi kta seca e heon; qa unkiś he ikiyadan aupi śni, helian unktepi kta e wiyeya unkanpi kta ce, eyapi.

16 Unkan hena nahmana iyape yukanpi kin he Palos tonśkaku kin nalion qehan, hehan ekta ye ça conkaśke kin timahen i, qa Palos okiyaka.

17 Hehan Palos akicita tancan wanji kico qa, Koślka kin de akicita tancan tanka kin ekta aya wo, taku okiyake kta ce, eya.

18 Unkan heon he icu, qa akicita tancan tanka kin ekta ai qa, 


\section{OHANYANPI QON.}

Palos kaślkapi un kin he mico, qa kośka kin de cicahi kta e econ maśi, taku oniciyalke kta keya ce, eya.

19 Hehan akicita tancan tanka he nape yuze ca kiciśnana icunom iyaye ça, Taku omayakidake kta he, eya iwanga.

20 Unkan, Heyakecinhan Juda wicaśta kin omniciye kin en $\mathrm{Pa}$ los au niśípi kta wanna konzapi, taku sanpa atayedan iwangapi kta seca e heon heyapi.

21 Tuka niś awicayupte śni wo. Wicaśta wikcemna tom aktonpi kin wokonze on wakiconzapi, Palos ktepi śni hehanyan takudan yutapi kte śni qa takudan yatkanpi kte śni, hena nakaha wiyeya yukanpi qa niś awicadupte kte cin he ape yukanpi, eya.

22 Hehan akicita tancan tanka kośka qon hdicu ye ça, Hena omayakidake cin he tuwedan sdonye ye śni wo, eciya.

$23 \mathrm{Qa}$ hehan akicita tancan nom wicakico qa, Akicita opawinge nom taku owasin on wicayuwiyeya po, qa nakun śuktanka alkan yotankapi kin wikcemna śakowin, qa wahukeza yuhapi kin opawinge nom, hanyetu kin oape iyamni, hehan Kesarea ekta yapi lista ce.

24 Nakun śuktanka wiyeya ehnaka po, Palos akan yankekiyapi kta, qa Phelika wicaśta itancan yanke cin he en tanyan aipi kta, ewicakiya.

25 Hehan wowapi wan kaken kaga :

26 Kladios Lusias, Phelika wicaśta itancan iyotan waśte kin wowapi kicaga ce. Wookiye.

27 Wicaśta kin de Juda wicaśta kin icupi, qa wanna ktepi kta hehan akicita hduha en wai, qa etanhan iwacu, he Roma wica226 śta kin heca e nawahon kin heon.

$28 \mathrm{Qa}$ taku iyaonpapi kin he tanyan sdonwaye kta wacin, heon iye omniciye yuhapi kin en awai ;

29 Qa iye wicoope hduhapi kin eciyatanhan wiwangapi kin iyaonpapi wanmdaka, tuka taku on ktepi, qa kaśkapi kta e iyaonpapi kin takudan iyewaye śni.

30 Qa wicaśta kin de Juda wicaśta nahimana iyape yukanpi kin he omakiyakapi qehan, kohanna niye ekta aye wicawaśi, qa tona taku en aupi kte cin hena hecen iyaonpeya cniciye wicawaśi. Tanyan yaun nunwe.

31 Hehan akicita iś econ wicaśi qon iyecen Palos hanyen icupi, qa Antipatris en aipi ;

32 Qa hanlianna hehan śuktanka akan yotankapi kin hena hetanhan ayapi kta e elipewicayapi, qa conkaśke kin ekta hdicupi. 33 Unkan hena iś Kesarea ekta ipi, qa wicaśta itancan wowapi qon qupi qelan, hehan Palos itolam ehdepi.

34 Unkan iye he yawa, qa makoce kin tukte e etanhan un hecinhan wiwanga; qa Kilikia etanhan un kin lie sdonya;

35 Hehan, Tohan tona taku iyanionpapi kin hena nakun hipi kinhan, hehan miś eya nacilion kta ce, eya; qa Herod woyaco tipi tawa qon he olna awanyag wicaśi.

\section{WICOWOYAKE 24.}

1 Unkan hetanhan anpetu zaptan, hehan Ananias wawayuśna itancan kin hee, qa hunkawicayapi kin om, ekta apamahde ya, nakun wicaśta ieksapa wan, Tertulos eciyapi, hena wicaśta itancan kin itokam Palos taku iyaonpapi kta. 
2 Unkan en kicopi qehan, Tcrtulos taku iyaonpe ca heya;

3 Niye enitanhan wookiye tanka unhapi ce, qa waawandake cin eciyatanhan oyate kin de en wicohan waśte econpi cce kin he ohinniyan qa owancaya unkicupi, qa on, Phelika iyotan waśte, wopida unkeniciyapi ce.

4 Tuka sanpa nagiyeciye kte śni, heon ceciciya ce, nitowaonśida kin on wicoie wanjikji naunyahonpi kta.

5 -Wicaśta kin de makośice, qa maka kin owancaya Juda oyate cante okiçuninwicaye cin, qa yuom decapi wan Nazareth etanhanpi, ewicakiyapi kin he en mdetahunkapi kin he wanji dee e iyeunyanpi ce.

6 He tipi wakan kin yuśape kta; unkan hehan unkiś unkicupi, qa wicoope unkitawapi kin eciyatanhan unyacopi kta;

7 Tuka Lusias akicita tancan tanka kin ekta i, qa wicowaśake tanka on unnapcpi kin etanhan eyaku.

8 Qa tona taku iyaonpeya eyapi kin hena niye kin ekta u wicaśi. Hena wiwicanunge cinhan taku iyaunkonpapi kin owasin atayedan sdonyaye kta ce.

9 Unkan Juda wicaśta kin iś waayuptapi qa, Hena hecetu ce, eyapi.

10 Hehan wicaśta itancan kin Palos ie kta e namkicawin, hehan waayupte; Oyate kin de en waniyetu ota wicaśta wayaco nanka e sdonwaya, heon etanhan nakaha iyuśkinyan wowahdaka.

11 Hecen Jerusalem ekta cekiya imdamde cin, hehantanhan wanna anpetu ake nomnana e sdonyaye kta oyakihi.

12 Qa tipi wakan kin ohna tuwe kici awakinica ecen wanma- yakapi śni, qa omniciye tipi kin ohna, qa otonwe kin cn, oyate kin wicayuomdeca waun śni.

13 Qa nakaha taku iyamaonpapi kin hena yasutapi kta e okihipi śni.

14 'Tuka de ociciyaka, yuomdecapi kin cajeyatapi qon he canku kin olna hunkake wicawaye cin Wakantanka tawapi kin ohowada, qa wicoope, qa wicaśta wokcan taku owapi qon hena owasin wicawada :

$15 \mathrm{Qa}$ Wakantanka wacinwaye, qa wicațe cin, waśte śica ko, ake woekicetu kte cin he ape waun, hc iś iyepi nakun hecen wicadapi.

16 Heon Wakantanka, qa nakun wicaśta kin en tawacin takudan iyaompepica śni waun kta e ohinniyan opimiçiya ce.

17 Unkan nakaha waniyetu ota iyohakam, mitaoyate kin taku qupi, wośnapi ko wicawecicau kta c wahdi.

18 Qa hecen tipi wakan kin ohna mihduska waun, wicota qa owodutaton kin unmana en owape śni, ecen Juda wicaśta Asia etanhan wanjikji wanmayakapi qon.

19 Hena eepi kin niye en nihiyupi, qa taku yuhapi kinhan iyamaonpapi kta tuka.

$20 \mathrm{Qa}$ dena iś, omniciye kin en nawajin kin, he icunhan taku śica ecamon kin taku sdonyapi kinhan oyakapi kta.

21 Tuka wicacokaya nawajin qehan, wicoie wanjidan taninyan epa, Anpetu kin de wicațe cin woekicetu kte cin heon mayadacopi, epe ciqon hee, eya.

22 Unkan Phelika hena nahon qehan, qa canku kin de on sanpa tanyan sdonye kta cin kin heon, hde wicaśi qa, Tohan Lusias akicita tancan tanka hi kinhan, 


\section{OHANYANPI QON.}

niyepi kin on taku owasin sdonwaye kta ce, eya.

$23 \mathrm{Qa}$ akicita tancan wan $\mathrm{Pa}$ los awanyag kiye ca, woozi yuhe kta, qa iye takuwicaye cin tuwe en hi qa okiye kta cin kinhan tehinda kte śni, keya.

24. Unkan anpetu tonana iyohakam, Phelika, qa tawicu Drusila eciyapi, Juda winohinca kin heca, he kici hi, hehan Palos kico, qa Messiya wacinyanpi kin on nation.

25 Unkan Palos wicoowotanna, qa iyatahde içiyapi śni, qa woyaco u kte cin hena oyake cin, he icunhan Phelika cancan, qa, Nakaha ekta hda wo, tohan tanyan iyomakipi kinhan ake cico kta ce, eya.

26 Palos kiyuśke kta e on mazaska qupi kta kecin, heon kico, qa kici wohdaka ece.

27 Tuka wanna waniyetu nonpa, hehan Phelika yanke cin en Porkios Phestos hiyotanka. Unkan Phelika Juda oyate kin iyokipi wicaye kta cin, qa heon Palos kaśkapi ecen elipeya kihda.

\section{WICOWOYAKE 25.}

1 Unkan Phestos makoce kin he en hiyotanke cin, iyohakam anpetu yamni, hehan Kesarea etanhan Jerusalexn elita itawankanhde iyaya.

2 Unkan wawayuśna itancan qa Juda wicaśta tokapapi kin, Palos on okiyakapi qa cekiyapi;

3 Cinkiyapi, Jerusalem ekta Palos au wicakiye kta, qa iyepi kin nalimana ktepi kta e tahepi iyapepi kita.

4 Tuka Phestos, Palos Kesarea ekta awanyakapi kta, qa iye kin ecadan ekta hde kta, keya.

$5 \mathrm{Qa}$ niyepi kin etanhan tona 228 waśakapi kinhan, apamahde om mde kta, qa wicaśta kin he taku en un kinhan wiyaonpapi kta ce, eya.

6 Unkan anpetu śahdogan, qa iś wikcemna hena om un kin, hehan Kesarea ekta hda, qa ihanlianna qehan woyaco oiyotanke kin akan kiyotanke cin, hehan Palos en au wicaśi.

7 He en hi, unkan hehan Juda wicaśta Jerusalem etanhan hipi qon lıena ihdukśan najinpi, qa wiyaonpapi tanka ota Palos en aupi, tuka yaecetupi okihipi śni.

8 Hehan iye wohdaka; Juda oyate wicoope hduhapi, qa tipi wakan kin en, qa nakun Kesar en takudan wawahtani śni ce, eya.

9 Tuka Phestos Juda oyate kin iyokipi wicaya cin, heon Palos ayupte ca heciya; Jerusalem ekta de ca, heciya mitokam dena on niyacopi kta tawatenyaya he, eya.

10 Hehan Palos, Kesar woyaco oiyotanke tawa kin en nawajin, hen mayacopi kta iyececa; Juda oyate kin taku śice cin takudan ecawicawecon śni e tanyan sdonyaya.

11 Wawahtani, qa taku śica on maktepi kta iyecece cin he ecamon kinhan, mate kta tawatenwaya tuka; tuka dena taku iyamaonpapi kin taku śni kinhan, tuwedan hena mayuhe wicakiye kte śni. Kesar cajemdata ce, eya.

12 Hehan Phestos, tona owicapeya wayaco iyotanke cin hena om wohdaka, hehan ayupta; Kesar cajedata, Kesar ekta de kta ce, eya.

13 Unkan anpetu tonana iyohakam, Agrippa wicaśtayatapi kin Bernike kici, Phestos nape yuzapi kta e on Kesarea en hipi. 
14 Qa anpetu ota hen yukanpi, hehan Phestos wicaśtayatapi kin Palos on okiyake ca heya, Wicaśta wan Phelika kaśka ehnake ciqon he nahanhin ecen yanka.

15 Jerusalem ekta wai qehan, wawayuśna tankapi, qa Juda hunka wicayapi kin hena he kapi, qa omakiyakapi, qa yacopi kta e cinpi.

16 Hena hecen awicamdupte ça, Wicaśta wan taku iyaonpapi kinhan, tona taku iyaonpeya eyapi kin wicitokam wiyaonpapi kin en waayupte iyowinkiyapi śni lkinhan, teyapi yacopi kin he Roma wicolian śni ce, epa.

17 Heon hena den hipi, unkan mdutehan śni, tuka ihanhanna hehan, woyaco oiyotanke kin akan wakimdotanke ça, wicaśta kin he en au wicawaśi.

18 Hehan tona taku iyaonpapi kin hena najinpi, tuka token wacami qon he iyecen takudan iyaonpapi śni.

19 Tuka iyepi wocekiye eyapi kin eciyatanhan wiwicawangapi kin wanjikji he en aupi, qa nakun wanji Jesus eciyapi kin he ța, tuka ake kini kin, Palos keya ece.

20 Unkan wiwicawangapi heca kin imdukcan śni heon, Jerusalem ekta de ça heciya dena on niyacopi $\mathrm{kta}$ tawațenyaya he, eya imunga.

21 Tuka Palos Sebastos cajeyate ça, he itokam yacopi kta keye cehan, Kesar ekta aye wicawakiye kta hehanyan awanyag wicawaśi.

22 Hehan, Miś eya wicaśta kin de nawahon kta wacin ce, Agrippa Phestos eciya. Unlzan iśs, Heyakecinhan nayahon kta ce, eya.

23 Unkan ihanhanna qehan,
Agrippa Bernike kici wokoyake waśteśte kiçun hipi, qa wanahon tipi kin en tin ipi ; qa nakun akicita tancanpi kin, qa otonwe en wicaśta tokapapi kin hena ko; hehan Phestos econwicaśi kin on Palos en ahipi.

24 Unkan Phestos heya, Agrippa wicaśtayatapi kin, qa wicaśta tona den om unkanpi kin, wicaśta kin on Jerusalem ektá, qa deciya nakun, Juda oyate kin owasin cemakiyapi qa, Sanpa ni kta e hecetu śni ce, eya hotaninpi gon, he dee wandakapi.

$25 \mathrm{He}$ taku sica econ kin, on țe kte cin, takudan iyewaye śni, qa iś iye Sabastos cajeyate cin, heon ekta ayapi skta e wakonza. 26 Tuka he on, mayuhe cin taku wecage kte cin atayedan takudan mduhe śni. He etanhan nakaha en cicahipi, wicaśtayatapi kin niye iniyotan, hecen tohan tanyan wiwangapi kinhan, token wakage kte cin taku mduhe kta.

27 Wicaśta wan kaśkapi kin he heciya aye śipi, qa wiyaonpapi kin takudan kagapi śni kinhan, he hecetu śni imdukcan ce, eya.

\section{WICOWOYAKE 26.}

1 Hehan Agrippa Palos heciya, Wanna woyahdake kte cin iyowinniciyapi ce. Unkan hehan Palos nape kin hdugate ça wohdaka.

2 Agrippa wicaśtayatapi kin, Juda oyate taku iyamaonpapi kin owasin nakaha nitokam wowahdake kte cin heon imduśkin. 3 Juda wicohan kin wiwangapi ko owasin tanyan hdukcan nanka sdonwaya, heon etanhan. Qa. hecen ceciciya ce, wacin tanka on namalion wo.

4 Toliaheya tanhan Jerusalem 229 


\section{OḦANYANPI QON.}

ekta mitaoyate kin om, homakśidan ehantanhan, token ohanyan waun qon Juda wicaśta kin owasin sdonyapi.

5 Token ohoundapi ece kin owotanna hince cin en opeya waun, Pharisee hemaca, hena e otokaheya tanhan sdonmayanpi, oyakapi cinpi unkanś.

6 Qa nakaha Wakantanka hunkake wicunyanpi kin iwahowicaye ciqon he apepi kin on, den mayacopi kin ecen nawajin.

7 De ekta wicoun unkitawapi ake nonpapi kin iyohipi kta e ape yukanpi, qa anpetu hanyetu ko celiyapi ece. Agrippa wicaśtayatapi kin, woape kin de on Juda wicaśta wimawangapi qon.

8 Wakantanka wicate cin ake ekicetu wicaye cin he tokeca e he taku wicadapica śni kecanipi he.

9 Miś eya Jesus Nazareth etanhàn caje kin on, taku ota kipajinyan ecamon kta iyececa kepce ciqon.

10 Jerusalem ekta hena hecen ecamon qon; qa wawayuśna tankapi kin wowaśake maqupi kin on, wicaśta owotanna kin ota wicakaślka tipi kin en iyewicawaye ca, te yapi kta wicayacopi qehan, miś eya iyecen epa ece.

11 Qa omniciye tipi kin iyaza otakiya wicawakaśtake ca, waośtehda wicawakiye ca, nina canniye wicawaye cin heon otonwe tokeca ekta śicaya wicawakuwa.

12 He icunhan wawayuśna tankapi kin wowaśake wowapi ko maqupi kin, he yuha Damaskos ekta imdamda.

13 Unkan tahepi iho, wicaśtayatapi, anpe cokaya kin hehantu, iyoyanpa wan anpetu wi kin sam iyeya wiyatpa kin he wan230 mdaka, he ihdukśan iỵyanpa maye ca tona om mawani kin hena nakun.

14 Unkan owasin makata elipeunkiçiyapi, hehan wicaho wan nawahion, Hebrew iapi on hecen emakiya, Salos, Salos, tokeca śicaya mayakuwa he, can pesto kin nayahtake cin he telika ce.

15 Unkan miś, Itancan, nituwe he, epa. Unkan, Jesus śicaya yakuwa kin he miye ce, eya.

16 Tuka inajin, qa nisiha kin on najin wo; token wandake ca, tokata taku wanyag ciciye kte cin hena en waayatanin, qa ookiye kin cicage kta e heon nakaha wanmayag ciciya.

17 Oyate kin etanhan ecihdaku kta, Ikcewicaśta kin nakun, hena e ekta nakaha ye ciśi.

18 Iśta wicadumdaye ça, otpaza etanhan iyoyanpa ekta, qa Satan towaśake kin etanhan Wakantanka ekta wicaduhomni kta; qa hecen wacinmayanpi kin on wahtanipi wicakicicajujupi kin, qa tona ska wicakagapi kin hena om woyuha yuhapi kta ce.

19 Hecen on, Agrippa wicaśtayatapi kin, malipiya wowanyake kin he cețunwahda śni.

20 Tuka tokaheya Damaskos en yakonpi, qa Jerusalem, qa Juda makoce kin owancaya, qa nakun Ikcewicaśta kin, ihduecetupi, qa Wakantanka ekta ihduhomnipi kta, qa ihduecetupi wicohan kin econpi kta hena hecen owicawakiyaka ece.

21 Hena on etanhan, Juda wicaśta kin tipi wakan kin ohna mayużapi, qa malktepi kta tuka.

22 Tuka Wakantanka omakiye cin heon anpetu kin dehanyan wani, qa tona ciqapidan tankinyanyan ko, wicawakiyaotanin, 
qa wicaśta wokcan kin Moses kici taku u kte cin oyakapi qon henana owicawakiyaka ece.

23 Messiya kakije kte ca iye tokaheya wicate cin etanhan najin kta, qa oyate kin Ikcewicaśta ko iyoyam wicaye kte cin.

24. Hena hecen eya, unkan Phestos hotankakiya ie ca, $\mathrm{Pa}$ los niyuhnaśkinyan, wowapi ota onspeniçiciye cin he wicayuhnaśkinyan en iyeniyan ce, eya.

25 Tuka iye, Phestos iyotan waśte kin, mayuhnaśkinye śni, tuka wowicake wicowahbadan oje kin epa ce, eya.

$26 \mathrm{Qa}$ wicaśtayatapi kin iś hena tanyan sdonya, heon itokam waditagya wowahdaka, hena taku kin iye kin anakilimanpi śni e mdukcan, okahmin en econpi śni nakaeś.

27 Agrippa wicaśtayatapi kin, wicaśta wokcan kin hena wicayada he. Wicayada e sdonwaya.

28 Hehan Agrippa Palos heciya; Kitan ecinyan Kristian hemaca kta e awacinmayaya ce.

29 Unkan Palos heya, Wakantanka cewakiya ce, niye niśnana śni, tuka tona anpetu kin de namahonpi kin owasin, kitan ecinyan hee śni, tuka ocowasin miś waun kin iyecen yaunpi kta, tuka icaśke kin dena ope kte śni.

30 Hena hecen eya, unkan hehan wicaśtayatapi, qa wicaśta itancan kin, Bernike kici, qa tona om iyotankapi kin hena ko najinpi ;

31 Qa icunom yapi qa, Wicaśta kin de taku on te kte ca, kaśkapi lste cin takudan econ śni ce, iyakitedan ekiciyapi.

32 Hehan, Wicaśta kin de Kesar cajeyate śni unkanś kiyuśkapi kta tuka, Agrippa Phestos eciya.

\section{WICOWOYAKE $2 \%$.}

1 Unkan Italia ekta watom unyanpi kta e wanna konzapi, hehan Palos qa wicakaśkapi tokeca kin, akicita tancan wan, Sebastos taobe kin etanhan, Julios eciyapi kin, he wicayuhekiyapi.

2 Unkan wita wata wan Adramutium etanhan, Asia makoce kin ekta tuktekten oihunni kte cin he en unkopapi, qa pacanan iyeunyanpi, qa Aristarkos Malkedonos, Thesalonike etanhan, he kici uryanpi.

3 Unkan ihanhanna qehan, Sidon en unkihunnipi. Unkan Julios Palos tanyan okicilian, takuwicaye cin ekta wicai, qa hena etanhan taku yuhe kta e iyowinkiya.

4 Unkan hetanhan pacanan iyeunyanpi, qa Kupros ihukuya watounpapi, tatoheya unyanpi kin heon.

5 Unkan Kilikia qa Pamphulia miniwanca kin unkihunnipi qehan, Lukia en Mura eciyapi kin he ekta unhipi.

6 Unkan hen wita wata wan Aleksandria etanhan, Italia ekta watom ye kte cin, he akicita tancan kin wanyake ca, he en ope unyanpi.

7 Unkan anpetu ota iwaśtedan watounpapi qa kitan se Knidos en iyotakons unkipi, tatoheya unyanpi nakaeś, hehan Krete ihukuya, Salmone iyotakons watounpapi.

$8 \mathrm{Qa}$ he kitan se kapeya unyanpi, hehan makoce wan $\mathrm{Oi-}$ hunni waśte eciyapi kin he en unkipi, he Lasea otonwe kin ikiyadan.

9 Wanna anpetu ota, qa watopapi kin okokipe aya, hamdepi tanka kin wanna enakiyapi, hehan Palos waholionwicakiya; 


\section{OHANYANPI QON.}

10 Qa hewicakiya, Wicaśta kin, watounpapi kte cin de on wita wata kin taku ohnaka ko yutakunipi kte śni, qa nakun unkiyepi kin iyotanhan iyeunkiyapi kta e imdukcan ce; eya.

11 Tuka akicita tancan kin, wahektape cin qa wita wata tawa kin hena anawicakicigoptan, qa Palos ie cin he wicada śni.

12 Unkan oihunni kin he en waniyetu osan yukanpi kte cin le waśte śni, heon hetanhan yapi, qa okihipi kinhan, Phenike ekta watom ipi, qa hen waniyetu owanji yukanpi kta, wicota Ikeyapi ; Krete en oihunni wan itokagatanhan, wiyohpeyata ohna waziyata etkiya huta kirı yuskomya wanke cin hee.

13 Unkan okaga tanhan tate kin iwaśtedan hiyu, qa hecen token wacinpi qon wanna ecetu kecinpi, hehan kiyuśkapi qa Krete ikiyedan watopapi.

14 Tuka iecadan, tateyanpa wan ohitike cin he en iyapa hiyu, he Tate-taja eciyapi.

15 Unkan wita wata kin nina iyapa, hecen tatoheya yapi okihipi śni, hehan kaha iyeunyanpi iyowinunkiy api.

16 Unkan wita wan Klauda eciyapi, he ikiyadan unyanpi, hehan kitan hin wata cistinna kin icu unkokihipi.

17 He yuwankan ehdakupi, qa icaśke kin unpi, qa wita wata kin opta yuskiskitapi, hehan wiyaka śice cin en iyolipeyapi kta kokipapi, qa heon śina on watopapi kin palipapi, qa hecen tate on kaha iyewicayapi.

$18 \mathrm{Qa}$ nina unkakihanśicapi, heon ihanhanna, hehan wata kin etanhan taku tankan elipeyapi.

19 Qa anpetu iciyamni hehan, unnapepi kin on, wita wata kin 232 en, taku wowinyunyanpi kin minin elipeunyanpi.

20 Unkan anpetu ota, anpetu wi wicanhpi ko tanin śni un, qa nina unkakihanśicapi, helian unnipi kta unkecinpi qon he wanna unkay uśtanpi.

21 Tuka tehan takudan yute śni unpi kin, hehan Palos wicacokaya najin, qa, Wicaśta kin, anamayagoptanpi qa Krete etanhan pacanan iyeyayapi kte śni iyececa, hecen taku śice ca wicakakije cin de nihiyahdepi kte śni tuka.

22 Qa nakaha ceciciyapi ce, cante waśte po ; tuwedan nițapi kte śni, tuka wita wata kin hecedan.

23 Wakantanka ohowada, qa iye mayuhe cin he taohnihde ye cin hinhan en mahinajin.

24 Qa, Palos kolipe śni wo: Kesar itokam inayajin lkta, unkan tona om watoyape cin owasin Wakantanka nicu ce, eya ce. 25 Heon etanhan, wicaśta kin, cante waśte po; Wakantanka wicawada, token emakiye cin lıe ecetu kte.

26 'Tuka wita wan en iyeunyanpi kta ce, eya.

27 Unkan otpaza iaketopa kin, hehan Adria miniwanca kin ohna kaha iyeunyanpi kin icunhan, hanye cokaya hehan watope wicakiyapi kin makoce wan ikiyadan upi kecinpi.

$28 \mathrm{Qa}$ hehan mazasu halionta ikoyake cin minin iyeyapi, qa akatinpi wikcemna nonpa e iyeyapi. Hetanhan kitanna kapeya yapi, hehan ake econpi, unkan wanna akatinpi ake zaptan.

29 Unkan imnija kin en iyewicayapi kta e kokipapi, heon wahektapa kin etanhan maza aspeiçiyapi tom minin iyeyapi, qa anpao kta e cinpi hinca. 
30 Unkan watope wicakiyapi kin wita wata kin etanhan najicapi kta akitapi, qa wata cistina qon he mini kin ekta yuhukun iyeyapi, watokapa tanhan maza aspeiçiyapi minin iyeya konzapi.

31 Unkan Palos akicita tancan kin akicita ko hewicakiya; Dena wita wata kin ohna yukanpi śni kinhan, yanipi oyakihipi kte śni ce.

32 Hehan alkicita kin wata cistina halionta ikoyake cin bapsakapi, qa kun iyeyapi.

33 Wanna anpao kta hehan $\mathrm{Pa}$ los owasin taku yutapi icu wicaśi, qa heya, Nakaha takudan yute śni yaunpi anpetu iake topa kin he dee, hehanyan takudan iyacupi śni.

34 Heon ceciciyapi ce, taku yutapi icu po, on waniśakapi kta ce, paha nitawapi sukaza wanjidan hinhpaye kte śni ce.

35 Hena hecen eya hehan aguyapi icu, qa owasin wicitokam Wakantanka cekiye ça, kiyuśpe ca yuta.

36 Hehan owasin cante waśtepi, qa woyute icupi.

37 Unkan wita wata kin ohna, wicanagi owasin opawinge nom sanpa wikcemna śakowin sanpa śakpe unyakonpi.

38 Unkan taku yutapi on imnanpi hehan wita wata kin yukapojedanpi, qa aguyapi su kin minin ehpeyapi.

39 Unkan wanna anpa kamdeze cehan, makoce kin iyekiyapi śni; tuka kahmin wan huta oihunni waśte kin he wanyakapi, qa okihipi kinhan, he en wita wata lrin aipi kta, keyapi.

40 Hehan maza aspeiciyapi kin bapsag iyeyapi, qa minin ehpeyapi, qa iyupse yuza icaśke kin kiyuśkapi; hehan śina watope- kiyapi tanka lin yuwankan iyeyapi, qa huta lsin ekta yapi.

4.1 Tuka tukten miniwanca nom lrokijuya hiyu kin he en iyohpayapi, qa hen wita wata kin azi yapi. Unkan watokapatanhan kin he sutaya ihan, tuka wahelktapatanhan kin taja on kamden iyeya.

42 Unkan akicita kin wicakaśkapi kin hena wicaktepi kta e konzapi, okinni wanjikji huta kin ekta niwanpi, qa najicapi kta, keyapi.

43 Tuka akicita tancan kin iś Palos nilkiye kta cin kin, heon taku econpi kte cin iyowicaki śni ; qa hehàn tona niwan okihipi kin hena tokaheya minin ehpeiçyapi qa huta lkin ekta niwanpi kta, keya.

44 Hehan tona nahahin en un kin hena, hunh canmdaska akan, qa hunh iś wita wata etanhan onśpaśpadan akan iyayapi. Unkan hecen owasin huta kin ekta tanyan iyohipi.

\section{WICOWOYAKE 28.}

1 Unkan elsta tanyan iyohipi hehan wita kin he Melite eciyapi e sdonyapi.

2 Unkan Ikcewicaśta kin nina taku waśte ecaunkiconpi, cetipi qa otoiyohi en unkicopi, nina magaju qa osni kin heon etanhan.

3 Unkan Palos can cikcistina ota pahi, qa peta en aonpa hehall, wamduśkadan wan iyokatapi kin etanhan hiyu, qa Palos nape kin en ikoyag iciya.

4. Unkan nape kin en taku ocinśice cin de ikoyake cin he Ikcewicaśta kin wanyakapi qehan, iyakitedan hekiciyapi ; Awicakehan wicaśta kin de tin wicakte kin heca, miniwanca kin 


\section{OḦANYANPI QON.}

etanhan ni hiyu eśta, taku owotanna kin he ni kta e iyowinkiye śni ce.

5 Tuka wamduśkadan qon nape kin etanhan peta kin en hdatata ehpeye ça tuktedan kiunniye śni.

6 Tuka kapo kta, qa iś ihnuhanna ta iyaye kta kecinpi. Qa tehan ekta etonwan yukanpi qa tuktedan kiunniye śni un kin wanyakapi, hehan tawacin kin tokeca hinhdapi qa, De taku wakan kin hee ce, eyapi.

7 Unkan hen ikiyedan, wita kin ohna wicaśta tokapa, Publios eciyapi kin he makoce onśpa yuha; unkan he tin hiyu unśipi, qa anpetu yamni hehanyan tanyan wo unqupi.

8 Unkan Publios atkuku kin wayazanka, tancan kate ca we ece iheya. Palos he en i, qa cekiye ça, nape on aputake ca asniyan.

9 Hecen econ, unkan hehan wita kin en tona wowayazan yuhapi kin hena en hipi, qa asni wicayapi.

10 Heon etanhan nina unkinihanpi, qa hetanhan unyanpi kte cin, hehan taku uncinpi kin owasin unqupi.

11 Unkan wi yamni, hehan wita wata wan Aleksandrea etanhan, he wita kin hen waniyetu wanke cin, he wiyokiyedan yuke cin, Jupiter cekpapi taw a kin hee, unkan he en unkopapi.

12 Qa Surakusa ekta unkihunnipi, qa anpetu yamni unyakonpi.

13 Unkan hetanhan ohomni unyanpi, qa Regium ekta unhipi. Qa anpetu wanjidan iyohakam, tate itokaga tanhan uya, qa anpetu inonpa hehan Puteoli en unkipi.

14 Hen hunkawanjin kiciyapi kin wanjikji iyewicunyanpi, qa anpeta śakowin om unyakonpi kta e cinpi. Hehan Roma ektakiya unyanpi.

15 Unkan heciyatanhan hunkawanjin kiciyapi kin unkiyepi eciyatanhan nahonpi, qa itkounkipapi au, Appiiphorum qa Owote tipi yamni kin hehanyan. Hena Palos wanwicayaka, unkan Wakantanka wopida eciye ça wakiśag ic̣iya.

16 Unkan Roma ekta unkipi, hehan akicita tancan qon wicakaśke cin hena wicaśtayatapi taakicita tancan kin qu, tuka Palos akicita wanjidan awanyag un kin he kiciśnana yanke kta $\mathrm{e}$ iyowinkiyapi.

17 Unkan anpetu yamni iyohakam, Palos Juda wicaśta tokapapi kin wicakico. Qa en mniciyapi hehan hewicakiya, Wicaśta hunkawanjinciyapi, atewicunyanpi oyate qa wicolian kin en taku śica takudan ecamon śni eśta, Jerusalem ekta makaśkapi ecen Roma oyate napepi kin en iyemayanpi.

18 Hena iś wimawangapi qehan makiyuśkapi kta, miye en taku on mate kte cin takudan iyeyapi śni.

19 Tuka Juda wicaśta kin nina tehindapi kin heon, Kesar caje mdate kta iyececa wadaka, mitaoyate lkin taku iyawicawaonpe kte cin takudan mduhe śni tuka qon.

20 Heon etanhan nakaka cicopi, wanciyakapi qa wociciyakapi kta, Israel oyate awacinpi kin he on maza icicaliitia kin de on makaślkapi ce, eya.

21 Hehan iś eya heciyapi, Juda makoce kin etanhan niye on wowapi unkahipi śni, qa hunkawanjin kiciyapi heciyatanhan hipi kin tuwedan taku śica iyanionpe ca oyake śni.

22 Tuka nis token nitawacin

234 


\section{WICOWOYAKE 1.}

kin naunnikonpi kta uncinpi, yuomdecapi kin de owancaya yaśicapi e sdonunyanpi ce.

23 Unkan anpetu wan cajeyatapi kin he en wicota Palos yanke cin en hipi; unkan iś Wakantanka tokiconze kin yaotanin, qa onspewicakiye ca, Moses toope kin wicaśta wokcan ko, etanhan Jesus on wahokonwicakiya, anpao kin etanhan otpaze cin hehanyan.

24 Unkan hunh taku oyakapi kin wicadapi, qa hunh iś wicadapi śni.

25 Unkan okonwanjidan awakicinpi śni kin, hehan kihdapi, Palos wicoie wanjidan oyake cin iyohakam; Woniya Wakan kin Isaya wicaśta wokcan kin en, hunkake wicunyanpi kin tanyan wowicakiyaka;

$26 \mathrm{Qa}$ heya, Oyate kin de ekta wicai, qa heya wo; Wanahonpi kin on nayahonpi kta tuka oyakahinigapi kte śni, qa wawanya- kapi kin on wandakapi kta tuka widukcanpi kte sni :

27 Oyate kin de cantepi kin cepa aye ca nogepi kin on kitan se wanahonpi, qa iśtapi kin olımus icupi; iśta kin on wawanyakapi kte śni, qa noge kin on wanahonpi kte śni, qa cante kin on okahnigapi kte śni, qa ihduecetupi kte śni, qa miś okizi wicawaye kte śni heon etanhan. 28 Hecen on he sdonya po, Wakantanka wiconi tawa kin Ikcewicaśta kin wicaqupi, unkan hena iś nationpi kta ce.

29 Hena hecen eya, unkan Juda wicaśta kin kihdapi, qa nina hdokinicapi.

30 Unkan Palos waniyetu nom tipi iyunwin ton odote cin he ohra yanke ça, tona en hipi kin owasin tin u wicaśi.

31 Wakantanka tokiconze kin yaotanin, qa Jesus Messiya Itancan taku tawa kin waditagya oyaka ece, tuwedan tehinda śni.

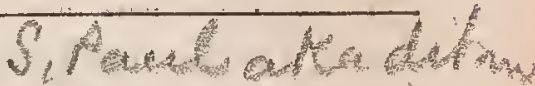

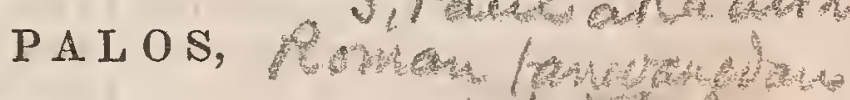 ROMA OYATE

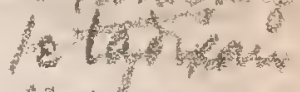

WOWAPI WICAKICAGE CIN. Ho

\section{WICOWOYAKE 1.}

1 Palos Jesus Messiya taokiye, yewicaśipi kin en kicopi, Wakantanka tawootanin waśte on kahnigapi ;

2 Ehanna wowapi wakan kin en wicaśta wokcan oyakapi qon hee;

3 Iye Cinhintku Jesus Messiya Itancan unyanpi kin heon etanhan; he qe wicacehpi eciyatanhan Dawid cinca kin hetanhan icaga ;
4 Qa wowaśake on Wakantanka Cinhintku kin hee e yuotaninpi, Woniya Wakan kin eciyatanhan, wiconte etanhan piya ikicage cin he en;

5 Iye kin eciyatanhan wowaśte unkicupi, qa oyate owasin wacinyanpi kin anagoptanpi kte cin heon yewicaśípi wicohan kin he nakun, iye caje kin on:

6 Hena ehna Jesus Messiya wicakico kin he niyepi.

7 Tona Roma ekta un kin owa235 
sin, Wakantanka waśte wicakida, yaowotanpidan kte cin on wicakico kin hena, wowapi cicagapi. Wowaonsida, wookiye ko, Wakantanka ateunyanpi, qa Jesus Messiya Itancan kin etanhan, nicipi un nunwe.

8 Tokaheya, Jesus Messiya eciyatanhan, niyepi owasin on etanhan, Wakantanka mitawa kin wopida ewakiya ece, niye wacinyayapi kin he maka kin. owancaya cajeyatapi kin heon.

9 Wakantanka minagi kin eciyatanhan ohowada, iye Cinhintku tawootanin waśte kin en, he sdonmayan, ayaśtan śni wocekiye epa eca, ohinniyan cajeciyatapi ece;

10 Qa nakaha Wakantanka tawacin kin eciyatanhan ekta ciupi owakihi kte cin heon cewakiya ece.

11 Wanciyakapi nina wacin, woniya etanhan taku wicaqupi wan cicupi kta, on niyusutapi kte cin heon etanhan.

12 Niyepi kin on iyuśkinmayanpi kte cin he waka, niś niye qa miś miye napin wacinunyanpi kin on etanhan.

13 Mihunkawanji, he de sdonye śni dukanpi kta wacin śni, wanna ota ekta ciupi kta kepca, tuka dehanyan iyomakipi śni, niyepi ekta waskuyeca wanjikji mduhe kta wacin, oyate toktokeca kin en matuhe cin he iyececa.

14 Helenes, Ikcewicaśta ko, ksapapi, wacintonpi śni ko, hena iyohi wicaweciye śni waun.

15 Hecen token owakihi kin, niyepi nakun Roma ekta yaunpi kin, wootanin waśte kin ociciyakapi kta wacin.

16 Messiya tawootanin waśte kin on imaśtece śni ; he niwicaye kte cin on W.akantanka to236 waśake kin hee, tona wicadapi kin hena en, Juda wicaśta kin he tokaheya, qa hehan Helenes wicaśta kin hena.

17 Heon etanhan Wakantanka owotanua ohanye cin he wacinyanpi kin etanhan wacinyanpi ekta yuotaninpi; wowapi qon iyececa, Tuwe owotanna kin he wacinyanpi on ni kta ce.

18 Mahpiya kin etanhan Wakantanka tocanniye kin yuotaninpi, woaktaśni owasin qa wicaśta woahtani econpi kin en, tona woahtani on wowicake kin anaptapi kin hena en.

19 Hena on Wakantanka token sdonyapi kte cin taninyan un; Wakantanka sdonye wicakiya nakaeś.

20 Maka kagapi kin hetanhan Wakantanka towakan qa towaśake otokahe wanice cin hena tanin, hena wanyagpica śni eśta, taku kage cin on etanhan sdonyapi ece; hecen on woanahibe yuhapi kte śni.

21 Qa hecen Wakantanka sdonyapi, tuka Wakantanka iyecen yutanpi śni, qa pidapi śni; tuka tawacinpi kin ekta wahanhanicida ayapi, qa iye cantepi wacintonśni kin otpas aya.

22 Kisapeiçidapi kin en icunhan witkotkoka icah ayapi.

23 Qa Wakantanka tepica śni towitan kin he wicaśta te kte cin ouncage kin iyecen yutokecapi, qa zitkadan, wahutopa, watutka kin hena koya.

24 Heon iye cantiheyapi śice cin en Wakantanka awicayuśtan, ihduśapapi kte ca, iye tancanpi kin kiciyuśihtinpi kte cin heon.

25 Hena Wakantanka wowicake tawa kin woitonśni kagapi, qa taku icahyapi kin he yuonihanpi qa ohodapi, waicah- 
ye cin he isanpa, he qe owihanke wanin yawaśtepi nunwe.

26 Heon etanhan wocantiheye śice cin en Wakantanka awicayuśtan; hecen winolinca wicayuhapi kin hena e wicolian owotanna kin yutokecapi, qa owotanna śni econpi ece.

27 Qa wicaśta kin iś eya winyan yuhapi kin he ayuśtanpi, qa iś iyepi otoiyohi winktapi on kicicinpi, wica kin wica kici wowiśtece okicilianyanpi, qa iye tancan mahen wahtanipi kin taku iyohi kte cin he yuhapi.

28 Qa Wakantanka wacin en yuze wacinpi śni kin he iyecen, Wakantanka iś eya wicotawacin walite śni kin en awicayuśtan, hecen taku wowiśtece kin hduśtanpi kta.

29 Hena e worhtani owasin, wiinahmanpi, wicośice, waicucupi kte cin, qa olinyanpi on iyatahdepi; winawizipi, tin wicaktepi, wakinicapi, wohnaye, qa wocanniye on ojupidan.

30 Waajijipi sa, waaiapi sa, Wakantanlsa śice dapika, wahianicidapi, wamnaiçidapi, ihdatanpi sa, taku śica kagapi sa, hunkakeyapi kin anawicagoptanpi śni ;

31 Wacintonpi śni, woope kicaksapi, wacantkiyapi śni, wacin tankapi śni, qa waonśidapi śni.

32 Hcna Wakantanka toope kin sdonyapi ; (tona hecen econpi kin hena wiconțe iyahdeiçiyapi,) tuka iś iye hecen econpi qa tona heconpi kin hena on iyokipipi ece.

\section{WICOWOYAKE 2.}

1 Heon etanhan wicaśta kin nituwe kaśta, wadaco kinhan iyaonpepica śni yaun kte śni. Taku on wicaśta tokeca daco kinhan he en niye nihdaco, wadaco tuka niś eya he kici taku okonwanjidan ecanon ece.

2 Tona wicolian hecen econpi kin hena Wakantanka wowicake eciyatanhan wicayaco kin he sdonunyanpi.

3 Qa wicaśta $\mathrm{kin}$, niś tona heconpi kin wicadaco, qa iyecen ecanon lkinhan, Walrantanka wicayaco kin he nayecipe kta kecanni he.

$4 \mathrm{Qa}$ iś iye towaśte wowijice kin ayakta śni he, wacintanka qa wawacinkta yuza tanka koya; qa Wakantanka towaśtc kin nihduecetu kta aniye cin hee e sdonyaye śni he.

5 Tuka cante nitehika, qa nihduecetu śni kin eciyatauhan, wocanniyc anpetu, qa Wakantanka owotanna wicayaco lin yutaninpi iyehantu kte cin en, wocantiyahde amnayaya he.

6 He wicaśta otoiyohi iye olian kin cciyatanhan wicakicu ece e kta:

7 Tona wicolian waśte en ape yukanpi, qa wowitar, woyuonihan, qa tepica śni kin akitapi ece kin hena wiconi owihanke wanice kta :

8 Tuka tona wakinin unpi, qa wowicake kin anagoptanpi śni, tuka woahitani awacinpi kin hena, wośihda, qa wocanniye,

9 Wośitkihda, qa wokakijc, wicaśta otoiyohi taku śica econ kin wicanagi kin kiciun kta, Juda wicaśta kin he tokaheya, qa liehan Helenes hee kta.

10 Tuka wowitan, woyuonihan, wookiye ko, tona waśteya olianye cin hena kiciun kta, Juda wicaśta kin he tokaheya, qa hehan Helenes hee kta.

11 Wakantanka wicaśta itohnake ekta etonwe śni ece.

12 Tona woope codan wahta237 
nipi kin hena woope codan yutakunipi kte śni, qa tona woope kin en walitanipi kin hena woope kin eciyatanhan wicayacopi kta.

13 Wakantanka ekta tona woope nahonpi kin hena owotanna kin eepi śni, tuka tona woope kin ecen econpi kin hena owotanna wicayawapi kta.

14 Tolıan Ikcewicaśta woope yuhapi śni kin hena iye cinka woope wicohan kin ecen econpi kinhan, woope yuhapi śni eśta, iś iye woope ihduhapi ece.

15 Hena iś iye cantepi kin ekta woope wicolian owapi kin he hdutaninpi, iye tawiyukcan kin nakun he yaotanin, qa tawacinpi kin otoiyohi tuktekten iyakicionpapi, qa akeś anakilimanpi ece.

16 Anpetu wan en, Wakantanka wo tanin waśte mitawa kin eciyatanhan, Jesus Messiya on, wicaśta taku nahmana awacinpi kin yaco kinhan.

17 Iho, Juda wicaśta eniciyapi, qa woope kin awacin nanke ca Wakantanka on nihdatan,

18 Qa iye tawacin liin sdonyaya, qa taku tona waśte kin idukcan, woope kin tanyan onnispe nakaeś ;

19 Qa tona iśta gongapi kin tanyan yewicayaye ça, otpaza ehna un kin iyoyamwicaye cin he niye kecanniçin ;

$20 \mathrm{Qa}$ wacinton śni kin hena waonspekiya niyuhapi, qa hokśiyoqopa kin onspewicayakiya ece, qa woope kin en wosdonye wowicake ko ouncage kin yuha yaü.

21 Heon etanhan wicaśta tokeca onspeyakiye cin niś niye onspeniciciye śni he. Wamanonpi kte śni keya odake cin, niś niye wamayanon he.
22 Wawicihahapi econpi kte śni kehe cin, niś niye tuwe wawiyakihaha he. Wakagapi kin wahiteyada śni kin niś niye tipi wakan kin etanhan wamayanon he.

23 Niś woope kin en iniwinkta kin, woope kicaksapi kin on Wakantanka wowiśtenyaya he. 24 Niyepi eciyatanhan Ikcewicaśta kin Wakantanka caje kin ośtehdapi ece ; wowapi en kagapi qon iyececa ;

25 Woope kin oyape cinhan, awicakehan bakilidayapi kin taku okihi ece; tuka woope yakaksa ece kinhan, banicihdayapi kin he bakihdayapi śni icaga.

26 Heon bakilidayapi śni kin he woope woowotanna kin tanyan ope cinhan, he bakihdayapi śni kin bakihdayapi kiciyawapi kte śni he.

27 Qa bakihdayapi śni ece kin he woope kin ope cinhan, he, niye oowa qa bakihdayapi kin eciyatanhan woope yecakse cin, niyaco kte śni he.

28 Tuwe akan tanhan ecedan hecece cin, he Juda wicaśta kin heca śni; qa bakihdayapi akan tanhan qa wicacelpi ecedan en un kin he hee śni;

$29^{\circ}$ Tuka tuwe mahen tanhan hecece cin he Juda wicasta kin hee, qa bakihdayapi kin he oowa kin en he śni, tuka wicanagi kin en un kin hee; he wowitan kin wicaśta akantu eciyatanhan śni, tuka Wakantanka eciyatanhan un.

\section{WICOWOYAKE 3.}

1 Hecen Juda wicaśta kin token iyotan un he, qa bakilidayapi kin he taku olkihi he.

2 Taku owasin on iyotan, tuka tokaheya Wakantanka oie kin wicaqupi qon he. 
3 Qa wanjikji wicadapi śni eśta, hecen tokeca lkta he. Hena wicadapi śni kin he Wakantanka wacinyanpi kin ecetu śni ye kta he.

4 Hiya, hecetu śni; Wakantanka wowicake kta, qa wicaśta otoiyohi iton lkte śni; wowapi en kagapi qon iyececa; Nioie kin on nihdaowotanna kta, qa niyacopi kin en ohiyaye kta ce.

5 Tuka waunhtanipi kin hena Wakantanka toowotanna yutanin kinhan token unkeyapi kta he; Wakantanka kakiświcaye cin he owotanna śni he ; (wicaśta akantu kin iyecen iwaa;)

6 Hiya hecetu śni ; hecetu kinhan token Wakantanka wicoicage kin wicayaco kta he.

7 Oiwaton sni kin on etanhan Wakantanka towicake kin he iye towitan kin hduotanin kinhan, token on etanhan wahtani sa iyecen mayacopi he.

8 Qa tokeca taku waśte u kte cin heon taku śica econkupi lite śni he, unkakicagapi qa wanjikji, unkeyapi ce, keyapi kin iyececa; hena wicayacopi kin he owotanna.

$9 \mathrm{He}$ etanhan tokeca kta; unkiye iyotan unwaśtepi he. Hiya, takuśnina on. Wanna Juda Helenes wicaśta ko, owasin woahtani ihukuya yukanpi kin he unyaotaninpi qon.

10 Wowapi en kagapi qon iyececa; Tuwedan owotanna un śni ;

11 Tuwedan okahinige śni ; tuwedan Wakantanka akite śni ;

12 Owasin icunonpa iyayapi, ptaya ihduśicapi ; tuwedan taku waśte econ śni, wanjidan eśta.

13 Dotepi kin wicahnakapi yuhdokapi kin hee; cejipi kin on wicahnayanpi ece, ihapi kin en sintehda tage yukan :
14 Ipi kin wicayaśicapi qa wicoie pa hinca ko on ojudan :

15 Sihapi kin iś weyapi kta e duzahanpi ;

16 Tacankupi kin ohna wicotakuni śni wicokakije ko yukan; 17 Qa wookiye canku kin he sdonyapi śni.

18 Qa iśtapi kin itokam Wakantanka kokipapi kin wanica ce.

19 Woope kin taku tona eye cinhan, tona woope ihukuya un kin hena hewicakiya ece e sdonunyanpi. Hecen wicai otoiyohi iohmus icupi kta, qa wicoicage owasin Wakantanka itokam wicayacopi kta.

20 Heon etanhan iye itokam, woope wicohan kin on, wicacehpi takudan yaowotanpi kte śni ; woope kin eciyatanhan woahtani sdonkiyapi ece.

21 Tuka nakaha woope codan Wakantanka toowotanna lzin yutaninpi; woope qa wicaśta wokcanpi kin he yaotaninpi ece.

22 Wakantanka toowotanna kin, Jesus Messiya wacinyanpi kin eciyatanhan u kin hee, tona wicadapi kin owasin en, qa owasin akan un, qa owasin akiyececapi.

23 Owasin hin wahtanipi, qa Wakantanka towitan kin iyohi śni unpi.

24 Qa iye towaonśida kin on iyunwin codan owotanna wicayawapi, wopekitonpi Jesus Messiya en un kin he eciyatanhan.

$25 \mathrm{He}$ Wakantanka toka qehan ehde, wokajuju kta, iye we kin wacinyanpi kin eciyatanhan, iye toowotanna hduotanin kte, woahtani ehanna econpi qon hena kajujupi kin on etanhan.

26 Wakantanka tehan ape un kin en, nakaha iye toowotanna 239 
kin hdutanin kta e heon; hecen iye kin owotanna un kta, qa nakun tuwe Jesus wacinye cinhan, he yaowotan kta.

27 Hehan ihdatanpi kin toki un he. He anaptapi. Woope kin tukte e on; wicolian kin hee he. Hiya, wacinyanpi woope kin hee on etanhan.

28 Heon woope wicohan codan, wacinyanpi on etanhan, wicaśta kin owotanna yawapi e he unkiyukcanpi.

29 Juda oyate iśnana Wakantanka tawapi he; qa Ikcewicaśta tawapi śni he. Hiya, nakun Ikcewicaśta tawapi.

30 Tuwe wacinyanpi on bakihidayapi kin yaowotanna kte, qa wacinyanpi eciyatanhan bakihidayapi śni kin owotanna yawa kte cin he Wakantanka wanjidan.

31 Hecen wacinyanpi kin on woope lin ecetu śni unyanpi he. Hiya, hecetu śni, tuka woope kin unyusutapi.

\section{WICOWOYAKE 4.}

1 Heon, Abraham ate unyanpi kin, wicacehpi esiyatanhan taku iyeye ciqon tukte e he, unkeyapi lita he.

2 Abraham wicohan on ihduowotanna kinhan taku iwinkta kte cin hduha, tuka Wakantanka itokamya śni.

3 Wowapi wakan kin token eya he. Abraham Wakantanka wacinyan, qa woowotanna kiciyawapi qon.

4. Tuwe htani kin he wokajuju kin wowaonśida iyecen kiciyawapi śni, tuka wopetonpi kin he iyececa.

5 Tuka tuwe ohanye śni, qa tuwe tona wahtanipi-kin wicayauwotanna kin he wacinye cin- han, wacinyanpi kin he wicoowotanna kiciyawapi ece.

6 Wicaśta kin tuwe wicohan codan Wakantanka wicoowotanna kiciyawa kin wowaśte yuhe cin he Dawid oyake ciqon iyececa:

7 Tona taku śica econpi qon wicakicicajujupi, qa walitanipi kin yutokan iyewicakiciciyapi kin hena wicayawaśtepi ece.

8 Wicaśta kin tuwe Itancan kin woalitani kiciyawa śni kin he yawaśtepi ce.

9 Unkan wowaśte kin de bakihdayapi kin ecedan en au he; qa iś bakihdayapi śni kin nakun en au he. Wacinyanpi kin he Abraham woowotamna on kiciyawapi ce, unkeyapi qon.

10 Token he on kiciyawapi he; bakihdayapi kin en un, qa iś bakihdayapi śni en. Bakihidayapi en un śni, tuka bakilidayapí śni kin en icunhan.

11 Qa nahanhin bakihdayapi śni un kin en icunhan, bakilidayapi kin he wowapetogton, wowacinye woow otanna ipuspe kin hee icu; liecen tona vacinyanpi kin owasin, bawicalidayapi śni eśta, he atcyapi lita, qa nakun hena e wicoowotanna wicakiciyawapi kta.

12 Qa tona bakilidayapi kin heceepidan śni, nakun Abraham ateunyanpi, hinahin bakilidaye śni, he ehan wacinye ciqon, tona he oye kin ohna manipi kinhan, hena iś eya bakilidayapi kin en ateyapi kte cin heon.

13 Maka kin tawaye kte cin, Abraham iye cinca kin om, iwahoyapi qon he woope kin eciyatanhan śni, tuka wacinyanpi on woowotanna kin eciyatanhan.

14 Tona woope kin eciyatanhanpi kin hena tarvayapi kte cin eepi hecinhan, wacinyanpi lin 
ecetuśniyapi, qa wahoyapi qon he nakun takudan okihi śni.

15 Woope kin he wocanniye lraga; tukten woope wanica hecinhan hen woalitani nakun wanica.

16 Heon etanhan, he wacinyanpi kin on, wowaonśida eciyatanhan kte cin heon, qa wahoyapi qon cincapi kin owasin en wicakiciyusutapi kta, tona woope eciyatanhan un kin heceedan śni, tuka tona Abraham, unkiye owasin ateunyanpi kin, wacinye ciqon en opapi kin hena koya.

17 Wowapi qon iyececa; Ikcewicaśta oyate ota ateniyanpi kta e ecihde ce; Wakantanka wicate cin ake ni wicaye ca, taku tona un śni kin hena taku un kin iyecen cajeyata ece kin he wacinyan kin heon etanhan.

18 Oyate ota ateyapi kte cin he apepica śni, tuka wacinyan un kin heon ape, taku eyapi qon iyececa; Nicinca iyenaka kta ce.

19 Qa waśagya wacinye cin heon iye tancan kin wanna kan he awakicin śni, wanna waniyetu opawinge ikiyedan, qa Sara tamni kin nakun wanna ecece śni tuka.

$20 \mathrm{Qa}$ Wakantanka wahoye cin he cetunhda kin on kaktihanhan śni ; tuka wacinyanpi on wakiśaka un, qa Wakantanka yatan.

21 Qa taku iwahoye cin he ecen econ kta okihi e he nina wicada.

22 Heon etanhan he wicoowotanna kiciyawapi.

$23 \mathrm{Qa}$ he hecen kiciyawapi kin he iye iśnana on wowapi kin en kagapi śni ;

24 Tuka unkiyepi on, he unkiciyawapi kte cin lieon etanhan, tuwe Jesus Messiya Itancan unkiyapi kin wiconțe etanhan na- jin kiye ciqon he wacinunyanpi kinhan.

25 Iye waunlitanipi kin on wicaqupi, qa owotanna unyawapi kte cin on ake najinkiyapi qon.

\section{WICOWOYAKE 5.}

1 Hecen wacinyanpi on owotanna unyawapi kin, heon etanhan Wakantanka ekta wookiye unhapi, Jesus Messiya Itancan unkiyapi kin eciyatanhan.

2 Qa he iye eciyatanhan en unhiyupi, wowaonśida en ahan naunjinpi kin en, wacinyanpi kin on etanhan, qa Wakantanka towitan kin ape unkiyuśkinpi ece.

3 Qa hecedan śni, nakun wokakije on unkiyuśkinpi ece ; wokakije kin he wacin tankapi kaga e sdonunyanpi;

$4 \mathrm{Qa}$ wacin tankapi kin the wiyukcanpi, qa wiyukcanpi kin he woape ;

5 Qa woape kin he iśten wicaye śni, Woniya Wakan unqupi kin he eciyatanhan, Wakantanka towaśte uncantepi kin en unkokicicaśtanpi kin he etanhan.

6 Takudan unkokihipi śni kin he icunhan, wanna iyehantu qehan Messiya wahtanipi sa kin on ta.

7 Tuwedan wicaśta owotanna kin on țe kte śni naceca; tuka okinni wicaśta waśte on tuwe te kta tawatenye kta nace.

8 Tuka wahtanipi sa heuncapi kin he icunhan, Messiya unkiyepi on te cin he en, Wakantanka token canteunkiyapi kin hduotanin.

9 Hecen sam iyeya, nalkaha iye we kin on owotanna unyawapi kin, iye eciyatanhan wocanniye kin etanhan eunhdakupi kta.

10 Toka unyakonpi qehan he 241 
icunhan, iye Cinhintku te cin eciyatanhan, Wakantanka kici okiciciye unyanpi hecinhan, he sam iyeya kici okiciciya unyakonpi kin, iye ni un kin eciyatanhan ni unyanpi kta.

11 Qa hecedan śni, tuka nakun Wakantanka kin en unkiyuśkinpi, Jesus Messiya Itancan unkiyapi kin eciyatanhan, he etanhan nakaha wookiye unhapi.

12 Heon etanhan wicaśta wanjidan eciyatanhan woalitani wicoicage kin wicehna hiyu, qa woahtani etanhan wiconte, qa hecen wiconte wicaśta owasin akan iwicayaya, owasin wahtanipi nakaeś.

13 Woope kin itokam woahtani wicaśta kin ehna un; tuka tukten woope wanice cinhan hen woahtani wicakiciyawapi śni ece.

14 Hececa eśta, Adam qa Moses iyotahedan wiconte ounyan, Adam tuwe u kte cin oowa kin hee wahtani kin tona iyecen wahtanipi śni kin hena eepi kaeś en ounyan.

15 Tuka woalitani qon he kici wowaonśida kin akidecece śni. Wicaśta wanjidan walitani qon he on wicota tapi hecinhan, he sam iyeya Wakantanka towaonśida, qa wowaonśida on ituwicakilianpi kin he wicaśta wanjidan, Jesus Messiya kin hee on wicota en wicakiciyuota kta.

$16 \mathrm{Qa}$ wicaśta wanjidan walitani qon eciyatanhan, wowaonsida on ituwicakilianpi kin he iyecece śni. Wanjidan on etanhan wiconte iwicayacopi ; tuka wowaonśida on ituhianpi kin he woahtani ota etanhan wicayaowotanna kta.

17 Wicaśta wanjidan walitani kin on etanhan wanjidan eciyatanhan wiconte ounyan hecintanhan wiconte
$\mathbf{2} 42$ han, he sam iyeya tona wowaonśida untkana, qa wicoowotanna wicaqupi kin icupi kin hena, wanjidan, Jesus Messiya kin hee, e eciyatanhan wiconi kin en ounyanpi kta.

18 Heon etanhan woahtani wanjidan eciyatanhan, wicaśta owasin en wiconte iwicayacopi kin aupi ; he iyecen woowotanna wanjidan on etanhan wiconi iwicayaowotanna kin he wicaśta owasin en aupi.

19 Wicaśta wanjidan waanagoptan śni un qon he etanhan wicota walitani sa wicakagapi kin, he iyecen wanji waanagoptan un kin eciyatanhan wicota owotanna wicakagapi kta.

20 Woahtani ihduota kte cin heon woope kin hi, tuka tukten woahtani yuota ikicage ciqon hen wowaonśiđa nina sam iyeya ihduota.

21 Hecen wiconte en woahtani ounye ciqon he iyecen wiconi owihanke wanica en wowaonśida ounye kta, Jesus Messiya Itancan unkiyapi kin eciyatanhan.

\section{WICOWOYAKE 6 .}

1 Hecen taku unkeyapi kta he. Wowaonśida yuotapi kte cin heon woalitani en unkitanpi kta he. 2 Hiya hecetu śni. Tona woahtani ekta untapi kin, token he en nakun unnipi kta he.

3 Tona Jesus Messiya en baptem wicaqupi kin hena iye te cin en baptem wicaqupi kin he sdonyayapi śni he.

4 Heon etanhan baptem eciyatanhan he kici unhnakapi, wiconte kin en, hecen iye Atkuku towitan kin Messiya wicate cin etanhan ekicetu ye ciqon be iyecen unkiś eya wiconi teca kin en maunnipi kta. 


\section{WICOWOYAKE 7.}

5 Qa iye te cin iyacinpi kin en kici huunhdepi hecinhan, nakun he piya ikicage cin en iyecen unyakonpi kta.

6 Qa wicaśta tanina unhapi kin he iye kici icipaweh okatanpi kin he sdonunkiyapi, hecen woahtani tancan lkin he ihangyapi kta, qa hetanhan woahtani wowidag unyanpi kte śni.

7 Tuwe te cinhan, he wanna woahtani etanhan kiyuślkapi.

8 Hecen Messiya kici unțapi hecinhan, kici unnipi kta e wacinunyanpi.

$9 \mathrm{Qa}$ Messiya wicate cin etanhan ekicetuyapi qon he ake icimana te kte śni e sdonunyanpi ; tokata wiconțe tohinni yuha okihi kte śni.

$10 \mathrm{He}$ te ciqon wancadan woahtani on ta; qa he kini kin Wakantanka on ni un.

11 He iyecen niś eya woahtani on te nihdawapi, tuka Wakantanka ekta ni yaunpi, Jesus Messiya Itancan unkiyapi kin eciyatanhan.

12 Heon nitancan te kte cin en woahtani ounye śni nunwe, hecen wocantiheye śice cin anayagoptanpi kte śni.

13 Qa nihuhapi kin hena wicolian śica en woahtani wowinyunye kta e qupi śni po, tuka wicate cin etanhan ni un kin iyecen Wakantanka içiçu po, qa nihuhapi kin wicoowotanna en Wakantanka wowinyunye kiya po.

14. Woahtani icimana inidakapi kte śni. Woope kin ihukuya yaunpi śni tuka wowaonśida kin ihukuya.

15 Hecen tokeca kta; woope kin ihukuya unyakonpi śni, tuka wowaonśida ihukuya unyakonpi kin heon etanhan waunhtanipi kta he. Hiya hecetu śni.
16 Tuwe anayagoptanpi kta inidake niçiyapi kin he anayagoptanpi kin on wowidake niyanpi kin he sdonyayapi śni he; he woahtani wiconte aiyahdeye cin hee, qa iś waanagoptanpi kin on wicoowotanna kta.

17 Tuka Wakantanka wopida kiciun nunwe, woahtani inidakapi tuka qon; tuka woonspe ecedan en yeniyanpi qon he cante eciyatanhan oyapapi.

18 Qa woahtani etanhan enihdakupi kin, heon wicoowotanna wowidake niyan yaunpi.

19 Wicaśta akantu kin iyecen iwaa ce, nicehipipi waśake śni kin heon etanhan. Wicośice en nihuhapi kin aśapapi qa woahtani wowinyunniyanpi kta e iyowinyakiyapi qon, he iyecen nakaha wicayuecedan kin en nihuhapi kin wicoowotanna inidakapi lkta e iyowinkiya po.

20 Woahtani wowidake niyanpi qehan, wicoowotanna inidakapi śni.

21 Hehan taku nakaha on iniśtecapi kin hena en taku waskuyeca duhapi qon he tukte e he; hena e ihanke kin he wiconte ee.

22 Tuka nakaha woahtani etanhan enihdakupi, qa Wakantanka wowidake niyanpi, heon wicayuecedan on waskuyeca duhapi, qa he ihanke kin owihanke wanin wiconi kin hee.

23 Woahtani wolkajuju kin he wiconte ee; tuka Wakantanka taku ituwicalihian kin he, Jesus Messiya Itancan unkiyapi kin eciyatanhan, owihanke wanin wiconi kin hee.

\section{WICOWOYAKE 7.}

1 Mihunkawanji, (tona woope sdonyapi kin hena owicawakiya); woope kin wicaśta kin wo243 
widag ya, tohanyan ni un kin hehanyan, he sdonyayapi śni he.

2 Winohinca kin tuwe hihna ton kinhan, hihnaku ni un kin hehanyan, toope kin on kaśkapi ; tuka hihnaku kin wanna te cinhan hehan hihnaku toope kin etanhan kiyuśkapi.

3 Hecen hihnaku ni un kin icunhan, wicaśta tokeca hihnaye cinhan, wawicihaliapi econ eciyapi kta; tuka hihnaku te cinhan hehan woope qon etanhan kiyuśkapi qa wicaśta tokeca hihnayan eśta he wawicihahapi econ śni.

4 Heon etanhan, mihunkawanji, niś eya Messiya tancan kin eciyatanhan woope kin ekta nitapi kin; hccen tuwe toleca hihnayayapi $\mathrm{kta}$, tuwe wicate cin etanhan ekicetuyapi qon he hee; heccn Wakantanka on waskuyeca icahunyanpi kta.

5 Wicacehpi kin en unyakonpi qehan, woahtani wocantiheye kin, woope kin eciyatanhan un kin, hena waskuyeca wiconte icahkiciciye kta e heon unhuhapi kin en ohanyan.

6 Tuka nakaha woope kin etanhan unkiyuśkapi, taku on unkaskapi qon he elta untapi kin; hecen oowa tanina kin en wowidag unyanpi kte śni, tuka woniya teca kin en.

7 Hecece cinhan token unkcyapi kta he: Woope kin he woalitani kin ee he: Hiya, hecetu śni; woope eciyatanhan śni unkanś, woahtani sdonwakiye kte śni tuka qon. Woope kin, Tokan tawa yacin kte śni ce, eye śni unkanś, wocantiheye śice cin sdonwaye kte śni tuka.

8 Hehan woahtani woahope kin wowinyunye ca, miye maheh wocantiheye śice cin owasin mi244 caga. Woope codan woahtani ta wanka.

9 Ehanna woope codan ni waun; tuka woahope kin he hi qehan, woahtani kini, qa miye mata.

10 Qa woahope kin wiconi kta e on yuśtanpi qon he wiconte kin ee imicicaga ce.

11 Woahtani woahope kin wowinyunyan, hecen mahnaye ça, on makte.

12 Hecen woope kin he wakan; qa woahope kin he nakun wakan, owotanna qa waśte.

13 Hecen taku waśte lin he wiconte imicicaga he. Hiya hecetu śni. Tuka woalitani kin he woahtani hee e tanin kta, heon taku waśte on etanhan wiconțe icali miciciya; hecen woahope kin eciyatanhan woalitani kin iyotan śice kta.

14 Woope kin he wakan sdonunyanpi; tuka miye qe wicacelipi kin hemaca, qa woahtani ihukuya wiyopemayanpi.

15 Taku ecamon ece kin he iyowinwakiye śni. Taku wacin lkin he ecen ecamon śni; tuka taku śice wadake cin he ecen ecarnon ece.

16 Taku wacin śni kin he ecen ecamon kinhan, woope kin he waśte c mduotanin.

17 Qa hecen tuwe hecon kin he miyc śni, tuka woahtani miye mahen un kin hee.

18 Miye en, micehipi kin he waka, taku waśte en maun śni e sdonwakiya. Ieiconzapi kin he iyemicihantu, tuka token taku waśte ecamon kte cin iyewakiye śni.

19 Taku waśte wacin kin he ecen ecamon śni; tuka taku śica wacin śni kin hee ecen ecamon ecc.

20 Hecen taku wacin śni kin 


\section{WICOWOYAKE 8.}

he ecen ecamon kinhan, tuwe hecon kin he miye śni, tuka woahtani miye mahen un kin he hecon.

21 Hecen woope wanji iyewakiya, taku waśte ecamon wacin eca, taku śica miye en miciun ece.

22 Wicaśta mahentu kin eciyatanhan Wakantanka toope kin on imduślkin :

23 Tuka mihuha kin en woope tokeca wan wanmdaka, he mitawacin toope kin kici kicize ca, woahtani woope mihuha kin en un kin he en wayaka amahda.

24 Wicaśta nina iyokiśice cin he miye; tuwe wiconte tancan kin de etanhan emahdaku kta he.

25 Wakantanka wopida ewakiya ece, Jesus Messiya Itancan unkiyapi kin hee on etanhan. Hecen miye mitawacin kin on Wakantanka toope kin wowidag mayan, tuka wicacehpi eciyatanhan woahtani woope kin he imadaka.

\section{WICOWOYAKE 8.}

1 Heon etanhan, nakaha tona Jesus Messiya en un kin, hena wiconte iwicayacopi wanin unpi, wicacehpi eciyatanhan manipi śni, tuka Woniya kin eciyatanhan.

2 Wiconi Woniya toope kin Jesus Messiya en un kin, he woahtani wiconte ko woope kin etanhan makiyuśka.

3 Woope kin wicacehpi eciyatanhan waśake śni un qon he taku okihi śni kin hee Wakantanka econ, iye Cinhintku wicacehpi wahtani ouncage kin en hiyuya, woahtani on, qa wicacehpi kin en woahtani yaco.

4 Hecen tona wicacehpi kin eciyatanhan maunnipi śni, tuka
Woniya eciyatanhan, unkiyepi en woope wicoowotanna kin ccetu kta.

5 Tona wicacehpi eciyatanhan un kin hena wicacehpi taku tawa kin awacinpi; qa tona Woniya kin eciyatanhan un kin hena taku Woniya tawa lkin awacinpi.

6 Wicacehpi awacinpi kin he wiconte kin ee; tuka Woniya awacinpi kin he wiconi qa wookiye kin hee.

\% Wicacehpi wicotawacin kin he Wakantanka toka ya. Ho Wakantanka toope kin ope śni, qa nakun ecaca okihi śni.

8 Hecen tona wicacelipi kin en un kin hena Wakantanka iyokipi yapi kta okitpanipi.

9 Tuka niyepi wicacehpi kin en yaunpi śni, Woniya kin en yaunpi, Wakantanka Taniya kin niyepi kin en ounye cinhan. Qa tuwe Messiya Taniya kin yuhe śni kinhan he tawa śni.

10 Qa Messiya en niunpi kinhan, wicatancan kin he woalitani eciyatanhan ța, tuka wicanagi kin he wiconi un, wicoowotanna eciyatanhan.

11 Tuwe Jesus wicate cin etanlhan najin kiye ciqon he Taniya kin niyepi en ounye cinhan, tuwe Messiya wicate cin etanhan najin kiye ciqon hee nitancan tapi kte cin ekicetu ye kta, iye Taniya Wakan niyepi en ounye cin he eciyatanhan.

12 Heon etanhan, mihunkawanji, wicacehpi kin takudan ikicazo śni unyakonpi, on etanhan wicacehpi kin eciyatanhan unnipi kta.

13 Wicacehpi kin eciyatanhan yanipi kinhan nițapi kta; tuka Woniya kin eciyatanhan wicatancan wicohan kin te yalkiyapi kinhan yanipi kta. 


\section{ROMA.}

14 Wakantanka Taniya kin tona yus awicaye cin hena Wakantanka cinca wicaye cin eepi.

15 Ake wokokipe kta e on ookiyeyapi woniya kin iyacupi śni, tuka cinhintkuyapi woniya kin he iyacupi, on Abba, Ate, eya hoyeunkiyapi ece.

$16 \mathrm{Qa}$ Wakantanka cinca kin heuncapi e unnagipi kin kici Woniya kin e unyuotaninpi.

17 Unkan uncincapi kinhan tawayapi kte cin heuncapi ; Wakantanka tawayapi ; Messiya kici tawayapi kte cin; kici unkákijapi kinhan, nakun kici wowitan unhapi kta ce.

18 De nakaha wicokakije kin he, wowitan unkiyepi en ecadan yutaninpi kte cin kici akiyecen yawapi kta e iyecece śni wadaka.

19 Wakantanka cincá wicayutaninpi kte cin on taku kagapi kin cantokpaniyan ape yanka.

20 Talu kagapi kin he woecetu śni kin en ihukuya ye kta cin śni, tuka tuwe woape on he wowidagye cin hee on etanhan.

21 Qa taku kagapi kin hee kaeś wicahwin wowidagyapi kin etanhan kiyuśkapi kta, qa Wakantanka cincawicaye cin ihduhapi wowitan kin en yeyapi kta.

22 Taku icage cin owasin dehanyan witaya comnihdazi, qa hokśiksuya un e sdonunyanpi.

23 Qa hecedan śni, tuka unkiś eya Woniya kin taku tokaheya icahya unhapi kin, unkiyepi qe comniunhdazipi, qa a tayedan cinca yapi kte cin unkapepi, unkitancanpi ekicetu ye kte cin hee.

24 Woape kin on niunyanpi; tuka woape wanyakapi kin he woape kin ee śni, tuwe taku wanyake cinhan tokeca e nahahin ape yanka he.

25 Tuka taku wanunyakapi śni 246 kin he unkapepi hecinhan, hehan wacintankapi on unkicupi kta e ape unyakonpi ece.

26 Qa nakun Woniya kin iś waunśakapi śni kin en icunhan ounkiyapi ece. Token wocekiye unkeyapi kte cin sdonunkiyapi śni, tuka Woniya kin e qe unkiyepi on wocekiye eya, howayapi oyagpica śni kin on.

27 Qa tuwe wicacante iyukcan kin he Woniya kin token wacin yuze cin sdonya, he Wakantanka tawacin kin eciyatanhan tona owotanna un kin hena on wocekiye eya ece.

28 Unkan Wakantanka waśte dakapi kin hena taku owasin tanyan owicakihan ece e sdonunyanpi, tona iye tawacin kin eciyatanhan wicakicopi kin hena.

29 Tona otokaheya tanhan sdonwicakiye ciqon hena wicakiconza, iye Cinhintku ouncage kin en icagapi kte cin heon, qa hecen he hunkawanjitku ota ehna tokapa kta.

$30 \mathrm{Qa}$ tona wicakiconze ciqon hena wicakico; qa tona wicakico qon hena wicayaowotanna; qa tona wicayaowotanna qon hena iś eya wicayutan.

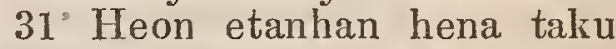
kin en taku unkeyapi kta he. Wakantanka unkicipi un kinhan, tuwe kaśeunyanpi kta he.

32 Tuwe iye Cinhintku kin kpatan śni, tuka unkiyepi owasin on kpagan qon, he iye kici taku owasin iyakiçuya unqupi kte śni he.

33 'Tona Wakantanka wicakahnige cin hena tuwe taku iyawicaonpe kta he. Wicayaowotanna kin he Wakantanka kin ee ce.

34 Tuwe wayaco kta he. Tuwe te ciqon he Messiya ee, qa ake 
piya ikicage cin hee, Wakantanka etapa kin eciyatanhan yanke ca, unkiyepi on wocekiye eciya. 35 Tuwe Messiya towaśte kin etanhan unyuśpapi kta he. Wicokakije, wośitkihda, wicokicize, wicaakihan, tancowicakadan, wokokipe, qa maza sagye okihi kta he.

36 (Owapi qon he hecetu, Niye kin on anpetu ohinniyan unktepi unyakonpi, tahinca ktepi kte cin iyecen unyawapi ce.)

37 Tuka hena taku kin owasin en, tuwe canteunkiyapi qon he eciyatanhan, tanyan ohiye unyakonpi.

38 Heon, wiconte, qa wiconi, qa mahpiya ohnihde cin hena, qa wokiconze kin, qa wicowaśake kin, qa taku nakaha un kin taku u kte cin ko;

39 Qa taku wankantuya un kin, qa taku mahentuya un kin, qa taku icage cin tokeca, takudan Wakantanka towaśte, Jesus Messiya Itancan unkiyapi en un kin, he etanhan unyuśpapi kte śni e sdonwaye hinca.

\section{WICOWOYAKE 9.}

1 Messiya eciyatanhall wowicake epe, itonśni mawanica, minagi kin hee e Woniya Wakan kin kici mahdutanin;

2 Nina iyomakiśice ca micante ekta ohinniyan iyokiśin waun.

3 Mihunkawanji qa tona wicacehpi eciyatanhan takuwicawaye cin hena on, Messiya etanhan mayacopi tawatenwaya owakihi tuka.

4 Hena Israel wicaśta kin hecapi, qa atayedan cinkśi wicaye cin hena eepi, qa wowitan, qa wicotakuye, qa woope wicaqupi - kin, qa wawokiyapi kin, qa wahoyapi kin hena tawapi.
5 Qa hunkake wicayapi kin etanhanpi kin hena eepi, qa hena etanhan wicacehpi eciyatanhan Messiya inapa, he owasin iwankam Wakantanka kin ee, owihanke wanin wowaśte yuha nunwe.

6 Wakantanka oie kin ecetu sni aye cin he wake śni. Tona Israel etanhanpi kin hena owasin Israel hecapi śni.

7 Qa Abraham etanhan icagapi kin heon owasin cincapi kin hecapi śni. Tuka, Isaak etanhanpi kin hena nicinca ewicakiyapi kta ce.

8 He kaketu, tona Wakantanka cinca wicaye cin hena wicacehpi eciyatanhan cinca wicayawapi qon heon etanhan śni, tuka tona cinca wahoyapi qon hena $\mathrm{e}$ wicowazi kin ee iyawapi.

9 Wahoyapi wicoie kin he decetu, Omaka dehantu kinhan wau kta, qa Sara cinhintku wan ton kta ce.

10 Qa heceyedan śni, tuka Rebeka nakun wanjidan on, Isaak ateunyandi kin hee on, ihduśake çehan,

11 Nahanhin tonpi śni, taku waśte taku śica ko unmana nahanhin econpi śni, Wakantanka tawacin kin wicakahnigapi kin en sutaya un kte cin heon, wicohan kin eciyatanhan śni, tuka tuwe wicalkico kin he eciyatanhan ;

12 Hehan heciyapi, Hakakta kin he tokapa kin wowidag ye kta ce.

13 Owapi qon he kaketu, Jakob waśte wadalka, tuka Esau śice wadaka ce.

14 Hecen token unkeyapi kta he. Wakantanka owotanna śni econ he. Hiya, hecetu śni.

$15 \mathrm{He}$ Moses heciya; Tuwe onśiwakida kte cin he onśiwaki247 
da kta, qa tuwe wacin en mduze kte cin he wacin en mduze kta ce.

16 Heon etanhan tuwe cin kin eciyatanhan śni, qa tuwe inyanke cin he nakun eciyatanhan śni, tuka Wakantanka onśi wicakida kin he eciyatanhan ece.

17 Wowapi wakan kin he Pharo heciya; He dee on etanhan icahciya, niye en mitowaśake kin wahduotanin kta, qa maka owancaya micaje kin oyakapi kte cin heon.

18 Heon etanhan tuwe kahnige cinhan, he onśikida ece; qa tuwe kahnige cinhan he yuteli ece.

19 Hecen hemayakiye kta nace; Tokeca e nahanhin wicaba he ; tuwe e iye tawacin kin ecetuśniya he.

20 Hiya, wicaśta kin, niś nituwe he, hecen Wakantanka i en hiyeyaya he. Taku kagapi kin he tuwe kage cin, Tokeca hecen mayakaga he, eciye kta he.

21 Maka wakśica kage cin heca maka kin okihi śni he, upśija omnaye wanjidan etanhan waksica wan woyuonihan, qa wanji iś wowiśtece on kage kte śni he.

22 Hecen Wakantanka wocanniye kin hdutanin kta cin, qa towaśake kin sdonye unkiyapi kta e on wocanniye wakśica wicotakuni śni kicagapi qon hena wawacinkta yuza tanka on wicakuwa kinhan;

$23 \mathrm{Qa}$ tona wowaonśida wakśica kin otokaheya tanhan wowitan on wiyeya ehnakapi qon, hena en iye towitan wowijice kin hduotanin kta.

24. He wicakico kin hena unkiyepi, Juda oyate etanhanpi kin hecedan śni, tuka nakun Ikcewicaśta etanhanpi.

25 Hose en heye ciqon iyececa; 248
'Tona mitaoyate śni kin hena mitaoyate ewicawakiye kta, qa waśte dakapi śni qon he waśte dakapi kin ewakiye kta.

26 Qa tukten, Mitaoyate kin he niyepi śni ce, ewicakiyapi qon, hen, Wakantanka ni un cinca kin ewicakiyapi kta ce, eya.

27 Isaya iś eya Israel oyate kin on hotanin; Israel cincapi kin miniwanca wiyaka sukaza iyecen ota yawapi eśta, tona okaptapi kin henana nipi kta ce, eya.

28 Wayawapi yuptecedan kta qa wicoowotanna en hduśtan kta; maka akan Itancan kin wayawapi ptecedan wan kage kta. $29 \mathrm{Qa}$ he itokam Isaya heye ciqon; Sabaoth Itancan kin wicowazi wan unkokicicaptapi śni unkanś, Sodom iyecen unyakonpi kta, qa Gomorra en unkiyoptapi kta tuka.

30 Hecen token unkeyapi kta he. Ikcewicaśta kin wicoowotanna iyeyapi ce, wacinyanpi kin eciyatanhan wicoowotanna kin hee.

31 Tuka Israel wiconwotanna woope odepi qon hena wicoowotanna woope kin iyohipi śni.

32 Tokeca he. Wacinyanpi kin eciyatanhan śni, tuka woope wicohan kin eciyatanhan odepi kin, heon etanhan. Inyan ibotopi kin de on siha ibotopi.

33 Wowapi kagapi qon iyececa; Wanyaka wo, Zion en inyan ibotopi, qa imnija on hicahanpi wan ewahde; qa tuwe he wacinye cinhan inihan kte śni.

\section{WICOWOYAKE 10.}

1 Mihunkawanji, micante cantiheye ca taku Wakantanka icewakiye cin, Israel oyate kin wi- • cani kte cin hee. 
2 Hena wicaweciyaotanin, Wakantanka wacin en yuzapi tuka he wosdonye eciyatanhan śni.

3 Wakantanka toowotanna kin sdonyapi śni, qa iye wicoowotanna tawapi kin hdusutapi akitapi, qa Wakantanka toowotanna kin he ihukuya iyeiçiyapi śni.

4 Tona wicadapi kin owasin en Messiya kin he wicoowotanna woope woyuśtan kin hee.

5 Woowotanna woope kin eciyatanhan kin he on Moses kaken owa qon; Wicaśta tuwe hena taku kin econ kinhan, hee hena on ni kta ce.

6 Tuka wacinyanpi eciyatanhan wicoowotanna un kin he kaken eya, Nicante kin mahen, Messiya kun ahi kte cin heon tuwe malpiya ekta wankan ye kta he ;

7 Qa, Wicate cin etanhan Messiya yuwankan au kte cin heon tuwe wośbe kin ekta ye kta he, eye śni wo.

8 Tuka taku eya he; Wicoie kin he nikiyedan un, nii kin ohna, qa nicante kin en un, wacinyanpi wicoie unkoyakapi kin he hee.

9 Nii kin on Jesus Itancan kin yahdaotanin, qa he wicate cin etanhan Wakantanka ekicetu ye cin he, nicante kin eciyatanhan wicayada kinhan, yani kta.

10 Wicoowotanna kta e wicacante on wicadapi, qa wiconi kta e wicai kin on wohdakapi ece.

11 Wowapi wakan kin he heya, Tuwe he wacinye cinhan, he wiśtece kte śni ce.

12 Juda qa Helenes oyate kin kici ocikpanipi wanica. Tuwe owasin en Itancan kin he tona hoyekiyapi kin hena owasin en wijinya un.

13 Tona Itancan caje kin ho- yekiyapi kinhan hena otoiyohi nipi kta.

14 Tuka tuwe kin wacinyanpi śni kinhan token on he hoyekiyapi kta he; qa he nahahin nahonpi śni kinhan token wacinyanpi kta he; qa tuwedan oyake śni kinhan token nahonpi kta he.

15 Qa ekta ye wicaśipi śni kinhan token oyakapi kta he. Owapi qon he hecetu; Tona wookiye wotanin waśte kin oyakapi, qa taku waśte en wotanin waśte oyakapi kin hena sihapi kin iyotan waśte ce.

16 Tuka owasin wotanin waśte kin anagoptanpi śni. Isaya heye ciqon; Itancan, tuwe unkoyakapi kin wicada he.

17 Heon etanhan wanahonpi kin eciyatanhan wicadapi kin $u$, qa wanahonpi kin he Wakantanka oie kin eciyatanhan.

18 Unkan miś, Hena nahonpi śni he, epe. Hiya, hena oqo kin makoce owasin ekta iyaye ca wicoie kin maka ihanke kin hehanyan $i$.

$19 \mathrm{Qa}$ Israel oyate kin he sdonyapi śni he. Tokaheya Moses heya, Tona oyate śni se un kin hena on iyocipaśtakapi kta, qa ikce oyate wacinton śni wan on śinhdaciyapi kta ce.

20 Tuka Isaya nina waditake ca heya; Tona omadepi śni qon hena e iyemayanpi, qa tona amakitapi śni qon hena en mihdutanin ce.

21 Qa Israel oyate kin hewicakiya, Anpetu osan oyate wanahon śni qa wacețunhdapi kin he nape wicawakiyugata ce.

\section{WICOWOYAKE 11.}

1 Heon hepa, Wakantanka taoyate kin ehpewicakiya he. Hiya hecetu śni. Israel wica- 
śta kin hemaca, Abraham cinca, qa Benjamin wicoun kin hematanhan.

2 Wakantanka taoyate kin tona otokaheya tanhan sdonwicakiye ciqon hena ehpewicakiye śni. Wowapi wakan Elijah on token eye cin sdonyayapi śni he; Wakantanka cekiye ca, Israel oyate kin cajeyata qa heya ;

3 Itancan, wicaśta wokcan nitawa kin hena wicaktepi, qa wahna wośnapi nitawa kin yujujupi, qa miye miśnana omakaptapi, unkan te mayanpi kta akitapi ce.

4 Tuka Taku Wakan kin token eya ayupta he; Nahahin Baal en canpeśka makehde inajinpi śni kin wicaśta kektopawinge śakowin wicawahduha ce.

5 He iyecen nakaha wowaonśida wicakahnigapi kin eciyatanhan hunh okaptapi.

$6 \mathrm{Qa}$ he wowaunsida eciyatanhan kinhan hehan wicolian eciyatanhan śni; hecetu śni kinhan, wowaonsida kin wanna wowaonsida heca śni. Qa wicohan eciyatanhan kinhan, hehan wowaonsida eciyatanhan śni ; hecece śni kinhan, wicohan kin wanna wicohan heca śni.

7 Hecen tokctu kta he; Israel taku akitc ciqon he iyeye śni, tuka tona wicakahnigapi kin hena e iyeyapi, qa unmapi kin iśta gonge wicayapi.

8 Wowapi qon iyececa; Wicaliba wicacante kin Wakantanla wicaqu, iśta on wiwanyakapi kte śni, qa noge on winahonpi kte śni, anpetu kin dehanyan.

9 Qa Dawid iś heya; Wahna wotapi tawapi kin he mazahtakiyapi, qa wihmunke, qa wonalnaye, qa iyunwin yuhapi kin hee kta ce.

10 Iśta kin aotpaswicayapi kte, heon wawanyakapi kte śni; qa cankahu ohinniyan patuś inajinpi nunwe.

11 Heon hepe; Hinhpayapi kta on hicahanpi he. Hiya, hecetu śni ; tuka hicahanpi kin on etanhan Ikcewicaśta kin wiconi iyowicahi, hena iś eya iyowicapaśtakapi kta e heon.

12 Hena e hicahanpi kin he wicoicage lin en wowijice kin hee, qa hena wicayuanptetu kin he Ikcewicaśta kin en wowijice kin hee hecinhan, wicayuecetupi kin he sanpa hince kta.

13 Ikcewicaśta henicapi kin hena hecen eciciyapi; Ikcewicaśta lin ekta yemaśipi waun, qa heon mitohtani kin wahdutan.

14 Hecen tona micehpi etanhanpi kin hena inalini wicawaye kta, kinhan wanjikji ni wicawaye kta.

15 Hena ehpewicayapi kin he wicoicage kin en wookiye kin hee hecinhan, hena ake wicayuecetupi kinhan he taku kta he; wiconte etanhan wiconi kin hee kta.

16 Taku tokaheya suton kin he wakan kinhan, ocowasin hecece kta; qa can huta kin he wakan kinhan, can adetka kin hena nakun hecece kta.

17 Unkan can adetka kin hena wanjikji yuksapi, qa niś can wihdi hu ikceka henica, tuka can adetka qon ehna can kin en icahniyanpi, qa can wihdi cepa can huta kin etanhan, en opeya yaun kinhan ;

18 Can adetka qon on ihdatan śni wo. Nihdatan keś can huia kin he duhe śni tuka can huta kin hee niyuha.

19 Hehan, Hen emahdepi kta on can adetka qon yuksapi ce, ehe kta naceca.

$20 \mathrm{Han}$, hecetu; wicadapi śni 
kin heon hena yuksapi, qa niye wacinyanpi on ecen nayajin ece. Wahaniçida śni qa kokipa wo.

21 Can adetka tawa qon hena Wakantanka kpatan śni hecinhan, okinni niś eya nikpatan kte śni.

22. Wakantanka towaonśida tocantiyahde ko wanyaka po ; tona hinhpayapi qon hena en wocantiyahde, qa niye en wowaonśida kin, wowaonśida kin he en nayajin kinhan, tuka hecanon śni kinhan niś eya nicaksapi kta.

23 Qa hena cețunhdapi ecen unpi śni kinhan, iś eya ake can kin en iyekiyapi kta. Ake can kin en Wakantanka icahwicaya okihi.

24 Qa niye can wihdi ikceya icage ciqon etanhan nicaksapi, qa inicage cin eciyatanhan śni, can wihdi waśte lin en iyeniyanpi kinhan, tona can kin he aicage ciqon liena iyotan ake can wihdi tawapi kin en iyeyapi kta.

25 Mihunkawanji, wowiyukcan kin de sdonye śni dukanpi kta wacin śni, okinni ksape niçidapi kta; Israel oyate kin apa iśta gonge aya, Ikcewicaśta kin ocowasin en hiyu kte cin hehanyan. $26 \mathrm{Qa}$ hecen Israel oyate kin owasin nipi kta. Wowapi en kagapi qon iyecetu ; Zion etanhan Wanikiya wan $u$, qa woalitani Jakob yutokan iyekiciciye kta.

$27 \mathrm{Qa}$ woahtani yutokan iyewicaweciciye cinhan hehan wicotakuye wicawaqu kte cin hee ce.

28 Wotanin waśte kin ekta, hena niyepi kin on etanhan toka wicayawapi, tuka wicakalinigapi lin ekta, hena e hunkakepi kin on etanhan waśte wicakidapi.
29 Wakantanka taku wicaqu qa wicakico kin hena iyopeiciyapi wanin un.

30 Unkan niś niyepi kin ehanna Wakantanka anayagoptanpi śni, tuka nakaha hena wicadapi śni kin on wowaonśida niçupi.

31 He iyecen nakaha hena iś eya anagoptanpi śni kin, niye nitowaonśida kin on etanhan onśiwicadapi kta.

32 Hena owasin witaya wicadapi śni kin en Wakantanka onawicataka, iyuhpa onśiwicada kta heon etanhan.

33 Wakantanka toksape qa wosdonye tawa wowijica ośbe kin lie tanka hinca. Woyaco tawa kin okahnilipica śni, qa tacanku kin iyeyepica śni.

34 'Tuwe Itancan tawacin kin sdonya he; qa tuwe okiya un he.

$35 \mathrm{Qa}$ tuwe he tokaheya taku qu he ; kinhan kicicajujupi kta. 36 Iye etanhan, qa iye eciyatanhan, qa iye ohna taku owasin un; owihanke wanin wowitan kiciun nunwe.

\section{WICOWOYAKE 12.}

1 Heon mihunkawanji, Wakantanka wacantohnake cin on etanhan eciciyapi ce, nitancanpi kin wośnapi ni un, wakan, qa taku Wakantanka iyokipi kin, hena hecen yaqupi kta, he wawoyakiyapi kte cin hecetu hinca.

2 Qa wicoicage kin de en iyecen awacanipi kte śni, tuka nitawacinpi yuecepidan kte cin on nihdutokecapi nunwe; hecen Wakantanka tawach waśte, oiyokipi, qa hduśtanpi kin he tukte e idukcanpi kta.

3 Wowaonśida maqupi kin he eciyatanhan; tona niyepi ekta un kin otoiyoli hecen eciciyapi; Tu251 
wedan token ilidukcan kte cin he isanpa ihdawa kte śni, tuka wacinksamya ihdukcan kta, Wakantanka otoiyohi wacinyanpi iyuta wicaqu kin he eciyatanhan.

4. Wicatancan wanjidan en wicahuha ota unhapi, qa huha kin hena owasin okonwanjidan ohanyc śni :

5 He iyecen unkiye unkotapi, tuka Messiya en tancan unwanjipidan, qa otoiyohi huha unkiciyapi.

6 Hecen wowaonśida otoiyohi yukinukanyan unqupi kin eciyatanhan wicookihi ociptetu unhapi; he wokcanpi kte cin hee hecinhan, wowacinye iyutapi kin on unkiyukcanpi ece e kta.

7 Qa waowicakiyapi kin hee hecinhan, wawicakiyapi kin en unyakonpi kta; qa tuwe waonspekiya okihi kinhan, he waonspekiyapi kin en un kta.

8 Tuwe wahokonwicakiye kte cin he wahokonwicakiyapi kin en un kte; tuwe taku wicaqu litc cin he ohanpiya econ kte; tuwe oyate wicayuhe cinhan he mnihenya ccon lita; qa tuwe onśiwicadake cinhan iyokipiya econ lkte.

9 Wacantkiyapi kin he wohnaye wanin un nunwe. Taku śice cin he sice dakapi, qa taku waśte kin he en iyailidaśka po.

10 Hunkawanjin kiciyapi waste kicidakapi kin he on otoiyohi cante cn kiciyuza po, qa woyuonitian on otoiyohi iyotan kicidaka po.

11 Wicohtani kin en kujapi śni, woniya on aiciciyapi, Itancan kin okiya po.

12 Woape en wiyuślkinpi; kakijapi kin en icunhan wacintankapi; qa wocekiye eya yukan po.
13 Tona owotanna un kin taku icakijapi kinhan owicakiya po. Wicaśta icimanipi kin ohaupiya ecawicakicon po.

14. Tona śicaya nicuwapi kin hena wicayawaśte po; wayawaśte po, qa wayaśicapi śni po.

15 Tona wiyuśkinpi kin hena om wiyuślkin po; qa tona ceyapi kin hena om ceya po.

16 Otoiyohi okonwanjidan awakicicin po. Taku wankan un kin hena ekta cwaciupi śni po; tuka tona kun ihdawa kin hena en opa po. Wacinksapeicidapi śni po.

17 Tuwedan taku śica on itkom śicaya econpi śni po. Wicaśta owasin itokamya taku owotanna ecedan kamnan po.

18 Tohanyan oyakihipi kinhan, wicaśta owasin om wookiye yakon po.

19 Waśte cicidapi kin, ihnuhan niye cinka watoyeconpi kin, tuka wocanniye nakicipa po. Hecen wowapi en kagapi, Tokiconpi kin he miye mitawa, wicawecu kta ce, Itancan kin eya. 20 Heon tuwe toka niye cin he wotektchda kinhan wo qu wo; qa ipuza kinhan mini qu wo; hecen ecanon kinhan, petaga kin he pa kin en eju ade lita.

21 Ihnulian taku sica on ohiniyanpi kin, tuka taku waśte e on taku śice cin ktepidan po.

\section{WICOWOYAKE 13.}

1 Wicanagi otoiyohi wicowaśake itancan kin hena ihukun iyeiçiye kte. Wicowaśake Wakantanka etanhan un kin hecedan yukan. Wicowaśake un kin hena Wakantanka kaga.

2 Heon etanhan tuwe wicowaśake kin kipajin kinhan he Wakantanka toope kin kipajin, 


\section{WICOWOYAKE 14.}

qa tona wawakipajin kin hena wicayacopi kta.

3 Wicaśtayatapi kin hena wicohan waśte kin en wakokipe wicakicagapi śni, tuka wicohan śice cin en. Hecen wicowaśake kin he koyakipe kte śni he. Taku waśte e econ wo, kinhan on niyatanpi kta ce.

4 He taku waśte on niye ekta Wakantanka taokiye kin hee. Tuka taku śica ecanon kinhan kokipa wo. He mazasagye ituh yuhe śni ; tuka Wakantanka taokiye kin heca, tuwe taku śica econ kinhan tokicon kta, wocanniye kin heon.

5 Heon etanhan anagoptan yaunpi kta, wocanniye kin hecedan on etanhan śni, tuka nakun wiyukcanpi kin heon.

6 Heon etanhan mazaska mnakiyapi kin en oyapapi ece: hena e Wakantanka taokiyepi kin ohinniyan taku kin de econ yulianpi.

7 Heon etanhan, owasin taku token wicaqupi kta iyecece cin wicaqu po: Tona iye taoyate kin etanhan mazaska mnayanpi kin he ecen wicaqu po; qa tona oyate tokeca etanhan mnayanpi lin he nakun ecen wicaqu po. Tona kinihanpi kte cin hena wicakinihan po, qa tona yuonihanpi kte cin hena wicayuonihan po.

8 Tuwedan takudan ikicazo śni un po, otoiyohi waśte yecidakapi kte cin hecedan. Tuwe wicaśta tokeca waśte wicadake cin he woahope kin tanyan yuha.

9 He decetu; Wawiciliahapi ecanon kte śni; Tin wicayakte kte śni; Wamayanon kte śni; Tuwedan en oitonśniyan wodalie kte śni; Taku tokan tawapi kin yacin lite śni; qa woahope tokeca yuke cinhan wicoie kin de en yuwitayapi; Wicaśta tokeca niye inihdacin waśte yadake kta ce.

10 Waśtewicadakapi kin he wicaśta tokeca taku śica takudan ecakicon śni, heon waśtewicadakapi kin he woahope yuecetupi kin hee.

11 Qa nakun kaketu, anpetu kin sdonunyanpi, woiśtinma etanhan unkiktapi $k$ te cin wanna iyehantu; nakaha eunhdakupi kte cin tokaheya wicaundapi qon he isanpa ikiyadan ilian ;

12 Hanyetu kin wanna ecadan unkihunipi kta, anpetu kin kohanna u kta, heon etanhan otpaza wicohan kin ehpeunyanpi, qa iyoyanpa wipe kin kounyakapi kta.

13 Qa iyokipiya maunnipi kta, anpetu kin en iyececa. Hanyetu wacipi qa witkopi kin en unkopapi kte śni; wicawihomnipi qa wicinpi, wakinicapi qa winawizipi kin hena en ounpapi kte śni.

14 Tuka niye Jesus Messiya Itancan kin he koyalka po, qa wicacehpi wocantiheye śice cin ecetu yapi kta e on takudan kicamnanpi śni po.

\section{WICOWOYAKE 14.}

1 Tuwe wicada kinhan waśake śni eśta iyowinkiya po, tuka akipam waakinicapi kin ekta śni.

2 Wicaśta wanjidan taku owasin yuta okihi awacin, qa wicaśta tokeca waśake śni kin he wahpe ece yuta.

3 T'uwe wote cinhan he tuwe wote śni kin ihaha kte śni; qa tuwe wote śni kinhan he tuwe wote cin yaco kte śni ; Wakantanka he iyowinkiya.

4 Nituwe he wicaśta tokeca taokiye kin daco he; he iye yu- 
he cin en najin kta qa iś hinhpaye kta. Qa he ecen najin kiyapi kta, Wakantanka najin kiye kta okihi.

5 Wicaśta wanji anpetu wanji iyotandaka, qa wicaśta tokeca wan anpetu owasin akiyecen yawa ece. Otoiyohi iye tawacin ekta sutaya awacin nunwe.

6 Tuwe anpetu wan awacin kinhan he Itancan kin on awacin ; qa tuwe anpetu kin awacin śni kinhan, Itancan kin ekta awacin śni. Tuwe wote cinhan, he Itancan kin on wota, qa Wakantanka wopida eciya; qa tuwe wote śni kinhan, Itancan kin on wote śni, qa Wakantanka wopida eciya ece.

7 Unkiyepi kin tuwedan iye hinca on ni śni, qa tuwedan iye hinca on te śni.

8 Unnipi kinhan Itancan kin on unnipi, qa unțapi kinhan Itancan kin on untapi. Hecen unnipi, qa untapi unma tukte hecinhan, Itancan kin tawa unyanpi.

9 Hecen on Messiya te ca ake kini, wicate ca wicani kin heniyoza en Itancan kta e heon.

10 Tokeca nihunkawanji daco he ; qa tokeca nihunkawanji iyakihiaka he. Owasin Messiya tawoyaco oiyotanke kin itokam inaunjinpi kta.

11 Hecen wowapi en kagapi; Miye wani kin, heon hupahu otoiyohi miye en canpeślka makehde inajin $\mathrm{kta}$, qa wicaceji otoiyohi Wakantanka en wohdala kta, Itancan kin eya.

12 Heon etanhan unkiyepi otoiyohi Wakantanka kin en wounhdakapi kta.

13 Hecen on, detanhan otoiyohi unkiciyacopi kte śni, tuka kaken e iyotan wakonza po, tuwedan taku en siha ibotopi, qa on hinhpaye kte cin he hunkawanjitku kin itokam ehde kte śni.

14 Miś sdonwaye ca Jesus Itancan kin en awacami, takudan atayedan śape śni ; tuka tuwe taku śapa kecin kinhan, he taku kin iye ek ta śapa ece.

$15 \mathrm{Qa}$ woyute kin on etanhan nihunkawanji iyokiśin yaye cinhan wowaśtedake eciyatanhan mayani śni. Messiya tuwe on te ciqon he woyute nitawa kin on ihang ye śni wo.

16 Ihnuhan taku waśte duhapi kin he yaśicapi kinhan.

17 Woyute qa woyatke kin Wakantanka tokiconze kin hee śni ; tuka wicoowotanna wookiye qa Woniya Wakan kin en wowiyuśkin kin hena ee.

18 Tuwe hena taku kin en Messiya wowidag ye cinhan, he Wakantanka iyokipi ye ca, wicaśta lin on waśte dakapi.

19 Heon etanhan taku tona wookiye kage cin hena unkakitapi kta qa nakun taku on otoiyohi icah unkiciciyapi kta.

20 Woyute kin on etanhan Wakantanka taku kage cin he ihang ye śni wo. Awicakehan taku owasin waśte; tuka tuwe taku yute ca on wahtani wicaye cinhan, he śica ece.

21 Waconica yutapi śni, qa mini śa yatkanpi śni, qa taku tokeca on etanhan nihunkaw anji hicahe ca, wahtani, qa waśale śni aye cin, hena econpi śni kinhan he waśte.

22 Wowacinye duha he: Niś niye Wakantanka itokamya hduha wo. Tuwe taku iyowinkiye cin on ihdaco śni kinhan, he yuwaśtepi cce.

23 Qa tuwe wicada śni kinhan he yute cinhan wanna yacopi, wacinyanpi kin on hecon śni, 
WICOWOYAKE 15.

heon etanhan. Taku owasin wacinyanpi eciyatanhan śni kin hena woalitani.

\section{WICOWOYAKE 15.}

1 Unkiye waunśakapi kin tona waśake śni un sutaya wacinyanpi śni kin on wacinuntankapi lita, qqa iyokipiunkiciyapi kte śni.

2 Otoiyohi wicaśta tokeca iyokipi unyanpi kta, wowaśte en icali yapi kte cin heon.

3 Messiya iye kaeś iyokipi ic̣iye śni, tuka wowapi en kagapi qon iyecen, Tona aniiapi kin waaiapi kin he miye aihpemayanpi qon.

4 Taku tona wowapi en kagapi kin hena onspeunkiyapi kte cin heon kagapi, hecen wicowalibadan, qa wowapi wakan kin en wicakicanpte cin eciyatanhan woape unhapi kta.

5 Wakantanka wicowahibadan, wicakicanpte ko yuhe cin he Jesus Messiya eciyatanhan, otoiyohi okonwanjidan wacin en kiciyus niyanpi nunwe.

$6 \mathrm{Qa}$ hecen wicotawacin wanjidan, qa wicai wanjidan on Wakantanka Jesus Messiya Itancan unkiyapi he Atkuku kin datanpi kta.

7 Heon etanhan otoiyohi iyowinkiciciya po, Wakantanka yutanpi kte cin heon Messiya iyowinunkiyapi qon iyececa.

8 Miye lkin kaken epe; Jesus Messiya bakilidayapi wicohan kin en ookiye ikicaga, Wakantanka towicake kin heon, qa hunkakepi kin wahowicayapi qon he hduecetu kte cin heon etanhan.

$9 \mathrm{Qa}$ hecen Ikcewicaśta kin Wakantanka yatanpi kta, iye towaonśida kin on; wowapi en kagapi qon iyececa, Heon etan- han Ikcewicaśta ehna ociyake ca nicaje kin iwadowan kta.

10 Qa ake hecen eya, Ikcewicaśta kin iyuśkin po, iye taoyate kin kici.

11 Qa ake, Ikcewicaśta kin owasin Itancan kin idowanpi, qa oyate kin owasin he yatan po.

12 Ake Isaya hecen eya, Jesse hutkan wanjidan qa tuwe Ikcewicaśta en wicaśtayatapi un kte cin he icage kta; he lkcewicaśta kin wacinyanpi kta.

13 Hehan woape Wakantanka kin he wicayadapi kin eciyatanhan wowiyuśkin owasin wookiye ko on imna niyanpi nunwe, Woniya Wakan towaśake kin eciyatanhan woape en nina inicagapi kte cin heon.

14 Mihunkawanji niyepi on awacin manka, wowaśte onijupidan, qa wosdonye owasin on oju niyanpi, hecen otoiyohi wahokonyeciciyapi kta oyakihipi.

15 Tuka mihunkawanji, hececa eśta waditagya wowapi cicagapi, hecen wicoie wanjikji kiksuye ciyapi kta, Wakantanka towaonśida maqupi kin he eciyatanhan ;

16 Ikcewicaśta ehna Jesus Messiya taokiye kin wanji he miye kta, Wakantanka tawootanin waśte kin on litawani, qa hecen Ikcewicaśta wahduśnapi kin he woiyokipi kta, Woniya Wakan kin on yuwakanpi kin heon.

17 Heon etanhan Jesus Messiya en taku Wakantanka tawa kin on wowinkta mduha.

18 Wicoie wicohan ko on Ikcewicaśta anagoptanpi kta e, miye eciyatanhan śni, Messiya taku econ qon hena cajemdate kte śni :

19 Tuka wowapetokeca tanka, 255 
qa woyuśinyaye econpi, Wakantanka Taniya Wakan towaśake kin eciyatanhan, hecen Jerusalem etanhan, qa ohomniyan Ilurikon ekta Messiya tawootanin waśte kin omdaka ece.

20 Qa hecen wotanin waśte kin aiciciya oyag uwate, tukten Messiya cajeyatapi kin hetu śni, okinni wicaśta tokeca taku ahde kin akan ti wakage kta naceca.

21 Tuka liecen wowapi en kagapi qon; Tona en cajeyatapi śni kin hena wanyakapi kta, qa tona nahonpi śni qon hena iyukcanpi kta.

22 Qa hecen on otakiya elkta ciupi kte cin okitpani mayanpi :

23 Tuka nakaha makoce kin dena en tuktedan omakan śni qa wanna waniyetu ota ekta ciupi leta nina wacin ;

24. Heon tohan Spania ekta mde cinhan hehan ekta ciupi kta. Heciya icimani mde cinhan tahepi wanciyakapi, qa ek ta ye mayayapi kta awacin manka, tokaheya niyepi kin on cistiyedan imamdeze cinhan.

25 Tuka nakaha Jerusalem elita mda, tona owotanna un kin hena owicawakiye kte cin heon.

26 Tona Makedonia qa Akeya en yukanpi kin hena, tona wahipanica wacinyanpi Jerusalem ekta yakonpi kin liena taku wicaqupi kta e iyokipipi.

27 Hecon iyolkipipi ; taku iwicakicazopi kin iyecen unpi nakaeś. Hena taku wakan yuhapi qon en Ikcewicaśta opapi hecinhan, taku wicacehpi ekta un kin hena on itkom owicakiyapi kta iyececa.

28 Heon etanhan tohan he mduśtan qa waskuyeca kin de hena en awicawecipuspe cin256 han, hehan niyepi iyoopteya Spania elsta imdarnde kta.

29 Qa ekta ciupi kinhan, Messiya tawootanin waśte woyuwaśte woojudan kin yuha wau kta sdonwakiya.

30 Hehan nakaha mihunkawanji ceciciyapi ce, Jesus Messiya Itancan kin caje on, qa Woniya Wakan towaśtedake kin on, miye kici Wakantanka nina cemiyeciciyapi kte cin he.

31 Tona Juda makoce kin ekta anagoptanpi śni kin hena etanhan emahdakupi kta, qa Jerusalem ekta wicohtani mitawa kin tona owotanna un kin iyokipi wicaye kte cin heon.

32 Qa hecen Wakantanka tawacin kin eciyatanhan wowiyuśkin yuha ekta ciupi, qa niyepi on imagaga miçiye kta.

33 Wookiye Wakantanka kin niyepi owasin en nicipi un llunwe.

\section{WICOWOYAKE 16.}

1 Phebe tawinohtinunyanpi, Kenkrea ekta okodakiciye kin en ookiye wanji hee cantohnake ciśipi.

2 He Itancan kin on iyowinyalkiyapi $\mathrm{kta}$, tona owotanna un kin iyececa, qa taku on i kin he en token ecayeconpi cin kinhan ecen okiya po. Iye kin wicota owicakiye ça miye nakun omakiya ece.

3 Priskula Akula kici, Jesus Messiya en mitawaśipi kin hena napewicayuza po.

4 Hena e minagi kin on iye tahupi kin ehnakapi, qa hena miye miśnana pidamakiyapi śni, tuka Ikcewicaśta ekta okodakiciye kin owasin pidawicayapi.

5 Qa iye tipi kin ohna okodakiciye kin napewicayuza po. Epinetus waśtewadake cin he 
WICOWOYAKE 16.

napeyuza po ; Akeya ekta Messiya en un qon tokaheya kin hee.

6 Mary nina ounkiyapi qon he napeyuza po.

7 Andronikus Junia kici mitakuye, qa om makaśkapi qon hena napewicayuza po; yewicaśipi kin owicapeya yuonihanpi, qa mitokapa tanhan Messiya en un qon hena.

8 Amplias Itancan kin en waśte wadake cin he napeyuza po.

9 Urbane Messiya en unkitawaśipi kin, qa Stacus waśte wakidake cin, hena napewicayuza po.

10 Apelles Messiya en yuecepidan kin he napeyuza po. Aristobulus takuwicaye cin hena napewicayuza po.

11 Herodian mitakuye kin napeyuza po. Narkisus takuwicaye cin Itancan en un kin hena napewicayuza po.

12 Truphena Truphosa kici Itancan kin on htanipi kin hena napewicayuza po. Persis waśte dakapi kin Itancan kin on nina litani kin he napeyuza po.

13 Ruphus Itanean kin on kalinigapi kin, qa iye hunku, nakun mitawa kin, hena napewicayuza po.

14 Asunkritus, Plegon, Ermas, Patrobas, Ermes, hena, qa tona om unpi hunkawanjin kiciyapi kin hena ko; napewicayuza po.

15. Philologus, Julia, Nereus, iye tawinohtin kici, Olumpas, qa hena om tona owotanna un kin hena owasin napewicayuza po.

16 Otoiyohi iiputakapi wakan kin on napekiciyuza po. Messiya taokodakiciye kin napeniyuzapi.

17 Mihunkawanji ceciciyapi ce, tona woonspe onspeniçiciyapi qon eciyatanhan śni yuomdecapi, qa wahtani wicayapi kin hena iwanwicayakapi, qa nawicakicipa po.

18 Tona hececapi kin Jesus Messiya Itancan unkiyapi kin wowidag wicaye śni, tuka iye tezipi kin hee, qa wicoie waśte, iapi iyokipi se ececa ko, oll wicaśta wacin ksape śni kin wicahnayanpi.

19 Niye waanayagoptanpi kin he wicaśta owasin sdonyapi. Heon niyepi kin on imduśkin. Tuka taku waśte e on yaksapapi kta, qa taku śica on wadupipi kte śni wacin.

20 Unkan kohanna Wookiye Wakantanka lkin niye nisihapi kin ihukuya Satan napan kta. Jesus Messiya Itancan unkiyapi towaonśida kin niyepi en nicipi un nunwe.

21 Timotheos mitawaśi, qa Lukius, Jason qa Sosipater mitakuye kin, hena napeniyuzapi.

22 Tertius wowapi kin de owa kin he miye, Itancan kin on napeciyuzapi.

23 Gayos wonmakiye ca, okodakiciye owasin hecawicakicon kin, he napeniyuzapi. Erastus otonwe kin en wicaśta itancan wan, qa Kuartus hunkawanjin kiciyapi wan, hena napeniyuzapi.

24 Jesus Messiya Itancan unkiyapi towaonśida kin niyepi owasin en nicipi un nunwe.

25 Tuwe wootanin waśte mitawa qa Jesus Messiya woyake cin he eciyatanhan niyusutapi kta okihi, wowiyukcan nakaha yuotaninpi kin eciyatanhan, he maka icage cin ehantanhan wicoicage kin en analimanpi yon; 26 'Tuka nakaha yuotaninpi, wicaśta wokcan tawowapi wakan kin heon, Wakantanka owi- 


\section{KORINTH.}

hanke wanin un, tawoahope kin eciyatanhan, qa oyate owasin wacinyan anagoptanpi lite cin heon oyakapi;
27 Jesus Messiya on etanhan, Wakantanka ecedan ksape cin hee wowitan owihanke wanin kicihan nunwe.

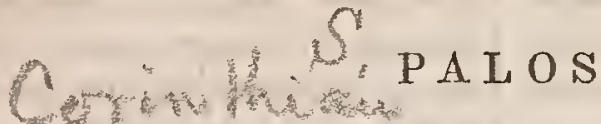 KORINTH OYATE}

\section{WOWAPI WICAKICAGE CIN:}

TOKAHEYA KIN.

\section{WICOWOYAKE 1.}

1 Palos Wakantanka tawacin kin eciyatanhan, Jesus yewicaśi kin heca e kahnigapi kin he, qa Sosthenes hunkawanjinunyanpi kici,

2 Wakantanka taokodakiciye Korinth ekta un qa Jesus Messiya on ecepidan kta, wakan wicakahnigapi kin hena eepi, qa tona makoce otoiyohi en Jesus Messiya iś iyepi kin Itancan kiyapi, qa unkiś eya Itancan unkiyapi caje kin hoyeyakiyapi kin wowapi unnicagapi.

3 Wowaonśida, wookiye ko, Wakantanka Ateunyanpi, qa Jesus Messiya Itancan kin etanhan niyepi en nicipi un nunwe.

4 Ohinniyan niyepi kin on Wakantanka mitawa wopida ewakiya, Wakantanka towaonśida Jesus Messiya eciyatanhan niçupi kin heon;

5 Taku henana owasin on, iye en, wijica nicagapi, wicoie owasin qa wosdonye owasin on.

6 Hecen niyepi kin en Messiya tawoyaatanin kin yusutapi.

7 Qa hecen wowaśte wanjidan aokpaniyan yaunpi śni, qa Jesus Messiya Itancan unkiyapi 258 ihdutanin kte cin he ape dukanpi.

8 He owihanketa hehanyan iyanionpepica śni niyusutapi kta, Jesus Messiya Itancan unkiyapi taanpetu kin etu.

9 Wakantanka wasinyepica, iye Cinhintku Jesus Messiya Itancan unkiyapi kin takodakiciyapi tawa kin en nicopi.

10 Mihunkawanji, Jesus Messiya Itancan unkiyapi cajeyan ceciciyapi ce; owasin okonwanjidan oie yetonpi, qa yuomdecapi wanin yaunpi kta, qa wicotawacin eca wanjidan wiyukcanpi ko wanjidan tanyan onspe yaunpi kte.

11 Mihunkawanji, Kloe etanhanpi kin hena omakiyakapi, niyepi kin en woakinica yulke cin he.

12 Hecen kaken epe; Niyepi otoiyohi, Palos opeya waun, qa, Apolos opeya waun, qa, Inyan opeya waun, qa Messiya opeya waun ce, ehapi ece.

13 Messiya yukinukan ehnakapi he; niyepi kin on Palos cansusbeca kin en okatanpi he; qa iś Palos caje on baptem nicupi he. 14 Wakantanka wopida ewakiya, enitanhanpi tuwedan ba- 


\section{WICOWOYAKE 2.}

ptem waqu śni, tuka Krispos qa Gayos heniyoza :

15 Tuwedan, miye micaje on baptem wicawaqu, keye kte śni heon.

16 Qa nakun Stephanas tiyohnaka kin baptem wicawaqu, qa tuwe tokeca baptem waqu kinhan sdonwaye śni.

17 Baptem wicawaqu kta e on Messiya ye maśi śni, tuka wotanin waśte omdake kte cin heon; wicoksape iapi kin ohna śni, okinni Messiya cansusbeca tawa lin taku śni yapi kta.

18 Cansusbeca oyalkapi kin he tona ihdutakunipi śni kin en witkotkoya iapi kin hee, tuka unkiyepi tona ni unyanpi kin en Wakantanka towaśake kin hee.

19 Kaken wowapi en kagapi; Tona wacin ksapa woksape tawa kin taku śni waye kta, qa tona okahnigapi kin hena wookahnige tawa kin mdutakuni kte śni ce.

20 Wacin ksape cin tokiya un he; wowapi kage cin heca tokiya un he ; wiconi kin de en wiyukcan kin tokiya un he; Wakantanka wicoicage kin de wicoksape kin yuwitkotko śni he.

21 Hecen Wakantanka toksape kin on, wicoicage kin de wicoksape eciyatanhan Wakantanka sdonyapi śni qehan, wowahokonkiye wowitkotkoke cin on, tona wacinyanpi kinhan hena ni wicaye kta Wakantanka iyokipi.

22 Juda oyate kin wowapetokeca dapi; qa Helenes oyate kin wicoksape akitapi ece.

23 Tuka unkiyepi kin Messiya cansusbeca en okatanpi kin he unkoyakapi; he Juda wicaśta kin ekta wonahnaye qa Helenes wicaśta kin ekta witkotkoya iapi kin hee.

24 Tuka tona wicakahnigapi,
Juda qa Helenes wicaśta kin hena ekta, Messiya Wakantanka towaśake qa Wakantanka toksape kin hee.

25 Wakantanka taku tawa withotkoke cin he wicaśta kin isanpa woksape; qa Wakantanka towaśake śni kin he wicaśta kin isanpa wowaśake.

26 Mihunkawanji, nicopi kin he wandakapi, wicacehpi eciyatanhan wacin ksapa ota śni, waśaka ota śni, qa wicaśta okinihan ota śni.

27 Tuka wicoicage kin en talku witkotkoke cin he Wakantanka kahniga, on taku ksape cin wiśten ye kta; qa wicoicage kin en taku waśake śni kin hena Wakantanka kahniga, on taku waśake cin wiśten ye kta.

$28 \mathrm{Qa}$ taku wicoicage kin en okinihan śni un kin he Wakantanka kaliniga, qa taku un śni kin hena ko, on taku un kin yutakuni kte śni.

29 Hecen iye itokam wicacehpi owasin ihdatan kte śni

30 Tuka iye eciyatanhan Jesus Messiya en unyakonpi; he unkiyepi on Wakantanka eciyatanhan woksape, qa woowotanna, qa woyuecedan, qa wokajuju ikicaga.

31 Hecen wowapi qon iyececa; Tuwe iwinkta kinhan Itancan kin on iwinkta kta.

\section{WICOWOYAKE 2.}

1 'Unkan mihunkawanji, ekta ciipi qehan, wicoie qa wicoksape oiyokipi kin yuha wai śni, tuka Wakantanka tawoyatanin kin ociciyakapi.

2 Niyepi kin ekta takudan sdonwaye kte śni e miciconza, Jesus Messiya hecedan, qa he cansusbeca kin en okatanpi. 


\section{KORINTH.}

3 Unkan waśake śni, wokokipeya nina macancan cicipi waun. 4. Qa mioic, qa womdake cin hena wicaśta toksape wicoie oiyokipi kin en un śni, tuka Woniya wayuotanin, qa wowaśake kin en un.

5 Hecen wicayadapi kin he wicaśta wicoksape tawa kin en ahe kte śni, tuka Wakantanka towaśake kin en.

6 Tuka tona ksapapi kin hena ekta woksape unkoyakapi ece; tuka wicoicage kin de en wicoksape kin hee śni, qa wicoicage kin de en wicaśtayatapi tolisape kin hee śni, hena atakuni śni aya.

7 Tuka wowiyukcan en Wakantanka toksape kin he unkoyakapi ece; eqe nahmanpi qon, woicage śni itokam wowitan unkitawapi kte cin heon, Wakantanka wakiconze ciqon.

$8 \mathrm{He}$ wicoicage kin de en wicaśta itancanpi kin tuwedan sdonye śni; sdonyapi unkans๋ Wowitan Itancan kin cansusbeca en okatanpi ktc śni tuka.

9 Tuka wowapi qon iyececa; Tona Wakantanka waśtedakapi kin taku wicakiciyuśtan kin he wiciśta kin tohinni wanyake śni, qa wicanoge on nahonpi śni, qa wicaśta cante kin mahen iyohpaye śni.

10 Tuka hena Wakantanka iye Taniya kin eciyatanhan unkokiyakapi; Woniya kin he taku owasin iyukcan, Wakantanka wośbe tawa kin sdonya.

11 Wicaśta kin ekta tuwe taku wicaśta tawa kin sdonya he, wicaśta taniya iye mahen un kin hee sdonkiya. He iyecen taku Wakantanka tawa kin tuwedan sdonye śni, tuka Wakantanka Taniya kin hecedan.

12 Unkan unkiye wicoicage 260 kin de en wicoksape kin hc unkicupi śni ; tuka Wakantanka eciyatanhan Woniya Wakan kin he unkicupi, hecen taku Wakantanka iyunwin codan unqupi kin hena sdonunyanpi kta.

13 Qa hena unkoyakapi, wicaśta wicoksape woonspe wicoie kin eciyatanhan śni, tuka Woniya Wakan woonspe tawa kin eciyatanhan, hecen wicoie wakan kin wicaśta wakan iyukcanwicunyanpi ece.

14 Tuka wicacehpi wicaśta kin he Wakantanka Taniya taku tawa kin icu śni, hena taku witkotkoke dake ça okahniga okihi śni, hena wakanyan iyukcanpi nakaeś.

15 Tuka tuwe wakanyan un kin he taku owasin iyukcan, tuka iye kin tuwedan on yukcanpi śni.

16 Itancan tawacin kin tuwe he iyukcan he, hecen onspekiye lkta. Tuka unkiye Messiya tawacin kin unhapi.

\section{WICOWOYAKE 3.}

1 Unkan mihunkawanji, miś wicaśta wakanpi kin iyecen ocjciyapi kta owakihi śni, tuka wicacehpi wicaśta kin heca, qa Messiya en hoksiyopa kin iyececa.

2 Pteasanpi yatke ciciyapi, qa waconica kin he cicupi śni; he ehan nahahin he oyakihipi śni, qa dehan nahahin nakun oyakihipi śni.

3 Nahahin wicacehpi wicaśta kin henicapi. Niyepi ekta winawizipi, qa wakinicapi, qa yuomdecapi yukan hecinhan, wicacehpi kin henicapi, qa wicaśta akantu iyecen mayanipi śni he.

4 Wanji, Palos opeya waun, 


\section{WICOWOYAKE 4.}

qa wanji iś, Apolos opeya waun, eye cinhan, wicacehpi wicaśta kin henicapi śni he.

5 Palos he tuwe, qa Apolos he tuwe he; wowidake unpi kin hecapi, on wicayadapi, otoiyohi Itancan kin wicaqu kin iyececa.

6 Miye wowaju, Apolos mini akaśtan, qa Wakantanka icahya.

7 Tuwe woju kin he taku śni, qa tuwe mini akaśtan kin kin he nakun, tuka Wakantanka waicahye cin hee tanka.

8 Tuwe woju qa tuwe mini akaśtan kin hena okonwanjipidan; otoiyohi iye tohtani kin iyacinyan iyunwin icupi kta.

9 Wakantanka kici htanipi kin hena unkiyepi; Wakantanka tamaga, qa Wakantanka tipi kage cin he niyepi.

10 Wakantanka towaonśida maqupi kin eciyatanhan wicaśta ticaga ksape cin iyecen, taku ahehde kin ewahde, qa he akan wicaśta tokeca tipi kaga; tuka otoiyohi token ticage cin he on waktaya un kte.

11 'Tuka ahehde kin tokeca tuwedan ehde kta okihi śni, taku ehdepi kin hecedan, Jesus Messiya hee.

12 Unkan tuwe ahehde kin de akan mazaskazi, qa mazaska, qa inyan okitanin, qa can, qa peji, qa cedi en kage cinhan,

13 Otoiyohi ohan kin atanin kta; anpetu kin he yuotanin kta, peta on yuotaninyan aye kta; qa peta kin otoiyohi ohan kin he taku kin iyukcan kta.

14 Tuwe ticage cin en ohan kin sutaya un kinhan he wokajuju icu kta.

$15 \mathrm{Qa}$ tuwe ohan kin huhnage cinhan he iwahpanice kta, tuka iye qe peta kin etanhan iyecen niyanpi kta.

16 Wakantanka tipi tawa kin he niyepi, qa Wakantanka Taniya kin onitipi kin he sdonyakiyapi śni he.

17 Tuwe Wakantanka ti kin aśamye cinhan he Wakantanka ihangye kta. Wakantanka ti kin he wakan, qa he niyepi.

18 Ihnuhan tuwe içihnaye cinhan. Tuwe niyepi ekta wicoicage kin de en ksapeicida kin lie witkotlroke icicaga nunwe; hecen ksape kta.

19 Wicoicage kin de en taku wolssape kin he Wakantanka witkotkoke daka. Kaken wowapi en kagapi ; Wicaśta ksape cin hena iye wohnaye tawapi kin on wicayuza ece.

20 Qa ake, Wicaśta ksape cin tawacinpi kin hena taku śni e, Itancan kin sdonya ce.

21 Hecen tuwedan wicaśta kin on iwinkta k te śni. Taku owasin nitawapi.

22 Palos, qa Apolos, qa Inyan, qa wicoicage kin, qa wiconi, qa wiconte, qa taku nakaha un kin, qa taku u kte cin hena owasin nitawapi ;

23 Qa Messiya tawa niyanpi, qa Wakantanka Messiya tawa ya.

\section{WICOWOYAKE 4.}

1 Wicaśta kin kaken ecanunkinpi kta, Messiya iwicadake cin qa Wakantanka tawowiyukcan awanyakapi kin heuncapi.

2 Nakun tona waawanyaliapi un kin hena owotanna olianyanpi kta iyececa wicadakapi ece.

3 Tuka mayadacopi, qa wicaśta akantu mayacopi eśta, he taku śni wadaka; qa nakun mihdaco śni.

4 Miye cinka takudan sdonwakiye śni, tuka heon owotanna mihdawa śni ; tuka tuwe mayukean kin Itancan kin hee. 


\section{KORINTH.}

5 Hecen iyehantu śni kin itokam takudan yacopi śni po, Itanean kin he hi śni kin hehanyan; he iye kin taku otpaza en nahmanpi qon aiyoyam ye kta, qa wicocante awacinpi.kin yuatanin kta; unkan hehan otoiyohi Wakantanka eciyatanhan idowanpi kta.

6 Mihunkawanji, niyepi on etanhan hena hecen Apolos kici miye imihdacin; hecen unkiyepi kin on wowapi en kagapi kin isanpa keeanwicayakinpi kte śni ornispepi kta, qa hecen otoiyohi on wahannicidapi kte śni.

7 Tuwe hecen iyotan nicaga he ; qa taku nicupi śni kin tukte e duha he. Qa niçupi kinhan tokeca nicupi śni kin iyecen on iniwinkta he.

8 Nakaha winipipi ; nakaha winijicapi ; unkiyepi unkicunonpa tanhan wicaśtaya tapi yaunpi : Unkan wicaśtayatapi yaunpi wacin, hecen niyepi kici wicaśtayatapi unyakonpi kta tuka.

9 Miś kaken wacin mduza; Wakantanka yewicaśipi unkiyepi kin ehake taninyan eunhdepi, wiconte iyacopi kin iyeceea; hecen wicoicage qa mahpiya ohnihde cin, qa wicaśta kin en woiwanyake kin he unkiyepikta.

10 Messiya on wiuntkotkopika, tuka niye Messiya en yaksapapi. Unkiye waunśakapi śni, tuka niye waniśakapi. Unkiye unyuonihanpi śni, tuka niye nicinihanpi.

11 Qa nakun anpe dehan wotekteunhdapi, qa iunpuzapi, qa tancounkapidan, qa unkaśtakapi, qa tuktedan tinsadan unyakonpi śni ;

12 Qa hununkitapi, unnapepi kin on htaunnipi. Unkaiapi keś wicunyawaśtepi ; śicaya unkuwapi keś wacin untankapi ;
13 Unyaśicapi keś ceunkiyapi ece. Wicoicage en watuśekśeca kin iyecen unkagapi, qa anpe dehanyan taku owasin etanhan taku kahin ehpeyapi kin hecen unyawapi ece.

14 Iśtenciyapi kta heon hena hecen wowapi wakage śni, tuka micinca waśte wicawakidake cin iyecen wahokonciciyapi.

15 Messiya en waonspekiyapi kektopawinge wikcemna duhapi eśta, ateyapi ota duhapi śni ; Jesus Messiya en wotanin waśte on cinca cicagapi.

16 Heon etanhan ceciciyapi ce, ecen omicipa po.

17 Heon niyepi kin ekta 'Timotheos ye waśi, he nalkun micinca hokśincantkiyapi, qa Itancan kin en wacinyepica, he mitacanku Messiya en un kin kiksuye niyanpi kta, ohinniyan okodakiciye otoiyohi en waonspewakiye cin iyececa.

18 Ekta ciupi kte śni se ececa, heon wanjikśi wahanhianiçidapi. 19 Tuka ecadan ekta ciupi kta, Itancan kin iyolkipi kinhan, qa tona wahaniçidapi token eyapi kin hee śni, tuka taku okihipi kin he sdonwaye kta.

20 Wakantanka tokiconze kin he wicoie en ahe śni, tuka wowaśake en han.

21 Cansakadan yuha ekta ciupi kta, qa iś wacantkiyapi cante alibayedan ko hduha wau kta, unma tukte yacinpi he.

\section{WICOWOYAKE 5.}

I Niyepi ekta wawicihahapi yukan keyapi ; qa wawicilialiapi kin he iyececa Ikcewicaśta kin ekta cajeyatapi śni, wanji iye atkuku tawicu kin yuze kte cin. 2 Unkan niye wahannicidapi; on iyoniciśicapi kta tuka; hecen 
tuwe hecon kin niyepi etanhan iyaye yapi kta.

3 Miye mitancan on ekta opeya ciunpi śni, tuka micante on en waun seca, heon en waun kin iyecen wanna mduśtan; tuwe hecon kin,

4 Mniniciyapi qa mitóksape duhapi kinhan, Jesus Messiya Itancan unkiyapi caje kin on, qa Jesus Messiya Itancan unkiyapi towaśake kin yuha,

5 Wicaśta kin he Satan yaqupi kta, cehpi yutakuni kte śni, hecen Jesus Itancan kin taanpetu kin en nagi kin ni kta.

6 Iniwinktapi kin he waśte śni. Taku on napoliyapi kin waniqadan aguyapi ocowasin napohya ece e sdonyayapi śni he.

7 On napoliyapi tannike cin ehpeya po; hecen aguyapi teca qa napohye śni yaunpi kta. Messiya wohduze unkitawapi kin he iye kaeś unkiyepi on wośnapi.

8 Heon etanhan wohduze kin en unkopapi kta, on napoliyapi wanakaja qa wocanniye wicośice ko on napoliyapi kin he unhapi kte śni, tuka wicoowotanna wowicake ko on napoliyapi kin hee unkunpi kta.

9 Wowapi en kaken cicagapi ; Wiinahmanpi kin om mayanipi kte śni.

10 Tuka wicoicage kin de en wiinalimanpi, qa tona waicucupi kte cin, qa wawicaki sa, qa tona wakagapi ohodapi kin hena e wicawake śni ; unkanś wicoicage kin de etanhan tankan dapi kta tuka.

11 Tuka nakaha kaken wowapi cicagapi, tuwe hunkawanjin kiciyapi eciyapi kin he wiinaliman, qa waicucukte cin, qa wakagapi ohoda, qa waaie sa, qa witko sa, qa wawicaki sa heca kinhan, kici yaunpi kte śni, ihnuhan kici wayatapi kinhan.

12 Tona tankan unpi kin hena token ecin wicamdaco kta he; tona timahen un kin hena wicadacopi śni he.

13 Tuka tona tankan un kin hena Wakantanka wicayaco ece. Hecen wawicihahapi econ qon he niyepi kin etanhan tankan iyeya po.

\section{WICOWOYAKE 6.}

1 Wicaśta wan taku icanśin niyanpi kinhan token ecin owotanna ohanye śni unpi kin en yukcanpi kta e ayaipi he, qa wicaśta wacinyan unpi kin en ayaipi śni.

2 Tona wacinyan unpi kin hena wicoicage kin yacopi kta e sdonyayapi śni he. Unkan niyepi wicoicage kin dacopi kta hecinhan, taku iyotan cistinna kin hena iyukcan iyenicecapi śni he.

3 Mahpiya ohnihde kin hena eepi kaeś wicunyukcanpi kta c sdonyayapi śni he. Hecen wiconi kin de en taku hiyeye cin sam iyeya unkokihipi.

4 Heon etanhan wiconi kin de en taku iyukcanpi kte cin duhapi kinhan, tona okodakiciye kin en yuonihanpi śni kin hena iyukcan wicaśi po.

5 Iniśtecapi kte cin heon hepa. Niyepi ekta wicaśta ksape ça tuwe hunkawanjitkupi kin wiyukcan okihi kte cin wanica he.

6 Tuka hunkawanjinkiciyapi kin taku iyakicionpapi ece, qa he nakun wacinyanpi śni kin wicitokam.

7 Hecen taku iyayecionpapi kin he sicaya ecanonpi ece. Tokeca e śicaya ecaniconpi kin ayatpaspapi śni he. Tolseca ta263 
ku nicipi kinhan wacin tanka yeçunpi śni he.

8 'Tuka niye śicaya ecanonpi, qa wawicayakipi ece, qa he nihunkawanjipi kin.

9 Tona owotanna śni un kin hena Wakantanka tokiconze kin tawapi kte sni e sdonyayapi śni he. Içihnayanpi śni po: wiinahmanpi, qa wakagapi ohodapi sa, qa wawicihahapi econpi sa, qa winyan içidapi kin, qa iwicahu sa,

10 Qa wamanonpi sa, qa waicucukte cin, qa witkopi sa, qa wawicakipi sa, henakiya Wakantanka tokiconze kin tawapi kte śni.

11 Qa niś niyepi kin hunh henicecapi tuka, tuka niyujajapi; tuka niyu wakanpi ; tuka niyuowotanpidan, Jesus Itancan kin cajeyan qa Wakantanka unkitawapi Taniya kin on.

12 Taku owasin ecamon kta e iyowinmakiyapi, tuka taku owasin ecamon kta iyecece śni ; taku owasin ecamon kta e iyowinmakiyapi, tuka takudan imadake kte śni wacin.

13 Woyute kin he wicatezi kin kicagapi, qa wicatezi kin iś woyute kicagapi, tuka henioza Wakantanka ilang ye kta. Unkan wicatancan kin he wiinahmanpi on kagapi śni, tuka he Itancan kin kicagapi, qa Itancan kin he iś wicatancan kin tawa kta.

14 Unkan Wakantanka wanna Itancan kin najin kiye ca, nakun iye towaśake kin on najin unkiyapi kta.

15 Nitancanpi kin hena Messiya huha kin ee sdonyayapi śni he. Hecen Messiya huha kin iwacu, qa witkowinna wan huha wakiye kta he. Hiya, hecetu śni.

16 Tuwe witkowinna wan en kici iyaihdaśke cin he kici tan264 can wanjidan, sdonyayapi śni he. Henioza cehpi wanjidan kta ce, eye ciqon.

17 Tuka tuwe Itancan kin en kici iyaihdaśke cinhan he iye kici woniya wanjidan.

18 Wiinalimanpi kin nakicipa po. Woahtani wicaśta econ kin owasin wicatancan kin itankan un, tuka tuwe wiinahman kinhan he iye tancan kin en waicihtani.

19 Tokeca, nitancanpi kin hena Woniya Wakan Wakantanka nicupi, qa niyepi en un kin he tipi tawa kin hee, qa hecen tawa nihdawapi śni e sdonyayapi śni he.

20 'Taku tanka iyopeniyanpi, heon nitancanpi ninagipi ko on Wakantanka yatan po, hena Wakantanka tawa.

\section{WICOWOYAKE 7 .}

1 Wowapi en miyecagapi kin he icalitagya, wicaśta kin winohinca yutan śni kin he waśte.

2. Tuka wiinahmanpi kte śni e heon etanhan wicaśta otoiyohi winohinca tawa hduhe kta, qa winohinca kin iś otoiyohi wicaśta hduha nunwe.

3 Unkan wicaśta kin iye tawicu kin wowaśte ecakicon kta, qa winohinca kin iś iye hihnaku kin nakun ecen ecakicon riunwe. 4 Winohinca kin iś iye tancan kin hduhe śni, tuka hihnaku kin he yuha, qa nakun wicaśta kin iś iye tancan kin hduhe śni, tuka tawicu kin he yuha.

5 Otoiyohi tancan ehpekiciyapi śni po, ptecedan hehanyan, napin wicayadapi kinhan heceedan, takudan yute śni qa wocekiye eyapi kin en ocowasin yeniçiyapi kte cin heon, kinhan ake napin yahdapi kta, unmana 
kinhan oiçihipi śni kin on Satan iyutanniyanpi kta naceca.

6 Hena hecon ciśipi kin e on hepe śni, tuka wahokonciciyapi kin heon.

7 Wicaśta owasin miye waun kin iyecen unpi kta wacin, tuka otoiyohi Wakantanka etanhan wicowaśake hduha un, wanji decetu, qqa wanji iśs kaketu.

8 'Tuka tona tanśna un qa tona wiwazicapi kin hena, miye waun kin iyecen unpi kte cin he waśte kta, kewicawakiya.

9 Tuka oiçihipi śni kinhan kiciyuzapi kta; wakan kiciyuzapi kin he waśte qa wacin itkon kin he śica.

10 Unkan tona kiciyuzapi kin hena kaken wahokonwicarvakiya, qa he miye cinka epe śni, I tancan kin he heya; Winohinca iye hihnaku kin ahduśtan kte śni.

11 Tuka ahduśtan kinhan tanśna un kta, qa iś ake hihnaku qon okiciye kta ; qa wicaśta kin iye tawicu kin ihnuhan ehpekiye cinhan.

12 Unkan ehake, Itancan kin hee śni, miye cinka hecen epe kta ; Hunkawanjin kiciyapi wan winohinca wicada śni kin heca yuhe ca, he kici un iyokipi kinhan, he ihnuhan ehpekiye cinhan.

$13 \mathrm{Qa}$ winohinca wan wicaśta wicada śni kin heca yuhe ca, he kici un iyolkipi kinhan, he iś nakun ehpekiye kte śni.

14 Wicaśta wicada śni kin he iye tawicu kin on yuwakanpi, qa winohinca wicada śni un kin he nakun iye hihnaku on yuwakan kagapi. Hecece śni unkanś nicincapi kin aśapapi kta, tuka nakaha hena e wakan.

15 'Tuka wicada śni un kin he unma kpagan kinhan hecen kpa- gan kta. Hena hecece cin en hunkawanjin kiciyapi, qa tawinohitin kiciyapi kin idakapi śni. Wakantanka wookiye en unkicopi.

16 Winohinca, niś taku sdonyaya he, okinni nihihna niyakiye kta. Qa wicaśta kin, niś token sdonyakiya he, okinni nitawin niyakiye kta.

17 Tuka otoiyoḥi token Wakantanka wicaqu qa Itancan kin otoiyohi wicakico kin he iyecen mani nunwe. Hecen okodakiciye owasin en econ wicawaśi ece.

18 Tuw bakilidayapi ecen kicopi hecinhan, bakilidayapi śni kin akite kte śni. Qa tuwe bakildayapi śni ecen kicopi hecinhan, he bakilidaye kte śni.

19 Bakilidayapi kin he taku śni, qa bakilidayapi śni kin he nakun taku śni, tuka Wakantanka tawoahope opapi kin he tanka.

20 Otoiyohi wicohtani en kicopi kin he en un kta.

21 Wowidake un kin henica ecen nicopi kinhan he ekta ewacin śni wo; tuka tawa niçiye kta e oyakihi kinhan he en oyahan de kta.

22 Tuwe wowidake un kin he Itancan kico kinhan, hee Itancan kin on tawa iciya; qa nakun tuwe tawa iciye cin hecen kicopi kinhan he Messiya wowidake tawa kin hee.

23 Tehiya openitonpi, heon wicaśta akantu wowidag niyanpi śni nunve.

24 Mihunkawanji, otoiyohi taku en un hecen kicopi kin he en Wakantanka kici un nunwe.

25 Unkan hokśiwinpidan icalitagya, Itancan kin eciyatanhan woope takudan niduhe śni. Tuka miye owotanna waun kta 265 


\section{KORINTH.}

Itancan kin ionśimakida, becen token wacin mduze cin owahdake kta.

26 Nakaha wicokakije kin on etanhan de waśte kepce, wicaśta kin ecen un kte cin he waśte.

27 Winohinca en iyanihdaśka he ; etanhan nihduśke lita akite śni wo. Qa winolinca en iyanihdaśke śni he; winohinca wan akite śni wo.

28 Tuka winohinca duza eśta wayahtani śni, qa hokśiwinna wan hihnaton eśta wahtani śni; tuka hena e wicacehpi ekta wicokakije yuhapi kta; hececa eśta ito itoncipapi.

29 Tuka mihunkawanji kaken epe kta; Wanna tokata ptenyedan; tona winohinca yuhapi kin hena winohinca tonpi śni kin iyecen unpi kte.

30 Tona ceyapi kin hena ceyapi śni kin iyececapi kte; tona iyuśkinpi kin hena iyuśkinpi śni kin iyececapi kta; qa tona wopetonpi takú yuhapi śni kin iyececapi kta.

$31 \mathrm{Qa}$ tona maka kin de wowinyunyanpi kin sicaya yuhapi kin iyececapi kte śni; wicoicage kin de ouncage kin atakuni śni aya.

32 Tuka takudan icante śice śni yaunpi kta wacin. Tuwe tawicu ton śni kin he Itancan taku tawa kin awacin yanka, token Itancan iyokipi ye kta.

33 Tuka tuwe tawicu ton kin he wicoicage kin en taku awacin yanka, token tawicu kin iyokipi ye kta.

34 Qa nakun winolinca hihna ton qa witanśna un kin hena kici akidecece śni. Hihna ton śni kin he Itancan taku tawa kin awacin yanka, tancan nagi ko wakan un kta heon. Tuka hihna ton kin he wicoicage en ta- ku un kin awacin yanka, token hihnaku kin iyokipi ye kta.

35 'Tanyan yaunpi kta heon hena hepa. Taku on hmunkapi kin nitokam ewahnake kta e hee śni ; tuka wacinktonja wanin, qa wiciyokipi kin en Itancan kin oyapapi kta e heon.

36 Tuka tuwe iye cunwintlku tanśna un kin tanyan ecakicon śni kecin, qa wanna wikośka hecinhan, qa he iyecetu kinhan, token cin kinhan ecen econ eśta, wahtani kte śni ; wakan kiciyuzapi kte.

37 'Tuka tuwe iye carite kin en sutaya un qa tokan tanhan hecon kiyapi śni, qa iye tawacin kin oicihi, qa cunwintku tanśna un hduhe lita e cante mahen iciconze cinhan he tanyan econ. 38 Hecen tuwe kiciyuzapi kin en wicaqu kinhan he tanyan econ, qa tuwe kiciyuzapi kin en wicaqu śni kin he sanpa tanyan econ ece.

39 Winohinca kin tohanyan hihnaku ni un kin hehanyan wicoope kin on kaśkapi ; tuka hihnaku kin te cinhan hehan tawaiciya, tuwe tokeca cin kinhan he hihnaye kta okihi, Itancan kin en un kin hecedan.

40 'Tuka ecen yanke cinhan he iyotan waśteya un kta imdukcan; qa Wakantanka Taniya kin he mduha kepce.

\section{WICOWOYAKE 8.}

1 Unkan taku wakagapi wakiyuśnapi kin icahtake, owasin wookahnige unhapi sdonunkiyapi. Wookahnige kin he tankaiçidapi kaga, tuka wacantkiyapi kin he tanka icahya.

2 Tuwe taku sdonya kecin kinhan he nahahin sdonye kte cin iyecen takudan sdonye śni. 
3 Tuka tuwe Walkantanka waśte dake cinhan Wakantanka he iye sdonya.

4 Taku wakagapi wakiyuśnapi kin yutapi kin he icahtake, wakagapi maka akan un kin he taku śni sdonunyanpi, qa Taku Wakan wanjidan, hecedan yukan.

5 Taku wakan eciyapi kin mahpiya ekta qa maka akan yukan eśta; taku wakan ota, qa itancan kiyapi kin nakun ota keyapi ece :

6 Tuka unkiye, Taku Wakan wanjidan Ateyapi kin hee unhapi, he etanhan taku owasin $u$, qa he en unkiye unyakonpi; qa Jesus Messiya Itancan kin wanjidan, he on taku owasin un qa he eciyatanhan unkiyepi.

7 Tuka owasin hecen okahnigapi śni. Wanjikji anpetu dehanyan wakagapi sdonyapi, taku wakagapi wakiyuśnapi kin iyacinyan yutapi ece; hecen nagi suta śni kin hena aśamyapi.

8 Taku untapi kin eciyatanhan Wakantanka iyokipi .unyanpi śni; wauntapi kinhan heon etanhan sanpa unwaśtepi śni, qa wauntapi śni kinhan heon sanpa unśicapi śni.

9 Tuka ihnuhan wawoyakihipi kin de tona waśake śni un kin hena en wonahnaye kte cin.

10 Niye osdonya yaun kin, wakagapi tipi kin en wota idotanke cin tuwe wanniyake cinhan, wicaśta nagi waśake śni un kin he taku wakagapi wakiyuśnapi kin yuta onspeyakiye krte śni he.

11 Hecen nihunkawanji waśake śni un kin, on Messiya te cin he ihangyapi kta, niye wasdonyaye cin heon etanhan.

12 Tuka hunkawanjin kiciyapi kin en hecen wayahtanpi, qa iye nagipi kin waśake śni kin nagiyeyayapi eca, Messiya en wayahtanipi ece.

13 Heon etanhan woyute kin mihunkawanji wahtaniye cinhan, ohinniyan tado wate śni waun kta, okinni mihunkawanji wahtaniwaye kta.

\section{WICOWOYAKE 9.}

1 Yewicaśipi kin hemaca śni he; tawa miciye śni he; Jesus Messiya Itancan unkiyapi he wanmdake śni he; Itancan kin en miohian kin he niyepi śni he. 2 Wicaśta tokeca ekta yewicaśipi kin hemaca śni eśta, niyepi kin ekta hemaca. Yewicaśipi kin en opeya waun kin ipuspe kin he niyepi, Itancan kin en.

3 Tona wimawangapi kin hena kaken awicamdupta ece :

4 Unkiye wauntapi qa waunyatkanpi unkokihipi sni he.

5 Yewicaśipi kin tokeca, qa Itancan hunkawanjinwicaye cin, qa Inyan iyececa, unkiye tankśiwicunyanpi wanji hdus unkayapi unkokihipi śni he.

6 Qa iś, miś miye Barnabas kiciśnana, wicohan ayuśtan owakihi śni he.

7 Tohantu kaśta tuwe iyunwin codan zuya iyaya he; tuwe hastanhanka iyuwi kin huhde qa etanhan waskuyeca yute śni he; qa tuwe tahinca optaye wan hduhe ca optaye kin etanhan asanpi yute śni he.

8 Wicaśta akantu eciyatanhan hena hecen epa he; qa woope kin he hena hecen eye śni he.

9 Moses toope kin en kaken kagapi, Tatanka aguyapi napan kin he iohmus icu yaye kte śni ce. Wakantanka tatanka ihawicakta he ;

$10 \mathrm{Qa}$ iś unkiyepi kin on hena $26 \%$ 


\section{KORINTH.}

hecen eya he. Unkiyepi kin on he wowapi en kagapi; hecen tuwe maliyumdu kinhan woape en maliyumdu kta, qa tuwe woape yuha aguyapi kapan kinhan lie kici woape en okicipe kta.

11 Unkiye ninagipi kin on taku unkonicijupi hecinhan, taku wicacelipi kin nitawapi kin he mnaunyanpi kte cin he taku tanka he.

12 Wicaśta tokeca niyepi ekta wookihi kin de en okicipapi kinhan, unkiye unkiyotanpi śni he. Tuka wookihi kin he unkunpi śni; taku owasin unkapaspapi, Messiya tawootanin waśte kin anaunptapi kte śni kin heon.

13 Tona tipi wakan kin el kicanyanpi kin hena tipi wakan kin etanhan nipi ece sdonyayapi śni he; qa tona wahna wośnapi kin ekta ohanyanpi kin hena wośnapi kin etanhan wotapi ece.

14 Hecen nakun Itancan kin woope Kaga; tona wotanin waśte oyakapi kin hena wotanin waśte kin on nipi kta.

15 Tuka hena taku kin takudan irvacu śni, qa hecen ecamiconpi kte cin heon hena hecen wowapi wakage śni; miye mate kte cin hee waśte, qa tuwe imawinkta kin de ecetu śni miciciye kte cin he sica.

16 Wotanin waśte kin omdaka keś, he wowinkta wahdawa śni ; waecon makiyapi, qa wotanin waśte omdake śni ehantanhan wokakije micihan.

17 Qa he iyokipiya ecamon kinhan on iyunwin mduhe kta, tuka iyomakipi śni eśta taku econmakiyapi wan maqupi.

18 Hecen taku mduhe kta he. Wotanin waśte omdake cinhan, Messiya tawootanin waśte kin iyunwin codan ewahde, qa he- cen wotanin waśte kin en wowakihi kin tanyan mduhe kta.

19 Wicaśta owasin ekta wowidake śni waun tuka owasin wowidake mayuhe wicawakiya, wanjikśi okihiwicawaye kte cin heon hecamon.

20 Juda oyate kin wicehna, Juda wicaśta kin hemaca, Juda oyate kin owicawakihi kte cin heon. Tona woope kin ihukuya un kin hena ekta woope yuha unpi kin hemaca, tona woope yuhapi kin hena owicawakihi kta.

21 Qa tona woope nicapi kin hena ekta woope codan unpikin hemaca ; - Wakantanka ekta woope codan waun śni tuka Messiya on woope yuha waun; tona woope nicapi kin hena owicawakihi kta.

22 Tona waśake śni kin hena en waśake śni se emaceca, tona waśake śni kin hena owicawakihi kta. Wicaśta owasin en taku owasin un kin hemaceca, wanjikji ni wicawaye kte cin heon.

23 Wotanin waśte kin he on hecamon ece; hecen he en miś cya owape kta.

24 He de sdonyayapi śni he; Tona oinyanke kin en inyankapi kin hena owasin inyankapi, tuka wicaśta wanjidan oqipe kin yekiya ece: Niś yeyakiyapi kta e iyecen inyanka po.

25 Tuwe kaśta kici econ kte cinhan taku owasin iyatahde ic̣iye śni un. Hena wateśdake atakuni kte śni kin yekiyapi kta, tuka unkiye wateśdake ohinniyan un kte cin he unkakitapi.

26 Heon ctanhan miś hecen waimnanka, toketu tanin śni kin he iyecece śni; hecen llape on econ waun, tuwe okaduza ape cin iyececc śni ; 
27 Tuka mitancan kin suta miçicage ça ținsmiçiya ; okinni wicaśta tokeca wotanin waśte kin owicawakiyaka eśta, miye qe heyata iyemayanpi lite cin he kowakipa.

\section{WICOWOYAKE 10.}

1 Mihunkawanji he de sdonye śni yaunpi kta wacin sni, hunkake wicunyanpi kin owasin malipiya śapa kin ihukuya yukanpi, qa owasin miniwanca kin opta hiyupi.

2 Qa owasin malipiya śapa qa miniwanca kin en Moses baptem wicaqu.

3 Qa owasin woyute wakan wanjidan etanhan yutapi.

4 Qa owasin woyatke wakan wanjidan etanhan yatkanpi. Imnija wakan kin wicihakam iyaye ciqon he etanhan yatkanpi. Imnija kin he Messiya hee.

5 Tuka hena wicota Wakantanka iwicayuśkin śni, hecen makoskan ihpayapi.

6 Unkan hena e waonspekiya unkitawapi kin eepi, hecen taku sica cantiheunyanpi kte śni, iś iye cantiheyapi gon iyececa.

7 Qa wakagapi ohoyadapi kte śni, iś iyepi wanjikji heconpi qon iyececa. Kaken wowapi en kagapi, Oyate kin wota qa wayatke iyotankapi, qa śkan najin hiyayapi.

8 Qa wiinahmanpi kin econkupi kte śni, iś iyepi wanjikji econpi qon ryececa; qa anpetu wanjidan en kektopawinge wikcemna nonpa sanpa kektopawinge yamni ihpayapi.

9 Qa Messiya iyutan unyanpi lkte śni, hena wanjikji iyutan yanpi qon iyececa; unkan wamduśka ihangwicayapi.

10 Qa wawiyahnupi lite śni, iś iyepi wanjikji wawihnupi qon iyececa, qa waihangye cin on yutalkunipi śni.

11 Hena taku kin owasin waonspekiyapi on en wicahiyahde, qa unkiyepi wicoicage owihanketa unyakonpi kin wahokonunkiyapi kte cin heon wowapi en kagapi.

12 Hecen tuwe tinsa najin kecin kinhan, he wakta un nunwe, okinni ithpaye kta.

13 Wowiyutanyan wan wicaśta sdonyapi śni ece kin en aniupi śni. Wakantanka wacinyepica, hecen wawoyakihipi kin isanpa iyutanniyanpi kta iyowinye kte śni, tuka wowiyutanyan kin he enakiye kta, hecen oyakihipi kta. 14 Heon etanhan waśte cicidapi kin, wakagapi ohodapi kin nakicipa po.

15 Wicaśta wacin ksape cin hena hecen ewicawakiya, token epe cin niś iyukcan po.

16 Woyawaśte wiyatke unyawaśtepi kin he Messiya we kin en kodakiciyapi kin hee śni he. Aguyapi unkiyuśpapi kin he Messiya tancan kin en kodakiciyapi kin hee śni he.

17 Unkiye unkotapi, tuka aguyapi unwanjipidan, qa tancan unwanjipidan; unkiye owasin aguyapi wanjidan en akiptan unkopapi.

18 Wicacehpi eciyatanhan Israel oyate kin ekta ewicatonwan po. Tona wośnapi kin etanhan yutapi kin hena wahna wośnapi kin en opapi śni he.

19 Heon token epe kta he: Wakagapi kin he taku, qa jś taku wakagapi wakiyuśnapi kin he taku tanka he : Hiya.

20 Tuka taku Ikcewicaśta kin wayuśnapi ece kin hena Wakan śica wakiyuśnapi, qa Wakantanka wakiyuśnapi śni. Unkan 269 


\section{KORINTH.}

taku wakan śicapi kin en oyecipapi kta wacin śni.

21 Itancan tawoyatke qa nakun taku wakan śicapi tawoyatke kin yatkan oyakihipi śni. Itancan kin wahna wotapi tawa kin en oyapapi, qa nakun taku wakan śicapi wahna wotapi tawapi kin en oyapapi kta oyakihipi śni.

22 Itancan kin sihda unyanpi kta he. Tye sam iyeya waunśakapi he.

23 Taku owasin iyemicihantu tuka owasin taku waśte icahye śni. Taku owasin iyemicihantu tuka owasin waonspekiye śni.

24 Tuwedan iśnana taku amnaiciye kte śni, tuka otoiyohi taku tokan tawa kte cin he akicicite lita.

25 Taku owasin tihocoka kin ekta wiyopeyapi kin he yuta po, qa wiyukcanpi kin on takudan iwangapi śni po.

26 Maka kin de taku ohnaka ko Itancan kin tawa.

27 Wicaśta wicadapi śni kin wanji nicopi, qa ekta dapi tawatenyayapi kinhan, taku nitokam ehdepi kin he yuta po, qa wiyukcanpi on wiwangapi śni po.

28 Tuka tuwe, De wakagapi wakiyuśnapi ce, eniciyapi kinhan, yutapi śni po, tuwe oniciyakapi qa wiyukcanpi kin hena on. Maka kin de taku ohnaka ko Itancan kin tawa.

29 Wiyukcanpi kin cajemdata, niye nitawa śni tuka tokan tawa kin he waka. Tokeca tawa miciye cin he wicaśta tokeca tawiyukcan kin on mayacopi kta he.

30 Wowaśte on en owape cinhan, tokeca e taku on wopida epe cin he on mayaśicapi he.

31 Heon wayatapi qa wadatkanpi, qa taku kaśta ecanonpi kin owasin Wakantanka yatanpi kta e on econ po.

32 Juda oyate, qa Helenes oyate, qa Wakantanka taokodakiciye tuwedan . icanśinyayapi kte śni.

33 Qa miye taku owasin on owasin iyokipi wicawaye cin he iyececa, taku mduhe kte cin he awakite śni, tuka taku wicota yuhapi kte cin, hecen nipi kta.

\section{WICOWOYAKE 11.}

1 Miye omicipa po, Messiya opeya waun kin iyececa.

2 Mihunkawanji, ciyatanpi ce, taku owasin on miyelssuyapi, qa woope ciçupi qon eceliin duhapi kin heon etanhan.

3 Tuka he de sdonyayapi kta wacin; Wicaśta otoiyohi en pa kin he Messiya ee; qa winohinca pa kin he wicaśta kin ee; qa Messiya pa kin he Wakantanka ee.

4. Wicaśta otoiyohi pa akalipe ton cekiye ca wiyukcan kinhan, he iye pa kin wowiśtenya ece.

5 Tuka winohinca otoiyohi pa akahipe ton śni cekiye ca, wiyukcan kinhan, he iye pa kin wowiśtenya; he paha yuśdapi kin kici akidececa.

6 Winohinca iś pa akalipe ton śni kinhan paha yuśdapi kta. Tuka winohinca paha yuśdapi, qa iś hdasanpi kin he wowiśtece kinhan, pa akalipe ton un kte.

7 Wicaśta kin iś pa akahipe ton un kin he hecetu śni, he Wakantanka touncage qa towitan kin hee; qa winohinca kin wicaśta towitan kin hee.

8 Wicaśta kin he winohinca kin etanhan śni ; tuka winolinca kin he wicaśta kin etanhan un.

9 Qa wicaśta kin he winohinca 
kicagapi śni, tuka winohinca kin iś wicaśta kin licagapi.

10 Heon winohinca kin pa kin akan wowaśake hduhe kta, malipiya ohnihde cin hena on etanhan.

11 Tuka nakun wicaśta kin winohinea codan un śni, qa winolinca kin iś wicaśta codan un śni, Itancan kin en.

12 Unkan winohinca kin he wicaśta kin etanhan un kin, he iyecen wicaśta kin iś winohinca kin eciyatanhan u ; qa talku owasin Wakantanka etanhan un.

13 Niś niyepi kin he iyukcan po; winohinca pa akahpe ton śni Wakantanka cekiye cinhan he hecetu he.

14 'Taku oiyokipi kin hee kaeś he onspeniciyapi śni he; wicaśta kin paha hanskaska hecinhan he wowiśtece.

15 Tuka winolinca kin iś paha hanskaska koyake cinhan he wowitan kin ee; paha kin he woakahpe qupi.

16 'Tuka tuwe waakinin un kinhan, wicolian kin de iyececa takudan unhapi śni, qa nakun Wakantanka taokodakiciye kin.

17 Unkan he de omdake ca ciyatanpi śni; taku sanpa waśte kin on mniniciyapi śni ; tuka taku aoptetu kin heon etanhan.

18 T'okaheya okodakiciye mniniciyapi eca yuomdecapi dukanpi keyapi nawalion; qa onśpa wicawada.

19 Waakinicapi niyepi en un $k t a$, hecen tona owotanna iniyukcanpi kin tanin kta.

20 Heon witaya mniniciyapi eca, he litayetu wotapi Itancan tawa kin yutapi kte cin hee śni ;

21 Wotapi kin en otoiyohi talku yute kte cin tokaheya icu ece; unkan wanji wotektehda qa wanji iś witko ece.
22 Tokeca, tipi ohna wayatapi, qa wadatkanpi kte cin yahduhapi śni he. Wakantanka taolzodakiciye kin ayaktapi śni, qa tona taku yuhapi śni kin hena iśtenwicayayapi he. Token eciciyapi kta he. Heon ciyatanpi lita he. Hiya, ciyatanpi śni.

23 Miye taku Itancan maqu qon he cicupi, Jesus Itancan liin, hanyetu wan iyopeyapi qon he en, aguyapi icu ;

24 Qa wanna wopida eye cin hehan kiyuśpe ca heya; Icupi qa yuta po ; mitancan kin niyepi on kiyuśpapi kin he dee; decen miksuya econ po.

25 Nakun iś eya iyecen wanna yatkan qehan, wiyatke kin icu qa heya; Mawe kin en wicotakuye teca kin he dee ce; decen econ po, tohanhan datkanpi eca, miksuya po.

26 Hecen tohan aguyapi kin de yatapi, qa wiyatke kin de datkanpi kinhan, henakiya Itancan te cin lie duotaninpi ece, tohanyan hi śni kin hehanyan.

27 Heon etanhan, tuwe aguyapi kin de iyacin śni yute ca, Itancan tawiyatke kin de yatke cinhan, he Itancan kin tancan, we ko, on yacopi.

28 Tuka wicaśta lin wihdukcan kta, hecen aguyapi kin de yute ca, wiyatke kin de etanhan yatke kta.

29 Tuwe iyacin śni yute ca yatke cinhan, he woyaco hdute ca hdatkan, Itancan tancan kin iyukcan śni kin heon.

30 Heon etanhan niyepi elita wicota waśakapi śni, qa wayazanpi sa, qa hunh iśtinmapi.

31 Unkiye unkihdukcanpi kinhan, tokan tanhan unkiyukcanpi kte śni.

32 Qa unkiyukcanpi kinhan, 271 


\section{KORIN'TH.}

Itancan kin wahokonunkiyapi, hecen wicoicage kin om unyacopi kte śni.

33 Hecen mihunkawanji tohan wota mniniciyapi kinhan, otoiyohi akicipe po.

$34 \mathrm{Qa}$ tuwe wotektehda kinhan, he tiyata wote kta; hecen woyaco en witaya yaipi kte śni. Qa taku nahahin ihe cin hena tohan wau kinhan, wiyeya ewahnake kta.

\section{WICOWOYAKE 12.}

1 Mihunkawanji, taku wakanyan wicaqupi kin iyukcan śni yaunpi kta wacin śni.

2 Ilkcewicaśta kin henicapi, wakagapi iapi okitpani kin en aniyanpi, ye niyanpi qon iyececa, he sdonyakiyapi.

3 Heon he sdonye ciciyapi; Tuwedan Wakantanka Taniya kin eciyatanhan ie ca, Jesus yaśice kte śni ; qa nakun tuwedan Jesus e Itancan eciye kta okihi śni, tuka Woniya Wakan ecedan eciyatanhan okihi ece.

4. Unkan taku wicaqupi kin hena okonwanjidan śni, tuka Woniya kin e wanjidan.

5 Qa waeconpi kin okonwanjidan śni, tuka Itancan kin e wanjidan.

6 Qa wicolian toktokeca; tuka Wakantanka kin e okonwanjidan, iye owasin en taku owasin owicakicilian.

7 Qa otoiyohi on iyopta ye kte cin heon Woniya yuotaninpi kin wicaqupi ece.

8 Woniya kin wicaśta wan woksape wicoie kin qu ece; qa wanji iś wosdonye wicoie kin, hena Woniya wanjidan etanhan.

$9 \mathrm{Qa}$ Woniya kin he wanji tokeca wowacinye qu ece; qa wanji tokeca iś wawokiziyapi qu ece, qa hena Woniya wanjidan etanhan.

$10 \mathrm{Qa}$ wicaśta wan wowapetokeca econpi, qa wanji tokeca wiyukcanpi, qa wanji tokeca taku wakan iyukcanpi, qa wanji tokeca iapi ocaje ota, qa wanji tokeca iapi ieska oyakapi kin hecekcen wicaqupi ece.

11 Tuka hena owasin Woniya wanjidan qa okonwanjidan un kin he olianye ca, token cin kin hecen wicaśta otoiyohi kiparnni wicaqu ece.

12 Wicatancan kin he wanjidan tuka wicahuha ota yukan; qa tancan kin en huha kin hena ota qeyaś tancan wanjidan ; Messiya kin iś he iyececa.

13 Woniya wanjidan on, unkiye owasin wicatancan wanjidan kta e baptem unqupi, Juda qa Helenes oyate, wowidake un kin, tona tawa içiyapi kin hena koya ; qa owasin Woniya wanjidan kin he en wayatke unkiyapi.

14 Wicatancan kin he huha wanjidan śni, tuka ota.

15 Hehan wicasiha kin he, Wicanapo kin lie miye śni heon wicatancan kin en opeya waun śni ce, eye cinhan, heon etanhan wicatancan kin en ope śni he.

$16 \mathrm{Qa}$ wicanoge kin he, Wicaiśta kin he miye śni qa heon wicatancan kin en opeya waun śni ce, eye cinhan, ehantanhan wicatancan kin en ope śni he.

17 Wicatancan kin ocowasin iśta kin ee unkanś, winahonpi kin tokiya un kta he; qa ocowasin winationpi kin ee unkanś, waomnapi kin tokiya un kta he.

18 Tuka nakaha Wakantanka token iyokipi kin hecen wicahuha kin otoiyohi wicatancan kin en ehde. 
13 Qa hena iyulipa wicahuha wanjidan unkanś, tancan kin tokiya un kta he.

20 Tuka nakaha wicahuha ota eśta, tancan wanjidan.

21 Hecen iśta kin he, Cicin śni ce, nape kin eciya okihi śni ; qa nakun pa kin he, Cicin śni ce, siha kin eciye kte śni.

22 Qa nakun sanpa, wicatancan en wicahuha kin tona waśake śni se un kin hena iyotan.

$23 \mathrm{Qa}$ wicatancan ekta tona yuonihan śni undakapi kin hena iyotan unhduonibanpi ece; qa tona owanyag śice cin hena iyo$\tan$ woiyokipi ece.

24 Tona waśte unyukanpi kin hena takudan sanpa cinpi śni; tuka Wakantanka wicatancan kin icikoyagya . kage ca, tukten iyokpaniyan un kin he en yuonihanpi tanka kaga.

25 Hecen wicatancan kin en yuomdecapi wanice kta; qa wicahuha kin hena otoiyohi awakicicinpi kta.

$26 \mathrm{Qa}$ hecen huha wanjidan iyotanhan iyekiye cinhan, he Kici huha kin iyuhpa iyotanhan iyekiyapi kta ; qa huha wanjidan yuonihampi kinhan he kici huha lin iyuhpa ịyuśkinpi kta.

27 Messiya tancan kin he niyepi, qa otoiyohi huha yecidukanpi.

28 Unkan okodakiciye kin en Wakantanka kakenken ewicahde, tokaheya yewicaśipi kin hena, qa iyokihe wokcanpi, qa iciyamni waonspekiyapi ; hehan wicowaśake, hehan waasniyanpi, waokiyapi, itancanpi, qa ceji toktokeca.

29 Owasin yewicaśipi kin hecapi he; owasin wokcanpi he; owasin waonspekiyapi he ; owasin wicowaśake kin heca he:

30 . Owasin waasniyanpi okihipi he; owasin ceji tokeca on iapi he; owasin ieskapi he.

31 Wookihi iyotan waśte kin hena nina akita po. Tuka hena isanpa canku waśte wan cicipazopi kta.

\section{WICOWOYAKE 13.}

1 Wicaśta akantu, qa mahipiya ohnihde cejipi kin on iwaa eśta, wacanwakiye śni ehantanhan maza śa snasna, qa taku ituya sna kin he iyemaceca.

$2 \mathrm{Qa}$ wowiyukcan mduhe ca, taku anahbeya un kin owasin, wookahnige owasin ko, sdonwaye ca, wowacinye owasin mduha, on he kin yutokan iyewaye kta owakihi eśta, wacanwakiye śni ehantanhan mataku śni.

3 Qa taku mitawa kin owasin won wicayapi kin en wahdusote ca, mitancan kin hee kaeś hulinage lita e waqu eśta, wacanwakiye śni ehantanhan takudan iciyopteya waun śni.

4 Wacantkiyapi kin he wicotawacin tanka qa wowaśte ocowasin. Wacantkiyapi kin he wawinawizi śni. Wacantkiyapi kin he wahanhaniçida śni, qa ihdatan śni ece ;

5 Wawiśtenya econ śni, qa iśnana takudan amnaiciye śni, wacinko śni, qa taku śica iyawicaonpe śni ;

6 Woahtani en iyuśkin śni, tuka wowicake kin en iyuśkin ;

7 Taku owasin kajuju; taku owasin wicada; taku owasin ape; qa taku owasin iyowinyan.

8 Wacantkiyapi kin he tohinni owihanke śni. Wokcanpi kin hena enakiyapi kta; ceji tokeca kin hena ayuśtan aye kta; qa wookahnige kin hee kaeś atakuni kte śni. 


\section{KORINTH.}

9 Apadan sdonunyanpi, qa apadan unkiyukcanpi.

10 Tuka tohan woyuśtan kin he hi kinhan tona woyuśtan śni kin hena ihanke kta.

11 Homakśiyoqopa qehan, hokśiyoqopa iyecen iwae ca, hokśiyoqopa iyecen wimdukcan, qa hokśiyoqopa iyecen awacin waun; tuka wicaśta imacaga hehan taku hokśíyoqopa tawa kin amduśtan.

12 Dehan ihdiyomdasin en iyecen aotpasya wawanunyakapi ece; tuka eqe hehan anog itolınake icitkokim wanunkiciyakapi kta. Nakaha taku kin tanyan sdonwaye śni; tuka e qe tokata sdonmayanpi kin iyecen tanyan sdonwaye kta e imicihan.

13 Unkan nakaha hena yamni en ounyan, wacinyanpi, qa apepi, qa wacantkiyapi; tuka wacantkiyapi kin he iyotan tanka.

\section{WICOWOYAKE 14.}

1 Wacantkiyapi kin he okipa po, qa taku wakanyan wicaqupi kin hena cin po; tuka widukcanpi kte cin he iyotan.

2 Tuwe ceji tokeca on ie cin he wicaśta kin takudan owicakiyake śni, tuka Wakantanka taku eciya; tuwedan nahon śni kin heon: hececa eśta Woniya kin eciyatanhan taku woanahibe kin oyaka tuka.

3 Tuka tuwe wiyukcan oyake cin he wicaśta kin wowicakiyaka, on icahyapi qa wahokonkiyapi qa kicanptapi.

4 Tuwe ceji tokeca on ie cin he iye ksamiciya; tuka tuwe wiyukcan oyake cinhan he okodakiciye kin ksamwicaya ece.

5 Niyepi kin owasin ceji toktokeca on iyaapi kta wacin, tuka widukcanpi kte cin he iyotan wacin. Tuwe wiyukcan kin he tanka qa tuwe ceji tokeca on ie cin he ihukuya, ieska oyake śni kinhan, hecen okodakiciye kin icahyapi kta tuka.

6 Unkan nakaha mihunkawanji, ceji tokeca on iwaa en ciupi kinhan, he token oniciyapi kta he; yaotaninpi, qa wookalinige, qa wiyukcanpi, qa woonspe kin unmana eciyatanhan eciciyapi śni kinhan.

7 Unkan taku ni un śni kin hena, cotanka qa iś candowankiyapi kin hotanin lkinhan, ho toktokeca śni kinhan, he taku on etanhan tukte e yajopi, qa tukte e dowankiyapi kin sdonyapi kta he.

8 Maza yahotonpi tanka kin ho okonwanjidan śni yahotonpi kinhan, tuwe zuyapi kta e wiyeya içicage kta he.

9 Ḧe iyecen niś niyepi, ceji on wicoie okahinil waśteśte iyaapi śni kinhan, token on etanhan taku oyakapi kin he taku ee sdonyapi kta he; okaduza kin en iyaapi kta.

10 Maka kin akan ho ocaje tokeca kanakiya naceca, qa owasin taku kapi.

11 Tuka miś ho kin taku kapi kin sdonwaye śni kinhan, tuwe ie cin he Ikcewicaśta kin kecanmakin kta, qa miś eya tuwe ie cin he Ikcewicaśta kin heca wadake kta.

12 Hecen wicolian wakan nina yacinpi kinhan, okodakiciye kin icahyapi kta e wadupipike kte cin he akita po.

13 Heon tuwe ceji tokeca on ie cinhan, he ieska oyake kta e icekiye kta.

14 Miye śaia cewakiye cinhan, minagi kin he cekiya; tuka mi tawacin e qe takudan icahye śni. 
WICOWOYAKE 14.

15 Hecen toketu he. Miye minagi kin eciyatanhan cewakiye kta, qa nakun mitawacin kin eciyatanhan cewakiye kta ; minagi kin on wadowan kta, qa nakun mitawacin kin on wadowan kta.

16 He hecece śni kinhan, ninagi on wopida eha eca, tuwe onspe śni en iyotanke cin he token, wopida ehe cin en, Amen eye kta he, taku ehe cin sdonye śni nakaeś.

17 Wopida tanyan eha, tuka iye kin okahnige śni.

18 Wakantanka mitawa wopida ewakiya ce, niyepi kin owasin sam iyeya ceji tokeca on iwaa ece.

19 Tuka okodakiciye kin en mitawacin kin eciyatanhan wicoie zaptanna iwae kta, on wicaśta tokeca onpsewicawakiye kte cin he iyotan waśte, qa ceji tokeca on wicoie kektopawinge wikcemna iwae kte cin he aoptetu.

20 Mihunkawanji, wookahinige en hokśiyoqopa unpi śni po; tuka wicotawacin śce cin en hokśiyoqopa po, qa wookahinige kin en wicaśta tanka un po.

21 Woope kin en kaken kagapi; Ceji tokeca, iha tokeca ko on oyate kin de owicaweciye lkta; tuka hececa eśta, namahonpi kte śni, Itancan kin eya.

22 Hecen ceji tokeca kin hena wowakta lkin hee, tona wicadapi śni kin hena eepi śni, tuka tona wicadapi kin hena on wowapetokeca kin hee.

23 Okodakiciye kin ocowasin witaya mniciyapi, qa owasin ceji tokeca on iapi kinhan, qa tona wacinton śni, wicada śni ko en hipi kinhan, winitkotkopika kecannicinpi kte śni he.

24 Tuka owasin wiyukcanpi, qa tuwe wicada śni wacinton śni un kin tin hiyu kinhan, he owasin on onspekiyapi, qa owasin on yacopi lita.

25 Qa hecen iye cante kin ekta taku analibe cin yutaninpi kta; hehan makipusdiya ehpeiciye ca Wakantanka ohoda kta, qa Wakantanka niyepi en nicipi un kin oyake kta.

26 Heon mihunkawanji toketu he: Mniniciyapi eca niyepi otoiyohi odowan duhapi, qa woonspe duhapi, qa ceji tolreca, qa yaotaninpi wan, qa ieska oyalkapi wan duhapi ece. Taku owasin waonspekiyapi kta e on econpi nunwe.

27 Tuwe ceji tolkeca on ie cinhan, wancadan wicaśta nomnom yamnimni, qa hena wanji itokto econpi kta, qa wanji ieska kiyapi kta.

28 'Tuka tuwe ieska kiyapi kte cin wanice cinhan, okodakiciye kin en inina yanke kta; iś iye oiçiciye ça Wakantanka okiye kta.

$29 \mathrm{Qa}$ wicaśta wokcan kin hena nomnom qa yamnimni oyakapi kta, qa unmanpi kin wiyukcan unpi kta.

30 Qa tuwe ikiyedan iyotanke cin he taku wakanyan okiyakapi kinhan, tokaheya kin he inina yanke kta.

31 Owasin wanji itokto widukcanpi kin odakapi kta oyakihipi ; hecen owasin onspekiyapi, qa owasin iwicakiyapi kta. 32 Qa wicaśta wokcanpi toksapepi kin wicaśta wokcanpi kin anawicakigoptanpi ece.

-33. Wakantanka kin he oqoyapi kin kage śni, tuka wookiye kin he tawa, oyate wakan okodakiciye kin owasin en un kin iyececa.

34 Unkan winolinca nitawapi 275 


\section{KORINTH.}

kin okodakiciye kin elita inina yankapi kta. Hena ie kta e iyowinyanpi śni, tuka anagoptan yukanpi kta, woope heye cin iyecen.

35 Qa tạku onspepi kta cinpi kinhan iye tipi ekta hihnakupi kin iwicawangapi kta. Okodakiciye kin en winohinca ie cin he wowiśtece.

36 Tokeca, niyepi kin etanhan Wakantanka oie kin hinanpa he; qa niyepi niśnanpidan ekta nihipi he.

37 Tuwe wicaśta wokcan, qa iś wicaśta wakan kin heca kecanicin kinhan, he taku wowapi en cicagapi kin hena Itancan tawoahope kin hee e yaotanin kta.

38 Qa tuwe iyukcan śni un kinhan, he iyukcan śni un nunwe.

39 Hecen mihunkawanji widukcanpi kte cin he akita po, qa ceji tokeca on iapi kte cin tehindapi śni po.

40 Qa taku oxrasin iyokipiya owotanria econpi nunwe.

\section{WICOWOY AKE 15.}

1 Tuka, mihunkawanji, wotanin waśte unkoniciyakapi, qa iyacupi qon en ayahanpi kin he sdonye ciciyapi.

2 He eciyatanhan nakun yanipi kta, wicoic unkoniciyakapi kin on, he tinsa duhapi kinhan, qa ecinśniyan awacanipi śni kinhan.

3 Otokaheya ekta taku iwacu qon he cicupi ; Waunhtanipi kin on Messiya te cin, wowapi wakan eye cin ecetu;

4 Qa hnakapi, qa anpetu ici yamni kin piya ikicage cin, wowapi wakan eye cin ecetu.

$5 \mathrm{Qa}$ Inyan wanhdake ca, hehan ake nonpapi kin wanhdakapi.
6 Hehan he iyohakam wancadan hunkawanjin kiciyapi opawinge zaptan wanyakapi, hena wicota nahahin ni unpi, tuka wanjikji iśtinmapi.

7 Qa he iyohakam Jakob wanhdaka; hehan yewicaśipi kin owasin wanhdakapi.

8 Qa eliake miś miye wanmdaka, okaślkan tonpi kin iyemaceca.

9 Yewicaśipi kin miye iyotan macistinna, qa Wakantanka taokodakiciye kin śicayn wakuwa qon heon yeśipi emakiyapi kte cin iyemacece śni.

10 Tuka tomakece cin Wakantanka towaonśida kin on hemaceca. Qa iye towaonśida mici un kin he taku śni wanica, tuka hena owasin iyakapeya nina htawani; qa he miye śni, Wakantanka towaonśida mici un kin hee.

11 Heon, he miś miye, qa iś iyepi kin, hecen unyaotaninpi, qa nakun hecen wicayadapi qon.

12 Unkan Messiya wiconte kin etanhan piya ikicage cin he oyakapi hecinhan, token on niyepi wanjikji, Wiconțe etanban piya wicoicage wanica ce, ehapi he.

13 Wicate cin piya wicoicage wanica hecinhan, Messiya piya ikicage śni.

14 Qa Messiya piya ikicage śni hecinhan, hehan unkoyakapi kin he taku śni qa wacinyayapi kin he nakun taku śni.

15 Qa Wakantanka en unlzitonpi śni kin; Wakantanka Messiya piya najin kiye cin he unkoyakapi; tuka he najin kiye śni, wicațe cin ake najinpi śni kinhan.

16 Wicate cin piya najin kiyapi kte śni kinhan, hehan Messiya nakun piya najin kiyapi śni. 17 Qa Messiya piya ikicage 
śni hecinhan, wacinyayapi kin lıe takudan okihi śni, qa nahahin wayahtanipi kin ehna yaunpi.

18 Hehan tona Messiya on iśtinma iyayapi qon hena nakun takunipi śni.

19 Wiconi kin de en ecedan Messiya on ape unyakonpi hecinhan, wicaśta owasin unkiye iyotan iyotanhan iyeunkiyapi ece.

20 Tuka wanna Messiya wiconțe etanhan piya ikicaga, qa tona iśtinmapi qon hena en taku tokaheya icage cin hee.

21 Wicaśta kin eciyatanhan wiconte u kin, he iyecen wicaśta kin eciyatanhan wiconte etanhan piya wicoicage kin $u$ ce.

22 Adam en owasin tapi kin, he iyecen Messiya en owasin piya ni yapi kta.

23 Tuka otoiyohi tohan iyeliantu kinhan; Messiya e tokaheya, qa hehan tona tawa kin hena, Messiya ake hi kinhan.

24 Qa hehan owihanke kta, tohan wokiconze kin Wakantanka Ateyapi kin kicu kta; wokiconze owasin, qa wicowaśake owasin, qa wookihi kin ihangye cinhan.

25 Tona tolka yapi kin hena owasin iye siha kin ihukun jyewicaye kte cin hehanyan ito wicaśtayatapi yanke kta.

26 Qa toka ehake ihangye kte cin he wiconte kin ee.

27 Te siha kin ihukuya taku owasin ekihnaka. Tuka tohan, He ihukuya taku owasin ehnakapi kin, eye cehan, tuwe he ihukuya taku owasin ehnake cin he okaptapi e tanin.

28 Qa tohan taku owasin he ihukun iyeyapi kinhan, hehan Cinhintku kin eqe tuwe taku owasin iye ihukun iyeye cin he ihukun un kta, hecen Wakantanka owasin en ocowasin un kta:

29 Wicate cin ecaca piya ikicagapi kte śni hecinhan, tona wicate cin on baptem wicaqupi kin hena taku tokonpi kta he; qa tokeca e wicate cin on baptem wicaqupi he.

30 Qa tokeca anpetu iyohi ta ikopeya unyakonpi he.

31 Jesus Messiya Itancan unkiyapi kin en widuśkinpi kin en owape cin he cajemdate ca, Anpetu iyohi mata ce, epa.

32 Wicaśta akantu iyecen, Ephesos ekta wamanica kici weciza eśta, on taku iyomapte kta he, wicate cin piya wicoicage śni kinhan. Wauntapi, qa waunyatkanpi kta, heyakecinhan unțapi kta ce.

33 Ic̣ihnayanpi śni po; kodakiciyapi śice cin hena wicolian waśte yuśica ece.

34. Wicohan owotanna on ihduhicapi, qa wahtanipi śni po. Wanjikji Wakantanka sdonyapi śni. Winiśtecapi kte cin heon hena hecen epe.

35 Tuka okinni tuwe heye kta ; Wicate cin token piya icahyapi, qa taku tancan kin yuha hiyupi he.

36 Winitkotkoka, taku oyaju kin he te śni kinhan, icage śni ece.

37 Qa taku oyaju kin he tancan icage kte cin he oyaju śni, tuka su ecedan, aguyapi qa iś taku toktokeca naceca.

38 Tuka Wakantanka token iyokipi kin hecen tancan qu, qa su ocaje otoiyohi tancan tawa kin kicu ece.

39 Cehpi owasin cehpi okonwanjidan śni, tuka wicacehpi kin he wanji ee, qa woteca celipi kin wanji hee, qa hogan ce- 


\section{KORINTH.}

hpi kin wanji hee, qa zitkadan cehpi kin wanji hee.

40 Unkan malipiyata tancan, qa makata tancan kin heca yukan. Tuka mahpiyata un kin he towitan kin wanjidan, qa makata un kin he towitan kin tokeca.

41 Anpetu wi towitan kin wanjidan, qa hanyetu wi towitan kin he tokeca, qa wicanhpi towitan kin he nakun tokeca; qa wicanhpi wanji wicanhipi tokeca kici wowitan en akidecece śni.

$42 \mathrm{He}$ iyecen wicate cin piya wicoicage. Tancan hwin ojupi, tuka lwin kte śni ekicetu yapi.

43 Wowihahaya ojupi, tuka wowitan ekicetu yapi. Wowaśake śni yan ojupi, tuka wowaśagya ekicetu yapi.

44 Wicacelipi tancan ojupi, tuka wowakan tancan ekicetu yapi. Wicacelpi tancan yukan, qa wowakan tancan kin yukan.

45 Kaken wowapi en kagapi qon, Adam wicaśta tokaheya kin he wicanagi ni un kin heca kagapi, qa Adam ehake un kin he Woniya Wanikiye cin hee.

46 Tuka taku wakan un kin he tokaheya śni, tuka taku cehpi kin hee, qa iyohakam taku wakan kin.

47 Wicaśta'tokaheya kin he maka etanhan un, qa maka kin heca; Wicaśta iyokihe kin he Itancan malipiya eciyatanhan un kin hee.

48 Maka kin heca un qon, tona maka hecapi kin hena hececapi ; qa malipiya tanhan un qon, tona mahpiya tanhan un kin hena hececapi.

49 Unkan makata un kin touncage kin unhapi kin, he iye- cen malipiya tanhan un kin touncage kin unhapi kta.

50 Mihunkawanji kaken epa; Wicacehpi qa wicawe kin Wakantanka tokiconze kin tawapi kta okihipi śni, qa nakun wicahwin wicahwin kte śni kin tawa kte śni.

51 Wanyaka po, wowiyukcan wan ociciyakapi; owasin unkiśtinmapi kte śni, tuka owasin unyutokecapi kta.

52 Ihnuhanna, iśta kakpapi se en maza yahotonpi tanka ehake kin tanin kinhan; maza yahotonpi tanka kin he yahotonpi kta, qa wicate cin liwin śni yan najin wicakiyapi kta, qa unkiye unyutokecapi kta.

53 Taku liwin kte cin de wicahwin śni koyake kta, qa te kte cin de tepica śni koyake kta.

$54 \mathrm{Qa}$ tohan taku liwin kte cin de wicahwin śni koyake ca, tancan wiconte kin de wiconi kin ogeton kinhan, hehan wicoie kin wowapi en kagapi qon ecetu kta; Wiconte kin he woohiye kin e ocowasin iocokaya.

55 Wiconte, wicape nitawa kin tokiya un he. Wicahnakapi, woohiye nitawa kin tokiya un he.

56 Wiconte icape kin he woalitani ee, qa woahtani wowaśake kin he woahope kin ee.

57 Tuka Wakantanka woohiye unqupi, Jesus Messiya Itancan unkiyapi kin eciyatanhan, he wopida yuha nunwe.

58 Hecen mihunkawanji waśte cicidapi kin, sutaya yahanpi, qa pahoho śni yaunpi nunwe; qa ohinniyan Itancan ohan kin en aiçiciya po, Itancan kin on litayanipi kin he taku śni wanice kta sdonyayapi kin heon etanhan. 


\section{WICOWOYAKE 16.}

1 Wicaśta owotanna un kin hena taku wicakicamnanpi kte cin, token. Galatia ekta okodakiciye kin econ wicawaśi qon, he iyecen niś econ po.

2 Anpetu tokaheya kin en niyepi otoiyohi token oyakihipi kin, iyacinyan eyahnakapi lita, hecen wau kinhan, wamnayanpi wanice kta.

$3 \mathrm{Qa}$ wau kinhan, tona wowapi on wicayakahinigapi kin, hena wicaqupi nitawapi kin he Jerusalem ekta aye wicawaśi lita.

4 Qa miye ekta mde kte cin he waśte kta naceca hecinhan hena om inde kta.

5 Unkan Makedonia opta mde cinhan, hehan ekta ciupi kta : qa Makedonia opta mde kte cin he hecetu.

6 Qa okinni cicipi waun kta, han, waniyetu ihunniwaye kta naceca; hecen hetanhan tokjya mde cinhan niye amayadapi kta.

7 Nakaha tahepi wanciyakapi kte śni, tuka Itancan kin iyokipi kinhan, cicipi owanji manke kta wacanmi.

8 Tuka anpetu iwikcemna zaptan kin hehanyan Ephesos en owanjidan manke kta.

9 Tiyopa tanka wawokihi kin makiyulidokapi, qa tona wakinicapi kin hena ota.

10 Unkan Timotheos ekta i kinhan, kokipeśniyan en niunpi lita e akita po: He nakun miye ecamon kin iyecen Itancan tolitani kin econ ece.

11 Heon tuwedan ihaha kte śni; tuka wookiye yuha au po, ekta mahiyu kta ; he hunkawanjin kiciyapi kin om u kta ape manka.

12 Apolos mihunkawanji, he hunkawanjin kiciyapi kin om ni- yepi elsta niipi kta e nina cinwakiya; tuka iye dehan ekta ye kta ecaca cin śni, tuka tohan cin kinhan ekta i kta.

13 Kiktahan un po, wowacinye en sutaya han po, wicaśta iyecen olianyan po, waśaka po.

14 Taku nitawapi kin owasin wacantkiyapi on econpi nunwe. 15 Mihunkawanji, ceciciyapi ce ; Stephanas tiyohnaka kin sdonyayapi, he Akeya en waskuyeca tokaheya icage cin hee, qa wicaśta owotanna owicalkiyapi kin en ye içiyapi ece kin;

16 'Tona hececapi, qa tona wawokiyapi, qa htanipi kin hena owasin wicihukuya ye niçiyapi kta.

17 Stephanas qa Phortunatus qa Akekus hipi kin heon imduśkin ; hena e taku ecamiyeconpi śni kin he ecetu miciciyapi.

$18 \mathrm{Qa}$ minagi kin magaga mayanpi, qa nitawapi nakun. Heon etanhan tona hececapi kin hena wicayuonihan po.

19 Asia ekta okodakiciye kin hena nape niyuzapi. Akula qa Priskila Itancan kin on sutaya nape niyuzapi, qa okodakiciye iye tipi ohna un kin hena koya.

20 Hunkawanjin kiciyapi kin owasin nape niyuzapi. Iiputakapi wakan kin on otoiyohi i ikiciputaka po.

21 Palos he miye nape kiciyuzapi kin de miye minape kin on wakaga.

22 Tuwe Jesus Messiya Itancan kin waśtedake śni kinhan, he anathema maran-atha un nunwe.

23 Jesus Messiya Itancan unyanpi towaonśida kin nicipi un nunwe.

24 Jesus Messiya on wacanwakiye cin niyepi owasin en nicipi un nunwe. Amen. 


\section{KORINTH OYATE}

\section{WOWAPI WICAKICAGE CIN;}

INONPA KIN.

\section{WICOWOYAKE 1.}

1 Palos, Wakantanka tawacin kin eciyatanhan, Jesus Messiya yeśi qon he, qa Timotheos hunkawanjinkiciyapi wan kici, Korinth ekta Wakantanka taokodakiciye kin, qa Akeya owancaya tona owotanna un kin hena koya, wowapi unnicagapi.

2 Wowaonśida, wookiye ko, Wakantanka Ateunyanpi, qa Jesus Messiya Itancan kin etanhan, nicipi un nunwe.

3 Walkantanka Jesus Messiya Itancan unkiyapi he Atkuku, qa waonśidapi en ateyapi, qa Wakantanka owasin wacintonhnag wicaye cin he wowaśte yuha nunwe.

4 He unkakijapi owasin cn wacintonhnag unyanpi, hecen tona wokakije owasin ehna un kin hena iwicunkiyapi unkokihipi kta, wacintonhnagyapi on Wakantanka unkikiyapi kin he eciyatanhan.

5 Hecen Messiya tokakije kin unkiyepi en unkiciyuotapi kin, he iyecen Messiya eciyatanhan wacintonhnag unyanpi kin he nakun unkiciyuotapi kta.

6 Kakiśunyanpi eca he wacintonhnag niyanpi, qa ni niyanpi kte cin heon; he wokakije on kakiśunyanpi kin hena yuha okihi wicayc kta c ohanyan ece; qa wacintonhnag unyanpi hecin280 han, hẹ wacintonhnake niyanpi, qa ni niyanpi kte cin heon.

7 Unkan niyepi kin on sutaya wacinunyanpi, wokakije kin en opeya yaunpi kin he iyecen wacintonhnakeyapi kin en oyapapi kta, sdonuny anpi.

8 Mihunkawanji, A sia en token wokakije unhiyahdepi qon he sdonye śni yaunpi kta uncinpi śni, iyotan nina unkakijapi, unkokihipi kin isanpa, hecen unnipi kta unkecinpi śni.

9 Tuka unkiyepi kin mahen wiconțe woyaco kin unhapi, hecen takudan wacinunkiçiyapi kte śni, tuka Wakantanka wicațe cin ekicetu wicaye cin he wacinunyanpi kta:

10 Iye wiconte hinsko tanka etanhan eunhdakupi, qa nakaha ehdaku ece; qa eeś nakun eunhdakupi kta wacinunyanpi :

11 Qa niś eya wocekiye on ounyakiyapi kta, hecen wicaśta ota etanhan, taku unqupi qon he on, unkiyepi on etanhan wicota wopida eyapi kta.

12 Unkiy uśkinpi kin he unnagipi hdaotanin kin hee, wowicake wowakan ko en okonwanjidan, wicacehpi wolrsape yuhe śni, tuka Wakantanka towaonśida yuha, wicoicage kin chna unnipi, qa niyepi kin ekta iyotan hececa.

13 Taku dawapi, qa sdonyayapi kin hecedan wowapi en un- 
WICOWOYAKE 2.

nicagapi; qa owihanke kin hehanyan wicayadapi kta kepce.

14 Onśpa sdonunyayapi, unkiyuśkinpi lin he niyepi, he iyecen wowitan nitawapi kin he unkiyepi, Jesus Itancan taanpetu kin en.

15 Hecen wacinmduza, tokaheya niyepi kin ekta ciupi kta tuka, on inonpa wowaśte duhapi kte cin heon.

16 Qa niyepi kin etanhan iyoopta Makedonia ekta mde ca, ake Makedonia etanhan niyepi kin ekta icicawin wahdi kta, qa Juda makoce kin ekta ye mayayapi kta wacin.

17 Hecen wacin mduze cehan, hehan iyowilian wacin mduza he; qa token miciconze cin he wicacehpi eciyatanhan miciconze ca hecen, Han, han, hiya, hiya, eya waun he.

18 Wakantanka wa"cinyepica, wicoie unkeniciyapi qon he, han qa hiya, hee śni.

19 Miye, qa Siluanos, qa Timotheos, Jesus Messiya Wakantanka Cinhintku kin unkoniciyakapi qon he, han, qa hiya, hee śni, tuka iye kin on, han ecedan.

20 Wakantanka iwahounyanpi kin hena owasin iye en, Han hee, qa iye en Amen, unliyepi kin etanhan Wakantanka yatanpi kte cin heon.

21 'Tuwe niyepi kici Messiya en unyusutapi, qa sdaunyanpi kin he Wakantanka kin ee.

22 Qa he unkapuspapi, qa uncantepi kin ekta Woniya tokaheya icupi ece kin he unqupi.

23 Unkan nakaha minagi kin ekta Wakantanka mayaotanin kta e cewakiya; Itoncipapi lita e on nahahin Korinth ekta wai śni.

24 Wacinyayapi kin on wowi- dag unniyanpi sni, tuka widuśkinpi kin en ounniciyapi kta. Wacinyanpi on sutaya nayajinpi ece.

\section{WICOWOYAKE 2.}

1 Miye qe hecen mihdulican, ake iyokiśinya ekta ciupi kte śni.

2 Miye iyokiśinciyapi kinhan, tuwe iyuśkinmaye kta he; iyokiśinwaye cin hee kta.

3 Hecen on he wowapi cicagapi, ekta wau kinhan tona on imduśkin kte cin hena on etanhan iyokiśin waun kte śni. Qa niyepi owasin wacinciyapi, mitowiyuśkin kin he en niyepi owasin oyapapi kta.

4 Wicokakije tanka, qa wicocanteśica, qa iśtamnihanpe ota ehna wowapi cicagapi qon, iyokiśinniyanpi lite cin heon hecamon śni ; tuka nina canteciciyapi kin he sdonyayapi kte cin heon.

5 Tuwe iyokiśicapi kage ciqon he miye iyokiśinmaye śni ; tuka niye nihunhanpidan, owasin cibapi kte śni.

$6 \mathrm{He}$ tuwe kin hecen yacopi qon, wicota econpi kin hecehnana kta.

7 Heon nakaha wicaśta kin he iyuśkinyayapi, ya yecanptapi kta, okinni nina iyokiśice cin on ktepidan kta.

8 Heon ceciciyapi ce, he cante en duzapi kin hduotanin po.

9 'Taku owasin on waanagoptan yaunpi hecinhan, iciyukcanpi kte cin heon wowapi cicagapi qon.

10 Tuwe taku yecicajujupi kinhan, miś eya hecamon, qa tuwe taku wecicajuju kin he niyepi kin on wecicajuju, Messiya itokam.

11 Hecen Satan ohiunyanpi 281 


\section{KORINTH.}

kte śni; iye token ic̣iconze cin sdonunyanpi.

12 'Troas ekta Messiya tawootanin waśte yuha wahi, qa Itancan kin tiyopa wan makiyuzamni ;

13 Hehan minagi elkta cante waśteya waun śni, Titos mihunkawanji kin hen iyewaye śni kin heon; hecen hena nape wicamduze ca, hetanhan Makedonia ekta imdamda.

14 Wakantanka, ohinniyan Messiya on ohiye unyanpi, qa ohinniyan unkiyepi kin on tawosdonye woomna yaotanin kin, hee wopida yuha nunwe.

15 Wakantanka ekta, Messiya on woomna waśte kin he unkiyepi, tona wiconi yuhapi kta, qa nakun tona yutakunipi kte śni kin hena en.

16 Dena iś tapi kta e wiconte oomna kin he unkiyepi, qa hena e nipi kta e wiconi oomna kin he unkiyepi; qa tuwe hena taku kin okihi he.

17 Wicota Wakantanka oie kin hnayan kuwapi kin hena iyeuncecapi śni ; tuka wowicake eciyatanhan, tuka Wakantanka eciyatanhan, qa Wakantanka itolaam, Messiya unkoyakapi ece.

\section{WICOWOYAKE 3.}

1 Ake unkihdatanpi unkayapi he; qa wicaśta wanjikji iyecen waayatanin wowapi nicagapi kte cin heca uncinpi he ; qa iś waayatanin wowapi unyecagapi kta uncinpi he.

2 Wowapi unkitawapi unkicantepi kin ekta owapi kin he niyepi, wicaśta owasin sdonyapi qa yawapi ;

3 Messiya tawowapi taninyan oyakapi unkiye unkagapi kin; mini sapa on kagapi śni, tuka 282
Wakantanka ni un kin Taniya kin on owapi ; inyan mdaska kin en un śni, tuka wicacante conica kin he en.

4 Unkan Wakantanka ekta Messiya eciyatanhan woawacin kin de unhapi.

5 Unkiye cinka taku unkiyukcanpi kta unkokitpanipi, unkiyepi atayedan, taku unkokihipi kin he Wakantanka eciyatanhan.

6 Iye wicotakuye teca kin en ookiye wawokihika unkagapi, oowa kin he en un śni, woniya kin hee en; oowa kin he wateya, tuka woniya kin he waniya ece.

7. Wiconte wawokiyapi kin he owapi, qa inyan akan pagopi qon he wowitan yuhe cin, on Israel cinca kin Moses itohnake wiyatpa kin he opahta yukanpi kta okihipi śni hecinhan, wowitan kin he enakiyapi tuka;

8 Hecen Woniya wawokiyapi kin he sam iyeya nina wowitan kte śni he.

9 Woyaco wawokiyapi kin he wowitan hecinhan, wicoowotanna wawokiyapi kin he sanpa nina wowitan kta.

$10 \mathrm{Qa}$ taku yuwitanpi qon he taku wanji on wowitan śni, taku iyakapeya wowitan kin hee on etanhan.

11 Qa taku enakiyapi qon he wowitan kin hee hecinhan, taku ecen he cin he iyotan wowitan kin ee.

12 Woape hececa unhapi kin heon nina wadiuntakapi ece.

13 Unkan Moses ite akahpe wan itohnake kin akahpe kiton kin he iyecece śni, taku enakiyapi kin owihanke kin hehanyan Israel cincapi kin ekta etonwan okihipi śni kin heon etanhan.

14 Tuka iye tawacinpi kin ao- 


\section{WICOWOYAKE 4.}

tpasyapi : qa anpetu kin dehanyan Wowapi Wakan tanina kin yawapi eca, ite akahpe kin he eyakupi śni ecen un; he Messiya eciyatanhan eyakupi tuka.

15 Tuka anpetu kin dehanyan, Moses yawapi eca, ite akahpe kin he iye cantepi kin akahpa.

16 Tuka tohan Itancan kin ekta yuhomnipi kinhan, hehan ite akahpe kin he yuzamnipi kta.

17 Woniya kin he Itancan kin ee, qa tukten Itancan taniya kin un kin, hen tawa içiyapi yukan.

18 Tuka unkiye owasin itohnake kin en takudan unkahpapi śni, ihdiyamdasin en wawanyakapi kin iyecen Itancan towitan kin wanunyakapi, qa iye ouncage kin iyecen unkagapi, wowitan sanpa wowitan kin en, Itancan Taniya kin on etanhan iyececa.

\section{WICOWOYAKE 4.}

1. Heon etanhan wawokiyapi kin de unhapi kin, wowaonśida unkicupi kin heon wacin ibounśakapi śni.

2 Tuka taku wowiśtece nahmana un kin hena ehpeunyanpi, qa wicahnayanpi kin en maunnipi śni, qa wohnaye yuha Wakantanka oie kin unkiciyuhapi śni, tuka wowicake yaotaninpi kin on wicaśta owasin wiyukcanpi kin ekta unhdaotaninpi, Wakantanka itokam.

3 Wootanin waśte unkitawapi kin he analimanpi hecinhan, tona takunipi śni kin hena anawicakilimanpi.

4 Hena ekta wicoicage kin de taku wakan tawa kin tona wicadapi śni wicotawacin kin wacinton śni wicakaga; Messiya Wakantanka ouncage kin hee, tawootanin waśte wowitan iyoyan- pa kin he iyoyamwicaye kte s̉ni heon etanhan.

5 Unkiye unkihdaotaninpi śni, tuka Messiya Jesus Itancan kin he unyaotaninpi ece; qa unkiye Jesus on ookiyewicayayapi kin. 6 Wakantanka iyoyanpa kin otpaza etanhan yege śi qon he uncantepi kin iyoyamunyanpi, Jesus Messiya itohnake kin eciyatanhan, Wakantanka towitan wosdonye iyoyanpa kin unqupi lite cin heon.

7 Tuka woyuha kin de maka cega ohna unhapi, hecen wowaśake tanka kin Wakantanka etanhan un kta, qa unketanhanpi kte śni.

8 Ihdukśantanhan aotinsunyanpi, tuka unkahdaskicapi śni : wacinhnuni unyanpi, tuka takudan wacinye śni kin heuncecapi śni.

9 Śicaya unkuwapi, tuka elipeunyanpi unyakonpi śni : makata ehpeunyanpi, tuka ihang unyanpi śni.

10 Ohinniyan untancanpi kin en Jesus Itancan te cin he yuha unkanpi, hecen Jesus wiconi tawa kin untancanpi kin en taninyan un kta.

11 Unkiye unnipi kin Jesus on ohinniyan wiconte en iyaye unyanpi, Jesus wiconi tawa kin uncehpipi te kte cin en atanin kte cir heon.

12 Hecen unkiyepi kin en wiconțe olianyan un, tuka niyepi kin en wiconi.

13 Unkiye wowacinye woniya kin de unhapi, wowapi en kagapi qon iyececa; Wicawada kin heon iwaa ce; unkiś eya wicaundapi kin heon unkiapi ece.

14 Qa tuwe Jesus Itancan kin piya ekicetu ye ciqon, he Jesus eciyatanhan, ake ekicetu unyanpi kta, qa niyepi kin om sutaya he unkiyapi kta e sdonunyanpi. 


\section{KORINTH.}

15 Niyepi kin on etanhan taku owasin ecen un, hecen wowaonśida tanka kin, wicota wopida eyapi kin on sanpa ota aye lita, Wakantanka towitan kin on.

16 Heon wacin ibounśakapi śni, tuka wicaśta akantanhan unkitawapi kin he takuni śni aya eśta, wicaśta mahen tanhan un kin he anpetu iyohi yutecapi.

17 Unkakijapi cistinna, qa ptecedan kin he wowitan ota hinca, ota hinca, owihanke wanin the hinca unkicagapi kta.

18 Icunhan taku wanyakapi kin hena ekta unketonwanpi śni, tuka taku tona wanyakapi śni kin hena ekta ewauncinpi. Taku tona wanyakapi kin hena ihanke kta, qa taku tona wanyakapi śni un kin hena owihanke wanica.

\section{WICOWOYAKE 5.}

1 Makata wakeya unkitawapi kin de yujujupi kinhan, Wakantanka ticage cin tipi nape codan kagapi kin, mahpiya ekta, owihanke wanin yanke cin he unhapi e sdonunyanpi.

2 Qa de ohna comniunhdazipi, qa tipi unkitawapi man̈piya eciyatanhan un kin he kounyakapi kta e cantounkpanipi.

3 He kounyakapi kinhan, tancodan unyakonpi kte śni.

4. Wakeya kin de ohna unkanpi kin hehanyan comniunhdazipi, unśakapi kin heon. Wokoyake unhduśdokapi kin he uncinpi śni, tuka kounyakapi kta: Hecen wiconi e wiconte ocowasin napce kta.

5 Tuwe he en icaliunyanpi kin he Wakantanka ee; qa he Woniya tokaheya kin wanna unqupi.

6 Heon ohinniyan cante un- waśtepi, qa tohanyan wicatancan kin ohna unkanpi kin hehanyan Itancan kin itehanyan unyakonpi kin he sdonunyanpi.

7 Wacinyanpi eciyatanhan maunnipi, qa wawanyakapi kin eciyatanhan śni.

8 Cante unwaśtepi, tuka tancan kin de ohna unkanpi śni, qa Itancan kin kici unyakonpi kta e he iyotan uncinpi ece.

$9 \mathrm{He}$ de aiçiciya unkakitapi, ekta kici unyakonpi, qa iś den unkanpi, unma tukte kaśta, iyokipiunyanpi kte cin he.

10 Owasin Messiya tawoyaco oiyotanke kin itokam taninyan inaunjinpi kta, otoiyohi taku iye tancun kin ohna ohanye cir hena icu kta, token econ qon eciyatanhan, he waśte qa śica unma tukte kaśta.

11 Itancan wohitike cin he sdonunyanpi kin, heon wicaśta cin wicunkiyapi; Wakantanka en unkihdaotaninpi, qa nakun widukcanpi kin en unkihdaotaninpi wacanmi.

12 Ake niyepi en unkihdatanpi śni, tuka unkiyepi on iniwinktapi kta e okihi unniyanpi; hecen tona cante kin eciyatanhan śni, tuka itohnake kin on iwinktapi kin hena ekta, taku on iniwinktapi kte cin duhapi kta.

13 Wacinunhnunipi hecinhan, he Wakantanka on etanhan; qa wacinunksapapi hecinhan, he niyepi kin on etanhan.

14. Messiya wacantkiye cin he iyounpaśtakapi ece.

$15 \mathrm{Qa}$ he de unkiyukcanpi, wanji owasin on te cinhan hehan owasin tapi tuka; qa owasin on te cin, heon tona nipi kin hena iś iyepi on nipi kte śni, tuka tuwe hena on te ca ake kini kin hee on nipi kta.

16 Heon etanhan, detanhan wi-

284 


\section{WICOWOYAKE 6 .}

cacelipi eciyatanhan tuwedan sdonunyanpi śni; han, Messiya liee wicacelipi eciyatanhan sdonunyanpi eśta, nakaha detanhan sdonunyanpi śni.

17 Hecen tuwe Messiya en un kill he wanna wicaśta teca; taku tannike cin hena enakiyapi; iho, taku owasin teca icaga.

18 Qa taku owasin Wakantanka etanhan un, he iye kici okiciciye unkiyapi, Jesus Messiya eciyatanhan, qa wookiye wawokiyapi kin he unqupi.

19 Hecen Wakantanka Messiya on, wicoicage kin, iye kici, okiciciye wicaye ca, walitanipi kin wicakiciyawa śni; qa wookiye wicoie kin he yuhe unkiyapi.

20 Heon Messiya on wawiciyapi kin unkiyepi, unkiyepi eciyatanhan Wakantanka ceniciyapi kin iyececa, Messiya caje on ceunniciyapi, Wakantanka kici okiciciya po.

21 Tuwe woalitani sdonkiye śni un qon he, unkiyepi on woalitani kagapi; hecen iye en Wakantanka toowotanna unkicagapi lita.

\section{WICOWOYAKS 6.}

1 Heon iye kici litaunnipi iyecen, niyepi nakun ceunniciyapi ce, ituh Wakantanka towaonśida kin iyacupi kte śni.

2 He iye kaken eya, Omaka oiyolipi kin en nacilion qa wiconi anpetu kin en ociciya: Iho nakaha omaka oiyokipi kin hee; nakaha wiconi anpetu kin he dee.

3 Takudan on tuwedan ibotounyanpi śni, hecen wawokiyapi kin he bapi kte śni :

4 Tuka Wakantanka taokiye unyanpi kin he taku owasin on unkihduotaninpi ece, wacintan- kapi kin en, wokakije en, wahpaya unnicapi kin en, woiyokiśice en ;

5 Apapi kin en, kaślka hnakapi kin en, yuomdecapi kin en, wicolltani kin en, kiktahan unpi kin en, wotektehdapi kin en ;

6 Ecedan unpi kin on, wosdonye kin on, tehan apepi kin on, wacantkiyapi kin on, Woniya Wakan kin on, awicakehan waśtedakapi kin on ;

7 Wowicake oie kin on, Wakantanka towaśake kin on, wicoowotanna wipe, etapa qa catkatanhan unpi kin on ;

8 Wowitan qa wowiśtece kin ohna; waaiapi qa yaonihanpi kin ohna; wicahnayanpi kin iyececa, tuka wicakapi ;

9 Sdonyapi śni un kin iyeuncecapi, tuka tankaya sdonunyanpi ; tapi se iyecen unyakonpi, tuka ito unnipi; unkaśtakapi, tuka nahanhin unktepi śni ;

10 Iyokiśicapi iyeuncecapi, tuka ohinniyan wiunyuśkinpi; wahpanicapi kin iyeuncecapi, tuka wicota watonka wicunkagapi ; takudan yuhapi śni kin iyeuncecapi, tuka taku owasin untonpi.

11 Korinth oyate kin, niyepi on i unhdukawapi, uncantepi kin tanka aya.

12 Unkiyepi en takudan aotins niyanpi śni, tuka niye nicantepi kin en oțins niçyapi.

13 Hehan iyunwin kin de mayaqupi lita; micinca kin iyecen ociciyapi ; niśs eya tankaya niçicagapi nunwe.

14 thnuhan tona awacin śni un kin hena en ecinśniyan iyanihdaśkapi kinhan. Token wicoowotanna woahtani kici tidan kta he; qa token iyoyanpa kin otpaza kici un kta he:

15 Qa Messiya Belial kici token okonwanjidan cante yuzapi 285 


\section{KORINTH.}

he; qa tuwe wicada lin he wicada śni un kin kici taku akiptan tawapi kta he;

16 Qa tipi wakan Wakantanka tawa kin he wakagapi kin kici token un kta he. Wakantanka ni un tipi wakan tawa kin he niyepi. Wakantanka heye ciqon lie iyececa; Hena om wati kta, qa om mawani kta. Hena Wakantanka mayanpi kta, qa mitaoyate kin hena eepi kta ce.

17 Heon etanhan hena cokaya tanhan tankan upi, qa icunonpa un po, Itancan kin eya, qa taku aśape cin oyutanpi śni po, kinhan miś tawa ciyapi kta;

18 Niye ate mayayapi kta, qa miye cinkśi ciyapi qa cunkśi ciyapi kta ce, Itancan Iyotan Waśake cin he hena hecen eya.

\section{WICOWOYAKE 7.}

1 Heon waśtecicidapi kin, waloyapi kin dena unhapi kin heon wicacehpi wicanagi ko taku aśape cin etanhan unhduecepidan, qa Wakantanka kokipeyahan wicoowotanna kin tanyan unhduśtanpi kta.

2 Anaungoptan po; unkiye tuwedan śicaya ecarvicunkiconpi śni, tuwedan wicunyuśicapi śni, tuwedan taku icakiś wicunyanpi śni.

3 He wayaco hepe śni; cante en unniyuzapi, ptaya unțapi qa iś unnipi kta ce, epe ciqon.

4 Niyepi on wadimatake hinca, niyepi on nina imawinkta; wocantohnake omajudan, unkakijapi owasin en nina imduśkin.

5 Makedonia en unhipi qehan, uncehpipi kin takudan oziiciye śni, tuka taku owasin on iyounkiśicapi; tankan tanhan wicokicize, qa mahen tanhan wokokipe.
6 Tuka Wakantanka tona iyokiśicapi iwicakiye cin he cantohnag mayan, Titos hen hdi kin heon :

7 Qa he hdi kin hecedan śni, tuka nakun niye cantohnagyayapi kin heon; nina wacin en yeyayapi, qa iyoniciśicapi, qa token cante en unyakiduzapi kin hena unkokiyakapi qehan, nina imduśkin.

8 Wowapi wan on iyokiśinciyapi eśta, heon nakaha iyopemiciye śni, iyopemiçiya tuka qon. He wowapi kin ptenyedan iyokiśinniyanpi wanmdaka.

9 Nakaha imduśkin, iyokiśinniyanpi kin heon etanhan śni, tuka nihduecetupi kta e ón iyoniciśicapi kin heon. Wakantanka eciyatanhan iyokiśinniciyapi, hecen talsudan icakiśs unniyanpi śni.

10 Wakantanka eciyatanhan iyowicakiśice cin he ihduecetupi on wiconi kta, on tohinni iyopeiciyapi śni kin he kaga; tuka wicoicage kin de en iyowicakiśice cin he wiconte icahya.

11 Wanyaka po, Wakantanka eciyatanhan iyoniciśicapi kin he itonpeya nicagapi, waayuptapi, qa wośihda, qa wokokipe, qa cante en hiyeyapi, qa winawizipi, qa hdajujupi kin hena niyepi en icaliniciyapi ; taku owasin on, he econpi qon $\mathrm{cn}$, takudan iyanionpapi śni yaunpi e nihduotaninpi.

12 Heon wowapi cicagapi, tuwe śicaya econ qon he on etanhan śni, qa tuwe sicaya ecakiconpi qun he on etanhan śni, tuka Wakantanka itokamya token wacin en unniyuzapi kin he niyepi en yutaninpi kta.

13 Heon etanhan iniciyapi kin on unkikiyapi ; qa Titos iyuślkin kin heon miś eya nina imduśkin, 


\section{WICOWOYAKE 8.}

iye nagi kin niyepi owasin eciyatanhan magagayapi kin heon etanhan.

14 Heon ciyatanpi qa owakiyaka eśta, on imaśtece śni ; tuka taku owasin eciyatanhan eciciyapi qon, he iyecen Titos en ciyatanpi kin he nakun wowicake.

15 Unkan niye owasin token anayagoptanpi, qa wokokipeya, cancanyan iyowinyakiyapi qon he kiksuya eca, iye iyotan cante en niyuzapi.

16 Heon etanhan, taku owasin on wicacidapi kin heon miś eya imduśkin.

\section{WICOWOYAKE 8.}

1 Mihunkawanji, Wakantanka towaonśida Makedonia okodakiciye kin en yutaninpi qon he sdonyeciciyapi.

2 Hena wokakije tanka en nina iyuśkinpi, qa iyotan wahpanica unpi kin he etanhan taku ota iyokipiya wicaqupi.

3 Okihipi kin iyacinyan, (miś mdaotanin, qa olihipi kin kapeya, iye cinka wicaqu wacinpi.

4 Qa wocekiye ota on ceunkiyapi, taku wicaqupi kin he, qa tona ecedan un kin wicakipamnipi wawokiyapi kin he nakun unkicupi kte.

5 Qa unkecinpi qon iyecece śni, tuka tokaheya iye tancanpi kin Itancan kin içiçupi, qa nakun Wakantanka tawacin kin eciyatanhan unkiyepi kin en ic̣içupi.

6 Hecen Titos ceunkiyapi, niyepi en wanna tokaheya hecon kin, he iyecen wowaonśida kin de nakun niciyuśtanpi kta.

7 Heon taku owasin on winijicapi, wacinyanpi, qa iapi, qa wosdonye, qa wicamniheca owasin, qa waśteunyakidapi ko, he iyecen wowaśte kin de on nakun winijicapi kta.

8 Wicoope eciyatanhan hena hepe śni, tuka wicaśta tokeca aiciciyapi kin heon; qa wacanyakiyapi kin he wowicake kinhan imdute kta.

9 Jesus Messiya Itancan unkiyapi towaonśida kin he sdonyayapi, watonka tuka niycpi on wahpaniiciya, hecen iye wahpanica kin on winijicapi kta.

10 Unkan he de on wahokonciciyapi ; niye decanonpi kta iyececa, wanihan sanpa hecanonpi kta e niçiconzapi, qa wiyeya yaunpi kin heon etanhan.

11 Nakaha woecon kin he hduśtan po, kohanna wicayadapi qon he iyecen taku duhapi kin etanhan ecen ecanonpi lita.

12 Tokaheya wicadapi yuke cinhan, wicaśta taku yuhe cin he eciyatanhan iyokipiya icupi ece, qa taku yuhe śni kin eciyatanhan śni.

13 Wicaśta tokeca oziiciyapi kta, qa niye duśakapi kte cin hee śni; tuka akiyecen unpi kta; heon nakaha wijinya yaunpi kin he hena taku iwicakakije cin en un kta;

$14 \mathrm{Qa}$ iś iyepi nakun wijicapi kinhan, wicakiśya yaunpi kin en oniciyapi kta; hecen akiyececapi yuke kta.

15 Wowapi en kagapi qon iyececa; Tuwe ota pahi kin he takudan ohdapte śni, qa tuwe waniqadan pahi kin he takudan icakiś un śni ce.

16 Tuka Wakantanka wopida yuha nunwe, he niyepi on Titos hecen cante yuslkiya.

17 Awicakehan wahokonkiyapi kin anagoptan; tuka iye hinca inalini, qa kohanna niyepi kin ekta ye wacin. 


\section{KORINTH.}

18 Unkan he kici hunkawanjinkiciyapi wan, wotanin waśte on okodakiciye owasin en yatanpi kin hee ekta ye unśipi.

$19 \mathrm{Qa}$ hecedan śni, tuka kici ohnihde unyakonpi kta e he okodakiciye kin kalinigapi, Itancan kin yatanpi kta, qa unkiye de iyokipiya econkupi kta e heon taku ituhanpi kin de en ounkiyapi kta.

20 Qa taku ota unpamnipi kin de on tuwedan unbapi kte śni e he unkakitapi.

21 Taku waśte ece unkihnipi kta, Itancan kin itokam, qa nakun wicaśta kin en.

22 Unkan hena om hunkawanjinunyanpi kin yeunśipi, he wanna ota unkiyukcanpi, owotanna olianyan ece, qa nakaha iyotan aiciciya, tanyan wacinniyanpi nakaeś.

23 Hecen Titos he kapi hecinhan, he mitawaśi qa niyepi on omakiye cin hee; qa hunkawanjinwicunyanpi kin hena eepi kinhan, okodakiciye yervicaśipi qa Messiya towitan kin hena eepi ce.

24 Heon etanhan, hena en, qa okodakiciye kin wicitokam, wacanyakiyapi kin, qa unniyatanpi qon he nakun wicakipazo po.

\section{WICOWOYAKE 9.}

1 Wicaśta owotanna owicakiyapi kte cin on wowapi cicagapi kta wanna iyecece śni.

2 Token wacin duzapi kin he sdonwaya, heon Makedonia etanhanpi kin hena en yaonihanyan cajeciyatapi, wanna waniyetu wanjidan hehanyan Akeya wiyeya yanka ce, qa inayahnipi kin he wicaśta ota iyowicapaśtaka.

3 Tuka hunkawanjin wicawa- ye cin hena ekta yewicawaśi, unniyatanpi qon he ecetu kta, qa epe ciqon iyecen wiyeya nankapi kta e heon.

4 Okinni Makedonia etanhan upi kin hena om elita wai, qa wiyeya yaunpi śni ecen wanunniyakapi kinhan, unkiye, niyepi epe śni, sutaya wacinyus unniyatanpi qon he on unkiśtecapi kta.

5 Heon hunkawanjin kiciyapi kin cewicawakiye kta wacami qon, tokaheya niyepi ekta ipi, qa wowaonśida nitawapi ehanna iwahoniyanpi qon he wiyeya ehnakapi kta, hecen awicake. han wowaonśida kta, qa watehindapi kin eciyatanhan kte śni.

6 'l'uka de; Tuwe waniqadan oju kin he waniqadan mnaye kta; qa tuwe iyakicuya oju kin he iyakicuya mnaye kta ce.

7 Otoiyohi iye cante kin ekta içiconze cin eciyatanhan qu kta, woiyokiśice on qa econ śipi kin on etanhan śni ; iyuśkinyan wicaqu kin he Wakantanka waśte daka.

8 Wakantanka wowaonśida owasin niyepi en niciyuotapi kta okihi; hecen taku owasin ota duhapi kin on wicohan waśte kin wadupipike kta.

9 Wowapi en kagapi qon iyecetu; Taku ehpeya, wahpaninica kin taku wicaqu ece kin he tonwotanna kin ohinniyan un kta.

10 Tuwe woju kin ojupi yuheliya ece kin he aguyapi woyute yayapi kte cin nicupi, qa takn oyajupi kin icah niciyapi, qa nitoowotanna waskuyeca kin niciyuotapi nunwe.

11 Qa taku owasin en wijinya yaunpi kta on iyotan oliannipipi kta, heon unkiye Wakantanka wopida unliekiciyapi. 
12 Wapamnipi wawoliyapi kin de tona owotalna un kin taku icakijapi kin enakiye kte ca, nakun Wakantanka wopida nina eciyapi kta e kaga.

13 Messiya tawootanin waśte kin en oyapapi e daotaninpi, qa hena taku ota wicayaqupi, qa owasin taku wicayaqupi kin he on wawokiyapi utapi kin de on etanhan Wakantanka yaonihanpi.

14 Qa niyepi on wocekiye eniciyapi kin heon nina cantonikpanipi, Wakantanka towaonśida tanka nicipi un kin heon etanhan.

15 Wakantanka taku yawapica śni unqupi kin he on wopida kiciun nunwe.

\section{WICOWOYAKE 10.}

1 Palos he miye, Messiya wahbayedan qa wacintanka heon ceciciyapi ce, niyepi ekta onśihanyan waun, tuka en waun śni ehantarhan, niyepi on wadimataka ;

2 Tuka ceciciyapi, en waun kinhan, wanjikji wicacehpi kin eciyatanhan maunnipi kecanunkinpi kin, hena en waditagya waun kta wacanmi kin he iyecen wadimatake kte śni.

3 Wicacelipi kin en maunnipi eśta, wicacehpi éciyatanhan unkicizapi śni.

4 Wipe unkunpi kin hena wicacehpi kin heca śni, tuka waśaka, Wakantanka on, inatake suta kin yujuju ece :

5 Wacinyuzapi kin kun ehpeya, qa taku tehan wankantuya un Wakantanka sdonyapi kin en wankan iyeiciye cin hena owasin, qa wicotawacin iyuhpa Messiya anagoptanpi kin en wayaka ai ece.
6 Qa wanahonpi śni kin tounkiconpi kta e wiyeya unkanpi; tohan anayagoptanpi kin hduśtanpi kinhan.

7 Wicaśta itohnake kin hecedan iwandakapi he. Tuwe Messiya tawa kecanicin kinhan, he ake kaken awacin kta; iś iye Messiya tawa kin heca he iyecen unkiś unkiyepi Messiya tawa unyanpi.

8 Wookihi Itancan unqupi kin, on icahunniyanpi $\mathrm{kta}$, qa on ihangunniyanpi kte śni, heou sanpa mihdatan eśta, imaśtece kte śni.

9 Wowapi on wakokipe cicagapi kta wacanmi śni.

10 He wowapi kage cin hena suta qa waśaka, tuka tancan en un kin he waśake śni qa ie cin he wowiha ce, eyapi.

11. Tuwe hecece cin he kaken awacin kta, en unkanpi śni wowapi wicoie kin on tounkecapi kin, he tohan en unkanpi kinhan, wicohan kin on heuncecapi kta.

12 Tona ihdatanpi kin hena unkihdacinpi, qa opeya unkihdawapi kta tawatenunyanpi śni; hena $e$ is iye on wihdutapi, qa iyepi on ihdukcanpi, qa hecen okahnigapi śni.

13 Unkiye wiunhdutapi kin isanpa unkihdatanpi kte śni; tuka wicohan iyutapi Wakantanka unqupi kin he eciyatanhan, iyutapi kin niyepi kin iyahde niyanpi.

14 Ekta iyahde unniyanpi kta e sanpa unkihdutitanpi śni. Tuka Messiya tawootanin waśte kin en niyepi kaeś ekta unniupi.

15 Wiunhdutapi kin ișanpa unkihdatanpi śni, wicaśta tokeca wicohtani kin en; tuka wicayadapi kin he icage cinhan, niyepi 289 


\section{KORINTH.}

on wiyutapi unhapi kin eciyatanhan sanpa unyuonihanpi kta unkapepi.

16 Hecen niye dukanpi kin akowapa wotanin waśte kin unkoyakapi kta, qa tuwe tokeca taku tawa wiyeya yanke cin en unkiwinktapi kte śni.

17 Tuka tuwe iwinkta kinhan Itancan kin on iwinlsta nunwe.

18 'Tuwe ihdaonihan kin he ecetudakapi kin ee śni, tuka Itancan kin tuwe yaonihan kin hee kta.

\section{WICOWOYAKE 11.}

1 Witkotkoya iwae cin cistiyedan iyowinmayakiyapi kta wacin. He iyowinmayakiyapi nakaeś.

2 Wakantanka winawizi kin iyecen inaciwizipi, wicaśta wanjidan kiciyus ciyapi, hokśiwinna ecedan un kin Messiya ekta aciipi kta heon.

3 Tuka he kowakipa, wamduśka wolnaye on Ewe hnaye ciqon he iyecen nitawacinpi kin yuśicapi kta, qa woyuecedan Messiya en un kin etanhan iyaye niy anpi kta naceca.

4 Tuwe en niupi kin he Jesus tokeca wan unkiye unkoyakapi śni kin he oyaka, qa Woniya tokeca, iyacupi śni qon, he nakaha iyacupi, qa wotanin waśte tokeca, anayagoptanpi śni qon he oniciyakapi kinhan, iyowinyakiyapi kta.

5 Miye yewicaśipi iyotan tanka kin takudan ihukuya waun śni, imdukcan.

6 Ho magahan eśta, wosdonye kin on waksapa; tuka niyepi kin en taku owasin on tanyan unkihdaotaninpi.

7 Wakantanka tawootanin waste kin iyunwin codan ociciyakapi, qa wankantuya iyeniyanpi kta $e$ on mihduhukuya kin he wawahtani he.

8 Okodakiciye tokeca kin taku wicawaki, qa opemicatonpi kin iwacu, ociciyapi kte cin heon; qa cicipi waun qa taku imakakije cehan, taku nitawapi kin mun śni :

9 Taku iyokpani waun qon he luunkawanjin kiciyapi Makedonia etanhan hipi kin máqupi, qa ohinniyan takudan inagiyeciyapi śni waun, qa eeś nakun hecen waun kta.

10 Messiya wowicake tawa miciun kin heon Akeya makoce kin en imawinkta kin de ecetu śni makiyapi kte śni.

11 Tokeca he ; waśte cicidapi śni kin heon etanhan he. Wakantanka sdonya.

12 Tuka taku ecamon kin he nakun ecamon kta, hecen tona wiyaonpapi akitapi kin hena etanhan wiyaonpapi kin emdaku kta, qa en iwinktapi kin he unkiyepi kin iyecen unpi kta.

13 Hena yewicaśipi itonśni kin hecapi, wohnaye ohanyanpi, qa Messiya yewicaśi qon hena iyecen ihduzapi.

14. Qa he taku wowinihan śni, Satan iye kaeś iyoyanpa olınihde kin iyecen ihduza.

15 Heon etanhan, iye taokiye kin hena wicoowotanna ookiye kin iyecen ihduzapi kte cin he taku tanka śni, hena iye wicohan kin eciyatanhan ihang yapi kta.

16 Ake hecen epa; Tuwedan witkotkoke madake kte śni. Tuka hecon kinhan witkotkoke cin iyecen iyowinmakiye $\mathrm{kta}$, cistiyedan mihdatan kta.

17 'Taku epe cin hena Itancan kin eciyatanhan epe śni, tuka nina ihdatanpi kin he on witkoya ie cin iyemaceca. 


\section{WICOWOYAKE 12.}

18 Wicota wicacehpi kin on ihdatanpi kin he iyecen miś eya mihdatan kta.

19 Witkotkopike cin hena iyokipiya iyowinwicayakiyapi ece, niye yaksapapi nakaeś.

20 'Tuwe wowidag' niyanpi, tuwe temniyanpi, tuwe wanicipi, tuwe wahanicida, tuwe ite ohna anipapi hecinhan, iyowinwicayakiyapi ece.

21 Waośtehdapi kin he waka, waunśakapi śni kin heon. Tuka taku on tuwe waditake cinhan, witkotkoya iwaa ce, he on miś eya wadimataka.

22 Hena Hebrew wicaśta kin hecapi he. Miś eya hemaca. Hena Israel etanhanpi he. Miś eya. Hena Abraham cinca he. Miś eya.

23 Hena e Messiya taokiye kin hecapi he; witkotkoke cin iyecen iwaa, miś eya imayotan. Wicolitani kin en imayotan; apapi kin en wopteca śni waun; kaśka hnakapi kin en imayotan, qa wiconte kin en otakiya waun. 24 Juda oyate zaptan akihde, apapi wikcemna topa wanjidan iyolkpani maqupi.

25 Yamni akihde cansakadan on amapapi; wancadan inyan on makininpi; yamni akihde wita wata micamdecapi ; anpetu wanjidan hanyetu iyahna ośbe kin mahen waun;

26 Nina icimawani, mini ota wokokipe kin en waun, wakipi sa okokipe kin en, mitaoyate on wokokipe kin en, Ikcewicaśta on wokokipe en, otonwe ekta wolkokipe en, hewoskantuya wokokipe en, miniwanca ekta wokokipe en, hunkawanjin kiciyapi itonpi śni kin hena ekta wokokipe en ;

27 Mdowakita qa nina hitawani kin on, ota kiktahan waun kin on, wotektewahda qa imapuza on, ijehan amakilian kin on, macuwita qa tancodan waun kin on; $28 \mathrm{Qa}$ tankan tanhan anpetu iyohi taku amaupi kin, okodakiciye kin owasin awanhdakapi kin he nakun.

29 Tuwe waśake śni un, qa miye wamaśake śni he; tuwe aiapi un, qa miye śinwahda śni he. 30 'Tuka imawinkta kta iyecece cinhan, wamaśake śni waun kin heon imawinkta kta.

31 Jesus Messiya Itancan unkiyapi he Wakantanka,tawa, qa Atkuku kin, owihanke wanin wowaste yuhe cin he sdonmayan, itonśni mawanica.

32 Damaskos en, Aretas wicaśtay a tapi yanke cin ihukuya, wicaśta itancan kin akicita ota wicayuha, Damaskos oyate otonwe tawapi kin awanhdake ça, maktepi kta akita.

33 Hehan conkaślke owanyeye wan etanhan, makanyankapi wan ohna, kun hiyumayanpi ; qa iye nape kin etanhan nawajica.

\section{WICOWOYAKE 12.}

1 Imawinkta kte cin he iyemicihantu śni, wowihanmde qa Itancan woyakapi tawa kin ekta wau kta.

2 Ehanna waniyetu ake topa sam iyeya, wicaśta wan Messiya en un kin he sdonwaya; (wicatancan kin ohna un kin sdonwaye śni, qa tanton śni un kinhan nakun sdonwaye śni, Wakantanka he sdonya;) wicaśta kin he mahpiya iciyamni kin ekta eyakupi;

3 Wicaśta kin he sdonwaya, (wicatancan kin ohna un sdonwaye śni, qa tanton śni un kinhan nakun sdonwaye śni, Wakantanka he sdonya;) 


\section{KORINTH.}

4 He Wakantanka ti kin ekta eyakupi, unkan wicoie oyagpica śni kin nahon, hena wicaśta oyake kta eśta okitpani kta.

5 Wicaśta kin hecece cin on imawinkta kta, qa miye qe on imawinkta kte śni, tuka wamaśake śni waun kin heceedan on. 6 Tuka imawinkta wacanmi keś wimatkotkoke kte śni, wowicake epe kta; tuka nakaha hecamon kte śni, tuwe wanmayake ca, token waun nalion kin he isanpa tanka kecanmakin kta he kowakipa.

7 Unkan woyakapi tanka kin on etanhan wahanmicida kte śni heon taku pepe wan micehpi kin en maqupi, Satan taokiye wanji hee, śicaya makuwa kta heon, wahanmiçida kte śni heon etanhan.

8 Qa heon Itancan kin yamni wocekiye ewakiya, he amayustan kte cin heon.

9 Hehan, Mitowaonśidá kin iyonihi kta, qa mitowaśake kin he wowaśake suni kin en hduśtanpi ce, emakiya. Hecen Messiya towaśake kin miye en miciun kte cin, heon makakije cin on iyuśkinyan imawinkta kta.

10 Heon étanhan wowayazan kin en imduśkin ece, qa waaiapi, qa iwicakakije, qa śicaya wicakuwapi, qa Messiya on wicokakije kin henakiya en; tohan wamaśake śni eca taku owakihi ece.

11 Iwinktapi kin on witkotkoka imacaga, niye hecon mayakiyapi, mayadaotaninpi kta tuka qon; yewicaśipi iyotan tankapi kin takudan en wicihukuya waun śni, mataku śni eśta.

12 Awicakehan niyepi ekta yewicaśipi wowapetokeca tawapi kin econkupi, wacintankapi owasin en, wapetog tokeca, taku wowinihan, qa- waśagya wicolian kin hena on.

13 Taku e on okodakiciye tokeca kin ihukuya yaunpi he ; taku nitawapi kin iwacu śni kin hee naceca. Heon wawahtani kin he micicajuju po.

14 Wanyaka po, iciyamni ekta ciupi kta wiy eya manka, qa nakun taku nitawapi kin iwacu kte śni. Taku yatonpi kin he awakite śni, tuka niyepi atayedan. Cinca yapi kin hunkakepi kin taku wicakihnakapi kin he hecetu śni, tuka hunkake yapi lin hena hokśiyoqopa kin on taku ekicihnakapi kta.

15 Ninagipi kin on htawani, qa mihdusote kte cin he iyomakipi; tuka iyotan canteciciyapi eśta, aoptetuya waśtemadakapi ece.

16 Tuka he hecetu, taku nitawapi kin iwacu śni; hececa eśta wamdupike cin heon wohnaye on ciyuzapi, keyapi.

17 Tona niyepi ekta ye wicawaśi qon hena eciyatanhan, taku mihdamna he.

18 'Titos cewakiye ca kici hunkawanjitku wan ekta ye waśi. Niyepi etanhan Titos taku kamna he. Wicoksape okonwanjidan en maunnipi śni he; oye okonwanjidan ohna maunnipi śni he.

19 Ake niyepi en unkihduwicakapi kecannipi he. Wakantanka itokamya Messiya kin en unkiapi ece. Waśtecicidapi kin, tanyan icahniyanpi kte cin heon taku owasin econkupi.

20 Ekta ciupi kinhan, niyepi token wacin kin hececa iyeciyapi kte śni, qa miye nakun token yacinpi śni kin hecen iyemayayapi kta kowakipa. Wakinicapi kin, winawizipi, canniyekici- 
yapi, wicokicize, waaiapi, waajijipi, wahanicidapi qa owodutaton kin henakiya en un kta kowakipa.

21 Qa okinni ekta wau kinhan, niyepi kin en Wakantanka mitawa kin iyokiśin maye kta, qa tona wanna woahtani econpi, qa taku aśape cin, wiinalimanpi, qa wicawihomnipi econpi kin on iyopeiçiyapi śni kin hena awicawakiceye kta naceca, he kowakipa:

\section{WICOWOYAKE 13.}

1 De iciyamni elkta ciupi ; waayatanin nonpa qa yamni ipi kin on wicoie owasin yasutapi kta.

2 Ociciyakapi qon he ake nakaha ociciyalkapi, icinonpa elta waun kin iyececa; qa. nakaha en waun śni, tona tolka wahtanipi, wicaśta tokeca ko orvasin, wowapi wicawecaga, ake ekta wai kinhan itonwicawape kte śni.

3 Miye mahen tanhan Messiya ie cin on wowapetokeca ayakitapi kin heon etanhan; he niyepi ekta taku okitpani śni, tuka niyepi kin en waśake hinca.

4 He iye wowaśake śni on can susbeca kin en okatanpi eśta, Wakantanka towaśake kin on ni un. Heon unkiśs eya waunśakapi śni, tuka niyepi kin ekta Wakantanka towaśake kin on kici unnipi kta.

5 Niś niyepi ihdukcan po, wacinyanpi kin en yaunpi hecinhan; tanyan wihduta po. Ehpeniyanpi śni kinhan, Jesus
Messiya niyepi kin mahen un, he sdonyakiyapi śni he.

6 Tuka elipewicayapi kin heuncapi śni e sdonyayapi kta imdukcan.

7 Taku śica takudan ecanonpi kte śni he Wakantanka icewakiya; unkiye unwaśtepi e unyaotaninpi kte cin hee śni, tuka wahtepi śni kin iyeuncecapi kta eśta, niye taku waśte ecanonpi lkte cin hee wacin.

8 Taku wowicalke kaśeye cin takudan unkokihipi śni, tuka wowicake kin on etanhan.

9 Unkiye waunśakapi śni, qa niye waniśakapi eca unkiyuśkinpi ece. Qa tanyan onihduśtanpi kte cin he uncinpi.

10 Heon etanhan en waun śni icunhan, hena wowapi en walkaga, okinni ekta waun kinhan yaśinya iapi kin ohna iwae kta naceca; wowaśake Itancan maqu lin eciyatanhan, he waicahyapi kin on maqupi, qa waihangyapi kin on etanhan śni.

11 Ehake, hunkawanjin ciyapi kin, wiyuśkin po; oiliduśtan un po, cante waśte po, okonwanjidan awacin po, wokiyapi kin en un po; qa wowaśte, wookiye ko Wakantanka kin nicipi un nunwe.

12 Otoiyohi iiputakapi wakan lin on i ikiciputaka po. Wicasta owotanna un kin owasin i iniputakapi.

13 Jesus Messiya Itancan towaonśida kin, Wakantanka towaśtedake kin, qa Woniya Wakan iwicakiye cin niyepi owasin en nicipi unpi nunwe. Amen. 293 


\section{PALOS \\ GALATIA OYATE KIN}

WOWAPI WICAKICAGE CIN.

\section{WICOWOYAKE 1.}

1 Palos yewicaśipi kin heca, wicaśta akantu kin etanhan śni, qa wicaśta on kagapi śni, tuka Jesus Messiya etanhan, qa Wakantanka Ateyapi kin, he wiconte etanhan najin kiye cin he nakun etanhan;

$2 \mathrm{Qa}$ hunkawanjin kiciyapi mici unpi kin owasin ko, Galatia ekta okodakiciye kin wowapi unnicagapi.

3 Wowaonśida, wookiye kro, Wakantanka Ateyapi kin, qa Jesus Messiya Itancan unkiyapi kin etanhan, nicipi un nunwe.

4. $\mathrm{He}$ iye waunhtanipi kin on icicu, wicoicage śice cin de etanhan eunhdakupi lkta, Wakantanka Ateunyanpi tawacin kin eciyatanhan :

5 He ohinniyan owihanke wanin wowitan kicihan nunwe. Amen.

6 Tuwe Messiya towaonśida kin inicopi kin kohanna aduśtanpi, qa wotanin waśte tokeca en oyapapi kin, heon imanihan manka.

$7 \mathrm{H}$, nakun tokeca śni, tuka wicaśta wanjikji nagiyeniyanpi, qa Messiya tawootanin waśte kin ayuśtan niyanpi kta tuka.

8 Tuka he unkiś unkiyepi, qa iś ohnihde wan malipiya kin eciyatanhan kin, wotanin waśte unkoniciyakapi qon he ohna śni, tuka tokeca oniciyakapi kinhan, wośnapi un nunwe.
9 Unkeyapi qon hecen ake nakaha epe, tuwe wotanin waśte iyacupi qon kici okonwanjidan śni kin oniciyakapi kinhan, he wośnapi un kte.

10 Wicaśta qa Wakantanka, unma tukte cinwicawakiya he; qa wicaśta kin iyokipiwicawaya awalkita he. Wicaśta kin iyokipiwicawaya unkanś, Messiya wowidagmaye kte śni tuka. 11 Mihunkawanji he de ociciyakapi, wotanin waśte omdake ciqon he wicaśta akantu lragapi śni ;

$12 \mathrm{Qa}$ wicaśta kin etanhan iwacu śni, qa iye omakiyakapi śni, tuka Jesus Messiya oyake cin eciyatanhan onmaspe.

13 Juda wicolian en opeya waun qehan, token owahianmde cin nayahonpi ; Wakantanka taokodakiciye kin nina śicaya wakuwa, qa mdujuju.

$14 \mathrm{Qa}$ mitaoyate lin en tona orn akinimaskokeca kin hena ota Juda wicohan kin en kawicawape ca, mihunkakepi wohdakapi qon iyotan nina tewahinda.

15 Tuka Wakantanka ina tezi kin etanhan imacu, qa iye towaonśida kin on mico kin he,

16 Iye Cinhintku kin sdonye mayan iyokipi qehan, Ikcewicaśta wotanin waśte kin owicawakiyake kte cin heon, kohanna wicacehpi, wicawe ko, kici wowahdake śni :

$17 \mathrm{Qa}$ Jerusalem ekta tona 
mitokapa tanhan yewicaśipi qon hena ekta wicawai śni, tuka Arabia ekta imdamde ca, ake Damaskos ekta icicawin wahdi.

18 Hehan waniyetu yamni iyohakam Inyan kici wowahdake kta e Jerusalem ekta wai, qa anpetu ake zaptan kici manka.

19 Tuka yewicaśipi kin tokeca tuwedan wanmdake śni, Jakob Itancan sunkaku kin heceedan.

20 Unkan wicoie wowapi en cicagapi kin de, iho, Wakantanka itokam itonśni mawanica.

21 He iyohakam Suria, qa Kilikia makoce kin en wahi.

22 Qa Juda makoce kin en okodakiciye kin Messiya en un kin, hena ekta ite kin sdonmayanpi śni waun.

23 Tuka, Tuwe śicaya unkuwapi qon he nakaha wowacinye ehanna yujuju qon he oyaka ce, hecedan nalionpi.

24 Unkan miye on Wakantanka yatanpi.

\section{WICOWOYAKE 2.}

$1 \mathrm{He}$ iyohakam waniyetu ake topa, hehan ake Titos yuha, Barnabas kici Jerusalem ekta itawankanhde mda.

2 Woyakapi on ekta mde ca, Ikcewicaśta ekta wotanin waśte omdake ciqon he hena owicawakiyaka, qa tona okinihanpi kin hena iśnaśnana owicawakiyaka; ituya waimnanke śni, qa taku śni on waimnanke kte śni lieon.

3 Tuka Titos mici un kin he Helenes heca keś bakihdayapi kta keyapi śni.

$4 \mathrm{Qa}$ he nakun hunkawanjinkiciyapi itonpiśni kin heca, hena unkiye token Jesus Messiya on tawa unkiçiyapi kin he iwanya- kapi kta e en hipi, wowidag unyanpi kte cin heon.

5 Tuka hena wihiyayedan oape wanjidan hehanyan wicihukuya unyakonpi śni, wotanin waśte wowicake kin nicipi un kte cin heon.

6 Unkan tona wicaśta wicakinihanpi seca, qa takupi kaśta he etanhan tokeca śni, Wakantanka wicaśta itohnake kin iwanyake śni, hena wicakinihanpi seca, tuka wicoie kin en takudan omakiyapi śni.

7 Tuka hececa eśta, tohan bakilidayapi śni wotanin waśte kin he maqupi e wanyakapi qehan, bakilidayapi wotanin waśte Inyan qupi qon he iyececa;

8 Tuwe bakihdayapi kin on yewicaśipi kin en Inyan okihiye cin hee Ikcewicaśta ekta okihimayan ;

$9 \mathrm{Qa}$ Jakob, Inyan qa Johannes kici, ipatanhdepi kin hecapi seca, wowaonśida maqupi kin he wanyakapi, hehan takodakiciyapi on Barnabas kici nape mayuzapi, unkiś Ikcewicaśta ekta unyanpi kta, qa iyepi kin bakilidayapi kin ekta yapi kta.

10 Tuka decedan, tona wahpanicapi kin wicunkiksuyapi kta keyapi, unkan miś nakun hecamon tawatenwaya.

11 Unkan Inyan Antiyoka ekta hi qehan, kici taninyan awakinica, iye iyaonpepica nakaeś.

12 Jakob etanhan wanjikji hipi śni kin he itokam Ikcewicaśta kin om wota ece; tuka wanna en hipi, hehan tona bakilidayapi qon hena kowicakipe ça, tokan iyaye ca ihdonica.

13 Qa he opeya Juda wicaśta tokeca kin iyecen analibeya econpi, hecen hena wohnaye kin on Barnabas nakun opeya yeyapi. 
14 Tuka wotanin waśte wowicake kin eciyatanhan owotanna manipi śni, he wanmdaka hehan owasin wicitokam Inyan hewaliya; Juda wicaśta henica, tuka Ikcewicaśta kin iyecen yani kinhan, qa Juda wicaśta kin iyecece śni, tokeca e Ikcewicaśta kin Juda wicohian kin en ope wicayaśi he.

15 Unkiye wicacelipi eciyatanlian Juda wicaśta kin heuncapi, qa Ikcewicaśta wahtanipi sa heuncapi śni ;

16 Wicaśta kin woope wicohan kin eciyatanhan yaowotanpi śni ece, tuka Jesus Messiya wacinyanpi kin on, he sdonunyanpi kin heon unkiś eya Jesus Messiya wacinunyanpi, Messiya wicadapi kin on unyaowotanpi kta e heon, qa woope opapi kin on etanhan śni; woope wicohan kin eciyatanhan wicacehpi owasin tuwedan yaowotanpi kte śni.

17 Tuka Messiya on unyaowotanpi kta unkakitapi kin he icunhan, wahtanipi sa heuncapi hecinhan, heon Messiya woahtani wowidake kin heca he. Hiya hecetu śni.

18 Taku mdujuju qon he ake piya wakage cinhan, wawahtani e mihdutanin.

19 Wakantanka on wani kta e heon, woope eciyatanhan, woope kin ekta mața ce.

20 Messiya kici icipaweh omakatanpi; tuka nahahin wani ; qa he miye śni tuka miye mahen Messiya ni un kin hee; qa wicacehpi kin en wani kin lie Wakantanka Cinhintku wacinyanpi kin eciyatanhan wani, he iye cantemakiye ca miye on iye icicu.

21 Wakantanka towaonsida kin mdujuju śni ; woope kin eciyatanhan wicoowotanna u kinhan, hehan Messiya ituya ța. 296

\section{WICOWOYAKE 3.}

1 Galatia wicaśta wacin tonpi śni kin, tuwe niyuhnaśkinyanpi, qa hecen wowicake kin anayagoptanpi kte śni he; niyepi kin en Jesus Messiya icipaweh okatanpi kin he niiśtapi kin itokam yuotaninpi.

2 Decedan omayakidakapi kta wacin, woope wicohan kin on, qa wacinyan anagoptanpi kin, unma tukte eciyatanhan Woniya kin iyacupi he.

3 Tokaheya Woniya kin on ecanonpi, qa ehake wicacehpi kin on nihduśtanpi kta he, qa wacinyatonpi kte śni.

4 Kana owasin taku śni on iyotanhan iyeyakiyapi he; he taku śni on etanhan hecinhan.

5 Tuwe Woniya kin yuhe niciyapi, qa niyepi en tankaya ohanye cin he woope wicohan kin on, qa wacinyan anagoptanpi kin unma tukte eciyatanhan he. 6 Hecen Abraham Wakantanka wicada, unkan he woowotanna kiciyawapi qon.

7 Heon etanhan, tona wacinyanpi kin en opapi kin hena Abraham cincawicaye cin eepi, he sdonya po.

8 Wacinyanpi kin on Wakantanka Ikcewicaśta kin wicayaowotanna kte cin, he Wowapi wakan otokaheya tanhan wanyake ca, hecen tokaheya Abraham wotanin waśte kin okiyaka; Niye en oyate kin owasin wicayawaśtepi kta ce.

9 Hecen tona wacinyanpi kin hena Abraham wacinye ciqon kici wowaśte yuhapi.

10 Tona woope wicolian kin etanhanpi kin hena woyaco kin en unpi. Hecen wowapi en kagapi ; Tona taku woope wowapi kin en kagapi kin owasin 


\section{WICOWOYAKE 3.}

opeya unpi śni, qa ecen econpi śni kin hena otoiyohi wanna wicayacopi ce.

11 Unkan woope kin eciyatanhan tuwedan Wakantanka itokam yaowotanpi śni e sdonunyanpi: Tona owotanna un kin hena wacinyanpi eciyatanhan nipi kta ce.

12 Unkan woope kin he wacinyanpi kin eciyatanhan śni; tuka, Wicaśta kin tuwe hena econ kinhan, hena on ni kta ce.

13 Woope woyaco kin etanhan Messiya eunhdakupi, unkiyepi on iye woyaco ikicaga. Wowapi en hecen kagapi, Tona can en otkeyapi kin hena owasin yacopi ce.

14 Hecen Jesus Messiya eciyatanhan Abraham wowaśte tawa kin he Ikcewicaśta kin en awicau kta; hecen wacinyanpi kin on Woniya iwahounyanpi kin he unkicupi kta.

15 Mihunkawanji, wicaśta a- kantu iyecen iwaa. Wicaśta akantu wicotakuye tawa kin hee kaeś, wanna hduśtanpi kinhall, tuwedan hdujuju śni, qa taku en akage śni ece.

16 Abraham iye cinca kici wahoyapi ; Cincapi kin eye śni, hehan ota kapi kta, tuka wanjidan, qa, Nicinkśi kin, he Messiya kin ee.

17 Unkan mis kaken epe kta; Otokahe ekta wicotakuye Messiya en Wakantanka hdusuta kin he, iyohakam waniyetu opawinge topa sanpa wikcemna yamni hehan woope $u$ kin he tokaheya kin yujuju, qa hecen wahoyapi qon ecetuśniye kta okitpani.

18 Taku tawapi kte cin he woope eciyatanhan un unkanś, wahoyapi kin eciyatanhan śni; tuka he Wakantanka Abraham iwahoya.
19 Hehan tokeca e woope kin yukan he. He woahtani on kagapi, cinca iwahoyapi qon u śni kin hehanyan, qa ohnihdewicayapi kin on yusutapi, iyotahedan un wan nape kin ohna.

20 Iyotahedan un kin he wanjidan eciyatanhan śni, tuka Wakantanka eqe wanjidan.

21 Heon woope kin he Wakantanka taku iwahowicaye cin he kaśeya he. Hiya hecetu śni. Woope wiconi kaga okihi unkanś, awicakehan woope kin eciyatanhan wicoowotanna $u$ kta tuka.

22 Tuka Wowapi Wakan kin he owasin woalitani ohdateya un wicayawa, qa hecen tona wicadapi kin hena, Jesus Messiya wacinyanpi wahoyapi qon he wicaqupi kta.

23 Wowacinye kin hi śni itokam woope kin on unkaśkapi ; qa wowacinye yuotaninpi kte cin en onauntakapi.

24 Hecen woope kin he wayeunkiyapi kin hee, Messiya en unkahipi kta, hecen wowacinye on unyaowotanpi kta e lieon.

25 Tuka wowacinye kin wanna hiyohi, hecen wanna wayekiyapi kin ihukuya unyakonpi śni.

26 Jesus Messiya wacinyanpi kin eciyatanhan owasin Wakantanka cinca kin heuncapi.

27 Tona Messiya caje on baptem nicupi kin hena Messiya kodakapi.

28 He en Juda qa Helenes wicaśta ko wanica; wowidake un tawaiciya ko wanica; wica winyan ko wanica; tuka Jesus Messiya ekta owasin okonniwanjipidan.

29 Messiya tawa niyanpi hecinhan, Abraham cinca kin henicapi, qa wahoyapi kin eciyatanhan tawayapi kte cin. 


\section{WICOWOYAKE 4.}

1 Unkan kaken epa; Tawaye kte cin tohanyan hokśiyopa un kin hehanyan wowidake kin takudan ocitpani śni, taku owasin on itancan un eśta.

2 Tuka tohan Atkuku ke cin hehanyan waonspekiye ca tipi awanyag wicakiyapi kin hena he yuhapi.

3 He iyecen unkiś eya hounkśiyopapi qehan, wicoicage woonspe tokaheya kin ihukuya unyakonpi.

4 Tuka wanna anpetu iyehantu qehan, Wakantanka iye Cinhintku kin $u$ śi, winohinca wan etanhan icage ça woope kin olidateya kagapi.

i 5 Hecen tona woope kin ihukuya un kin hena opewicakiton kta, atayedan cinhintkuyapi kin unhapi kte cin heon etanhan.

6 Unkan cinkśiniyanpi kin heon Wakantanka iye Cinhintku Taniya kin nicantepi kin en niu śipi, he, Abba, Ate, eya howaya ece.

7 Hecen wowidake kin henica śni, tuka cinhintku kin henica. Qa cinhintku hecinhan, Messiya eciyatanhan Wakantanka ekta tawaye kte cin heca.

8 Tuka Wakantanka sdonyayapi śni qehan, taku wakan śni kin hena wowidag niyanpi.

9 Unkan nakaha Wakantanka sdonyayapi, qa iś Wakantanka sdonniyanpi yaunpi kin, hecen token ake taku tokaheya hunkeśni qa takudan okihi śni un kin ekta nihdohdapi, qa hena ake wowidag niyanpi kta yacinpi he.

10 Anpetu qa wi kin, omaka waniyetu ko awacanipi.

11 Kocicipapi, taku śni on niyepi kin htacicinipi epca, heon etanhan.
12 Mihunkawanji, ceciciyapi ce; Miś waun kin iyecen un po; niye yaunpi kin iyecen waun, taku śica takudan ecamiyeconpi śni.

13 Tokaheya elkta wicacehpi waśake śni on wotanin waśte kin ociciyakapi kin he sdonyayapi.

14 Qa micehpi en taku iyutanmaye cin he śiceyadapi śni, qa paha iyeyayapi śni, tuka Wakantanka ohnihde ye cin iyecen, qa Jesus Messiya iyecen tin ye mayaśipi.

15 Hecen wowaśte duhapi qon tukte e he. Kaken ciyaotaninpi, okihipica unkanś niiśtapi kin yahduśdokapi qa mayaqupi kta wicayadapi kta tuka.

16 Wowicake ociciyakapi kin heon toka mayayapi he.

17 Hena e tanyan canteniciyuzapi seca, tuka he taku waśte on etanhan śni, heyata iyeunyanpi kta cinpi, hecen niye tanyan cantewicayakiyuzapi kta.

18 Tuka ohinniyan taku waśte on tanyan canteyuzapi kin he waśte, qa niyepi ekta waun kin hecedan śni.

19 Micinca, niyepi kin en Messiya icahyapi kte cin heon ake hokśiksuya waun.

20 Niyepi kin on, token wacin mduza tanin śni, heon nakaha ekta cicipi waun qa miho kin wahdatokeca kta wacin.

21 Tona woope ihukuya yaunpi yacinpi kin, woope kin nayahonpi śni hecinhan omakiyaka po.

22 Wowapi en hecen kagapi; Abraham cinhintku nonpa, unma wowidake winohinca etanhan, qa unma iś winolinca tawaiciye cin etanhan.

23 Tuka wowidake winohinca etanhan un kin he wicacehpi 
eciyatanhan tonpi, qa tawaiciye cin etanhan un kin wahoyapi kin on tonpi.

24 Hena e wiyacinpi wan ee; qa wicotakuye nonpa kin heepi. Unma Sina lie kin etanhan $u$, he wowidag yapi kaga, Hagar hee.

$25 \mathrm{Qa}$ Hagar he Arabia ekta Sina lie kin hee, qa Jerusalem wanna he cin kici akidecen un, qa cinca kici wowidake un.

26 Tuka Jerusalem wankan tanhan $u$ kin he tawaiciya, he owasin ina unyanpi kin ee.

27 Hecen wowapi en kagapi; Cinca oyakitpani qa cinca ton śni yaun kin iyuśkin wo; tohinni bokśinyaksuye śni:kin panpan qa dowan wo; tuwe iśnana un kin he cinca ota, qa hihnaton kin he cinca tonana.

28 Mihunkawanji, Isaak iyecen wahoyapi cinca kin heuncapi.

29 'Tuka he ehan tuwe wicacehpi eciyatanhan tonpi kin he Woniya eciyatanhan un kin lee śicaya kuwa qon, iś eya dehan iyececa.

30 Tuka Wowapi wakan kin token eya he ; Winohinca wowidake un kin cinca kici tankan iyeya wo; wowidake un cinca kin he tawaiçiya cinca kin kici akidecen tawaye kte śni.

31 Hecen mihunkawanji, wowidake un cinca kin ee śni, tuka tawaiciya cinca kin unkiyepi.

\section{WICOWOYAKE 5.}

1 Hecen tawaiçiyapi kin on Messiya tawaiciye unyanpi kin he en tinsadan un po, qa ake wowidake yapi wanapin kin on iyaihdaśkapi śni po.

2 Wanyaka po, Palos he miye e kaken eciciyapi; Banicilidayapi kinhan Messiya takudan on oniciyapi kte śni ce.
3 Wicaśta bakilidayapi kin otoiyohi kaken ewakiya ece; Woope kin ocowasin econ kta iyececa tuka ce.

4 Tona woope kin on niyaowotanpidan keniciyapi kin wanna Messiya elipeyayapi, qa wowaonśida etanhan nihinhipayapi kin.

5 Unkiye Woniya kin eciyatanhan wicoowotanna woape kin wacinyanpi on unkapepi ece.

6 Jesus Messiya en bakilidayapi kin he takudan okihi.śni, qa nakun bakihdayapi śni kin; tuka wacinyanpi wowaśte en ohanye cin he taku okihi ece.

7 Tanyan yainankapi; tuwe iyoncipi śni kin, hecen wowicake kin wicayadapi kte śni he.

8 Woawacin kin de tuwe nicopi kin eciyatanhan u śni.

9 On napohyapi cistinna kin he aguyapi ocowasin napoliya.

10 Itancan kin eciyatanhan wacinciyapi, takudan tokeca awacanipi kte sni; qa tuwe kaśta nagiyeniyanpi kinhan he woyaco hduhe kta.

11 Mihunkawanji, miś miye bakihdayapi kin ieyanwapaha kinhan tokeca e śicaya makuwapi he; kinhan, can susbeca iwośihda kin he enakiyapi tuka.

12 Tokin tona cante okicunin niyanpi kin hena yuśpa iyewicayapin.

13 Mihunkawanji, tawaiciyapi kin en nicopi, tuka tawaiciyapi kin he wicacelipi iyokipi kta e wowinyunyanpi śni po; tuka wowaśte on otoiyohi okiciciya po.

14. Woahope kin ocowasin wicoie wanjidan en ecetu, he dee, Niś niye inihdacinyan wicaśta aiyog yaun kin canteyakiye kta ce.

15 Tuka yecidahtakapi qa tem- 


\section{GALATIA.}

yeciyapi kinhan, on wakta po, okinni ihangyeciyapi kta naceca.

16 Miye kaken epe; Woniya kin on mani po, hecen wicacehpi on cantiheye cin yahduśtanpi kte śni.

17 Wicacehpi kin he Woniya kin inakiwizi, qa Woniya kin iś wicacehpi kin inakiwizi, qa henaozakiya anokatanhan kicizapi, hecen taku token yacinpi śni kin hena ecanonpi ece.

18 Tuka Woniya kin yus aniyanpi hecinhan, woope kin ihukuya yaunpi śni.

19 Wicacelipi wicohan kin taninyan un; Wawicihaliapi, wiinalimanpi, waaśapapi, wicaśtapi śni ;

20 Wakagapi kin ohodapi, wapiyapi, śice kicidapi, wakinicapi, wawihnupi, wacantahdepi, wicokicize, wawakipajinpi, yuomdecapi,

21 Winawizipi, tin wicaktepi, witkopi, qa witkoya wacipi, qa taku tona hecece cin hena ee; wanakaja ociciyakapi kin he iyecen nakaha eciciyapi, Tona taku hececa econpi kin hena Wakantanka tokiconze kin tawapi kte śni.

22 Tuka Woniya kin waskuyeca tawa kin he, wowaśtedake, wowiyuśkin, wookiye, wacintankapi, wicowalibayedan, wowaśte, wowacinye ;

23 Wicotawacin waśte, qa iyatahde içiyapi śni hena ee; qa taku hecece cin woope kin tuktedan iyamya un śni.

24. Unkan tona Messiya tawa wicaye cin hena wicacelipi kin icipaweh okatanpi, qa wacinyuzapi cantiheyapi koya.

25 Woniya kin unkicinipi kinhan, Woniya kin unkopapi kta.

26 Otoiyohi śihdakiciyapi, qa winakiciwizipi, qa ecinśniyan iwinkta iciyapi wanin unyakonpi nunwe.

\section{WICOWOYAKE 6 .}

1 Mihunkawanji, wicaśta wan ihnuhanna taku śica en iyolipaye cinhan, niye tona owotanna yaunpi kin woksape wowahbayedan yuha wicaśta kin he ake piya opeya maniya po; qa awanihdaka wo, okinni taku wawiyutanyan un kin en niu nun ce.

2 Otoiyohi waqinpi kin kicicin po, hecen Messiya toope kin tanyan ecetu yayapi kta.

3 Tuwe taku śni un eśta, taku içida kinhan, he icihnayan.

4 Tuka otoiyohi iye olian kin awakicin kta, hecen iye qe eciyatanhan, qa tokan tanhan śni, iyuśkin kta.

5 Otoiyohi waqinpi tawa kin kicin kta.

6 Tuwe wicoie kin eciyatanhan onspekiyapi kinhan, he waonspekiye cin taku waśte kin owasin etanhan okinikiya nunwe.

7 Ihnuhan nihnayanpi kin; Wakantanka uncapi kte śni ce. Wicaśta kin taku oju kinhan, he yukse kta ce.

8 Tuwe iye cehpi kin wokiciju kinhan, he wicacelipi kin etanhan wicotakuni śni mnaye kta; qa tuwe Woniya kin woliciju kinhan, he Woniya kin eciyatanhan wiconi owihanke wanica mnaye kta.

9 Taku waśte econ unkapinpi śni nunwe; hu unstakapi śni kinhan tohantu kte cin mnaunyanpi kta.

10 Heon tohanyan unkokihipi kinhan, wicaśta owasin taku waśte ecawicunkiconpi kta, qa wacinyanpi tiyohnaks: kin hena iyotan. 


\section{WICOWOYAKE 1.}

11 Wowapi hinsko tanka miye minape on cicagapi $\mathrm{e}$ wandakapi.

12 Tona wicacehpi ekta waśte ihdutaninpi cinpi kin hena banicihdayapi kta cinniciyapi, Messiya can susbeca tawa kin on śicaya kưwapi kte śni kin heon heconpi.

13 Tona bakilidayapi kin hena e qe nakun woope kin tanyan yuhapi śni, tuka baniçihdayapi kta cinpi, nicehpipi kin on iwinktapi kta heon.

14 Tuka takudan on imawinkta śni nunwe, Jesus Messiya Itancan unkiyapi can susbeca tawa kin hecedan on, he on miye ekta wicoicage kin icipaweh okatanpi, qa wicoicage kin ekta icipaweli omakatanpi.

15 Jesus Messiya en bakihdayapi kin he takudan okihi śni, qa bakilidayapi śni kin he nakun, tuka wicaśta teca kin.

16 Tona woope kin de eciyatanhan manipi, Israel Wakantanka tawa wicaye cin hena ko, wookiye, wowaonśida lio, wicakici unpi nunwe.

17 Detanhan tuwedan nagiyemayc śni nunwe; Jesus Messiya towapetogton kin mitancan kin en yuha waun.

18 Mihunkawanji, Jesus Messiya Itancan unkiyapi towaonśida kin ninagipi kin kici un nunwe. Amen.

\section{PALOS}

\section{EPHESOS OYATE KIN}

\section{WOWAPI WICAKICAGE CIN.}

\section{WICOWOYAKE 1.}

1 Palos, Wakantanka tawacin kin eciyatanhan, Jesus Messiya yeśi qon hee, Ephesos ekta tona owotanna un, qa Jesus Messiya wacinyanpi kin hena wowapi cicagapi:

2 Wowaonśida, wookiye ko, Wakantanka Ateunyanpi kin etanhan, qa Jesus Messiya Itancan unkiyapi kin etanhan niyepi en nicipi un nunwe.

3 Jesus Messiya Itancan unkiyapi Wakantanka tawa, qa Atkuku kin yawaśtepi nunwe; he iye Messiya en taku mahpiya tanhan un kin wowaśte wakan ocaje owasin on unyuwaśtepi :
4 He en maka kagapi kin itokam unkahnigapi qon iyececa, unwakanpi kta qa iye itokam wowaśte en iyaonpepica śni unyalkonpi kte cin heon:

5 Qa iye en, Jesus Messiya eciyatanhan cinca unyanpi, iye tawacin oiyokipi kin eciyatanlıan:

6 Iye towaonśida wowitan yatanpi kte cin on, he iye Waśtekidake cin en waśteundakapi :

$7 \mathrm{He}$ on wopekitonpi kin unhapi, iye we kin on, woahtani kajujupi kin hee, iye tow aonśida tanka kin eciyatanhan:

$8 \mathrm{He}$ iye unkiciyuotapi, wicoksape owasin qa wookahnige ko on :

9 Qa iye toiyokipi kin eciyatanhan, iye tawacin wowiyukcan 


\section{EPHESOS.}

kin sdonyeunkiyapi, he iye en iciconze ciqun he iyecen :

10 Hecen tohan iyehantu kinhan, wokonze kin eciyatanhan taku owasin Messiya en witaya mnaye kta, taku mahpiya ekta un, qa taku maka akan un kin hena, iye en.

11 Qa tuwe iye tawacin wokonze kin eciyatanhan, taku owasin kage cin he otokaheya tanhan unkahnigapi kin, he iye en woailipeye kin unhapi.

12 Hecen unkiye tokaheya Messiya wacinunyanpi qon, iye towitan kin yatan unyakonpi kte cin heon.

$13 \mathrm{He}$ niś niyepi nakun, wowicake oie kin nayahonpi qehan, wotanin waśte on ni niyanpi kte cin hee, he iye wacinyayapi, unkan Woniya wahoyapi kin he on wapetognitonpi :

14 He taku unhapi kte cin tokaheya wicaqu ece kin hee, taku opetonpi hdamnanpi kin $u$ kte cin hehanyan, iye towitan yatanpi kte cin heon.

15 Hecen miś eya, Jesus Messiya wacinyayapi kin he, qa tona owotanna un kin owasin waśte wicayadakapi kin nawahon kin,

16 Ehantanhan niyepi on wopida eya amdaśtan śni, qa wocekiye epa eca, ciksuyapi ece :

17 Jesus Messiya Itancan unkiyapi Wakantanka tawa, qa wowitan Ateyapi kin hee woksape wowiyukcan ko oniya kin niçupi kta, he iye sdonyayapi kta e heon:

18 Nitawacinpi iśta kin iyoyam niyanpi kta, hecen nicopi wowacinye kin he taku kin e sdonyayapi kta, qa tona owotanna un kin taku tawapi kte cin wowitan wijica kin he taku;

$19 \mathrm{Qa}$ tona wicrundapi kin en iye towaśake tanka hinca woptecaśni kin he taku kin nakun, iye towaśake waśagya ohanye cin eciyatanhan.

20 He Messiya okicihan, wiconțe etanhan najin kiye çehan, qa mahpiya ekta iye etapa kin en ekihde:

21 Wokiconze owasin, qa wowaśake, qa wookihi, qa itancankiyapi, qa wicacaje caje yatapi kin owasin, wiconi kin de en hecedan śni, wiconi u kte cin he nakun, hena iwankam tehan wankantuya ekihde:

$22 \mathrm{Qa}$ iye siha kin ihukuya taku owasin ekihnaka, qa taku hiyeye cin owasin iwankam okodakiciye kin en he pa kin kaga ;

23 He iye tancan kin hee, tuwe taku owasin on owasin ojuye cin woojudan kin hee.

\section{WICOWOYAKE 2.}

1 Unkan niś niyepi kin woahitani wicohan śica ko en niṭapi :

2 Hena ehna ehanna mayanipi, wicoicage kin de wiconi kin eciyatanhan, qa okotonyan wowaśake kin itancan kin eciyatanhan, he taku wakan woakta śni cinca kin en olianye cin hee.

3 Hena om, unkiyepi owasin ehanna unnipi, uncelipipi wacin yuze cin en, wicacehpi wicotawacin ko wacinpi kin ecetu unyanpi, qa unmanpi kin iyecen wocantiyahde cinca kin heuncapi.

4 Tuka Wakantanka wowaonsida kin on wijica kin he towaśtedake tanka kin on waśte undakapi kin heon;

5 Unkiye wicohan śica ehna untapi qehan, Messiya kici niunkiyapi ; wowaonśida on wiconi duhapi ece : 


\section{WICOWOYAKE 3.}

6 Qa Jesus Messiya en witaya najin unkiyapi, ya malipiya kin ekta kici kiyotang unkiyapi ;

7 Hecen wiconi u kte cin en, Jesus Messiya on cantounhnakapi kin on, towaonśida woyuha wopteca śni lkin hduotanin kta.

8 Wowaonśida kin on yanipi, wacinyanpi kin eciyatanhan, qa he niyepi kin etanhan śni, Wakantanka taku niçupi kin hee.

9 Wicohan kin eciyatanhan śni, okinni tuwe ihdatan kta tuka.

10 Iye taku kage cin he unkiyepi, Jesus Messiya en wicolian waśte aicah unyanpi, hena ohna maunnipi kte cin heon otokaheya tanhan Wakantanka wiyeya ehnaka.

11 Hecen ehanna wicacelipi ekta Ikcewicaśta henicapi kin he kiksuya po, qa wicacehpi bahdayapi eyapi, nape on econpi kin he bakihidayapi śni eniciyapi qon :

$12 \mathrm{He}$ ehan Messiya yuhe śni yaunpi; Israel wokiconze kin iyenicihantupi śni, qa wahoyapi wicotakuye kin sdonyayapi śni, wowacinye ninicapi, qa wicoicage kin en Wakantanka codan yaunpi.

13 Tuka nakaha Jesus Messiya en, niś niyepi ehanna itehanyan yaunpi qon, wanna Messiya we kin on kiyadan anihipi.

14 Unkan iye wookiye unkitawapi kin hee, iye qe napin yuwanjidan, qa cokahnagya ehnakapi qon he yujuju;

15 Qa iye cehpi lin en, tokakiciyapi kin woahope woope kin econ wicaśipi qon he yutakuni śni; iś iye en nonpa etanhan wicaśta wanjidan teca icaliye kta, hecen wookiye kage kte cin heon etanhan.

16 Qa tancan wanjidan on, can susbeca kin eciyatanlian, hena napin Wakantanka kici okiciciye wicakiye ca, heon tokakiciyapi kin kte.

17 Qa tona tehan dukanpi qon kiyadan un kin hena ko wookiye oniciyakapi hi.

$18 \mathrm{He}$ eciyatanhan Woniya wanjidan on, napin Ateunyanpi kin ekta kiyedan ipi kin he unhapi.

19 Heon etanhan, detanhan togkicihdapi, qa oyate tokeca kin henicapi śni; tuka tona owotanna unpi kin hena om yatipi, qa Wakantanka tiyohnaka tawa kin he niyepi :

20 Qa yewicaśipi wicaśta wokcan ko taku ahehde tawapi kin akan tinicagapi, Jesus Messiya he oise en otancanke kin hee.

21 Iye ohna tipi kin ocowasin ayucoco ehnakapi, qa Itancan kin en tipi wakan icaga.

22 He ohna niy epi nakun ptaya tinicagapi, Woniya kin eciyatanhan, Wakantanka tipi ye kte cin heon.

\section{WICOWOYAKE 3.}

1 Heon Ikcewicaśta kin niyepi on etanhan, Palos, Jesus Messiya on kaśka hnakapi kin he miye.

2 Niyepi on Wakantanka towaonśida wicohtani maqupi kin he nayahonpi hecinhan;

3 'Token woyakapi kin on wowiyukcan kin he sdonye makiya; he wanna wicoie tonana on wakaga :

$4 \mathrm{He}$-dawapi kin ehantanhan Messiya tawowiyukean kin token imdukcan kin sdonyayapi kta.

$5 \mathrm{He}$ wicoicage tokeca kin en wicaśta cinca kin owicakiyakapi śni, tuka nakaha Woniya kin eciyatanhan, yewicaśipi owotanna qa wicaśta wokcan tawa303 
wicaye cin hena owicakiyakapi kin iyecen :

6 Hecen Ikcewicaśta kin nakun kici tawayapi kte ca, kici tancan wanjipidan kta, qa Messiya tawotanin waśte kin eciyatanhan wahoyapi qon he en opapi kta.

$7 \mathrm{He}$ on ookiye makagapi, Wakantanka towaonśida maqupi kin he eciyatanhan, iye towaśake ohanye cin on.

8 Miye tona owotanna un kin owasin en iyotan macistinna, tuka wowaonśida kin de maqupi, Ikcewicaśta kin ekta Messiyá tawoyuha okahinilipica śni kin he omdake kte cin hee.

9 Qa wowiyukcan kin otokahe wanin ehantanhan Wakantanka, taku owasin Jesus Messiya on kage cin, he en nahmanpi qon hee takodakiciyapi kin he owasin sdonye wicawakiye kta.

10 Hecen nakaha okodakiciye kin eciyatanhan, wokiconze wowaśake ko, mahpiya ekta un kin en, Wakantanka toksape wopteca śni kin atanin kta.

11 Wokonze otokahe wanin ehantanhan kage ciqon he eciyatanhan, Jesus Messiya Itancan unkiyapi kin en ;

12 Iye wacinunyanpi kin eciyatanhan, kokipeśniyan ekta unliipi, qa waditagya unkiapi ece.

13 Heon etanhan niyepi kin on kakiś mayanpi kin heon canwannikapi kte śni wacin.

$14 \mathrm{Qa}$ heon Jesus Messiya Itancan unkiyapi he Atkuku kin en, anpetu iyohi canpeśka makehde inawajin.

$15 \mathrm{He}$ eciyatanhan wicowazi mahpiya ekta un, qa maka akan un kin, ocowasin caśtonpi.

16 Hecen iye towitan woyuha kin eciyatanhan taku nicupi kta, iye Taniya kin eciyatanhan, wo- was̉ake on wicaśta mahen tanhan waśagniyanpi kta.

17 Hecen wacinyanpi on nican tepi kin mahen Messiya ounye kta;

18 Hecen wowaśtedake on hutkan niyusutapi, qa sutaya hu nihdepi kta; qa tona owotanna un kin owasin om, ohdakinyan qa ohanske cin, omahentuya qa obosdatu kin he țaku kin iyư.kcan oyakihipi kta;

19 Qa Messiya towaśte okalinilipica śni kin he sdonyayapi kte, hecen Wakantanka toojudan kin ocowasin ojuniyanpi kte.

20 Tuwe nina waśagya econ okihi, unkiś unkidapi qa awauncinpi kin isanpa, wowaśake kin on onicihianyanpi kin he eciyatanhan;

21 He iye okodakiciye kin en, Jesus Messiya on, ohinniyan owihanke wanin wowitan kicihan nunwe.

\section{WICOWOYAKE 4.}

1 Heon etanhan miś miye Itancan caje on makaślkapi, ceciciyapi ce, Wicakicopi on nicopi kin he iyacinyan mayanipi kte.

2 Wicotawacin waśte ocowasin qa wicowahbadan, wacin tankapi ko yuha, wowaśtedake en otoiyohi iyowinyeciciyapi kta :

3 Qa wookiye icaśke kin on Woniya okonwanjidan kill he yahduhapi kta e aiçiciya po.

4 Wicatancan wanjidan qa Woniya nakun wanjidan, nicopi kin en wowacinye wanjidan inicopi kin he iyececa;

5 Itancan wanjidan, wowacinye wanjidan, baptem wanjidan,

6 Taku Wakan wanjidan, he owasin Ateyapi kin, owasin 
iwankam un, qa owasin opta un, ga niyepi owasin en niunpi kin.

6 Tuka Messiya iyunwin codan wicaqu iyutapi kin eciyatanhan unkiyepi otoiyohi wowaonśida unqupi.

8 Heon kaken eya; Wakantkiya kihde cehan, wayaka ahdapi qon he ocowasin ahde ca wicaśta kin taku wicaqu ce.

9 Wankan kihde cin he taku he ; tokaheya maka mahentuya kin iyaye cin hee.

10 Tuwe kun hi qon he taku owasin ojuye kte cin heon, he mahpiya kin ako sanpa tehan wankantuya kihde cin.

11 Unkan apa yewicaśipi kin heca wicaqu, qa wicaśta wokcan wanjikji, qa wotanin waśte oyakapi kte cin wanjikji, qa waawanhdakapi waonspekiya ko wanjikji ;

12 Tona owotanna un kin hena wicayuecedan $\mathrm{kta}$, wawokiyapi wicohan kin on, qa Messiya tancan icahyapi kte cin heon:

13 Tohan owasin okonwanjidan wacinyanpi, qa Wakantanka Cinhintku sdonyapi kin ekta, qa wicaśta tanka Messiya toojudan kin iyehankeca iyutapi kin ekta iyounhipi kte cin hehanyan.

14 Hecen detanhan hokśiyoqopa aptanptan iyeyapi, qa woonspe tate owasin on kaha iyeyapi kin heuncecapi kte śni, wicaśta ośkiśkeya olanyanpi, qa wohnaye on wicahnayanpi wicohan kin eciyatanhan;

T5 Tuka wowaśtedake en iewicaunkapi kta, hecen tuwe wicapa kin ee, Messiya hee en taku owasin on unkicagapi kta.

16 Iye eciyatanhan tancan kin ocowasin wayupiya iciyakaśkapi, qa okihe kin owasin wawokiyapi on icicazontapi, huha kin otoiyohi iyacinyan icage cin he iyecen tancan kin icahyapi, wowaśtedake en icage kte cin heon.

17 Heon kaken epe ca Itancan kin on mdaotanin; Ikcewicaśta tokeca wicotawacin witkotkopi kin en manipi kin he iyecen mayanipi kte śni.

18 Hena wicotawacin aotpasyapi, qa Wakantanka wiconi tawa kin itehanyan unpi, wosdonye śni iye tanmahen un kin eciyatanhan, wicocante tehika yuhapi kin heon;

19 Hena iyasaka icah ayapi, qa wicaśtapi śni kin en iyohpeiçiyapi, taku aśapapi kin owasin wohitiya econpi kte cin heon.

20 Tuka niyepi e qe hecen Messiya kin onspeniciciyapi śni :

21 He nayalionpi, qa he onspeniciyapi hecinhan, Jesus en wowicake kin iyececa.

22 Wicohan tokaheya kin en, wicaśta tannike cin, wohnaye wocantiheye kin on liwin kin hee yahduśdokapi kta;

23 Qa nitawacinpi woksape kin en niyutecapi kta;

24 Qa wicaśta teca woowotanna, qa wowicake wakan kin en, Wakantanka eciyatanhan icahyapi kin he kodakapi kta.

25 Heon etanhan, itonpi śni kin ayaśtanpi, qa otoiyohi wicaśta tokeca kici wowicake ece ohdaka po ; otoiyohi huha yeciciyapi kin heon.

26 Śsinyahidapi eśta, wahtanipi śni po. Canniniyanpi ecen wi iyaye śni nunwe.

27 Qa Wakanśica kiyukanpi śni po.

28 Tuwe wamanon kin he ake manon kte śni, tuka litani kta, nape on taku waśte econ kta, hecen walipanica kin he taku wicaqu kte cin yuhe kta. 


\section{EPHESOS.}

29 Wicoie sice cin owasin takudan niipi kin etanhan hinanpe śni nunwe, tuka taku waśte, on icali yapi kta, tona nahonpi kinhan wowaonśida wicaqu kte cin he ecedan.

30 Qa Wakantanka Taniya Wakan kin iyokiśinyapi śni po; he on wopekitonpi anpetu kin aiyahdeya askam niyanpi.

31 Wicokige owasin, qa wocantiyahde, qa wośihda, qa iwaśicunpi, qa waaiapi canniyekiciyapi ko owasin elipeyayapi nunwe.

$32 \mathrm{Qa}$ otoiyohi okiciciyapi, qa cantekiciyapi, qa otoiyohi kicicajuju po, Wakantanka Messiya on nicicajujupi kin he iyecen.

\section{WICOWOYAKE 5.}

1 Heon hokśiyopa cantekiyapi kin iyecen Wakantanka opa po.

2 Qa wacantkiyapi kin en mani po ; Messiya canteunkiyapi, qa unkiyepi on Wakantanka en içicu, qa waihduśna, woomua iyokipi kin he iyececa.

3 'Tuka wiinahmanpi, qa waaśapapi, qa waicucupikte cin, niyepi ekta caje yatapi śni nunwe, tuka tona owotanna un taku iyokipi wicaye cin hena.

4 Qa wawiśtenyapi, qa wicoie ecinśniyan kin, qa iapi śica hena hecetu śni ; tuka wopida eyapi kin he waśte.

$5 \mathrm{He}$ de sdonyayapi, tuwe wawicihaliapi econ, qa tuwe waaśape cin, qa tuwe waicucukte cin, he wakagapi cekiya ece, hena wanjidan eśta Messiya qa Wakantanka kici tokiconze kin en takudan tawapi kte śni.

6 Iapi ecinśniyan on ihnuhan tuwe nihnayanpi kin; wicohan kin hena on Wakantanka tocan- niye kin woakta śni cincapi kin en awicau kta.

7 Heon etanhan hena en opeya unpi śni po.

8 Ehanna otpaze cin henicapi, tuka wanna Itancan kin en iyoyanpa kin henicapi; iyoyanpa cinca kin iyecen mani po :

9 Woniya tawaskuyeca kin he wowaśte owasin, qa wicoowotanna, qa wowicake kin en un :

10 Qa Itancan kin taku iyokipiye kte cin he akita po.

11 Qa otpaza wicolian, on taku waśte icage śni kin hena en okicipapi śni po, tuka hena iyopeya po.

12 Hena taku nahrnana econpi kin caje yatapi kin he wowiśtece.

13 Tuka taku tona iyopeyapi kin hena owasin iyoyanpa kin yuotanin; qa taku owasin yuotanin kin he iyoyanpa kin ee.

14 Heon etanhan kaken eya; Tuwe iśtinbe cin kikta, qa wiconțe etanhan najin wo, Messiya iyoyamniye kta ce.

15 Hecen on wakta po, itonpeya mayanipi ktá, witkotkoke cin iyecece śni, tuka wicaśta wacin ksape cin iyececa.

16 Qa omaka kin tanyan hduha po, anpetu kin śice cin heon etanhan.

17 Heon etanhan wacin ksapa po, qa Itancan token tawacin kin sdonya po.

$18 \mathrm{Qa}$ miniśa on witkopi śni po, he on iyatahdepi ece; tuka Woniya kin on ojudan un po.

19 Qa Psalm, qa odowan, qa wakan dowanpi kin on wahokonkiciciya po ; ahiyayapi qa nicantepi kin ekta Itancan kin idowan po.

20 Qa ohinniyan, taku owasin on, Wakantanka Ateyapi kin wopida eciya po, Jesus Messiya Itancan unkiyapi caje kin on: 


\section{WICOWOYAKE 6.}

21 Qa Wakantanka kokipeyahan otoiyohi tokan ihukun iyeiciya po.

22 Winohinca, nihihnapi kin wicihukun iyeiciya po; Itancan kin en iyececa.

23 Wicaśta kin he iye tawicu pa-kin hee, Messiya okodakiciye pa kin hee he iyececa; qa he iye wicatancan kin wanikiye cin hee.

24. Heon okodakiciye kin Messiya ihukuya yanke cin he iyecen winohinca kin iye hihnakupi kin taku owasin on ihukun unpi kta. 25 Wicaśta, winohinca nitawapi kin cantewicakiya po, Messiya okodakiciye kin waśte lkidake ca, on iye ic̣içu kin he iyecen ;

26 Hecen iye oie kin eciyatanhan, mini on hduecedan, qa hduska kta;

$27 \mathrm{Qa}$ hecen iye ekta ehdaku kta, wowitan okodakiciye kin heca, woaśape qa yuśkipi, qa taku hececa wanica; hecen wakan, qa waaśape śni un kta.

$28 \mathrm{He}$ iyecen, wicaśta kin iye tancanpi kin iyecen tawicupi kin waśte wicakidakapi kta ; tuwe tawicu kin waśte kidake cinhan he waśte icidaka.

$29 \mathrm{Qa}$ tohinni tuwedan iye cehipi kin śice kidake śni, tuka icahikiye ça, tanyan hduha, Messiya okodakiciye hduhe cin iyececa.

30 He tancan, qa celipi, qa huhu kin en, huha kin he unkiyepi.

31 Heon etanhan wicaśta kin, atkuku, hunku ko ehpewicakiye ca, tawicu kin en iyaihdaśke kta, qa heniyos wicacehpi wanjidan kta.

32 He wowiyukcan wan tanka, tuka Messiya qa okodakiciye kin hena wicawaka.
33 Heon niś niyepi otoiyohi niye inihdacin nitawin waśte yakidake kta, qa winohinca kin iś iye hihnaku kin kinihan kte.

\section{WICOWOYAKE 6 .}

1 Hokśiyoqopa, nihunkakepi kin anawicakigoptan po, Itancan kin en, he owotanna.

2 Niyate nihun kici wicahduonihan wo; woahope on taku wahoyapi tokaheya kin hee;

3 Hecen tanyan yaun, qa maka akan tehan yani kta.

4 Qa ateniyanpi kin, nicincapi kin wacinko wicakiyapi śni po, tuka Itancan waicaliye ca woonspe tawa kin en icaliwicakiya po.

5 Qa wowidake yaunpi kin, tona wicacehpi ekta niyuhapi kin wokokipeya cancaniçiya anawicakigoptan po, Messiya kin iyececa, nicantepi okonwanjidan on :

6 Wanniyakapi ecedan śni, wicaśta iyokipiwicayapi kin iyececa; tuka Messiya taokiye kin iyececa, nagi kin eciyatanhan, Wakantanka tawacin kin ecen ecanonpi kta.

7 Iyokipiya wawokiya po, Itancan kin ekta hecetu, qa wicaśta kin en iyecece śni.

$8 \mathrm{Qa}$ tuwe taku waśte econ kinhan, he on Itancan kin eciyatanhan wayuhe kta, wowidake un, qa iś tawaiçiya un kin, unma tukte lkaśta, he sdonyayapi.

9 Qa tona itancan yaunpi kin, niś eya he iyecen ecawicayeconpi kta, qa wakokipe wicayecagapi kte śni. Malipiya kin ekta iś iyepi qa niś niyepi nakun Itancan yakiyapi kin yanke ca, he ekta wicaśta itohnake yuonihanpi wanica, he sdonyayapi.

10 Unkan ehake mihunkawanji, 307 
Itancan kin en waśagya un po, iye towaśake wicookihi kin on.

11 Wakantanka tawipe kin ocowasin un po, hecen Wakanśica wohnaye ohanye cin itkokim inajin oyakihipi kta.

12 Wicawe, qa wicacehpi kin kici unkicizapi śni, tuka wokiconze, qa wicowaśake, qa wiconi kin de en otpaza itancanpi kin, qa taku wakan śicapi wankantuya yukanpi kin hena om.

13 Heon etanhan Wakantanka tawipe kin ocowasin icu po, hecen anpetu śice cin en najin oyakihipi kta, qa taku owasin hduśtan nayajinpi kta.

14 Heon wowicake ipiyag kitonpi ecen najin po, qa wicoowotanna maza maku akalipe kin koyaka po.

15 Qa nisihapi kin en wookiye wotanin waśte ohduśtan kin okihan po.

$16 \mathrm{Qa}$ owasin akapa tanhan wowacinye wahacanka kin icu po, he on wicaśta śica tunwanhinkpe ide kin owasin yusni oyakihipi kta.

17 Qa wiconi maza wapaha kin he icu po, qa nakun Woniya tamazasagye kin, Wakantanka oie kin hee: 18 Wocekiye qa wokidapi owa- Amen. sin on Woniya kin en, ohinniyan tona owotanna un kin on cekiya po, qa heon woape owasin wokidapi ko yuha kiktahan un po: 19 Qa nakun miye on, iapi owakihi kte cin heon, hecen waditagya i wahdukawa, qa wotanin waśte wowiyukcan kin omdake kta. 20 Heon wahośiya un wan kaśka hnakapi kin hemaca, hecen on token iwae kte cin he iyecen waditagya iwae kta.

21 Tuka taku mitawa kin, token waun kin he nakun, sdonyayapi kta e heon Tucikos hunkawanjin kiciyapi wan waśte dakapi, qa Itancan kin en ookiye owotanna kin he taku kin owasin oniciyakapi kta:

22 Heon etanhan niyepi. ekta ye waśi, unkiyepi ekta taku kin sdonyayapi kta, qa he nicantepi kin wacintonhnag niyanpi kte cin heon.

23 Hunkawanjin kiciyapi kin en wookiye un nunwe, qa wowaśte, wowacinye ko, Wakantanka Ateyapi, qa Jesus Messiya Itancan kin eciyatanhan.

24 Tona awicakehan Jesus Messiya Itancan unkiyapi waśte dakapi kin hena owasin en, wowạonśida wicakici un nunwe.

\section{PAL O S \\ PHILIPPI OYATE \\ WOWAPI WICAKICAGE CIN.}

\section{WICOWOYAKE 1.}

1 Palos qa Timotheos, Jesus Messiya trokiyewicaye cin, tona Philippi ekta Jesus Messiya 308 en owotanna un kin hena, qa waawanhdake cin hunka wicayapi ko, wowapi unnicagapi:

2 Wakantanka Ateunyanpi, qa Jesus Messiya Itancan unki- 


\section{WICOWOYAKE 1.}

yapi kin etanhan, wowaonśida, wookiye ko niyepi kin en nicipi un nunwe.

3 Ciksuyapi eca, Walkantanka mitawa wopida ewakiya ece.

4 Ohinniyan mitawocekiye otoiyohi en, niyepi owasin on iyuśkinyan cewakiya ece:

5 Anpetu tokáheya kin eliantanhan, qa dehanyan, wotanin waśte kin en kodayeciyapi kin heon.

6 Unkan he sdonwaya; tuwe tokaheya olian waśte nicicagapi kin he niciyuśtanpi kta, Jesus Messiya anpetu tawa kin hehanyan.

7 Hecen niyepi owasin wacin en ciyuzapi kte cin he hecetu, cante en mayaduzapi kin heon; makaśkapi kin, qa wotanin waśte omdake ca mdasuta kin he en, qa nakun wowaśte mitawa kin en niyepi owasin oyapapi ece.

8 Wakantanka sdonmayan, Jesus Messiya cante kin on owasin cantocikpanipi.

$9 \mathrm{Qa}$ he de icewakiya, waśte yadakapi kin he wosdonye wiyukcanpi ko owasin en, sanpa qa sanpa tankaya aye kta:

10 Kinhan, taku waśte kin hena idukcanpi kta, hecen okonwanjidan hicahe śni yaunpi kta, Messiya anpetu tawa kin en ;

11 Qa wicoowotanna waskuyeca kin on ojudan yaunpi nunwe, hena Jesus Messiya on Wakantanka woyatan qa wowitan tawa kin ee.

12 Mihunkawanji, taku token ecamiconpi kin he on wotanin waśte kin sanpa otanin aye cin he sdonyayapi kta wacin.

13 Hecen wicaśtayatapi tahocoka kin ekta, qa owancaya Messiya on makaśkapi kin he otanin.
14 Qa Itancan kin en, hunkawanjin kiciyapi kin ota, miye makaśkapi kin on, sanpa nina waditakapi, qa kokipeśniyan wicoie kin taninyan oyakapi ece.

15 Hunh waakinicapi qa winawizipi kin on, qa hunh iś waśtedakapi kin on Messiya oyakapi ece.

16 Hena e waakinicapi kin on, wowicake eciyatanhan śni Messiya kin oyakapi, qa makaśkapi kin en wokakije kokijuya aupi kta kecinpi ;

17 Qa unmapi kin iś waśtekidapi kin on etanhan, wotanin waśte mdusuta kta e on emahdepi kin sdonyapi.

18 Ecin to ; toketu kaśta, wohnaye on etanhan, qa iś awicakehan, unma tukte kakeś, oyakapi kin he on imduśkin, qa eeś nakun imduśkin kta.

19 Nitawocekiyepi, qa Jesus Messiya Taniya wawokiye cin on, he wiconi emicicetu kta sdonwaya.

20 Qa nina awacanmi qa ape manka, hecen takudan on imaśtece kte śni, tuka ohinniyan waditagya waun kin he iyecen nakaha mitancan kin on Messiya yuonihanpi kte, wani qa mate kte cin unma tukte kaśta on etanhan.

21 Wani kin he Messiya ee, qa mate cinhan sanpa tanyan waun kita.

22 Wicacelipi kin en opeya wani kinhan, miohan waskuyeca kin hee, tuka unma tukte wacin nun tanin śni.

23 Taku nom aoṭins mayanpi; ekta mde ca Messiya kici waun kte hinca, he iyotan waśte.

24 Tuka wicacelipi kin en opeya waun kin he niyepi on wawolkiyapi kin hee.

25 Hecen wahdukcan, qa heon 309 
wani, qa niyepi owasin om waun kta sdonwakiya, sanpa inicagapi qa wacinyan iduśkinpi kte cin heon.

$26 \mathrm{Qa}$ hecen akeś ekta ciupi kinhan miye on, Jesus Messiya en sanpa nina iduśkinpi kta.

27 Tuka Messiya tawootanin waśte kin eciyatanhan yanipi nunwe; hecen wanciyakapi wau kinhan, qa iś wau śni, unma tukte kaśta, niohanpi kin nawahon $\mathrm{kta}$, wicotawacin wanjidan en sutaya yaunpi, qa wicanagi wanjidan yuha wotanin waśte kin wacinyan oyecipapi kin.

$28 \mathrm{Qa}$ tona toka niyanpi kin hena on, ihnuhan taku ininihanpi kin : hee hena en wicotakuni śni au kta yuotanin, tuka heon niyepi kin en wiconi Wakantanka etanhan au kta otanin.

29 Messiya he ayuco niçupi, iye wacinyayapi kte ça, nakun iye on kakiśniyanpi kte cin.

30 Qa makakije cin wanmayadakapi qon, qa nakaha en mau nayahonpi kin, he iyecen wolkakije duhapi kta.

\section{WICOWOYAKE 2.}

1 Heon Messiya on iwicakiyapi yuke cinhan, qa wowaśtedake en imagagaieiyapi, qa Woniya kin en kodakiciyapi yuke cinhan, qa wacantkiyapi wowaonśida ko yuke cinhan,

2 Mitowiyuśkin kin miciyuśtan po, akiyecen awacannipi, qa wowaśte okonwanjidan duhapi, qa wicotawacin wanjidan yaunpi kta.

3 Waakinicapi qa ihdatanpi kin on takudan ecanonpi kte śni, tuka walibayedan yaunpi, ga otoiyohi niye isanpa tokan waśte yecidakapi kta.

4 Otoiyohi wicitawa kin ece- dan ekta etonwanpi śni po, tuka nakun otoiyohi tokan tawa kin ekta.

5 Jesus Messiya token tawacin qon he iyecen wacin duzapi kta. $6 \mathrm{He}$ Wakantanka ouncage kin en un, qa Wakantanka kici akidecen yanka keiciye cin he wawicakipi śni kecin.

7 Tuka ihduśdoke ca wowidake un kin ouncage. kin icu, qa wicaśta kin iyecen ihdus icaga;

$8 \mathrm{Qa}$ wicaśta ouncage kin en un qehan, ihduhukuya qa wiconte ekta aiyahdeya wanahon yanka, can susbeca en te cin he ehan.

9 Heon etanhan Wakantanka he tehan wankantuya hduwankan icu, qa caje wan wicacaje owasin isanpa waśte kin he qu ; 10 Hecen Jesus caje kin on, hupahu kin owasin canpeśka makehde enajin kta, mahpiya ekta un, qa maka akan un, qa maka kin ihukuya un kin henakiya.

11 Qa Wakantanka Ateyapi kin yatanpi kte cin on, Jesus Messiya he Itancan kin ee ce ; wicaceji owasin eya oyakapi kta.

12 Heon waśte cicidapi kin, ohinniyan nayahonpi kin he iyecen, en cicipi waun kin hecedan śni, tuka nakaha en waun śni eśta, sanpa wokokipeya cancaniçiya yanipi kte cin hduśtan po.

13 Tuwe cinniciyapi, qa econniciyapi kin he Wakantanka ee, iye toiyokipi kin eciyatanhan niyepi kin en ohanyan.

14 Wihnupi qa waakinicapi wanin taku owasin econ po.

15 Hecen iyaonpepica śni, qa owotanna, wicoicage wan pemni, qa yukśankśanyan un kin ehna Wakantanka cinca ecedan 310 


\section{WICOWOYAKE 3.}

yaunpi kta; hena en maka petijanjan iyecen iyoyanpa yaunpi ;

$16 \mathrm{Qa}$ wiconi wicoie kin pazo yaunpi: Hecen Messiya anpetu tawa kin en taku on imawinkta kin he dee, taku śni on waimnanke śni, qa taku śni on htawani śni kin hee.

17 Unkan wacinyayapi wośnapi wawokiyapi kin en, amihdapson eśta nakun imduśkin, qa niyepi owasin om imduśkin kta. 18 Heon niś niyepi nakun iduśkinpi qa miyeci iniwinktapi ece.

19 Tuka niyepi kin ekta Timotheos kohanna yewaśi kta wacanmi, Jesus Messiya Itancan kin on, hecen tohan token yaunpi kin sdonwaye cinhan, hehan miś nakun iyokipi waun kta.

20 Tuwe iyecen wacin yuze ca iye cinka taku nitawapi kin ihakta kte cin mduhe śni.

21 Owasin wicitawa kin akitapi, qa taku Jesus Messiya tawa kin akitapi śni.

22 Tuka he iyutapi kin sdonyayapi, hoksidan wan atkuku kici un kin he iyecen wotanin waśte kin en micica.

23 Iye kohanna ye waśi kta, tohan token waun kte cin sdonwakiya.

24 Qa miś eya ecadan wau kta e Itancan kin wacinwaya.

25 Tuka Epaphroditos misunka qa mitawaśi, qa kici akicita waun, tuwe u yaśipi, qa tuwe taku imakakije cin omakiye cin, he niyepi ekta hde waśi kta iyececa epca.

26 He niyepi owasin cantonikpanipi, qa iyokiśica un, wayazanke cin nayakilionpi kin heon.

27 He qeś wayazanke ça wan- na te kta ikiyedan, tuka Wakantanka onśikida, qa he iśnana śni, tuka miye nakun, hecen woiyokiśice icitakihna mduhe kte śni e heon.

$28 \mathrm{Qa}$ heon itonpeyahan hde waśi, ake wandakapi kinban iduśkinpi kta, qa miś aopten iyokiśica amaye kta.

29 He Itancan kin on etanhan wopida owasin yuha tin u śi po, qa tona hececapi kin wicayuonihan po.

$30 \mathrm{He}$ Messiya wicohtani tawa kin on wanna aśkadan te kta tuka, wiconi tawa kin itonkipe śni, miye en wawokiyapi aokpaniyan ecanonpi kin he niciyuśtanpi kte cin heon etanhan.

\section{WICOWOYAKE 3.}

1 Ehake mihunkawanji, Itancan kin en iyuśkin po. Taku kin dena on wowapi cicagapi kin he miye tehike wadake śni, qa niyepi kin on waśte.

2 Sunka kin on waktaya un po; tona śicaya econpi kin on wakta po ; wayuśpapi kin on waktaya un po.

3 'Tona bakihdayapi, qa wicocante on Wakantanka ohodapi, qa Jesus Messiya en iwinktapi, qa wicacehpi wacinyanpi śni kin heuncapi.

4 Miś nakun, taku on wicacehpi kin wacinwaye kte cin mduha tuka. 'Tuwe wicacehpi wacinyan okihi kecin kinhan he isanpa owakihi.

5 Anpetu iśahdogan hehan bamakihdayapi, Israel oyate qa Benjamin tawicoun kin hematanhan, Hebrew etanhan $\mathrm{He}$ brew kin, qa woope kin eciyatanhan Pharisee kin hemaca.

6 Ohodapi kin eciyatanhan okodakiciye kin śicaya wakuwa, 311 


\section{PHILIPPI.}

qa woope woowotanna kin he eciyatanhan takudan iyaonpepica śni waun.

7 Tuka taku tona mihdamna qon hena Messiya on takuśni wadake :

8 Han, awicakehan taku owasin taku śni wadake, Jesus Messiya Itancan mitawa kin wosdonye oiyokipi kin on, iye on taku owasin toki elipewaya, qa hena tacesdi ece mdawa, Messiya iyowahi kte cin heon;

9 Qa he en waun kta, mitoowotanna wicoope kin eciyatanhan un kin he wahduhe śni, tuka taku Messiya wacinyanpi eciyatanhan un kin, Wakantanka toowotanna wacinyanpi kin on icage cin hee wacin.

10 Hecen he iye sdonwaye kta, qa piya ikicage cin wowaśake kin, qa iye kakije cin okicipapi kin hena ko, iye te cin ouncage kin en imacage kta :

11 Hecinhan, wiconte etanhan woekicetu kin en iyowahi kte linca.

12 Wanna dehan iyowahi, qa wanna mayuśtanpi kin ee śni ; tuka kuwa amde, okinni taku on Jesus Messiya mayuze cin hee mduze kta.

13 Mihunkawanji, nahanhin mduze mihdawa śni ;

14 Tuka taku wanji ecamon, taku hekta un kin hena akiktonś waun, qa taku tokata un kin hena ekta nape yewakiya ece ; hecen oinajinta awaimnanka, Jesus Messiya on Wakantanka wankantuya wicakico kin en oqipe kin yewakiye kte cin heon etanhan.

15 Heon tona unyuśtanpi kin hecen awauncinpi kta, qa taku on togye wacin duzapi hecinhan, he nakun Wakantanka oniciyakapi kta.
16 Tuka tohan iyounhipi kin he en woope wanjidan eciyatanhan maunnipi, qa okonwanjidan awauncinpi kte.

17 Hunkawanjin ciyapi kin, omicipa po, qa tona hecen manipi kin hena iwanwicayaka po, waonspekiya unyaduhapi kin he ijsececa.

18 Wanna ota ociciyakapi, qa nakaha ake ceya omdake, wicaśta ota Messiya can susbeca tawa kin tokaye cin iyecen manipi ece.

19 Hena owihanke kin he wicotakuniśni ; iye tezipi kin Wakantanka yapi, wowiśtece kin en iwinktapi, qa taku makata un kin awacinpi ece.

20 Tuka okodalkiciye unkitawapi kin he mahpiya kin ekta un, qa hetanhan Wanikiya Jesus Messiya Itancan kin hi kta unkapepi.

21 Iye untancanpi hanye cin he yutokeca kta; qa iye tancan wakan kin ouncage kin en icahye kta, towaśake on taku owasin ohiye kta okihi kin heon etanhan.

\section{WICOWOYAKE 4.}

1 Heon etanhan mihunkawanji waśte cicidapi, qa cantocikpanipi kin, wowiyuśkin mitawa qa wateśdake mitawa kin, Itancan kin en sutaya najin po, waśte cicidapi.

2 Yuodiyas cewakiye ca, Suntuce cewakiya, Itancan kin en okonwanjidan awacinpi kta.

3 Qa mitawaśi wicake cin ceciciya ce, winohinca tona wotanin waśte kin en mici olianye cin hena owicakiya wo, qa Klement, qa mitawaśi unmapi, cajepi kin wiconi wowapi kin en un kin hena koya.

4 Ohinniyan Itancan kin en 
iyuśkin po; qa ake, Iyuśkin po, epe.

5 Niwahbapidan kin he wicaśta owasin sdonyapi kta. Itancan kin kiyadan un.

6 Takudan iyokiśinya awacinpi śni po, tuka taku yacinpi kin owasin on, wocekiye, wokidapi qa wopida eyapi ko on Wakantanka oyakidakapi kta.

7 Qa Wakantanka tawookiye okahnihipica śni kin he nicantepi qa nitawacinpi kin Jesus Messiya en niyuhapi kte.

8 Unkan ehake mihunkawanji, taku wicakapi kin, taku yuonihanpi kin, taku owotanna kin, taku wakan kin, taku waśte dapica kin, taku waśte eyapi kin, wowaśte qa wowitan yuke cinhan, hena awacin po.

9 Taku tona onnispepi, qa iyacupi, qa nayahonpi, qa miye en wandakapi kin hena econ po, qa Wookiye Wakantanka kin niyepi kin en nicipi un nunwe.

10 Itancan kin en nina imduśkin, nakaha unhanketa awamayacinpi kin he piya hca aya heon. He de en ihamayaktapi, tuka token on ecamiyeconpi nun tanin śni.

11 Taku imakakije cin he wake śni; taku en micihé cin hena on iyokipiya waun kta e onspemiçiciya.

12 Token wahpaniya waun kte cin he sdonwakiya, qa token wijinya waun kte cin he nakun sdonwakiya; ohinniyan qa taku owasin on, wimapi, qa wotektewahda kta, wimajica qa mawahpanica kte cin onspemakiyapi.

13. Messiya waśagmaye cin he eciyatanhan taku owasin owakihi.

14 Tuka makakije cin en omayakiyapi kin he tanyan ecanonpi.

15 Philippi wicaśta, he de sdonya po, wotanin waśte otokahe ekta, Makedonia etanhan imdamde cehan, waqupi qa icupi kin icahtagya okodakiciye kin niye niśnana omayakiyapi.

16 Thessalonike ekta waun qon he icunhan, taku imakakije cin on wancadan qa nonpa taku mayakaipi.

17 Taku maqupi kta wacin kin hee śni, tuka waskuyeca niyepi on sanpa niciyuotapi kte cin he wacin.

18 Tuka owasin mduhe ca wijica waun; niyepi etanhan taku Epaphroditos maqu kin hena iwacu, taku waśtemna wośna waśte, qa Wakantanka iyokipi kin heca he iwamaśeca.

19 Taku inicakijapi kin owasin Wakantanka mitawa niçupi kta, iye woyuha wowitan kin en un kin hetanhan, Jesus Messiya eciyatanhan.

20 Wakantanka Ate unyanpi kin wowitan owibanke wanin kicihan nunwe. Amen.

21 Tona Messiya en owotanna un kin hena nape wicayuza po. Hunkawanjin kiciyapi om waun kin hena nape niyuzapi.

22 Tona owotanna un kin hena owasin nape niyuzapi, Kesar tiyohnaka kin hena iyotan.

23 Jesus Messiya Itancan unkiyapi towaonśida kin niyepi owasin en nicipi un nunwe. Amen. 


\section{PALOS \\ K O L O S S E O Y A T E}

WOWAPI WICAKICAGE CIN.

\section{WICOWOYAKE 1.}

1 Palos, Wakantanka tawacin kin eciyatanhan, Jesus Messiya yeśi qon he, qa Timotheos hunkawanjin unyanpi kin kici,

2 Kolosse ekta tona owotanna un, qa hunkawanjin kiciyapi Messiya wacinyanpi kin hena, wowapi cicagapi. Wowaonśida wookiye ko, Wakantanka Ate unyanpi, qa Jesus Messiya Itancan kin etanhan, niyepi kin en nicipi un nunwe.

3 Ohinniyan wocekiye unkeniciyapi, qa on Wakantanka Jesus Messiya Itancan unkiyapi Atkuku kin wopida unkekiciyapi.

4 Jesus Messiya wacinyayapi, qa tona owotanna un kin owasin waśte wicayadakapi kin he naunhonpi ;

5 Woape mahpiya kin ekta enicihnakapi kin on, he ehanna wotanin waśte wicoie wicakapi kin en nayahonpi kin;

6 He en nihipi, maka kin owancaya iyaye cin he iyececa, qa waskuyeca aicaga, niyepi kin en nakun, anpetu kin en nayahonpi, qa awicakehan Wakantanka towaonśida iyacupi qon ehantanhan.

7 Epaphras unkitawaśipi waśte, qa niyepi on Messiya taokiye wicake cin he hena hecen onspeniciyapi ;

$8^{\circ}$ Qa Woniya kin en wacanyakiyapi kin he nakun unkokiyakapi.
9 Heon anpetu kin he naunlionpi kin ehantanhan niyepi on celíya unkayaśtanpi śni, qa woksape owasin, wookahinige ko en, iye tawacin sdonyapi kin on, onijupidan kte cin he unkidapi ece.

10 Hecen taku iyokipi kin owasin on, Itancan kin en tanyan mayanipi kta, wicohan waśte owasin en, waskuyeca icahyayapi, qa Wakantanka sdonyapi kin en sanpa inicagapi kta.

11 Iye towitan wowaśake kin eciyatanhan, wicookihi kin owasin on waśagniyanpi kta, wadiwicatake cin owasin on etanhan, qa wacin tankapi, wiyuśkinpi kici :

12 Qa Ateyapi kin wopida eyakiyapi lita, he iyoyanpa ekta tona owotanna un kin woailipeye tawapi kte cin en ounpapi kta e iyehantu unyanpi :

13 He otpaza wowaśake kin etanhan unkiyuśpapi, qa iye Cinhintku waśte kidake cin tokiconze kin en ounye unkiyapi :

$14 \mathrm{He}$ en, iye we kin eciyatanhan, wopekitonpi unhapi, woahtani kajujupi kin hee:

15 Wakantanka wanyagpica śni un kin ouncage kin hee, qa taku kagapi kin owasin en tokapa kin hee :

16 Iye eciyatanhan taku owasin kagapi, mahpiya ekta un qa maka akan un kin hena, taku wanyakapi qa taku wanyagpica śni, oiyotanke kin, wokiconze 
kin, wicośkanśkan kin, qa wicowaśake kin ; taku owasin iye on icahyapi kin hena iye icicaga:

17 Qa taku owasin itokatam un kin he iye, qa he on taku owasin ecen han :

18 Qa iye tancan kin okodakiciye kin hee, qa pa kin he iye, qa otokahe kin, wiconte etanhan tokaheya tonpi kin hee, hecen iye taku owasin en tokapa un kta.

19 Iye ohna taku ocowasin ounye kte cin he Atkuku kin iyokipi.

20 Qa iye can susbeca we kin eciyatanhan wookiye kage ca, heon etanhan taku owasin iye kici okiciciye wicaye kta, taku maka akan un, qa taku malipiya ekta un kin hena, iye on.

21 Qa niś niyepi, ehanna oyate nitokecapi, qa nitawacinpi kin en toka lín henicapi, wicohan śice cin on, tuka nakaha he kici wokiyeniyan nicagapi ;

22 Iye cehpi tancan kin en țe cin eciyatanhan, hecen iye itokam owotanna, qa waaśapapi śni qa takudan iyaonpepica śni, inajin niyanpi kta:

23 Wacinyayapi kin en nayajinpi kinhan, sutaya yaunpi, qa tinsa nayajinpi, qa wotanin waśte woape nayahonpi kin etanlıan nihinḷpayapi śni kinhan; he mahpiya kin ihukuya wicaśta hiyeye cin owasin owicakiyakapi, qa he en Palos he miye ookiye makagapi:

24 Nakaha niyepi kin on etanhan makakije cin heon imduśkin, qa Messiya wokakije tawa, taku micehpi kin en yuśtanpi śni kin hena iye tancan kin, okodakiciye kin hee, on weciyuśtan kta.

$25 \mathrm{He}$ en ookiye makagapi, Wakantanka wokonze kin eci- yatanhan, he niyepi on maqupi, Wakantanka oie kin mduecetu kte cin heon.

26 Taku oyakapi śni kin, wicoicage qa wicoun ota kin ehantanhan anahmanpi kin, he nakaha tona owotanna iye tawa wicaye cin hena owicakiyaka.

27 Ikcewicaśta kin ekta taku otanin śni kin de wowitan woyuha kin tinskokeca kin, he Wakantanka hena sdonye wicakiye kta, he niyepi kin en Messiya wowitan woape kin hee.

$28 \mathrm{He}$ unkiye unyaotaninpi, wicaśta kin owasin wahokonwıcunkiyapi qa wicaśta owasin onspewicủnkiyapi ece, woksape owasin on; hecen Jesus Messiya en wicaśta otoiyohi owotanna inajin wicunkiyapi kta.

29 Heon miś eya hitawani, iye ohan kin miye en waśagya ohanye cin he eciyatanhan inawahini.

\section{WICOWOYAKE 2 .}

1 Niyepi kin on, qa Laodikia ekta un kin hena, qa tona wicacehpi on miite kin wanmayakapi śni kin on token htawani kin he sdonyayapi kta wacin :

2 Hecen iye cantepi kin kicanptapi kta, qa wacantkiyapi kin on kicicazontapi, qa wosdonye tanyan okahnigapi kta wowijice kin owasin on, qa Wakantanka towiyukcan sdonyapi kte cin on, Ateyapi qa Messiya tawa kin hee.

3 Iye en woksape, qa wookahnigc woyuha kin owasin nalimanpi.

4 Tuwedan wicoie skuya kin on nihnayanpi kte śni heon hena hepa.

5 Wicacehi on, ekta waun śni eśta, wolksape kin on cicipi waun, imduśkin, niohanpi owo315 
tanna kin, qa Messiya sutaya wacinyayapi kin he wanyag waun.

6 'Token Jesus Messiya Itancan kin iyacupi qon he iyecen ohna mani po.

7 Iye en sutaya hu nihdepi, qa en tinicagapi, qa wowacinye kin ținsa ayahanpi kta, onspeniciyapi qon he iyececa, qa en wopida eyapi kin sanpa ota ecanonpi kta.

8 Ihnuhan wicoksape eyapi kin on, qa ikce wohnaye kin on tuwe nihnayanpi kinhan, wicaśta hitunkakanpi oyakapi kin on, qa wicoicage en woonspe tokaheya eciyatanhan, qa Messiya eciyatanhan śni :

9 Iye kin ohna tancanyan $\mathrm{Ta}$ ku Wakan kin ocowasin ounyan.

10 Qa iye en niś niyepi kin yuecetu yaunpi, qa he wokiconze wowaśake ko owasin Itancan kin hee.

11 Qa he on banicilidayapi, bakilidayapi nape codan econpi kin he on, Messiya bakihdayapi tawa kin eciyatanhan, wicacehpi woahtani tancan kin ehpekiyapi kte cin heon.

12 Baptem on iye kici nihnakapi, qa nakun kici ekicetu niyanpi, Wakantanka wicate cin etanhan najin kiye cin he wowaśake kin wicayadapi kin heon etanhan.

13 Qa niyepi ehanna wayahitanipi, qa nicelipi banicilidayapi śni kin en nițapi qon, wanna wayahtanipi kin owasin nicicajujupi qa niniyanpi.

14 Qa wicoope kin taku nape on owapi qon, unkiyuśepi qa kaśeunyanpi kin he pajuju, qa can susbeca tawa kin en okatan, qa hecen yutokan iyeya.

$15 \mathrm{Qa}$ wokiconze, wicowaśake 316 ko yutakuni śni iyeye çehan, taninyan wicakipazo, qa iye linca on iwakte hdi.

16 Heon etanhan, woyute, qa woyatke, qa omniciye wakan, qa wi teca, qa anpetu okihpapi kin hena on ihnuhan tuwe niyacopi kin :

17 Hena e taku u kte ciqon ohanzi kin hee, tuka tancan kin he Messiya etanhan.

18 Tuwe walipaniiciye ca, malipiya ohnihde kin ohowicada kin ihnuhan he nihnayanpi kin: he taku wanyake śni kin en yeiciye ca, iye celipi tawacin kin on ecinśniyan ihdatan;

19 Qa Pa kin he yuze śni; he on tancan kin ocowasin okihe en iyakaśkapi kin on woyute oiyahdeyapi, qa iciyakaślkapi, qa hecen Wakantanka oicage kin on icaga ece.

20 Heon etanhan, wicoicage kin en woonspe tokaheya kin ekta Messiya kici nițapi hecinhan, tokeca e wicoicage kin en yanipi kin iyecen wicoope kin oyapapi he :

21 Oyutanpi śni, utapi śni, qa nape on yuzapi śni po, eyapi ece : 22 Hena owasin urpi kin on yasotapi ece; wicașta akantu wicoope qa woonspe kin eciyatanhan.

23 Wicolian kin he wicotawacin ohodapi kin en taku woksape iyecen yuhe ca, wicowahbadan, qa wicatancan itonkipapi śni kin hena en; tuka wicacehpi iyokipiyapi kin he woyuonihan śni ece.

\section{WICOWOYAKE 3.}

1 Heon etanhan, Messiya kici piya inicagapi hecinhan, taku wankantuya Wakantanka etapa kin eciyatanhan, Messiya 
WICOWOYAKE 3.

kiyotanke cin hen, un kin hena akita po.

2 Taku wankan un kin hena awacin po, qa taku makata un kin hena awacinpi śni po.

3 Nitapi, qa nitowiconi kin he Messiya kici, Wakantanka en nalimanpi.

4 Tohan Messiya wiconi unkitawapi kin taninyan hiyu kinhan, hehan wowitan en, kici niyuotaninpi kta.

5 Heon etanhan, nihuhapi makata un kin hena kikte po : wiinalimanpi, waaśapapi, wawihnupi, tawacin śicapi, waicucupikte cin, he wakagapi ohodapi kin hee.

6 Hena on etanhan Wakantanka tocanniye kin woakta śni cincapi kin cn awicau ece.

7 Ehanna hena ehna yanipi qehan, opeya mayanipi.

8 Tuka nakaha hena owasin ayuśtan po; wocanniye, wośihda, wocantiyahdepi, waakagapi, qa iapi śice cin niipi kin etanhan.

9 Ihnuhan oiyecitonpi śni kin; wicaśta tannike cin iye wicolian ko yahduśdokapi ;

$10 \mathrm{Qa}$ wicaśta teca kin he wosdonye kin on yutecapi, waicalya ouncage kin eciyatanhan, he kodakapi.

11 He en, Helenes, qa Juda wicaśta wanica; bakilidayapi qa bakilidayapi śni kin wanica; Ikcewicaśta qa Skuthes wicaśta kin wanica; wowidake un kin heca qa tawaiçiyapi kin wanica, tuka Messiya he ocowasin, qa owasin en un.

12 Heon etanhan, owotanna yaunpi qa waśtenidakapi kin, Wakantanka wicakahnige cin iyecen, wocantekiye koyaka po, qa wowaonśida, wahanicidapi śni, wicowahbadan, qa wacintankapi kin hena ko.
13 Otoiyohi iyowinyeciciyapi, qa otoiyohi yecicajujupi kta, tuwe wicaśta tokeca on taku śihda kinhan, Messiya kin nicicajujupi kin he iyecen niś econ po.

14. Qa taku owasin akantanhan wocantekiye kicun po, he wicoowotanna iyakaśke cin ee.

15 Qa Walkantanka tawookiye kin nicantepi kin en waniconzapi nunwe, he en wicatancan wanjidan en nicopi lkin pida un po.

16 Messiya oie kin wijinya en niunpi nunwe; woksape owasin on, Psalm, qa odowan, qa wakan dowanpi kin en, otoiyohi wahokonyeciciyapi, qa onspeyeciciyapi kta, qa nicantepi kin en wowaonśida yuha Itancan kin iyadowanpi kta.

17 Qa taku ecanonpi kinhan, wicoie qa wicohan kin unma tukte kaśta, Jesus Itancan kin caje on owasin econ po, qa on Wakantanka Ateyapi kin wopida eciya po.

18 Qa winohinca kin, nihihnapi kin wicihukuya un po, Itancan kin en hecetu kin he iyececa.

19 Wicaśta kin, nitawinpi kin waśte wicakida po, qa wicakigepi śni po.

20 Śceca kin, nihunkakepi kin taku owasin anawicakigoptan po, he Itancan kin iyolkipi.

21 Qa ateyapi kin, nicincapi kin śihdawicayapi śni po, okinni wacin ibośakapi kte.

22 Wowidake yaunpi kin, tona wicacehpi eciyatanhan niyuhapi kin anawicakigoptan po ; wicaśta iyokipiwicayapi kin iyecen, iśtapi kin itokam wawokiyapi kin hee śni, tuka nicantepi ocowasin on Wakantanka kokipa po. $23 \mathrm{Qa}$ taku ecanonpi kin owasin nicantepi kin eciyatanhan econ 317 
KOLOSSE.

po, Itancan kin on etanhan, qa wicaśta kin on etanhan śni.

24. Itancan kin etanhan woaihpeye iyunwin kin iyacupi kta he sdonyayapi ; Messiya Itancan kin wowidake niyanpi nakaeś.

25 'Tuka tuwe śicaya econ kinhan, he eciyatanhan icu kta, qa wicaśta itohnake iwanyakapi kin wanica.

\section{WICOWOYAKE 4.}

1 Unkan, itancan yaunpi kin, tona wowidake wicaduhapi kin hena, taku owotanna qa iyecetu kin wicaqu po; mahpiya ekta wanji Itancan yayapi yanke cin he sdonyayapi.

2 Ohinniyan cekiya un po, qa he en wopida eya wakta po.

$3 \mathrm{Qa}$ unkiye on wocekiye unkeyeciyapi kta, Wakantanka iapi tiyopa wan unkiciyuhdokapi kta, Messiya tawowiyukcan kin unloyakapi kta, on makaśkapi kin hee:

4 Hecen on, taku token epe kte cin ecen mdaotanin kta.

5. Tona tankan unpi kin hena on ksamyahan mani po, qa anpetu kin opekiton po.

6 Ohinniyan iyaapi kin wowaonśida en un, qa miniskuya on skuyeyapi kta, hecen wicaśta otoiyohi token awicaduptapi kte ein sdonyayapi kta.

7 Tucikos hunkawanjin kiciyapi waśtedakapi, qa waawanhdake owotanna, mici Itancan taokiye kin hee token waun kin owasin oniciyakapi kta.

8 Heon etanhan niyepi kin ekta ye waśi, token yaunpi kin he sdonye ca, nicantepi kin iyuśkin niyanpi kta;

9 Qa he kici Onesimos ye waśi, he hunkawanjin kiciyapi owo318 tanna, qa waśte enitanhampi kin. Heniyoza taku owasin den un kin oniciyakapi kta.

10 Aristarkos kici makaślkapi kin he nape niyuzapi, qa Markos Barnabas tonśkaku nakun. He iwahoniyanpi, niyepi ekta niipi kinhan ti mahen u śi po:

11 Qa.Jesus, he nakun Justus eciyapi kin; hena e bakilidayapi kin etanhanpi. Henana Wakantanka tokiconze kin en tawaśi wicawaye ca, hena iyuśkin mayanpi.

12 Epaphras enitanhanpi kin, Messiya taokiye kin hee nape niyuzapi ; he ohinniyan wocekiye en, niyepi on nina cekiya, Wakan tanka tawacin kin owasin en, owotanna, qa ecedan nayajinpi kte cin heon.

$13 \mathrm{Qa}$ he omdaka, niyepi on, tona Laodikia ekta un, qa tona Hierapolis ekta un kin, hena koya on nina htaniiciya.

14 Lukas, pejihuta wicaśta waśte kin, Demas kici, nape niyuzapi.

15 Hunkanwanjin kiciyapi Laodikia elsta unpi kin hena nape wicayuza po, qa Numphas, qa iye ti kin ohna okodakiciye kin he nakun.

16 Wowapi kin de niyepi ekta yawapi kinhan, okodakiciye Laodikia ekta un kin hena nakun yawa wicakiya po, qa niś niyepi kin wowapi Laodikia etanhan kin he nakun yawa po.

17 Qa Arcippos kaken eciya po, Nitohtani Itancan kin etanhan iyacu qon he awakicin wo, yahduśtan kta ce.

18 Palos he miye, nape kiciyuzapi kin de minape kin on wakaga. Makaśkapi kin kiksuya po. Wowaonśida niyepi en nicipi un nunwe. Amen. 


\section{PAL O S \\ THESSALONIKE OYATE}

\section{WOWAPI WICAKICAGE CIN;}

\section{TOKAHEYA KIN.}

\section{WICOWOYAKE 1.}

1 Palos, qa Siluanos, qa Timotheos, Thessalonike ekta okodakiciye kin, Wakantanka Ateyapi kin en, qa Jesus Messiya Itancan kin en un kin, wowapi wicakicagapi: Wowaonśida, wookiye ko, Wakantanka Ateunyanpi etanhan, qa Jesus Messiya Itancan kin nakun, niyepi en nicipi un nunwe.

2 Niyepi owasin on Wakantanka ohinniyan wopida unkekiciyapi, qa wocekiye unkeyapi kin en caje unniyatapi ece.

3 Qa wowacinye wicohan nitawapi kin, qa wacantkiyapi en litayanipi kin, qa Wakantanka Ateunyanpi kin itokam, Jesus Messiya Itancan unkiyapi kin en ape dukanpi kin hena ohinniyan unkiksuyapi.

4 Mihunkawanji waśte, Wakantanka nicahnigapi kin he sdonunyanpi.

5 Wotanin waśte unkitawapi en niipi kin he wicoie ecedan en un śni, tuka wowaśake nakun en, qa Woniya Wakan, qa tanyan sdonyapi kin en; niyepi ehna, qa niyepi on etanhan wicaśta tounkecapi kin sdonyayapi.

6 Wicokakije ota ehna, qa Woniya Wakan wowiyuśkin kin yuha wicoie kin iyacupi, hecen Itancan kin oyapapi, qa nakun unkiyepi.
7 Hecen Makedonia qa Akeya en, tona wacinyanpi kin owasin waonspekiye niyuhapi :

8 Qa niyepi kin etanhan Itancan oie kin oyakapi, Makedonia qa Akeya ekta, qa becedan śni, tuka owancaya Wakantanka wacinyayapi kin he ieyanpahapi, qa hecen taku unkeyapi kta e iyehantu śni.

9 Hena e qe niye nicapi, qa niyepi ekta tukten canku kin ohna unkiyolipayapi kin he oyakapi, qa token wakagapi kin etanhan Wakantanka ekta nihduhomnipi, Wakantanka ni un qa wicake cin ohoyadapi kte cin heon :

10 Qa iye Cinhintku kin mahpiya eciyatanhan $u$ ayapepi kta, he wiconțe etanhan najin kiya, Jesus wocanniye u kte cin etanhan eunhdakupi kin hee.

\section{WICOWOYAKE 2.}

1 Mihunkawanji, niyepi ekta token unkipi kin niye e sdonyayapi, ituya unkipi śni.

2 'Tuka Philippi ekta kakiśunyanpi, qa unkiliahapike cin niye sdonyayapi; qa he iyohakam Wakantanka unkitawapi kin on wadiuntakapi, qa waakinicapi ota ehna wotanin waśte kin unkoniciyakapi.

3 Qa wowahokonkiye unkitawapi kin he wohnuni, qa woaśa319 


\section{THESSALONIKE.}

pe eciyatanhan śni, qa wohnaye en un śni ;

4 Tuka token wotanin waśte kin unhapi kta e Wakantanka iyokipi kin he iyecen unkiapi ece ; wicaśta iyokipiwicayapi kin iyecece śni, tuka Wakantanka cante unkiyukcanpi kin he.

5 Tohinni iskuya wicoie kin unkeyapi śni e niye sdonyayapi, qa waicucupikte cin śina unkinpi śni e Wakantanka sdonya.

6 Qa wicaśta kin etanhan wowitan unkakitapi śni, niyepi, qa wicaśta tokeca nakun etanhan śni, Messiya yewicaśi kin iyecen waeconwicunkiyapi kta iyececa tuka.

7 'Tuka niyepi cokaya wahibadan unkanpi, azinkiya wan cinca icahwicakiye cin he iyecen.

8 Hecen nina cante en unniyuzapi, qa waśteunnidakapi kin heon Wakantanka tawootanin waśte kin hee, qa unnagipi kin hena ko unniçupi kta tawatenunyanpi tuka.

9 Mihunkawanji, unkohanyanpi ga htaunnipi kin yeksuyapi, hanyetu anpetu ko htaunnipi, wotanin waśte Wakantanka tawa unkoniciyakapi kin he icunhan, tuwedan treya waqinunniciyapi kte śni uncinpi kin heon etanhan.

10 Niye sdonyayapi, qa Wakantanka nakun, tona wicayadapi kin en, wakanyan, owotanna, qa iyaonpepica śni unyakonpi.

11 Qa wicaśta cinca wahokonkiye cin he iyecen, niyepi otoiyohi wahokonunniciyapi, qa token unnicihnapi qa econ unniśipi kin he sdonyayapi;

12 Wakantanka iye tokiconze, qa towitan kin en nicopi kin, he iyokipiya mayanipi kte cin heon.

13 Qa heon ohinniyan Wakantanka wopida unkekiciyapi; 320
Wakantanka oie kin unkiyepi etanhan nayahonpi, qa iyacupi qehan, wicaśta oie kin iyecen iyacupi śni, tuka awicakehan Wakantanka oie kin hee, niye tona wicayadapi kin en ohányan ece kin iyececa.

14 Mihunkawanji, niś niyepi kin Wakantanka taokodakiciye, Juda makoce kin ekta, Jesus Messiya en un lin hena owicayecipapi ; niś niye takuwicayayapi kin eciyatanhan kakiśniyanpi kin, iś iye Juda oyate kin eciyatanhan kakiświcayapi qon iyececa :

15 Hena e qe Jesus Itancan kin litepi, qa wicaśta wokcan wicayuhapi qon hena nakun, qa unkiye śicaya unkuwapi, Wakantanka iyokipiyáni śni, qa wicaśta owasin kaśewicayapi :

$16 \mathrm{Qa}$ Ikcewicaśta nipi kte cin iwahokonwicunkiyapi kta he tehindapi, qa hecen ohinniyan wahtanipi kin hduśtanpi kta. Wocantiyahde owihonke aiyahdeya wanna en awicau.

17 Tuka mihunkawanji, unkiś niyepi kin etanhan ptecedan eunyakupi, itohnake kin on, cante kin he wake śni, heon nitohnake wanunniyakapi kta nina cantokpaniyan unkakitapi.

18 Heon etanhan ekta unnikupi kta uncinpi, Palos miye hinca, wancadan qa nonpa, tuka Şatan iyounkipi śni.

19 Woape, qa wowiyuśkin, qa wowinkta wateśdake unkitawapi kin he taku he ; he niś niyepi śni he; Jesus Messiya Itancan unkiyapi hi kinhan he itokam.

20 Wowitan qa wowiyuśkin unkitawapi kin he niyepi.

\section{WICOWOYAKE 3.}

1 Heon etanhan sanpa tinsiciya unkokihipi śni qehan, $\dot{A}$ - 


\section{WICOWOYAKE 4.}

thenas ekta unkiśnana unyakonpi kta e iyounkipipi :

2 Qa Timotheos hunkawanjin unyanpi, Wakantanka taokiye, qa Messiya tawootanin waśte kin en unkitawaśipi kin hee, ye unśipi, sutaya icalniyanpi, qa wacinyayapi kin eciyatanhan wahokonniciyapi kte cin heon.

$3 \mathrm{Qa}$ hecen wicokakije kin dena on tuwedan pahohopi kte śni, hena ehna eunhdepi kin niye sdonyayapi.

4 Unkan niyepi kin opeya unyakonpi qehan, wokakije unhapi kta unkoniciyakapi, qa he ecetu kin sdonyayapi.

5 Heon etanhan ținsmiçiye kta owakitpani, hehan token wacinyayapi kin sdonwaye kta $\mathrm{c}$ on he ekta ye waśi, okinni taku wawiyutanyan un kin iniyutanpi naceca, qa hecen htaunnipi kin taku śni ye kta.

6 Tuka nakaha Timotheos niyepi kin etanhan en unhdipi, qa wacinyayapi wacanyakiyapi ko on wotanin waśte unkahipi, qa nakun ohinniyan waśte unyadakapi kin heon wanunyadakapi kte linca, unkiś wanunniyakapi kte cin iyececa;

7 Heon etanhan, mihunkawanji, wokakije wicocante śica ko unlapi kin owasin ehna niyepi on unkicicanptapi, wacinyayapi kin heon.

8 Nakaha unnipi, niś niyepi kin Itancan en sutaya yaunpi kinhan.

9 Unkan niyepi kin on, Wakantanka unkitawapi kin itokam, wowiyuśkin owasin on unkiyuśkinpi kin he on, ake niyepi on, taku wopida Wakantanka eciya unkokihipi kta he;

10 Hanyetu anpetu ko nina ceunkiyapi, nitohnake kin wanunniyakapi kta, qa wacinyayapi kin en taku nahahin ocowasin śni un kin he tanyan unniciyuśtanpi kta.

11 Wakantanka iś iye, Ateunyanpi kin, qa Jesus Messiya Itancan unkiyapi kin, niyepi ekta canku kin ohna tanyan ye unyanpi nunwe.

12 Qa otoiyohi waśteyecidakapi, qa owasin waśtewicayadakapi kin he en, Itancan kin icaliniyanpi, qa niciyuotapi nunwe, unkiś waśteunnicidakapi kin he iyececa.

$13 \mathrm{Qa}$ heon wowakan en nicantepi kin iyaonpepica śni niciyusutapi kta, Wakantanka Ateunyanpi kin itokam, tohan Jesus Messiya Itancan unkiyapi kin tona wicayuwakan kin owasin om hi kinhan.

\section{WICOWOYAKE 4.}

1 Unkan mihunkawanji, Jesus Itancan kin on etanhan cinunniciyapi qa ceunniciyapi, token mayanipi, qa Wakantanka iyokipiyayapi kte cin, unkiyepi etanhan iyacupi qon he iyecen sanpa iyoniptapi kte.

2 Jesus Itancan kin etanhan woahope kin tona unniçupi kin sdonyayapi.

3 Wakantanka niyuecepidan kin he tawacin kin he dee, wiinahmanpi kin en nihdoyapi kte śni ;

4 Tuka otoiyohi nitancanpi kin yuecepidan, qa yuonihanpi kin en, yahduhapi kte cin sdonyakiyapi kta.

5 Qa wicotawacin sica cantiheyapi kin he en unpi kte śni; Ikcewicaśta Wakantanka sdonyapi śni kin hena iyececapi.

6 Taku en tuwedan hunkawanjitku kin kape ca hnaye kte śni ; tona heconpi kin owasin 321 


\section{THESSALONIKE.}

Itancan kin tokicon ece, unkoniciyakapi qa unniciyaotaninpi qon iyececa.

7 Waaśapapi kin ekta Wakantanka unkicopi śni, tuka unyuecepidan kte cin heon.

8 Hecen tuwe waaktaśni kinhan, he wicaśta paha iyewicaye śni, tuka Wakantanka iye Taniya Wakan unqupi kin hee aktaśni.

9 Hunkawanjinkiciyapi waśtekicidakapi kin on wowapi cicagapi kta iyecece śni. Otoiyohi waśte yecidakapi kta he niś niye Wakantanka etanhan onnispepi.

$10 \mathrm{Qa}$ Makedonia owancaya hunkawanjinkiciyapi kin owasin hecawicayeconpi ece. Tuka mihunkawanji, ceunniciyapi ce, nakun sanpa nina ecanonpi kte.

11 Ito owanji dukanpi kta, qa taku tin nicihe cin he yahduśtanpi, qa niye ninapepi kin on litayanipi kta e aiciciya po, econ unniśipi qon iyecen;

12 Hecen tona tankan unpi kin hena itokam owotanna mayanipi kta, qa takudan icakije śni yaunpi kta.

13 Tuka mihunkawanji, tona iśtinmapi kin hena on sdonye śni dukanpi kta, qa iyoniciśicapi kta, wicaśta tokeca wowacinye nicapi kin iyececa, e he wacin śni.

14 Jesus te ça ake piya ikicage cin he wicaundapi kinhan, he iyecen tona Jesus en iśtinmapi kin hena iye kici Wakantanka uwicakiye kta.

15 Unkan Itancan oie kin eciyatanhan he de unkoniciyakapi, unkiye tona unnipi, qa Itancan bi kte cin iyounhipi kinhan, tona iśtinmapi kin hena wicitoIram unyanpi kte śni.

16 Iyaśapi wan qa malipiya 322 ohnihde itanean wan ho kin, qa Wakantanka maza yahotonpi tanka tawa ko Itancan kin hduha, mahpiya kin eciyatanhan kun u kta ; unkan tona Messiya en tapi qon hena tokaheya najinpi kta.

17 Hehan unkiye tona unnipi, qa he iyounhipi kinhan, hena om malipiya śapa ekta eunhdakupi kta, okotonyan kin ehna Itancan kin itkounkipapi kta; qa hecen ohinniyan Itancan kin kici unyakonpi kta.

18 Wicoie kin hena on otoiyohi wahokonkiciciya po.

\section{WICOWOYAKE 5.}

1 Mihunkawanji, omaka qa makoncage kin on wowapi cicagapi kta yacinpi śni ;

2 Hanyetu en wamanon sa kin he iyecen Itancan taanpetu kin $u$, niś niyepi kin tanyan sdonyayapi.

3 Tohan, Wokiyapi qa tanyan yukanpi ce, eyapi kinhan, hehan íhnuhanna wicotakuni śni en awicau kta, winohinca cinca ton kte cin yazan hinhde cin he iyececa, qa najicapi kte śni.

4. Tuka mihunkawanji, niś niyepi kin otpaza ehna yaunpi śni, qa hecen anpetu kin he wamanon sa iyecen en nilipi kte śni.

5 Niyepi owasin, iyoyanpa cinca kin henicapi qa anpetu cincapi kin. Unkiyepi hanyetu otpaza kici unketanhanpi śni.

6 Heon etanhan wicaśta tokeca iyecen unkiśtinmapi kte śni ; tuka kiktahan witko śni unyakonpi kta.

7 'Tona iśtinmapi kinhan, hena hanyetu icunhan iśtinmapi ; qa tona witkopi kinhan, hena hanyetu en witko unpi ece. 
WICOWOYAKE 5.

8 Tuka unkiye anpetu kin unketanhanpi, qa heon witko śni unyakonpi kta, wowacinye wacantkiyapi ko maku akahpe lrin unkicunpi kta, qa wiconi apepi kin he maza wapaha unyanpi kta.

9 Wakantanka wocanteptanye en eunhdepi śni, tuka Jesus Messiya Itancan unkiyapi kin eciyatanhan wiconi unkicupi lite cin.

10 He iye, unkiyepi on ta, hecen kiktahan unyakonpi, qa iś unkiśtinmapi, unma tukte kaśta, kici unnipi kta.

11 Heon etanhan otoiyohi wahokonkiciciyapi, qa onspekiciciya po, ecanonpi kin hececa.

12 Hunkawanjin unniyanpi kin, ceunniciyapi ce; tona niyepi en htanipi, qa Itancan kin on awanniyakapi, qa wahokonniciyapi kin hena sdonwicaya po.

$13 \mathrm{Qa}$ wicolian yuhapi kin on etanhan, wacantkiyapi on sutaya cante en wicayuza po. Okiciciya yakon po.

14 Hunkawanjin unniyanpi kin, ceunniciyapi ce; tona yuśkiśkeya unpi kin hena iyopewicaya po, tona wacin cistinna kin hena wicakihna po, tona waśake śni un kin hena owicakiya po, qa wicaśta owasin on wacin tanka po.

15 Ihnuhan tuwe taku śica on itkom taku śica econ kinhan, tuka ohinniyan niś niyepi kin otoiyohi on, qa wicaśta owasin on taku waśte ecedan akita po.

16 Ohinniyan wiyuśkin po.

17 Ayaśtan śni cekiya po.

18 Taku owasin on wopida eya po. He niyepi on Jesus Messiya en Wakantanka tawacin kin hee.

19 Woniya kin kasnipi śni po.

20 Wiyukcanpi kin ihahapi śni po.

21 Taku owasin iyukcanpi, qa taku waśte kin he tinsa yuha po. 22 'Taku śica ocaje owasin nakicipa po.

23 Qa wookiye Wakantanka kin niyepi ocowasin niyuecepidan nunwe; hecen ninagipi kin ocowasin, qa nitawacinpi, qa nitancanpi kin iyaonpepica śni niyuhapi kta, Jesus Messiya Itancan unkiyapi kin hi kte cin hehanyan.

24 Tuwe nicopi kin he wacinyepica, qa ecen econ kta.

25 Mihunkawanji, unkiyepi kin on cekiya po.

26 Iiputakapi wakan kin on hunkawanjin kiciyapi kin owasin i iwicaputaka po.

27 Itancan kin on hecon ciśipi, hunkawanjin kiciyapi wakan kin owasin ekta wowapi kin de yawapi kta.

28 Jesus Messiya Itancan unkiyapi kin towaonśida kin nicipi un nunwe. Amen. 


\section{PA L O S \\ THESSALONIKE OYATE}

WOWAPI WICAKICAGE CIN,

INONPA KIN.

\section{WICOWOYAKE 1 .}

1 Palos, qa Siluanos, qa Timotheos, Thessalonike ekta okodakiciye Wakantanka Ateunyanpi, qa Jesus Messiya Itancan kin en un kin hena, wowapi wicakicagapi;

2 Wowaonsida, wookiye ko Wakantanka Ateunyanpi kin, qa Jesus Messiya Itancan kin etanhan nicipi un nunwe.

3 Mihunkawanji, wacinyayapi kin he nina icage cea, wacanyakiyrpi kin otoiyohi en nina niciyuotapi kin, heon ohinniyan niyepi on, Wakantanka wopida unkekiciyapi kte cin he hecetu.

4 Hecen niyepi on, unkiye Wakantanka okodakiciye tawa kin en unkiwinktapi ece; śicaya nicuwapi kin owasin, qa wokakije duhapi kin en, wacinyan wakiśagya yaunpi kin heon.

5 Wakantanka tawoyaco owotanna wowapetokeca kin he dee, nicakijapi kin heon Wakantanka tokiconze kin iy ehantu niyawapi kta.

6 Tona kakiśniyanpi kin hena itkom Wakantanka wokakije wicaqu kte cin he owotanna.

7 Qa tona kakiśya yaunpi kin, niś niyepi kin oziiciya unkici yaunpi kta; tohan Jesus Itancan kin towaśake ohnihde wicaye cin om mahpiya kin eciyatanhan ihdutaninyan u kinhan,
8 Peta ide kin en, tona Wakantanka sdonyapi śni, qa Jesus Messiya Itancan unkiyapi tawotanin waśte anagoptanpi śni kin hena wośitkihda wicaqu kta ;

9 Hena e Itancan itohnake kin etanhan, wicotakuni śni owihanke wanica on kakiświcayapi kta.

10 Tohan oyate owotanna tawa kin en ihduwitan kta, qa tona iye wacinyanpi kin hena owasin on yatanpi kta e on hi kinhan; anpetu kin he en taku unniciyaotaninpi qon he wicayadapi kin heon.

$11 \mathrm{Qa}$ heon etanhan ohinniyan niyepi on ceunkiyapi, Wakantanka wicakico kin de iyehantu niyawapi kta, qa niyepi en iye towaśte oiyokipi kin ocowasin, qa wowaśake on wacinyanpi wicohan kin he nakun hduśtan kta.

12 Hecen Jesus Messiya Itancan unkiyapi caje kin niyepi on yuonihanpi kta, qa iye on, niś eya niyuonihanpi kta, Wakantanka unkitawapi, qa Jesus Messiya Itancan towaonśida kin eciyatanhan.

\section{WICOWOYAKE 2.}

- 1 Mihunkawanji, Jesus Messiya Itancan unkiyapi u kte cin he, qa en mniunkiciyapi kte cin hena on ceunniciyapi ce; 
WICOWOYAKE 3.

2 Ihnuhan wicanagi qa wicoie qa wowapi unnicagapi kin on ihnuhanna nitawacinpi ekta nihuhuzapi qa ininihanpi kin, Messiya taanpetu kin kiyadan u kin hececa.

3 Ihnuhan tuwe taku on nihnayanpi kin; woayuśtan wan tokaheya u kta, qa woahtani wicaśta kin wicotakuni śni cinhintku kin hee e yutaninpi kta.

4 He iye taku Wakan eyapi kin, taku ohodapi ko owasin iwankam yeiçiye ça kici kiciza, hecen iye tipi wakan Wakantanka tawa kin en, Wakantanka iyecen iyotanke ca Wakantanka keiciya oihdaka.

5 Cicipi waun qehan hena ociciyakapi qon he yeksuyapi śni he.

6 Unkan nakaha taku iyowicaki śni ece kin he sdonyayapi; hecen tohantu kinhan taninyan hiyu kta.

y Woope aktaśni wowiyukcan kin he wanna ohanyan ece, tuka tuwe he iyoki śni kin he, tohanyan tokan iyeyapi śni kin, hehanyan iyowicaki kte śni.

8 Unkan hehan woope aktaśni kin he tanin kta, Jesus Itancan kin Taniya, iye i kin etanhan hiyu kin on, qa ihdutanin wiyatpa kin on, he ihangye kta.

9 Tuwe Satan ohan kin eciyatanhan wicookihi owasin, qa wowapetokeca, qa wowinihan wicohan itonśni kin yuha u,

$10 \mathrm{Qa}$ tona awihnuni kin hena en woahtani wohnaye owasin koya; lıena wowicake waśte dakapi kin on nipi kte cin he aktapi śni, heon etanhan.

11 Qa heon etanhan hena en Wakantanka wohnaye wawokihi kin awicau kta, hecen woitonśni kin wicadapi kta.

$12 \mathrm{Qa}$ hecen tona wowicake wicadapi śni, qa woahtani en iyuśkinpi kin hena owasin wicayacopi kta.

13 'Tuka mihunkawanji, Itancan waśtenidakapi kin, niyepi on unkiye ohinniyan Wakantanka wopida unkekiciyapi kta, otokaheya tanhan Woniya wicayuecedan, qa wowicake wicadapi kin on, Wakantanka yanipi kta e on nicahnigapi kin heon etanhan.

14 Qa he en nicopi, wotanin waśte unkitawapi kin on, hecen Jesus Messiya Itancan unkiyapi towitan kin iyacupi kta.

15 Heon etanhan, hunkawanjinciyapi kin, sutaya najin po, qa woope oyakapi wicoie, qa wowapi unkitawapi kin eciyatanhan onspeniciyapi kin hená tinsaya yuha po.

16 Qa Jesus Messiya Itancan unkiyapi kin hee, qa Wakantanka Ateunyanpi kin iye waśte undakapi, qa wicimdeza owihanke wanice cin wowaonśida eciyatanhan woape waśte ko unqupi kin,

17 He nicantepi kin mdesniyanpi, qa wicoie, wicohan ko waśte kin owasin en niyusutapi nunwe.

\section{WICOWOYAKE 3.}

1 Unkan ehake mihunkawanji, unkiyepi on cekiya po, Itancan oie kin inyanke ca, wowitan kta, niyepi kin ehna iyececa.

2 Qa wicaśta śice ça wicaśtapi śni kin etanhan eunhdakupi kta; owasin wowacinye yuhapi śni kin heon.

3 Tuka Itancan kin he wacinyepica, he niyusutapi, qa taku śica etanhan enihdakupi kta.

4 Itancan kin en niyepi on kaken awauncinpi, token econ un325 


\section{TIMOTHEOS.}

niśipi qon ecen ecanonpi, qa eeś nakun ecanonpi kta.

5 Qa Itancan kin nicantepi kin Wakantanka waśtedakapi, qa Messiya wacintanka kin he en ope niyanpi nunwe.

6 Unkan mihunkawanji, Jesus Messiya Itancan unkiyapi caje kin on ceunniciyapi ce, hunkawanjin kiciyapi wan ecinśniyan mani, qa wicoope unniçupi qon eciyatanhan śni kinhan, he nakicipa po.

7 Token unkoyecipapi kte cin niye sdonyayapi ; niyepi en ośkiśkeya maunnipi śni.

8 Qa tuwedan aguyapi tawa iyunwin codan untapi śni, tuka wicohtani, wicokakije ko ehna, hanyetu, anpetu ko htaunnipi, tuwedan takudan unkinicazopi kte śni heon.

9 Unkokihipi śni kin heon etanhan śni ; tuka unkiye waonspekiya yuheunniciyapi, qa unkoyapapi kte cin heon.

10 Nicipi unyakonpi qehan, he de unkoniciyakapi, 'Tuwe litanipi tawațenye śni kinhan, he takudan yute kte śni ce.

11 Enitanhanpi wanjikji ecinśniyan manipi, qa ecaca hitanipi śni, tuka waaie sa hecapi kin he naunlionpi.

12 Tona hececapi kin hena Jesus Messiya Itancan unkiyapi kin etanhan wahokonwicunkiyapi, qa econ wicunśipi, ainina hitanipi, qa aguyapi tawapi kin ecedan hdutapi kta.

13 Unkan mihunkawanji, niś niyepi kin taku waśte econpi kin econ kapinpi śni po.

14. Qa tuwe wowapi kin de en, unkoiepi kin anaungoptanpi śni kinhan, he iwanyakapi, qa kici okiciciyapi śni po, hecen iśtece kta.

15 Tokayapi śni po, tuka hunkawanjinkiciyapi kin iyecen wahokonkiya po.

16 Qa ohinniyan, taku owasin on etanhan wookiye Itancan kin wookiye niçupi nunwe. Itancan kin niyepi owasin en nicipi un nunwe.

17 Palos he miye, napekiciyuzapi kin de minape on owawa, he wowapi otoiyohi en wowapetokeca kin hee, hecen wakaga ece.

18 Jesus Messiya Itancan unkiyapi kin towaonśida kin niyepi owasin en nicipi un nunwe.

\section{PALOS}

\section{TIMOTHEOS WOWAPI KICAGA;}

\section{TOKAHEYA KIN.}

\section{WICOWOYAKE 1.}

1 Palos Jesus Messiya yeśi qon, Wakantanka Wanikiya unkitawapi, qa Jesus Messiya Itancan wacinunyanpi kin he toope kin eciyatanhan,
2 Timotheos, wacinyanpi kin eciyatanhan micinkśi kin hee, wowaśte, wowaonśida, wookiye ko, Wakantanka Ateunyanpi, qa Jesus Messiya Itancan unkiyapi kin hena etanhan kiciun nunwe. 
3 Makedonia elkta mde cehan, Ephesos en owanji yanke ciśi, wanjikji woonspe tokeca oyakapi kte śni e iwahokonwicayakiye kte cin heon ;

4 Qa hitunkakanpi, wicoicage owihanke śni oyakapi ko awacinpi kte śni; hena taku kin wiwangapi eceedan okihi, qa on Wakantanka wacinyanpi lin en icahyapi śni.

5 Wicacante ecedan, wicanagi -waśte, qa wa cinyanpi wicakapi kin etanhan wacantkiyapi kin he woahope ecetuyapi kin hee.

6, Tona he icunonpa yapi kin hena takuśniśni akinicapi kin en yeiciyapi.

7 Woope kin tokan onspewicakiyapi kta cinpi; tuka taku eyapi, qa taku waditagya oyakapi kin he iyukcanpi śni.

8 Woope kin he waśte sdonunyanpi, tuwe woope kin iyecen yuhe cinhan.

9 Qa he de sdonunyanpi, woope lin he wicaśta owotanna kicagapi śni, tuka tona woope opapi śni, qa anagoptanpi śni lkin hena wicakicagapi, tona Taku Wakan ohodapi śni, qa wahtanipi sa, waonśidapi śni qa wayaśicapi, atkuku hunku kici wicakiktepi kin, qa wicaśta wicakațapi kin hena,

10 Wawicihahapi econpi, waihuhupi sa, wicaśta wiyopewicaye cin hena, itonpiśni qa woope kicaksapi kin hena on woope lagapi ece; qa taku tokeca woonspe zank kin kaśeye cinhan he nakun :

11 Wakantanka wacantkiya wotanin waśte wowitan yuhe maśi kin he eciyatanhan.

12 Unkan Jesus Messiya Itancan unkiyapi he okihimaye ca, wacinyepica madake ça, wico- htani kin de en opemaye ciqon he wopida ewakiya ece.

13 He itokam waośtewahda, qa śicaya wicawakuwa, qa wahanmicída; tuka wicawada śni icunhan hena iyukcan śni ecamon kin heon onśimadapi.

14. Qa Itancan unkiyapi towaonśida kin he wacinyanpi qa wacantkiyapi Jesus Messiya en nina yuotapi.

15 Wicoie kin de wacinyepica, qa owancaya icupi kta iyececa, Jesus Messiya wahtanipi sa niwicaye kta e on makata hi ; hena en miye imayotan.

16 Tuka heon etanhan onśimadapi, hecen miye en tokaheya Jesus Messiya wawacinkta yuza tanka ow asin hdutanin kta, wicani kta e on wicadapi kte cin hena waonspewicawakiye kta e hcon. 17 Hecen Wicaśtayatapi owihanke wanica, tepica śni, qa wanyakepica śni un kin, Taku Wakan ecedan ksape cin hee, woyuonihan, wowitan ko, owihanke wanin kicihan nunwe.

18 Timotheos micinkśi, woope kin de ciçu, niye on wokcanpi tokaheya ekta un qon hena eciyatanhan, hecen hena on ozuye waśte en akicita oyape kta ;

19 Wowacinye qa wicanagi waśte sutaya yahduhe kte cin heon. Wanjikśi hena elipeyapi qa on wacinyanpi ekta kamden iyeiciyapi.

20 Yumeneos qa Aleksander kici, hena hececapi, hena Satan waqu, waośtehdapi kte śni onspeiçiciyapi kte cin heon.

\section{WICOWOYAKE 2.}

1 Heon, taku owasin itokam, wicaśta kin owasin on, wokidapi, wocekiye, waiciyapi wicoie, wopida ko eyapi kta wacin. 


\section{TIMOTHEOS.}

2 Wicaśtayatapi kin hena on, qa tona itancan unpi kin owasin on, hecen woohodupi owasin qa wicohan owotanna kin en, owanjidan unkanpi qa wikope śni unnipi kta.

$3 \mathrm{He}$ waśte, qa Wakantanka Wanikiya unkitawapi kin itokam woiyokipi kin hee.

$4 \mathrm{He}$ iye wicaśta owasin nipi, qa wowicake sdonyapi kin en hipi kte cin he cin.

5 Taku Wakan wanjidan, qa Taku Wakan qa wicaśta kici wiciyotahedan un kin wanjidan, Jesus Messiya wicaśta kin hee.

6 He iye atayedan owasin opewicakiton kta e icicu, anpetu tawa kin waayatanin kte cin heon.

$7 \mathrm{He}$ en eyanpaha qa yeśipi kin heca makagapi ; Messiya en wowicake epa, itonśni mawanica; wowacinye wowicake ko Ikcewicaśta onspe wicawahiye kte cin heon.

8 Heon, wicaśta kin owancaya, wocanniye, waakinicapi ko wanin nape aśape śni kiyugan celkiyapi kta wacin.

9 Qa winohinca kin iś eya iyecen wokoyake oiyokipi kin kicunpi kta, qa tawacin waśte, wacin tankapi koya; qa paha sonpi, mazaskazi, inyan okitanin kin, qa wokoyake taku ota iyopeyapi kin hena ko unpi kte śni;

10 Tuka wicolian waśte kin hena, taku winohinca tona Wakantanka awacinpi kin iyokipipi kin hee.

$11 \mathrm{Qa}$ winohinca kin inina un, qa taku owasin en wanahion yanke ca on onspeiciciye kta.

12 Tuka winohinca kin iś waonspekiye.ca wicaśta kin iwankam un kta e iyowinwakiye śni, he inina yanke kta.

13 Adam he tokaheya kagapi, qa hehan Ewe.
14 Qa Adam hnayanpi śni, tuka winohinca kin iye hnayanpi, qa hecen woahtani hiyahdeya.

15 Tuka iś eya cinca tonpi kin en niyanpi kta, wacinyanpi, wacantkiyapi, wicohan waśte qa wacintankapi kin hduha yukanpi kinhan.

\section{WICOWOYAKE 3.}

1 Iapi kin he wowicake; tuwe waawanhdake unpi cin kinhan he wicohan waśte wan cin.

2 Tuka waawanhdake cin he iyaonpepica śni un kta, winohinca wanjidan yuze kta, waktaya un kta, wacinksapa, walibayedan, ohanpi, wowicakiyaka ;

3 Miniśa en iyeiciye śni, waawicape śni, mazaska nina akite śni ; tuka wacintanka, wacinko śni, waicucu wacin śni ;

4 Iye tiyohnaka kin tanyan hduhe kta, qa wicowahbadan owasin on cinca kin anagoptanwicakiye kta.

5 Tuwe tiyolnaka tawa kin tanyan hduha okihi śni kinhan, ecin token Wakantanka taokodakiciye kin tanyan yuhe kta he.

6 Aśkatudan en opapi kin hecece kte śni, he heca kinhan, okinni wahanicida kin on Wakanśica tawoyaco kin ekta iyaye kta.

7 Qa tona itankan yakonpi kin hena tanyan oyakapi un kta, he hecetu śni kinhan, wowihahaya un kta, qa Wakanśica wihmunke tawa kin en iyohpaye kta.

8 Iś eya hunkayapi un kin hena nakun wacinksapapi kta, ceji jatapi śni, miniśa en iyeiçiyapi śni, qa mazaska nina akitapi kte śni : 9 Wicanagi ecedan ohna wowacinye wowiyukcan kin yuhapi kta. 
10 Hena e tokaheya wicayukcanpi kta, qa hehan takudan iyaonpepica śni kinhan, hunkayapi un kin en iyeyapi kta.

11 Nakun is tawicupi kin wahbapidan kta, waaie śni unpi kta, waktaya unpi kta, taku owasin on owotanpidan.

12 Hunkayapi un kin iś tawicu wanjidan kta, cinca wicayapi qa tiyohnaka tawapi kin tanyan hduhapi kta.

13 Tona hunkayapi un wicohan kin tanyan yuhapi kin hena wowitan waśte hdamnanpi, qa Jesus Messiya wacinyanpi kin en waditaka icicagapi.

14 Dena hecen wowapi cicaga, kohanna niye en ciu kta wacin.

15 Tuka tehan wai śni kinhan, deciya tanhan on token Wakantanka ti kin ohna opiniciye kta sdonyaye kta, he okodakiciye Wakantanka ni un tawa kin ee, wowicake ihupa qa taku ahe cin hee.

16 Unkan he taku aiepica śni, Wakantanka awacinpi kin he wowiyukcan tanka; Wakantanka wicacehpi en ihdutanin qon, Woniya kin on yaowotanna kagapi, mahpiya ohnihde kin wanyakapi, Ikcewicaśta kin ekta oyakapi, wicoicage kin en wicadapi, qa wowitan kin ekta wankan ehdakupi.

\section{WICOWOYAKE 4.}

1 Woniya kin atayedan heya, Anpetu ihankeya kin en, wanjikśi wacinyanpi kin ayuśtanpi kta, qa woniya śapa wahnaye sa, qa taku wakan śica tawoonspe ko anagoptanpi kta.

2 Wicahnayanpi on itonśni iapi kta, nagipi kin maza kata on guguyapi kin heon.

3 Winohinca wicayuzapi kin he tehindapi, qa woyute, tona wowicake wicadapi, qa sdonyapi kin hena wopida eya icupi kta e Wakantanka kaga, hee yute śni wicaśipi lita.

4 Taku Wakantanka kage cin he owasin waśte, qa wopida yuha icupi kinhan, takudan oliinyanpi kte śni.

5 He Wakantanka oie, wocekiye ko on yuwakanpi.

6 Hena taku kin hunkawanjinkiciyapi kin kiksuyewicayaye cinhan, Jesus Messiya taokiye waśte yaun kta, wacinyanpi, wicoie, qa woonspe waśte ekta iyohiyaye cin hena en tanyan icaliniyanpi kta.

7 Tuka iapi śice cin, wakanka hitunkakanpi yuhapi ko nakicipe ca, Wakantanka awacinpi kin he amniheiciya wo.

8 Wicatancan yeiciyapi kin he taku cistinna okihi; tuka Wakantanka awacinpi lin he taku owasin okihi, wiconi kin de, qa wanji u kte cin iwahoyapi kin en un.

9 Wicoie kin he wowicake, qa owancaya anagoptanpi kta iyececa ;

10 Wakantanka ni un wicaśta owasin Wanikiya tawapi, qa iyotan tona wicadapi kin hena, he wacinunyanpi kin heon htaunnipi, qa ośtehdapi unyakonpi.

11 Hena taku kin iwahokonwicakiye ca onspewicakiya wo.

12 Koniśka yaun kin heon tuwedan iwahtenida kte śni; tuka tona wicadapi $i_{\text {kin }}$ hena waonspewicalkiya wo, wicoie kin en, wicohan kin en, wacantkiyapi kin en, woksape kin en, wacinyanpi kin en, woyuecedan kin en.

13 Wowapi yawapi, qa wahokonwicakiyapi, waonspewicakiyapi ko, hena econ wo, en waj śni kin hehanyan. 


\section{TIMOTHEOS.}

14. Taku wawokihi en niun kin, wokcanpi, qa waawanhdake unpi, nape aniputakapi kin on niçupi qon he akiktonje śni wo.

15 Hena taku kin awacin, qa ocowasin en yeiciya wo, hecen onspeniciciye cin owasin sdonyapi kta.

16 Atayedan awaiçicin, qa woonspe nitawa kin awakicin wo, he hduha wo. Hecanon kinhan niniçiye ca, tona nanihonpi kin hena nakun niwicayaye kta.

\section{WICOWOYAKE 5.}

1 Hunkayapi wan iyopeye śni wo, tuka ateyapi un kin iyecen wahokonkiya wo, qa tona hakaktapi kin hena nihunkawanji iyececapi kta;

2 Winohinca tankapi kin hena is inawicayaye cin iyecen, qa tona aśkatudan unpi kin hena. tawinohtinwicayaye cin iyececapi kta, woyuecedan owasin en.

3 Wiwazicapi kin, tona awicakehan wiwazicapi un kin hena wicayuonihan wo.

4 Tuka wiwazica wan cinca wicaye cin, qa takojakpaku yuke cinhan, hena tokaheya wicowazi tawapi kin en wowaśte econ onspeiciciyapi kta, qa hunkake wicayapi kin iyunwin wicakicupi kta; taku waśte qa Wakantanka itokam oiyokipi kin hee.

5 Tuwe awicakehan wiwazica un kin he iśnana ehpeyapi un, Wakantanka wacinye ca ohinniyan, hanyetu anpetu ko, wocekiye eyapi, qa hoyekiyapi kin en un.

6 Tuka tuwe iyokipi içiya un kinhan, he ni un icunhan wanna ta.

7 Hena hecen iwahokonwica330 kiya wo, hecen iyaonpepica śni unpi kta.

8 Qa tuwe tona wicayuhe cin, qa iyotan tiyohnaka tawa kin, taku wicakicamna śni kinhan, he wacinyanpi kin wanna ayuśtan, qa waawacin "śni kin sam iyeya wicaśta śica.

9 Wiwazica waniyetu wikcemna sakpe ihukuya un kin he kahinigapi kte śni. He wicaśta wanjidan hihnaye ciqon,

10 Wicohan waśte on tanyan oyakapi, cinca icahwicakiya, oicimani kin wonwicaya, tona owotamna un siha wicakiyujaja, tona kakijapi kin owicakiya, qa wicohan waśte owasin amniheiçiya en opa hecinhan.

i1 Tuka wiwazica tona aśkatudan wotapi kin hena wicakahnige śni wo; tohan Messiya en śkehanhan aye cinhan ake hihna tonpi kta;

12 Qa tokaheya wacinyanpi qon he ayuśtanpi kin heon woyaco aihduhapi kta.

13 Qa nakun kujapi, qa ti iyaza yakonpi; qa kujapi hecedan śni, nakun taku oyakapi sa, qa waaiapi sa, taku eyapi kte śni hin hena eyapi ece.

14 Heon, tona nahahin wikośkapi kin hena hihna tunpi kta, cinca tonpi kta, qa tiyohnalka hduhapi kta wacin, hecen toka kin taku iyawicaonpe kta okihi śni kagapi kta.

15 Wanna wanjikji icunonpa Satan ihakam iyayapi.

16 Wicaśta wanji, qa iś winohinca wanji wacinyan un kin, he wiwazica hduhe cinhan okiye kta; hecen okodakiciye kin tkeya qinpi kte śni, qa tona awicakehan wiwazica unpi kin hena ece owicakiyapi kta.

17 Waawanhdake un kin, tona tanyan awanhdakapi kinhan, he- 
na wowitan icitakihna wicakiciyawapi kta, tona wicoie, waonspekiyapi ko, en litanipi kin hena iyotan.

18 Wowapi wakan kin hecen eya, 'Tatanka aguyapi napan kin he iohmus sutaya iyacu kte śni. Qa ake, Tuwe htani kin he taku on opetonpi qon iyohi ece.

19 Waayatanin nonpa, qa yamni duhe śni kinhan, hunka yapi un kin wanji en wiyaonpapi aupi kin anagoptan śni wo.

20 Tona walitanipi kinhan hena owasin wicitokam taninyan iyopewicaya wo, hecen unmapi kin kokipapi kta.

21 Wakantanka itokam, qa Jesus Messiya Itancan kin, mahpiya ohnihde wicakahnigapi kin hena ko wicitokam wahokonciciya, dena taku kin ecinśniyan wiyukcanpi wanin ecanon kta, qa wicaśta iwanyakapi kte cin on etanhan takudan ecanon kte śni.

22 Ohankoya tuwedan nape aputake śni wo; qa wicaśta tokeca wahtanipi kin en ope śni wo. Ecedan ihduha wo.

23 Tokata mini ecedan yatke śni wo, nitezi kin on, qa waniyazan sa kin heon, miniśa waniqadan yatkan wo.

24. Wicaśta wanjikji wahtanipi kin taninyan han, qa itokam woyaco ekta awicaya; qa hunh wicihakam tanin ece.

$25 \mathrm{Qa}$ he iyecen wicolian waśte wanjikśi taninyan un, qa tona hecece śni eśta anahbepica śni.

\section{WICOWOYAKE 6 .}

1 Wowidake un kin tona can napinpi kin ihukuya yukanpi kin hena itancan wicayawapi kin wicakinihanpi owasin okihi wicahdawapi kta, hecen Wa- kantanka caje qa tawoonspe kin ośtehdapi kte śni.

2 Qa tona itancan wacinyan unpi kin heca ihukuya unpi kinhan, hena śicewicakidapi kte śni, om hunkawanjinkiciyapi kin heon; tuka hena wacinyan unpi, qa waśtewicadakapi, qa taku waśte kin en owicakicipapi kin heon sanja owicakiyapi kta. Hena hecen onspewicakiye ca wahokonwicakiya wo.

3 Tuwe wicoie. waśte, Jesus Messiya Itancan unkiyapi oie kin, wicohan ecedan kin eciyatanhan un kin hena wicada śni kinhan, qa togye waonspewicakiye cinhan,

$4 \mathrm{He}$ wahanicida, qa takudan sdonye śni un, tuka wakinicapi wiwangapi wicoie kin en cante kin ocowasin iyeiçiya; hena etanhan winawizipi, wakinicapi, waośtehdapi, woawacin śica ko icaga.

5 Qa wicaśta tawacïn śicapi kin ośkiślkeya hdokinicapi, wowicake yuhapi śni, qa taku kamnanpi kinhan, he Wakantanka ohodapi kin ee kecinpi. Tona hececapi kin hena awicayuśtan wo.

6 Wakantanka ohodapi kin woiyokipi kici he woyuha tanka.

7 Maka kin de en takudan unkahipi śni, qa awicakehan hctanhan takudan unkahdapi kte śni.

8 Hecen woyute, wokoyake ko unhapi kinhan, hena iyounkipipi kte.

9 Tona watonpika cinpi kinhan, hena taku wawiyutanyan un, qa mazahtakiyapi en iyohpayapi, qa witkotkoya cantiheyapi śica ota on kiuniyanpi ece kin hena en, hena e waihangyapi, wicotakuni śni ko mahen wica- 


\section{TIMOTHEOS.}

śta kin ocowasin iyolipewicayapi ece.

10 Taku śica owasin hutkan kin he mazaska waśtedakapi kin hee : he wanjikji inalinipi, qa on wacinyanpi kin etanhan iyaye yapi, qa wicokakije ota on caicipapi.

11 Tuka Wakantanka wicaśta tawa kin he niye, hena taku kin nakicipa wo; wicoowotanna, Wakantanka ohodapi, wacinyanpi, wacantkiyapi, wacintankapi qa wowahbadan, hena akita wo.

12 Wowacinye okicize waśte kin he en econ wo, owihanke wanin wiconi kin he icu wo, he en nicopi qa waayataninpi ota itokam wohdakapi waśte ecen oyahdake ciqon.

13 Wakantanka taku owasin nikiye cin he itokam, qa Jesus Messiya Pontios Pilate itokam owotanna wohdake ciqon he on ceciciya ce ;

14 Áśape śni qa iyạonpepica śni, woahope kin de duhe kta, Jesus Messiya Itancan unkiyapi taninyan u kte cin hehanyan.

15 He tohan iyehantu kinhan, tuwe yawaśtepi, qa ecedan Wawokihi kin he taninyan hiyuye kta, wicaśtayatapi kin en Wi- , Amen. caśtayatapi, qa itancanpi kin en Itancan kin hee.

16 He iśnana wiconi hduhe ca, iyoyanpa en hiyolipica śni kin lie en ounyan, wicaśta kin tuwedan he wanyake śni, qa nakun wanyaka okihi kte śni; he wowitan, qa wowaśake owihanke wanin kiciun nunwe.

17 Tona maka kin den watonpike cin wahokonwicakiya wo, watankaicidapi kte śni, qa wo. yuha atakuni śni aya ece kin wacinyanpi kte śni, tuka Wakantanka ni un taku iyounkipipi kta e iyakicuya unqupi kin hee;

18 Taku waśte ece econpi kta, wicohan waśte ijicapi kta, wapamnipi, qa wawicaqupi tawatenyapi kta;

19 Taku tokata ihe cin he ekta ahehde waśte wan içihnakapi kta, hecen wiconi owihanke wanica yuhapi kta.

20 Timotheos, taku yuheciciye cin hena tanyan yuha wo, qa ikcekceya ecinśniyan wakinicapi kin he nakicipa wo, wosdonye woitonśni akinicapi kin he nakun.

21 Wanjikji he en opapi, qa wacinyanpi kin icunonpa iyayapi. Wowaonśida niciun nunwe.

\section{PALOS}

\section{TIMOTHEOS WOWAPI KICAGA,}

\section{NON P $\triangle \mathrm{KIN}$.}

WICOWOYAKE 1.

1 Palos, Wakantanka tawacin kin on, Jesus Messiya yeśi qon, Jesus Messiya wiconi iwahounyanpi qon he eciyatanhan, 332
2 'Timotheos, hokśincantkiyapi mitawa kill, wowapi cicaga. Wowaonśida, wowaśte, wookiye ko, Wakantanka Ateyapi kin etanhan, qa Jesus Messiya Itancan unkiyapi kin etanhan. 


\section{WICOWOYAKE 2.}

3 Wakantanka, mihunkake kin etanhan nagi ska hduha ohowada kin, he wopida ewakiya, anpetu, hanyetu ko wocekiye epa eca, ayaśtan śni ciksuya waun ;

4. Wanciyaka nina wacin, iśtamnihanpe ota aniu qon hena weksuya, hecen wowiyuśkin omajudan lita;

5 Tohan wowacinye anahbepica śni niye en niun kin he weksuye cinhan, he tokaheya nikunśi Lowis en un, qa nakun nihun Yunike en un, qa he niye en niun kin sdonwaya.

6 Heon etanhan kiksuyeciye kta, Wakantanka taku niçu kin, nape aciputake cin eciyatanhan, he amniheniçiye kta.

7 Wakantanka wokokipe woniya kin unqupi śni ; tuka wowaśake, wacantkiyapi, qa wacinksapapi woniya kin.

8 Hecen Itancan unkiyapi tawootanin kin on iśtece śni wo, qa nakun miye makaśkapi tawa waun kin; tuka Wakantanka towaśake kin eciyatanhan wotanin waśte en wokakije kin en okicipa wo:

9 He iye ni unyanpi, qa wicakicopi wakan kin on unkicopi, unkolianpi kin eciyatanhan śni, tuka iye tawacin, qa towaonśida, maka icage cin itokam Jesus Messiya on unqupi qon he eciyatanhan:

10 Tuka wanna hdutanin, Jesus Messiya Wanikiya unkitawapi taniniciye cin on, he wiconțe ihangye ca, wotanin waśte kin en, wiconi qa tepica śni unpi kin iyoyanpa en au.

11 Heon eyanpaha qa yeśipi kin heca makagapi, Ikcewicaśta wowicawakiyake kte cin heon.

$12 \mathrm{Qa}$ he etanhan hena taku on iyotanhan iyewakiya; tuka imaśtece śni. Tuwe wacinwakiye cin he sdonwakiye ca, taku ehnake waśi kin hena anpetu wan u kte cin hehanyan miciyuha okihi sdonwaya.

13 Wicoie on zaniyan unpi nahonciye ciqon, wowacinye, wowaśte ko, Jesus Messiya en un kin he tinsa yuza wo.

14. Woniya Wakan unkicipi un kin eciyatanhan, wicolian waśte niçupi qon he tanyan yuha wo.

15 Tona Asia en un kin owasin amayuśtanpi kin he sdonyaya; Phugellos qa Hermogenes he en opapi.

16 Onesiphoros tiyohnaka tawa kin Wakantanka onśilkida nunwe ; iye ota micanpte ca makaśkapi kin on iśtece śni.

17 Qa Roma ekta hi qehan, nina omade qa iyemayan.

$18 \mathrm{He}$ anpetu kin he en Itancan kin etanhan wowaonśida iyeye kta e Itancan okihikiya nunwe; Ephesos ekta taku tona on omakiye ciqon he tanyan sdonyaya.

\section{WICOWOYAKE 2.}

1 He etanhau, micinkśi, Jesus Messiya towaonśida kin he en wakiśaka wo.

2 Qa miye etanhan taku nayahon, wicota yaotaninpi qon, hena wicaśta wacinyepica, tona tokan onspewicakiya okihipi kin, hena wicaqu wo.

3 Qa niye nakun Jesus Messiya taakicita waśte kin iyecen taku tehika en waditaka wo.

4 Tuwe ozuye econ kinhan, he taku wiconi lin de wicohan kin en ihdupemni śni, tuwe taakicitaye cin he iyokipiye kte cin heon.

5 Qa tuwe ohiye wacin econ kinhan, qa wakiconzapi kin ecen 333 


\section{TIMOTHEOS.}

econ śni kinhan, he wateśdaglikicatonpi śni ece.

6 Wicaśta wakicanye cin he litani kinhan, iye tokaheya taku icage cin etanhan yute kta.

7 Taku epe cin he awacin wo. Qa Itancan kin taku owasin okahihihniyan nunwe.

8 Wotanin waśte mitawa kin eciyatanhan, Jesus Messiya, Dawid wicowazi tawa kin etanhan, he wiconte etanhan elsicetuyapi kin he kiksuya wo.

9 Qa iye on wicaśta wabotice cin iyecen kakiśmayanpi, makaśkapi kin he en; tuka Wakantanka oie kin he kaśkapi śni.

10 He etanhan tona wicakahinigapi qon hena on taku owasin iyowinwaya, hena e wiconi Jesus Messiya en un kin he yuhapi kta, qa nakun owihanke wanin wowitan kin.

11 Wicoie kin de wicakapi; Iye kici unțapi kinhan, nakun kici unnipi kta;

12 Iye on unkakijapi kinhan, kici wicaśtayatapi unyalkonpi kta. Qa unkiye elipeunyanpi kinhan, iś eya ehpeunyanpi kta.

$13 \mathrm{Qa}$ wacinunyanpi śni eśta, iye wacinyepica un, ihduecinśniyan kta okihi śni ce.

14 Hena taku kin kiksuyewicaye ça, Itancan kin itokam, wicoie wawokihi śni, tona nahonpi kin wicahnaye cin hena on akinicapi kte sni e iwahokonwicakiya wo.

15 Wakantanka iyokipiyaye kta, wicaśta wakage cin iśtece lsta iyecece śni kin heca e nihdutanin kta, qa wowicake wicoie kin owotanna yapamni kta e aiciciya wo.

16 Tuka iapi śica ecinśniyan kin he nakicipa wo, he iyotan wicośice ekta icah aye kta.

17 Unkan wicoie tawapi kin 334 śiyaka śica wan iyecen waihangye kta; Yumeneos qa Philetos hena hececapi ;

18 Hena wowicake kin icunonpa iyayapi qa, Woekicetu kin wanna yuśtanpi ce, eyapi, qa heon wanjikji wacinyanpi kin etanhan wicayahomnipi.

19 Hececa eśta, Wakantanka ahehde tawa kin sutaya he ca ipuspe kin de en un; Itancan kin tona tawa wicaye cin hena sdonwicakiya. Qa nakun, Tuwe Messiya caje kin hoyekiye cinhan, he woahtani ayuśtan kta ce.

20 Tuka tipi tanka wanji ohna mazaskazi, qa mazaska wakśica hecedan śni, nakun can, qa maka wakśica ko yukan, hunh taku waśte, qa hunh taku śica on yuhapi.

21 Hecen on, tuwe hena taku kin etanhan itpakinte cinhan, he wakśica waśte kin heca kta, ecedan, qa Itancan kin wowiny unye kta e iyokipi, qa wicohan waśte owasin on yuśtanpi.

22 Kośka cantiheyapi śice cin hena nakicipa wo; tuka wicoowotanna, wowacinye, wacantkiyapi, wooki ye ko, henakiya tona cante ecedan on Itancan cekiyapi kin hena om en opa wo.

23 Tuka wiwangapi okahnilipica śni ecinśniyan kin hena en yeiçiye śni wo, hena wicokicize kaga e sdonyaya.

24. Unkan Itancan taokiye kin he wicakis un kte śni; tuka owasin en wahbayedan un kta, waonspekiye ça, wawacinkta yuza tanka kta:

25 Qa tona tokaiçiya unpi kin hena iwahnana wahokonwicakiye kta, okinni Wakantanka ihduecetupi wicaqu lita, hecen wowicake kin iyukcanpi kta.

$26 \mathrm{Qa}$ hecen, tona Wakanśica 


\section{WICOWOYAKE 4.}

tohan cin eca, wayaka awicahda ece kin hena mazahtakiyapi tawa kin etanhan ihduśpapi kta.

\section{WICOWOYAKE 3.}

1 He de sdonya wo, anpetu ihankeya kin en taku tehika e u kta.

2 Hehan wicaśta kin waśteicidapi kta, mazaska waśtedakapi, wamnaicidapi, wahanicidapi, hunkakeyapi kin anawicakigoptanpi śni, wapidapi śni, owotanpidan śni ;

3 Cinca cantekiyapi śni, woope kicaksapi, waaiapi sa, iyatahdeiçiyapi sa, ohitiiçidapi, qa taku waśte śicedapi ;

4 Wicahnayanpi sa, wacirkopi, watankaiçidapi, magagaiciyapi kin he iyotandapi, qa Wakantanka iyotandapi śni.

5 Wakantanka awacinpi kin wiyacinpi kin yuhapi, tuka wowaśake kin he wicadapi śni ; tona hececapi kin hena etanhan ihdamna wo.

6 Hena hececapi kin tipi nahmana tin ipi, qa winohinca wacintonpi śni, woahtani hduśakapi, qa śicaya wacin yuzapi on icunonpa ayapi ;

7 Ohinniyan waonspeiciciyapi keś wowicake sdonyapi kin chan ipi okihipi śni kin wayaka awicayapi ece.

8 Unkan, Jannes qa Jambres hena Moses kici akinicapi qon he iyecen, dena iś eya wowicake kin en ibotopi, wicaśta tawacin śicapi, qa wacinyanpi kin ayuśtanpi.

9 Tuka dena e sanpa iyoopta aye kte śni, witkotkopike cin owasin en tanin kta, hena e qe econpi qon iyececa.

10 Tuka niye wowaonspekiye mitawa kin tanyan sdonyaya, miolian kin, token miciconze cin, wacinwaye cin, wawacinkta mduza tanka kin, wacanwakiye cin, wahbayedan awape kin,

11 'Tehiya makuwapi kin, makakije cin, tona mahiyahde kin, Antiyoka ekta, Ikoniyon ekta, Lustra ekta, hena śicaya makuwapi kin mahiyahde; unkan owasin etanhan Itancan kin emahdaku.

12 Qa tona Jesus Messiya en owotanna olianyan cinpi kin hena owasin sicaya wacakuwapi kta.

13 Tuka wicaśta śica, wicahnayanpi sa ko, hena sanpa śicapi kta, wicahnayanpi qa nakun hnayanpi kta.

14 Hececa tuka niye qe taku onnispe, qa wicayada kin hena en un wo, tokiya tanhan onnispe kin he sdonyaya.

15 Qa honikśiyoqopa kin ehantanhan Wowapi wakan sdonyaya, he niniyan on ksamniye kta okihi, Jesus Messiya wacinyanpi kin eciyatanhan.

16 Wowapi wakan ocowasin Wakantanka Taniya kin eciyatanhan, qa waonspekiyapi, wiyopeyapi, owotanna kagapi, qa wicohan waśte en onspewicakiye cin he okihi.

17 Hecen Wakantanka wicaśta tawa kin ecedan un kta, qa wicohan waśte owasin on hduśtanpi kta.

\section{WICOWOYAKE 4.}

1 Heon, Wakantanka itokam, qa Jesus Messiya Itancan kin, tona nipi tapi ko wicayaco kta, tohan wokiconze hduha hi kinhan, he nakun itokam wahokonciciya ;

2 Wicoie kin oyaka wo, tohan 335 


\section{II. 'TIMOTHEOS.}

oiyokipi, qa nakun oiyokipi śni eśta, handitaiciya, wo. Owotanna wicakage wacin, iyopewicaya, wahokonwicakiya wo, woonspe qa wawacinkta yuzapi tanka kin on.

3 Anpetu wan $u$ kte cin he tohan hi kinhan, woonspe waśte kin aktapi kte śni, tuka iye wicotawacin śice cin eciyatanhan waonspekiyapi kin awicakitapi kta, noge yaśpuyapi se iyececa heon etanhan.

4 Qa nogepi kin wowicake kin etanhan hduhomnipi, qa hitunkakanpi kin ekta ihdoyapi kta.

5 Tuka niye qe taku owasin en wakta yaun kta, wicokakije en waditaka wo; wotanin waśte oyakapi wicolian kin ecen econ wo; wicohan duhe cin he hduśtan wo.

6 Wanna wamihduśna kta iyemicihantu, qa wahde kte cin he wanna kiyadan ihan.

7 Okicize waśte kin ecamon, woinyanke kin ihuniwakiya, wacinyanpi kin he hduha waun ;

$8 \mathrm{Qa}$ tokata, woowotanna wateśdake wan mihnakapi, he Itancan wayaco owotanna kin maqu kta, anpetu kin he en; qa miye miśnana śni, tuka tona iye taniniciye kte cin waśtedakapi kin hena owasin koya.

9 Kohanna en mayahi kta nina aciciya wo.

io Demas he amayustan, wiconi kin de waśtedake ca Thessalonike ekta kihda, Kreskes Galatia ekta, qa Titos Dalmatia ekta.

11 Lukas kiciśnana waun; Markos icu qa kici u wo, he iye wicohan kin de en omakiya ece. 336
12 Tucikos Ephesos ekta ye waśi.

13 Yau kinhan, Troas ekta Karpos śina emicihnake waśi qon he au wo, qa nakun wowapi kin hena, tahasaka owapi kin hena iyotan.

14 Aleksander mazazi kage cin he taku śica ota iyamaonpa; iye ohan kin on Itancan kin kicicajuju nunwe.

15 He on waktaya un wo; wicoie unyaotaninpi qon he nina kipajin.

16 Tokaheya wowahdake ciqon he ehan tuwedan micica śni, qa owasin inamatanpi, he wicakiciyawapi śni nunwe.

17 Tulia hececa eśta, Itancan kin mici inajin, qa waśagmaye, ca hecen miye on woyakapi kin tanyan sdonyapi kta, qa Ikcewicaśta kin owasin nahonpi kta; qa mnaja i kin etanhan emahdakupi.

18 Nakun Itancan kin wicohian śica owasin etanhan emahdaku kta, qa wokiconze mahipiya ekta un kin he en nimaye kta. He iye wowitan owihanke wanin kiciun nunwe.

19 Priskila qa Akula napewicayuza wo, qa nakun Onesiphoros tiyohnaka tawa kin.

20 Erastos Korinth ekta yanka. Miletos ekta Trophimos wayazanka ehpeya wahdicu.

21 Nahahin waniyetu śni u kte hinca wo. Yubulos nape niyuza, qa nakun Pudens, qa Linos, qa Klauda, qa hunkawanjin kiciyapi kin owasin.

22 Jesus Messiya Itancan kin ninagi kici un nunwe. Wowaonśida nicipi un nunwe. Amen. 


\section{TITOS WOWAPI KICAGE CIN.}

\section{WICOWOYAKE 1.}

1 Palos Wakantanka taokiye, qa Jesus Messiya yeśi qon, tona Wakantanka wicakaliniga, wacinyanpi kte cin heon, qa wowicake wicolian waśte en un kin sdonyapi kte cin heon;

2 Qa owihanke wanin wiconi opapi kte cin, Wakantanka itonśni okitpani, maka kagapi kin itokam iwahounyanpi qon he on ;

3 'Tuka wanna iyehantu qehan, eyanpahapi kin on oie kin hdaotanin, he miye maqupi, Wakantanka Wanikiya unkitawapi kin he eciyatanhan;

4 Titos, witaya wacinyanpi kin he eciyatanhan cinkśiwaye cin; Wowaśte, wowaonśida, wookiye ko, Wakantanka Ateyapi kin etanhan, qa Jesus Messiya Itancan Wanikiya unhapi kin eciyatanhan kiciun nunwe.

$5 \mathrm{He}$ etanhan Krete en yanke ciśi, taku tona yuśtanpi śni kin hena wiyeya eyahnake $\mathrm{kta}$, qa otonwe otoiyohi en hunkayapi wicayaliage kta e on; token ccon ciśi qon iyececa.

6 Tuwe iyaonpepica śni kinhan, wicaśta tawicu wanjidan, qa cinca wacinyanpi wicahduhe cin, wicakis unpi ewicakiyapi qa wanahonpi śni kin hececapi śni :

7 Waawanhdake cin he takudan iyaonpepica śni un kta, he Wakantanka taokiye kin ee; iśnana wacin en ihduze śni, wa- cinko śni,miniśa en iyeiçye śni, ohitiicida śni, qa mazaska nina akite kte śni ;

8 Tuka oicimani kin onśiwicada kta, qa taku waśte kin waśtedake kta, wacin ksapa un kta, qa owotanna, wacantkiya, qa iyatahdeiçiye śni un kta;

9 Qa token onspekiyapi qon he iyecen wicoie oyakapi kin sutaya hduhe kta, hecen woonspe ecedan kin on wahokonwicakiya okihi kta, qa tona kipajiniçiyapi kin hena iyopewicaye kta.

10 Wicaśta wicaśtapi śni, qa witkoya iapi, qa wicahnayanpi kin heca ota, tona bakilidayapi etanhanpi kin hena iyotanpi.

11 Hena iohmus wicayapi kta iyececa; hena e qe tiyohnaka ocowasin yaptanyan iyewicayapi, qa mazaska wahiteśni kin on taku iyecetu śni kin onspewicaliiyapi ece.

12 Iyepi kin etanhan waayate wan heye ciqon; Krete oyate kin ohinniyan itonpiśni, wamanica ocinśice cin, qa wicatezi kuja kin hecapi ce.

13 Wayaotanin kin he wowicalke. He etanhan nina iyopewicaya wo, hecen wacinyanpi kin en ecedan unpi kta :

14 Qa hecen Juda oyate hitunkakanpi yuhapi kin, qa wicaśta akantu woope kagapi kin wowicake kaśeye cin hena awacinpi kte śni.

15 Tona ecedan unpi kin hena taku owasin ecedan yuhapi; qa tona aśapapi qa wicadapi śni kin 337 
hena taku ecedan kin takudan yuhapi śni, tuka tawacinpi nagipi ko hena ee kaeś aśapapi.

16 Hena iś Wakantanka sdonyapi keyapi, tuka wicohan on aktapi śni, walitepi śni, wanahonpi śni, qa wicolian waśte owasin en wacintonpi śni.

\section{WICOWOYAKE 2.}

1 Tuka niye ge taku woonspe ecedan en iyokipi kin hena ecen oie ya wo.

2 Wicaśta tankapi kin hena waktaya unpi kta, mdesahan wacin ksamya unpi kta, wacinyanpi, wacanthiyapi, wacintankapi ko en ecedan unpi kta.

3 Qa winohinca tankapi kin iś eya winohinca waśte iyecen olianyanpi kta, waaiapi kte śni, miniśa ota en iyeiçiyapi kte śni, qa taku waśte onspe wicakiyapi kta :

4 Hecen wikośka kin ksamwicayapi kta, wicaśta yuhapi cinca ko waśte wicakidapi kta;

5 Wacin ksapapi kta, ecepidan, tipi awanhdakapi kta, waśteya unpi $\mathrm{kta}$, hihnakupi kin anawicakigoptanpi kta; hecen Wakantanka oie kin yaśicapi kte śni.

6 Kośka kin iś nakun wacin ksapapi kte cin iwahokonwicakiya wo.

7 Qa taku owasin on wicohan waśte kin en waonspelkiya nihdutanin kta; woonspe kin en wayuśice śni, wacinlssapa, owotanna;

$8 \mathrm{Qa}$ iapi ecedan qa iyaonpepica śni kin he nun kta; hecen tuwe itkopatanhan un kinhan, he iśtece kta, taku śica takudan aniie kta okihi śni kin heon.

9 Tona wowidake un kin hena itancan wicayuhapi kin anawi- cakigoptanpi kta, qa taku owasin wakinicapi wanin iyokipi wicayapi kta;

10 Takudan nahmana icupi śni, qa wacinyanpi waśte ocowasin en ihdutaninpi kta. Hecen taku owasin on, Wakantanka Wanikiya unhapi kin tawoonspe kin he tanyan yuhapi kta.

11 Wakantanka towaonśida wicaśta owasin wiconi wicakahi kin he wanna tanin;

12 Wakantanka aktapi śni, qa wicoicage cantiheyapi kin hena ehpeunyanpi kta, qa nakaha wiconi kin en, wacinksamya, owotanna, wakanyan unnipi lita e onspeunkiyapi.

$13 \mathrm{Qa}$ wacinyanpi waśte ape unyakonpi kta, Wakantanka iyotan tanka, qa Jesus Messiya Wanikiya unhapi kin he wowitan hduha taniniçiye kte cin hee.

14. He unkiyepi on içiçu, woahtani owasin etanhan opeunkitonpi kta, qa oyate tawawicaye kte cin wicohian waśte nina hin akitapi kte cin hena wicahduska kta e heon.

15 Hena wicoie kin hecen oie ye ça, wahokonwicakiye ça iyopewicaya wo. Ihnuhan tuwe śicenidake cinhan.

\section{WICOWOYAKE 3.}

1 He kiksuyewicaya wo, taku itancanpi qa wicowaśake kin hena ihukun iyeiçiyapi kta, wicaśtayatapi kin anawicagoptanpi kta; qa wicolian waśte owasin econpi kta, on wiyeya yukanpi kta.

2 Tuwedan yaśicapi kte śni, qa wicakizapi kte śni, tuka wahibayedan unpi, qa wicaśta owasin on wacin tankapi kta.

3 Ehanna unkiś eya wacin untonpi śni, qa waanaungoptanpi 


\section{PHILEMON.}

śni, unhnayanpi, qa cantiheyapi śica ota, qa iyokipiciciyapi kin en wowidake unyakonpi, wocanniye, icekinpi ko en unnipi, walite śni unyakonpi, qa otoiyohi śiceunkicidakapi.

4 Tuka hehan Wakantanka Wanikiya unkitawapi kin he towaśte, qa wacantkiye cin wicaśta kin en yutaninpi qehan ;

5 Wicolian owotanna econkupi kin eciyatanhan śni, tuka iye towaonśida kin eciyatanhan niunyanpi ; teca wicatonpi woyujaja kin on, qa Woniya Wakan wicayuteca kin heon etanhan;

6 He Jesus Messiya Wanikiya unkitawapi kin eciyatanhan iyakiçuya unkakaśtanpi kin.

7 Hecen iye towaonśida kin on etanhan unyaowotanpidan kinhan, wiconi owihanke wanica unkapepi kin eciyatanhan, taku iye tawa kin he tawaunyanpi kta.

8 Iapi kin he wowicake; qa heon hena taku kin ohinniyan yasutaya iyae kta wacin; tona Wakantanka wicadapi kin hena wicohan waśte wacin ksamya opapi kta. Hena taku waśte, qa wicaśta kin en wawokihi. 9 Tuka witkoya wiwangapi, qa Amen. wicoicage oyakapi, qa wawakipajinpi, qa woope akinicapi kin hena okamnayan ya wo ; hena takudan okihi śni qa ecinśniyan un.

10 Wicaśta waakinica un kin he wancadan, qa inonpa iyopeyapi kin, ihakamya lieyata iyeya wo.

11 Tuwe hecece cinhan, he wanna yuptanyanpi, qa woahtani econ, qa iye atayedan ihdaco kin he sdonyaya.

12 Tohan niye ekta Artemas, qa iś Tucikos ye waśi linhan, kohanna Nikopolis ekta en mahi kte hinca wo; hen waniyetu waun kta miciconza.

13 Zenas woope aie kiyapi kin he, qa Apollos icimani ipi kinhan, takudan icakije śni tanyan iyoopteya yewicakiya wo.

14 Qa tona unkicicapi kin hena wicohan waśte aiciciya econpi kta, wicakijapi kin owicakiyapi kta, qa hecen waskuyeca icahye śni yukanpi kte śni.

15 Tona micicapi kin hena owasin nape niyuzapi. Tona wacinyanpi kin eciyatanhan waśteundakapi kin hena nape wicayuza wo. Wowaonśida niyepi owasin en nicipi un nunwe.

\section{PALOS}

\section{PHILEMON WOWAPI KICAGE CIN.}

1 Palos, Jesus Messiya on, kaśka hnakapi kin he, qa 'Timotheos sunkaunyanpi kici, Philemon waśte unkidapi qa ounkiyapi ece kin,

2 Qa nakun Apphia waśteundakapi kin, Arcippos kici ama- kicita kin he kici, qa yati ohna okodakiciye kin, witaya wowapi unnicagapi :

3 Wowaonsida, wookiye ko, Wakantanka A teunyanpi, qa Jesus Messiya Itancan kin etanhan, nicipi un nunwe. 


\section{PHILEMON.}

4 Wocekiye epa eca, ohinniyan ciksuye ca, on Wakantanka wopida ewakiya ece :

5 Jesus Itancan kin wacinyaye ca waśte yadaka, nakun tona owotanna un kin owasin, he nawahon kin heon;

6 Hecen wacinyaye cin owicayaqu kin he sanpa waśaka aye kta, on taku waśte owasin, Jesus Messiya eciyatanhan, unkiyepi en un kin he yutaninpi kta.

7 Mihunkawanji, wacanyakiye cin heon nina unkiyuśkinpi, qa unkicanptapi, tona ow otanna un kin hena cantepi kin iyuśkinwicayaye cin heon etanhan.

8 Qa heon taku iyokipi kin Messiya en itancanyan econ ciśi kta owakihi :

9 Tuka wacantkiyapi kin eciyatanhan ceciciya ce, Palos he miye, wanna wimacahinca, qa nakaha Jesus Messiya on kaślka mahnakapi waun.

10 Hecen Onesimos micinkśi, makaśkapi kin icunhan micinca wakage cin hee on ceciciya.

11 He ehanna takudan nicamna śni, tuka nakaha miye omakiye ca nakun niye oniciya okihi. He nakaha hde waśi.

12 Niye he iyowinkiya wo, minagi kin hee kaeś iyececa.

13 He yuha waun kta wacin, wotanin waśte kin on etanhan makaślkapi icunhan niye eekiya omakiye kte cin heon.

14 Tuka token yacin kin sdonye śni waun takudan ecamon kte śni ; taku waśte econ niśipi kin he iyecen ecanon kte śni, tuka niye cinka econ wacani kta he wacin.

15 He iye anpetu tonana ani340 yuśtan, ake ohinniyan yahduhe kta heon etanhan hecon naceca.

16 Wanna wowidake unpi kin iyecece śni, tuka wowidake kin ikapeya waśte, hunkawanjinkiciyapi waśtekidapi kin iyecen ; miś hecen wacin wakiyuze cin, niye iyotan hecen wacin yakiduze kta, wicacehpi qa Itancan kin nakun eciyatanhạn hunkawanjinyaye kta.

17 Kodayapi iyecen mayaduha hecinhan, he miye iyecen ake ehdaku wo.

18 Taku icakiśniye ca, taku iyohiniciciye śni hecinhan, he miciyawa wo.

19 Palos he miye, minape kin on de owawa, he miye wakajuju kta. Qa taku miyecicajuju śni, niye atayedan ocowasin yaun kin he caje mdate śni.

20 Han, mihunkawanji, Itancan kin en niye on imduśkin -kta; Itancan kin en micante kin iyuśkinmayan wo.

21 Wanayagoptan kta imdukcan, heon wowapi cicaga; qa epe cin sam iyeya ecanon kta sdonwaya.

22 'Tipi tukten manke kte cin he nakun wiyeya emicihnaka wo; wocekiye ehapi kin on etanhan niyepi on makiyuśkapi kta epca waun.

23 Epaphras, Jesus Messiya on, kici makaśkapi kin he nape niyuza ;

24 Qa Markos, Aristarkos, Demas, qa Lukas micihtanipi kin hena nakun.

25 Jesus Messiya Itancan unkiyapi towaonśida kin ninagi kin kici un nunwe. Amen. 


\section{PALOS \\ H E B R E W O Y A T E}

WOWAPI WICAKICAGE CIN.

\section{WICOWOYAKE 1.}

1 Ehanna, Wakantanka toktogye, otakiya wicaśta wokcan kin eciyatanhan hunkake wicunyanpi kin wowicakiyake ciqon, he, anpetu ehake kin dena icunhan, iye Cinhintku kin eciyatanhan wounkiyakapi ;

$2 \mathrm{He}$ on woicage kin owasin kage ça, he taku owasin tawaye kta e ehde:

$3 \mathrm{He}$ iye towitan okitanin, qa ouncage okagapi hince cin hee, iye towaśake oie kin on taku owasin hduha yanka, he iś iye eciyatanhan waunhtanipi wopakinte kage cehan, Taku iyotan tanka wankan yanke cin he etapa kin en kiyotanka.

4 He iye caje wan mahpiya ohnihde kin isanpa waśte ailipeyapi qa yuhe cin, he iyecen sam iyeya waśte.

5 Micinkśi kin he niye, anpetu kin de cinca cicaga ce, mahipiya ohnibde kin tukte wanji eciya he. Qa ake, He atemaye kta, qa miye cinkśsiwaye kta ce.

6 Unkan, tokapa kin he wicoicage kin ekta hiyuye çchan hecen eya, Wakantanka ohnihde wicaye cin owasin he itokam canpeśka makehde enajin kta ce.

7 Qa mahpiya ohnihde kin hena on kaken eya, He tateyanpa kin ohnihdeye ca, peta ide kin wowidakeya ece.

8 Tuka Cinhintku kin heciya,.
Wakantanka, oiyotanke nitawa kin he owihanke wanica; nitokiconze mazasagye kin he mazasagye owotanna;

9 Woowotanna waśteyadake cin heon Wakantanka, nita Wakantanka kin, tona nitawaśi kin hena isanpa wowiyuśkin wihdi on sdaniyan ce.

10 Qa, Itancan, otokahe ekta maka ahe cin eyahde, qa ninape kage cin mahpiya kin hee;

11 Hena yutakuni śni aye kta, tuka niye ecen yaun; owasin onliohda wan iyecen kuke kta;

$12 \mathrm{Qa}$ śina wan iyecen pehan eyahnake ca, hecen yutokecapi kta ; tuka niye okonniwanjidan, qa waniyetu nitawa kin owihanke kte śni ce.

13 'Tuka ohnihdewicaye cin tukte wanji e, Mietapa kin en iyotanka wo, tona toka niyanpi kin hena nisiha woahe kin en ewicawahnake kta ce, eciya he.

14 Hena owasin taku wakan wawokiya, tona wiconi ailipewicayapi, qa tawapi kte cin hena owicakiyapi kta e u wicaśipi qon hena eepi śni he.

\section{WICOWOYAKE 2.}

1 Heon etanhan taku naunhonpi kin hena tohinni unkikusepi kte śni e on sutaya awauncinpi kta.

2 Wicoie mahpiya ohnihde oyakapi qon he suta, qa tona anagoptan śni, qa he kaśeye cin 


\section{HEBREW.}

hena owasin iyacinyan woyaco owotanna icupi hecinhan;

3 Unkiye wiconi hinsko tanka kin unkaktapi śni kinhan, token naunpapi kta he. He tokaheya Itancan kin oyake ca, tona nalionpi kin hena atayedan unkokiyakapi :

4 Wakantanka iś eya, iye tawacin kin eciyatanhan, wicakiciyaotanin, wowapetokeca taku on yuśinyewicaye cin hena ko, waśagya econpi ota, qa Woniya Wakan wicakipamni kin hena on etanhan.

5 Wicoicage u kte cin cajeunyatapi kin he mahpiya ohnihde kin yuhewicakiye śni.

6 Tuka wanji tukten yaotanin qa heya, Wicaśta kin he taku he, on yelssuye kta he; qa wicaśta cinhintlku kin, hecen awanyahdaka he:

7 Mahipiya ohnihde kin litanna ihukuya yakage ca, wowitan, woyuonihan ko wateśdake yecaton, qa taku nape on yakage cin hena yuheyakiya:

8 Qa iye siha kin ihukuya taku owasin eyahnaka. Taku owasin iye ihukuya ehnake cin, hecen taku ihukuya ehnakapi śni kin takudan okapte śni. Tuka nakaha nahahin taku owasin ihukuya ehnakapi kin wanunyakapi śni.

9 Tuka Jesus wiconte kakijapi kin on, mahpiya ohnihde kin kitanna ihukuya kagapi kin he wowitan, woyuonihan ko wateśdag kiton kin he wanunyakapi, hecen iye, Wakantanka towaonśida kin eciyatanhan, wicaśta kin owasin on wiconte sdonye kta.

10 Tuwe iye tan tanhan taku owasin un, qa iye on taku owasin un kin he cinca ota wowitan elkta wicahdoye kte cin on, wi- coni tawapi kte cin mdetahunka kin he wokakije on ecedan kage kta e iyokipi.

11 Hecen tuwe wicayuecedan, qa tona yuecepidan kin hena owasin okonwanjipidan; heon etanhan hena hunkawanjin wicakiya kewicalkiye kta iśtece śni ;

12 Qa hecen eya, Nicaje kin mihunkawanji owicawalkiyake kta, qa okodakiciye kin cokata icidowan kta ce.

13 Qa ake, He wacinwaye kta ce. Qa ake, Iho, miye, qa cinca Wakantanka maqu kin hena.

14. Cinca kin hena wicacehpi, qa wicawe kin en opapi kin heon iś iye hena en opa; hecen tuwe wiconte wowaśake yuhe cin, Wakanśica hee, e ihangye kta;

15 Qa tona tohanyan ni unpi kin hehanyan wiconte ikopapi kin on wowidake unpi kin hena ewicahdaku kta.

16 Awicakehan malipiya ohnihde kin he koyake śni, tuka Abraham cinca kin hecen ihduza.

17 Heon etanhan taku owasin en, iye hunawanjinwicaye cin iyecen ikicage kte cin he hecetu; hecen taku Wakantanka tawa kin en, Wawayuśna Itancan waonśida, qa wicake kta, qa oyate walitanipi kin on wookiye wicakicage kta.

18 Iye, iyutanyanpi kin on, atayedan kakije cin, heon etanhan tona iyutanwicayapi kin hena ewicahdaku kta okihi.

\section{WICOWOYAKE 3.}

1 Heon, mihunkawanji wakan, mahpiya tanhan wicakico kin en oyapapi kin, wounhdakapi kin on Jesus Messiya yeśipi qa Wa- 
wayuśna Itancan kin he awacin po.

2 Moses tipi ocowasin ohna owotanna un kin, he iyecen iye qe tuwe econ śi qon owotanna ecakicon.

3 Tuwe ticage cin he tipi kin sam iyeya yuonihanpi ece kin, he iyecen he qe Moses kapeya wowitan tanka yuha.

4 Tipi otoiyohi tuwe kaga; tuka tuwe taku owasin kage cin he Wakantanka ee.

5 Moses wowidake un kin heca, tipi tawa ocowasin ohna wacinyepica un, taku oyakapi kte cin hena yaotanin kte cin heon.

6 Tuka Messiya, iye ti kin ocowasin ohna, Cinhintkuyapi kin iyececa; tipi tawa kin he unkiyepi, waditagya wounhdakapi, qa wowiyuskinyan wacinunyanpi kin he owihanketa tinsa unhapi kinhan.

7 Heon etanhan, Woniya Wakan kin kaken eya, Anpe dehan iye ho kin nayationpi kta hecinhan;

8 Cante ihdutehipi śni po, hepuza en, anpetu wawiyutanyan un kin en, wawakipajinpi qon iyecen ;

9 Nihunkakepi iyutanmayanpi, qa mayukcanpi, qa waniyetu wikcemna topa hehanyán miohan kin wanyakapi ce.

10 Heon etanhan wicoicage kin he on iyomayake ca, hepa, Cantepi kin ekta ohinniyan nunipi, qa mitacanku kin sdonyapi śni ce.

11 Hecen śinwahda qa, Okihipapi mitawa kin en yapi kte śni ce, eya wakonza.

12 Mihunkawanji, wakta po, okinni cante wicada śni, qa śice cin en niunpi, on Wakantanka ni un kin aduśtanpi kta.
13 Tuka anpetu otoiyohi wahokonkiciciya po, anpe dehan eyapi kin hehanyan; okinni woahtani wohnaye kin on wanji tukte niyutehipi kta.

14. Tokaheya wicaundapi kin he owihanketa sutaya unhapi kinhan, Messiya en ounpapi.

15 Anpe delian, iye ho kin nayahonpi kinhan, cante hdutehipi śni po, wawakipajinpi qon en iyececa ce, eyapi kin hehanyan. 16 Tona nahonpi qon hena kipajinpi he: tuka Moses tona Egupta etanhan awicau qon hena owasin śni he.

17 Tona waniyetu wikcemna topa hehanyan iyoyakeyapi qon hena tuwepi he ; tona wahtanipi, qa hepuza en tancan ihpayapi qon hena eepi śni he.

$18 \mathrm{Qa}$ okilipapi tawa kin en yapi kte śni, konza kewicakiye ciqon hena tuwepi he; tona wicadapi śni qon hena eepi śni he.

19 Hecen wicadapi śni kin heon en yapi kta okihipi śni e wanunyakapi.

\section{WICOWOYAKE 4.}

1 Hecen okihpapi kin de en unkopapi kta e iwahounyanpi kin, heon niyepi kin wanjikśi iyoyahipi kte śni se ececa e ikounpapi kta.

2 Wotanin waśte kin unkokiyakapi, iś iyepi kin iyececa; tuka wicoie nahonpi qon he owicakiye śni, tona nahonpi kin wowacinye icicahiyapi śni kin heon etanhan.

3 Tuka tona wicaundapi kin okihpapi kin en ounpapi kta ; heye ciqon iyececa; Śsinwahda on okihpapi mitawa kin en opapi kte śni ce, epa ce; maka icage cin hehantanhan wicolian kin yuśtanpi tuka. 


\section{HEBREW.}

4 Tukten anpetu iśakowin kin he ke ça hecen eya, Anpetu iśakowin kin he Wakantanka okihipa, taku kage cin owasin etanhan.

5 Qa hen ake, Okilipapi mitawa kin en opapi kte śni.

6 Heon etanhan, hunli en opapi kte cin he tokata ihan, qa tona tokaheya wotanin waśte owicakiyakapi qon hena wicadapi śni kin heon en opapi śni.

7 Ake anpetu wan ke ca Dawid en heya, Anpe dehan, iye ho kin nayahonpi kinhan, cante hdutehipi śni po.

8 Jośuwa okilipe wicakiya unkanś, he iyohakam anpetu tokeca caje yate kte śni tuka.

9 Hecen Wakantanka taoyate kin okihpapi wan wicakicihan.

10 Tuwe okihpapi en ope cin he wanna tohtani kin ayuśtan, Wakantanka iye tawa ayuśtan qon iyececa.

11 Heon etanhan wookilipe kin he en unkopapi kta e amniheunkiciyapi kta, okinni wicadapi śni qon he iyecen tuwe ihpaye kta naceca.

12 Wakantanka oie kin he ni un, wawokihi, qa mazasagye anog ope kin he isanpa pe hinea, wicanagi, wicaniya kici iyotahedan capa iyaye ca, okihepi cupe ko kicakse ça, wicotawacin wicacante wacin yuze cin hena koya iyukcan ece.

13 Qa taku kagapi kin takudan iye iśta kin en anahbepica śni; tuwe oicazo unqupi kin he iśta kin en taku owasin tancodan qa śdayehnahan yanka.

14 Heon Wawayuśna Itancan tanka wan, Jesus Wakantanka Cinhintku, malipiya kin ekta iyaye cin hee unhapi kin heon wounhdakapi kin he sutaya unhduzapi kta.
15 Wawayuśna itancan kin, unkiye unkakijapi kin en unkicanptapi kta okihi śni kin heca unhapi śni, tuka iye taku owasin on iyutanyanpi, unkiye iyeuncecapi, tuka woahtani codan un.

16 Heon etanhan wowaśte oiyotanke kin en waditagya unkupi kta, hecen wowaonśida unkicupi kta, qa tohan uncinpi eca wowaśte wawokihi kin iyeunyanpi ece e kta.

\section{WICOWOYAKE 5.}

1 Wawayuśna itancan otoiyohi, wicaśta etanhan icupi kin, he Wakantanka taku tawa kin en, wicaśta on etanhan ehdepi, taku kiciçupi, woahtani on wośnapi kin hena ko wayuśna kta e heon.

2 Tona onspepi śni, qa canku icunom yapi kin hena onśiwicada kta okihi, iś iye wookihi śni aohduteyapi nakaeś.

$3 \mathrm{Qa}$ heon oyate kin on wośna ece kin, he iyecen iś iye woahtani econ kin on taku wayuśna ece kta.

4 Qa tuwedan iye cinka wowitan kin de icu śni, tuka, Aaron iyecen, Wakantanka tuwe kico kin hecedan.

5 He iyecen, Messiya iś eya Wawayuśna itancan kta e ihdutan śni; tuka tuwe, Micinkśi kin he niye, anpe dehan cinca cicaga ce, eciye ciqon hee hecon.

6 Qa nakun tokan en kaken eya; Melkizedek ouncage kin iyecen Wawayuśna owihanke śni kin henica ce.

$7 \mathrm{He}$ qe anpetu wicacelipi en un qehan, wocekiye, wokidapi ko eye ça, iśta mnigaga nina hoyekiye ça, tuwe wiconțe etanhan ehdaku kta okihi kin he ce- 


\section{WICOWOYAKE 6 .}

kiye ca, kokipeyahan un kin, heon nahonpi.

8 Qa Cinhintku kin hee kaeś, taku token kakije cin on woanagoptan onspeiciciya.

9 Qa iye yuśtanpi kin heon tona anagoptanpi kin owasin owihanke wanin wiconi kin wicakicaga.

10 Melkizedek ouncage kin iyecen, Wawayuśna Itancan, Wakantanka keciye cin.

11 Wicaśta kin he on taku ota unkoniciyakapi kta, tuka oyakapi tehika, wanation yahunkapi śni nakaeś.

12 Hinnakaha waonspekiya henicapi kta, tuka nahahin tuwe Wakantanka oie oowa tokaheya kin hena akta onspeniciyapi kta e iyenihantupi, qa tona asanpi ece cinpi, qa waconica yun okihipi śni kin hee en iyoniptapi.

13 Tuwe kaśta asanpi ece yute cinhan, he wicoowotanna wicoie kin en wayupike śni, nahahin hokśiyoqopa nakaeś.

14 Tuka tona tankapi, qa wanna taku utapi on tanyan ihduśtanpi, taku waśte, taku śica ko iyukcanpi kin, hena waconica yutapi ece.

\section{WICOWOYAKE 6 .}

1 Heon etanhan Messiya woonspe tokaheya tawa kin he hekta ehpeunkiyapi, qa woyuśtan ekta unyanpi kta; wiconte wicohan on iyopeiciyapi, qa Wakantanka wacinyanpi kin taku ahe cin akta eunhdepi kte śni;

2 Baptem eyapi woonspe kin, qa nape on awicaputakapi, qa wicate cin ekicetuwicayapi, qa woyaco owihanke wanice cin hena koya.

3 Unkan Wakantanka iyowin- unkiyapi kinhan, hecen econkupi kta.

4 Tona wancadan iyoyanpapi, qa malipiya tanhan taku wicaqupi kin he utapi, qa Woniya Wakan kin etanhan okinipi ;

5 Qa Wakantanka oie waśte kin he utapi, wiconi n kte cin wowaśake kin nakun ;

6 Hena ayuśtanpi hecinhan, ake piya iyopeiciye wicakiyapi kte cin he okihipica śni; iye atayedan Wakantanka Cinhintku can susbeca en piya okatanpi, qa taninyan wowiśtenyapi kin heon etanhan.

7 Maka kin ota amagaju kin he yuliepe. ca, tona kicanyanpi kin hena on taku peji iyecetuya aicage cin he Wakantanka yuwaśte.

8 Tuka taku takanheca hu, qa wapepeka aicage cin he ehpeyapi, qa yaśicapi kte cin kiyadan ihan, he owihanketa huhnage inta.

9 Tuka, waśtecicidapi kin, hena hecen unkeyapi eśta, niyepi kin on taku sanpa waśte qa wiconi en ope cin hena en niunpi e sdonunyanpi.

10 Wakantanka owotanna, qa heon wicolitani, qa wowaśte wokicanye nitawapi, iye caje kin on ecanonpi, tona owotanna un owicayakiyapi, qa nahahin owicakiya dukanpi kin he akitonje kte śni.

11 Unkan niyepi otoiyohi owihanketa, woape woyuśtan kte cin hehanyan iyecen amniheniciyapi kta uncinpi.

12 Qa nikujapi kte śni, tuka tona wicadapi, qa wacintankapi on, taku iwahowicayapi qon wanna yuhapi kin, hena owicayapapi kta.

13 Wakantanka Abraham taku iwahoye çehan, taku sanpa tan345 


\section{HEBREW.}

ka cajeyate kta wanica, heon iye atayedan cajeihdata ;

14 Qa heya, Awicakehan wowaśte on ciyawaśte kta, qa yuotapi kin on yuotaciye kta ce.

15 Unkan wahbayedan ape yanke cin he iyohakam taku iwahoyapi qon elkicicetu.

16 Wicaśta akantu taku iyotan tanka cajeyatapi ece; qa wokonze woyusuta kte cin he waakinicapi owasin en woanapte kin hee.

$17 \mathrm{He}$ en, Wakantanka, tona taku iwahoyapi qon tawapi ste cin hena en, iye tawacin yuhohopica śni kin he iyotan sdonye wicakiye kta cin, qa heon wokonze on hdusuta.

18 Heon taku nom yutokecapica śni, en Wakantanka itonśni okihipica śni kin hena taku kin on etanhan, tona woape unkitokam ehdepi kin en onaunpapi kin wicakicanpte wan waśaka unhapi kta.

$19 \mathrm{He}$ unhapi, wicanagi taku on aspeiciya, wicakapi qa suta kin hee, taku tipi cokaya hnakapi kin mahen tanhan un kin he en aspeiciya.

20 Heciya Jesus kahin tokam ye cin unkiyepi on timahen iyaya, he Melkizedek ouncage kin iyecen Wawayuśna Itancan kin heca kagapi.

\section{WICOWOYAKE 7.}

1 Melkizedek, Salem Wicaśtayatapi, qa Wawayuśna Wakantanka Iyotan wankantu kin he tawa, hee, Abraham, wicaśtayatapi wicakaśtake cin, etanhan hdicu kin he itkokipe ça yawaśte.

2 Unkan Abraham taku owasin etanhan iwikcemna kin he qu. Wicaśta kin he tokaheya caje oyakapi kin on, Woowotanna wicaśtayatapi kin hee, qa hehan, Salem wicaśtayatapi kin, he Wookiye wicaśtayatapi kin hee. 3 Atkuku wanica, hunku wanica, qa wicoicage oyakapi kin wanica, anpetu tokaheya wanice ca wiconi owihanke wanica, Wakantanka Cinhintku kin iyecen icahyapi, qá Wawayuśna ohinniyan yanka.

4 Wicaśta tanka kin de wanyaka po, Abraham, hunkakeyapi un kin hee kaeś, taku iwakte ahdi kin etanhan iwikcemna kin he qu.

5 Tona Lewi cinca kin etanhanpi, wośnapi wicohan yuhapi kin hena, wicoope kin eciyatanhan iye hunkawanjitkupi oyate kin etanhan taku icupi kta e iwahowicayapi; hena Abraham nite kin etanhan inanpapi tuka heconpi.

6 Tuka tuwe hena etanhan wicoicage hdawa śni kin he Abraham etanhan taku iwikcemna kin icu, qa wahoyapi kin yuhe ciqon he yawaśte.

7 Unkan he de akinicapi śni, tuwe ihukuya un kin he iyotan waśte kin on yawaśtepi ece.

$8 \mathrm{Qa}$ deciya wicaśta țapi kte cin hena taku iwikcemna kin icupi ece; tuka heciya, he ni un e yaotaninpi.

9 Unkan he de heyepica; Lewi taku iwikcemna kin icu ece kin, he iś eya Abraham eciyatanhan taku iwikcemna kin wicaqu ;

10 Melkizedek itkokipe cehan he nahahin atkuku nite kin mahen un.

11 Heon etanhan, Lewi wośnapi wicohan yuhe cin eciyatanhan woyuśtan en au unkanś; he en oyate kin wicoope yuhapi ; tokiyatanhan wawayuśna 
WICOWOYAKE 8.

tokeca wan icage kta, Melkizedek ouncage kin eciyatanhan, qa Aaron ouncage kin eciyatanhan caje yatapi kte śni he.

12 Wośnapi wicolian kin he yutokecapi kinhan, heon etanhan wicoope kin nakun yutokecapi kta.

13 Qa tuwe on hena hecen eyapi qon he wicoun tokeca etanhan inanpa; he etanhan tuwedan wahna wośnapi kin ekta olianye śni.

14 Itancan unlkitawapi kin he Juda wicoun kin etanhan icaga e sdonunyanpi; qa wicoun kin he wośnapi kagapi kta e Moses takudan eye śni.

$15 \mathrm{Qa}$ de iyotan atanin, Melkizedek ouncage kin iyecen, Wawayuśna tokeca wan icage kta ;

16 He wicacehpi wicoie woope kin eciyatanhan śni, tuka wiconi owihanke wanica wowaśake kin eciyatanhan icahyapi.

17 Melkizedek ouncage kin iyecen, Wawayuśna ohinniyan nanka ce, eya yaotaninpi qon.

18 Unkan nakun woahope tokaheya ye cin he yutokan iyeyapi, waśake śni qa taku okihipica śni kin heon etanhan.

19 Woope kin he takudan yuśtan śni, tuka wowacinye iyotan waśte kin he en au; qa heon Wakantanka kin en ikiyedan unkupi.

20 Iye wokonze oie kin codan wawayuśna kin kagapi śni ; dena e wokonze oie codan wawayuśna wicakagapi ;

21 Tuka he qe wokonze wicoie liin eciyatanhan; tuwe, Melkizedek ouncage kin iyecen Wawayuśna ohinniyan nanka ce, Itancan kin heye ca iyopeiciye kte śni ce, eciye ciqon hee hecon.

22 He iyecen wicotakuye iyo- tan waśte kin en Jesus wayuecetu wan kagapi.

23 Wiconte kin on, ohinniyan en un okitpanipi kin, heon hena wawayuśna kin otapi.

24 Tuka he qe owihanke wanin yanke cin, heon wośnapi wicohan yutokecapica śni wan yuha.

$25 \mathrm{Qa}$ hecen tona iye eciyatanhan Wakantanka en upi kin hena owasin niwicaye kta okihi, hena wocekiye ewicaliciye kta e ohinniyan ni un kin heon. 26 Wawayuśna Itancan kin hececa he iyounkipipi, wakan, iyaonpepica śni, waaśape śni, qa wahtanipi sa wicicunonpa un, qa mahpiya kin sanpa wankantuya icahyapi.

27 He iye, wawayuśna kin hena iyecen, anpetu iyohi, tokaheya iye wahtani kin on, qa hehan oyate wahtanipi kin on etanhan wośna kte cin he iyecece śni; wancadan hecon, iye atayedan waihduśna qon he ehan.

28 Woope kin he wicaśta waśakapi śni kin hena wawayuśna wicakaga ece; tuka wokonze wicoie kin, woope iyohakam un kin, he Cinhintkuyapi, owihanke wanin yawaśtepi kin he kaga.

\section{WICOWOYAKE 8.}

1 Taku nakaha unkeyapi yuptahnakapi kin he dee; Wawayuśna Itancan kin hececa unhapi, mahipiya ekta Otancanke oiyotanke etapa kin en kiyotanka;

2 Tipi wakan kin he, qa wakeya wicakapi kin, Itancan ticaga qa wicaśta akantu econpi śni, kin he en ookiye kin hee.

3 Wawayuśna otoiyohi taku kiciçupi, wośnapi ko wayuśna kta e kagapi ; heon wicaśta kin 347 


\section{HEBREW.}

de iś eya taku wayuśna hduhe kta e hecetu.

4 Maka akan un unkanś, Wawayuśna kin heca kte śni ; wawayuśna woope kin eciyatanhan taku kiciçupi kin wayuśnapi ece kin hena yukanpi kin heon.

5 Hena oowa, qa taku mahpiyata un kin ohanzi kin en ohanyanpi ece; Moses wakeya qon kage kta hehan Wakantanka okiye ca, Awacin wo, he kin ekta token wanyag niciyapi qon he iyecen owasin yakage kta ce, eciya.

6 Tuka nakaha iye wawokiyapi iyotan waśte wan yuha, wicotakuye waśte hinca, wahoyapi iyotan waśte kin en ahe cin he en wiciyotahedan un kin, heon etanhan.

7 Wicotakuye tokaheya kin he iyaonpepica śni unkanś, iyokihe kin he tuktedan kiyukanpi kte śni tuka.

8 Hena taku iyawicaonpe ca heya, Wanyaka wo, anpetu kin u kta, hena en Israel tiyohnaka, qa Juda tipi kin wicotakuye teca wicawecage kta, Itancan kin eya :

9 Egupta makoce kin etanhan awicawau kta e hena hunkakewicayapi kin nape wicamduze ciqon anpetu kin he en, wicotakuye wicawecage ciqon he iyecece śni; wicotakuye mitawa kin he miciyuhapi śni; qa heon miś ihawicawakta śni ce, Itancan kin eya.

10 Anpetu kin hena. iyohakam wicotakuye wicawecage kte cin he dee; Hena wicotawacin kin en mitoope kin wicawaqu kta, qa cantepi kin akan owawa lita, qa miye Wakantanka mayanpi kta, qa miś hena mitaoyate wicawaye kta ;

11 Qa wicaśta otoiyohi tuwe ikiyadan ti, qa otoiyohi hunkawanjitku kin onspewicakiyapi, qa, Itancan kin sdonya wo, eyapi kte śni; tuka owasin sdonmayanpi kta, wohakakta tankinkinyanpi ko:

12 Taku ecinśniyan econpi kin hena onśiwicawada kta, qa walitanipi, wicolian śica econpi ko, icimana wicaweciksuye kte śni ce, Itancan kin eya.

13 Taku teca cajeyate cin he tokaheya kin yutanina kin heon heya. Unkan taku tanina, qa takuni śni aye cin, he tanin śni iyaye kta kiyadan ihan.

\section{WICOWOYAKE 9.}

1 Hèhan awicakehan tokaheya qon he en woohoda wicohan kin yukan, qa nakun makata tipi wakan.

2 Wakeya wan kagapi, tokaheya kin he ohna petijanjan ihupa, qa wahna wotapi, qa aguyapi taninyan ehnakapi kin, he tipi wakan eciyapi.

3 Qa ti cokahnakapi icinonpa mahen tanhan, he wakeya kin iyotan wakan eciyapi :

4 Hen mazaskazi oizinyapi kin, qa wicotakuye can koka kin akapatanhan owancaya mazaskazi akalipeton, hee ohna mazaskazi cega manna ohnakapi kin, qa can sakadan Aaron tawa kin hica aye ciqon, qa wicotakuye inyan mdaska kin hena. 5 Qa he iwankapatanhan, wowitan Cerubim wowaonśida ohe kin aohanziyapi ; hena on nakaha atayedan taku unkeyapi kte śni.

6 Hena taku kin hecen yuśtanpi qchan, wawayuśnapi kin wakeya tokaheya kin en yapi, qa ohodapi wicolian kin econpi ece. 7 Tuka icinonpa kin he en Wa-

348 
wayuśna itancan kin ecedan, waniyetu otoiyohi wancadan mahen i, qa we codan i śni, he iś iye atayedan on, qa oyate taku ecinśniyan econpi ko on en ai.

8 Heon Wonrya Wakan kin hecen oyaka;' Wakeya tokaheya lkin nahahin ecen he cin icunhan, tipi iyotan wakan kin ekta canku kin nahahin yuotaninpi śni :

$9 \mathrm{He}$ ito kayehan wiyacinpi un, qa en taku rvicaqupi wośnapi ko aipi, tuka hena e tuwe hecon kin nagi kin yuecedan kta okihi śni.

10 Hena e woyuecetu kte anpetu kin hehanyan, woyute, qa woyatke, qa woyujaja ota, econ wicaśipi kin.

11 Tuka Messiya, taku waśte u kte cin en, Wawayuśna Itancan ihdutanin, wakeya iyotan waśte qa yuśtanpi, nape on kagapi śni, wicoicage kin de etanhan śni, kin he ohna;

12 Tatokadan qa ptejicadan we kin yuhe śni, tuka wopekitonpi owihanke wanin kage ca, iye we kin hduha wancadan tipi iyotan wakan kin timahen iyaya.

13 Hecen tatanka qa tatokadan we kin, qa pte heyuktanna ca-hota amnimnipi kin, tona waaśape cin wicayuecedan, qa wicacehpi yuska kta e okihi hecinhan ;

14 Sam iyeya Messiya, Woniya owihanke wanice cin eciyatanhan, Wakantanka en iyaonpepica śni waihduśna qon, he iye we kin, wiconte wicohan kin etanhan nagi nipakintapi kta, Wakantanka ni un kin wowidag niyanpi kte cin heon.

15 Qa heon etanhan, Wicotakuye Teca kin en Iyotahedan un kin he iye, hecen wicotakuye tokaheya ihukuya wahtanipi qon hena kajuju kta e on te cin, he eciyatanhan tona wicakico kin owihanke wanin taku tawapi kte cin iwahowicayapi qon he yuhapi kta.

16 Tukten wicotakuye wanji yukan hecinhan, hen wicotakuye kage cin he wanna ta e yutaninpi.

17 Taku tapi kin hena on wicotakuye wowapi kin suta ece; tuka wicotakuye kage cin nahanbin ni un kimhan, he takudan okihi śni.

18 Unkan heon wicotakuye tokaheya qon he we codan yuecepidan śni.

19 Woope kin eciyatanhan, Moses woahope kin owasin oyate kin owicakiyaka yaśtan qehan, ptejicadan, qa tatokadan we kin icu, qa mini, talinca hin duta, peji hota ko, qa wowapi kin oyate kin owasin ko, awicamnimni ;

20 Qa heya, Wicotakuye Wakantanka econ niśipi kin we kin he dee ce.

21 Qa nakun wakeya lin, takin wakśica wowinyunyanpi kte cin owasin ko we amnimni.

22 Woope kin eciyatanhan taku owașin se we on pakintapi; qa we papsonpi śni ehantanhan wokajuja wanica.

23 Heon etanhan, hena taku kin on, taku mahpiya ekta un iyacinpi kin pakintapi kta e hecetu; tuka taku mahpiya ekta un kin hena e qe taku sanpa waśte wośnapi kin on.

24 Messiya tipi wakan nape on kagapi kin he mahen i śni, tipi wakan hinca iyacinpi kin hena ee ; tuka mahpiya kin e qe mahen, qa heciya unkiyepi on Wakantanka itokam ihdutanin kta.

25 Qa wawayuśna itancan kin he owanka wakan lin en, taku 349 


\section{HEBREW.}

tokeca we kin yuha, waniyetu otoiyohi wancadan mahen i ece kin he iyecen ota akihde waihduśna kta iyecece śni ;

$26 \mathrm{He}$ iyececa unkanś, maka kagapi kin ehantanhan, wanna ota akihde kakije kta tuka; tuka nakaha woicage ihanke kin en, iye waihduśna kin eciyatanhan, woahtani yutokan iyeye kta e on taniniçiya.

27 Unkan wicaśta kin wancadan țapi kte cin he iwahowicayapi, qa he iyohakam woyaco kta :

$28 \mathrm{He}$ iyecen Messiya, wicota wahtanipi kin yuhe kta e wancadan wayuśnapi; unkan, tona he wanyakapi kta ape yukanpi kin hena niwicaye kta e, woahtani codan, inonpa ihdutanin kta.

\section{WICOWOYAKE 10.}

1 Taku waśte u kte cin ohanzi kin he woope kin en un, qa hena atayedan taku kin ouncage kin hee śni ; heon etanhan, waniyetu otoiyohi wośnapi ohinniyan aipi kin he tona en hipi kin wicayuecedan kta okihi śni.

2 He olkihi unkanś hena taku kin wayuśnapi ayuśtanpi kte śni he. Tona en opapi wancadan pakintapi kinhan icimana woahtani sdonkiyapi kte śni tuka:

3 Tuka hena on waniyetu otoiyohi woahtani kiksuyapi ece.

4 Tatanka tatokadan kici hena we kin woahtani yutokan iyeye kta okihipica śni.

5 Heon etanhan, makata hi qehan, Wośnapi, taku wicaqupi ko yacin śni, qa tancan wan miyecaga ce, eya.

6 Peta wośnapi, qa woalitani on wośnapi kin hena iyonicipi s̉ni ;

7 Hehan, Ito wau, wowapi 350 tanka en miye on hecen owapi ce ; Wakantanka, nitawacin kin ecen ecamon kta ce, epa.

8 Wankan, Wośnapi, taku wicaqupi, peta wośnapi, qa woahitani on wośnapi kin; woope eciyatanhan ahi wayuśnapi kin ; hena yacin śni, qa iyonicipi śni, eye cehan ;

9 Hंehan nakun, Wakantanka, nitawacin kin ecen ecamon kta wau ce, eye cin. Tokaheya kin he yutokan iyeya, icinonpa kin he hdusuta kte cin heon.

$10 \mathrm{He}$ iye tawacin kin ee on unyuecepidan, Jesus Messiya tancan wancadan wayuśnapi kin he eciyatanhan.

11 Wawayuśna otoiyohi anpetu iyohi, en ohoda inajin, qa ohinniyan taku wośnapi ece kin hena ake ecekcen wayuśna, hena woahtani kajuju kta tokadan okihi śni.

12 'Tuka wicaśta kin de, woahitani on wośna wanjidan wahduśna, hehan Wakantanka etapa kin eciyatanhan owihanke wanin kiyotanka ;

13 Qa hetanhan tona toka yapi kin hena owasin iye siha kin ihukun iyewicayapi lita ape yanka.

14 Wośnapi wanjidan on etanhan tona wicayuecepidan kin hena owihanke wanin wicahduśtan.

15 Woniya Wakan kin he nakun unkiciyaotaninpi; tokaheya eye ciqon he iyohakam;

16 Anpetu kin hena iyohakam, wicotakuye wicawecage kte cin he dee kta, Itancan kin eya; Mitoope kin hena iye cantepi kin en wicewaqu kta, qa tawacinpi kin en owakiwa kta ;

$17 \mathrm{Qa}$ iye wahtanipi, taku ecinśniyan econpi ko, ícimana weksuye kte śni. 
18 Hena owasin kajujupi kinhan, woahtani on wośnapi kin wanice kta.

19 Heon, mihunkawanji, Jesus we kin eciyatanhan tipi iyotan wakan kin en waditagya unyanpi kte ;

20 Canku wan teca qa.ni un, ti cokaya hnakapi, iye cehpi kin hee, ohna unkicagapi kin he eciyatanhan.

$21 \mathrm{Qa}$ Wakantanka ti kin ohna Wawayuśna Itancan wan unhapi ;

22 Heon cante wicake hinca hduha śogya wacinunyanpi qa ikiyedan unkupi kta, wicanagi śice cin etanhan uncantepi kin unkamnimnipi ;

23 Qa mini ska kin on untancanpi kin unyujajapi ; qa wacinunyanpi unhdaotaninpi kin he yuhohopi śni unhduhapi kta; tuwe wahounyanpi kin he wacinyepica :

$24 \mathrm{Qa}$ otoiyohi awaunkicicinpi kte, wacantkiyapi, qa wicohan waśte en iyounkicipaśtakapi kte cin heon.

25 Qa wanjikji econpi kin iyecen mniunkiciyapi ece kin he unkayuśtanpi kte śni ; tuka wahokonunkiciciyapi kta; qa anpetu kin he kiyadan $u$ wandakapi kinhan, sanpa nina ecanonpi kta.

26 Wowicalke sdonyapi kin unkicupi kin he iyohaham iyokipiya waunhtanipi kinhan, woahtani on wośnapi kte cin wanica.

27 Tuka woyaco inihanyan apepi kin he, qa wocanniye ide kin, tona tokayapi kin hena ihangwicaye kta.

28 Tuwe Moses toope kin akta śni, nonpa qa iś yamni yaotaninpi kinhan, he onśidapi śni ța ece ;
29 Hecen tuwe Wakantanka Cinhintku we kin amani, qa wicotakuye we kin on yuecepidan qon he wakan śni dake ça, Wowaśte Woniya kin he taku i en hiyeye cinhan, he wokakije iyotan tanka hiyahde kte śni lrecannipi he.

30 Tokiconpi kin he mitawa, towicawecicon kta ce, Itancan kin eya ce, tuwe eye ciqon be sdonunyanpi. Qa ake, Itancan taoyate kin wicayaco kta ce.

31 Wakantanka ni un nape kin en ohinhpayapi kin he wokokipe.

32 Anpetu tokaheya kin kiksuya po, hena en iyoyamniyanpi, qa hehan wicokakije okicize tanka wan iyahdeniciyapi.

$33 \mathrm{He}$ icunhan waaiapi, wicokakije ko on iwanniyakapi kta enihdepi ; qa nakun tona hecawicakiconpi kin hena tawaśi wicayayapi.

34 Miye makaśkapi kin icunhan onśimayakidapi, qa woyuha duhapi qon nicipi kin he on widuślkinpi, mahpiya ekta woyuha iyotan waśte ohinniyan ye kte cin he duhapi sdonyakiyapi kin heon etanhan.

35 Wadinitakapi qon he ayuśtanpi śni po, he woyuha tanka okihi kta.

36 Wacinnitankapi kta iyececa; hecen Wakantanka tawacin kin ecen ecanonpi kinhan, taku iwahounyanpi kin he iyacupi kta.

37 Wanna aślkadan tuwe u kte cin he hi kta, qa yutehan kte śni.

38 Tuwe owotanna un kin he wacinyan un kin on ni kta. Tuka tuwe namni kinhan he minagi kin iyokipi kte śni ce.

39 Tuka tona wicotakuni śni ekta icicawin hdapi kin heunca351 


\section{HEBREW.}

pi śni ; tona wacanagi ni kta e on wicadapi kin hena unkiyepi.

\section{WICOWOYAKE 11.}

1. Wacinyanpi kin he taku apepi oahe kin ee, qa taku wanyakapi śni un yuotanin kin hee.

$2 \mathrm{He}$ on hunka wicayapi kin yuonihanyan ihdaotaninpi qon.

3 Wacinyanpi kin eciyatanhan, Wakantanka oie kin on woicage kin icahyapi kin he sdonunyanpi, taku wanyakapi un kin hena taku tanin kin etanhan kagapi śni.

4 Wacinyanpi kin eciyatanhan Abel Qain kape ca, wośnapi iyotan waśte wan Wakantanka wakiyuśna qon he on owotanna e hduotanin; Wakantanlia nakun hecen yaotanin, taku qu kin heon, qa he eciyatanhan ța keś nahahin ia ece.

5 Wacinyanpi kin eciyatanhan Henok wiconțe wanyake kte śni e tokan ayapi, qa tuktedan iyeyapi śni, Wakantanka tokan aye cin heon. Tokan ayapi śni itokam Wakantanka iyokipiye cin he okiyakapi.

6 Tuka wacinyanpi codan tuwedan iyokipiye kta okihi śni. Tuwe en $u$ kinhan he Wakantanka ni un, qa tona aicieiya akitapi kin hena iyunwin wicaqu ece kin he wicada kta.

ry Wacinyanpi kin eciyatanhan Nowa Wakantanka ihamna, taku nahahin wanyalkapi śni kin hena, qa itonkipa un kin heon tiyohnaka tawa ni kte cin on wata tanka wan kaga; qa iye olian kin on oyate kin wicayaco, qa hecen woowotanna wacinyanpi eciyatanhan un kin he tawa kta e icaga.

8 Wacinyanpi kin eciyatanhan Abraham, makoce wan yuhe kte 352 cin he ekta ye sipi qehan, anagoptan, qa tokiya ye kte cin tanyan sdonkiye śni iyaya.

9 Wacinyanpi kin eciyatanhan makoce iwahoyapi qon he en ounyan, makoce tawa śni kin en iyeeeca, Isaak qa Jakob taku iwahoyapi qon om tawaye kte cin hena om wakeya ohna ti ece.

10 Otonwe wan taku ahde kin ahe cin he ape yanka, ticage ca icahye cin he Wakantanka kin ee.

11 Wacinyanpi kin eciyatanhan, Sara iye qe cinca yuhe kta e wowaśake icu, qa wanna waniyetu ece kin sanpa hiyu tuka cinca ton, tuwe iwahoye ciqon he wicaka e awacin yanke cin heon.

12 Heon wanjidan etanhan, qa he ta nun seca, tuka he etanhan wicota icaga, mahpiya wicanhi kin iyenaka; qa miniwanca kahda wiyaka sukaza kin iyecen yawapica śni.

13 Dena owasin wacinyan tapi, taku iwahowicayapi qon nahahin icupi śni, tulka tehan tanhan wanyakapi, qa wicadapi, qa adoksohan icupi, qa maka akan oyate tokeca qa unhdaka unpi kin heea ihdaotaninpi.

14 Tona taku hececa eyapi kin hena malioce wanji akitapi e taninyan ohdakapi.

15 Unkan awicakehan makoce lsin etanhan hiyupi qon he wacin en yuzapi unkanś, heciya ake elsta hdapi kta okihipi tuka.

16 Tuka nakaha wanji iyotan waśte mahipiya ekta wanke cin hee akitapi. Heon etanhan $\mathrm{Wa}-$ kantanka hena Wakantanka tawapi kin eciyapi kta iśtece śni, qa otonwe wăn wiyeya ewicakicihnalka.

17 Wacinyanpi kin eciyatan- 
han, Abraham, iyutanyanpi qehan, Isaak wahduśna, qa tuwe taku iwahoyapi qon wicada kin he cinhintku ecedan kin wahduśna.

18 Isaak etanhan wicoicage nitawa kin cajeyatapi kta ce, eciyapi tuka qon.

19 Wiconte ee kaeś etanhan Wakantanka ekicetuya okihi he wicada; qa hetanhan nakun wiyacinpi on ehdaku.

20 Wacinyanpi kin eciyatanhan Isaak, Jakob qa Esa wicahdawaśte, taku u kte cin heon.

21 Wacinyanpi kin eciyatanhan Jakob, wanna țe kta hehan, Joseph cinhintku napin wicahdawaśte, qa cansagye oinkpa kin akan wocekiye eya.

22 Wacinyanpi kin eciyatanhan Joseph, wanna te kta hehan, Israel cincapi kin hetanhan hdapi kte cin he caje yate ça, iye huhu kin aye wicaśi.

23 Wacinyanpi kin eciyatanhan, Moses tonpi kin, hehan hunkakepi kin wi yamni hehanyan anakilimanpi, hokśidan waśte e wanhdakapi, qa wicaśtayatapi toope kin kokipapi śni.

24 Wacinyanpi kin eciyatanhan Moses wanna tanka jcage cehan, Pharo cunwintku cinca eciyapi kta wicada śni ;

25 Wakantanka taoyate kin om kakija un kte cin he hdaliniga, qa woalitani woiyokipi kin ho ptenyedan yuhe kta e cin śni ;

26 Messiya on aiapi kin he, woyuha Egupta en un kin sam iyeya wowijice tanka daka; wokajuju iyunwin kin he awacin kin heon etanhan.

27 Wacinyanpi kin eciyatanhan Egupta makoce kin ayuśtan, qa wicaśtayatapi tocanniye kin kokipe śni. Tuwe wanyag- pica śni kin he wanyaka se ape yanka.

28 Wacinyanpi kin eciyatanhan wośnapi wohduze, qa we amnimnipi kin hena kaga, tuwe cinca tokapapi kin ihangwicaye cin he iye owicayutan kte śni heon.

29 Wacinyanpi kin on Mde-śa kin he opta iyayapi, puze cin iyecen. Unkan Egupta oyate kin hecon utapi qehan, minin tapi.

30 Wacinyanpi kin eciyatanhan Jeriko conkaśke kin makata ihpaya, anpetu śakowin ohomni unpi qehan.

31 Wacinyanpi kin on, Rahab witkowinna kin, tona anagoptanpi śni kin om ihangyapi śni, makoce iwanyag hipi kin hena wookiye on iyowinwicakiye cehan. 32 Qa taku sanpa epe kta he; Gideon, qa Barak, qa Samson, qa Jeptha, qa Dawid, qa Samuel, qa wicaśta wokcanpi kin owasin owicamdake kta anpetu kin. iyehankeca śni.

33 Hena wacinyanpi kin eciyatanhan wokiconze kin ohiyapi, wicohan owotanna ohanyanpi, wahoyapi kin icupi, mnaja kin iohmusyapi ;

34. Peta wohitike cin kasnipi, maza sagye ope kin nakicipapi, wowaśake śni etanhan waśagic̣iyapi, okicize en waditaka icicagapi, qa Ikcewicaśta ozuye kin napewicayapi.

35 Winohinca kin cinca kitapi kin ekicetuyapi ewicahdakupi. Qa wanjikji nina kakiśyapi, tuka etanhan ehdakupi kta cinpi śni, woekicetu iyotan waśte kin yuhapi kte cin heon etanhan.

$36 \mathrm{Qa}$ wanjikji waaiapi, qa nina wicakaśtakapi kin on iyutanpi, qa nakun maza icicahiha, kaśka wicahnakapi ko on. 


\section{HEBREW.}

37 Inyan on wicalininpi, canibakse on bawicaksapi, iyutanwicayapi, mazasagye on wicaktepi ; tahinca ha qa tatokadan ha koyag manin unpi; taku owasin icalijapi, kakijapi un qa śicaya wicakuwapi.

38 Hena maka kin de iyewicakicihantu śni ; hewoskan manin unpi, he kin akan, taku itu unpi otipi, qa makolidoka kin ohna.

39 Hena owasin wacinyanpi kin eciyatanhan tanyan ihdutaninpi, qa taku wahoyapi kin ocowasin icupi śni.

40 Wakantanka taku sam iyeya waśte unkiyepi on elınaka, hecen hena e unkicunonpa tanhan wicahduśtanpi kte śni.

\section{WICOWOYAKE 12.}

1 Heon etanhan waayataninpi mahipiya śoka wan hinsko tanka aohdute unyanpi kin, hecen taku tkeya qinpi kin owasin eunhnakapi kta, woahtani ohiunyanpi ece kin he koya, qa oinyanke unkitokam wanke cin he wacin tankaya unkinyankapi kta.

2 Qa Jesus wacinunyanpi kin toka kage ca hduśtan kin hee ekta euntonwanpi kta. He iye, wowiyuśkin ìtokam ehdepi qon he on, can susbeca kin hee kaeś iyowinkiye ca, wowiśtece kin he taku da śni, qa Wakantanka oiyotanke etapa kin eciyatanhan kiyotanka.

3 Tuwe wahtanipi sa taku i en hiyeyapi kin he iye en wahbayedan iyowinye ciqon he awacin po, hecen nitawacinpi kin ekta iconimnipi kte śni, qa wacin iboyaśakapi kte śni.

4 Woahtani kici yecizapi kin en nahahin we kin elkta aiyahdeya ecanonpi śni.
5 Qa wowahokonkiye cinca iyecen oniciyapi kin he ayektonjapi he; Micinkśi, Itancan wicakapsinpsinte cin he śice dake śni wo, qa iyopeniye cinhan wacin ibośake śni wo, eye cin.

6 Itancan kin wicaśta waśtekida kinhan, he iyopekiye ça, cinca iyowinwicakiye cin otoiyohi hdapsinpsinta ece.

7 Wiyopeniyanpi kinlıan, cinca kin iyecen Wakantanka ecaniconpi; tukte cinca kin he atkuku iyopekiye śni he.

8 Tuka owasin en opapi ece kin iyecen iyopeniyanpi śni kinhan, tuwe cinca tanin śni kin lienicapi, qa cinlintkuyapi un kin henicapi śni.

9 Unkan, wicacelipi eciyatanhan atewicunyanpi kin hena iyopeunkiyapi ece, unkan wicunhduonihanpi hecinhan, sam iyeya wicanagi Ateyapi kin he anaungoptanpi kte ça on unnipi kta. 10 Hena e anpetu tonana hehanyan, token iyokipipi kin hecen iyopeunkiyapi; tulka he qe unwaśtepi kte cin he on, hecen iye towakan kin en ounpapi kta. 11 Wiyopewicayapi kin owasin en icunhan woiyokipi śni, tuka woiyokiśice; tuka ihakam tona hecawicakiconpi kin en wicoowotanna waskuyeca wookiye kin icahwicakiya ece.

12 Heon etanhan wicanape kaozezeya un kin hena yuwankan icu po, qa hupahu waśake śni kin hena koya;

13 Qa nisihapi canku mdaya kicaga po, taku huśte kin he icunonpa iyeyapi kte śni, tuka okiziyapi kte cin heon.

14 Owasin en wookiye akita po, qa nakun wowakan kin, he tuwe yuhe śni kinhan Itancan kin wanyake kte śni.

$15 \mathrm{Qa}$ tuwe Wakantanka to- 
waonśida kin iyohi kte śni naceca, heon aiciciya iwanihdaka po, okinni can hute paza icage ça, nagiyeniyanpi kta, qa hecen wicota aśamyapi kta.

16 Okinni wicawihomni wan yuke kta, qa taku wakan ohoda śni, Esa iyececa, he watokapa kin taku yutapi onśpadan iyopekiya.

17 Qa iyohakam woyawaśte kin hduhe kta, tuka heyata iyeyapi, he sdonyayapi, qa token tawacin yutokeca kte cin he iyeye śni, iśtarnnihañpe on nina akita tuka.

18 Paha oyutanpica, qa peta on idc qon he ekta yahipi śni, qa taku iyotan samya wanke cin, qa otpaza, qa nina icamna kin;

19 Qa nakun mazayahotonpi tanka hoton, wicoie ho kin hena koya; he tona nahonpi qon wicoie kin he akta owicakiyakapi kte śni e icekiyapi.

20 Taku econ wicaśipi quon he okitpanipi; Woteca wan hee kaeś paha kin yutan kinhan he inyan on kininpi kta, qa iś wanhinkpe on katpa iyeyapi kta ce.

21 Unkan he wanyakapi kin nina wowinihan, qa heon Moses, Nina maliniyanyan qa macancan ce, eya.

22 Tuka Zion Paha kin ekta yahipi, Wakantanka ni un otonwe tawa kin, Jerusalem mahpiya tanhan $u$ kin, qa ohnihde unpi kin kektopawinge ota ekta;

23 Omniciye tanka, qa tokaheya wicatonpi okodakiciye kin, mahipiya ekta caje owicawapi kin hena, qa Wakantanka owasin wicayaco kin he, qa wicaśta owotanna yuśtanpi nagipi kin hena ekta;
$24 \mathrm{Qa}$ Jesus wicotakuye teca kin en iyotahedan un kin hec ekta, qa we amnimnipi kte cin, Abel sam iycya taku waśte eye cin he ekta.

25 Ihnuhan tuwe ie cin anayagoptanpi śni kinhan; tona tuwe maka akan ic ciqon he anagoptanpi śni qon hena najicapi śni hecinhan, sam iyeya unkiye naunjicapi kte śni, tuwe mahpiya tanhan ie cin ihaunktapi śni kinhan.

26 Iye ho kin he chan maka kin yahuhuza, tuka nakaha wahounyanpi qa heya, Ake wancadan maka ecedan mdahuhuza kte śni, tuka nakun malipiya kin.

27 Unkan, Ake wancadan, eye cin he taku tona yahuhuzapi kte cin hena taku kagapi kin iyecen yutokan iycyapi lkta, hecen taku tona yahuhuzapica śni kin hena eccn he kta, he kapi.

28 Heon etanhan wokiconze yuhohopica śni kin unkicupi, wowaonśida unhapi kta, hecen woyuonihan, wokokipe ko yuha Wakantanka iyokipiya ohoundapi kta.

29 Wakantanka unkitawapi kin he peta wahulinahye cin heca.

\section{WICOWOYAKE 13.}

1 Hunkawanjinkiciyapi waśtekicidapi kin he ecen un nunwe.

2 Oicimani kin wonwicaya akiktonjapi śni po; heconpi kin on wanjikśi malipiya ohnihde kin sdonwicaye śni won wicayapi.

3 Tona kaśka wicahnakapi kin hena om nicaśkapi kin iyecen wicakiksuya po; qa tona kakiśyapi un kin hena koya, wicatancan kin ohna dukanpi kin heon. 


\section{HEBREW,}

4 Owasin en wakan kiciyuzapi kin he woyuonihan, qa ohehdepi aśamyapi śni kin; tuka wicawihomnipi qa wawicihahapi econpi kin hena Wakantanka wicayaco kta.

5 Waicucupikte cin wanin oyaliandapi kta, qa taku duhapi kin hena on iyonicipipi nunwe. Tohinni aciyuśtan kte śni qa icimana ehpeciye kte śni, eye ciqon heon etanhan.

6 Heon, Itancan kin omakiye cin hee, qa he etanhan taku wicaśta akantu ecamiconpi kte cin kowakipe lte śni ce, waditagya unkeyapi kta.

7 Tona awannihdakapi, Wakantanka oie kin oniciyakapi kin hena wicakiksuya po, hena wicohan ihanke kin he awacin po, qa wacinyanpi qon he en opa po :

8 Jesus Messiya, litanihan qa nakaha okonwanjidan, qa owihanke wanice kta.

9 Ihnuhan woonspe otakiya qa toktokeca on yutokan iyeniyanpi kinhan; woyute tona he awacinpi qon takudan okihiwicaye śni kin he wicacante kin yusuta śni, tuka wowaonśida kin on yusutapi kin he waśte.

10 Wahna wośnapi wan unhapi kin he etanhan tona wakeya qon ohna ohanyanpi kin hena yutapi kta iyececapi śni.

11 Woteca, tona we kin woahtani on, wawayuśna itancan kin tipi wakan kin timahen ai qon, hena tancanpi kin tihocoka kin itankan hulinaliyapi ece.

12 Heon Jesus iś eya, iye we kin on oyate kin wicayuecedan kta e otonwe tiyopa kin itankan kakiśyapi qon.

13 Hecen iye on aiapi qon aihpeunyanpi kin yuha ekta wicoti kin tankan unyanpi kta.
14 Degrya otonwe ohinniyan eren he cirs unhapi śni, tuka wanji u ate cin he unkakitapi.

15 Heon etanhan, iye caje on, ohinniyan Wakantanka en wowitan wośnapi Irin unkaupi kta, mkihapi etanhan waskuyeca, iye caje kin on, wounhdakapi kin hee.

16 Taku waśte econpi, qa wacantkiyapi kin hena akiktonjapi šni po, hena wośnapi kin Wakantanka iyokipi ece.

1 17 Tona wahoronniciyapi kin hena anawicakigoptan po, qa wicihukuya yeiciya po, hena ninagipi kin on awanniyakapi, ondaliapi kte cin he iyecen; hecen wowiyuśkinyan econpi $\mathrm{kta}$, qa woiyokiśnya econpi kte śni; he niyepi kin on waśte.

18 Worekiye mkekiciya po; wicanagi waśte anhapi unkecinpi, talku owasin en owotanna unkohanyanpi lita uncinpi.

19 Trka hecrnonpo kta e iyotan ceciciyapi, Kohanna niyepi ekta u mayanpi kte cin heon.

20 Unkan, Wookiye Wakantanka kin, Jesus Itancan unkiyapi, tahinca awanwicahdake tanka kin he, wiconte etanhan ake hiyuye ciqon he wicotakuye owihanke wanica we kin eciyatanhan;

21 Iye tawacin kin ecen ecanonpi kta e, wicohian waśte owasin en niyusutapi kte, qa taku iye iśta kin en oiyokipi kin ecaniconpi kta, Jesus Messiya eciyatanhan; he wowitan owihanke wanica kicihan nunwe.

22 Mihunkawanji, ceciciyapi ce, wowahokonkiye wicoie kin iyowinkiya po, wicoie tonana on wowapi cicagapi.

23 Timotheos hunkawanjin unyanpi kin wanna kiyuśkapi e 
WICOWOYAKE 1.

sdonya po, he ecadan u kinhan kici wanciyakapi kta.

24. Tona niyepi en itancanpi kin hena owasin, qa tona owotanna un kin owasin nape wica- men.

\section{J A K OB}

\section{TAWOWAPI KIN .}

\section{WICOWOYAKE 1.}

1 Jakob, Wakantanka taokiye, qa Jesus Messiya Itancan tawa kin he, wicowazi akenonpapi enanakiya eyaye cin hena wowapi wicakicaga; wookiye.

2 Mihunkawanji, taku wawiyutanyan un kin ota ayakipapi kinhan, he wowiy uśkin ocowasin hdawa po:

3 Wacinyayapi iyutanpi kin he wacintankapi kaga e sdonyayapi.

4 Tuka wacintankapi kin he tohan kin tanyan hduśtan kte; hecen niyuśtanpi qa ocowasin yaunpi kta, qa takudan iyonitpanipi kte śni.

5 Niyepi etanhan tuwe woksape nice cinhan, Wakantanka kida kta, iye owasin iyakicuya wicaqu ece, qa iyopewicaye śni ; unkan qupi kta.

6 Tuka takudạn cețunhda śni wacinyan kida kta ; tuwe wacetunhda kin he miniwanca taja kin iyececa, tateyanpa kaśkanślkan qa kahoya elipeye cin.

7 Wicaśta kin he Itancan kin etanhan taku icu kta kecin kta iyecece śni.

8 Wicaśta tawacin nonpa kin he tacanku owasin ohna kaktihanhan un ece.

9 Hunkawanjinkiciyapi wahpa- yuza po. Italia etanhanpi kin hena nape niyuzapi.

25 Wowaonśida lzin niyepi owasin en nicipi un nunwe. A- 
han un, qa iyoyanpa Ateyapi kin eciyatanhan kun u ece; iye qe tokeca śni, qa yuhomnipica śni hinca.

18 Iye tawacin kin eciyatanhan, wowicake oie kin on cinca unkagapi, hecen taku kage cin ehna unkiye waskuyeca tokaheya iyeuncecapi kta.

19 Heon etanlian, mihunkawanji waśte, wicaśta otoiyohi wanahon ohanko kta, ie kapin kta, qa wacintanka lite:

20 Wicaśta śinhdapi kin he Wakantanka toowotanna kin icahye śni.

21 Heon etanhan, wicowahteśni kin owasin, qa taku śica iyatahdepi kin elipeya po; qa wicoie ninagipi niye kta okihi kin he onśiciciya icu po.

22 Tuka wicoie kin ecen econ po, qa iye içihnayanpi, nahonpi ecedan, unpi śni po.

23 Tuwe wicoie nahon eśta ecen econ śni kinhan, he wicaśta wan ihdiyomdasin on ite iwanihdake cin iyececa;

24. Iwanihdake ca tankan iyaya, unkan hehan hinnakaha ite tokeca kin he akiktonja ece.

25 Tuka tuwe wicoope on tawaiçiyapi yuśtanpi kin he opahta yanke ca, en un kinhan, tuwe nahon qa akiktonje cin heca śni, tuka wicohan econ kin heca, hee e taku econ kin en yawastepi kta.

26 Tuwe niyepi kin etanhan woohodapi kin en opa se ececa hecinhan, qa ceji hdaśke śni, tuka iye cante içihnaye cinhan, he woohodapi ope cin he taku śni.

27 Wakantanka qa Ateyapi kin itokam woohodapi opapi ecedan qa aśape śni kin he dee; Wamdenica qa wiwazica kakijapi eca en wicahipi, qa wicoi- cage kin en taku iyaonpepica śni ildduhapi kin hee.

\section{WICOWOYAKE 2.}

1 Mihunkawanji, Jesus Messiya, wowitan Itancan wacinyanpi kin, on wicaśta iwandakapi kte cin he yuhapi śni po.

2 Nitomniciyepi kin en, wicaśta wan mazaskazi napcupe yuhe ca wokoyake waśte kiçun kin en hi kinhan; qa nakun wicaśta wahpanica wan heyake śica un kin en hi kinhan;

3 Qa tuwe wolroyake waśte kicun kin he tanyan ayatonwanpi, qa heyakiyapi, Oiyotanke waśte kin de en hiyotanka wo; qa wicaśta wahpanica qon, Hen najin wo, qa iś, siha wahde kin ihukuya hiyotanka wo, eyakiyapi kinhan ;

4 Otoiyohi tanyan canteyeciyuzapi śni, qa wicotawacin śica eciyatanhan widukcanpi śni he.

5 Nahon po, mihunkaw anji waśte; Wakantanka maka akan wahpanicapi kin hena wicakaliniga he, wacinyanpi on wijicapi, qa tona iye waśtedakapi wokiconze iwahowicaye ciqon he tawayapi kte cin.

6 Tuka niye wahpanicapi kin wahtewicayadapi śni. Wijicapi kin hena iyotanhan iyeniciyapi śni, qa woyaco oiyotanke kin en aniyanpi śni he.

7 Wicacaje waśte on cajeniyatapi ece kin he yaśicapi śni he.

8 Wowapi kin eciyatanhan, woope iyotan waśte, Nikiyedan un kin niye inihdacin waśteyadake kta ce, eyapi kin he ecen ecanonpi kinhan, tanyan ecanonpi kta.

9 Tuka wicaśta token ihduze cin ekta eyatonwanpi kinhan, woahtani ecanonpi, qa woope 
kin on śicaya econ oniyakapi ece.

10 Tuwe woahope owasin yuha eśta, wanjidan kicakse cinhan, he ocowasin on yacopi.

11 'Tuwe, Wawicihahapi ecanon kte śni ce, eye ciqon he iś nakun, Wamayanon kte śni ce, eya. Unkan wawicihahapi takudan ecanon śni eśta, wamayanon kinhan, wanna woope kin kiyecaksa.

12 Tona woope on tawaiçiyapi kin on wicayacopi kte cin hena iyecen iapi, qa econ po.

13 Tuwe waonśida śni kin he wowaonśida wanin woyaco ecedan qupi lita; qa wowaonśida woyaco kin kapeya iyuśkin ece.

14 Mihunkawanji, tuwe wacinyan keya eśta, iyecen wicohan yuhe śni kinhan, he taku iyokihi kta he. Wacinyan un kin he niyan okihi he.

15 Nihunkawanji qa nitawinohtin tancokapidan, qa anpetu iyohi woyute nicapi kinhan;

16 Qa wanji hewicayakiyapi, Wookiye on hdapo, cosiciyapi qa wipiiciya po; tuka taku wicatancan icakije cin hena wicayaqupi śni ehantanhan, taku iwaśtepi kta he.

17 He iyecen wacinyanpi kin, wicohan codan kinhan, he wanna ța, iśnana un kin heon.

18 Han, tuwe heye kta; Niye wacinyan yaun, qa miye wicohan mduha; hecen wacinyaye cin he niolian codan makipazo wo, kinhan miohan kin on wacinwaye cin he cicipazo kta ce.

19 Taku Wakan kin wanjidan he wicayada; he tanyan ecanon; taku wakan śicapi kin iś eya wicadapi qa cancanpi ece.

20 Tuka, wicaśta wacinton śni kin, wacinyanpi wicohan codan kin he ta e sdonyaye kta he.
21 Abraham ateunyanpi kin he, cinhintku Isaak wahna wośnapi akan wahduśna qon he ehan, wicohan on yaowotanpi śni he.

22 Qa iye ohan kin kici wacinye cin token ohanye ca, wicohan on wacinye ciqon he yuśtanpi e wandaka he.

23 Unkan hecen wowapi kin, Abraham Wakantanka wicada, unkan he woowotanna kiciyawapi, qa he Wakantanka takicuwa eciyapi ce, eyapi qon he yuecetupi.

24 Heon, wacinyanpi ecedan on etanhan śni, tulra wicohan kin on wicaśta owotanna yawapi ece he wandakapi.

25 Nakun Rahab witkowinna kin ohnihde unpi qon hena iyowinwicakiye ca canku tokeca ohna hde wicaśi qehan, iś eya wicohan on owotanna yawapi śni he.

26 Wicatancan kin woniya codan un kin he ta ece, he iyecen wacinyanpi kin wicohan codan kinhan, he nakun ta ece.

\section{WICOWOYAKE 3.}

1 Mihunkawanji, wicota waonspekiya unpi śni po; woyaco iyotan tanka unkicupi kta e sdonyayapi.

2 Owasin taku ota on waunhtanipi ece.' Tuwe oie on wahtani śni kinhan, he wicaśta yuśtanpi qa tancan ocowasin hdaśke kta okihi.

3 Wanyalka po, suktanka anaungoptanpi kta e heon iikiyuwi i kin en iyewicunkiciyapi; qa tancanpi ocowasin wicunyuhomnipi ece.

4. Wita wata kin hena nakun wanyaka po, tankinkinyan qa tate ohitika on kaha iyeyapi eśta, iyupse yuza cistinna on 
token itancan kin cin eca, yuhomnipi ece.

$5 \mathrm{He}$ iyecen wicaceji kin taku cistinna, tuka tanka icida ece. Iho, peta cistinna keś can tanka ideya ece.

6 Wicaceji kin he peta, qa woahtani iyatahdepi hecen wicaceji unhuhapi kin ehna un, wicatancan kin ocowasin aśamya, qa wicoicage ohomniyan aideye ca, iś eya wakanśica ti kin etanhan ideiciya.

7 Woteca ocaje owasin, qa taku kinyan unpi, taku sdohan unpi qa miniwanca mahen unpi kin, henakiya etanhan wicaśta wanunyanpi wicakage ca wicayuha:

8 Tuka wicaceji kin he tuwedan yuwalibaka okihi śni; he wośice wicaśta śni; pejihuta śica on wicața ece kin he ojudan.

9 He on Wakantanka Ateyapi kin unyawaśtepi; qa he on wicaśta akantu, Wakantanka ouncage iyecen kagapi kin hena wicunyaśicapi ece.

10 Wicai wanjidan etanhan wayawaśtepi qa wayaśicapi kin hinanpa ece. Mihunkawanji, hena hecetu śni.

11 Miniyowe wan oenape wanjidan etanhan, mini waśte qa nakun mini pa hinca hiyuya he.

12 Mihunkawanji, suken can wan Oliwe waskuyeca aicaga, qa iś hastanyanka iyuwi wan suken waskuyeca okihi he; he iyecen miniyowe wan mini waśte qa nakun mini. pa linca hiyuye śni ece.

13 Enitanhanpi tuwe wicaśta ksape ca wiyukcan un he; woksape wowalibadan on tanyan aihduhe cin eciyatanhan iye ohan waśte hdutanin kte.

14 Tuka winawizipi pa linca, qa wakinicapi nicantepi kin en duhapi kinhan, ihnuhan widuśkinpi qa wowicake kin on iyatonpi śni kin.

15 Wicolsape kin he wankantanhan u śni; tuka maka kin etanhan; wicacelipi etanhan, qa wakanśica iyececa.

16 Tukten winawizipi qa wakinicapi yuke cinhan, wicotoketu tanin śni qa wicolian śica owasin hen un ece.

17 Tuka woksape wankantanhan un kin he tokaheya ecedan, hehan wokiyapi kaga, walibadan, waanagoptan, wowaonśida qa waskuyeca waśte on ojudan, owotanna wiyukcan qa owotanna wohdaka ece.

18 Unkan tona wookiye kagapi kin hena woowotanna waskuyeca kin wookiye en owicakicijupi ece.

\section{WICOWOYAKE 4.}

1 Niyepi ekta wicokicize qa wakinicapi kin tokiyatanhan u he; cantiheyayapi śica nihuhapi en wakis un kin hena eciyatanhan śni he.

2 Cantiheyayapi tuka duhapi śni; wicayaktepi qa yuhe wacannipi, tuka icu oyakihipi śni ; wicayakizapi qa zuya dapi, tuka taku duhapi śni, yadapi śni kin heon etanhan.

3 Yadapi tuka iyacupi śni ece, ecinśniyan yadapi kin on, cantiheyayapi śica on dusotapi kte cin heon.

4 Wiinayahmanpi qa wawicihahapi ecanonpi kin, wicoicage kin de waśtedapi kin lie Wakantanka śicedaka e sdonyayapi śni he. Heon tuwe wicoicage kin de wotakuye wacin kinhan, he Wakantanka tokaya.

5 Wowapi wakan kin, Woniya en unyakonpi kin he winawizipi 
cantiheya ce, ituya eya idukcanpi he.

6 Tuka sanpa wowaonśida wicaqu ece; heon heya, Wakantanka wahaniçidapi kin wicakipajin, tuka tona onśikitianpi kin hena wowaonśida wicåqu ece.

'7 Heon etanhan, Wakantanka ihukuya iyeiciya po. Wakanśica kipajin po, kinhan nanicipapi kta.

8 Wakantanka ikiyedan u po, hecen iś nikiyepidan u kta. Wahtanipi sa, nape hdujaja po; qa wacin ninonpapi kin, cante hduecepidan po.

9 Iyokiśicapi, waśihdapi qa ceya po; iyahapi kin he wicaceya ee kta, qa widuśkinpi kin he woiyokiśice kta.

10 Itancan kin itokam makata elipeiciya po, kinhan yuwankan inicupi kta.

11 Mihunkawanji, otoiyohi akiciiapi śni po. Tuwe hunkawanjitku aie ẹ hunkawanjitku hdaco kinhan, he woope kin aie ca woope kin yaco; tuka woope kin daco kinhan, woope kin ecen econpi kin henica śni, tuka wayaco kin henica.

12 Woope kage cin he Wanjidan, he wanikiye ca nakun waihangya okihi. Wicaśta tokeca daco kin niśs nituwe he.

13 Tho po, nakaha tona hehapi ; Dehan, qa iś heyakecinhan, otonwe kin kae elita unyanpi qa waniyetu wanji en unyakonpi kta, wopeuntonpi qa wiyopeunyanpi qa taku unkamnanpi kta ce.

14 Tuka heyakecinhan taku u kte cin sdonyayapi śni; yanipi kin he talku he, opo wan cistiyedan tanin qa hehan atanin śni kin he heca.

15 Kaken ehapi kta iyececa; Itancan kin iyowinunkiyapi qa unnipi kinhan, decen qa iś $\mathrm{ka}$ ken econkupi kta ce.

16 Tuka nakaha wahanniçidapi kin on iduśkinpi; hecen wiyuśkinpi kin owasin śica.

17 Heon etanhan, tuwe taku waśte econ kta sdonkiya tuka econ śni eca, he woalitani içicaga ece.

\section{WICOWOYAKE 5.}

1 Iho po, tona wijica yaunpi kin, wokakije nihiyahdepi kte cin hena on ceyapi qa śicahowaya po.

2 Woyuha nitawapi kin kuka, qa nitawokoyake kin wamduśkadan yapotapi.

3 Mazaskazi qa mazaska nitawapi kin gigi aya; qa gigi kin he niyutaninpi kta, qa nicehpipi kin ihangye kta, peta iyececa. Woyuha anpetu ehake kin amnaniciyapi.

4 Wanyaka po, tona maga nitawapi abaksapi wokajuju hnaye ayanicapi kin he howaya; qa tona aguyapi baniciksapi qon hoyeyapi kin he Sabaoth Itancan noge kin en hiyolipaya.

5 Maka akan woiyokipi imnahan yaunpi qa tanyan wayatapi ece; wapatapi anpetu lin en iyecen cante cemyakiyapi.

6 Wicaśta owotanna kin he dacopi qa yaktepi ; tuka nicipajinpi śni.

7 Heon etanhan, mihunkawanji, Itancan kin hi kte cin hehanyan wacintanka po. Wanyaka po, wicaśta woju ece kin he maka waskuyeca waśte aicage cin hena ape yanke ca, on tehan wacintanka, magaju tokaheya qa nakun ehake kin ahinhe kte cin hehanyan.

8 Niś eya wacintanka po; cante hdusuta po; Itancan kin u kte cin lıe wanna kiyedan ihan. 361 


\section{INYAN.}

9 Mihunkawanji, otoiyohi okicihinyanpi śni po, okinni niyacopi kta. Wanyalka po, Wayaco kin he tiyopa kin itokam inajin.

10 Mihunkawanji, wicaśta wokcan Itancan caje kin on iapi qon hena kakijapi qa wacintankapi on waonspekiya wicayuha po.

11 Wanyaka po, tona wacintankaya unpi kin hena waśte kecanwicunkinpi ece. Job token wacintanka qon he nayahonpi, qa Itancan owihankeye cin he wandakapi; Itancan kin waonśida qa wawacinkta yuza tanka.

12 Tuka mihunkawanji, taku owasin isanpa, Taku namahon ce, eyapi śni po, mahipiya kin he, qa maka kin de, qa taku tokeca cajeyatapi ece kin nakun; tuka, han, ehapi kin he han ecedan kta, qa, hiya, ehapi kin he hiya ecedan kta; okinni woyaco kin en iyoyahipadapi nun ce.

13 Niyepi kin etanhan wanji kakija he; wocekiye eye kta. Wanji wiyuśkin he; odowan ahiyaye kta.

14 Niyepi kin ekta wanji wayazanka he ; okodakiciye kin en hunkawicayapi kin wicakico
Kta; on cekiyapi qa Itancan caje kin on wihdi on sdayapi kta.

15 Unkan wacinyan cekiyapi kin he wayazanke cin asniye kta; qa Itancan kin he najinkiye kta; qa woahtani econ hecinhan, hena kicicajujupi kta.

16 Wayahtanipi kin otoiyohi okiciciyakapi qa otoiyohi wocekiye ekiciciya po, hecen anisnipi kta. Wicaśta owotanna nina waśagya cekiye cin he taku tanka okihi ece.

17 Elijah wicaśta wacinyuza iyeuncecapi, qa wocekiye on magaju kte śni e icekiya; unkan waniyetu yamni sanpa wi śakpe hehanyan maka kin ahinhe śni.

18 Hehan ake wocekiye eya, unkan mahpiya kin magaju hiyuye ça, maka kin waskuyeca icaliya.

19 Mihunkawanji, wanji enitanhanpi wowicake kin etanhan nuni, qa tuwe he ake ekta yuhomni kinhan,

20 Tuwe wicaśta wahtani sa wan canku onuni kin etanhan yuhomni kinhan, he wicanagi wan wiconte etanhan eyaku kta, qa woahtani ota akahpe kta e he sdonkiye kta.

\section{IN Y A N}

\section{TAWOWAPI TOKAHEYA KIN.}

\section{WICOWOYAKE 1.}

1 Inyan, Jesus Messiya yeśi qon, wicaśta toktokeca enanakiya unpi, Pontus, Galatia, Kappadokia, Asia qa Bithunia ekta, 2 Wakantanka A teyapi kin otokaheya tanhan sdonwicaye cin 362 eciyatanhan, qa Woniya wicayuecedan kta on wicakaliniga, anagoptanpi kta, qa Jesus we kin awicamnimni kta e on, hena wowapi wicakicaga: Wowaonśida en niunpi, qa wookiye niciyuotapi nunwe.

3 Wakantanka, Jesus Messiya 


\section{WICOWOYAKE 1.}

Itancan unyanpi Atkuku kin he yawaśtepi nunwe ; iye towaonśida ota eciyatanhan Jesus Messiya wicate cin etanhan kinikiye ciqon he on wowacinyan ni un kin en piya icahunyanpi ;

4 Woaihpeye wan hwinpica śni, aśapapi śni, qa ohinniyan un kta, mahpiya ekta enicihnakapi kin on;

5 Wacinyanpi eciyatanhan Wakantanka towaśake kin on wiconi anikpatanpi, eqe anpetu ehake kin en yuotaninpi kta.

6 Heon nina iduśkinpi ece, tuka nakaha ptenyedan, he hecetu naceca, taku wawiyutanyan un kin ota on iyoniciśicapi :

7 Hecen wacinyayapi iyutanpi kin, he mazaskazi peta ece on śdoyapi keś atakuni śni ece, sam iyeya nina tehike cin he woyaonihan,'wicakinihanpi qa wowitan on tanin kta, tohan Jesus Messiya ihdutanin kinhan.

8 He wandakapi śni eśta waśteyadakapi ; qa nakaha wanyake śni yaunpi keś wacinyayapi kin on iduśkinpi, wowiyuśkin oyagpica śni qa wowitan ojudan kin on :

9 Wacinyayapi ihanke kin iyacupi, ninagipi ni kte cin hee.

10 Wiconi kin he on, wicaśta wokcan wowaonśida en niunpi kin he oyakapi qehan, aic̣iciya akitapi qa wiwangapi;

11 Messiya Taniya iyepi en un kin; tohantu qa anpetu kin tukte e ka e iwangapi, Messiya kakije kte ca wowitan ihakam un kte cin hena itokam oyake ciqon he ehan.

12 Unkan, iś iyepi kte śni, tuka tona wotanin waśte, Woniya Wakan mahpiya eciyatanhan lzun u śipi kin iyahna sdonyeniyanpi kin eqe nakaha taku oniciyakapi kin hena unkiyepi on yuhapi e owicakiyakapi; taku kin hena mahpiya ohnihde kin en eyokasin wacinpi.

13 Heon etanhan, nitawacin nisehu kin opta ipiyagkiton po, waktaya un po, qa Jesus Messiya ihdutanin kinhan wowaśte nicaupi kte cin he ape yukan po, owihanke kin hehanyan.

14 Wicacinca waanagoptanpi kin iyenicecapi kta, qa ehanna wasdonye śni yaunpi qehan cantiheyayapi śica alke iyecen niçicagapi kte śni.

15 Tuka tuwe nicopi kin he wakan, he iyecen niś eya oyahandapi kin ocowasin en niwakanpi nunwe.

16 Heon hecen owapi qon, Wakanyan un po, miye mawakan kin heon etanhan.

17 Qa Ateyapi kin wicaśta itohnake iwanyake śni wicaśta otoiyohi.iye ohan kin eciyatanhan wicayukcan ece kin he hoyeyakiyapi kinhan, tohanyan den unhdaka yaunpi kin kokipeyahan un po.

18. Witko tkoya oyahandapi lkin, nihunkakepi wohdakapi eciyatanhan duhapi qon he etanhan, taku atakuniśni ece, mazaskazi qa mazaska on openicitonpi śni e sdonyakiyapi ;

19 Tuka Messiya we tehike cin he on, tahin wanunyanpi cincadan, taku iyaonpepica śni qa ecedan un kin iyececa.

20 Iye qe maka kin de kagapi śni itokam wakiconzapi, tuka anpetu ihankeya kin dena en yutaninpi, niyepi kin on ;

21 Wakantanka wicațe cin etanhan najinkiye ca wowitan qu qon he wicayadapi, iye eciyatauhan; hecen wicayadapi qa wacinyayapi kin he Wakantanka en un kta.

22 Woniya kin eciyatanhan wowicake anayagoptanpi kin 363 


\section{INYAN.}

heon nagi nihduskapi, hunkawanjinkiciyapi kin awicakehan waśtewicayadakapi kta, heon cante ecedan on otoiyohi nina waśtekicidaka po.

23 Piya icahniyanpi, oicage lruke kte cin etanhan śni, tuka kukepica śni kin on, Wakantanka oie kin ni un qa owihanke wanice kte cin hee on.

24 Wicacelipi owasin peji iyececa, qa wicaśta towitan kin owasin peji lica kin iyececa. Peji kin he śniś aye ca wahica kin hinhpaya ece;

25 Tuka Itancan oie kin he owihanke wanin un kta. Unkan wotanin waśte oniciyakapi kin wicoie $k$ in he hee.

\section{WICOWOYAKE 2.}

1 Heon etanhan, wocanniye owasin, qa wohnaye ocowasin, qa woanakihbe, qa wowinawizi, - qa iapi śica iyuhpa ehpeya po ;

$2 \mathrm{Qa}$ holkśiyoqopa nakaha tonpi kin iyecen, wicoie asanpi wohnaye wanin un kin he cin po, hecen on inicagapi kta:

3 Itancan kin wacantkiya e uyatapi hecinhan.

4 He en yahipi, inyan wan ni un kin iyececa, wicaśta akantu ehpeyapi, tuka Wakantanka e kahnige ca tehike daka;

5 Unkan niśs eya, inyan ni un kin iyececa, tipi wakan wan en icah̉niyanpi, wawayuśnapi wakan kin, hecen wośnapi wakan qa Jesus Messiya eciyatanhan Wakantanlia iyokipi kin waduśnapi kta.

6 Heon etanhan nakun wowapi en kagapi qon, Wanyaka po, Zion en inyan wan oise pa hinca ewahde, kahnigapi, tehika; unkan tuwe he wacinye cinhan wiśtece lite śni ce.
7 Niye wacinyayapi kin he teliike yadapi; tulka tona waanagoptanpi śni kin hena, inyan tipi kagapi ehpeyapi qon he oise pa hinca icaga, inyan on siha ibotopi qa imnija on śinhdapi ece kin hee ;

8 Tona wicoie kin on śinhdapi qa waanagoptan śni unpi kin hena on; heon hecen ewicahdepi. 9 Tuka wicoicage kahnigapi, wawayuśnapi wicaśtayatapi, wicoun wakan, qa oyate opewicakitonpi kin he niyepi ; hecen tuwe otpaza etanhan, iye iyoyanpa wakan tawa kin en nicopi kin he olian waśte kin odakapi kta.

10 Ehanna oyate henicapi śni, tuka nakaha Wakantanka taoyate yaunpi ; he ehan onśinidapi śni, tuka wanna wowaonśida iyoyahipi.

11 Waśtecicidapi, ceciciyapice, oyate tokeca qa unhdaka unpi kin iyecen wicacehpi cantiheyapi kin, wicanagi kin kici kicize cin, hena ayuśtan po:

12 Qa Ikcewicaśta ehna owotanna olianyan po; hecen śicaya econ keniciyapi keś, wiwicawangapi anpetu kin en, niohanpi waśte wanyakapi kte cin hena on Wakantanka yaonihanpi kta.

13 Itancan kin on etanhan, wicaśta akantu wakonzapi kin owasin anagoptan po ; wicaśtayatapi kin he iyotan tanka;

14 Qa wicaśta itancanpi kin iś tona śicaya ohanyanpi śitkihdawicayapi kta, qa tona tanyan econpi wicayaonihanpi kta e on u wicaśi kin hena.

15 Hecen Wakantanka tawacin, tanyan oyahandapi kin on wicaśta witkotkoka wiyukcan śni kin hena inina yankewicayakiyapi kta. 


\section{WICOWOYAKE 3.}

16 Nihduhapi, tuka tawaniciyapi kin he wocanniye onhohida iyecen yecunpi kte śni, tuka Wakantanka tawowidake kin iyececa.

17 Owasin wicayuonihan po. Hunkawanjinkiciyapi kin waśtekida po. Wakantanka kokipa po. Wicaśtayatapi kin yuonihan po.

18 Wowidake yaunpi kin, tona niyuhapi kin ocowasin kokipeyahan anawicagoptan po, tona waśte qa wahbadan kin hecedan śni, tuka tona ksizece cin hena nakun.

719 Tuwe Wakantanka awacin un kin on, hecetu śni eśta kakiśyapi qa woiyokiśice yuha un kinhan, he wowaste.

20 Wayahtanipi kin on anipapi, qa wacinnitankapi kinhan taku iyokihi kta he ; tuka tanyan ecanonpi kin on kakiśniyanpi kinlan, wacinnitankapi hecinhan, he Wakantanka iyokipi.

21 Unkan he on nicopi ; Messiya unkiyepi on kakije ca woonspe aihpeunkiyapi kin he iyecen niś eya iye owe kin en opa dapi kta.

22 Iye qe woahtani takudan econ śni, qa i kin ohna wohnaye takudan iycyapi śni;

23 Kigepi qehan itkom wicakige śni ; kakiśyapi he ehan owawicakiye śni ; tuka tuwe owotanna wiyukcan ece kin he en ihdoya.

24 Tye hinca can akan un qehan, iye tancan kin en waunhitanipi kin yuha; hecen unkiye woahtani ehpeunyanpi woowotanna en unnipi kta: Iye apapi qon on asniniyanpi ce.

25 Tahin wanunyanps nunipi kin iyenicecapi; tuka wanna tuwe wonniyanpi qa nagi awanniyakapi kin he ekta yahdipi.

\section{WICOWOYAKE 3.}

1 He iyecen, winolincapi kin, nihihnapi wicihukun un po; hecen tona wicoie kin anagoptanpi śni kin hena, wicoie codan eśta, iye tawicupi ohampi kin on iyakapewicayapi kta;

2 Niohanpi waśte wokokipe iyahna wanyakapi kin icunhan.

3 Ihduzapi kin he akantanhan, paha kisonpi, qa mazaskazi oinpi, qa wokoyake unpi kin hee kte śni :

4 Tuka wicaśta mahentanhan cante kin elkta, taku hwinpica śni kin, tawacin wahbadan qa inina un, he Wakantanka itokam taku tehike cin ee.

5 Iś eya, wanakaja, winohinca waśteśte Wakantanka wacinyanpi qon hena e hecen kicunpi, qa hihnakupi kin wicihukun unpi.

6 Hecen Sara Abraham anakigoptan, qa, Itancan, eciya cce; he cunkśiwicayc cin hena niyepi, tanyan ecanonpi qa wokokipe takudan on niyuśinyayapi śni kinhan.

7 Nakun wicaśta yaunpi kin, wosdonye eciyatanhan om yukan po, nitawinpi wakśica wankadan iyececa, qa om wiconi wowaśte kin tawaniçiyapi kta iyecen wicahduonihan po; hecen wocekiye nitawapi kin kagipi kte śni.

8 Ehake, owasin cante wanjidan hduwitaya onśikicida po; hunkawanjinkiciyapi kin iyecen waśtekicida po, waonśidapi qa wacantkiya po.

9 Taku śica on itkom taku śica econpi śni po; qa nicigepi eśta wicakigepi śni po; tuka itkopatanhan wayawaśte po heon nicopi, hecen woyawaśte tawayayapi kta e sdonya po. 


\section{INYAN.}

10 Tuwe wiconi waśtedake ca anpetu waśte wanyaka cin kinhan, iye ceji kin taku śica en yeye kte śni, qa iha kin wohnaye takudan ie kte śni.

11 Taku śice cin okamna kta, qa taku waśte e econ kta; wookiye akite ca kuwa aye kta ce.

12 Jehowa iśta kin wicaśta owotanna awicatonwan, qa wocekiye eyapi kin he anohwicakiciya ; tuka tona śicaya econpi kin Jehowa itohnake kin itkowicakipa ece.

13 Unkan taku waśte kin pasi yaunpi kinhan, tuwe e kiunniniyanpi kta he.

14 Tuka owotanna ohanyanpi kin on śicaya nicuwapi kinhan, he wowaśte; hecen wokokipe tawapi kin kokipapi śni, qa cante hahayedán yankapi śni po:

15 'Tuka nicantepi kin en Walantanka Itancan kin wakanda po; qa tuwe wowacinye en niunpi kin on iniwangapi kinhan, wahbayedan kokipeyahan aduptapi kta e ohinniyan wiyeya yanka po ;

16 Wawiyukcan waśte hduha po; hecen śicaya olianyanpi iyecen tona aniiapi kin hena iśtecapi kta, Messiya on niolianpi waśte kin itonśniyan oniyakapi kin.

17 Wakantanka hecen tawacin hecinhan, taku śica econpi kin hee śni, tuka taku waśte econpi kin on kakiśniyanpi kinhan hee waśte kta.

18 Messiya iś eya wancadan woalitani on kakija, owotanna un kin owotanpidan śni kin hena on, Wakantanka ekta unkaupi kta e heon, wicacelipi kicun ktepi, tuka Woniya kin on kiniyanpi.

19 Nakun he on ekta ye ca wi366 canagi wicakaśka tipi ohna unpi kin hena wowicakiyaka;

20 Hena e ehanna waanagoptanpi śni, Wakantanka wawacinkta yuza tanka tehan awicape, Nowa taanpetu kin en, wita wata kagapi kin icunhan, he ohna tonana, wicanagi śahdoganna mini on nipi.

21 Qa wiyacinpi kin he iyececa, baptem nakaha nimyanpi ece; wicacelipi aśape cin yutokall iyeyapi kin hee śni, tuka Wakantanka ekta wawiyukcan waśte waayupte cin hee, Jesus Messiya ihduekicetu kin heon etanhan;

22 Iye qe malipiya kin ekta kihde ca Wakantanka etapa kin eciyatanlıan yanka; mahpiya ohnihde, qa wicookihi, qa wowaśake kin owasin iye ihukun iyewicayapi.

\section{WICOWOYAKE 4.}

1 Heon etanhan, Messiya wicacehpi en unkiyepi on kakije cin heon, niś eya iyecen tawacin kicun po: tuwe wicacehpi kin en kakije cin he wanna woahtani ayuśtan :

2 Hecen, hetanhan icimana wicacehpi eciyatanhan ni kte śni, wicaśta taku śica cantiheyapi kin en, tuka Wakantanka tawacin kin eciyatanhan.

3 Hekta unnipi kin, Ikcewicaśta tawacinpi kin ecen econkupi kin he ota, wicohan śica, wocantiheye śca, miniśa ota, wakan wacipi, witkopi, qa wakagapi wakandapi śice cin hena omaunnipi qehan.

$4 \mathrm{He}$ en iśs iye witkoya śkanpi kin on iyecen iyatahdeniçiyapi śni kin heon tognihdapi qa niyaśicapi ece ;

5 Hena e tuwe ni unpi tapi ko- 


\section{WICOWOYAKE 5.}

ya wicayaco kta wiyeya yanke cin hee itokam wohdakapi kta.

6 Heon etanhan, nakun tona țapi kin hena wotanirı waśte owicakiyakapi qon, hecen wicaśta wicacehpi en un kin iyecen wicayacopi eśta woniya en Wakantanka eciyatanhan nipi kta.

7 Tuka taku owasin ecadan owihanke kta. Hcon etanhan wacinksapa po, qa wocekiye on waktaya un po.

8 Qa taku owasin isanpa niye iyakitedan nina cantekiciya po ; wacantkiyapi kin he woahtani ota akahpe kta.

9 Iapi śica wanin otoiyohi wonkiciya po.

10 Taku wicaqupi kin wicaśta otoiyohi icu kin he iyecen iyakitedan okinikiciciya po, tona Wakantanka towaonśida ocaje ota tanyan yuhapi kin iyececa.

11 Tuwe ie cinhan Wakantanka oie kin iyecece kta; qa tuwe waokiya hecinhan wookihi Wakantanka qu kin he eciyatanhan kte; hecen taku owasin on Wakantanka y atanpi kta, Jesus Messiya eciyatanhan; iye qe woya$\tan$ qa wookihi owihanke wanin kiciun nunwe. Amen.

12 Waśtecicidapi kin, peta on iyutanpi kin on iniyutanpi kte cin he on yuśinyayapi śni po, taku tokeca aya!ripapi iyececa.

13 Tuka Messiya kakije ciqon en oyapapi kin heon iyuśkin po; hecen tohan towitan tanin kinhan nina iduśkinpi kta.

14 Messiya caje kin on niyaśicapi kinhan, niyuwaśtepi ece; wowitan qa Wakantanka Taniya kin en niunpi kin heon. Hena e eciyatanhan ośtehdapi, tuka niye eciyatanhan yatanpi ece.

15 Tuka ihnuhan wanji tin wicakte sa, qa wamanon să, qa taku śica econ sa, qa tokan tawa i en iyewicakiciciye cin iyecen kakiśniyanpi kinhan.

16 Hececa eśta tuwe Messiya tawa kin on kakiśyapi kinhan iśtece kte śni; tuka heon Wakantanka yatan kta.

17 Wakantanka tiyohnaka tawa kin wicayukcanpi kte cin wanna iyehantu. Unlkan he unkiyepi etanhan aye cinhan, tona Wakantanka tawootanin waśte wicadapi śni kin hena token owihankepi kta he.

$18 \mathrm{Qa}$ wicaśta owotanna lin kitan se ni kinhan, tuwe śicaya econ qa wahtani sa kin hena tukten yakonpi kta he.

19 Heon etanhan, tona Wakantanka eciyatanhan kakijapi kin hena, waśte ece econpi kin en, Wawicahlye wacinyepica kin iyecen iye nagipi kin yuhekiyapi kte.

\section{WICOWOYAKE 5.}

1 Miye hunkayapi kin hemaca, qa Messiya kakije ciqon wanmdake ça, wowitan yutaninpi kte cin he en owapa, tona hunkayapi yaunpi kin ceciciyapi ce ; 2 Wakantanka optaye tawa ehna yaumpi kin he wonwicaya po; econ niciyapi kin iyecece śni, tuka iyokipiya awanwicadakapi kta; woyuha śica kin on etanhan śni, tuka tawacin waśte on ecanonpi kta :

3 Wakantanka taku tawa lince cin en itancanpi kin iyenicecapi kte śni, tuka optaye kin en waonspekiya yaunpi kta.

4 Hecen Waawanhdake Itancan kin tohan ihdutanin kinhan, hehan wowitan wateśdake wan śnije kte śni kin he iyacupi kta.

5 Nakun, tona aśkatudan yaunpi kin, hunkawicayapi liin anawicagoptan po: Han, owasin 
otoiyohi anakicigoptan po, qa|sus Messiya eciyatanhan, wowiwowahbadan koyaka po. Tona wahaniçidapi kin hena Wakantanka wicakipajin, tuka tona onśikihanpi kin hena wowaonśida wicaqu ece.

6 Heon etanhan, Wakantanka nape waśake cin ihukuya onśikilian po ; hecen tohan iy ehantu kinhan yuwankan inicupi kta :

7 Wacin akipam duzapi kin owasin iye en aihpeya po; iye qe awanicinpi ece.

8 Witkopi śni po, waktaya un po; Wakanśica tokaniyanpi kin he mnaja hoton ece kin iyecen omani un, tuwe yapote kte cin he akita ece.

9 Sutaya wacinyayapi kin on he kipajin po; nihunkawanjipi maka akan unpi kin hena en wokakije kin dena iyececa yuśtanpi ece e sdonyayapi.

10 Tuka Wakantanka wowaonśida ocowasin hduhe cin, Je-

tan owihanke wanica iye tawa kin en unkicopi kin he, tohan cistiyedan nicakijapi kinhan, niyuśtanpi kte, pahohośni enihnakapi, niyuwaśakapi, qa sutaya nihdepi nunwe.

11 Iye qe wowitan qa wokiconze kin owihanke wanin tawa nunwe. Amen.

12 Siluanos nihunkawanjipi wan wacinyepica wadake cin on wowapi ptecedan cicagapi ; wahokonciciyapi qa wociciyakapi, Wakantanka towaśte wicakapi en nayajinpi kin he dee ce.

13 Babulon ekta tona om nicahnigapi kin hena nape niyuzapi, qa nakun Markos micinkśi kin.

14 Wacantkiyapi iiputakapi kin on otoiyohi nape kiciyuza po. 'Tona Jesus Messiya en yaunpi kin owasin wookiye nicipi un nunwe. Amen.

\section{IN Y A N}

\section{'TAWOWAPI INONPA KIN.}

\section{WICOWOYAKF 1.}

1 Simon Inyan, Jesus Messiya tawowidake qa yeśi qon he, tona Wakantanka unkitawapi toowotanna kin on, qa Jesus Messiya Wanikiye cin eciyatanhan, unkiye unhapi kin iyecen wowacinye tehika yuhapi kin hena wowapi wicakicaga.

2 Wowaonśida, wookiye kis, Wakantanka sdonyapi kin en, qa Jesus Itancan unkiyapi kin on niciyuotapi nunwe.

3 Iye towaśake wakan kin he taku on wiconi qa woohodapi kte cin owasin unqupi kin he iyececa, tuwe wowitan qa wookihi on unkicopi kin he sdonyapi kin eciyatanhan.

4 Hena on taku tanka hinca, qa taku tehike hinca iwahounyanpi ; hecen hena on Taku Wakan ouncage kin en oyapapi kta, wiconi kin de en wocantiheye śica on wicośice nayecipapi kin.

5 Qa nakun he on nina mniheiciya po, wacinyayapi kin en waditakapi kin ikoyagya po; qa waditakapi liin en wosdonye ;

$6 \mathrm{Qa}$ wosdonye kin en iyatahdeiçyapi śni; qa iyatahdeic̣i- 
yapi śni kin en wacintankapi; qa wacintankapi kin en taku wakan iyececapi ;

7 Qa taku wakan iyececapi kin en waśtekicidapi ; qa waśtekicidapi kin en wacantkiyapi ikoyagya po.

8 Hena taku kin en niunpi qa niciyuotapi kinhan, Jesus Messiya Itancan unkiyapi sdonyapi kin en taku icahye ça waskuyeca aicage cin henicapi kta e nicagapi.

9 Tuka tuwe dena yuhe śni kinhan, he iśtagonge ca tehan wawanyaka okihi śni, qa wahtani qon hena kipakintapi kin he akiktonja.

10 Heon etanhan, mihunkawanji, nicopi qa nicahnigapi kin aiciciya hdusuta po; hena hecen ccanonpi kinhan icimana nihinhpayapi kte śni.

11 Qa nakun heon Jesus Messiya Itancan qa Wanikiya unkitawapi tokiconze owihanke wanice cin en yaipi kte cin wijinya niçupi kta.

12 Hcon etanhan taku hececa ohinniyan kiksuyeciciyapi kta awcktonje kte śni, hcna niye hinca sdonyayapi, qa nakaha wowicake kin de en sutaya nihdepi eśta.

13 Tohanyan wakeya kin de ohna waun kin hehanyan kiksuyeciciyapi kin on iyocipaśtakapi kte cin he hecetu wadake:

14 Wakeya mitawa kin de aśkadan wehnake kta sdonwakiya, Jesus Messiya Itancan unkiyapi omakiyake ciqon iyececa.

15 Tuka nakun detanhan imdamde cin iyohakam, taku kin dena ohinniyan yeksuya oyakihipi kta c awakite kta.

16 Jesus Messiya Itancan unkiyapi towaśake qa hi late cin hena unkoniciyalkapi qehan, hi2俱 tunkakanpi ksamya kagapi kin unkunpi śni, tulka iye tanka hince cin he iśta on wanunyakapi.

17 Wakantanka Ateye cin eciyatanhan woyuonihan wowitan ko icu, wowitan waśte kin etanhan wicaho kin de hiyu qehan, Micinkśi waśtewakidakc cin hc dee, on iyomakipi hinca ce.

18 Unkan wicaho kin he mahipiya eciyatanhan u kin he naunhonpi, paha wakan kin akan kici unyakonpi qchan.

19 Tuka wokcanpi oie iyotan suta wan unhapi; he qe anayagoptanpi kinhan tanyan ecanonpi kta, petijanjan tukten otpaze cin en ijanjan kin iyececa, nicantepi kin ekta anpao kamdeze ca, en anpao wicanhpi kin hinanpe kte cin hehanyan.

$20 \mathrm{Qa}$ de tokaheya sdonya po, wowapi wakan kin en wokcanpi kin owasin tuwedan iye cinka oyake śni.

21 Wokcanpi kin wanakajatanhan wicaśta tawacin kin eciyatanhan u śni ; tuka wicaśta wakan Wakantanka tawa Woniya Wakan kin token iewicakiye ciqon hena hecen eyapi ece.

\section{WICOWOYAKE 2.}

1 Tuka he ehan oyate kin ehna wicaśta wokcan itonpi śni yukan qon, he iyecen niyepi kin ehna waonspekiya itonpi śni kin heca yuke kta, nahiniana wohnuni woihangye kin en aupi kta, Itancan opewicakiton qon he elipeyapi, qa wicotakuniśni olianko amnaiçiyapi kta.

2 Qa wicohian śjca econpi kin en wicota opapi kta; hena on wowicake canku kin yaśicapi ece e kta.

3 Qa waicu wacinpi kin eciya369 


\section{INYAN.}

tanhan wicoie skuya on wiyopeniyanpi kta; hena e wicayacopi kin wanakajatanhan en awicau, qa ihangwicayapi kte cin he liba śni.

4. Wakantanka mahpiya ohnihde wahtanipi gon hena wicakpatan śni, tuka wakanśica ti kin ekta iyayewicaya, qa woyaco kte cin hehanyan wicayuhapi kta e otpaza maza icicahiha on wicakaśka hecinhan;

5 Qa maka tannike cin kpatan śni, tuka Nowa wicaśta wiciśahdogan wicoowotanna oyake cin he kpatan, qa wicoicage walitanipi sa amnitanwicaya;

6 Qa Sodom qa Gomorra otonwe kin caliota kahya ehpewicaye, woihangye on wicayaco, qa tona tokata wahtani sa unpi kte cin hena woonspe wicakicaga ;

7 Qa Lot owotanna un, wicaśta wahtepi śni ohan śicapi kin wa.cin iyowicakiśica ece kin he elidaku :

8 Wicaśta owotanna kin he hena wicehna ti, qa taku wanyaké ca nahion kin on, anpetu eca iye nagi owotanna ekta wacin iyowicakiśica ece, śicaya ohanyanpi kin on :

9 Itancan kin wicaśta waśte wowiyutanye kin etanhan token ewicahdaku kta sdonya, qa nakun wicaśta owotanna śni kin woyaco anpetu kin hehanyan wicayuhe kta, śitkihdawicayapi kta e heon:

10 'Tuka tona woaśa mye cantiheyapi kin en wicacehpi eciyatanhan manipi, qa wokiconze wahtedapi śni kin hena iyotan : hena walianicidapi qa wamnaicidapi ece; qa tona tankapi kin ivicayaśicapi kokipapi śni.

11 Mahpiya ohnihde kin, wookihi qa wowaśake on iyotanpi qeyaś, Itancan kin itokam wa370 yaśicapi woyaco kin en awicayapi śni ece.

12 Tuka dena iś, woteca iyececa, wicayuzapi qa ihangwicayapi kta e on wicakagapi kin, taku okahinigapi śni keś he yaśicapi ece; qa wicotakuniśni tawapi kin en ihangiciyapi kta;

13 Qa anpetu icunhan tanyan wotapi kin he woiyokipi dakapi kin heon woahtani iyunwin kin icupi kta. Hena om wayatapi icunhan wohnayeiciyapi kin on iyuśkinpi, woaśape qa taku samya wanke cin hecapi ;

14 Iśta yukanpi wiinahmanpi kin on ojudan qa woahtani ayuśtanpica śni ; wicanagi kacegya unpi kin hena wicahnayanpi ece; cante wawicakipi e ecewakta kin heca yuhapi, wicotakuniśni cincapi lkin :

15 Canku owotanna kin ayuśtanpi, qa onuniyata unpi, Balaam Bosor cinhintku woahtani iyunwin waśtedake ciqon he tacanku kin en opapi :

16 Iye taku śica econ qon on iyopeyapi ; śonśonna kin ia okitpani ece wicaho on ie ca, wicaśta wokcan witkotkoya ohanye cin he kiśica.

17 Miniyowe mini wanica, malipiya śapa tate iyumni on toke cincin eyaye cin hena hecapi ; hena on otpaza sape hinca owihanke wanica wicakicihnakapi.

18 Wahanhaniçidaya takuśniśni eyapi eca, wicacelpi cantiheyapi kin eciyatanhan, witkoya ohanyanpi kin on, tona wohnaye en ounyanpi kin kitan ecinyan nawicakicipapi qon hena iyakapewicayapi ece.

19 Tawaiciyapi kin wicaqupi kta keyapi, tuka iyepi kaeś wicotakuniśni iwicadaka. Taku on tuwe iyalsapeyapi kinhan, he on wowidagyapi ece. 
20 Itancan qa Wanikiya Jesus Messiya sdonyapi kin eciyatanhan, wicoicage lin de en woaśape kin nakipapi eśta, ake hena on iyakapeyapi qa en ihdaśkapi kinhan, hena otokahe ekta waśte qa owihankepi lin he śica ece.

21 Woowotanna canku kin he sdonyapi śni unkanś he waśte kta tuka, qa he sdonyapi qa woahope wakan wicaqupi kin ayuśtanpi kin he śice linca.

22 Tuka wiyacinpi eyapi wicakapi kin he iyecen waakipapi ; Sunka taku hiyuye cin he ake hduta ce; qa, Kukuśe yujajapi qon he ake upśija en iwanka ce.

\section{WICOWOYAKE 3 .}

1 Waśtecicidapi kin, de nakaha wowapi inonpa cicagapi ; hena en nitawacinpi mdesahan kiksuyapi on iyocipaśtakapi.

2 Hecen wicaśta wokcan wakan token oieyapi qon, qa nakun unkiye Itancan qa Wanikiya yeunśipi qon token econ unniśipi kin hena yeksuyapi kta.

3 Qa de tokaheya sdonya po, anpetu ehake kin en wailhahapi, iye cantiheyapi śica eciyatanhan manipi kin heca hipi kta;

$4 \mathrm{Qa}$ heyapi kta; Hi kta keyapi qon he tukte e he; wicahunkake iśtinma ihpayapi ehantanhan otokaheya tanhan taku orvasin tokeca śni wanka ce.

5 Tuka he de sdonyapi śni, Wakantanka oie kin eciyatanhan mahpiya kin ehanna un, qa maka kin mini mahen qa nakun mini akan han;

6 Qa nakun he eciyatanhan, maka ehanna un qon he mini apaśbog hiyaye ca ihangya.

7 Tulka mahipiya qa maka nakaha un kin hena iye oie kin on kihnakapi, peta on ayuhapi, wo- yaco anpetu qa wicaśta śica śitkihdawicayapi kte cin hehantu.

8 Tuka, waśtecicidapi kin, he de sdonye śni yukanpi śni po, Itancan kin ekta ampetu wanjidan waniyetu kek topawinge iyececa, qa waniyetu kektopawinge anpetu wanjidan iyececa ece.

9 Itancan kin talsu wahowicaye cin yutehan śni, wicaśta wanjikśi yutehanpi eyapi kin iyececa; tuka unkiyepi on wawacinkta yuza tanka, tuwedan arvihnuniiçiye kte śni, tuka owasin woiyopeiçiye en ihdoupi kta cin.

10 Tuka Itancan taanpetu kin wamanon sa wan hanyen hi ece kin he iyecen $u$ lkta, hehan mahiya kin hmunlimunyan hinhda on tokan iyaye kta, qa taku kin peta hinca on skan lita; qa maka kin wicolian ohna un kin hena hulinage kta.

11 Hena taku kin owasin hecen skan kta hecinhan, wicohan waśle qa Taku Wakan iyececapi kin en taku wicaśta yaunpi kta iyececa he :

12 Wakantanka taanpetu u kte cin he ayapepi qa on inayahinipi kta; he en malipiya kin aide ecen atakuni kte śni, qa taku lin peta tanka hinca on skan lita.

13 Hececa eśta, unkiye, iye wahounyanpi kin eciyatanhan, mahpiya teca qa maka teca en woowotanna ounye cin he wanunyakapi kta e unkapepi.

14 Heon etanhan, waśtecicidapi kin, taku hececa ayapepi kin heon aiciciya po, hecen iye wookiye en aśape śni qa iyaonpepicaśni iyeniyanpi kta.

$15 \mathrm{Qa}$ Itancan wawacinkta yuza tanka kin he wiconi kecankin po; Palos, hunkawanjinun371 


\section{JOHANNES.}

yanpi waśte kin, iś eya wolssape qupi kin eciyatanhan, wowapi nicagapi kin iyececa;

16 He wowapi lkage cin owasin en taku hececa cajeyata ece; hena wanjikśi okahinili tehika, tona onspepi śni qa kaktihanhan unpi kin yatokecapi, wowapi unma kin iyececa, iye ihdutakunipi kte śni heon.

17 Heon etanhan, waśtecici- dapi kin, niś niyepi hena itokam sdonyayapi, heon ihnuhan wicaśta śica wohnaye kagapi kin nihnayanpi, qa sutaya nihdepi kin etanhan nihinhpayapi kinhan.

18 Tuka wowaśte kin en, qa nakun Itancan unyanpi Wanikiya Jesus Messiya sdonyapi kin en icaga po. Iye kin wowitan nakaha qa owihanke wanica kiciun nunwe. Amen.

\section{JOHA N NES}

\section{TAWOWAPI TOKAHEYA KIN.}

\section{WICOWOYAKE 1.}

1 Taku otokaheya tanhan un, taku naunhonpi, qa iśta on wanunyakapi, opaunlitapi qa nape on unzapi, Wiconi Oie kin hee.

2 Wiconi kin he ihdutanin; unkan Wiconi Owihanke Wanica Ateyapi kin kici un, qa unkiyepi kin en ihdutanin qon he wanunyakapi, qa unyaotaninpi qa unkoniciyakapi.

3 Taku wanunyakapi qa naunhonpi kin he unkoniciyakapi, hecen niye unyecicapi kta; unkan awicakehan Ateyapi kin kici okiciciya unyakonpi, qa nakun iye Cinhintku Jesus Messiya kici.

4. Unkan nitowiyuśkinpi kin yucopi kta e heon dena decen wowapi unnicagapi.

5 Hehan wotanin waśte iye eciyatanhan naunhonpi qa unkoniciyakapi kin, Wakantanka he iyoyanpa, qa otpaza takudan en un śni kin he hee.

6 Iye kici okiciciya unyakonpi ce, unkeyapi qa otpaza lin oma- unnipi kinhan, unkitonpiśni qa wowicake kin econkupi śni ece. 7 Tuka iye iyoyanpa en un kin he iyecen iyoyanpa kin en maunnipi kinhan, otoiyohi okiciciya unyakonpi, qa iye Cinhintku Jesus Messiya we kin- he woahtani owasin unkipakintapi kta.

8 Woahtani unnicapi ce, unkeyapi kinhan, unkiçihnayanpi qa wowicake kin unkiyepi en un śni.

9 Tuka waunhtanipi kin unkohdakapi kinhan, iye kin wacinyepica qa owotanna un, hecen woalitani yutokan iyeunkiciciyapi kta, qa taku śica owasin unkipakintapi kta.

10 Eca waunlitanipi śni ce, unkeyapi kinhan, iye itonśni undakapi, qa iye oie kin unkiyepi en un śni ece.

\section{WICOWOYAKE 2.}

1 Micinca cikcistinpidån, wayahitanipi kte śni e heon dena decen wowapi cicagapi. Unkan tuwe wahtani kinhan Ateyapi kin elkta Wawiciya wan unhapi,

$3 \% 2$ 
Jesus Messiya owotanna kin hee.

2 Unkan waunhtanipi wokajuju kin he iye; qa unkiye unkitawapi kin hecedan śni, tuka mala kin owancaya woalitani econpi kin hena.

3 Unkan deciyatanhan iye kin wanna sdonunyanpi e sdonunkiyapi, tawoahope unkokicipapi kinhan.

4 'Tuwe, He sdonwaya ce, eye ca tawoahope okicipe śni kin he itonśni, qa wowicake kin en un śni.

5 Tuka tuwe iye oie kin okicipe cinhan, awicakehan he en Wakantanka waśtedake cin yuecetupi. Heciyatanhan iye kin en unyakonpi kin sdonunkiyapi ece.

6 Tuwe he kici un keye cinhan, iye token omani qon iś iyecen omani k ta iyececa.

7 Mihunkawanji, woahope teca wan cicagapi śni, tuka woahope tanina, otokaheya tanhan duhapi qon hee. Woahope tanina kin wicoie otokahe elrta nayalionpi qon he hee.

8 Hehan akeś woahope teca wan cicagapi; he iye kin en wowicake, qa nakun niyepi kin en ; otpaze cin he wanna henakeca, qa iyoyanpa wicake cin he wanna tanin kin heon etanhan.

9 Tuwe iyoyanpa kin en un keiciye ca, hunkawanjitku kin śicekidake cin he dehantu otpaza kin en un.

10 Tuwe hunkawanjitku wastekidake cin he iyoyanpa kin en un ece, qa wokaśeye takudan en un śni.

11 Tuka tuwe hunkawanjitku sicekidake cin he otpaza en un, qa otpaza omani, qa tokiya ye cin sdonkiye śni, iśta kin otpaza kaśeya, heon etanhan.
12 Hokśiyopa cikcistinpidan, iye caje kin on wayahtanipi kin nicicajujupi kin heon wowapi cicagapi.

13 Hunkakeniyanpi kin, tuwe otokaheya tanhan un kin he sdonyayapi kin heon wowapi cicagapi. Kośkapi kin, tuwe śice cin he ohiy ayapi kin heon wow api cicagapi.

14 Hokśiyopa cikcistinpidan, Ateyapi kin sdonyayapi kin he etanhan wowapi cicagapi. Hunkakeyapi kin tuwe otokaheya tanhan kin he iye sdonyayapi kin heon wowapi cicagapi. Kośkapi kin, waniśakapi, qa Wakantanka oie kin en niunpi, qa tuwe śice cin he yaktepidan kin he etanhan wowapi cicagapi.

15 Wicoicage kin de waśtedapi śni po, qa taku wicoicage kin ekta un kin hena nakun. Tuwe wicoicage kin de waśtedake cinhan, Ateyapi kin waśtedake cin en un śni.

16 Taku wicoicage en un kin owasin, wicacehi cantiheye cin, wicaiśta cantiheye cin, qa" wiconi kin den walianiçidapi kin, hena Ateyapi kin etanhan śni, tuka wicoicage kin de etanhan.

17 Unkan wicoicage kin de atakuni kte śni, qa wocantiheye kin nakun; tuka tuwe Wakantanka tawacin kin yuhe cinhan, he owihanke wanin ounye kta.

18 Hokśiyopa cikcistinpidan, anpetu ihankeya kin wanna hiyohi ; unkan, Messiya kipajin kin he u kta ce, nayationpi qon he iyecen Messiya kipajinpi kin ota wanna hipi; heon de anpetu ehake kin sdonunyanpi.

19 Unkiyepi kin etanhan inanpapi, tuka hena unkicicapi śni. Hena unkicicapi unkanś, unkici. 373 


\section{JOHANNES.}

pi un wacinpi kta: tuka owasin unketanhanpi śni tanin kta e heon etanlian hececa.

20 Tuka Wanji Wakan kin he niye sdaniyanpi, qa heon taku owasin sdonyayapi.

21 Wowicake kin sdonyayapi śni kin heon wowapi cicagapi śni ; tuka he sdonyayapi, qa woilonśni takudan wowicake kin eciyatanhan u śni, heon etanhan.

22 Tuwe itonśni he ; tuwe Jesus he Messiya kin ee śni, keye cin hee; Messiya kipajin kin hee, Ateyapi qa nakun Cinhinthu kin anawicakihbe cin hee.

23 Tuwe Cinhintkuyapi kin wicada śni kin he Ateyapi kin yuhe śni : tuka tuwe Cinhintkuyapi kin oyake cinhan, he Ateyapi kin nakun yuha ece.

24 Taku otokaheya tanhan nayahonpi qon he yuha po. Taku atokahe ekta nayahonpi qon en niunpi kinhan, Cinhintkuyapi kin en yaunpi $\mathrm{kta}$, qa nakun A teyapi kin en.

25 Unkan wahoyapi qon taku iwahounyanpi kin, owihanke wanin wiconi kin hee.

26 Tona nihnayanpi kta cinpi kin hena on decen wowapi cicagapi.

27 Tuka sdaniyanpi, iye eciyatanhan duhapi kin he nicipi un; qa tuwe waonspeniciyapi kta iyenicecapi śni ; tuka sdaniyanpi kin he taku owasin onspeniciyapi, he wowicake qa woitonśni wanica; he taku onspeniciyapi kin iyecen, iye kin en un po.

28 Unkan dehan, hokśiyopa cikcistinpidan, iye kici un po; hecen tohan iye ihdutanin kinhan wacinunyanpi kta, qa taninyan hinajin kinhan, itokam unkiśtecapi kte śni.

29 Iye owotanna un sdonyaya- pi kinhan, tuwe woowotanna econ kin he iye kin eciyatanhan wanna teca nien tonpi e nakun sdonya po.

\section{WICOWOYAKE 3.}

1 Wanyaka po, Ateyapi kin wocantekiye hinsko tanka unqupi kin, Wakantanka cinca unkekiciyapi kta : lieon etanhan wicoicage kin sdonunyanpi śni, iye kin sdonyapi śni qon iyececa.

2 Waśtecicidapi kin, warma Wakantanka cinca kin heuncapi; qa tounkecapi kte cin hinahin tanin śni: tuka iye kin ihdutanin kinhan, iyeuncecapi kta e sdonunkiyapi ; he token un kin wanunyakapi kta nakaeś.

3 Unkan tuwe hecen wacinyan canteyuze cinhan ihduecedan ece, iye kin ecedan un kin iyecen.

4 Tuwe woalitani econ kin he woope kin kicaksa ece; woahtani lie woope kicaksapi kin hee.

5 Unkan iye ihdutanin qon, waunhtanipi kin yutokan iyeye kta e heon, he sdonyayapi; qa iye kin woahtani takudan en un śni.

6 Tuwe iye en un kin he wahitani śni ; tuwe wahtani ece kin he iye kin tohinni wanyake śni, qa nakun sdonye śni.

7 Hokśiyopa cikcistinpidan, ihnuhan tuwe nihnayanpi kinhan; tuwe taku owotanna econ ece kin he owotanna, iye kin owotamna un kin iyececa.

8 'Tuwe woalitani econ ece kin he wakanśica eciyatanhan un; otokaheya tanhan wakanśica kin walitani ece. Heon etanhan Wakantanka Cinhintku kin ihdutanin, wakanśica ohan kin ihangye kte cin heon.

9 Tuwe Wakantanka eciyatan- 
han teca nien tonpi kin he woalitani econ śni, taku on icahye cin he en un kin heon; unkan wahtani okihi śni, Wakantanka eciyatanhan teca nien tonpi kin he etanhan.

10 Detanhan Wakantanka cinca kin sdonwicayapi, qa nakun wakanśica cinca kin; tuwe taku owotanna ece econ śni kin he Wakantanka eciyatanhan śni, qa nakun tuwe hunkawanjitku tanyan cante kiyuze śni kin.

11 Otokaheya tanhan taku iwahokonunkiciyapi nayahonpi qon he dee, otoiyohi waśteunkicidakapi kte cin.

12 Qain taku śice cin eciyatanhan un, qa iye sunkaku kikte qon iyecece śni. Unkan taku on kikte he. Iye kin olian śica, qa sunkaku kin waśte kin heon etanhan.

13 Mihunkawanji, wicoicage lkin śicenidapi eśta inihanpi śni po.

14 Hunkawanjinkiciyapi waśtewicunkidapi kin heon wiconte etanhan wiconi e ekta unkiyohipi sdonunkiyapi ; tuwe hunkawanjitku waśtekida śni kin he wiconte ehna un.

15 'Tuwe hunkawanjitku śicekidake cin he tinwicakte kin heca; unkan tuwe tinwicakte kin he wiconi owihanke wanica aihduhe śni e sdonyayapi.

16 Deciyatanhan wacantkiyapi kin sdonunyanpi; iye kin unkiyepi on wiconi ehpekiye cin heon; hecen unkiś eya hunkawanjinkiciyapi kin on wiconi ehpeunkiyapi kta iyececa.

17 Tuka tuwe maka akan woyuha ton, qa hunkawanjitku walipanica wanhdake ca, cantekiye cin anakipte cinhan, token Wakantanka waśtedake cin en un kta he.
18 Micinca cikcistinpidan, wicoie qa wicaceji ecedan on wacanunkiyapi kte śni, tuka wicolian qa wowicake kin on.

19 Unkan deciyatanhan wowicake eciyatanhan unyakonpi, qa iye itohnake kin en cante unhdutinzapi kta e sdonunkiyapi.

20 Uncantepi kin unhdacopi kinhan, Wakantanka uncantepi kin sam iyeya tanka, qa taku owasin sdonya un.

21 Waśtecicidapi kin, uncantepi kin unhdacopi śni kinhan, Wakantanka ekta wowaditake kin unhapi :

22 Qa taku unkidapi kin owasin unkicupi ece, tawoahope kin unkiciyuhapi, qa taku iye iśta kin en iyolkipi kin he econkupi, heon etanhan.

23 Unkan tawoahope kin he dee, Tye Cinhintku Jesus Messiya caje kin wacinunyanpi, qa otoiyohi waśteunkicidapi kta, econ unśipi qon iyececa.

24 Tuwe tawoahope kin opecinhan, he Wakantanka kici un, qa iś iye kin en un; unkan Woniya unqupi kin he eciyatanhan iye unkicipi un kin he sdonunyanpi ece.

\section{WICOWOYAKE 4.}

1 Waśtecicidapi kin, ihnuhan woniya owasin wicayadapi kinhan; tuka woniya kin wicayukcan po, Wakantanka etanhan unpi kinhan; wicaśta wokcan itonpiśni ota wanna wicoicage kin ehna iyayapi.

2 Deciyatanhan Wakantanka Taniya kin sdonyayapi kta; woniya kin tona Jesus Messiya wicacehpi un hi oyake cin hena Wakantanka eciyatanhanpi ece.

3 Qa woniya kin tona Jesus Messiya wicacehpi un hi oyake śni kin hena Wakantanka eciya375 


\section{JOHANNES.}

tanhanpi śni; tuka Messiya kipajin u kta nayahonpi qon he dee, wanna wicoicage kin en un. 4 Kokśiyopa cikcistinpidan, Wakantanka eciyatanhan yaunpi, qa hena ohiwicayayapi; tuwe en niunpi kin he tuwe wicoicage en un kin isanpa tanka.

5 Hena e wicoicage kin etanhanpi ; heon wicoicage kin iyecen iapi, unkan wicoiçage kin anawicagoptan ece.

6 Unkiye Wakantanka unketanhanpi; tuwe Wakantanka sdonye cin he unkiye anaungoptanpi ece; qa tuwe Wakantanka eciyatanhan śni kin he anaungoptanpi śni. Deciyatanhan wowicake woniya qa wohnaye woniya kin napin sdonunyanpi.

7 Waśtecicidapi kin, otoiyohi canteunkiciciyapi kte; wacantkiyapi kin he Wakantanka eciyatanhan un; qa tuwe wacantkiye cin he Wakantanka eciyatanhan tonpi, qa Wakantanka sdonya.

8 T'uwe wacantkiye śni kin he Wakantanka sdonye śni; Wakantanka he wacantkiyapi kin hee.

9 Wakantanka canteunkiyapi kin he deciyatanhan tanin, Wakantanka iye Cinhintku iśnana icage cin he makata $u$ śi, heciyatanhan unnipi kta e heon.

10 Wacantkiyapi kin he deciyatanhan, unkiye Wakantanka waśteundakapi kin hee śni, tuka iye canteunkiyapi, qa Cinhintku waunhtanipi yutokan iyeye k.ta e on $u$ śi qon hee.

11 Waśtecicidapi kin, Wakantanka hecen canteunkiyapi kinhan, unkiś eya otoiyohi canteunkiciciyapi kta iyececa.

12 Tuwedan tohinni Wakantanka wanyake śni. Otoiyohi canteunkiciciyapi kinhan Wa- kantanka unkicipi un, qa iye canteunkiyapi kin unkiyepi en tanyan yuśtanpi ece.

13 Heciyatanhan unkiye kici unyakonpi, qa iye unkicipi un kin sdonunyanpi ece, iye Taniya unqupi kin heon.

14 Unkan wanunyakapi, qa Ateyapi kin Cinhintku ukiya, wicoicage kin ekta, Wanikiya hee kta $\mathrm{e}$ he unyaotaninpi.

15 Tuwe Jesus he Wakantanka Cinhintku kin hee e oyake cin he kici Wakantanka un, qa iś eya Wakantanka en un ece.

16 Wakantanka canteunkiyapi kin he sdonunyanpi qa wicaundapi. Wakantanka wacantkiyapi kin hee : qa tuwe wacantkiyapi en un kin he Wakantanka kici un, qa Wakantanka iś nakun iye en un ece.

17 Deciyatanhan wocantekiye unkiyepi en unkiciyuśtanpi ece, hecen woyaco anpetu kin en wowaditake unhapi kta : iye un kin unkiś iyecen wicoicage kin den unyakonpi ece.

18 Wacantkiyapi kin en wokokipe wanica, tuka wacantkiyapi yuśtanpi kin he wolkokipe tankan ehpeya ece; wokokipe kin he wicocante śica yuha ece; tuwe walkokipe cin he nahanhin wacantkiyapi kin en yuśtanpi śni.

19 Iye tokaheya canteunkiyapi kin heon unkiye Wakantanka waśteundakapi.

20 Tuwe, Walkantanka waśtewadaka ce, eye ça hunkawanjitku śicekidake cinhan, he itonśni. Tuwe hunkawanjitku wanhdake cin he waśtekidake śni kinhan, token ecin Walkantanka wanyake śni kin waśtedaka okihi kta he.

21 Unkan iye etanhan woahope kin de unhapi; tuwe Wakan- 
tanka waśtedake cin lie hunkawanjitku kin iś nakun waśtekidake kta ce.

\section{WICOWOYAKE 5.}

1 Tuwe Jesus he Messiya kin ce e wicada kin he Wakantanka eciyatanhan tonpi ; qa tuwe waicahye cin waśtedake cinhan, he tuwe iye eciyatanhan teca ni en tonpi kin he nakun waśtedaka ece.

2. Deciyatanhan Wakantanka cinca kin waśtewicundakapi kin sdonunkiyapi, Wakantanka waśteundakapi qa tawoahope kin tanyan unliciyuhapi kinhan.

3 Wakantanka waśtedakapi kin he dee, tawoahope lin tanyan ounpapi kin he; unkan tawoahope kin wośitkihda śni.

4 Tuwe Wakantanka eciyatanhan tonpi kin he wicoicage kin ohiya ece; unkan woohiye wicoicage ohiye cin he dee, wacinunyanpi kin hee.

5 Wicoicage ohiya ece kin he tuwe he; tuwe Jesus he Wakantanka Cinhintku kin ee e wicada kin hee.

6 Tuwe mini qa we eciyatanhan hi qon he Jesus Messiya ee; mini ecedan on hi śni,"tuka mini we ahna. Unkan wayrotanin kin he Woniya kin hee; Woniya kin he wowicake, heon etanhan.

7 Mahpiya kin ekta yamni wayaotaninpi ece, Ateyapi kin, Wicoie kin, qa Woniya Wakan kin; unkan hena yamni wanjipidan.

8 Nakun maka akan taku yamni wayaotanin ece, Woniya kin, qa mini kin, qa we kin ; unkan hena yamni wanjidan en un.

9 Wicaśta akantu taku yaotaninpi kin he unkicupi kinhan, Wakantanka taku yaotanil kin he iyotan tanka; Wakantanka taku yaotanin kin he dee, iye Cinhintku hdaotanin qon hee.

10 Tuwe Wakantanka Cinhintku kin wicada kin he wayaotanin iye atayedan yuha un. 'Tuwe Wakantanka wicada śni kin he itonśni daka ece; wayaotanin kin on Wakantanka iye Cinhintku hdaotanin qon he wicada śni kin heon.

11 Unkan wayaotanin qon he dee, Wakantanka wiconi owihanke wanica unqupi kin he; unkan wiconi kin he iye Cinhintku kin en un.

12 Tuwe Cinhintkuyapi kin yuhe cin he wiconi yuha; qa tuwe Wakantanka Cinhintku kin yuhe śni kin he wiconi yuhe śni.

13 Wakantanka Cinhintku caje kin wacinyayapi kin dena wowapi cicagapi kin; wiconi owihanke wanica duhapi e sdonyakiyapi kta, qa Wakantanka Cinhintku caje kin wacinyayapi kta e heon.

14 Unkan iye ekta wowaditake unhapi kin he dee, Iye tawacin kin eciyatanhan taku unkidapi eca naunkihonpi ece :

15 Qa taku unkidapi eca naunkihonpi ece e sdonunkiyapi kinhan, taku tona unkidapi kin hena unhapi e nakun sdonunkiyapi ece.

16 Tuwe hunkawanjitku wahtani wanhdake cinhan, woalitani kin he wiconte ekta śni kinhan, wocekiye ekiciye kta, unkan. Wakantanka wiconi qu kta, tona wahtanipi wiconte ekta iyahde śni hena on. Woahtani wanji wiconte elta; he on wocekiye eye kta epe śni.

17 Taku śica econpi kin owasin woahtani; unkan woahtani wiconte iyohi śni kin heca yukan.

18 'Tuwe Wakantanka eciyatanhan tonpi kin he wahtani śni 


\section{JOHANNES.}

ece e sdonunyanpi; tuka tuwe Wakantanka eciyatanhan icage cin he tanyel ihduhe ca hecen taku śice cin he oyutan śni ece.

19 Wakantanka eciyatanhan unyakonpi kin he sdonunkiyapi; qa wicoicage kin ocowasin woalitani en wanka.

20 Unkan Wakantanka Cinhintku kin he hi, qa wicoksape unqupi, on tuwe wicake cin iyeunkiyapi kta e solonunyanpi ; unkan tuwe wicake cin he en unyakonpi, iyo Cinhintku Jesus Messiya hee. Wowicake Wakantanka qa owihanke wanin wiconi kin hee.

21 Hokśiyopa cikcistinpidan, taku wakagapi kin itehan ihduha po. Amen.

\section{JOHANNES}

\section{TAWOWAPI INONPA KIN.}

1 Hunkayapi un kin, Kuria kalinigapi qon wowapi kicaga; iye cinca kin, wowicake eciyatanhan waśtewicawadake cin hena om, qa miśnana śni, tuka nakun tona wowicake sdonyapi kin hena owasin ;

2 Wowicake kin en unyakonpi, qa owihanke wanin unkicipi un kte cin heon etanhan.

3 Wowaśte, wowaonśida, wookiye ko, Wakantanka Ateyapi kin eciyatanhan, qa nakun Jesus Messiya Itancan, Ateyapi kin he Cinhintku kin eciyatanhan, wowicake wacantkiyapi ko en, nicipi un nunwe.

4 A teyapi kin woahope unqupi qon iyecen, nicinca wowicake eciyatanhan manipi iyewicawakiye cehan nina inıduśkin.

5 Unkan nakaha, Kuria, ceciya ce, woahope teca wan cicage cin iyecece śni, tuka wanji otokahe ekta unhapi qon, otoiyohi canteunkicieiyapi kte cin hec.

$6 \mathrm{Qa}$ wacantkiyapi kin he dee, tawoahope kin eciyatanhan maunnipi kte cin hee. Woahope kin he dee, Otokahe ekta taku nayalionpi qon he ohna mayanipi kte cin.
7 Wanna wicahnaye sa ota wicoicage kin ehna iyayapi, hena Jesus Messiya wicacehpi un hi qon oyakapi śni. He wicahnaye sa qa Messiya kipajin kin heca.

8 Iwanihdaka po, hecen taku unkamnanpi kin toki ehpeunyanpi kte śni, tuka wokajuju iyakicuya unkicupi kta.

9 Tuwe wahtani qa Messiya tawoonspe kin en un śni kin he Wakantanka yuhe śni. Tuwe Messiya tawnonspe kin en un kin he Ateyapi kin yuhe ça nakun Cinhintku kin.

10 Tuwe en niipi qa woonspe kin de yuhe śni kinhan, tin u śipi śni po, qa wookiye eciyapi śni po.

11 Tuwe wookiye eciye cin he iye ohan śice cin en opa.

12 Taku ota wowapi cicagapi kta mduha eśta, minihuha minisapa ko on ecamon kte śni: tuka en ciupi qa i eciyatanhan ociciyakapi kta e ecin cante mduza; hecen wowiyuśkin unkitawapi kin ojudan kta.

13 Nitanka kahnigapi qon cincawicaye cin hena nape niyuzapi. Amen. 


\section{JOHANNES}

\section{TAWOWAPI IYAMNI KIN.}

1. Hunkayapi waun kin, Gayos waśtedakapi kin, wowicake en waśtervadake cin he, wowapi cicaga.

2 Waśtecicida, taku owasin isanpa, tanyan iyoopte de kta qa zaniyan yaun kta e cewakiya ece, ninagi iyoopta ye cin iyececă.

3 Hunkawanjinkiciyapi kin den bipi, qa wowicake en niun kin he oyakapi qehan, wowicake eciyatanhan mayani kin heon nina imduśkin.

4 Micinca wowicake kin eciyatanhan manipi nawahon kin heon wowiyuśkin iyotan tanka mduha, takudan kape śni.

5 Waśtecicida, taku hunkawanjinkiciyapi kin, qa nakun wicaśta toktokeca ecawicayecon kin he tanyeli ecanon:

6 Hena e okodakiciye kin en wacanyakiye cin tanyan oyakapi : hena icimanipi en, Taku Wakan iyecen, owicayakiye cinhan, tanyan ecanon kta.

7 Iye caje kin on iyayapi, qa Ikcewicaśta kin etanhan takudan icupi śni, heon etanhan.

8 Heon tona hececapi kin hena iyowinwicunkiyapi kta iyececa; hecen wowicake kin ounkiyapi kta.

9 Okodakiciye kin wowapi kin cajeyan nape wicayuza wo. wicawecaga; tuka hena ehna Diotrephes itancan içida kin he iyowinunkiyapi śni.

10 Heon etanhan wau kinhan, iye ohan kin, oie śica on unkaiapi ece lin hena weksuye kta. Unkan he on imnan śni, tuka iye hinca hunkawanjinkiciyapi kin iyowinwicaliye śni, qa tona hecon wacinpi kin iyowicaki śni, qa okodakiciye kin etanhan tankan iyayewicaya ece.

11 Waśtecicida, taku śice cin he en ope śni wo, tuka taku waśte kin. Tuwe taku waśte econ kin he Wakantanka eciyatanhan un, tuka tuwe taku śica econ kin he Wakantanka wanyake śni.

12 Demetrios owasin on tanyan oyakapi, qa wowicake kin nakun eciyatanhan; unkan unkiś eya he unyaotaninpi, qa waunyaotaninpi kin he wowicake e sdonyayapi.

13 Taku ota ociciwa kta mduha tuka, tuka minisapa wiyaka ko on hena wowapi cicage kte śni : 14 Tuka ecadan wanciyake kta, qa i eciyatanhan unkokiciciye kta wacin.

15 Wookiye niye en niciun nunwe. 'Takukiciyapi kin hena nape niyuzapi. Takukiciyapi 379 


\section{JUD A S}

\section{T A W O W A P I K N .}

1 Judas, Jesus Messiya tawowidake kin, qa Jakob sunkaku kin, tona Walkantanka Ateyapi kin en wicayuecepidan, qa Jesus Messiya en wicakpatanpi, qa wicakicopi kin hena wowapi wicalicaga.

2 Wowaonśida, qa wookiye, wacantkiyapi ko niyepi en niciyuotapi nunwe.

3 Waśtecicidapi kin, wiconi ptaya unhapi kin on aiciciya wowapi cicagapi eca, wowacinye ehanna wicaśta wakan wicaq̨upi qon he nina yakitampi kta e on wowapi cicagapi, qa wahokonciciyapi kta iyececa.

4 Wicaśta wanjikśi nahmana en opapi, wanakajatanhan woyaco kin de on owicawapi ; hena Taku Wakan iyececapi śni, qa Wakantanka unkitawapi towaonśida kin he wicowicaśtaśni kagapi, qa Wakantanka ecedan Itancan kin he anahmanpi, qa nakun Jesus Messiya Itancan unyanpi kin.

5 Heon etanhan, taku, ehanna sdonyayapi eśta, he ake kiksuyeciciyapi kta wacin, Itancan kin oyate kin Egupta etanhan ehdaku, qa iyohakam tona wicadapi śni kin hena ihangwicaye cin.

$6 \mathrm{Qa}$ nakun malipiya ohnihde otokahe unpi kin kpatanpi śni, tuka iye tipi ehpekiyapi qon, hena otpaza ihulkuya, owihanke wanin mazaicicahilia kin on, anpetu tanka woyaco kin he en awicayuha.

7 Sodom qa Gomorra iyececa, 380 qa otonwe hena ihdukśan wanke cin, hena iś eya iyecen wiinahmanpi kin en içiçupi, qa wicacelipi toktokeca akitapi ece qon liena e woonspe on ehdepi, qa peta owihanke wanica on wośitkihda en unpi.

$8 \mathrm{He}$ iycceca, wihamnanpi sa kin dena wicacehpi kin așamyapi, qa wokiconze wahtedapi śni, qa taku tanka yaśicapi ece.

9 Hececa, tuka Mikael malipiya ohnihde itancan kin he wakanśica kin kici econ, qa Moses tancan kin on akinicapi qehan, woośtehda woiyaonpe wan en aya tawațenye śni, tuka, Itancan kin iyopeniyan nunwe, eciya.

10 Tuka dena e qe taku sdonyapi śni kin hena yaśicapi ece : tuka iye icagapi kin eciyatanhan taku sdonyapi kin hena on ibduśicapi, woteca ia okitpanipi kin iyececa.

11 Wotehi wicakiciun nunwe; Qain tancanku kin ohna yapi, qa wokajuju on, Balaam tawohnaye kin nina ainyankapi, qa Kore wicakipajin qon en ihangiçiyapi.

12 Hena woaśape kin heca, wacantkiya wayatapi kin en opeya wotapi, qa wikopeśniyan cemic̣iyapi ; mahpiya śapa mini wanica tate on ayapi ece kin; can kin ptanyetu eca śeca aya, waskuyeca codan, inonpa śeca, qa hute eciyatanhan yujunpi kin hena hececapi.

13 Miniwanca taja ohitika, iye wowiśtece tawapi kin minitaga 


\section{WICOWOYAKE 1.}

iyecen hiyuyapi ece; wicanlipi onuniyata unpi, on otpaza sape linca owihanke wanin wicakicihnakapi.

14 Henok Adam etanhan wicaśta iśakowin qon he itokam liena owicayake ca heya; Wanyaka po, Itancan kin wicaśta waśte wicayuhe cin woyawa tanka om u ce ;

15 Owasin wicayaco kta, qa tona Taku Wakan ohodaśni kin hena wicohan śikśica owasin ohodaśniyan econpi qon hena sdonkiyewicaye kta, qa wicoie canksiksi owasin wahtanipi sa Taku Wakan ohodapi śni oie kicatonpi ece qon hena nakun.

16 Hena e wihnupi sa, wawiyaonpapi sa, iye cantiheyapi kin eciyatanhan manipi ece; qa i eciyatanhan walianlianicidaya iapi, qa taku on etanhan wicaśta itohnake kin waśtedakapi ece.

17 Tuka waśtecicidapi kin, niyepi, Jesus Messiya Itancan unyanpi yewicaśi qon hena oiepi ehanna oyakapi qon hena kiksuya po ;

18 Anpetu ihankeya kin en wicaśta wawilialiapi kin yuke kta, iye cantiheyapi śice cin eciya- tanhan manipi kin, he oniciyakapi qon.

19 Ihdutokanpi kin dena eepi, witantanpi, qa Woniya kin yuhapi śni.

20 Tuka waśtecicidapi kin, niś niyepi wowacinye iyotan wakan nitawapi kin he ohna icaliceiyapi, qa Woniya Wakan kin eciyatanhan wocekiye eya po;

21 Wakantanka waśtedakapi kin en ihduha po ; qa Jesus Messiya Itancan unyanpi towaonśida wiconi owihanke wanica iyahde kte cin he ape yukan po.

22 Unkan hunl wicayukcanyan onśiwicada po ;

$23 \mathrm{Qa}$ hunh iś wokokipe on niwicaya po, peta etanban iwicacupi kin iyececa; qa wokoyake wicarelipi on aśapapi kin he. na ee kaeś śicedaka po.

24 Unkan tuwe woahtani econ śni niyuhapi kta okihi, qa wowiyuśkin yuha iye towitan kin en iyaonpepica sni enihdepi kte cin,

25 Wakantanka ecedan ksapa, Wanikiya unkitawapi kin hee, Jesus Messiya Itancan unyanpi kin eciyatanhan, wowitan, woyaonihan, wookihi qa wokiconze, dehantu qa wicoicage owasin hehanyan, tawa nunwe. Amen.

\section{JOHANNES}

\section{WAYUOTANIN TAWA KIN.}

\section{WICOWOYAKE 1.}

1 Jesus Messiya wayuotanin tawa, taku ecadan $\mathrm{u}$ kta $\mathrm{e}$ he tawowidake kin sdonyewicakiye kta Wakantanka qu qon he dee; unkan taohnihde wan u śi, qa tawowidake Johannes he okiyaka:

$2 \mathrm{He}$ Wakañtanka oie kin, qa Jesus Messiya wohdake cin, taku tona wanyake cin hena yaotanin.

3 Tuwe wokcan oie kin de ya381 
wa, qa tona nationpi, qa taku en kagapi kin anagoptanpi kin, hena wowaśte yuhapi. Wanna kiyadan ihan.

4 Johannes, Asia ekta okodakiciye śakowinpi kin wowapi wicakicaga. Wowaonśida, wookiye ko, tuwe un, qa un qon, u kte ein hetanhan niyepi en nicipi un nunwe; qa Woniya śakowinpi oiyotanke tawa kin itokam unpi kin hena eciyatanhan;

$5 \mathrm{Qa}$ Jesus Messiya etanhan, he waayatanin wicaka, wiconte etanhan tokaheya tonpi, qa maka wicaśtayatapi kin Itancan kin hee. Tuwe waśte unkidapi, qa waunhtanipi kin etanhan iye we kin en unyujajapi,

6 Qa Atkuku Wakantanka ekta wicaśtayatapi, wośna kagapi ko unkagapi kin hee, wowitan, wowaśake ko owihanke wanin tawa nunwe. Amen.

7 Wanyaka po, mahipiya śapa kin akan $\mathrm{u}$; unkan wiciśta owasin wanyakapi kta, qa tona he capapi qon hena nakun, qa iye on oyate maka ohnaka kin owasin aicipapi kta. Hecetu nunwe. Ämen.

8 Alpha qa Omega, otokahe qa owihanke kin he miye ce; Itancan un, qa un qon, u kta, iyotan waśake cin he hena hecen eya.

9 Johannes hunkawanjinyayapi, wokakije, qa wokiconze, wowalibadan ko, Jesus Messiya tawa kin en, tawaśi yayapi kin he miye; Wakantanka oie qa Jesus Messiya wohdake cin on etanhan, wita wan, Patmos eciyapi, hen waun.

10 Itancan taanpetu kin en Woniya kin kici waun; unkan mihektapa tanhan wicaho wan tanka, maza yahotonpi tanka wan iyececa, nawahion; 382
11 He kaken eya, Alpha qa Omega, tokaheya qa ehake kin he miye ce. Taku wandake cin wowapi ecen kage ca, $\Lambda$ sia ekta okodakiciye kin iwahowicaya wo; Ephesos, qa Smurna, qa Pergamos, qa Thuatira, qa Sardis, qa Philadelphia, qa Laodikia.

12 Unkan wicaho omakiye cin he wanmdake kta e ekta mihdamna. Unkan mihdamna qehan mazaskazi petijanjan ihupa śakowin wanmdaka:

13 Qa petijanjan ihupa śakowin kin cokaya Wanji Wicaśta Cinhintku kin iyececa, tawokoyake kin siha kin ekta iyahdeya, qa maku kin opta mazaskazi ihduskita.

14 Qa pa kin paha ko ska, tahinca wanuyanpi hin kin iyecen ska, qa wa iyececa, qa iśta kin peta ide kin iyececa ;

$15 \mathrm{Qa}$ siha kin iś mazazi ecedan iyececa, peta tanka en itkonyahe seca; qa ho kin he mini ota ihalia kin iyececa.

16 Qa nape etapa kin ohna wicanlipi śakowin hduhe ça, i kin eciyatanhan maza sagye anog ope kin heca wan hiyu, qa itohnake kin he anpetu wi nina iyege cin iyececa.

17 He wanmdaka, unkan ta wanke cin iyecen siha hde kin en mahinlipaya. Hehan iye nape etapa kin on omayutan qa, Ihnuhan koyakipe cin, emakiya; Tokaheya qa ehake kin he miye;

18 Tuwe țe ça ake kini, qa ito owihanke wanin ni waun; Amen; qa Wakanśica ti kin wiconte ko tiyopa iyuhdoke kin yuha manka ce.

19 Taku wandake cin he wowapi ecen kaga wo, taku nakaha un qa dena iyohakam taku u kte cin hena. 


\section{WICOWOYAKE 2.}

20 Wicanhipi śakowin minape etapa kin ohna wandake ciqon wowiyukcan kin hee, qa mazaskazi petijanjan ihupa kin hena nakun. Wicanhipi śakowin qon okodakiciye śakowinpi ohnihde yuhapi kin hena eepi ; qa petijanjan ihupa śakowin wandake cin okodakiciye śakowinpi kin hena eepi.

\section{WICOWOYAKE 2.}

1 Ephesos ekta okodakiciye ohnihde yuhapi kin he ecen wowapi kicaga wo; Tuwe wicanhipi śakowin nape etapa kin ohna hduhe ca, mazaskazi petijanjan ihupa śakowin cokaya omani kin he hecen eya;

2 Niohan, qa nitohtani, qa wacinnitanka kin he sodonwaya; qa tona śicapi kin hena iyowinwicakiya oyakihi śni; qa tona yewicaśipi kin hecapi keiçiyapi kin hena wicadukcan qa itonpiśni kin heca iyewicayaya.

3 Wacinnitanka, qa ayape, qa micaje kin on htayani, qa oyakite śni.

4 Tuka taku cistinna iyacionpa, tokaheya waśteyadake cin he aduśtan.

5 Heon tokiya tanhan nihinhipaye cin he kiksuye, ça ihduecetu, qa niohan tokaheya kin hduśtan wo. Hecanon śni kinhan kohanna ekta ciu, qa petijanjan nitawa kin yutokan iyeciciye kta, nihduecetu śni kinhan.

6 'Tuka he duha, Nikoleton wicohan kin śiceyadake cin, hena miś eya śicewadaka.

y Tuwe noge yukan hecinhan, taku Woniya kin okodakiciye kin ewicakiye cin he nahon kta. Tuwe ohiye cinhan lie can wiconi, Wakantanka tamaga co- kaya he cin, hetanhan yun wakiye kta.

8 Unkan Smurna ekta okodakiciye ohnihde yuhapi kin he ecen wowapi kicaga wo; $\mathrm{Tu}$ we tokaheya qa ehake un kin, te ca ake kini kin hee hecen eya;

9 Niohan kin sdonwaya, wokakije wowahpanica ko nitawa kin, (tuka winijica;) qa tona Juda wicaśta keiciyapi qa hecapi śni, tuka Satan omniciye tawa kin etanhanpi, hena waaiapi kin nakun sdonwaya.

10 Token nicakije kte cin ihnuhan taku koyakipe cin. Iho, Wakanśica niyepi wanjikji wicakaśka tipi kin en iyeniyanpi $\mathrm{kta}$, iniyukcanpi kta e heon; unkan anpetu wikcemna hehanyan wokakije duhapi kta; tuka wicate cin hehanyan wicaka wo, kinhan wiconi wateśdake wan cicu kta.

11 Tuwe noge yukan hecinhan, taku Woniya kin okodakiciye kin ewicakiye cin he nahon kta. Tuwe ohiye cinhan he wiconte icinonpa kin on kiuniyanpi kte śni.

12 Unkan Pergamos ekta okodakiciye ohnihde yuhapi kin he ecen wowapi kicaga wo ; Tuwe maza sagye anog ope kin hduhe cin hee hecen cya;

13 Niolian kin sdonwaya, qa tukten Satan oiyotanke tawa yanke cin hen yati; qa micaje kin yuha yaun, qa wacinmayanpi kin aduśtan śni, anpetu kin Antipas waayatanin wicaka mitawa kin niyepi kin en, tukten Satan ti kill, hen ktepi qon he ehan.

14. Tuka taku wanjikjidan iyacionpa;. Balaam hee Balak Israel cinca kin taku on wahtani ic̣iyapi kte cin wicitokam ehde 383 
kin he onspekiye ca, taku wakagapi wakiyuśnapi kin yutapi, qa wiinahmanpi econpi $\mathrm{kta}$, he tawoonspe tona yuhapi kin hena wicaduha ;

15 Qa nakun Nikoleton tawoonspe yuhapi kin hena wicaduha, he śicewadaka tuka.

16 Ihduecetu wo; hecanon śni kinhan kohanna ekta ciu, qa mii maza sagye kin on hena kici wecize kta.

17 Tuwe noge yukan hecinhan, taku Woniya kin okodakiciye kin ewicakiye cin he nahon kta. Tuwe ohiye cinhan he manna kihnakapi qon he etanhan yunwakiye ça inyan ska wan waqu kta, inyan kin he en wicacaje teca wan kagapi kin he tuwedan sdonye śni, tuka tuwe icu kte cin hecedan.

18 Unkan Thuatira ekta okodakiciye ohnihde yuhapi kin he ecen wowapi kicaga wo; Wakantanka Cinhintku, iśta peta ide kin iyecen hduhe ca, sihà kin mazazi ecedan iyececa hee hecen eya ;

19 Niohan kin sdonwaya, wacanyakiye, ca htayani, qa wicayada, qa ayape, qa niohan kin, qa ehake kin hena tokaheya kin sam iyeya ota.

20 Tuka taku tonana iyacionpa, winohinca wan Jezebel eciyapi, winohinca wokcanka keiciye $\operatorname{cin}$ he mitaokiye kin wicalinaye ça, wiinahmanpi econpi, qa taku wakagapi wakiyuśnapi kin yutapi kta e onspe wicakiye cin hee iyowinyakiya.

21 Unkan he wawicihahapi econ kin on iyopeiçiye lita e anpetu waqu, tuka iyopeiciye śni.

22 Ito, owinja wan akan ewaonpe kta, qa tona kici wawicihalapi econpi kin hena nakun, wo384 kakije tanka en; wicohan kin on iyopeiçiyapi śni kinhan.

23 Qa iye cinca kin wiconte on wicawakte kta; qa okodakiciye kin owasin, tuwe wicapakśin wicacante ko iyukcan kin he miye e sdonyapi kta. Qa niyepi kin otoiyohi niohanpi kin eciyatanhan cicupi kta.

24 Tuka niyepi, qa tona Thuatira ekta woonspe kin he yuhapi śni, qa Satan ośbe tawa eyapi kin sdonye śni yaunpi kin kaken eciciyapi ; Waqinpi tokeca acihnakapi kte śni ;

25 Tuka taku duhapi kin he tinsa hduha po, hibu kte cin hehanyan.

26 Qa tuwe ohiye ça owihanke kin iyahdeya miohan kin yuha un kinhan, oyate kin en wowaśake waqu kta.

27 Qa maza icapsinte wan on wicayuhe ca maka wakśica iyecen wicakamdece kta ce; hecen Ate etanhan hduha waun.

$28 \mathrm{Qa}$ anpao wicanhipi kin he waqu kta.

29 Tuwe noge yukan hecinhan taku Woniya kin okodakiciye kin ewicakiye cin he nahon kta.

\section{WICOWOYAKE 3.}

1 Unkan Sardis ekta okodakiciye ohnihde yuhapi kin he ecen wowapi kicaga wo; Tuwe Wakantanka Taniya śakowin kin hena, qa wicanhipi śakowin hduhe cin be hecen eya; Niolian kin sdonwaya, caje wan duha, yani kin hee, tuka nița.

2 Kiktahan un, qa taku tona nahahin en un qa te kte cin hena hduwaśaka wo; Wakantanka itokam niohan kin owotanna e iyewaye śni.

3 Heon token iyacu, qa nayahon kin kiksuye ça, ținsa yuze 


\section{WICOWOYAKE 3.}

ca, ihduecetu wo. Kiktahan yaun śni kinhan wamanon sa iyecen ekta ciu kta, qa tohan ekta cihi kte cin he sdonyakiye kte śni.

4 Tuka Sardis ekta .wicacaje tonana duha, hena e tawolkoyalke kin hduśapapi śni ; qa ska ihduzapi ecen mici manipi kta; he iyehantupi nakaeś.

5 Tuwe ohiye cinhan he wokoyake ska koyake kta, qa iye caje kin wiconi wowapi kin etanhan wapajuju kte śni, tuka lie caje kin Ate itokam, qa ohnihde wicaye cin hena wicitokam omdake kta.

6 Tuwe noge yukan hecinhan taku Woniya kin okodakiciye kin ewicakiye cin he nation kta.

7 Unkan Philadelphia ekta okodakiciye ohnihde yuhapi kin he ecen wowapi kicaga wo; Tuwe wakan qa wicaka un, qa Dawid tiyopa iyulidoke kin yuhe ca, yulidoka eca tuwedan ecen iyeye śni, qa ecen icu eca tuwedan yuhdoke śni ece lkin hee hecen eya;

8 Niohan kin sdonwaya, tiyopa wan yulidokapi ecen he cin he nitokam ewahde, qa tuwedan ecen iyeya okihi śni ; wowaśake cistinna duhe cea mioie kin yuha yaun, qa micaje kin ehpemayaye śni.

9 Ito, Satan omniciye tawa kin etanhanpi Juda wicaśta keic̣iyapi, tuka hecapi śni, qa itonpi śni kin, hena iho, upi, qa nisiha kin itokam canpeśka makehde inajin wicawakiye kta, qa waśtecicida e sdonyapi kta.

10 Woape oie mitawa kin he awandake cin, heon tohan taku wawiyutanyan un kin tona maka owancaya unpi kin iyutanwicaye kta u kinhan, hehan awanciyake kta.
11 Iho ecadan wau kta; taku duhe cin he tinsa yuza wo, hecen wateśdake nitawa kin tuwedan nici kte śni.

12 Tuwe olniye cinhan, he tipi wakan mita Wakantanka tawa kin ohna can ihupa wan wakage kta, qa icimana tankan ye kte śni ; qa he akan mita Wakantanka caje kin owawa kta, qa mita Wakantanka otonwe tawa caje kin, Jerusalen teca kin hee, mahipiya kin etanhan Wakantanka mitawa kin eciyatanlian u kin hee, qa nakun micaje teca kin.

13 Tuwe noge yukan hecinhan, taku Woniya kin olkodakiciye kin ewicakiye cin he nation kta.

14 Unkan Laodikia elata okodakiciye ohnihde yuhapi kin he ecen wowapi kicaga wo; Tuwe Amen eciyapi, waayatanin owotanna, qa wicaka, Wakantanka woicage tawa otokahe kin hee hecen eya ;

15 Niohan kin sdonwaya, sni qa ipiga unmana ecen yaun śni, sni qa ipiga unma tukte e ecen yaun kta wacin ;

16 Tuka initeca, qa sni ipiga kici unmana ecen yaun śni, heon mii kin etanhan icihdepe kta.

17 Wimajica, qa woyuha ota waton, qa takudan imakakije śni, keha ce; qa inicakije ça, onniśike ça, niwalipanica, qa iśta nigonge ça, tanconikadan e sdonyakiye śni.

18 Kaken wahokonciciya ce, mazaskazi peta on yuecedan kagapi kin he opemicaton wo, winijica kta ce; qa wokoyake ska, kodake kta e heon, hecen tanconikadan on winiśtece cin he tanin kte śni; qa wiciśta wihdi kin, niiśta iyakiun $\mathrm{kta}$, hecen wawandake kta.

19 Tona cantewicawakiye cin

385 


\section{WAYUOTANIN.}

hena wahokonwicawakiye ca, iyopewicawakiya ece. Heon mniheiciye ca ihduecetu wo.

20 Ito, tiyopa kin en nawajin qa wakabubu ece; tuwe miho kin nahon, qa tiyopa hdulidoke cinhan, he ekta tin hibu, qa kici wawate kta, qa iś eya mici wote kta.

21 Tuwe ohiye cinhan he oiyotanke mitawa kin akan mici iyotanke wakiye kta, miye ohiwaye ça Ate oiyotanke tawa kin akan weci imdotanke cin he iyecen.

22 Tuwe noge yukan hecinlian, taku Woniya kin okodakiciye kin ewicakiye cin he nahon kta.

\section{WICOWOYAKE 4.}

1 Hena iyohakam wanmdake ca ito malipiya kin ekta tiyopa wan yulidokapi; qa wicaho tokaheya nawahon kin he maza yahotonpi tanka kin iyececa, he omakiye ca heya, Deciya u wo, dena iyohakam taku $u$ kte cin hena wanyagciciye kta ce.

2 Unkan kohanna Woniya kin en waun; qa ito, mahpiya kin ekta oiyotanke wan ecen hdepi, qa tuwe akan iyotankehan yanlia.

3 Tuwe akan iyotanke cin he iwanyakapi kin inyan tehika, iyaspis qa sahdinos iyececa; qa oiyotanke kin ihdukśan wihmunke wan hmihbeya yanka, smahagdos inyan kin iyececa.

$4 \mathrm{Qa}$ oiyotanke kin ihdukśan oyanke wikcemna nonpa sam topa wanka; qa oyanke kin hena en hunkawicayapi wikcemna nonpa sam topa iyotankapi, qa wokoyake ska koyakapi e wanmdaka; qa pa kin en mazaskazi teśdakapi.
5 Unkan oiyotanke kin etanhan wakanhdi qa wakinyan lotonpi qa wicaho hiyu; qa oiyotanke kin itokam petijanjan śakowin peta ece ideyapi, hena Wakantanka taniya śakowin kin heepi.

6 Unkan oiyotanke kin itokam mde wan wanka janjan seca, cahtowata iyecen kohdi; qa oiyotanke kin cokaya, qa oiyotanke kin ihdukśan, taku ni un topa kin yukanpi, hena e tokapatanhan qa hektapatanhan ko iśta ojupidan.

y Unkan taku ni un tokaheya kin he mnaja ouncage kin iyececa; qa taku ni un iyokihe kin he tatanka ouncage kin iyececa; qa taku ni un iciyamni kin he wicaśta itohnake kin iyecen yuha; qa taku ni un icitopa kin he wanmdi kinyan un ouncage kin iyececa.

8 Qa taku ni un topa kin otoiyohi hupahu śakpelspe yuhapi, qa ihdukśan, qa mahen tanhan iśta ojupidan; qa anpetu hanyetu ko ayaśtan śni hecen eyapi, Wakan, Wakan, Wakan, Itancan Wakantanka Iyotan waśaka, tuwe un, qa un qon, u lite cin hee ce.

9 Qa tohan taku ni un topa kin, tuwe oiyotanke kin akan kiyotanke ca owihanke wanin ni un kin he wowitan, qa wootanin, qa wopida qupi kinhan;

10 Hehan hunkawicayapi wikcemna nonpa sam topa kin hena, tuwe oiyotanke kin akan kiyotanke cin he itokam makata ehpeiciyapi, qa tuwe ohinniyan ni un kin ohodapi, qa wateśdake tawapi kin oiyotanke kin itokam ehpekiyapi, qa hecen eyapi ;

11 Itancan, wowitan, qa wootanin, qa wowaśake iyacu kta e iyenihantu, taku owasin yaka- 


\section{WICOWOYAKE 5.}

ge ca nitoiyokipi kin on owasin kagapi qa eçen han.

\section{WICOWOYAKE 5.}

1 Unkan tuwe oiyotanke kin akan kiyotanke cin he etapa kin eciyatanhan wowapi wan mahen tanhan qa akapatanhan owapi, qa ipuspe śakowin on apuspapi kin he wanmdaka.

2 Qa mahpiya ohnihde waśaka wan wanmdaka, he hotankakiya hotanin qa, Wowapi kin he tuwe yumdaye ça ipuspe śakowin kin yuhdoke lita okihi he, eya.

3 Unkan mahpiya kin ekta, qa maka kin akan, qa maka kin ihukuya tanhan tuwedan wowapi kin he yumdaye ça, en wanyaka olihi śni.

4 Hehan nina waceya, tuwe wowapi kin yumdaye ca yawa, qa en wanyake kta iyekicihantu kin tuwedan iyeyapi śni kin heon etanhan.

5 Unkan hunkawicayapi kin wanji hemakiya; Ceye śni wo, ito Juda wicoun kin etanhan, mnaja kin, Dawid hutkan kin hee wowapi kin yumdaye ca, ipuspe śakowin kin yuhdoke kta okihi.

6 Hehan wanmdake ca, iho, oiyotanke kin cokaya, qa taku ni uñ topa, hunkawicayapi ko wicacolaya Amnos wan najin, wośnapi iyececa, he śakowin iśta śakowin ko yukan, Wakantanka taniya śakowin maka kin owancaya ye wicayapi kin hena eepi.

7 He en hi, qa tuwe oiyotanke kin akan kiyotanke cin nape etapa kin etanhan wowapi qon icu.

8 Unkan wowapi kin he icu qehan, taku ni un topa kin, qa hunkawicayapi wikcemna nonpa sam topa kin hena Amnos kin itokam makata ehpeiçiyapi, otoiyohi candowankiyapi hduhapi, qa nakun mazaskazi wiyatke taku waśtemna on ojudan, wicaśta waśte wocekiye tawapi kin hee:

9 Qa odowan teca wan ahiyayapi, qa heyapi; Wowapi kin iyacu, qa ipuspe kin dulidoke kta e iyenihantu ce, waniyuśnapi qa Wakantanka ekta niwe kin on opeunyakitonpi, wicoun otoiyohi etanhan, qa iapi kin, qa wicowazi kin, qa oyate kin otoiyohi etanhan;

10 Qa Wakantanka ekta wicaśtayatapi wawayuśnapi ko unyakagapi ; unkan maka kin oununyanpi kta ce.

11 Unkan wanmdake ça, oiyotanke kin ihdukśan, qa taku ni unpi kin hunkawicayapi ko wicihdukśan, malipiya ohnihde woyawa tanka ota, qa kektopawinge ece kektopawinge, hena hopi kin nawahon ;

12 Hotankakiya heyapi, Amnos wośnapi qon he wowaśake, qa woyuha, qa woksape, qa wicookihi, qa wootanin, qa wowitan, qa wowaśte henakiya icu kta okihi ce.

13 Unkan taku mahpiya ekta un, qa taku maka akan un, qa taku maka ihukuya tanhan un, qa taku miniwanca mahen un, hena taku ohnaka ko owasin kaken eyapi nawahon; Tuwe oiyotanke kin akan kiyotanke cin Amnos kin kici wowaśte kin, wootanin kin, wowitan kin, qa wowaśake kin henakiya owihanke wanin tawapi nunwe.

14 Unkan taku ni un topa kin hena, Hecetu nunwe, eyapi ; qa hunkawicayapi wikcemna nonpa sam topa kin hena makata elipeiciyapi, qa tuwe ohinniyan ni un kin he ohodapi. 
WICOWOYAKE 6.

1 Unkan Amnos kin ipuspe tokaheya yulidoke cehan wanmdaka; unkan taku ni un topa kin wanji, wakinyan hotonpi kin iyecen hotanin qa, U qa wanyaka wo, eya nawahon.

2 Unkan wanmdake ca, ito, śuktanka ska wan tuwe akan iyotanke cin he itazipa wan yuha; qa wateśdake wan qupi ; unkan ohiye ca, ohiye kta e iyaya.

3 Unkan ipuspe inonpa yuhdoke cehan, taku ni un icinonpa kin he, U qa wanyaka wo, eya nawahion.

4. Unkan śuktanka wan tokeca hin śa kin he hinanpa; unkan tuwe akan iyotanke cin he maka kin etanhan wookiye icu kta e qupi, hecen otoiyohi kiciktepi kta; qa nakun maza sagye wan tanka qupi.

5 Unkan ipuspe iyamni kin yuhoke cehan, taku ni un wiciyamni kin he, U qa wanyaka wo, eya nawalion. Unkan wanmdake ca ito śuktanka sapa wan; qa tuwe akan iyotanke cin he nape ohna taku on tke utapi wanji yuha.

6 Unkan taku ni un topa kin cokaya tanhan wicaho wan nawahion, Aguyapi iyutapi wanji he kaśpapidan wanjidan, qa śuktanka tawote iyutapi nonpa he kaśpapidan wanjidan kta; qa wihdi miniśa ko ihnuhan kiuniyaye cin, eya.

7 Unkan ipuspe itopa kin yuhoke cehan, taku ni un icitopa kin he ho kin nawalion; U qa wanyaka wo, eya.

8 Unkan wanmdake ça ito śuktanka wan hin san; tuwe akan iyotanke cin he Wiconțe eciyapi, qa Wicahapi kin he ihakam iyaya. Unkan maka hanke ito388 pa kin hehanyan maza sagye, qa wicaakilian, qa wiconțe, qa wamanica maka akan un kin hena on wicaśta wicakte kta e wowaśake qupi.

9 Unkan ipuspe izaptan kin yuhidoke cehan, wahna wośnapi kin ihukîya tona Wakantanka oie, qa iye waayataninpi kin he on wicaktepi qon hena nagipi kin wanwicamdaka.

10 Hena hotankakiya hotaninpi qa heyapi, Itancan wakan, qa wicaka, tohanyan tona maka kin akan yukanpi kin wicadaco śni qa we unkitawapi kin on toyeçon śni he.

11 Unkan hena otoiyohi wokoyake ska wicaqupi, qa ptecedan ape yukanpi kta, tona om taokiye unpi qon, qa iye hunkawanjitkupi kin, iś iyepi iyecen wicaktepi kte cin he yuśtanpi kta hehanyan, kewicakiyapi.

12 Unkan ipuspe iśakpe kin yulidoke cehan, wanmdake ça ito maka śkanśkan tanka, qa anpetu wi kin sapa aya, wahinske ojuha kin iyececa, qa hanyetu wi kin iś we iyecen icaga.

13 Qa mahpiya wicanlipi kin makata hinhpayapi, witanśna hu wan tateyanpa tanka kahuhuza eca waskuyeca śtunkadan keś palipa ece kin he iyececa.

14 Qa wowapi pehan ehnakapi kin he iyecen malipiya kin tanin śni iyaye ça, lie otoiyohi, wita otoiyohi ko yutokan iyeyapi.

$15 \mathrm{Qa}$ maka wicaśtayatapi, qa wicaśta tankinkinyanpi, qa wicaśta watonpika, qa wicaśta itancanpi, qa wicaśta waśakśakapi, qa wicaśta wowidake unpi kin otoiyohi, qa ihduhapi kin otoiyohi, makohdoka qa he imnija kin ehna naicihmanpi ;

16 Qa inyan he imnija ko hena heciyapi, Unkahinhpayapi, qa tu- 


\section{WICOWOYAKE 7.}

we oiyotanke kin alkan kiyotanke cin itohnake kin etanhan, qa Amnos kin wocanniye tawa kin etanhan anaunliman po:

$17 \mathrm{He}$ wośihda tawa anpetu tanka kin wanna hi ; unkan tuwe najin okihi kta he.

\section{WICOWOYAKE $\%$}

1 Unkan hena iyohakam mahpiya ohnihde topa maka oise topa kin en inajinpi kin wanwicamdaka, maka tate ouye topa kin yuhapi, hecen maka qa miniwanca qa can hiyeye cin wanjidan atateyanpa kte śni.

2. Hehan mahpiya ohnihde tokeca wan wiyohiyanpa tanhan wankantkiya u kin wanmdaka, he Wakantanka ni un ipuspe tawa kin yuhe ca mahpiya ohnihde topa maka miniwanca ko kiuniyanpi kta e wicaqupi qon hena hotankakiya hoyewicakiye ;

3 Qa heya, Maka, qa miniwanca, qa can hiyeye cin kiuniyanpi sni po, Wakantanka unkitawapi taokiye wicaye cin itepi kin en wapetog wicuntonpi śni kin hehanyan.

4 Unkan tona wapetog wicatonpi wicayawapi kin nawahon, Israel cincapi wicoun owasin etanhan kektopawinge opawinge sam kektopawinge wikcemna topa sanpa kektopawinge topa wapetog wicatonpi.

5 Juda wicoun kin etanhan kektopawinge ake nom wapetog wicatonpi; qa Ruben wicoun kin etanhan kektopawinge ake nom wapetog wicatonpi ; qa Gad wicoun kin etanhan kektopawinge ake nom wapetog wicatonpi ;

6 Aser wicoun kin etanhan kektopawinge ake nom wapetog wicatonpi ; qa Nepthalim wicoun kin etanhan kektopawinge akenonpa wapetog wicatonpi; qa Manasse wicoun kin etanhan kektopawinge ake nom wapetog wicatonpi ;

7 Sumeon wicoun kin etanhan kektopawinge ake nom wapetog wicatonpi ; qa Lewi wicoun kin etanhan kektopawinge ake nom wapetog wicatonpi ; qa Isakar wicoun kin etanhan kektopawinge ake nom wapetog wicatonpi ;

8 Zabulon wicoun kin etanhan kektopawinge ake nom wapetog wicatonpi; qa Joseph wicoun kin etanhan kektopawinge ake nom wapetog wicatonpi; qa Benjamin wicoun kin etanhan kektopawinge ake nom wapetog wicatonpi.

9 Hena taku kin iyohakam wanmdake ca, iho, wicota hinca, wicayawa kta tuwedan okihi śni, oyate hiyeye cin, qa wicoun kin, wicowazi kin, iapi ko owasin otoiyohi etanhanpi, oiyotanke kin itokam, qa Amnos kin itokam najinpi, wokoyake ska ece koyakapi, qa can wapa nape ohna yuhapi ;

10 Qa hotankakiya hotaninpi qa heyapi, Wakantanka unkitawapi oiyotanke kin akan kiyotanke cin hee, Amnos kin kici, niwicaye yuha nunwe.

11 Unkan mahpiya ohnihde owasin oiyotanke kin okśan, qa hunkawicayapi taku ni un topa kin hena ko wicihduRśan inajinpi, qa oiyotanke kin itokam ite makipusdiya ehpeiciyapi, qa Wakantanka ohodapi ;

12 Qa heyapi, Amen; Wowaśte, qa wowitan, qa woksape, qa wopida, qa woyuonihan, qa wowaśake, qa wicookihi henatkiya Wakantanka unkitawapi kin 
owihanke wanin yuha nunwe. Amen.

13 Hehan hunkawicayapi kin etanhan wanji waayupte ca, Wokoyake ska koyakapi kin hena tuwepi, qa tokiya tanhan upi he, emakiya.

14 Unkan, Itancan, niye sdonyaya ce, epa. Unkan hemakiya; Wicokakije tanka etanhan upi qon dena eepi, unkan wokoyake hdujajapi, qa Amnos we kin on hduskapi.

15 Heon etanhan Wakantanka oiyotanke kin itokam yakonpi, qa tipi wakan iye tawa kin ohna anpetu hanyetu ko ohodapi ece; qa tuwe oiyotanke kin akan kiyotanke cin he hena wicacokaya ounye kta.

$16 \mathrm{Qa}$ tohinni wotektehdapi kte śni, qa ipuzapi kte śni, qa anpetu wi kin qa taku kata aśpanwicaye kte śni.

17 Tuka Amnos kin, oiyotanke cokaya un kin he, hena wonwicaye ça, minilidoka ni un kin icahda yus awicaye kta : qa hena iśtapi kin etanhan Wakantanka iśtamnihanpe kin owasin wicakipakinte kta.

\section{WICOWOYAKE 8.}

1 Unkan ipuspe iśakowin kin yuhidoke cehan, mahpiya ekta wihiyayedan wancadan ape cin hanke hehanyan inina yukanpi.

2 Unkan mahpiya ohnihde śakowin Wakantanka itokam najinpi kin wanwicamdaka ; qa maza yahotonpi tanka sakowin wicaqupi.

3 Hehan malipiya ohnihde wan tokeca u qa wahna wośnapi kin ekta hinajin, mazaskazi oizinye wan yuha: Unkan taku waśtemna ota qupi kin he, tona owotanna un kin owasin wocekiye tawapi kin ahna, mazaskazi wahna wośnapi kin oiyotanke kin itokam wanke cin he akan wośna kta e heon.

4 Unkan mahpiya ohniḩde nape kin etanhan śota izite cin, tona owotanna un wocekiye tawapi kin hena ko, Wakantanka itokam wankantkiya aya.

5 Unkan malipiya ohnihde kin he oizinye kin icu, qa wahna wośnapi peta kin etanhan oju, qa maka kin ekta ehpeya; unkan iho, wicaho, qa wakinyan hotonpi, qa wakanhdi, qa maka śkanśkan.

6 Unkan mahipiya ohnihde śakowinpi maza yahotonpi tanka yuhapi kin hena yahotonpi kta e hduwiyeyapi.

7 Unkan malipiya ohnihde tokaheya kin yahoton, qa wasu peta ko we icicahiyapi malka kin ahinhe: Qa can iyamnimni kin huhnaga, qa wato kin iyulipa huhnaga.

8 Unkan mahpiya ohnihde iyokihe kin he yahoton, qa taku he wan tanka iyececa peta on itkonyahe cin he miniwanca kin en iyoheyapi : Qa miniwanca kin hanke iyamni we icaga:

9 Qa taku miniwanca oicage ni un kin hanke iyamni țapi, qa wita wata kin iyamnimni kin owihanke yapi.

10 Unkan malipiya ohnihde iciyamni kin he yahoton, qa wicanhpi wan tanka ideyapi petijanjan iyececa, mahpiya kin eciyatanhan hinhpaye ca wakpa iyamnimni kin ahinhipaye ça mini hdoka kin en.

11 Wicanlipi kin he, Yatkepicaśni, eciyapi. Unkan mini yatkepica śni icage ca wicaśta ota mini kin on tapi, pa icage cin heon.

12 Unkan mahpiya ohnihde 
icitopa kin he yahoton; unkan anpetu wi hanke iyamni, ga hanyetu wi hanke iyamni, qa wicanhpi iyamnimni kin apapi, qa hecen hanke iyamni otpaza icu, qa anpetu kin hanke iyamni hehanyan iyoyanpa śni un, qa hanyetu kin nakun iyececa.

13 Hehan wanmdake ca nawahon, mahpiya ohnihde wan malipiya cokaya tanhan kinyan iyaye ca hotankakiya heya, Wicokakije, wicokakije, wicokakije, tona maka ounyanpi kin hena en, maza yahotonpi tanka hotonpi kin mahpiya ohnihde yamni yahotonpi kte cin heon etanhan.

\section{WICOWOYAKE 9.}

1 Unkan mahpiya ohnihde icizaptan kin he yahoton, qa iho, wacanhipi mahpiya kin eciyatanhan maka kin ahinhpaya wanmdaka; unkan he makohdoka ceteta wanica tiyopa iyuhdoke kin qupi.

2 Qa makohdoka ceteta wanica he tiyopa kin yuhdoka, unkan makolidoka kin etanhan śta hiyu, peta on maza śdoyapi tanka hinca etanhan śota uye cin iyececa; qa wośbe etanhan śota kin he on anpetu wi kin olkotonyan kici aotpaza.

3 Unkan śota kin etanhan psipsicadan au, maka kin ekta, qa wicowaśake wicaqupi, wamduśka maka akan un kin wicowaśake yuhe cin he iyececa.

4 Qa wato maka aicage cin, taku toya un, qa can kin owasin kiuniye śni wicaśipi, tuka wicaśta kin tona itepi kin en Wakantanka tawowapetogton yuhapi śni kin henana.

5 Qa hena wicaktepi kta e wicaqupi śni, tuka wi zaptan hehanyan kakiświcaye kta e wi- caqupi. Unkan kakiśyapi kin he wamduśka wicaśta cawicape cinhan kiuniyanpi kin he iyececa.

6 Unkan anpetu kin hena en wicaśta wiconte akitapi kta, tuka iyeyapi kte śni, qa țapi cinpi kta, tuka wiconte e nawicakicipe kta.

7 Psipsicadan kin hena ouncage kin śuktanka ozuye on wiyeya ehnag najinpi kin he iyececa ; qa pa akan mazaskazi wateśdake iyececa wapaha kitonpi, qa itepi kin wicaśta ite kin iyececa.

8 Qa paha kin he winolinca paha iyecen yuhapi, qa hi kin hena mnaja hi kin iyececa.

9 Qa maza sapa maku akahpe iyececa maku akahpe tonpi ; qa liupahu hmulimunyan he ozuye canpahmihma, qa śuktanka ota ozuye ekta nawang, yapi kin he iyececa.

10 Wamduśka kin iyecen sinte yuhapi, qa sinte kin en icape yuke ca, wi zaptan hehanyan wicaśta kin kiuniwicaye kta okihipi.

11 Unkan wicaśtayatapi wan yuhapi, wośbe ceteta wanica etanhan ohnihde, Hebrew iapi kin eciyatanhan Abaddon eciyapi, qa Helenes iapi kin eciyatanhan Apoluon eciyapi kin hee.

12 Wicokalkije kin wanji wanna henakeca, qa ito he iyohakan wicokakije nom u kta ce.

13 Unkan hehan malipiya ohnihde iciśakpe kin yahoton, qa mazaskazi wahna wośnapi Wakantanka itokam wanke cin he he topa kin etanhan wicaho wan taninyan nawalion:

14 He mahpiya ohnihde iciśakpe maza yahotonpi tanka yuhe cin he heciya; Mahipiya ohnihde 391 
topa wakpa tanka Yuphrate kin ekta wicakaśkapi kin hena wicakiyuśka wo.

15 Hehan mahipiya ohnihde topa kin wicakiyuśkapi; hena e wancadan ape cin, qa anpetu wanji, qa wi wanji, qa omaka wanjidan hehanyan wiyeya ehnag unpi, wicaśta iciyamnimni kin wicaktepi kta e heon.

16 Unkan ozuye kin śuktanka akan iyotankapi kin hena kektopawinge kektopawinge opawinge nonpa; hena wicayawapi kin nawalion.

$17 \mathrm{Qa}$ wowanyake kin en śuktanka kin hecen wanwicamdaka, qa wicaśta akan iyotankapi kin hena maku akalipe śaśa, toto, qa zizi yuhapi. Unkan śuktanka pa kin he mnaja pa kin iyececa, qa ipi kin etanhan peta, qa śota, qa cahdi on kagapi kin hiyu.

18 Unkan hena yamni, peta, qa sota, qa calidi on kagapi, hena ipi kin etanhan hiyu qon he on wicaśta iciyamnimni kin wicaktepi.

19 Wowaśake yuhapi kin he jpi kin ohna qa sintepi kin ohna; sintepi kin hena wamduśka kin iyececa, qa pa yukan ece, qa liena on wakiuniyapi ece.

20 Unkan wicaśta tona wicokakije kin hena on wicaktepi śni kin hena iye napepi wicohan kin on iyopeiciyapi śni, hecen taku wakan śicapi kin ohowicadapi, qa mazaskazi, qa mazaska, qa mazazi, qa inyan, qa can walkagapi ohodapi ece kin ayuśtanpi śni; hena wawanyake śni, qa winahon śni qa mani okihi śni.

21 Qa nakun tinwicaktepi, qa wapiyapi, qa wiinahmanpi, qa wamanonpi econpi kin on iyopeiçiyapi śni.

\section{WICOWOYAKE 10.}

1 Unkan hehan mahpiya ohnihde wan tokeca waśake hinca mahpiya kin eciyatanhan kunthiya u, mahpiya śapa hdowin kin he wanmdaka; wihmunke wan wapaha kiye ca, ite kin he anpetu wi kin iyececa, qa siha kin peta wankantkiya ide kin iyececa.

2 Qa nape kin on wowapi wan cistinna yumdayeya yuha. Qa siha etapa kin miniwanca ahekiye ca, sicatka kin iś maka kin akan.

$3 \mathrm{Qa}$ hotankakiya hotanin, mnaja wan hoton kin iyececa. Unkan hotanin hehan wakinyan śakowin hotonpi.

4 Unkan wakinyan śakowin kin hotonpi hehan wowapi wakage kta, tuka wicaho wan nawahón, mahpiya kin eciyatanhan hemakiya, Taku wakinyan śakowin eyapi kin he sutaya apuspa wo, qa wowapi en kage śni wo.

5 Unkan mahpiya ohnihde miniwanca maka ko akan najin wanmdake ciqon he nape kin mahpiya kin ekta hdugate;

6 Qa tuwe ohinniyan ni un, qa mahpiya kage ca, taku ohnaka ko, malka qa taku ohnaka ko, miniwanca qa taku ohnaka ko kage cin hee on konza, Anpetu kin wanna henakeca kta;

7 Tuka mahpiya ohnihde iciśakowin yahoton kinhan, anpetu kin hena en Wakantanka tawowiyukcan henakeca kte cin hehanyan, iye taokiye, wicaśta wolkcan kin owicakiyake ciqon he iyecetu ce.

8 Unkan wicaho mahpiya kin eciyatanhan tanin qon he ake hoyemakiye ca heya, Mahpiya ohnihde miniwanca maka ko akan najin liin he ekta ye ca, 
wowapi cistinna yumdayapi kin nape en un kin he icu wo.

9 Hehan mahpiya ohnihde kin he en wai qa hewakiya, Wowapi cistinna kin he maqu wo. Unkan, Icu qa yuta wo, tezi kin pa niye kta, tuka nii kin ohna tuhmaga canhanpi kin iyecen skuye kta ce, emakiya.

10 Unkan wowapi cistinna kin mahpiya ohnihde nape kin etanhan iwacu, qa temwaya; qa mii kin en tuhmaga canhanpi kin iyecen skuya, tuka nawapca hehan tezi kin pa mayan.

11. Hehan, Oyate, qa Ikcewicaśta, qa iapi kin, qa wicaśtayatapi ota en wicayakidukcan kta ce, emakiya.

\section{WICOWOYAKE 11 .}

1 Unkan cedi wan cansakadan iyececa he maqupi ; unkan mahpiya ohnihde kin najinhan najin qa heya; Najin, qa tipi wakan Wakantanka tawa kin iyuta wo, qa wahna wośnapi, qa tona en cekiyapi ece kin hena nakun.

2 'Iuka hocoka kin, tipi wakan itankan, he tankan iyeye ca iyute śni wo; he Ikcewicaśta kin wicaqupi ; unkan otonwe wakan kin amanipi kta, wi wikcemna topa sam nonpa hehanyan.

3 Unkan waayataninpi nom mitawa kin wahinske ojuha oihdugepi waayatapi kta e wicawaqu kta, anpetu kektopawinge sanpa opawinge nonpa sanpa wikcemna śakpe hehanyan.

4 Hena e Oliwe can nonpa qa petijanjan nom, Wakantanka maka yuhe cin itokam the cin hena eepi.

5 Unkan tuwe hena kiuniwi.. caye kta cin kinhan, ipi kin etanhan peta hiyu, qa toka wi- cayapi kin ihang wicaye kta: Tuwe kiuniwicaya cin kinhan he hecen ktepi kta.

6 Hena e mahpiya kin onataka okihipi, hecen magaju kte śni, waayatapi anpetu kin icunhan, qa mini kin we icahyapi kta okihipi, qa tohan cinpi eca wokakije owasin on maka kin kakiśyapi ece.

7 Unkan taku yaotaninpi kin wanna yuśtanpi kta hehan, wośbe kin etanhan wamanica wan wankantkiya hiyu, qa hena azuwicaye kta, qa ohiwicaye ça wicakte Kta.

8 Unkan hena tancan tapi kin otonwe tanka canku kin olına wanke kta, he iapi wakan kin eciyatanhan Sodom qa Egupta eciyapi, qa tukten Itancan unkitawapi kin icipaweh okatanpi qon.

9 Unkan wicaśta kin, qa wicowazi kin, qa iapi kin, qa oyate kin, hena tancan tapi kin wanwicayakapi kta, anpetu yamni qa wanji hanke hehanyan, qa tancan tapi kin maka mahen wicahapi kte cin iyowinwicakiyapi kte śni.

10 Unkan hena on etanhan tona makoce kin en ounyanpi kin magagaiçiyapi, qa wiyuśkinpi kta, qa talku kiciçupi kta, waayatapi nom tona maka akan ni un kin kakiświcayapi qon hena on etanhan.

11 Unkan anpetu yamni qa wanji hanke iyohakam, wiconi oniya kin Wakantanka eciyatanhan hena mahen iwicayaya; qa siha on najinpi, unkan tona wanwicayakapi kin nina yuśinyayapi.

12 Unkan hena wicaho wan tanka nahonpi, mahpiya kin eciyatanhan hewicakiya; Deciya wankan u po, eya. Unkan ma393 
hpiya śapa wan akan mahpiya kin ekta iyayapi, qa tona toka wicayapi qon hena wanwicayakapi.

13 Unkan wihiyayedan ape cin he icunhan maka śkanśkan wan tanka, qa otonwe kin hankedan iwikcemnamna kin hinhpaya, qa maka śkanśkan kin on tona țapi kin hena wicaśta kektopawinge śakowin. Qa unmapi kin yuśinyayapi qa mahpiya Wakantanka kin wowitan qupi.

14 Wicokakije inonpa kin wanna henakeca, qa ito wicokakije iyamni kin kohamna $\mathrm{u} \mathrm{k}$ ta ce.

15 Hehan mahpiya ohnihde iciśakowin kin yahoton, qa mahipiya kin ekta wicaho tanka hotaninpi, qa heyapi, Itancan unkitawapi qa Messiya tawa kin, maka wokiconze kin tawaye ca, ohinniyan en wicaśtayatapi un kta ce.

16 Hehan hunkawicayapi wikcernna nonpa sanpa topa, Wakantanka itokam oiyotanke kin akan kiyotankapi kin, hena makata ehpeiçiyapi, qa Wakantanka ohodapi;

17 Qa heyapi, Itancan Wakantanka Iyotan waśake cin, tuwe un, qa un qon, u kte cin he niye ce, pidaunyayapi, nitowaśake tanka kin iyekcu, qa wicaycconza ce.

18 Qa oyate kin sinhdapi, qa wocantiyahde nitawa kin wanna hi ; qa tohan wicațe cin wicayacopi kta, qa ookiye wicayaye cin wicaśta wokcan, qa owotanna, qa tona nicaje kin kokipapi, cistinpidan tankinkinyan ko, taku wicayaqu kta, qa tona maka napiśtanyanpi kin hena napiśtanwicayaye kte cin.

19 Hehan malipiya kin ekta tipi wakan Wakantanka tawa kin yuhdokapi, qa tipi walran 394 kin en wicotakuye can koka kin wanyakapi. Unkan ito wakanhdi, qa wicaho, qa wakinyan hotonpi, qa maka śkanśkan, qa wasu tanka.

\section{WICOWOYAKE 12.}

1 Unkan mahipiya kin ekta wowapetokeca tanka wan tanin : Winohinca wan anpetu wi kin koyake ca, hanyetu wi kin siha kin ihukuya, qa pa kin en wicanhipi ake nonpa wapaha kiya.

2 He ihduśake ça, hokśiksuye ça howaya, cinca ton kta e wayazanka.

3 Unkan hehan mahpiya kin ekta wowapetokeca tokeca wan tanin; wamduśka duta tanka wan, he pa śakowin, qa he wikcemina yulıa, qa pa śakowin kin en wateśdake śakowin teśdaka.

4. Qa sinte kin on mahpiya wicanhipi iyamnimni kin iwicacu, qa maka kin ekta elipewicaya. Unkan wamduśka kin he winohinca hokśiksuye cin itokam najin, cinca ton kinhan temye kta. 5 Hehan cinca ton, wica kin, oyate hiyeye cin owasin maza icapsinte wan on wicayuhe lite cin hee. Unkan hoksiyoqopa kin he Wakantanka, qa iye oiyotanke kin ekta eyakupi.

6 Unkan winohinca kin he hewoskan makoce kin elkta napa, heciya Wakantanka taku wiyeya ekicihnaka, qa hen anpetu kektopawinge sanpa opawinge nonpa sam wikcemna śakpe hehanyan wonyapi kta.

7 Hehan mabipiya kin ekta ozuye kagapi; Mikael qa iye taokiye kin wamduśka kin kici kicizapi. Unkan wamduśka lin qa iye taokiye kin kicizapi ;

8 Qa ohiwicayapi śni; hecen 


\section{WICOWOYAKE 13.}

hetanlian malipiya kin ekta tuktedan wicakiyukanpi śni.

9 Unkan wamduśka tanka kin, wamduśka wanakaja tanhan un kin hee, Wakanśica qa Satan eciyapi, wicaśta hiyeye cin owasin wicahnaye cin hee, makata ehpeyapi, qa tona ookiye wicaye cin hena nakun kici tankan iyewicayapi.

10 Unkan mahpiya kin eciyatanhan wicaho wan tanka nawation, hecen eya, Wanna unkita Wakantankapi wiconi tawa, qa towaśake qa tokiconze kin u ; qa Messiya wicookihi tawa kin nakun ; tuwe hunkawanjin wicunkiyapi kin taku iyawicaonpe cin, Wakantanka unkitawapi kin itokam, anpetu hanyetu ko awicaie cin hee kun elipeyapi kin heon etanhan.

11 Unkan hena Amnos we kin he on, qa waayataninpi oie tawapi kin he on ohiyapi, qa wiconte hehanyan wiconi tawapi kin waśte kidapi śni.

12 Heon etanhan, mahpiya kin iyuśkin po, qa tona ohna tipi kin. Tuka tona maka kin miniwanca ko elkta yakonpi kin hena wicokakije yuhapi kta; niyepi kin ekta Wakanśica kun iyaye ca wośihda tanka yuha, aśkayedan kta e sdonkiye cin heon etanhan.

13 Unkan wamduśka kin maka kin ekta ehpeyapi kin he wanhdaka, hehan winohinca cinca ton qon he śicaya kuwa.

14 Unkan winohinca kin he wanmdi tanka hupahu tawa nom qupi, on liewoskan makoce tukten ti kte cin heci kinyan iyaye kta, heciya waniyetu yamni qa wanji hanke hehanyan wonyapi kta, wamduśka itohnake kin itehanyan.

15 Unkan wamduśka kin iye i kin etanhan mini hiyuya, wakpa wan iyececa, qa winolinca kin ihakam hiyayeya, minin teye kta e heon.

16 Unkan maka kin winohinca kin he okiye ca, i hdukawa, qa wakpa wamduśka i kin etanhan hiyuye cin he yahepa.

17 Unkan wamduśka kin he winohinca kin on śinhda, qa winohinca cinca wicaye cin, tona Wakantanka tawoahope kin ahopapi, qa Jesus Messiya wootanin tawa yuhapi kin hena azuwicaya.

\section{WICOWOYAKE 13.}

\section{Unkan hehan miniwanca} kahda wiyaka kin en nawajin. Qa miniwanca kin etanhan wamanica wan hiyu wanmdaka, pa śakowin, qa he wikcemna yuha, qa he kin akan wacinhe wikcemna, qa pa kin akan waośtehdapi caje kin.

2 Unkan wamanica wanmdake cin he inmu tanka iyececa, qa siha kin hena wahanksica siha kin iyececa, qa i kin mnaja i kin iyececa. Unkan wamduśka qon towaśake kin he qu, qa oiyotanke tawa kin, qa wicookili tanka.

3 Unkan wanmdake ca, ito pa kin wanji kiuniyanpi, on te kta tuka; unkan kiuniyanpi kin on te kte cin he okizi, qa wicaśta kin owasin wamanica kin on yuśinyayapi.

4. Qa wamduśka kin towaśake kin wamanica qu qon he ohodapi, qa wamanica kin he nakun ohodapi, qa heyapi, Tuwe wamanica kin iyececa he, qa tuwe he kici kicize kta okihi he.

5 Unkan i wan qupi, he on taku tanka, qa iapi śice cin eye kta, qa wowaśake qupi, wi wikcemna topa sanpa nonpa he395 
hanyan, ozuye econ kte cin heon.

6 Unkan i hdukawa qa Wakantanka yaśica, caje, qa walkeya tawa kin, qa tona mahpiya kin ekta etipi kin hena taku awicalkicaga.

7 Qa tona owotanna un kin hena azuwicaye ca wicaktedan kta e qupi, qa wicowazi, qa iapi kin, qa oyate kin owasin wicayuhe kta e wowaśake qupi.

8 Unkan tona maka akan unpi cajepi kin wiconi wowapi Amnos, maka kagapi kin hehantanhan wośnapi qon, he tawa kin en owapi śni kin hena owasin wamanica kin ohodapi.

9 Tuwe noge yukan hecinhan, he nahon kta.

10 Tuwe wayaka awicahdi kinhan he wayaka ayapi kta, qa tuwe maza sagye on wicakte kin he maza sagye on ktepi kta ce. He en wicaśta owotanna apepi qa wacinyanpi kin tanin.

11 Unkan wamanica wan tolreca maka kin etanhan hiyu kin he wanmdaka; he he nom yuha tahin wanuyanpi cincadan iyececa, qa wamduśka iyecen ia.

12 Unkan wamanica tokaheya kin he towaśake kin ncowasin yuha, qa maka kin, tona akan un kin hena ko wamanica tokaheya kin kiuniyanpi, qa on te kta, tuka okizi kin he ohodawicakiya.

$13 \mathrm{Qa}$ wowapetokeca tanka econ, wicaśta wicitokam malipiya kin eciyatanhan peta malka kin ahinlipaye ya.

$14 \mathrm{Qa}$ tona maka akan unpi kin wicahnayan, wowapetokeca wamanica kin itokam econ kta e qupi qon hena on. Qa tona maka akan unpi kin wamanica maza sagye on capapi tuka kini 396 qon he iyacinpi wan kagapi kta e iwahokonwicakiya.

$15 \mathrm{Qa}$ wamanica iyacinpi kin he oniya qu okihi, hecen on wamanica iyacinpi kin ie kta, qa tona iyacinpi kin ohodapi śni kin hena wicakte kta okihi.

16 Unkan owasin, cistinpidan tankinkinyanpi ko, wijicapi qa wahpanicapi, tona ihduhapi, wowidake unpi kin hena ko, nape etapa kin en, qa iś itepi kin en wowapetogton tawa kin yuhe wicakiya.

17 Unkan tuwedan wopeton kte śni, tuka tona wamanica towapetogton, caje kin, qa caje ihdawa kin he wanji tukte e yuhe cin henana.

18 De en woksape yukan. Tuwe ksape cin he wamanica tona ihdawa kin he yawa kta. He wicaśta iyawapi kin hee, ihdawa kin he opawinge śakpe sanpa wikcemna śakpe sanpa śakpe.

\section{WICOWOYAKE 14.}

1. Hehan wanmdake ca, iho, Amnos wan Zion Paha kin akan najin, qa kektopawinge opawinge sanpa kektopawinge wikcemna topa sam kektopawinge topa kici najinpi, iye Atkuku caje kin itepi kin en owapi qon.

2 Unkan malipiya kin eciyatanhan wicaho wan nawation, mini ota ihalia kin iyececa, qa wakinyan tanka hoton kin iyececa. Qa dowankiyapi sa candowankiyapi on dowanpi wicahotanin nawation.

3 Qa oiyotanke kin itokam, qa taku ni un topa kin, qa hunkawicayapi kin wicitokam odowan teca se ahiyayapi. Odow an kin he tuwedan onspe kta okihi śni, tuka kektopawinge opawinge sam kektopawinge wikcemna 
WICOWOYAKE 14.

topa sam kektopawinge topa maka kin etanhan opewicatonpi qon hena.

4. Winohinca on aśamwicayapi śni kin hena eepi, tanśna unpi nakaeś. Tona Amnos kin tokiya ye cinhan iyahna yapi kin hena eepi. Wicaśta kin etainhan Wakantanka qa Amnos kin ekta opewicatonpi tokaheya kin hena eepi

5 Qa hena ipi kin en wohnaye takudan iyeyapi śni; qa Wakantanka oiyotanke kin itokam takudan iyaonpepica śni unpi.

6 Hehan mahpiya ohnihde tokeca wan mahipiya cokaya kinyan iyaye sin he wanmdaka, wotanin waśte owihanke wanin un kin, tona maka akan unpi kin, wicaśta kin, qa wicowazi, qa iapi, qa oyate hiyeye cin owasin ieyanpaha owicakiyake $k$ ta yuha iyaya.

7 Qa hotankakiya heya, Wakantanka kinihanpi qa wowitan qu po ; woyaco anpetu kin wanna hi ce; qa tuwe mahipiya, maka, miniwanca, qa wakoniya kin kage cin he itokam canpeśka makehde inajin po.

8 Unkan mahpiya ohnibde icinonpa kin he ihakam ye ca heya, Hinhpaya ce, Babulon otonwe tanka kin he hinhpaya; wawicilahapi wocantiyahde mini śa tawa kin etanhan oyate hiyeye cin owasin yatke wicakiye cin he dee.

9 Unkan mahpiya ohnihde iciyamni kin he liena wicihakam ye ça hotankakiya heya, Tuwe wamanica, qa iyacinpi kin ohoda, qa wowapetogton tawa kin ite, qa iś nape kin en yuhe cinhan;

$10 \mathrm{He}$ Wakantanka wocantiyahde miniśa tawa kin wocanteptanye miniyatke tawa kin en okaśtan kin hetanhan yatke kta, qa mahpiya ohnihde owotanna un kin hena wicitokam, qa Amnos kin itokam, peta, cahdi ko en kakiśy api kta.

11 Qa kakiświcayapi śota kin owihanke wanin wankan iyaya ece ; qa tona wamanica iye iyacinpi ko ohodapi, qa tuwe he caje wapetogtonpi yuhe cin hena anpetu hanyetu ko oziyapi wanin unpi.

12 Wicaśta owotanna apepi kin he de en un, qa tona Wakantanka tawoahope opapi qa Jesus wacinyanpi kin hena.

13 Hehan mahpiya kin eciyatanhan wicaho wan nawahon, hecen emakiya, Wowapi ecen kaga wo; Detanhan wicațe cin tona Itancan kin en tapi kin hena wicayawaśtepi ece. Han, Woniya kin eya, Wicohtani kin ayuśtanpi kta, qa wicolian kin wiciyahna iyaya ece.

14 Hehan wanmdake ça, iho, mahpiya ska wan, qa wanji Wicaśta Cinhintku kin iyececa akan iyotanka, he mazaskazi wapaha wan wapaha kiye ça, nape etapa kin on isan śkopa pe hinca yuha.

15 Unkan malipiya ohnilide tokeca wan tipi wakan kin etanhan $u$, qa tuwe mahpiya ska kin akan iyotanke cin he panyanhan heciya; Isan śkopa nitawa kin en iyekiye ca aguyapi baksa wo. Mnayanpi kte cin wanna iyehantu; maka woksapi kin wanna śeca ce.

16 Unkan mahpiya ska akan iyotanke cin he isan śkopa tawa kin maka ekta yekiya, unkan maka kin baśdapi.

17 Hehan mahpiya ohnihde wan tokeca tipi wakan mahpiya kin ekta he cin hetanhan u, he nakun isan śkopa pe hinca yuha. 


\section{WAYUOTANIN.}

18 Unkan malipiya ohnihde tokeca wan wahna wośnapi kin ctanhan $u$, he peta on wowaśake yuha. Qa he tuwe isan śkopa pe linca yuhe cin he panyanhan hoyekiye ca heya, Isan śkopa pe linca nitawa kin ekta yekiye ca maka hastanhanka kin mnayan wo; hastanhanka iyage kin wanna sapa ce.

19 Unkan malipiya ohnihde kin he isan śkopa tawa kin maka ekta yekiye ca, maka hastanhanla iyuwi kin mnaye ca, Wakantanka wocantiyahde miniśa iyuśkice tawa tanka kin he en iyohpeya.

20 Unkan otonwe kin itankan mini śa iyuśkice kin napanpi, qa mini śa iyuśkice kin etanhan we hiyu, suktanka i iyuwi kin hehanyan, stadiya kektopawinge sam opawinge sakpe hehanyan.

\section{WICOWOYAKE 15.}

1 Unkan malipiya kin ekta wowapetokeca tanka, qa wowinihan wanmdaka; mahpiya ohnihde śakowin wicokakije śakowin ehake kin yuhapi, hena en Wakantanka wocantiyahde tawakin henalieca kta.

2 Unkan mde wan janjan peta kici icicahiyapi seca wanmdaka, qa tona ohiya, wamanica qon, iye iyacinpi kin qa wowapetogtun kin, qa tona caje iyawapi kin, ohiye cigon hena janjan mde kin alkan najinpi, qa Wakantanka candowankiyapi tawa kin heca yuhapi.

3 Qa Moses Wakantanka taokiye tadowan, qa Amnos kin tadowan kin ahiyayapi, qa heyapi, Itancan, Wakantanka Iyotan waśake, cin niolian kin tanka, qa wowinihan, tona owotanna un kin en Wicaśtayatapi kin he niye ce, nitacanku kin owotanna qa wicaka.

4 Itancan, tuwe lronicipe kte śni, qa nicaje kin inidowan kte śni he; niśnana oniowotanna; qa oyate hiyeye cin owasin upi, qa nitokam canpeśka makehde enajin kta; woyaco nitawa kin wanna tanin kin heon etanhan.

5 Unkan he iyohakam wanmdake ca, iho, malpiya kin ekta waayataninpi wakeya wakan kin he yuzamni han.

6 Qa mahpiya ohnihde śakowin wicokakije śakowin yuhapi kin hena tipi wakan kin etanhan inanpapi, miniliuha ska wiyatpa kin heca koyakapi, qa maraskazi on maku opta ihduskitapi.

7 Unkan taku ni un topa kin wanji mahipiya ohnihde śakowin kin mazaskazi wiyatke kin heca śakowin wicaqu; hena Wakantanka ohinniyan ni un wocantiyahde tawa kin on ojudan.

8 Unkan Wakantanka towitan, qa towaśake kin eciyatanhan tipi wakan kin śota ojudan. Qa tipi wakan kin tuwedan timahen ye kta okihi śni, wicokakije śakowin mahipiya ohnihde śakowin yuhapi kin henakeca kte śni hehanyan.

\section{WICOWOYAKE 16.}

1 Unkan tipi wakan kin etanhan wicaho wan tanka nawahion, mahipiya ohnihde śakowinpi qon hewicakiya, Yapi, qa wiyatke Wakantanka wocantiyahde tawa kin hena maka kin en akaśtan po, eya.

2 Hehan mahpiya ohnihde tokaheya kin $u$, qa wiyatke tawa kin maka kin en akaśtan. Unkan wicaśta tona wamanica wowapetogton tawa kin yuhapi, qa iyacinpi kin ohodapi kin he-

398 


\section{WICOWOYAKE 16.}

na en śiyaka o tanka qa śica awicahinhipaya.

3 Unkan malipiya ohnihde icinonpa kin wiyatke tawa kin miniwanca kin en akaśtan; unkan he we icaga, wicate cin we kin iyececa, qa miniwanca kin en taku niyake un kin owasin tapi.

4. Unkan mahpiya ohnihde iciyamni kin wiyatke tawa kin wakpa qa wakoniya kin hena en akaśtan ; unkan hena we icaga.

5 Hehan nıahpiya ohnihde wan mini kin yuhe cin he kaken eya, nawalion ; I tancan, tuwe un, qa un qon, $u$ kte cin he niye, oniowotanna, hecen wicadaco:

6 Wicaśta owotanna, qa wokcanpi kin hena we kin wicakipapsonpi; qa hecen we yatke wicayakiya, he iyekicihantupi nakaeś.

7 Unkan wanji tokeca wahna wośnapi kin eciyatanhan kaken eya, nawation ; Itancan, Wakantanka Iyotan waśaka, wadaco kin he ohinniyan owotanna qa wicaka ce.

8 Hehan malipiya ohnihde icitopa kin wiyatke tawa kirı anpetu wi kin en akaśtan. Unkan he wicaśta kin peta on wicagu kta e qupi.

9 Qa iyokatapi tanka kin wicaśta wicagu, qa hecen Wakantanka wicokakije hena yuhe cin he caje kin ośtehdapi, qa wowitan qupi kta on ihduecetupi śni.

10 Unkan niahpiya ohnihde icizaptan kin wiyatke tawa kin wamanica oyanke tawa kin en akaśtan; unkan wokiconze tawa kin otpaza ojudan, qa wayazanpi on cejipi kin hdahtahtakapi.

$11 \mathrm{Qa}$ wicokakije qa śiyaka o kin on etanhan mahpiya Wakantanka kin yaśicapi, qa iye olianpi kin on iyopeiçiyapi śni.

12 Unkan malipiya ohnihde iciśakpe kin wiyatke tawa wakpa tanka kin Yuphrate, he en akaśtan; unkan mini kin puza aya, hecen wicaśtayatapi wiyohiyanpa tanhan au kin canku kin wicakiciyumdayapi kta.

13 Unkan wamduśka i kin etanhan, qa wamanica i kin etanhan, qa woitonśni wokcan kin he i kin etanhan, taku wakan śica yamni, hnaśka iyececa, hena hiyu wanmdaka.

14. Hena taku wakan śica taniyapi kin hecapi, wowapetokeca econpi, qa maka sitomniyan wicaśtayatapi kin, qa wicoicage ocowasin ekta yapi, Wakantanka Iyotan waśake cin anpetu tawa tanka kin en zuya awicaupi kta e heon.

15 Iho, wamanon sa wan iyecen wau ce. Tuwe kiktahan un, qa wokoyake kpatan kin he yawaśtepi ece, hecen tancodan mani kte śni, qa wiśtece cin wanyakapi lite śni.

16 Unkan Hebrew iapi kin eciyatanhan tukten Armagedon eciyapi, hen witaya u wicaya.

17 Hehan mahipa ohnihde iciśakowin kin wiyatke tawa kin okaduze cin en akaśtan. Unkan mahipiya kin ekta tipi wakan kin etanhan, qa oiyotanke kin etanhan, wicaho tanka wan tanin, qa heya, Wanna henakeca. 18 Unkan tho wicaho taninpi, qa wakinyan hotonpi, qa wakanhdi tanin, qa maka ślkanśkan wan tanka, wicaśta kin maka akan iwicacage cin ehantanhan maka śkanśkan iyecen tanka wanica.

19 Qa otonwe tanka kin he yamni kiyuśpapi; qa otonwe Ilcewicaśta tawapi kin hena hinhpayapi. Qa Babulon tanka kin he Wakantanka itokam kiksuyapi, wocanniye ohitike cin 


\section{WAYUOTANIN.}

miniśa wiyatke kin qu kta heon.

20 Unkan wita kin owasin najica, qa he kin hena tuktedan iyeyapi śni.

21 Unkan malipiya kin eciyatanhan wasu tanka wicaśta kin awicahinhe, otoiyohi taku tke utapi opawinge wanjidan inskokeca. Unkan wasu wicokakije kin on wicaśta kin Wakantanka yaśicapi ece, he wokakije iyotan tanka kin heon.

\section{WICOWOYAKE 17.}

1 Hehan mahpiya ohnihde śakowin wiyatke śakowin yuhapi qon etanhan wanji $\mathrm{u}$, qa omakiye ca hemakiya, Deciya u wo, wawicihahapi econ sa tanka mini ota akan iyotanke cin he token yacopi kin cicipazo kta ce.

2 He kici wicaśtayatapi maka akan unpi kin wawicihahapi cconpi ece, qa tona maka akan unpi kin. wawiciliahapi tawa miniśa kin on witkopi ce.

3 Hehan Woniya kin on hewoskan makoce ekta amayan. Unkan winohinca wan wamanica duta wan akan iyotanke cin he wanmdaka; he waostehdapi wicacaje kin ojudan, qa pa śakowin, he wikcemna ko ikoyaka.

4 Unkan winohinca kin he taku stan duta ko koyake ca, mazaskazi, inyan teliika, oinpidan ko hena on wiyatpa un, qa nape kin ohna mazaskazi wiyatke wan yuha waośtehdapi, wawicihahapi ko aśamyapi kin on ojudan.

5 Qa ite kin en caje wan owapi, Woanahbe kin, Babulon tanka, maka kin akan wawicihahapi, qa wayaśicapi hunku kin hee.

6 Unkan wicaśta owotanna we kin winohinca kin he iwitko 400 wanmdaka, qa tona Jesus yaotaninpi kin hena we kin on. $\mathrm{He}$ wanmdake ça nina imanihan waun.

" Hehan mahpiya-ohnihde kin he, Tokeca e ininihan he, emakiya; Winolinca woanahbe kin he ociciyake kta, qa wamanica akan iyotanke cin, pa śakowin, qa he wikcemna hduhe cin he nakun.

8 Wamanica wandake cin he un qon, qa un śni kin; qa wośbe cetete wanica etanhan hiyu kta, qa wicotakuni śni ekta hde kta. Qa tona maka akan unpi, maka kagapi kin ehantanhan, cajepi kin wiconi wowapi kin en owapi śni, hena wamanica kin wanyakapi kinhan yuśinyayapi kta, he un qon, qa un śni, qa hinahin un kin heon etainhan.

9 Unkan wicotawacin wicoksape yuhe cin he den un. $\mathrm{Pa}$ śakowin kin hena paha śakowin winohinca kin akan iyotanke cin hee ;

$10 \mathrm{Qa}$ wicaśtayatapi śakowin kin heepi, zaptan wanna hinhpayapi, qa wanji nahahin en yanka, qa wanji iś hinahin hi śni ; he hi kinhan ptecedan he kta.

$11 \mathrm{Qa}$ wamanica wandake ciqon he un qon, qa un śni kin, he iciśahdogan kin ee, śakowinpi kin he etanhan, qa wicotakuni śni ekta hde kta.

$12 \mathrm{Qa}$ he wikcemna wandake ciqon hena wicaśtaya tapi wikcemna kin eepi, nahahin wokiconze yuhapi śni, tuka wihiyayedan wancadan ape cin wicaśtayatapi iyecen wamanica kin kici wicowaśake icupi kta.

13 Hena wicotawacin wanjidan yuhapi, qa wicowaśake wicookihi ko tawapi kin wamanica kin qupi.

14 Qa hena Amnos kin kici ki- 


\section{WICOWOYAKE 18.}

cizapi kta, tuka Amnos kin wicaktedan lita, he itancanpi kin Itancan, qa wicaśtayatapi kin en Wicaśtayatapi kin hee, qa tona om un kin hena wicakicopi, qa wicakalinigapi, qa wicakapi.

15 Hehan hecen emakiya, Mini wandake ciqon tukten wawicihaliapi econ sa iyotanke cin he oyate, qa wicota, qa wicaśta, qa iapi kin eepi.

$16 \mathrm{Qa}$ he wikcemna wandake ciqon, wamanica ikoyake cin hena, wawiciliahapi econ sa kin śice kidakapi kta, qa liewoskantuya tancodan iyaye yapi kta, cehpi kin temyapi, qa peta on liulinalyyapi kta.

17 Hena e Wakantanka tawacin kin ecen econpi kta, qa wicotawacin wanjidan unpi kta, ca wokiconze tawapi kin wamanica qupi kta e wacin yus wicakiya, Wakantanka oie kin yuecetu kte cin hehanyan.

$18 \mathrm{Qa}$ winohinca wandake ciqon he otonwe tanka kin makata wicaśtayatapi kin iwicadakc cin hee.

\section{WICOWOYAKE 18.}

1 Unkan hena taku kin iyohakam mahpiya ohnihde wan mahpiya kin eciyatanhan kutkiya u wanmdaka, wicowaśake tanka yuha; qa he towitan kin on maka kin iyoyanpa.

2 Unkan he waśagya hotankakiya hotanin, qa heya, Hinhpaya ce, Babulon tanka kin he hinhipaya; qa taku wakan śicapi en ounyanpi, qa wanagi aśape cin owasin he otipi, qa zitkadan śice cin śicedapi kin owasin he hohpi yapi ce.

3 Wawicihahapi wocantiyahde miniśa tawa kin hetankan oyato hiyeye cin owasin yatkanpi; qa he kici wicaśtaya tapi maka akan unpi kin wawicihahapi econpi, qa taku teliika ota yuhe cin on wopeton maka akan unpi kin wijiniçiyapi ce.

4 Hehan mahpiya eciyatanhan wicaho wan tokeca nawalion, kaken eya, Mitaoyate kin tankata inanpa po, hecen woahtani tawa kin ehna yaunpi kte śni, qa wicokakije tawa kin en oyapapi kte śni.

5 He wahtani kin mahpiya kin ekta iyahdeya, qa taku śica econ kin hena Wakantanka kiksuya un.

6 Token ecaniconpi qon he iyecen ecakicon po, qa iye ohan kin etanhan nonpa akihde kicu po, wiyatke ojudan ye cin he ohna nonpa akihde okicicaśtan po.

7 Token wahaniçida qa takudan icakije śni un qon he iyecen wicokakije, qa iyokiśicapi kin qu po. Iye cante kin ekta kaken eya; Wicaśtayatapi winohinca heca iyotankehan waun, qa wiwazica hemaca śni, qa iyokiśicapi kin icimana wanmdake kte śni ce.

8 Heon etanhan anpetu wanjidan en wicokakije tawa kin hiyahde kta, wiconțe, qa iyokiśicapi, qa wicaakihan, qa ocowasin peta on hulinage kta; tuwe yaco kin Itancan kin Wakantanka hee waśaka ce, heon etanhan. 9 Unkan wicaśtayatapi maka ohnaka kin kici wawicilialiapi econpi, qa takudan icakije śni unpi kin hena he aceyapi kta, qa nihinciyapi $\mathrm{kta}$, he huhnage cin śota kin wanyakapi kinhan;

10 Wicokakije kin ikopapi kin on itehanyan inajinpi, qa heyapi kta, Hchehe, hehehe, Babulon otonwe tanka kin, otonwe waśake cin, ihnuhanna niyacopi kin anihinhpaya ce. 


\section{WAYUOTANIN.}

$11 \mathrm{Qa}$ heon wopeton maka akan unpi kin nihinciyapi, qa ceyapi kta, hetanhan maza tawapi kin tuwedan opewicakicaton lite śni :

12 Maza kin he mazaskazi, qa mazaska, qa inyan tehika, qa oinpidan, qa minihuha waśte, qa śina to, qa minihuha zipzipedan, qa śina śa, qa can waśtemna ocaje owasin, qa huhu wakśica owasin, qa can tehika, qa mazazi, qa mazasapa, qa inyan ska wakśica;

13 Qa canha śa, qa taku waśtemna, qa pejihuta pa, qa canśin, qa miniśa, qa wihdi, qa aguyapi mdu, qa woteca, qa tahin wanuyanpi, qa śuktanka, qa canpahmihma, qa wicatancan, wicanagi ko henakiya.

14. Waskuyeca kin ninagi waste dake cin he wanna herrakeca, qa woyute napin qa taku wicimdeza, hena owasin aniyuśtan, qa hena ake tohinni iyeyaye kte śni.

15 Unkan wopeton tona taku hececa wiyopeyapi ece, qa on wijiniciyapi kin hena itehanyan inajinpi kta, he wokakije kin ikopapi, nihinciyapi, qa ceyapi ;

16 Qa heyapi; Hehehe, hehelie, otonwe tanka kin miniliuha waśte, śa stan, śa ko koyake ca, mazaskazi, inyan tehika oinpidan ko kohdaka; qa ihnuhanna wiwicajica tanka kin he yutakuni śni.

17 Unkan tona wita wata iyupse yuzapi, qa tona ohna yapi, qa tona watopapi, qa tona miniwanca ohna wopetonpi ece kin hena owasin itehanyan inajinpi;

$18 \mathrm{Qa}$ he huhnage cin śota kin wanyakapi qehan, hotaninpi qa heyapi ; Otonwe kin tukte wanji e otonwe tanka kin he iyececa he.
19 Qa pa kin akan maka ahdatapi, qa hotaninyan nihinciyapi, qa ceyapi, qa heyapi; Hehehe, hehehe, otonwe tanka kin he eciyatanhan tona miniwanca kin ohna wita wata yuhapi kin hena owasin watonpika, talku telika opeton qon heon, he ihnuhanna hewoskan kagapi ce.

20 Heon, mahpiya kin, iyuśkin wo, qa ohnihde owotanna yaunpi, qa wokcanpi kin nakun, niyepi kin on Wakantanka tokicon kin he etanhan.

21. Hehan malipiya ohnihde waśake linca wan, inyan wan, inyan wiyutpan tanka iyececa, he icu, qa miniwanca kin en elipeye ca heya, Hecen wowaśake on Babulon otonwe tanka kin jujuwahan ehpeyapi kta, qa tohinni iyeyapi kte śni ce.

22 Unkan niye kin en candowankiyapi sa, qa dowanpi sa, qa cotanka yajopi sa; qa maza yahotonpi sa wicaho kin tohinni nahonpi kte śni; qa taku kage sa, ocaje owasin, niye kin en iyeyapi kte śni, qa wiyutpan hmun kin niye en hetanhan tohinni nahonpi kte śni.

$23 \mathrm{Qa}$ petijanjan ojanjan kin icimana iyoyamniye kte śni, qa niye en hihnayanpi qa tawicuyapi wicaho kin tohinni nahonpi kte śni. Wicaśta wopeton nitawa kin hena maka akan wicaśta itancanpi kin hecapi; qa wicayahmunge cin on oyate kin owasin wicayahnayan.

24 Unkan he ohna wicaśta wokcan wicaśta owotanna ko we kin iyeyapi, qa tona maka akan ktepi kin hena nalrun.

\section{WICOWOYAKE 19.}

1 Unkan hena iyohakam mahpiya kin ekta wicota hotanka- 
WICOWOYAKE 19.

kiya hotaninpi nawahon, kaken eyapi, Jehowa yatan po, niwicaye ca, wowitan, qa wootanin, qa wowaśake, Itancan Wakantanka unkitawapi kin he tawa nunwe.

2 Wayaco kin owotanna qa wicaka; witkowin tanka, wawicihahapi econ kin on maka lkin yuśice cin he yaco, qa taokiye kin hena we kin he nape kin en tokicon.

3 Hehan inonpa heyapi, Jehowa yatan po. Unkan he śota kin owihanke wanin wankantkiya iyaya.

4 Hehan hunkawicayapi wikcemna nonpa sanpa topa, qa taku ni un topa kin hena makata elipeiciyapi, qa Wakantanka oiyotanke kin akan kiyotanke cin he ohodapi, qa heyapi, Amen, Jehowa yatan po.

5 Hehan oiyotanke kin etanhan wicaho wan taninyan heya, Taokiye niyanpi kin owasin, qa tona cekiyapi ece, cistinpidan tankinkinyanpi ko, Wakantanka nitawapi kin yatan po.

6 Unkan nawahon, wicota hotaninpi kin iyececa, qa mini ota ihalia kin iyececa, qa wakiny an tanka hotonpi kin iyececa, kaken eyapi, Jehowa yatan po; Itancan Wakantanka Iyotan Waśake cin he Wicaśtayatapi un.

7 Heon etanhan piundapi, qa unkiyuśkinpi kta, qa wowitan unqupi kta; Amnos kin tohan tawicu ton kte cin wanna iyehantu, qa winolinea kin taku owasin wiyeya ekihnaka.

8 Unkan he minihuha ska wiyatpa kin heca koyake kta e qupi; minihuha ska kin he tona waśte woowotanna yuhapi kin hee.

9 Hehan hecen emakiya, Ka- ken wowapi kaga wo, Tona Amnos kin winoliinca yuze. kta omniciye kin en wicakicopi lin hena wicayawåśtepi ce. Hehan ake hecen emakiya, Wakantanka oie wicake cin hena ee.

10 Unkan hehan he siha hdepi kin en makata ehpemiçiye ca ohowada kta ; tuka iye hemakiya, Thnuhan hecanon kin, miś cici ookiye waun, qa nihunkawanji Jesus yaotanin yuhapi kin hemaca; Wakantanka ohoda wo. Jesus yaotanin kin he wokcanpi woniya kin hee.

11 Unkan mahppiya kin yuzamnipi e wanmdaka, qa ito śuktanka ska wan, qa tuwe akan iyotanke cin he Wicalke ca Owotanna eciyapi, he owotanna wicayaco qa ozuye econ ece.

12 Iśta kin peta ide kin iyececa, qa pa kin akan wateśdalke ota yukan; qa caje kin owapi, tuka he tuwedan sdonye śni, iye iśnana sdonkiya.

$13 \mathrm{Qa}$ wokoyake we en oputkanpi kin heca koyaka: Qa Wakantanka Oie kin eciyapi.

14 Unkan mahpiya wicobe kin owasin he ihakam yapi, śuktanka ska akan iyotankapi qa miniliuha waśte, ska, qa wakaśote śni kin heca koyakapi.

15 Qa iye i kin etanhan maza sagye anog ope kin hiyu, heon oyate kin awicape kta, qa maza icapsinte wan on wicayuhe kta; qa Wakantanka Iyotan Waśake cin he wocantiyahde ohitike cin miniśa iyuśkice kin he naśkica.

$16 \mathrm{Qa}$ onhohda ceca sani ko akan caje kin owapi, Wicaśtayatapi kin en Wicaśtayatapi, qa. itancanpi kin Itancan kin he dee.

17 Hehan mahpiya ohnihde wan anpetu wi kin en najin kin he wanmdaka; he hotankakiya 403 
hotanin, qa taku kinyanpi kin owasin, okotonyan kin en kinyan unpi kin hea hewicakiya; Upi qa Wakantanka he wotapi tanka tawa kin en mniciya po ;

18 Wicaśtayatapi ceĥpi kin yatapi kta, qa wicaśta itancanpi cehpi kin, qa tona waśakśakapi celipi kin he, qa śuktanka celpi, qa tona akan iyotankapi kin, wicacehpi ocaje owasin, wowidake unpi, qa ihduhapi kin, cistinpidan tankinkinyanpi koya.

19 Unkan wamanica kin wanmdaka, qa wicaśtayatapi maka akan unpi kin akicita wicayuhapi ko witaya mniciyapi, qa tuwe suktanka akan iyotanke cin akicita wicayuhe cin ko, kici kicizapi kte cin heon.

20 Hehan wamanica kin he niyake yuzapi, qa woitonśni wokcan kin he nakun, he wamanica kin itokam wowapetokeca econ, qa on tona wamanica wowapetogton tawa yuhapi, qa iyacinpi kin ohodapi kin hena wicahnaye cin hee. Unkan hena napin niyake peta mde wan inyan zi on itkonyahe cin he en iyohpewicayapi.

21 Unkan unmanpi kin owasin tuwe śuktanka akan iyotanke cin he maza sagye tawa kin on wicaktepi, mara sagye he i kin etanhan hiyu qon hee on. Unkan hena cehpi kin on taku kinyan un kin owasin imnanpi.

\section{WICOWOYAKE 20.}

1 Hehan mahpiya ohnihde wan mahpiya kin eciyatanhan kun u wanmdaka, he wośbe ceteta wanica tiyopa iyuhdoke kin yuhe ça, nape ohna maza icicahilia wan tanka:

2 Qa he wamduśka kin iyahpaya, sintehda wanakaja tanhan 404 un kin, Wakanśica qa Satan hee, qa kaśka ehnaka, waniyetu kektopawinge hehanyan;

3 Qa wośbe ceteta wanica he en ehpeye ça onataka, qa ipuspe wan on apuspa, hecen oyate kin wicahnaye lite śni, waniyetu kektopawinge kin ihuniyanpi śni kin hehanyan. Unkan he iyohakam ptenyedan kiyuśkapi kta. 4 Unkan oiyotanke kin heca wanmdaka, qa tona akan iyotankapi kin hena woyaco yuhapi. Unkan tona Jesus oyakapi kin on, qa Wakantanka oie kin on pa bawicaksapi qon, wamanica kin wakagapi ko ohodapi śni, qa wowapetogton tawa kin itepi, qa napepi kin en yuhapi śni, hena nagipi kin wanwicamdaka, ake kinipi qa Messiya kin kici ounyanpi, waniyetu kektopawinge hehanyan.

5 Tuka wicate cin unmanpi kin ake kinipi śni, waniyetu kektopawinge kin henakeca śni kin hehanyan. Piya iwicacage tokaheya kin hee.

6 Tuwe piya iwicacage tokaheya kin en ope cinhan he yawaśtepi qa owotanna un. Hena wiconte inonpa kin iyowicahi kte śni, tuka Wakantanka qa Messiya kin ekta wawayuśnapi kin heca wicayawapi kta, qa kici ounyanpi kta, waniyetu kektopawinge hehanyan.

7. Unkan waniyetu kektopawinge kin henakeca kinhan, Satan wicakaślka tipi kin etanhan kiyuśkapi kta.

8 Qa oyate kin wicahnaye kta e makata tate ouye topa kin ektakiya ye kta, qa Gog Magog kici zuya u wicaśi kta; hena miniwanca wiyaka sukaza kin iyecen wicota.

9 Unkan maka ohdakinyan ohna yapi, qa wicaśta owotanna 
ewanke cin ihdukśan hinajinpi, qa otonwe wakan kin nakun. Unkan mahpiya kin etanhan Wakantanka eciyatanhan peta hiyu, qa huhnahwicaya.

10 Unkan Wakanśica, hena wicahnaye cin he peta qa inyanzi mde kin en iyohpeyapi, heciya wamanica qa woitonśni wokcan kin unpi, qa anpetu hanyetu ko owihanke wanin kakijapi kta.

11 Hehan oiyotanke ska tanka wan wanmdaka, qa tuwe akan iyotanke cin he ite kin etanhan maka mahpiya ko najicapi ; qa tukten wanke kte cin iyeyapi śni.

12 Qa wicate ciqon, cistinpidan tankinkinyanpi ko, hena Wakantanka itokam najinpi, wanmdaka. Unkan wowapi kin yumdayapi ; qa wowapi tokeca wan yumdayapi, wiconi wowapi kin hee: Qa wicate ciqon token wowapi kin en kagapi kin he iyecen wicayacopi, iye wicohan kin eciyatanhan.

13 Unkan miniwanca kin wicate cin tona en un kin hena hiyuwicaya, qa wiconte Hades ko wicate cin tona en unpi kin hena hiyuwicayapi. Qa iye wicoḥan kin eciyatanhan otoiyohi wicayacopi.

14 Unkan wiconțe qa Hades kin hena peta mde kin en iyohpewicayapi. Wiconte inonpa kin hee.

15 Unkan tuwe wiconi wowapi kin en owapi iyeyapi śni kin he peta mde kin en .iyohpeyapi ece.

\section{WICOWOYAKE 21.}

1 Hehan mahpiya teca qa malia teca wan wanmdaka. Mahpiya tokaheya qa maka tokaheya kin hena yutokan iyeyapi, qa miniwanca wanica.
2 Unkan Johannes he miye e otonwe wakan kin wamdaka, Jerusalem teca kin hee mahpiya kin etanhan, Wakantanka eciyatanhan kun $u$, winohinca wan wicaśta hihna ton kte cin on wiyeya un kin he iyececa.

3 Unkan mahpiya kin eciyatanhan wicaho wan tanka nawalion, kaken eya, Wanyaka wo, Wakantanka wakeya tawa kin wicaśta kin ehna han, qa iye hena om ti kta, qa hena e oyate tawawicaye kta, qa Wakantanka hince cin hena om un kta, qa Wakantanka tawapi kta. 4 Qa wiciśta kin etanhan Wakantanka iśtamnihanpe owasin wicakipakinte kta, qa hetanhan wiconte wanice $\mathrm{kta}$, wicocante śica, wicaceya, wicokakije ko wanice $\mathrm{kta}$, taku tokaheya un kin wanna henakeca.

5 Unkan tuwe oiyotanke kin akan kiyotanke cin he, Ito taku owasin teca wakaga ce, eya. Hehan, Wowapi ecen kaga wo, hena oie kin wicake ca owotanna, emakiya.

6 Qa ake kaken emakiya, Wanna henakeca. Alpha qa Omega, otokahe qa owihanke kin he miye ce. Tuwe ipuza kinhan he wiconi mini hdoka kin etanhan iyunwin codan yatke wakiye kta.

7 'Tuwe ohiye cinhan he taku owasin tawaye kta, qa iye Wakantanka maye kta, qa miś he cinkśiwaye kta.

8 Tuka tona wikopapi, qa wicadapi śni, qa wahtepi śni, qa tin wicaktepi, qa wawicihahapi econpi sa, qa wapiyapi, qa wakagapi ohodapi sa, qa oitonpiśni kin hena owasin mde wan peta qa inyan zi on itkonyahe cin he ohna taku tawapi kta, wiconțe inonpa kin hee. 
9 Hehan mahpiya ohnihde śakowin wiyatke śakowin wicokakije ehake śakowin kin on ojudan yuhapi qon hena etanhan wanji hi, qa omaliye ca heya, $U$ wo, winohinca wan Amnos kin tawicuye kte cin he cicipazo lita ce.

10 Unkan Woniya kin on paha wan tanka tehan wankantuya kin ekta amaye ca, otonwe tanka kin, Jerusalem wakan, mahpiya kin etanhan Wakantanka eciyatanhan kutkiya u kin he makipazo ;

11 Wakantanka wowitan tawa kin yuha, qa wiyatpa kin he inyan tehika iyececa, inyan wan acah̉śdaya iyececa :

12 Qa ihdukśan aconkaśkapi tanka qa tehan wankantu kin, qa tiyopa ake nonpa yukan, qa tiyopa kin hena en mahpiya ohnihde ake nonpa yulsanpi, qa akan wicacaje owapi, Israel cincapi wicoun ake nonpa kin hena eepi.

13 Wiyohiyanpa tanhan tiyopa yamni; qa waziyapa tanhan tiyopa yamni; qa itokaga tanhan tiyopa yamni; qa wiyohpeya tanhan tiyopa yamni.

14 Qa otonwe conkaśke kin taku akan he cin hena ake nonpa, qa Amnos kin yewicaśi ake nonpa cajepi kin hena en un.

15 Unkan tuwe omakiye rin he mazaskazi cedihu wan yuha, on otonwe, qa tiyopa kin, conkaśke ko iyute kta.

16 Otonwe kin oise tom yukan, qa ohdakinyan kin ohanske cin kici akidehankeca. Unkan cedihu kin on otonwe kin iyuta, wiyu tapi kek topawinge ake nom; ohdakinyan ohanske cin obosdatu ko akidecen han.

17 Qa conkaśke kin he iyuta, wiciśpa opawinge sanpa wikce. 406 mna topa sam topa, wicaśta ihduta, mahpiya ohnihde ihdute cin hee.

18 Conkaśke kin he iyaspis inyan tehika on kagapi, qa otonwe lin he mazaskazi ecedan, janjan ecedan iyececa.

19 Qa otonwe conkaślze kin taku akan he cin he inyan tehika ocaje owasin en un. Taku akan he cin tokaheya kin he iyaspis ; inonpa kin he saphiros ; iyamni kin he kalkedon; itopa lin he smaragdos;

20 Qa izaptan kin he sardonis ; iśakpe kin he sardius; iśakowin kin he krusolitos; iśahdogan kin he berulos; inapcinwanka kin he topaz; iwikcemna kin he krusophrasos; iakewanji kin he huakinthos; qa iakenonpa kin he amathustos.

21 Tiyopa ake nonpa kin hena inyan tehilka ocaje ake nom, tiyopa otoiyohi inyan tehika wanjidan; qa otonwe ocanku kin he mazaskazi ecedan janjan kin iyecen wiyatpa.

22 Qa he en tipi wakan wanica; Itancan Wakantanka Iyotan Waśake cin, qa Amnos kin hena tipi wakan kin eepi.

$23 \mathrm{Qa}$ otonwe kin he anpetu wi, hanyetu wi ko en iyoyanpapi lita cinpi śni; Wakantanka wowitan tawa kin iyoyanpa, qa Amnos kin he petijanjan kin hee.

24. Qa oyate kin tona ni wicayapi kin hena iyoyanpa kin he en manipi kta; qa wicaśtayatapi maka akan unpi kin hena wowitan qa wootanin tawapi kin en ahipi kta.

$25 \mathrm{Qa}$ tiyopa kin hena anpetu icunhan ecen iyeyapi kte śni; qa heciya hanyetu wanica.

26 Oyate hiyeye cin wowitan, qa wootanin kin en ahipi kta. 
27 Qa taku wiśamye aśamye cin he en i kte śni, qa taku śicaya econ, qa itonśni kin; tuka tona cajepi kin wiconi wowapi Amnos tawa kin en owicawapi kin henanapidan.

\section{WICOWOYAKE 22.}

1 Helian wakpa wan wiconi mini ska kin, calitowata iyecen ska, he makipazo, he Wakantanka qa Amnos oiyotanke tawa kin etanban kaduza.

2 Unkan canku cokaya, qa wakpa kin anokatanhan wiconi can kin icaga, he waskuyeca ocaje ake nonpa aicaga, wi otoiyohi waskuyeca aicahya ece; qa can wapa kin hena oyate kin okizi wicaya ece.

3 Unkan heciya wicayuśice wanice kta; tuka Wakantanka oiyotanke tawa lin he, qa Amnos tawa kin hen he kta, qa taokiye kin hena tawoahope kin ecen econpi kta :

4. Qa itolmake kin wanyakapi kta, qa caje kin iye itepi kin en yuhapi kta.

$5 \mathrm{Qa}$ heciya hanyetu wanice kta; qa petijanjan qa anpetu wi iyoyanpa kin he cinpi śni ; Itancan Wakantanka iyoyamwicaya ece; qa ohinniyan owihanke wanin wicaśtayatapi ounyanpi kta.

6 Hehan hecen emakiya, Wicoie kin dena wacinyepica qa wicakapi. Qa Itancan Wakantanka wicaśta wokcan wakan tawapi kin hee, taku ecadan $\mathrm{u}$ kte cin hena taokiye kin owicaliyake kta e on ohnihde tawa kin u śi.

7 Iho ecadan wau kta. Tuwe wowapi kin de wowiyukcan oie kin tanyan yuhe cinhan he yawaśtepi ce.
8 Unkan Johannes he miye e hena wanmdake ca nawahon. Hena nawahon, qa wanmdaka; unkan malipiya ohnihde hena wanyagmakiye cin he siha kin en makata ehpemiçiye, ohowada lita tuka.

9 Hehan hemalkiya, Ihnuhan hecanon kin; miś eya ookiye cici waun, qa nihunkawanji wicaśta wokcanpi kin etanlıan waun, qa tona wowapi kin de oie yuhapi kin hena owicawapa; Wakantanka ohoda wo.

10 Hehan ake hemakiya, Wowapi kin de wowiyukcan oic kin hena sutaya apuspe śni wo. Wanna ecadan kta ce.

11 Tuwe owotanna śni kin ohinniyan owotanna kte śni, qa tuwe aśape cin he ohinniyan aśapa un nunwe; qa tuwe owotanna kin he ohinniyan owotanna kta, qa tuwe ecedan kin he ohinniyan ecedan un nunwe.

12 Unkan iho, kohanna wau kta, qa taku mitawa kin hduha waun, otoiyohi iye wicohan kin eciyatanhan wicawaqu kta.

13 Alpha qa Omega, tokapa qa hakakta, otokahe qa owihanke kin he miye ce.

14. Tona tawoahope kin ecen econpi kin hena wicayawaśtepi, hecen wiconi can kin en ya okihipi kta, qa tiyopa kin ohna otonwe kin timahen yapi kta.

15 Tuka śunka, qa tona wapiyapi sa, qa wiinahmanpi sa, qa wawicihahapi econpi sa, qa walragapi ohodapi sa, qa tuwe itonśni waśtedake ça econ kin hena tankan yakonpi ece.

16 Jesus he miye ce, okodalkiciye kin ekta niyepi kin en hena oniciyakapi kta e heon ohnihde mitawa kin u waśi. Dawid can hutkan adetka ko he miye, qa anpao wicanhipi nina iyege cin. 407 
17 Unkan Woniya kin tawicu- $\mid$ wapi oie kin dena yuaoptetu yapi kin kici, Kuwa, eyapi. Qa tuwe nahon kinhan he, Kuwa, eye kta. Qa tuwe ipuza kinhan, he u kta. Qa tuwe cin kinhan he iyunwin codan wiconi mini kin etanhan icu nunwe.

18 Tona wowapi kin de wowiyukcan oie kin nahonpi kin hena kaken ewicawakiya; Tuwe wicoie kin dena en taku sanpa kage cinhan, he wicokakije wowapi kin de en cajeyatapi kin Walantanka qu kta: $19 \mathrm{Qa}$ tuwe wowiyukcan wo- Amen. 408 kinhan, he taku tawa kte cin wiconi wowapi kin etanhan Wakantanka icu kta, qa otonwe wakan kin etanhan, qa taku tona wowapi kin de en kagapi kin he etanhan.

20 Tuwe hena yaotanin kin he kaken eya, Iho, kohanna wau kta ce. Amen. Itancan Jesus hecen yau nunwe.

21 Jesus Messiya Itancan unkitawapi kin he towaonśida kin niyepi owasin en nicipi un kta.

JESUS ITANCAN KIN TATANPI NUNWE.

\section{Microiormad by}

\section{Presenvition}

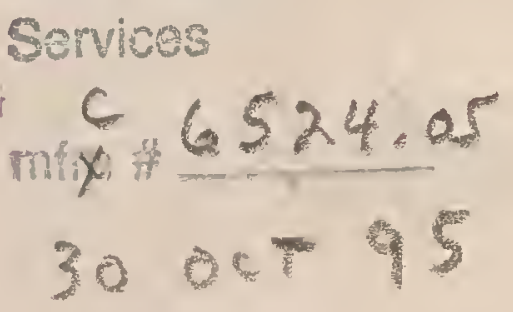










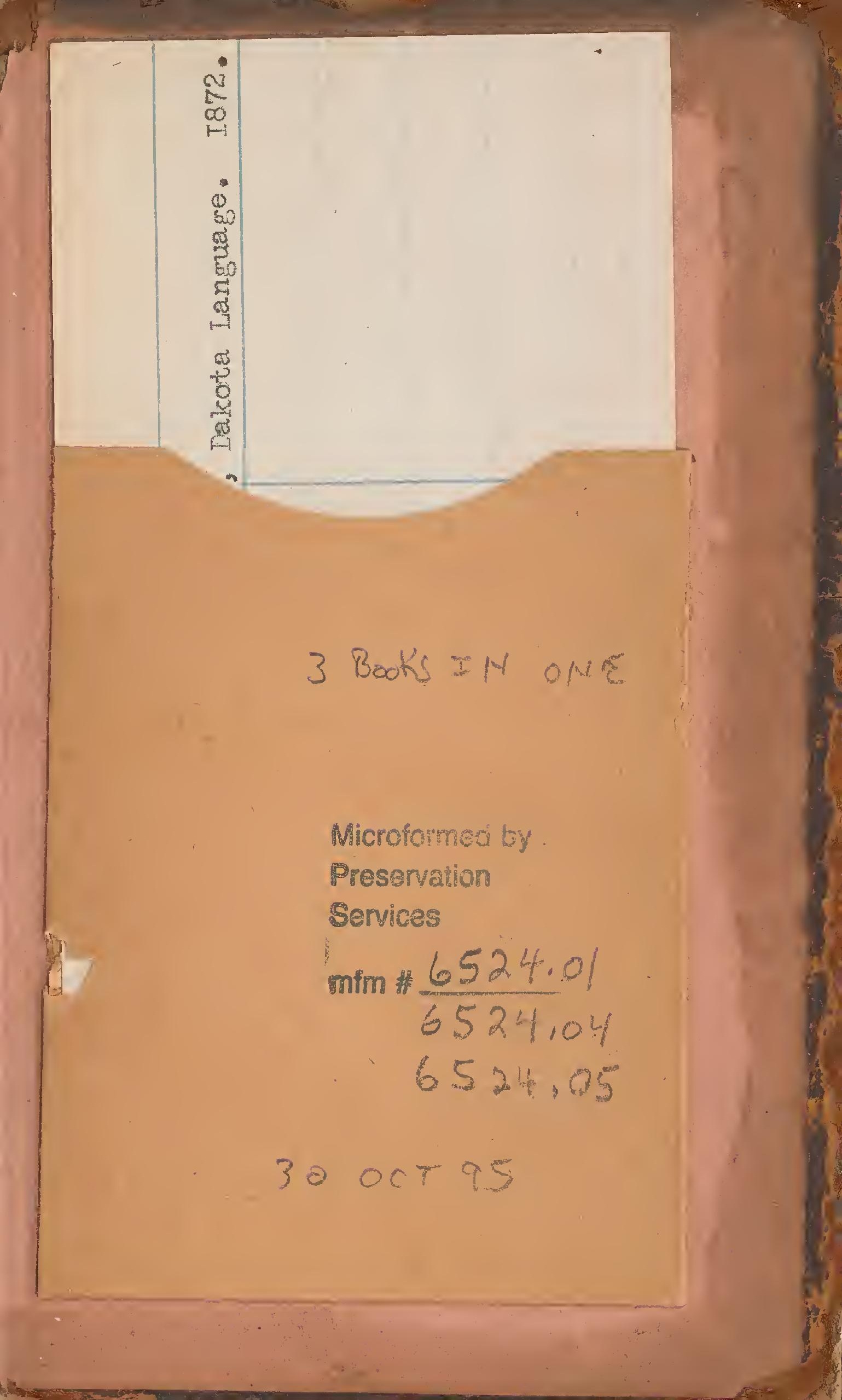




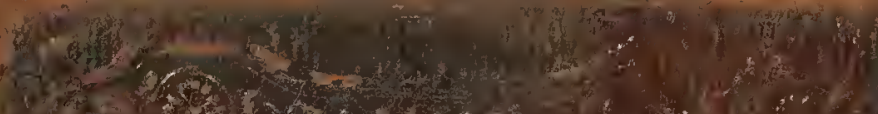

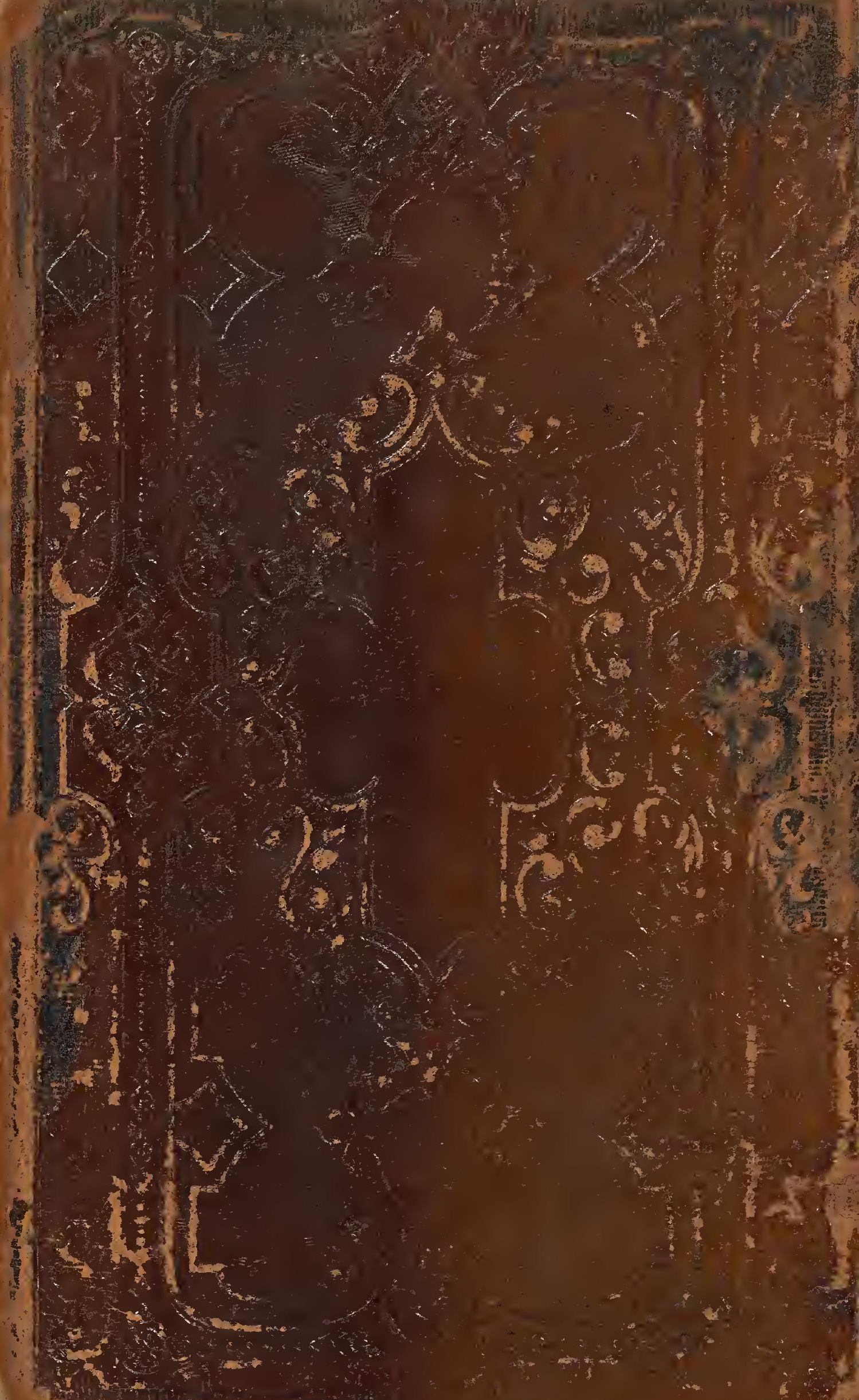
47 bi 

